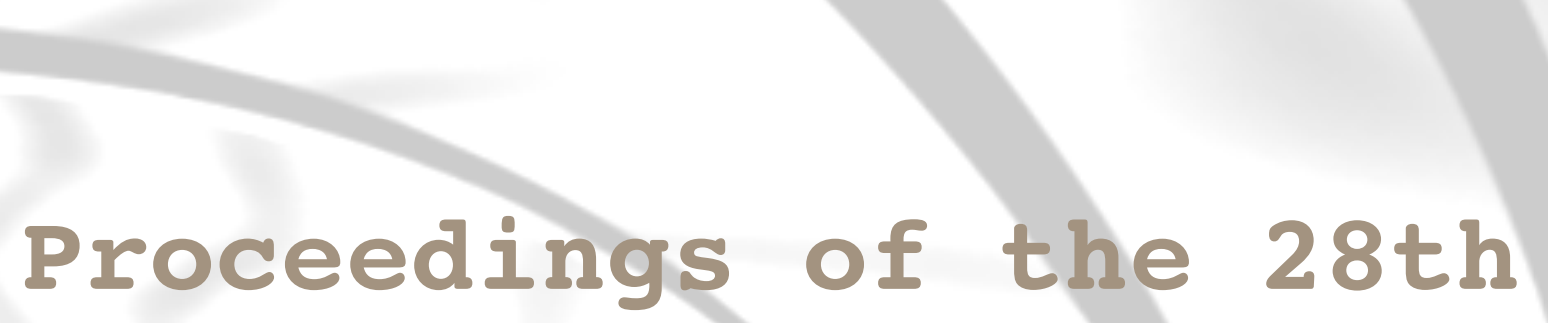

\title{
International Meshing Roundtable
}

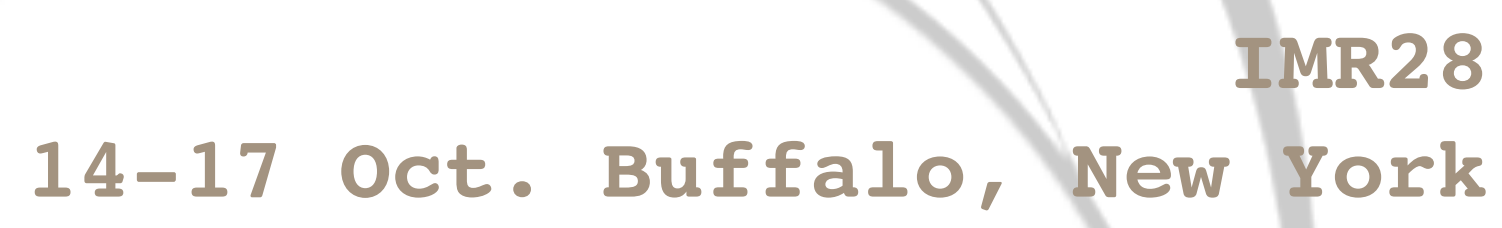




\title{
Proceedings of the 28th International Meshing Roundtable
}

\author{
Suzanne Shontz \\ Joaquim Peiró \\ Ryan Viertel
}


28th International Meshing Roundtable

Buffalo, NY, USA, October 14-17, 2019

\section{Editors:}

Suzanne Shontz, University of Kansas

Joaquim Peiró, Imperial College London

Ryan Viertel, Sandia National Laboratories*

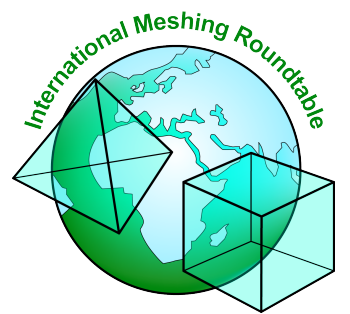

http://imr.sandia.gov

Cover art adapted from the winning "Meshing Maestro" entry by Siemens PLM Software. Boat model from https://grabcad.com/library/007-qboat-1

*Sandia National Laboratories is a multi-mission laboratory managed and operated by National Technology \& Engineering Solutions of Sandia, LLC., a wholly owned subsidiary of Honeywell International, Inc., for the U.S. Department of Energy's National Nuclear Security Administration under contract DE-NA0003525.

Copyright (c)2019 held by the authors of the individual papers.

Distribution of the material in this volume is permitted under the Creative Commons Attribution 4.0 International License.

SAND2019-14295 B

ISBN: 978-1-7334890-0-3

DOI: $10.5281 /$ zenodo.3653101 


\section{Contents}

Application of Tensor Factorisation to Analyse Similarities in CAD Assembly Models

Flavien Boussuge, Christopher M. Tierney, Trevor T. Robinson, and Cecil G. Armstrong

Subdivided Linear and Curved Meshes Preserving Features of a Linear Mesh Model

Albert Jiménez-Ramos, Abel Gargallo-Peiró, and Xevi Roca

A Parallel Variational Mesh Quality Improvement Method for Tetrahedral Meshes

Suzanne M. Shontz, Maurin A. Lopez Varilla, and Weizhang Huang

Updating and Re-Meshing Virtually Decomposed Models Benoit Lecallard, Christopher M. Tierney, Trevor T. Robinson, Cecil G. Armstrong, Declan C. Nolan, and Alexander E. Sansom

Anisotropic Error Estimate for High-Order Parametric Surface Mesh Generation Rémi Feuillet, Olivier Coulaud, and Adrien Loseille

Anisotropic Goal-Oriented Mesh Adaptation in Firedrake Joseph G. Wallwork, Nicolas Barral, David A. Ham, and Matthew D. Piggott

Higher-Order Accurate Meshing of Non-Smooth Implicitly Defined Manifolds Jakob W. Stanford and Thomas-Peter Fries

Automatic 2D Abstraction and Hexahedral Meshing by Sorting a Delaunay Mesh

Reza Taghavi

Topography Adapted Mesh Generation for Atmospheric Boundary Layer Flow Simulation

Abel Gargallo-Peiró, Matias Avila, and Arnau Folch

Certified Functions for Mesh Generation

Andrey N. Chernikov

Discrete Mesh Optimization on Surface and Volume Meshes

Daniel Zint, Roberto Grosso, and Florian Lunz 
Coarse Quad Layouts Through Robust Simplification of Cross Field Separatrix Partitions

Ryan Viertel, Braxton Osting, and Matthew Staten

Feature-Aligned Poly-Square Mapping of Large-Scale 2D Geometries for Semi-Structured Quad Mesh Generation

Celong Liu, Kelin Hu, Qin Chen, and Xin Li

Accelerating the Exact Evaluation of Geometric Predicates with GPUs Marcelo de Matos Menezes, Salles Viana Gomes de Magalhães, Matheus Aguilar de Oliveira, W. Randolph Franklin, and Rodrigo Eduardo de Oliveira Bauer Chichorro

Bicameral Mesh Anisotropy Nilanjan Mukherjee, Jonathan Makem, and Jean Cabello

Pre-Conditioning and Continuation for Parallel Distributed Mesh Curving Eloi Ruiz-Gironés and Xevi Roca

Naturally Curved Quadrilateral Mesh Generation Using an Adaptive Spectral Element Solver Julian Marcon, David A. Kopriva, Spencer J. Sherwin, and Joaquim Peiró

Untangling High-Order Meshes Based on Signed Angles Mike Stees, Myra Dotzel, and Suzanne M. Shontz

Multiple Approaches to Frame Field Correction for CAD Models Maxence Reberol, Alexandre Chemin, and Jean-François Remacle

A Regularization Approach for Automatic Quad Mesh Generation Julia Docampo-Sánchez and Robert Haimes

Guaranteed Quality-Driven Hexahedral Overlay Grid Method Nicolas Le Goff, Franck Ledoux, Jean-Christophe Janodet, and Steven J. Owen

Reviving the Search for Optimal Tetrahedralizations Célestin Marot, Kilian Verhetsel, and Jean-François Remacle

GMSHs Approach to Robust Mesh Generation of Surfaces with Irregular Parametrizations Jean-François Remacle and Christophe Geuzaine

CAD Defeaturing Using Machine Learning Steven Owen, Timothy M. Shead, and Shawn Martin

Building Direction Fields on the Medial Object to Generate 3D Domain Decompositions for Hexahedral Meshing Dimitrios Papadimitrakis, Cecil G. Armstrong, Trevor T. Robinson, Alan Le Moigne, and Shahrokh Shahpar

Dual-Based User-Guided Hexahedral Block Generation Using Frame Fields Simon Calderan, Guillaume Hutzler, and Franck Ledoux 
Multi-Block Decomposition and Meshing of 2D Domain Using Ginzburg-Landau PDE 



\section{Preface}

The papers in this volume were peer-reviewed and selected for presentation at the 28th International Meshing Roundtable (IMR), held October 14-17, 2019 in Buffalo, New York, USA. The International Meshing Roundtable was started by Sandia National Laboratories in 1992 as a small meeting of organizations striving to establish a common focus for research and development in the field of mesh generation. Now after 28 consecutive years, it has become clear that the International Meshing Roundtable has become the recognized international focal point for stateof-the-art meshing research collaboration spanning research and development from universities, commercial companies, and government laboratories.

The 28th International Meshing Roundtable consisted of presentations of peerreviewed technical papers, research abstracts, keynote and invited talks, short course presentations, a poster session and competition, a meshing contest, and an open spaces session. This year we have made the proceedings openly accessible by hosting them on Zenodo under a creative commons license. The Program Committee would like to express our appreciation to all who participate in making the International Meshing Roundtable a successful and enriching experience.

The papers in these proceedings present novel contributions that range from the theoretical to practical. This year, the committee selected twenty-seven papers based on the input from peer reviewers. Reviewers assesed the papers based on quality, originality, and appropriateness to the theme of the International Meshing Roundtable. We would like to thank all who submitted papers. We also extend our appreciation to the colleagues who provided reviews of the submitted papers. Their efforts were essential to the process of selecting papers for the International Meshing Roundtable. The names of the reviewers are acknowledged in the following pages.

The conference received travel support from the National Science Foundation (NSF) for student and postdoctoral attendees from the U.S. institutions and additional travel support from Pointwise, csimsoft, and Sandia National Laboratories. We deeply acknowledge their support. We extend special thanks to Kathy Loeppky of Sandia National Laboratories for her time and effort to make the 28th International Meshing Roundtable a success.

October 2019,

28th IMR Program Committee 



\section{List of Reviewers}

Cecil Armstrong

Romain Aubry

Ken Blake

David Bommes

Flavien Boussuge

Jean Cabello

Marcel Campen

Philip Caplan

Brian Carnes

Andrey Chernikov

Brett Clark

Thierry Coupez

Emily Donahue

Jan Eichstaedt

José Escobar

Nicola Ferro

Harry Fogg

Vincent François

Mark Gammon

Shuming Gao

Xifeng Gao

Rao Garimella

Oubay Hassan

Nancy Hitschfeld

Xiangmin Jiao

Steve Karman
Avary Kolasinski

David Kopriva

Jean-François Lagüe

Franck Ledoux

Vijay Mahadevan

Ahmed Mahmoud

Jonathan Makem

Loïc Maréchal

Julian Marcon

Carianne Martinez

David McLaurin

Scott Mitchell

Ketan Mittal

David Moxey

Walter Nissen

Steven Owen

David Palmer

Stefano Paoletti

Michael Park

Joaquim Peiró

Per-Olof Persson

Stefano Pippa

William Quadros

Alex Rand

Navamita Ray

Jean-François Remacle 


Xevi Roca
Mael Rouxel-Labbé
Eloi Ruiz-Gironés
Josep Sarrate
Shankar Sastry
Ruben Sevilla
Suzanne Shontz
Hang Si
Dmitry Sokolov
Matthew Staten
Thomas Toulorge
Chaman Singh Verma
Nicholas Vining
Kenneth Weiss
Shoudong Xu
Jessica Zhang
Paul Zhang
Daniel Zint




\section{Committee Members}

Suzanne Shontz - University of Kansas - Committee Chair

Joaquim Peiró - Imperial College London - Papers Chair

Scott Canann - Siemens PLM Software

John Verdicchio - Cambridge Flow Solutions Ltd.

Trevor Robinson - Queen's University Belfast

Nilanjan Mukherjee - Siemens PLM Software

Angela Herring - Los Alamos National Laboratory

Vladimir Tomov - Lawrence Livermore National Laboratory

Daniele Panozzo - New York University

Ryan Viertel - Sandia National Laboratories 


\title{
APPLICATION OF TENSOR FACTORISATION TO ANALYSE SIMILARITIES IN CAD ASSEMBLY MODELS
}

\author{
Flavien Boussuge ${ }^{1}$, Christopher M. Tierney ${ }^{1}$, Trevor T. Robinson ${ }^{1}$, Cecil G. Armstrong \\ ${ }^{1}$ Queen's University Belfast, Belfast, U.K., f.boussuge@qub.ac.uk \\ ${ }^{2}$ Queen's University Belfast, Belfast, U.K., christopher.tierney@qub.ac.uk \\ ${ }^{3}$ Queen's University Belfast, Belfast, U.K., t.robinson@qub.ac.uk \\ ${ }^{4}$ Queen's University Belfast, Belfast, U.K., c.armstrong@qub.ac.uk
}

\begin{abstract}
Generating fit-for-purpose CAD models from complex assemblies is time consuming for analysts. Tedious tasks include to identify and isolate the components of interest for the analysis, remove duplicate components, or correct inconsistent components' interfaces are common for large assemblies during the product development process. In this paper a new approach to help engineers analyse the consistency of CAD assembly models is proposed. The method utilises a tensor factorisation technique developed for relational machine learning and applies it on B-Rep topological and geometrical relations. The generated decomposition is used to identify which entities in the assembly are similar (within a threshold) to a selected input entity. The factorisation model regards globally all input relationships, e.g. the connections between components, to identify similar entities based on their relationships in the relational domain. It is shown that a hierarchical clustering method can group entities based on the similarities of their attributes and relationships.
\end{abstract}

Keywords: CAD/CAE Integration, Relational Learning, Computer Aided-Design, Assembly Representation,

\section{INTRODUCTION}

The pre-processing of digital mock-ups for multi-physics simulation requires identification of components anticipated not to have an influence on the desired results and removing them from the simulation model. Before starting the geometric adaption and simplification of the CAD objects, for large assembly the user should initially extract and determine the components of interest for the analysis. Often, CAD assemblies extracted from Product Lifecycle Management systems contain data inconsistencies such as missing components, duplicated entities, misalignments of parts, or poor-quality B-Rep geometry and topology. Consequently, designers and analysts spend considerable time correcting the CAD input assembly in order to obtain a model that is fit-for-purpose for finite element analysis and ready to be meshed. In addition, identifying similar geometric configurations in assembly is highly beneficial to avoid repeating pre-processing tasks.

With the rapid growth of relational and network data in social media modeling, bioinformatics and the semantic web, relational machine learning methods [1] have been proposed to learn from information represented in the form of relations between entities. The objective of relational learning is to build a model of the relational domain, where the data can be incomplete, noisy or contain false information [2]. The data is in the form of a graph, where nodes represent entities and edges the relations between the entities. Typical applications are the prediction of links in social networks or the identification of duplicated or missing entities in incomplete knowledge bases. In particular, tensor factorization techniques $[1,3]$ have shown capabilities to process large multi-relational knowledge bases from the semantic web Linked Open Data [4,5], consisting of millions of entities, hundreds of relations and billions of known facts on a standalone computer.

The CAD assemblies produced by most industrial CAD systems (e.g. CATIA or Siemens NX) are comprised of BRep component models which provide direct topological and geometrical information. These can be represented in the form of a graph. A B-Rep topological graph, as shown in Figure 1, can be transformed into RDF triples [6] (e.g. Faceis_bounded_by-Edge) and used as input data for relational learning methods. Geometrical attributes, such as face type, edge convexity, etc... can similarly be transferred as known facts (e.g. Edge - has_convexity - Convex). The combination of these topological and geometrical attributes with information on connections between components provides meaningful descriptors to interrogate assembly configurations. Thus, applying relational machine learning techniques on relational data from large CAD assembly models provides an opportunity to rapidly identify similar components and geometric regions as well as to visualize duplicated, missing or inconsistent relationships. Currently, 
the size and complexity of large assembly structures make their analysis time-consuming. For example, a full mechanical aero-engine model can contain 5000 solids, $0.3 \mathrm{M}$ faces and $1 \mathrm{M}$ edges. This data will represent millions of entities and known facts making tensor factorisation, such as the RESCAL algorithm proposed by Nickel et al.[3], an ideal candidate to experiment with.
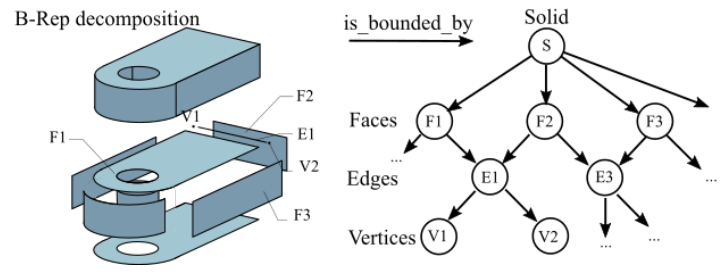

Figure 1. The topology of a Boundary Representation (B-Rep) Model can be represented as a graph.

This paper demonstrates the suitability of tensor factorization techniques as a relational learning approach for CAD assembly models. Our objective is to show how the scalability of these techniques can provide designers and analysts with rapid feedback on the configuration and consistency of the models they are generating or modifying.

Thus, it is illustrated how to learn efficiently from simple relational information available in CAD assembly models, e.g. adjacency between faces and solids, components' connectivity for tasks like:

- Entity resolution: (also known as object identification or instance matching). Here, the entity resolution task consists in identifying which entities (i.e. components, B-Rep faces and edges) in the CAD assembly refer to a similar input entity considering the B-Rep structure as well as the components connectivity within the assembly.

- Link-based clustering: entities (components, B-Rep faces and edges) are sorted into groups based on the similarity of their attributes and relationships. By iterating on the generated clusters, the user can create groups of similar components in order to filter the assembly. In addition, identical components which may not appear in the same cluster can expose assembly model inconsistencies where neighbouring relationships are not consistent between components.

Finally, by demonstrating how to efficiently look for similarity in the CAD models, the aim is to reduce the currently tedious and highly manual tasks when extracting and preparing simulation analysis models from a large assembly model.

\section{RELATED WORK}

\section{$2.13 D$ shape retrieval}

The approach in this paper is related to $3 \mathrm{D}$ shape retrieval methods. Literature on 3D model retrieval is vast and a comprehensive literature survey has be provided by Tangelder \& Velkampt [7]. A brief overview on this topic is given here. Feature-based methods use shape descriptors to match similar shapes. For example, using spherical harmonics shape descriptors, the 3D search engine of Funkhouser et al. [8] retrieve similar objects from a database of $3 \mathrm{D}$ shapes. Robust and efficient feature-based methods convert the entire geometry model to global descriptors $[7,9]$. Kazhdan et al. proposed a rotation invariant descriptor based on spherical harmonics [10]. Graph-based methods evaluate the similarity between two 3D models by extracting a graph structure from the shape's geometry. The extracted graph links the components of the shape together. This graph, examples of which include a model graph [9], a reeb graph [11] or a skeleton graph [12,13], is used to compare shapes. Local model retrieval aims at finding similar subparts of different 3D models. For design reuse, Bai [9] extracts hierarchical descriptors from a CAD model feature tree to compare subparts of CAD models. Although this model graph method allows quick database queries, it relies on a CAD feature tree which is not unique for a given shape and is not always available [14]. To retrieve partial correspondences in CAD model, You and Tsai[15] define graphs corresponding to the B-Rep structure enriched with geometric attributes such as surface types, curve types and edge convexity. Although these $3 \mathrm{D}$ retrieval techniques could be applied to identify similar components in a CAD assembly, they do not consider the relationships between components within the assembly. Two components can share identical shapes but can be connected to completely different components in an assembly or have different connectivity configurations. Our objective is to identify similarities considering all available relationships in the CAD assembly.

\subsection{CAD Assembly analysis}

Assembly retrieval methods have been proposed to search and reuse complex mechanical assembly models. In [16], Chen et al. proposed a multilevel assembly descriptor to distinguish $\mathrm{CAD}$ assemblies. The descriptor is a combination of the hierarchical assembly structure, the interactions between components and global shape descriptors of components and sub-assemblies. A graph matching algorithm is then used to identify similar assemblies. To accelerate the matching process, an indexing mechanism is introduced. However, only the assembly level is considered (not the individual components' B-Rep structure). In addition, the hierarchical structure is not always available to an analyst for a large assembly [17] or not adapted to analysis requirements [18]. Hu et al. [19] uses a vector space model (mostly employed in document retrieval) for lightweight assembly retrieval. Although achieving interactive results and allowing partial matching, CAD components are transformed into simplified meshes (losing topological and geometrical information) and interactions between parts are not considered. Wang et al. [20] propose an assembly retrieval method efficiently comparing assembly models, represented as point sets using an Earth Mover's Distance-based matching method [21] to evaluate the dissimilarity between signatures, however, does not consider the relationships between parts in an assembly in the matching. The enriched assembly model EAM of Lupinetti et al. [22,23] contains (among other descriptors) patterns of repeated components as well as an interface layer 
encoding the relationships between the different parts in an assembly model. EAMs, represented as graphs, are then compared by solving sub-graph isomorphism problems. The interface layer is also not considered when identifying the repeated components.

Regarding CAD assembly analysis, the tools available in CAD systems are mostly limited to clash/clearance analysis between components. Using Boolean operations, Shahwaan et al. [24] extracts and classifies interfaces between components to qualitatively identify the functional designation of components. This method considers the kinematic links between components to infer information, however, it requires a consistent model. Misaligned or missing components stop the application of inference rules. In addition, inferring on a large assembly is time consuming, making this approach difficult to use for quickly identifying inconsistencies. To overcome the scalability issue of Boolean operations in a CAD kernel, Jourdes et al. [25] has demonstrated how to efficiently extract assembly interfaces using a GPU ray casting approach. In the ontology-based approach of Vilmart et al. [18], information on repetitions of components and sub-assemblies serves as a basis to apply inference rules and deduce new assembly information, such as component designation. However, here too, components are grouped based on a one-to-one comparison using common shape descriptors such as symmetries. Due to the combinatory problem, such an approach is also difficult to scale on large assembly models. To the best of our knowledge, approaches to analyse the consistency of an assembly model which consider the relationships between components have not been proposed in literature.

\subsection{Statistical Relational Learning}

Statistical Relational Leaning (SRL) is concerned with domain models where entities are interconnected by multiple relations. In their review of Relation Machine Learning for Knowledge Graphs, Nickel \& Murphy [1] classify SLR methods in two main classes: graph feature models and latent features models.

The first class captures the correlation using statistical models based on observable properties of the graph. For example, an unknown relation can be derived from the existence of a path in the graph. Initially, local similarity techniques were used to analyse the nearest neighbourhood of entities. In [26], Adamic \& Adar proposed the frequencyweighted common neighbours index to identify similarity of entities by counting the common items between neighbours. As mentioned in [1], local similarity techniques scale well on large graphs, however, they are limited to single relationships where similarity is identified locally based on the direct neighbourhood. To consider that two entities can be similar without having common attributes, global similarity indices have been proposed. Among them, the Leicht-Holme-Newman index [27] analyses the ensemble of paths between entities. Although, the predictions are improved on graphs when relationships are non-local, these techniques might require more computation time $[1,28]$. For multi-relational knowledge graphs, where each edge is labelled to denote the type of relationship between the two vertices, Lao \& Mitchell [29] propose a Path Ranking
Algorithm to infer new beliefs in an imperfect knowledge base by predicting the probability of missing edges. Unlike latent feature models, this technique provides an easily interpretable model of the extracted features on the observable data. For further literature, Lu et al. [28] provides a survey on similarity indices used for link prediction, determining whether a particular relationship exists or retrieving relationships by their likelihood.

The second SLR class captures the correlation between the node/edges using latent features. Unlike graph-feature models which use features observed in the data, the latent features associated to entities have not been observed in the data, but are assumed to be hidden causes for the observables features [2]. Similar entities are derived from operations on these latent features. The objective of this SRL class is to infer the latent features automatically from the data. Tensor factorisation models have been proposed for learning from multi-relational knowledge graphs. Among them, Franz et al. [30] propose the TripleRank method to rank and produce richer description of linked data on the semantic web. The RESCAL factorisation model of Nickel et al. [2,3,31] has successfully demonstrated its ability to predict unknown triples on large knowledge bases consisting of millions of entities and known facts. RESCAL captures similarities of entities in the relation domain via interactions of the latent features. Jenatton et al [32] proposed a tensor factorisation model for highly multi-relational data, where the number of different relations is large. The reference paper of Kolda and Bader [33] provides an extensive review on tensor decomposition models and their applications.

Finally, as mentioned in [1], it is difficult to determine if a relational latent feature model or graph feature model is better for learning knowledge graphs. Authors in [1] agree that latent feature models are suited for data showing global relational patterns. Tensor factorization techniques are computationally efficient on a large database when relations can be explained using a small number of latent variables. Graph feature models are best suited when graphs patterns appear locally. For example, the Path Ranking Algorithm [29] is efficient when relationships can be explained from short paths in the graph.

In the context of this work, we propose to transcribe CAD models (described as a B-Rep model) as knowledge graphs to learn from. In this work, the RESCAL[3] tensor factorisation model is used as the learning model. This choice is based on the following assumptions:

- $\quad$ Similar entities (i.e. CAD components, B-Rep faces and edges) are not necessarily close in an adjacency graph. For example, standard components (bolts, nuts...) can appear in various locations in a mechanical structure. The path linking those components based on component adjacency might be long, thus making the graph feature model less efficient. On the other hand, the shared entity representation in RESCAL captures global dependencies due to the shared latent representations.

- One objective is to provide quick feedback to the designer/analyst on modelling errors, allowing quick design iteration to correct the CAD model. In [2], the authors demonstrated that the RESCAL model scales 
linearly with the number of entities. Then, once the factorised model is computed, the user can quickly query if a specific relationship exists or not, essentially in real-time.

- $\quad$ Another objective is to automatically generate clusters of CAD components to help the user filter their assembly for a specific analysis. Depending on the input relationships selected by the user, the latent representations of entities can be used by clustering algorithms to automatically generate groups of components.

The novelty of this paper is the use of a latent-features method based on tensor factorisation to compare B-Rep entities (solid, faces and edges) in a CAD assembly. B-Rep entities and their connections within the assembly are extracted and stored in a large knowledge base to learn from. By capturing the interactions of the latent features, the tensor factorisation considers all the input relationships between the B-Rep entities. Entity similarities are derived from operations on these latent features, which are then used to detect design or assembly inconsistencies.

\section{TENSOR FACTORIZATION OF CAD ENTITIES}

\subsection{Proposed pipeline}

The method proposed in this work to analyse the consistency of CAD assembly models is decomposed into the following three main steps (refer to Figure 2).

\section{Step 1. Pre-processing: extract data and fill the tensor}

The first step extracts the data from the CAD assembly models. This data is used to populate a three-way tensor $X$ of size $\mathrm{n} \times \mathrm{n} \times \mathrm{m}$ which will be factorised in Step 2. Similar to [3], the entries of the tensor $X$ on the two first dimensions correspond to the combined entities to analyse. In this work, all the solids $S$, faces $F$ and edges $E$ of the B-Rep models BRep are defined as entities. Additional geometrical types (e.g. surface type, edge type), interfaces and convexity attributes are also entities.

The third dimension contains the $\mathrm{m}$ different types of relations between the entities. For each existing known relationship of a $\mathrm{k}^{\text {th }}$ relation between the $\mathrm{i}^{\text {th }}$ entity and $\mathrm{j}^{\text {th }}$ entity, a tensor entry $x_{i j k}=1$ is added to $X$. Otherwise, for non-existent relationships, the entry $x_{i j k}$ is set to 0 . Hence, for each kth relation, a frontal slice $X_{k}=X_{:,, i k}$ is generated. This slice contains all the existing relationships (i.e. defined as semantic triples $i^{\text {th }}$ entity $-\mathrm{k}^{\text {th }}$ predicate $-\mathrm{j}^{\text {th }}$ entity in the $\mathrm{RDF}$ format) linking the entities through this $\mathrm{k}^{\text {th }}$ relation.

In order to provide a first set of descriptors of the solid models, we propose to transfer the internal B-Rep structure into the slices of the tensor. As highlighted by You and Tsai [15], the benefit of using the B-Rep structure is its invariance to geometric transformation. Alignment of objects is not required before extracting the geometric signatures.

The following four main tensor slices are generated in this work:
- The first frontal slice $X_{0}$ of $X$ contains the topological relations between solids, faces and edges of the B-Rep model. For example, if a topological relation " $S_{i}$ is_bounded_by - $F_{j}$ " exists, an individual tensor entry $x_{i j 0}=1$ of $X$ is generated linking the $\mathrm{i}^{\text {th }}$ entity (solid $S_{i}$ ) to the $\mathrm{j}^{\text {th }}$ entity (face $F_{j}$ ).

- The second slice contains the relations between the faces/edges and their geometrical and convexity type. For example, the fact that a face is planar is described by the relation " $F_{j}$ - is_type - GeomType $p$ " translated by the entry $x_{j p 1}=1$ in the tensor slice $X_{I}$.

- The third slice contains the interface relationships between solids. If a solid $S_{i}$ is touching/penetrating another solid $S_{l}$, or if there is a gap between the solids (smaller than a user-defined distance) the two relations " $S_{i}$ - has_interface - $I_{o}$ " and " $S_{l}$ has_interface - $I_{0}$ " are added as new entry $x_{i l 2}=1$ in the slice $X_{2}$. The relation between the interface and the interface type (interference, contact or gap) is also added.

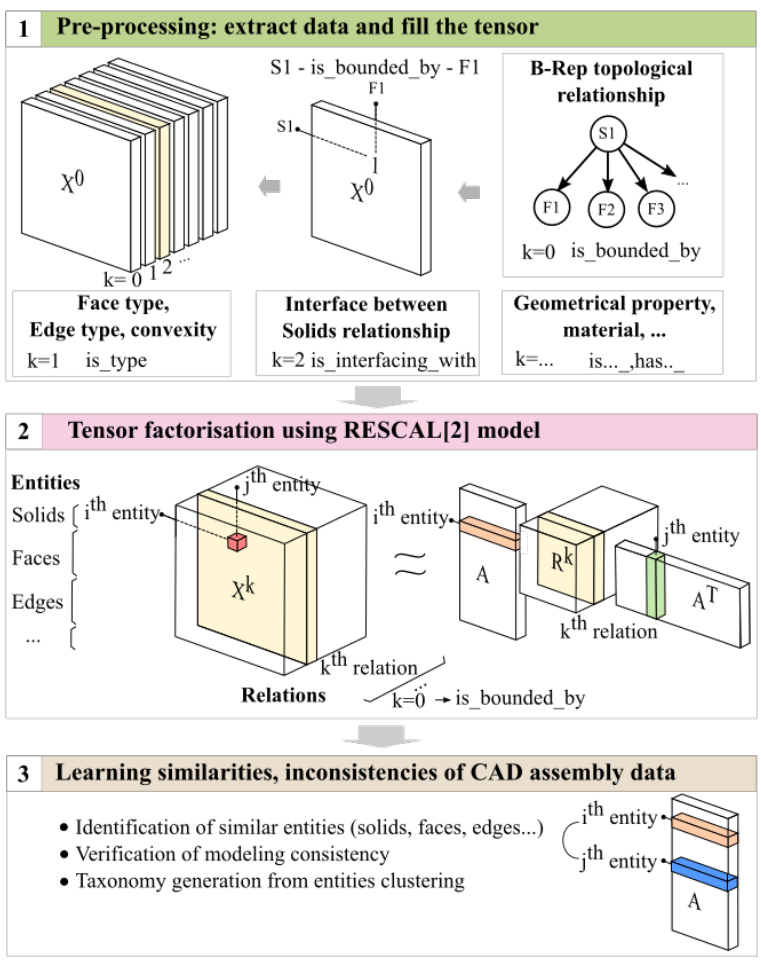

Figure 2 Overview of the proposed approach to learn similarities and inconsistencies in CAD assembly model using the RESCAL[3] tensor factorisation model.

The number of slices of the tensor model is not limited, new slices can be added (see discussions section 6). The objective of this paper is to demonstrate the applicability of the tensor factorisation on CAD assembly models. The current approach is limited to topological relationships and straightforward geometrical parameters which are directly available from the CAD system. Additional parameters generated by the user, such as material properties or 
simulation attributes can be added in the model. Adding indepth shape descriptors to better describe freeform surfaces or graph structures (e.g. medial object or reeb graph) is discussed in section 6 and left for future work.

\section{Step 2. Tensor factorisation using RESCAL[3]}

Given the tensor $X$ of size $\mathrm{n} \times \mathrm{n} \times \mathrm{m}$ built in step 1, the RESCAL factorisation model proposed by Nickel et al. in [3] was used to compute a factorisation of $X$. RESCAL factorises each frontal slice $X_{k}$ of $X$ into the following matrix product:

$$
X_{k} \approx A R_{k} A^{T}, \quad k \in 1 . . m
$$

where $A$ is a $\mathrm{n} \times \mathrm{r}$ factor matrix, $R_{k}$ is $\mathrm{r} \times \mathrm{r}$ matrix which denotes the kth frontal slice of an adjacency $\mathrm{R}$ tensor. (RESCAL jointly factorises these adjacency matrices $R_{k}$, such that $A$ is common for all frontal slices of $X$ ), $\mathrm{r}$ is a userdefined parameter defining the number of latent components (or common factors) in the matrix $A$. The matrix $A$ can be viewed as an embedding of the entities in the r-dimensional latent space [2]. The matrices $A$ and $R_{k}$ are computed by solving a regularized minimisation problem as described in [3].

Step 3. Learning similarities, inconsistencies of CAD assembly data.

Given the factorization of the initial tensor $X$, the essential feature of the RESCAL method is that the latent space $A$ reflects the similarity of entities in the relational domain [2]. Here the similarity of entities refers to the similarities of their relationships. For example: if two solids are bounded by the same type of faces, which are bounded by the same type of edges (slice $X_{0}$ ); if these two solids are also connected to other objects having similar topology, and so on...; there might be evidence that the two solids are identical within the assembly structure. Hence, two entities $e_{i}$ and $e_{j}$ can be compared by looking at their individual latent representations $a_{i}$ and $a_{j}$ in $A$. These latent representations not only measure the common attributes between the entities but also consider the similarity of related entities and relations involved in the relationships of the $\mathrm{i}^{\text {th }}$ and $\mathrm{j}^{\text {th }}$ entity.

The RESCAL model has been initially developed to perform relational learning tasks on large sets of relational data from the semantic web's Linked Open Data [31]. This paper illustrates how the approach can be used on large CAD assembly models to extract components or component entities in a range of scenarios. As described in Section 1, to demonstrate the applicability of tensor factorisation, the relational learning tasks of entity resolution and link-based clustering are performed. Section 4 develops these specific tasks and applies them to CAD assembly data.

\section{USAGE SCENARIOS FOR CAD/CAE INTEGRATION}

The objective of relational learning is to build a model of the domain from relational data that can be incomplete, noisy or even contain false information, thus avoiding the need for expensive user clean-up operations on CAD assembly data. From this relational model, specific learning tasks can be performed. In this section, the benefit of deriving a factorized model of a CAD assembly to analyse the consistency of the design will be described. The following usage scenarios are proposed in the context of CAD/CAE integration to help analysts understand and correct the input CAD models with a view to generating simulation models.

\subsection{Entity resolution: retrieving similar entities in the CAD assembly}

To simplify the approach, a CAD component is assumed to be modelled as a B-Rep solid. Following the data extraction of a CAD assembly (see Step 1 in Section 3.1) a tensor is generated containing the B-Rep solids, faces and edges. The tensor is then factorised (see Step 2 in Section 3.1) and produces a matrix $A$ reflecting the relational similarity of entities.

The scenario is then to input an entity: a solid, a face or an edge and to identify which entities are similar to it in the CAD assembly. As mentioned in Section 3.1, similarity refers to the data contained in all relations in the initial tensor. As the topology relations of the CAD components are input in the tensor, together with the connection between the components, two CAD components (i.e. solids) are similar not only because they share a similar topological graph, but also because their similarity is propagated through their connections with the other components in the assembly which also have the same topological graph.

Hence, to compare an input entity $e_{i}$, given by the user, to any other entity $e_{j}$, a ranking of all entities is computed using their latent representation: the vectors $a_{i}$ and $a_{j}$ corresponding the $\mathrm{i}^{\text {th }}$ and $\mathrm{j}^{\text {th }}$ row in $A$.

Following the approach of Nickel [2], to compare two entities, the function $\mathrm{k}$ is calculated as such:

$$
k\left(a_{i}, a_{j}\right)=\exp \left(-\frac{\left\|a_{i}-a_{j}\right\|^{2}}{\delta}\right),
$$

where $\delta$ is an additional user-given parameter to exponentially scale the similarity value.

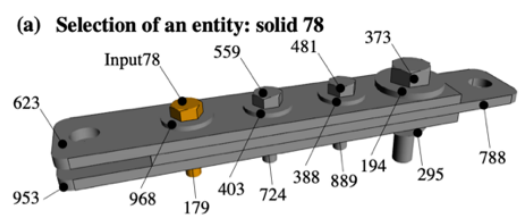

(b) Similar entities to solid 78

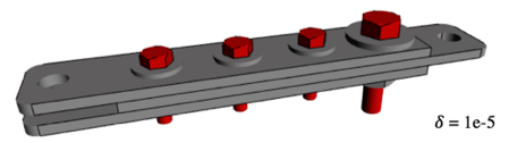

\begin{tabular}{|c|c|}
\hline Solids & $\mathrm{k}$ \\
\hline $\mathbf{7 8}$ & 1.0 \\
\hline 373 & 1.0 \\
\hline 481 & 1.0 \\
\hline 559 & 1.0 \\
\hline 179 & 0.0 \\
\hline 295 & 0.0 \\
\hline 889 & 0.0 \\
\hline 724 & 0.0 \\
\hline 788 & 0.0 \\
\hline 623 & 0.0 \\
\hline 953 & 0.0 \\
\hline 194 & 0.0 \\
\hline 388 & 0.0 \\
\hline 403 & 0.0 \\
\hline 968 & 0.0 \\
\hline
\end{tabular}

Figure 3 Retrieving similar solid entities. (a) The solid 78 is given as input and compared with all other solids in the assembly. (b) Solids are coloured based on their similarity value with solid 78. In Red, the solids 373, 481 and 559 are exactly similar to 78 . 
Figure 3 shows an example of the entity resolution on a simple model containing 15 solids. Solid 78 (one of the bolts) is given as an input and $\mathrm{k}$ is calculated for all the solids. For all the other bolts the same $k$ value is returned. This result is expected as the bolts have the same topology, same face type, same edge type and convexity type. In addition, their faces have the same number of singularities. Most importantly, they are connected to the same components which are also identical. Their relations in the tensor $X$ are symmetric which is reflected by having identical latent representations. Here, the data given as input in the tensor is not sufficient to distinguish the bolt 373 (largest bolt) being less similar than the same-size bolts 559 and 481 . Indeed, depending on the designer/analyst need, additional shape characteristics should be added and evaluated.

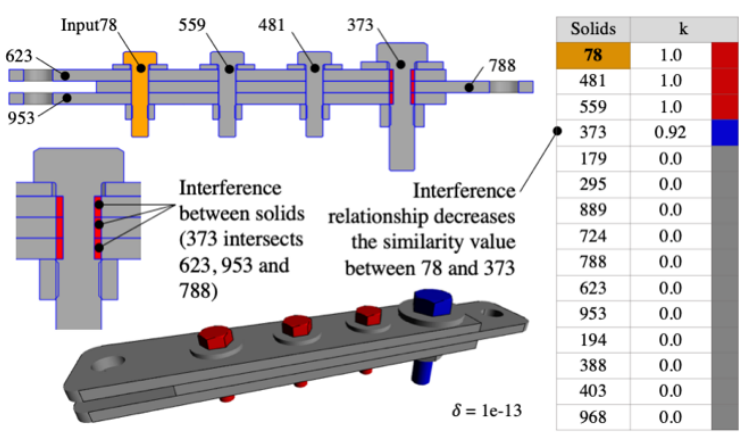

Figure 4 Example of the consideration of interference relationship between solids. The bolt 373 intersects the plates 623, 953 and 788 . This configuration differs from the bolts 78,559 and 481 having the same diameter as the plate holes (touching interface). 373 is less similar to 78 than $559 / 481$ to 78 due to the consideration of the interference relationships.

In Figure 4 the bolted junction model is modified by changing the diameter of the holes in the plate bodies (623, 788 and 953) in order to introduce an interference between the bolt 373 and the plates (the diameter of bolt 373 being bigger than the holes diameter). These interferences modify the relationships of the bolt 373. Now, as shown in Figure 4, bolt 373 is still very similar to input 78 but slightly less similar than the bolts 373 and 481 . This result illustrates how the RESCAL factorisation is able to consider all the relations as opposed to pairwise comparing entity to entity.

Once the factorisation is computed (see Section 0 for computation time), interrogating the $A$ matrix is quick $(<1 \mathrm{~s}$ for an $A$ matrix of size $175000 \times \mathrm{n}$, corresponding to an assembly with 1000 solids, see Table 1 car engine model). Hence, the user can easily select a component in the assembly and ask which components are similar. By changing the $\delta$ parameter and adding a threshold for k (e.g. $\mathrm{k}>1 \mathrm{e}-10$ ), the user can quickly filter the entities from the more similar to the less similar. Figure 5 illustrates entity resolution results for different values of the $\delta$ parameter. In the prototype implementation used to test the applicability of the proposed method, a slider component has been designed allowing the user to interactively discover similar objects for a given input. As shown in Figure 5(a), when setting $\delta$ to a low value, the method returns only the most similar bolt components. When increasing $\delta$, more components are displayed until all components pass the threshold for $\mathrm{k}$. Here, the scenario is to use this approach to quickly select similar components to keep/remove for the final simulation model. For example, in Figure 5(b), the 10 camshaft fingers (shaded component) are found distinctively even when $\delta$ is high (relative to this specific CAD model). This can be explained by the relatively distinct shape of the component within the assembly.

The input entities are not limited to solids but can also be faces or edges. On Figure 5(d) a planar face lying on the head of the bolt of the bolted junction model is selected. Similar faces are not only found on the same solid but also on all the similar bolts, showing that topological relations and components' interfaces are simultaneously considered in the factorisation.

\subsection{Set of entity resolution: finding similar features}

In this section, it will be illustrated how the entity resolution can be extended to sets of entities, still using the property of the $A$ factor matrix in addition to the face adjacency graph from the B-Rep model. A usage scenario is to find similar features of a B-Rep solid, given one as an input. Here a feature refers to a set of B-Rep faces in a solid. Hence, the user inputs a set of adjacent faces and the algorithm returns a list of sets of faces considered similar. The following Algorithm 1 details the procedure. The returned list of features is ranked by summing $k$ for each element of the feature ( $k$ has been previously calculated for each similar entity of each face of the given set of faces). Hence, the similar features can be visualized by the user in a similar manner to the entity resolution of Section 4.1. 

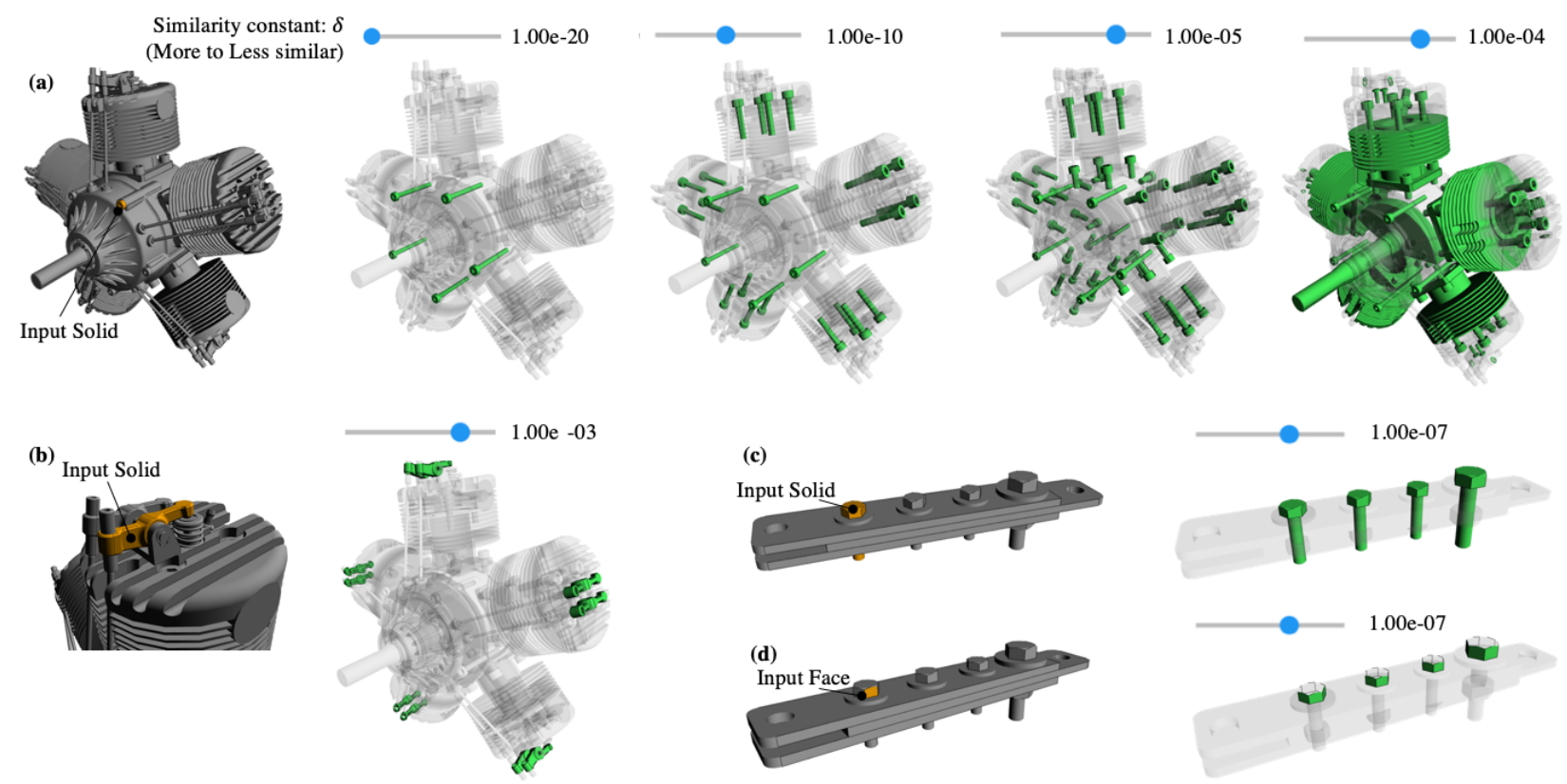

Figure 5 Examples of the entity resolution learning task. For a given solid or face entity, the user can modify the similarity constant in order to display more to less similar entities.
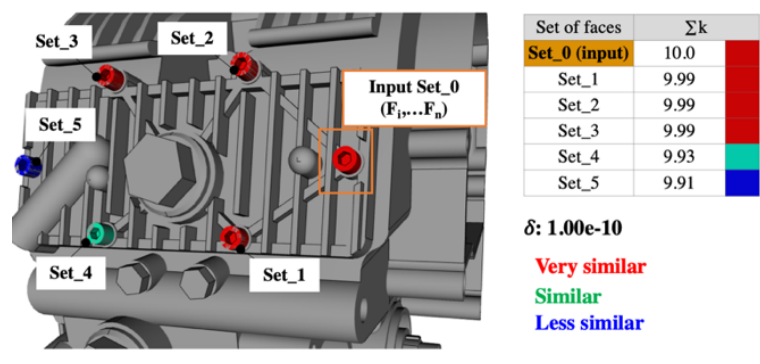

$\delta: 1.00 \mathrm{e}-10$

Very similar

Similar

Less similar

Figure 6 Entity resolution on set of faces. The user can visualize similar sets of faces on a B-Rep component given an input set of faces.

Figure 6 shows an example of entity resolution on sets of faces. The displayed model is a standalone B-Rep solid which has been created from the Boolean union of multiple individual components. The analysts' workflow is to remove small features, such as bolt heads considered as not relevant for the simulation they are to carry out. Given as an input the Set_0(F1,..Fn), shown highlighted in the box, the approach herein returns 5 similar features for a given $\delta$. Similar to Section 4.1, the user can vary the value of $\delta$ to have more or less similar features returned. The processing time, however, is higher as more operations are performed compared to Section 4.1. For the example shown in Figure 6, Set 4 and Set 5 are ranked as "less similar" than 1,2 and 3. Although, the latent factors in $A$ cannot be interpreted directly, the difference can be explained by looking at the surrounding faces of the features in the model. Here Set 4 and 5 are connected to faces which are less similar to the faces

surrounding the input Set 0 than the faces connected to Set 1,2 and 3 .

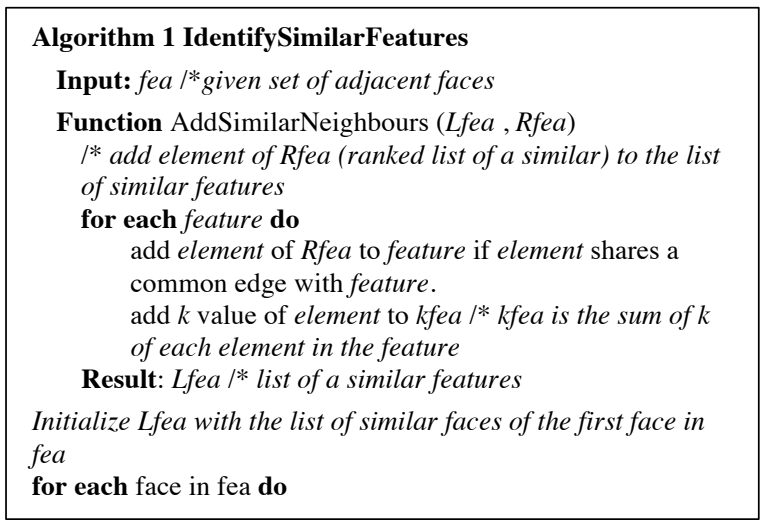

Algorithm 1 Identification of similar features given an input set of adjacent face.

\subsection{Link-based clustering: building a taxonomy}

In [31], authors have shown the capability of RESCAL to perform link-based clustering on large Linked Open Data databases containing millions of entities and known facts. As mentioned in Section 1, the objective is to partition entities into groups based on the similarities of relationships. In this section, a hierarchical clustering algorithm is used to cluster the entities using their latent representation in $A$. In this latent-component space, each row of $A$ defines a vector of dimension $r$. This set of vectors is given as input to a 
hierarchical clustering algorithm. In this work, the algorithm provided by the SciPy Python library [34] is used.

Figure 7 illustrates the result of the hierarchical clustering for the bolted junction model. The corresponding dendogram chart is shown. Here a threshold distance set to 0.05 returns 4 clusters corresponding to the 4 types of solids present in the model. By changing the threshold distance value, the user can visualize different cluster configurations.

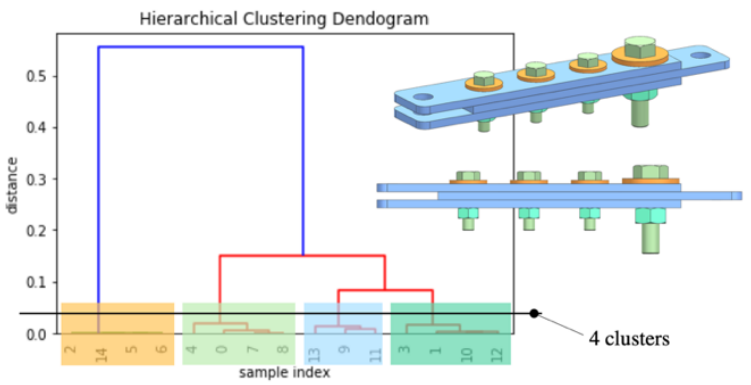

Figure 7 Hierarchical clustering of the bolted junction model. By setting a threshold, the user can quickly iterate different clusters configurations.

In a $\mathrm{CAD} / \mathrm{CAE}$ context, the identification of similar components and similar regions of space is meaningful to save time in the FEM meshing operations and boundary

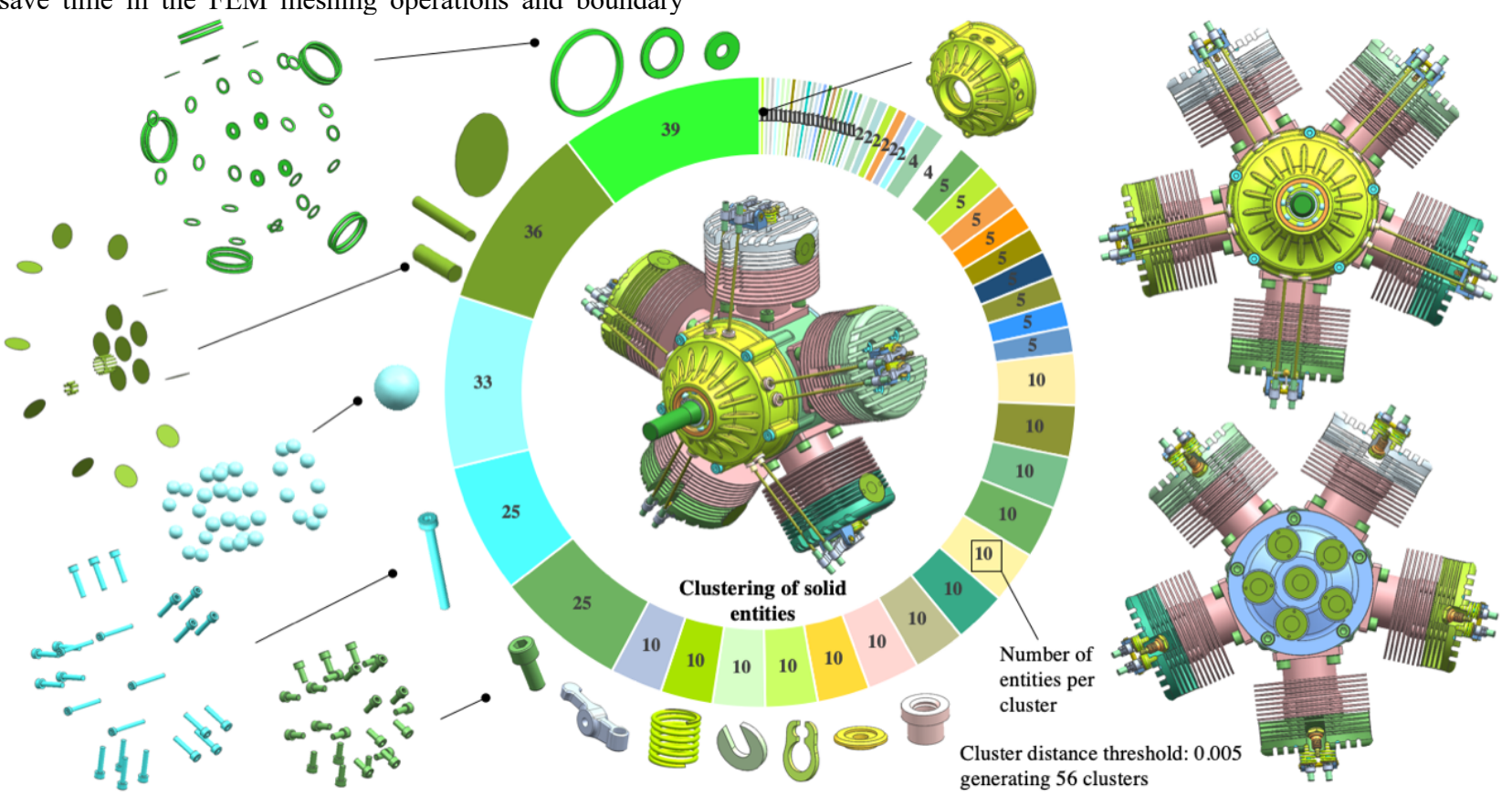

Figure 8 Application of a hierarchical clustering algorithm. The $A$ matrix is used as input for the clustering algorithm. For this model, with a distance threshold of $0.005,56$ clusters of solid entities are generated condition application. Although a CAD assembly tree may exist, as explained by Vilmart [18], its structure might not be adapted for simulation purposes. Indeed, depending on the design protocol and user decisions, similar components can appear in multiple branches of the assembly tree. They are not part of the same instance. A typical example is the assembly of standard junctions. When multiple suppliers are involved in the assembly design, they will use their own junction models which will appear as different subassemblies in the final model. As mentioned in [31], an application of the link-clustering is the automatic generation of taxonomies. In the context of this work, under the supervision of the user, a CAD assembly model can be analysed and similar entities (components, faces, or edges) can be grouped. Figure 8 shows the result of the clustering for the radial engine model [35] (containing 374 solids) when the threshold is set to 5e-3. The largest clusters contain standard components which are repeated multiple times in the assembly. In this model generating these clusters helps an analyst to easily filter the assembly components. For assembly meshing requiring conformal meshes, when the components' interfaces imprints are also given as input, the user will need only to mesh one instance for each cluster and copy it to the groups of similar entities. 


\subsection{Identification of modelling inconsistencies}

A practical application of our approach concerns the identification of modelling inconsistencies in a CAD assembly. Indeed, when displaying the generated clusters, some entities might not appear in the expected cluster. Figure 9 shows examples of inconsistencies found in CAD assemblies when analysing the clusters. In Figure 9(a), two bolts (in orange) are not identified as being part of the bolt cluster. This is due to the connectivity with neighboring components which is not consistent compared to the other bolts in the component. Their positionings generate interferences with the casings changing their relationships in the global tensor. In Figure 9(b), two groups of components having the same shape are connected differently to the casing. Here too, this configuration generates different relationships for the two groups of components. Although, clash management is usually handled by automatic scripts in CAD software, it obeys strict rules conventionally defined by the user. Here, this approach could complement the clash detection analysis as it doesn't follow any predefined rules and could be apply as a visual quality check tool.

(a)

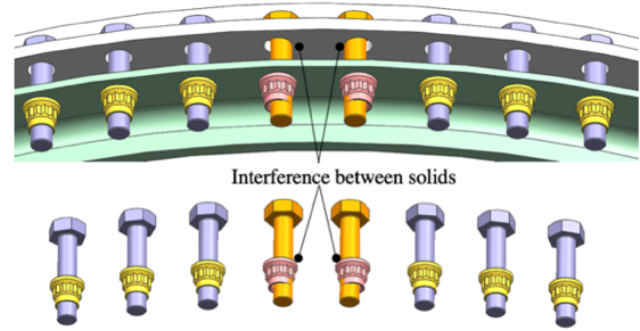

(b)

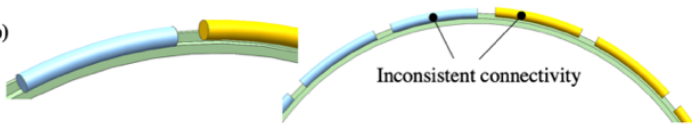

(c)

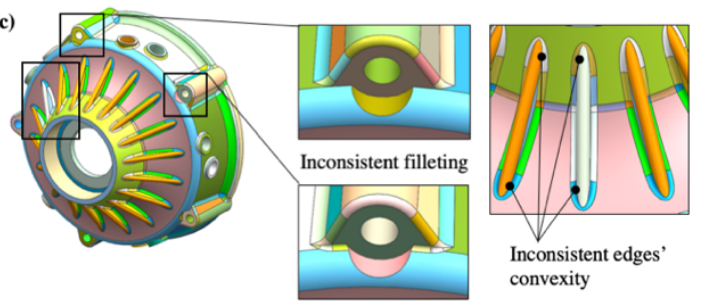

Figure 9 Example of modelling inconsistencies found following the link-based clustering task.

In Figure 9(c), the clustering is applied on face entities and displayed (one colour represents one cluster of faces). The symmetries of the model are not totally reflected in the colouring applied to the faces. An inconsistent filleting modifies the object's topology and an inconsistent edge convexity type modifies the convexity relationships of the cyclic stiffeners. These inconsistencies break the cyclic symmetry property. By using a relational learning approach, the user can visualize the similarities between the input entities and then identify the inconsistencies which need to be corrected. Having a consistent CAD assembly is highly valuable for simulation and manufacturing. For example, a finite element simulation of an assembly requires all the contacts between components to be defined. Running a process on a large assembly to automatically apply these contacts requires a clean input model, where all the components are correctly positioned. This approach provides a low-cost method to help the designer identify modelling issues upfront, without having to interrogate an entire assembly model component by component.

Figure 10 shows another example of modelling inconsistencies found when trying to identify similar features (see Section 4.2). Initially, a bolt head is given as the input set of faces. Choosing a small value for $\delta$ to display the most similar features, one of the bolt heads is not listed (see BoltHead_8 in Figure 10(a)). Increasing $\delta$ makes BoltHead 8 appear with a similarity $k$ value significantly different from the other bolt heads. Looking closely at the CAD model for BoltHead_8, it appears that the bolt is intersecting the casing component before the Boolean union. This intersection results in BoltHead_8 having a slightly different topology from the other bolt heads, which is reflected in the latent factors of $A$.

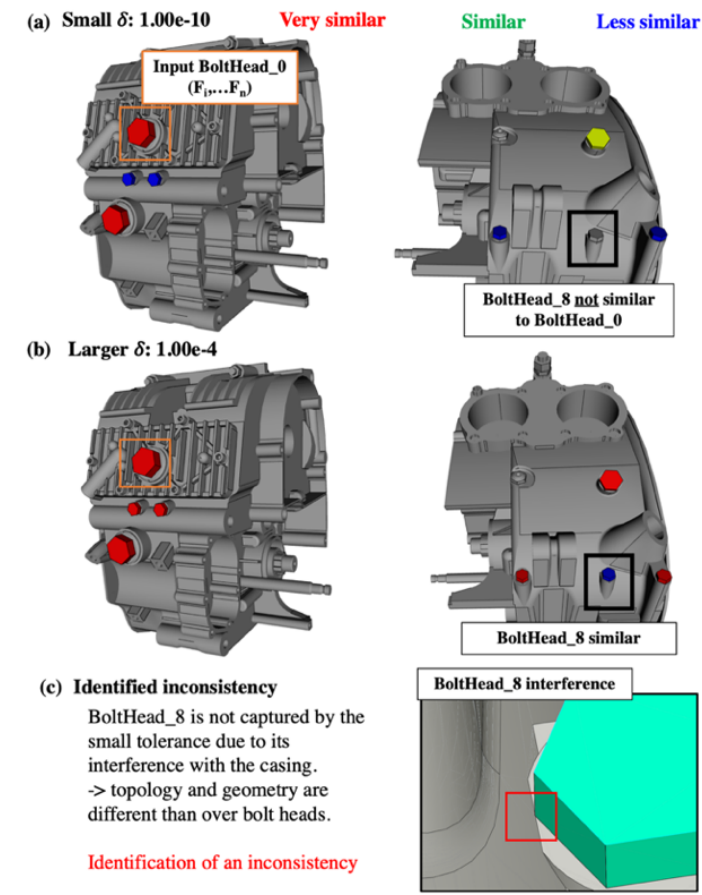

Figure 10 Inconsistency in the modelling of Bold Heads. Increasing the scale value of the similarity analysis reveals that the similarity of BoltHead_8 to BoltHead_0 is higher than the others bolt heads. (a) Entity resolution with small $\delta$, (b) same entity resolution with a larger $\delta$, (c) Bolthead_8 is intersecting the casing resulting in different geometry and topology.

Identifying inconsistent geometrical regions of a CAD component is relevant for simulation. It saves time for an analyst to filter the regions to simplify or remove before meshing for finite element modelling. In this paper, the 
identification of similarities and the link-based clustering can be considered as an interactive tool for designer/analyst to verify the consistency of the CAD models. Ideally, processes could be developed to automate this identification.

\section{EVALUATION ON LARGE CAD ASSEMBLIES}

\subsection{Scalability of the tensor factorisation}

In [3], the authors designed the RESCAL factorisation model to be able to scale to large knowledge bases consisting of millions of entities, hundreds of relations and billions of known facts. In the context of this work, scalability is important as analysts tend to simulate increasingly larger assembly models containing thousands of components [17]. Even the design of standalone components can become highly complex in industry. For example, an intercase component in an aero-engine model contains around 10,000 CAD faces and 25,000 edges. It is essential to consider the running time of an analysis tool on such a large model.

RESCAL has been designed to scale linearly with the number of entities (see [2] giving details on the computational complexity). In this section, the scalability of the model is evaluated by running the proposed approach on various CAD assembly models. In this work, RESCAL was integrated with PythonOCC [36] (a Python version of the open-source geometric kernel OpenCascade [37]). The extraction of topological data and the interface detection is performed using the Siemens NX 11 API [38] and the Parasolid [39] library. Similar data extraction can be performed in other CAD systems.

The factorisation and clustering was carried out on a windows workstation with 4 Intel i7-6700 $3.40 \mathrm{GHz}$ CPUs, 32 GB RAM. Table 3 presents the computation time for different CAD models shown in Figure 16. The models in Figure 8 and Figure 16(a,e,f) are provided by the GrabCAD [35] community. These examples were used to verify the applicability of the proposed approach and its scalability to large models. As expected, the timing of the factorisation is proportional to the number of entities. For the largest model, the car engine, the factorisation time is less than 1 minute with a $r$, the number of latent components (or common factors) in the matrix $A$, equals to 100 .

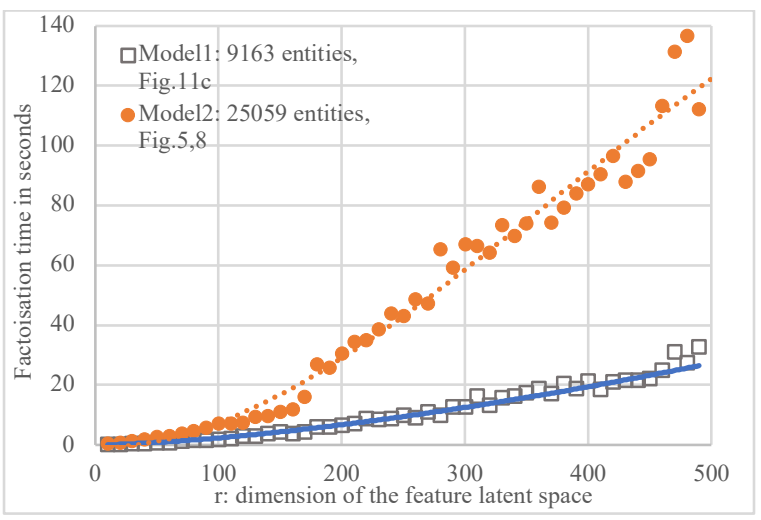

Figure 11 Evolution of the dimension of the feature latent space on the computation time to factorise the tensor.

As mentioned in [2], the tensor factorization computational complexity grows cubically with $r$. Error! Reference source not found. illustrates this evolution on two use-cases for different value of $r$. Depending on the size of the model, choosing this number can have an influence on the total computation time. The number of latent features $r$ to consider is difficult to evaluate. Although in-depth evaluation has not be done in this work, our tests on the usecases of Figure 16 using $r$ equals to 100 allowed to generate clusters of expected similar shapes. Our observations suggest using a low rank (e.g. between 20 and 50) for small assembly ( $<200$ solids) to avoid overfitting the model and a larger rank for larger assemblies to include more features and avoid underfitting. Once the factorization is computer, the clustering operation is almost instantaneous, making it possible to test different cluster variants. Figure 16 shows the cluster variant corresponding to $\delta=1 \mathrm{e}-5$ for each assembly model.

\subsection{Comparison to graph-based and spherical harmonics-based methods}

The tensor factorization approach enables the user to quickly interrogate the factorisation matrix when performing an entity resolution task. In this section we compared our approach for this task to two similar methods from the literature: the spherical harmonics approach of Kazhdan et al. [10] and the graph matching approach similar to [15] and $[22,23]$. For this evaluation, our use-case is the Vesta model: a large industrial CAD assembly provided by Rolls-Royce Plc and containing 6503 solid entities (see Figure 12 (b)). In Table 1, the runtime of the entity resolution task has been recorded for the three methods on five different components. For the spherical harmonics method, we use the executable of Kazhdan[40] with 17 spherical functions having 32 spherical harmonics. For the graph matching method, we extract the B-Rep topological face-edge graph of each solid and enrich it with the same geometrical information as the tensor slices, see Section 3.1, i.e. surface/edge type and edge convexity. To perform the sub-graph isomorphism, we use the VF2 algorithm implemented in the python library NetworkX[41]. 
(a) Component query

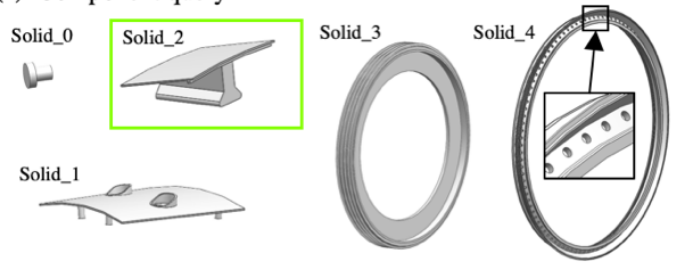

(b) CAD assembly

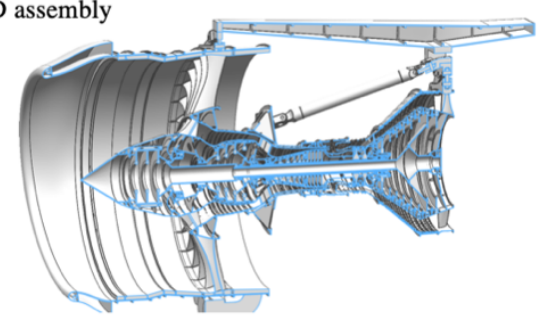

(c) Results of the query for Solid_2
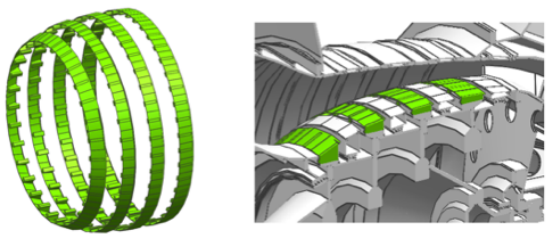

Figure 12 Entity resolution evaluation task on large assembly (Vesta model, courtesy of Rolls-Royce PIc). (a) query components, (b) the CAD assembly with 6503 solids, (c) example of a query using the tensor factorization-based method.

Table 1 Entity resolution timing comparison for the 5 components of Figure 12.

\begin{tabular}{|c|c|c|c|}
\hline Method & $\begin{array}{l}\text { Global } \\
\text { descriptor: } \\
\text { Spherical } \\
\text { Harmonics } \\
{[10]}\end{array}$ & $\begin{array}{l}\text { Graph } \\
\text { matching: } \\
\text { Subgraph } \\
\text { Isomorphism }\end{array}$ & $\begin{array}{l}\text { Relational } \\
\text { Learning: } \\
\text { Tensor } \\
\text { factorisation }\end{array}$ \\
\hline B-Rep stats & \multicolumn{3}{|c|}{$\begin{array}{l}\text { Nb Solids: } 6503, \mathrm{Nb} \text { Faces: } 128638 \text {, } \\
\text { Nb Edges: } 319500\end{array}$} \\
\hline CAD data extraction & \multicolumn{3}{|c|}{$3 \operatorname{mins} 01 \mathrm{~s}$} \\
\hline Interface detection & \multicolumn{3}{|l|}{10 mins $15 \mathrm{~s}$} \\
\hline \multirow[t]{2}{*}{$\begin{array}{l}\text { Specific Data } \\
\text { preparation task }\end{array}$} & $\begin{array}{c}\text { Shape } \\
\text { descriptors } \\
\text { computation }\end{array}$ & $\begin{array}{c}\text { Graph } \\
\text { generation. }\end{array}$ & $\begin{array}{c}\text { Tensor } \\
\text { Factorisation } \\
\text { (rank: } 100)\end{array}$ \\
\hline & $1 \mathrm{~min} 42 \mathrm{~s}$ & $0.77 \mathrm{~s}$ & $2 \operatorname{mins} 18 \mathrm{~s}$ \\
\hline \multicolumn{4}{|l|}{ Entity Resolution task } \\
\hline $\begin{array}{l}\text { Solid_0 } \\
5 \text { faces, } 4 \text { edges }\end{array}$ & $0.14 \mathrm{~s}$ & $0.06 \mathrm{~s}$ & $0.07 \mathrm{~s}$ \\
\hline $\begin{array}{l}\text { Solid_1 } \\
20 \text { faces, } 28 \text { edges }\end{array}$ & $0.12 \mathrm{~s}$ & $3.11 \mathrm{~s}$ & $0.07 \mathrm{~s}$ \\
\hline $\begin{array}{l}\text { Solid_2 } \\
19 \text { faces, } 48 \text { edges }\end{array}$ & $0.14 \mathrm{~s}$ & $15 \mathrm{mins} 43 \mathrm{~s}$ & $0.07 \mathrm{~s}$ \\
\hline $\begin{array}{l}\text { Solid_3 } \\
36 \text { faces, } 36 \text { edges }\end{array}$ & $0.11 \mathrm{~s}$ & 12 mins $36 \mathrm{~s}$ & $0.07 \mathrm{~s}$ \\
\hline $\begin{array}{l}\text { Solid_4 } \\
141 \text { faces, } 241 \text { edges }\end{array}$ & $0.12 \mathrm{~s}$ & $2 \operatorname{mins} 18 \mathrm{~s}$ & $0.07 \mathrm{~s}$ \\
\hline
\end{tabular}

As shown in Table 1, the spherical harmonics approach requires a specific data preparation task to calculate the descriptor. Similarly, our approach requires to compute the factorisation of the tensor. The graph matching method can directly operate on the B-Rep graph, however, depending on the sub-graph configuration of the query component to match in the large assembly graph, the entity resolution task can be time consuming. This is particularly true when the combinatory is high, such as for the repeated components Solid 2 and Solid_3 corresponding to the numerous blades and seals present in the assembly. In this case, the matching algorithm has to look for a medium-size sub-graph pattern repeated several times into a large graph. The other two approaches, on the other hand, can directly compare the entities by looking at their factorised/shape descriptor vectors. Table 2 summarises the pros and cons of the three methods.

\section{Table 2 Advantages and disadvantages of} the evaluated methods

\begin{tabular}{|c|c|c|}
\hline Method & Pros & Cons \\
\hline $\begin{array}{l}\text { Global } \\
\text { descriptor: } \\
\text { Spherical } \\
\text { Harmonics } \\
{[10]}\end{array}$ & $\begin{array}{l}\text { Efficient to identify } \\
\text { components having } \\
\text { globally similar shapes. } \\
\text { Query components can } \\
\text { be stored in a database. } \\
\text { Not sensitive to CAD } \\
\text { topological variation. }\end{array}$ & $\begin{array}{l}\text { Do not detect subpart } \\
\text { (feature) in shape. Do } \\
\text { not consider CAD } \\
\text { topological } \\
\text { segmentation. Do not } \\
\text { consider interfaces } \\
\text { between components. }\end{array}$ \\
\hline $\begin{array}{l}\text { Graph } \\
\text { matching: } \\
\text { Subgraph } \\
\text { Isomorphism }\end{array}$ & $\begin{array}{l}\text { Can match sub-shapes } \\
\text { from a database to a new } \\
\text { model. }\end{array}$ & $\begin{array}{l}\text { Require an initial } \\
\text { segmentation. Sensitive } \\
\text { to CAD topological } \\
\text { variation. Sensitive to } \\
\text { the combinatory of } \\
\text { different entities. }\end{array}$ \\
\hline $\begin{array}{l}\text { Relational } \\
\text { Learning: } \\
\text { Tensor } \\
\text { factorisation } \\
\text { (our } \\
\text { approach) }\end{array}$ & $\begin{array}{l}\text { Efficient to interactively } \\
\text { compare entities within } \\
\text { the same model. Able to } \\
\text { analyse all relationships, } \\
\text { considering interfaces } \\
\text { between components. } \\
\text { Quick query. }\end{array}$ & $\begin{array}{l}\text { Require an initial } \\
\text { segmentation. Cannot } \\
\text { compare subpart stored } \\
\text { in a database. Sensitive } \\
\text { to CAD topological } \\
\text { variation }\end{array}$ \\
\hline
\end{tabular}

To evaluate the clustering task performance, we built up a ground truth model of the Vesta assembly containing annotated components to compare with the clustering results obtained from our approach. Figure 13 (a-c) illustrates this model. 6218 solids out of 6503 have been grouped into 148 classes to represent a model an analyst would usually start with when setting up a FEM simulation on such large model. Indeed, an intuitive approach is to group similar repeated components along the aeroengine rotation axis. We then computed two cluster performance metrics: the adjusted rand index and the completeness scores using the scikit-learn python library[42]. The adjusted rand index measures from 0 to 1 ( 1 been the perfect score) the similarity between two clusters. The completeness metrics, measuring from 0 to 1 ( 1 been the perfect score), are divided into a homogeneity score (measuring how much each cluster contains only members of a single class) and a completeness score (measuring how much all members of a given class are assigned to the same cluster). The V-measure is the harmonic mean of the homogeneity and completeness scores[43]. 

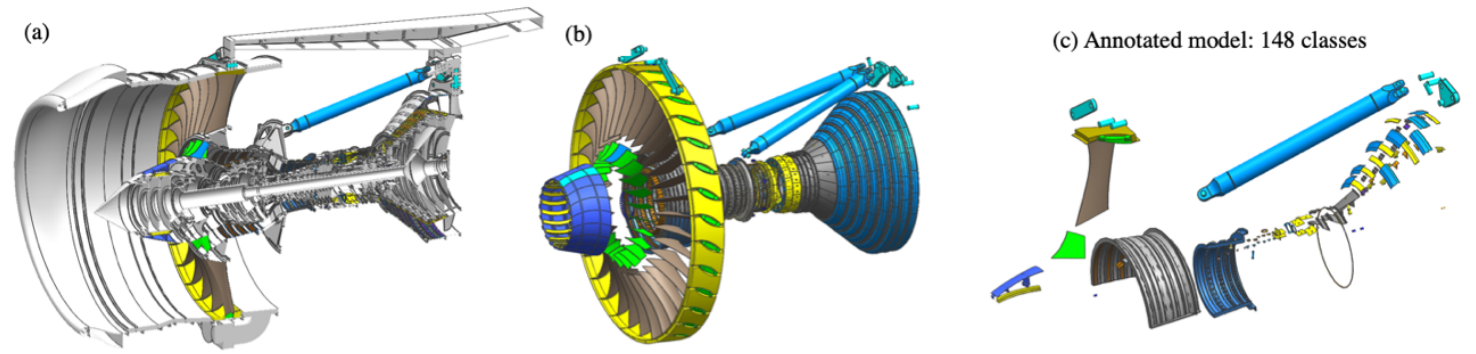

(d) Spherical harmonics-based clustering: 175 clusters

(e) Tensor-based clustering: 186 clusters

(f) Tensor-based clustering: 336 clusters
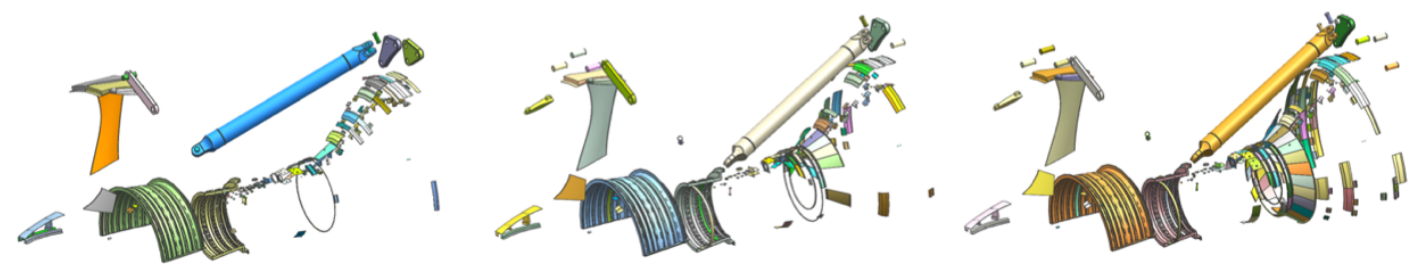

Tensor-based clustering (adjusted rand index)
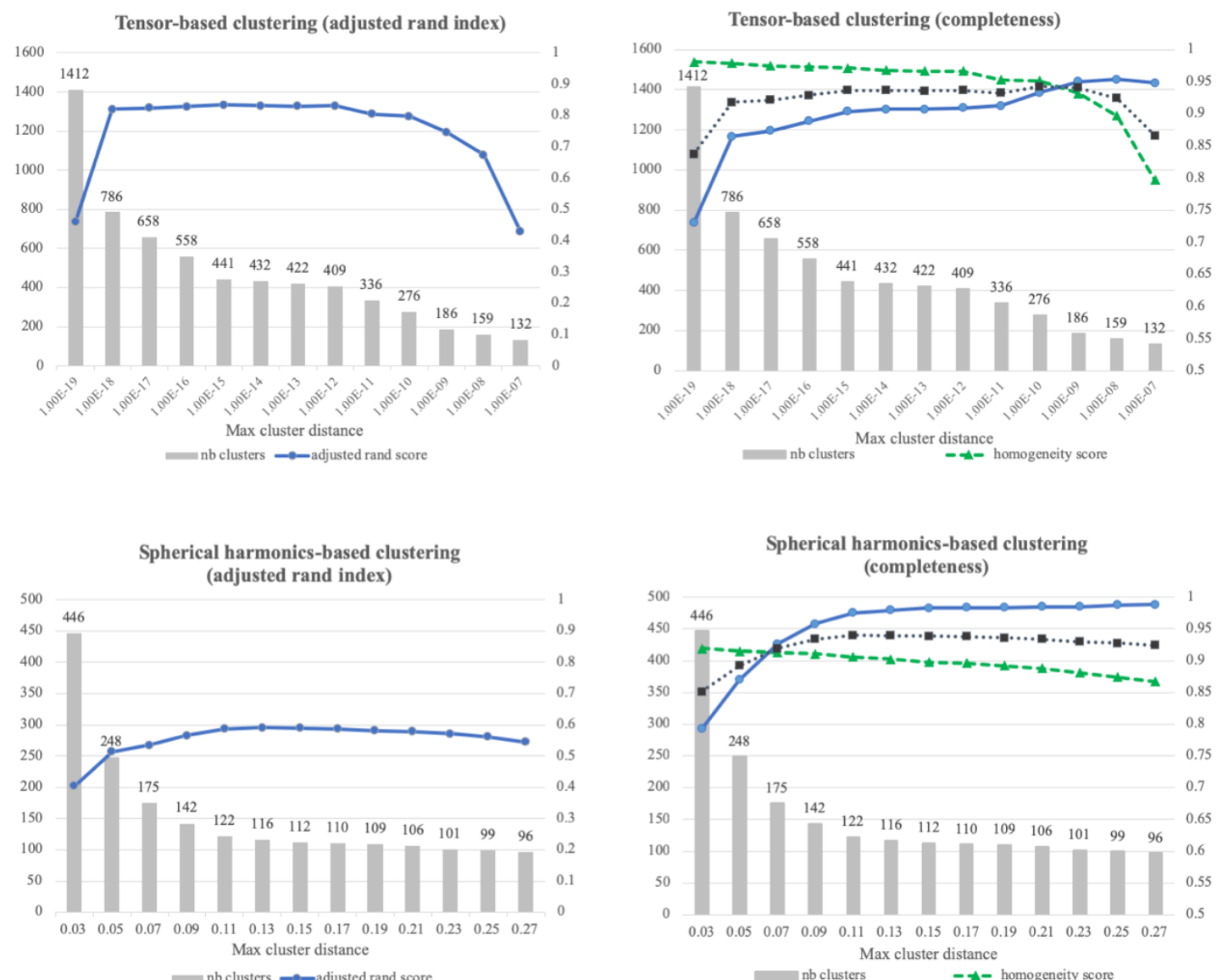

Figure 13 Evaluation of the clustering task on the Vesta model (courtesy of Rolls-Royce PIc) using the adjusted rand score and completeness score metrics: (a) the initial annotated model containing 6218 annotated solids (in color) of 6503; (b) display of the annotated solid; (c) Ground truth model containing 148 classes (one displayed component corresponds to one class), (d) clustering result based on spherical harmonics descriptors [10], (e-f) clustering result using our approach. 
As shown in Figure 13, our approach scores over 0.7 for the adjusted rand index and over 0.9 for the $\mathrm{V}$-measure. However, it never reaches the ground truth classes configuration. This can be explained by the fact that our approach does not consider the position of components along the axis of rotation. Indeed, the ground truth model separates blades and vanes components depending on their axial stage position in the aeroengine. This information is not given as an input to the tensor. However, by considering the interfaces between components, it starts separating the blade/vanes components into the compressors and turbines areas. This separation cannot be reached when using the spherical harmonics approach as it compares components individually. As discussed in the following section, future work is dedicated to improving these metrics by incorporating additional information in the initial tensor.

\section{DISCUSSION AND FUTURE WORK}

In this work, the advantages of the tensor factorization approach to learn similarities and inconsistencies of CAD assembly data are shown to be:

The factorisation model considers all relationships in the model and use this global information when creating groups of components. This is compared to the initial assembly tree structure where the components are grouped independently from their connectivity within the assembly.

The computation time scales linearly with the number of entities avoiding any combinatory scaling issues (see Table 1). The user can interactively explore similarities in the model, given the low computation time for clustering the matrix $A$.

Additional relations can be extracted from the data and added as slices in the initial tensor.

The approach relies on the boundary decompositions of the CAD models. The comparison of B-Rep shapes should be independent of the modeling process and topological constraints of geometric modelers [14,18]. As shown in Figure 14, a given shape can have different boundary decompositions. This may be due to the difference between the underlying architectures of different $\mathrm{CAD} / \mathrm{CAE}$ packages, where periodic faces, such as cylindrical faces, will be represented with or without edges at the periodic seams. To make our approach less CAD modeler dependent, the virtual topology operators of Tierney [44] were used to merge faces and edges having the same underlying geometry. This is similar to the maximal topology concept of Vilmart et al. [18].

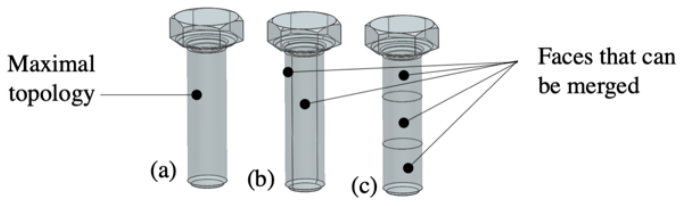

Figure 14 Examples of different boundary decomposition for the same shape. (a) The maximal topology (all faces and edges having same underlying geometry are merged). (b) Topology convention where cylinders are divided into half cylinders, (c) Particular segmentation to represent the bolt's thread.

One limitation of the proposed approach is that the factorisation model considers all relations as equal [3]. A relation containing a large number of relationships can influence the impact of an under-represented relation. Future research will look to improve the factorisation model to introduce weights to the relations while maintaining the scalability performance. This weighting will allow the user to adapt the influence of the relations for a particular application and provide a mechanism to automatically control and normalise the similarity threshold for different applications.

Another limitation of the approach is that it is challenging to make $\delta$ and $k$ meaningful from an engineering perspective and therefore, depending on the shape descriptors given as input, determining the best threshold may necessitate multiple user iterations. However, these iterations are nearly instantaneous and finding a combination that works could be achieved through trial and error approaches. (a)

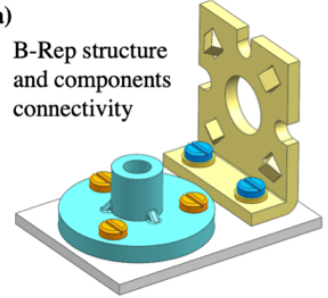

(b)

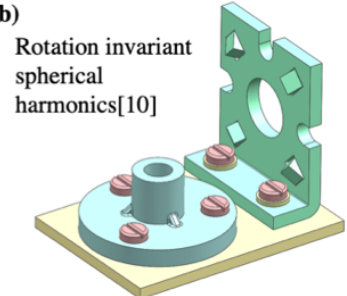

Figure 15 Cluster components (a) based on the BRep structure and the interfaces between components and (b) based on shape descriptors [10]

Extracting additional shape descriptors to enhance the identification of similarities is future work. Currently the topological and geometrical information from the B-Rep structure is used as shape descriptor. However, the current extracted information (see section 3.1) cannot capture all the characteristics of complex CAD models and is sensitive to small topological changes. For example, freeform surfaces will need additional descriptors to the ones proposed in this paper. Clearly, additional descriptors are needed to enhance the similarities. Because the factorisation model can deal with a large number of relationships, the challenges are to determine the correct set of shape descriptors to distinctively identify entities and to manage the conflict between different descriptors. Figure 15 shows the results of clustering components based on the current approach considering the connectivity between components ( Figure 15a) and based on rotation invariant shape descriptor using spherical harmonics[10] calculated on each solid (Figure 15b). It can be seen that the interfaces between components does not impact the similarity identification and therefore the screws in Figure 15 (a) that were grouped into two similarity groups have been reduced to one group in Figure 15 (b). The shape descriptors using spherical harmonics are computationally efficient to identify similar shapes independently (see Table 
1). Shape descriptors are usually described by discrete values, e.g. a grid of spherical harmonic coefficients for different radii of spherical functions[10]. This information could be integrated directly into the tensor as new entities or attributes (attributes can be added to the factorisation using RESCAL-ALS[2], an extended version of RESCAL). For example, an attribute will be added when two solids have the same coefficient for a particular harmonic of a particular spherical function. However, this direct integration results in a large number of additional relationships. For example, the hydraulic jack model would require 35422 new attributes or entities compared to the 255 interfaces between components. This number 35422 corresponds to the use of 17 spherical functions with 32 spherical harmonics. A tolerance of 1e-4 is used to consider two harmonics coefficients as equal. Decreasing this tolerance reduces the number of new relationships but decreases the similarity threshold between shapes. These new relationships globally influence the factorisation, i.e. the connectivity between components relationships could not be significant enough compared to the large number of shape descriptors relations. Integrating new descriptors is challenging and should be considered together with the introduction of weighting factors or to down-select relationships based on their overall contribution. This down-selection may vary for different applications, for example for feature removal applications certain relationships may be ignored that directly relate to feature insignificant for a particular analysis.

Graph models such as Reeb graphs[11] or skeletons [12,13] can be added to the initial tensor to better describe the internal structure of objects without adding a large number of attributes. Here the computation time to extract these should be investigated to not penalise the current efficient computing time for building the input to the tensor method. Indeed, although adding new shape descriptors will improve the similarities of entities, it is important to consider the scalability of the extraction algorithm for large assembly models. Nevertheless, the data extraction process does not require the intervention of the user and is independent from the factorisation. It can be performed once. The output data can be stored and updated for any modified components only.

Non-geometric information from the PLM system such as material properties can also be added as attributes to the factorisation.

Finally, the proposed approach using the proposed shape characteristics is efficient and scalable on B-Rep models. Tessellated models or FE results can also be an entry point for simulation. There is also an opportunity to apply a similar approach, given the right set of shape descriptors, to detect inconsistencies on such discrete models.

The work presented in this paper offers a number of potential benefits for meshing workflows:

1. Geometry preparation: Identifying inconsistencies in assemblies to aid downstream conformal meshing. The grouping of similar components enables geometry clean-up operations and boundary condition applications to be applied to only one component in the group and propagated to the remaining components, significantly reducing the amount of tedious manual operations.

2. Mesh generation: One instance in a group of similar components can be meshed with this mesh transformed to all instances. This enables identical meshes to be assigned to repeated components for certain analyses.

3. Post-processing: Knowing the relationships between components, sub-assemblies and assemblies will make it easier to visual, interpret and utilize results for very large assembly models, where subsets of space can be easily accessed by the used.

\section{CONCLUSIONS}

The preparation of fit-for-purpose CAD models for the efficient simulation of assemblies can be time consuming for the analyst. In this paper, the applicability of relational learning by tensor factorisation is shown to help engineers analyse the consistency of CAD assembly models. From this work, the following conclusions can be drawn:

- The RESCAL tensor model [3] is capable of generating a latent space reflecting the similarity of entities in the relational domain.

- The latent space is used to perform entity resolution on CAD assemblies: i.e. identifying which CAD entities (B-Rep solids, faces and edges) are identical to a given input entity. Entity resolution is extended to group of faces through an algorithm taking advantages of the latent features and face adjacency.

- Clustering of entities is performed in the latent space in order to generate groups of similar entities used to filter the components of interest for the analysis. The clusters can also be analysed by the user to identify inconsistencies between components, or to filter components considered as non-pertinent for a specific application.

- The proposed CAD model analysis approach can scale to large assembly models due to the scalability property of RESCAL. The user can quickly iterate a CAD assembly and test different cluster variants.

\section{. ACKNOWLEDGEMENTS}

The authors wish to acknowledge the financial support provided by the European Commission via the MUMPS project (704557), a H2020-MSCA-IF-2015 founding scheme and also the support provided by Innovate UK via GEMinIDS (project 113088), a UK Centre for Aerodynamics project. The authors acknowledge RollsRoyce Plc for granting permission to publish this paper and for providing the Vesta aeroengine use-case. 
Table 3 Statistics of the examples used in this paper. The RESCAL factorisation is performed with 10 iterations and a rank $r$ of 100 . *Shape descriptors[10] are here for timing comparison, they are not used in the factorisation

\begin{tabular}{|c|c|c|c|c|c|c|c|c|c|c|c|}
\hline \multirow[b]{2}{*}{ Model } & \multicolumn{3}{|c|}{ B-Rep } & \multirow{2}{*}{$\begin{array}{c}\text { Interfa } \\
\text { ces }\end{array}$} & \multirow{2}{*}{$\begin{array}{l}\text { Tensor } \\
\text { entities }\end{array}$} & \multirow{2}{*}{$\begin{array}{c}\text { CAD } \\
\text { data } \\
\text { extraction }\end{array}$} & \multirow{2}{*}{$\begin{array}{l}\text { Interface } \\
\text { detection }\end{array}$} & \multirow{2}{*}{$\begin{array}{c}\text { Shape } \\
\text { descript } \\
\text { ed*[10] }^{*}[1\end{array}$} & \multirow{2}{*}{$\begin{array}{l}\text { RESCAL } \\
\text { factorisati } \\
\text { on }\end{array}$} & \multirow{2}{*}{$\begin{array}{l}\text { Hierarchi } \\
\text { cal } \\
\text { Clustering }\end{array}$} & \multirow{2}{*}{$\begin{array}{c}\text { Clusters } \\
\delta=1 \mathrm{e}- \\
5\end{array}$} \\
\hline & Solids & Faces & Edges & & & & & & & & \\
\hline $\begin{array}{l}\text { Fused engine, } \\
\text { Fig. } 6,10,11 a\end{array}$ & 1 & 1636 & 4312 & N/A & 5949 & $3 \mathrm{~s}$ & N/A & N/A & $1.19 \mathrm{~s}$ & N/A & $\mathrm{N} / \mathrm{A}$ \\
\hline $\begin{array}{l}\text { Hydraulic } \\
\text { pump, Fig. } 11 \mathrm{~b}\end{array}$ & 71 & 1725 & 3603 & 180 & 5579 & $3 \mathrm{~s}$ & $3 \mathrm{~s}$ & $5 \mathrm{~s}$ & $1.25 \mathrm{~s}$ & $0.004 \mathrm{~s}$ & 26 \\
\hline $\begin{array}{l}\text { Hydraulic jack, } \\
\text { Fig. 11c }\end{array}$ & 126 & 2620 & 6129 & 255 & 9130 & $4 s$ & $6 \mathrm{~s}$ & $8 \mathrm{~s}$ & $3.05 \mathrm{~s}$ & 0.002 & 54 \\
\hline Stapler, Fig. 11d & 122 & 5697 & 14385 & 396 & 20600 & $7 \mathrm{~s}$ & $2 s$ & $7 \mathrm{~s}$ & $7.37 \mathrm{~s}$ & $0.004 \mathrm{~s}$ & 58 \\
\hline $\begin{array}{l}\text { Radial engine, } \\
\text { Fig. } 5,8\end{array}$ & 374 & 7963 & 16722 & 992 & 26051 & $10 \mathrm{~s}$ & $23 \mathrm{~s}$ & $27 \mathrm{~s}$ & $7.56 \mathrm{~s}$ & $0.014 \mathrm{~s}$ & 56 \\
\hline $\begin{array}{l}3 D \text { printer, Fig. } \\
11 e\end{array}$ & 878 & 32674 & 71778 & 3442 & 108772 & $55 \mathrm{~s}$ & $28 \mathrm{~s}$ & $42 \mathrm{~s}$ & $31 \mathrm{~s}$ & $0.06 \mathrm{~s}$ & 119 \\
\hline $\begin{array}{l}\text { Car engine, Fig. } \\
\text { llf }\end{array}$ & 997 & 49369 & $\begin{array}{c}12544 \\
8\end{array}$ & 2857 & 178671 & $1 \min 42 \mathrm{~s}$ & $\begin{array}{c}2 \mathrm{mins} \\
05 \mathrm{~s}\end{array}$ & $\begin{array}{c}1 \mathrm{~min} \\
17 \mathrm{~s}\end{array}$ & $47 \mathrm{~s}$ & $0.09 \mathrm{~s}$ & 142 \\
\hline
\end{tabular}


(a)
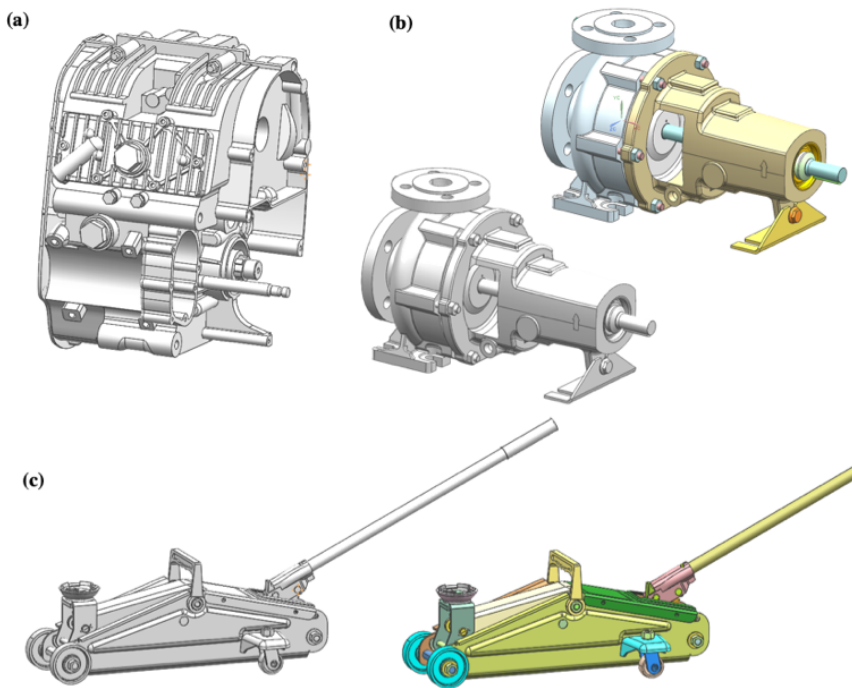

(d)

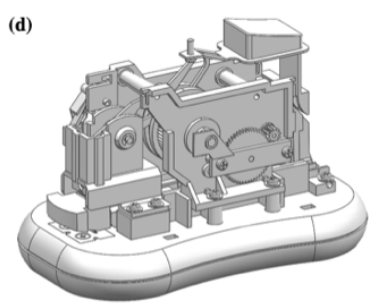

(e)

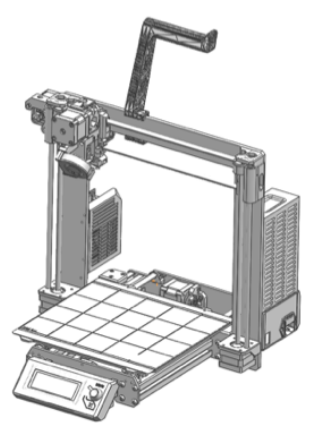

(f)

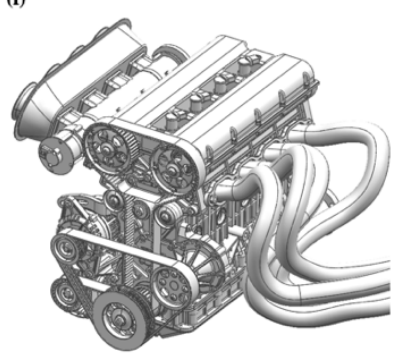

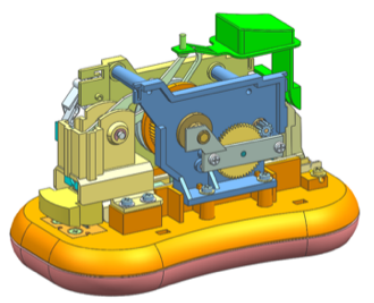
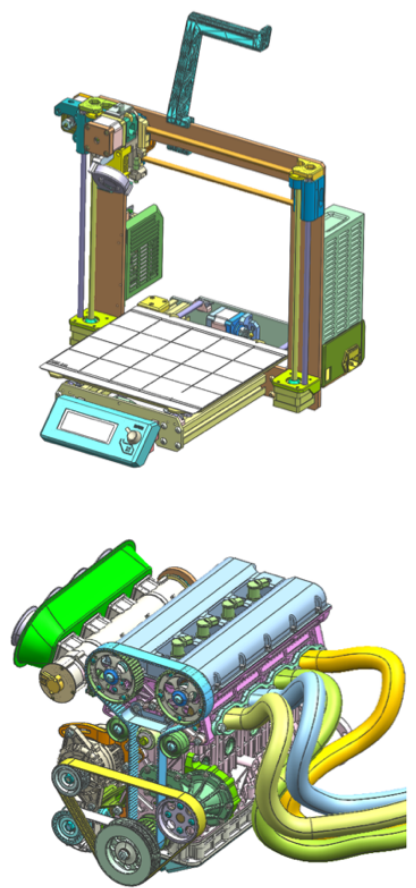
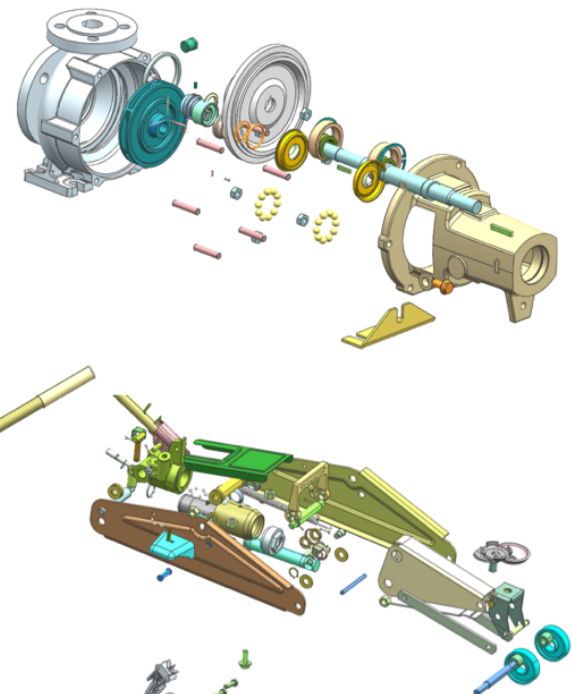

Figure 16 Results of the link-based clustering task on CAD assembly models. Left column: Initial models imported from Step files. Middle column: cluster variant with $\delta=1 e^{-5}$, one colour corresponds to one cluster. Right column: exploded view of the components' clusters. 


\section{REFERENCES}

[1] M. Nickel, K. Murphy, V. Tresp, E. Gabrilovich, A review of relational machine learning for knowledge graphs, Proc. IEEE. 104 (2016) 11-33.

[2] M. Nickel, Tensor factorization for relational learning, Ludwig-Maximilians-Universität München, n.d.

[3] M. Nickel, V. Tresp, H.-P. Kriegel, A Three-Way Model for Collective Learning on Multi-Relational Data., in: ICML, 2011: pp. 809-816.

[4] C. Bizer, T. Heath, T. Berners-Lee, Linked data: The story so far, in: Semant. Serv. Interoperability Web Appl. Emerg. Concepts, IGI Global, 2011: pp. 205227.

[5] W3C, Linked data, (n.d.). https://www.w3.org/standards/semanticweb/data (accessed February 1, 2019).

[6] W3C Working Group, Resource Description Framework Schema 1.1, (2014).

[7] J.W.H. Tangelder, R.C. Veltkamp, A survey of content based 3D shape retrieval methods, in: Shape Model. Appl. 2004. Proc., IEEE, 2004: pp. 145-156.

[8] T. Funkhouser, P. Min, M. Kazhdan, J. Chen, A. Halderman, D. Dobkin, D. Jacobs, A search engine for 3D models, ACM Trans. Graph. 22 (2003) 83105.

[9] J. Bai, S. Gao, W. Tang, Y. Liu, S. Guo, Design reuse oriented partial retrieval of CAD models, Comput. Des. 42 (2010) 1069-1084.

[10] M. Kazhdan, T. Funkhouser, S. Rusinkiewicz, Rotation invariant spherical harmonic representation of 3 d shape descriptors, in: Symp. Geom. Process., 2003: pp. 156-164.

[11] D. Bespalov, W.C. Regli, A. Shokoufandeh, Reeb graph based shape retrieval for CAD, in: ASME 2003 Int. Des. Eng. Tech. Conf. Comput. Inf. Eng. Conf., American Society of Mechanical Engineers, 2003: pp. 229-238.

[12] H. Sundar, D. Silver, N. Gagvani, S. Dickinson, Skeleton based shape matching and retrieval, in: Shape Model. Int. 2003, IEEE, 2003: pp. 130-139.

[13] A. Tagliasacchi, T. Delame, M. Spagnuolo, N. Amenta, A. Telea, 3d skeletons: A state-of-the-art report, in: Comput. Graph. Forum, Wiley Online Library, 2016: pp. 573-597.

[14] F. Boussuge, J.-C. Léon, S. Hahmann, L. Fine, Extraction of generative processes from B-Rep shapes and application to idealization transformations, Comput. Des. 46 (2014) 79-89.

[15] C.-F. You, Y.-L. Tsai, 3D solid model retrieval for engineering reuse based on local feature correspondence, Int. J. Adv. Manuf. Technol. 46 (2010) 649-661.

[16] X. Chen, S. Gao, S. Guo, J. Bai, A flexible assembly retrieval approach for model reuse, Comput. Des. 44 (2012) 554-574.

[17] F. Boussuge, J.-C. Léon, S. Hahmann, L. Fine, An analysis of DMU transformation requirements for structural assembly simulations, in: Int. Conf.
ECT2012, Proc. Eighth Int. Conf. Eng. Comput. Technol. Dubrovnik, Croat. 4-7 Sept. 2012, 2012.

[18] H. Vilmart, J.-C. Léon, F. Ulliana, From CAD Assemblies toward Knowledge-based Assemblies using an Intrinsic Knowledge-based Assembly Model, in: Proc. CAD'17, 2017: pp. 374-378.

[19] K.-M. Hu, B. Wang, J.-H. Yong, J.-C. Paul, Relaxed lightweight assembly retrieval using vector space model, Comput. Des. 45 (2013) 739-750.

[20] P. Wang, Y. Li, J. Zhang, J. Yu, An assembly retrieval approach based on shape distributions and Earth Mover's Distance, Int. J. Adv. Manuf. Technol. 86 (2016) 2635-2651.

[21] Y. Rubner, C. Tomasi, L.J. Guibas, The earth mover's distance as a metric for image retrieval, Int. J. Comput. Vis. 40 (2000) 99-121.

[22] K. Lupinetti, F. Giannini, M. Monti, J.-P. Pernot, Multi-criteria retrieval of CAD assembly models, J. Comput. Des. Eng. 5 (2018) 41-53.

[23] K. Lupinetti, F. Giannini, M. Monti, J.-P. Pernot, Automatic extraction of assembly component relationships for assembly model retrieval, Procedia CIRP. 50 (2016) 472-477.

[24] A. Shahwan, J.-C. Léon, G. Foucault, M. Trlin, O. Palombi, Qualitative behavioral reasoning from components' interfaces to components' functions for DMU adaption to FE analyses, Comput. Des. 45 (2013) 383-394.

[25] F. Jourdes, G.-P. Bonneau, S. Hahmann, J.-C. Léon, F. Faure, Computation of components' interfaces in highly complex assemblies, Comput. Des. 46 (2014) $170-178$

[26] L.A. Adamic, E. Adar, Friends and neighbors on the web, Soc. Networks. 25 (2003) 211-230.

[27] E.A. Leicht, P. Holme, M.E.J. Newman, Vertex similarity in networks, Phys. Rev. E. 73 (2006) 26120.

[28] L. Lü, T. Zhou, Link prediction in complex networks: A survey, Phys. A Stat. Mech. Its Appl. 390 (2011) 1150-1170.

[29] N. Lao, T. Mitchell, W.W. Cohen, Random walk inference and learning in a large scale knowledge base, in: Proc. Conf. Empir. Methods Nat. Lang. Process., Association for Computational Linguistics, 2011: pp. 529-539.

[30] T. Franz, A. Schultz, S. Sizov, S. Staab, Triplerank: Ranking semantic web data by tensor decomposition, in: Int. Semant. Web Conf., Springer, 2009: pp. 213-228.

[31] M. Nickel, V. Tresp, H.-P. Kriegel, Factorizing yago: scalable machine learning for linked data, in: Proc. 21st Int. Conf. World Wide Web, ACM, 2012: pp. 271-280.

[32] R. Jenatton, N.L. Roux, A. Bordes, G.R. Obozinski, A latent factor model for highly multi-relational data, in: Adv. Neural Inf. Process. Syst., 2012: pp. 3167-3175.

[33] T.G. Kolda, B.W. Bader, Tensor decompositions and applications, SIAM Rev. 51 (2009) 455-500.

[34] SciPy.org, SciPy - hierarchical clustering, (2018). https://docs.scipy.org/doc/scipy/reference/cluster.hie 
rarchy.html (accessed February 1, 2019).

[35] Stratasys, GrabCAD, (2018).

https://grabcad.com/library/radial-engine-163;

https://grabcad.com/library/prusa-i3-mk3-

solidworks-1\%0D,

https:/grabcad.com/library/engine-2-0-liter-4-

cylinder-88mm-bore-x-80mm-stroke-1\%0D.

[36] T. Paviot, PythonOCC - 3D CAD for python, (2017). http://www.pythonocc.org/ (accessed February 1, 2019).

[37] OpenCascade - Opensource 3D CAD kernel, (2019). https://www.opencascade.com/ (accessed February 1, 2019).

[38] Siemens plm software, NX, (2018). http://www.plm.automation.siemens.com/en_us/pro ducts/nx/index.shtml (accessed June 5, 2017).

[39] Siemens plm software, Parasolid, (2018). https://www.plm.automation.siemens.com/en_us/pro ducts/open/parasolid/ (accessed June 5, 2017).

[40] Michael Kazhdan, Rotation Invariant Shape Descriptors, (n.d.).

http://www.cs.jhu.edu/ misha/Code/ShapeSPH/Sha peDescriptor/ (accessed August 16, 2019).

[41] NetworkX - Isomorphism - VF2 algorithm, (2019). https://networkx.github.io/documentation/latest/refer ence/algorithms/isomorphism.vf2.html.

[42] Scikit-learn, Clustering evaluation, (2019). https://scikit-

learn.org/stable/modules/clustering.html\#clusteringperformance-evaluation (accessed August 14, 2019).

[43] A. Rosenberg, J. Hirschberg, V-measure: A conditional entropy-based external cluster evaluation measure, in: Proc. 2007 Jt. Conf. Empir. Methods Nat. Lang. Process. Comput. Nat. Lang. Learn., 2007: pp. 410-420.

[44] C.M. Tierney, L. Sun, T.T. Robinson, C.G. Armstrong, Using virtual topology operations to generate analysis topology, Comput. Des. 85 (2017) 154-167.

doi:https://doi.org/10.1016/j.cad.2016.07.015. 


\title{
SUBDIVIDED LINEAR AND CURVED MESHES PRESERVING FEATURES OF A LINEAR MESH MODEL
}

\author{
Albert Jiménez-Ramos ${ }^{1} \quad$ Abel Gargallo-Peiró ${ }^{1} \quad$ Xevi Roca $^{1}$ \\ ${ }^{1}$ Barcelona Supercomputing Center, 08304, Barcelona, Spain. xevi.roca@bsc.es
}

\begin{abstract}
To provide straight-edged and curved piece-wise polynomial meshes that target a unique smooth geometry while preserving the sharp features and smooth regions of the model, we propose a new fast curving method based on hierarchical subdivision and blending. There is no need for underlying target geometry, it is only needed a straightedged mesh with boundary entities marked to characterize the geometry features, and a list of features to recast. The method features a unique sharp-to-smooth modeling capability not fully available in standard CAD packages. The goal is to obtain a volume mesh that under successive refinement leads to smooth regions bounded by the corresponding sharp features. The examples show that it is possible to refine and obtain smooth curves and surfaces while preserving sharp features determined by vertices and polylines. We conclude that the method is well-suited to curve large quadratic and quartic meshes in low-memory configurations.
\end{abstract}

Keywords: mesh curving, surrogate geometry, geometry modeling, subdivision, blending

\section{INTRODUCTION}

In flow simulations for wind energy, transport of pollutants, and bio-engineering the boundary of the computational domain is usually represented by a straightedged mesh obtained by sampling real data. This straight-edged mesh approximates the geometry, at different scales, corresponding to the viscous surfaces to analyze such as topography $[1,2]$, urban areas [3], and human organs. The mesh also presents a series of sharp features, vertices, polylines bounded by vertices, that the method should preserve, and that bound the smooth regions of the computational model.

The resolution to approximate the geometry could be insufficient for the required flow analysis, and thus, additional refinement of the boundary mesh would be required. However, a standard refinement approach, when no target geometry is available, could be inadequate for flow simulation in a twofold way. First, the refined mesh might reproduce precisely the geometry of the first straight-edged mesh and thus, introduce artificial flow artifacts close to initially non-smooth features that should be smooth. Second, the refined mesh might target a smooth surface geometry, implicitly determined by the initial straight-edged mesh, but without adequately respecting after successive refinement the sharp features, curves, and vertices, of the computational model. Ideally, vertices should remain fixed, and polylines should target a smooth limit curve.

Solving these issues is essential for those flow analyses that start from a mesh obtained by sampling real data where the computational model presents smooth regions bounded by sharp features. Even they can be useful in aeronautical applications where only legacy data, in a format of vertices, and polyline and surface meshes, is available. In some applications, practitioners might also need, a non-standard but flexible sharp-to-smooth modeling capability, to remove some sharp features ensuring that surrounding regions become smooth along with the removed feature.

Intending to provide piece-wise linear meshes or curved piece-wise polynomial meshes that target a unique smooth geometry while preserving the sharp features of the model, our contribution is to propose a new fast curving method based on hierarchical sub- 
division and blending with sharp-to-smooth modeling capabilities. Our approach only needs an initial straight-edged mesh with boundary triangles marked with surface identifiers, and a list of features to recast. There is no need for underlying target geometry. The goal of the method is to obtain a volume mesh of the flow domain that under successive refinement leads to smooth regions bounded by the sharp features determined after recasting. The recasting operation is devised to implement a sharp-to-smooth modeling capability. We favored a fast and explicit curving method, based on subdivision and blending, to an implicit approach formulation that features validity guarantees or untangling capabilities, based on boundary curving and optimization, but slower and more memory demanding. This favoring is so since the appearance of invalid elements is small compared with the scale of the generated meshes, and fast local untangling can repair those invalid elements. This work details our mesh curving methodology and illustrates its application with the included numerical examples.

The proposed fast curving method based on hierarchical subdivision and blending with sharp-to-smooth modeling capabilities is novel in many aspects:

- In mesh curving it is standard to have explicit access to the boundary of the target geometry $[4,5,6,7,8,9,10,11,12]$ however, fewer works have considered the case when the target geometry is not explicitly available. The work presented in [13] proposes two curving methods based in weighted least squares approximations and piece-wise polynomial fittings to generate curved meshes of the target surfaces. Both techniques guarantee $\mathcal{C}^{0}$-continuous curved surface meshes whereas, in this work we approximate with at least $\mathcal{C}^{0}$-continuous meshes a surrogate geometry composed of feature surfaces with an interior that is $\mathcal{C}^{1}$-continuous and $\mathcal{C}^{2}$-continuous almost everywhere. Furthermore, herein, the $\mathcal{C}^{0}$ continuity of the surface mesh is increased to $\mathcal{C}^{2}$ continuity when using regular configurations of quartic elements to interpolate the limit surface.

- The curved surface meshes provided by the method in [13] can be used to bound the mesh volumes and thus, to generate curved high-order meshes when the boundary surfaces are not explicitly available. Furthermore, it is possible to remove sharp features by selecting one-by-one the mesh entities defining it [14]. Besides the surface mesh curving method, the main differences with the latter work are that herein we use: a hierarchical blending approach to curve the mesh volume; and an all-in-one feature selection to perform sharp-to-smooth recasting.
- In mesh generation, Loop's subdivision surfaces have been used before to define surrogate geometry [15] and also to curve quadratic and quartic surface meshes [16] but not to obtain volume meshes with curved boundaries. Later, the butterfly subdivision scheme [17] has been used to relocate the boundary nodes when the geometry is unavailable [18], but the volume is not curved using hierarchical blending. Note that previous methods use blending for mesh curving but in the specific case of curving boundary layer elements when the target geometry is available [19].

- There are alternative subdivision methods to generate curved volume meshes, featuring parallel implementations, but they need as input a curved mesh to define the surrogate geometry [20].

The organization of the rest of the paper is as follows. First, in Sect. 2, we present the problem statement and the methodology used in this work to solve it. Second, in Sect. 3, we present some preliminary results on subdivision methods required to develop the main contributions of this paper. We detail these contributions in Sect. 4 and Sect. 5, where we present the method to generate curved high-order surface and volume meshes from a given linear mesh when the target geometry is unavailable. Following, in Sect. 6, we present several results to illustrate the capabilities and main features of the presented methods. To conclude, in Sect. 7, we present some concluding remarks, to sum up the main contributions of this work.

\section{PROBLEM STATEMENT AND METHODOLOGY}

\subsection{Problem Statement}

The input data is a tetrahedral mesh with its boundary entities marked to characterize the geometry features, and a list of the features to be recast. A geometry feature is characterized by a set of entities of the mesh with the same identifier. Specifically, a vertex feature describes a vertex point to preserve, and it is characterized by the global identifier of the point to be preserved. A curve feature describes a curve to preserve. Each curve feature is characterized by a list of edges with the same curve identifier. Finally, a surface feature describes a surface to preserve. A list of boundary triangles with the same identifier characterizes a surface feature.

Alternatively, we can obtain the geometry features by considering a tetrahedral mesh when only the surface features are described. That is, if only the boundary triangles are marked, we can retrieve the feature curves from the intersection of the boundary of two or more feature surfaces. Similarly, vertex features can 


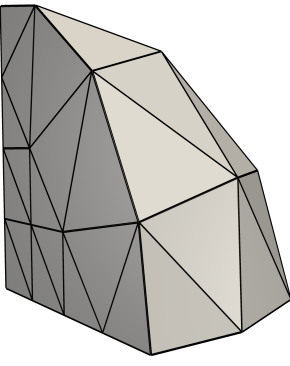

(a)

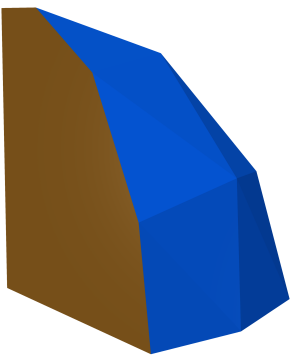

(c)

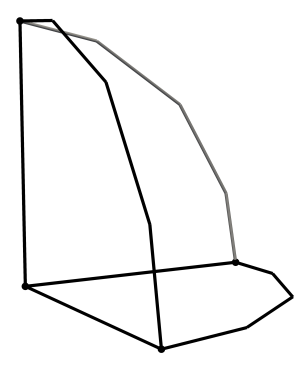

(b)

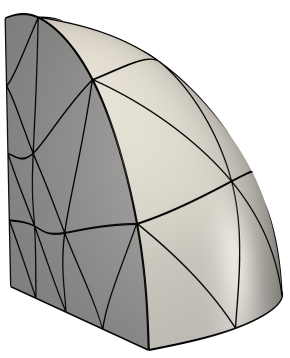

(d)
Figure 1: Method: (a) a linear tetrahedral mesh, with marked boundary (b) curves and (c) surfaces, is curved to obtain (d) a curved tetrahedral mesh of polynomial degree four.

be determined by the intersection of two or more feature curves.

In addition to the tetrahedral mesh and the geometry features, we have an optional input of which geometry features to recast. Recasting a geometry feature consists in removing it from the list of features to preserve and merging adjacent regions to obtain a smooth model along the removed feature. That is, if we recast a curve, we remove the curve feature and merge the two adjacent surfaces. While when we recast a vertex, we remove the vertex feature and merge the curves incident to the vertex. Since each geometry feature is associated with a unique identifier, the list of features to be recast is a sub-sequence of these unique identifiers.

Given the input modeled tetrahedral mesh, the output of this work is a high-order tetrahedral mesh of polynomial degree $p$ with a boundary preserving the marked sharp features and satisfying three properties. First, high-order element vertices interpolate the initial linear mesh nodes. Second, the nodes of the highorder edges that belong to a feature curve and are not adjacent to a feature vertex (inner curve edges) interpolate a cubic $\mathcal{C}^{2}$-continuous curve. Third, the nodes of the high-order elements that belong to a feature surface and are not adjacent to a feature curve or vertex (inner surface elements) interpolate an almost everywhere quartic $\mathcal{C}^{2}$-continuous surface. These properties provide regularity guarantees in the output mesh that are discussed in Sect. 4.3.

\subsection{Method: Hierarchical Subdivision and Blending}

The curved high-order mesh generation procedure proposed in this work is composed of four main steps:

1. Approximate a surrogate boundary. Given a linear tetrahedral mesh, Fig. 1(a), we extract its boundary. The boundary is a linear triangular mesh with its entities marked, see Fig. 1(b) and Fig. 1(c), and by means of the hierarchical subdivision of its elements we generate a curved high-order triangular surface mesh. The curved surface mesh approximates a surrogate boundary composed of feature surfaces with an interior that is $\mathcal{C}^{1}$-continuous and $\mathcal{C}^{2}$-continuous almost everywhere.

This surrogate is determined by the subdivision of the curves and surfaces, and preserves the sharp features and smooth regions marked on the boundary of the initial volume mesh. See details in Sect. 4.

2. Substitute the boundary of the volume mesh. We increase the polynomial degree of the volume mesh and replace the straight-sided boundary of the current high-order volume mesh by the high-order surface mesh obtained in the first step. See details in Sect. 5.1.

3. Accommodate the curvature of the boundary. We accommodate the curvature of the curved surface mesh to the boundary volume elements using an explicit hierarchical blending, see Fig. 1(d). See details in Sect. 5.2.

4. Local untangling. If necessary, we optimize the inverted elements locally following the approach detailed in $[8,9]$.

In some applications, it may be desired to perform a sharp-to-smooth modeling of the geometry. Therefore, as a preprocess, we can recast the non-desired geometry features accordingly to the list of features to remove provided as input to obtain a smoother surrogate geometry. The recasting process is detailed in Sect. 5.1.

\section{PRELIMINARIES: CURVE AND SURFACE MESH SUBDIVISION}

In this section, we present the approximative subdivision algorithms that we use to refine the boundary 
of a tetrahedral volume mesh. The boundary mesh is composed of vertices, curves, and surfaces. Then, the subdivision is performed hierarchically, that is, vertices remain fixed, curves are refined using a curve subdivision scheme and surfaces are subdivided using a surface subdivision scheme. Our goal is to generate a finer boundary mesh that targets a smooth limit surface. Therefore, the curve subdivision scheme has to generate new points consistently with the subdivision surface scheme.

In Sect. 3.1, we detail the curve subdivision algorithm we use in this work, and Sect. 3.2 recalls Loop's subdivision surface scheme. Although these methods are approximative, in Sect. 3.3 we explain how they can be modified to interpolate the initial mesh points preserving the same continuity properties.

\subsection{Curve Subdivision Scheme}

The curve subdivision scheme used in this work was originally presented in [21]. Successive applications of the algorithm generate finer polygons, all of them with the same limit curve determined by the initial mesh, denoted as control mesh. This curve is parametrized by a polynomial of degree three and it is $\mathcal{C}^{2}$-continuous.

Given a polygon, the curve subdivision scheme consists of three steps: generate a new node for each edge, update the position of the original nodes, and define the new elements of the finer mesh. A new edge point is generated at the midpoint of the two endpoints of the segment. Specifically, at refinement level $l+1$, the edge connecting nodes $i$ and $i+1$ is divided and the position of the new edge node, $\boldsymbol{x}_{(i, i+1)}$, is given by

$$
\boldsymbol{x}_{(i, i+1)}=\frac{1}{2}\left(\boldsymbol{x}_{i}^{l}+\boldsymbol{x}_{i+1}^{l}\right),
$$

where $\boldsymbol{x}_{j}^{k}$ denotes the position of node $j$ at level $k$.

The position of the original nodes is also modified and they are relocated to a linear combination of their position and the position of their neighboring nodes at level $l$,

$$
\boldsymbol{x}_{i}^{l+1}=\frac{1}{8}\left(\boldsymbol{x}_{i-1}^{l}+6 \boldsymbol{x}_{i}^{l}+\boldsymbol{x}_{i+1}^{l}\right) .
$$

As subdivision proceeds, the refined polygons tend to a cubic $\mathcal{C}^{2}$-continuous curve. We remark that the initial control mesh determines the limiting curve, so all the refined polygons converge to the same curve. Furthermore, it is possible to compute the position in the limit curve of the nodes at any refinement step from the following expression

$$
\boldsymbol{x}_{i}^{l, \infty}=\frac{1}{6}\left(\boldsymbol{x}_{i-1}^{l}+4 \boldsymbol{x}_{i}^{l}+\boldsymbol{x}_{i+1}^{l}\right),
$$

where $\boldsymbol{x}_{j}^{k, \infty}$ denotes the limiting position of the node $j$ at refinement level $k$.

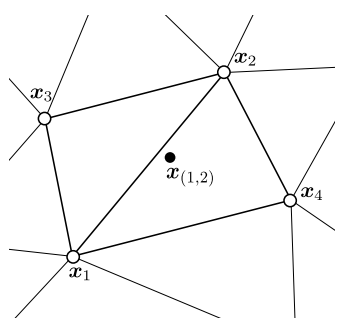

(a)

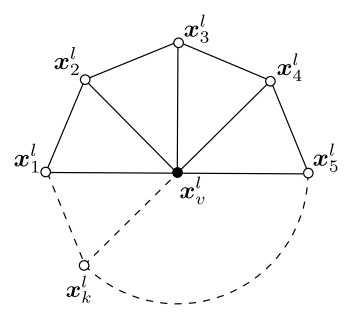

(b)
Figure 2: Edge and point configurations for Loop's subdivision surfaces. (a) A new node, $\boldsymbol{x}_{(1,2)}$, is generated on the edge $(1,2)$. (b) Neighbor nodes $\left\{\boldsymbol{x}_{i}^{l}\right\}$ of a node $\boldsymbol{x}_{v}^{l}$.

\subsection{Surface Subdivision Scheme}

In this work, we subdivide a given triangular surface mesh using Loop's subdivision algorithm [22]. Loop's subdivision scheme consists of three steps: generate a new node for each edge, update the position of the original nodes, and refine the original triangle into four smaller ones. Successive applications of the algorithm generate finer triangular meshes, all of them with the same limit surface determined by the initial control mesh. This surface is $\mathcal{C}^{2}$-continuous almost everywhere.

Before detailing Loop's subdivision process, we present several definitions related to neighbor elements and nodes. The neighbor elements of a node $v$ are the elements incident to $v$, and the neighbor nodes of $v$ are the vertices of these elements. For the case of simplices, there exists an equivalent definition based on edges. The neighbor edges of a node $v$ are the edges incident to $v$, and the neighbor nodes of $v$ are the vertices of these edges. We say that a surface node is regular if it has six neighbor nodes. Otherwise, we say the node is irregular. Around regular nodes, the limit surface is parametrized by a polynomial of degree four and is $\mathcal{C}^{2}$-continuous, while on irregular nodes it is of class $\mathcal{C}^{1}[23]$.

In the Loop subdivision algorithm, a new node is generated for each edge. Note that in a surface mesh an edge connects two nodes and belongs to two elements. Let $\boldsymbol{x}_{1}, \ldots, \boldsymbol{x}_{4}$ be the position of four points in $\mathbb{R}^{3}$ that define two triangles $(1,2,3)$ and $(1,4,2)$. These triangles share the edge $(1,2)$, as shown in Fig. 2(a). In this edge, a new node, with position denoted by $\boldsymbol{x}_{(1,2)}$, is generated using the expression

$$
\boldsymbol{x}_{(1,2)}=\frac{3}{8}\left(\boldsymbol{x}_{1}+\boldsymbol{x}_{2}\right)+\frac{1}{8}\left(\boldsymbol{x}_{3}+\boldsymbol{x}_{4}\right) .
$$

In addition to generating the new mid-edge nodes, Loop's scheme also modifies the location of the vertices of the initial mesh. At refinement level $l$, the 


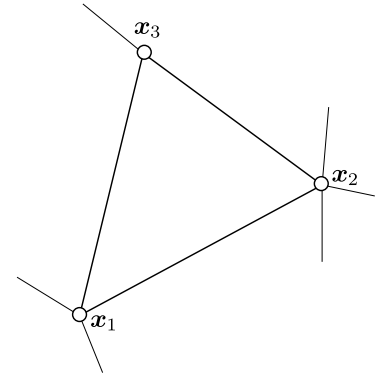

(a)

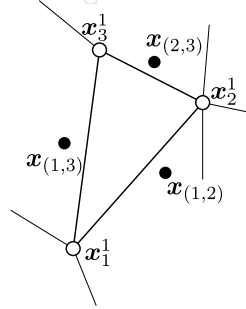

(b)

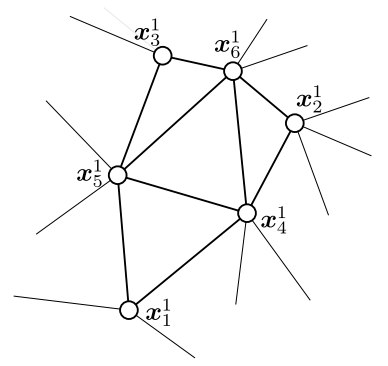

(c)

Figure 3: Subdivision process: (a) element of the original mesh; (b) original element with updated vertex nodes (white dots), and new edge nodes (black dots); and (c) subdivided element after one iteration of the process.

position of an existing node, $\boldsymbol{x}_{v}^{l}$, with neighbor nodes $\left\{\boldsymbol{x}_{i}^{l}\right\}_{i=1, \ldots, k}$, Fig. 2(b), is modified according to

$$
\boldsymbol{x}_{v}^{l+1}=\left(1-k \omega_{k}\right) \boldsymbol{x}_{v}^{l}+\omega_{k} \sum_{i=1}^{k} \boldsymbol{x}_{i}^{l}
$$

where $\omega_{k}$ is defined as

$$
\left.\omega_{k}=\frac{1}{k} \quad \frac{5}{8}-\left(\frac{3}{8}+\frac{1}{4} \cos \left(\frac{2 \pi}{k}\right)\right)^{2}\right) .
$$

If we connect and relabel the three new nodes generated for each edge, the subdivided surface mesh is obtained, see Fig. 3. Note that all the nodes generated on the edges of the mesh are regular. This is so since each edge is shared by two triangles, and the new elements are defined by connecting the mid-edge nodes. Therefore, since the percentage of regular nodes increases with each iteration, the discontinuity on the derivatives around irregular nodes can be mitigated applying successively the scheme, if desired.

We remark that the subdivision scheme defines a hierarchy of control meshes, all of them converging to the same limit surface. Moreover, we can compute the limiting location for the nodes at any refinement level. In particular, given a linear mesh, the limit position for the node $v$ at any refinement level $l$, denoted by $\boldsymbol{x}_{v}^{l, \infty}$, is

$$
\boldsymbol{x}_{v}^{l, \infty}=\left(1-k \chi_{k}\right) \boldsymbol{x}_{v}^{l}+\chi_{k} \sum_{i=1}^{k} \boldsymbol{x}_{i}^{l},
$$

where $\left\{\boldsymbol{x}_{i}^{l}\right\}_{i=1, \ldots, k}$ are the positions of the neighbor nodes of $v$ at level $l$, and where the weights are computed as

$$
\chi_{k}=\frac{1}{k+\frac{3}{8 \omega_{k}}} .
$$

We highlight that there exists an explicit parametrization of the limit surface on the elements in which all the nodes are regular. This parametrization is indeed a piecewise polynomial of degree four, and its explicit expression can be found in $[15,24]$.

\subsection{Non-interpolative to Interpolative}

Given a control mesh, the schemes in Sect. 3.1 and Sect. 3.2 for mesh subdivision generate a hierarchy of subdivided meshes all of them tending to the same limit manifold (curve or surface). These schemes do not preserve the position of the initial vertices of the mesh. However, a new control mesh can be computed so that the limit manifold contains the nodes of the initial mesh [15]. That is, new points can be found such that the limit curves and surfaces interpolate the given data points.

Specifically, let $\varphi$ be the operator that maps the position of the initial mesh nodes, $\boldsymbol{X}_{0}$, onto their limit position. Then, we compute a new control mesh, with nodes position denoted by $\boldsymbol{X}_{C}$, such that

$$
\varphi\left(\boldsymbol{X}_{C}\right)=\boldsymbol{X}_{0} .
$$

In the case of the subdivision schemes considered in this work, $\varphi$ is a linear application with rows given by the coefficients in Eq. (3) or Eq. (6), depending on the point to be updated. This matrix is sparse and the solution of the linear system can be computed using a sparse direct solver. In our Python implementation, we call the sparse direct solver of the SuperLU library $[25,26]$ through the Python SciPy package [27]. Recall that this operation is performed on the boundary of a tetrahedral mesh. Therefore, the dimension of the linear system is of the order of the number of boundary nodes and not of the order of nodes of the volume mesh.

\section{APPROXIMATE SURROGATE GEOMETRY: CURVED SURFACE MESH}

In this section, we detail the procedure based on subdivision that we propose in this work to generate a 


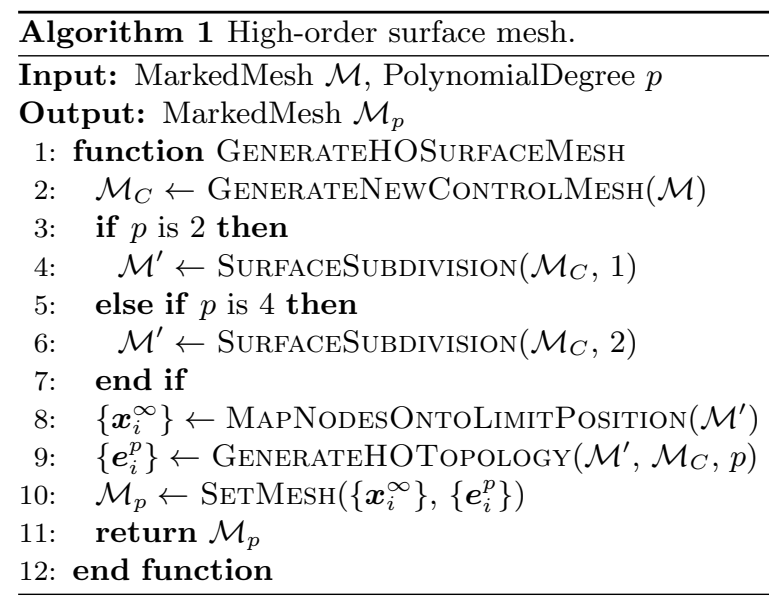

curved surface mesh that approximates a smooth surrogate boundary.

In Algorithm 1, we describe the main steps of this process. First, in Line 2, we cast the triangular surface mesh to a new control mesh to ensure that the location of the initial vertices is preserved in the high-order mesh, as detailed in Sect. 3.3. Given the new control mesh, the subdivision method determines a limit manifold that in this work corresponds to the surrogate geometry for the curving procedure. In addition, we exploit the structure of the subdivision surface method to determine the new high-order elements. In particular, four subelements of a linear triangle, once subdivided, determine one element of polynomial degree $p=2$. Similarly, sixteen linear elements obtained after applying two iterations of the subdivision algorithm to a linear element determine a unique element of polynomial degree $p=4$. Therefore, in Lines 4 and 6 we call the function SurfaceSubdivision to perform the number of subdivision required for the generation of the high-order mesh. The surface subdivision process with feature preservation proposed in this work, SurfaceSubdivision, is detailed in Algorithm 2 from Sect. 4.1. Following, the nodes are mapped onto its limit position, Line 8 , interpolating the surrogate geometry. Finally, in Line 9, the subdivided mesh is cast to a high-order mesh by reinterpreting the children of each element as a new high-order element. The casting of the subdivision to a high-order mesh and the approximation of the surrogate geometry are detailed in Sect. 4.2.

We highlight that the surrogate geometry for the generation of the high-order mesh is determined by the initial linear mesh and the given geometry features. However, as it will be detailed in Sect. 4.3, prior subdivision steps to the ones performed in Lines 4 and 6 improve the smoothness of the curved high-order mesh that approximates the surrogate. Therefore, if desired, after computing the new control mesh in Line 2 a new finer straight-sided mesh could be generated applying several subdivision steps. From this point, the curving procedure would continue as detailed in Algorithm 1.

In this work, we have favored a subdivision scheme that preserves the location of the vertices of the original linear mesh. To this aim, in Line 2 of Algorithm 1 a new control mesh for the subdivision procedure is computed. If the standard approximative version of the subdivision schemes is preferred, the same procedure applies but the generation of the new control mesh can be omitted.

\subsection{Surrogate Geometry: Surface Mesh Subdivision}

This work is devoted to curving linear meshes when no geometry is available. Thus, surface mesh subdivision converges to a limit manifold that determines the surrogate geometry for the mesh curving problem. In addition, in the interior of the feature curves it is $\mathcal{C}^{2}$ continuous, whereas in the interior of the feature surfaces it is $\mathcal{C}^{1}$-continuous becoming even $\mathcal{C}^{2}$-continuous in regular regions of the mesh. In this section, we propose a hierarchical subdivision process that allows preserving the sharp features marked in the mesh.

We consider a triangular surface mesh with its entities characterizing the geometry features to preserve. Following Algorithm 2, three main steps are performed. First, in Line 5, we generate the points of the subdivided mesh. Next, in Line 6 , the elements of the finer mesh are generated. Finally, the subdivided mesh inherits the marks from the coarse mesh, Line 8 . These steps are repeated for all the specified subdivision iterations.

The generation of the points of the hierarchical subdivision scheme is performed looping through the unique edges and nodes of the mesh, as detailed in Algorithm 3 . Since each node of the mesh is given a unique identifier, an edge is uniquely defined by the nodes it connects. Consider an edge $e=(i, j)$. If the edge belongs to a feature curve, the position of the new edge node, $\boldsymbol{x}_{e}^{\text {new }}$, is at the midpoint of the edge points $\boldsymbol{x}_{i}$ and $\boldsymbol{x}_{j}$, see Eq. (1), Line 5. In contrast, if $e$ belongs to a feature surface, in order to generate the new edge point, we need to evaluate Eq. (4) and thus, we need access to the nodes of the elements sharing the edge $e$ but opposite to it, Line 9 . We denote the position of these nodes by $\boldsymbol{x}_{t_{1}}$ and $\boldsymbol{x}_{t_{2}}$. Therefore, the position of the new edge node is given accordingly to Line 10 .

In order to update the position of the original nodes, we loop through the nodes of the mesh. Consider a node $v$. If $v$ is a feature vertex, its position does not change, so $\boldsymbol{x}_{v}^{\text {new }}=\boldsymbol{x}_{v}$, see Line 16 . To update the position of a point that belongs to a curve by the evaluation of Eq. (2), we need access to the two neighbor 


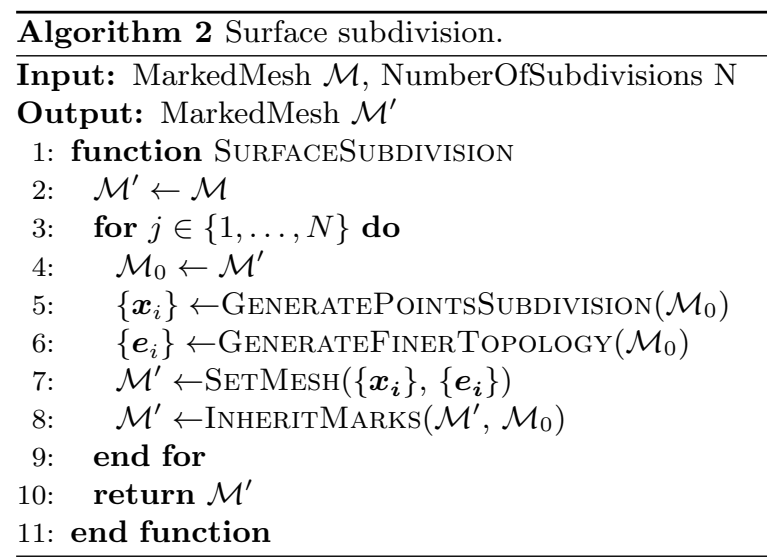

nodes in the curve. We denote the position of these two nodes by $\boldsymbol{x}_{i}$ and $\boldsymbol{x}_{j}$, see Line 19. Now, the updated position of node $v$ is given accordingly to Line 20. Analogously, we update the position of a node that belongs to a surface. In Line 24 , we get the position of its neighbor nodes $\left\{\boldsymbol{x}_{i}\right\}_{1 \ldots k}$, and the position of node $v$ is updated using Eq. (5) in Line 25.

We highlight that Algorithm 3 combines two types of subdivision algorithms. Therefore, the limit manifold, which determines our surrogate geometry, inherits different smoothness guarantees from the original subdivision algorithms that are discussed in Sect. 4.3.

\subsection{Cast Subdivision to Curved High-order Surface Mesh and Interpolate Surro- gate Geometry}

The hierarchical subdivision procedure presented in Sect. 4.1 generates a new point for each edge of the mesh and four elements for each refined linear triangle. Therefore, in the curving process in Algorithm 1, we exploit this structure and reinterpret the topology from the children of each original element into a new high-order element. An element of polynomial degree two is obtained after one application of the subdivision algorithm to a linear element. Two iterations of the subdivision scheme lead to a quartic element. Therefore, high-order element generation based on subdivision defines elements of polynomial degree $p=2^{k}$, where $k$ is the number of iterations of the subdivision schemes performed. The limit surface is parametrized by a polynomial of degree four around regular nodes, therefore, we focus on quartic meshes since then the limit surface and its regularity is exactly captured around regular nodes for this polynomial degree.

The main steps of the reinterpretation of the subdivided elements as a unique high-order element are stated in Algorithm 4. For each element of the initial linear mesh, $\boldsymbol{e}_{i}$, we obtain the elements of the finer mesh generated from the subdivision of $\boldsymbol{e}_{i}$, Line 3 .

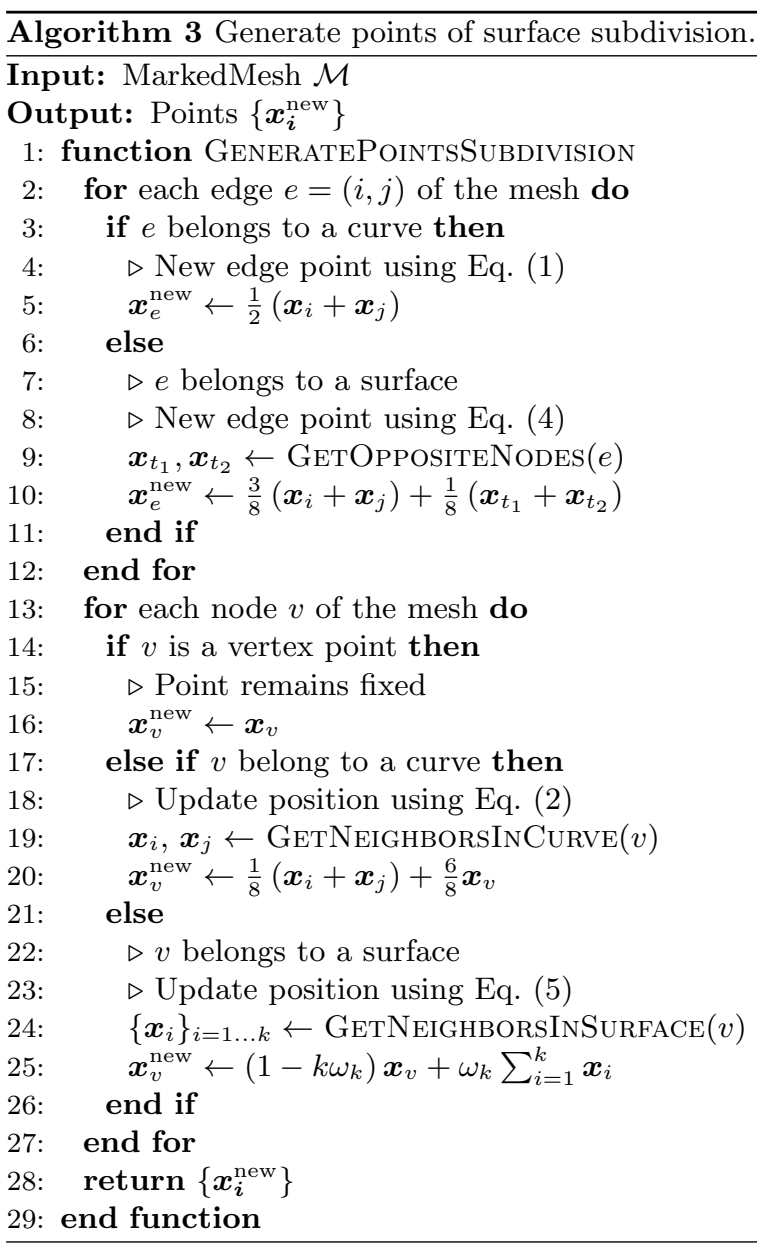

When used to generate meshes of polynomial degree two, this set contains four linear elements, whereas when used to generate a new mesh of degree four, this set contains sixteen elements. Finally, in Line 4, this set of linear elements is reinterpreted as a unique highorder element.

Specifically, given an element of the linear mesh, Fig. 4(a), one iteration of the hierarchical subdivision scheme generates three edge nodes and modifies the position of the vertices, Fig. 4(b). We denote by 4,5 and 6 the edge nodes created from the subdivision of edge $(1,2),(1,3)$ and $(2,3)$, respectively. Now, the nodes are mapped onto its limiting position using Eq. (3) or Eq. (6). Finally, instead of connecting the edge nodes and subdividing the element as in Fig. 4(c), a curved element of polynomial degree two is defined by considering the new generated nodes as the midedge nodes that are required to define an element of polynomial degree two, see Fig. 4(d).

This process is repeated for all the elements, obtaining a curved surface mesh of polynomial degree two that approximates the surrogate geometry with third order 


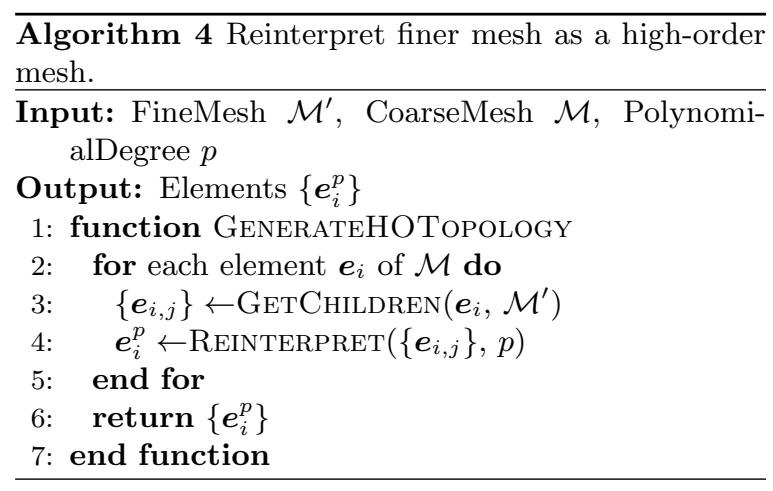

accuracy, see Sect. 4.3 for more details on the regularity of the high-order mesh. This high-order mesh has the same number of nodes as the subdivided mesh but it has the same number of elements as the original linear mesh.

For quartic polynomial degree, $p=4$, each element is defined by fifteen nodes. The procedure to generate the curved mesh of degree four from a given linear mesh is similar to the quadratic case. Applying twice the subdivision process fifteen new nodes are obtained, see Fig. 5(c). Next, these nodes are mapped onto its limiting position using Eq. (3) or Eq. (6). Finally, we reinterpret the topology of the sixteen elements from the subdivision into a unique element of polynomial degree four, see Fig. 5(d). Specifically, nodes 7, 8, 9, $10,11,12,13,14$ and 15 are the edge nodes created from the subdivision of edges $(1,4),(1,5),(2,4),(2,6)$, $(3,5),(3,6),(4,5),(4,6)$ and $(5,6)$, respectively.

This process is repeated for all the elements, obtaining a curved surface mesh of polynomial degree four with the same number of nodes as after applying two times the subdivision scheme to the original linear mesh, although the number of elements is the same than in the linear mesh.

\subsection{Smoothness of the Surrogate Geome- try and Curved Surface Mesh}

In this section, we analyze the smoothness of the surrogate geometry and of the high-order meshes obtained using the approach presented in Sect. 4.2.

The surrogate boundary is composed of the union of the limit curves and the limit surfaces determined by the control mesh. On the one hand, the curve subdivision scheme, see Sect. 3.1, ensures that in the inner edges of the feature curves (not adjacent to a feature vertex) the limit curve is cubic and $\mathcal{C}^{2}$-continuous. On the contrary, curve edges of the control mesh that are incident to a vertex point determine the region where the limit curve is of class $\mathcal{C}^{0}$.

On the other hand, Loop's subdivision scheme, see

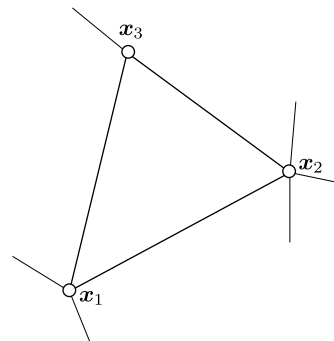

(a)

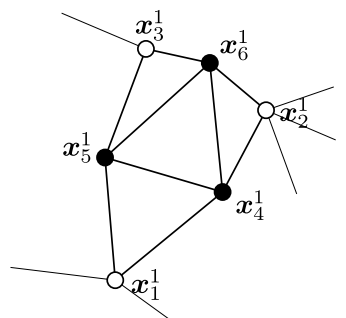

(c)

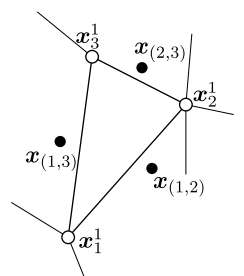

(b)

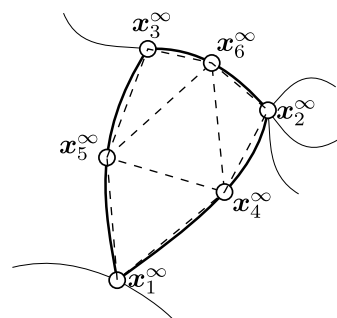

(d)
Figure 4: Generation of an element of polynomial degree two from a linear one. (a) Straight-sided element. (b) Original element once new nodes (black dots) have been generated and the position of the old ones (white dots) have been modified by the hierarchical subdivision scheme. (c) Subdivision of the linear element. (d) Curved element of polynomial degree two, displaying with dashed lines the four elements from the subdivision scheme.

Sect. 3.2, ensures that in the inner triangles of the feature surfaces (not adjacent to a feature curve or vertex), the limit surface is $\mathcal{C}^{1}$-continuous, and $\mathcal{C}^{2}$ continuous almost everywhere. In particular, in the inner triangles, this surface is of class $\mathcal{C}^{2}$ everywhere except at the position of the irregular vertices of the initial control mesh. At these points, the surface is strictly $\mathcal{C}^{1}$-continuous. The surface triangles that are adjacent to a vertex point or a curve determine the region where the limit surface is $\mathcal{C}^{0}$-continuous. Moreover, in those triangles where the limit surface is of class $\mathcal{C}^{2}$, it can be parametrized by quartic polynomials. We remark that this discontinuity in the derivatives is confined. Fig. 6(a) shows a regular mesh featuring inner triangles in dark gray and triangles adjacent to a feature curve (bold) in light gray. In this configuration, the limit surface is of class $\mathcal{C}^{2}$ only on the dark gray region. The limit curve determined by the bold edges is also of class $\mathcal{C}^{2}$.

Regarding the smoothness of the obtained high-order meshes, curve (surface) meshes of polynomial degree $p=2$ approximate the cubic (quartic) limit curve (surface) with third-order accuracy. That is, meshes of polynomial degree 2 are strictly $\mathcal{C}^{0}$-continuous, and no 


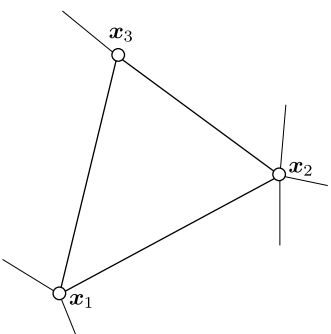

(a)

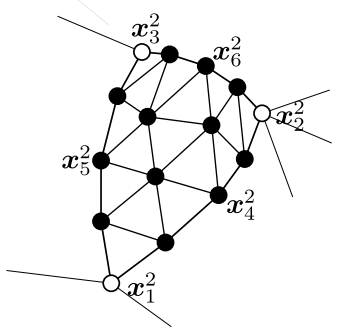

(c)

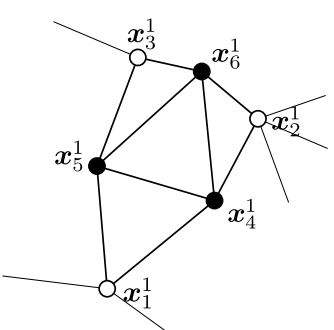

(b)

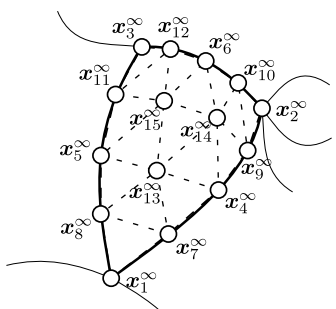

(d)
Figure 5: Generation of an element of polynomial degree four from a linear one. (a) Straight-sided element. (b) First subdivision step. (c) Second subdivision step. (d) Curved element of polynomial degree four, displaying with dashed lines the sixteen elements from the subdivision scheme.

guarantees of the $\mathcal{C}^{2}$-continuity are given by the proposed subdivision-based curving method. However, some prior subdivisions can be applied to the initial linear mesh using the subdivision method presented in Sect. 4.1. Next, this refined mesh can be curved with the subdivision-based curving method proposed in Algorithm 1. This new finer high-order mesh determines a better approximation of the surrogate geometry, and consequently, its smoothness is also improved.

Following, we analyze the smoothness of the meshes of polynomial degree four. First, the inner edges of the feature curves of the high-order mesh exactly capture the $\mathcal{C}^{2}$-continuous limit curve. This is so since the limit curve is parametrized by a third degree polynomial, while the elements are described by shape functions of degree four. Similarly to the curve case, the nodes of the surface mesh also interpolate the limit surface. However, the surface mesh does not inherit the smoothness of the limit surface straight-forwardly as in the curve case. This is so since the limit surface is parameterized element-wise, but the parameterization is of degree four only in a regular element, that is, in an element where its three vertices have six neighbors.

In the interior of an element, the mesh is of class $\mathcal{C}^{\infty}$. Therefore, the smoothness of the surface mesh has to be analyzed along the edges (interfaces between two in-

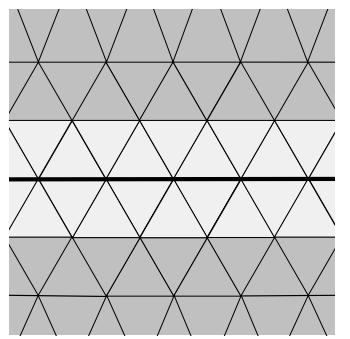

(a)

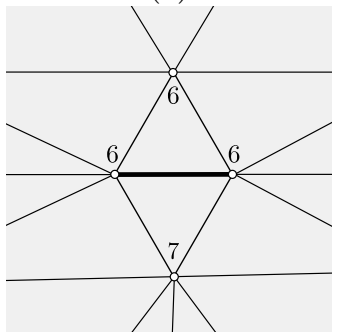

(c)

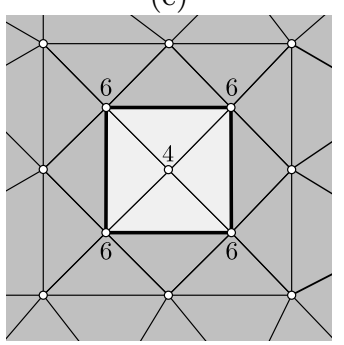

(e)

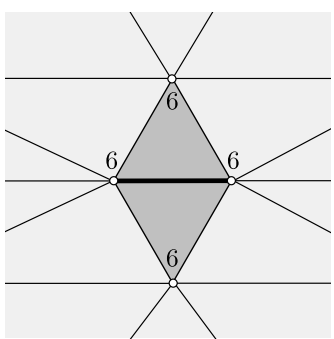

(b)

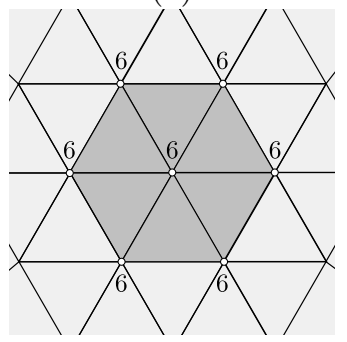

(d)

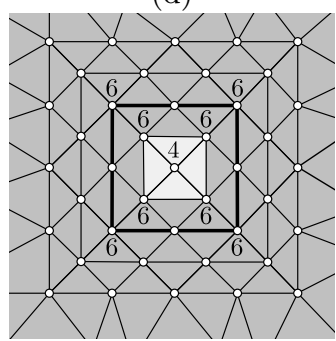

(f)
Figure 6: Dark gray regions indicate $\mathcal{C}^{2}$ smoothness, while light gray color indicate regions where $\mathcal{C}^{2}$-continuity cannot be ensured. (a) Regular mesh around a feature curve (bold). (b) Mesh with a regular edge (bold). (c) Mesh with an irregular edge (bold). (d) Regular patch around a regular vertex. (e) Irregular patch (light gray) around an irregular vertex. (f) Mitigation of the irregular patch after subdividing the mesh.

ner surface elements) and vertices (interfaces between more than two inner surface elements). We first analyze the case between two elements that share an edge. We say an edge is a regular edge if all the vertices of the two triangles that share such edge are regular, Fig. 6(b), i.e. if the vertices have six neighbors. In this case, the two elements of degree four that share the edge interpolate exactly the quartic limit surface. Hence both elements are exactly equal to the limit surface and, since the limit surface is $\mathcal{C}^{2}$-continuous, the interface (edge) between the two elements also is. In general, no guarantee of the continuity of the derivatives can be deduced along an edge that is not regular, see Fig. 6(c).

Following, we analyze the smoothness of the mesh 
around the vertices of the inner surface elements. Given a regular vertex (with six neighbors), if all the edges incident to it are regular then the surface mesh is of class $\mathcal{C}^{2}$ around such vertex. In particular, if all the edges incident to a regular vertex are regular, then all its neighbor vertices are also regular, as observed in Fig. 6(d). In such regions, colored in Fig. 6(d) in dark gray, the surface mesh captures exactly the limit surface and inherits all its features. The presence of an irregular vertex, as illustrated in Fig. 6(e), implies the surface mesh to approximate the limit surface, rather than exactly capturing it. Therefore, on the one hand, around regular patches, we are able to interpolate and exactly capture the limit surface and obtain a $\mathcal{C}^{2}$-continuous surface mesh. On the other hand, around irregular patches, the limit surface is interpolated and approximated with fifth order accuracy but not matched exactly.

In order to improve the smoothness of the high-order mesh and mitigate this issue, the structure induced in the mesh by Loop's subdivision process can be exploited. All the new vertices generated by Loop's subdivision are regular. Therefore, the linear mesh, Fig. 6(e), can be subdivided before generating the mesh of degree four with the presented procedure. As observed in Fig. 6(f) in contrast to Fig. 6(e), the light gray irregular region where the limit surface (and subsequently its smoothness) is not exactly captured is reduced. Exploiting prior refinements of the linear mesh, the regions where the high-order surface mesh is not $\mathcal{C}^{2}$-continuous can be successively reduced.

\section{CURVED VOLUME MESH APPROXIMATING A SURROGATE BOUNDARY}

In this section, we detail how a linear tetrahedral mesh with marked boundary entities is curved while preserving the sharp features. In Sect. 5.1, we detail the sharp-to-smooth modeling of the geometry features and the replacement of the straight-edged boundary of the linear mesh by the curved boundary mesh. In Sect. 5.2, the curvature on the boundary is accommodated to the interior using a blending technique. This procedure leads to a high-order tetrahedral mesh where its boundary approximates a surrogate geometry composed of feature surfaces with an interior that is $\mathcal{C}^{1}$-continuous and $\mathcal{C}^{2}$-continuous almost everywhere. In addition, the vertices of the high-order mesh are kept in the same position than in the initial linear mesh.
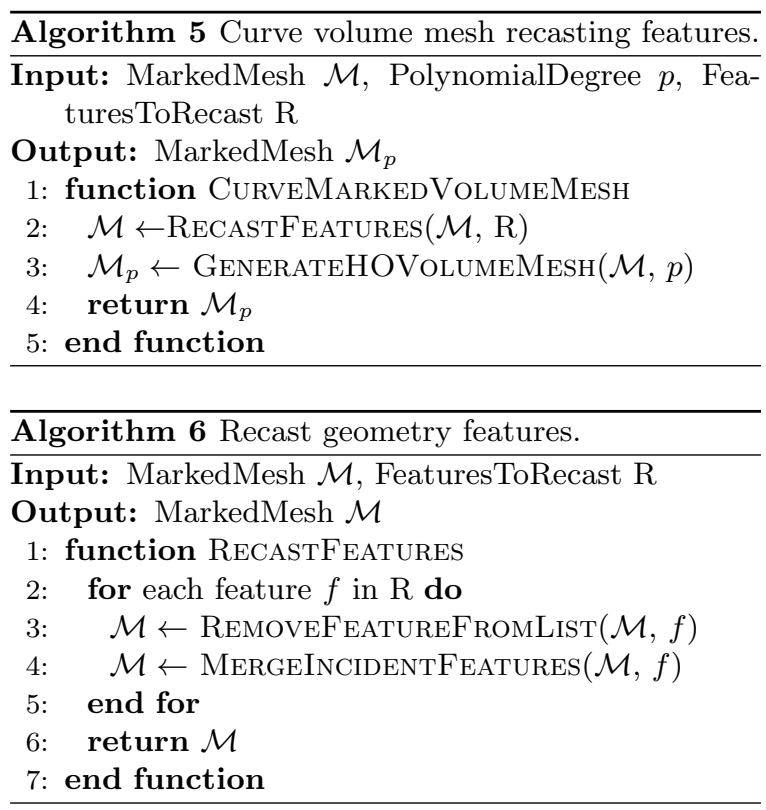

\subsection{Substitute the Boundary of the Vol- ume Mesh}

In this section, we detail the subdivision-based curving of a high-order mesh. This process is presented in Algorithm 5. First, in Line 2, we recast the desired feature entities. Then, in Line 3, we generate a high-order volume mesh preserving the sharp features provided by the new model once the original entities have been recast. These processes, denoted as RecastFeatures and GenerateHOVolumeMesh, are next detailed in Algorithms 6 and 7 .

The first step to curve the volume mesh is to recast, if necessary, the geometry features present in the original model. Recall that vertices, curves, and surfaces are characterized by a unique identifier. Thus, in order to recast a sharp feature, it is enough to provide its identifier. That is, in Algorithm 6, the variable FeaturesToRecast contains a list of the identifiers of the feature vertices and curves to be recast. Specifically, the recasting of a feature is composed of two steps: remove the feature from the list of features to preserve, Line 3; and merge the features incident to such feature, Line 4. Since each feature is described by a unique identifier, the process of merging the incident features reduces to assigning the same identifier to these features.

Next, the curving method based on hierarchical subdivision and blending is performed. The generation of a high-order volume mesh is described in Algorithm 7. First, given a linear tetrahedral mesh with the recast features, Fig. 7(a), we extract its boundary, Line 2 . The boundary is a surface mesh that inherits the geometry features of the volume mesh. Next, in Line 


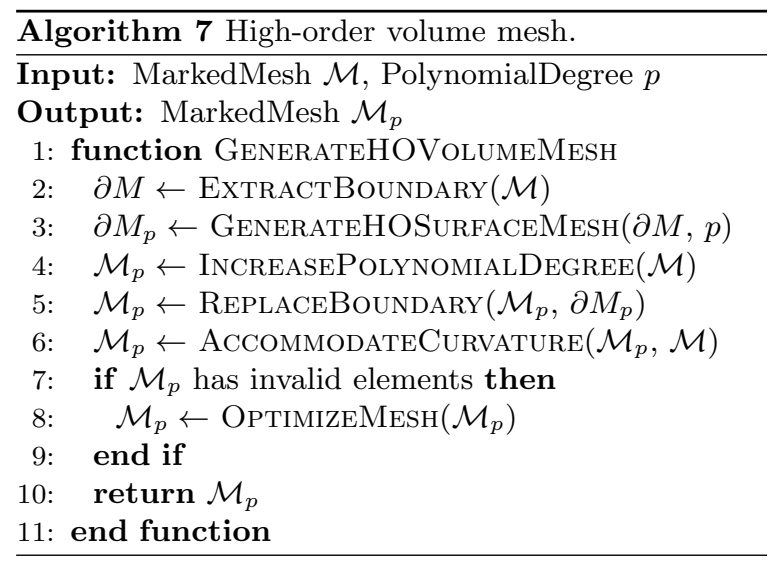

3, we call the function GenerateHOSurfaceMesh described in Algorithm 1 to generate a surface mesh of polynomial degree $p$ preserving the sharp features. Third, we generate a straight-edged high-order volume mesh, Line 4, illustrated in Fig. 7(b). Following, in Line 5, we replace the boundary of the straight-edged mesh by the curved surface mesh, see Fig. 7(c). Then, in Line 6, the curvature of the surface is accommodated to the elements adjacent to boundary, using a blending technique to be described in Sect. 5.2. Finally, if the mesh contains tangled elements, it is optimized using $[8,9]$, see Line 8 .

The methodology proposed in this work can also be used to generate, given an initial linear mesh, finer linear meshes that successively improve the approximation of the surrogate geometry. To do so, the generated high-order mesh can be reinterpreted as a linear mesh by the decomposition of each high-order element into linear elements. Specifically, the reference highorder element is decomposed into several structured linear elements determined by the high-order nodes. In particular, each quadratic element is decomposed into eight linear tetrahedra, while an element of polynomial degree four leads to sixty-four linear elements. If the linear mesh contains tangled elements, the optimization procedure described in [8] is applied to ensure a valid mesh.

\subsection{Accommodate the Curvature of the Boundary}

In Algorithm 7, after replacing the curved boundary in the straight-sided high-order mesh, Line 5, the obtained high-order mesh may contain low-quality or tangled elements, see Fig. 8(a) and 8(b). Since the curvature information is provided by the surface mesh, only boundary tetrahedra are affected and therefore, the number of invalid elements is small compared with the scale of the generated meshes. Thus, similarly to [19], as an attempt to improve the mesh quality

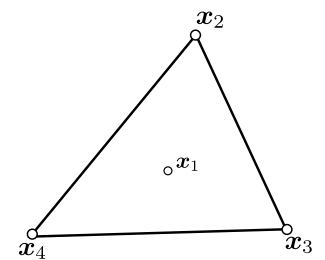

(a)

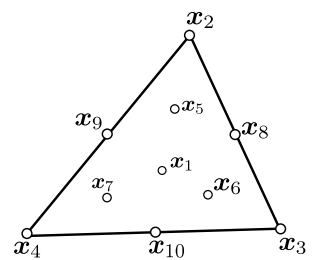

(b)

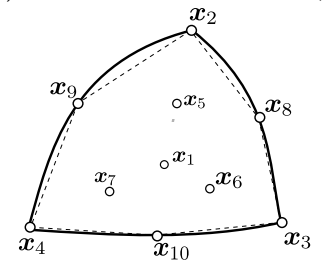

(c)

Figure 7: Curving of the boundary for a boundary element of polynomial degree $p=2$. (a) Linear physical element, where the face (2 34 ) belongs to the boundary. (b) Straight-edged physical element of polynomial degree two. (c) Curved boundary element of polynomial degree two, displaying with dashed lines the four elements from the subdivision scheme applied to the boundary.

in a fast and explicit manner, in Line 6 in function AccommodateCurvature, we use Transfinite Interpolation (TFI) [28] to accommodate the curved surface to those entities of the boundary elements not present in the surface mesh. Specifically, given a boundary element, we use transfinite interpolation hierarchically on its entities to accommodate the curving of the boundary. That is, first we relocate the nodes on edges, then nodes on faces, and finally, nodes in the interior of tetrahedra.

First, consider an edge of a high-order boundary element with one of its endpoints on the curved surface. The new location of the edge nodes is given by the linear isoparametric mapping between the onedimensional reference domain and the physical edge, denoted as $\phi^{1}$. Specifically, the new position of the $k$ th node of the edge, $\boldsymbol{x}_{k}$, for $k=0, \ldots, p$, is determined as

$$
\boldsymbol{x}_{k}=\phi^{1}\left(\boldsymbol{\xi}_{k}\right)=\sum_{l=0}^{1} \boldsymbol{x}_{v_{l}} N_{v_{l}}\left(\boldsymbol{\xi}_{k}\right)
$$

where $\boldsymbol{\xi}_{k}$ is the position of the $k$ th node of the reference domain, $\boldsymbol{x}_{v_{l}}$ is the position of the $l$ th endpoint of the edge, and $N_{v_{l}}$ is the linear nodal shape function of the interval associated to the $l$ th endpoint. In Fig. 8(c), we illustrate the relocation of the nodes of the edges in a triangle when one of its edges (in bold) is on the boundary.

Now, we are interested in relocating the nodes on the interior of a face of a boundary tetrahedron. On the 


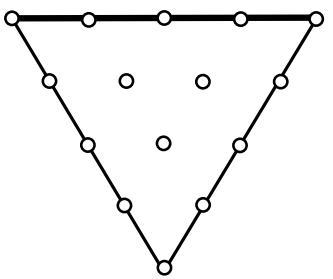

(a)

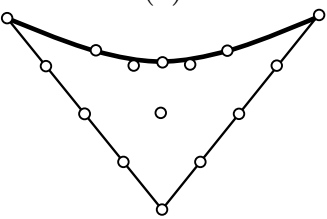

(c) (b)

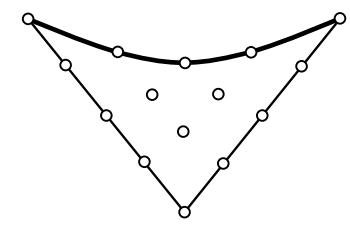

(d)

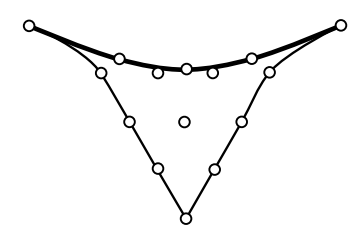

Figure 8: Accommodating the curvature to a triangular element of polynomial degree $p=4$. (a) Straightedged triangle with a boundary edge (in bold). (b) Triangle with curved boundary. (c) Transfinite interpolation applied to the edges, (d) and to the face.

one hand, if such face belongs to the boundary, its nodes have been already relocated by the subdivision scheme, see bold edge in Fig. 8(c). On the other hand, if such face does not belong to the boundary, its edge nodes have been modified using the transfinite interpolation for edges explained above, see non-bold edges in Fig. 8(c). For the latter, we apply the transfinite interpolation for faces, that is, we accommodate the deformation of the curving of the edges to the interior of the triangular faces.

We denote by $f_{i}$ the edge of the triangle opposed to vertex $i, i=1,2,3$. Let us denote as $\boldsymbol{x}_{f_{i}, k}$ the coordinates of the $k$ th node of the edge $f_{i}$ in the physical element, $k=0, \ldots, p$. These nodes are fixed now, since as previously detailed their location has already been computed. Therefore, each curved or relocated edge can be parametrized using the restriction to such edge of the two-dimensional isoparametric mapping of degree $p, \phi^{p}$, as:

$$
\phi_{f_{i}}(\boldsymbol{\xi}):=\left.\phi^{p}\right|_{f_{i}}(\boldsymbol{\xi})=\sum_{k=0}^{p} \boldsymbol{x}_{f_{i}, k} N_{f_{i}, k}(\boldsymbol{\xi})
$$

where $N_{f_{i}, k}(\xi)$ is the high-order nodal shape function of the triangle associated to the $k$ th node of the edge $f_{i}$. In particular, the boundary of the triangle is fixed and parameterized by the three mappings $\left\{\phi_{f_{i}}\right\}_{i=1,2,3}$.

Consider a point $\boldsymbol{x}$ in the physical triangle, to which we want to compute its displaced position in terms of the location of the boundary edge nodes. Denote by $\boldsymbol{\xi}$ the position of the point in the reference triangle expressed in cartesian coordinates such that $\phi^{p}(\boldsymbol{\xi})=\boldsymbol{x}$. Now, denote by $\boldsymbol{\lambda}$ the same point in the reference domain expressed in barycentric coordinates $\left(\lambda_{1}, \lambda_{2}, \lambda_{3}\right)$, $\sum_{i=1}^{3} \lambda_{i}=1$. Following [28], we compute the projection of the point to the edges. A point on an edge can be parametrized as a function of the barycentric coordinates of the two vertices of the triangle defining the edge. Therefore, two different projections $\left(\boldsymbol{\lambda}_{f_{*}}^{j}\right.$ for $j \in f_{*}$ ) are computed for each one of the three edges of the triangle (rows, $\boldsymbol{\lambda}_{f_{i}}^{*}$ for $i=1,2,3$ ):

$$
\begin{gathered}
f_{1}=(2,3):\left\{\begin{array}{l}
\lambda_{f_{1}}^{2}=\left(0,1-\lambda_{3}, \lambda_{3}\right), \\
\lambda_{f_{1}}^{3}=\left(0, \lambda_{2}, 1-\lambda_{2}\right),
\end{array}\right. \\
f_{2}=(1,3):\left\{\begin{array}{l}
\lambda_{f_{2}}^{1}=\left(1-\lambda_{3}, 0, \lambda_{3}\right), \\
\lambda_{f_{2}}^{3}=\left(\lambda_{1}, 0,1-\lambda_{1}\right),
\end{array}\right. \\
f_{3}=(1,2):\left\{\begin{array}{l}
\lambda_{f_{3}}^{1}=\left(1-\lambda_{2}, \lambda_{2}, 0\right), \\
\lambda_{f_{3}}^{2}=\left(\lambda_{1}, 1-\lambda_{1}, 0\right) .
\end{array}\right.
\end{gathered}
$$

Note that $\boldsymbol{\lambda}_{f_{i}}^{j}$ belongs to edge $f_{i}$ and has the $j$ th component expressed as a function of the others. Then, we express these six projections of the point at the edges, computed in barycentric coordinates, back in the reference coordinates $\boldsymbol{\xi}$. As previously remarked, we denote the change from barycentric coordinates of a point $\boldsymbol{\lambda}_{f_{i}}^{j}$ to reference coordinates as $\boldsymbol{\xi}_{f_{i}}^{j}$, for $i=1,2,3$. Since these points are on the edges of the triangle, they can be mapped onto the physical triangle through the mappings $\phi_{f_{i}}$ of the edges, $i=1,2,3$, presented in Eq. (7) as:

$$
\boldsymbol{x}_{f_{i}}^{j}:=\phi_{f_{i}}\left(\boldsymbol{\xi}_{f_{i}}^{j}\right)
$$

We highlight that given a point $\boldsymbol{x}, \boldsymbol{x}_{f_{i}}^{j}$ corresponds to the coordinates on the physical element of the projection $j$ of the point to the edge $f_{i}, i=1,2,3$.

Finally, the new position of point $\boldsymbol{x}$ in the physical triangle, denoted as $\hat{\boldsymbol{x}}$, is given in [28] as:

$$
\begin{aligned}
\hat{\boldsymbol{x}} & =\lambda_{1}\left(\boldsymbol{x}_{f_{2}}^{1}+\boldsymbol{x}_{f_{3}}^{1}-\boldsymbol{x}_{v_{1}}\right)+\lambda_{2}\left(\boldsymbol{x}_{f_{1}}^{2}+\boldsymbol{x}_{f_{3}}^{2}-\boldsymbol{x}_{v_{2}}\right) \\
& +\lambda_{3}\left(\boldsymbol{x}_{f_{1}}^{3}+\boldsymbol{x}_{f_{2}}^{3}-\boldsymbol{x}_{v_{3}}\right)
\end{aligned}
$$

where $\boldsymbol{x}_{v_{j}}$ is the position of the $j$ th vertex of the physical triangle. We highlight that the transfinite interpolation for triangles can be expressed as a function of the isoparametric mapping of the edges and the location of the vertices of the triangle.

In order to relocate the nodes in the interior of the high-order physical faces, the steps detailed above are applied to the nodes in the interior of the high-order reference triangle, see Fig. 8(d). This procedure is repeated for all the faces with a boundary node or edge.

Lastly, we follow an analogous approach to modify the position of the nodes in the interior of the boundary tetrahedra. The boundary of a tetrahedron is composed of four faces and six edges. These faces and edges have already been curved with the procedures 
detailed above. Therefore, we relocate the interior nodes according to the curved boundary already accommodated to the edges and faces. Similarly to the triangle case, the transfinite interpolation for tetrahedra can be expressed as a function of the isoparametric mapping of the edges, the isoparametric mapping of the faces, and the location of the vertices of the tetrahedron.

Denote by $T$ the set of vertices of a tetrahedron. We define the entity $f_{i_{1}, \ldots, i_{k}}$ as the entity of dimension $d-k, d=3$, with vertices given by the nodes $T \backslash$ $\left\{i_{1}, \ldots, i_{k}\right\}$. Note that the face opposed to node $i$ is denoted by $f_{i}$, and the edge shared by the faces $f_{i}$ and $f_{k}$ is $f_{i k}$. Given the three-dimensional isoparametric mapping of degree $p, \phi^{p}$, analogously to Eq. (7), we denote the restriction to the face $f_{i}$ as $\phi_{f_{i}}$, and the restriction to the edge $f_{i k}$ as $\phi_{f_{i k}}$.

Similarly to the two-dimensional case, consider a point $\boldsymbol{x}$ in the physical tetrahedron, and denote by $\boldsymbol{\xi}$ and $\boldsymbol{\lambda}$ its preimage in the reference tetrahedron expressed in cartesian and barycentric coordinates, respectively. Now, denote by $\boldsymbol{\lambda}_{f_{i}}^{j}$ the projection of the point to the face $f_{i}$ that has the $j$ th component expressed as a function of the others. $\boldsymbol{\lambda}_{f_{i k}}^{j}$ denotes the projection of the point to the edge $f_{i k}$ that has the $j$ th component expressed as a function of the others. These projections in the reference domain expressed in cartesian coordinates are denoted by $\boldsymbol{\xi}_{f_{i}}^{j}$ and $\boldsymbol{\xi}_{f_{i k}}^{j}$, respectively.

Since these points are on the faces and edges of the tetrahedron, they can be mapped onto the physical element through the mappings $\phi_{f_{i}}$ on the faces and $\phi_{f_{i k}}$ on the edges as:

$$
\boldsymbol{x}_{f_{i}}^{j}:=\phi_{f_{i}}\left(\boldsymbol{\xi}_{f_{i}}^{j}\right), \quad \boldsymbol{x}_{f_{i k}}^{j}:=\phi_{f_{i k}}\left(\boldsymbol{\xi}_{f_{i k}}^{j}\right)
$$

Finally, the new position of point $\boldsymbol{x}$ in the physical triangle, denoted as $\hat{\boldsymbol{x}}$, is given in [28] as:

$$
\begin{aligned}
\hat{\boldsymbol{x}} & =\lambda_{1}\left(\boldsymbol{x}_{f_{2}}^{1}+\boldsymbol{x}_{f_{3}}^{1}+\boldsymbol{x}_{f_{4}}^{1}-\boldsymbol{x}_{f_{23}}^{1}-\boldsymbol{x}_{f_{24}}^{1}-\boldsymbol{x}_{f_{34}}^{1}+\boldsymbol{x}_{v_{1}}\right) \\
& +\lambda_{2}\left(\boldsymbol{x}_{f_{1}}^{2}+\boldsymbol{x}_{f_{3}}^{2}+\boldsymbol{x}_{f_{4}}^{2}-\boldsymbol{x}_{f_{13}}^{2}-\boldsymbol{x}_{f_{14}}^{2}-\boldsymbol{x}_{f_{34}}^{2}+\boldsymbol{x}_{v_{2}}\right) \\
& +\lambda_{3}\left(\boldsymbol{x}_{f_{1}}^{3}+\boldsymbol{x}_{f_{2}}^{3}+\boldsymbol{x}_{f_{4}}^{3}-\boldsymbol{x}_{f_{12}}^{3}-\boldsymbol{x}_{f_{14}}^{3}-\boldsymbol{x}_{f_{24}}^{3}+\boldsymbol{x}_{v_{3}}\right) \\
& +\lambda_{4}\left(\boldsymbol{x}_{f_{1}}^{4}+\boldsymbol{x}_{f_{2}}^{4}+\boldsymbol{x}_{f_{3}}^{4}-\boldsymbol{x}_{f_{12}}^{4}-\boldsymbol{x}_{f_{13}}^{4}-\boldsymbol{x}_{f_{23}}^{4}+\boldsymbol{x}_{v_{4}}\right)
\end{aligned}
$$

We remark that this method does not guarantee to repair the invalid elements, neither ensures an increase of the element quality. However, it is an explicit and fast formulation, which in practice represents a good initial condition for mesh curving methods when no geometry is available $[9,29,12,30,4]$. In all the tested applications, see Sect. 6 , the procedure improves significantly the quality of the meshes. Once the TFI-based relocation process is finalized, if low quality or inverted elements are present, we perform the non-linear quality optimization procedure presented in [9].

\begin{tabular}{lccc}
\hline & Min Q & \# inv & Time \\
\hline Boundary & 0.93 & 0 & $283 \mathrm{~s}$ \\
Volume (no TFI) & 0 & 169 & $961 \mathrm{~s}$ \\
Volume (TFI) & 0.80 & 0 & $367 \mathrm{~s}$ \\
& & & $1611 \mathrm{~s}$ \\
\hline
\end{tabular}

Table 1: Quality statistics of a mesh of polynomial degree $p=4$ for Sierra del Escudo (Spain).

\section{RESULTS}

In this section, we present several examples to illustrate the main features of the methods presented in this work. As a proof of concept, the proposed algorithms have been developed in Anaconda Python [31]. The prototyping code is sequential (one execution thread) and non-vectorized. All the examples have been run on a MacBook Pro (with one dual-core Intel Core i5 CPU, a clock frequency of $2.3 \mathrm{GHz}$, and a total memory of 16 GBytes).

Although not specified in the previous sections, if desired, we perform a straight subdivision in straight curves and planar surfaces. The straight subdivision scheme does not modify the position of a node and generates the new mid-edge nodes at the midpoint of the edge to subdivide.

In all the examples, we validate both the high-order boundary and volume meshes using the Jacobianbased distortion measure proposed in $[32,8]$. In particular, the quality of a high-order element is computed with respect to its straight-sided original element in the linear mesh.

\subsection{Regular Mesh: Matching the $\mathcal{C}^{2}$ Sur- rogate Topography}

In this example, we illustrate the features of our method with a linear mesh that discretizes a real topography. The original data is provided as a level curve map and thus, a CAD model is not available.

We consider a tetrahedral mesh composed of a discretization of a topography that defines the bottom surface and a planar top surface located at the desired height. The tetrahedral mesh is regular and is generated using the mesher presented in $[1,2]$. Thus, all the nodes of the surface mesh are regular. From the linear mesh, we generate a curved high-order mesh of polynomial degree four using the procedure detailed in Sect. 5. Since the surface nodes are regular, the high-order topography surface mesh is $\mathcal{C}^{2}$-continuous.

In Fig. 9(a), we show the initial linear mesh composed of $4.8 \cdot 10^{5}$ nodes and $2.6 \cdot 10^{6}$ tetrahedra. The highorder boundary mesh is composed of $1.1 \cdot 10^{6}$ nodes and $1.4 \cdot 10^{5}$ triangles, does not contain tangled ele- 


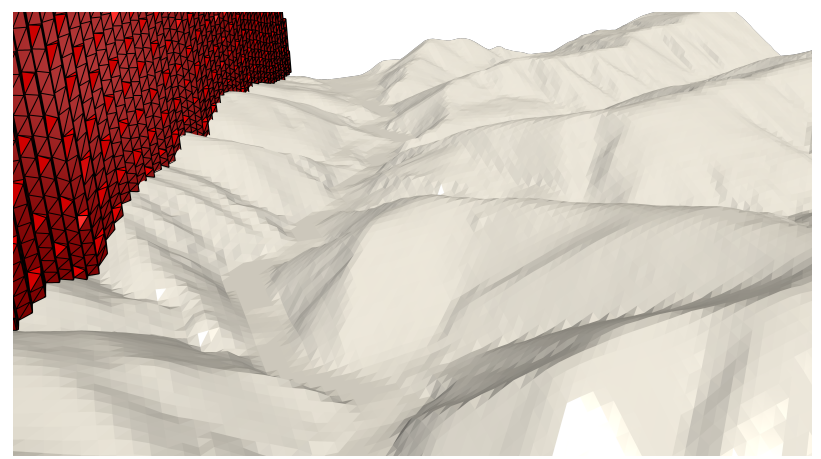

(a)

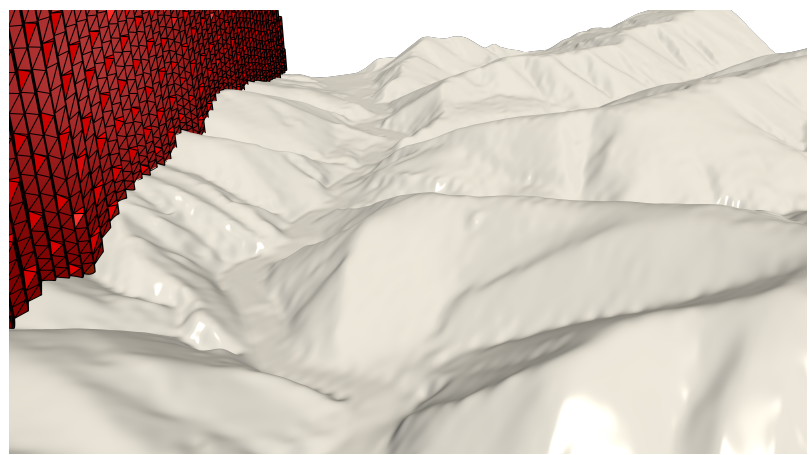

(b)

$\begin{array}{lllll}0 & 0.25 & 0.5 & 0.75 & 1\end{array}$

Figure 9: Curving of a tetrahedral mesh of Sierra del Escudo (Spain). Elements of the volume meshes are colored with their elemental quality. (a) Linear mesh. (b) Curved mesh of polynomial degree $p=4$ with no invalid elements.

\begin{tabular}{lccc}
\hline & Min Q & $\#$ inv & Time \\
\hline Boundary & 0.56 & 0 & $191 \mathrm{~s}$ \\
Volume (no TFI) & 0 & 358 & $611 \mathrm{~s}$ \\
Volume (TFI) & 0 & 24 & $237 \mathrm{~s}$ \\
Volume & & 0 & $60 \mathrm{~s}$ \\
(TFI + Optimization) & 0.70 & 0 & $1099 \mathrm{~s}$ \\
\hline
\end{tabular}

Table 2: Quality statistics of a mesh of polynomial degree $p=4$ for a Falcon aircraft.

ments, and is generated in 283 seconds, see Table 1. The curved high-order volume mesh is generated in 961 seconds and contains 169 inverted elements. The process of accommodating the curvature of the boundary detailed in Sect. 5.2 is performed to $4.1 \cdot 10^{5}$ elements abutting the boundary. This blending takes 367 seconds and untangles all the invalid elements, attaining a minimum quality of 0.8. Finally, in Fig. 9(b), we show the mesh of polynomial degree four composed of $2.9 \cdot 10^{7}$ nodes and $2.6 \cdot 10^{6}$ elements.

\subsection{Sharp-to-smooth Modeling}

In this example, we illustrate the capability of our method to perform a sharp-to-smooth modeling in different regions of the geometry. In particular, we recast some of the features entities present in the original model, and thus provide a new model improving the smoothness of the surrogate geometry. Each feature vertex (node of the mesh), curve (set of edges of the mesh) and surface (set of triangles of the mesh) is associated with a unique identifier. Therefore, to recast a feature, it is enough to know its identifier.

We consider a linear tetrahedral mesh from a CAD legacy model of a simplified Falcon aircraft. The boundary entities are marked defining 28 surfaces, 54 curves, and 34 vertices. As shown in Fig. 10(a) and Fig. 10(b), the main part of the fuselage is composed of two surfaces and a curve. However, such curve is not desirable since, ideally, we would desire a smooth mesh along each section of the fuselage. Therefore, we recast the curve indicating its unique identifier. The first step consists in removing the curve from the list of feature curves. Following, the two surfaces initially incident to this curve, see Fig. 10(a), are merged by identifying the id's of the two surfaces as a unique one, see Fig. 10(c). Thus, the whole fuselage is modeled as a smoother virtual surface.

Similarly, we observe that each section of the wing is described by two surfaces: one at the top and one at the bottom; and two curves: one on the leading edge and one on the trailing edge. In order to obtain a model with an improved smoothness on the leading edge, we decide to recast the feature curve describing the leading edge. This way, the surface at the top and the bottom are merged, and join smoothly in the front part of the wing. We highlight that the curve describing the trailing edge is maintained, and thus this sharp feature is preserved.

Note that the lateral wing joins the fuselage in a profile described by two curves (top and bottom) and two vertex points (front and back). We recast the feature vertex in the front. Therefore, this vertex is removed from the list of feature vertices, and the two curves are merged by identifying their id's as a unique one. As a result, we obtain a single closed curve with a sharp endpoint on the trailing edge.

Similar changes are made in similar parts of the mesh to generate a more convenient mesh model for flow simulation, see Fig. 10(c) and Fig. 10(d). As highlighted in Sect. 5.1, once the id's of all the features to recast are identified, the recasting process is straight- 


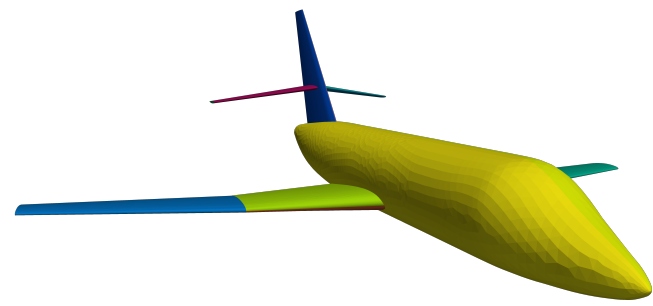

(a)

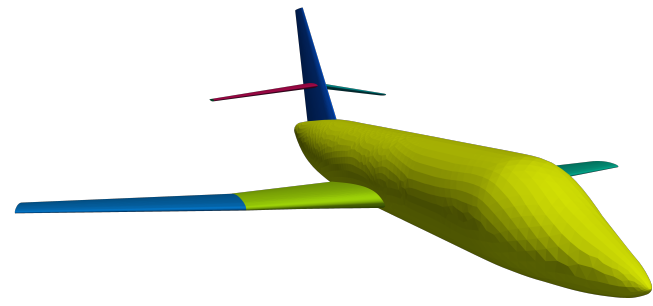

(c)

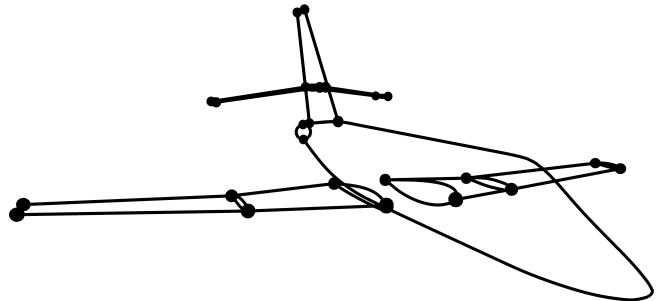

(b)

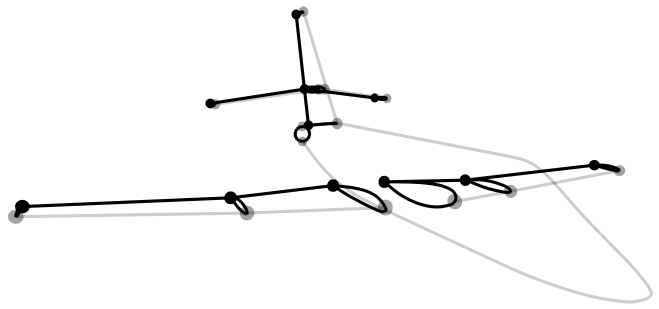

(d)

Figure 10: Initial and final linear mesh model of a Falcon aircraft. Marks on boundary entities of the initial model: (a) boundary triangles, and (b) curve and vertex features. Marks of the final model: (c) virtual surface features, (d) curve and vertex features recast (gray) and preserved (black).

forward. Given the list of identifiers, the recasting process consists in removing these features from the list of features to preserve and automatically identify the features adjacent to the recast feature as a single one. We remark that the recasting of some of the geometry features does not modify the mesh, only the number of vertex, curve, and surface features changes. Specifically, the original model of the presented Falcon aircraft contains 34 vertex points, 54 curves, and 28 surfaces; while the model with the recast features contains 20 vertices to preserve, 32 curves, and 20 surfaces.

In order to illustrate the difference between these two models, we take a close look at the leading edge of the wing. In Fig. 11(a), we show the mesh of polynomial degree $p=4$ generated with the initial marks. We observe a discontinuity in the normal vector of the wing along the leading edge. In Fig. 11(b), we show a mesh generated with a model in which the leading edge has been recast. The nodes originally present in the leading edge are still on the leading edge, but the new points are generated to interpolate the almost everywhere $\mathcal{C}^{2}$-continuous surrogate geometry. Those regions where the features have been recast are smoother in the second mesh model than in the original one. The leading edge now belongs to the interior of the surface, and therefore, all the nodes interpolate a $\mathcal{C}^{1}$. continuous surface.
A summary of the mesh quality can be found in Table 2 . The linear mesh is composed of $1.3 \cdot 10^{5}$ nodes and $1.7 \cdot 10^{6}$ tetrahedra, and the volume mesh of polynomial degree $p=4$ is generated in 611 seconds. This mesh, prior to the blending technique, contains 358 tangled elements. In this example, there are $3.0 \cdot 10^{5}$ boundary elements and the TFI reduces to 24 the number of invalid elements, that is, in 237 seconds a $93 \%$ of the invalid elements have been untangled. Now, we apply the optimization technique presented in $[8,9]$ to optimize locally the quality of the inverted elements. Since the mesh after the TFI is close to be optimal, it is a good initial condition for the implicit optimization and in 60 seconds the mesh becomes valid achieving a minimum quality of 0.7 . In Fig. 12, we show the valid curved tetrahedral mesh of polynomial degree $p=4$ composed of $1.8 \cdot 10^{7}$ nodes and $1.7 \cdot 10^{6}$ elements.

\section{CONCLUDING REMARKS}

The obtained results show that we can generate, from an initial straight-edged mesh, successively refined piece-wise linear, quadratic and quartic meshes, that target smooth curves and surfaces, while preserving the initially marked sharp features and smooth regions. The interior of the obtained limit curves is of class $\mathcal{C}^{2}$, and the interior of the surfaces is at least $\mathcal{C}^{1}$ continuous, being of class $\mathcal{C}^{2}$ when the surface mesh is structured. 


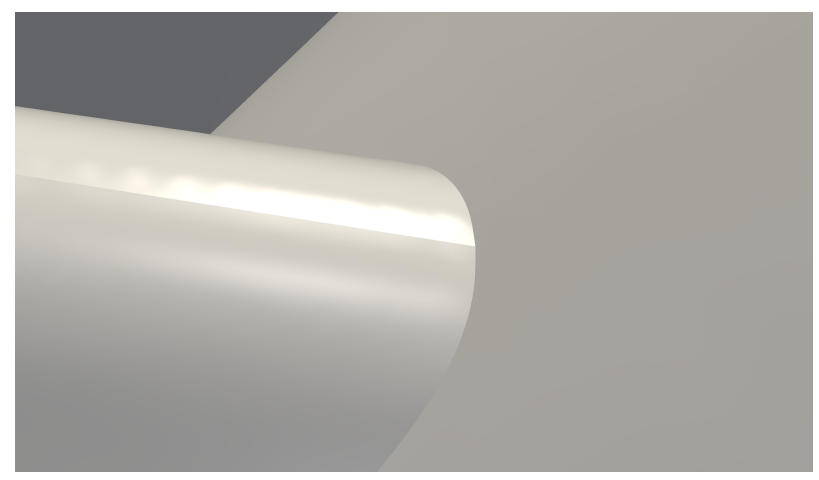

(a)

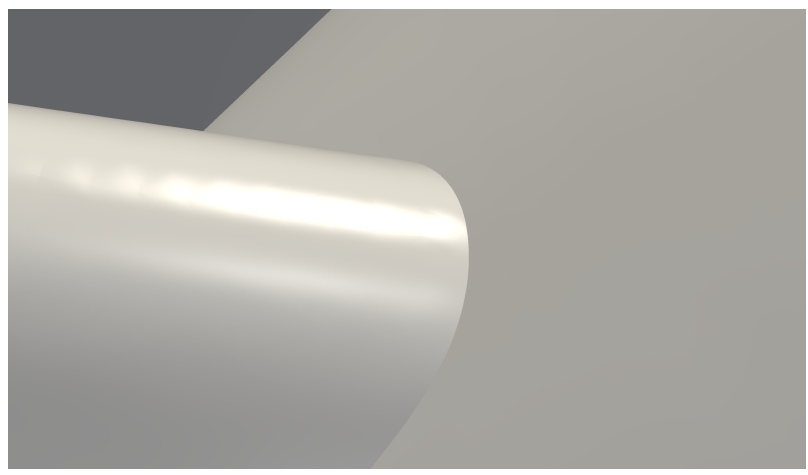

(b)

Figure 11: Close look at the wing of a Falcon aircraft. Mesh of polynomial degree four with (a) the initial model, and (b) the final model with the leading edge recast.

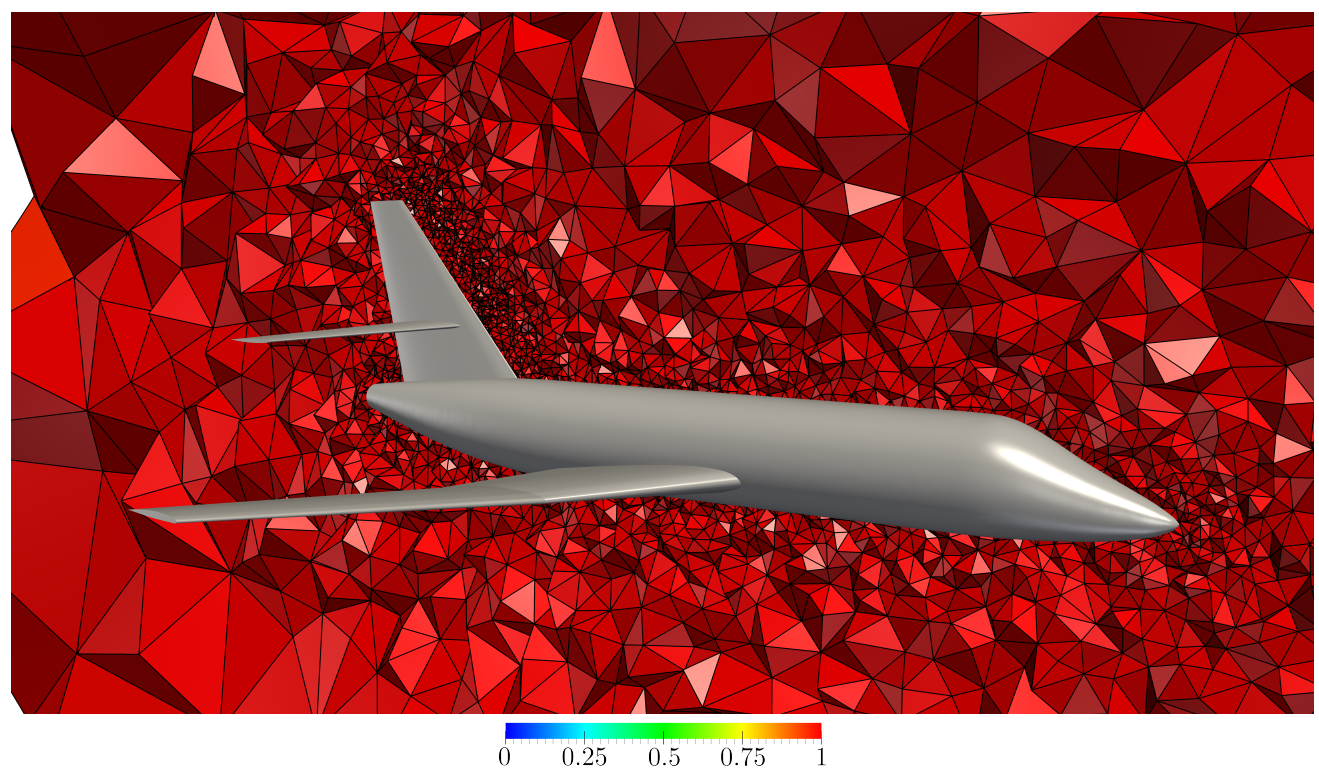

Figure 12: Curved tetrahedral mesh of polynomial degree $p=4$ of a Falcon aircraft with no invalid elements.

Our method incorporates a unique sharp-to-smooth modeling capability not fully available in standard CAD packages. This capability allows removing sharp features, i.e. vertices and curves, and smoothly merge the incident entities, i.e. curves and surfaces. The resulting surrogate geometry features $\mathcal{C}^{1}$-continuity along the merging region. On the contrary, standard CAD packages use NURBS curve and surface modeling and thus, do not feature all these sharpto-smooth modeling combinations. Note that with NURBS, it is possible to impose different levels of continuity between adjacent NURBS curves (surfaces) sharing a common point (curve) but only when using non-trimmed NURBS. Furthermore, NURBS modeling does not allow determining $\mathcal{C}^{1}$-continuity on a point shared by more than four non-trimmed quadri- lateral surfaces.

Recall that in topographical applications, it is standard to have a structured mesh representation, and thus, this methodology leads to $\mathcal{C}^{2}$-continuous topography surface meshes. We also found that since the method is fast and explicit, even a non-vectorized Anaconda Python implementation, is competitive with whole mesh curving methods that might need parallel implementations for fine meshes.

The sharp curves and surfaces of the initial mesh determine the target geometries, and thus, when higher is the refinement level, closer to the target geometries is the resulting mesh. This capability of matching limit curves and surfaces is required to perform convergence studies to validate and verify the in-house flow solver. 
If after successive refinement, the computational geometry would not lead to a limit geometry, the flow solution would not converge, too. Note that, after successive refinement, the meshes become nested and thus, the incorporation of a geometrical multigrid capability into the flow solver could be adequate.

The proportion of invalid elements is small compared to the size of the meshes, and thus, we showed that it can be fixed using local untangling and curving without the need for a global solver. That would not be the case for meshes for viscous laminar flow where highly stretched elements are needed to resolve boundary layers. Nevertheless, the meshes obtained with the proposed approach are well suited for those applications where isotropically but graded meshes are needed, such as in inviscid flow simulation and unsteady large eddy simulation.

In conclusion, it is possible to refine and curve a volume mesh and obtain smooth surfaces while preserving sharp features determined by vertices and polylines, the latter targeting smooth limit curves. For manifolds with boundaries, only a straight-edged mesh with boundary triangles marked with surface identifiers is required. Then, the method automatically computes the boundary curves and vertices from the triangle marks. Finally, the technique is well-suited to curve large quadratic and quartic meshes in low-memory configurations, e.g. curving a topographic mesh composed of two million and a half quartic elements using a laptop equipped with 16 GBytes of main memory.

\section{ACKNOWLEDGMENTS}

This project has received funding from the European Research Council (ERC) under the European Union's Horizon 2020 research and innovation programme under grant agreement No 715546. This work has also received funding from the Generalitat de Catalunya under grant number 2017 SGR 1731. The work of the third author has been partially supported by the Spanish Ministerio de Economía y Competitividad under the personal grant agreement RYC-2015-01633. Special thanks to Eloi Ruiz-Gironés.

\section{References}

[1] Gargallo-Peiró A., Avila M., Owen H., Prieto L., Folch A. "Mesh Generation for Atmospheric Boundary Layer Simulation in Wind Farm Design and Management." Procedia Engineering, vol. 124, 239-251, 2015

[2] Gargallo-Peiró A., Avila M., Owen H., PrietoGodino L., Folch A. "Mesh generation, sizing and convergence for onshore and offshore wind farm Atmospheric Boundary Layer flow simulation with actuator discs." Journal of Computational Physics, vol. 375, 209-227, 2018

[3] Gargallo-Peiró A., Folch A., Roca X. "Representing urban geometries for unstructured mesh generation." Procedia engineering, vol. 163, 175-185, 2016

[4] Persson P.O., Peraire J. "Curved Mesh Generation and Mesh Refinement using Lagrangian Solid Mechanics." 122008

[5] Chaurasia H., Roca X., Persson P., Peraire J. "A coarse-to-fine approach for efficient deformation of curved high-order meshes." Research Notes, 21st Int. Meshing Roundtable, Springer International Publishing, pp. 1-5, 2012

[6] Johnen A., Remacle J.F., Geuzaine C. "Geometrical validity of curvilinear finite elements." Journal of Computational Physics, vol. 233, 359-372, 2013

[7] Gargallo-Peiró A. Validation and generation of curved meshes for high-order unstructured methods. Ph.D. thesis, Universitat Politècnica de Catalunya. Departament de Matemàtica Aplicada III, July 2014

[8] Gargallo-Peiró A., Roca X., Peraire J., Sarrate J. "Distortion and quality measures for validating and generating high-order tetrahedral meshes." Engineering with Computers, vol. 31, no. 3, 423437, Jul 2015

[9] Gargallo-Peiró A., Roca X., Peraire J., Sarrate J. "Optimization of a regularized distortion measure to generate curved high-order unstructured tetrahedral meshes." International Journal for $\mathrm{Nu}$ merical Methods in Engineering, vol. 103, no. 5, 342-363, 2015

[10] Ruiz-Gironés E., Sarrate J., Roca X. "Generation of curved high-order meshes with optimal quality and geometric accuracy." Procedia engineering, vol. $163,315-327,2016$

[11] Ruiz-Gironés E., Roca X., Sarrate J. "Highorder mesh curving by distortion minimization with boundary nodes free to slide on a 3D CAD representation." Computer-Aided Design, vol. 72, 52-64, 2016

[12] Moxey D., Ekelschot D., Keskin Ü., Sherwin S., Peiró J. "High-order curvilinear meshing using a thermo-elastic analogy." Computer-Aided Design, vol. 72, 130 - 139, 2016. 23rd International Meshing Roundtable Special Issue: Advances in Mesh Generation 
[13] Jiao X., Wang D. "Reconstructing high-order surfaces for meshing." Engineering with Computers, vol. 28 , no. 4, 361-373, Oct 2012

[14] Ims J., Duan Z., Wang Z.J. "meshCurve: an automated low-order to high-order mesh generator." 22nd AIAA computational fluid dynamics conference, p. 2293. 2015

[15] Persson P.O., Aftosmis M.J., Haimes R. "On the Use of Loop Subdivision Surfaces for Surrogate Geometry." P.P. Pébay, editor, Proceedings of the 15th International Meshing Roundtable, pp. 375-392. Springer Berlin Heidelberg, Berlin, Heidelberg, 2006

[16] Jiménez Ramos A. Incorporating curvature to the boundary of linear and highorder meshes when a target geometry is unavailable. Master's thesis, Universitat Politècnica de Catalunya, 2018

[17] Dyn N., Levine D., Gregory J.A. "A butterfly subdivision scheme for surface interpolation with tension control." ACM transactions on Graphics (TOG), vol. 9, no. 2, 160-169, 1990

[18] Yang H.Q., Zhou X., Harris R.E., Yang S. "An Open Source, Geometry Kernel Based HighOrder Element Mesh Generation Tool." AIAA Scitech 2019 Forum, p. 1719. 2019

[19] Johnen A., Roca X., Toulorge T., Remacle J. "A new framework for curving structured boundary-layer meshes." International Conference on Adaptive Modeling and Simulation. 2018

[20] Gargallo-Peiró A., Houzeaux G., Roca X. "Subdividing triangular and quadrilateral meshes in parallel to approximate curved geometries." Procedia Engineering, vol. 203, 310 - 322, 2017. 26th International Meshing Roundtable, IMR26, 18-21 September 2017, Barcelona, Spain

[21] Lane J.M., Riesenfeld R.F. "A theoretical development for the computer generation and display of piecewise polynomial surfaces." IEEE Transactions on Pattern Analysis and Machine Intelligence, , no. 1, 35-46, 1980

[22] Loop C. Smooth Subdivision Surfaces Based on Triangles. Master's thesis, Department of Mathematics, The University of Utah, Masters Thesis, January 1987

[23] Zorin D. "A method for analysis of C 1-continuity of subdivision surfaces." SIAM Journal on $\mathrm{Nu}$ merical Analysis, vol. 37, no. 5, 1677-1708, 2000

[24] Stam J. "Evaluation of Loop Subdivision Surfaces." 011998
[25] Demmel J.W., Eisenstat S.C., Gilbert J.R., Li X.S., Liu J.W.H. "A supernodal approach to sparse partial pivoting." SIAM J. Matrix Analysis and Applications, vol. 20, no. 3, 720-755, 1999

[26] Li X., Demmel J., Gilbert J., iL. Grigori, Shao M., Yamazaki I. "SuperLU Users' Guide." Tech. Rep. LBNL-44289, Lawrence Berkeley National Laboratory, September 1999. http://crd.lbl. gov/ xiaoye/SuperLU/. Last update: August 2011

[27] Jones E., Oliphant T., Peterson P., et al. "SciPy: Open source scientific tools for Python.", 2001. [Online; last accessed September-2018]

[28] Perronnet A. "Interpolation transfinie sur le triangle, le tétraèdre et le pentaèdre. Application à la création de maillages et à la condition de Dirichlet." Comptes Rendus de l'Académie des Sciences-Series I-Mathematics, vol. 326, no. 1, $117-122,1998$

[29] Ruiz-Gironés E., Gargallo-Peiró A., Sarrate J., Roca X. "Automatically imposing incremental boundary displacements for valid mesh morphing and curving." Computer-Aided Design, 2019

[30] Toulorge T., Geuzaine C., Remacle J.F., Lambrechts J. "Robust untangling of curvilinear meshes." Journal of Computational Physics, vol. 254, 8-26, 2013

[31] Van Rossum G., Drake Jr F.L. Python reference manual. Centrum voor Wiskunde en Informatica Amsterdam, 1995

[32] Gargallo-Peiró A., Roca X., Peraire J., Sarrate J. "A distortion measure to validate and generate curved high-order meshes on CAD surfaces with independence of parameterization." International Journal for Numerical Methods in Engineering, vol. 106, no. 13, 1100-1130, 2016 


\title{
A PARALLEL VARIATIONAL MESH QUALITY IMPROVEMENT METHOD FOR TETRAHEDRAL MESHES
}

\author{
Suzanne M. Shontz ${ }^{1} \quad$ Maurin A. Lopez Varilla ${ }^{2} \quad$ Weizhang Huang $^{3}$ \\ ${ }^{1}$ Department of Electrical Engineering and Computer Science, Bioengineering Program, Information \\ and Telecommunication Technology Center, University of Kansas, Lawrence, KS USA \\ ${ }^{2}$ Husky Injection Molding Systems Ltd., Luxembourg, Luxembourg \\ ${ }^{3}$ Department of Mathematics, University of Kansas, Lawrence, KS USA
}

\begin{abstract}
There are numerous large-scale applications requiring mesh adaptivity, e.g., computational fluid dynamics and weather prediction. Parallel processing is needed for simulations involving large-scale adaptive meshes. In this paper, we propose a parallel variational mesh quality improvement algorithm for use with distributed memory machines. Our method parallelizes the serial variational mesh quality improvement method by Huang and Kamenski. Their approach is based on the use of the Moving Mesh PDE method to adapt the mesh based on the minimization of an energy functional for mesh equidistribution and alignment. This leads to a system of ordinary differential equations (ODEs) to be solved which determine where to move the interior mesh nodes. An efficient solution is obtained by solving the ODEs on subregions of the mesh with overlapped communication and computation. Strong and weak scaling experiments on up to 128 cores for meshes with up to $160 \mathrm{M}$ elements demonstrate excellent results.
\end{abstract}

Keywords: parallel mesh quality improvement, variational method, tetrahedral mesh, distributed computing

\section{INTRODUCTION}

There are numerous large-scale scientific applications requiring adaptive meshes with millions to billions of elements, e.g., [1, 2, 3, 4]. Such large computational simulations are possible due to the availability of massively parallel supercomputers which integrate central processing units (CPUs) and accelerators, such as graphics processing units (GPUs), Phi coprocessors, and field programmable gate arrays (FPGAs). New parallel mesh generation, parallel mesh adaptation, and parallel mesh quality improvement algorithms have been developed to take advantage of these novel architectures.

Although there are numerous parallel mesh generation algorithms [5], only a few parallel mesh quality improvement algorithms have been developed $[6,7$,
$8,9,10]$. The methods presented in $[6,7,8,11]$ are solely devoted to improving the mesh quality, whereas the ones in $[9,10]$ combine mesh untangling and mesh quality improvement procedures. However, to the best of our knowledge, no parallel variational mesh adaptation methods have been developed.

Since very few parallel mesh quality improvement algorithms and no parallel mesh adaptation algorithms have been proposed, we also review the serial methods developed for such purposes. The vast majority of sequential mesh quality improvement and mesh adaptation methods employ optimization techniques to improve the mesh quality or to adapt the mesh to changes in the geometry or the physics of the application. Optimization-based mesh quality improvement and mesh adaptation algorithms adjust the po- 
sitions of the node while preserving the mesh topology $[12,13,14,15,16,17,18,19,20]$.

Variational methods for mesh adaptation and mesh quality improvement have recently received considerable attention from the meshing community (e.g., [19, $20,21,22,23,24])$. Whereas most optimization-based mesh quality improvement algorithms use gradientbased techniques to minimize an objective function, Huang and Kamenski [19, 20] instead use the Moving Mesh PDE (MMPDE) method to discretize and find the minimum of an appropriately constructed meshing functional $[25,26,27]$. The minimizer of the meshing functional is a bijective mapping which generates an improved quality mesh as an image of the initial mesh.

In this paper, we present a novel, efficient parallel variational mesh quality improvement algorithm and the corresponding implementation for distributed memory machines. Our parallel method is based on the sequential method by Huang, Ren, and Russell [21] and the recent implementation by Huang and Kamenski [20]. The method finds the minimizer of a meshing functional by solving a system of ordinary differential equations (ODEs) for the nodal velocities. We first review the key concepts of variational mesh methods and the implementation of the sequential MMPDE method in Section 2. In Section 3, we describe our parallel variational mesh quality improvement method for distributed memory systems, along with the implementation. Our method employs a domain decomposition approach in order to divide the workload among the cores. We reorganize the computation within each subregion in order to facilitate the overlap of communication with computation. We analyze the computational complexity of the method in Section 4 . We carry out numerical experiments on tetrahedral meshes and determine the strong and weak scaling properties of the proposed method. The numerical experiments and the associated results are discussed in Section 5. We present our conclusions on the work and several directions for future work in Section 6.

\section{VARIATIONAL MESH ADAPTATION METHODS}

In this section, we present an overview of variational mesh adaptation and the corresponding methods. In the variational approach, an adaptive mesh is generated as the image of a reference mesh under a coordinate transformation. The coordinate transformation is determined as the minimizer of a meshing functional. The mesh concentration is typically controlled through a scalar or a matrix-valued function. This is referred to as the metric tensor or monitor function. Monitor functions are defined based on error estimates and/or physical considerations.
Several authors have reported on variational mesh adaptation methods with various types of meshing functionals. For example, Winslow [28] developed an equipotential method based on variable diffusion. Brackbill and Saltzman developed a method combining mesh concentration, smoothness, and orthogonality [29]. Dvinsky developed another approach based on the energy of harmonic mappings [30]. Variational methods based on the conditioning of the Jacobian matrix of the coordinate transformation were developed by Knupp [15] and Knupp and Robidoux [26]. More recently, equidistribution and alignment conditions were used by Huang [31] and Huang and Russell [32] to develop mesh adaptation methods.

The Moving Mesh PDE (i.e., MMPDE) method, which was proposed by Huang, Ren, and Russell in 1994 [21] is the basis upon which many other variational mesh adaptation methods have been developed. In 2015, Huang and Kamenski developed a more efficient implementation of the serial MMPDE method [20].

\subsection{New implementation of the variational mesh adaptation method}

In this subsection, we focus on Huang and Kamenski's new implementation of the MMPDE method [20]. Consider a domain $\Omega \subset \mathbb{R}^{d}(d \geq 1)$ and $\mathcal{T}_{h}=\{K\}$ be a simplicial mesh containing $N$ elements and $N_{v}$ vertices on $\Omega$. Denote the affine mapping $F_{K}: \hat{K} \rightarrow K$ and its Jacobian matrix by $F_{K}^{\prime}$, where $\hat{K}$ is the master element. Let the edge matrices for $K$ and $\hat{K}$ be $E_{K}$ and $\hat{E}$. Assume that a metric tensor (or a monitor function) $\mathbb{M}=\mathbb{M}(\mathbf{x})$ is given on $\Omega$ which provides directional and magnitude information for the elements.

A key idea of the MMPDE method is to view an adaptive mesh as a uniform one in the metric $\mathbb{M}$ such that the following two properties hold. First, the size of all elements $K$ in the metric $\mathbb{M}_{K}$ is the same. Second, all elements $K$ in the metric $\mathbb{M}_{K}$ are similar to $\hat{K}$.

These two properties give rise to the equidistribution and alignment conditions:

$$
\begin{aligned}
& \left.|K| \sqrt{\operatorname{det}\left(\mathbb{M}_{K}\right.}\right)=\frac{\sigma_{h}}{N}, \quad \forall K \in \mathcal{T}_{h} \\
& \frac{1}{d} \operatorname{tr}\left(\left(F_{K}^{\prime}\right)^{T} \mathbb{M}_{K} F_{K}^{\prime}\right)=\operatorname{det}\left(\left(F_{K}^{\prime}\right)^{T} \mathbb{M}_{K} F_{K}^{\prime}\right)^{\frac{1}{d}}, \\
& \forall K \in \mathcal{T}_{h},
\end{aligned}
$$

where $|K|$ is the volume of $K$ and $\sigma_{h}=$ $\sum_{K}|K| \sqrt{\operatorname{det}\left(\mathbb{M}_{K}\right)}$.

Then an energy function for the equidistribution and 
alignment conditions is given by

$$
\begin{aligned}
I\left[\mathcal{T}_{h}\right] & =\sum_{K}|K| G_{K}, \text { where } \\
G_{K} & =\frac{1}{3} \sqrt{\operatorname{det}\left(\mathbb{M}_{K}\right)}\left(\operatorname{tr}\left(\mathbb{J M}_{K}^{-1} \mathbb{J}^{T}\right)\right)^{d} \\
& +\frac{1}{3} d^{d} \sqrt{\operatorname{det}\left(\mathbb{M}_{K}\right)}\left(\frac{\operatorname{det}(\mathbb{J})}{\left.\sqrt{\operatorname{det}\left(\mathbb{M}_{K}\right.}\right)}\right)^{2} \text { and } \\
\mathbb{J} & =\left(F_{K}^{\prime}\right)^{-1}=\hat{E} E_{K}^{-1} .
\end{aligned}
$$

Minimization of the energy function will result in a mesh that closely satisfies the equidistribution and alignment conditions.

The MMPDE moving mesh equation is then defined as the (modified) gradient system of $I\left[\mathcal{T}_{h}\right]$, i.e.,

$$
\frac{d \mathbf{x}_{i}}{d t}=-\frac{\operatorname{det}\left(\mathbb{M}_{i}\right)^{\frac{1}{d}}}{\tau} \frac{\partial I\left[\mathcal{T}_{h}\right]}{\partial \mathbf{x}_{i}}, \quad i=1, \ldots, N_{v},
$$

where $\tau>0$ is a parameter for adjusting the response time scale of mesh movement to the change in $\mathbb{M}$.

For mesh quality improvement, we choose $\mathbb{M}=\mathbb{I}$, which means we want the mesh to be as uniform as possible in the Euclidean space. In this case, the moving mesh equation reads as

$$
\frac{d \mathbf{x}_{i}}{d t}=\sum_{K \in \omega_{i}}|K| \mathbf{v}_{i_{K}}^{K}, \quad i=1, \ldots, N_{v},
$$

where $\omega_{i}$ is the element patch associated with $\mathbf{x}_{i}, i_{K}$ is the local index for $\mathbf{x}_{i}$ on $K$, and $\mathbf{v}_{i_{K}}$ is the local nodal velocity contributed by $K$ to the node $x_{i}$. The analytical formula of the local nodal velocities is given in [20]. In the case when $\mathbb{M}=\mathbb{I}$,

$$
\begin{aligned}
{\left[\begin{array}{c}
\left(\mathbf{v}_{1}^{K}\right)^{T} \\
\vdots \\
\left(\mathbf{v}_{d}^{K}\right)^{T}
\end{array}\right] } & =-G_{K} E_{K}^{-1}+E_{K}^{-1} \frac{\partial G_{K}}{\partial \mathbb{J}} \hat{E} E_{K}^{-1} \\
& +\frac{\partial G_{K}}{\partial \operatorname{det}(\mathbb{J})} \frac{\operatorname{det}(\hat{E})}{\operatorname{det}\left(E_{K}\right)} E_{K}^{-1}, \\
\left(\mathbf{v}_{0}^{K}\right)^{T} & =-\sum_{j=1}^{d}\left(\mathbf{v}_{j}^{K}\right)^{T} .
\end{aligned}
$$

The partial derivatives in equation (2) are obtained by substituting $\mathbb{M}=\mathbb{I}$ into the formula for $G_{K}$ shown above and then differentiating with respect to $\mathbb{J}$ and $\operatorname{det}(\mathbb{J})$.

The nodal velocities of the boundary nodes are set to 0 . They can also be modified to let the boundary nodes slide along the boundary.

To determine the locations of the interior nodes, Equation (1) is then solved using the adaptive fourth-order Runge-Kutta-Fehlberg ODE solver (RKF45). It has been shown analytically and numerically in [33] that the mesh governed by the MMPDE moving mesh equation will stay nonsingular (i.e., no crossing nor tangling will occur) if it is nonsingular initially.

\section{PARALLEL VARIATIONAL MESH QUALITY IMPROVEMENT ALGORITHM}

In this section, we present our novel parallel algorithm and implementation for distributed memory systems based on the moving mesh method described in the previous section.

\subsection{Sequential algorithm}

For the sequential algorithm, the adaptive fourthorder Runge-Kutta Fehlberg ODE solver (RKF45) with fifth-order error estimator is employed to solve (1) (see [34] for details). The RKF45 method approximates the solution of an ODE system in the form

$$
\frac{d y}{d t}=f(t, y)
$$

using a non-constant, optimal step size $d t$ in each iteration. The method determines the step size $d t$ in each iteration by comparing a fourth-order approximation, $y_{i+1}$, and a fifth-order approximation, $z_{i+1}$, to the solution. These approximations are given by

$$
y_{i+1}=y_{i}+\frac{25}{216} k_{1}+\frac{1408}{2565} k_{3}+\frac{2197}{4104} k_{4}-\frac{1}{5} k_{5},
$$

and

$$
\begin{aligned}
z_{i+1} & =y_{i}+\frac{16}{135} k_{1}+\frac{6656}{12825} k_{3}+\frac{28561}{56430} k_{4} \\
& -\frac{9}{50} k_{5}+\frac{2}{55} k_{6},
\end{aligned}
$$

respectively. Here

$$
\begin{aligned}
& k_{1}= d t f\left(t_{i}, y_{i}\right), \\
& k_{2}= d t f\left(t_{i}+\frac{1}{4} d t, y_{i}+\frac{1}{4} k_{1}\right), \\
& k_{3}=d t f\left(t_{i}+\frac{3}{8} d t, y_{i}+\frac{3}{32} k_{1}+\frac{9}{32} k_{2}\right), \\
& k_{4}=d t f\left(t_{i}+\frac{12}{13} d t, y_{i}+\frac{1932}{2197} k_{1}-\frac{7200}{2197} k_{2}\right. \\
&\left.\quad+\frac{7296}{2197} k_{3}\right), \\
& k_{5}=d t f\left(t_{i}+d t, y_{i}+\frac{439}{216} k_{1}-8 k_{2}+\frac{3680}{513} k_{3}\right. \\
&\left.\quad-\frac{845}{4104} k_{4}\right), \\
& k_{6}=d t f\left(t_{i}+\frac{1}{2} d t, y_{i}-\frac{8}{27} k_{1}+2 k_{2}-\frac{3544}{2565} k_{3}\right. \\
&\left.\quad+\frac{1859}{4104} k_{4}-\frac{11}{40} k_{5}\right) .
\end{aligned}
$$


The error is given by the $\infty$-norm of the difference between the two solutions, i.e., err $=\left\|z_{i+1}-y_{i+1}\right\|_{\infty}$. If err is smaller than a given tolerance, tol, then the solution $y_{i+1}$ is accepted. One can show that the optimal step size is given by $q * d t$, where

$$
q=0.84\left(\frac{t o l * d t}{e r r}\right)^{\frac{1}{4}}
$$

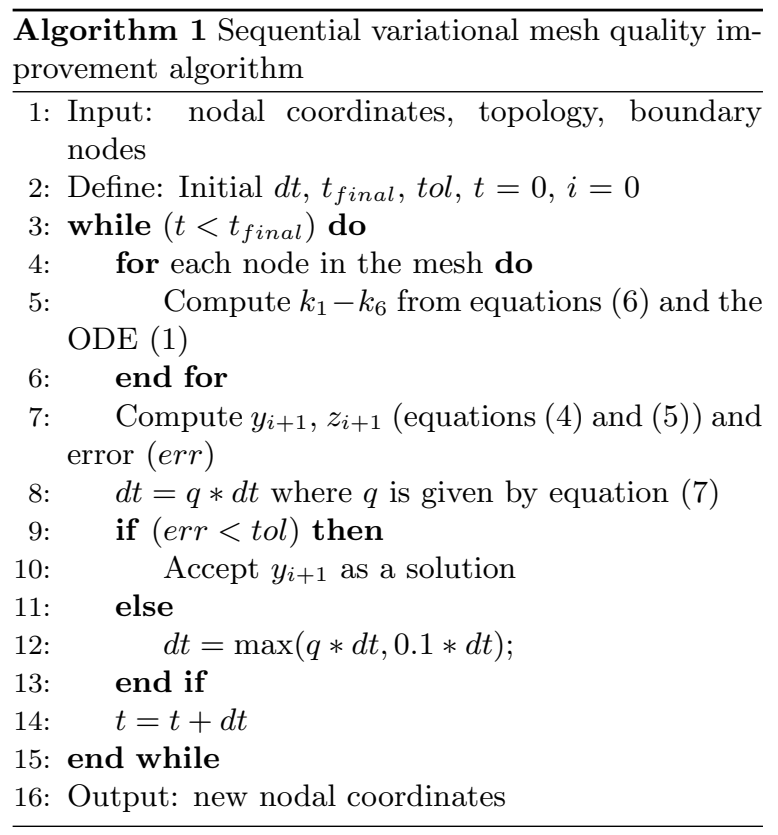

In Algorithm 1, (i.e., the algorithm for the method proposed in [20]), the calculation of the nodal velocities is directly related to the calculation of the $k_{i}$, which is the most computationally-intensive step (i.e., step 5). To calculate the values, $k_{i}$, in the RKF45 method, the algorithm loops over all elements calculating partial nodal velocities for each node. This requirement is the basis for our distributed data approach in the parallel algorithm.

\subsection{Overview of the parallel algorithm}

In this subsection, we highlight three important aspects of our parallel algorithm (Algorithm 2): the distribution of the workload, the communication strategy, and the termination criteria. Although there exist multiple strategies to distribute the work among cores, we employ a domain decomposition approach in which we divide the domain into $p$ regions, where $p$ is the number of cores (i.e., steps 4 and 5 in Algorithm 2). Each region is (ideally) composed of one connected component. We use this approach because according to Equation (2) the nodal velocity of a particular node $\mathbf{x}_{m}$, such as the one in Fig. 1, is calculated based on the edge matrices of elements $E_{1}$,
$E_{2}, E_{3}, E_{4}$, and $E_{5}$. Therefore, a decomposition of the elements of the domain into regions is the strategy that yields the best performance. To accomplish this, we use METIS [35], which is a library for partitioning meshes and graphs. We employed the mpmetis scheme to partition the mesh into regions so that each region has roughly the same number of elements and the number of interfaces between regions is minimized.

Once we have the mesh partition, core $P_{0}$ reads and distributes the information concerning the topology and nodal coordinates to the rest of the cores. In this step, each core creates a list (SharedNodes_p []) whose size is equal to the number of nodes along partition boundaries (i.e., the number of shared nodes). Each core stores partial nodal velocities to specific locations in the list and fills-in the rest with zeros.

Whereas each core computes the new nodal positions for the interior nodes of its corresponding region, the new nodal positions for nodes along partition boundaries (corresponding to the shared nodes) requires communication and verification steps (i.e., steps 17 and 19 in Algorithm 2). In our parallel algorithm, all communication steps are reduction operations. To compute the new nodal positions for shared nodes, we perform a reduction operation (summation) over the list SharedNodes_p [] in which we store the partial nodal velocities of the shared nodes. We also need communication steps to calculate the global error (i.e., step 23 in Algorithm 2) and the average mesh quality (i.e., step 31 in Algorithm 2). To calculate the global error, we require a reduction operation to calculate the maximum. We also require a summation reduction for the average mesh quality.

Finally, we employ a tetrahedral mesh quality metric in order to evaluate the quality of the mesh on each iteration. We utilize the mesh quality information in the termination criteria. The mesh quality metric implemented in our algorithm is given by

$$
q=\frac{C R}{3 * I R},
$$

where $C R$ is the circumsphere radius and $I R$ is the inscribed sphere radius. For this metric, $q \in[1, \infty)$ where $q=1.0$ is the optimal mesh quality. We terminate the parallel variational mesh quality improvement algorithm when the difference in the average mesh quality on two consecutive iterations is small (i.e., less than a specified tolerance). Also, note that we do not use unnecessary synchronization calls (MPI_Barrier) in the algorithm. However an implicit synchronization might be performed (if necessary) with MPI_Wait. MPI_Barrier is only used for timing purposes. 


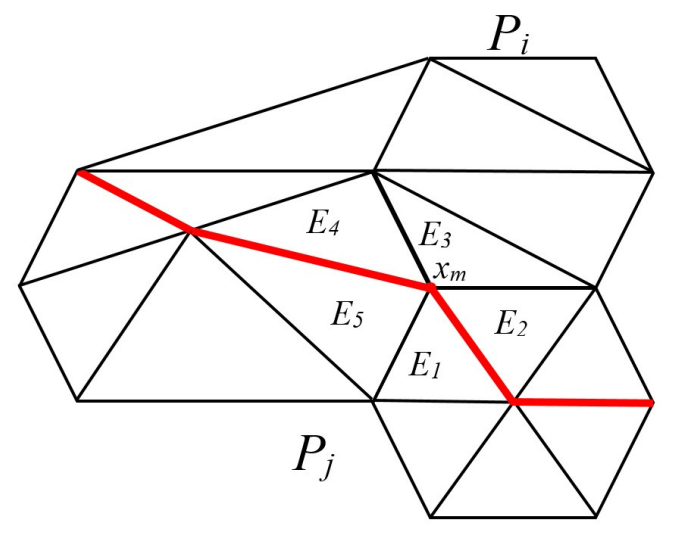

Figure 1: Patch of elements with $\mathbf{x}_{m}$ as one of its vertices.

\subsection{Overlapping communication with computation}

As we mentioned before, the most computationallyintensive step in the parallel variational mesh quality improvement algorithm is the computation of the nodal velocities. For the case in Fig. 1 , core $P_{i}$ is unable to compute the nodal velocity for node $\mathbf{x}_{m}$ because the core does not have access to elements $E_{1}$ and $E_{5}$. Therefore, $P_{i}$ calculates only a portion of the nodal velocity at this node. The same is true for core $P_{j}$.

According to the previous description, we design the parallel algorithm such that every core $P_{i}$ loops once over its own elements to calculate the nodal velocities for the interior nodes within a region. However, for shared nodes, the nodal velocities are incomplete. Therefore, in this case, e.g., for $\mathbf{x}_{m}$ in Fig. 1, cores $P_{i}$ and $P_{j}$ store the partial nodal velocities in the SharedNodes_p [] list. Finally, the nodal velocities for the shared nodes require a reduction operation (summation) over SharedNodes_p [] and a verification step (i.e., steps 17 and 19 in Algorithm 2).

It is possible to overlap the communication and computation and reduce the overall run time by reorganizing the data in the data structures. To this end, each core splits the list of local elements Elements_proc [] into two new lists, i.e., Elements_proc1[] and Elements_proc2 [] (i.e., step 7 in Algorithm 2). The algorithm stores the elements that contain at least one shared node in the data structure Elements_proc1 [], whereas the elements whose nodes are interior nodes are stored in Elements_proc2[]. Thus, we calculate the nodal velocities in two steps (i.e., steps 15 and 18 in Algorithm 2). After the first step, the algorithm will have partial nodal velocities for the shared nodes. Therefore, we can initiate the communication using the non-blocking collective command

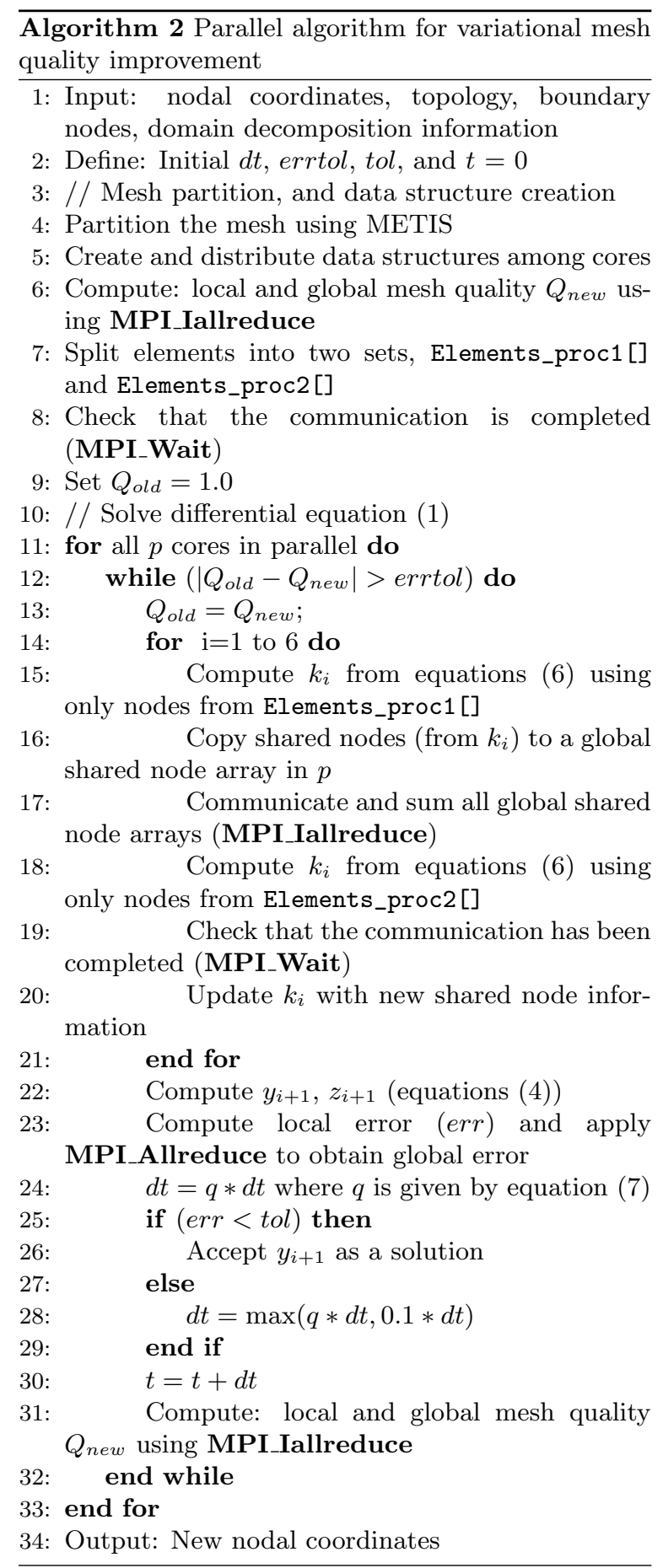

MPI_Iallreduce which immediately calculates the nodal velocities for the interior nodes (i.e., step 18 in Algorithm 2). Once the algorithm finishes the calculation of nodal velocities for the interior nodes, the algorithm checks to see if the communication has completed using MPI_Wait (i.e., step 19 in Algorithm 2). Finally, the algorithm updates the nodal velocities for 
the shared nodes (i.e., step 20 in Algorithm 2).

\section{PARALLEL RUNTIME ANALYSIS}

In this section, we discuss the runtime performance of the parallel algorithm described in the previous section. In particular, we analyze the average parallel runtime.

First, we assume the partition of the initial mesh is given to the algorithm as input data. Recall that we use METIS to accomplish this step. Once the algorithm reads the input data, core $P_{0}$ distributes the information among cores according to the partition file. This overhead computation is performed sequentially and occurs just once throughout the execution of the algorithm. We assume this step takes $t_{N}$ time.

Since we performed the mesh partitioning step over the elements of the mesh, assuming that $N$ is the total number of mesh elements, each core contains (ideally) $\lceil N / p\rceil$ elements. With this information, the splitting operation performed within each region to overlap communication with computation takes $\lceil N / p\rceil(d+1)$ operations, where $d$ is the dimension. This step is also performed once in the algorithm.

For the next step, we solve the differential equation (1) by calculating the values $k_{i}$. To calculate $k_{i}$, the algorithm loops over the mesh elements. If the maximum time to calculate the nodal velocity for each node is $t_{v n}$, then the total serial time to calculate the nodal velocities is $N(d+1) t_{v n}$. Therefore, the parallel time is $\lceil N / p\rceil(d+1) t_{v n}$. Moreover, we define the number of elements containing at least one shared node in the region corresponding to core $P_{i}$ as $N_{s h}^{\left(P_{i}\right)}$, and the number of elements containing only interior nodes as $N_{i n t}^{\left(P_{i}\right)}$. Note that $\lceil N / p\rceil=N_{s h}^{\left(P_{i}\right)}+N_{i n t}^{\left(P_{i}\right)}$. For the communication process, first, we extract the local shared nodes. Assuming the time to copy one node from the local to the global list is $t_{c}$ and the number of shared nodes in the mesh is $V_{s h}$, then this step takes $V_{s h} t_{c}$ time. Similarly, assuming that the time to send a vector with $V_{s h}$ nodes is $t_{s}$, the communication process takes $\log _{2}(p) t_{s}+p V_{s h} t_{c}$. Note that this was implemented as a non-blocking communication process using the computation time $N_{i n t}^{(p)}(d+1) t_{v n}$ to overlap communication and computation. Thus, the time to compute these two processes is $T c_{\text {total }}$, where

$$
T c_{\text {total }}= \begin{cases}T_{\text {int }}, & \text { if } T_{\text {int }}>T_{\text {comm }} \\ T_{\text {int }}+\left|T_{\text {int }}-T_{\text {comm }}\right|, & \text { otherwise. }\end{cases}
$$

Here $T_{i n t}=N_{i n t}^{(p)}(d+1) t_{v n}$ and $T_{\text {comm }}=\log _{2}(p) t_{s}+$ $p V_{s h} t_{c}$.

To copy the information from the global to the local list in each core costs $V_{s h} t_{c}$. Assuming that the time to compute the error in each coordinate of each node is $t_{e}$, the total serial time is $N(d+1) d t_{e}$. In parallel, it is $\lceil N / p\rceil(d+1) d t_{e}$ plus the time to calculate the maximum error among cores which is $\log _{2}(p)$. Finally, if $t_{q}$ is the time to calculate the quality of one element, then $N t_{q}$ is the time to calculate the quality for the serial algorithm, and $\lceil N / p\rceil t_{q}$ is the time for the parallel one. The time to calculate the average quality among cores is $\log _{2}(p)$. With this information, the total parallel time per iteration is

$$
\begin{aligned}
T_{P}= & p V_{s h} t_{c}+2 \log _{2}(p)+(d+1)\left(\lceil N / p\rceil_{s h} t_{v n}\right. \\
& \left.+\lceil N / p\rceil d t_{e}\right)+\lceil N / p\rceil t_{q}+T c_{\text {total }} .
\end{aligned}
$$

The major source of overhead due to communication in (10) is $T c_{\text {total }}$ and $p V_{s h} t_{c}$ due to the fact that the number of shared nodes always increases with the number of mesh elements and the number of cores, therefore $p V_{s h} t_{c}$ increases. Hence, excellent timing results are expected in the cases for which the number of interior nodes in each partition is large compared with the number of shared nodes in the mesh.

\section{NUMERICAL EXPERIMENTS}

Our algorithm was implemented in $\mathrm{C} / \mathrm{C}++$ using the message-passing interface (OpenMPI version 1.8.7). We ran our experiments on the high performance computing cluster available to us through the Advanced Computing Facility ( $\mathrm{ACF}$ ) at the University of Kansas. More specifically, we ran the experiments on twenty-one Dell R730 servers, each of them equipped with $2 \mathrm{x}$ dodeca-core Intel Haswell processors running at $2.5 \mathrm{GHz}$ with $128 \mathrm{~GB}$ of RAM, 1TB HDD, and FDR Infiniband.

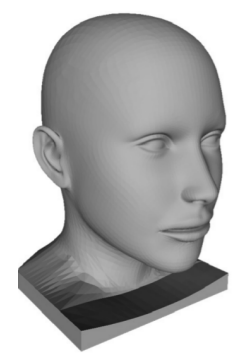

(a)

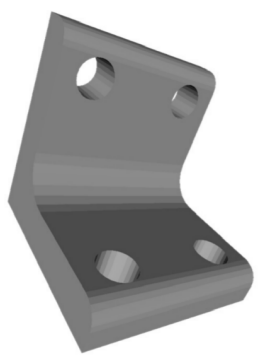

(b)

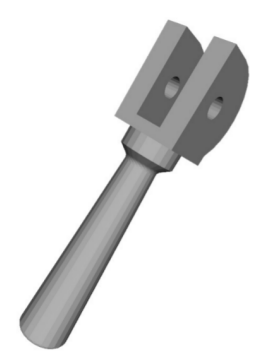

(c)
Figure 2: Domains used to test the parallel algorithm: (a) bust, (b) bracket and (c) double cam tool

To test the performance of our parallel algorithm, we constructed several tetrahedral meshes based on three geometries from various applications and with different characteristics. Figure 2 illustrates the threedimensional domains used in our experiments. We chose the geometries from different online databases, Fig. 2(a) is part of the 3dcadbrowser project [36], 
while Fig. 2(b) and (c) are part of the French Institute for Research in Computer Science and Automation (INRIA) databases [37]). We used GHS3D [37] and MeshLab [38] to generate a new surface mesh and to scale the domain to meet our needs. Based on these surface meshes, we generated tetrahedral volume meshes using Tetgen [39] with the numbers of elements specified in Tables 1 and 2. Finally, we randomly perturbed the nodes of each mesh to reduce their quality. The resulting tetrahedral meshes are used to test the performance of our parallel variational mesh quality improvement (Parallel VMQI) algorithm.

We used the meshes for the bust and the double cam tool domains to test the algorithm for strong scaling and the meshes for the bracket domain to test weak scaling.

Table 1: Size of tetrahedral meshes for the bust and the double cam tool domains

\begin{tabular}{|crr|}
\hline Mesh & \# Nodes & \# Elements \\
\hline bust & $12,895,493$ & $80,000,012$ \\
double cam tool & $7,089,753$ & $41,405,684$ \\
\hline
\end{tabular}

Table 2: Various mesh sizes for the bracket domain

\begin{tabular}{|c|rr|}
\hline Mesh & \# Nodes & \# Elements \\
\hline & 450,960 & $2,500,032$ \\
& 864,028 & $5,000,025$ \\
bracket & $1,716,222$ & $9,999,990$ \\
& $3,269,784$ & $19,999,978$ \\
& $6,497,224$ & $40,000,000$ \\
& $12,957,609$ & $80,000,037$ \\
& $24,177,335$ & $159,745,245$ \\
\hline
\end{tabular}

For our first experiment, we employed a tetrahedral mesh with approximately $80 \mathrm{M}$ elements for the bust domain (see Fig. 3). We ran the algorithm with different numbers of cores using $d t=10^{-14}$, errtol $=10^{-5}$, and $t o l=0.001$ as input parameters (see Algorithm 2). These values guarantee that the algorithm will run until convergence with an error of errtol $=10^{-5}$. Figure 4 shows the average mesh quality versus the number of iterations. This demonstrates the ability of the algorithm to improve the average mesh quality.

Figures 5(a) and 5(b) show that for a small number of cores, the runtime, and speedup achieved by the parallel algorithm are very close to the ideal ones. A small deviation in the speedup for a larger number of cores is also observed. The deviation is clearer at sixteen cores and it does not grow much for a higher number of cores. It is clear that the pre-processing step (distribution of nodes, elements and boundary nodes

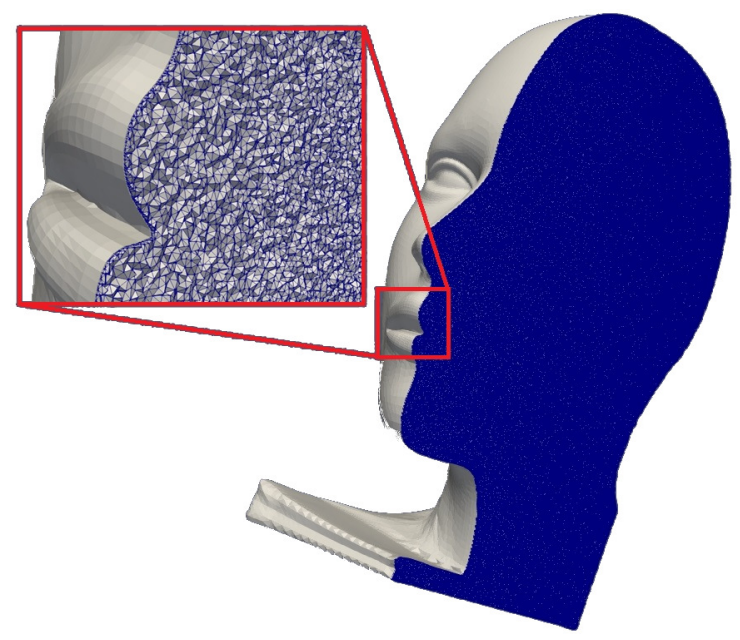

Figure 3: $80 \mathrm{M}$ element tetrahedral mesh of the bust domain

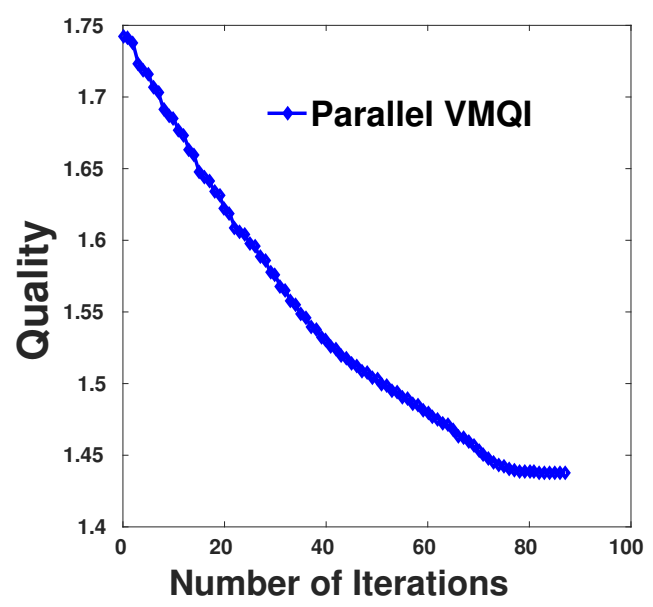

Figure 4: Average quality versus number of iterations for the $80 \mathrm{M}$ element tetrahedral mesh of the bust domain

and identification of shared nodes) is a major source of overhead that significantly contributes to the discrepancy between the calculated and ideal speedup. On the other hand, when the number of interior nodes on each core is high compared with the number of shared nodes, it is more likely that the communication steps (when solving the differential equation) overlaps with the calculations of the nodal velocities for the interior nodes; therefore, the communication steps contribute less to the performance degradation in such a case. The runtimes reported in Fig. 5 are the average of five experiments.

Our second experiment is a strong scaling experiment using the double cam tool domain and a tetrahedral mesh with approximately 40M elements (see Fig. 6), 


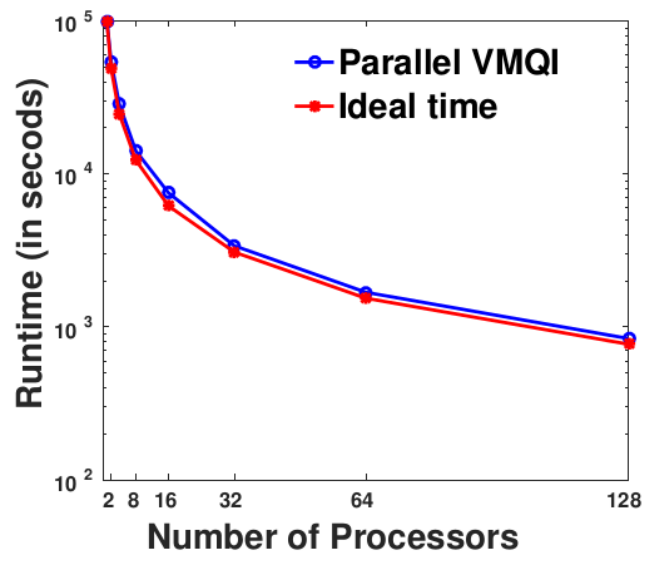

(a)

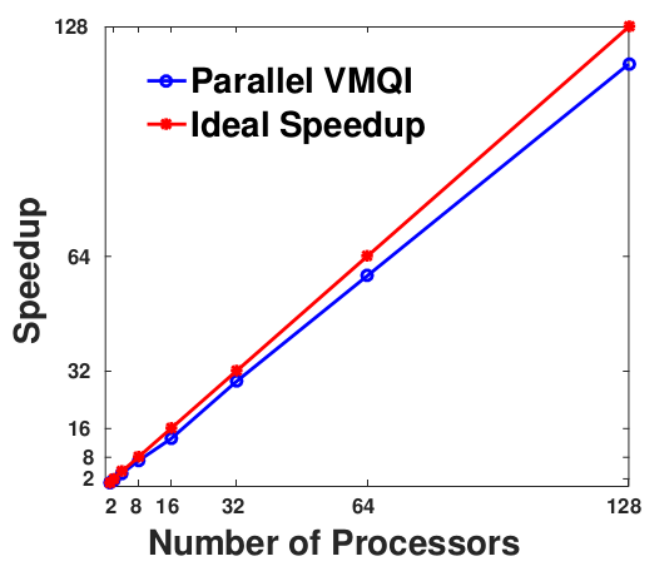

(b)

Figure 5: (a) Total runtime and (b) speedup for the Parallel VMQI algorithm for the $80 \mathrm{M}$ element tetrahedral mesh of the bust domain

which is approximately half the number of elements used for the first experiment. We decided to include this test case so as to measure the performance of the algorithm when the number of interior nodes per core is reduced. In this case, it may be more challenging to overlap communication with computation in an effective manner. The initial parameters (dt, errtol, tol) are the same as in the first strong scaling test. Figure 7 shows the average mesh quality versus the number of iterations for this tetrahedral mesh.

Figures $8(\mathrm{a})$ and $8(\mathrm{~b})$ show the total runtime and speedup for the $40 \mathrm{M}$ element mesh of the double cam tool. The results are in general similar to the ones for the first test case. This demonstrates that our parallel algorithm scales very well with the resources used at the University of Kansas. It is worth mentioning that the maximum number of cores report in our experiments is limited by our accessibility to the cluster.

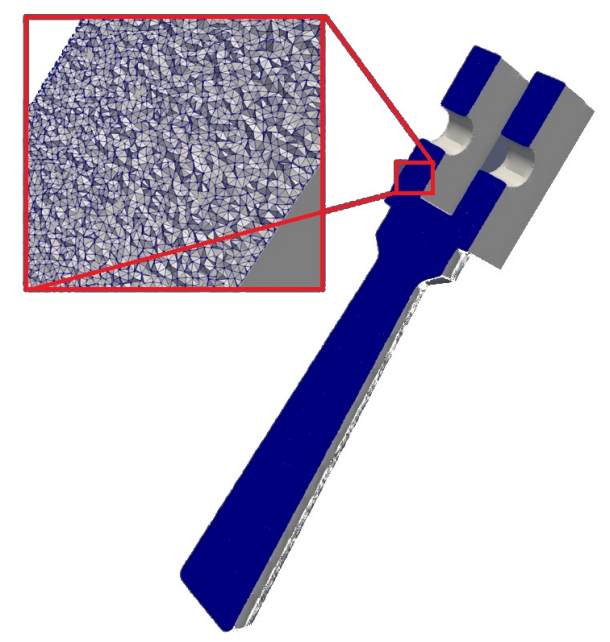

Figure 6: $40 \mathrm{M}$ element tetrahedral mesh of the double cam tool domain

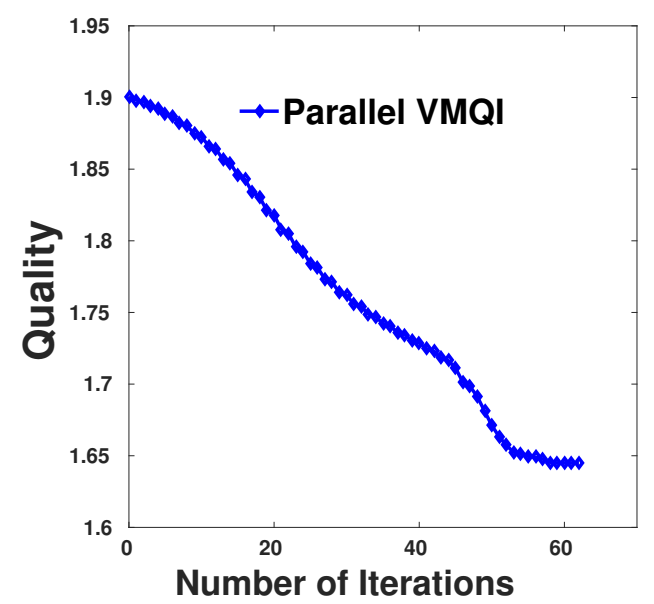

Figure 7: Average quality versus the number of iterations for the $40 \mathrm{M}$ element tetrahedral mesh of the double cam tool domain

We attribute the good results from the previous two examples to the the ability of our parallel algorithm to overlap communication with computation thus reducing the runtime. When this is possible, the major source of performance degradation, i.e, $T c_{\text {total }}$ from equation (9), is reduced. Figures 9(a) and 9(b) show the computation and communication time for the bust and double cam tool test cases. The figure shows the time employed by one core to calculate the nodal velocities for the interior nodes in its own region (computation). The communication time is the time employed to communicate the shared nodes. Note that, for these two cases, the computation time is always significantly higher than the communication time, which guarantees a good performance of the algorithm. Also, it is 


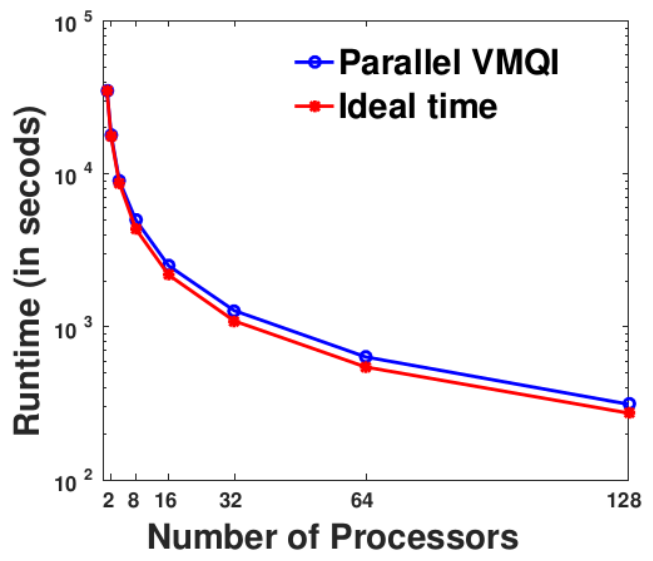

(a)

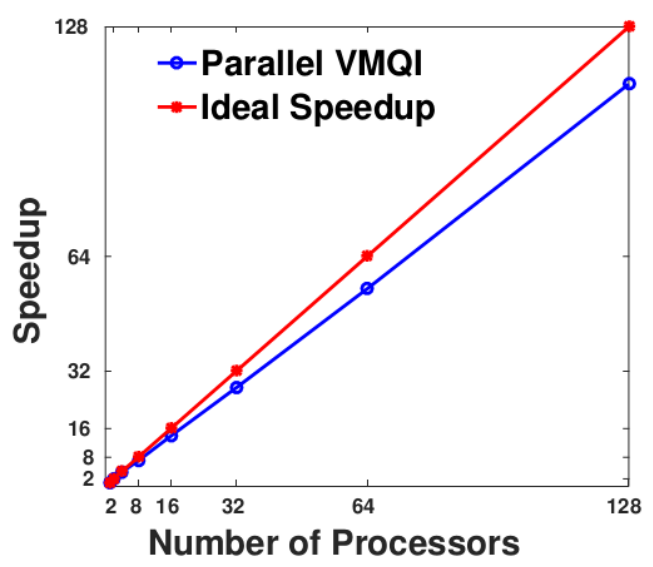

(b)

Figure 8: (a) Total runtime and (b) speedup for the Parallel VMQI algorithm for the $40 \mathrm{M}$ element tetrahedral mesh of the double cam tool domain

expected that the computation time is reduced by half each time we double the number of cores. However, the communication time does not show a clear growth pattern. Theoretically, for the ideal case, the communication time should exhibit logarithmic growth, but in practice this is not the case. For our case, the communication time is related to the architecture of the cluster and with the distribution and availability of nodes and cores at runtime.

We also performed a weak scaling test, which investigates how the solution time changes with respect to the number of cores (and assuming a constant workload per core), using various tetrahedral meshes for the bracket domain (see Tab. 2 and Fig. 10). For this experiment, we used the same parameters as in the previous test case, except for the initial $d t$ value, which was $d t=10^{-6}$ for this case. We made this change to better control the number of iterations in

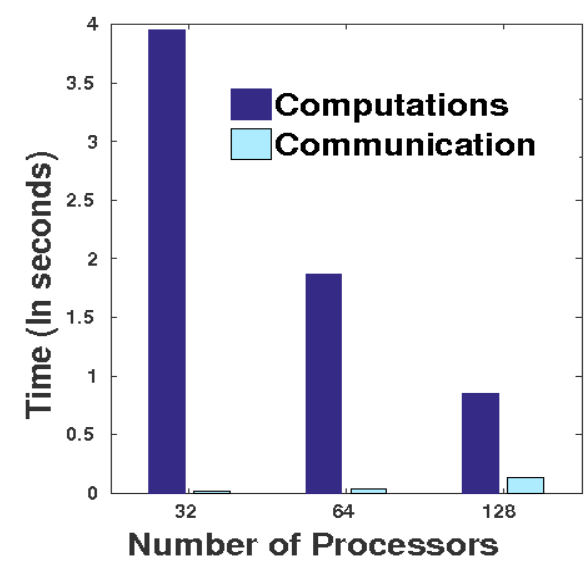

(a)

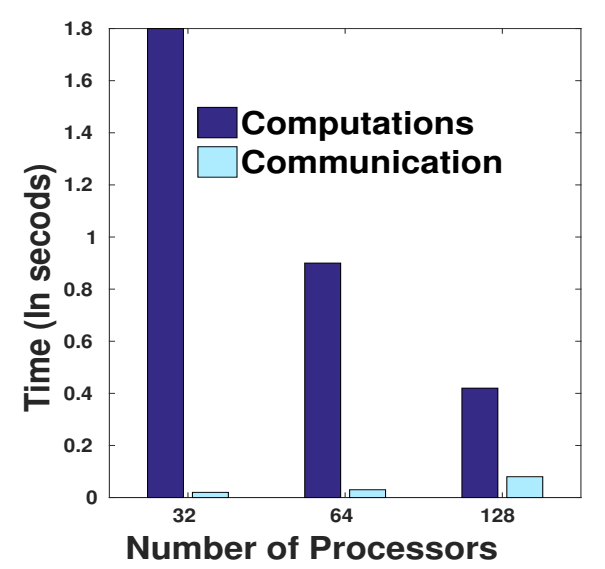

(b)

Figure 9: Communication and computation times employed for one iteration to calculate the nodal velocities in one region of (a) $80 \mathrm{M}$ element mesh of the bust domain and a (b) $40 \mathrm{M}$ element mesh of the double cam tool domain

each computational simulation. Figure 11 shows the weak scaling result for the algorithm. We observe a small deviation in the runtime for various numbers of cores. This deviation is at most six seconds, which is a deviation of less than $5 \%$ from the mean value. This behavior is a typical weak scaling result on unstructured mesh computations, as it is very difficult to double exactly the problem size as the number of cores is doubled. Also, since the number of iterations for each simulation might be different (see Fig. 12), the results from Fig. 11 correspond to the time the algorithm takes to run only ten iterations. 


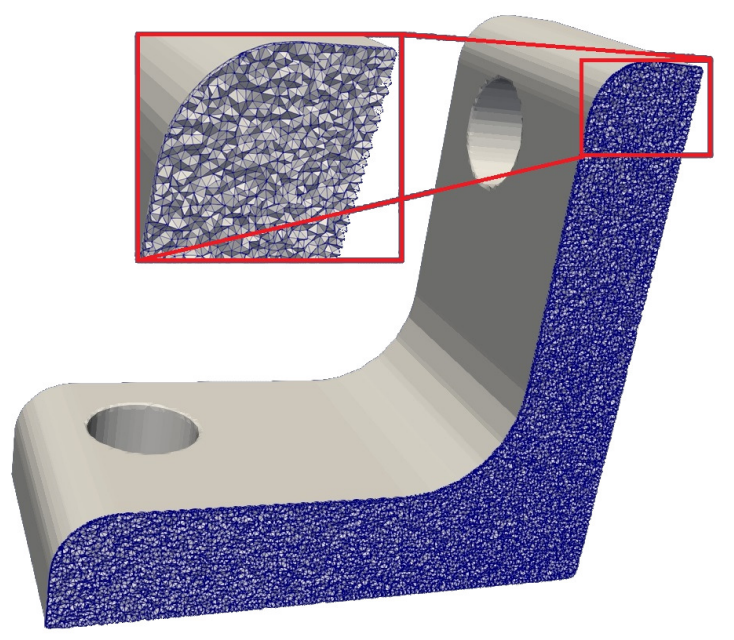

Figure 10: $20 \mathrm{M}$ element tetrahedral mesh of the bracket domain

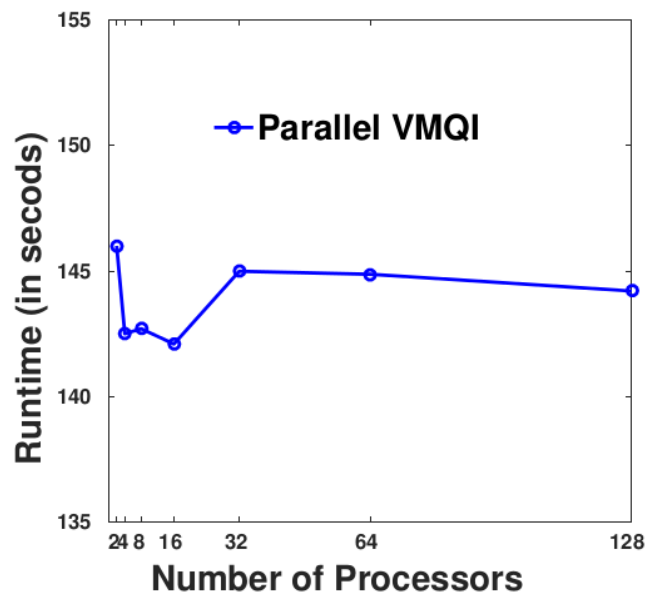

Figure 11: Runtime versus number of cores to test the weak scaling efficiency

\section{CONCLUSIONS AND FUTURE RESEARCH}

We proposed a parallel variational mesh quality improvement algorithm and an associated implementation for the method in $[20,21]$ for distributed memory machines. To distribute the workload among cores, we use METIS to generate a mesh partition composed by regions of connected elements. The algorithm identifies the elements in each region that contain at least one node that is shared by multiple regions (shared nodes). After distribution of the data (nodal coordinates, topology, boundary nodes), each core organizes its corresponding elements into two sets, i.e., the elements composed of only interior nodes and the elements which have at least one shared node.

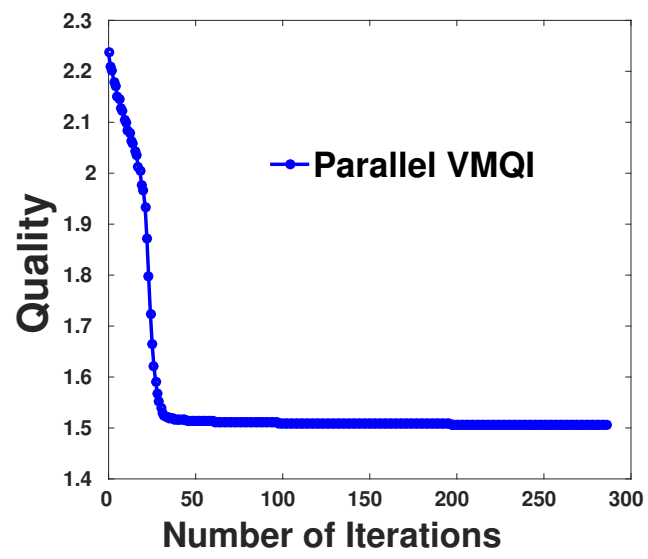

(a)

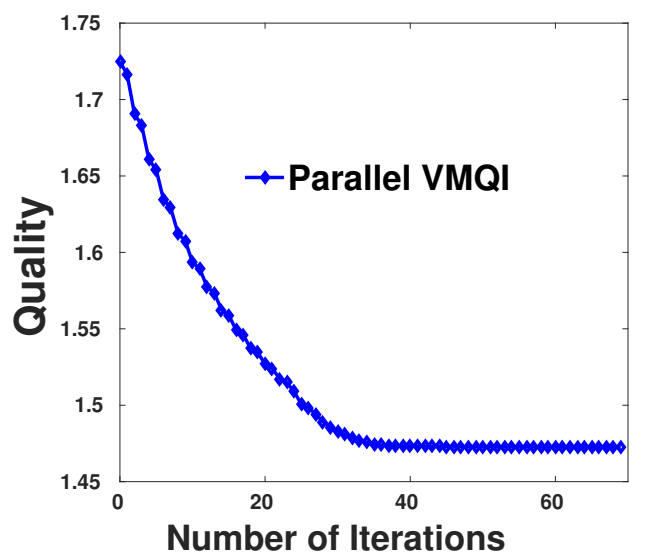

(b)

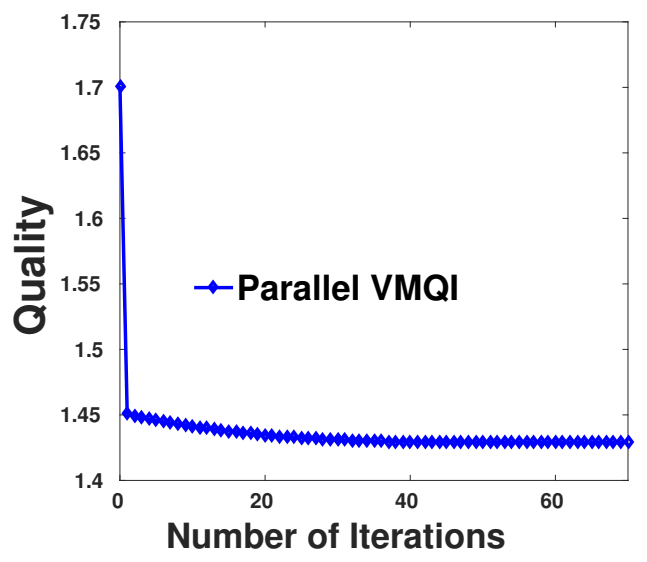

(c)

Figure 12: Quality versus number of iterations for the bracket domain with (a) $2.5 \mathrm{M}$, (b) $10 \mathrm{M}$, and (c) $40 \mathrm{M}$ elements

We employed the RKF45 method to solve the system of ODEs associated with the interior nodes. For this 
process, the parallel algorithm loops over all elements on each core to calculate the nodal velocities for each interior node. Whereas each core is able to calculate the nodal velocities for the interior nodes within its region, computing the nodal velocities of the shared nodes requires communication among cores. To do this efficiently, the algorithm first calculates the nodal velocities for elements containing at least one shared node. Then we communicate the partial nodal velocities of the shared nodes using a non-blocking collective instruction to overlap communication with computation of the nodal velocities for the interior nodes. When the number of interior nodes per core is high, a total overlap of communication and computation is achieved. Finally, the algorithm calculates the average quality of the mesh in each iteration and uses this information to terminate the computations when no significant improvement of the average mesh quality is observed.

We tested our parallel variational mesh quality improvement algorithm on three different 3D domains which were discretized using tetrahedral meshes. The results of our numerical experiments show good strong scalability and speedup for the meshes with $80 \mathrm{M}$ and $40 \mathrm{M}$ elements on up to 128 cores. The efficiency observed in the experimental results is the consequence of the complete overlap of communication and computation when calculating the nodal velocities (see Fig. 9 ). For the test cases presented in this paper, the major source of overhead occurs in the pre-processing step, i.e., where $P_{0}$ distributes the data and identifies the shared nodes. In addition to this, if the number of interior nodes on each core is relatively small compared with the total number of shared nodes, then the communication time among cores increase relative to the runtime. Hence we obtain a performance degradation, as a complete overlap of communication and computation is not possible. The weak scaling results we obtained are typical for unstructured meshes.

For future research, we plan to explore different communication strategies to minimize the memory consumption and communication time. A local-blocking communication strategy might decrease the performance for small number of cores, but it will perform better for a larger number of cores. In addition, a parallel pre-processing step will reduce the runtime and memory consumption for $P_{0}$. Another possible avenue for research is the adoption of a different domain decomposition strategy such as node coloring. In regards to applications, one can extent the same ideas presented in this paper to the variational mesh adaptation algorithms such as the one in [20].

\section{ACKNOWLEDGMENTS}

The work of the first author was supported in part by NSF grants OAC-1500487 (formerly OAC-1330056 and OAC-1054459), CCF-1717894, and OAC-1808553. The work of the second author was supported by NSF grant OAC-1500487. The work of all three authors was supported through instrumentation funded by the Army Research Office under contract W911NF-15-10377 .

\section{References}

[1] Lei J., Wang X., Xie G., Lorenzini G. "Turbulent flow field analysis of a jet in cross flow by DNS." J. Eng. Thermophys., vol. 24, 259-269, 2015

[2] Aliabadi S., Johnson A., Abedi J., Zellars B. "High Performance Computing of FluidStructure Interactions in Hydrodynamics Applications Using Unstructured Meshes with More than One Billion Elements." Proc. of the 2002 International Conference on High Performance Computing (HiPC 2002), vol. 2552, pp. 519-533. Springer Berlin Heidelberg, 2002

[3] Chan H., Lu Z., Chi X. "Large-scale parallel simulation of high-dimensional American option pricing." J. Algorithm Compt. Technol., vol. 6, $1-16,2012$

[4] Tian F., Dai H., Luo H., Doyle J., Rousseau B. "Fluid-structure interaction involving large deformations: 3D simlations and applications to biological systems." J. Comput. Phys., vol. 258, 451-469, 2014

[5] Chrisochoides N. "A survey of parallel mesh generation methods." A. Bruaset, A. Tveito, editors, Numerical Solution of Partial Differential Equations on Parallel Computers, pp. 237-264. Springer Berlin Heidelberg, 2006

[6] Freitag L., Jones M., Plassmann P. "A parallel algorithm for mesh smoothing." SIAM J. Sci. Comput., vol. 20, 2023-2040, 1999

[7] Jiao X., Alexander P. "Parallel feature-preserving mesh smoothing." Computational Science and its Applications ICCSA, vol. 3483, pp. 1180-1189. 2005

[8] Gorman G., Southern J., Farrell P., Piggott M., Rokos G., Kelly P. "Hybrid OpenMP/MPI anisotropic mesh smoothing." Procedia Computer Science, vol. 9, pp. 1513-1522. 2012

[9] Bentez D., Rodrguez E., Escobar J., Montenegro R. "Performance evaluation of a parallel algorithm for simultaneous untangling and smoothing 
of tetrahedral meshes." Proc. of the $23^{\text {rd }}$ International Meshing Roundtable, pp. 579-598. 2014

[10] Sastry S., Shontz S. "A parallel log-barrier method for mesh quality improvement and untangling." Eng. Comput., vol. 30, 503-515, 2014

[11] Cheng Z., Shaffer E., Yeh R., Zagaris G., Olson L. "Efficient parallel optimization of volume meshes on heterogeneous computing systems." Eng. Comput., vol. 33, 717-726, 2017

[12] Branets L., Carey G. "A local cell quality metric and variational grid smoothing algorithm." Eng. Comput., vol. 21, 19-28, 2005

[13] Zhang Y., Hamza A. "PDE-based smoothing from 3D mesh quality improvement." Electro/Info Tech IEEE Int. Conf., pp. 334-339. 2006

[14] Freitag L., Knupp P. "Tetrahedral mesh improvement via optimization of the element condition number." Int. J. Numer. Meth. Eng., vol. 53, 13771391, 2002

[15] Knupp P. "Jacobian-weighted elliptic grid generation." SIAM J. Sci. Comput., vol. 17, 14751490, 1996

[16] Shontz S., Vavasis S. "A robust solution procedure for hyperelastic solids with large boundary deformation." Eng. Comput., vol. 28, 135-147, 2012

[17] Kim J., Panitanarak T., Shontz S. "A multiobjective mesh optimization framework for mesh quality improvement and mesh untangling." Int. J. Numer. Meth. Eng., vol. 94, 2042, 2013

[18] Freitag L., Knupp P., Munson T., Shontz S. "A comparison of two optimization methods for mesh quality improvement." Eng. Comput., vol. 22, 6174,2006

[19] Huang W., Kamenski L., Si H. "Mesh smoothing: An MMPDE approach.", 2015. Research note at the $24^{\text {th }}$ International Meshing Roundtable

[20] Huang W., Kamenski L. "A geometric discretization and a simple implementation for variational mesh generation and adaptation." J. Comput. Phys., vol. 301, 322-337, 2015

[21] Huang W., Ren Y., Russell R. "Moving mesh partial differential equations (MMPDEs) based upon the equidistribution principle." SIAM J. Numer. Anal., vol. 31, 709-730, 1994

[22] Huang W., Kamenski L., Russell R. "A comparative study of meshing functionals for variational mesh adaptation." J. Math. Study, vol. 48, 168186, 2015
[23] Alliez P., Cohen-Steiner D., Yvinec M., Desbrun M. "Variational tetrahedral meshing." $A C M$ Trans. Graph, vol. 24, 617-625, 2005

[24] Hachem E., Feghali S., Codina R., Coupez T. "Anisotropic adaptive meshing and monolithic variational multiscale method for fluid-structure interaction." Comput. Struct., vol. 122, 88-100, 2013

[25] de Almeida V. "Domain deformation mapping: Application to variational mesh generation." SIAM J. Sci. Comput., vol. 4, 12521275 , 1999

[26] Knupp P., Robidoux N. "A framework for variational grid generation: Conditioning the Jacobian matrix with matrix norms." SIAM J. Sci. Comput., vol. 21, 2029-2047, 2000

[27] Liao G. "Variational approach to grid generation." Numer. Meth. PDE, vol. 8, 143-147, 1992

[28] Winslow A. "Adaptive mesh zoning by the equipotential method." Tech. Rep. UCID-19062, Lawrence Livermore National Laboratory, 1981

[29] Brackbill J., Saltzman J. "Adaptive zoning for singular problems in two dimensions." J. Comput. Phys., vol. 46, 342-368, 1982

[30] Dvinsky A. "Adaptive grid generation from harmonic maps on Riemannian manifolds." J. Comput. Phys., vol. 95, 450-476, 1991

[31] Huang W. "Variational mesh adaptation: Isotropy and equidistribution." J. Comput. Phys., vol. 174, 1643-1666, 2005

[32] Huang W., Russell R. Adaptive Moving Mesh Methods. Springer, 2011

[33] Huang W., Kamenski L. "On the mesh nonsingularity of the moving mesh PDE method." Math. Comp., vol. 87, 1887-1911, 2018

[34] Mathews J., Fink K. Numerical Methods Using MATLAB. Prentice Hall, third edn., 1999

[35] Karypis G., Kumar V. "A fast and highly quality multilevel scheme for partitioning irregular graphs." SIAM J. Sci. Comput., vol. 20, 359392, 1999

[36] 3D Models, CAD Solids - 3D CAD Browser, 2001-2019 (accessed 6/17/2019). URL http://www.3dcadbrowser.com/

[37] GAMMA3: Automatic mesh generation and adaptation methods, (accessed 6/17/2019). URL https://team.inria.fr/gamma3/ 
[38] MeshLab, (accessed 6/21/2019). URL http://www. meshlab.net

[39] Si H. Tetgen: A quality tetrahedral mesh generator and three-dimensional Delaunay triangulator, (accessed 6/17/2019). URL http://wias-berlin.de/software/ 


\title{
UPDATING AND RE-MESHING VIRTUALLY DECOMPOSED MODELS
}

\author{
Benoit Lecallard ${ }^{1}$, Christopher M. Tierney ${ }^{1}$, Trevor T. Robinson1ㅜ, Cecil G. Armstrong ${ }^{1}$, \\ Declan C. Nolan ${ }^{1}$, Alexander E. Sansom² \\ 'Queen's University Belfast, Belfast, U.K. t.robinson@qub.ac.uk \\ ${ }^{2}$ Rolls-Royce Plc, Alexander.Sansom@rolls-royce.com
}

\begin{abstract}
Generating hexahedral meshes is often an expensive process, which limits the use of high-fidelity numerical simulation methods for design. Hexahedral meshes can be generated by decomposing a geometric model into simpler meshable regions, but robustly propagating design modifications to the decomposed representation makes any attempt to update the mesh very challenging. In this paper, a virtual topology workflow enabling automatic generation of hex-dominant meshes is extended to propagate parametric modifications and feature changes to the decomposition and resulting mesh. Geometric and topological modifications are identified and linked to the decomposition through virtual topology relationships. Modified regions are localized and reasoning on the virtual decomposition enables their definition and associated meshing strategy to be updated. Instead of starting the meshing process from the beginning, only modified cells are re-meshed. This provides an efficient and automated method to propagate design changes down to the analysis model.
\end{abstract}

Keywords: Automatic decomposition, mesh generation, mesh update, hexahedral, parametric models, virtual topology

\section{INTRODUCTION}

The increasing use of finite element analysis throughout a product lifecycle is limited by the ability to generate appropriate simulation models. This is especially true for the simulation of complex events, such as crash or large displacement analyses, where generating the hexahedral (hex) elements preferred for this task is very user-intensive work. Simulation-based design depends on the ability to quickly generate analysis models for many design variations to run an optimization procedure. This is incompatible with the manual analysis set up required in a typical hex meshing workflow. Analysis requirements are also prone to change, especially within coupled multi-physics analyses where analysis results from one domain dictate updates for another. For example, if a model is deformed by wear or thermal expansion and is used as an input for subsequent analysis these changes must be reflected in the downstream analysis model.

Many tools have been developed during the last decades in an attempt to automate hex meshing with various results [1]. They include direct methods such as Whisker Weaving [2] and Plastering [3], as well as indirect methods such as tetcombination [4]. Decomposition-based methods partitioning the design geometry into smaller sub-regions for which a simple meshing strategy can be found are the most widely used methods. These meshing strategies include mapping [5], sub-mapping [6] and sweeping [7], where a quad mesh of a source face is swept in order to generate $3 \mathrm{D}$ hex elements. Even if these tools fail to tackle generic geometries, they can be integrated in an incremental decomposition workflow to significantly alleviate the workload of generating meshes. Virtual topology-based decomposition, coupled with cellular modelling for meshing workflows, has shown promising results and flexibility [8][10]. The benefits of using virtual topology (VT) for generating meshes without altering the CAD model definition were first presented by Sheffer et al. [11]. In the context of this work, a cellular model is a decomposition of space into cells of analysis significance. The interfaces in the model are robustly captured and are cells in their own right [12]. This structure means that the links between the decomposed virtual representations and the design model can be robustly maintained [13]. In addition, as a model is decomposed for meshing, the cellular representation maintains connections between the subset domains that enable both the automation of downstream meshing and the localization of modified cells after design changes.

After design changes, the mesh needs to be updated to remain an accurate representation of the model. In the Computer-Aided Design (CAD) environment, updating the 
design is straightforward, either by changing parameters associated to the model or by adding/removing features in the construction tree. However, applying the same design modification to geometrically identical models that are constructed with different feature orders may produce unexpected differences that may be inadvertently propagated to the analysis model.

Direct geometric editing (synchronous technology) is also available, where the user can interactively manipulate geometric entities without requiring access to the construction tree, allowing modifications of the model outside of the design environment. However, the analysis model is often constructed in a separate Computer-Aided Engineering (CAE) package, where such manipulations will break the link to the original CAD model, and CAD-CAE integration is a major bottleneck toward automation[14]. As a result, the mesh cannot be as easily updated as the construction tree and parameters are lost during the transfer across packages. The user is often tasked to update the CAD model before exporting it to the meshing environment where many pre-processing activities, such as clean-up operations, carried out to create the previous analysis model must be repeated. Furthermore, most automated tools for hex-mesh generation [2]-[4] do not offer the ability to efficiently update the mesh and require the whole decomposition process to be repeated. One challenge is that decomposition tools are often used to partition the design model geometrically to create a decomposed representation fit for hex meshing. These geometric partitions usually break the links between the design model and the decomposed representation, meaning that even if design changes can be identified there are no relationships that can be exploited in order to robustly update the decomposition. The major challenges to automatically updating a hex-dominant mesh created from a decomposition via the approach proposed herein are to:

- Identify the geometric and/or topological changes resulting from a design update.

- Reflect design amendments on the analysis model by exploiting the virtual topology relationships stored when generating the initial decomposition.

- $\quad$ Update the decomposition used for meshing and ensure it remains valid.

- $\quad$ Minimize the computational expense by re-using as many existing elements as possible.

- $\quad$ Maintain mesh quality after update.

This work proposes to extend a virtual topology decomposition workflow to address this problem, by using an integrated cellular model to reflect parametric and feature changes on the mesh. This paper first describes the virtual topology framework used for automatic decomposition and hex-dominant meshing. Then, handling feature and parametric perturbations for re-meshing is presented. This is done by first localizing the modifications, then updating the analysis topology and finally updating the mesh locally. The main contribution is the implementation of a cellular mesh and interface management to enable mesh updates even when the topology of the model is significantly altered. Considerations on meshing strategies are presented to help update the decomposition and the mesh.

\section{RELATED WORK}

Mesh update has been a topic of research for many years and includes several domains such as mesh morphing, mesh adaption and re-meshing. Mesh morphing [15] consists of mapping an initial mesh onto a new geometry which is similar to the initial geometry, either to account for the model deformation, or to re-use an existing mesh on a similar geometry (e.g. a design update). The mapping of nodes requires knowledge of both the entities mapping between the geometries and the node to entity associativity in the original mesh. The mesh topology must remain constant to ensure a correct mapping.

Mesh adaption [16] consists of modifying a mesh against known quantities. It can be an iterative process to minimize the simulation error while solving the computational problem iteratively. Parametric modification in an optimization loop can also drive mesh adaption procedures. The mesh can be locally refined or coarsened, with or without connectivity (or number of elements) modifications. Sheffer and Üngör have proposed a dual representation using both the boundary and a parametric representation to link design modifications and mesh adaption procedures [17]. In particular, the history of virtual operations applied for simplification is retained and automatically mapped on the updated design model before meshing. Mesh updating is done by moving the elements to the new geometry, and then adjusting the mesh quality by using techniques such as whisker-sheet operations. Feature displacements have been investigated recently by Shen et al. [18], using mesh deformation and the mesh is refined using density fields extracted from the initial mesh. However, adaption approaches are limited to simple parametric perturbations, as the mesh topology needs to be consistent to be mapped.

Parametric perturbations can induce topological modifications on a model. To this extent, Van Der Meiden and Bronsvoort have defined a method to relate the range of parameters to topological entities, therefore identifying critical parameters of interest [19]. Sun et al. have proposed a method using virtual topology to deform a surface mesh in the presence of simple topological perturbations [20].

Re-meshing is employed whenever mesh generation methods need to be re-applied either locally or globally to the model. For example, fully re-meshing a model can be avoided after feature insertion if the feature is meshed with new elements and connected to the existing mesh. Smit and Bronsvoort have successfully implemented a cellular modelling-based approach to tetrahedral re-meshing [21]. After capturing the feature differences between two models and their interactions, all the valid original nodes are mapped to the new model, and new elements are created to fill the gaps. However, in the absence of any link or equivalence between elements and geometric cells, all the nodes need to be classified and processed for re-meshing, which can be slow for very large meshes. 

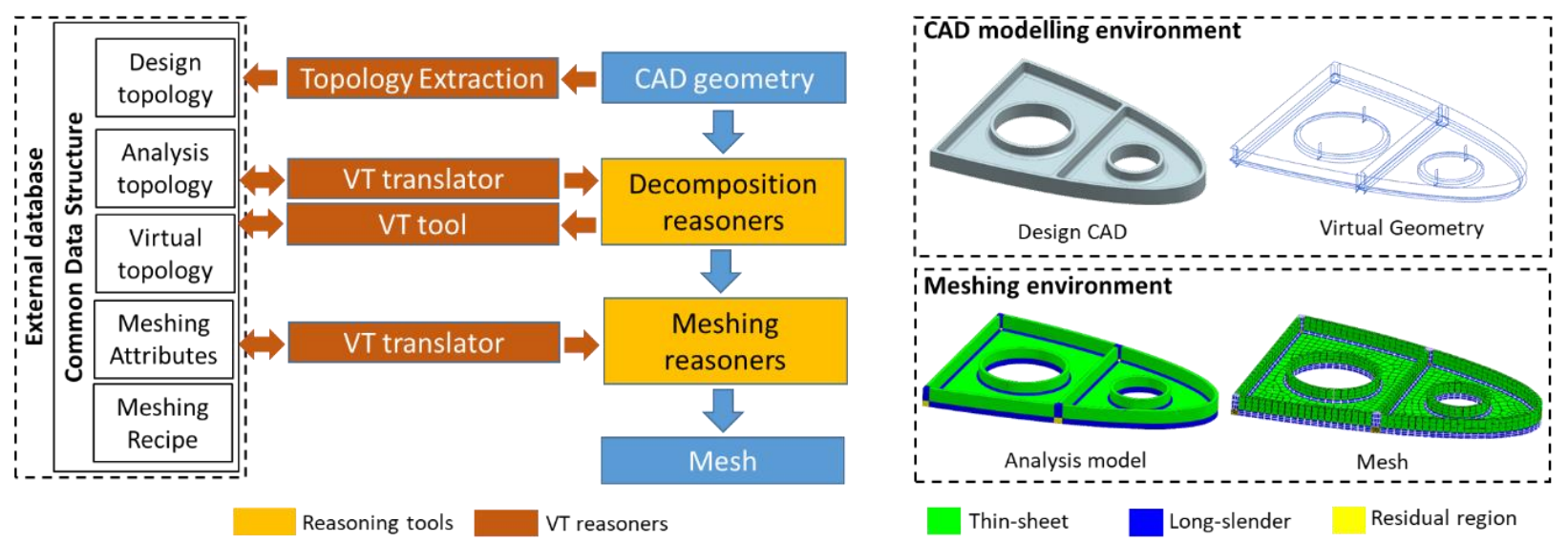

Figure 1. Virtual topology-based workflow for automatic decomposition and meshing.

In this paper, hex-dominant re-meshing is addressed using a cellular representation of a virtually decomposed model. Only the sub-regions that need to be modified to accommodate the design change are identified and remeshed, while maintaining the constraints of a structured mesh. This allows design variations to be automatically propagated throughout a virtual topology workflow and reflected on the final mesh (Figure 4).

\section{VIRTUAL TOPOLOGY DECOMPOSITION AND MESHING}

This section describes a virtual topology-based workflow that integrates various reasoners for automatic decomposition and meshing (see Figure 1).

The process takes a CAD model as an input and has two main outputs. The first is a topological description of the decomposed model called analysis topology, contained in a Common Data Structure (CDS). The second is a mesh in a CAE environment that is exported to a neutral format file. The original topology is first extracted from the CAD to the CDS, and a series of virtual topology split operations are applied to create the analysis topology. This analysis topology contains the virtual decomposition and is linked to the original design topology through virtual topology relationships stored in the CDS. Once the model has been virtually decomposed, a mesh can be automatically generated by using the meshing recipe reasoner (section 3.4) and the mesh reasoner (section 3.5). Virtual topology allows more freedom for preparing a model for meshing and provides much more flexibility since the actual geometry is not modified. Instead of storing partitioning surfaces, only the method to construct them is stored (along with virtual geometry curves, curves that are not connected to the B-Rep design model but exist in the CAD environment, see section 3.3) and passed to the meshing environment to generate the mesh.

\subsection{Design topology extraction to CDS}

The role of the common data structure (CDS) is to convey information between the different steps and packages involved in the automated virtual workflow. It is based on an external SQL relational database, similar to the one presented by Tierney et al. [22], [23]. The relations relevant to virtual topology decomposition and meshing are shown in Figure 1. Other data that does not fit within the CDS is transferred using neutral formats. For example, virtual geometry can be transferred using STEP or Parasolid format. Meshes can be transferred using formatted text files, such as bulk data files that contain nodal information and element connectivity. The CDS contains the links to connect these various representations.

The CDS is initialized by querying all the entities contained in the CAD model through a topology extraction tool (Figure 1). Each entity is assigned a unique identifier when added to the database, which is linked to the name and/or tag attribute from the CAD system. Geometric attributes such as coordinates for vertices or mid-points for edges are also stored to aid tracking entities. Higher dimension entities can also be identified from their bounding entities.

The topological definition of the CAD model is extracted and stored in a design topology relation, which is duplicated in an analysis topology relation. Both topology relations contain a cellular representation of the model, with each topologic cell defined recursively from lower dimension elements forming their boundaries, along with the relative orientation between the bounded cell and its boundary.

To enable virtual topology manipulations, a virtual topology relation stores the history of the virtual operations applied on the design topology to create the decomposition in the analysis topology. The virtual topology relation contains the link between the virtual entities (subset or superset depending on the virtual split or merge operation) and their host entities.

\subsection{Decomposition reasoners}

Regions suitable for hex mesh generation are identified using a sequence of decomposition reasoners within the proposed virtual topology workflow. A decomposition reasoner encapsulates an algorithm to identify regions suitable for a specific meshing method (e.g. sweeping, mapping, template...) in a generic way. Implementation specific routines are handled outside of the reasoner, which 
can be seen as a black box from the point of view of the process. The input is a design model with its topology extracted in the CDS. The output is splitting information which is used by the virtual topology tool to partition the domain, and a meshing strategy attribute specific to the reasoner. The power of this virtual topology approach is that multiple decomposition reasoners (sweep, multi-sweep, 3D block, 2D block...) can be integrated seamlessly within one workflow

All the geometric queries of the reasoners are made through a VT translator tool, which uses information stored in the $\mathrm{CDS}$ and the combination of the CAD and virtual geometry to query the analysis topology model and not the design model. Hence, the reasoner can operate on a model that has been virtually decomposed beforehand. This ensures that reasoners can be used one after another and in any order, but also allows virtual defeaturing to be carried out before the decomposition and not just as a final step before meshing. This ability to operate in the presence of virtual topology is critical for a robust analysis workflow.

After the reasoners have identified which region to extract, the splitting information is created. This is done by identifying which entities need to be partitioned, and what existing entities can be used to do so. Necessary geometric information such as points or curves to complete the definition of a split are also created by the decomposition reasoners.

\subsection{Virtual Topology reasoners}

Virtual topology uncouples the topological definition of a model from the geometrical one. This enables virtual generation of a meshed analysis model without altering the design model. Two VT reasoners are used in this process. The VT translator transfers geometric information between the real CAD model and the virtually decomposed model in the CDS. The VT tool manipulates topological representations in the CDS to clean or decompose a model, by applying operators as described in [8], [11]. These operators ensure that the analysis topology remains valid after topological manipulation, with the relative orientation of the virtual entities and the modifications properly recorded and updated in the CDS.

The VT translator processes the geometrical splitting information from the decomposition reasoner to create in the $\mathrm{CAD}$ environment all the curves required to define partitioning faces. These curves are referred to as virtual geometry, since they only exist as a layer of geometry independent from the design model in the CAD environment. Virtual geometry curves are used as an input by the VT tool to virtually partition the model to create the analysis topology. This analysis topology is linked to the design topology by a series of VT relationships resulting from the application of VT operators, stored in the CDS.

Virtual topology requires definition of virtual entities to formalize the relationship between virtual entities and their host entities (if any) [11]:

- Parasite entities: entities that did not exist in the original topology but lie on an existing entity of higher dimension (i.e. an edge lying on the face it splits or a face that lies in the interior of a body).

- $\quad$ Subset entities: subsets of host entities that are split by a parasite entity of lower dimension.

- Orphan entities: entity without host (e.g. an edge in the interior of a body bounding only parasite faces).

A virtual split operation uses a parasite entity on a host entity of higher dimension to create subset entities. For example in Figure 2(a), a face $f 1$ which is a bounded portion of a geometric surface can be split by adding a parasite edge $e l$ which is a bounded portion of a curve. The parasite edge lies on the host shape to divide it into two subset faces $f 2$ and $f 3$, but a single surface definition remains.

A virtual merge operation on the other hand groups multiple host entities into one by removing lower dimensional entities common to the hosts at their interfaces. For example, in Figure 2(b), a vertex $v 2$ bounding only two edges can be removed to generate one superset edge e4. A merge operation is required to update a modified decomposition since it enables the recombination of adjacent cells locally, without having to undo the whole decomposition operation. It can also be used to simplify and clean the model definition to facilitate mesh generation. a)
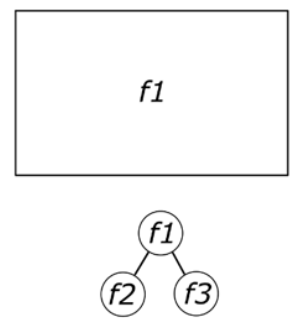
Virtu

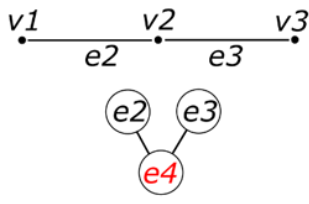

Virtual Topology relation
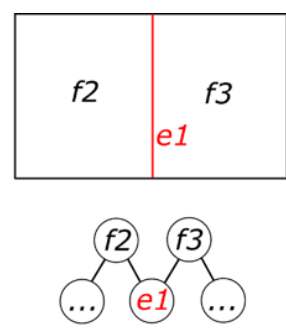

Analysis Topology relation

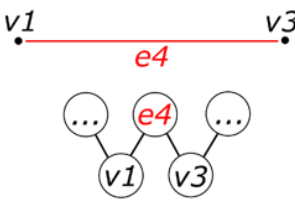

Analysis Topology relation
Figure 2. (a) The face $f 1$ is virtually split by inserting the parasite edge e1, and (b) edges $e 2$ and $e 3$ are merged into $e 4$ by virtually removing v2. Red entities are virtual geometry.

Virtual parasites, subsets and orphan edges are superimposed on the CAD design model as virtual geometry. All their links with the analysis model only exist in the CDS, and their purpose is to provide geometric information that does not exist in the original design model. They are created by a decomposition reasoner and can be easily transferred between packages using a neutral format such as STEP. Once added in the CDS, they can be used to aid virtual topology manipulations.

\subsection{Meshing recipe reasoner}

The meshing recipe reasoner is used to translate the different meshing strategies identified by the decomposition 
reasoners into compatible mesh controls for hex-dominant mesh generation. The input is a CDS containing the analysis topology of the decomposed model along with the meshing strategy identified for each volume cell, and geometric information, such as aspect ratios of regions and curve lengths, previously extracted from the CAD. The reasoner outputs optimized division numbers for every curve and meshing methods for faces to the CDS.

Different decomposition reasoners can be applied to the model which will result in different meshing strategies with different priorities. The specific reasoners used in this work sought to create an anisotropic hex meshing recipe which was conformal throughout.

Sweeping strategies are converted into constraints on the number of elements following the methods applied in [9]. Source faces of sweepable regions can either be paved or mapped, while wall faces must be mapped meshed. Figure 3 shows how the meshing constraints propagate through the model, and the resulting mesh. Soft or hard goals on division numbers are applied on each edge of the model. A hard goal ensures a fixed division number will be applied (e.g. number of elements through thickness), while soft goals are optimized to meet the constraints. Constraints are checked to remove overly constraining mapping equalities. All the necessary geometric information is contained in the CDS, hence this reasoner is package independent.

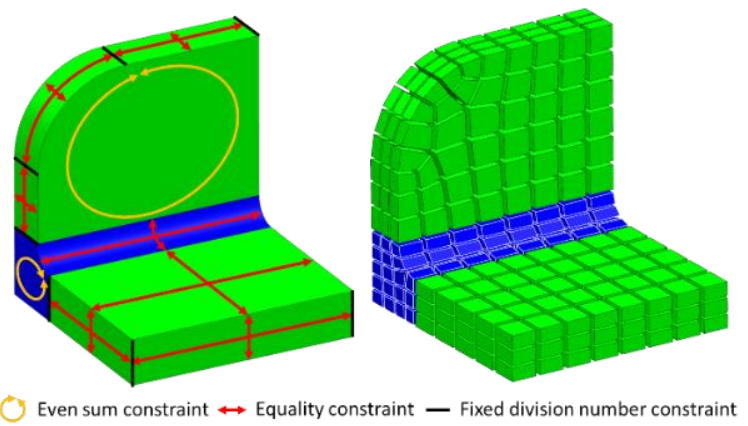

Figure 3. Flow of meshing constraints and associated mesh.

The LPSolve [24] package is used to optimize each individual number of elements on curves, by implementing a revised simplex algorithm. As a result, the mesh is fully constrained, which ensures order independence during the meshing step, and guarantees a conformal mesh will be obtained at interfaces.

\subsection{Mesh reasoner}

Once the meshing recipe has been generated, the mesh can be generated in a CAE environment using the meshing reasoner. The input to the mesh reasoner is the CAD model and the CDS containing the meshing recipe. The output is a conformal mesh.

The virtual partitioning surfaces are explicitly rebuilt from the virtual geometry and used to split the geometry of the model, hence becoming interfaces between sub-regions. Depending on the package used, the model is transferred to the meshing environment before or after the geometric decomposition which will create all the analysis topology entities. Mesh densities contained in the meshing recipe are applied on each curve.

All the interfaces are checked and meshed first to ensure a conformal mesh is obtained. The 2D surface meshes of all the interfaces are stored in a common neutral format file, with elements grouped by interface identifiers. This step is required to enable mesh manipulation later, but it also offers the possibility of the 3D meshes being generated on the individual cells in parallel. All the source faces of sweepable regions are meshed first, and hex elements are created by sweeping. Residual regions, where there is no known hexmeshing strategy identified by the reasoners, are tet-meshed at the end, after a layer of pyramid elements has been inserted to conform to the quad mesh of the interfaces. If the decomposition reasoners have identified hex-meshing strategies other than sweeping, the relevant meshing algorithms can be applied by the meshing reasoner.

The mesh is then exported in a neutral format file such as a Nastran input deck. This format enables the mesh to be transferred into different meshing packages, and to be edited simply by editing the mesh file.

\subsection{Integrated workflow}

The choice and sequence of decomposition reasoner to apply is left to the user, while pre-defined workflows can be identified for specific classes of geometries. This sequence will define which meshing methods will be used, since the same region could be identified by different reasoners for different hex-meshing methods. An example of a virtual decomposition workflow for automatic meshing is shown in Figure 1. It includes a thin-sheet decomposition reasoner for identifying thin regions which can be sweep-meshed through their thickness and a long-slender decomposition reasoner for identifying regions with one large dimension suitable for sweeping. Models of thin-walled components are suitable for thin-sheet extraction, where regions with one small dimension compared to the other two offer a simple sweepmeshing strategy [25]. Pairs of large parallel faces are discretized and imprinted one onto another in order to calculate their intersection in the parametric space. The result is then projected back on the boundary representation to identify appropriate partitioning geometry, which will be used to create the virtual geometry and the virtual split operations for sweep meshing through the thickness. This integrated virtual topology workflow effectively demonstrates multi-sweeping in thin-walled components, with explicit interfaces in the decomposed cellular model facilitating multi-directional sweeping.

Truss-like structures, or thin-walled structures with their thin sheet removed, feature a lot of long-slender regions. A similar approach to Sun's method [26] is used to extract such regions. Long edges with a large aspect ratio relative to the width of the faces they bound are identified and grouped into loops. These loops are then used to find loops of mappable faces, which verify the conditions for sweep meshing. Cap faces are identified as a loop of virtual edges. There may also be an offset applied if the geometry is prone to the existence of skewed elements. This virtual geometry is then used to help virtually split the analysis model. 

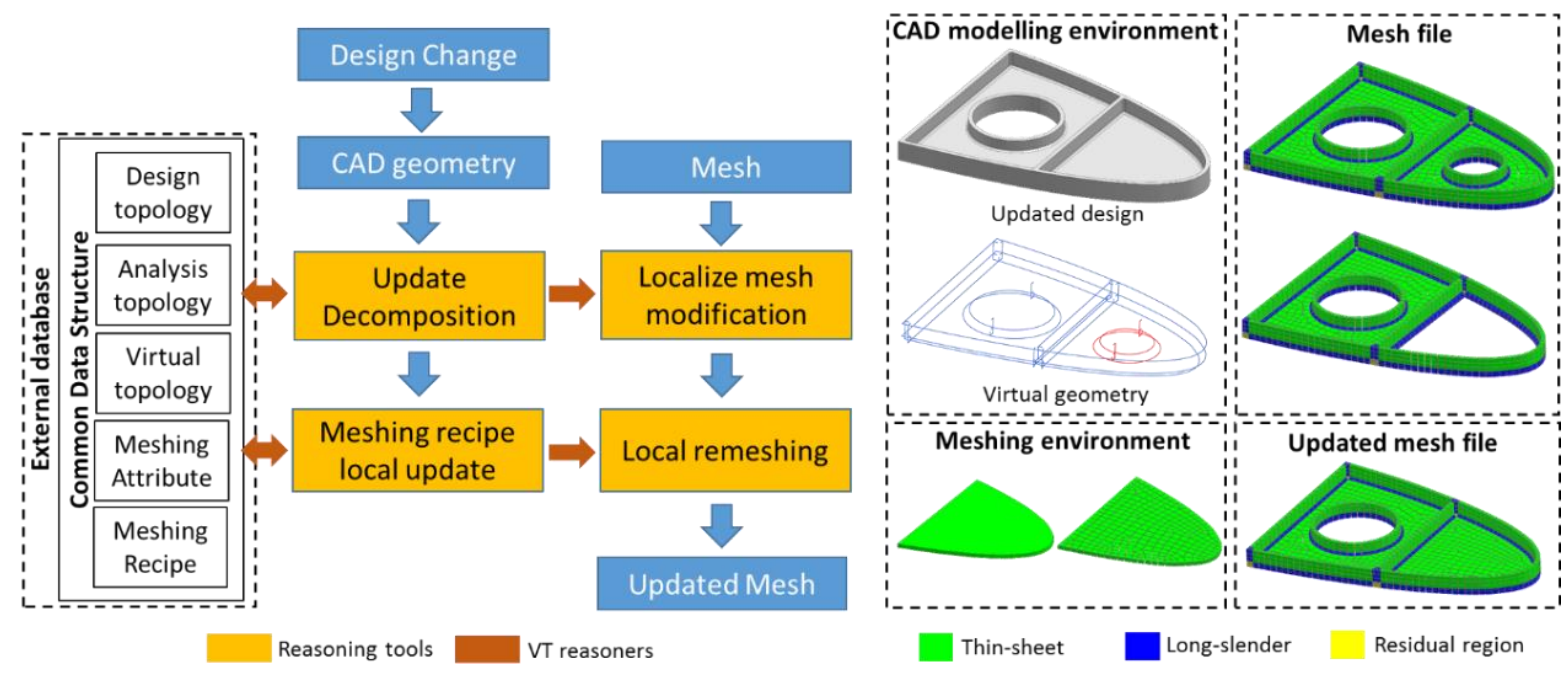

Figure 4. Workflow for updating the decomposition and mesh after design change.

Region attributes such as whether a region is thin-sheet or long-slender are stored in the mesh recipe relation of the CDS. This relation, along with the cellular model of the analysis topology, informs the reasoner tools and enables automatic identification of the meshing recipe. The meshing recipe is then stored in the CDS, describing face and edge meshing constraints in terms of size or number of elements.

Other decomposition reasoners have also been developed to identify simple sweepable regions or to decompose models into axisymmetric regions and repeated cyclic sectors, providing a minimal meshable representation [27].

\section{UPDATING THE DECOMPOSITION}

Figure 4 shows how the virtual workflow described in the previous section can be extended to handle design modifications to update the decomposition and ultimately the mesh. This section describes first how design modifications are identified by comparing the new design with the one stored in the CDS. Then, the constraints stemming from the hex-meshing strategies assigned to regions guide the update of the virtual geometry and the analysis topology. This reasoner takes a CAD model with a design change and the CDS associated with the previous version of the design as an input, and outputs an updated CDS for the new design (with updated virtual geometry), which can be used to update the mesh.

Modifications of the design can have various effects on the boundary representation of a model, especially for decomposed models where the number of boundary entities is increased. Figure 5 shows an example of a model decomposed for sweep-meshing undergoing various design modifications. Any design changes on a model can be classified into the following types:

- Topology only modifications, where the boundary representation is modified but not the shape. For example, introducing imprints on a face subdivides the face but the underlying surface geometry remains the same.
- Geometric only modifications, e.g. Figure 5(c) where only the geometry of the design is modified by changing the part length. All topology remains unchanged.

- Geometry and topology modifications, e.g. where new features, such as bosses, fillets etc. are added or removed from a model, Figure 5 (d), or where a parametric perturbation results in an additional topology change.

In order to update the decomposition, it is necessary to propagate the aforementioned modifications to the analysis topology. More specifically, the parasite entities used to virtually decompose the model must be modified (if necessary) alongside the virtual geometry in order to enable the mesh to be updated. In this work, design modifications can affect:

- Only the analysis model geometry. In this case it is necessary to determine if the virtual geometry needs to be updated, e.g. in Figure 5 (c) where the change in part length $\mathrm{L}$ requires the invalid virtual geometry (dashed red lines) to be morphed to the new model boundary.

- Both the analysis topology and virtual geometry, e.g. feature modifications will trigger geometric and topological modifications to propagate to the analysis model, such as removing the fillet in Figure 5 (d).

If the parametric perturbation has modified the design topology, then the analysis topology is also modified. However, it is possible the topological connectivity of parasite entities can be modified without changing the design topology. For example, the thickness $t$ of the bottom pad is increased in Figure 5 (e), resulting in parasite entities whose configuration is now altered. The two parasite faces were disconnected in the original decomposition. However, in the updated decomposition, Figure 5 (e), they now share a common edge (in dashed bold). These changes can be subtle but will have a profound impact on updating the mappings required to update the mesh automatically. 
a)

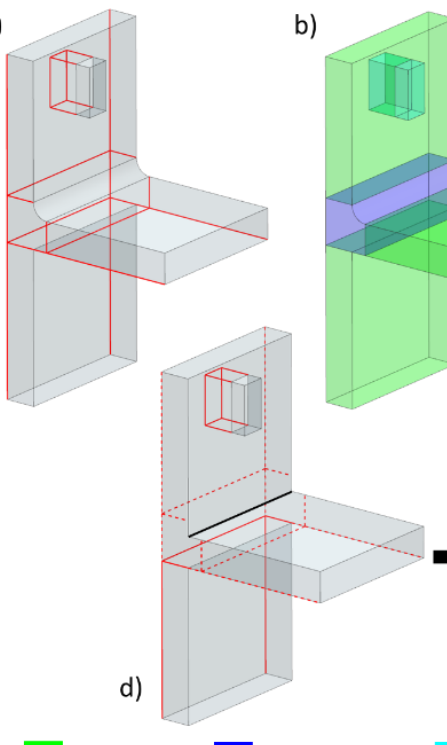

Thin-sheet
)

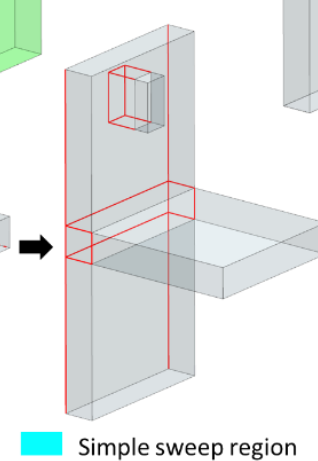

c)

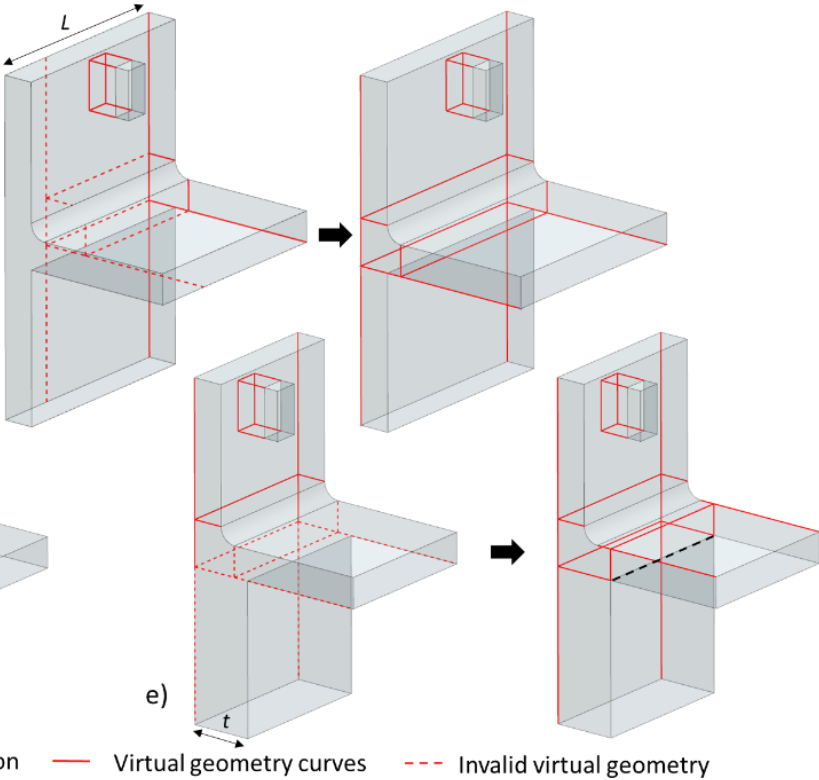

Figure 5. (a) initial decomposition, (b) corresponding meshing strategies, (c) geometric only change, (d) topological modification and (e) only the analysis topology is modified, one edge has an invalid projection.

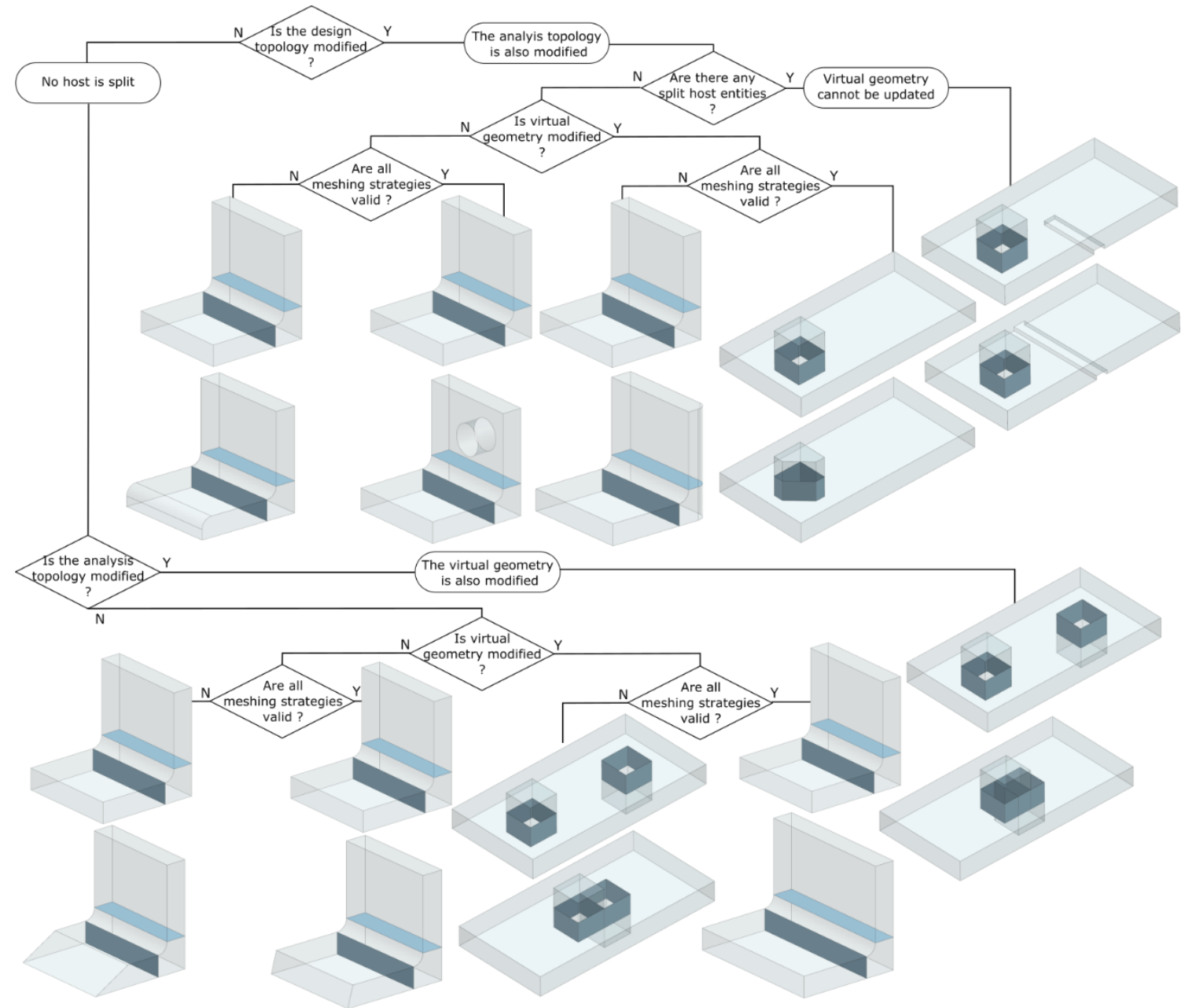

Figure 6. Analysis topology before and after design modification for various configurations. 
Figure 6 shows procedures used in this work to determine the classification for the various geometric and topological configurations for decomposition update that can arise upon design modification (virtual faces shown in dark grey for visualization). This structure has been determined to be the most suitable for the mesh types being used in this paper, however a different structure or ordering may be better suited to different models, or the requirements of different analysts. Although only design changes involving geometrical modifications are used to illustrate the workflow, topological only modifications are handled in the same way. Some configurations are easy to update, e.g. for a purely geometric update where both the boundary topology and virtual geometry have not changed. However, the top right configuration is very challenging to update, as the bottom host face on which the boss edges were projected has become two unconnected faces due to the extension of the pocket. This is related to the persistent naming problem [28], where parametric modifications trigger topology changes that modify the underlying geometry.

The workflow in Figure 9 describes the method used to identify the aforementioned design changes and to update a virtually decomposed model after such design changes. Topological and geometrical modifications are identified from the CAD model. After the design modifications have been identified at the design topology level, analysis model modifications need to be identified. This is done by checking if the virtual decomposition history can be mapped on the new design, by checking if all the virtual splitting entities still lie within their hosts. Mapping constraints inferred from hex-meshing strategies are checked to ensure they are still valid and can inform the update of projected virtual geometry. Finally, all the candidate bodies for re-meshing are identified.

\subsection{Tracking parametric and feature modifications}

The CDS contains a representation of both the analysis topology and the original design topology independently from the CAD environment and also stores the virtual topology relationships required to transform one into the other. The original topology in the CDS is used to identify and classify both geometric and topological modifications after the CAD model has been updated. The VT relationships provide the link to map the changes in design to the analysis topology.

This section will describe how changes to the model in the CAD environment are propagated to the original topology in the CDS and then to the analysis topology describing the decomposition. The key point is that all entities in the original topology and analysis topology are linked to those in the $\mathrm{CAD} / \mathrm{CAE}$ environment through two different attributes:

1) Name attributes attached to entities in the $C A D$ environment. Any unique identifier offered by the CAD system (name, tag, color ...) can be used, provided that is can be assigned to an entity, queried and will persist between different modelling sessions.
2) Geometric attributes defining unique geometric identifiers of entities in the CAD environment, e.g. the center point of the edges, as well as the coordinates of its end vertices.

Both attributes are necessary, as structured interrogation of them allows the geometric and topological modifications to the design to be determined as outlined in the following sections. Once modified entities have been identified, each entity is mapped to an entity in the analysis topology through a series of VT relationships and topological queries. This enables the modifications to be identified and the entities of the analysis topology to be classified.

This classification is done from lower dimension entities to higher dimension ones, since any modification on the boundaries of an entity will propagate to the entity, while an entity can be modified without having its boundaries modified. While some CAD packages offer the ability to attach name attributes to vertices, other packages have not implemented this capability. Coordinates used as geometric attributes are not enough to classify vertices in the absence of name attribute, as a design change can move a vertex to the location of a different vertex that is also modified. However, the matching of the geometric attribute for edges includes checking the coordinates of both the mid-point and the bounding vertices. Therefore, the edge classification is based on the vertex classification, but not only as the midpoint factors as well. In this implementation, edges are classified first, so that vertex classification can be guided by the bounded edges classification.

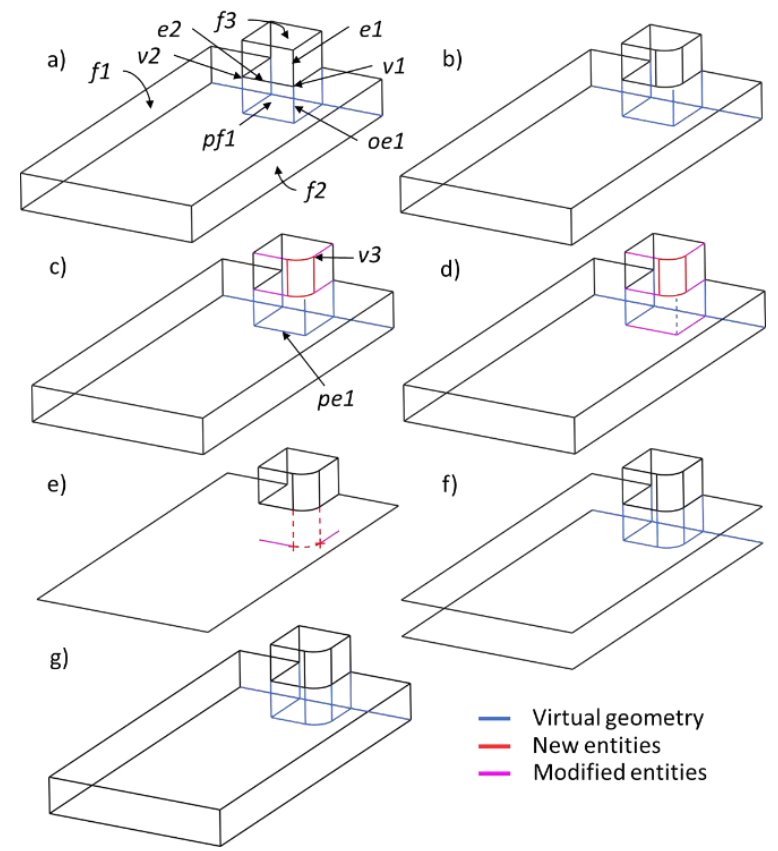

Figure 7. a) Original decomposition, b) the decomposition is not updated after a fillet is added, c) original entities classification, d) analysis entities classification, e) open design loops are closed and new virtual entities are identified, f) open analysis loops are closed and g) updated decomposition. 


\subsubsection{Edge classification}

After parametric or feature modification, all the original edge entities in the CAD model are compared to the ones stored in the CDS and classified as follows:

- Original edge: If both the name and geometric attributes match, then it is assumed that the edge has not been modified (black edges Figure 7(c)).

- Modified edges: if the name is matched but not the geometric attributes, it is assumed that the edge has moved and is modified (purple edges in Figure 7(c)).

- New edges: Edge from the CAD whose name is not matched in the CDS is assumed to be a new edge (red edges in Figure 7(c)).

- Deprecated edges: Edges from the design topology in the CDS not matched to entities in the updated CAD model are assumed to be deprecated (edge $e l$ in Figure $7(\mathrm{a}))$.

After adding the fillet, the two faces bounded by $e 1$ are bounded by two new edges, but some CAD systems will create just one new edge and reuse $e l$ as the name attribute for the other. In previous workflows, this would prevent the automatic update of analysis models. Since $e 1$ was bounding two faces in the original model that are not connected in the updated model, el must be classified as deprecated to remove any variability in the name attribute assignment.

New edges in the CAD model are grouped by the faces they bound, by querying the CAD model topology. This will be used later to simplify the identification of candidate for updating face topologies. Face tags that do not exist in the database help identify new CAD faces.

At this stage in the process, only the modifications to the design model have been identified. If only the geometric attributes of edges have changed, the geometry has changed and is updated as described in section 4.2. Otherwise, new or missing edges indicates that the original topology has changed. Further classification is required to identify modified, new and deprecated parasite entities in order to update the analysis topology.

Analysis topology modifications are inferred by their connectivity with original entities. For example in Figure 7, the parasite face $p f l$ connected to the modified edge $e 2$ is classified as modified, and the mesh-mapping constraint on pfl indicates that the opposing edge pel of the edge $e 2$ is also modified (purple in Figure 7(d)). This enables meshing constraints to be easily propagated to the updated topology.

\subsubsection{Vertex classification}

To accommodate the absence of name identifier on vertices in the geometric kernel used in this work, the classification of vertices is helped by the classification of the edges they bound. Vertices are classified as follow:

Original vertex: vertices that bound original edges, or modified edges if there are matching geometric attributes and topological connectivity (vertex $v 2$ in Figure 7(a)).
- Modified vertex: vertex bounding modified edges with consistent topological connectivity.

- New vertex: vertices bounding a new edge that are not original or modified vertices (vertex $v 3$ in Figure 7(c)).

- Deprecated vertex: vertices bounding deprecated edges or modified edges (vertex $v 1$ in Figure 7(a)).

For each deprecated original edge, the list of edges connected to its bounding vertices is queried from the CDS. If these edges still exist and share the vertex in the new CAD design, the vertex still exists, otherwise it is deprecated. In this last case, a new vertex is created for each connected edge, and connected edges are stored along with their relationship to the old and new vertex to update the design topology at a later stage.

Analysis topology edges connected to the vertex are also checked and their relationship is stored. In the case where the vertex was bounding a parasite or orphan edge aligned with the sweep direction of a region, the eventual parasite or orphan edge connected is identified as deprecated. Its end vertices are stored to attempt to identify any new parasite edges. This is because these edges link the source and target faces of swept regions, and topological modification on one face can help identify analysis topology modification on the other face. For example, the orphan edge oel in Figure 7(a) is classified as deprecated since the vertex $v l$ is deprecated, hence the parasite edges lying on face $f 2$ are classified as modified.

\subsubsection{Face classification}

Topologically modified faces in both the original design and analysis topology are identified based on their bounding entities, since their geometric definition is more expensive to query. Identification of the geometric modifications of faces is kept for a later stage of the process, as it will be used to assess the validity of meshing strategies. Faces are classified as follows:

- $\quad$ Original topology faces: Faces with all their bounding edges classified as original or modified.

- $\quad$ Modified topology faces: Faces bounded by deprecated edges or disconnected modified edges (face $f 3$ in Figure $7(\mathrm{a})$ ). Hence the boundary definition is incomplete in the CDS and will need to be updated.

- New faces: Face with a new name attribute, bounded by new edges. These are identified after updating the original topology in the CDS.

- Deprecated faces: Faces with less than two edges not deprecated.

Faces with an incomplete boundary definition are marked as open loops and stored as a sequence of edges, with all vertices bounding only one edge marked as open ends. Open loops appear when an original or a virtual edge is deprecated. Two modified edges bounded by a common deprecated vertex also identify an open loop, and the vertex is stored as a double open-end until it is replaced by the new vertices generated at each end of the modified edges. Furthermore, a face that has all its bounding edges deprecated is also considered deprecated. However, new parasite faces cannot be identified at this stage since the new parasite edges will 
be identified when updating the design topology (see section 4.2).

New faces bounded by an existing or modified edge indicates that the face has been subdivided as a result of the design modification, similar to the persistent naming problem described previously (top right case in Figure 6). By looking at the classification of the edges that were connected to the edge that is matched, the other sub-faces can be identified, and split edges are re-classified to account for the fact that their bounding entities have been modified. Faces that are merged as a result of parametric modification are processed like other topology-modified faces, and the boundary entities of the deprecated face are used as candidates to complete the boundary definition of the modified one. The example in Figure 8 features both split and merged configurations where $f l$ and $f 3$ are merged while $f 2$ becomes two faces.

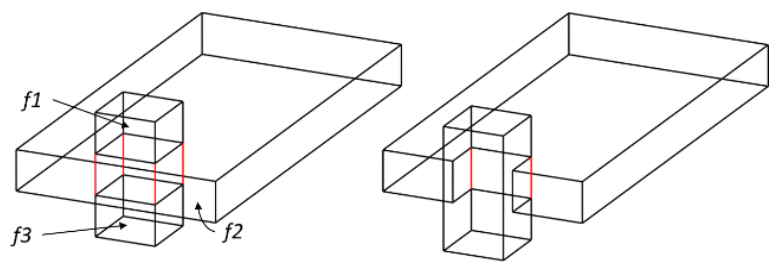

Figure 8. Face definition can be split or merged by parametric modifications.

\subsubsection{Body classification}

Original body classification is derived from the classification of its bounding entities. Analysis body classification is based on the virtual topology relationship along with original bodies and meshing strategies checks described in section 4.4. They are classified as follow:

- $\quad$ Original body: Bodies with all their faces, edges and vertices classified as original.

- Modified body: Analysis bodies bounded by modified entities that can be re-meshed.

- $\quad$ Modified topology body: body with deprecated faces

- Invalid body: Modified body with an invalid meshing strategy.

- New body: New original body with a name attribute not referenced in the CDS.

- Deprecated body: Bodies with all their bounding entities removed.

Modified volume subsets in the analysis topology can be preliminarily identified from the open loop faces in their boundaries. Further identification is done during the update of the analysis topology. Invalid bodies describe a set of modified bodies for which the meshing strategy has become invalid and is identified at a later stage (see section 4.4). These bodies are hence deprecated in the decomposition and will be merged to roll back the decomposition, enabling a local decomposition update to be performed if necessary.

\subsection{Original topology update}

Once all the entities in the original topology have been classified, they can be updated in the CDS to match the new design contained in the CAD model, according to Figure 9.

Deprecated and new entities in the CAD model indicate that the design topology has been modified. In that case, all the deprecated entities are removed from the CDS. Deprecated end vertices are matched against existing vertices, and a new vertex entity is created if no valid vertex is found. Bounding/bounded relationships and relative orientations are updated in the original topology relation. New edges and their vertices that are not matched by any existing vertex are added in the entity relation, and the bounding/bounded relation between edges and vertices is added to both topology relations.

In the case where all the entities are matched, without any deprecated or new entities, only the geometry has changed. The design topology can be updated by simply updating the geometrical attributes in the CDS. Mid-points of the modified original edges are updated in the entity relation of the CDS, along with the coordinates of modified vertices. Updating the topology before the geometry attributes enables new entities to be sorted and avoids having the same geometric attribute for multiple entities.

At this point, only the edges and vertices of the design topology are updated. Edges and vertices of the analysis topology need to be updated (in the next section) before updating the original face topology, since analysis topology faces are updated simultaneously with the original faces. The reason is that some new virtual edges can be identified when closing loop of edges on the face (see section 4.3.2).

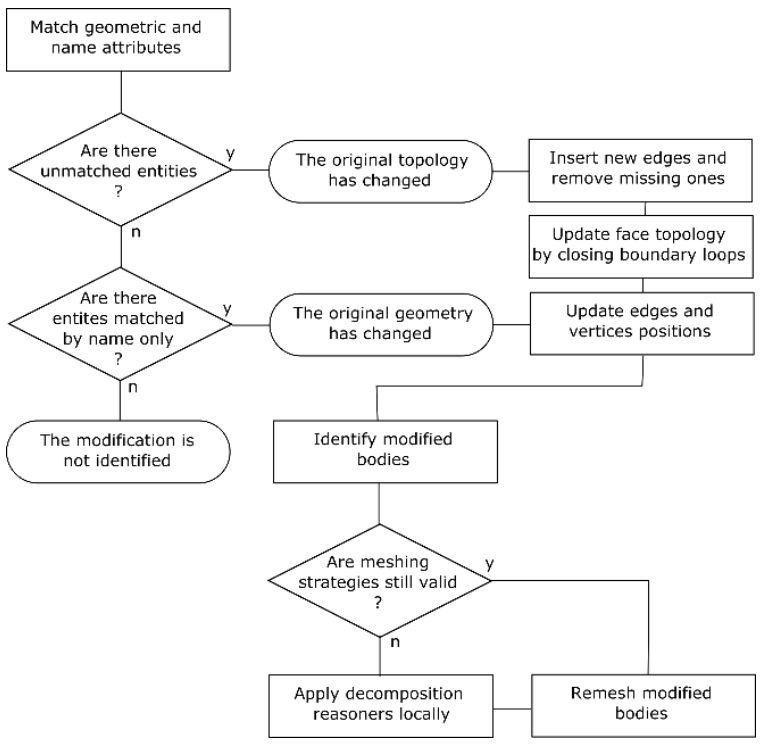

Figure 9. Geometry and topology update workflow. 


\subsection{Virtual geometry and analysis topology update}

The next step after the design modifications have been identified and classified is to update the virtual geometry, and the topological connectivity of modified entities in the analysis topology, within the CDS. Deprecated virtual geometry vertices and edges are removed from the topological description.

\subsubsection{Virtual geometry update}

Modified original edges in the analysis topology have already been updated when updating the design topology. Here, the virtual geometry subset and parasite edges are updated by querying the entities that have been modified and propagating the changes to the host entities on which they rely.

In situations where parasite edges have been modified their virtual geometry needs to be updated. If a parasite edge is bounded by a vertex in the original topology, then a one-way projection is sufficient to capture the update virtual geometry. End vertices which were projected to create parasite edges need to be re-projected, e.g. in Figure 10(a), vertices $A$ and $B$ are created by projecting existing vertices to the original face $f l$ and edge $e l$ respectively.

A more complicated scenario arises when a modified parasite/orphan edge is bounded by two parasite vertices. In Figure 10(b), which corresponds to the model in Figure 14, the vertices $C$ and $D$ are the result of the reciprocal projection between the original edges $e 2$ and $e 3$, hence a two-way projection is required. Point containment and angles are used to check whether the projection has succeeded, and the pair of vertices are stored to identify new potential parasite or orphan entities. If the projection has failed, parasite faces bounding the parasite edges bounded by the vertices are classified as deprecated, and the connected bodies become invalid.

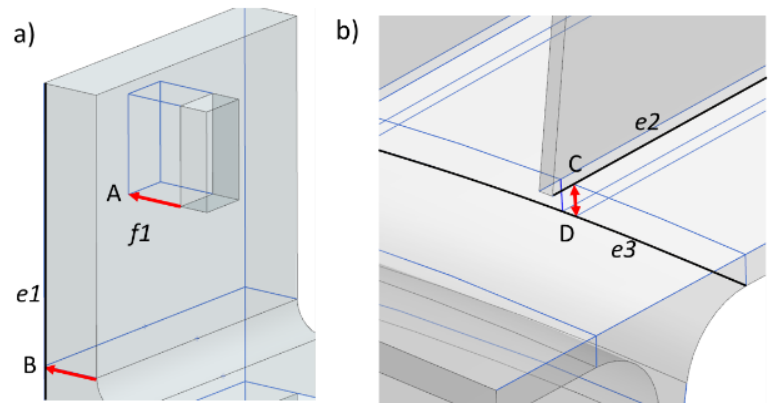

Figure 10. Projection of parasite vertices.

All the parasite vertices lying on modified edges and faces are also re-projected in order to update their coordinates. This checks the validity of subset edges and faces. Virtual geometry is used to assess the point containment of parasite vertices splitting parasite edges. Parasite face validity is inferred from the validity of their boundary entities.

\subsubsection{Modified face topology update}

At this stage, all the necessary information is available to update the topology of faces with an incomplete boundary definition or open loops. Design topology and analysis topology faces are updated at the same time, as updating the design topology to match the CAD topology will guide the update of the analysis topology. Suitable candidates to close open loops in the design topology are found in the list of new boundary edges in the updated design. Candidates for the original and parasite loops of the analysis topology include new boundary edges and new virtual geometry edges. Subset faces are updated using the relationships previously identified between edges and their bounded faces.

Gaps in open loops are filled iteratively by adding candidate edges at the open-end vertices and updating the open end until another open end is found. The process terminates when there are no more open ends, and all the edges belong to closed loops. Since this method can handle several disconnected gaps in the boundary definition of the loop, both inner and outer loops can be processed. In particular, the outer loop can absorb a previous inner loop if a feature on the face is moved to its boundary (see Figure 16 for example, where the rod is moved to the boundary of the middle rib).

In the case of analysis topology, mapping information from the attached meshing strategy is used to identify matching loops between source and target faces, and identify what entities can be projected to complete the opposite loops. This highlights the importance of the traceability between design topology, analysis topology and meshing attributes. For example, if a new edge is added in the loop of a source face for a thin-sheet region, this edge can be projected to identify a new parasite edge on the target face (Figure 7(e)). If the projection succeeds, then a parasite face is created, otherwise the thin-sheet meshing strategy needs to be reassessed.

Once all the open loops have been closed (Figure 7(f)), leftover virtual geometry entities are traversed to identify the smallest loops and infer new parasite faces. The analysis bodies can then be updated, and a valid topological representation of the analysis model is obtained (Figure $7(\mathrm{~g}))$.

\subsection{Updating meshing strategies}

Since the main objective of the decomposition is to identify meshing strategies for hex meshing, checks are implemented after the analysis topology update to make sure the model can still be meshed automatically. Angles are checked to make sure no skew elements will be introduced. Wall faces of swept regions, such as face $f 3$ in Figure 12(a), also need to remain mappable for the sweep mesh generation to be successful. The CDS contains the geometrical and topological information to perform these tests.

If the hex meshing strategy becomes invalid, the body is classified as residual and connected residual regions are merged into a single superset. Since virtual topology is used for the decomposition, merging is done by altering entity connectivity and orientation in the analysis topology contained in the CDS, as described in [8]. Merging retains 
all the correct imprints from other neighbor cells that would be lost by undoing the split operations, hence the meshing attributes of the neighbor cells remain valid. In Figure 11(a), a plate with two opposite bosses is decomposed into a thinsheet and two general sweepable regions. After parametric modifications, the two blocks are merged into a single residual region while the thin-sheet strategy of the plate remains valid, Figure 11(b).

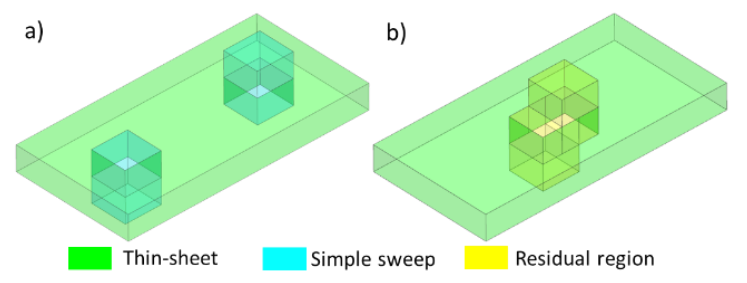

Figure 11: 2 volume cells are merged into one residual region after parametric perturbation.

After a valid analysis topology has been recovered, reasoning tools for decomposition are 'locally' used to recover deprecated meshing strategies or process new residual regions. In the case where a region had a previous hex-meshing strategy that has been identified as invalid, the reasoner related to this particular meshing strategy is used first. This also enables to re-use information provided by tools external to the framework, such as face-pair information. In Figure 12(a-b), a plate with a boss is decomposed into a thin-sheet and a general sweepable region. Adding a fillet to a bounding edge of the source face of the thin sheet makes the sweeping strategy invalid as $f 3$ is not mappable anymore, Figure 12 (c). The face pair $f 1-f 2$ initially used to identify the thin-sheet region is re-used to identify a thin-sheet and a new residual region, Figure 12 (d). The long-slender reasoner is automatically applied to classify this residual as sweepable, and the model is once again fully hex-meshable Figure 12 (e-f).

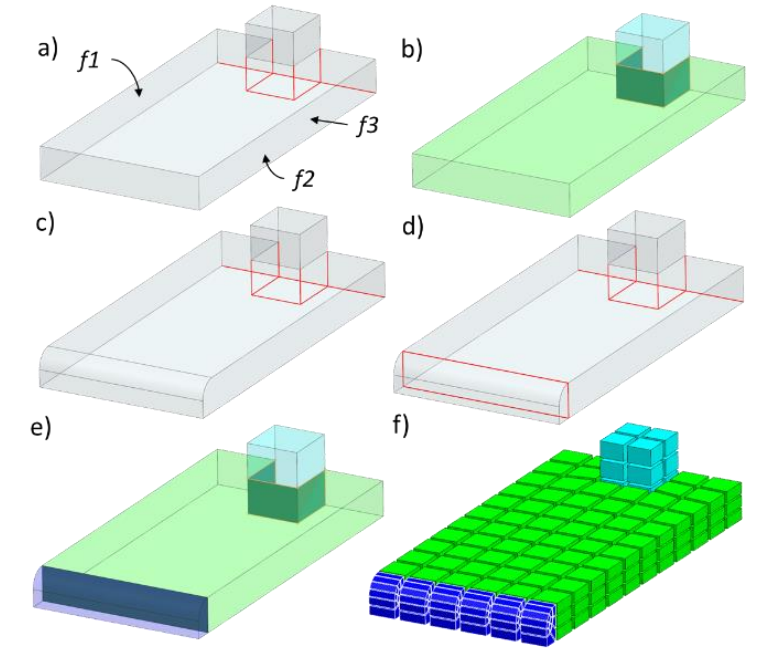

Figure 12. Thin-sheet strategy made invalid by a fillet insertion is recovered, and a new residual is processed to recover a fully hex-meshed model.
Alternatively, residual regions may become eligible for a hex-meshing strategy after a design change and can be reassessed. In the case where several invalid bodies have been grouped in a single residual superset, the sequence of reasoners used to decompose the original design model can be re-used on the updated design. This ability to localize changes reduces the rework required to generate a valid analysis decomposition.

\section{UPDATING THE MESH}

Since all the modification have been localized, the mesh can be updated only where necessary, thus significantly reducing the expense of re-meshing. The original mesh was created from a decomposed model; hence every volume cell in the analysis topology is linked to a collection of mesh elements. Therefore, only the elements associated to a modified volume cells need to be altered. This collection of elements is referred to as a mesh collector. Upon first generation, all the interface meshes between sub-regions are stored in a separate mesh file, allowing interface information to be maintained when locally modifying the mesh. Management of interfaces in this manner also provides the ability to parallelize the meshing and re-meshing processes. Figure 13 shows an overview of the re-meshing process. Only the modified sub-regions are exported to the meshing environment, and a meshing recipe is automatically identified, taking into consideration constraints from the neighboring meshes that are not modified. Finally, the deprecated elements are replaced by the new ones directly in the input deck file of the solver, hence there is no need to load the whole mesh.

\subsection{Meshing recipe update}

The meshing recipe is updated to inform the re-meshing process of the modified regions. In simple cases with small deformations, the same meshing recipe can be reused. However, large modifications require the meshing division numbers and constraints to be adapted to the new geometry by locally updating the meshing recipe. To ensure compatibility with the rest of the mesh, the original recipe is interrogated to extract the controls at the interfaces with the modified regions. A new integer programming problem is created, using the number of elements division on interface curves as fixed constraints. Meshing constraints directing the flow of elements are recovered from the CDS and new constraints are added. The problem is solved using LPSolve [24] as before, and the resulting division numbers are used to update the meshing recipe. If no feasible solution can be found then the constraints are relaxed where possible, or a larger portion of the model needs to be re-meshed. Alternatively, further decomposition can be carried out on the modified volume cells in order to create the necessary transition zones to remove over constraints. 


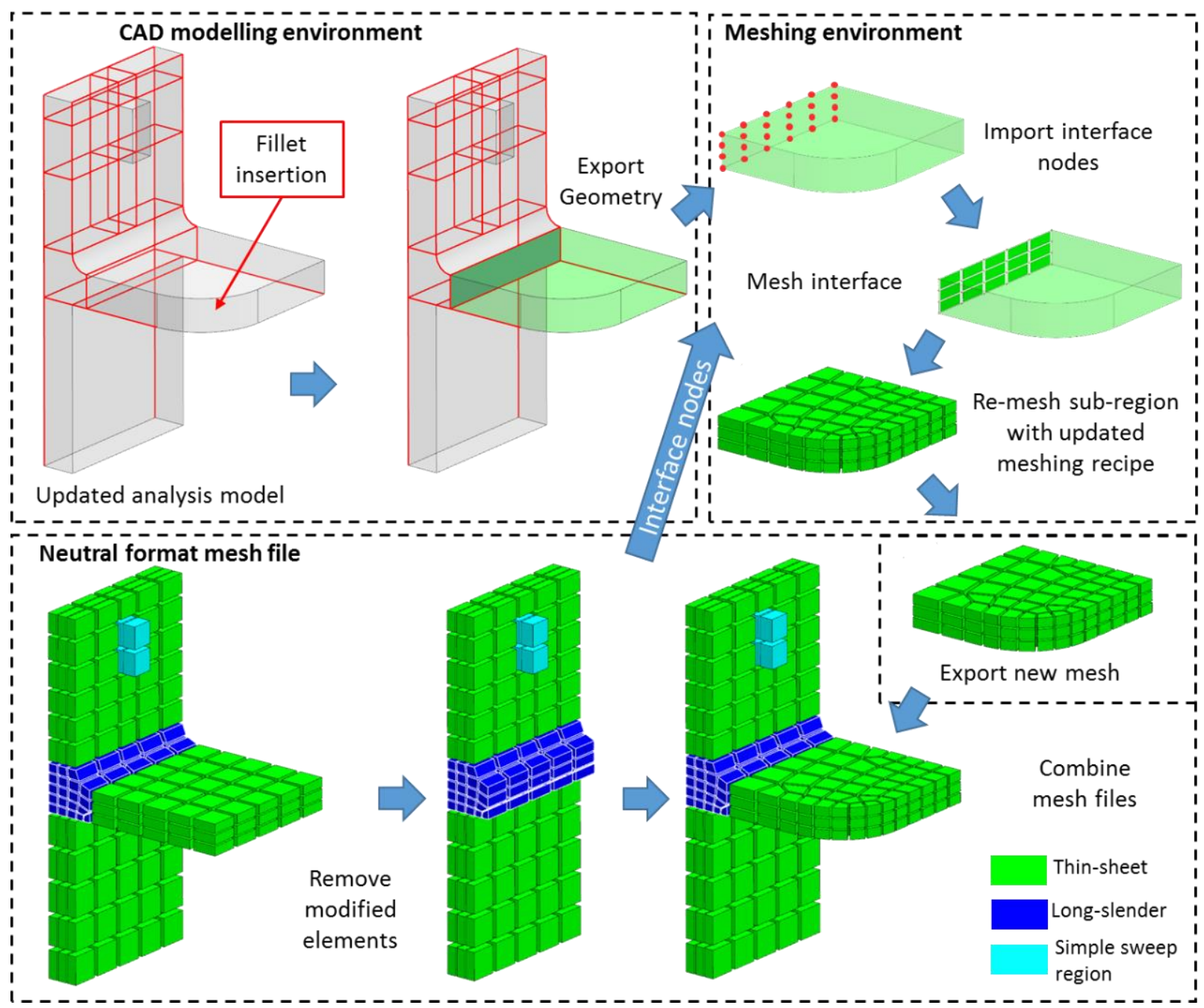

Figure 13. Local re-meshing workflow. Only the modified sub-regions are extracted from the virtual decomposition and re-meshed in the meshing environment, after the meshing recipe is locally updated and the interface nodes are recovered. Then the new mesh is re-assembled in the main mesh file

\subsection{Local body extraction}

Efficient re-meshing of a modified region is achieved by transferring only the modified regions to the meshing environment. This implies that any sub region can be geometrically extracted from the virtual decomposition in the analysis without having to decompose the whole model. This is possible through the robust connections that exist between the analysis topology and the geometric definitions in the CAD and CAE environments. To achieve this, all the interface entities can be identified from the analysis topology relation in the CDS. These parasite faces are created, grouped into connected sets and sewed together to make them usable for CAD geometry split. The result can then be extracted and exported, before the split is undone and the cutting faces deleted, to keep the CAD design model unaltered. Geometric modifications are only required for the mesh generation. The residual region in Figure 14 (c) can be extracted without having to remove thin-sheet and long slender regions first, with all the appropriate imprints from neighboring regions.

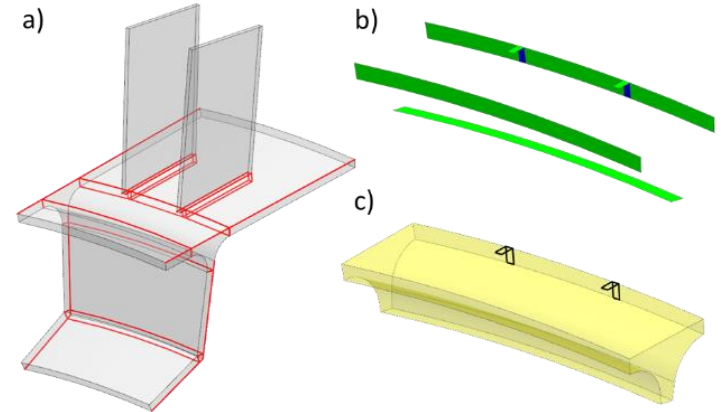

Figure 14. (a) virtually decomposed model, (b) parasite faces of the residual regions created and sewed, and (c) extracted residual with imprints.

\subsection{Meshing sub-regions}

All the nodes lying on the interfaces between the sub-region being re-meshed and the rest of the analysis model are recovered from the mesh files of the interfaces. These nodes 
are imported into the meshing environment as frozen (locked in space) mesh points and associated with the corresponding face geometry in order to rebuild the surface mesh of the interface. The labels of interface nodes and elements are then updated to match the original mesh labelling. This ensures that the interface nodes will not be modified by the 3D meshing algorithm and that the new elements can be connected to the unmodified part of the analysis model mesh. If the re-meshing involves several connected bodies, all the new interface nodes generated will be added to the interface mesh file. Any deprecated nodes and elements are removed.

After the local mesh controls have been applied from the meshing recipe, the 3D elements are generated by the meshing reasoner described in section 2.2 , and a mesh file containing all the new mesh for the concerned subsets is exported as a neutral mesh file. Nodes and elements indexing are automatically managed by setting the start index as the largest value in the current mesh file to ensure compatibility with the existing mesh and conformity at the interface.

\subsection{Mesh manipulations}

After the modified bodies have been re-meshed, the main mesh file needs to be updated. Since all the elements are grouped by sub-region into mesh collectors, the neutral mesh file can be re-written to include new nodes and elements. The updated mesh file is first created by copying the headers until the section containing the nodes is reached. Nodes are read and copied to the new file, removing deprecated nodes and updating the modified ones. All the new nodes are inserted at the end. Then mesh collectors of each of the bodies are transferred, with elements and their nodal connectivity replaced in the case where bodies are remeshed. New collectors are copied from the mesh file containing new subset meshes, and finally the material properties section is updated and copied.

\section{RESULTS AND DISCUSSION}

The proposed method is run automatically on a large number of test models to ensure that the decomposition and meshing update can handle many configurations. Figure 15 shows the resulting meshes after a design change on a L-shaped bracket. Modifications to the L-shaped bracket include parametric changes by modifying wall thicknesses, and more advanced topological changes by adding fillets etc. to the model. The initial hex mesh in Figure 15 (a) is automatically updated to fully conforming hex meshes after all design modifications. In particular, thin-sheet decompositions are updated without having to interrogate the face pairs again, which is a costly part of the initial process.

The model in Figure 16 is taken from [21] in order to assess the performance of the method presented here on a complex design modification. The model is automatically decomposed in 12 seconds, and the meshing requires 16 seconds. After perturbation of the parameter ' $d$ ' in Figure 16(c), the analysis topology and virtual geometry are updated in 3.8 seconds, and the model is re-meshed in 11 seconds, recovering a full hex mesh. This corresponds to a
$47 \%$ gain of time compared to running the automatic meshing procedure form the beginning.
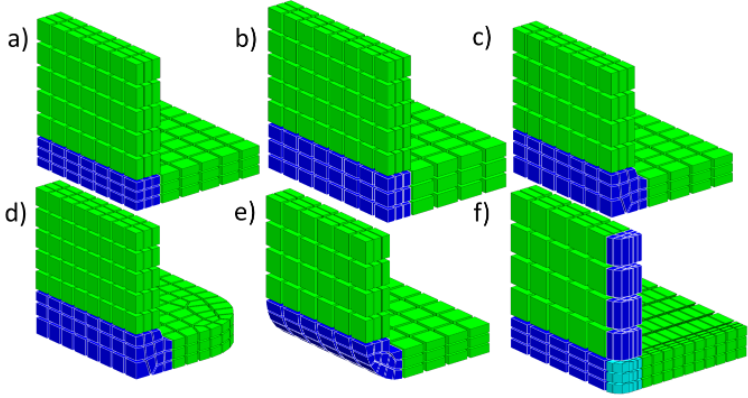

Figure 15. L-bracket re-meshed after various design modifications.

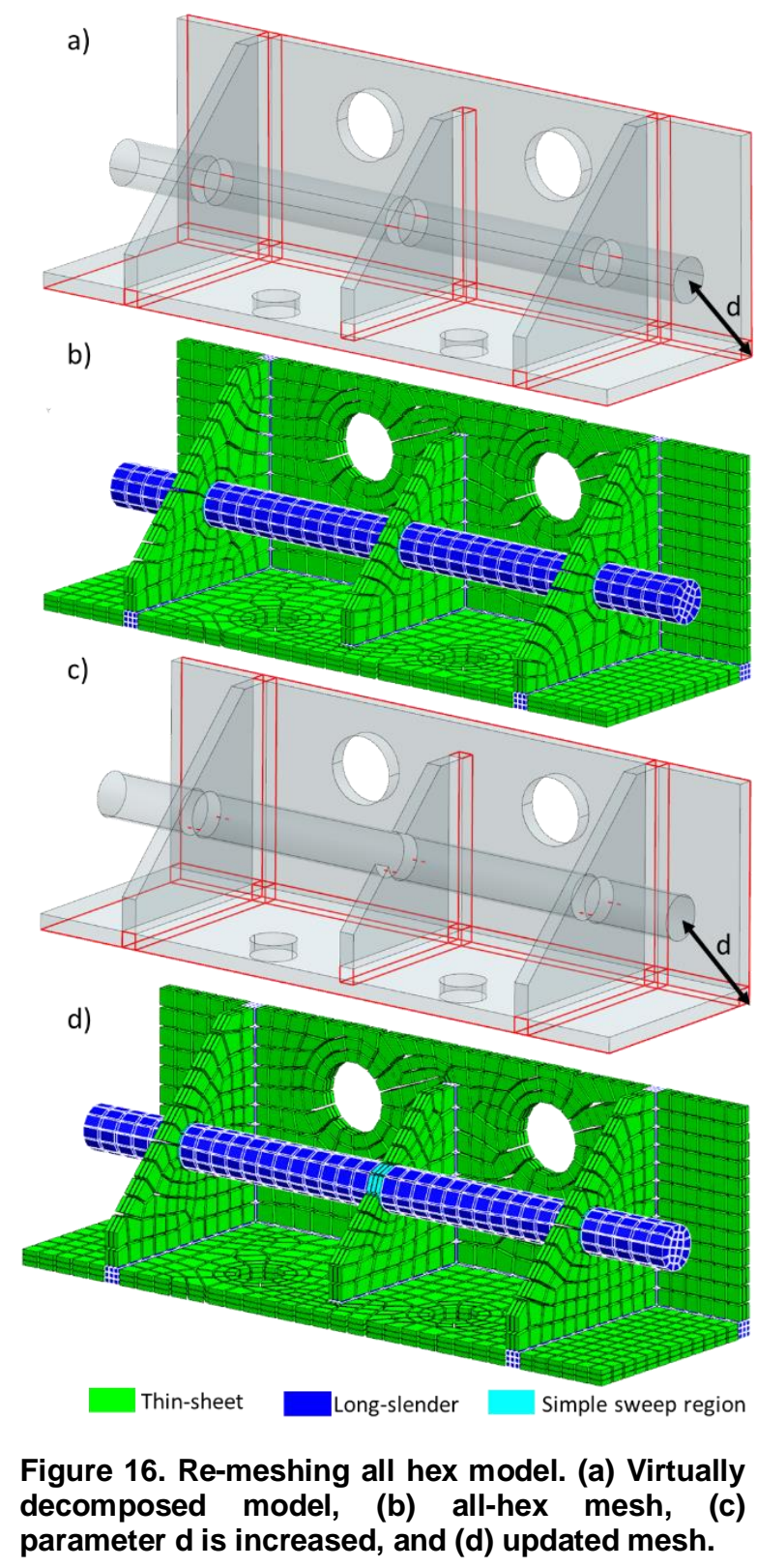




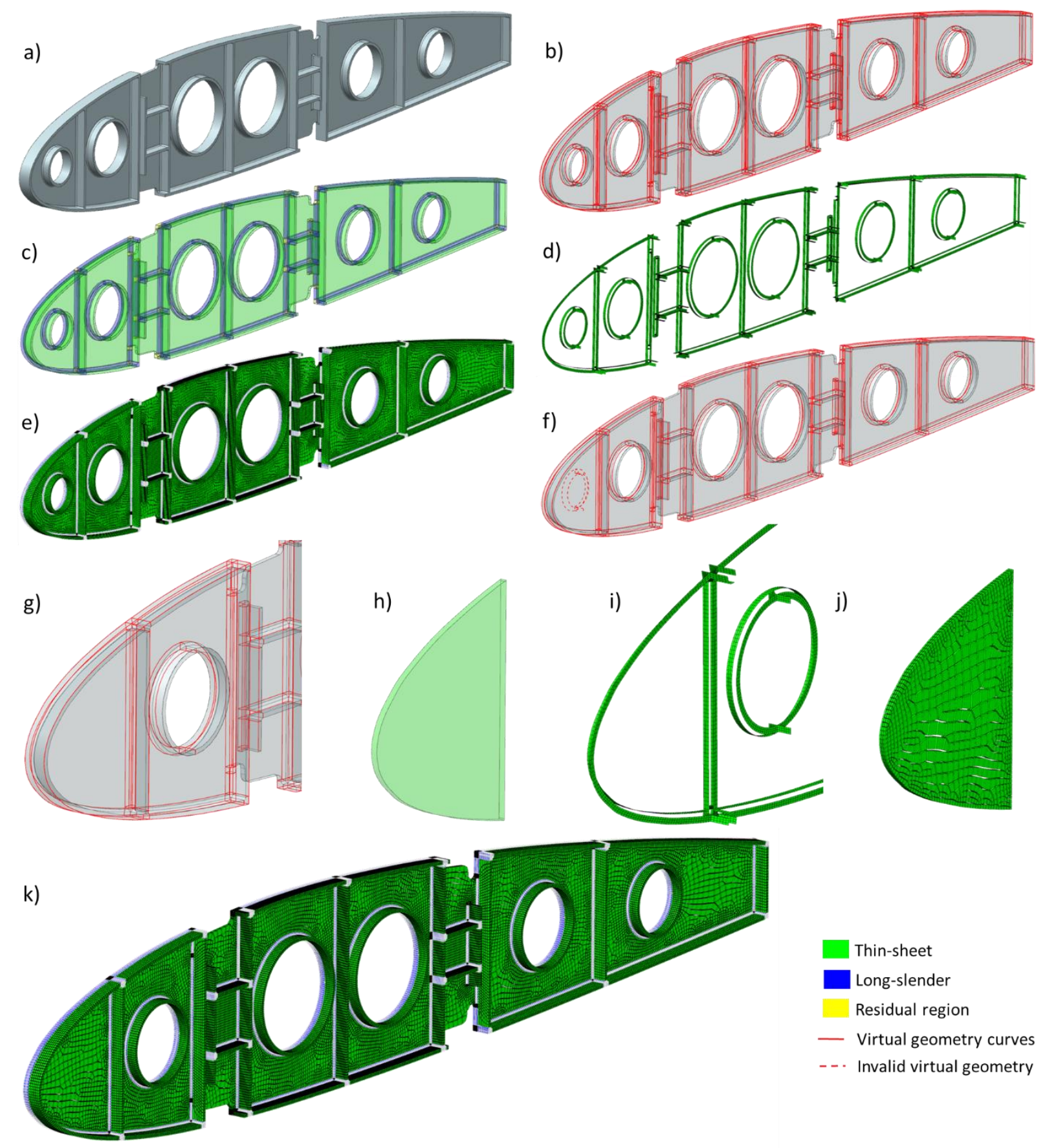

Figure 17. Automatic meshing and re-meshing of a rib model. (a) Original design, (b) decomposed analysis topology, (c) equivalent geometric decomposition, (d) interfaces are meshed first, (e) resulting mesh, (f) design modification with topological changes, (g) updated analysis topology, (h) modified cell extraction, (i) updated interfaces meshes and interfaces nodes import, (j) re-meshed cell and (k) updated mesh.

While the proposed method offers significant time reduction compared to a process involving manual decomposition and meshing for large models, the re-meshing process is not as beneficial for small analysis models, where a design modification can require re-meshing of most of the subregions.
An example of a larger model is given in Figure 17. Successive reasoners are used to decompose the model within 2 minutes (on a windows workstation with a $3.7 \mathrm{GHz}$ Intel Xeon E5-1630 CPU with 32GB RAM) in Figure 17(b), and the geometric decomposition is generated in 1 minute, Figure $17(\mathrm{c})$. The mesh is automatically generated within 5 minutes in Figure 17(e), with 235,000 hexahedra and 78,000 
tetrahedra. The interface mesh file (Figure 17(d)), contains 19,800 quad elements allowing individual manipulation of each of the 174 meshed sub-regions. After changing the design by removing a hole and its protrusion near the leading edge, the virtual decomposition is updated in 3.5 seconds (Figure $17(\mathrm{~g})$ ), and the model is locally re-meshed (Figure $17(\mathrm{~h}-\mathrm{k})$ ) in 19.4 seconds. 7850 elements of the previous mesh are replaced by 2430 new hexes. The normal remeshing in the CAE environment for this modification takes approximately 6 minutes, which is only for re-meshing. This does not include the manual time required to update the decomposition and link it to the meshed model.

A critical scenario for this approach arise when an original entity hosting many subsets is modified. In Figure 18, all the projections need to be updated to ensure they are still valid after perturbation of the bottom face; hence the modification propagate to a large portion of the analysis model. Although applying a front propagation technique that stops once the modified subsets have been updated is future work, the current implementation still outperforms any manual intervention.

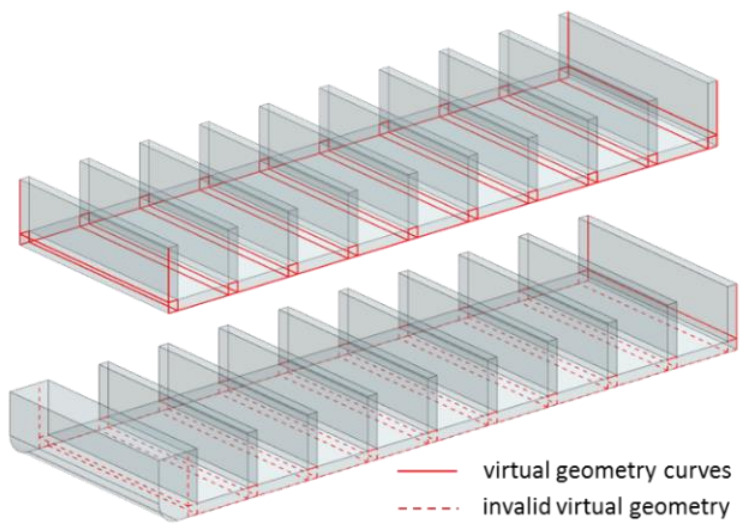

Figure 18. All the projection must be checked after the bottom face is modified.

In the event of rigid body motion, the whole decomposition is modified. In the absence of topological modification, the analysis model is easily updated. However, all bodies are modified, hence the model needs to be fully re-meshed in the current implementation. This could be easily handled by any mesh deformation algorithm in future work.

One important challenge when re-meshing is to ensure a good quality mesh will be re-generated, as moving features can create small faces that will drive down the size of elements and increase computation time. In Figure 19, virtual geometry is moved in close proximity to original entities. Detection of close entities is carried out using the medial axis while taking into account the virtual geometry, since the proximity is a result of the decomposition. This information will be used in future work to decide merging operations and define whether topological updates are required to the parasite topologies.

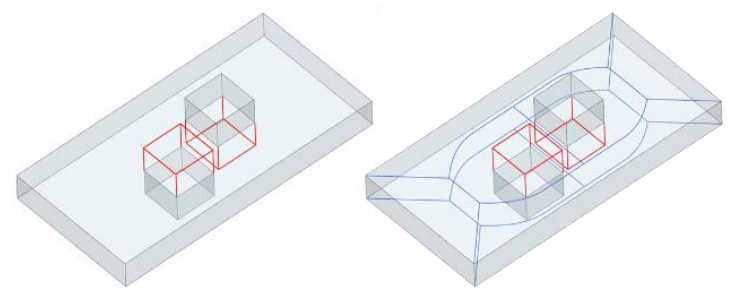

Figure 19. Medial axis (in blue) on thin sheet source and target faces to identify close entities.

Whilst the automated workflow in this paper has been demonstrated around the use of thin-sheet and long-slender decomposition reasoners, the same process is valid for any decomposition reasoner that introduces meshing strategy which creates mapping constraints between entities. For example, if a reasoner identifying regions suitable for transfinite meshing methods were applied, the mapping between opposite pairs of faces and edges would help to propagate the design modification. The reason is that meshing constraints are used to guide the update of the decomposition, and the proposed approach is capable of automatically updating these meshing attributes to match design modifications.
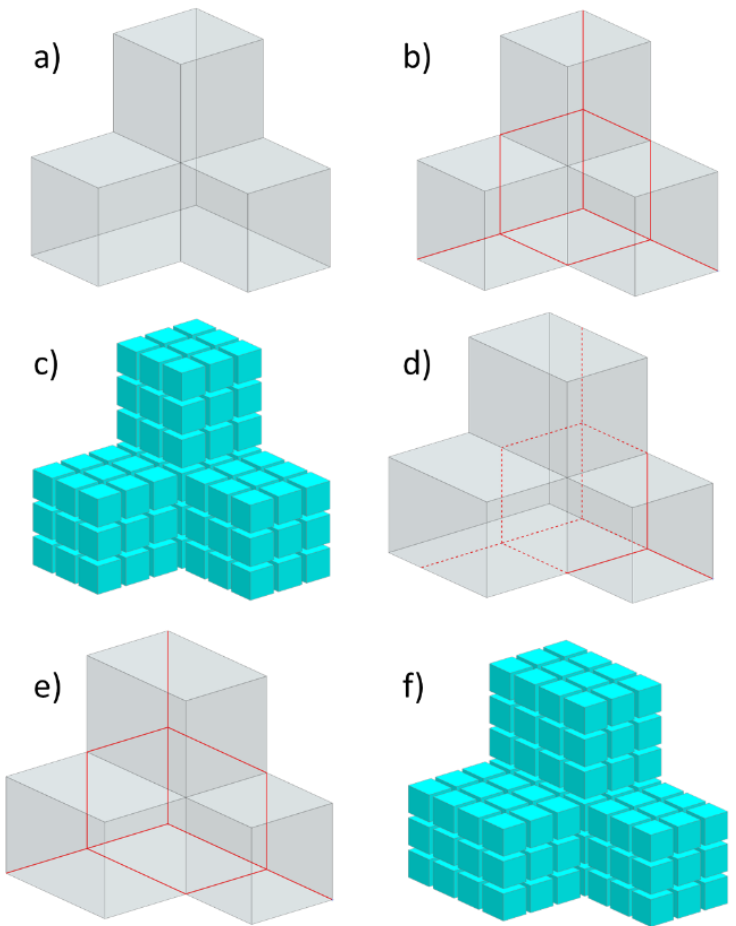

Figure 20. Manually defined block can be updated. (a) original model, (b) sweepable blocks defined manually, (c) automatic mesh, (d) parametric modification, (e) automatically updated decomposition and (f) updated mesh.

Virtual block decomposition defined manually can also be updated if meshing strategies have also been defined by the user (Figure 20). Limiting the update to only those blocks that have been modified provides analysts with the assurance they will not have to perform a block decomposition for all 
subsequent design changes. This enables an analyst to focus only on the regions of interest that have been modified. This is important in the context of large analysis models where a manual block decomposition could be performed on a modified region with all interfaces, meshing constraints and re-meshing automatically handled by the work presented here.

The identification of modified regions relies on the postulate that all the splitting entities connectivity can be traced back to original entities, hence they can be identified by manipulating the topological graphs of the models and the virtual topology relation. If the modifications cannot be processed with the method described here, the model can still be automatically meshed using the virtual topology workflow presented from the beginning. The user-defined parameters such as the sequence of the reasoners and the target aspect ratios are automatically recovered and re-used. However, if a bump was to appear on a face without moving any of the points sampled for the identification of geometrical modification nor changing the topology, the current method would be unable to identify this modification and no modification would be made to match the new design. Alternative shape descriptors could be used to identify these localized geometric updates and feed this information into the workflow.

\section{CONCLUSION}

A virtual topology workflow has been extended to handle design changes in automatic meshing workflows, by using the cellular description of the mesh to manipulate and update it. Significant time reduction is achieved by automatically controlling the re-meshing process, especially since only parts of the mesh are loaded and updated. This approach has achieved the following objectives:

- $\quad$ The model can be re-meshed after large parametric modifications or feature changes, and all design modifications are propagated to the analysis model.

- The mesh structure is maintained as much as possible by taking into account hexahedral meshing constraints.

- $\quad$ The mesh update process is fully automated and more efficient than traditional approaches.

\section{FUTURE WORK}

The presented method is limited by the variety of reasoners applied in the work, and further work is required to improve and validate the proposed approach. This includes:

- Implementing more decomposition reasoners. Only reasoners that identifies region hexmeshable with a one-to-one sweeping method have been used. Reasoners dedicated to the identification of mappable cube-like region can be used within the proposed method, as the meshing constraints are similar and can be used to guide the update of the decomposition.

- Many-to-many sweeping is currently not handled since the mesh reasoner is limited to simple oneto-one sweeps. Many-to-many sweeping introduces different constraints on entities than mapping or sweeping techniques, and therefore will require additional reasoning to help the update of the decomposition.

- The decomposition reasoners used provide good results on thin models, but introducing different blocking tools could enhance further the method. Special care will be required if singularity lines are introduced, since the mapping of entities through meshing constraints will become more difficult.

\section{ACKNOWLEDGMENTS}

The authors wish to acknowledge the financial support provided by Innovate UK via GEMinIDS (project 113088), a UK Centre for Aerodynamics project. The authors acknowledge Rolls-Royce for granting permission to publish this paper.

\section{REFERENCES}

[1]

]

J. Sarrate, E. Ruiz-Gironés, and X. Roca, "Unstructured and Semi-Structured Hexahedral Mesh Generation Methods," Comput. Technol. Rev., vol. 10, pp. 35-64, 2014.

T. Tautges, T. Blacker, and S. Mitchell, "The whisker weaving algorithm: A connectivity-based method for constructing all-hexahedral finite element meshes," Int. J. Numer. Methods Eng., vol. 39, no. 19, pp. 3327-3349, 1996.

T. Blacker and R. Meyers, "Seams and wedges in plastering: a 3-D hexahedral mesh generation algorithm," Eng. Comput., vol. 9, no. 2, pp. 83-93, 1993.

G. F. Carey, "Hexing the Tet," Commun. Numer. Methods Eng., vol. 18, no. 3, pp. 223-227, Jan. 2002.

L. E. Eriksson, "Generation of BoundaryConforming Grids Around Wing-Body Configurations Using Transfinite Interpolation," Aiaa J., vol. 20, no. 10, pp. 1313-1320, 1982.

E. Ruiz-Gironés and J. Sarrate, "Generation of structured meshes in multiply connected surfaces using submapping," Adv. Eng. Softw., vol. 41, no. 2, pp. 379-387, Feb. 2010.

D. R. White and T. J. Tautges, "Automatic scheme selection for toolkit hex meshing," Int. J. Numer. Methods Eng., vol. 49, pp. 127-144, 2000.

C. M. Tierney, L. Sun, T. T. Robinson, and C. G. Armstrong, "Using virtual topology operations to generate analysis topology," Comput. Des., vol. 85, pp. 154-167, 2017. 
[9] B. Lecallard et al., "Automatic HexahedralDominant Meshing for Decomposed Geometries of Complex Components," Comput. Des. Appl., vol. 16 , no. 5, pp. 846-863, 2019.

[10] D. C. Nolan, C. M. Tierney, C. G. Armstrong, and T. T. Robinson, "Defining Simulation Intent," Comput. Des., vol. 59, pp. 50-63, 2015.

[11] A. Sheffer, M. Bercovier, T. Blacker, and J. Clemets, "Virtual Topology Operators for Meshing," Int. J. Comput. Geom. Appl., vol. 10, no. 03, pp. 309-331, Jun. 2003.

[12] R. Bidarrat, K. Jan de Kraker, and W. F. Bronsvoort, "Representation and management of feature information in a cellular model," Comput. Des., vol. 30, no. 4, pp. 301-313, 1998.

[13] C. M. Tierney, "Managing equivalent representations of design and analysis models," Queen's University Belfast, 2014.

[14] G. P. Gujarathi and Y.-S. Ma, "Parametric $\mathrm{CAD} / \mathrm{CAE}$ integration using a common data model," J. Manuf. Syst., vol. 30, pp. 118-132, 2011.

[15] M. L. Staten, S. J. Owen, S. M. Shontz, A. G. Salinger, and T. S. Coffey, "A Comparison of Mesh Morphing Methods for 3D Shape Optimization," in Proceedings of the 20th International Meshing Roundtable, Berlin, Heidelberg: Springer Berlin Heidelberg, 2011, pp. 293-311.

[16] P. Knupp, “Applications of mesh smoothing: copy, morph, and sweep on unstructured quadrilateral meshes," Int. J. Numer. Methods Eng., vol. 45, no. 1, pp. 37-45, 1999.

[17] A. Sheffer and A. Üngör, "Efficient Adaptive Meshing of Parametric Models," J. Comput. Inf. Sci. Eng., vol. 1, no. 4, p. 366, Dec. 2001.

[18] C. Shen, R. Wang, S. Gao, and H. Maehama, "An approach to feature moving of hexahedral mesh," Comput. Des., vol. 107, pp. 12-22, Feb. 2019.

[19] H. A. Van Der Meiden and W. F. Bronsvoort, "Tracking topological changes in parametric models," Comput. Aided Geom. Des., vol. 27, pp. 281-293, 2010.

[20] L. Sun, T. T. Robinson, C. G. Armstrong, S. Marques, and W. Yao, "Surface Mesh Deformation in CAD-based Shape Optimization," in AIAA Scitech 2019 Forum, 2019, p. 2360.

[21] M. Sypkens Smit and W. F. Bronsvoort, "Efficient tetrahedral remeshing of feature models for finite element analysis," Eng. Comput., vol. 25, no. 4, pp. 327-344, Nov. 2009.

[22] C. M. Tierney, D. C. Nolan, T. T. Robinson, and C. G. Armstrong, "Managing Equivalent Representations of Design and Analysis Models," Comput. Aided. Des. Appl., vol. 11, no. 2, pp.
193-205, Mar. 2014.

[23] C. Tierney, F. Boussuge, and D. C. Nolan, "Tolerant geometric extraction of fluid domains to assist CFD analyses of aero-engines," in AIAA Scitech 2019 Forum, 2019, p. 1720.

[24] "LPSolve Mixed Integer Linear Programming." [Online]. Available: http://lpsolve.sourceforge.net/5.5/. [Accessed: 25Mar-2019].

[25] L. Sun, C. M. Tierney, C. G. Armstrong, and T. T. Robinson, "Decomposing complex thin-walled CAD models for hexahedral-dominant meshing," Comput. Aided Des., vol. 103, pp. 118-131, Dec. 2018.

[26] L. Sun, C. M. Tierney, C. G. Armstrong, and T. T. Robinson, "An enhanced approach to automatic decomposition of thin-walled components for hexahedral-dominant meshing," Eng. Comput., pp. 1-17, Nov. 2017.

[27] F. Boussuge, C. M. Tierney, T. T. Robinson, and C. G. Armstrong, "Symmetry-based decomposition for meshing quasi-axisymmetric components," Procedia Eng., vol. 203, pp. 375387, 2017.

[28] J. Kripac, “A mechanism for persistently naming topological entities in history-based parametric solid models," Comput. Des., vol. 29, no. 2, pp. 113-122, Feb. 1997. 


\title{
ANISOTROPIC ERROR ESTIMATE FOR HIGH-ORDER PARAMETRIC SURFACE MESH GENERATION
}

\author{
Rémi Feuillet $^{1} \quad$ Olivier Coulaud $^{2} \quad$ Adrien Loseille $^{1}$ \\ ${ }^{1}$ GAMMA Team, INRIA Saclay, 1 Rue Honoré d'Estienne d'Orves, 91120 Palaiseau, France. \\ $\{$ remi.feuillet, adrien.loseille\} @inria.fr \\ ${ }^{2}$ Cenaero, Rue des Frères Wright 29, 6041 Gosselies, Belgium. \\ olivier.coulaud@cenaero.be
}

\begin{abstract}
Parametric surface mesh generation is one of the crucial step of the computational pipeline. Standard techniques, that are now mature, control the deviation to the tangent plane by using intrinsic quantities as the minimum and maximum curvatures. However, for high-order meshes, deriving intrinsic quantities that have the ability to control the mesh generation process is much more challenging. Indeed, those provided by the first and second fundamental forms of a surface are not sufficient when high order curved meshes are employed. In this paper, we introduce a new set of error estimates for high-order surface mesh generation. It is based on performing a Taylor expansion of the underlying surface in the tangent plane. The independence to the parametric space is obtained by using an inversion formula. High-order terms of this expansion are then used to derive an optimal metric by using the log-simplex approach. Examples are shown to prove the efficiency of the method.
\end{abstract}

Keywords: Surface mesh generation, High-order error estimates, High-order mesh generation.

\section{INTRODUCTION}

In industrial applications, the definition of the computational domain (or of a design) is provided by a continuous description composed by a collection of patches using a CAD (Computer Aided Design) system. If several continuous representations of a patch exist via an implicit equation or a solid model, we focus on the boundary representation (BREP). In this description, the topology and the geometry are defined conjointly. For the topological part, a hierarchical description is used from top level topological objects to lower level objects, we have:

$$
\text { model } \rightarrow \text { bodies } \rightarrow \text { faces } \rightarrow \text { loops } \rightarrow \text { edges } \rightarrow \text { nodes }
$$

Each entity of upper level is described by a list of entities of lower level. This is represented in Figure 1 for an Onera M6 model, where a face, a loop and corresponding edges are depicted. Note that most of the time, only the topology of a face is provided, the topol-

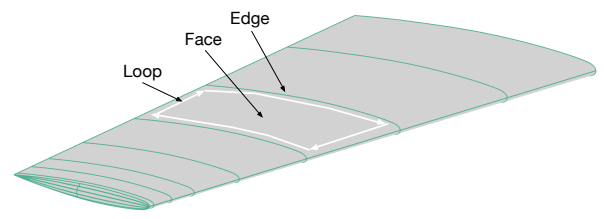

Figure 1: Topology hierarchy (Face, Loop, Edge) of the continuous representation of model using the Boundary REPresentation (BREP).

ogy between all the faces (patches) needs to be recovered. This piece of information is needed to have a watertight valid surface mesh on output for the whole computational domain. This step makes the surface mesh generation of equal difficulty as volume mesh generation and have been shown to be not trivial [1]. 


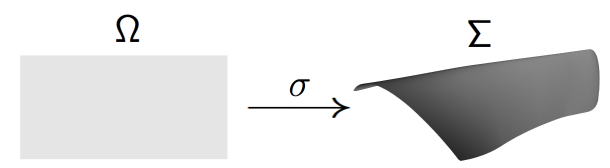

Figure 2: Mapping between the parametric and physical space.

For node, edge, and face, a geometry representation is also associated to the entity. For node, it is generally the position in space, while for edge and face a parametric representation is used. It consists in defining a mapping from a bounded domain of $\mathbb{R}^{2}$ onto $\mathbb{R}^{3}$ such that $(x, y, z)=\sigma(u, v)$ where $(u, v)$ are the parameters (Figure 2).

Generally, $\sigma$ is a NURBS function (Non-uniform rational B-spline) as it is a common tool in geometry modeling and CAD systems [2]. From a conceptual point of view, meshing a parametric surface consists in meshing a $2 \mathrm{D}$ domain in the parametric space.

The linear case has been widely studied for years $[3,4$, $5,6,7,8,9,10]$ and some approaches consist of meshing the 2D domain according to a curvature-based metric [11]. The use of this metric enables an independence to the used parameters space as the curvature is an intrinsic data. The generation of highorder meshes is on the contrary relatively new. The most common idea is to generate a linear mesh and then to project the high-order nodes on the geometry. However, the position of the nodes may not be suitable for a high-order representation of the boundary. For this reason, optimization procedures are applied to the mesh to improve its shape $[12,13,14,15,16]$. The procedure can be done by solving an optimization problem or performing a spring analogy. This can also be performed directly in the parameters space [17] by minimizing a distortion measure. In all these cases, this is r-adaptation which is performed. The intent of the presented work is to provide tools for high-order parametric surface mesh generation. The use of the high-order estimates developed in [18] will provide a node distribution which will be specifically tailored for the high-order with a given threshold. Note that high order surface meshes have a large set of applications. It can be naturally used as an input for the generation of $3 \mathrm{D}$ curved meshes. In our case, high-order surface mesh is used advantageously as a surrogate CAD (geometry) model. Indeed, it provides fast forward and inverse evaluation as required in classic linear mesh adaptation.

The first section briefly recalls the framework used for the high-order error estimates and how a metric can be deduced from it. The second section shows how the standard parametric surface mesh generation can be extended to the high-order using this framework.
Finally examples are shown to highlight the process.

\section{HIGH ORDER METRIC BASED MESH ADAPTATION}

\subsection{Continuous mesh framework}

In this section, some basic tools concerning the continuous mesh framework are recalled. For further details about these techniques, one refers to $[19,20]$ and the references therein. The main idea underlying the metric based mesh adaptation is to change the way distances are computed, via a continuous metric field $\mathbf{M}=(\mathcal{M}(x))_{x \in \Omega}$, where for all $x \in \Omega$, the matrix $\mathcal{M}(x)$ is a metric of $\mathbb{R}^{n}$, namely a symmetric definite positive matrix of $\mathbb{R}^{n}$. As it is explained below, the notion of unit mesh with respect to a metric field establishes a link between a metric field and a mesh, which reduces the problem of finding an optimal mesh to the problem of finding an optimal metric field. First of all, the scalar product induced by a constant metric $\mathcal{M}$ is given by

$$
\langle x, y\rangle_{\mathcal{M}}={ }^{t} x \mathcal{M} y, \text { for all } x, y \in \mathbb{R}^{n} .
$$

The following distance results from this scalar product.

$$
\|x\|_{\mathcal{M}}=\sqrt{{ }^{t} x \mathcal{M} x} \text {, for all } x \in \mathbb{R}^{n} .
$$

Likewise, in the Banach space $\left(\mathbb{R}^{n},\|\cdot\|_{\mathcal{M}}\right)$, the distance between two points $a, b \in \mathbb{R}^{n}$ is given by

$$
\ell_{\mathcal{M}}(a b)=\sqrt{{ }^{t} a b \mathcal{M} a b},
$$

where $a b$ stands for the vector linking $a$ to $b$. In the same way, if $\mathbf{M}=(\mathcal{M}(x))_{x \in \Omega}$ is a smooth metric field on $\Omega$, the length of the segment between $a$ and $b$ can be computed by using the integral formula

$$
\ell_{\mathbf{M}}(a b)=\int_{0}^{1} \sqrt{{ }^{t} a b \mathcal{M}(\gamma(t)) a b} d t,
$$

where $\gamma(t)=(1-t) a+t b$.

As the next definition shows, there is a strong correspondence between continuous metric spaces and meshes, through the notion of unit mesh with respect to a metric field.

Definition 1.1 A mesh $\mathcal{H}$ of a domain $\Omega \subset \mathbb{R}^{n}$ is said to be unit with respect to a continuous metric field $\mathbf{M}=(\mathcal{M}(x))_{x \in \Omega}$ if the edges lengths of its elements equal 1. If $\left\{e_{1}, \ldots e_{n+1}\right\}$ are the edges of an element $K$ of $\mathcal{H}$, then

$$
\ell_{\mathbf{M}}\left(e_{i}\right)=1, \quad \text { for all } i \in\{1, \ldots, n+1\} .
$$

In most of the metric based mesh adaptation methods, the problem is reduced to an optimization problem of 
finding the optimal metric field for which unit meshes minimize the interpolation error of the solution. In this article, the problem of surface approximation is seen as a 2D high order metric based mesh adaptation in the parameters space.

\subsection{Error model}

The examined error model is the on studied in [18]. Let $u$ be a smooth solution on the domain $\Omega, \mathcal{H}$ be a mesh of $\Omega$ and $k$ be an arbitrary positive integer. In what follows, $\Pi_{k} u$ denotes the projection of $u$ onto the finite elements space $\mathbb{P}^{k}(\mathcal{H})$, whose functions are polynomials of degree $k$ on each element $K$ of $\mathcal{H}$. For all $x_{0} \in \Omega$, it is well known that there exists a positive constant $C$ such that, for all $x_{0} \in \Omega$ and $x \in \mathbb{R}^{n}$,

$$
\begin{array}{r}
\left|u(x)-\Pi_{k} u(x)\right| \leq C\left|d^{(k+1)} u\left(x_{0}\right)\left(x-x_{0}\right)\right| \\
+\mathcal{O}\left(\left\|x-x_{0}\right\|^{k+2}\right),
\end{array}
$$

where $d^{k+1} u\left(x_{0}\right)$ is the differential form of $u$ of order $k+1$ at $x_{0},|$.$| is the absolute value function$ and $\|$.$\| denotes the Euclidean norm of \mathbb{R}^{n}$. In the high order case, the main idea is to replace the right hand side of (5) by a term governed by a metric field $\mathbf{Q}=(\mathcal{Q}(x))_{x \in \Omega}$, which approximates the $k+1$ differential form of $u$. More precisely, we are looking for $\mathbf{Q}$ such that for all $x_{0} \in \Omega, x \in \mathbb{R}^{n}$

$$
\left|d^{k+1} u\left(x_{0}\right)\left(x-x_{0}\right)\right| \leq\left({ }^{t}\left(x-x_{0}\right) \mathcal{Q}\left(x_{0}\right)\left(x-x_{0}\right)\right)^{\frac{k+1}{2}}
$$

The main issue is to find the metric field $\mathbf{Q}$ such that the inequality (6) is as optimal as possible. From a geometrical point of view, the local problem is to find the largest ellipse in 2D (or the largest ellipsoid in 3D) included into the domain surrounded by the level set of level 1 of $d^{(k+1)} u\left(x_{0}\right)$. Indeed, let $\mathcal{B}_{\mathcal{Q}}=\left\{x \in \mathbb{R}^{n}:{ }^{t} x \mathcal{Q} x \leq 1\right\}$ be the unit ball of a metric $\mathcal{Q}$, which is an ellipse in 2D (an ellipsoid in 3D). Now assume that, for all $x \in \mathbb{R}^{n}$ such that $d^{(k+1)} u\left(x_{0}\right)(x)=1$, one has ${ }^{t} x \mathcal{Q} x \geq 1$. Let $x \in \mathbb{R}^{n}$ and $y=\frac{x}{\left|d^{(k+1)} u\left(x_{0}\right)(x)\right|^{\frac{1}{k+1}}}$. In particular $d^{(k+1)} u\left(x_{0}\right)(y)=1$, and:

$$
d^{(k+1)} u\left(x_{0}\right)(y) \leq{ }^{t} y \mathcal{Q} y .
$$

Since $d^{(k+1)} u\left(x_{0}\right)$ is a homogeneous polynomial of degree $k+1$, it comes

$$
1 \leq \frac{{ }^{t} x \mathcal{Q} x}{\left|d^{(k+1)} u\left(x_{0}\right)(x)\right|^{\frac{2}{k+1}}},
$$

and consequently

$$
\left|d^{(k+1)} u\left(x_{0}\right)(x)\right| \leq\left({ }^{t} x \mathcal{Q} x\right)^{\frac{k+1}{2}}, \quad \text { for all } x \in \mathbb{R}^{n} .
$$

The purpose of the next section is to solve this minimization problem.

\subsection{Log-simplex method}

The high order mesh adaptation method which we use in this paper is the log-simplex method, introduced in [18]. It can be seen as an extension of the method described in $[19,20]$ to the high order case. The main difference between the $P^{1}$ and the $P^{k}$ adaptation methods relies on the fact that $\mathbf{Q}$ is directly given by the Hessian matrix of $u$ when dealing with $P^{1}$ adaptation, whereas it is mandatory to find a suitable metric field satisfying (6) for the $P^{k}$ adaptation. The log-simplex algorithm is a way to compute such a metric field. It is based on a sequence of linear problems written in terms of the logarithm matrix $\mathcal{L}=\log (\mathcal{Q})$. In this section, the highlights of this method are recalled. For a full description of the algorithm, see [18].

Given a homogeneous polynomial $p$ of degree $k+1$ on $\mathbb{R}^{n}$ which stands for $d^{(k+1)} u\left(x_{0}\right)$, a set of points $\left\{x_{1}, \ldots, x_{m}\right\}$ of $\mathbb{R}^{n}$ such that $p\left(x_{i}\right)=1$ for all $i \in\{1, \ldots, m\}$ is considered. The optimization problem that the log-simplex method solves is the following.

Find a metric $\mathcal{Q}$ such that

$$
\begin{aligned}
& \operatorname{det}(\mathcal{Q}) \text { is minimal, } \\
& { }^{t} x_{i} \mathcal{Q} x_{i} \geq 1, \quad \text { for all } i \in\{1, \ldots, m\} .
\end{aligned}
$$

The first line of (7) translates the fact that we are looking for the metric with the largest area (or volume in $3 \mathrm{D}$ ). Since the cost function of this problem is nonlinear, one rewrites it as a problem in $\mathcal{L}=\log (\mathcal{Q})$. Notice that $\mathcal{L}$ is not a metric but only a symmetric matrix. This formulation also allows the discrete counterpart of the problem to be well-posed. Indeed, in [18], it is shown that the discrete form of (7) is ill-posed. For $\operatorname{det}(\mathcal{Q})=\exp (\operatorname{trace}(\mathcal{L}))$, a linear cost function is recovered by replacing $\mathcal{Q}$ by $\mathcal{L}$ in $(7)$. On the contrary, the constraints which are linear on $\mathcal{Q}$ become nonlinear when writing them in terms of $\mathcal{L}$. This can lead to really expensive computations. To avoid this problem, the convexity property of the exponential is used and replaces these constraints by approximated linear ones. More precisely, through the classic convexity inequality, if $x \in \mathbb{R}^{n}$ satisfies ${ }^{t} x \mathcal{L} x \geq-\|x\|^{2} \log \left(\|x\|^{2}\right)$, it ensures that ${ }^{t} x \mathcal{Q} x \geq 1$. By this way, the following linear optimization problem is obtained.

Find a symmetric matrix $\mathcal{L}$ such that

$$
\begin{aligned}
& \operatorname{trace}(\mathcal{L}) \quad \text { is minimal, } \\
& { }^{t} x_{i} \mathcal{L} x_{i} \geq-\left\|x_{i}\right\|^{2} \log \left(\left\|x_{i}\right\|^{2}\right), \quad \forall i \in\{1, \ldots, m\} .
\end{aligned}
$$

Since this problem is linear in $\mathcal{L}$, it can be solved by a simplex method (see for instance [21]). Unfortunately, in most of the cases, solving (8) once does not provide 
accurate metrics, in the sense that the unit ball of the obtain metric $\mathcal{Q}=\exp (\mathcal{L})$ can be far from the level set of $p$ (see Figure 3 ).
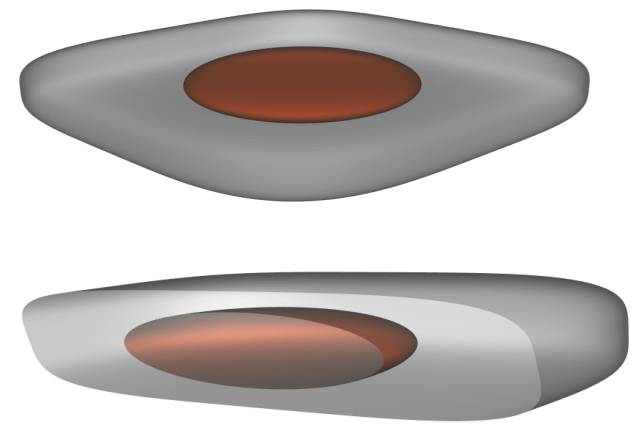

Figure 3: Illustration of the log approximation for the constraints for an error level 1 (in grey). The optimal metric (in red) is far from the boundary of

the error due to the convexity approximation.

This issue is dealt by an iterative process. More precisely, once we have computed the solution of (8) and recovered $\mathcal{Q}=\exp (\mathcal{L})$, we apply the mapping $x \rightarrow \mathcal{Q}^{\frac{1}{2}} x$ by replacing $p$ by $q=p \circ \mathcal{Q}^{-\frac{1}{2}}$. Then, we take a new set of points $\left\{x_{1}, \ldots, x_{m}\right\}$ such that $q\left(x_{i}\right)=1$, for all $i \in\{1, \ldots, m\}$ and solve again (8). Finally, the log-simplex algorithm is the following.

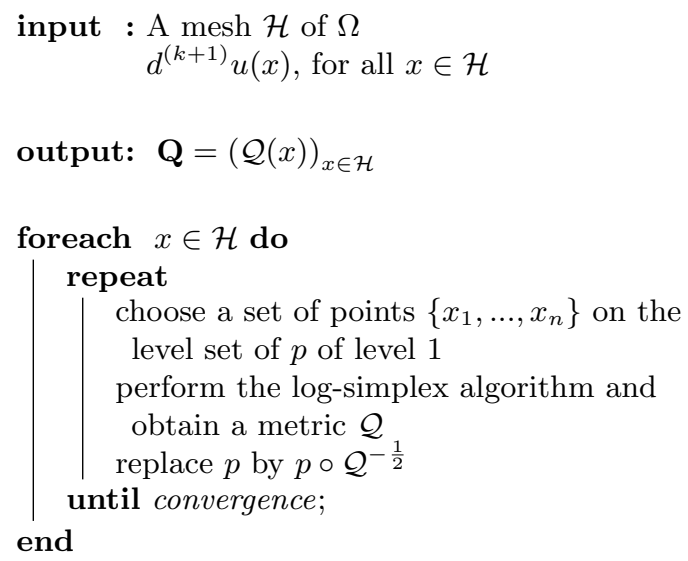

In order to implement numerically the log-simplex method, notice that this algorithm must contain a polynomial reduction so that the possible infinite branches in the level set of $d^{(k+1)} u$ disappear. All the theoretical and numerical issues of the log-simplex method are described in details in [18].

The objective of the following section is to find the function $u$ on which the high-order error model should be applied and then to deduce a metric on which mesh adaptation will be performed in the parameters space.

\section{HIGH-ORDER SURFACE MESH GENERATION}

\subsection{Metrics for linear surface mesh gener- ation}

In the case of parametric surface meshing, the whole problem is to find a suitable metric thanks to which a mesh adaptation process in the parameters space will be performed. First let us have a look on the case of curve meshing.

\subsubsection{Metrics for curve meshing}

When dealing with meshing of parametric curves, it is frequent to perform a local analysis on it. To do so, let us have a look on a Taylor expansion of a parametric curve $t \rightarrow \gamma(t) \in \mathbb{R}^{3}$, that we will assume smooth enough, in the vicinity of $t_{0}{ }^{1}$

$\gamma(t)=\gamma\left(t_{0}\right)+\gamma^{\prime}\left(t_{0}\right)\left(t-t_{0}\right)+\frac{\gamma^{\prime \prime}\left(t_{0}\right)}{2}\left(t-t_{0}\right)^{2}+\mathcal{O}\left(\left(t-t_{0}\right)^{3}\right)$.

Now if we consider a change of variable with $s$ being the curvilinear abscissa such that $s\left(t_{0}\right)=0$ and $\frac{d s}{d t}=$ $\left\|\gamma^{\prime}(t)\right\|$ then the Taylor expansion becomes:

$$
\gamma(s)=\gamma(0)+s \mathbf{T}+\frac{1}{2} \kappa\left(t_{0}\right) s^{2} \mathbf{N}+\mathcal{O}\left(s^{3}\right)
$$

where $\mathbf{T}=\frac{\gamma^{\prime}(t)}{\left\|\gamma^{\prime}(t)\right\|}$ and $(\kappa, \mathbf{N})$ are such that $\frac{d \mathbf{T}}{d s}=$ $\kappa \mathbf{N}$. $(\kappa, \mathbf{T}, \mathbf{N})$ are intrinsic data $[22] . \quad \kappa$ is called the curvature and can be computed with:

$$
\kappa(t)=\frac{\left\|\gamma^{\prime}(t) \times \gamma^{\prime \prime}(t)\right\|}{\left\|\gamma^{\prime}(t)\right\|^{3}} .
$$

By setting $\mathbf{B}=\mathbf{T} \times \mathbf{N},(\mathbf{T}, \mathbf{N}, \mathbf{B})$ defines an orthornormal basis, of so-called Frénet frame.

Note that if we denote $(x, y, z)$ the components of $\gamma(t)$ in the Frénet frame defined in $t_{0}$ with $\gamma\left(t_{0}\right)=$ $\left(x_{0}, y_{0}, z_{0}\right)$ in this frame, we have:

$$
\left\{\begin{array}{l}
y=y_{0}+\frac{1}{2} \kappa\left(t_{0}\right)\left(x-x_{0}\right)^{2}+\mathcal{O}\left(\left|x-x_{0}\right|^{3}\right) \\
z=z_{0}+\mathcal{O}\left(\left|x-x_{0}\right|^{3}\right)
\end{array}\right.
$$

Based on the Frénet frame, and on the curvature, a $3 \mathrm{D}$ metric tensor can be deduced via:

$\mathcal{M}_{1}=\left({ }^{t} \mathbf{T}^{t} \mathbf{N}^{t} \mathbf{B}\right)\left(\begin{array}{ccc}\frac{1}{(2 \sqrt{\epsilon(2-\epsilon)} \rho(t))^{2}} & 0 & 0 \\ 0 & \lambda & 0 \\ 0 & 0 & \lambda\end{array}\right)\left(\begin{array}{c}\mathbf{T} \\ \mathbf{N} \\ \mathbf{B}\end{array}\right)$

where $\lambda \in \mathbb{R}$ is an arbitrary constant ${ }^{2}, \rho(t)=\frac{1}{\kappa(t)}$ is the radius of curvature, and $2 \sqrt{\epsilon(2-\epsilon)}$ is a scaling

\footnotetext{
${ }^{1}$ In the case of NURBS, it is easy to split the NURBS according the smooth parts [2].

${ }^{2}$ It sets the size in the normal plane to the curve
} 
coefficient which guarantees, for a second order approximation of the curve, to maintain a deviation gap between the mesh elements and the curve geometry of $\epsilon[23]$. As the metric relies on only intrinsic data, it is independent of the parameterization.

The metric can be mapped back to the parameter space via the following formula:

$$
\tilde{\mathcal{M}}_{1}={ }^{t} \gamma^{\prime}(t) \mathcal{M}_{1} \gamma^{\prime}(t)
$$

In this case, the formula simplifies to $\tilde{\mathcal{M}}_{1}=\frac{1}{h_{1}^{2}}=$ $\frac{\left\|\gamma^{\prime}(t)\right\|^{2}}{(2 \sqrt{\epsilon(2-\epsilon)} \rho(t))^{2}}$.

Once the metrics for curves are set, the next step is to define metrics for the surfaces.

\subsubsection{Metrics for surface meshing}

The meshing process of parametric surfaces is a bit more complex. It relies on some differential geometry notions [22]. For this purpose, let us consider a parametric surface $(u, v) \rightarrow \sigma(u, v) \in \mathbb{R}^{3}$ that we will assume smooth enough. In this case, the first fundamental form $I(d u, d v)$ is defined as follows:

$I(d u, d v)=(d u d v)\left(\begin{array}{cc}\left\|\sigma_{u}\right\|^{2} & \left(\sigma_{u}, \sigma_{v}\right) \\ \left(\sigma_{u}, \sigma_{v}\right) & \left\|\sigma_{v}\right\|^{2}\end{array}\right)\left(\begin{array}{c}d u \\ d v\end{array}\right)$,

where $(d u, d v)$ is an elementary displacement, and $\sigma_{u}$ (resp. $\sigma_{v}$ ) the partial derivative of $\sigma$ w.r.t. $u$ (resp. $v$ ). The first fundamental form explains how the three dimensional distances are perceived in the two dimensional space. In particular, it provides a two-dimensional riemannian structure to the surface with a metric tensor defined as:

$$
\mathcal{M}_{I}=\left(\begin{array}{cc}
\left\|\sigma_{u}\right\|^{2} & \left(\sigma_{u}, \sigma_{v}\right) \\
\left(\sigma_{u}, \sigma_{v}\right) & \left\|\sigma_{v}\right\|^{2}
\end{array}\right) .
$$

In the same framework, comes also the second fundamental form $I I(d u, d v)$ that is defined as follows:

$I I(d u, d v)=\left(\begin{array}{c}d u \\ d v\end{array}\right)\left(\begin{array}{cc}\left(\sigma_{u u}, \mathbf{N}\right) & \left(\sigma_{u v}, \mathbf{N}\right) \\ \left(\sigma_{u v}, \mathbf{N}\right) & \left(\sigma_{v v}, \mathbf{N}\right)\end{array}\right)\left(\begin{array}{c}d u \\ d v\end{array}\right)$,

where $\left(\sigma_{u u}, \sigma_{u v}, \sigma_{v v}\right)$ are the second derivatives of $\sigma$ w.r.t $(u, v)$ and $\mathbf{N}=\frac{\sigma_{u} \times \sigma_{v}}{\left\|\sigma_{u} \times \sigma_{v}\right\|}$ is the normal vector to the surface. The second fundamental form expresses the gap of a surface to its tangent plane at the order two.

Based on these two quadratic forms and their matrices, we are able, for a given point of the surface, to define the principal curvatures $\left(\kappa_{i}\right)_{i=1,2}$ and principal directions $\left(\mathbf{V}_{i=1,2}\right.$ (in $\left.3 \mathrm{D}\right)$ as solution of the generalized eigenvalue problem:

$$
\left\{\begin{array}{rl}
\mathcal{M}_{I I} \mathbf{v}_{i} & =\kappa_{i} \mathcal{M}_{I} \mathbf{v}_{i} \\
\mathbf{V}_{i} & =\frac{\left(\sigma_{u} \sigma_{v}\right) \mathbf{v}_{i}}{\left\|\left(\sigma_{u} \sigma_{v}\right) \mathbf{v}_{i}\right\|}
\end{array} i=1,2\right.
$$

with $\mathcal{M}_{I I}$ being the symmetric matrix associated to the second fundamental form. These quantities are independent of the parameterization and when $\kappa_{1} \neq \kappa_{2}$, $\left(\mathbf{V}_{1}, \mathbf{V}_{2}\right)$ forms an orthonormal basis of the tangent plane. If we complete the basis with $\mathbf{N}$, they form a local basis $\left(\mathbf{V}_{1}, \mathbf{V}_{2}, \mathbf{N}\right)$ of $\mathbb{R}^{3}$. Note that if we denote $(x, y, z)$ the components of $\sigma(u, v)$ in this local basis defined in $\left(u_{0}, v_{0}\right)$ with $\sigma\left(u_{0}, v_{0}\right)=\left(x_{0}, y_{0}, z_{0}\right)$ in this basis, we have:

$$
\begin{aligned}
z & =z_{0}+\frac{1}{2}\left(\kappa_{1}\left(u_{0}, v_{0}\right)\left(x-x_{0}\right)^{2}+\kappa_{2}\left(u_{0}, v_{0}\right)\left(y-y_{0}\right)^{2}\right) \\
& +\mathcal{O}\left(\left\|\left(x-x_{0}, y-y_{0}\right)\right\|^{3}\right) .
\end{aligned}
$$

Now, thanks to this basis and on the curvatures, we can define the following 3D metric tensor:

$$
\begin{aligned}
& \mathcal{M}_{2}=\left({ }^{t} \mathbf{V}_{1}{ }^{t} \mathbf{V}_{2}{ }^{t} \mathbf{N}\right) \times \\
& \left(\begin{array}{ccc}
\frac{1}{\left(c_{1} \rho_{1}(u, v)\right)^{2}} & 0 & 0 \\
0 & \frac{1}{\left(c_{2} \rho_{2}(u, v)\right)^{2}} & 0 \\
0 & 0 & \lambda
\end{array}\right)\left(\begin{array}{c}
\mathbf{V}_{1} \\
\mathbf{V}_{2} \\
\mathbf{N}
\end{array}\right)
\end{aligned}
$$

where $\lambda \in \mathbb{R}$ is an arbitrary constant, $\rho_{i}(u, v)=\frac{1}{\kappa_{i}(u, v)}$ for $i=1,2$ are the radii of curvature, with the convention $\rho_{1}(u, v) \leq \rho_{2}(u, v)$ and $c_{1}$ and $c_{2}$ are scaling coefficients. For the direction of greater curvature (e.g. the direction given by $\mathbf{V}_{1}$ ), we want to control the deviation under a threshold $\epsilon$ which comes down to set $c_{1}$ to the value of $2 \sqrt{\epsilon(2-\epsilon)}$. Now, as we want the same threshold in all the directions in the tangent plane, the coefficient $c_{2}$ is set to $2 \sqrt{\epsilon \frac{\rho_{1}}{\rho_{2}}\left(2-\epsilon \frac{\rho_{1}}{\rho_{2}}\right)}$ [24]. Similarly to curves, the metric relies only on intrinsic data and is therefore independent of the parameterization Now, the metric $\mathcal{M}_{2}$ can be mapped back to the parameters space by applying the first fundamental form:

$$
\tilde{\mathcal{M}}_{2}=\left({ }^{t} \sigma_{u}{ }^{t} \sigma_{v}\right) \mathcal{M}_{2}\left(\begin{array}{c}
\sigma_{u} \\
\sigma_{v}
\end{array}\right) .
$$

This is the metric that will be used as an anisotropic metric for the mesh adaptation in the parameters space.

\subsection{Computation of higher-order metrics}

The object of this section is to deal with the extension of the previous framework for higher-order elements. In particular, we seek for parameterization independent Taylor expansion similar to (9) and to (10) with terms of degree greater than 2. First let us have a look on the case of the curve.

\subsubsection{Case of the curve}

As seen previously, the metric should rely on intrinsic data to be independent of the parameterization. A way to do so is to have a look at the formula (9). This 
formula gives a Taylor expansion of the gap of a curve to the straight edge at the order two and shows that it is driven by the curvature. Moreover, this expansion is done with the physical coordinates which naturally gives an independence with respect to any parameterization. A natural idea is therefore to extend the previous Taylor expansion to get higher-order terms and deduce metrics that will be fitted to high-order approximation.

To do so, let us write $\gamma(t)$ in the Frénet frame $\left(\mathbf{T}_{0}, \mathbf{N}_{0}, \mathbf{B}_{0}\right)$ associated to $t_{0}$, a regular point of $\gamma$. If we note $X=x-x_{0}, Y=y-y_{0}, Z=z-z_{0}$, we have:

$$
\left\{\begin{array}{l}
X=\left(\gamma(t)-\gamma\left(t_{0}\right), \mathbf{T}_{0}\right) \\
Y=\left(\gamma(t)-\gamma\left(t_{0}\right), \mathbf{N}_{0}\right) \\
Z=\left(\gamma(t)-\gamma\left(t_{0}\right), \mathbf{B}_{0}\right)
\end{array}\right.
$$

Let us note $\phi(t)=\left(\gamma(t)-\gamma\left(t_{0}\right), \mathbf{T}_{0}\right)$. If $t_{0}$ is a regular point of $\gamma$ then $\gamma^{\prime}\left(t_{0}\right) \neq 0$ and therefore $\phi^{\prime}\left(t_{0}\right) \neq 0$. The inversion function theorem can thus be applied and there exists a function $\psi$ such that $\psi(X)=\psi(\phi(t))=t-t_{0}$ in the vicinity of $t_{0}$. Moreover, if $\phi$ is $C^{k+1}$ then $\psi$ is $C^{k+1}$ and $\psi^{\prime}(X)=\frac{1}{\phi^{\prime}(t)}$ with $X=\phi(t)$.

Based on this statement, it is thus possible to get a Taylor expansion of $t-t_{0}$ with respect to $X$ up to order $k$. To do so, let us compute the higher-order derivatives of $\psi$ in $t_{0}$. As $\gamma$ ( and consequently $\phi$ ) is an analytical function issued from CAD model, all its derivatives can be computed using the implementation details of [2]. The derivatives of $\psi$ are then deduced using the following result [25]:

Theorem 2.1 (Faà di Bruno's Formula) Let us consider $f, g: \mathbb{R} \rightarrow \mathbb{R}$ of class $C^{k+1}$ with $k+1 \geq n$, then

$$
\begin{aligned}
& \frac{\mathrm{d}^{n}}{\mathrm{~d} x^{n}}(g(f(x)))= \\
& \sum_{E} \frac{n !}{m_{1} ! . . m_{n} !} g^{\left(m_{1}+\ldots+m_{n}\right)}(f(x)) \prod_{j=1}^{n}\left(\frac{f^{(j)}(x)}{j !}\right)^{m_{j}}
\end{aligned}
$$

where $E=\left\{\left(m_{1}, . ., m_{n}\right) \in \mathbb{N}^{n} \mid \sum_{k=1}^{n} k \cdot m_{k}=n\right\}^{3}$.

In our case, we set $g=\psi$ and $f=\phi$ and it comes that $\frac{\mathrm{d}^{n}}{\mathrm{~d} x^{n}}(g(f(x)))=0$ for $k+1 \geq n \geq 2$.

This brings us to that for $k+1 \geq n \geq 2$ :

$\left(\phi^{\prime}(t)\right)^{n} \psi^{(n)}(x)=F\left(\psi^{\prime}(x), . ., \psi^{(n-1)}(x), \phi^{\prime}(t), . ., \phi^{(n)}(t)\right)$,

where $F$ is a function that can be directly deduced from Theorem 2.1.

This result means that as long as $\phi^{\prime}(t) \neq 0$, then $\psi^{(n)}(x)$ can be recursively computed given its previous

\footnotetext{
${ }^{3}$ Note that in practice, the set $E$ can be precomputed once for all for the range of values of $n$ that are used.
}

derivatives and the derivatives of $\phi$.

Thanks to this, we can now write:

$$
t=t_{0}+\sum_{n=1}^{k+1} a_{n} X^{n}+\mathcal{O}\left(|X|^{k+2}\right),
$$

where $a_{n}$ have been computed with the derivatives of $\psi$. Now if we recall that $\gamma$ is $C^{k+1}$, we also have:

$$
\left\{\begin{array}{l}
Y=\sum_{n=1}^{k+1} b_{n}\left(t-t_{0}\right)^{n}+\mathcal{O}\left(\left|t-t_{0}\right|^{k+2}\right), \\
Z=\sum_{n=1}^{k+1} c_{n}\left(t-t_{0}\right)^{n}+\mathcal{O}\left(\left|t-t_{0}\right|^{k+2}\right) .
\end{array}\right.
$$

By composition of both Taylor expansion, we then obtain a Taylor expansion of $Y$ and $Z$ in $X$ at the order $k+1 \geq 2$, which is independent of the parameterization and a generalization of (9).

From this, we have an intrinsic information of the gap of a curve to the straight edge up to the order $k+1$. We can then write:

$$
\left(\begin{array}{c}
Y \\
Z
\end{array}\right)=F_{k}(X)+A_{k+1} X^{k+1}+\mathcal{O}\left(|X|^{k+2}\right),
$$

where $F_{k}(X)$ is a polynomial of degree $k$ in $\mathrm{X}$ and $A_{k+1} \in \mathbb{R}^{2}$. For an approximation of a curve at the degree $k$, the leading term of the error is therefore $A_{k+1} X^{k+1}[19,20,18]$. Thus, if we control $\left\|A_{k+1} X^{k+1}\right\|$, the error of approximation will be controlled. Now, if we note that:

$$
\left\|A_{k+1} X^{k+1}\right\|=\left(\left\|A_{k+1}\right\|^{\frac{2}{k+1}} X^{2}\right)^{\frac{k+1}{2}},
$$

we can then set $\kappa_{k+1}=2\left\|A_{k+1}\right\|^{\frac{2}{k+1}}$ and reuse the metrics used for the linear meshing with $\rho=\frac{1}{\kappa_{k+1}}$ for radii of curvature and $\epsilon_{k+1}=\epsilon^{\frac{2}{k+1}}$ for threshold. This way, the classic formula is found for $k=1$ and a generalization is proposed for $k \geq 2$. Note that in some configurations, the found size for the order $k$ can be significantly lower than the found size for the order $k+1$. In this case the size given by order $k+1$ is preferred.

Now, let us interest to the more complex case of the surfaces.

\subsubsection{Case of the surface}

Like for curves, the idea is to start from an intrinsic representation of the surface. For this purpose, let us have a look at formula (10). This formula gives a Taylor expansion of the gap of a surface to its tangent plane in physical coordinates which is an intrinsic representation. As previously, our idea is to extend this Taylor expansion to higher-order terms.

To do so, let us note $\left(\mathbf{V}_{1,0}, \mathbf{V}_{2,0}, \mathbf{N}_{0}\right)$ the local basis 
defined for a regular point $\left(u_{0}, v_{0}\right)$ of the surface. If we note $X=x-x_{0}, Y=y-y_{0}, Z=z-z_{0}$, we have:

$$
\left\{\begin{array}{l}
X=\left(\sigma(u, v)-\sigma\left(u_{0}, v_{0}\right), \mathbf{V}_{1,0}\right) \\
Y=\left(\sigma(u, v)-\sigma\left(u_{0}, v_{0}\right), \mathbf{V}_{2,0}\right) \\
Z=\left(\sigma(u, v)-\sigma\left(u_{0}, v_{0}\right), \mathbf{N}_{0}\right)
\end{array}\right.
$$

Now, if we define:

$$
\Phi(u, v)=\left(\begin{array}{c}
\left(\sigma(u, v)-\sigma\left(u_{0}, v_{0}\right), \mathbf{V}_{1,0}\right) \\
\left(\sigma(u, v)-\sigma\left(u_{0}, v_{0}\right), \mathbf{V}_{2,0}\right)
\end{array}\right)
$$

and if $\left(u_{0}, v_{0}\right)$ is a regular point of $\sigma$, then its jacobian matrix $J_{\Phi}$ is invertible in $\left(u_{0}, v_{0}\right)$. Consequently, the inverse function theorem can be applied and there exists a function $\Psi$ such that $\Psi(X, Y)=\Psi(\Phi(u, v))=$ $\left(u-u_{0}, v-v_{0}\right)$ in the vicinity of $\left(u_{0}, v_{0}\right)$. Moreover, if $\Phi$ is $C^{k+1}$ then $\Psi$ is $C^{k+1}$ and $J_{\Psi}(X, Y)=J_{\Phi}(u, v)^{-1}$ with $(X, Y)=\Phi(u, v)$.

With this statement, we know that we can have a Taylor expansion of $\left(u-u_{0}, v-v_{0}\right)$ with respect to $(X, Y)$ up to order $k+1$. For this purpose, let us compute the higher-order derivatives of $\Psi$. As $\sigma$ (and therefor $\Phi)$ is an analytical function issued from a CAD model, all its derivatives can be computed using the recipes in [2]. The derivatives of $\Psi$ can be then deduced using the following result [26]:

\section{Theorem 2.2 (2D Faà di Bruno's Formula)}

Let us consider $f, g: \mathbb{R}^{2} \rightarrow \mathbb{R}^{2}$ of class $C^{k+1}$ with $k+1 \geq|\alpha|$, then

$$
\begin{aligned}
& \frac{\partial^{|\alpha|}}{\partial x^{\alpha}}(g(f(x)))=\sum_{|\sigma|=1}^{n} \alpha ! \frac{\partial^{|\sigma|}}{\partial x^{\sigma}}(g(f(x))) \times \\
& \sum_{E_{\sigma}} \prod_{i=1}^{2} \prod_{A_{\alpha}} \frac{1}{e_{i \alpha^{i}} !}\left(\frac{1}{\alpha^{i} !} \frac{\partial^{\left|\alpha^{i}\right|}}{\partial x^{\alpha^{i}}}\left(f_{i}(x)\right)\right)^{e_{i \alpha^{i}}},
\end{aligned}
$$

where $f(x)=\left(f_{1}(x), f_{2}(x)\right), \alpha=\left(\alpha_{1}, \alpha_{2}\right) \in \mathbb{N}^{2}, n=$ $|\alpha|=\alpha_{1}+\alpha_{2}, \sigma=\left(\sigma_{1}, \sigma_{2}\right) \in \mathbb{N}^{2}$

$E_{\sigma}=$

$\left\{\left(e_{1 \alpha^{1}}, e_{2 \alpha^{2}}\right) \in \mathbb{N}^{2},\left|\alpha^{i}\right| \in \llbracket 1, n \rrbracket,\left(\sum_{\left|\alpha^{i}\right|=1}^{n} e_{i \alpha^{i}}=\sigma_{i},\right)_{i=1,2}\right\}$,

and $^{4}$

$$
\begin{aligned}
& A_{\alpha}= \\
& \left\{\left(\alpha^{1}, \alpha^{2}\right):\left|\alpha^{i}\right| \in \llbracket 1, n \rrbracket, i=1,2, \sum_{i=1}^{2} \sum_{\left|\alpha^{i}\right|=1}^{n} e_{i \alpha^{i}} \cdot \alpha^{i}=\alpha\right\} .
\end{aligned}
$$

In our case, we set $g=\Psi$ and $f=\Phi$ and it comes that $\frac{\partial^{|\alpha|}}{\partial x^{\alpha}}(g(f(x)))=0$ for $k+1 \geq|\alpha| \geq 2$.

\footnotetext{
${ }^{4}$ Note that in practice, the sets $E_{\sigma}$ and $A_{\alpha}$ can be precomputed once for all for the range of values of $\alpha$ and $\sigma$ that are used.
}

If we consider all the $n+1 \alpha$ such that $|\alpha|=n$, then we have a system of equations of the kind:

$$
\begin{aligned}
& A\left(\frac{\partial^{|\alpha|} \Phi}{\partial x^{\alpha}}\right)_{|\alpha|=n} \times\left(\frac{\partial^{|\alpha|} \Psi}{\partial x^{\alpha}}\right)_{|\alpha|=n}= \\
& F\left(\left(\frac{\partial^{|\alpha|} \Psi}{\partial x^{\alpha}}\right)_{|\alpha|<n},\left(\frac{\partial^{|\alpha|} \Phi}{\partial x^{\alpha}}\right)_{|\alpha| \leq n}\right),
\end{aligned}
$$

where $A$ is $(n+1) \times(n+1)$ matrix, $\left(\frac{\partial^{|\alpha|} \Psi}{\partial x^{\alpha}}\right)_{|\alpha|=n}$ is a vector of size $n+1$ containing the $n+1$ derivatives of $\Psi$ of order $n$ and $F$ is vector function of size $n+1$ that can be deduced from theorem 2.2. Moreover, it is shown in [26] that $|A|=\left|J_{\Phi}\right|^{n}$, which proves that the system has always a solution if the inverse function theorem is successfully applied.

This way, a recursive method to compute all the derivative of $\Psi$ in $\left(u_{0}, v_{0}\right)$ is set and the computation of the Taylor expansion is therefore possible:

$$
\left(\begin{array}{c}
u-u_{0} \\
v-v_{0}
\end{array}\right)=\sum_{n=1}^{k+1} \sum_{i+j=n} A_{i j}^{n} X^{i} Y^{j}+\mathcal{O}\left(\|(X, Y)\|^{k+2}\right),
$$

where $A_{i j}^{n} \in \mathbb{R}^{2}$ and is defined thanks to the partial derivatives of $\Psi$.

But, as $\sigma$ is $C^{k+1}$, we also have:

$$
\begin{aligned}
& Z=\sum_{n=1}^{k+1} \sum_{i+j=n} c_{i j}^{n}\left(u-u_{0}\right)^{i}\left(v-v_{0}\right)^{j} \\
& +\mathcal{O}\left(\left\|\left(\left(u-u_{0}\right),\left(v-v_{0}\right)\right)\right\|^{k+2}\right) .
\end{aligned}
$$

By composition of both Taylor expansions, we then obtain a Taylor expansion of $Z$ in $(X, Y)$ at the order $k+1 \geq 2$ which is independent of the parameterization and a generalization of the formula (10).

The gap to the tangent plane is thus expressed up to the order $k+1$ :

$$
Z=F_{k}(X, Y)+R_{k+1}(X, Y)+\mathcal{O}\left(\|(X, Y)\|^{k+2}\right),
$$

where $F_{k}$ is a polynomial of degree $k$ and $R_{k+1}$ is an homogeneous polynomial of degree $k+1$. For an approximation of the surface at the degree $k$, the leading term of the error is $R_{k+1}(X, Y)[19,20,18]$. So, if we want to control the $P^{k}$ approximation, we need to control $\left|R_{k+1}(X, Y)\right|$.

By applying the log-simplex algorithm explained in the previous section, we are able to find a metric that satisfies an inequality like (6), that is to say, we are able to compute a matrix $Q_{k+1}$ such that:

$$
\left|R_{k+1}(X, Y)\right| \leq\left(\frac{1}{2}(X Y) Q_{k+1}\left(\begin{array}{c}
X \\
Y
\end{array}\right)\right)^{\frac{k+1}{2}},
$$

where $Q_{k+1}$ is the optimal symmetric matrix (in a sense explained in the first section) that verifies this inequality. 
If we note $\left(\kappa_{i, k+1}, \mathbf{v}_{i, k+1}\right)_{i=1,2}$, the eigenvalues and eigenvectors of $Q_{k+1}$, we can then reuse the metrics used for the linear meshing with $\rho_{i}=\frac{1}{\kappa_{i, k+1}}$ the radii of curvature, $\mathbf{V}_{i, k+1}=\left(\mathbf{V}_{1,0} \mathbf{V}_{2,0}\right) \mathbf{v}_{i, k+1}$ the principal directions in the tangent plane and $\epsilon_{k+1}=\epsilon^{\frac{2}{k+1}}$ the threshold. This way, the classic formula is found for $k=1$ and a generalisation is proposed for $k \geq 2$. Like for curves, in some configurations, found sizes for the order $k$ can be significantly lower than found sizes for the order $k+1$. In this case sizes given by order $k+1$ are preferred.

\subsection{Meshing process}

The mesh generation process is based on the classical unit-mesh concept, where a metric field, as $\mathcal{M}_{2}$ or $\tilde{\mathcal{M}}_{2}$, is used to drive the orientation and sizing of the elements. In the context of parametric surface meshing, several approaches are typically devised to generate a final 3D surface mesh. Full 2D methods are a convenient way to avoid $3 \mathrm{D}$ surface meshing and inverse projection to the geometry. However, a special care is needed to handle degenerated points, periodicity, highly non uniform (even discontinuous) parameterization or degenerated edges. Approaches that mix $2 \mathrm{D}$ and $3 \mathrm{D}$ methods tend to reduce the impact of the parametric space to the final mesh.

In the paper, we consider a rather classical approach. The core steps of the procedure are decomposed as follows:

\section{For each Edge}

1.1 Generate a 3D adaptive mesh using $\mathcal{M}_{1}$

2. For each Face

2.1 Generate a fast $(u, v)$-aligned tessellation,

2.2 Compute High-order metric $\tilde{\mathcal{M}}_{2}$ on the tessellation,

2.3 Project 3D Edges of Loops as parametric curves and generate a $2 \mathrm{D}(u, v)$ mesh forming the boundary of the patch,

2.4 Re-cycle points from the tessellation : insert points from the tessellation onto the current mesh,

2.4 Move to $3 \mathrm{D}$, convert $\tilde{\mathcal{M}}_{2}$ to $\mathcal{M}_{2}$ to adapt the mesh,

2.5 Generate high-order mesh.

The EGADS API [27] is used to perform the CAD linking and the planar mesh generation process is performed using a Delaunay triangulation-based algorithm[28].

\section{EXAMPLES}

We illustrate our approach on several examples. To compute error indicates, the computation of an exact Haussdorf distance would be too expansive. Therefore, a normalized and an absolute error in distance are computed. For each triangle of the generated surface mesh, a sampling of the underlying parameters space is performed and the distance between a point of the curved mesh and its surface counterpart is performed:

$\delta(\tilde{u}, \tilde{v}, \tilde{w})$
$=\left\|\sigma\left(\sum_{i=0}^{n} \phi_{i}(\tilde{u}, \tilde{v}, \tilde{w})\left(u_{i}, v_{i}\right)\right)-\sum_{i=0}^{n} \phi_{i}(\tilde{u}, \tilde{v}, \tilde{w}) \sigma\left(u_{i}, v_{i}\right)\right\|$,

where $\left(u_{i}, v_{i}\right)$ is the set of points of the geometry used to define the high-order triangle $\phi_{i}$ the associated shape functions and $(\tilde{u}, \tilde{v}, \tilde{w})$ are the barycentric coordinates of the triangle.

To get the normalized error in distance, the absolute distance is normalized by multiplying it by the local curvature of greater value.

The worst of all these errors then gives the overall errors of the meshing process.

Note that the relative error can be higher than the absolute one. This process can be explained for several reasons. Firstly, the absolute error, is dependent on the system of units. In the case of the shuttle, the average order of magnitude for the bounding box is $10^{2}-10^{3}$ while it is of $10^{0}-10^{1}$ in the case of the torus. Secondly, the point of worst value that determines the error is not necessarily the same for both errors. The worst value of the absolute error can occur in high stretching zones where the curvature is high, which emphasizes the value. Thus, it provides levels of error of greater magnitude.

Note that all the figures are obtained using Vizir [29].

Sphere example. Despite its simplicity, the unit sphere example is used to illustrate that our error estimate is independent to the parameterization of the model. A classic boundary representation of a sphere is to consider a surface of revolution, where the two pole maps to two degenerated edges. In the vicinity of these points, depending on the underlying CAD kernel, the definition of normals or more generally the definition of the derivatives of $u, v$ are either undefined or unstable.

We illustrate the obtained Taylor expansion on the point of the sphere using the inversion formula given by (11). Note that the sphere is parameterized as a 
circle of revolution. far from the pole, we have :

$$
\begin{aligned}
Z= & -0.5 Y^{2}-0.5 X^{2}-0.125 Y^{4} \\
& -0.25 X^{2} Y^{2}-0.125 X^{4} \\
& -0.0625 Y^{6}-0.1875 X^{2} Y^{4} \\
& -0.1875 X^{4} Y^{2}-0.0625 X^{6} \\
& +\mathcal{O}\left(\|(X, Y)\|^{7}\right) .
\end{aligned}
$$

In the vicinity of the two poles, the expansion is

$$
\begin{aligned}
Z= & -0.5 Y^{2}-0.5 X^{2}-0.125 Y^{4} \\
& -0.25 X^{2} Y^{2}-0.125 X^{4} \\
& -1.80444 e^{-9} X Y^{4}+1.9886 e^{-9} X^{3} Y^{2} \\
& -0.0625 Y^{6}-0.187501 X^{2} Y^{4} \\
& -0.187499 X^{4} X^{2}-0.0625 X^{6} \\
& +\mathcal{O}\left(\|(X, Y)\|^{7}\right) .
\end{aligned}
$$

We observe that a numerical noise appears while an almost perfect expansion is obtained as in regular points. As expected, the second order terms reveal half the principal curvatures. This numerical noise is a consequence of the manipulation of the huge values at stake in the vicinity of the apex. As the value of the derivative of the parameters is going to 0 , the normalization can lead to arbitrary huge values. In the end, everything is simplified but the numerical computations are a bit impacted by this.

Torus. The torus example is based on a NURBS representation is based on two NURBS of degree 5 both defined by 6 control points and 12 knots. $P^{1}$, $P^{2}$ and $P^{3}$ meshes are generated for this case. The point-wise normalized and absolute error in distance are reported in Table 1 . The meshes and point-wise errors are reported in Figures 4 and 5 .

\begin{tabular}{|c|c|c|c|}
\hline Order & DOF & Normalized & Absolute \\
\hline$P^{1}$ & 2787 & 0.0371489 & 0.275027 \\
\hline$P^{2}$ & 666 & 0.0287625 & 0.18465 \\
\hline$P^{3}$ & 252 & 0.0137922 & 0.0883003 \\
\hline
\end{tabular}

Table 1: Deviation to the geometry for torus geometry for $P^{1}$ to $P^{3}$ meshes.

Also, to show the efficiency of the method on this case, a study has been made between a method where the $P^{1}$ metric will be used and ours where a $P^{k}$ metric is used to generate a meh of degree $k$. For various absolute error levels, $P^{1}$-metric based and $P^{k}$-metric based meshes are generated and comparison are done with regards to the number of needed degrees of freedom. In Table 2 (resp 3 ), a comparison between a $P^{1}$-metric and a $P^{2}$-metric (resp. $P^{3}$-metric) is done. It shows in particular, that for a given error, the number of requested degrees of freedom is higher using the $P^{1}$ error than the $P^{2}$ (resp. $P^{3}$ ) one. If we have a look at the meshes with the point-wise error in Figure 6 (resp 7), we explain it by the fact that the high-order metric is able to detect high-order features that the $P^{1}$-metric does not detect. In particular, we see that the error due to the presence of an annulus on the torus is mostly canceled. And from a more global point a view, the high-order metric provides a mesh with a more equitable distribution of the error with a lower number of degrees of freedom.

\begin{tabular}{|c|c|c|}
\hline Error & $P^{1}$-metric \#DOF & $P^{2}$-metric \#DOF \\
\hline 0.5 & 347 & 156 \\
\hline 0.2 & 687 & 242 \\
\hline 0.03 & 1556 & 1036 \\
\hline
\end{tabular}

Table 2: Comparison between the number of needed degrees of freedom to get a given error between a $P^{1}$-metric and a $P^{2}$-metric.

\begin{tabular}{|c|c|c|}
\hline Error & $P^{1}$-metric \#DOF & $P^{3}$-metric \#DOF \\
\hline 0.1 & 255 & 238 \\
\hline 0.002 & 6171 & 1848 \\
\hline 0.0002 & 11329 & 5362 \\
\hline
\end{tabular}

Table 3: Comparison between the number of needed degrees of freedom to get a given error between a $P^{1}$-metric and a $P^{3}$-metric.

Shuttle. The shuttle geometry is based on two NURBS of degree 3 defined by 8 (resp. 13) control points and 12 (resp. 17) knots with strong variation in the parametric space. The $P^{1}, P^{2}$ and $P^{3}$ meshes are depicted in Figures 8 and 9. The error to the geometry are reported in Table 4.

\begin{tabular}{|c|c|c|c|}
\hline Order & DOF & Normalized & Absolute \\
\hline$P^{1}$ & 1647 & 254.551 & 197.814 \\
\hline$P^{2}$ & 1690 & 61.6727 & 58.3507 \\
\hline$P^{3}$ & 1791 & 7.02513 & 22.217 \\
\hline
\end{tabular}

Table 4: Deviation to the geometry for the shuttle geometry. 

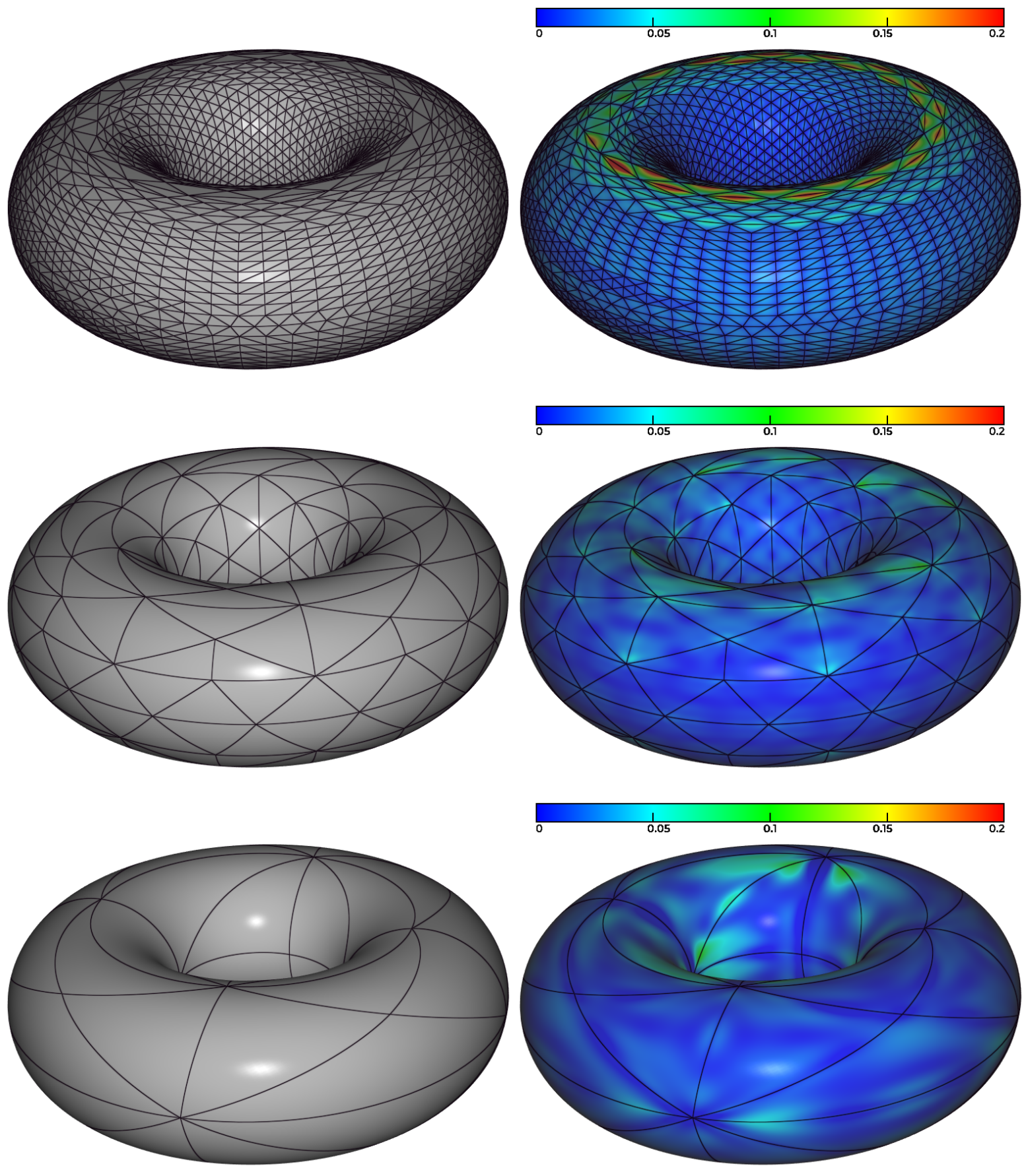

Figure 4: $P^{1}$ (top), $P^{2}$ (middle) and $P^{3}$ (bottom) meshes for the torus geometry.
Figure 5: $P^{1}$ (top), $P^{2}$ (middle) and $P^{3}$ (bottom) point-wise distance to the torus geometry. 

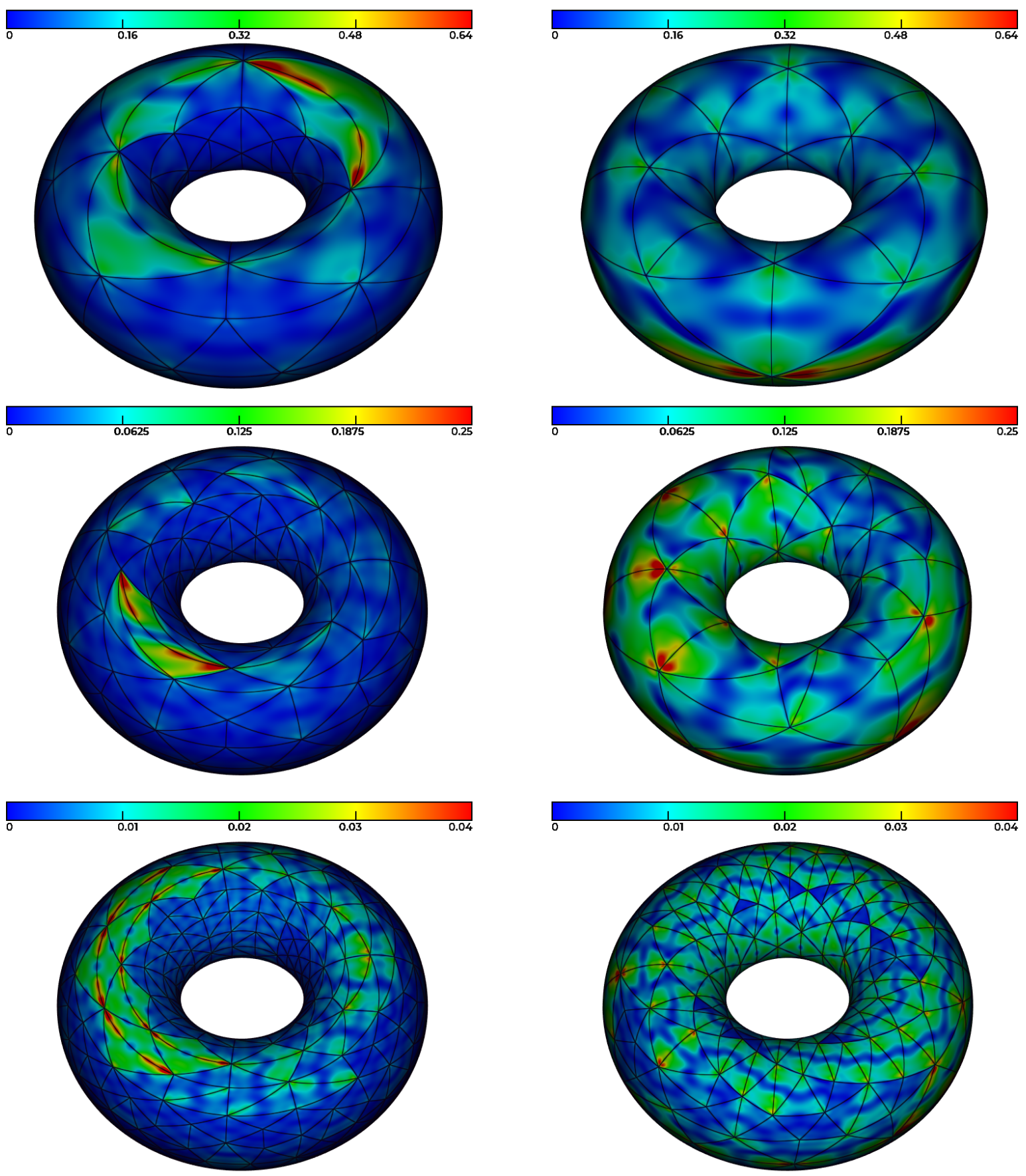

Figure 6: $P^{2}$ meshes generated with a $P^{1}$-metric (left) and $P^{2}$-metric (right) for various error levels: 0.5 (top), 0.2 (middle), 0.03 (bottom) 

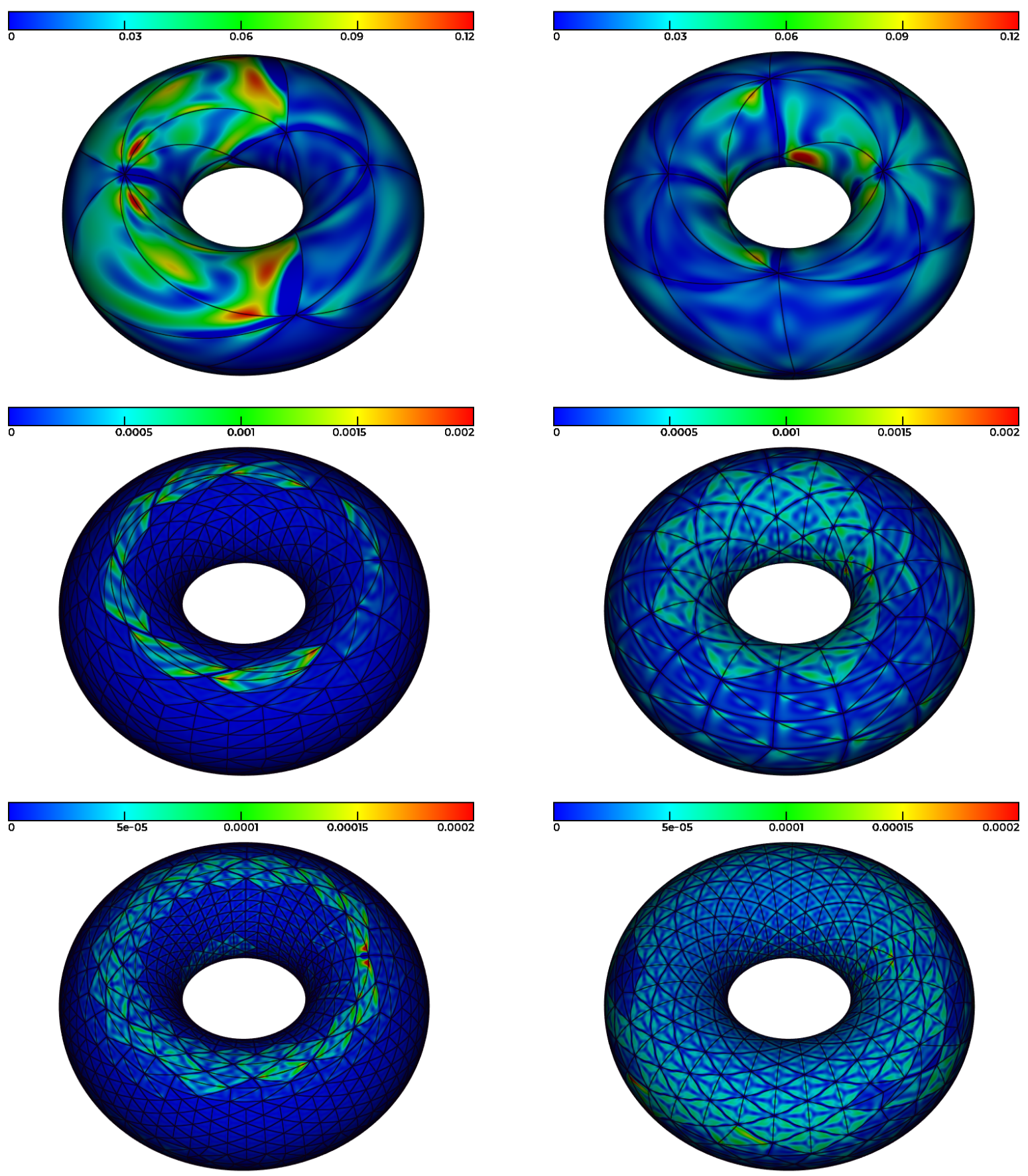

Figure 7: $P^{3}$ meshes generated with a $P^{1}$-metric (left) and $P^{3}$-metric (right) for various error levels: 0.1 (top), 0.002 (middle), 0.0003 (bottom) 

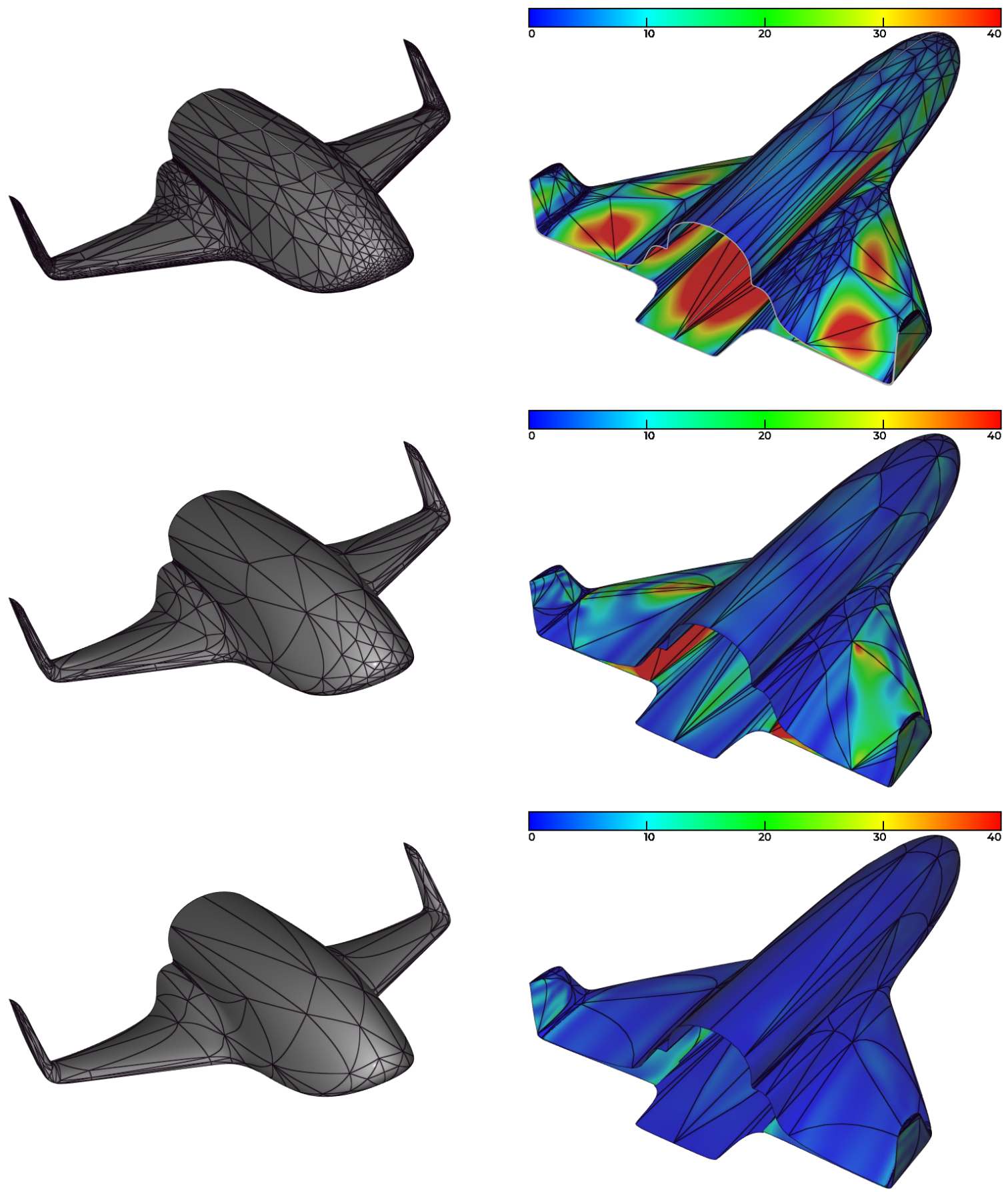

Figure 8: $P^{1}$ (top), $P^{2}$ (middle) and $P^{3}$ (bottom) meshes for the shuttle geometry.
Figure 9: $P^{1}$ (top), $P^{2}$ (middle) and $P^{3}$ (bottom) point-wise distance to the shuttle geometry. 


\section{CONCLUSION}

Generating high-order curved surface meshes from a geometry require the derivation of intrinsic quantities of the surface in order to guarantee the approximation. In the paper, we have used a Taylor expansion coupled with an inversion formula to derive a local approximation of the underlying surface in the Frénet frame. To extend the notion of principal curvature, a log-simplex approach is used to approximate optimally the variation of the polynomial by a quadratic function. The eigenvalues and eigenvectors can be viewed as "high-order" curvatures. As a metric field is naturally derived, these estimates can be used directly in any adaptive anisotropic mesh generation process.

Natural perspectives are possible for this work. Notably, this work could be extended to the implicit surface meshing. Indeed, once the implicit function theorem is applied, it is possible to apply the previous framework in order to get a Taylor expansion in any point of the surface and consequently deduce a metric. The only limitation to this meshing method would be to find the set of points where the surface is evaluated, which is the same problem as in linear meshing of implicit surfaces.

Also, the parametric mesh generation process could be improved by performing metric based curvilinear mesh adaptation to optimize the position of the high-order nodes. This problem is currently under investigation.

\section{ACKNOWLEDGMENTS}

This work was supported by the following public grants : Investissement d'avenir project, reference ANR-11-LABX-0056-LMH, LabEx LMH and ANR IMPACTS, reference ANR-18-CE46-0003.

The authors are also grateful to Bob Haimes (MIT) for giving some advice about the use of the EGADS API for high-order differentiation.

\section{References}

[1] Alleaume A. Automatic Non-manifold Topology Recovery and Geometry Noise Removal, pp. 267279. Springer Berlin Heidelberg, Berlin, Heidelberg, 2009

[2] Piegl L., Tiller W. The NURBS Book (2nd Ed.). Springer-Verlag New York, Inc., New York, NY, USA, 1997

[3] de Cougny H.L., Shephard M.S. "Surface meshing using vertex insertion." Proceedings of the 5th International Meshing Roundtable, pp. 243-256. 1996
[4] Tristano J.R., Owen S.J., Canann S.A. "Advancing front surface mesh generation in parametric space using a riemannian surface definition." Proceedings of the 7th International Meshing Roundtable. 1998

[5] Miranda A.C., Martha L.F. "Mesh generation on high-curvature surfaces based on a background quadtree structure." Proceedings of the 11th International Meshing Roundtable. 2002

[6] Wang D., Hassan O., Morgan K., Weatherill N. "EQSM: An efficient high quality surface grid generation method based on remeshing." Computer Methods in Applied Mechanics and Engineering, vol. 195, no. 41-43, 5621-5633, 2006

[7] Laug P. "Some aspects of parametric surface meshing." Finite Elements in Analysis and Design, vol. 46, no. 1-2, 216 - 226, 2010

[8] Siqueira D., Cavalcante-Neto J.B., Vidal C.A., da Silva R.J. "A Hierarchical Adaptive Mesh Generation Strategy for Parametric Surfaces Based on Tree Structures." 2010 23rd SIBGRAPI Conference on Graphics, Patterns and Images, pp. 79-86. IEEE, 2010

[9] de Siqueira D.M.B., Freitas M.O., CavalcanteNeto J.B., Vidal C.A., da Silva R.J. "An Adaptive Parametric Surface Mesh Generation Method Guided by Curvatures." Proceedings of the 22nd International Meshing Roundtable. 2014

[10] Aubry R., Dey S., Mestreau E., Karamete B., Gayman D. "A robust conforming NURBS tessellation for industrial applications based on a mesh generation approach." Computer-Aided Design, vol. $63,26-38,2015$

[11] Borouchaki H., George P.L. "Maillage de surfaces paramétriques. Partie I: Aspects théoriques." Research Report RR-2928, INRIA, 1996

[12] Toulorge T., Lambrechts J., Remacle J.F. "Optimizing the geometrical accuracy of curvilinear meshes." Journal of Computational Physics, vol. $310,361-380,2016$

[13] Ruiz-Gironès E., Sarrate J., Roca X. "Defining an $L^{2}$-disparity Measure to Check and Improve the Geometric Accuracy of Non-interpolating Curved High-order Meshes." Procedia Engineering, vol. 124, 122-134, 2015

[14] Ruiz-Gironès E., Roca X., Sarrate J. "High-order mesh curving by distortion minimization with boundary nodes free to slide on a 3D CAD representation." Computer-Aided Design, vol. 72, 52 64, 2016. 23rd International Meshing Roundtable Special Issue: Advances in Mesh Generation 
[15] Ruiz-Gironès E., Sarrate J., Roca X. "Generation of curved high-order meshes with optimal quality and geometric accuracy." Procedia engineering, vol. 163, 315-327, 2016

[16] Turner M. "High-order mesh generation for CFD solvers." PhD Dissertation Thesis, Imperial College, 2018

[17] Gargallo-Peiró A., Roca X., Peraire J., Sarrate J. "A distortion measure to validate and generate curved high-order meshes on CAD surfaces with independence of parameterization." International Journal for Numerical Methods in Engineering, vol. 106, no. 13, 1100-1130, 2016

[18] Coulaud O., Loseille A. "Very High Order Anisotropic Metric-Based Mesh Adaptation in 3D." Procedia Engineering, vol. 163, 353-364, 2016

[19] Loseille A., Alauzet F. "Continuous mesh framework part I: well-posed continuous interpolation error." SIAM J. Numer. Anal., vol. 49, no. 1, 38-60, 2011

[20] Loseille A., Alauzet F. "Continuous mesh framework part II: validations and applications." SIAM J. Numer. Anal., vol. 49, no. 1, 61-86, 2011

[21] Dantzig G.B., Thapa M.N. Linear Programming 2: Theory and Extensions. Springer-Verlag, 2003

[22] Do Carmo M.P. Differential Geometry of Curves and Surfaces: Revised and Updated Second Edition. Courier Dover Publications, 2016

[23] Frey P.J. "About surface remeshing." Proceedings of the 9th international meshing roundtable, 2000

[24] George P.L., Borouchaki H., Alauzet F., Laug P., Loseille A., Maréchal L. Meshing, Geometric Modeling and Numerical Simulation 2: Metrics, Meshes and Mesh Adaptation. John Wiley \& Sons, 2019

[25] Faà di Bruno F. "Note sur une nouvelle formule de calcul différentiel." Quarterly J. Pure Appl. Math, vol. 1, no. 359-360, 12, 1857

[26] Encinas L.H., Masque J.M. "A short proof of the generalized Faà di Bruno's formula." Applied mathematics letters, vol. 16, no. 6, 975-979, 2003

[27] Haimes R., Drela M. "On The Construction of Aircraft Conceptual Geometry for High-Fidelity Analysis and Design." 50th AIAA Aerospace Sciences Meeting including the New Horizons Forum and Aerospace Exposition. 2012

[28] George P.L., Borouchaki H. Delaunay triangulation and meshing. Hermes, 1998
[29] Loseille A., Feuillet R. "Vizir: High-order mesh and solution visualization using OpenGL 4.0 graphic pipeline." 2018 AIAA Aerospace Sciences Meeting, pp. AIAA 2018-1174. 2018 


\title{
ANISOTROPIC GOAL-ORIENTED MESH ADAPTATION IN FIREDRAKE
}

\author{
Joseph G. Wallwork ${ }^{1} \quad$ Nicolas Barral ${ }^{2} \quad$ David A. Ham ${ }^{3} \quad$ Matthew D. Piggott $^{4}$ \\ ${ }^{1}$ Imperial College London, London, U.K. j.wallwork16@imperial.ac.uk \\ ${ }^{2}$ Imperial College London, London, U.K. n.barral@imperial.ac.uk \\ ${ }^{3}$ Imperial College London, London, U.K. david.ham@imperial.ac.uk \\ ${ }^{4}$ Imperial College London, London, U.K. m.d.piggott@imperial.ac.uk
}

\begin{abstract}
We consider metric-based mesh adaptation methods for steady-state partial differential equations (PDEs), solved using the finite element method in Firedrake. In this work, a number of mesh-adaptive methods are implemented within this framework, each enabling accurate approximation of a scalar quantity of interest (QoI). Through the QoI we define adjoint equations, with which we may gain understanding of its sensitivities to aspects of the PDE solution. Dual weighted residual type error estimation techniques are utilised in order to enable a goal-oriented strategy. Isotropic and anisotropic approaches are considered, both of which are able to achieve the same relative error in approximating the QoI as with uniform refinement, but using fewer elements. For validation purposes, we compare QoI values resulting from these approaches against analytical values which may be extracted for a particular advection-diffusion based test case. Potential applications in desalination plant outfall modelling are discussed.
\end{abstract}

Keywords: anisotropy, mesh adaptation, error estimation, dual weighted residual, Firedrake

\section{INTRODUCTION}

In computational fluid dynamics (CFD), it is often the case that we are required to do more than just approximate the solution of a PDE. For many problems, what is most important is the accurate approximation of some functional, relating to a quantity of interest (QoI). For example, the QoI could be the drag on an aeroplane wing [1], the profit or power output of a tidal turbine array [2], or the ocean surface elevation near to important coastal infrastructure in the approach of a tsunami [3]. One method for accurately approximating such a quantity is to base a mesh adaptation routine around achieving this goal, as investigated in this work.

Mesh adaptation relies upon an appropriate choice of error estimator. Commonly utilised strategies include gradient-based methods [4], Hessian-based methods [5] and 'explicit' estimators derived from a posteri- ori bounds [6]. For example, mesh adaptation under Hessian-based error estimation seeks to minimise the interpolation error with respect to some scalar field. The scalar field may be one which is related to the fluid flow, such as free surface height. We use the term goaloriented mesh adaptation to refer to strategies which seek meshes permitting PDE solutions which minimise the error accrued in evaluating a QoI, whilst minimising the usage of computational resources.

The classical approach to goal-oriented error estimation uses the adjoint equation to compute sensitivities of the QoI to aspects of the PDE solution. In the pioneering works of $[7,8]$, the dual weighted residual (DWR) method was developed, providing linear and quadratic approximations to the QoI error, in terms of PDE residuals and the forward and adjoint solutions.

Dual weighted residual error estimation has been used to drive effective meshing strategies for a number of CFD applications (such as $[9,10]$ ). The majority of 
codes using DWR estimation do not incorporate directionality; an element is typically refined wherever the local DWR indicator breaches a pre-specified tolerance. This approach is particularly suited to quadtree and oct-tree adaptive mesh refinement strategies, where there is an inherent hierarchical mesh structure (such as in [3]).

Metric-based mesh adaptation routines take a different approach, with meshes interpreted in a continuous sense using Riemannian metric fields [11]. An advantage of metric-based methods is that they can be used to produce anisotropic meshes, enabling the accurate resolution of strongly direction-dependent aspects of the fluid flow using relatively few elements. That is, the shape and orientation of mesh elements are taken into consideration, as well as their size. In its classical formulation, the DWR error estimator provides a scalar field, from which we are not able to immediately extract anisotropic information. However, a number of strategies have been proposed to achieve this (see, for example, $[12,13,14])$.

The work of [1] includes an alternative goal-oriented error analysis to the classical DWR formulation, providing an error estimator expressed in terms of an interpolation error, weighted by the adjoint solution. Within the metric-based framework, it is possible to produce anisotropic meshes from this formulation.

This paper includes a literature review of anisotropic approaches to goal-oriented mesh adaptation, including those referenced above. The main focus of this paper is a successful implementation of one isotropic and two anisotropic approaches to goal-oriented mesh adaptation within the Firedrake finite element solver framework. To the best of the authors' knowledge, this work provides the first implementation of these three approaches in the same code framework. Preliminary numerical experiments show that each approach is able to achieve a wide range of tolerated errors using fewer elements than would be required in a uniform mesh.

The organisation of this paper is arranged as follows. The metric-based mesh adaptation strategy is introduced in Section 2. The theory of goal-oriented error estimation is outlined in Section 3, along with a literature review of different strategies for using such estimators to construct anisotropic metric fields. Section 4 outlines the way in which a metric-based, goaloriented mesh adaptation strategy has been implemented in this work, which is then used for numerical experiments in Section 5. These numerical experiments concern the TELEMAC-2D 'Point Discharge with Diffusion' steady advection-diffusion test case, for which an analytical solution exists [15]. This enables us to validate the goal-oriented mesh adaptation strategies considered. Finally, conclusions are drawn and outlook discussed in Section 6 .

\section{METRIC-BASED MESH ADAPTATION}

In this work we utilise a metric-based approach. This means that the mesh adaptation process is driven by a Riemannian metric field of dimension $n \times n$, where $\Omega \subset \mathbb{R}^{n}$ is the PDE domain.

A Riemannian metric field, or metric, denoted $\{\mathcal{M}(\mathbf{x})\}_{\mathbf{x} \in \Omega}$, is a collection of symmetric positive definite (SPD) linear forms defined pointwise, which contain local information on distances. Anisotropic edge lengths and element volumes can be derived from a metric. The main idea of metric-based mesh adaptation, introduced for the first time in [16], is to use a Riemannian metric space within the mesher to compute the necessary geometrical quantities, and to generate a unit mesh with respect to this Riemannian metric space.

A major advantage of the metric-based approach is that it enables control of mesh anisotropy, meaning that not only element size, but also shape and orientation may be dictated.

Given some error estimator $\eta$, our aim is to find a mesh $\mathcal{H}$ of the domain $\Omega$ such that the numerical solution of a PDE either: (a) achieves a certain level of error $[12,17]$; or (b) minimises the interpolation error for a given number of mesh vertices $[18,1]$. In this work we follow the former approach. For this, we need to establish the error estimator and express it as a metric.

Throughout this paper, we use the notation $\mathcal{H}$ when referring to meshes and $K$ to denote elements thereof. Occasionally, the cell diameter function $h$ is explicitly mentioned as a subscript, $\mathcal{H}_{h}$, if instructive. The edge set of element $K$ is denoted $\partial K$, whose normal vectors are denoted $\widehat{\mathbf{n}}_{K}$. The indicator function which is unity on element $K$ and zero elsewhere is denoted $\mathbb{1}_{K}$.

\subsection{Hessian-Based Metric}

Suppose $u$ is a (sufficiently smooth) scalar field of interest whose Hessian may be approximated in an element $K$ as $H$. The interpolation error associated with a linear approximation $\mathcal{I}_{h} u$ is related to $H$ through the truncated Taylor expansion of $u$ by [19]

$$
\left\|u-\mathcal{I}_{h} u\right\|_{\mathcal{L}_{\infty}(K)} \leq \gamma \max _{\mathbf{x} \in K} \max _{\mathbf{e} \in \partial K} \mathbf{e}^{T}|H(\mathbf{x})| \mathbf{e},
$$

where $\gamma>0$ is a constant related to the spatial dimension. Since $H$ is symmetric, it has an orthogonal eigen-decomposition, $H=V \Lambda V^{T}$, meaning it makes sense to take the absolute value as

$$
|H|=V|\Lambda| V^{T} .
$$

A metric tensor $\{\mathcal{M}(\mathbf{x})\}_{\mathbf{x} \in \Omega}$ may be defined as [17]

$$
\mathcal{M}(\mathbf{x})=\frac{\gamma}{\epsilon}|H(\mathbf{x})|,
$$


where $\epsilon>0$ is the tolerated error level.

Since we take the absolute value for all eigenvalues, this ensures $\mathcal{M}(\mathbf{x})$ is SPD. Doing so is justified by the fact that we primarily care about the magnitude of errors and not their sign. The constant $\gamma$ does not play an important role in the adaptation and is difficult to compute in practice. We follow [17] by setting $\gamma=1$.

By specifying smaller values for the desired error $\epsilon$ in (3), we allow heightened mesh complexity in return for reduced interpolation error.

Since in practice the field $u$ is only known in a discrete sense, its Hessian must be approximated using a recovery technique, which typically involves solving an auxiliary PDE. In this work, we use a double $\mathcal{L}_{2}$ projection approach (see [17] for details).

For further details on Hessian-based mesh adaptation, see $[17,19,20]$.

\subsection{Combining Metric Information}

Suppose now that we have two Riemannian metrics, $\mathcal{M}_{1}$ and $\mathcal{M}_{2}$, each of which captures some aspect of the PDE solution, or an error estimate thereof. For instance, we may consider the Hessians of the solutions of both a PDE and its adjoint.

The most straightforward way to combine these metrics is to consider a convex combination

$$
\mathcal{M}:=\alpha \mathcal{M}_{1}+(1-\alpha) \mathcal{M}_{2},
$$

for a parameter $\alpha \in(0,1)$. That (4) is indeed a metric follows from the definition of positive-definiteness:

$$
\mathbf{y}^{T} \mathcal{M}(\mathbf{x}) \mathbf{y}>0, \forall \mathbf{y} \neq \mathbf{0} .
$$

An advantage of using (4) is that the parameter $\alpha$ can be used to weight the resulting metric towards $\mathcal{M}_{1}$ or $\mathcal{M}_{2}$, as appropriate. In this work we consider exclusively the case where $\alpha=0.5$, which we refer to as metric averaging. The geometrical meaning of the metric averaging is not intuitive. However, it is simple to implement and the numerical experiments in Section 5 illustrate that it can be an effective means of combining metric information in practice.

Alternatively, metrics can be combined using superposition (also known as intersection). This method of combining metric information will always yield meshes with more elements than those which would arise from the constituent metrics. Unlike with combination by (4), this approach is not weighted towards one metric, although the intersection order is in general noncommutative. For details on metric superposition, see pp.3778-3779 of [17].

An investigation into the properties of metric superposition and averaging was considered on pp.131-138 of [21]. In the context of unsteady adaptation applied to advection problems, metric superposition was found to deal better with shocks. However, metric averaging was found to be more effective at resolving sharp angles and small scale features.

\subsection{Metric Normalisation}

It is necessary to scale metrics before combining them using the methods outlined in Subsection 2.2. In the case where two metrics have been constructed from the Hessians of different fields, for example, there is no guarantee that these second derivative matrices are of the same order of magnitude. Normalising before combination means that the Hessians may be averaged or superimposed in a meaningful way.

For a Hessian $H$ defined over a domain $\Omega \subset \mathbb{R}^{n}$ and fixed $p \in[1, \infty), \mathcal{L}_{p}$ normalisation is defined by [22]

$$
\mathcal{M}_{\mathcal{L}_{p}}=\frac{n}{\epsilon}\left(\int_{\Omega} \operatorname{det}(|H|)^{\frac{p}{2 p+n}} \mathrm{~d} \mathbf{x}\right)^{\frac{1}{p}} \operatorname{det}(|H|)^{\frac{-1}{2 p+n}}|H|,
$$

where $\epsilon$ is the tolerated error level. Taking the limit $p \rightarrow \infty$ yields the strategy referred to as $\mathcal{L}_{\infty}$ normalisation. This approach corresponds to the case where (6) reduces to (3), with constant $\gamma=n$. Whilst commonly used, the $\mathcal{L}_{\infty}$ normalisation strategy is not able to fully capture discontinuities and will in many cases use very high levels of mesh refinement surrounding such features. A low order $\mathcal{L}_{p}$ normalisation strategy, such as $p=1$ or $p=2$, is more suited to resolving such features and also permits multiscale mesh adaptation.

\subsection{Metric Gradation}

We use a metric gradation procedure to ensure that the sizes prescribed by the metric at neighbouring vertices do not vary by more than a specified threshold, taken here as $\beta=1.4$. That is, the ratio of the prescribed sizes is bounded from above by $\beta$. This has the effect that meshes resulting from the metricbased mesh adaptation routine do not have sudden changes in resolution, which could act as artificial internal boundaries in the flow. For details on the metric gradation algorithm used, see [18].

\section{GOAL-ORIENTED ERROR ESTIMATION}

\subsection{Forward and Adjoint Equations}

Consider a PDE, referred to as the forward equation,

$$
\Psi(u)=0,
$$

defined upon a domain $\Omega$ with piecewise smooth boundary $\partial \Omega$. For the purposes of this paper, suppose $\partial \Omega$ may be decomposed into the disjoint union of 
open boundary, Dirichlet and Neumann components as $\partial \Omega=\partial \Omega_{\text {open }} \cup \partial \Omega_{D} \cup \partial \Omega_{N}$.

For a function space $V$ containing the exact (forward) solution, $u$, for (7), consider a functional

$$
J: V \rightarrow \mathbb{R}
$$

Here $J$ describes an important scalar quantity related to the flow - the so-called quantity of interest (QoI). Let $\langle\cdot, \cdot\rangle$ denote the usual $\mathcal{L}_{2}$ inner product on $\Omega$ and the $\mathcal{L}_{2}$ inner product over other spaces $S$ be denoted $\langle\cdot, \cdot\rangle_{S}$. We restrict attention to quantities of interest which can be written as

$$
J(v)=\langle g, v\rangle,
$$

where $g$ is some kernel function on $\Omega$.

The variational formulation of (7) is given by

$$
-\rho(u, v)=\langle\Psi(u), v\rangle=0, \quad \forall v \in V .
$$

We refer to $\rho(\cdot, v)$ as the weak residual of the PDE. Typically, integration by parts is applied in deriving this weak residual, so that Neumann boundary conditions may be applied and only first order derivatives appear. This also becomes important for the construction of goal-oriented error estimators in Subsection 5.2.

For a finite dimensional subspace $V_{h} \subset V$, we have the Galerkin approximation

$$
-\rho\left(u_{h}, v_{h}\right)=\left\langle\Psi_{h}\left(u_{h}\right), v_{h}\right\rangle=0, \quad \forall v_{h} \in V_{h} .
$$

The (continuous) adjoint equation associated with (7) is given by

$$
\left(\frac{\partial \Psi}{\partial u}(u)\right)^{T} u^{*}={\frac{\partial J^{T}}{\partial u}}^{T}
$$

where $u^{*}$ is the corresponding adjoint solution. The weak residual $\rho^{*}(\cdot, v)$ for the adjoint equation satisfies

$$
\rho^{*}\left(u^{*}, v\right)=0, \quad \forall v \in V,
$$

where

$$
\rho^{*}\left(u^{*}, v\right)=\left\langle\frac{\partial J}{\partial u}, v\right\rangle-\left\langle\left(\frac{\partial \Psi}{\partial u}(u)\right)^{T} u^{*}, v\right\rangle,
$$

with appropriate application of integration by parts; similarly for the Galerkin approximation to (12).

Due to assumption (9) on the form of the QoI, we may rewrite the adjoint weak residual (14) as

$$
\rho^{*}\left(u^{*}, v\right)=J(v)-\left\langle\left(\frac{\partial \Psi}{\partial u}(u)\right)^{T} u^{*}, v\right\rangle .
$$

\subsection{Estimate for Error in Qol}

Due to the goal-oriented error analysis of [8], we have the classical result

$$
J(u)-J\left(u_{h}\right)=\rho\left(u_{h}, u^{*}-u_{h}^{*}\right)+R,
$$

where the remainder term $R$ is quadratic in the forward and adjoint errors $e=u-u_{h}$ and $e^{*}=u^{*}-u_{h}^{*}$. A 'second-order' result is also derived, given by

$J(u)-J\left(u_{h}\right)=\frac{1}{2} \rho\left(u_{h}, u^{*}-u_{h}^{*}\right)+\frac{1}{2} \rho^{*}\left(u_{h}^{*}, u-u_{h}\right)+\bar{R}$,

where the remainder term $\bar{R}$ is cubic in the forward and adjoint errors. The remainder terms in (16) and (17) vanish if we have both a linear PDE and a quadratic QoI [8].

Goal-oriented error estimators may be derived from (16) and (17), as outlined in the following subsections.

Local error indicators $\eta_{K}$ on each element $K$ of a mesh $\mathcal{H}$ may be extracted from (16) by evaluating

$$
\eta_{K}=\left|\rho\left(u_{h}, u^{*}-u_{h}^{*}\right)\right|_{K} \mid .
$$

and from (17) by evaluating

$$
\eta_{K}=\left|\frac{1}{2} \rho\left(u_{h}, u^{*}-u_{h}^{*}\right)\right|_{K}+\left.\frac{1}{2} \rho^{*}\left(u_{h}^{*}, u-u_{h}\right)\right|_{K} \mid .
$$

Summing over all elements yields a (global) error estimator

$$
\eta:=\sum_{K \in \mathcal{H}} \eta_{K}
$$

\subsection{Isotropic Goal-Oriented Error Estima- tion}

As mentioned in Subsection 3.1, integration by parts over $\Omega$ is typically applied when deriving the variational formulation (10) of (7). Evaluating error indicators of the form (18) typically involves integrating by parts again, over each element, $K$, yielding

$$
\begin{aligned}
\eta_{K}=\mid & -\left\langle\Psi_{h}\left(u_{h}\right), u^{*}-u_{h}^{*}\right\rangle_{K} \\
& +\left\langle\psi_{h}^{N}\left(u_{h}\right), u^{*}-u_{h}^{*}\right\rangle_{\partial K \cap \partial \Omega_{N}} \\
& +\left\langle\psi_{h}^{\text {flux }}\left(u_{h}\right), u^{*}-u_{h}^{*}\right\rangle_{\partial K \backslash \partial \Omega} \mid,
\end{aligned}
$$

where $\psi_{h}^{N}$ is the residual of the Neumann boundary conditions and $\psi_{h}^{\text {flux }}$ corresponds to flux terms between elements. For continuous Galerkin methods, the flux terms in $\psi_{h}^{\text {flux }}$ arise purely due to the Neumann condition. For discontinuous FEM (such as discontinuous Galerkin (DG) methods [23]), there are also contributions due to the flux terms incorporated into the weak residual $\rho(\cdot, \cdot)$. 
A similar expression to (21) results from applying integration by parts to (17).

As discussed in Section 1, classical DWR approaches are not usually used to give anisotropic meshes. In the case of metric-based mesh adaptation, the simplest way to create a metric $\{\mathcal{M}(\mathbf{x})\}_{\mathbf{x} \in \Omega}$ from (18) is to scale the identity matrix by an appropriate scalar field. However, one drawback of this approach is that (18) is piecewise constant and discontinuous across element interfaces $(\mathbb{P} 0)$, whereas we seek a piecewise linear metric which is continuous across element interfaces $(\mathbb{P} 1)$. Given an appropriate projection operator $\Pi_{1}: \mathbb{P} 0 \rightarrow \mathbb{P} 1$, we may define an isotropic metric by

$$
\mathcal{M}=\left[\begin{array}{ll}
\Pi_{1} \eta^{\mathbb{P 0}} & \\
& \Pi_{1} \eta^{\mathbb{P 0}}
\end{array}\right], \quad \eta^{\mathbb{P 0}}:=\sum_{K \in \mathcal{H}} \eta_{K} \mathbb{1}_{K}
$$

in the $2 \mathrm{D}$ case. One projection approach is to use a nodewise construction, taking averages of the values of $\eta_{K}$ over adjacent cells, weighted by the area thereof [9]. Extension of (22) to the 3D case is trivial.

Metrics of the form (22) allow us to control the size of elements under mesh adaptation, but not their shape or orientation. The normalisation methods outlined in Subsection 2.3 should be applied to such isotropic metrics, as well as the anisotropic ones introduced in the following subsection.

\subsection{Anisotropic Goal-Oriented Error Esti- mation}

The literature contains a number of approaches for extending the goal-oriented error estimation framework to obtain anisotropic meshes. Some notable contributions are summarised in the following.

The approach advocated in [13] uses one mesh corresponding to each spatial dimension, which is refined uniformly in that direction alone. Local DWR indicators (18) are computed on each mesh and interpolated onto the base mesh, where a single anisotropic metric is formed. This approach essentially involves the computation of as many residuals as there are spatial dimensions, with the corresponding DWR estimators combined in an appropriate way so as to yield a single Riemannian metric field.

An alternative approach to DWR error estimation is considered in [1]. Therein, the result

$$
J(u)-J\left(u_{h}\right)=\left\langle\left(\Psi_{h}-\Psi\right)(u), u^{*}\right\rangle+\widetilde{R}
$$

is derived, where the remainder term $\widetilde{R}$ involves interpolation errors on $V_{h}$ and the adjoint error, $e^{*}$. Here we have what may be interpreted as an interpolation error in the PDE, weighted by the adjoint solution.

If the PDE can be written in a conservative form,

$$
\Psi(u)=\nabla \cdot \mathcal{F}(u)=0,
$$

then, by integration by parts in (23) and an application of the Cauchy-Schwarz inequality, [1]

$$
\begin{aligned}
\left|J(u)-J\left(u_{h}\right)\right| & \leq\left\langle\left|\left(\mathcal{F}-\Pi_{h} \mathcal{F}\right)(u)\right|,\left|\nabla u^{*}\right|\right\rangle_{\Omega} \\
& +\left\langle\left|\widehat{n} \cdot\left(\overline{\mathcal{F}}-\Pi_{h} \overline{\mathcal{F}}\right)(u)\right|,\left|u^{*}\right|\right\rangle_{\partial \Omega},
\end{aligned}
$$

where $\overline{\mathcal{F}}$ embodies the boundary conditions and terms resulting from the integration by parts.

Since (25) involves interpolation errors in $\overline{\mathcal{F}}$ and $\mathcal{F}$, it is argued that we may use the interpolation error bound (1) to obtain an anisotropic metric by scaling components of the Hessians of these quantities by components of the adjoint and its gradient. That is, we define Riemannian metric fields

$$
H^{\text {volume }}=\sum_{i=1}^{n} \sum_{j=1}^{m}\left|H\left(\mathcal{F}_{i}(u)\right)\right|\left|\frac{\partial u_{i}^{*}}{\partial x_{j}}\right|
$$

on the whole domain and

$$
H^{\text {surface }}=\sum_{i=1}^{n}\left|u^{*}\right|\left|H\left(\sum_{j=1}^{m} \overline{\mathcal{F}}_{j}(u) \cdot n_{j}\right)\right|
$$

on the domain boundary. Again, the modulus sign indicates that the Hessian eigenvalues are considered with a positive sign, to ensure positive-definiteness. The metrics resulting from (26) and (27) are superposed on the boundary in order to give a globally defined metric field. For further details, see [1], where this approach is shown to be effective in solving aerospace problems using the Euler equations. For the extension to unsteady problems, see $[24,25]$.

The approach of [12] also makes use of the Hessian to form an anisotropic metric. However, in this case, interpolation errors in the adjoint solution are considered, as opposed to interpolation errors in the PDE residual. We may interpret the error representation (16) as

$$
J(u)-J\left(u_{h}\right) \approx\left\langle\Psi_{h}\left(u_{h}\right), u^{*}-\Pi_{h} u^{*}\right\rangle ;
$$

similarly

$$
J(u)-J\left(u_{h}\right) \approx\left\langle\Psi_{h}^{*}\left(u_{h}^{*}\right), u-\Pi_{h} u\right\rangle
$$

for the corresponding expression involving the adjoint residual.

An element-based anisotropic metric may then be constructed from (28) by weighting the Hessian of the adjoint solution with the residual in the forward equation:

$$
\widetilde{\mathcal{M}}(K)=\left\|\Psi_{h}\left(u_{h}\right)\right\|_{K}\left|\bar{H}\left(u^{*}\right)\right|_{K}
$$

and similarly, for (29):

$$
\widetilde{\mathcal{M}^{*}}(K)=\left\|\Psi_{h}^{*}\left(u_{h}^{*}\right)\right\|_{K}|\bar{H}(u)|_{K} .
$$

Here $\bar{H}$ indicates the average Hessian computed on a particular element. 
As noted, (30) and (31) are defined elementwise, whereas we seek a (continuous) nodewise metric. In practice, we apply the same weighted-average projection operator as in (22) in order to yield a nodewise definition of the strong residual. Denoting these averaged quantities by $\left|\overline{\Psi_{h}}\right|$ and $\left|\overline{\Psi_{h}^{*}}\right|$, we may construct metrics $\{\mathcal{M}(\mathbf{x})\}_{\mathbf{x} \in \Omega}$ and $\left\{\mathcal{M}^{*}(\mathbf{x})\right\}_{\mathbf{x} \in \Omega}$ by

$$
\begin{aligned}
\mathcal{M}(\mathbf{x}) & =\left|\overline{\Psi_{h}}\right|_{\mathbf{x}}\left|H\left(u_{h}^{*}\right)\right|_{\mathbf{x}}, \\
\mathcal{M}^{*}(\mathbf{x}) & =\left|\overline{\Psi_{h}^{*}}\right|_{\mathbf{x}}\left|H\left(u_{h}\right)\right|_{\mathbf{x}} .
\end{aligned}
$$

Note that in (32) Hessians in the forward and adjoint solutions are approximated by Hessians of the corresponding finite element solutions.

Another notable contribution in the field of anisotropic goal-oriented mesh adaptation is the work of [14]. The authors make use of upper bounds for interpolation errors of the forward and adjoint solutions (and gradients thereof) which are expressed in terms of the anisotropic quantities used by a metric-based mesh adaptation routine. As with the approaches of [1] and [12], metrics are defined using Hessians, due to their relation (1) with interpolation error.

Each of the approaches discussed above (along with many others which exist in the literature) have been used to provide logical, effective anisotropic extensions for goal-oriented mesh adaptation; these approaches are certainly worth further investigation. However, for simplicity of the presentation of this paper, we henceforth focus on the anisotropic approaches introduced in [12] and [1], along with the isotropic approach defined by $(22)$.

Another reason for comparing the anisotropic approaches of [12] and [1] is that the former uses a posteriori error estimation, whilst the latter uses a priori error estimation. In this classification, we follow [1] in interpreting an a posteriori standpoint as assuming knowledge of errors accrued on an existing mesh and an a priori standpoint as assuming knowledge of the PDE solution. That is, the a posteriori metrics (30)(31) involve residuals evaluated on the current mesh, whereas the a priori metrics (26)-(27) involve exact solutions for the forward and adjoint PDEs and make no reference to finite element solutions. In practice, these exact values are of course approximated by finite element solutions, given by replacing instances of $u$ and $u^{*}$ with $u_{h}$ and $u_{h}^{*}$, respectively. However, the difference in classification has to do with the derivation of the methods, rather than implementation details.

For the reasons discussed above, we henceforth refer to meshes adapted using metrics (30)-(31) (and combinations thereof) as a posteriori and metrics derived from (26)-(27) as a priori. The isotropic approach is also a posteriori.

It is worth remarking that, whilst the anisotropic methods considered are inspired by the work of [12] and [1], there are some notable differences between the original implementations and those used in this work. Further details regarding these differences are discussed in Subsection 5.2.

\section{IMPLEMENTATION}

\subsection{Error Estimate Evaluation}

An important aspect of goal-oriented error estimate evaluation is the treatment of forward and adjoint error terms, $e=u-u_{h}$ and $e^{*}=u^{*}-u_{h}^{*}$, since these terms are clearly unknown. In the following, we assume the PDE is to be solved in $\mathbb{P} 1$ space, although the same logic applies to other finite element spaces.

One approach for approximating $e^{*}$ involves an auxiliary adjoint PDE. Given the current mesh $\mathcal{H}_{h}$, consider an iso- $\mathbb{P} 2$ refinement, denoted $\mathcal{H}_{h / 2}$. That is, we insert vertices wherever there would be quadrature nodes of a piecewise quadratic element, effectively quadrupling the number of elements. Henceforth, we refer to this as uniform refinement.

The adjoint PDE is solved on $\mathcal{H}_{h / 2}$ in $\mathbb{P} 2$ space, yielding an approximation $\widehat{u_{h / 2}^{*}}$ to the adjoint solution $u^{*}$. On the same mesh, we obtain a linear approximation by projection, meaning the adjoint equation need only be solved once in order to approximate this error. We obtain an estimate for the adjoint error by projecting the difference back onto $\mathcal{H}_{h}$. In summary:

$$
u^{*}-u_{h}^{*} \approx \widehat{u_{h / 2}^{*}}-\Pi_{1} \widehat{u_{h / 2}^{*}}
$$

where $\Pi_{1}$ is the projection operator into $\mathbb{P} 1$ space.

This approach requires considerable additional computational resources, since the adjoint equation must be solved again on a fine mesh, in a higher order space. However, we illustrate in Section 5 that it provides proof of concept that the DWR error estimation strategy can be effectively deployed within our framework to yield high quality meshes. For nonlinear PDEs, one could argue that additional adjoint solves are less problematic than additional forward solves, since the adjoint PDE is always only linear.

An alternative approach performs local patch-based interpolation, using a supermesh of $\mathcal{H}_{h}$. The approximate adjoint solution $u_{h}^{*}$ may then be interpolated onto the supermesh (see pp.870-871 of [14] for details).

Both of the strategies referred to above may also be applied to approximation of the primal error $e$, through additional solves of the forward PDE.

For the a posteriori anisotropic approach, we do not actually need to evaluate the adjoint error terms, since this is approximated using the Hessian. As such, the 
above discussion is not of concern. In the a priori case, we follow the authors of [1] in approximating gradients in the exact adjoint solution by gradients in the numerical approximation thereof. As such, approximation of $e$ and $e^{*}$ is only required for isotropic metrics. In these cases, we make use of the space enrichment approach, providing a benchmark to which the anisotropic strategies may be compared.

\subsection{Mesh Adaptation Approach}

In this subsection the mesh adaptive solver routine is outlined for stationary PDEs.

Given an initial mesh $\mathcal{H}_{0}$ and a desired error level $\epsilon>0$, we construct a sequence of meshes $\left\{\mathcal{H}_{i}\right\}_{i \in \mathbb{N}}$ by iteratively solving the PDE and its adjoint, evaluating an appropriate error estimator and extracting an associated metric field. See Algorithm 1 for a workflow representation.

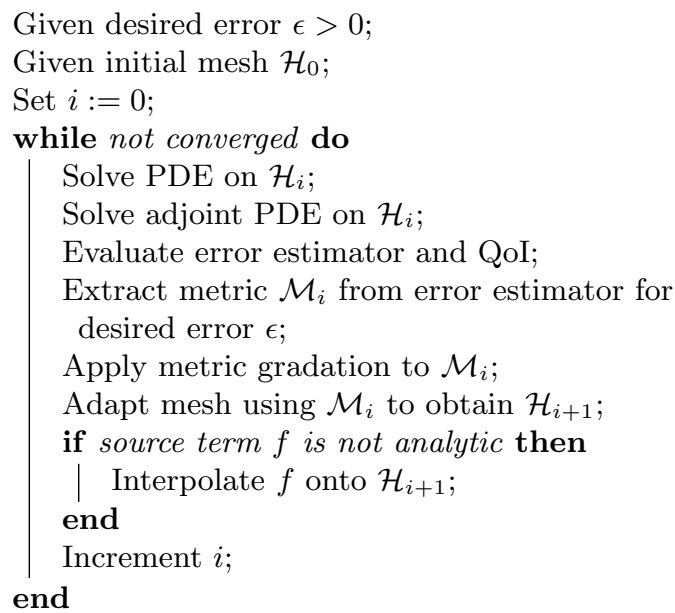

Algorithm 1: Mesh adaptation routine.

Convergence is attained when either the change in QoI or the number of mesh elements falls below some relative tolerance, both taken here as $0.5 \%$, as recommended in [12]. A maximum iteration count of 35 is imposed. In practice, assuming that $\mathcal{H}_{0}$ is relatively isotropic, we find that at least three mesh iterations are required in order to introduce anisotropy into meshes resulting from Algorithm 1. As such, we require that at least three iterations are performed.

Under the assumption that source terms are described analytically, there is no need to interpolate across meshes, since the source term can be defined in a mesh-independent way. This is the case considered in the numerical experiments of Section 5. If the source term is defined using data then interpolation will be required whenever a new mesh is considered.

In this work we restrict attention to steady problems, with unsteady problems to be considered in the future. In such problems, solutions must be transferred from one mesh to another between timesteps.

\subsection{Software}

Firedrake is a Python based finite element package with a high-level interface which is intuitive from the perspective of a mathematician [26]. PDEs in weak form are expressed using Unified Form Language (UFL) [27] in a very similar way as they would be written by hand. Firedrake uses sophisticated code generation technology to produce efficient lowlevel code. This automatically generated $\mathrm{C}$ code uses PETSc $[28,29]$ to solve the resulting linear and nonlinear systems.

Anisotropic mesh adaptation is achieved in Firedrake using the Pragmatic library [30]. This C++ library takes as input an arbitrary mesh and a metric field over it and returns a mesh that is adapted to the metric. The adapted mesh is derived from the input one through a series of mesh modifications (edge splits, collapses and swaps and vertex smoothing in the 2D case) that optimise the lengths of the edges as well as a certain quality functional. More detail can be found in $[31,32,33]$. Pragmatic and Firedrake are interfaced through PETSc $[34,35]$. High-level error estimate information is passed to Pragmatic using Riemannian metric fields constructed in Firedrake using the FEM solutions of the PDE and its adjoint.

Conservative projection [36] between meshes in Firedrake is enabled by the libsupermesh library [37, 38]. Evaluating DWR error estimators using the higher order finite element solution (33) requires these projection operators to transfer information to and from the iso- $\mathbb{P} 2$ refined space, upon which the approximation to $u^{*}-u_{h}^{*}$ is constructed.

For the PDE considered in the Section 5 of this paper, the continuous form adjoint is easily derived and may be solved in the same manner as the forward equation. For more complex PDEs or boundary conditions, Firedrake also supports the automatic generation and solution of the adjoint PDE in a discrete adjoint type approach, using pyadjoint $[39,40]$. Note that stabilising the continuous adjoint equation using the same strategy as for the forward PDE is not in general equivalent to generating the discrete adjoint equation from the stabilised PDE. For example, these discretisations for the adjoint PDE are not equivalent for SUPG stabilisation applied to advection-diffusion problems, which we consider in Section 5.

The Firedrake and PETSc versions used in this work are archived using Zenodo as [41]. The simulation code used to generate results in Section 5 is archived as [42]. 


\section{NUMERICAL EXPERIMENTATION}

\subsection{Forward and Adjoint Equations}

For the purposes of numerical experimentation, consider steady advection-diffusion of a passive tracer $\phi$ immersed in a fluid with velocity $\mathbf{u}$ and diffusivity $\nu$ :

$$
\left\{\begin{aligned}
\mathbf{u} \cdot \nabla \phi-\nabla \cdot(\nu \nabla \phi) & =f & & \text { in } \Omega \\
\nu \widehat{\mathbf{n}} \cdot \nabla \phi & =g_{N} & & \text { on } \partial \Omega_{N} \\
\phi & =g_{D} & & \text { on } \partial \Omega_{D},
\end{aligned}\right.
$$

where $f$ is a source term. For a function space $V$, consider the weak formulation

$$
a(\phi, v)=L(v), \quad \forall v \in V,
$$

where

$$
\begin{aligned}
a(\phi, v):= & \langle\mathbf{u} \cdot \nabla \phi, v\rangle+\langle\nu \nabla \phi, \nabla v\rangle \\
& -\left\langle\nu \widehat{\mathbf{n}}_{K} \cdot \nabla \phi, v\right\rangle_{\partial \Omega \backslash \partial \Omega_{N}}, \\
L(v):= & \langle f, v\rangle+\left\langle g_{N}, v\right\rangle_{\partial \Omega_{N}} .
\end{aligned}
$$

Thus $\rho(\cdot, v) \equiv L(v)-a(\cdot, v)$. Stabilisation terms are usually added to (35) in order to control under- and over-shoots that are typical with advection-dominated problems. In this work we use (continuous) Lagrange $(\mathbb{P} 1)$ finite elements and Streamline Upwind Petrov Galerkin (SUPG) stabilisation, under which an additional term $\langle\Psi(\phi), \mathbf{u} \cdot \nabla v\rangle$ is used, where

$$
\Psi(\phi)=f-\mathbf{u} \cdot \nabla \phi+\nabla \cdot(\nu \nabla \phi)
$$

denotes the strong residual for (34). For further details on the stabilisation strategy, see [43].

The continuous adjoint equation associated with (34) may be derived as

$$
\left\{\begin{aligned}
-\nabla \cdot\left(\mathbf{u} \phi^{*}\right)-\nabla \cdot\left(\nu \nabla \phi^{*}\right) & =\frac{\partial J}{\partial \phi} & & \text { in } \Omega \\
\phi^{*} & =0 & & \text { on } \partial \Omega \backslash \partial \Omega_{N} \\
\nu \widehat{\mathbf{n}} \cdot \nabla \phi^{*}+\phi^{*} \mathbf{u} \cdot \widehat{\mathbf{n}} & =0 & & \text { on } \partial \Omega \backslash \partial \Omega_{D} .
\end{aligned}\right.
$$

Note that the derivative of the QoI with respect to the forward solution provides a source term for the adjoint equation. SUPG stabilisation applied to the adjoint equation requires the adjoint strong residual,

$$
\Psi^{*}\left(\phi^{*}\right)=\frac{\partial J}{\partial \phi}+\nabla \cdot\left(\mathbf{u} \phi^{*}\right)+\nabla \cdot\left(\nu \nabla \phi^{*}\right) .
$$

\subsection{Goal-Oriented Metrics}

Formulating the DWR estimator amounts to applying integration by parts on each element. For the stabilised version of the CG formulation given in (35), this yields

$$
\begin{aligned}
&\left.\rho_{h}\left(\phi_{h}, \phi^{*}-\phi_{h}^{*}\right)\right|_{K} \\
&=\left\langle\Psi\left(\phi_{h}\right), \phi^{*}-\phi_{h}^{*}\right\rangle_{K} \\
&+\left\langle\nu \widehat{\mathbf{n}}_{K} \cdot \nabla \phi_{h}-g_{N}, \phi^{*}-\phi_{h}^{*}\right\rangle_{\partial K \cap \partial \Omega_{N}} \\
&+\left\langle\nu \widehat{\mathbf{n}}_{K} \cdot \nabla \phi_{h}, \phi^{*}-\phi_{h}^{*}\right\rangle_{\partial K \backslash \partial \Omega} \\
&+\left\langle\Psi\left(\phi_{h}\right), \mathbf{u} \cdot \nabla\left(\phi^{*}-\phi_{h}^{*}\right)\right\rangle_{K},
\end{aligned}
$$

where the terms on the RHS correspond to those given in (21), along with the stabilisation error term. Observe that if $\phi_{h}$ is replaced with the (assumed smooth) exact solution of (34) then this estimator vanishes. Similarly, if $\phi^{*}-\phi_{h}^{*}$ is replaced with the adjoint finite element solution $\phi_{h}^{*} \in V_{h}$ then the estimator vanishes, because $\phi_{h}$ is a solution in the finite element space $V_{h}$. When summed over all elements of the mesh, the inner product over $\partial K \backslash \partial \Omega$ in (40) corresponds to a flux jump term.

For the stabilised adjoint equation, we have the DWR estimator

$$
\begin{aligned}
& \left.\rho_{h}^{*}\left(\phi_{h}^{*}, \phi-\phi_{h}\right)\right|_{K} \\
& \quad=\left\langle\Psi^{*}\left(\phi_{h}^{*}\right), \phi-\phi_{h}\right\rangle_{K} \\
& \quad+\left\langle\nu \widehat{\mathbf{n}}_{K} \cdot \nabla \phi_{h}^{*}+\phi_{h}^{*} \mathbf{u} \cdot \widehat{\mathbf{n}}_{K}, \phi-\phi_{h}\right\rangle_{\partial K \backslash\left(\partial \Omega \backslash \partial \Omega_{D}\right)} \\
& \quad+\left\langle\Psi^{*}\left(\phi_{h}^{*}\right), \mathbf{u} \cdot \nabla\left(\phi-\phi_{h}\right)\right\rangle_{K},
\end{aligned}
$$

where the boundary and flux terms have been combined due to their similarity.

Isotropic metrics may be constructed from (40) and (41) using (22). The strong residual in the forward PDE required for constructing the anisotropic metric (30) is given by (37), whilst the strong residual in the adjoint PDE required for (31) is given by (39).

For metrics constructed from (26)-(27) to make sense, we require the PDE (34) to be in conservative form. Due to the constant velocity field, it may be expressed in terms of a potential functional $\mathcal{F}$ as

$$
\nabla \cdot \mathcal{F}(\phi)=f, \quad \mathcal{F}(\phi)=\mathbf{u} \phi-\nu \nabla \phi .
$$

However, the source term $f$ requires special treatment. In this work we neglect the boundary metric terms arising from (27) and construct a metric by summing (26) and the Hessian of the source term:

$$
\begin{aligned}
\mathcal{M}= & \left|H\left(\mathcal{F}_{1}(\phi)\right)\right| \frac{\partial \phi^{*}}{\partial x}|+| H\left(\mathcal{F}_{2}(\phi)\right)|| \frac{\partial \phi^{*}}{\partial y} \mid \\
& +|H(f)|\left|\phi^{*}\right| .
\end{aligned}
$$

A similar strategy is used for the corresponding adjoint metric. That is, for a potential functional $\mathcal{G}$ satisfying

$$
\nabla \cdot \mathcal{G}\left(\phi^{*}\right)=g, \quad \mathcal{G}\left(\phi^{*}\right)=-\mathbf{u} \phi^{*}-\nu \nabla \phi^{*},
$$

we define an adjoint metric

$$
\begin{aligned}
\mathcal{M}= & \left|H\left(\mathcal{G}_{1}\left(\phi^{*}\right)\right)\right|\left|\frac{\partial \phi}{\partial x}\right|+\left|H\left(\mathcal{G}_{2}\left(\phi^{*}\right)\right)\right|\left|\frac{\partial \phi}{\partial y}\right| \\
& +|H(g)||\phi|,
\end{aligned}
$$

recalling that $g=\frac{\partial J}{\partial \phi}$. To the best of the authors' knowledge, no a priori error result for the adjoint equation equivalent to (23) exists in the literature. 
Nonetheless, extending the a priori metric construction by combining the forward metric (43) with the adjoint metric (45) is shown in Section 5 to provide meshes upon which the QoI may be accurately approximated.

Following (6), we use $\mathcal{L}_{1}$ metric normalisation for all goal-oriented mesh adaptation strategies. Using $\mathcal{L}_{1}$ normalisation, as opposed to the more commonly applied $\mathcal{L}_{\infty}$ normalisation, allows us to better capture the discontinuities associated with the source terms in the forward and adjoint equations.

It is worth remarking that, whilst stabilisation errors have been accounted for in (40) and (41), they are not considered in either of the anisotropic metric techniques considered in this paper. An investigation of how best to integrate the stabilisation error into these anisotropic metrics is something to be considered in future work. However, stabilisation errors are accounted for in the anisotropic metrics constructed in [14].

\subsection{Point Source Test Case}

For this work we consider the 'Point Discharge with Diffusion' test case from TELEMAC-2D validation document version 7.0 [15], whose source is a delta function at the point $\mathbf{x}_{0}=\left(x_{0}, y_{0}\right)=(1,5)$ in the domain $\Omega=[0,50] \times[0,10]$.

The fluid velocity $\mathbf{u}=\left(u_{x}, u_{y}\right) \equiv(1,0)$ is entirely in the $x$-direction and the diffusivity $\nu \equiv 0.1$ is constant. An inflow condition of $\phi \equiv 0$ is imposed at the boundary where $x=0$, along with free-slip conditions for $y \in\{0,10\}$ and an open boundary at $x=50$. The only difference in the test case considered here is that we consider the point source to be located at $\mathbf{x}_{0}=(2,5)$, so that it is not as close to the inflow boundary. We consider the same initial mesh as in [15], comprised of a uniform grid of 4,000 right-angled triangles.

The quantities of interest considered take the form

$$
J_{i}(\phi)=\int_{\Omega} \mathbb{1}_{R_{i}} \phi \mathrm{d} x=\int_{R_{i}} \phi \mathrm{d} x
$$

where $R_{i} \subset \Omega$ are 'receiver' regions, for $i \in\{1,2\}$. That is, each kernel $g=\mathbb{1}_{R_{i}}$ is an indicator function. Goal-oriented mesh adaptation seeks a mesh which permits an accurate approximation of the tracer concentration over the receiver region. Both (46) and (34) are linear, meaning that the error estimates provided by (16) and (17) are exact [8].

For a related application in environmental science, consider the inlet and outlet pipes of a desalination plant. The former pipe brings sea water into the plant, extracting from region $R_{i}$. Salt is extracted from this water and the remaining salt residues are released back into the ocean through the latter pipe, depositing at $\mathbf{x}_{0}$. An undesirable (but entirely possible) situation is the one in which a significant quantity of salt from the outlet pipe is later taken back into the plant at the inlet, making the task of desalination more difficult.

For this application we do not necessarily care about the wider dispersal of high salinity water, but rather achieving an efficient and accurate calculation of the salinity at the inlet pipe. If salt is interpreted as a passive tracer in the fluid flow, we can model its advection and diffusion using (34) and quantify its concentration at the inlet using (46). Through goal-oriented mesh adaptation, we are able to select a mesh which gives a good approximation to the salinity at the inlet, whilst retaining a relatively small number of elements.

As in the similar steady-state advection-diffusion test case examined in [12], we consider two scenarios: one where the receiver is directly downstream from the source and one where it is offset to one side of the channel. Given the definition of the disc

$$
B_{\epsilon}(\mathbf{y})=\left\{\mathbf{x} \in \Omega \mid\|\mathbf{x}-\mathbf{y}\|_{2} \leq \epsilon\right\}
$$

we choose $R_{1}=B_{\frac{1}{2}}((20,5))$ in the former instance and $R_{2}=B_{\frac{1}{2}}((20,7.5))$ in the latter.

As documented in [15], the analytical solution for this problem is given by

$$
\phi_{\text {exact }}(\mathbf{x})=\frac{q}{2 \pi \nu} \exp \left(\frac{u_{x} x}{2 \nu}\right) K_{0}\left(\frac{u_{x}\left\|\mathbf{x}-\mathbf{x}_{0}\right\|}{2 \nu}\right),
$$

where $q$ denotes the inflow discharge at the source and $K_{0}$ is the modified Bessel function of the second kind and order zero.

Evaluating the QoI amounts to integrating (48) over region $R_{1}$ or $R_{2}$, as appropriate. Due to the presence of the modified Bessel function, we are not able to perform this integration analytically and hence resort to applying numerical quadrature on a sequence of increasingly refined uniform meshes. For the cases of both centred and offset receiver regions, we attain convergence to five decimal places on a mesh with 1,024,000 elements, as illustrated in Table 1. The close agreement between QoI values under analytical and finite element solutions validates our $\mathbb{P} 1$ SUPG FEM solution strategy.

Thus the benchmark values $J_{1}^{\text {exact }}=0.16344$ and $J_{2}^{\text {exact }}=0.06959$ are obtained using the analytical solution evaluated on this mesh. The analytical solution (48) is presented in Figure 1a on this mesh, along with finite element approximations to the forward and adjoint solutions. In each case, $\mathbb{P} 1$ elements are used.

An initial qualitative observation to be made from Subfigures $1 \mathrm{a}-1 \mathrm{~b}$ and Table 1 is that the piecewise linear finite element approximation on the fine uniform 


\begin{tabular}{|l|l|l|l|l|}
\hline Elements & $J_{1}(\phi)$ & $J_{1}\left(\phi_{h}\right)$ & $J_{2}(\phi)$ & $J_{2}\left(\phi_{h}\right)$ \\
\hline 4,000 & 0.20757 & 0.20547 & 0.08882 & 0.08901 \\
16,000 & 0.16904 & 0.16873 & 0.07206 & 0.07205 \\
64,000 & 0.16263 & 0.62590 & 0.06924 & 0.06922 \\
256,000 & 0.16344 & 0.16343 & 0.06959 & 0.06958 \\
$1,024,000$ & 0.16344 & 0.16345 & 0.06959 & 0.06958 \\
\hline
\end{tabular}

Table 1: Convergence of QoIs $J_{1}$ and $J_{2}$ under analytical and finite element solutions on a sequence of uniform meshes. Columns labelled $J_{i}(\phi)$ correspond to analytical solutions, whilst columns labelled $J_{i}\left(\phi_{h}\right)$ correspond to finite element solutions.

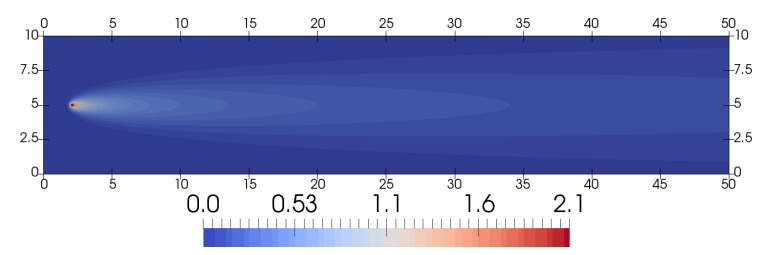

(a) Analytical solution (48).

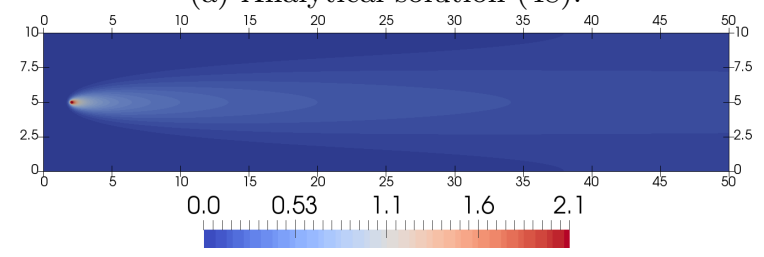

(b) Finite element forward solution.

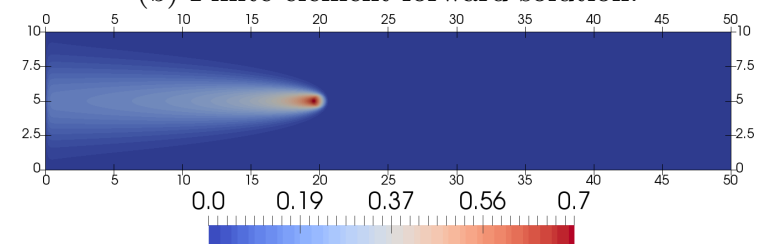

(c) Finite element adjoint solution corresponding to QoI $J_{1}$ and receiver $R_{1}$.

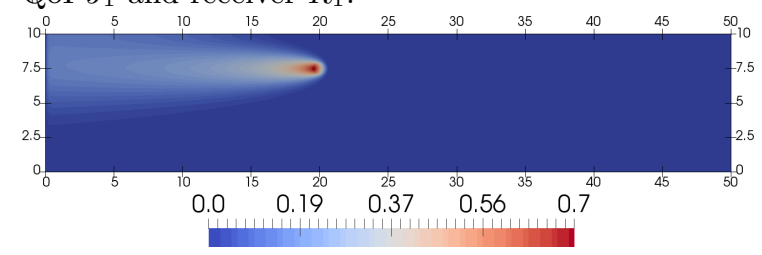

(d) Finite element adjoint solution corresponding to QoI $J_{2}$ and receiver $R_{2}$.

Figure 1: Analytical and finite element solutions for the TELEMAC-2D 'Point Discharge with Diffusion' validation experiment [15], along with adjoint solutions corresponding to QoIs $J_{1}$ and $J_{2}$. Each field is presented on a 1,024,000 element uniform mesh.

mesh provides an excellent approximation to the analytical solution. It appears from Subfigures 1a-1b that the only region where there is a noticeable difference between the analytical and finite element solutions is near the boundary for $x \in[30,50]$. This is in agreement with what may be observed in the TELEMAC$2 \mathrm{D}$ solution given in [15].

Whilst (34) has a point source term at $\mathbf{x}_{0}=(2,5)$, the corresponding adjoint equations have source terms in regions $R_{1}$ and $R_{2}$, which are discs with radius 0.5 . As such, we observe from Subfigures $1 \mathrm{c}-1 \mathrm{~d}$ that the source regions are indeed larger than in the forward PDE. Whilst in the forward PDE information flows in the direction of positive $x$, we observe from Subfigures $1 \mathrm{c}-1 \mathrm{~d}$ that information flows in the opposite direction for the adjoint PDE, as is to be expected.

The purpose of the numerical experimentation described in this subsection is to obtain meshes from goal-oriented error metrics, upon which $J_{1}^{\text {exact }}$ and $J_{2}^{\text {exact }}$ may be accurately approximated (for some desired error level $\epsilon$ ), under the condition that the meshes have as few elements as possible. By fulfilling this purpose, we illustrate the successful implementation of goal-oriented mesh adaptation.

We do not intend to make a rigorous performance analysis, since the problem size considered is relatively small; larger scale problems are required for testing run-time. Instead, we capitalise on the existence of the analytical solution (48), using it to validate our approach and thereby imply that the mesh adaptation strategies considered here may be readily applied to more complicated PDE problems.

\subsubsection{Centred Receiver Region}

Firstly, we consider the case of $R_{1}$, wherein the receiver is directly downstream of the source. We consider three different goal-oriented approaches for residuals in the forward PDE: isotropic mesh adaptation based on (22); a posteriori anisotropic mesh adaptation based on (30); and a priori anisotropic mesh adaptation based on (43). Henceforth, we shall use these names when referring to the three approaches.

Figure 2 illustrates a selection of meshes adapted using these strategies. In each mesh, we observe that there is coarse resolution downstream of the receiver location. Given that this problem is advection-dominated, this is to be expected, as it indicates the insensitivity of the tracer concentration at the receiver to what happens downstream from there. Additionally, in each case there is a region of high resolution surrounding the point source, which enables us to accurately capture the dynamics thereof.

As expected, we observe elements with significantly more anisotropy in the meshes displayed in Subfigures $2 \mathrm{~b}$ and $2 \mathrm{c}$ than in $2 \mathrm{a}$. Note that a posteriori anisotropic metrics constructed using (30) contain the Hessian of the adjoint solution. The influence of 


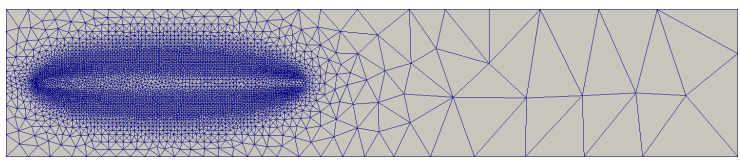

(a) Mesh with 12,246 elements resulting from isotropic adaptation using (22).

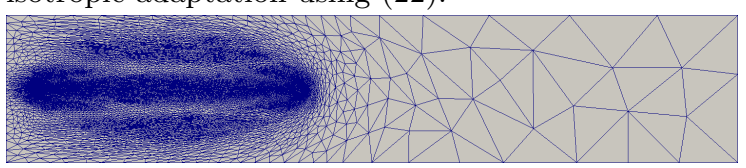

(b) Mesh with 16,407 elements resulting from a posteriori anisotropic adaptation using (30).

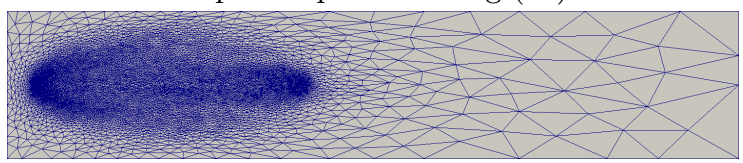

(c) Mesh with 44,894 elements resulting from a priori anisotropic adaptation using (43).

Figure 2: Meshes generated using goal-oriented metrics as indicated, for receiver region $R_{1}$.

this Hessian is visible as the three bands propagating against the flow in Subfigure 2b, relating to regions where the second derivatives are most significant.

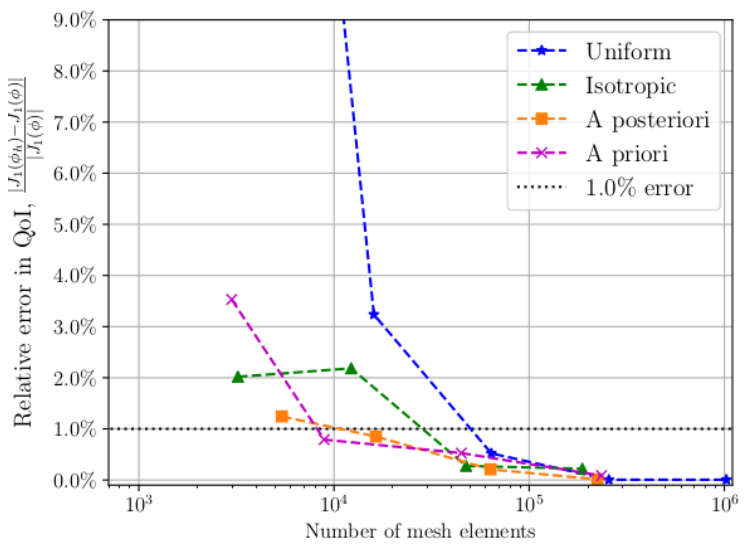

Figure 3: Relative error in $J_{1}^{\text {exact }}$ for finite element solutions computed on meshes arising from: uniform refinement; isotropic adaptation; a posteriori anisotropic adaptation; and a priori anisotropic adaptation.

Figure 3 plots the relative error in approximating $J_{1}^{\text {exact }}$ for a sequence of mesh adaptations. This sequence is generated using decreasing values of $\epsilon>0$, to specify the desired error level. For large values of $\epsilon$, the resulting goal-oriented meshes are often extremely coarse, with an insufficient number of elements to capture the QoI at all, leading to a zero value. These data are excluded from the figures displayed in this work.

Table 1 shows convergence of the QoI to its analytical value, $J_{1}^{\text {exact }}$, on a sequence of uniform meshes. Figure 3 illustrates that this also occurs under all three goaloriented mesh adaptive approaches.

For a given accuracy level relative to the converged QoI value, all three goal-oriented meshing strategies are able to achieve this using fewer elements than required using uniform meshes. Further, the anisotropic approaches outperform the isotropic method in the sense that they require fewer elements to reach the $1 \%$ accuracy level. However, the isotropic approach attains $2 \%$ accuracy using as few as 3,000 elements and the three methods yield fairly similar results overall.

Whilst it appears from Figure 3 that the anisotropic metrics do not lead to significant gains over isotropic ones, it is important to recall that the latter approach requires an auxiliary FEM solve in a higher order, iso$\mathbb{P} 2$ refined space. In particular, the construction of the isotropic metric involves approximating the adjoint solution in an enriched space. This additional FEM solve is inherently more computationally intensive than solving the adjoint equation in the unenriched space, meaning a significant computational cost is accrued. This motivates using anisotropic methods, for which no such auxiliary FEM solves are required.

Next we consider combining the metrics used above with the corresponding metrics associated with the adjoint equation. For this, we use the metric averaging and superposition operators defined in Subsection 2.2. For the isotropic approach, we combine the forward metric used above with an isotropic metric constructed using (41) and (22). The a posteriori anisotropic approach combines (30) and (31), whilst the a priori approach combines (43) and (45).

In each case illustrated in Figure 4, we observe a degree of symmetry in the region $x \in[2,20]$ about the line $x=11$, which marks halfway between the source and receiver. This symmetry follows from the similarity of the forward and adjoint solutions referred to in Subfigures $1 \mathrm{~b}$ and 1c. One noticeable break in this symmetry is exhibited in Subfigures 4e and $4 \mathrm{f}$, where the different forms of the forward and adjoint source terms (point source and disc) are clearly visible. The high element count in Subfigure 4e is due to many elements being deployed in order resolve the discontinuous source terms in the forward and adjoint equations - a feature which is also visible in Subfigure 4f.

As observed in Figure 2, the goal-oriented strategies advocate low resolution downstream of the receiver, since the QoI is insensitive to what happens there.

A first observation to be made from the relative error plot in Figure 5 is that all goal-oriented meshing strategies considered succeed in yielding solutions with the same level of error as is attained on a uniform 


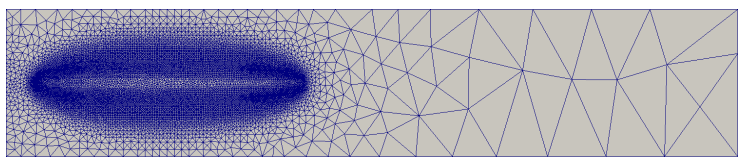

(a) Mesh with 26,792 elements resulting from averaging isotropic metrics.

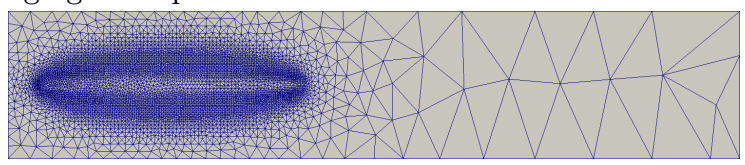

(b) Mesh with 12,300 elements resulting from superposing isotropic metrics.

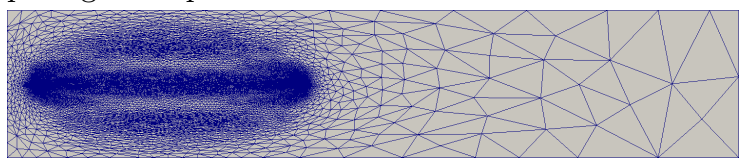

(c) Mesh with 16,538 elements resulting from averaging a posteriori anisotropic metrics.

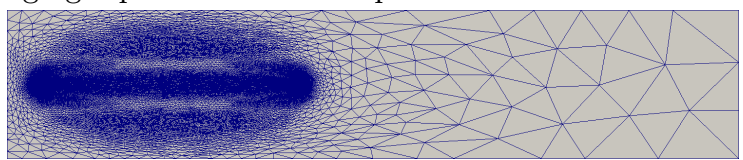

(d) Mesh with 26,116 elements resulting from superposing a posteriori anisotropic metrics.

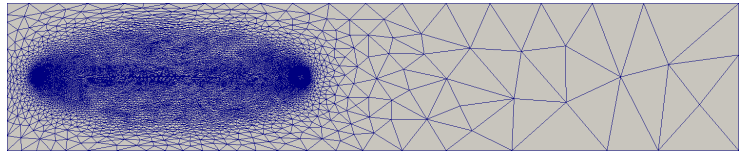

(e) Mesh with 45,108 elements resulting from averaging a priori anisotropic metrics.

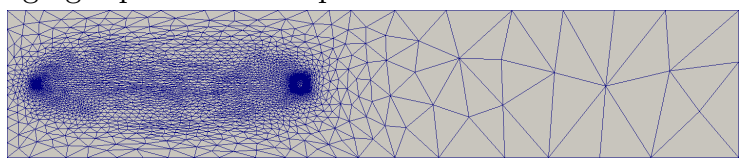

(f) Mesh with 18,383 elements resulting from superposing a priori anisotropic metrics.

Figure 4: Meshes generated using combined goaloriented metrics as indicated, for receiver region $R_{1}$.

mesh, but with fewer elements.

Compared with the results shown in Figure 3, we observe in Figure 5 that both superposing and averaging isotropic metrics provide meshes upon which the $1 \%$ error level may be attained using fewer elements. We deduce that the isotropic adjoint error metric contains information which improves the error estimation.

For the anisotropic approaches, there does not appear to be any clear improvement attained under metric combination for the centred receiver case. One possible explanation for this is that the forward anisotropic metrics already recommend relatively high resolution in the directly downstream of the source, as is visible in Subfigures $2 \mathrm{~b}$ and $2 \mathrm{c}$. As such, the information as-

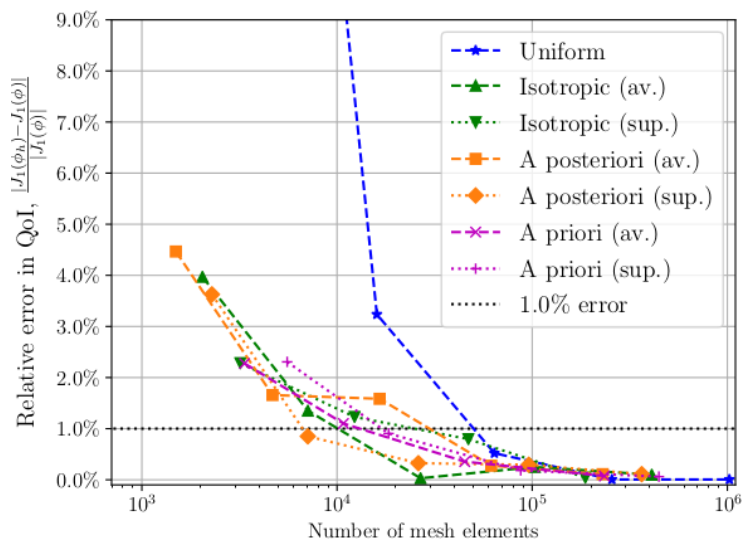

Figure 5: Relative error in $J_{1}^{\text {exact }}$ resulting from mesh adaptation routines which combine forward and adjoint error metrics. Here av. and sup. denote 'average' and 'superposition', respectively.

sociated with the adjoint metric is not so important in the case of a centred receiver region.

However, the superposition and averaging of metrics are shown to yield meshes with 2,000-3,000 elements, upon which the QoI error is less than 5\%. This is in contrast with the initial 4,000 element uniform mesh, upon which the corresponding error is $25.7 \%$.

In conclusion, the above experiments validate the three goal-oriented mesh adaptation implementations in the case where the receiver is directly downstream from the source.

\subsubsection{Offset Receiver Region}

Secondly, we consider the case with an offset receiver region, indicated by $R_{2}$.

Again, all three meshes shown in Figure 6 use coarse resolution downstream of the receiver region. Additionally, relatively coarse resolution is used in the lower part of the domain. This is to be expected, since the sensitivity of the QoI to what happens in this region is negligible, as indicated in Subfigure 1d.

Whilst the isotropic metric recommends heightened mesh resolution in a band spanning the region between source and receiver (as seems reasonable for a goal-oriented strategy), both anisotropic strategies prefer to use high resolution surrounding the source and receiver. In the case of the a priori anisotropic metric, this is likely due to the Hessians of the source terms. In the forward equation, the source term is a delta function, whilst in the adjoint equation it is an indicator function. As such, both of these source terms are discontinuous (and hence non-differentiable) on the edges of the source regions. However, our ap- 


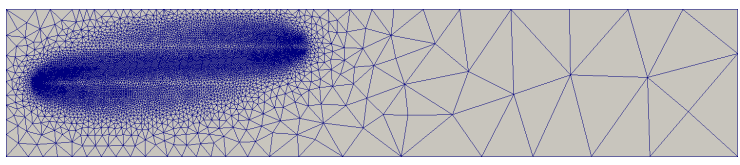

(a) Mesh with 19,399 elements resulting from isotropic adaptation using (22).

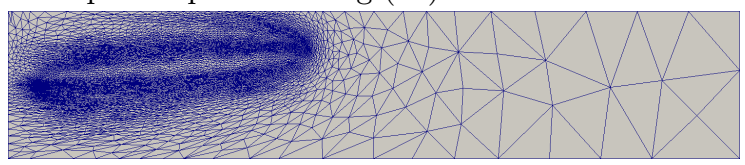

(b) Mesh with 9,868 elements resulting from anisotropic adaptation using (30).

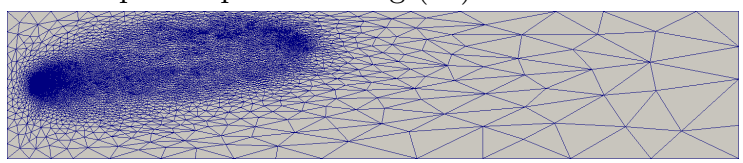

(c) Mesh with 29,143 elements resulting from anisotropic adaptation using (43).

Figure 6: Meshes generated using goal-oriented metrics as indicated, for receiver region $R_{2}$.

plication of $\mathcal{L}_{1}$ normalisation means that the resulting goal-oriented meshes are able to capture these discontinuous features. In the context of QoI error estimation, we accept additional mesh resolution surrounding region $R_{2}$, since this ultimately permits a more accurate approximation.

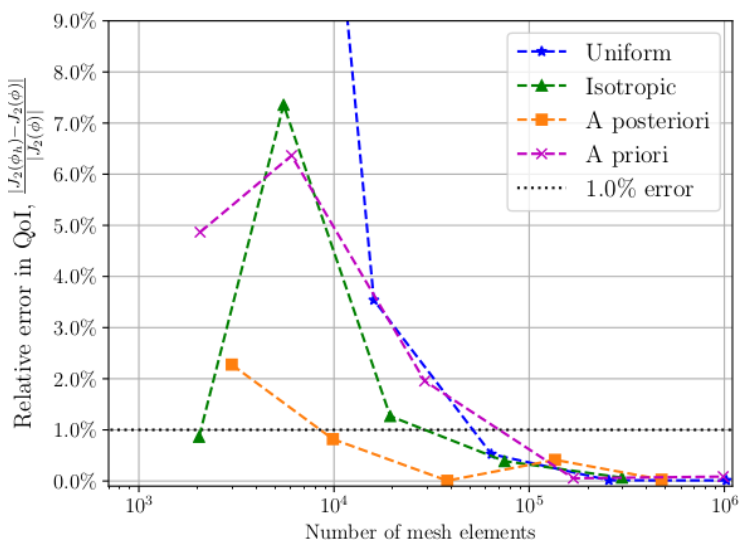

Figure 7: Relative error in $J_{2}^{\text {exact }}$ and error estimator (16) evaluated on meshes arising from: uniform refinement; isotropic adaptation; a posteriori anisotropic adaptation; and a priori anisotropic adaptation.

As with the centred receiver, Figure 7 illustrates all three goal-oriented approaches eventually converging to $J_{2}^{\text {exact }}$. Additionally, both a posteriori goal-oriented approaches require fewer elements to attain the $1 \%$ relative error level than on a uniform mesh. The anisotropic a posteriori approach requires particularly few elements to attain this threshold - around 10,000.
The a priori approach offers a reduction in error over uniform meshing if 10,000 elements or fewer are to be used. On the other hand, this strategy appears to offer no significant improvement over using uniform meshes if more than 10,000 elements are to be used. It is likely that the a priori anisotropic approach suffers from over-resolving the source region at the expense of under-resolving the receiver, for the reasons discussed above. Examining Subfigure 6c, it appears that mesh resolution is more focused on the source than the receiver, supporting this argument. That the a priori approach uses high resolution around the (discontinuous) source term is due to the inclusion of its Hessian.

The leftmost data point for the isotropic approach indicates an error less than $1 \%$ using even fewer elements: 2,035. However, the next data point suggests that the first is in fact a QoI error which is anomalously small. On closer examination of the 2,035 element mesh, the receiver is covered by only very few elements, suggesting that the QoI value may only be coincidentally close to the analytical value. Indeed, this potential anomaly warrants further investigation in future work.

Finally, we consider combined forward and adjoint metrics related to the case of a receiver which is offset from the centre of the channel.

Figure 8 illustrates many of the phenomena which have already been discussed, including the lack of unnecessary downstream resolution, as well as large numbers of elements used near to source and receiver regions in Subfigures $8 \mathrm{e}$ and $8 \mathrm{f}$.

As in the previously considered cases, Figure 9 shows all goal-oriented approaches achieving relative errors below the $1 \%$ level using fewer elements than required on uniform meshes. Further, all three goal-oriented approaches seem to be improved by inclusion of adjoint error estimator information, because smaller errors may be attained using the same number of elements as previously. These improvements are in contrast to Figure 5, where the additional information from the adjoint metric does not significantly improve the QoI approximation. For receiver regions which are offset from the centre of the flow, it appears that the adjoint metric provides useful information for obtaining meshes which yield accurate QoI estimates.

The superposition of a posteriori anisotropic metrics results in small errors using particularly few elements, as does the averaging of isotropic metrics. The anomaly observed in Figure 7 for the isotropic metric seems to also appear here, although to a lesser extent.

In Subsection 5.2 we remark that it is not known whether the adjoint metric associated to the a priori approach relates to a rigorous error estimate. How- 


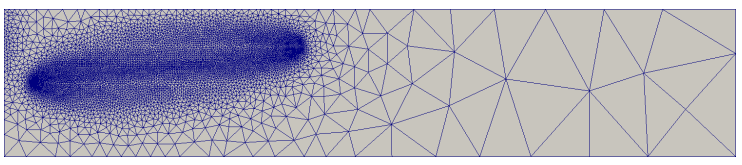

(a) Mesh with 13,980 elements resulting from averaging isotropic metrics.

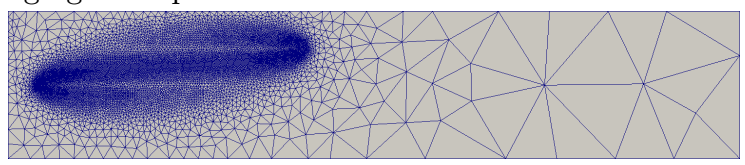

(b) Mesh with 19,588 elements resulting from superposing isotropic metrics.

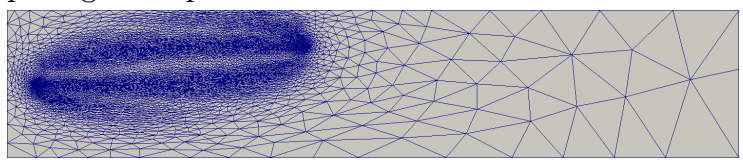

(c) Mesh with 9,289 elements resulting from averaging a posteriori anisotropic metrics.

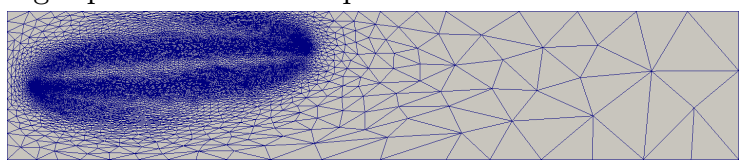

(d) Mesh with 14,470 elements resulting from superposing a posteriori anisotropic metrics.

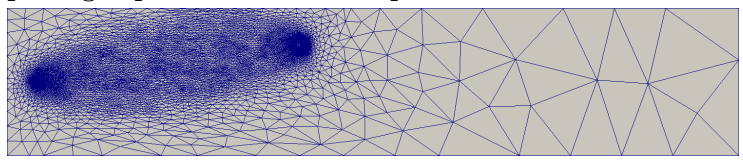

(e) Mesh with 25,204 elements resulting from averaging a priori anisotropic metrics.

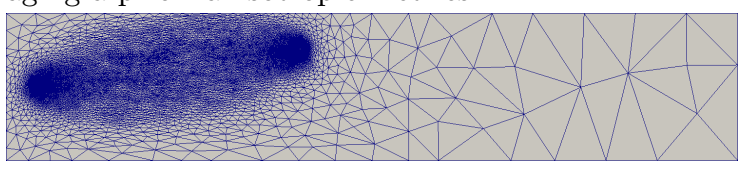

(f) Mesh with 49,793 elements resulting from superposing a priori anisotropic metrics.

Figure 8: Meshes generated using combined goaloriented metrics as indicated, for receiver region $R_{2}$.

ever, the fact that the error may be reduced by inclusion of this information indicates that it certainly contains practical value.

That the relative error is so consistently small for each anisotropic method - using only very few elements - illustrates the great potential of anisotropic goal-oriented mesh adaptation.

For the a posteriori anisotropic and averaged isotropic methods, we observe that the QoI approximation accuracy 'saturates' at around $0.5 \%$, for meshes with over 4,000 elements. Uniform meshing requires around 64,000 elements in order to achieve this error threshold. As such, the saturation of the errors resulting from these goal-oriented approaches indicates that the

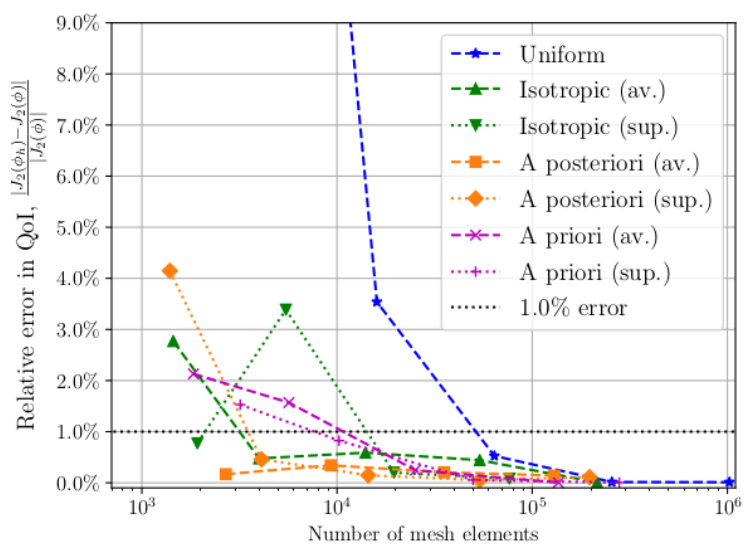

Figure 9: Relative error in $J_{2}^{\text {exact }}$ resulting from mesh adaptation routines which combine forward and adjoint error metrics. Notation as in Figure 5.

high resolution required by a 64,000 element mesh is being achieved in the important regions under these strategies. In particular, sufficient resolution is used in the region of interest. However, lower resolution is used elsewhere, where it is unnecessary.

The major success is that all goal-oriented metrics illustrate convergence to $J_{2}^{\text {exact }}$ using (often significantly) fewer elements than required on uniform meshes. That is, we demonstrate the capability of our implementation of goal-oriented mesh adaptation to accurately approximate QoIs of the form of $J_{1}$ and $J_{2}$ for model steady-state advection-diffusion problems.

Having validated the three methods for the centred and offset receiver cases, we argue that they may be utilised in more complex velocity fields in future work.

\subsection{Three Dimensional Problems}

Whilst serving their purpose as validation experiments, two dimensional approximations are insufficient for many realistic CFD applications, such as the desalination outfall problem discussed in Subsection 5.3. Figure 10 illustrates the extension of the goaloriented methodology to such problems.

The offset receiver tracer transport test case examined in Subsection 5.3 is extended to three dimensions in the natural way by considering source location $\mathbf{x}_{0}=$ $(2,5,5)$, region of interest centred at $(20,7.5,7.5)$ and fluid velocity $\mathbf{u}=(1,0,0)$.

The 3D mesh plot shown in Figure 10 exhibits a wide range of scales, from high resolution near to the source and receiver to very coarse resolution downstream of the receiver region. The cell volumes range from $1.25 \times$ $10^{-10}$ cubic units to 4.81 cubic units, covering more than ten cubic orders of magnitude. That is, there 


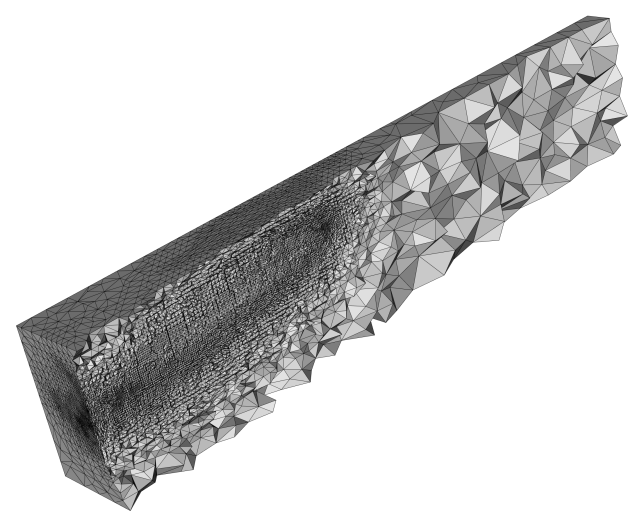

Figure 10: Clip of a mesh with 1,776,396 elements resulting from goal-oriented mesh adaptation for a $3 \mathrm{D}$ extension of the TELEMAC-2D test case discussed in Subsection 5.3. The metric is obtained by averaging forward and adjoint a posteriori anisotropic metrics.

are variations of a factor of as much as 1,000 in each direction. Clearly, using a uniform mesh with cells of volume $1.25 \times 10^{-10}$ is completely prohibitive.

That the goal-oriented adaptation strategy exhibited in Figure 10 is capable of producing multi-scale meshes is essential when moving to large-scale realistic 3D applications, where the region of interest is tiny in comparison to the domain size.

It is also worth noting that, for PDE problems with quantities of interest of the form used in this work, the advantages associated with goal-oriented mesh adaptation improve with spatial dimension. The ratio between the volume/area of the region of interest to the volume/area of the domain decreases when we generalise the $2 \mathrm{D}$ problem as described in Subsection 5.3 to the $3 \mathrm{D}$ problem considered here.

Consider the slice plots shown in Figure 11, which compare isosurfaces of finite element solutions computed on a uniform mesh and the goal-oriented adaptive mesh shown in Figure 10. The impact of the low downstream resolution is visible in Subfigure 11b, where grid-scale features are apparent in the isosurface contours. In contrast, the contours of the uniform mesh isosurfaces shown in Subfigure 11a are relatively smooth across the entire domain. Given the location of the receiver, the downstream resolution applied in the uniform mesh case is unnecessary, as observed in Subsection 5.3 .

Another clear advantage of the goal-oriented mesh is that it is able to accurately capture the point source. This cannot be said for the uniform mesh case, where the area surrounding the source appears to have suffered from numerical diffusion effects. Accurately ap-

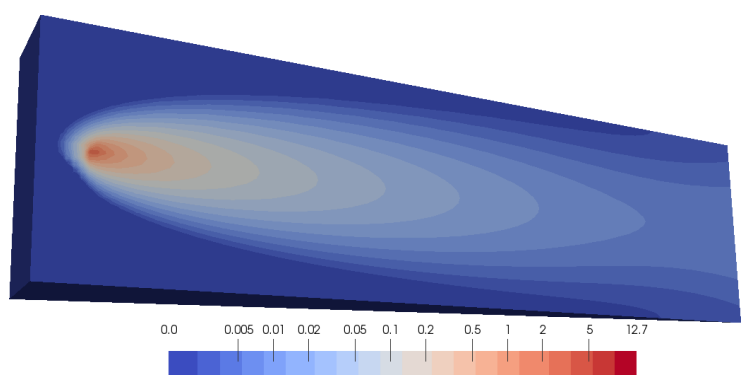

(a) Finite element solution on a uniform mesh with 1,920,000 elements.

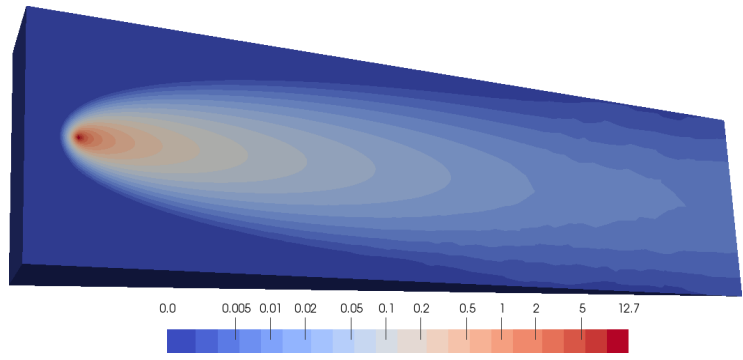

(b) Finite element solution on a 1,766,396 element mesh which was obtained by adaptation with respect to averaged forward and adjoint a posteriori metrics.

Figure 11: Slices of the solution to the 3D extension of the TELEMAC-2D test case discussed in Subsection 5.3, presented on meshes as indicated. Slices were taken on the plane intersecting source and receiver which is orthogonal to the $z$-axis.

proximating the source term is essential for capturing the resultant downstream dynamics.

\section{CONCLUSION}

The main achievement of this work is the successful implementation of three approaches to goal-oriented mesh adaptation in the finite element package Firedrake. To the best of the authors' knowledge, this is the first comparison of these rather different approaches within a single framework.

An established advection-diffusion test case with a known analytical solution is considered in Section 5 . The fact that an analytical solution exists means that the implementation may be validated by showing that a quantity of interest converges to its exact value under each goal-oriented approach. The experiments in Section 5 illustrate that all three goal-oriented strategies enable convergence of two different quantities of interest to their analytical values using (often significantly) fewer elements than would be required for uniform meshes. Whilst no clear conclusions may be drawn concerning the optimality of one particular goal-oriented approach, this work motivates fur- 
ther investigation of each of these strategies within the Firedrake framework.

In a number of instances, it is shown that combining forward and adjoint goal-oriented error metrics, by superposition or averaging, can lead to smaller errors in QoI estimation, for the same number of elements. In particular, this work contains a novel anisotropic metric construction strategy, based on the extension of a known a priori metric to an equivalent metric corresponding to the adjoint equation. To the best of the authors' knowledge, it has not yet been shown that this metric relates to a rigorous error estimator. However, the numerical experiments in Section 5 show that combining this metric with the established a priori metric can result in meshes with relatively few elements upon which the QoI may be accurately approximated. Future work will seek a rigorous error estimate associated with this metric.

The numerical experiments performed in Subsection 5.3 may be considered as simplified interpretations of a desalination outfall modelling scenario, with idealised domains, fluid velocities and diffusivity coefficients. Such idealised problems are useful for validation purposes, due to the existence of analytical solutions.

Moving to more realistic applications comes with a number of additional challenges. Namely, realistic desalination outfall problems are inherently three dimensional, tide-dependent and multi-scale, with the diameters of the inlet and outlet pipes, the scales of key mixing processes, and the size of the ocean domain varying across multiple orders of magnitude. Future work will extend the goal-oriented adaptation approach discussed in this paper to unsteady problems with realistic domains and tidal forcing within the Thetis coastal ocean modelling framework [44] which is built on top of Firedrake. That this work extends to three dimensions is illustrated in Subsection 5.4.

Due to the relatively small sizes of the experiments considered in Subsection 5.3, performance analyses were not considered in this paper. Larger scale test cases should be investigated in future work, wherein such experiments can be made rigorous.

Additionally, we intend to investigate goal-oriented mesh adaptation in the context of more complicated finite element discretisations. One increasingly popular discretisation is the discontinuous Galerkin (DG) space family [23], within which additional interelement flux terms arise in the formulation of dual weighted residual error estimators.

\section{Acknowledgements}

Many thanks to members of Imperial College London's Applied Modelling and Computation Group (AMCG) and to the developers of the Firedrake and Thetis projects for their useful recommendations regarding this work. Further thanks to staff and students of the Mathematics of Planet Earth Centre for Doctoral Training (MPE CDT) for their ongoing support and advice. This work was funded by the Engineering and Physical Sciences Research Council (EPSRC) under grants EP/L016613/1 and EP/R029423/1.

\section{References}

[1] Loseille A., Dervieux A., Alauzet F. "Fully anisotropic goal-oriented mesh adaptation for 3D steady Euler equations." Journal of computational physics, vol. 229, no. 8, 2866-2897, 2010

[2] Funke S.W., Kramer S.C., Piggott M.D. "Design optimisation and resource assessment for tidalstream renewable energy farms using a new continuous turbine approach." Renewable energy, vol. 99, 1046-1061, 2016

[3] Davis B.N., LeVeque R.J. "Adjoint methods for guiding adaptive mesh refinement in tsunami modeling." Global Tsunami Science: Past and Future, Volume I, pp. 4055-4074. Springer, 2016

[4] Zienkiewicz O.C., Zhu J.Z. "A simple error estimator and adaptive procedure for practical engineering analysis." International journal for numerical methods in engineering, vol. 24, no. 2, 337-357, 1987

[5] Loseille A., Alauzet F. "Optimal 3D highly anisotropic mesh adaptation based on the continuous mesh framework." Proceedings of the 18th International Meshing Roundtable, pp. 575-594. Springer, 2009

[6] Ainsworth M., Oden J.T. "A posteriori error estimation in finite element analysis." Computer methods in applied mechanics and engineering, vol. 142, no. 1-2, 1-88, 1997

[7] Becker R., Rannacher R. A feed-back approach to error control in finite element methods: basic analysis and examples. IWR, 1996

[8] Becker R., Rannacher R. "An optimal control approach to a posteriori error estimation in finite element methods." Acta numerica, vol. 10, 1-102, 2001

[9] Venditti D.A., Darmofal D.L. "Anisotropic grid adaptation for functional outputs: application to two-dimensional viscous flows." Journal of Computational Physics, vol. 187, no. 1, 22-46, 2003

[10] Wang Y., Ragusa J.C. "Standard and goaloriented adaptive mesh refinement applied to radiation transport on 2D unstructured triangular 
meshes." Journal of Computational Physics, vol. 230, no. 3, 763-788, 2011

[11] Loseille A., Alauzet F. "Continuous mesh framework part I: well-posed continuous interpolation error." SIAM Journal on Numerical Analysis, vol. 49, no. 1, 38-60, 2011

[12] Power P., Pain C.C., Piggott M., Fang F., Gorman G.J., Umpleby A., Goddard A.J., Navon I. "Adjoint a posteriori error measures for anisotropic mesh optimisation." Computers $\mathcal{E}$ Mathematics with Applications, vol. 52, no. 8-9, 1213-1242, 2006

[13] Rogé G., Martin L. "Goal-oriented anisotropic grid adaptation." Comptes Rendus Mathématique, vol. 346, no. 19-20, 1109-1112, 2008

[14] Carpio J., Prieto J.L., Bermejo R. "Anisotropic goal-oriented mesh adaptivity for elliptic problems." SIAM Journal on Scientific Computing, vol. 35, no. 2, A861-A885, 2013

[15] Riadh A., Cedric G., Jean M. "TELEMAC modeling system: 2D hydrodynamics TELEMAC-2D software release 7.0 user manual." Paris: RESD, Electricite de France, p. 134, 2014

[16] George P., Hecht F., Vallet M. "Creation of internal points in Voronoi's type method. Control and adaptation." Adv. Eng. Software, vol. 13, no. 5-6, 303-312, 1991

[17] Pain C., Umpleby A., De Oliveira C., Goddard A. "Tetrahedral mesh optimisation and adaptivity for steady-state and transient finite element calculations." Computer Methods in Applied Mechanics and Engineering, vol. 190, no. 29-30, 3771-3796, 2001

[18] Alauzet F. "Size gradation control of anisotropic meshes." Finite Elements in Analysis and Design, vol. 46, no. 1-2, 181-202, 2010

[19] Frey P.J., Alauzet F. "Anisotropic mesh adaptation for CFD computations." Computer methods in applied mechanics and engineering, vol. 194, no. 48-49, 5068-5082, 2005

[20] Alauzet F., Loseille A. "A decade of progress on anisotropic mesh adaptation for computational fluid dynamics." Computer-Aided Design, vol. 72, 13-39, 2016

[21] Barral N. Time-accurate anisotropic mesh adaptation for three-dimensional moving mesh problems. Ph.D. thesis, Université Pierre et Marie Curie, 2015
[22] Alauzet F., Olivier G. "An L $p$-L $\infty$ space-time anisotropic mesh adaptation strategy for time dependent problems." Proceedings of ECCOMAS CFD. 2010

[23] Reed W.H., Hill T. "Triangular mesh methods for the neutron transport equation." Tech. rep., Los Alamos Scientific Lab., N. Mex.(USA), 1973

[24] Alauzet F., Belme A., Dervieux A. "Anisotropic goal-oriented mesh adaptation for time dependent problems." Proceedings of the 20th international meshing roundtable, pp. 99-121. Springer, 2011

[25] Belme A., Dervieux A., Alauzet F. "Time accurate anisotropic goal-oriented mesh adaptation for unsteady flows." Journal of Computational Physics, vol. 231, no. 19, 6323-6348, 2012

[26] Rathgeber F., Ham D.A., Mitchell L., Lange M., Luporini F., McRae A.T., Bercea G.T., Markall G.R., Kelly P.H. "Firedrake: automating the finite element method by composing abstractions." ACM Transactions on Mathematical Software (TOMS), vol. 43, no. 3, 24, 2016. URL http://arxiv.org/abs/1411.2940

[27] Alnæs M.S., Logg A., Ølgaard K.B., Rognes M.E., Wells G.N. "Unified form language: A domain-specific language for weak formulations of partial differential equations." ACM Transactions on Mathematical Software (TOMS), vol. 40, no. 2, 9, 2014

[28] Balay S., Abhyankar S., Adams M.F., Brown J., Brune P., Buschelman K., Dalcin L., Dener A., Eijkhout V., Gropp W.D., Kaushik D., Knepley M.G., May D.A., McInnes L.C., Mills R.T., Munson T., Rupp K., Sanan P., Smith B.F., Zampini S., Zhang H., Zhang H. "PETSc Users Manual." Tech. Rep. ANL-95/11 - Revision 3.11, Argonne National Laboratory, 2019. URL http://www.mcs.anl.gov/petsc

[29] Balay S., Gropp W.D., McInnes L.C., Smith B.F. "Efficient Management of Parallelism in Object Oriented Numerical Software Libraries." E. Arge, A.M. Bruaset, H.P. Langtangen, editors, Modern Software Tools in Scientific Computing, pp. 163202. Birkhäuser Press, 1997

[30] Rokos G., Gorman G. "PRAgMaTIc-parallel anisotropic adaptive mesh toolkit." Facing the Multicore-Challenge III, pp. 143-144. Springer, 2013

[31] Ibanez D., Barral N., Krakos J., Loseille A., Michal T., Park M. "First Benchmark of the Unstructured Grid Adaptation Working Group." Procedia engineering, vol. 203, 154-166, 2017 
[32] Park M.A., Barral N., Ibanez D., Kamenetskiy D.S., Krakos J.A., Michal T.R., Loseille A. "Unstructured Grid Adaptation and Solver Technology for Turbulent Flows." 2018 AIAA Aerospace Sciences Meeting, p. 1103. 2018

[33] Park M.A., Balan A., Anderson W.K., Galbraith M.C., Caplan P., Carson H.A., Michal T.R., Krakos J.A., Kamenetskiy D.S., Loseille A., et al. "Verification of Unstructured Grid Adaptation Components." AIAA Scitech 2019 Forum, p. 1723. 2019

[34] Barral N., Knepley M.G., Lange M., Piggott M.D., Gorman G.J. "Anisotropic mesh adaptation in Firedrake with PETSc DMPlex." arXiv preprint arXiv:1610.09874, 2016

[35] Dalcin L.D., Paz R.R., Kler P.A., Cosimo A. "Parallel distributed computing using Python." Advances in Water Resources, vol. 34, no. 9, 1124-1139, 2011. New Computational Methods and Software Tools

[36] Farrell P., Piggott M., Pain C., Gorman G., Wilson C. "Conservative interpolation between unstructured meshes via supermesh construction." Computer Methods in Applied Mechanics and Engineering, vol. 198, no. 33, 2632 - 2642, 2009

[37] Farrell P.E., Maddison J.R. "Conservative interpolation between volume meshes by local Galerkin projection." Computer Methods in Applied Mechanics and Engineering, vol. 200, no. 14, 89-100, 2011

[38] Maddison J.R., Farrell P.E., Panourglas I.P. "Parallel supermeshing for multimesh modelling." Tech. Rep. eCSE03-08, ARCHER, May 2016. URL https://www . archer.co.uk/community/eCSE/eCSE03-08/eCSE03-08-TechnicalReport.pdf

[39] Farrell P.E., Ham D.A., Funke S.W., Rognes M.E. "Automated derivation of the adjoint of high-level transient finite element programs." SIAM Journal on Scientific Computing, vol. 35, no. 4, C369C393, 2013

[40] Mitusch S.K. An Algorithmic Differentiation Tool for FEniCS. Master's thesis, University of Oslo, 2018

[41] "Software used in 'Anisotropic Goal Oriented Mesh Adaptation in Firedrake'.", June $2019 . \quad$ URL https://doi.org/10.5281/zenodo. 3250888

[42] "Simulation code used in 'Anisotropic Goal Oriented Mesh Adaptation in Firedrake'.", Aug. $2019 . \quad$ URL https://doi.org/10.5281/zenodo. 3358565
[43] Donea J., Huerta A. Finite element methods for flow problems. John Wiley \& Sons, 2003

[44] Kärnä T., Kramer S.C., Mitchell L., Ham D.A., Piggott M.D., Baptista A.M. "Thetis coastal ocean model: discontinuous Galerkin discretization for the three-dimensional hydrostatic equations." Geoscientific Model Development, vol. 11, no. $11,4359-4382,2018$

[45] Hendrickson B., Leland R. "A multilevel algorithm for partitioning graphs." Supercomputing '95: Proceedings of the 1995 ACM/IEEE Conference on Supercomputing (CDROM), p. 28. ACM Press, New York, 1995

[46] Amestoy P.R., Duff I.S., L'Excellent J.Y., Koster J. "A fully asynchronous multifrontal solver using distributed dynamic scheduling." SIAM Journal on Matrix Analysis and Applications, vol. 23, no. 1, 15-41, 2001

[47] Amestoy P.R., Guermouche A., L'Excellent J.Y., Pralet S. "Hybrid scheduling for the parallel solution of linear systems." Parallel Computing, vol. 32 , no. 2, 136-156, 2006

[48] Mitchell L., Müller E.H. "High level implementation of geometric multigrid solvers for finite element problems: applications in atmospheric modelling." Journal of Computational Physics, vol. 327, 1-18, 2016. URL http://arxiv.org/abs/1605.00492 


\title{
HIGHER-ORDER ACCURATE MESHING OF NON-SMOOTH IMPLICITLY DEFINED MANIFOLDS
}

\author{
Jakob W. Stanford ${ }^{1} \quad$ Thomas-Peter Fries $^{2}$ \\ 1,2 Institute of Structural Analysis, Graz University of Technology, Graz, Austria. \\ \{stanford/fries\}@tugraz.at
}

\begin{abstract}
A higher-order accurate meshing algorithm for non-smooth surfaces defined via Boolean set operations from smooth surfaces is presented. Input data are a set of level-set functions. This geometry definition allows an easy detection of non-smooth features such as corners and edges. Furthermore, edges can be treated as intersection curves. At first, a linear reconstruction of the surface is subsequently generated with octree decomposition, corner- and edge-detection and surface meshing. Based on this, first the edges and then the interior nodes of higher-order Lagrangian elements are projected onto the implicit surface. Various projection methods are studied for nodes of edge elements and surface elements. Special attention is paid to the accurate meshing of tangential intersection curves. Optimal convergence properties for approximation problems are confirmed in numerical studies.
\end{abstract}

Keywords: higher-order finite elements, curved surface meshing, implicit surface, intersection problems, element lifting

\section{INTRODUCTION}

For finite element analyses, the discretization of the computational domain by means of a mesh is an essential prerequisite that can vastly influence the quality of the results. For analysis with higher-order methods such as the $p$-FEM, the mesh quality plays an even more critical role $[1,2,3,4]$. It is not only important to accurately consider boundary features such as corners or edges. Also the placement of the inner- or higher-order element nodes is crucial for achieving the expected rates of convergence. For geometries created within a solid modeling environment, curved meshes for such methods are often created based on their parametric boundary representation. However, numerous challenges are encountered for geometries where trimming and ill-parametrization are involved, which frequently is the case in practical applications. Therefore, a promising alternative is to create such meshes based on the implicit description of the solid model, rather than just its boundary. This implies that boundary features such as corners, edges and surfaces are to be reconstructed in that order (specifically) for the purpose of mesh generation.

This contribution concentrates on the automated creation of surface meshes and edge meshes suitable for higher-order accurate finite element methods such as the $p$-FEM, for the case that geometry definition is available through a purely implicit representation. More specifically, the geometry description consists of multiple solid bodies implicitly described through analytical functions. While the boundary of each body itself must be smooth, their combination through Boolean operations - as usual in the concept of constructive solid geometry (CSG) [5] - may contain $C^{0}$ and $C^{1}$ continuous edges, hence, leading to non-smooth surfaces. The inclusion of $C^{1}$ continuous edges is necessary: They are commonly found in typical engineering geometries, where surface segments of different curvature meet along a line, for example in pipe bends or car bodies. Ignoring them, or not considering them accurately enough, introduces a geometry discretization error dominating and diluting the 
convergence behavior of the finite element method as shown in [6]. Their accurate discretization has already been covered in [7].

\subsection{Previous Work}

Most commonly, higher-order surface meshes for geometries defined within a solid modeling software are created by using the parametric mapping provided by the boundary-representation (B-rep, usually described through NURBSs) of the geometry to map a mesh from some reference domain onto the surface patch. See [8] or text books such as [9, 10] for general B-rep meshing and e.g., $[11,12,13,14,15]$ and the references cited therein for (curved) higher-order meshing.

An alternative to using the parametric B-rep of a solid model for mesh generation is to use the implicit description. Basically, this amounts to the task of triangulating implicit surfaces. For this task, existing methods are split according to [16] into spatial partitioning methods, such as the marching cubes algorithm [17], that discretize the ambient space to then intersect this discretization with the implicit surface and into continuation methods. These start by finding a point on the surface (or edge) and then continuously add new points to the triangulation. The marching triangles algorithm [18] or advancing front method [19, 20] belong to this category. See also [21] for a review on surface triangulation methods.

These methods mentioned, however, do not explicitly consider non-smooth surfaces, i.e., those containing corners, edges and transition regions. Additional work to consider sharp features include for example [22, 23]. These, however, do not make use of the multi-surface definition assumed in this paper, and it is not clear how accurate these methods can resolve $C^{1}$ continuous edges. When considering this special geometry definition, sharp features such as edges or corners can be defined as intersection of the smooth sub-surfaces. Their detection leads to a surface-surface intersection (SSI) problem, which has also been widely studied in literature $[24,25]$. Works such as [26, 27, 28] consider the intersection of two implicitly defined surfaces and [29] even considers tangential intersections resulting in $C^{1}$ continuous edges.

So far, only linear geometry discretization was discussed. For higher-order mesh generation, approaches may be divided into direct methods that use the parametrization of a solid model to directly create a curved mesh. A posteriori methods, start with a linear mesh and lift inner nodes into the curved surface. For an overview see [30] and the references therein. Most research in that field assumes the geometry description to be available as B-rep. Regarding the curved meshing of implicitly defined geometries, the series of works $[31,32,33,34,6]$ deals with the generation of curved higher-order elements based on a purely implicit description. Therein, a special issue is how to place inner nodes such that a smooth isoparametric mapping is implied and that optimal rates of convergence are achieved.

\subsection{The Contributions of this Publication}

The novelty of this publication is that it presents a way to create a curved mesh of non-smooth implicitly defined geometries that is usable for higher-order methods such as the $p$-FEM in a sense that the created meshes allow for optimal rates of convergence.

The presented approach is a two-staged bottom-up approach, that first discretizes corners, edges and surfaces in that order, and then in a second stage subsequently creates higher-order meshes for edges and surfaces. On the first glance it might seem as if that could also be achieved by employing any of the existing algorithms for implicit surfaces with simply projecting the higher-order nodes onto the surface a posteriori. But already the linear discretization needs to be created with the goal of higher-order accuracy in mind. Furthermore, not all methods of projecting element nodes onto the surface yield optimal rates of convergence. Therefore this paper studies several options to lift inner nodes onto the surface to achieve optimal rates of convergence.

The proposed method builds upon a linear-but accurate - discretization of the solid domain. As detailed in Sec. 3, this is achieved by an octree decomposition to find corners and points on edges. With a continuation method edges - defined as intersection curves - are traced and finally the surface is triangulated using a combination of marching cubes and advancing front method. This linear mesh is stored in a data structure where surface elements are defined in terms of edges and edges in terms of nodes. With this, edges and elements (faces) may be lifted separately as shown in Sec. 4. Sec. 2 of this paper introduces the notation used and Sec. 5 compares the discussed lifting methods and applies the procedure to some boundary value problem on different geometries.

\section{PRELIMINARIES}

Behind the manifolds (surfaces or edges) to be meshed lays a solid domain $\Omega$,

$$
\Omega=\left\{\boldsymbol{x} \in \mathbb{R}^{3} \mid \phi_{\Omega}(\boldsymbol{x})<0\right\}
$$

where $\phi_{\Omega}(\boldsymbol{x}): \mathbb{B}_{\Omega} \subset \mathbb{R}^{3} \rightarrow \mathbb{R}$ is a level-set function and $\mathbb{B}_{\Omega}$ its respective bounding box such that $\Omega \subset \mathbb{B}_{\Omega}$. The 


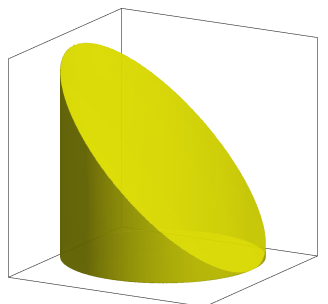

(a)

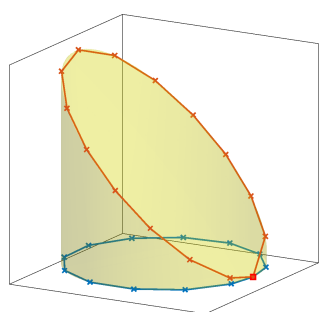

(e)

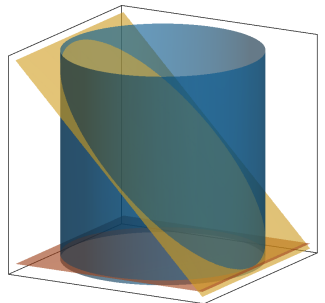

(b)

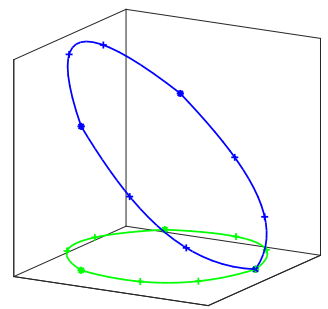

(f)

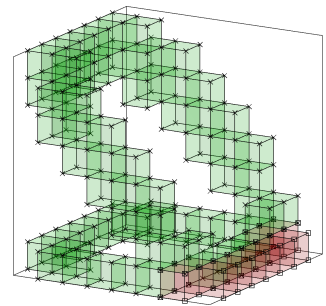

(c)

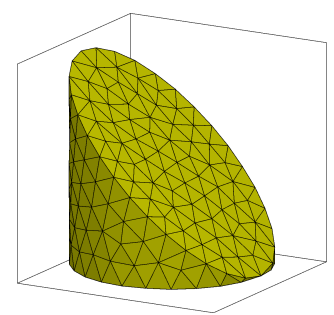

(g)

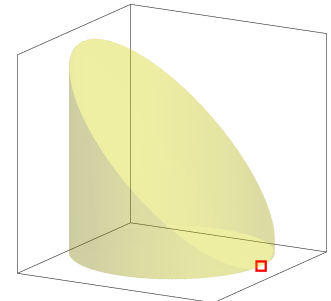

(d)

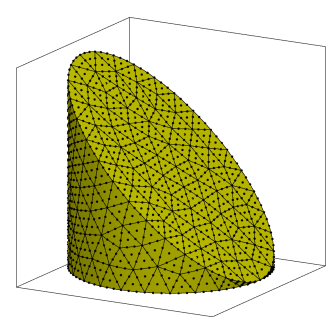

(h)

Figure 1: Overview of the process: (a) solid model under consideration, (b) constitutive surfaces, (c) octree decomposition (green cells: 2 intersections, red: $\geq 3$ intersections, other cells not shown), (d) detected corners, (e) detected corners and edges, (f) higher-order edge mesh, (g) linear surface mesh, (h) curved surface mesh.

boundary of $\Omega$ is defined with the same concept as

$$
\Sigma_{\Omega}=\partial \Omega=\left\{\boldsymbol{x} \in \mathbb{R}^{3} \mid \phi_{\Omega}(\boldsymbol{x})=0\right\}
$$

We refer to $\Sigma_{\Omega}$ as the final surface to be meshed. Per the following definitions, it is a closed 2-manifold. There exist also a number of analytic $C^{\infty}$-smooth level-set functions $\phi_{i}(\boldsymbol{x}): \mathbb{B}_{i} \subset \mathbb{R}^{3} \rightarrow \mathbb{R}$ to define smooth surfaces (or simply surfaces)

$$
\Sigma_{i}=\left\{\boldsymbol{x} \in \mathbb{R}^{3} \mid \phi_{i}(\boldsymbol{x})=0\right\},
$$

where $\mathbb{B}_{i}$ are again the corresponding bounding boxes to each $\phi_{i}(\boldsymbol{x})$. Herein, it is assumed that each $\phi_{i}(\boldsymbol{x})$ describes a distinct surface, that is $\Sigma_{i} \neq \Sigma_{j} \forall i, j, i \neq$ $j$. Each $\Sigma_{i}$ is also equipped with a surface normal vector

$$
\boldsymbol{n}_{i}(\boldsymbol{x})=\frac{\nabla \phi_{i}(\boldsymbol{x})}{\left\|\nabla \phi_{i}(\boldsymbol{x})\right\|} .
$$

Similar to the concept of constructive solid geometry, these level-set functions and surfaces together make up the final domain or surface, such that

$$
\partial \Omega=\left.\bigcup_{i} \Sigma_{i}\right|_{\partial \Omega} \quad \text { with }\left.\Sigma_{i}\right|_{\partial \Omega}=\Sigma_{i} \cap \partial \Omega,
$$

where $\left.\Sigma_{i}\right|_{\partial \Omega}$ are denoted as the surface segments. Also note that in practice, $\phi_{\Omega}(\boldsymbol{x})$ is created from $\phi_{i}(\boldsymbol{x})$ through Boolean operations. If it is desired to have $\phi_{\Omega}(\boldsymbol{x})$ available as an analytic function, R-functions [35] may be used to implement the Boolean operations. The intersection $\Gamma_{i j}$ describes the point set

$$
\Gamma_{i j}=\Sigma_{i} \cap \Sigma_{j} \cap \Sigma_{\Omega},
$$

which can contain regular and tangential intersection curves forming $C^{0}$ and $C^{1}$ continuous edges respectively. It may also contain branching points of intersection curves, which are characterized by

$$
\left\|\nabla \phi_{i}(\boldsymbol{x}) \times \nabla \phi_{j}(\boldsymbol{x})\right\|=0 .
$$

$\Gamma_{i j}$ can however not contain surfaces, due to the limitations on $\phi_{i}(\boldsymbol{x})$ set above. Additionally, as per [36, Theorem 3], it is a closed, smooth space curve. Regular corners are described by the point set

$$
\Gamma_{i j k}=\Sigma_{i} \cap \Sigma_{j} \cap \Sigma_{k} \cap \Sigma_{\Omega} .
$$

Together with branching points, these form the sets of corners and are denoted by $\partial \Gamma_{c}$. To facilitate tracing of edges, also edge segments $\Gamma_{e}$ are defined. These go from corner to corner. Furthermore, edge segments exclude surfaces intersections that do not form an edge on $\Sigma_{\Omega}$. Because $\Sigma_{i}$ are smooth and branching points are included in the definition of $\partial \Gamma_{c}$, an edge segment is a smooth space curve, free from branching points and therefore easy to trace with a continuation method. An edge-segment is $C^{1}$ continuous or tangential if the tangent vector

$$
\boldsymbol{t}_{i j}(\boldsymbol{x})=\boldsymbol{n}_{i}(\boldsymbol{x}) \times \boldsymbol{n}_{j}(\boldsymbol{x})
$$

vanishes, that is $\left\|\boldsymbol{t}_{i j}\right\|=0$. In such a case, $\boldsymbol{t}_{i j}$ points in the direction where the normal curvatures of both surfaces are equal.

Regarding the finite element technology used in this paper, Lagrangian elements of order $p$ with equidistantly spaced element-nodes $\boldsymbol{x}_{\ell}$ are used. With the 
help of the isoparametric mapping

$$
\chi(\boldsymbol{r}): \Omega_{\tau}^{\mathrm{ref}} \subset \mathbb{R}^{d} \rightarrow \Omega_{\tau} \subset \mathbb{R}^{3} \quad d=\{1,2\},
$$

the Jacobian (in this case the Gram's determinant) is defined as

$$
|\mathbf{J}(\boldsymbol{r})|=\sqrt{\operatorname{det}\left((\nabla \chi(\boldsymbol{r}))^{\top} \cdot \nabla \chi(\boldsymbol{r})\right)} .
$$

\section{LINEAR RECONSTRUCTION}

The linear discretization lays the foundation for the eventual lifting process and it relies heavily on the concept of geometry definition laid out in Sec. 2.

\subsection{Corner Detection and Edge Tracing}

With octree decomposition of the bounding box $\mathbb{B}_{\Omega}$ and parallel intersection testing with respect to each $\Sigma_{i}$, roots for potential intersection curves, branching points and regular corners are isolated. The roots are then located using (6), (7) and (8) respectively. These equations can be solved with a standard NewtonRaphson scheme. With the multi-level-set concept it is clear that only cells intersected by three or more $\Sigma_{i}$ may contain regular corners and the same can be said for branching points and intersection curves with two or more $\Sigma_{i}$-intersections. Hence, only a small subset of cells needs to be checked for roots. Edge segments are then traced starting on respective points using a predictor-corrector-like scheme: First, a start value $\boldsymbol{x}_{E, e+1}^{0}$ near the next edge-point is created based on the tangent vector of the intersection curve:

$$
\boldsymbol{x}_{E, e+1}^{0}=\boldsymbol{x}_{E, e}+\boldsymbol{t}_{i j}\left(\boldsymbol{x}_{E, e}\right) h_{t r} .
$$

This start value is then projected onto the intersection curve by solving the system of equations:

$$
\begin{aligned}
\phi_{i}\left(\boldsymbol{x}_{E, e+1}\right) & =0 \\
\phi_{j}\left(\boldsymbol{x}_{E, e+1}\right) & =0 \quad \text { with } \phi_{\Omega}\left(\boldsymbol{x}_{c}\right)=0, \\
\phi_{\circ, e}\left(\boldsymbol{x}_{E, e+1}\right) & =0
\end{aligned}
$$

where zero-level set of $\phi_{\circ, e}$ describes a sphere, centered at $\boldsymbol{x}_{E, e}$ with radius $h_{t r}$, which is the predefined steplength. In practice, $h_{t r}$ is set to a value smaller than the minimal distance between two branching points. If no solution to (13) is found, $h_{t r}$ may recursively be halved. Tracing terminates if $\boldsymbol{x}_{E, e+1}$ is closer than $h_{t r} / 2$ to a corner or to the start point of the initial point $\left(\boldsymbol{x}_{E, 0}\right)$ on the edge. (13) is solved with the classical Newton-Raphson method using $\boldsymbol{x}_{E, e+1}^{0}$ as initial guess.

In case a tangential intersection curve is being traced, the approach to solve (13) with a Newton-Raphson scheme would fail or would produce inaccurate results at least. Therefore it is suggested to minimize the objective function

$$
F(\boldsymbol{x})=\phi_{i}(\boldsymbol{x})^{2}+\phi_{j}(\boldsymbol{x})^{2}+\phi_{\circ, e}(\boldsymbol{x})^{2}+\tau_{i j}(\boldsymbol{x})
$$

with the tangentiality constraint

$$
\tau_{i j}(\boldsymbol{x})=\left\|\nabla \phi_{i}(\boldsymbol{x}) \times \nabla \phi_{j}(\boldsymbol{x})\right\|^{2} .
$$

instead. The tangentiality constraint ensures an accurate solution by specifically enforcing tangentiality. Note that this constraint may also be used to quantify the tangentiality of a given intersection curve. Because $\tau_{i j}$ is simply a term added to the objective function, it is technically still an unconstrained optimization problem that is solved with the gradient descent method using the step length described in [37].

\subsection{Linear Surface Meshing}

In a nutshell, surface segments $\left.\Sigma_{i}\right|_{\partial \Omega}$ are discretized with the following steps: 1) Discretization from a marching cubes scheme. This triangulates the whole $\Sigma_{i}$, i.e., also segments not part of $\Sigma_{\Omega}$. 2) Therefore, simplices not completely part of $\left.\Sigma_{i}\right|_{\partial \Omega}$ are removed. 3) The resulting triangulation is linked with the edgepolygons by an advancing front algorithm, extended for direct meshing of surfaces similar to [38] or [39]. 4) To avoid elements with undesired inner angles, the mesh is smoothed using an extension of distmesh [40] for curved surfaces. At the end of this process, a linear surface mesh $\Sigma_{i}^{h}$ with specified edge-length $h$ is available for linear FEM analyses or for subsequent higher-order meshing.

The combination of advancing front method and marching cubes is needed in order to accommodate for closed surfaces (where there exists no boundary that can serve as initial front) and bounded surfaces (where the edges need to be considered explicitly).

\section{HIGHER-ORDER MESHING}

\subsection{Higher-Order Edge Meshing}

For lifting a linear edge mesh $\Gamma_{e}^{h}$ to a curved, higherorder mesh $\Gamma_{e}^{h, p}$, multiple alternatives may be considered. The first one is to distribute the required amount of higher-order mesh nodes along $\Gamma_{e}$ equidistantly and project those nodes onto $\Gamma_{i j}$. This yields a curved mesh with approximately equidistant nodal spacing. By using the resulting curved mesh, one can iteratively improve the nodal positions, which leads to a variational approach: To equidistantly place nodes on a curved mesh, an auxiliary boundary value problem (BVP) - specifically the Laplace Beltrami equation (as later defined in (22)) - is solved using the finite element method. The idea is to use the solution 


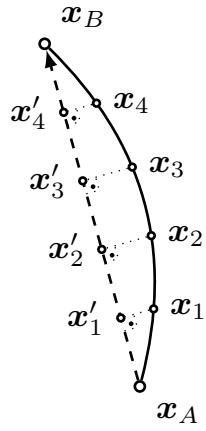

Figure 2: Illustration of equidistant node projection.

of the BVP as arc-length parametrization (approximate and scaled by a constant factor) to place the higher-order mesh nodes. For that, on both ends of the edge Dirichlet boundary conditions (the values 0 and 1 respectively) are set and the BVP is solved. The solution

$$
u^{h}(\boldsymbol{x}): \Gamma_{e}^{h} \longrightarrow\left[\begin{array}{ll}
0 & 1
\end{array}\right]
$$

is then used to place nodes $\hat{\boldsymbol{x}}$ at desired positions $\hat{u}$ in the arch-length domain by finding $\hat{\boldsymbol{x}}$ such that $u^{h}(\hat{\boldsymbol{x}})=\hat{u}$. The node positions obtained this way, lay on the mesh $\Gamma_{e}^{h}$ used in the BVP and hence need to be projected onto $\Gamma_{e}$ again.

An arguably simpler method is to element-wise lift inner nodes onto $\Gamma_{i j}$ such that their normal projection onto the linear connection between the end nodes is equidistant, as illustrated in Fig. 2. For this, only the following system of equations is solved for $\boldsymbol{x}_{e}$ :

$$
\begin{aligned}
\phi_{i}\left(\boldsymbol{x}_{e}\right) & =0 \\
\phi_{j}\left(\boldsymbol{x}_{e}\right) & =0, \\
\phi_{\perp}\left(\boldsymbol{x}_{e}^{\prime}, \boldsymbol{v}_{A B}, \boldsymbol{x}_{e}\right) & =0
\end{aligned}
$$

where the zero iso-line of $\phi_{\perp}\left(\boldsymbol{x}_{e}^{\prime}, \boldsymbol{v}_{A B}, \boldsymbol{x}_{e}\right)$ defines a plane normal to the vector $\boldsymbol{v}_{A B}=\boldsymbol{x}_{B}-\boldsymbol{x}_{A}$ and going through $\boldsymbol{x}_{e}^{\prime}$, which is the normal projection of $\boldsymbol{x}_{e}$ onto $\overline{A B}$. See Fig. 2 for a sketch.

\subsection{Higher-Order Surface Meshing}

The remaining task is to lift an existing linear surface mesh to a specified order $p$. Essentially, this is accomplished by projecting additional mesh-nodes onto the surface. While this sounds trivial, multiple variants exist and not all lead to optimal results. Specifically, there are choices to be made regarding a starting point and the projection method to be used. It is assumed that the linear surface mesh is represented through a hierarchical data structure where elements are defined in terms of element-edges and element-edges in terms of nodes:

$$
\text { Element } \rightarrow \text { Edge } \rightarrow \text { Node }
$$

This way, element-edges and elements can be lifted separately. This is important, because edges of the original edge-mesh, from hereon denoted boundary edges, require a different lifting than the other elementedges, herein denoted interior edges. The following discussion of edge-lifting hence only concerns interior edges.

The task of node lifting is to determine the coordinates of a higher-order element node $\boldsymbol{x}_{\ell}$ in the physical domain. This mapping is split into two stages:

$$
\boldsymbol{r}_{\ell} \stackrel{\chi(\boldsymbol{r})}{\longrightarrow} \boldsymbol{x}_{\ell}^{(0)} \stackrel{\mathcal{P}(\boldsymbol{x})}{\longrightarrow} \boldsymbol{x}_{\ell}
$$

as depicted in Fig. 3. In the first stage, $\chi(\boldsymbol{r})$ maps the node from the reference domain of the given higherorder element onto some intermediary element in $\mathbb{R}^{3}$. This provides the start value $\boldsymbol{x}_{\ell}^{(0)}$ for the projection operator $\mathcal{P}(\boldsymbol{x})$, which lifts the nodes to their final location on the surface. The intermediary element in the simplest case can be a linear element yielding the mapping $\chi^{\text {lin }}(\boldsymbol{r})$. For surface-elements, it can also be an element that is curved based on its already lifted edges, as created with transfinite interpolation [41, 42] denoted by $\chi^{\text {bldg }}(\boldsymbol{r})$ in Fig. 3. Hence, the kind of intermediate element determines the location of the start value of the subsequent projection.

For the projection operator $\mathcal{P}(\boldsymbol{x})$ there are two alternatives: One is a line-search method: Find $\alpha$ such that

$$
f(\alpha):=\phi_{i}\left(\boldsymbol{x}_{\ell}^{(0)}+\alpha \boldsymbol{v}\right)=0,
$$

where the vector $\boldsymbol{v}$ is the search direction and $\alpha$ a scaling parameter, which is to be determined by a univariate Newton-Raphson scheme. The final node position is then $\boldsymbol{x}_{\ell}=\boldsymbol{x}_{\ell}^{(0)}+\alpha \boldsymbol{v}$. For the search direction $\boldsymbol{v}$, possibilities are:

1. The surface gradient at the starting point: $\boldsymbol{v}=$ $\nabla \phi_{i}\left(\boldsymbol{x}_{\ell}^{(0)}\right)$, or

2. the surface normal of the intermediate element $\boldsymbol{v}=\boldsymbol{n}_{\tau}$, or, for edges, the average of the normal vectors of the adjacent elements $\boldsymbol{v}=\boldsymbol{n}_{\tau, 1}+\boldsymbol{n}_{\tau, 2}$.

The other projection method considered here would be to change the search direction $\boldsymbol{v}$ in each iteration. This leads to an iteration scheme like

$$
\boldsymbol{x}_{\ell}^{(k+1)}=\boldsymbol{x}_{\ell}^{(k)}-\phi_{i}\left(\boldsymbol{x}_{\ell}^{(k)}\right) \frac{\nabla \phi_{i}\left(\boldsymbol{x}_{\ell}^{(k)}\right)}{\left\|\nabla \phi_{i}\left(\boldsymbol{x}_{\ell}^{(k)}\right)\right\|^{2}} .
$$

In this case, the gradient of $\phi_{i}(\boldsymbol{x})$ at each iteration step can be interpreted as search direction. 


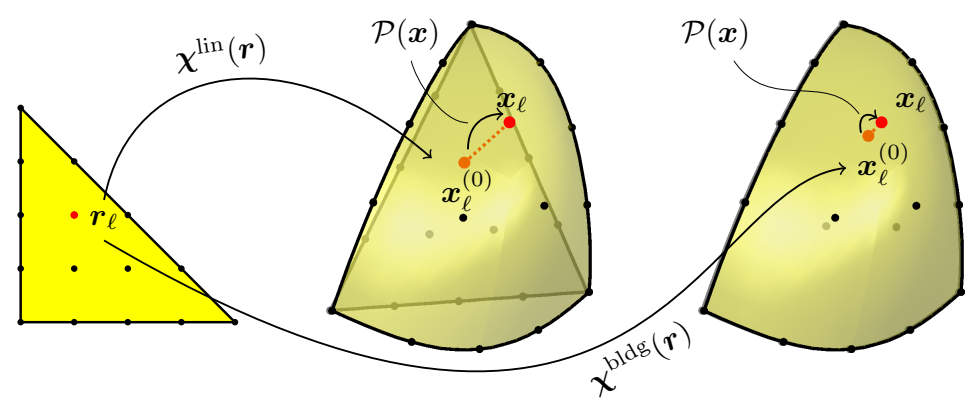

Figure 3: Two-staged mapping procedure for the surface mesh with linear intermediate element (gray, middle figure) and blended intermediate element (right figure).

\section{NUMERICAL STUDIES}

\subsection{Study of Edge Lifting Variants}

In this study, the effect of the two discussed possibilities to create higher-order edge meshes on the mesh quality is assessed. For this purpose, a convergence study is run on the geometry shown in Fig. 4a and Fig. 4d. The study was done only on the upper edge.

Referring to the alternatives discussed in Sec. 4.1, the variants studied are as follows:

$V_{E, 1}$ lift with equidistant projection,

$V_{E, 2}$ mesh with variational approach.

In the study, the error in the approximated edgelength

$$
\varepsilon_{L, e}=\left|\frac{L_{e}^{h}-L_{e}^{r e f}}{L_{e}^{r e f}}\right|
$$

is measured (here, $|\cdot|$ denotes the absolute value of a scalar). Also, the ratio of the Jacobi determinant (as defined in (11)) within an element is computed, which is hence an element-wise measure, as proposed, e.g., in [2]. As mesh-wide measure, the worst of these ratios is taken:

$$
\rho_{J}=\max \frac{\min |\mathbf{J}(\boldsymbol{r})|}{\max |\mathbf{J}(\boldsymbol{r})|} \quad \forall \boldsymbol{r} \in \Omega_{\tau}^{\mathrm{ref}} .
$$

The possible values range from 1 (best) to 0 (worst). In the study, $|\mathbf{J}(\boldsymbol{r})|$ was evaluated on fine sub-intervals in each element.

The results in Fig. 4 show slightly better convergence behavior with a lower constant for $\varepsilon_{L, e}$ in $V_{E, 2}$. Also for $\rho_{J}, V_{E, 2}$ yields better results: Here, the rate of convergence is also dependent on $p$, whereas in $V_{E, 1}$, it seems to only depend on the element size $h$. As result, $V_{E, 2}$ is chosen as edge-lifting variant for the following studies.

\subsection{Study of Surface Lifting Variants}

The various options on lifting surface elements shall be studied on a full sphere (denoted $G_{2}$ ) and a hollow sphere intersected by two cylinders (denoted $G_{3}$ ) as originally shown in [43]. See Fig. 5 for the coarsest meshes for each geometry.

The options discussed in Sec. 4.2 are summarized and assigned the following variants: For the mapping onto the intermediate element resulting in the start value $\boldsymbol{x}_{\ell}^{(0)}$

$V_{\chi, 1}$ linear element using $\chi^{\text {lin }}(\boldsymbol{r})$,

$V_{\chi, 2}$ transfinite interpolation from edges with $\chi^{\mathrm{bldg}}(\boldsymbol{r})$,

and for the choice of search direction

$V_{\boldsymbol{v}, 1}$ line search with $\boldsymbol{v}=\boldsymbol{n}_{\tau}$ for surface elements, or $\boldsymbol{v}=\boldsymbol{n}_{\tau, 1}+\boldsymbol{n}_{\tau, 2}$ or for edges,

$V_{\boldsymbol{v}, 2}$ line search with $\boldsymbol{v}=\nabla \phi_{i}\left(\boldsymbol{x}_{\ell}^{(0)}\right)$,

$V_{\boldsymbol{v}, 3}$ gradient-descent method with variable search direction.

For each possible combination of the variants above, an $h p$ convergence study is conducted. As boundary value problem the Laplace-Beltrami problem, as described in $[44,45]$, was considered. Find $u: \Gamma \mapsto \mathbb{R}$ such that:

$$
\begin{aligned}
-\Delta_{\Gamma} u & =f & & \text { on } \Omega \\
u & =g_{D} & & \text { on } \partial \Omega_{D},
\end{aligned}
$$

where $\Delta_{\Gamma}$ is the surface Laplace-operator. In this case $\Omega=\left.\bigcup_{i} \Sigma_{i}\right|_{\partial \Omega}, \partial \Omega_{D}=\bigcup_{e} \Gamma_{e}$ and

$$
g_{D}=u^{e x}=\sin (2 x+3 / 2 y+7 / 10 z) .
$$

The source term $f$ as chosen such that $u=u^{e x}$ on $\Omega$ as done in the method of manufactured solutions. 


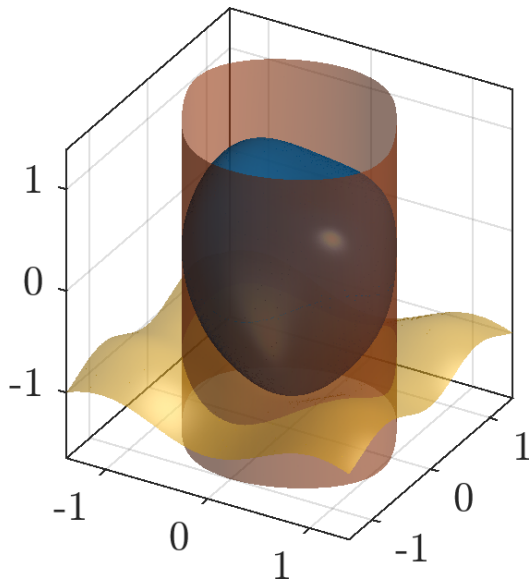

(a) Input Geometry

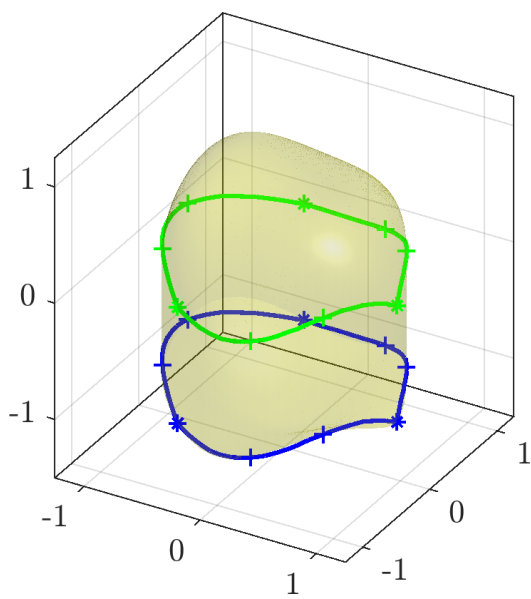

(d) Edge Meshes (the upper one was considered in the study)

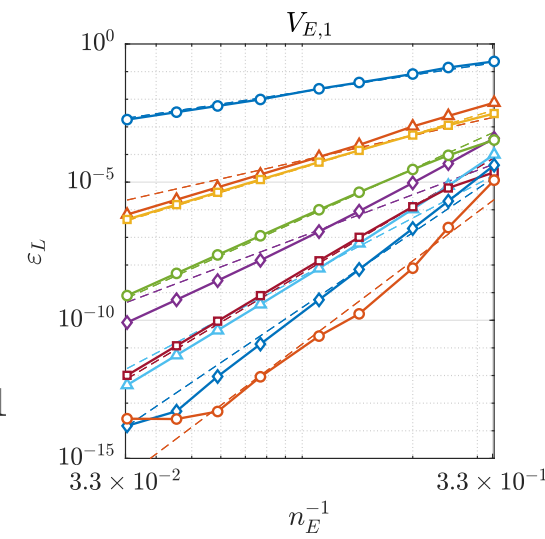

(b)

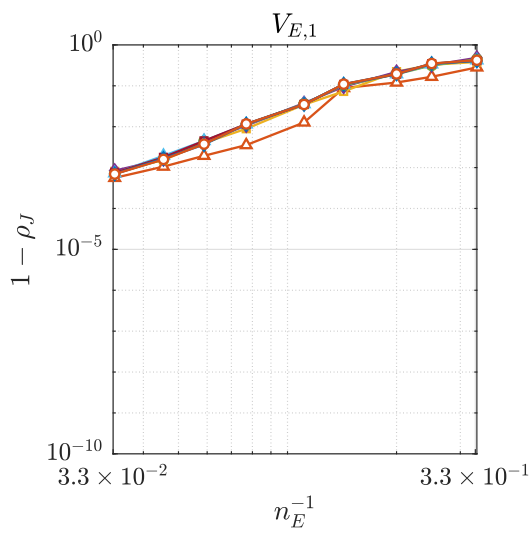

(e)

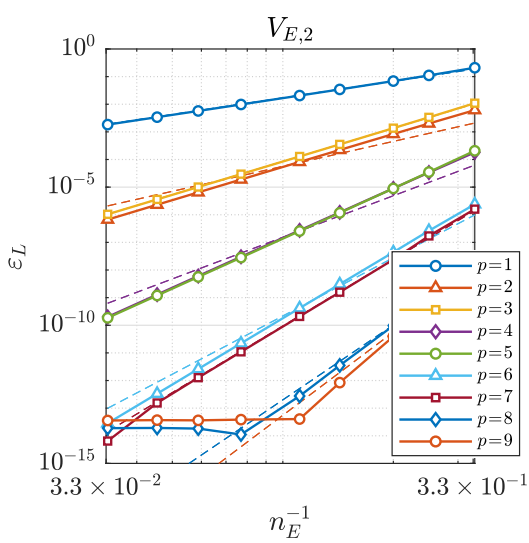

(c)

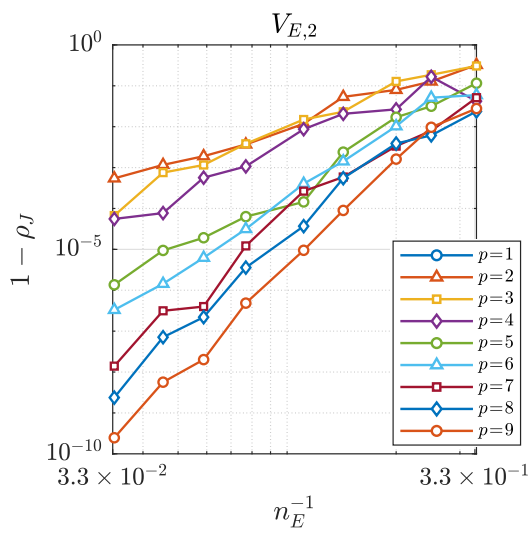

(f)

Figure 4: Results for the Edge Lifting Variants: Error in approximated edge lengths $\varepsilon_{L}$ for both variants (b, c). Jacobian ratio $\rho_{J}$ for both variants $(e, f)$. 
The closed form of $f$ is not given here, because the resulting expression is rather lengthy, as its computation involves the surface Laplace-operator, which in turn involves the surfaces normal vector field of $\phi_{\Omega}(\boldsymbol{x})$.

The continuous weak form of $(22)$ is: Find $u \in H^{1}(\Omega)$ such that:

$$
\int_{\Omega} \nabla_{\Gamma} u \cdot \nabla_{\Gamma} v \mathrm{~d} x=\int_{\Omega} f v \mathrm{~d} x \quad \forall v \in H_{0}^{1}\left(\partial \Omega_{D}\right) .
$$

where $\nabla_{\Gamma}$ is the surface gradient operator, $H^{1}(\Omega)$ is the usual Sobolev space containing the all square integrable functions with first derivatives in $\Omega$ and $H_{0}^{1}\left(\partial \Omega_{D}\right)=\left\{u \in H^{1}|u|_{\partial \Omega_{D}}=0\right\}$. The weak form (23) is discretized with a standard continuous BubnovGalerkin method. The resulting linear system of equations is solved using a direct solver for sparse, symmetric systems.

The error of the residual of the strong form of the BVP

$$
\varepsilon_{S F}^{2}=\sum_{\ell}\left\|\Delta_{\Gamma} u+f^{2}\right\|_{L_{2}\left(\Omega_{\ell}\right)}
$$

serves as measure for the quality of the approximated solution.

To assess the regularity of the iso-parametric mapping, again $\rho_{J}$ as defined in (21) is taken.

The results for $\varepsilon_{S F}$ in Fig. 6 attest optimal rates of convergence for $V_{\chi, 2}$. For $V_{\chi, 1}$ only elements of $p=2$ converge as expected. Since this is also the highest possible order for which standard Lagrangian elements to not have an inner node, it demonstrates the importance of choosing a suitable start valueachieved through a curved intermediate element-for surface projection. For the search direction, there is no clear conclusion from the error plots, especially for $G_{3}$, where the error levels seem to be almost identical across the variants. A noteworthy exception is $V_{\boldsymbol{v}, 3}$ for $G_{2}$. The sphere $G_{2}$ is smooth and hence is free from boundary edges, which would be lifted with a different method. From that it can be concluded that closed, smooth surfaces can also be lifted with this method.

\subsection{Boundary Value Problem on Surface Mesh}

In this example, the geometries shown in Fig. 8 are considered for an $h p$ convergence study. The geometry $G_{18}$ is considered in [46] as test case due to the branching point created by the intersection of the sphere with the cylinder. $G_{24}$ features some curved, $C^{1}$ cont. edges and $G_{31}$ shows a typical engineering geometry. Again, the Laplace-Beltrami problem (22) is solved. In addition to $\varepsilon_{S F}$ also the relative Jacobian

$$
|\mathbf{J}(\boldsymbol{r})|^{\mathrm{rel}}=\frac{|\mathbf{J}(\boldsymbol{r})|}{2 \int_{\Omega_{\tau}} 1 \mathrm{~d} \boldsymbol{x}}
$$

is observed. Values range from 0 to $\infty$ with 1 being the optimal value indicating a quasi-affine iso-parametric mapping.

The results of the study are collected in Fig. 10. In a nutshell, $\varepsilon_{S F}$ reaches optimal rates of convergence for all tested geometries after some pre-asymptotic behavior. In Fig. 9c, the relative Jacobian is plotted for a specific mesh used in the study. It can be seen that the Jacobian varies especially in elements that are part of boundary edges. This behavior might be reduced by optimizing interior edges, for example by Winslowbased smoothing $[47,48]$ extended to manifolds.

\section{CONCLUSION AND OUTLOOK}

It was shown how curved, higher-order accurate meshes for non-smooth surfaces can be created. The non-smooth surfaces are the result of set-theoretic operations on smooth surfaces that are defined implicitly with algebraic equations. Special focus was paid to provide meshes that allow optimal rates of convergence in the $p$-FEM, even for $C^{1}$ continuous edges. By defining the final geometry via multiple smooth implicit surfaces, the detection of non-smooth surfaces features (such as corners and edges) is significantly simplified: In detail, the presented method starts with an octree decomposition of the embedding space for root isolation. Then, by root-finding, corners and points on intersection curves are found, which are then traced to arrive at a polygonized version of the edge. Smooth surfaces are first being discretized using marching cubes, then reduced to the faces part of the final surface and then finally, together with the edge mesh, connected using the advancing front method. The result is a linear surface mesh that is lifted by projecting element nodes onto the surface. Different variants for the surface meshing procedure are studied. Numerical examples show optimal rates of convergence for boundary value problems on different geometries.

The presented work can serve as basis for volume meshing of implicit geometries where the constructed higher-order surface meshes as described herein are used as a starting point. It may also be extended to incorporate variational methods to smooth surface meshes. 


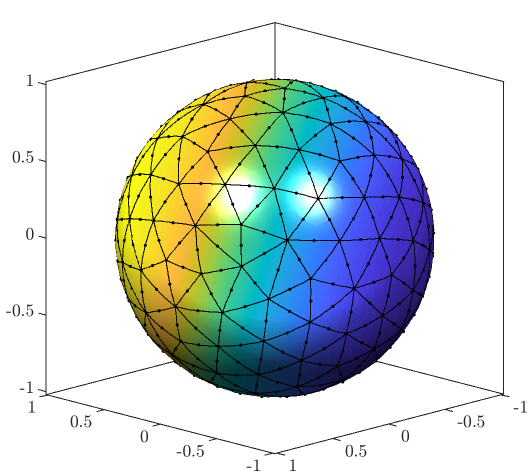

(a) $G_{2}$

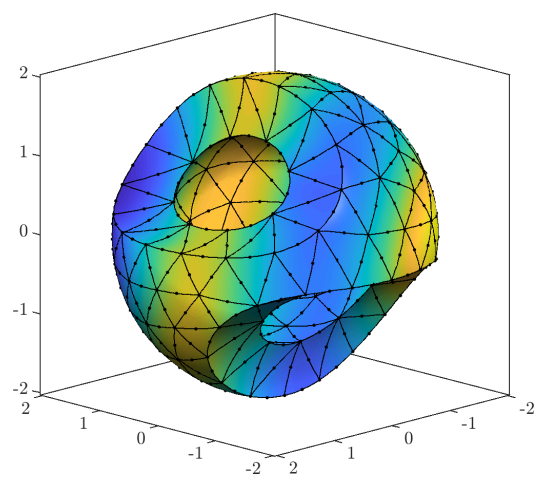

(b) $G_{3}$

Figure 5: Coarsest meshes and solution to the Laplace-Beltrami problem (with $p=2$ ) used in studies. 

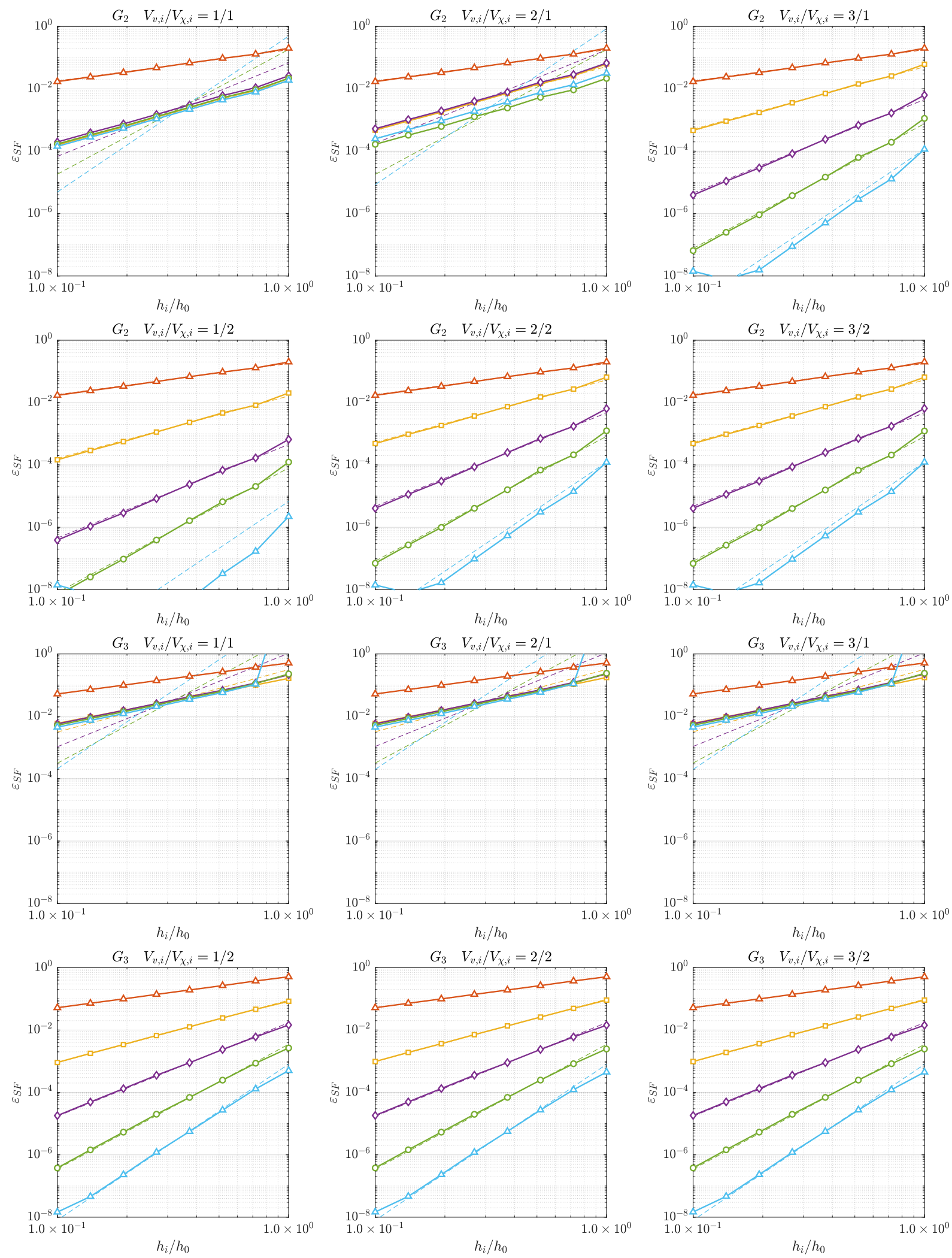

Figure 6: Residual error $\varepsilon_{S F}$ for all surface lifting variants on both geometries. 

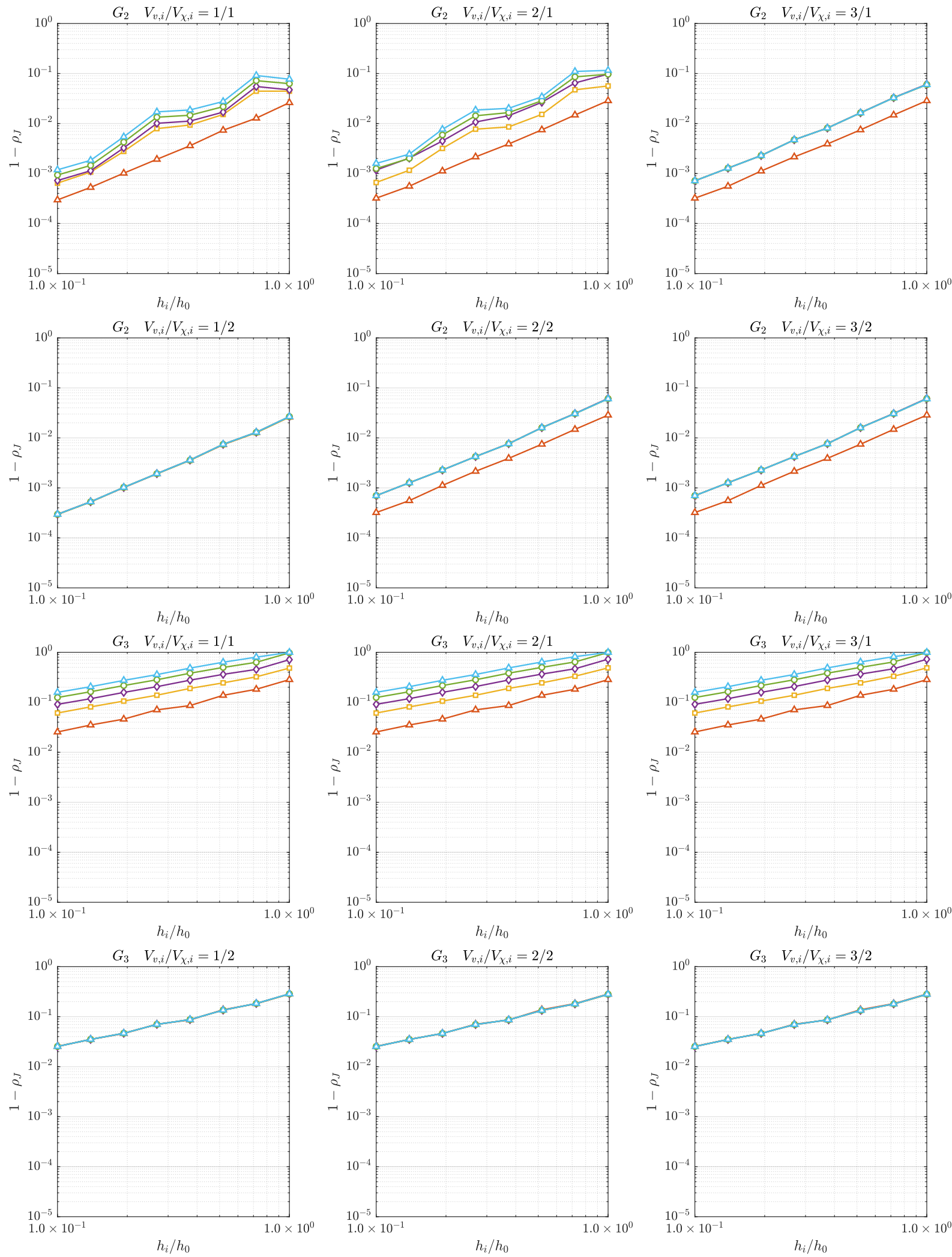

Figure 7: Jacobian ratios $\rho_{J}$ for all surface lifting variants on both geometries. 


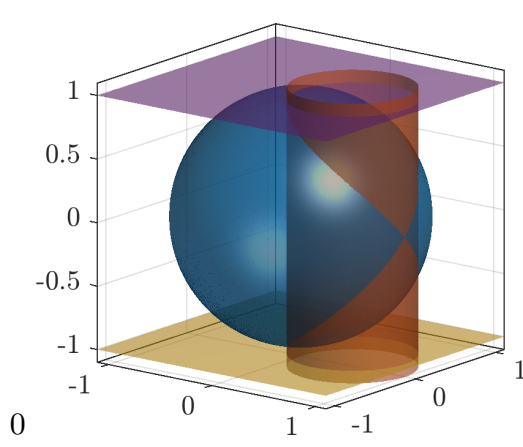

(a) $G_{18}$

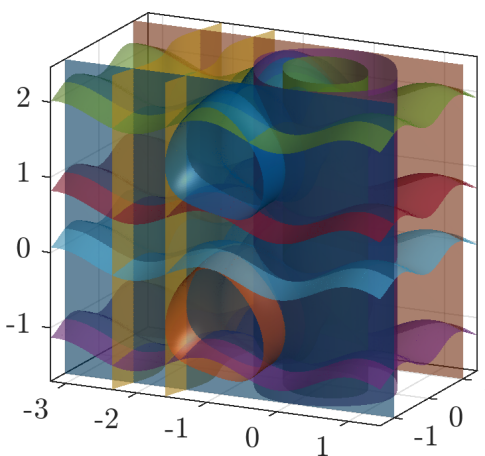

(b) $G_{24}$

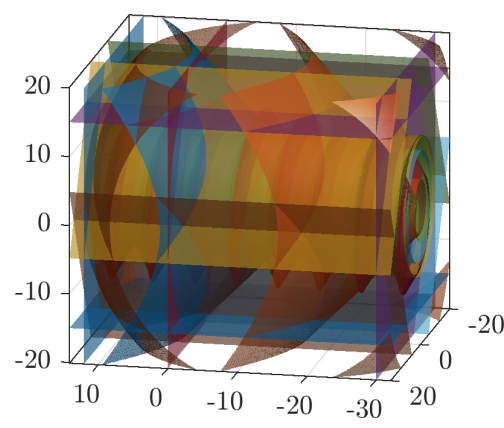

(c) $G_{31}$

Figure 8: Input Geometries for the problem considered in Sec. 5.3.

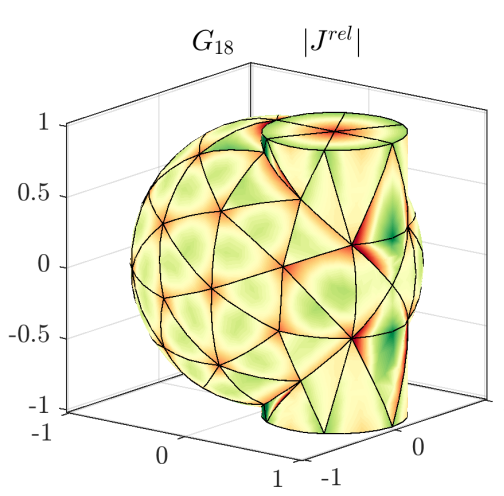

(a)

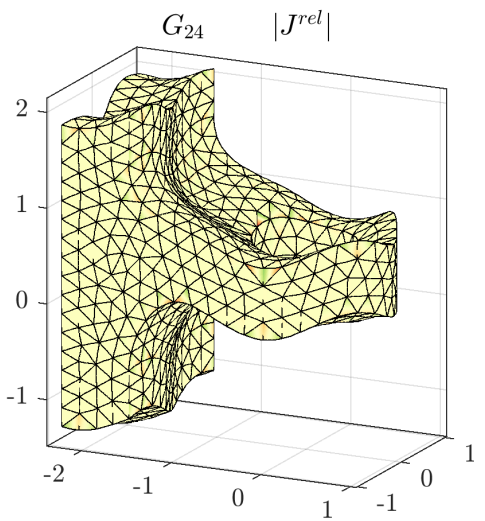

(b)

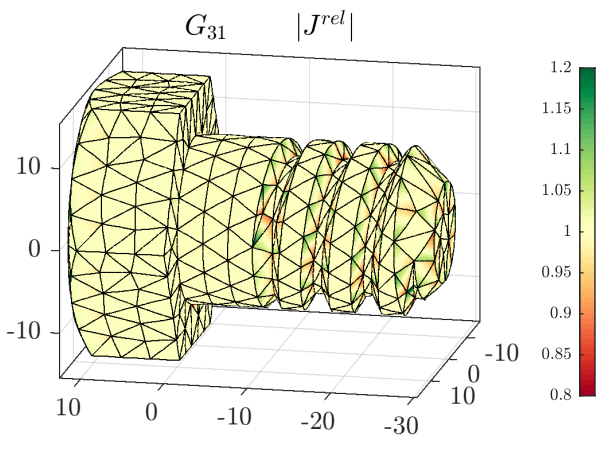

(c)

Figure 9: Approximated solution, residual error and scaled Jacobian for coarsest mesh with $p=6$ for the problem considered in Sec. 5.3.

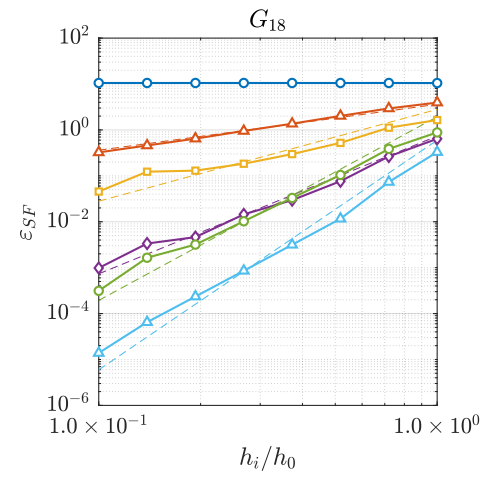

(a) Condition Number $\kappa$

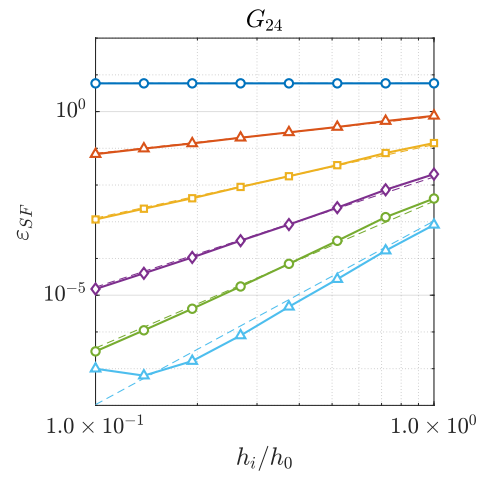

(b) Residual Error $\varepsilon_{S F}$

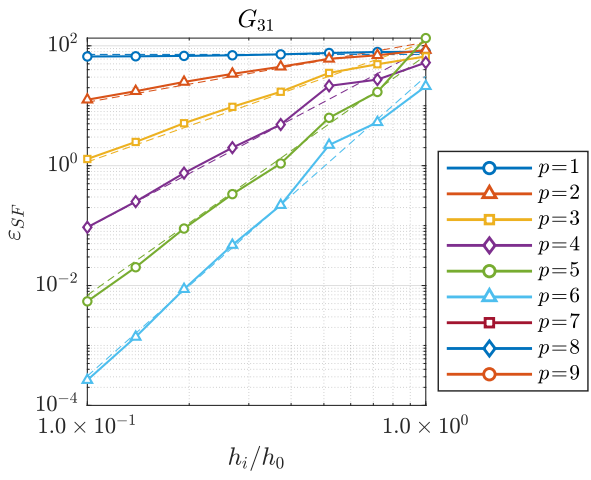

(c) Jacobian Ratio $\rho_{J}$

Figure 10: Results for the convergence study considered in Sec. 5.3. 


\section{References}

[1] Dey S., Shephard M.S., Flaherty J.E. "Geometry representation issues associated with p-version finite element computations." Comp. Methods Appl. Mech. Engrg., vol. 150, no. 1, 39-55, 1997

[2] Dey S., O’bara R.M., Shephard M.S. "Curvilinear Mesh Generation in 3D." Proceedings of the 8th International Meshing Roundtable. 1999

[3] Solin P., Segeth K., Dolezel I. Higher-Order Finite Element Methods (Studies in Advanced Mathematics). CRC Press, Boca Raton, FL, 2003

[4] Botti L. "Influence of Reference-to-Physical Frame Mappings on Approximation Properties of Discontinuous Piecewise Polynomial Spaces." Journal of Scientific Computing, vol. 52, no. 3, 675-703, 2011

[5] Requicha A.A. "Representations of rigid solid objects." Computer Aided Design Modelling, Systems Engineering, CAD-Systems, pp. 1-78. Springer, Berlin, 1980

[6] Stanford J., Fries T. "A higher-order conformal decomposition finite element method for plane Brep geometries." Comp. \& Struct., 2018

[7] Stanford J., Fries T. "Higher-order accurate meshing of implicitly defined tangential and transversal intersection curves." Lect. Notes in Comp. Sci. and Engrg. Springer, 2019

[8] Sherwin S.J., Peiró J. "Mesh generation in curvilinear domains using high-order elements." Internat. J. Numer. Methods Engrg., vol. 53, no. 1, 207-223, 2002

[9] Frey P., George P.L. Mesh Generation: Application to Finite Elements. John Wiley \& Sons, Chichester, 2008
[10] Lo D.S. Finite Element Mesh Generation. CRC Press, Boca Raton, FL, 2014

[11] Gargallo-Peiró A., Roca X., Sarrate J. "A surface mesh smoothing and untangling method independent of the CAD parameterization." Comput. Mech., vol. 53, no. 4, 587-609, 2013

[12] Geuzaine C., Remacle J.F. "Gmsh: A 3-D finite element mesh generator with built-in preand post-processing facilities." Internat. J. $\mathrm{Nu}$ mer. Methods Engrg., vol. 79, no. 11, 1309-1331, 2009

[13] Ruiz-Gironés E., Sarrate J., Roca X. "Generation of Curved High-order Meshes with Optimal Quality and Geometric Accuracy." Procedia Engineering, vol. 163, 315-327, 2016

[14] Roca X., Gargallo-Peiró A., Sarrate J. "Defining quality measures for high-order planar triangles and curved mesh generation." Proceedings of the 20th International Meshing Roundtable. 2011

[15] Sevilla R., Fernández-Méndez S., Huerta A. "NURBS-enhanced finite element method (NEFEM)." Internat. J. Numer. Methods Engrg., vol. 76 , no. 1, 56-83, 2008

[16] Gomes A. Implicit curves and surfaces : mathematics, data structures and algorithms. Springer, Dordrecht New York, 2009

[17] Lorensen W.E., Cline H.E. "Marching cubes: A high resolution $3 \mathrm{D}$ surface construction algorithm." ACM SIGGRAPH Computer Graphics, vol. 21 , no. 4, 163-169, 1987

[18] Hartmann E. "A marching method for the triangulation of surfaces." The Visual Computer, vol. 14 , no. 3, 95-108, 1998

[19] Lo S. "A new mesh generation scheme for arbitrary planar domains." International Journal for Numerical Methods in Engineering, vol. 21, no. 8, 1403-1426, 1985

[20] Löhner R., Parikh P. "Generation of threedimensional unstructured grids by the advancingfront method." Int. J. Numer. Methods Fluids, vol. 8 , no. $10,1135-1149,1988$

[21] de Araújo B.R., Lopes D.S., Jepp P., Jorge J.A., Wyvill B. "A Survey on Implicit Surface Polygonization." ACM Comput. Surv., vol. 47, no. 4, 60:1-60:39, 2015

[22] Belokrys-Fedotov A.I., Garanzha V.A., Kudryavtseva L.N. "Generation of Delaunay meshes in implicit domains with edge sharpening." Comput. Math. Math. Phys., vol. 56, no. 11, 1901-1918, 2016 
[23] Ohtake Y., Belyaev A.G. "Dual/primal mesh optimization for polygonized implicit surfaces." Proceedings of the seventh ACM symposium on Solid modeling and applications, pp. 171-178. ACM, 2002

[24] Patrikalakis N.M. "Interrogation of Surface Intersections." Geometry Processing for Design and Manufacturing, pp. 161-185. Society for Industrial and Applied Mathematics, 1992

[25] Patrikalakis N. "Surface-to-surface intersections." IEEE Computer Graphics and Applications, vol. 13, no. 1, 89-95, 1993

[26] Abhyankar S.S., Bajaj C.J. "Automatic parameterization of rational curves and surfaces IV: algebraic space curves." ACM Transactions on Graphics, vol. 8, no. 4, 325-334, 1989

[27] Asteasu C. "Intersection of arbitrary surfaces." Computer-Aided Des., vol. 20, no. 9, 533-538, 1988

[28] Bajaj C.L., Hoffmann C.M., Lynch R.E., Hopcroft J.E.H. "Tracing surface intersections." Comput. Aided Geom. Des., vol. 5, no. 4, 285307, 1988

[29] Düldül B.U., Düldül M. "Can we find Willmorelike method for the tangential intersection problems?" J. Comput. Appl. Math., vol. 302, 301311,2016

[30] Turner M. High-Order Mesh Generation For CFD Solvers. Ph.D. thesis, 2017

[31] Fries T., Omerović S. "Higher-order accurate integration of implicit geometries." Internat. J. $\mathrm{Nu}$ mer. Methods Engrg., vol. 106, no. 5, 323-371, 2016

[32] Fries T.P., Omerović S., Schöllhammer D., Steidl J. "Higher-order meshing of implicit geometries - Part I: Integration and interpolation in cut elements." Comp. Methods Appl. Mech. Engrg., vol. 313, 759-784, 2017

[33] Fries T., Schöllhammer D. "Higher-order meshing of implicit geometries, Part II: Approximations on manifolds." Comp. Methods Appl. Mech. Engrg., vol. 326, 270-297, 2017

[34] Fries T.P. "Higher-order conformal decomposition FEM (CDFEM)." Comp. Methods Appl. Mech. Engrg., vol. 328, 75-98, 2018

[35] Pasko A., Adzhiev V., Sourin A., Savchenko V. "Function representation in geometric modeling: concepts, implementation and applications." The Visual Computer, vol. 11, no. 8, 429-446, 1995
[36] Hu C.Y., Maekawa T., Patrikalakis N.M., Ye X. "Robust interval algorithm for surface intersections." Computer-Aided Design, vol. 29, no. 9, 617-627, 1997

[37] Barzilai J., Borwein J.M. "Two-Point Step Size Gradient Methods." IMA J. Numer. Anal., vol. 8, no. 1, 141-148, 1988

[38] Nakahashi K., Sharov D. "Direct surface triangulation using the advancing front method." AIAA paper, pp. 95-1686, 1995

[39] Lan T., Lo S. "Finite element mesh generation over analytical curved surfaces." Computers $\&$ Structures, vol. 59, no. 2, 301-309, 1996

[40] Persson P.O., Strang G. "A Simple Mesh Generator in MATLAB." SIAM Review, vol. 46, no. 2, 329-345, 2004

[41] Gordon W.J., Hall C.A. "Construction of curvilinear co-ordinate systems and applications to mesh generation." Internat. J. Numer. Methods Engrg., vol. 7, no. 4, 461-477, 1973

[42] Gordon W.J., Hall C.A. "Transfinite element methods: Blending-function interpolation over arbitrary curved element domains." Numerische Mathematik, vol. 21, no. 2, 109-129, 1973

[43] Schöberl J. "NETGEN An advancing front 2D/3D-mesh generator based on abstract rules." Computing and Visualization in Science, vol. 1, no. 1, 41-52, 1997

[44] Burman E., Hansbo P., Larson M.G., Massing A. "Cut Finite Element Methods for Partial Differential Equations on Embedded Manifolds of Arbitrary Codimensions." ESAIM: Math. Model Numer. Anal., 2018

[45] Dziuk G. "Finite elements for the Beltrami operator on arbitrary surfaces." S. Hildebrandt, R. Leis, editors, Partial Differential Equations and Calculus of Variations, vol. 1357 of Lecture Notes in Mathematics, pp. 142-155. Springer, Berlin, 1988

[46] Patrikalakis N.M., Maekawa T. Shape Interrogation for Computer Aided Design and Manufacturing. Springer, Berlin, 2002

[47] Knupp P.M. "Winslow Smoothing on TwoDimensional Unstructured Meshes." Engineering with Computers, vol. 15, no. 3, 263-268, 1999

[48] Fortunato M., Persson P.O. "High-order unstructured curved mesh generation using the Winslow equations." J. Comput. Phys., vol. 307, 1-14, 2016 


\title{
AUTOMATIC 2D ABSTRACTION AND HEXAHEDRAL MESHING BY SORTING A DELAUNAY MESH
}

\author{
Reza Taghavi \\ SIW Corp., Saint Paul, MN 55114 USA. taghavi@siw.com
}

\begin{abstract}
A framework is presented for the automatic 2D abstraction, decomposition and block-structured hexahedral meshing of a volume defined by a closed triangular mesh. No proofs, necessary or sufficient conditions are provided. Instead, constructive definitions and instructions describe a procedure for automatically building a 2D abstraction and an all-hexahedral mesh. First, a constrained Delaunay tetrahedralization of the interior of the object is built and its tetras are partitioned into groups based on their internal edge and face counts. Through labeling, organized structures are observed where 64-tetras (6 internal edges and 4 internal faces) bookend stacks of 54-tetras (5 internal edges and 4 internal faces). Stacks of 54-tetras, connected four-to-each 64-tetra, form a network of primary prism that populate the entire object near and along its edges. The exposed edges of the primary prism form rails that partition the input mesh into two categories of patches. Source and target patch pairs that define extrudable sub-volumes, and edge patches that define secondary prism. Degeneracies are remedied through affine transformations and an efficient local mesh manipulation process. The mid-mesh of the extrusion pairs is then computed and extended in order to obtain a 2D manifold that is a far simpler, albeit incomplete, 2D abstraction of the object than a customary mid-surface/medial axis representation. The result is a partition of the volume into extrudable, prismatic and tetrahedral blocks that trivially leads to a single block-structured hexahedral mesh.
\end{abstract}

Keywords: Block-decomposition, 2D abstraction, mesh generation, tetrahedra, hexahedra, Delaunay, extrusion

\section{INTRODUCTION}

Block-structured hexahedral meshes are of interest for both their geometrical and computational qualities. If available, a block-structured hexahedral mesh is often the preferred choice for non-linear plasticity and CFD analysis due to its ability, among other things, to ensure near- $90^{\circ}$ element corner angles while maintaining excellent orientation with respect to boundaries. Furthermore, in thin parts, commonplace in aerostructures, electronics and generative design, the density of hexahedral meshes are, in general, less affected by element aspect ratio than in the case of tetrahedral meshes. As a result, block-structured hex meshes of such parts may feature up to two orders of magnitude fewer elements than equivalent tetrahedral meshes, thus resulting in substantial computational gains.

\section{PREVIOUS WORK}

Attention to the special role played by 3-triangles (Delaunay triangles featuring 3 internal edges) and 64-tetras (Delaunay tetras featuring 6 internal edge and 4 internal faces) in identifying special morphologies in closed 2D and 3D regions was first reported in the context of approximating medial axes and mid-surfaces using meshes [1] [2] [3]. But while only in 2D may one approximate the "inside" mid-object of a closed loop as the locus of the circumcenters of its 3-and 2-triangles, in $3 \mathrm{D}$, the circumcenters of 64- and 54-tetras may not be used as an approximation of the mid-surface [4]. In his LayTracks3D approach to meshing general solid, [5] identifies entire prismatic blocks along the sharp edges of objects and calls them tracks and rails. He then goes on to create an allhexahedral mesh. We will use a similar concept in this work. 


\section{NOMENCLATURE AND CONSTRUCTION}

We present a method for automatically building a 2D abstraction of an object that is simpler than a midsurface/medial axis, with the final goal of generating a blockstructured hexahedral mesh. Our approach is based on the observation that a constrained Delaunay tetrahedralization of a closed and finely meshed triangular surface mesh, without the addition of any internal nodes, yields only a handful of a certain type of tetras that we call 64-tetras (meaning, 6 internal edges and 4 internal faces). We speculate that the number and placement of the 64-tetras is robust, invariant and characterizes the morphology of an object. Without providing any proof nor any necessary or sufficient conditions, we suggest that the exposed edges of the 54-tetras ( 5 internal edges and 4 internal faces) partition the triangular surface of the mesh into patch pairs delineating extrudable volumes. We then use the patch pairs and their bordering primary prisms to compute a $2 \mathrm{D}$ abstraction of the object that leads us to a final hexahedral block-structured mesh.

\subsection{Definition of 64- and 54-tetras}

Figure 1 shows a finely triangulated surface mesh representing a test object that we will use throughout this presentation. This object is obtained by boring two partially offset and partially intersecting cylinders through a brick.

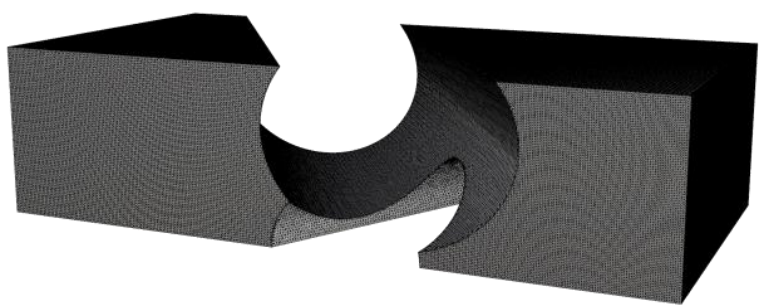

Figure 1: Surface mesh of test object

A fine triangulation of the test object followed by Delaunay tetrahedralization produces 236,242 tetras, only 15 of which have 6 internal edges and 4 internal faces and thus qualify as 64-tetras. For the sake of definition, in the context of a tetrahedral mesh, an internal face is defined as one that is shared by 2 tetras, and an internal edge is defined as one that borders only internal faces. We call such tetrahedra 64-tetras. 64-tetras play an important role in the present method. Figure 2 represents all the 64-tetras generated in our test. We speculate, without proof, that for a "fine enough" initial triangular mesh, the number and general placement of 64tetras remain unaffected by the level of refinement.

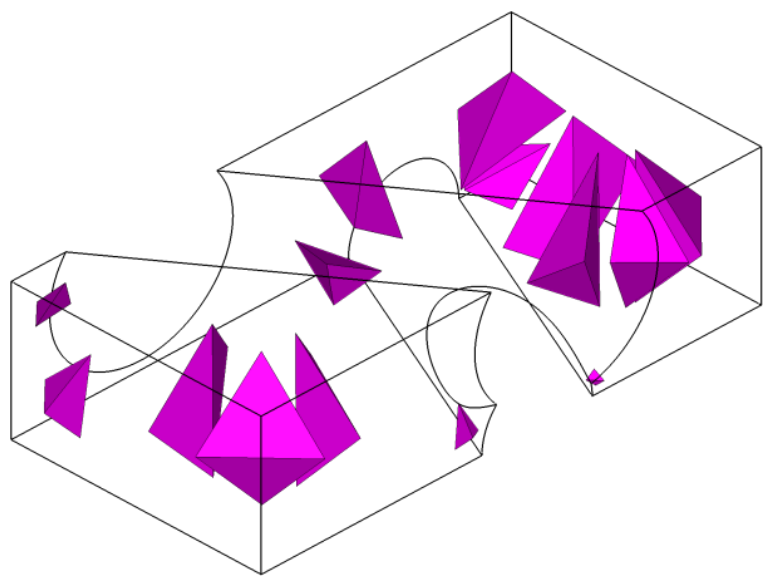

Figure 2: 64-tetras

A practical way of differentiating tetras is by labeling them with a decimal number where the tens represent the number of internal edges and the units represent the number of internal faces of that tetra. In this fashion, a 64 tetra will be labelled as 64 for its 6 internal edges and 4 internal faces. Let's now consider 54-tetras. In Figure 3 they appear organized as prismatic structures (stacks of tetrahedra) throughout the mesh. 54-tetras have 4 internal faces but only 5 internal edges, thus leaving one exposed edge on the surface. 54-tetras appear as stacks of tetras bookended by 64-tetras. In Figure 3, 64tetras are rendered as solids while 54-tetras are rendered as gray wire meshes.

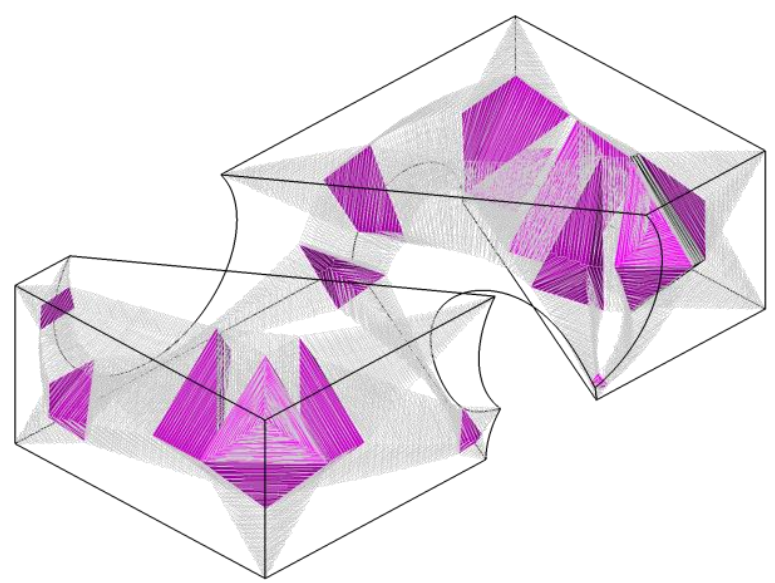

Figure 3: 64- and 54-tetras

\subsection{Primary prisms}

By sorting 54-tetras into individual stacks starting and ending at 64-tetras, in the present example, 38 individual sets are found, organized as jagged sets of connected tetras linking the triangular faces of pairs of opposite 64-tetras. We refer to these sets as primary prisms because they represent a volume homeomorphic to a prism. In Figure 4 different colors are assigned to each primary prism to help distinguish them from one another. Note that 64-tetras are not visible in this solid rendering as all 4 faces of 64-tetras are covered by primary prisms. 


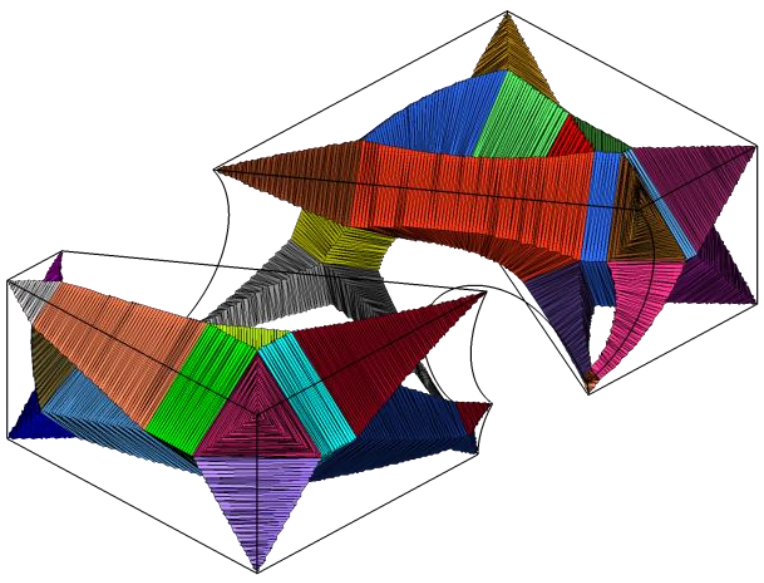

Figure 4: Colored primary prisms composed of connected 54-tetras linking opposite 64-tetras (not visible)

\subsection{Regular, loop and degenerate primary prisms}

Primary prisms are made of 54-tetras. In general, primary prisms originate and end at the face of a 64-tetra but there are exceptions: loop primary prisms and degenerate primary prisms.

1. Loop primary prisms which occur around holes are composed solely of 54-tetras. Figure 5 shows a closeup view of two loop primary prisms that occur in the Landing Gear example depicted in Figure 39.

2. Degenerate primary prisms originate at a 64-tetras but terminate at 34-tetras and are generally located near the corners of the object. Figure 6 shows a degenerate primary prism in the present example.

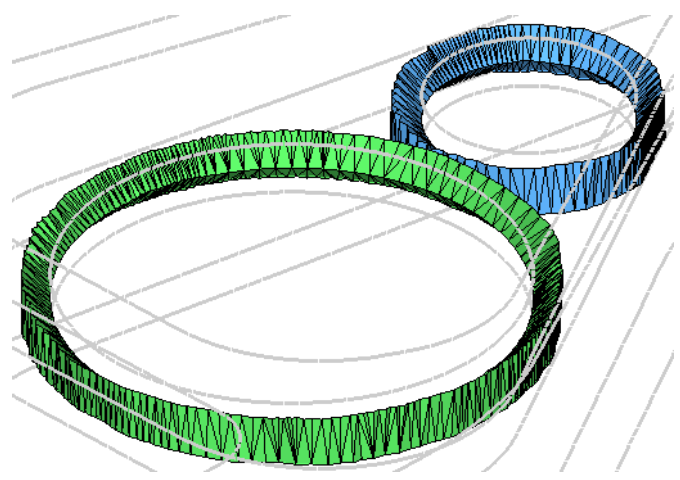

Figure 5: Two loop primary prisms produced in the Landing Gear example. Features of the model appear in gray
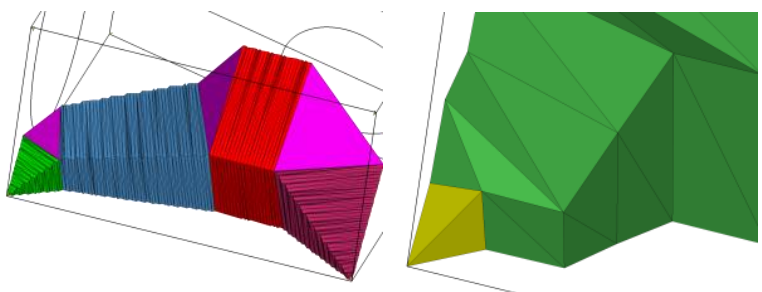

Figure 6: A degenerate primary prism (left) and its closeup view (right).

By definition, a 54-tetra possesses one exposed edge on the surface of the mesh. The exposed edges of the 54-tetras in a primary prism form 3 jagged curves on the surface mesh. We call these curves rails. Figure 7 shows one such prism and its 3 rails, as well as the pair of 64-tetras that bookend it.
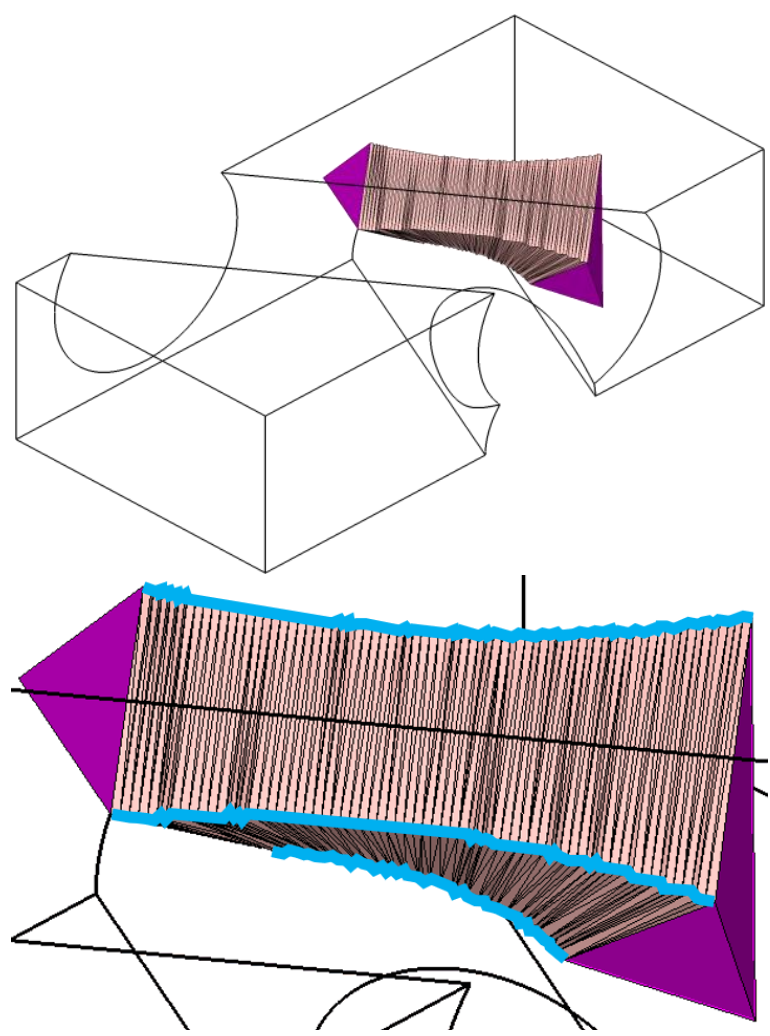

Figure 7: A single primary prism bookended by two 64-tetras (top) and its 3 rails highlighted in light blue (bottom)

\subsection{Partition of the surface into extrusion and edge patches}

Rails are connected sets of exposed 54-tetra edges. Again, without proof, we suggest that rails for closed loops on the surface mesh and as such partition the surface mesh into a number surface patches. In the present example, 40 patches are created as shown in Figure 8. 


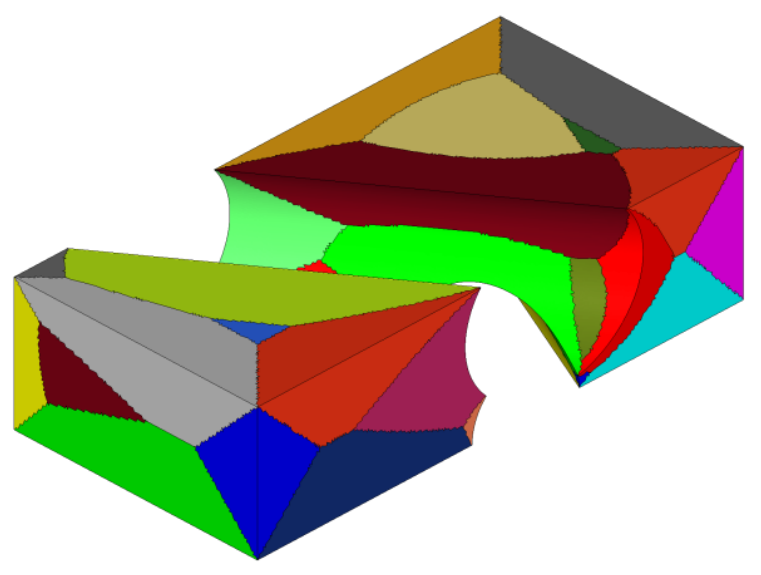

Figure 8: Surface patches resulting from the partition of the surface faces by rails

Surface patches may be further classified into two categories: extrusion patches and edge patches. Extrusion patches are formed when loops of primary prisms carve out two opposing patches from the surface mesh. Two such loops are shown in Figure 9: Two primary prism loops (top) and their associated extrusion patch pairs are shown in the bottom image.

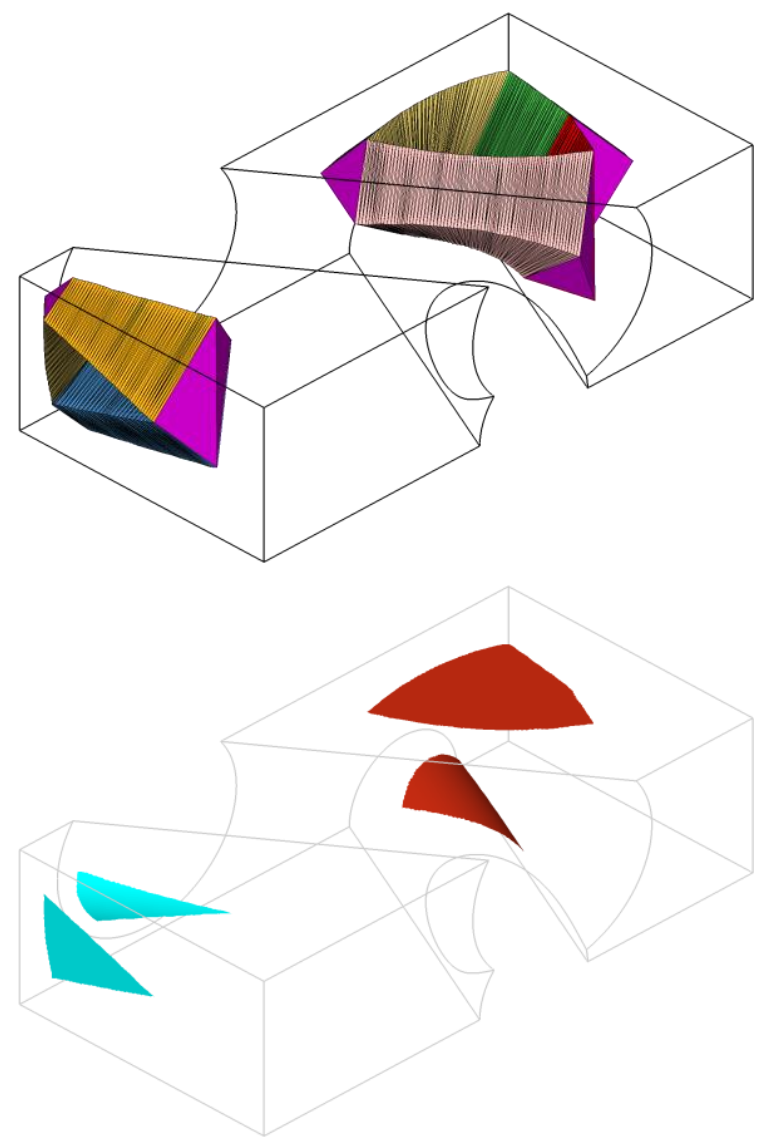

Figure 9: Two primary prism loops (top) and their associated extrusion patch pairs (bottom)
Extrusion patches always appear in pairs. Figure 10 shows the 7 pairs of extrusion patches produced in the present example. For the sake of clarity, patch pairs are colored identically.

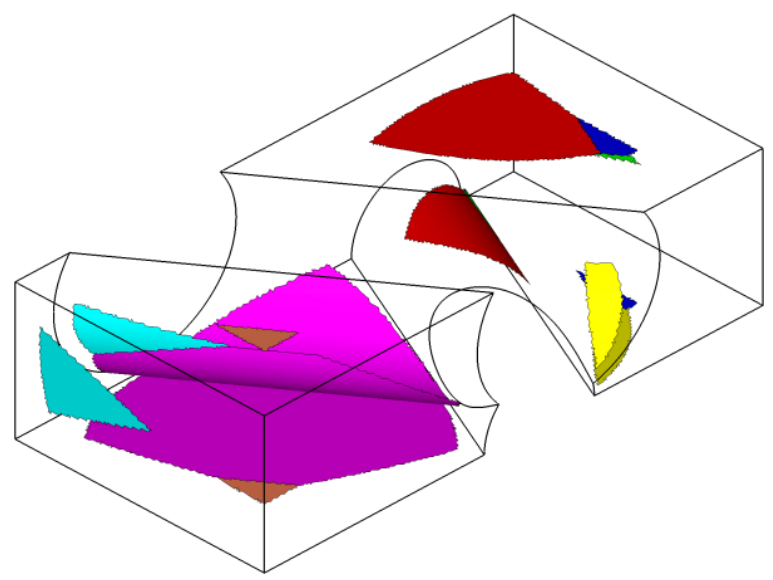

Figure 10: Extrusion patches

Extrusion patch pairs, along with the lateral walls of the primary prisms surrounding them carve out swaths of extrudable volumes from the object. Figure 11 shows the two earlier patch pairs and their associated sidewalls extracted from the primary prism loops that surround them.

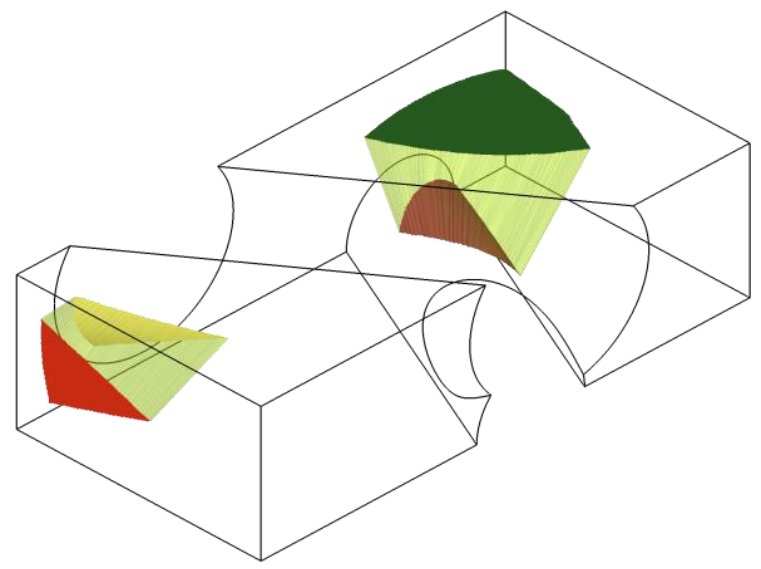

Figure 11: Two extrusion pairs and the sidewalls surrounding them

The remaining non-extrusion patches are called edge patches. Figure 12 shows the edge patches in the current example. Masked extrusion patches appear as holes. 


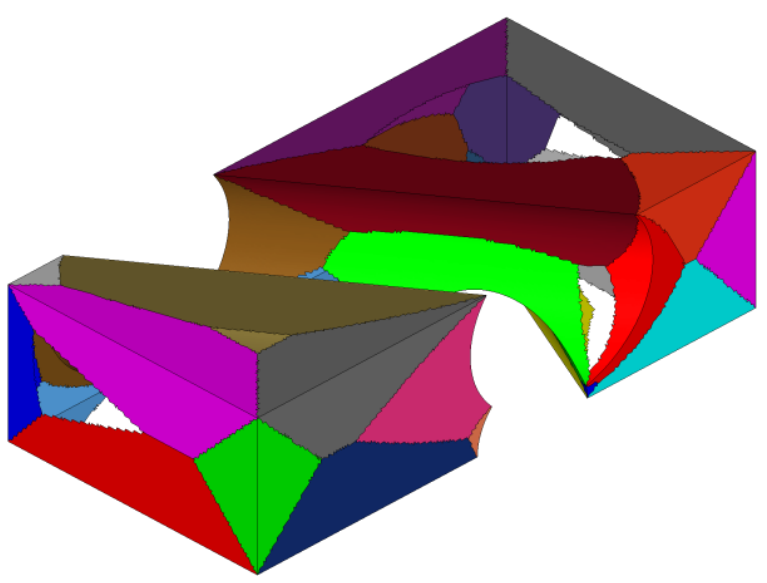

Figure 12: Edge patches

\subsection{Edge patches and secondary prisms}

Secondary prisms are a new type of prism enclosed by edge patches and the internal walls of one or more primary prisms. Remembering that an edge patch is one that is not an extrusion patch, the two edge patches (out of a total of 26) shown in Figure 13. The boundaries of these edge patches are composed of primary prism rails.

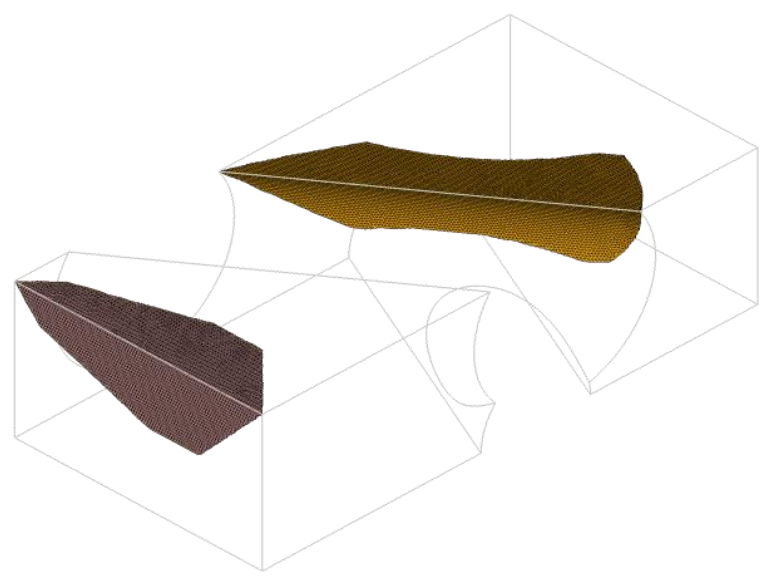

Figure 13: Two edge patches

Consider all primary prism that shares two rails with these two edge patches. These prisms are shown in Figure 14 where, for the sake of clarity, the edge patches are not shown .

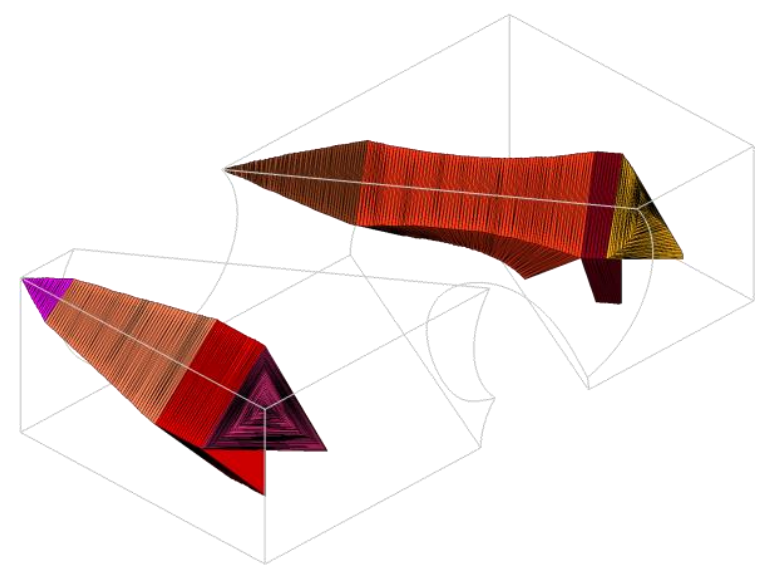

Figure 14: Primary prisms that share 2 rails with the two edge patches

Finally, consider the sidewalls of these primary prisms that face the edge patches which, along with the edge patches, form two closed volumes. these volumes are called secondary prisms and are shown in Figure 15 from a different angle for the sake of clarity. Like primary prisms, secondary prisms define precise volumes (blocks) within the object but unlike primary prisms, secondary prisms are not made of 54-tetras. Instead (without further development) they are mostly made of 33- and 44-tetras.

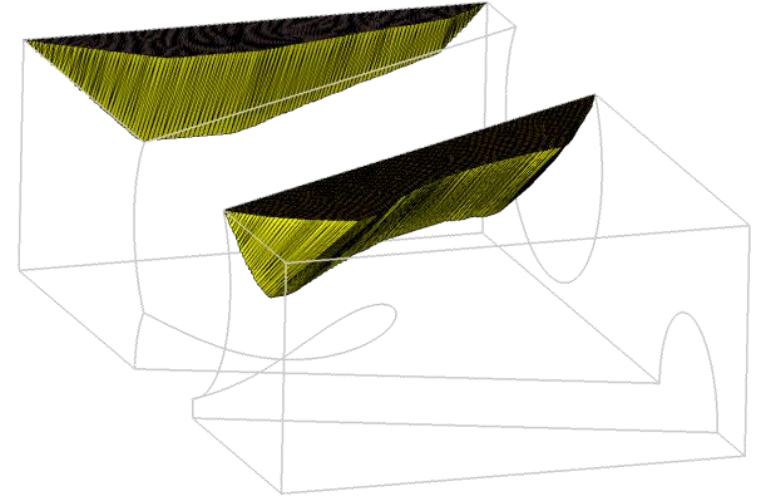

Figure 15: Edge patches and the primary prism sidewalls that define secondary prisms

\subsection{Calculation of the mid-mesh of extrusion patch pairs}

Without any proof, we assert that extrusion patch pairs are connected through 33- and 44-tetras only. 33-tetras have one exposed face and 3 internal edges. 44-tetras have no exposed faces but 2 exposed edges, in general opposed to one another in the tetra and belonging to opposite patches. There is no easy way to represent the 33-and 44-tetras that join each patch pair. Figure 16 shows the leftmost extrusion patch pair depicted earlier in Figure 10, with a section cut through the 33- and 44tetras that connect the patch pair. It can be noted that 33- (in blue) and 44-tetras (in yellow) form, by far, the majority of the tetras in a Delaunay mesh with no internal nodes obtained from a "fine enough" surface mesh. 


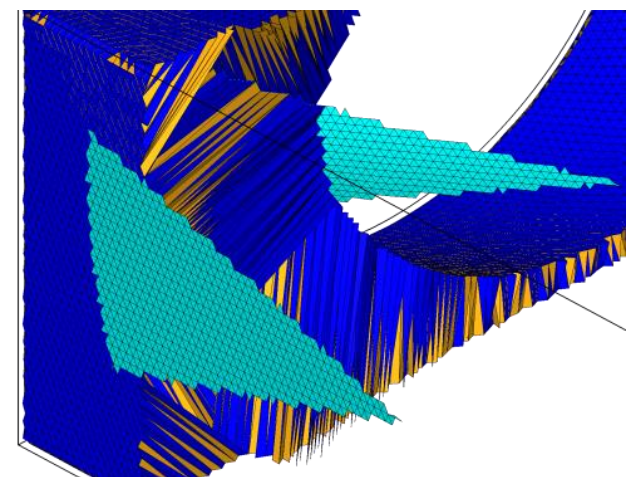

Figure 16: Cut through 33-and 44-tetras

The 33- and 44-tetras that connect the 2 patches can be easily identified as the set of 33- and 44-tetras that have either a surface face and its corresponding opposed node or, opposed edges, on each patch of the pair. Consequently, we can accurately construct the mid-mesh of such extrusion patch pairs. The process is described later in detail. Figure 17 represents a 2D abstraction of the example model composed of the 7 smoothed and "extended" (details below) mid-meshes of the 7 extrusion patch pairs depicted earlier in Figure 10.

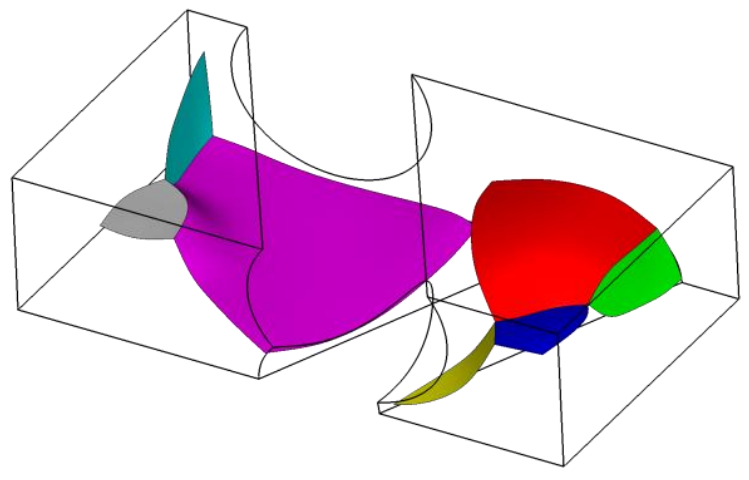

Figure 17: Smoothed 2D abstraction (extended midmeshes) of extrusion pairs

Smoothed 2D abstractions may now be re-meshed as quad meshes using any quad meshing meshed such as advancing front or paving (Figure 18).

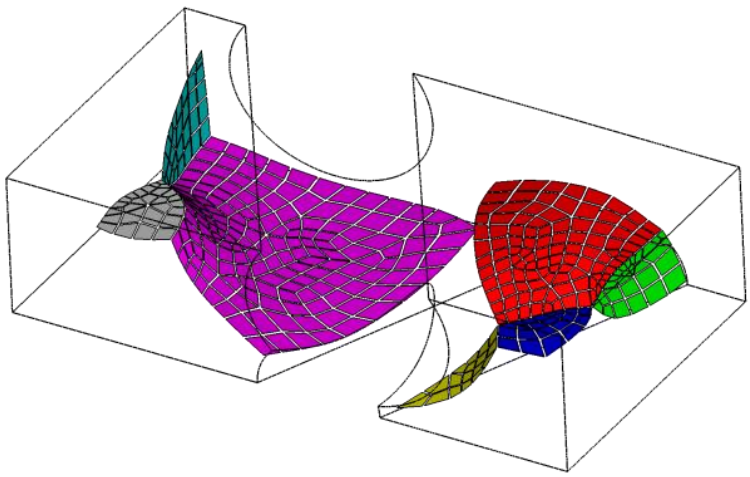

Figure 18: Quad-meshed mid-meshes

\section{HEX MESHING}

Without proof, we suggest that the volume of the object, and more specifically the tetras composing the Delaunay mesh, are partitioned into 4 sets:

\section{64-tetras}

2. Primary prisms, composed of 54- and a few 34 tetras

3. Extrudable sub-volumes, composed of 33- and 44tetras

4. Secondary prisms, composed 33- and 44-tetras almost exclusively

These sets are all primitives and as such can be treated as blocks and meshed and combined as a compatible allhexahedral mesh.

\subsection{Hex meshing of extrusions}

The vertices of quad-meshed $2 \mathrm{D}$ abstractions may be extruded along the internal edges of the 33- and 44-tetras that connect each patch pair. It can be noted that wherever two extruded mesh blocks come into contact, their grids are compatible by construction (detailed later) because their abstracted patches are adjacent. Figure 19 shows the hex meshes resulting from all 7 extrusions and subdivided in 2 along the extrusion. 


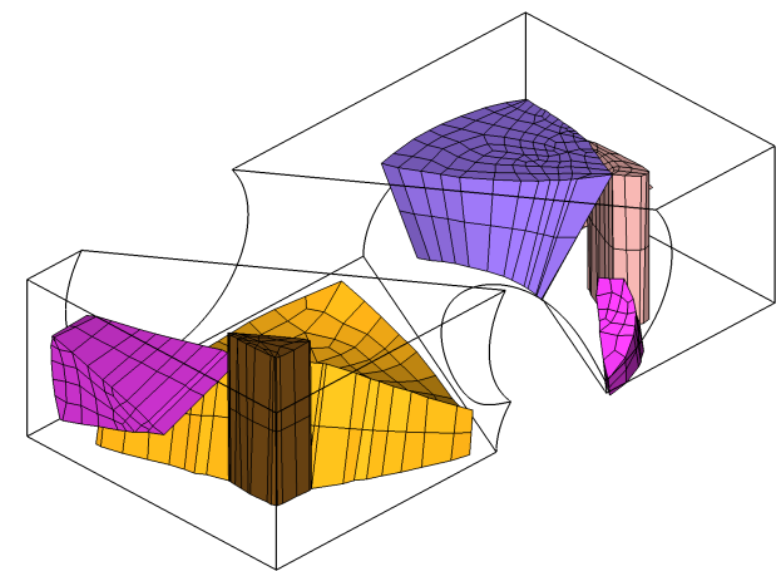

Figure 19: Extruded mid-mesh quads

\subsection{Hex Meshing of primary prism blocks and blocks defined by 64-tetras (64-blocks)}

The blocks defined by primary prisms, referred to hereafter as primary prism blocks are meshed as hexes by first subdividing them longitudinally, then azimuthally, by splitting their triangular cross-section into 3 quads. The boundary nodes of the quad meshed abstractions dictate the slicing locations of the primary prism blocks in their longitudinal direction thus ensuring that the extruded mesh and the hex-meshed prism blocks share compatible nodes. 64-tetras and degenerate primary prism blocks, which are also homeomorphic to tetras, are tetrahedral blocks and are split each into 4 hexas each (Figure 20).

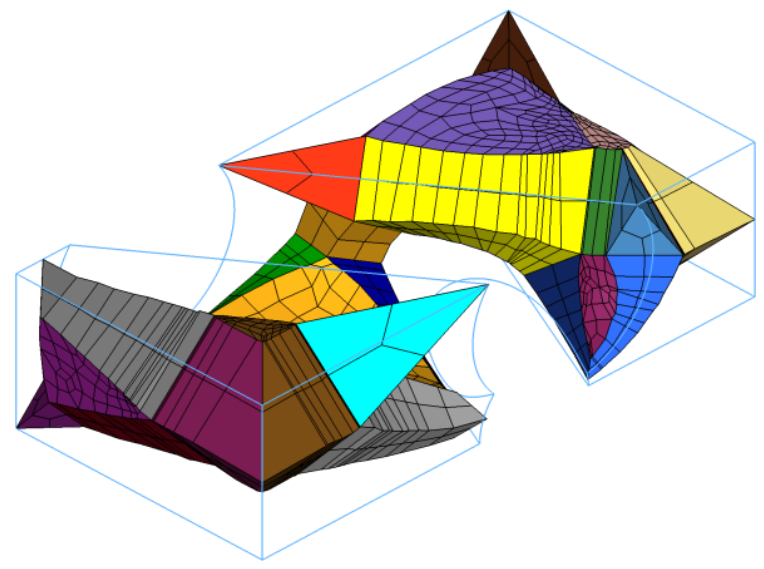

Figure 20: Hex-meshed extrusion, primary prisms and 64-blocks

\subsection{Hex meshing of secondary prism blocks}

Secondary prisms are defined by edge patches and the sidewalls of primary prisms. Therefore, the blocks they represented can be subdivided following the longitudinal subdivision of the primary prism blocks whose sidewall they share. Figure 21 shows the completed mesh composed of the extrusions, split in 2 in the direction of the extrusion, matching the primary and secondary prisms split into hexas and the 64tetras also split into 4 hexas.

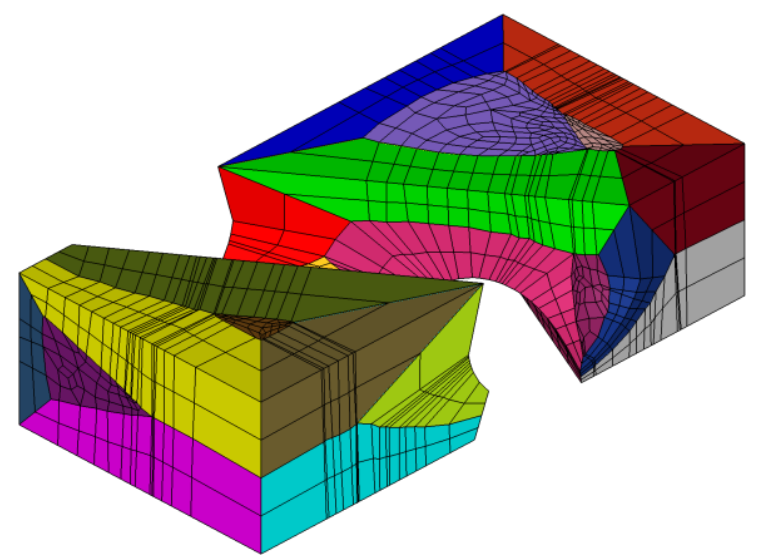

Figure 21: Final lock-structured hexahedral mesh

\section{DETAILS}

The details of the construction of the $2 \mathrm{D}$ abstraction which ensures the compatibility of the extruded meshes, the data structure needed to maintain a link between the tetra and hexa meshes and other fine points are addressed in this section.

\subsection{Construction of a $2 D$ abstraction that ensures the compatibility of the extruded hex meshes}

Extrusion source and target patch pairs connect 33- and 44tetras. For each pair, the mid-mesh can be constructed by assembling the individual 2D elements obtained when a 33tetra is split with a plane across its 3 internal edges (producing a triangle) and a 44-tetra is split across its 4 internal edges (producing a quadrilateral). For the sake of example, assuming that the source patch is at the bottom and the target patch on top, Figure 22 illustrates how 33- and 44-tetras contribute to the mid-mesh. The contribution of a 33-tetra whose exposed face belongs to the target patch (blue triangle shown in A), the contribution of a 33-tetra whose exposed face belongs to the source patch (blue triangle shown in B), and the contribution of a 44-tetra with one exposed edge on the source and another on the target patch (blue quadrilateral shown in C) are shown. 


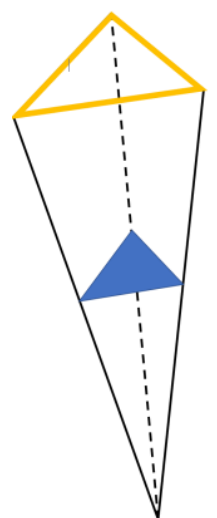

(A)

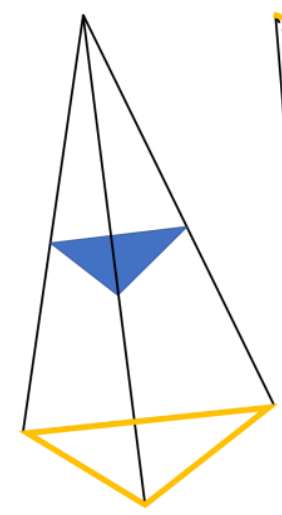

( B )

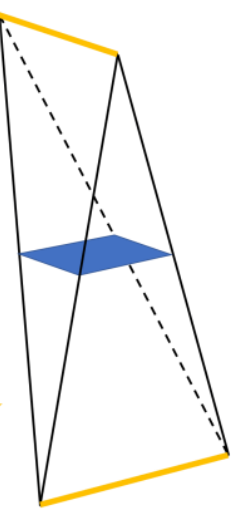

(C)
Figure 22: Contributions of 33-and 44-tetras to the mid-mesh. Surface edges are highlighted in thick gold color.

By assembling the individual triangles and quads (in turn, split into triangles along their shortest diagonal) the mid-mesh of any patch pair is built. A closeup of the mid-mesh can be seen in Figure 23 as the fine jagged light pink mesh where, for the sake of visibility, one of the primary prisms bounding the extrusion is shown as a wire mesh and a 64-tetra ending it is rendered in solid. It can be noted that the boundary of the midsurface stops short of reaching the center of the prism and stops at the mid-points of the edges of the 54-tetras that form the primary prism.

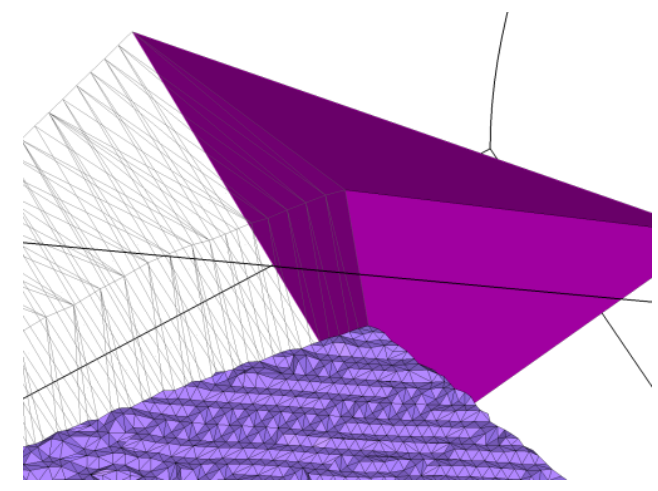

Figure 23: Closeup view of the partially constructed mid-mesh

At this stage, the set of mid-meshes would resemble what is depicted in Figure 24, i.e. a collection of isolated and unconnected surface meshes. Smoothing and quad-meshing these separate mid-meshes would result in a series of independent quad mesh patches which, once extruded, would result in a set of hex meshes that would not have any common nodes, unlike what was shown earlier in Figure 19, incompatible. Earlier, in Figure 17, a set of smoothed 2D abstractions were shown that did feature quad mesh compatibility (Figure 18) and compatibility of the resulting hex meshes

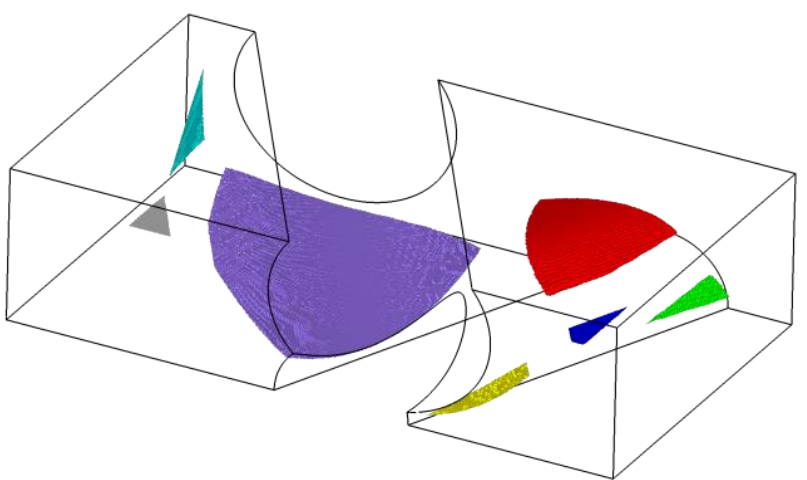

Figure 24: Incomplete mid-meshes

To ensure compatibility of the hex meshes resulting from extrusion, we introduce what we call a 2D abstraction by expanding each individual mid-mesh and augmenting them, peripherally, all the way to the center of the primary prisms and 64-tetras that surround them. By adding additional triangles, patches will meet at the center of primary prisms and 64-tetras. The added triangles are visible in Figure 25 where, for the sake of improved visibility, the corner 64-tetra is rendered transparent and one of the surrounding primary prisms is rendered as a wire mesh. The added triangles, visible as elongated light pink triangles, are constructed between the boundary vertices of the mid-patches and additional vertices placed at the center of faces (of 54-tetras) inside the primary prisms. In addition, two large triangles are also visible (resulting from two mid-face vertices and the center vertex of the 64-tetra) which complete the expansion of the mid-meshes into a 2D abstraction. After smoothing, the 2D abstraction, shown earlier in Figure 17, is ready for quad-meshing.

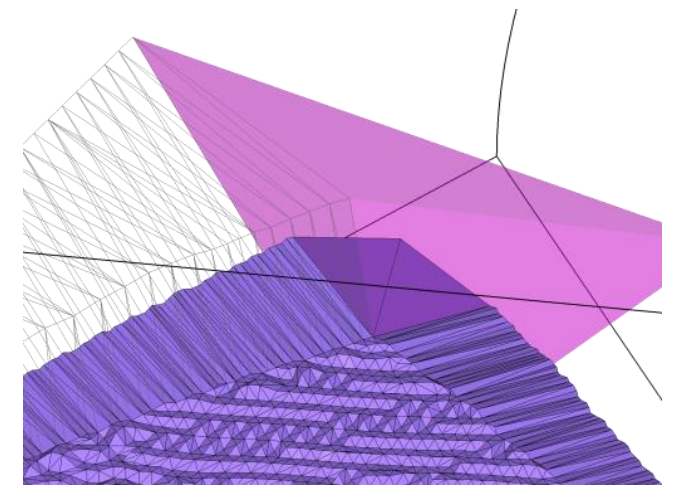

Figure 25: Completed unsmoothed extended midmesh (2D abstraction)

\subsection{Data structure and sequence of operations}

An internal data structure guarantees a two-way connection between the nodes of the initial Delaunay tetra mesh and the nodes of the final hex mesh as the Delaunay mesh is partitioned and the hex mesh is created through the following stages:

1. Initial closed triangular mesh 
2. Constrained Delaunay tetrahedral mesh

3. Primary prisms and their rails

4. Secondary prisms

5. Patch pairs resulting from the partitioning of the surface mesh by the rails

6. Mid-patches computed from the patch pairs and extended to the centerline of prisms to form $2 \mathrm{D}$ abstractions

7. Smoothing and quadrilateral meshing of 2D abstractions and their extrusion into hexahedral mesh blocks

8. Meshing by slicing of primary prism blocks along marks dictated by the quad mesh boundary nodes

9. Meshing by slicing of secondary prism blocks along slices dictated by the slices of the primary prism blocks

10. Meshing of 64-tetras

\subsection{Meshing of primary prism blocks}

Each node of the quad-meshed 2D abstraction points to a node of the triangular 2D abstraction from which it stems. This is enforced by the quad meshing software component utilized in this work, namely, MeshGems-SurfOpt, developed by DISTENE [6]. In turn, each node of the triangular 2D abstraction is, by construction, the mid-point of a 33- or 44tetra internal edge which connects one node of the source to one node of the target patch. Therefore, along the boundary of the quad mesh, each node also sits at the center of an internal face of a 54-tetra belonging to a primary prism bordering the extrusion.

Every boundary node of the quad mesh, shown in Figure 26, is represented by a black dot. By construction, each border node is at the center of an internal face of the primary prism that borders it. Therefore, each border node defines a slice of the primary prism block.

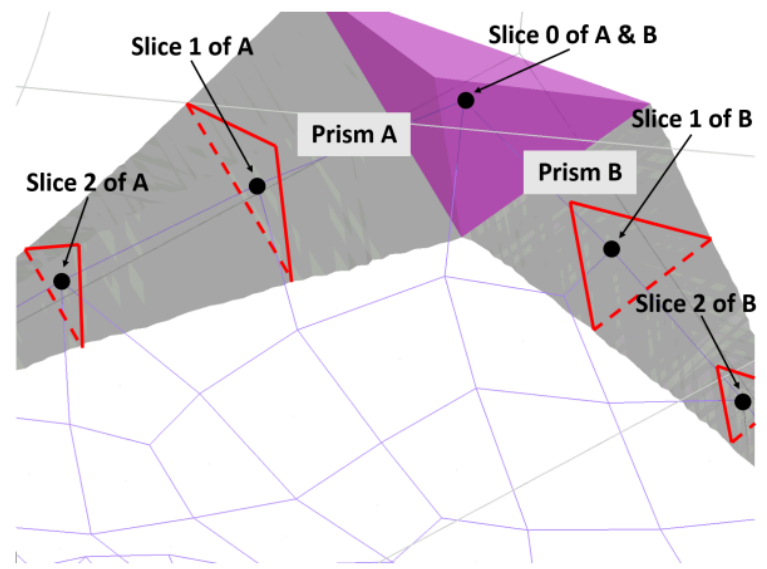

Figure 26: Quad border vertices define slices in bordering prism blocks
Primary prism blocks may now be sliced accordingly to create prism elements compatible with the extruded hexahedral blocks. In Figure 19, the extruded hex meshes have been split in 2 along the direction of the extrusion and the primary prism block slices have been split into 3 hexas each, and 64-tetra blocks and degenerate primary prisms blocks have been split into 4 hexas each.

\subsection{Meshing of secondary prism blocks}

Secondary prisms are bound by edge patches and flanked by primary prims. As with primary prism blocks, they can be split into prism elements and subsequently split into 3 hexas along the same slices as the primary prisms. Degenerate secondary prism blocks are treated as 64-tetras and meshed into 4 hexas accordingly.

\subsection{Ducts and cables for handling non- manifold 2D abstractions}

Sometimes, multiple primary prisms may be bunched together where multiple extrusions converge from different directions. Figure 27 shows such an example where two prisms, shown in light blue and gold, share a wall and act as one. In such cases, the data structure is further extended to include two new categories. Ducts, which are composed of multiple primary prisms sharing 2 rails, and cables which are a set of rails sharing all their edges.
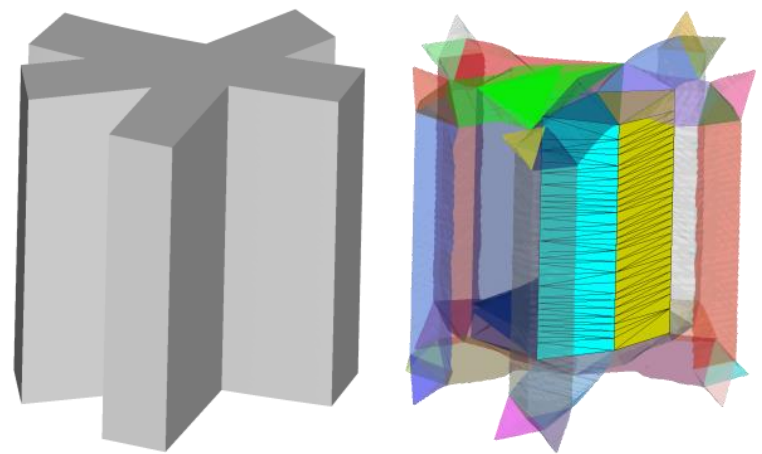

Figure 27: Bunching of two prisms into a duct. Solid model (left) and highlighted duct composed of 2 prisms colored light blue and gold

In the presence of a duct, during the process of building 2D abstractions, the mid-meshes are extended all the way to the centerline of ducts instead of the centerline of individual prisms. This ensure node compatibility among all the extrusions converging at the duct.

\subsection{Saddle Points and 144-tetras}

At geometry saddle-points, a different type of 44-tetras may be created where the exposed segments of the tetra are adjacent instead of being opposed, as encountered earlier. We call these 144-tetras. These define a new category of primary prism that start at 64-tetras and terminate at 144-tetras. We refer to these primary prism as knife primary prism. 


\subsection{Computation of geodesics on the Mesh}

In many instances, including the meshing of secondary prism blocks it may be necessary to compute distances and geodesics along the edges at the surface of the initial surface mesh. To this end Dijkstra's algorithm is used as implemented by [7].

\subsection{Smoothing prior to quad meshing}

By construction, a 2D abstraction is generally very noisy as seen in Figure 25. Therefore, smoothing is necessary prior to quad-meshing of the 2D abstraction in order to improve signalto-noise ratio and avoid the creation of tiny quads. A shrinkproof smoothing of the 2D abstraction and its boundary is used [8]. After smoothing, MeshGems-Surfopt [6], which incorporates its own mesh smoothing, is applied to obtain a quad mesh suitable for extrusion.

\section{DEGENERACIES}

Several types of degeneracies occur in this procedure. They are addressed by a series of local mesh manipulations, including edge collapse, edge split, edge flips [9], fast data structure updates and topology recalculation. The iterative resolution of these degeneracies uses a considerable portion of the computational work before the network of 64-tetra and primary prisms is finalized and 2D abstraction can proceed. These degeneracies are:

1. Symmetry degeneracies. These are Delaunay degeneracies which occur when a Delaunay mesh is created from an input triangular mesh that features local symmetries

2. Degeneracies resulting in 1- and 12-tetra proliferation. These degeneracies are related to symmetry degeneracies and occurs in bulky parts along convex curved surfaces.

3. Overlapping and folding rails which may also result in captive isolated faces between rails.

4. Convex curvature degeneracies which are also related to symmetry degeneracies.

5. Occurrence of convex sharp edges adjacent to more than one tetra or adjacent to a tetra other than a 12-tetra.

6. Formation of very small prisms made up of only a handful of 54-tetras or prisms with one or two zero-length rails.

7. Formation of entire captive patches. These occur when either te source or target patch in an extrusion pair is absent.

8. Occurrence of fused segments between otherwise distinct rails.

\subsection{Symmetry degeneracies}

The most common type of degeneracy is, referred to hereafter symmetry degeneracy, is one in which the surface mesh of an object featuring local symmetries is tetrahedralized using
Delaunay. Due to the occurrence of multiple equidistant options for the formation of a Delaunay tetra, neighboring tetras may form in a seemingly chaotic fashion. For instance, the 4 vertical connector columns in the electronic component shown in Figure 28 degenerate into fragmented and chaotically oriented primary prisms.

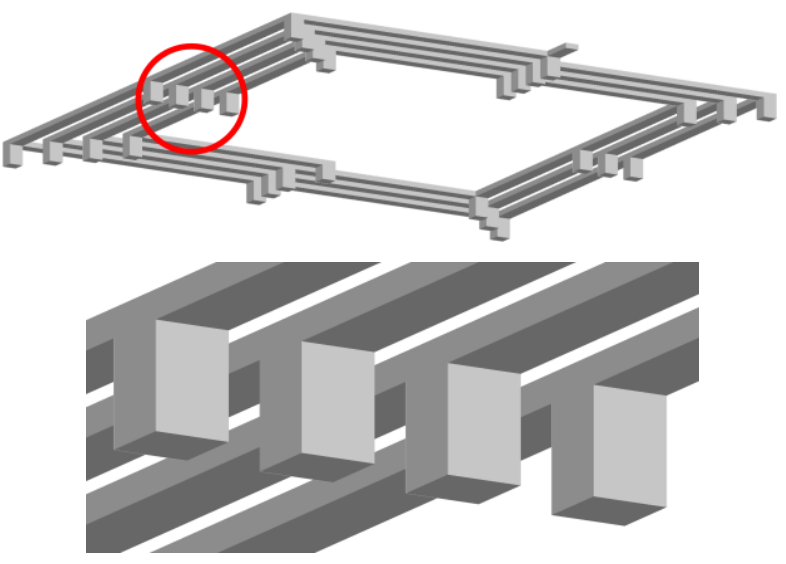

Figure 28: Circuit board (top) and detail (bottom) where Delaunay degeneracy occurs

Without special treatment, degeneracy leads to fragmented primary prisms in the vertical connectors (shown as stacks of small multicolor primary prisms in the columns, in Figure 29, top). An ad-hoc symmetry-breaking affine transformation $\Phi$ is applied to all the vertices of the surface mesh prior to Delaunay meshing. After the hex mesh is built, the reverse transform, $\Phi^{-1}$, is applied to the vertices of the hex mesh in order to recover the true geometry. Here, we use a simple transform that stretches the model by a factor 1.1 in the $\mathrm{x}-$, 1.2 in the $y$ - and 1.3 in the z-direction. The degeneracy is removed and single primary prisms are ensured in the vertical columns of the model as shown in Figure 29, bottom.

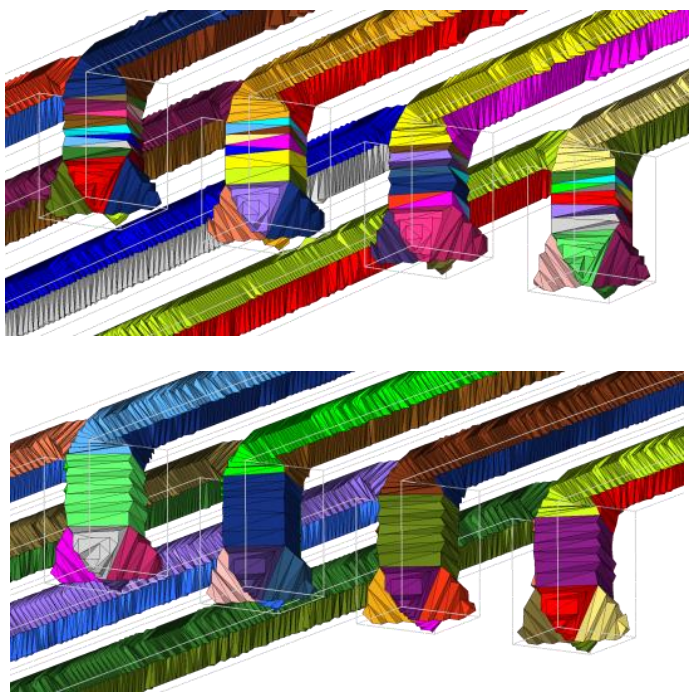

Figure 29: Fragmentation of prisms without affine transform (top). After removal of degeneracy through transform (bottom) 
The Delaunay degeneracy may occur in any object. Therefore, the affine transformation is systematically applied as part of the general algorithm as shown in the pseudocode further below.

\subsection{Degeneracies resulting in 1- and 12- tetra proliferation}

The proliferation of 1- and 12-tetras are resolved by the systematic removal of such tetras. Figure 30, top, shows a cut constant-velocity boot (automotive, CV boot) which features large extents of slightly convex curved surfaces. Delaunay tetrahedralization results in flat 12-tetras, appearing in red, which, if not removed, would result in the creation of ghost primary prisms and the subsequent failure of the procedure.
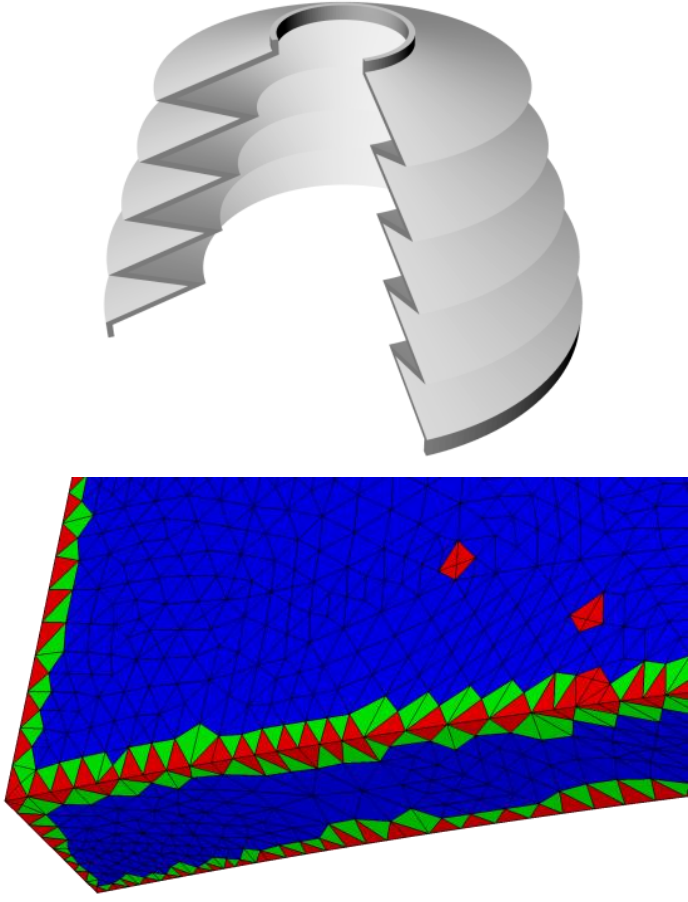

Figure 30: A cut CV-boot (top) results in spurious flat 1- and 12-tetras (in red, bottom) which are removed. Non-flat 12-tetras remain along edges

\subsection{Overlapping and folding rail degeneracies}

The coarser the input triangular mesh, the more likely it is that rails may overlap, especially in the vicinity of their starting nodes which are 64-tetra corner nodes. Figure 31 shows two such overlapping rails found in the CV boot example shown earlier. The 3 overlapping edges of the rails, shown with the 4 node marks, are collapsed in order to remove this degeneracy.
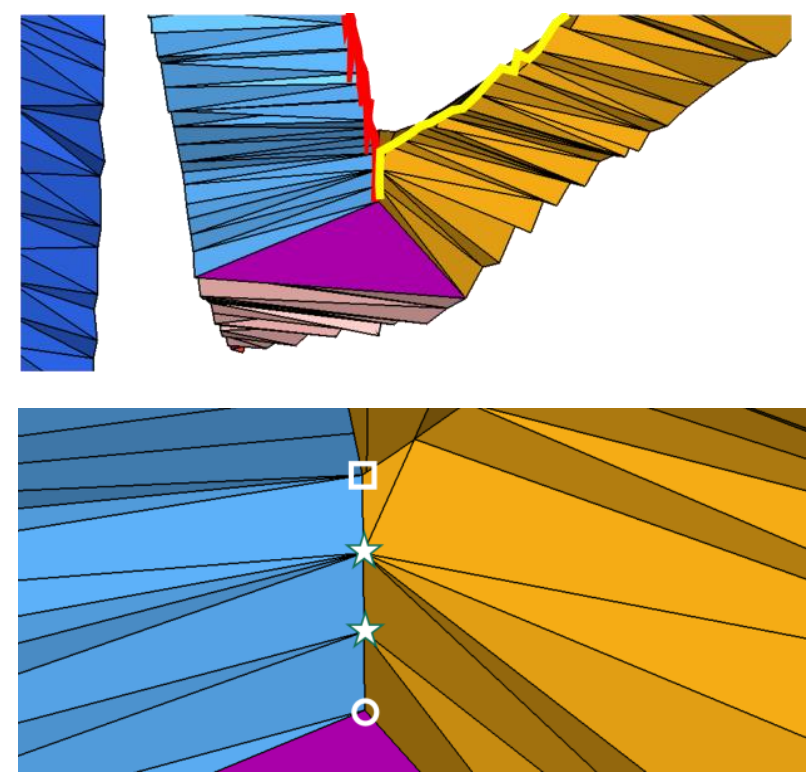

Figure 31: Overlapping rails shown in red and yellow in the top figure. The bottom figure shows how the rails must separate at the circle mark instead of the square.

\section{4. "Peel rails or discard captive faces" degeneracies}

Figure 32 shows how two neighboring primary prisms may share nodes (tagged as white circles) resulting in overlapping rails and/or the creation of captive faces. If a captive face (shown as a transparent yellow triangle) is removed through edge collapse, two separate and valid extrusions are created on the left and right side of the overlapping nodes. But if the overlapping rails are eliminated through a combination of edge split and edge collapse one single extrusion is created that surrounds the two loop primary prisms surrounding the 2 holes of the model. The latter is the solution that is automatically retained based on aspect ratio considerations, and can be seen in the Examples section.

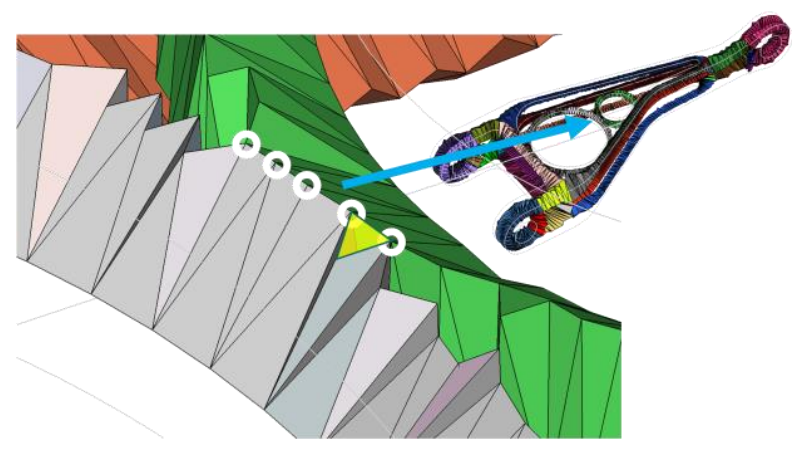

Figure 32: Rail peel and captive face degeneracy 


\subsection{Convex curvature degeneracies}

Figure 33 shows a type of degeneracy caused by noisy convex and featureless surfaces. They also result in a proliferation and concentration of 1- and 12-tetras in the curved areas. Their systematic removal would leave behind an intractable primary prism structure that would result in the failure of the procedure.

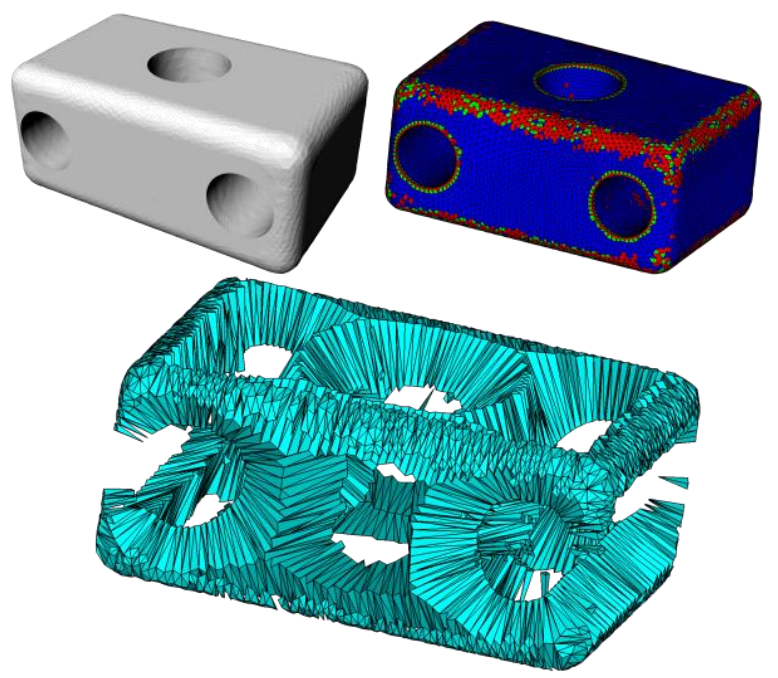

Figure 33: Convex curvature degeneracy

\section{AUTOMATION}

The following pseudocode (algorithm) is performed on a closed triangulated surface mesh.

\section{Read Input surface mesh}

2. Process surface mesh

2.1. Improve mesh sizing

2.2. Identify feature angles

3. Affine transform $\Phi$ of the input surface

4. Build 2D Abstraction of input as a quad mesh

4.1. Build Delaunay tetrahedral mesh

4.2. Iterative removal of other (than symmetry) degeneracies

4.2.1. Discard Isolated 01- and 12-tetras that have "flat" dihedral angles

4.2.2. Ensure 12-tetras at convex features (flip)

4.2.3. Identify and label tetras as $64,54,33,44$, etc.

4.2.4. Identify primary prisms and their rails

4.2.5. Correct Folding rails (collapse)

4.2.6. Discard captive faces between rails (collapse)

4.2.7. Discard "tiny" prisms (collapse)

4.2.8. Move 64-tetra corners to closest concave node (collapse)

4.2.9. Partition surface mesh into patches

4.2.10. Identify extrusion patch pairs
4.2.11. Remove captive patches when one of the 2 extrusion patches is missing (collapse)

4.2.12.Peel rails apart (edge splitting)

4.2.13. Identify ducts

4.2.14. Ensure that all prisms in a duct have the same number of 54-tetras (collapse)

4.3. Build mid-meshes of extrusion patch pair

4.4. Extend mid-meshes and obtain $2 \mathrm{D}$ abstraction

4.5. Smooth and quad mesh $2 \mathrm{D}$ abstraction

5. Build mixed hexa, prism and tetra mesh

5.1. Build extrusion blocks (hex elements)

5.2. Build 64-blocks (single tetra element)

5.3. Build degenerate primary prism blocks (single tetra element)

5.4. Build non-degenerate primary prism blocks (stacks of prism elements)

5.5. Build degenerate secondary prism blocks (single tetra element)

5.6. Build non-degenerate secondary prism blocks (stacks of prism elements)

6. Split mixed mesh to get all-hex mesh

6.1. Split tetras into 4 hexas

6.2. Split prisms into 3 hexas

6.3. Split extrusion hexas into 2 along the extrusion

\section{Inverse transform $\Phi^{-1}$ of hex mesh}

\section{EXAMPLES}

The following figures represent 2D abstractions and coarse hex meshes obtained for 5 examples. Once again, it can be noted that these 2D abstractions are simpler and contain fewer branches than mid-surface/medial axis abstractions of similar objects.

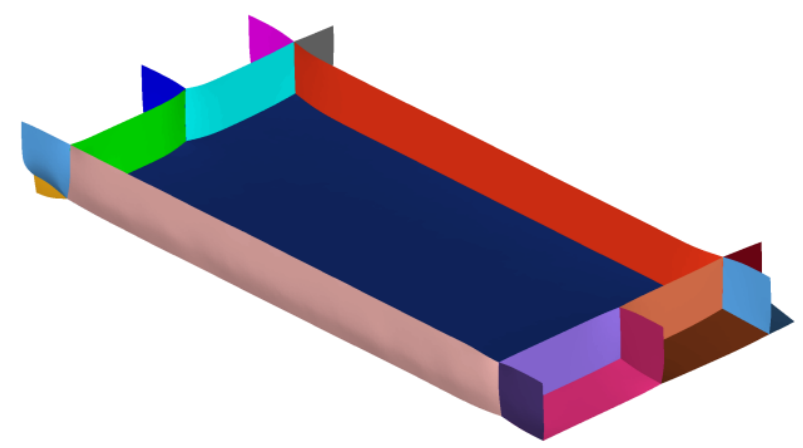

Figure 34: Heatsink 2D abstraction 


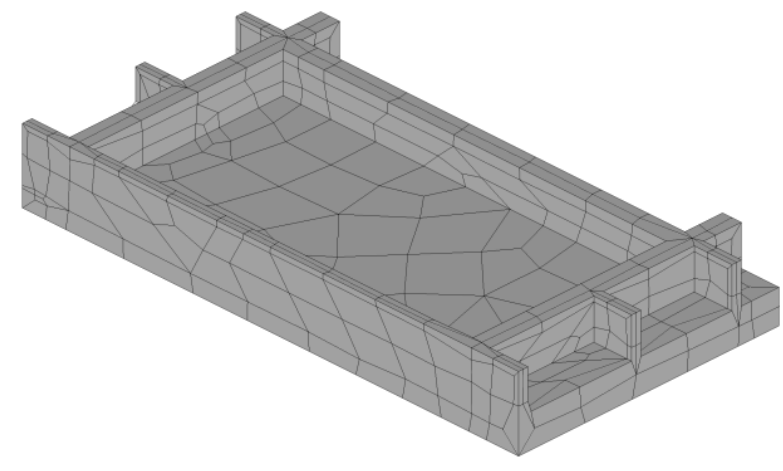

Figure 35: Heatsink hexa mesh

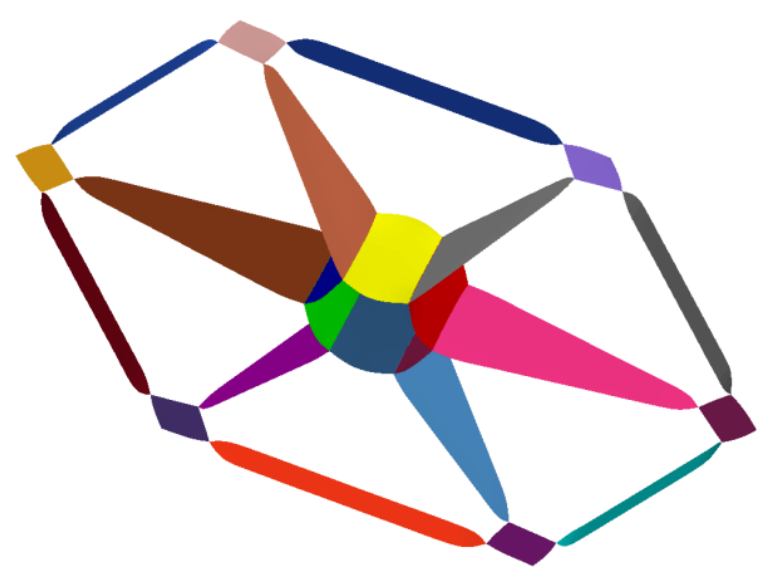

Figure 36: Flywheel 2D abstraction

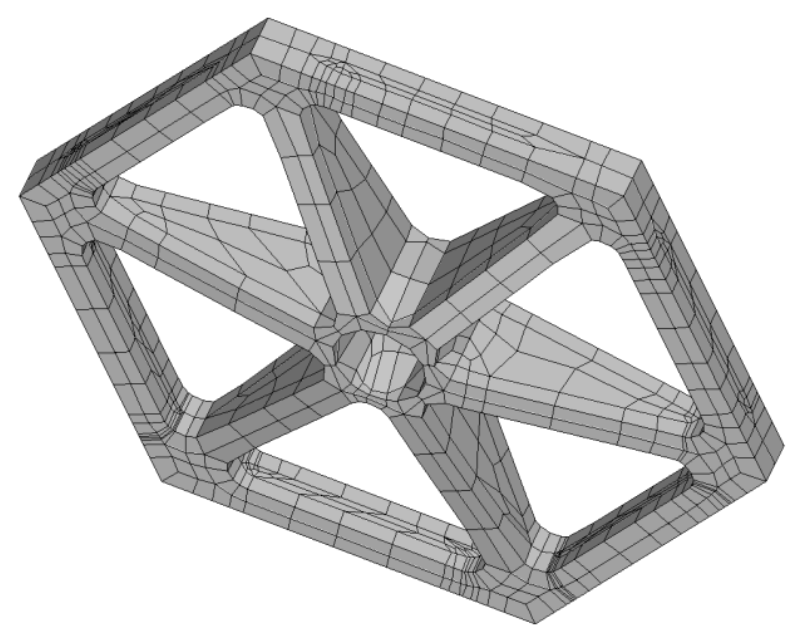

Figure 37: Flywheel hexa mesh

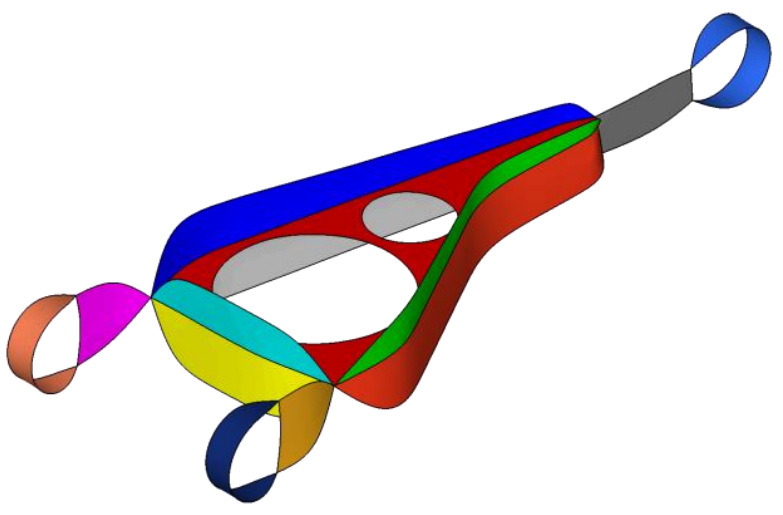

Figure 38: Landing gear 2D abstraction

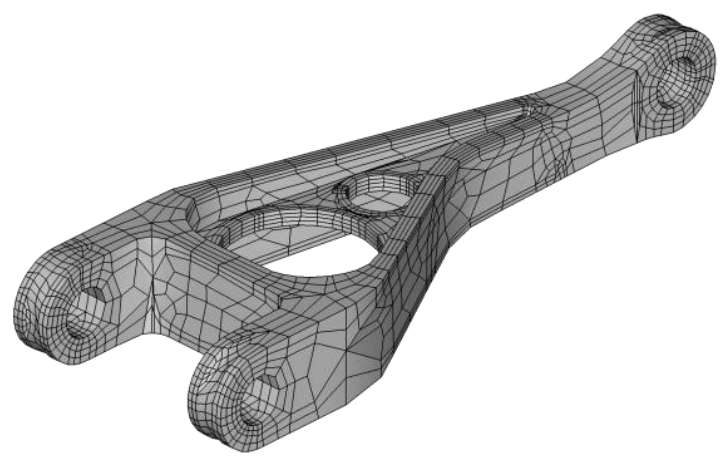

Figure 39: Landing gear hexa mesh

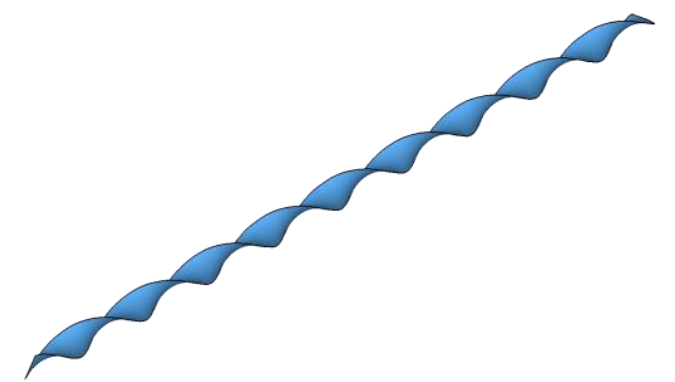

Figure 40: Drill bit 2D abstraction

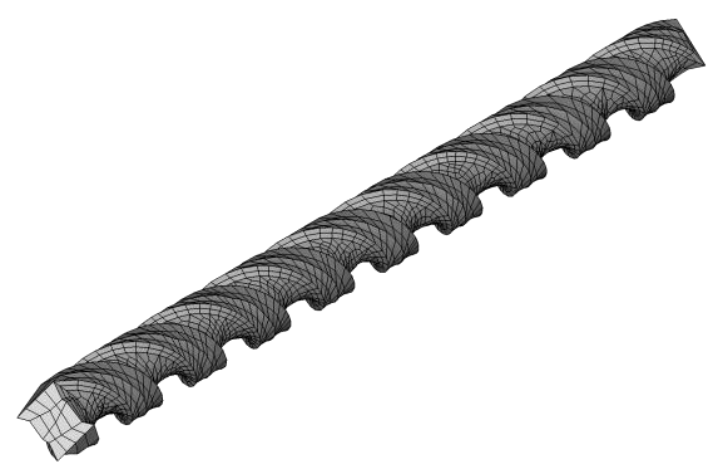

Figure 41: Drill bit hexa mesh 


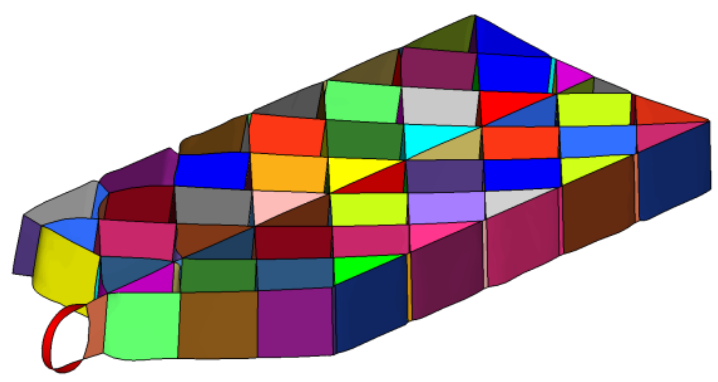

Figure 42: SpaceX Falcon-Heavy center booster half-gridfin 2D abstraction

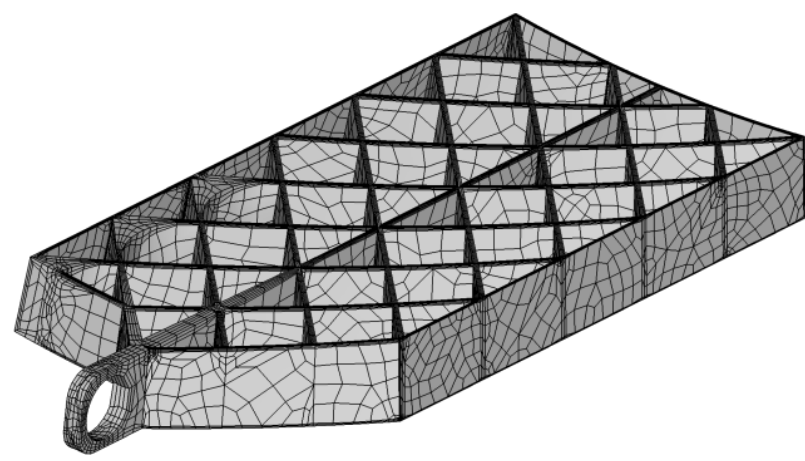

Figure 43: SpaceX Falcon-Heavy center booster half-gridfin hexa mesh

\section{CONCLUSION AND REMARKS}

An automatic hexahedral meshing framework is presented that uses the optimal properties of a Delaunay tetrahedralization to create a 2D abstraction and a block-structured hexahedral mesh. Several open questions remain including how to deal more effectively with various types of degeneracy. A few useful outcomes of this work are:

1. The proposed 2D abstraction is simpler, has fewer branches than a mid-surface/medial axis and, a-priori, requires no pruning. It essentially lets the branching be handled by the network of tetra and prism blocks. It could possibly replace semi-automatic abstraction tools used in today's CAD systems.

2. The block-structured hex mesh has the special property that the grid layering along extrusions and that along the prism blocks do not interfere and thus allow for the independent assignment of extrusion mesh density and mesh resolution across the extrusions and azimuthally within the prism and tetra blocks. This also allows for the implementation of local and compact hex mesh refinement schemes for local mesh refinement.

3. The hexahedral mesh layering near holes is adequately oriented along hole features; which is a desirable quality of FEA meshes.

4. A potential application of this type of combined abstraction/hex meshing is in meshing ultra-thin objects where vast swaths of extrusions coexists with a few highly $3 \mathrm{D}$ zones. This would enable the creation of mixed shell and solid meshes from the same input object.

\section{ACKNOWLEDGMENTS}

The author wishes to thank Altair Engineering, Inc. (USA), InTechSolutions Co., Ltd (Korea) and NEC Solution Innovators, Ltd. (Japan) for their support.

\section{REFERENCES}

[1] K. Y. Lee, Analysis Model Derivation from Design Geometry, School of Mechanical \& Manufacturing Engineering Faculty of Engineering The Queen's University of Belfast, 2004.

[2] R. Taghavi, "Automatic clump generation based on mid-surface," in Continuum and Distinct Element Numerical Modeling in Geomechanics, Melbourne, 2011.

[3] C. Armstrong, Personnal Communication, 2018.

[4] N. Amenta, S. Choi and R. K. Kolluri, "The power crust,unions of balls, and the medial axis transform," Comput. Geom. Theory and Applications, vol. 19, 2001.

[5] W. R. Quadros, "LayTracks3D: A new approach for meshing general solids using medial axis transform," Computer-Aided Design, vol. 72, pp. 102-117, March 2016.

[6] DISTENE S.A.S., "MeshGems-SurfOpt Version 2.7 API Manual," Bruyères-le-Chatel, 2019.

[7] M. Yan, "Dijkstra's Algorithm," 2015. [Online]. Available: http://math.mit.edu/ rothvoss/18.304.3PM/Presentation s/1-Melissa.pdf. [Accessed 3 August 2015].

[8] J. Vollmer, R. Mencl and H. Müller, "Improved Laplacian Smoothing of Noisy Surface Meshes," EUROGRAPHICS, vol. 18, no. 3, 1999.

[9] J. R. Shewchuk, Delaunay Refinement Mesh Generation, Pittsburgh, PA: School of Computer Science Computer Science Department Carnegie Mellon University , 1997, pp. 22-24. 


\title{
TOPOGRAPHY ADAPTED MESH GENERATION FOR ATMOSPHERIC BOUNDARY LAYER FLOW SIMULATION
}

\author{
Abel Gargallo-Peiró Matias Avila Arnau Folch \\ Computer Applications in Science and Engineering Department \\ Barcelona Supercomputing Center \\ C/ Jordi Girona 29, 08034 Barcelona, Spain \\ \{abel.gargallo, matias.avila, arnau.folch\} @bsc.es
}

\begin{abstract}
We present a new hybrid meshing procedure specifically designed to simulate Atmospheric Boundary Layer (ABL) flows featuring Coriolis effects for onshore applications. Two new meshing contributions for ABL flow simulation are proposed. First, a new metric-based adaptive meshing process to discretize the topography is presented. The adaptivity process is specifically written to deal with the discrete nature of topographic data, reparemeterizing smoothly the topography to allow computing the first and second order derivatives required by the desired metrics. Second, we present a new hybrid mesh generation procedure to discretize the ABL. We generate a prismatic boundary layer that captures the gradients of the Surface Boundary Layer, and following, an unstructured mesh is generated to discretize the rest of the domain. We show examples to illustrate the reduction in the element count with respect to standard semi-structured approaches, and we present the simulation result obtained on an onshore topographical scenario.
\end{abstract}

Keywords: Topography, Atmospheric Boundary Layer Flows, Hybrid Meshes, Mesh adaptation

\section{INTRODUCTION}

Simulation of Atmospheric Boundary Layer (ABL) flows is of interest to various scientific disciplines and related applications including, among others, meteorology, atmospheric transport of pollutants, or wind energy. In the ABL, orographic gradients, ground surface drag, and atmospheric thermal instabilities from radiative forcing, can generate turbulence and strong wind shear (vertical velocity gradients) in the so-called Surface Boundary Layer (SBL), which extends up to a $10-20 \%$ of the total ABL depth $[1,2,3,4,5]$. The need to capture these high gradients impose requirements to the mesh generators employed in ABL simulation. The requirement to reproduce the highgradients of the SBL has been translated in most mesh generators for ABL simulation in a fully struc- tured mesh in the normal direction to the surface $[6,7,8,9,10,11]$.

In addition to the structure along the vertical direction, most mesh generators specifically designed to discretize the ABL $[6,7,8,9]$ are also semi-structured in the surface and do not feature adaptivity to the terrain. They feature hexahedra and use block structured strategies to provide a fine resolution in the zone of interest and less resolution far away from this region. However, since in each block the mesh is structured, inevitably finer resolution of the interest areas is extended to the rest of the domain. Although having the drawback of increasing the required number of elements of the mesh, this structured strategies have been exploited in offshore cases (analyzing the wind resource on the sea) or in topographic scenarios that do not feature high complexity or high gradients in or- 
der to align the mesh with the wind inflow direction, generating one mesh for each simulated wind inflow direction.

Herein, we are interested in generating meshes in complex topographic scenarios, see for instance results in Section 6.2. In those scenarios, although when simulating without Coriolis there is a dominant wind direction, the complexity of the topography does not allow a priori to determine the best alignment of the mesh with the flow in the interest close-to surface region. In addition, herein we model the Coriolis effect in the flow, which translates in the fact that, even in offshore scenarios, the wind direction close to the ground is different than the wind direction in the top of the domain. The change of the wind direction determined by the Coriolis effect depends on the latitude of the scenario but in general can impose a change in the direction around 20 degrees from the ground to $2 \mathrm{~km}$ over the topography. Thus, taking into account the Coriolis force, not even in offshore cases there is a unique wind direction, since it changes according to the height. Taking into account both that the wind direction changes at different heights and also due to the influence of the topography, in this work we propose to use an unstructured mesh on the surface.

Herein, we propose two new meshing contributions for Atmospheric Boundary Layer flow simulation. First, we propose an adaptive process to the topography, to have elements of the desired length on the topography and capture the curvature of the features present in the terrain. Second, we propose to generate the volume mesh in a different approach than the standard in simulation of ABL flows. First, we generate a prismatic boundary layer that captures the gradients of the Surface Boundary Layer, and following using an unstructured mesh to discretize the rest of the ABL. We take into account that away from the ground the vertical velocity gradient is small and the flow is almost isotropic. In addition, the influence of the topography is minor and a coarser mesh can capture the same flow features than the structured fine mesh that we have generated close to the topography to capture its geometrical features. Thus, tetrahedral elements deliver a geometrical flexibility that can be exploited to increase the element size in all the directions.

The rest of the paper is organized as follows. First, in Section 2 we present the previous works on the field of mesh generation for ABL flows. Second, in Section 3 it is presented the modeling of the discrete input topography and the two metrics used to adapt the surface mesh to the topography. Third, in Section 4 the surface mesh adaptation procedure is presented. Next, in Section 5 the combined structured and unstructured volume mesh generation process is detailed. Finally, in Section 6 different topographic scenarios are discretized with the presented methodology and the generated meshes are applied to simulate Atmospheric Boundary Layer flows.

\section{RELATED WORK}

The standard approaches for Atmospheric Boundary Layer simulation feature structured or semi-structured meshes. The use of structured grids was first used in Finite Difference and Finite Volumes applications, introducing the effect of the topography by means of a change of coordinates in the formulation of the problem instead of discretizing the topography with the mesh $[12,13]$. Also in Finite Element and Finite Volume applications the use of structured hexahedral meshes represents the current most standard procedure. This semi-structured meshing strategy exploits the advantage of hexahedra to align the mesh with the flow on offshore applications and also exploits the tensor structure of hexahedra to reproduce the close-tosurface boundary layer. Several mesh generation approaches have based on solving a system of hyperbolic Partial Differential Equations (PDEs) to improve the orthogonality of the mesh and the desired element volume $[14,6,7]$. Similarly, the use of elliptic PDEs has been explored to determine the location of the mesh nodes [15]. In contrast with these approaches, in $[9,16]$ it is proposed a procedure that combines sweeping the quadrilateral surface mesh with a quality-based mesh optimization, to determine the best configuration of the nodes of the mesh according to the chosen quality measure.

There are several alternatives to the use of hexahedral elements. First, regarding the simulation of ABL flows, in [11] a fully prismatic mesh is generated to discertize the boundary layer. This work takes advantage of generating a triangle surface mesh to avoid extending the finer mesh size in the interest region to the rest of the domain and, simultaneously, takes advantage of the tensor direction of the prisms to discretize the boundary layer. As an alternative to structured or semi-structured approaches, different approaches featuring tetrahedra have been followed for problems that require a mesh conformal with the topography but that do not require a boundary layer close to the surface $[17,18,19,20,21,22,23]$.

In this work, we aim to develop an specific-purpose approach to deal with ABL flows featuring Coriolis effects on complex topographies. In contrast with previous works, we aim to generate an hybrid prismatic and tetrahedral mesh that features the main advantages of both structured and unstructured meshes for our target CFD model. First, in the surface mesh generation process, we exploit the flexibility of the wellestablished triangle meshers to generate a triangle surface mesh adapted to the topography. Second, as pre- 
viously highlighted, in the first $10-20 \%$ of the volume, a boundary layer is required. Thus, we take advantage of the tensor structure of prismatic elements to discertize the ABL with the desired growing ratio. Finally, once in a region with smaller vertical gradients, we take advantage of the flexibility of tetrahedra to discertize the domain with the desired element size, and not limited by the structure of the mesh in the first region of the domain close to the surface.

\section{TOPOGRAPHY GEOMETRY MODELING}

In this section, it is detailed the strategy followed herein to model the geometry data for topographic landscapes. Topographic geometries are discrete due to the existent data extraction processes. Thus, first we present the approach to obtain a smooth geometry representation that allows computing first and second order derivatives of the underlying topography, see Section 3.1. Following, in Section 3.2, the two metrics used in this work to adapt to the geometry are presented, particularizing them for the case of topographic geometries.

\subsection{High-order approximation of the to- pography}

This work is devoted to generate meshes conformal with the terrain. The geometry corresponds to real measured data that can be given in many formats, such as contour topography maps, Cartesian grids or point clouds. Herein, all the input frameworks are converted into a triangle mesh that is used as a geometry representation. Following, we define the proposed parameterization of the target surface (topography). Due to the existent data extraction procedures, topographic data for wind resource assessment in this work always ensures that each point in the plane has a unique height value. This is so since the triangle mesh that represents the geometry can be interpreted as a planar triangle mesh with a height value in each of the points of the mesh. In particular, we define $\Pi_{\Sigma}$ as the parametric plane, the region in $\mathbb{R}^{2}$ where the topography is defined (region of the plane where the topography data has been measured). Thus, given a point $\mathbf{x} \in \Pi_{\Sigma}$ the height function of this point $\mathrm{z}_{h}(\mathbf{x})$ on the topography is defined as

$$
\begin{array}{cccc}
\mathrm{z}_{h}: & \Pi_{\Sigma} \subset \mathbb{R}^{2} & \longrightarrow & \mathbb{R} \\
& \mathbf{x}=(x, y)^{t} & \longmapsto & \mathrm{z}_{h}(\mathbf{x}),
\end{array}
$$

where $\mathrm{z}_{h}(\mathbf{x})$ is computed by finding the triangle to which this point belongs and interpolating the height value in the triangle. The function $\mathrm{z}_{h}$ unequivocally determines a parameterization $\boldsymbol{\varphi}_{h}$ of the topography surface $\Sigma$ as

$$
\begin{array}{cccc}
\boldsymbol{\varphi}_{h}: & \Pi_{\Sigma} \subset \mathbb{R}^{2} & \longrightarrow & \Sigma \subset \mathbb{R}^{3} \\
& \mathbf{x}=(x, y)^{t} & \longmapsto & \mathbf{z}=\left(x, y, \mathrm{z}_{h}(\mathbf{x})\right)^{t} .
\end{array}
$$

This parameterization maps a point in the parametric plane to a point in the topography. As highlighted, it is a discrete parameterization that finds the surface triangle to which the point belongs, and computes the exact location of this point in the topography. To compute for instance the curvature of the target surface (Section 3.2) in the mesh adaptation process (Section 4 ), it is required to perform queries of the first and second order derivatives of the geometry. However, since topography representation is a piece-wise linear triangle geometry, the derivatives of the topography geometry are not well defined. On the one hand, there are discontinuities of the first order derivative in the edges between the elements of the topography geometry. On the other hand, since the geometry mesh features piece-wise linear elements, the curvature of each triangle configuring the geometry is null. Thus, similarly to $[24,25]$, herein we propose to reconstruct local high-order approximations of the geometry that allow queries of the derivatives of the surface representation.

To generate the high-order approximation in a point $\mathbf{x} \in \Pi_{\Sigma}$, first it is located the triangle that contains this point in the mesh that defines the geometry. Following, several layers of elements adjacent to the container elements are gathered. Each new layer is computed as the triangles neighboring to the current considered elements. Then, we compute the least squares approximation of the desired order of the cloud of points determined by the neighborhood of elements around the target point. Specifically, given a set of topography points $\left\{\mathbf{z}_{1}, \ldots, \mathbf{z}_{n_{p}}\right\}$ the high-order approximation used in this work is written as

$$
\mathrm{z}_{p}(x, y)=\sum_{\{i, j\} \in I_{p}} a_{i j} x^{i} y^{j}
$$

where $p$ is the desired polynomial degree, $I_{p}=$ $\{\{i, j\} \mid i, j \geq 0$ and $i+j \leq p\}$ is the set of indexes lower or equal to $p$, and $\left\{a_{i j}\right\}_{\{i, j\} \in I_{p}}$ are the coefficients of the polynomial $\mathrm{z}_{p}(x, y)$ on $x$ and $y$. In particular, we seek the approximation that better fits the cloud of points in the least squares sense:

$$
\left\{a_{i j}\right\}_{\{i, j\} \in I_{p}}=\underset{\substack{a_{i j} \in \mathbb{R} \\\{i, j\} \in I_{p}}}{\operatorname{argmin}} \sum_{k=1}^{n_{p}}\left(\mathrm{z}_{p}\left(x_{k}, y_{k}\right)-z_{k}\right)^{2},
$$

where $\mathbf{z}_{k}=\left(x_{k}, y_{k}, z_{k}\right)$ are the cloud point coordinates.

Note that if there are less points than parameters $a_{i j}$ the problem stated in Eq. (4) is ill-conditioned. Thus, by default $p$ levels of elements around the container element are considered. Once all the neighboring points 
are gathered, they are counted to ensure that the number of points in the cloud is higher or equal than the number of parameters. Otherwise, an extra layer of neighboring elements is included, repeating this process until the number of points ensures a well-posed minimization problem. In particular, if we have the exact same number of parameters we would obtain a surface containing all the points of the cloud.

Once computed the smooth high-order approximation, the new parameterization $\boldsymbol{\varphi}_{p}$ is defined as

$$
\begin{array}{cccc}
\boldsymbol{\varphi}_{p}: & \Pi_{\Sigma} \subset \mathbb{R}^{2} & \longrightarrow & \mathbb{R}^{3} \\
& \mathbf{x}=(x, y)^{t} & \longmapsto & \mathbf{z}=\left(x, y, \mathrm{z}_{p}(x, y)\right)^{t} .
\end{array}
$$

Note that this parameterization has well defined first and second order derivatives that will allow to compute the desired metrics on the geometry in Section 3.2. The order of approximation used in this work in the presented examples is three, and, accordingly to what has been previously stated, the number of levels of adjacency to compute the approximation is also three (without requiring in any example to automatically computing extra layers of neighbors).

\subsection{Metrics over the topography geometry}

The main objective of this work is to generate meshes that feature the desired edge length on the surface and that at the same reproduce the curvature of the geometry. Thus, the edge lengths of the surface mesh will be measured taking into account two different metrics: the metric of the tangent space, and the metric derived from the Hessian of the parameterization.

First, some notation is stated regarding the measurement of edge lengths in surfaces with respect to a given metric. Following Section 3.1, we denote a general parameterization of a surface $\Sigma$ as:

$$
\begin{gathered}
\boldsymbol{\varphi}: \Pi_{\Sigma} \subset \mathbb{R}^{2} \longrightarrow \Sigma \subset \mathbb{R}^{3} \\
\mathbf{x}=(x, y)^{t} \longmapsto \mathbf{z}=\boldsymbol{\varphi}(\mathbf{x}) .
\end{gathered}
$$

We define the curve $\gamma(t)$ between two points $\mathbf{z}_{1}$ and $\mathbf{z}_{2}$ on $\Sigma$ in terms of the parameterization $\boldsymbol{\varphi}$ and the edge $\left[\mathbf{x}_{1}, \mathbf{x}_{2}\right]$ on the parametric space as

$$
\begin{aligned}
\gamma:[0,1] & \longrightarrow \Gamma \subset \Sigma \subset \mathbb{R}^{3} \\
t & \longmapsto \varphi(\mathbf{x}(t)),
\end{aligned}
$$

where $\mathbf{x}(t)$ is defined as

$$
\begin{aligned}
\mathbf{x}:[0,1] & \longrightarrow\left[\mathbf{x}_{1}, \mathbf{x}_{2}\right] \subset \Pi_{\Sigma} \subset \mathbb{R}^{2} \\
t & \longmapsto \mathbf{x}_{1}+t\left(\mathbf{x}_{2}-\mathbf{x}_{1}\right),
\end{aligned}
$$

and with $\gamma$ fulfilling that $\gamma(0)=\mathbf{z}_{1}$ and $\gamma(1)=\mathbf{z}_{2}$. The length of the curve on the surface is

$$
l\left(\mathbf{z}_{1}, \mathbf{z}_{2}\right)=\int_{0}^{1} \sqrt{<\frac{\partial \boldsymbol{\gamma}}{\partial t}(t), \frac{\partial \boldsymbol{\gamma}}{\partial t}(t)>} \mathrm{d} t
$$

where $\langle\mathbf{a}, \mathbf{b}\rangle=\mathbf{a} \cdot \mathbf{b}$ is the scalar product between the vectors $\mathbf{a}$ and $\mathbf{b}$.

Next, following the ideas in [26], the chain rule is used in the definition of the curve in Eq. (7) and Eq. (8):

$$
\frac{\partial \boldsymbol{\gamma}}{\partial t}=\left(\frac{\partial \boldsymbol{\varphi}}{\partial x} \frac{\partial \boldsymbol{\varphi}}{\partial y}\right) \cdot \frac{\partial \mathbf{x}(t)}{\partial t}=\nabla \boldsymbol{\varphi} \cdot\left(\mathbf{x}_{2}-\mathbf{x}_{1}\right)
$$

and rewrite the length of the curve in Eq. (9) in terms of the parametric coordinates of the two surface nodes:

$$
\begin{aligned}
& l_{\mathcal{M}_{1}}\left(\mathbf{z}_{1}, \mathbf{z}_{2}\right)= \\
& =\int_{0}^{1}\left(\left(\mathbf{x}_{2}-\mathbf{x}_{1}\right)^{t} \cdot \nabla \boldsymbol{\varphi}^{t} \cdot \nabla \boldsymbol{\varphi} \cdot\left(\mathbf{x}_{2}-\mathbf{x}_{1}\right)\right)^{1 / 2} \mathrm{~d} t \\
& =\int_{0}^{1}\left(\left(\mathbf{x}_{2}-\mathbf{x}_{1}\right)^{t} \mathcal{M}_{1}(\mathbf{x}(t))\left(\mathbf{x}_{2}-\mathbf{x}_{1}\right)\right)^{1 / 2} \mathrm{~d} t
\end{aligned}
$$

where

$$
\begin{aligned}
& \mathcal{M}_{1}(\mathbf{x}(t)) \equiv \mathcal{M}_{1}= \\
& =\nabla \varphi^{t} \cdot \nabla \boldsymbol{\varphi}=\left(\begin{array}{ll}
\frac{\partial \varphi}{\partial x} & \frac{\partial \varphi}{\partial y}
\end{array}\right)^{t} \cdot\left(\begin{array}{ll}
\frac{\partial \varphi}{\partial x} & \frac{\partial \varphi}{\partial y}
\end{array}\right),
\end{aligned}
$$

is the matrix expression of the first fundamental form of the surface $\Sigma$ at the point on the parametric space $\mathbf{x}(t)$, see Eq. (8). In particular, for isotropic mesh generation with a desired length $h$ of an edge known in each region of the domain, we define the tangent metric in terms of $\mathcal{M}_{1}$ as

$$
\mathcal{M}_{T}:=\frac{1}{h^{2}} \mathcal{M}_{1}
$$

and the corresponding length measure as

$$
\begin{aligned}
& l_{\mathcal{M}_{T}}\left(\mathbf{z}_{1}, \mathbf{z}_{2}\right):= \\
& \int_{0}^{1}\left(\left(\mathbf{x}_{2}-\mathbf{x}_{1}\right)^{t} \cdot \mathcal{M}_{T}(\mathbf{x}(t)) \cdot\left(\mathbf{x}_{2}-\mathbf{x}_{1}\right)\right)^{1 / 2} \mathrm{~d} t .
\end{aligned}
$$

Note that we would ideally like the edges of the mesh to have measure 1 with the metric $\mathcal{M}_{T}$. In this manner, in the adaptive procedure that will be presented in Section 4, Eq. (13) will be used to compute the length of the curve on the surface in terms of the coordinates of the nodes in the parametric space. Next, these elements with measure greater than one (with a safety factor) will be refined until all edges fulfill the desired metric.

In addition to measuring the edge length of the mesh taking into account the length of the curve described in the surface, we also want to capture the curvature of the surface. That is, the second order derivatives of the surface will be also taken into account in the adaptive procedure. To do so, we explicitly exploit that the parameterization $\boldsymbol{\varphi}$ in Eq. (6) for topographic geometries can be rewritten as $\boldsymbol{\varphi}_{p}(x, y)=\left(x, y, \mathrm{z}_{p}(x, y)\right)$, as detailed in Eq. (3) and Eq. (5) from Section 3.1. Thus, the parameterization can be also understood as 
a field $z_{p}$ over a $2 \mathrm{D}$ mesh on $x$ and $y$. Hence, herein we propose to adapt the mesh to capture the curvature of the geometry using techniques for 2D mesh adaptation to reduce the interpolation error of $\mathrm{z}_{p}(x, y)$.

Following the ideas presented in $[27,28]$, from the Hes$\operatorname{sian} \mathcal{H}$ of the topography at a point,

$$
\mathcal{H}=\left(\begin{array}{ll}
\frac{\partial^{2} z_{p}}{\partial x \partial x} & \frac{\partial^{2} z_{p}}{\partial x \partial y} \\
\frac{\partial^{2} z_{p}}{\partial y \partial x} & \frac{\partial^{2} z_{p}}{\partial y \partial y}
\end{array}\right),
$$

the following metric is defined:

$$
\mathcal{M}_{C}=\mathbf{V} \mathbf{D} \mathbf{V}^{t}
$$

where $\mathbf{V}=\left(\mathbf{e}_{1}, \mathbf{e}_{\mathbf{2}}\right)$ is the matrix composed by the eigenvectors $\mathbf{e}_{1}$ and $\mathbf{e}_{2}$ of $\mathcal{H}, \mathbf{D}=\beta \operatorname{diag}\left(\left|\lambda_{1}\right|,\left|\lambda_{2}\right|\right)$ a diagonal matrix with its absolute value of the eigenvalues, and $\beta$ a curvature discretization parameter. Herein, $\beta$ has a default value of 1 , which has been determined experimentally for the average metrics that arise in topographic applications after testing several topographic scenarios. However, it can also be specified by the user to discretize the curvature of the geometry with the desired accuracy, or computed according to $[29,30,31]$ to provide an estimate of the number of vertices in terms of the metric complexity. Similarly to Eq. (13), the length of the curve between $\mathbf{z}_{1}$ and $\mathbf{z}_{2}$ with respect to the metric $\mathcal{M}_{C}$ is defined as

$$
\begin{aligned}
& l_{\mathcal{M}_{C}}\left(\mathbf{z}_{1}, \mathbf{z}_{2}\right):= \\
& \int_{0}^{1}\left(\left(\mathbf{x}_{2}-\mathbf{x}_{1}\right)^{t} \cdot \mathcal{M}_{C}(\mathbf{x}(t)) \cdot\left(\mathbf{x}_{2}-\mathbf{x}_{1}\right)\right)^{1 / 2} \mathrm{~d} t
\end{aligned}
$$

where $\mathbf{x}(t)$ is defined in Eq. (8). In particular, if the user has prescribed an element size of $h$, the edges of a mesh should ideally fulfill $l_{\mathcal{M}_{T}}=1$ and $l_{\mathcal{M}_{C}}=1$. However, these two conditions may not be achievable simultaniously since, for instance, the curvature of the geometry may demand $l_{\mathcal{M}_{T}}<<1$. Following, in Section 4 it is detailed the adaptive mesh generation proposed in this work, based on the two presented metrics. First, it will be generated a coarse topography mesh, which will be locally refined it until no edges of the mesh have length greater than 1 , or if the length of the edges is lower than a minimum value set by the user. With these two conditions, the edge length of the surface mesh is controlled and it is ensured that the mesh accurately reproduces the curvature of the geometry.

\section{SURFACE MESH: UNSTRUCTURED TOPOGRAPHY MESH GENERATION}

In this section, we detail our surface mesh adaptation procedure for topographic geometries. The topography is parameterized according to the technique proposed in Section 3.1, and the edge lengths of the mesh

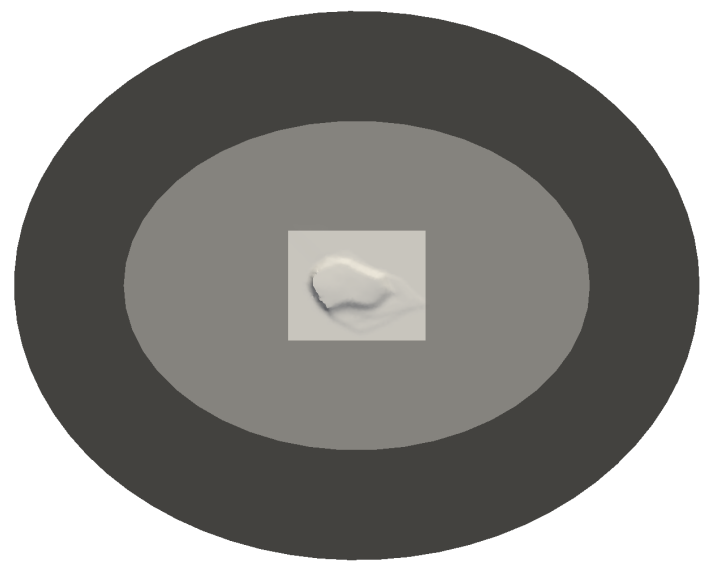

Figure 1: Synthetic example illustrating the domain regions: farm (light gray), transition (gray) and buffer (dark grey).

are measured according to the tangent and curvature metrics, Eq. (13) and Eq. (15) respectively, both detailed in Section 3.2.

In this work, the topography is divided into three regions, with three different levels of resolution illustrated in Figure 1: the interest wind farm area (higher resolution, light gray), a transition area (gray), and an elliptical buffer area to impose the boundary conditions (lower resolution, dark gray). First, the farm area is meshed. The farm is a quadrilateral domain featuring the region of interest in the simulation (for instance, area where a wind farm is to be designed). The adaptive process to the topography is applied in the farm region, where high geometric accuracy is required to discretize the features of the topography. The transition area is an elliptical domain that encircles the farm region and is meshed with a triangle mesh that smoothly matches the fine mesh of the transition with the element size of the buffer, the outer region. Finally, it is defined an additional elliptic region with a coarse element size to impose the boundary conditions. Herein, the outer boundary is chosen to be elliptical to avoid the discontinuities that the corners of a quadrilateral domain can induce in ABL simulations with Coriolis and to impose in a continuous manner the input/output flow conditions.

\subsection{Adaptive mesh generation process}

To generate a surface mesh adapted to the topographic features, two inputs are required: the maximum element size $h_{\max }$ and the minimum allowed size $h_{\min }$. First, a planar triangle mesh with the maximum element size is generated using the Triangle mesh generator [32]. In Figure 2(a) the initial mesh with constant 


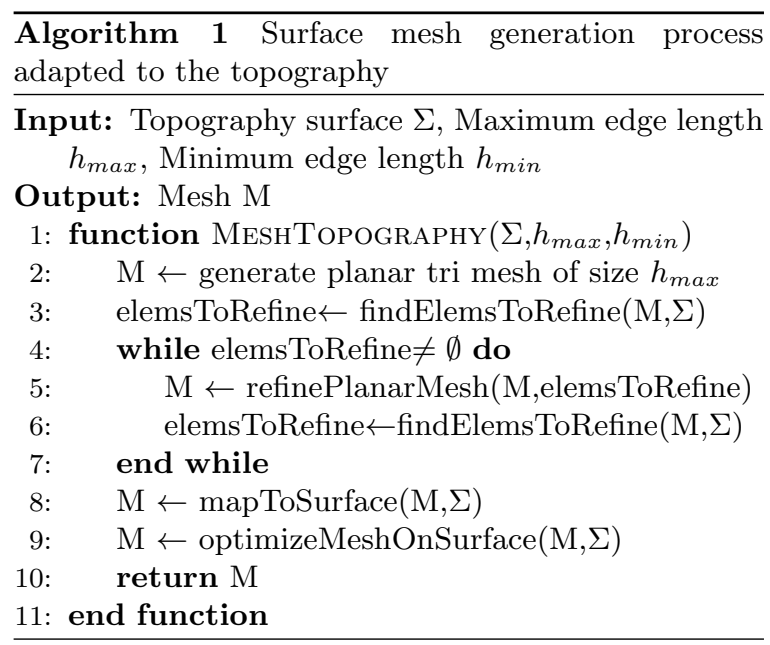

element size is illustrated. This mesh constitutes the starting point for the adaptive procedure. Following, the edge lengths of all the elements of the mesh are measured using the tangent and curvature metrics. If an edge of an element is greater than 1, this implies that the edge is longer than desired with the metric. The condition to accept the element as it is or refine it is relaxed to those elements with lengths greater than $\sqrt{2}$, see $[24,26]$. Thus, this element is included in a list of elements to be refined.

Once all the mesh elements have been checked, we locally refine the mesh where it is required. The refinement is performed by using the previous mesh as background mesh and asking to those elements included in the refinement list to have half of the size of the triangle. This process is repeated until all the elements have length below the desired threshold for both metrics, or if the minimum edge length is below the desired minimum length. We highlight that the minimum length is checked using the euclidean metric to control the minimum length in the mesh for the simulation.

The iterative procedure to perform the mesh refinement is detailed in Algorithm 1. In particular, while there are elements to refine, Line 4, the mesh is refined as previously detailed. Every time that the mesh is refined, the edge lengths with respect the two metrics are computed. Once the edge lengths are computed, the elements with edges not fulfilling the desired edge lengths are listed again to be refined, Line 6. However, to control the minimum allowed element size and to avoid obtaining excessively refined meshes, those elements with length under the euclidian metric higher than $h_{\text {min }}$ are not included again in the refinement process. The process concludes when no elements violate any metric, or when the elements violating the metrics have already achieved the minimum allowed edge length. At this point, the nodes of the planar triangle mesh are mapped to the topography in Line

\section{8, discretizing it.}

In Figure 2 the adaptive process is applied to generate a mesh on the Bolund peninsula geometry. In this illustrative example, the input mesh sizes are $h_{\max }=$ $5 \mathrm{~m}$ and $h_{\min }=0.5 \mathrm{~m}$. First, in Figure 2(a) a planar mesh of constant element size is generated, Line 2 in Algorithm 1. Then Figures 2(b) to 2(h) illustrate the successive refinements of the mesh according to Line 5. Finally, in Figure 2(i) the final surface mesh is illustrated. In the procedure, the initial number of elements is 5089, and the final one is 9037 .

Once the adaptive process is finalized, it is obtained a mesh that has elements of the desired size on the surface, and that reproduces the curvature of the geometry up to the minimum allowed mesh size. Up to this point local mesh refinement has been performed in order to improve the accuracy of the geometric approximation. However, in this process the quality of the generated mesh for simulation has still not been assessed. Thus, to conclude the generation of the topography surface mesh, in Line 9 a quality optimization of the surface elements is performed. Following, in Section 4.2 we detail the quality measure that will be used in this work to validate the generated meshes, and the current mesh configuration will be improved by means of reallocating the nodes on the surface to improve the mesh quality.

\subsection{Surface mesh optimization}

The final step of the generation of the surface mesh is an optimization of the location of the mesh nodes on the exact topography to obtain a mesh which minimizes the elemental distortion (maximizes the quality). For each element on the surface, its ideal is defined as an equilateral triangle of the desired size. The optimization procedure targets that each surface element reassembles the ideal as much as possible. It has to be taken into account that this ideal configuration can not in general be achieved since the mesh topology is now fixed and since the surface elements have their nodes tied to the geometry.

To measure if an element is valid, and to quantify how much it differs from the desired configuration, we use a distortion measure (see Knupp [33] for a review of measures). A distortion measure quantifies in the range $[1, \infty)$ the deviation of an element with respect to an ideal configuration (for instance, the equilateral triangle with the desired size for the triangle case). In this work, the distortion of an element with nodes $\mathbf{z}_{1}, \ldots, \mathbf{z}_{n_{p}}$ is denoted as $\eta\left(\mathbf{z}_{1}, \ldots, \mathbf{z}_{n_{p}}\right)$. The distortion takes value 1 when the element presents the desired configuration, and tends to infinity when the element degenerates. Following the ideas for high-order elements in $[34,35]$, the distortion measure used in 


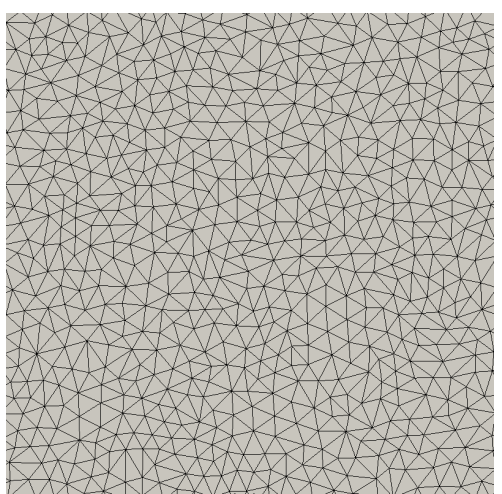

(a)

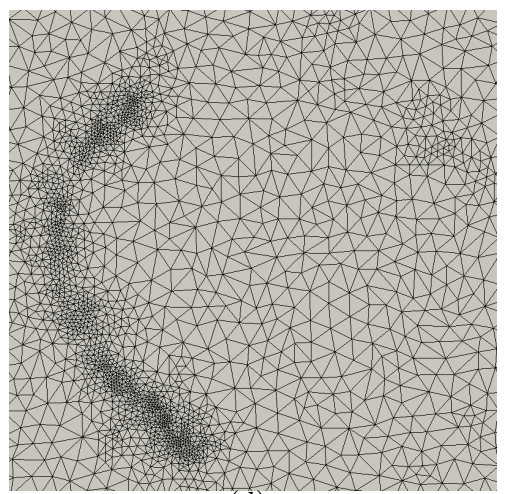

(d)

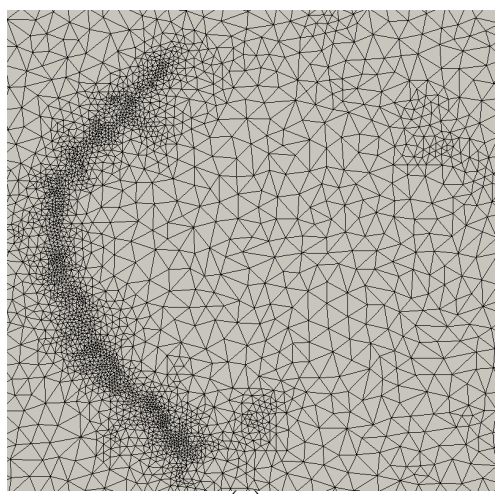

(g)

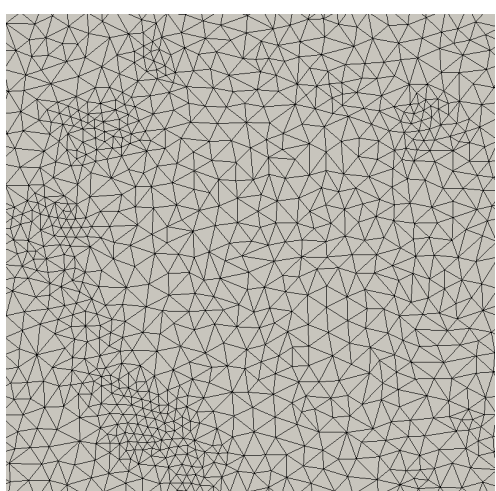

(b)

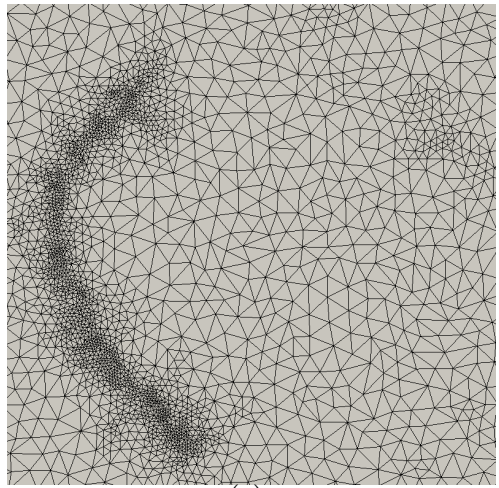

(e)

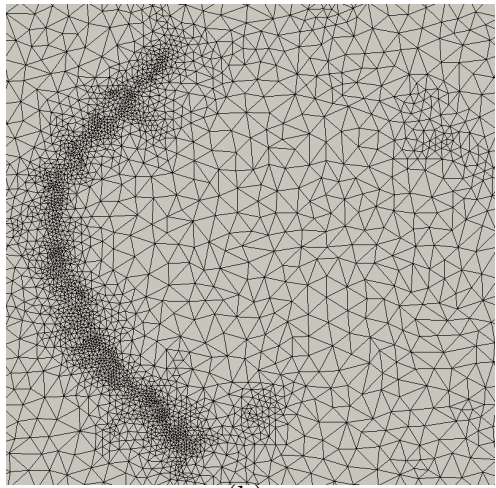

(h)

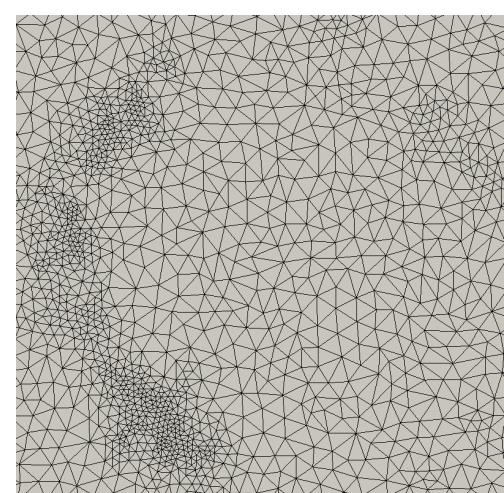

(c)

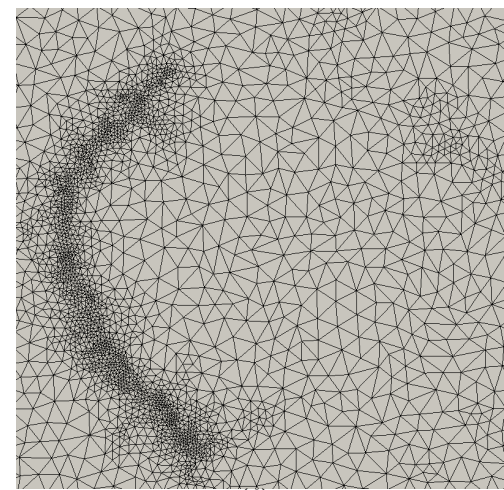

(f)

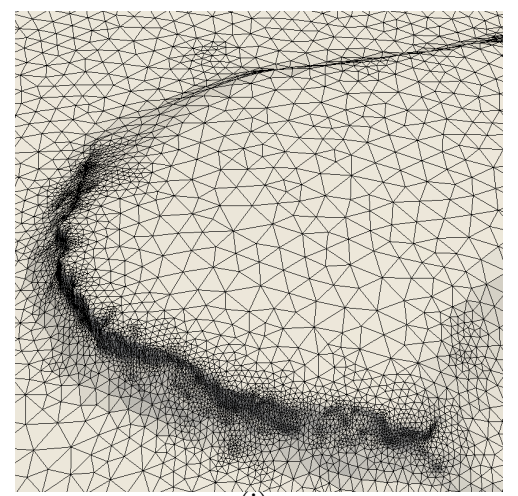

(i)

Figure 2: (a)-(h) Mesh adaptive process for the Bolund geometry illustrated in Figure 1. (i) Final adapted topography surface mesh. 
this work can be written for any given element with nodes $\mathbf{z}_{1}, \ldots, \mathbf{z}_{n_{p}}$ as:

$$
\eta\left(\mathbf{z}_{1}, \ldots, \mathbf{z}_{n_{p}}\right):=\frac{\left\|\eta_{s h}(\mathbf{D} \phi)\right\|_{E^{I}}}{\|1\|_{E^{I}}},
$$

where $\eta_{s h}$ is defined in [33] as

$$
\eta_{s h}(\mathbf{D} \phi):=\frac{\|\mathbf{D} \phi\|_{F}^{2}}{d|\operatorname{det} \mathbf{D} \phi|^{2 / d}}
$$

where $\phi\left(\mathbf{z}_{1}, \ldots, \mathbf{z}_{n_{p}}\right)$ is the mapping between the ideal $E^{I}$ and physical elements, $\mathbf{D} \phi$ is its Jacobian, $\|\cdot\|_{F}$ is the Frobenius norm, $\|\cdot\|_{E^{I}}$ is the $\mathcal{L}^{2}$ norm on the ideal element, $\|1\|_{E^{I}}$ is the measure of the ideal element, and $d$ is the dimension of the element $(d=2$ for planar and surface meshes, and $d=3$ for volumetric meshes). In particular, the quality of an element is the inverse of the distortion:

$$
q:=\frac{1}{\eta} \in[0,1]
$$

which gives us a value in $[0,1]$, being 0 an invalid configuration, and 1 the desired one. For the surface case, we consider as ideal the equilateral triangle with the desired size. In Figure 3(a) we show the initial surface mesh, coloring the elements with respect to their quality. It can be observed that non-regular lower quality elements are present in areas with big slopes of the topography.

To optimize the nodes on the topography [36, 37], we use the surface parameterization to rewrite the distortion in Eq. (16) for an element $E$ with nodes $\mathbf{z}_{1}, \ldots, \mathbf{z}_{n_{p}}$, in terms of the parametric coordinates of the nodes $\mathbf{x}_{1}, \ldots, \mathbf{x}_{n_{p}}$ as:

$$
\begin{aligned}
\eta^{\boldsymbol{\varphi}}\left(\mathbf{x}_{1}, \ldots, \mathbf{x}_{n_{p}}\right): & =\eta\left(\boldsymbol{\varphi}\left(\mathbf{x}_{1}\right), \ldots, \boldsymbol{\varphi}\left(\mathbf{x}_{n_{p}}\right)\right) \\
& =\eta\left(\mathbf{z}_{1}, \ldots, \mathbf{z}_{n_{p}}\right) .
\end{aligned}
$$

In the optimization process it is found the location of the nodes on the parametric plane $\Pi_{\Sigma}$ such that provide minimal elemental distortion (maximum quality) of the surface elements in the least squares sense. In particular, it is sought $\left\{\mathbf{x}_{1}^{*}, \ldots, \mathbf{x}_{n_{N_{s}}}^{*}\right\} \subset \Pi_{\Sigma}$ such that:

$$
\begin{aligned}
& \left\{\mathbf{x}_{1}^{*}, \ldots, \mathbf{x}_{n_{N_{s}}}^{*}\right\}= \\
& \underset{\mathbf{x}_{1}, \ldots, \mathbf{x}_{n_{N_{s}}} \in \Pi_{\Sigma}}{\operatorname{argmin}} \sum_{e=1}^{n_{E_{s}}}\left(\eta^{\varphi}\left(\mathbf{x}_{e_{1}}, \ldots, \mathbf{x}_{e_{n_{p}}}\right)\right)^{2},
\end{aligned}
$$

where $n_{N_{s}}$ is the number of surface nodes, $n_{E_{s}}$ is the number of surface elements, $e_{i}$ denotes the global node id of the $i t h$ node of element $e$, and $\eta_{e}^{\varphi}$ denotes the distortion of element $e$. We highlight that to deal with inverted elements ( $\operatorname{det} \mathbf{D} \phi \leq 0$ ), and specially to untangle meshes in the optimization procedure, the regularization of the determinant $\operatorname{det} \mathbf{D} \phi$ presented in
Table 1: Shape quality statistics (minimum, maximum, mean and standard deviation) for the triangle meshes presented in Figure 3.

\begin{tabular}{|c|c|c|c|c|}
\hline Mesh & Min.Q. & Max.Q. & Mean Q. & Std \\
\hline Fig. 3(a) & 0.17 & 1.00 & 0.89 & 0.12 \\
Fig. 3(b) & 0.50 & 1.00 & 0.96 & 0.05 \\
\hline
\end{tabular}

$[38,39]$ is used. In particular, $\operatorname{det} \mathbf{D} \phi$ in Eq. (16) is replaced by $r(\operatorname{det} \mathbf{D} \phi)$, where

$$
r(x)=\frac{1}{2}\left(x+\sqrt{x^{2}+4 \delta^{2}}\right),
$$

where $\delta$ is a numerical parameter that we determine following the approach presented in [40].

Once the optimal location in the parametric space $\left\{\mathbf{x}_{1}^{*}, \ldots, \mathbf{x}_{n_{N_{s}}}^{*}\right\}$ is obtained, the nodes are mapped back to the topography by means of the parameterization as $\left\{\mathbf{z}_{1}^{*}, \ldots, \mathbf{z}_{n_{N_{s}}}^{*}\right\}=\left\{\boldsymbol{\varphi}\left(\mathbf{x}_{1}^{*}\right), \ldots, \boldsymbol{\varphi}\left(\mathbf{x}_{n_{N_{s}}}^{*}\right)\right\}$. For the optimization process, we use the input piece-wise linear parameterization in order to ensure that the final location of the mesh nodes is on the input topography.

The topography mesh generation process for the Bolund hill (Denmark) is shown in Figure 3. In addition, in Table 1 we illustrate the mesh quality statistics resulting from the detailed procedure. Figure 3(a) shows the initial distorted surface mesh, which has a minimum quality of 0.17 . In contrast, Figure $3(\mathrm{~b})$ shows the optimized mesh, with a minimum quality that has been increased to 0.50 , and where it can be observed that the distorted elements from the initial mesh have become almost regular all over the domain. We highlight that the quality of the surface mesh is of major importance for the volume mesh generation. The surface mesh defines the boundary of the volume mesh, and therefore, an invalid (or low-quality) surface mesh will derive in a low-quality (or invalid) volume mesh.

\section{VOLUME MESH: ATMOSPHERIC BOUNDARY LAYER MESH GENERATION}

The Atmospheric Boundary Layer mesh is generated in a domain enclosed by the topography, a planar ceiling at the desired height (user input defaulted as $2 \mathrm{~km}$ over the highest topography point), and an elliptic lateral wall. The elliptic lateral boundary is defined by extruding vertically the boundary of the $2 \mathrm{D}$ domain, see Figure 1, up to the desired height.

This volumetric domain is meshed following Algorithm 2. First, in Line 2, the triangle surface mesh of the topography is swept (extruded) to generate a structured prismatic mesh close to the ground. This process is de- 


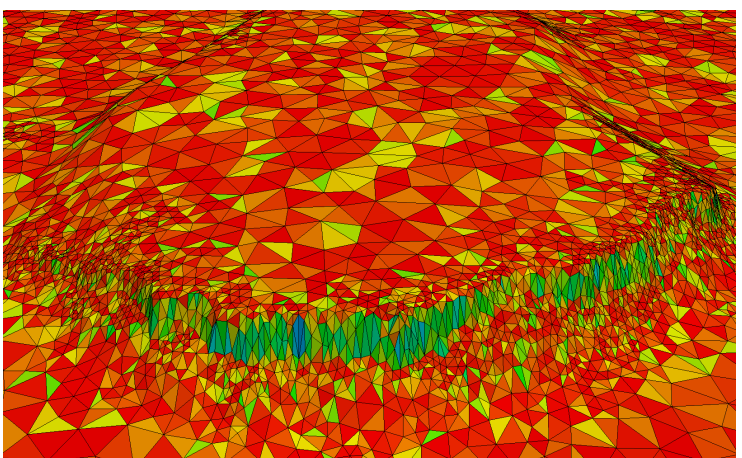

(a)

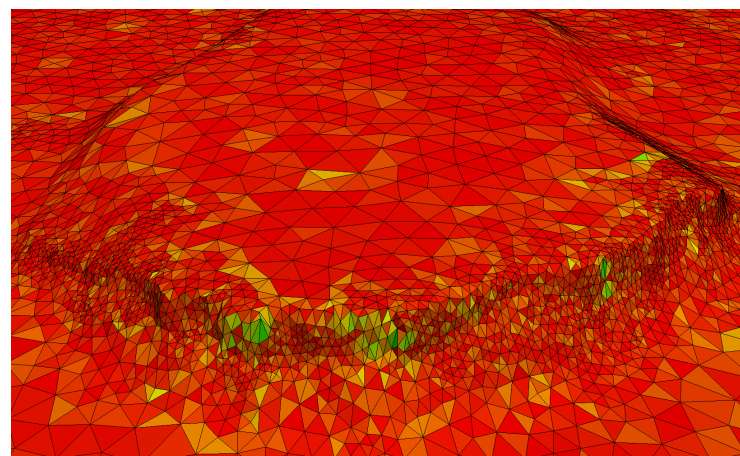

(b)

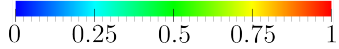

Figure 3: Topography mesh generation for the Bolund hill: (a) initial distorted surface mesh, and (b) optimized high-quality surface mesh. The surface elements elements are colored according to their quality, Eq. (17).

tailed in Section 5.1. As input for the prismatic meshing process, it is required to provide the initial height of the elements $\left(h_{0}\right)$, the growing ratio $(r)$, a maximum elemental height for the boundary layer $\left(h_{1}\right)$, and the height until which the boundary layer extends $\left(z_{B L}\right)$. Herein, the growth factor of the boundary layer is chosen in the interval $[1.05,1.25]$, and the anisotropy in the first layer is set between the order of $1 / 100$ or $1 / 1000$, depending on the CFD case and the region of the domain. In addition, the maximum height of the structured boundary layer region is set by default at the $20 \%$ of the total ABL (400 meters over $2 \mathrm{~km}$ ), see $[1,2,3,4,5]$. If the elements become isotropic before reaching the height of the structured region, the sweeping is continued keeping constant the size along the vertical direction.

Once the prismatic mesh is finished, in Line 3 an unstructured tetrahedral mesh is generated to fill the rest of the domain with tetrahedra: from the last prismatic layer to the flat ceiling located at height $z_{t o p}$. The generation of the tetrahedral mesh relies on TetGen [41], to which a surface mesh is provided together with an element size field. The element size of the triangles from the prismatic mesh are assigned with their own size, while the elements in the top ceiling are assigned with the top ceiling size given by the user $\left(h_{2}\right)$. The elements on the elliptical lateral boundary are assigned with a smooth linear size gradation from the prismatic mesh to the top ceiling height. The prismatic layer together with the tetrahedral mesh compose an hybrid mesh, Line 4, and following, the resulting hybrid mesh is optimized, see Line 5 . The generation of the unstructured mesh and the optimization of the hybrid mesh are detailed in Section 5.2.

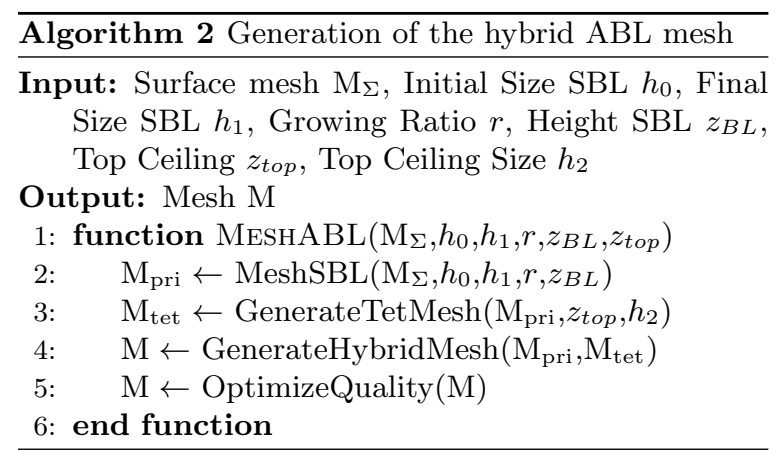

\subsection{Structured prismatic meshing using an optimization-based sweeping}

Given a initial triangle surface mesh generated with the approach presented in Section 4, the volume mesh of the close to the surface region is generated by means of an iterative sweeping procedure that requires two main steps to compute each new sweeping layer. First, given a layer of triangle elements, a new layer of triangles is generated. These two triangle meshes determine the new prismatic layer by connecting each node on the initial layer with the corresponding swept node in the extruded layer. Second, once a new layer of prisms has been generated, it is performed a local optimization of the low quality elements that have been generated up to this point.

Given a layer of triangle elements, we generate a new layer of prisms by means of sweeping each node computing a new extruding length and an extruding direction. The current extrusion length is computed in a standard manner using a geometrical law based on a user input growing ratio, herein defaulted as 1.15. As input for the user it is also asked a maximum element size (defaulted as the isotropic configuration of 
the swept element). Once this size is reached or if the element becomes isotropic, the extruding length is kept constant and not further increased with the growing ratio.

To determine the extruding direction, we compute the pseudo-normal $[42,43]$ of the nodes adjacent to each swept node. In particular, given a node $\mathbf{z}$ with $n_{l}$ neighboring nodes $\left\{\mathbf{z}_{1}, \ldots, \mathbf{z}_{n_{l}}\right\}$, the pseudo-normal $\mathbf{n}_{\mathbf{z}}$ is defined as

$$
\begin{aligned}
\mathbf{n}_{\mathbf{z}} & :=\frac{\sum_{i=1}^{n_{l}} \mathbf{z}_{i} \times \mathbf{z}_{i+1}}{\left\|\sum_{i=1}^{n_{l}} \mathbf{z}_{i} \times \mathbf{z}_{i+1}\right\|} \\
& =\frac{\sum_{i=1}^{n_{l}}\left(\mathbf{z}_{i}-\mathbf{z}\right) \times\left(\mathbf{z}_{i+1}-\mathbf{z}\right)}{\left\|\sum_{i=1}^{n_{l}}\left(\mathbf{z}_{i}-\mathbf{z}\right) \times\left(\mathbf{z}_{i+1}-\mathbf{z}\right)\right\|},
\end{aligned}
$$

where we consider $\mathbf{z}_{n_{l}+1} \equiv \mathbf{z}_{1}$. The pseudo-normal is used to maximize the orthogonality of the extruded layer with respect to the previous one. The main property of the pseudo-normal $\mathbf{n}_{\mathbf{z}}$ is that it defines the plane that maximizes the area of the projection of the polygon defined by $\left\{\mathbf{z}_{1}, \ldots, \mathbf{z}_{n_{l}}\right\}$, see $[42,43]$, reducing the chances of generating invalid extruded elements. We highlight that the pseudo-normal is blended with the vertical direction in order to enforce that the mesh grows towards the ceiling and that it gets to the top orthogonally to the planar ceiling.

It must be remarked that although the use of the pseudo-normal reduces the possiblity of obtaining inverted or low quality elements, this is still not guaranteed in the extruding process. Thus, it is required to assess the quality of the mesh during the procedure to ensure its validity and, if possible, improve its quality with a similar approach to the one used for the surface mesh in Section 4.2. To compute the quality of a given prismatic element, an ideal element is required. We set the ideal of each prism in terms of the best prism that we would get if no topography was present. That is, the ideal element of a given physical prism is defined as the initial surface element extruded orthogonally with the computed extrusion length at the current layer. This is indeed the element that we would desire to generate, since it is extruded orthogonally and has the best configuration on the surface (it has been optimized for the target topography in Section 4.2). Once we have set the ideal prism, we use Eq. (16) to compute the distortion of the elements and assess its validity.

Once a new prismatic layer is generated, it is optimized to improve its configuration with respect to the defined ideal elements. Recall that although it has been extruded using the best projection plane, the constraint of coming from a non-planar mesh prevents the mesh from being optimal. In particular, only a mesh with a planar topography would be optimal with respect to the ideal configuration. Therefore, before generating a new layer of elements, the current layer is op-
Table 2: Shape quality statistics (minimum, maximum, mean and standard deviation) for the meshes presented in Figure 4.

\begin{tabular}{|c|c|c|c|c|}
\hline Mesh & Min.Q. & Max.Q. & Mean Q. & Std \\
\hline Fig. 4(a) & 0.10 & 1.00 & 0.98 & 0.10 \\
Fig. 4(b) & 0.10 & 1.00 & 0.85 & 0.23 \\
Fig. 4(c) & 0.20 & 1.00 & 0.86 & 0.19 \\
\hline
\end{tabular}

timized. Since the mesh is still being generated and taking into account the computational efficiency of the process, during the sweeping process we only optimize the lowest quality elements on the mesh. The quality threshold for this local optimization is defaulted in our applications as 0.2 . To allow those elements to have room for improvement, several layers of neighboring nodes are included in the optimization process. The rest of nodes are kept fixed and are not optimized. In all the examples of this work, two levels of neighboring nodes are considered. In particular, following a Gauss-Seidel approach, we solve for each free node the non-linear minimization problem:

$$
\mathbf{z}_{i}=\underset{\mathbf{z}_{i} \in \mathbb{R}^{3}}{\operatorname{argmin}} \sum_{e=1}^{n_{E}^{i}} \eta_{\hat{e}}^{2}\left(\mathbf{z}_{e_{1}}, \ldots, \mathbf{z}_{e_{n_{p}}}\right),
$$

where $\{\hat{e}\}_{e=1, \ldots, n_{E}^{i}}$ denotes the set of adjacent elements to node $\mathbf{z}_{i}, \hat{e}$ denotes the global id of the $e$-th adjacent element of node $\mathbf{z}_{i}, \eta_{\hat{e}}$ is the distortion of the $e$-th element, and $e_{i}$ corresponds to the global node id of the $i$ th node of the eth neighbor element.

\subsection{Unstructured mesh generation and hy- brid mesh optimization}

Once the sweeping process is finalized, the unstructured tetrahedral mesher TetGen [41] is used to generate a volume mesh that fills the rest of the domain up to the desired height. To do so, the target volume is defined in terms of a planar ceiling, an elliptical surface that encloses all the perimeter of the initial surface mesh, and the top boundary of the prismatic mesh. Each triangle of the boundary that encloses the volume is assigned with an element size field that determines the size of the tetrahedral mesh. In particular, the triangles from the swept prismatic mesh are assigned a size that ensures a smooth matching between the prismatic and the tetrahedral mesh. The triangles of the planar top ceiling are assigned the element size chosen by the user. Finally, the triangles from the elliptic lateral are assigned an element size computed using a linear blend between the size at the swept surface and the size at the planar top ceiling.

Once all the volume mesh has been generated, only the prismatic elements have been optimized in terms of the 


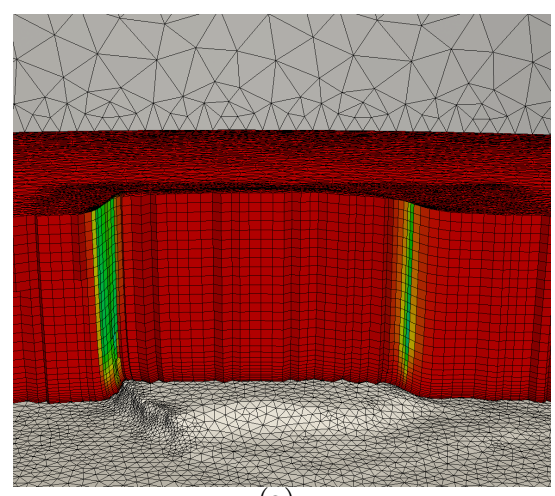

(a)

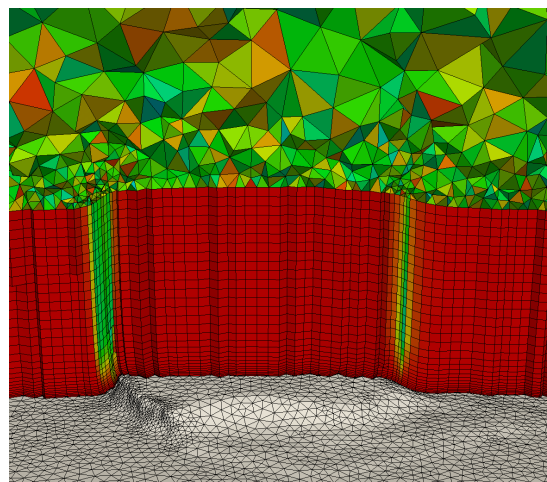

(b)

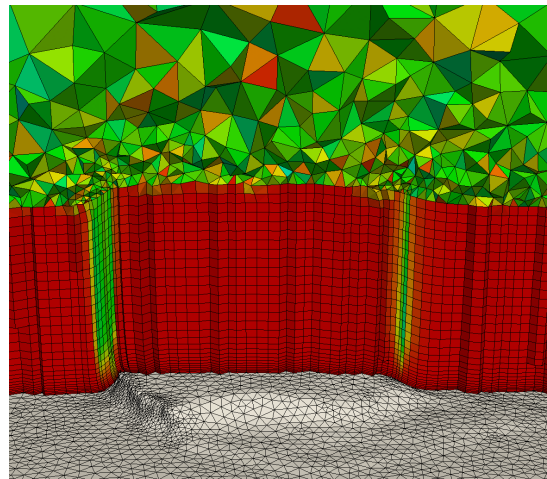

(c)

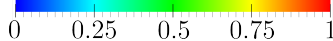

Figure 4: Main meshing steps to generate the mesh of the Atmospheric Boundary Layer for the Bolund hill: (a) prismatic mesh, (b) hybrid prismatic-tetrahedral mesh, and (c) globally optimized hybrid mesh. The elements are colored according to their quality.

quality presented in Eq. (17), and only locally in the sweeping process to improve the previous configuration before a new surface sweep. Therefore, the mesh is still not globally optimal in terms of the defined quality (distortion) measure. Thus, after the topology of the final mesh has been set, we perform a final mesh optimization to compute the coordinates of the mesh nodes that deliver minimal distortion. To do so, we optimize all the nodes except the topography surface ones. The nodes on the topography are kept fixed since they are already optimal in the parameterized topography geometry. The volume nodes are free to move in $\mathbb{R}^{3}$, whereas the rest of boundary nodes are allowed to move on their boundary surfaces.

In particular, we find the coordinates of the nodes that do not belong to the topography that minimize the mesh distortion in the least-squares sense [34], by optimizing in a Gauss-Seidel approach the objective function

$$
f\left(\mathbf{z}_{1}, \ldots, \mathbf{z}_{n}\right)=\sum_{e=1}^{n_{E}} \eta_{e}^{2}\left(\mathbf{z}_{e_{1}}, \ldots, \mathbf{z}_{e_{n_{p}}}\right)
$$

where $n_{N}$ is the number of nodes of the mesh, $n_{E}$ is the number of elements in the mesh, $\eta_{e}$ is the distortion of the $e$-th element, and $e_{i}$ corresponds to the global node id of the $i t h$ node of element $e$.

Figure 4 illustrates the generated hybrid volume mesh from the topography surface mesh illustrated in Figure 3(b). Figure 4(a) shows the first step of the process, the prismatic mesh obtaining by sweeping the topography surface mesh. Figure 4(b) shows the hybrid mesh obtained after meshing with tetrahedra the domain between the top triangles mesh from the prismatic sweep mesh and the top ceiling mesh. Finally in Figure 4(c) we illustrate the final hybrid mesh obtained after the performed quality optimization. We highlight that the domain has been designed to clearly illustrate both the topography adaptation, the prismatic mesh and the unstructured mesh in the same image. That is, the sizes of the farm, transition and buffer areas, and the region featuring the boundary layer are not realistic for an ABL flow simulation. A complete Bolund scenario is meshed in Section 6.1. Table 2 details the quality of the meshes presented in Figure 4. The minimum quality of the final mesh, Figure $4(\mathrm{c})$, is 0.2 . The minimum is obtained in prismatic mesh in the region extruded close to the almost vertical wall from the geometry, where the mesh has been more refined. We highlight that without the final mesh optimization the minimum quality of the mesh is 0.1 , presenting a significant quality improvement due to the presented optimization process. In addition, if no volumetric optimization is present during the extruding procedure (Sec. 5.1), the mesh becomes invalid during the sweeping process and the mesh cannot be generated. This issue stresses the importance of the quality optimization framework, specially in regions where the topography features high gradients. 


\section{RESULTS}

In this section, we present two examples to illustrate the capabilities of the presented hybrid mesh generation approach adapted to the topography. First, in Section 6.1 it is performed an analysis of the meshes obtained using the hybrid process in contrast with the standard semi-structured hexahedral meshers for ABL flows. In particular, the same mesh sizing parameters are used to analyze the main differences between the semi-structured and the unstructured approaches. Second, in Section 6.2 the meshing process is illustrated for the topography encircling the Badaia wind farm, located in Spain.

In Section 6.2, the generated mesh is used to solve the Reynolds Averaged Navier-Stokes (RANS) equations with a $\mathrm{k}-\varepsilon$ turbulence model adapted to the Atmospheric Boundary Layer [44] and featuring Coriolis effects. The model was presented and developed in $[10,45]$ and implemented in the finite-element multiphysics parallel solver Alya [46, 47]. However, the proposed meshing algorithm has been implemented in the external model-independent pre-process code WindMesh $[10,9,16,48]$. As a result, meshes generated with this utility can be used to simulate both with RANS or Large-Eddy Simulation (LES) turbulence models and, in addition, are also valid for solvers based on other numerical methods such as Finite Volumes. The simulation illustrated in Figure 8 has been run in the supercomputer MareNostrum4 [49] using 512 cores.

For the different examples, we detail the computational time of the mesh generation process performed on a MacBook Pro with one dual-core Intel Core i7 $\mathrm{CPU}$, a clock frequency of $3.0 \mathrm{GHz}$, and a total memory of 16 GBytes. In Section 6.1, the computational time to generate the semi-structured mesh in Figure 5 (a) is 9 seconds, and the time to generate the hybrid mesh in Figure 5(b) is $17 \mathrm{~s}$. In Section 6.2, the CPU time to generate the hybrid mesh in Figure 7 is 246 seconds.

\subsection{Bolund peninsula}

The objective of this work is to propose a new mesher to simulate ABL flows featuring Coriollis effects in complex topographies. In this context, the motivation of this section is to present a simple example to illustrate the advantages of using the proposed approach against a standard semi-structured ABL mesher in terms of the resulting element count and the flexibility to attain the desired element sizes in the domain. We highlight that standard meshers are devoted for problems without Coriollis. In those cases there is a dominant large-scale wind direction at the top ceiling, and this is exploited by means of gener- ating semi-structured hexahedral meshes aligned with this inflow direction. However, in cases featuring complex topographies, the direction of the wind close to the ground is not dominated by a particular direction. As previously highlighted, this problem is increased when Coriollis effects are present, where even without topography the wind direction changes depending on the height with respect to the ground. Thus, in contrast with standard meshers, we have developed a specific-purpose mesher for complex topographies and simulations featuring Coriollis effects.

The comparison of this section is performed with the aim to illustrate the advantages of the meshing approach proposed in this work for the specific-purpose for which it has been developed. One of the objectives of the hybrid mesher is to reduce the degrees of freedom required to discretize a complex topography and the Atmospheric Boundary Layer in simulations featuring Coriollis effects. Hence, we will measure the reduction of degrees of freedom of the proposed mesher in contrast with the standard approach $[6,7,8,9,16,10,11]$. However, we highlight that semi-structured meshers have the advantage of being aligned with the wind inflow direction in problems without Coriollis effects or without complex topographies.

To perform this comparison, we use our in-house semistructured code WindMesh $[9,16]$, that generates a semi-structured cross-type mesh, see Figure 5(a), which constitutes the standard in the field of ABL flow simulation $[6,7,8,9,10,11,16]$. As previously highlighted, these semi-structured meshers are devoted to generate an hexahedral mesh aligned with the inflow direction. Thus a structured quadrilateral mesh is generated in the interest regions, and then an elliptical domain is meshed using a semi-structured approach increasing the mesh size towards the exterior of the domain. Next, this mesh is extruded to discretize the boundary layer, see $[9,16]$ for further details.

Figure 5 illustrates the obtained meshes. Figure 5(a) shows the generated semi-structured mesh, while Figure 5(b) we illustrate the hybrid mesh generated with exactly the same parameters than the semi-structured one. That is, the imposed mesh sizing is exactly the same. In this particular test case, featuring a small domain with a small topographic scenario around Bolund hill (a small peninsula in Denmark), the input sizes are: 5 meters in the farm region and 25 in the buffer region. Regarding the vertical discretization, the initial cell size is 0.5 and the growing ratio is 1.15 . We highlight that in this example, we do not adapt the hybrid mesh to the topography, to perform a fair comparison with the semi-structured approach. However, the adaptive procedure can also be exploited to locate the degrees of freedom where they are really required 


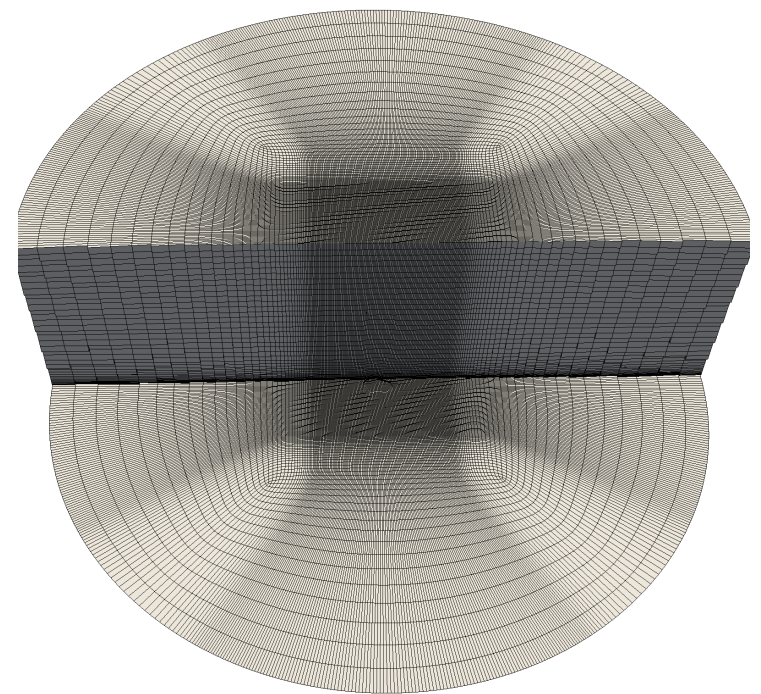

(a)

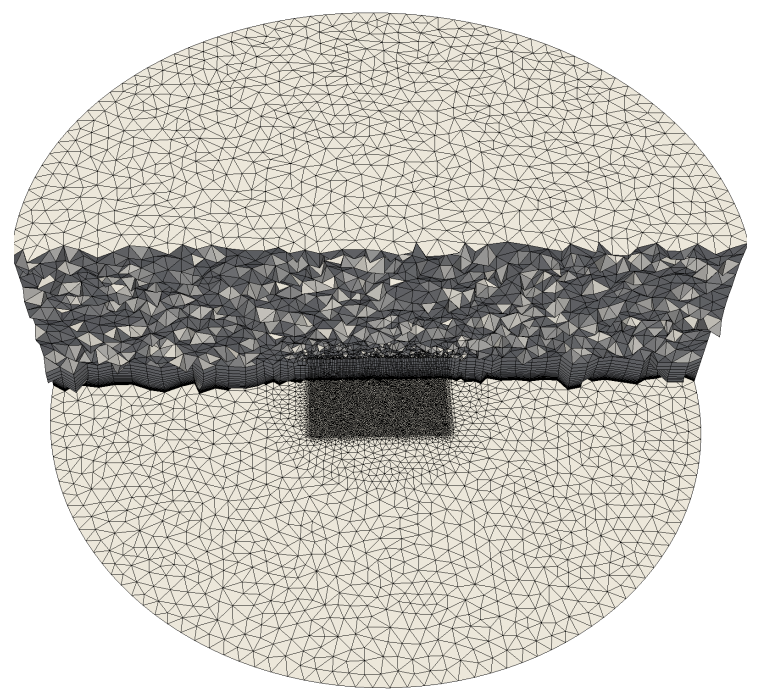

(b)

Figure 5: Meshes generated on Bolund hill: (a) standard semi-structured mesh and (b) hybrid mesh.

and reduce even more the total element count.

Regarding the number of elements and nodes of the generated meshes, the hexahedral mesh in Figure 5(a) is composed by $1407780(1.41 \mathrm{M})$ nodes and 1362756 $(1.36 \mathrm{M})$ elements. In contrast, the meshes in Figure $5(\mathrm{~b})$ is composed by $268698(0.27 \mathrm{M})$ nodes and $767444(0.77 \mathrm{M})$ elements, from which 405680 (0.41M) are prisms and $361764(0.36 \mathrm{M})$ tetrahedra. In particular, we obtain a mesh with half of the elements and a fifth of the number of nodes but attaining the same required resolution than in the structured approach. Recall that the computational cost of a given simulation depends on the number of nodes, since the nodes determine the number of unknowns to solve. Thus, a fifth of the number of nodes is a significant reduction of the computational cost and of the size of the matrices involved in the solvers, which have a dimension of the order of the number of nodes. However, we have the additional advantage of being able to adapt the mesh to ensure that the element size on the surface is the desired and to capture the curvature of the geometry.

The difference in the element count derives from the flexibility of the new approach to attain the mesh sizes prescribed by the user in the different regions. In contrast, the semi-structured approach extends the highresolution areas towards the rest of the domain, both in the surface mesh and in the volume mesh when extruding the boundary layer. It must be highlighted again that, in applications with a dominant wind direction and not introducing the Coriolis effect on the RANS equations, the semi-structured mesh presents a clear advantage, which is the alignment of the elements with the flow direction. However, we are in- terested on onshore problems featuring Coriolis, and thus the combination of the effect of complex topographic scenarios and a wind direction that changes according to the height reduces the advantages from a semi-structured approach for our objectives. Thus, in our application, without a unique wind direction, the hybrid approach reduces to one fifth the degrees of freedom of the simulation and simultaneously we reduce the complexity of generating a different mesh for each inflow direction.

Alternatively to the unstructured hybrid approach presented in this work, an unstructured quadrilateral surface mesh and an unstructured hexahedral mesh could also be an alternative to the standard semistructured approach. However, the existence of mature Delaunay-based meshers such as Triangle [32] and TetGen [41], has allowed us in this work to focus on dealing with the input topography data, the geometry re-parameterization, the adaptation process and the boundary layer meshing. Together with the lack of a unique wind direction, we have favored a fully unstructured triangle approach on the surface that is adapted to the topography features, relying on existent mature technology provided by Triangle. Regarding the volume mesh, we have exploited the vertical structure of the SBL and generated first a prismatic region. Finally, we have generated an unstructured tetrahedaral mesh to allow a size gradation in all the directions out of the SBL, relying on the mature technology provided by TetGen. 


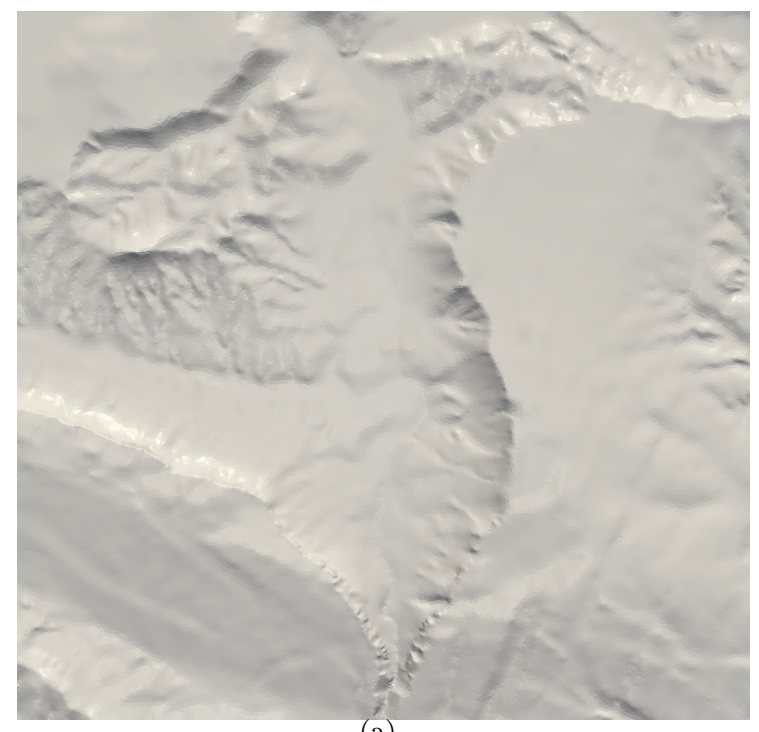

(a)

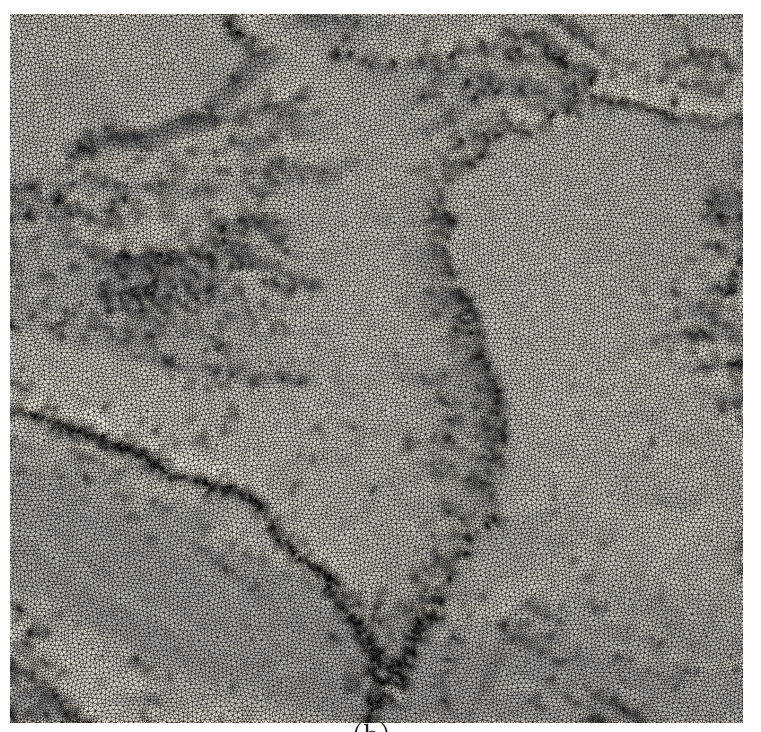

(b)

Figure 6: (a) Badaia topography, and (b) adapted surface mesh.

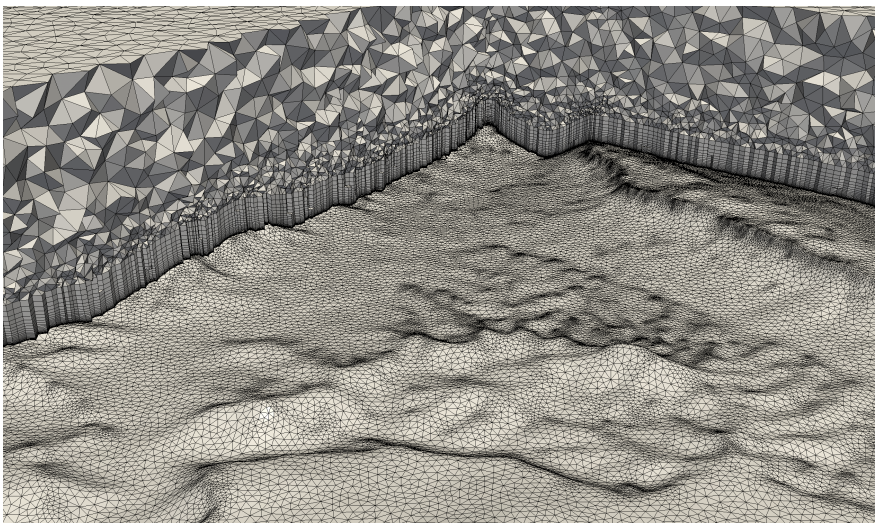

(a)

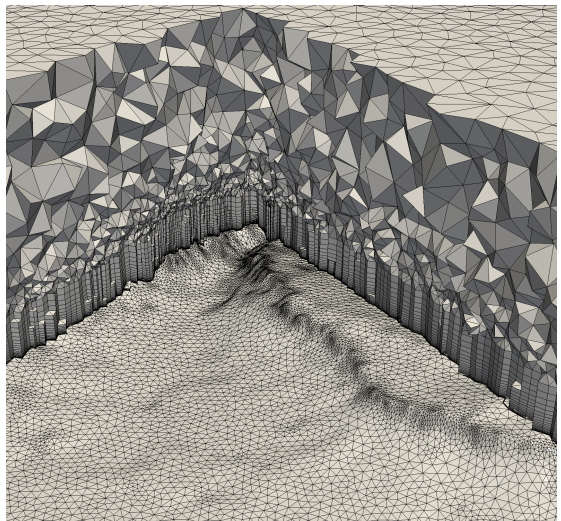

(b)

Figure 7: ABL hybrid mesh on Badaia topography: (a) overview and (b) close-up.

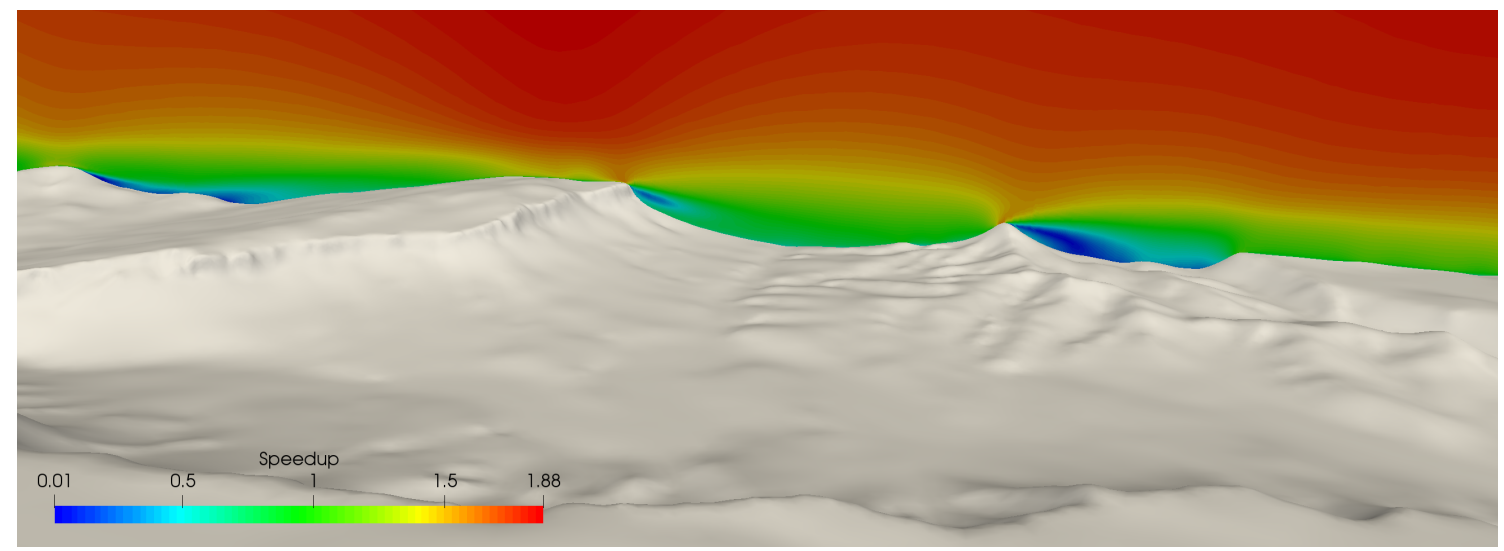

Figure 8: Velocity speedup with respect to a reference point upwind over Badaia topography. 


\subsection{Badaia topography}

In this section, we present the adapted mesh generated on the Badaia topographic scenario, Figure 6(a), located in Spain. The main objective is to illustrate both the adaptive surface technique and the hybrid volume mesher in a real scenario. This topography features a valley surrounded by several plateaus and mountains with orographic steps up to 700 meters. The minimum height of the topography is 388 meters over the sea level, and the maximum 1098 meters. The area of the target meshed domain is of $20 \times 20 \mathrm{~km}^{2}$, and the top ceiling of the mesh is located at $2 \mathrm{~km}$ over the highest topographic point.

The surface meshing procedure generates a triangle surface mesh with elements featuring edges of at most 75 meters, which are allowed to be refined up to 10 meters to capture the geometry curvature. The generated surface mesh is presented in Figure 6(b). The initial mesh is composed by 41531 nodes and 82379 elements. The mesher performs 4 cycles of the refinement procedure presented in Section 4.1. The final surface mesh is composed by 62469 nodes and 124172 elements. After the adaptive procedure the obtained mesh has a minimum elemental quality of 0.2 , which is improved up to 0.24 after performing the optimization procedure presented in Section 4.2.

In Figure 7 two different slices of the volume mesh are presented. The generated volumetric mesh is composed by $2.2 \mathrm{M}$ nodes and $5.6 \mathrm{M}$ elements, from which $3.7 \mathrm{M}$ are prisms and $1.9 \mathrm{M}$ are tetrahedra. The mean quality of the generated mesh is 0.84 and the minimum is 0.08. After the mesh optimization, the minimum mesh quality is improved up to 0.11 , whereas the mean remains is 0.84 . In Figure 8 we illustrate the applicability of the generated meshes for simulation. In particular, we illustrate the wind velocity speedup with respect to a reference point upwind. The wind inflow direction is from left to right of the domain.

Although out of the scope of this paper, in the future we would like to perform an actual study of the benefits of the topography adapted mesh on the solution of the ABL flow. The fact that the mesh can be adapted to the topography may allow us to reduce the degrees of freedom, augmenting the mesh size in large flat regions, and increasing the resolution around the features of the topography that require it.

\section{CONCLUDING REMARKS AND FUTURE WORK}

In this work, we have presented a new meshing strategy to simulate ABL flows on onshore topographical scenarios. The first contribution is an adaptive procedure to attain the desired mesh size on the topography surface and to capture the curvature of the geome- try. Since topographic geometries are discrete (point clouds, contour lines or triangle meshes in contrast to CAD geometries), to compute the required metrics to perform the mesh adaptivity, a new approach to compute local smooth parameterizations of the geometry is presented. In particular, the geometry is locally approximated by a high-order polynomial of the desired degree (by default 3 ) that allows to compute a local continuous first and second order derivatives. Using those derivatives, we compute the metric of the tangent space and a curvature metric. These metrics allow us to refine the elements of the mesh to attain the desired edge length of the surface mesh and to discretize the curvature of the geometry with the desired accuracy.

The second contribution is the new hybrid volume mesh generation procedure. On the first $10-20 \%$ of the domain a prismatic mesh is generated to capture the Surface Boundary Layer flow. On the rest of the domain, the flexibility of tetrahedra is exploited to avoid extending the fine mesh on the interest region to the rest of the domain. The result is an hybrid mesh that resolves the Surface Boundary Layer using prisms, and then discretizes the rest of the domain with the desired resolution using tetrahedra. The tetrahedral elements allow imposing a smooth size transition between the finer element size of the prismatic layer and the coarser element size desired in the top planar ceiling.

To illustrate the advantages in terms of the number of generated degrees of freedom, in Section 6.1 a standard semi-structured mesh is compared to the new strategy. In addition, Section 6.2 illustrates the applicability of the mesher to discretize complex topographical scenarios producing valid meshes for simulation. We discretize the Badaia topography with the hybrid approach and we use the generated mesh to perform a RANS simulation using the model presented in $[45,10]$.

In the near future, we will perform a detailed analysis of the advantages and disadvantages of the use of an hybrid approach to simulate ABL flows against the standard semi-structured approach, both in terms of the simulation accuracy and the number of degrees of freedom of the simulation. In addition, we would like to study the possibility to use the topography adaptation procedure to reduce the degrees of freedom of the mesh, by means of increasing the mesh size in flat areas and only requiring a finer mesh size around the topography features. Regarding the application of the mesher for wind farm assessment, we would also like to explore using the generated hybrid mesh to discretize wind farms modeled with the actuator disc model with a similar approach to the one presented in $[9,16]$. 


\section{ACKNOWLEDGMENTS}

This work has been partially funded by the EU H2020 Energy oriented Center of Excellence (EoCoE) for computer applications, the New European Wind Atlas (NEWA) and the High Performance Computing for Energy (HPC4E) projects. We thank Iberdrola Renovables [50] for their collaboration and for providing the topography data.

\section{References}

[1] Köpp F., Schwiesow R., Werner C. "Remote measurements of boundary-layer wind profiles using a CW Doppler lidar." Journal of climate and applied meteorology, vol. 23, no. 1, 148-154, 1984

[2] Garratt J.R. "The atmospheric boundary layer." Earth-Science Reviews, vol. 37, no. 1-2, 89-134, 1994

[3] Blocken B., Stathopoulos T., Carmeliet J. "CFD simulation of the atmospheric boundary layer: wall function problems." Atmospheric environment, vol. 41, no. 2, 238-252, 2007

[4] Wizelius T. Developing wind power projects: theory and practice. Earthscan, 2007

[5] Stull R.B. An introduction to boundary layer meteorology, vol. 13. Springer Science \& Business Media, 2012

[6] Sørensen N.N. "HypGrid2D a 2-D mesh generator." Tech. rep., Denmark. Forskningscenter Risoe. Risoe-R; No. 1035(EN), 1998

[7] Stefan I., Mikkelsen R., Sørensen N.N., Henningson D. "Validation of methods using EllipSys3D." Tech. rep., Engelska, 2008

[8] Weller H. https://openfoam.org/, 2018

"OpenFOAM."

[9] Gargallo-Peiró A., Avila M., Owen H., Prieto L., Folch A. "Mesh Generation for Atmospheric Boundary Layer Simulation in Wind Farm Design and Management." Procedia Engineering, vol. 124, 239-251, 2015

[10] Avila M., Gargallo-Peiró A., Folch A. "A CFD framework for offshore and onshore wind farm simulation." Journal of Physics: Conference Series, vol. 854, no. 1, 012002, 2017

[11] B. Bichet T.F., Alexandre P. "Comparison of two CFD tools against measurements on complex terrain." 1st Symposium OpenFOAM dedicated to Wind Energy, pp. 1-19. 2013
[12] Haltiner G.J., Williams R.T. Numerical prediction and dynamic meteorology. Wiley, 1980

[13] Arakawa A., Konor C.S. "Vertical differencing of the primitive equations based on the CharneyPhillips grid in hybrid $\sigma$-p vertical coordinates." Month. Weather Rev., vol. 124, no. 3, 511-528, 1996

[14] Michelsen J.A. Block structured Multigrid solution of $2 D$ and $3 D$ elliptic PDE's. Department of Fluid Mechanics, Technical University of Denmark, 1994

[15] Marras S. Variational multiscale stabilization of finite and spectral elements for dry and moist atmospheric problems. Ph.D. thesis, Universitat Politècnica de Catalunya, 2012

[16] Gargallo-Peiró A., Avila M., Owen H., PrietoGodino L., Folch A. "Mesh generation, sizing and convergence for onshore and offshore wind farm Atmospheric Boundary Layer flow simulation with actuator discs." Journal of Computational Physics, vol. 375, 209-227, 2018

[17] Montero G., Montenegro R., Escobar J. "A 3-D diagnostic model for wind field adjustment." Journal of Wind Engineering and Industrial Aerodynamics, vol. 74, 249-261, 1998

[18] Montero G., Rodrıguez E., Montenegro R., Escobar J.M., González-Yuste J.M. "Genetic algorithms for an improved parameter estimation with local refinement of tetrahedral meshes in a wind model." Advances in Engineering Software, vol. 36 , no. $1,3-10,2005$

[19] Escobar J.M., Montero G., Montenegro R., Rodríguez E. "An algebraic method for smoothing surface triangulations on a local parametric space." Int. J. Numer. Meth. Eng., vol. 66, no. 4, 740-760, 2006

[20] Montenegro R., Montero G., Escobar J.M., Rodríguez E., González-Yuste J.M. "Tetrahedral Mesh Generation for Environmental Problems over Complex Terrains." P. Sloot, A. Hoekstra, C. Tan, J. Dongarra, editors, Computational Science ICCS 2002, vol. 2329 of Lecture Notes in Computer Science, pp. 335-344. Springer Berlin Heidelberg, 2002

[21] Behrens J., Rakowsky N., Hiller W., Handorf D., Läuter M., Päpke J., Dethloff K. "amatos: Parallel adaptive mesh generator for atmospheric and oceanic simulation." Ocean modelling, vol. 10, no. 1-2, 171-183, 2005 
[22] Oliver A., Rodríguez E., Escobar J., Montero G., Hortal M., Calvo J., Cascón J., Montenegro R. "Wind Forecasting Based on the HARMONIE Model and Adaptive Finite Elements." Pure and Applied Geophysics, vol. 172, no. 1, 109-120, 2015

[23] Gargallo-Peiró A., Folch A., Roca X. "Representing Urban Geometries for Unstructured Mesh Generation." Procedia Engineering, vol. 163, 175-185, 2016. 25th International Meshing Roundtable

[24] Frey P.J. "About Surface Remeshing." Proc. 9th Int. Meshing Roundtable, 2000

[25] Frey P., Borouchaki H. "Surface meshing using a geometric error estimate." International journal for numerical methods in engineering, vol. 58, no. 2, 227-245, 2003

[26] Frey P., George P. Mesh Generation: Application to Finite Elements. Wiley, 2008

[27] Peraire J., Vahdati M., Morgan K., Zienkiewicz O.C. "Adaptive remeshing for compressible flow computations." J. Comput. Phys., vol. 72, no. 2, $449-466,1987$

[28] Frey P.J., Alauzet F. "Anisotropic mesh adaptation for CFD computations." Computer methods in applied mechanics and engineering, vol. 194, no. 48-49, 5068-5082, 2005

[29] Loseille A., Alauzet F. "Optimal 3D highly anisotropic mesh adaptation based on the continuous mesh framework." Proc. 18th Int. Meshing Roundtable, pp. 575-594. Springer, 2009

[30] Loseille A., Alauzet F. "Continuous mesh framework part I: well-posed continuous interpolation error." SIAM Journal on Numerical Analysis, vol. 49, no. 1, 38-60, 2011

[31] Loseille A., Alauzet F. "Continuous mesh framework part II: validations and applications." SIAM Journal on Numerical Analysis, vol. 49, no. 1, 6186,2011

[32] Shewchuk J.R. "Triangle: Engineering a 2D quality mesh generator and Delaunay triangulator." Applied computational geometry towards geometric engineering, pp. 203-222. Springer, 1996

[33] Knupp P.M. "Algebraic mesh quality metrics for unstructured initial meshes." Finite Elem. Anal. Des., vol. 39, no. 3, 217-241, 2003

[34] Gargallo-Peiró A., Roca X., Peraire J., Sarrate J. "Optimization of a regularized distortion measure to generate curved high-order unstructured tetrahedral meshes." Int. J. Numer. Meth. Eng., vol. 103, 342-363, 2015
[35] Gargallo-Peiró A., Roca X., Peraire J., Sarrate J. "Distortion and quality measures for validating and generating high-order tetrahedral meshes." Eng. Comput., vol. 31, 423-437, 2015

[36] Gargallo-Peiró A., Roca X., Peraire J., Sarrate J. "A distortion measure to validate and generate curved high-order meshes on CAD surfaces with independence of parameterization." Int. J. Numer. Meth. Eng., vol. 106, no. 13, 1100-1130, 2015

[37] Gargallo-Peiró A., Roca Navarro X., Peraire J., Sarrate J. "High-order mesh generation on CAD geometries." Adaptive Modeling and Simulation 2013, pp. 301-312. 2013

[38] Garanzha V., Kaporin I. "Regularization of the Barrier Variational Method." Computational mathematics and mathematical physics, vol. 39, no. 9, 1426-1440, 1999

[39] Escobar J.M., Rodríguez E., Montenegro R., Montero G., González-Yuste J.M. "Simultaneous untangling and smoothing of tetrahedral meshes." Comput. Meth. Appl. Mech. Eng., vol. 192, no. 25, 2775-2787, 2003

[40] Gargallo-Peiró A., Roca X., Sarrate J. "A surface mesh smoothing and untangling method independent of the CAD parameterization." Comput. Mech., vol. 53, no. 4, 587-609, 2014

[41] Si H. "TetGen, a Delaunay-Based Quality Tetrahedral Mesh Generator." ACM Trans. Math. Softw., vol. 41, no. 2, 11:1-11:36, 2015

[42] Roca X. Paving the path towards automatic hexahedral mesh generation. Ph.D. thesis, Universitat Politècnica de Catalunya, 2009

[43] Roca X., Sarrate J. "An automatic and general least-squares projection procedure for sweep meshing." Eng. Comput., vol. 26, no. 4, 391-406, 2010

[44] Apsley D., Casrto I. "A limited-length-scale k- $\varepsilon$ model for the neutral and stably-stratified Atmospheric Boundary Layer." Bound.-Lay. Meteorol., vol. 83, no. 1, 75-98, 1997

[45] Avila M., Folch A., Houzeaux G., Eguzkitza B., Prieto L., Cabezón D. "A Parallel CFD Model for Wind Farms." Procedia Computer Science, vol. 18, no. 0, 2157 - 2166, 2013. 2013 International Conference on Computational Science

[46] Houzeaux G., Vázquez M., Aubry R., Cela J. "A massively parallel fractional step solver for incompressible flows." J. Comput. Phys., vol. 228, no. $17,6316-6332,2009$ 
[47] Vázquez M., Houzeaux G., Koric S., Artigues A., Aguado-Sierra J., Arís R., Mira D., Calmet H., Cucchietti F., Owen H., Taha A., Burness E., Cela J., Valero M. "Alya: Multiphysics engineering simulation toward exascale." Journal of Computational Science, vol. 14, 15-27, 2016

[48] Gargallo-Peiró A., Houzeaux G., Roca X. "Subdividing triangular and quadrilateral meshes in parallel to approximate curved geometries." Procedia Engineering, vol. 203, 310-322, 2017

[49] Barcelona Supercomputing Center. "MareNostrum 3." https://www.bsc.es/marenostrum, 2018

[50] Dpt. E.R. "Iberdrola Renovables." http://www.iberdrolarenovablesenergia.com, 2001 


\title{
CERTIFIED FUNCTIONS FOR MESH GENERATION
}

\author{
Andrey N. Chernikov \\ Old Dominion University, Norfolk, VA, U.S.A. achernik@odu.edu
}

\begin{abstract}
Formal methods allow for building correct-by-construction software with provable guarantees. The formal development presented here resulted in certified executable functions for mesh generation. The term certified means that their correctness is established via an artifact, or certificate, which is a statement of these functions in a formal language along with the proofs of their correctness. The term is meaningful only when qualified by a specific set of properties that are proven. This manuscript elaborates on the precise statements of the properties being proven and their role in an implementation of a version of the Isosurface Stuffing algorithm by Labelle and Shewchuk. This work makes use of the Calculus of Inductive Constructions for defining executable functions, stating their properties, and proving these properties via a direct examination of these functions (the property of liveness). The certificate is made available for inspection and execution using the Coq proof assistant.
\end{abstract}

Keywords: guaranteed quality mesh generation, formal methods, correct-by-construction, certified software

\section{INTRODUCTION}

Formal methods have undergone a rapid development in recent years. There are a number of reasons for this phenomenon. First, as algorithms and their software implementations are becoming increasingly sophisticated, they require a corresponding increase in the efforts to argue about the properties of the results they produce. For a number of complex algorithms, like unstructured guaranteed quality mesh generation, the complexity of stating and proving their specifications necessitates multiple manuscripts and pushes the limits of the traditional paper-and-pencil approach. Second, parallel algorithms, which have been developed for virtually all areas of computing, often bear another order-of-magnitude intricacy factor over the corresponding sequential algorithms due to numerous ways a parallel system can process and move data. Third, as automated systems are becoming increasingly integrated, a failure of one component has ramifications for the whole software-hardware system and can lead to costly and/or dangerous consequences. A number of major organizations, including Amazon [1], Microsoft [2], Twitter [3], Intel [4], MIT [5,6], and
Inria $[7,8]$, have made progress in the development and the use of formal methods and functional programming towards managing software correctness and maintainability.

This work is the second formal verification in the area of mesh generation, to the best of the author's knowledge, that exhibits the property of liveness [9], i.e., being connected to the implementation via machinechecked proofs. The property of liveness increases the level of confidence in the correctness, as the proofs make use of the properties of the actual operations used in the code, not just abstracted models of those operations. The first work was published by Chernikov and $\mathrm{Xu}[10]$ on the correctness of a version of a Marching Cubes algorithm [11]. The present work proves certain correctness properties of the Isosurface Stuffing algorithm [12], and also differs from the previous [10] in two major aspects. The first one is that it works with tetrahedra, not just triangular faces. The second difference is in the proof approach. The previous work [10] used proofs via computation, i.e., the properties being proven were embedded in the functions computed as part of the proof. The present work sep- 
arates the proofs into two parts: propositional specifications that state the properties being proven, and the actual proofs that establish that these specifications hold. As a result, the present approach is easier to read and maintain. Another known work, in formally proving properties of a plane Delaunay triangulation algorithm, is that of Dufourd and Bertot [13]. The work [13] does not exhibit the property of liveness as it relies only on axiomatization, not implementation, of real numbers. The underlying number type in the present work is integers, which is implemented in the standard library of the Coq environment [8] being used, and suitable due to the lattice-based nature of the algorithm being studied. When required by the algorithm, the intersections with the surface are computed using integer arithmetic to within a finite precision.

The development presented herein resulted in certified executable functions for mesh generation. The term certified means that their correctness is established via an artifact, or certificate, which is a statement of these functions in a formal language along with the proofs of their correctness. The term is meaningful only when qualified by a specific set of properties that are proven. The functions whose properties are being proven and the precise formal statements of their certified properties are elaborated further down in this manuscript. The certificate in the form of an accompanying Coq script is made available [14]. As can be seen from the Venn diagram in Figure 1, only a subset of the entire implementation is involved in the proof of the properties stated below. A certificate with a formal proof of another set of properties may involve a different subset of functions.

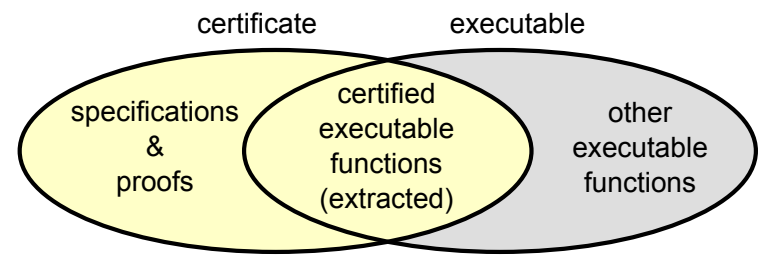

Figure 1: A Venn diagram for the components of certified executable software. The parts described here are in yellow.

Published in 2007 by Labelle and Shewchuk [12], the Isosurface Stuffing (IS) algorithm solved a longstanding problem in tetrahedral meshing of geometrically complex shapes, that of achieving practically significant guaranteed dihedral angle bounds. Among other favorable properties of this algorithm are its fast execution, mesh gradation, placement of mesh boundary vertices exactly (to within a tolerance) onto the domain boundaries, as well as certain fidelity guarantees. Conceptually, the IS algorithm is similar to the well-known Marching Cubes (MC) algorithm [11]. Both algorithms employ a regular cubical grid to cover the region of interest. The IS algorithm uses a body centered cubic (BCC) lattice that is tessellated into a structured tetrahedral grid. Both the MC and the IS algorithms evaluate each vertex of the corresponding grid using a given cut function, which returns one of three values, corresponding to the vertex being inside, outside, or on the surface of the shape defined by this function. Depending on the local combination of these values in the vertices of a cube, the MC algorithm outputs one of the predetermined stencils, which is a set of triangles that approximates the surface inside this cube. The IS algorithm evaluates the cut function in the vertices of a structured tetrahedron and outputs one of predefined sets of stencil tetrahedra (referred to as unstructured below). Both algorithms also make use of approximated locations of zero values of the cut function along the edges of the respective cubes or tetrahedra.

What distinguishes the IS algorithm from the MC and its variations is the presence of proofs that guarantee $a$ priori, i.e., before the execution, that the dihedral and certain other angles will be bounded by known constant values. Due to a large number of cases to examine and, most importantly, to the unknown locations of the zero values within respective edges, these proofs would be next to impossible to construct manually. Instead, the IS authors implemented and described a computer-assisted approach where the computation of the angle bounds is done in software with the use of interval arithmetic and of recursive bisection of the parameter space. What was not described, however, is an a priori proof of correct connectivity between the adjacent tetrahedral stencils. Such a proof is important as a precedent is known with the original MC algorithm, wherein symmetry was incorrectly used to reduce the number of stencils, thus causing topological holes in the approximated surface [10]. The present work offers a formal statement of the connectivity properties of the IS stencils along with the proofs that these properties hold.

Figure 2 shows two example meshes obtained with the certified implementation discussed here. The implementation uses the same stencils in the uniformly refined area next to the domain boundary as the original IS algorithm. However, unlike the IS algorithm, it does not implement the octree optimization with additional stencils that allow for fewer tetrahedra in the interior of the domain by omitting the creation of certain octree leaves. These additional octree stencils are left for future work.

The number of IS connectivity cases to be verified, when unfolded, grows rapidly. Every two adjacent structured tetrahedra that share a triangular face in- 

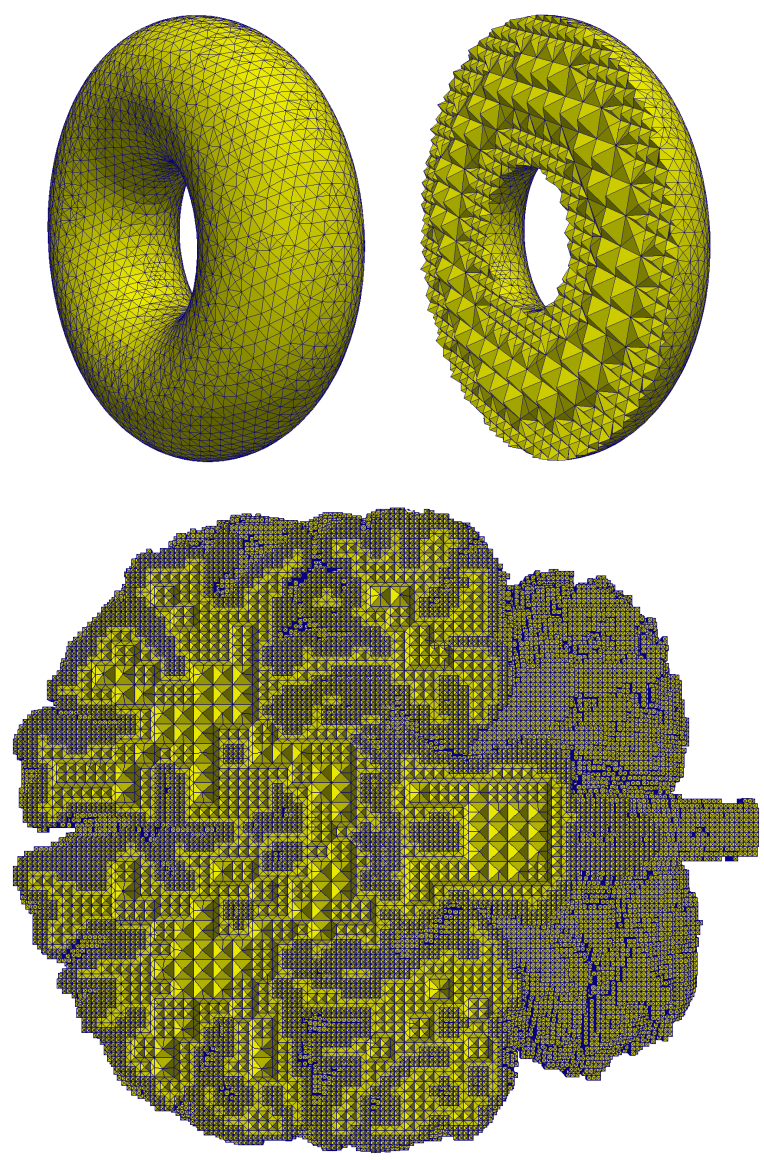

Figure 2: Top: the surface and a cross-section of a mesh of a torus defined by an analytical function (61,528 tetrahedra, $20.2^{\circ}$ minimum output dihedral angle). Bottom: a cross-section of a mesh of a human brain atlas [15] defined by an image $\left(9,788,808\right.$ tetrahedra, $30^{\circ}$ minimum output dihedral angle).

volve 5 vertices (see Figure 3) and, since each vertex can evaluate the cut function to one of 3 values, the number of 5 -vertex sign assignments is $3^{5}=243$. If a higher confidence in the stencils is aimed at, as is the case in the proofs that follow, each of the four faces of a structured tetrahedron needs to be examined separately, which leads to a multiplier of $4: 243 \cdot 4=972$. Also, if formulas for each case of a translation and a rotation of a structured tetrahedron differ, these formulas need to be looked at separately as well. In this development, 12 variations of a formula are used, which raises the number of cases to $972 \cdot 12=11,664$. Moreover, each case involves several (0 to 3 on each side of the shared face) unstructured tetrahedra that subdivide either of the adjacent structured tetrahedra, and every edge of each of these unstructured tetrahedra needs to be examined with respect to the subdivision of the shared face from the other side. A manual enumeration and examination of all such cases becomes highly time-consuming and error-prone. Another important consideration is the ability to modify and extend this algorithm without the need for a repeated manual analysis. Once an automatic (i.e., not depending on the stencils) verification procedure is developed, it can be trivially applied to another set of stencils.

As an example, Figure 3 shows two structured tetrahedra, $(f 1, f 2, f 3, p)$ and $(f 1, f 2, f 3, q)$, that share face $(f 1, f 2, f 3)$. The symbols in vertex positions show the signs of the cut function: "+" symbolizing the vertex being inside the domain, "-" being outside, and " 0 " lying on the boundary. The signs of vertices $f 1, f 2$, f3, $p$, and $q$ are computed by direct evaluation of the cut function. The positions of vertices $u, v$, and $w$ are computed by iterative bisection of the edges $(q, f 1)$, $(f 3, f 1)$, and $(f 2, f 1)$, respectively, in order to find (to within a tolerance) the zero values of the cut function, i.e., the intersections of these edges with the domain boundary. Applying the corresponding stencils [12] to subdivide the structured tetrahedron $(f 1, f 2, f 3, p)$, one obtains three unstructured tetrahedra: $(f 1, p, w$, $v),(f 3, p, w, v)$, and $(w, f 3, f 2, p)$. Similarly, for the structured tetrahedron $(f 1, f 2, f 3, q)$, one obtains four unstructured tetrahedra: $(f 1, u, v, w),(f 3, u, v, w)$, $(w, f 2, u, f 3)$, and $(q, f 2, u, f 3)$. Tetrahedra $(f 1, p$, $w, v)$ and $(f 1, u, v, w)$ are classified as located outside the domain, and are not considered in this work. The remaining unstructured tetrahedra, three on one side of the shared face and two on the other side, are classified as lying within the domain and are considered further.

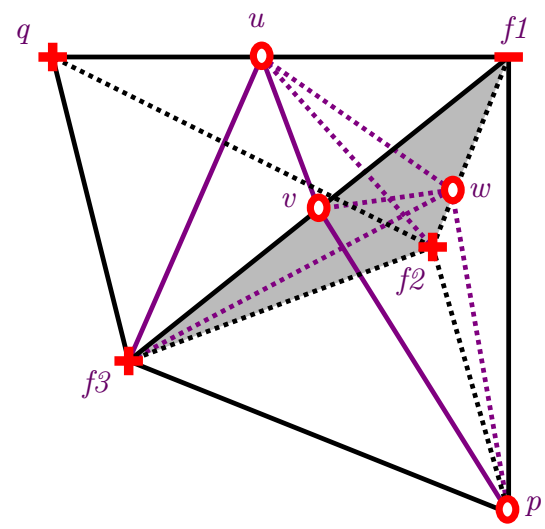

Figure 3: An example of two face-adjacent structured tetrahedra, $(f 1, f 2, f 3, p)$ and $(f 1, f 2, f 3, q)$ shown with black lines, a sign assignment to their vertices, and a subdivision into unstructured tetrahedra shown with purple lines. The shared face $(f 1, f 2, f 3)$ is shaded.

Via an examination of all edges of the unstructured tetrahedra classified as lying inside the domain, it can be concluded that the two sets of edges created inside the two respective structured tetrahedra are consistent 
within the shared face. In the rest of this work, formal conditions for such a verification are developed and proven.

Section 2 introduces the methodology used in this work. Section 3 describes the executable functions whose properties are certified. Section 4 presents the formally stated properties of the function that constructs structured tetrahedra and the proof of these properties. Section 5 certifies the function that constructs unstructured tetrahedra, considered separately, in a similar progression. In Section 6 the properties of both functions are proven together, which is necessary since unstructured tetrahedra are constructed out of the structured ones. Section 7 concludes the exposition with the summary of the presented work and expected extensions.

\section{METHODOLOGY}

The Curry-Howard correspondence is a fundamental concept in proof theory that considers proving to be a kind of programming. Indeed, while a computer program is a sequence of transformations of input data to output data, a proof is a sequence of transformations that takes the input proposition (premise) and converts it to the output proposition (conclusion). In this sense, implementing an algorithm as a function is similar to proving a theorem.

Computer-assisted proving involves a human user who guides the process through formulating intermediate steps and choosing proof techniques. The user interacts with a software program that manages the state of the proof, verifies the validity of the typed commands, and executes those commands.

Proof automation allows for finding proofs in special cases by invoking predefined proof search strategies. Once the user identifies that at a certain stage in the proving process a particular proof goal is suitable for some predefined automatic procedure, that procedure can be invoked in order to reduce the number of manually guided steps.

Purely functional programming is a programming methodology that views the subroutines to be similar to mathematical functions, whose return value depends only on the values of the parameters, and that produce no side effects. This methodology, when enforced in a 'pure' language, often translates to restricting the programming constructs so that there is no memory aliasing via pointers, parameters to functions are passed and returned 'by value', and mutable global values are not allowed. Eliminating side effects and aliasing makes it much easier to argue about the return value of a function, and therefore a pure functional programming language is a suitable choice for a proof assistant. Functional programming is contrasted to imperative programming that changes state with commands, such as, for example, an assignment.

Extraction is a methodology that separates the executable part of a program's specification from its logical part and saves it in a language that supports compilation and deployment.

The Coq formal proof management system [8] was chosen for this development. Coq combines a purely functional programming language called Gallina, which is based on a formal language Calculus of Inductive Constructions, and a vernacular language of commands that allow for stating software specifications and proving mathematical theorems. Some of the Coq's salient features that are relevant to the current exposition are briefly reviewed below. A thorough treatment can be found in relevant texts $[7,16,17]$.

Coq's Calculus of Inductive Constructions is a general theory that defines a typed programming language that can serve as constructive foundation of mathematics. Inductive definitions are in the core of Coq's language. For example, a basic number type used in this development, binary integer numbers $(Z)$, are defined inductively in Coq's standard library as follows.

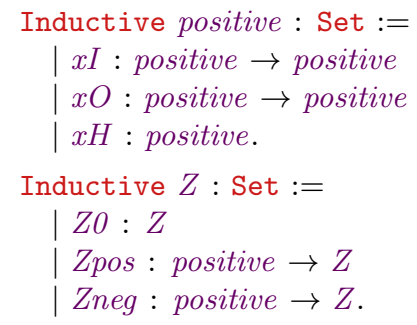

Type $Z$ can be thought of as a wrapper around the positive type, with its constructors corresponding to number 0 , some positive number, or some negated positive number, respectively. The positive number type is defined via constructor $x H$, representing binary digit 1 in the most significant position, and two constructors, $x O$ and $x I$, that add a least significant digit, 0 or 1 respectively, to a given positive number. For example, Zneg $(x O(x I x H))$ represents -6 . The arrow operator $\rightarrow$ is a special case of a universal quantifier and is used in a variety of contexts, including listing function and constructor arguments, as well as logical implication.

Polymorphism is a concept in programming that stands for providing a single interface to elements of different types. Polymorphism is fully supported in Coq by declaring undefined types as variables. In the current development, for example, the concrete representation of the Vertex data structure is irrelevant to the proof of unstructured stencil correctness. As a result, in the Coq script it was abstracted as an unspecified type: 
Variable Vertex : Type.

The unstructured tetrahedron data structure is then defined as four Vertex data elements appearing as parameters to the tetrahedron constructor $U T$ :

$$
\begin{aligned}
\text { Inductive UnstructuredTet } & := \\
\text { UT }: \text { Vertex } & \rightarrow \text { Vertex } \rightarrow \\
\text { Vertex } & \rightarrow \text { Vertex } \rightarrow \text { UnstructuredTet } .
\end{aligned}
$$

Closure of a function is a function that carries bindings to all the data structures referenced within it [18]. Along with polymorphism, functional closure allows for abstracting implementation details that are not needed for the proof part. One example of closure used in this development is the cut function named GetSign, which is bound to the definition of the domain to be meshed in the executable part of the implementation. However, the proof part only needs to know that this function takes a parameter of type Vertex and returns its Sign:

Variable GetSign : Vertex $\rightarrow$ Sign.

Tactics are predefined commands that are executed in the current proof environment to transform and eventually discharge the proof goal. Coq offers a collection of tactics that can accomplish a variety of logical transformations, such as applying a previously proven theorem (apply), proof by case enumeration (destruct), substitution of previously defined expressions (unfold), and many more. Some tactics exhibit a high degree of automation to ease the development effort on the part of the user. For example, one of the tactics used hereby, eauto, combines a Prolog-like resolution procedure auto with deferred instantiation of existential variables.

Tactical language in Coq is called Ltac. It contains a number of high-level commands that allow for applying tactics in various arrangements, including sequences, loops, and branches. The current development makes use of some of the features of this language, such as repeated application of tactics, catching exceptions, defining automated reusable proof procedures, and matching the shape of the current goal against a given pattern.

\section{CERTIFIED EXECUTABLE FUNCTIONS}

\subsection{Function for Constructing Structured Tetrahedra}

The IS algorithm uses structured tetrahedra to fill in the region of space to be meshed in the proximity of the domain boundary. The data structure for structured tetrahedra contains not only their vertex information, but also a sufficient amount of data to query all four of its neighbors adjacent via the faces, along with the vertices of the neighbors that correspond to the vertices of the given tetrahedron.

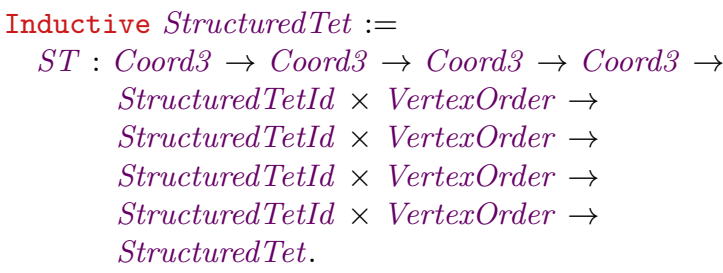

Here the Coord3 parameters to the constructor $S T$ are the three-dimensional coordinates of its vertices. The Coord3 data type simply combines three $Z$ values corresponding to each of the Euclidean dimensions.

$$
\begin{aligned}
& \text { Inductive Coord3 }:= \\
& \qquad \text { C3 }: Z \rightarrow Z \rightarrow Z \rightarrow \text { Coord3. }
\end{aligned}
$$

As mentioned above, the data structure for unstructured tetrahedra uses a variable, i.e., unspecified, type Vertex. When it is necessary to make the vertex types used in the proofs match, the Coord3 type is passed as a parameter that instantiates the Vertex type.

The type StructuredTetId is defined as follows.

$$
\begin{aligned}
& \text { Inductive StructuredTetId }:= \\
& \text { STId }: \text { Coord3 } \rightarrow \text { StructuredTetCase } \\
& \rightarrow \text { StructuredTetId. }
\end{aligned}
$$

The Coord3 parameter to the constructor STId is the three-dimensional coordinate of the reference, or anchor, that defines the position of the tetrahedron.

The type StructuredTetCase defines one of 12 possible orientations that are enumerated as constructors of this type:

$$
\begin{aligned}
& \text { Inductive StructuredTetCase : } \\
& \text { Tet_I1 | Tet_I2 | Tet_I3 | Tet_I4 | } \\
& \text { Tet_J1 | Tet_J2 | Tet_J3 | Tet_J4 | } \\
& \text { Tet_K1 | Tet_K2 | Tet_K3 | Tet_K4. }
\end{aligned}
$$

The VertexOrder parameters to the $S T$ constructor specify the ordering of the vertices of each neighbor tetrahedron, so that the corresponding vertices can be known (see Section 6). It is a function that returns a permutation of four vertices:

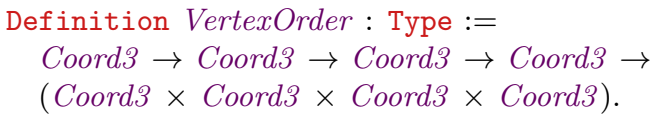

Figure 4 illustrates some of these data elements. The cube drawn with black lines has even-coordinate corners, and the one drawn with blue lines has oddcoordinate corners. The corner marked $i j k$ is the reference. The other corner labels indicate the offset from the reference coordinate. The red lines show the edges that are added to form four tetrahedra shown: $(220,222,311,331),(220,222,311,111)$, 
$(220,222,131,331),(220,222,131,111)$. These tetrahedra correspond to cases Tet_K1, Tet_K2, Tet_K3, and Tet_K4, respectively. Another set of four tetrahedra (not shown) is constructed around edge $(202,222)$. The third set of four tetrahedra (also not shown) is constructed around edge $(022,222)$. Twelve tetrahedra correspond to each reference coordinate $i j k$. The executable function GetStructuredTet returns one of these tetrahedra, depending on the case requested.

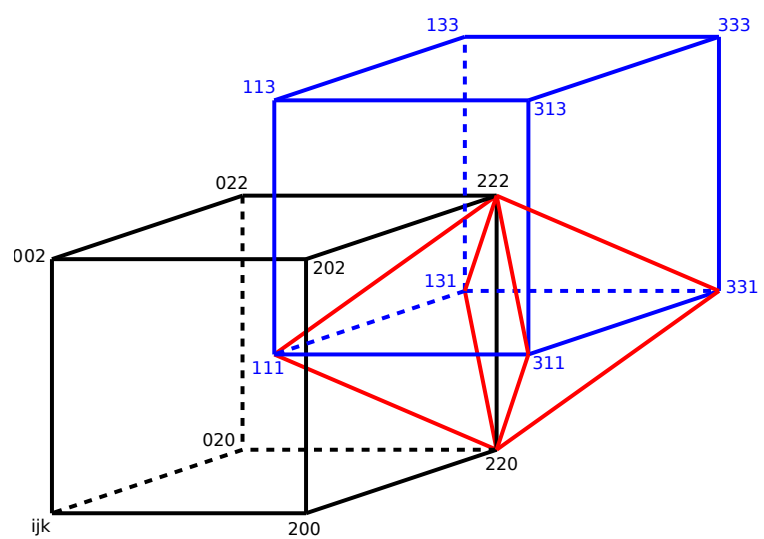

Figure 4: The vertices used by the function that constructs structured tetrahedra.

The structured tetrahedra can be defined by a simple formula that returns their connectivity with respect to the vertices of the BCC grid. This formula will translate and rotate a reference tetrahedron to define all other similar tetrahedra. Such a formula was encoded into an executable function named GetStructuredTet.

Definition GetStructuredTet (tid: StructuredTetId)

[omitted]

: StructuredTet :=

\subsection{Function for Constructing Unstruc- tured Tetrahedra}

The executable function GetUnstructuredTets returns a list of tetrahedra that subdivide a given structured tetrahedron. The parameters to this function $v 1$, v2, v3, v4 are vertices of the given structured tetrahedron. This function enumerates all of the 81 possible combinations of values GetSign v1, GetSign v2, GetSign v3, and GetSign v4, and returns a corresponding predefined list of unstructured tetrahedral stencils adopted from the original publication [12].

Definition GetUnstructuredTets

$$
\begin{aligned}
& (\text { v1 v2 v3 v4 : Vertex }) \\
& \text { : list UnstructuredTet }:=\text { [omitted] }
\end{aligned}
$$

\section{CERTIFICATE FOR STRUCTURED TETRAHEDRA}

\subsection{Specification}

The specification for the correctness of the structured tetrahedron returned by function GetStructuredTet states the following requirement. Let $v_{1}, v_{2}, v_{3}, v_{4}$ be the vertices of this tetrahedron, and nei_tid and $n e i_{-}$order be the identificator and the order of vertices of its $i$-th neighbor $(i=1,2,3,4)$. A convention is used that the $i$-th neighbor is the one that is across from the $i$-th vertex. Then, if the same function GetStructuredTet is called for nei_tid, and the returned vertices u1, u2, u3, u4 are permuted by function nei_order, then the vertices with the corresponding positions are equal, except for the vertices in position $i$ that are not equal. The Section mechanism of Coq allows for moving the frequently used parameters of the constructs defined within this section to its Variables clause, thus improving readability.

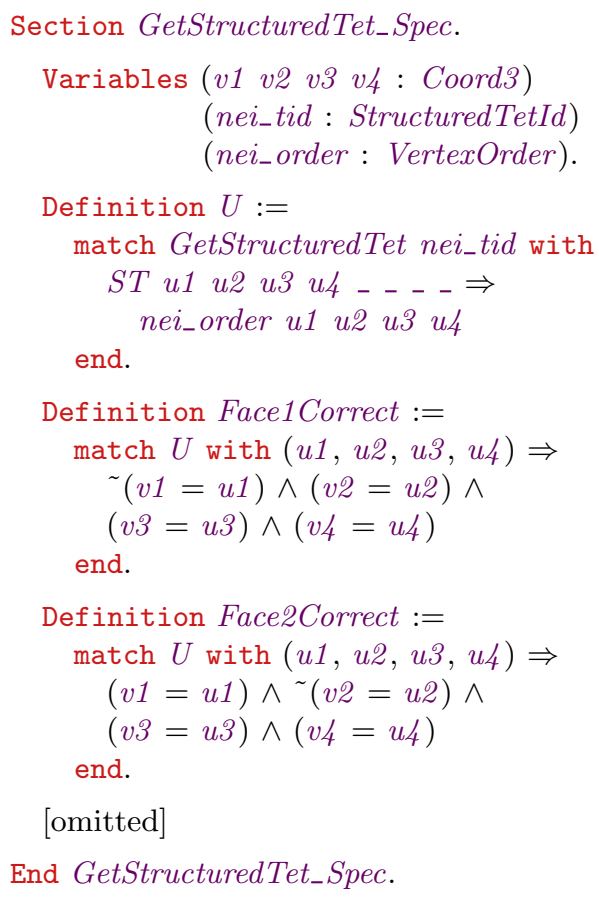

\subsection{Proof}

The following theorem proves the specification above.

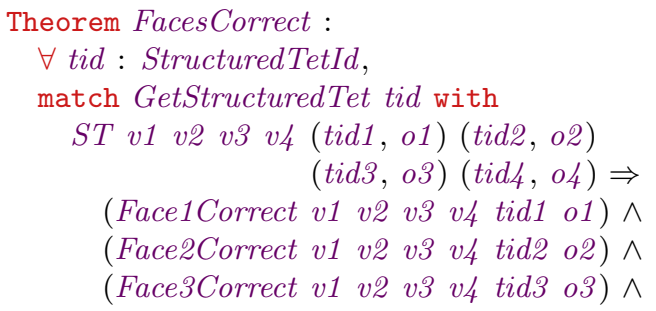




\author{
(Face4Correct v1 v2 v3 v4 tid4 o4) \\ end. \\ Proof. [omitted] Qed.
}

\section{CERTIFICATE FOR UNSTRUCTURED TETRAHEDRA}

\subsection{Specification}

Given two edges, each belonging to the stencils on either side of the shared face, these edges are considered compatible if they do not intersect in their interiors. A set of conditions is developed below that allow for verifying this requirement.

\subsubsection{Barycentric Coordinate System}

Let $v_{1}, \ldots, v_{n}$ be the vertices of a simplex in Euclidean space $\mathbb{R}^{3}$, given as triples of coordinates. For the present exposition, the relevant values of $n$ are 3 and 4, corresponding to a triangle or a tetrahedron, respectively. For some point $u \in \mathbb{R}^{3}$, the real numbers $a_{1}, \ldots, a_{n}$, not all equal to zero, such that $\left(a_{1}+\cdots+a_{n}\right) u=a_{1} v_{1}+\cdots+a_{n} v_{n}$, are called barycentric coordinates of $u$ with respect to the simplex with vertices $v_{1}, \ldots, v_{n}$. Barycentric coordinates with $a_{1}+\cdots+a_{n}=1$ will be used below. For the proofs presented, only three values of a barycentric coordinate are relevant: zero, some unknown value strictly between zero and one, and one. These are defined symbolically by the following respective constructors of type BCoord:

Inductive BCoord $:=$ Zero | Interior | One.

\subsubsection{Barycentric Coordinates Within a Triangle}

Consider the barycentric coordinate system defined by the shared face $(f 1, f 2, f 3)$. Then the following proposition FCoord lists the barycentric coordinates of vertices $f 1, f 2, f 3$, as well as of the vertices computed on the edges of this face by function GetIntersection:

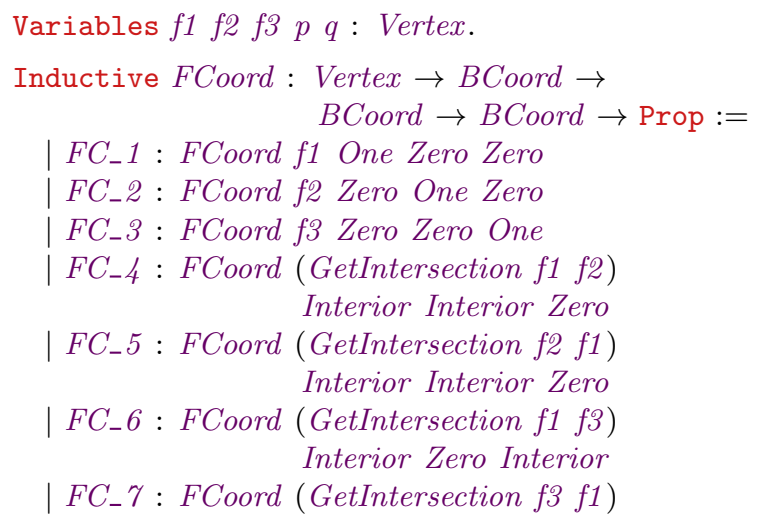

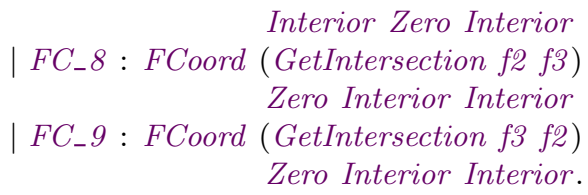

\subsubsection{Barycentric Coordinates Within a Tetrahedron}

For the tetrahedron $(f 1, f 2, f 3, p)$, the barycentric coordinate system is defined similarly. For the proof that follows, however, the full coordinates of the vertices are not needed. The only needed piece of information is which vertices lie off the shared face $(f 1, f 2, f 3)$, as evidenced by their non-zero last barycentric coordinate (i.e., the one corresponding to vertex $p$ ). The proposition below makes this information available.

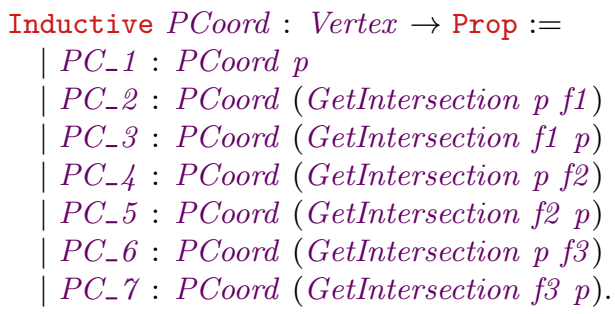

The predicate QCoord is defined similarly to PCoord, only with respect to vertex $q$ of tetrahedron $(f 1, f 2$, f3, q).

\subsubsection{Barycentric Non-Intersection in the Shared Face}

Given a point $p$ and a segment $\left(q 1, q^{2}\right)$, both lying in face $(f 1, f 2, f 3)$, a proposition can be stated that guarantees $p$ not being in the interior of $(q 1, q 2)$. Let the barycentric coordinates of points $q 1, q 2, p$ with respect to vertex $f 1$ be $a, b, c$, respectively, see Figure 5 . Then, if $c$ is above or below $a$ and $b$, as specified precisely by the propositions that follow, this requirement can be formalized.

Proposition Below enumerates the cases (i.e., combinations of values of its parameters) that assure the required property of segment-point non-intersection, where the first parameter is the coordinate of the point, while the second and the third parameters are the coordinates of the segment's vertices. Care is taken not to make conclusions based on comparing two Interior coordinates, since their specific values are unknown before they are computed. 


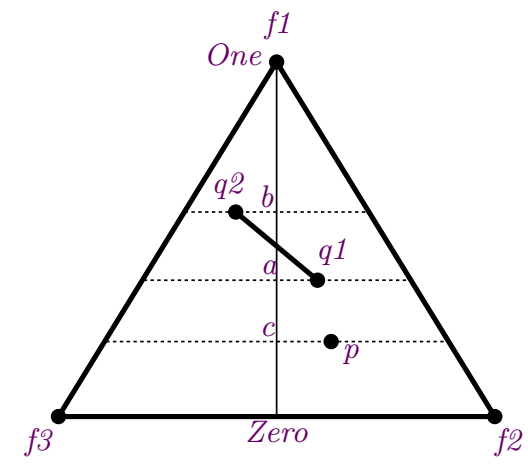

Figure 5: Point $p$ with barycentric $f 1$-coordinate $c$ not lying in the interior of segment $(q 1, q 2)$ whose vertices have barycentric $f 1$-coordinates $a$ and $b$.

\section{| B_4 : Below Zero Interior One.}

Proposition Above is stated similarly.

$$
\begin{gathered}
\text { Inductive Above : BCoord } \rightarrow \text { BCoord } \rightarrow \\
\text { BCoord } \rightarrow \text { Prop := } \\
\mid \begin{array}{c}
A_{-} 1: \text { Above One Zero Interior } \\
A_{-} \text {: Above One Zero One } \\
\text { A_3: Above One Interior Interior } \\
A_{-4}: \text { Above One Interior One. }
\end{array}
\end{gathered}
$$

In order to allow the vertices of the segment to appear in an arbitrary order, symmetric versions of propositions Below and Above are defined. They simply refer to these propositions with both orderings of parameters.

$$
\begin{aligned}
& \text { Section Below_Above_Symm. } \\
& \text { Variables c a } b: \text { BCoord. } \\
& \text { Inductive BelowSymm : Prop := } \\
& \text { | BS_1: Below c a } b \rightarrow \text { BelowSymm } \\
& \text { |BS_2 : Below c } b \text { a } \rightarrow \text { BelowSymm. } \\
& \text { Inductive AboveSymm : Prop := } \\
& \mid \text { AS_1: Above c a } b \rightarrow \text { AboveSymm } \\
& \text { AS_2 : Above c } b \text { a } a \rightarrow \text { AboveSymm. }
\end{aligned}
$$

End Below_Above_Symm.

The proposition SPNI1D below states that BCoord value $c$ is either above or below values $a$ and $b$ (regardless of the ordering of $a$ and $b$ ), which guarantees that a point with coordinate $c$ does not lie in the interior of the segment whose vertices have corresponding coordinates $a$ and $b$.

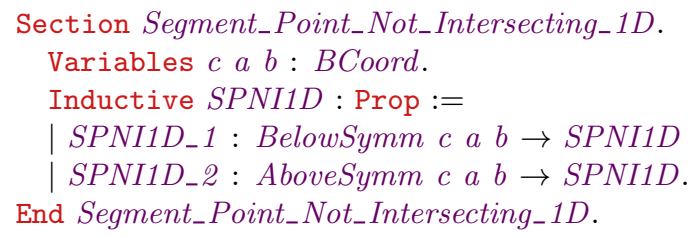

If the non-intersection condition SPNI1D holds with respect to at least one barycentric coordinate, then the vertex with barycentric coordinates $(c 1, c 2, c 3)$ does not intersect the interior of the segment whose vertices have coordinates $(a 1$, a2, a3) and $(b 1$, b2, b3):

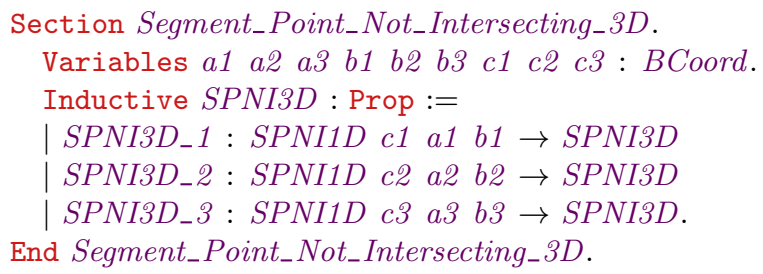

A similar approach is used in the case of two segments lying in face $(f 1, f 2, f 3)$, see Figure 6.

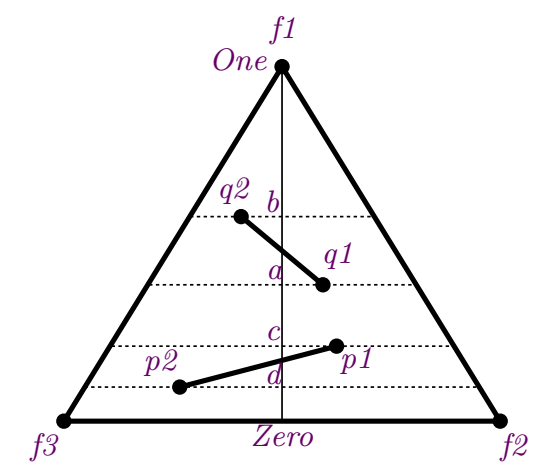

Figure 6: Segment $(p 1, p 2)$ whose vertices have barycentric $f 1$-coordinates $c$ and $d$ and segment $(q 1, q 2)$ whose vertices have barycentric $f 1$-coordinates $a$ and $b$ not intersecting in their interiors.

First, a proposition is stated that establishes a one-dimensional barycentric condition of their nonintersection in the interiors. Given that the coordinates of the first segment's vertices are $a$ and $b$, and the coordinates of the second segment's vertices are $c$ and $d$, the condition requires that either both vertices of the first segment are below the vertices of the second segment or vice versa:

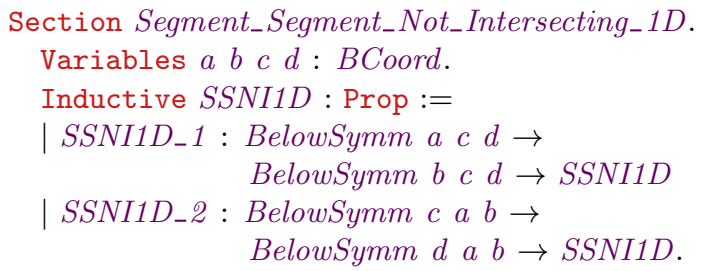

Then a proposition is stated requiring that the onedimensional condition holds with respect to at least one of three barycentric dimensions.

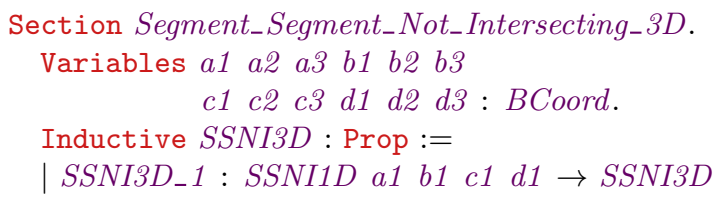


| SSNI3D_2 : SSNI1D a2 b2 c2 d2 $\rightarrow$ SSNI3D

| SSNI3D_3 : SSNI1D aз b3 сз d3 $\rightarrow$ SSNI3D. End Segment_Segment_Not_Intersecting_3D.

\subsubsection{Barycentric Non-Intersection Within Adjacent Tetrahedra}

In this section, a condition is established that formally defines a sufficient condition for two edges, each incident on the irregular tetrahedra on either side of the shared face, to be compatible, i.e., not intersecting in their interiors. This condition is given by the proposition TwoEdgesCompatible stated below. Two edges, $(p 1, p 2)$ and $(q 1, q 2)$, are considered compatible if at least one of the following conditions is satisfied:

- Vertex $p 1$ is in a special position (proposition SpecialVertex described further below) with respect to edge $\left(q 1, q^{2}\right)$ and vertex $p^{2}$ is not in the interior of edge $(q 1, q 2)$ (proposition NotIn described further below).

- The condition above holds if vertices $p 1$ and $p 2$ are interchanged.

- The two conditions above hold if edges $(p 1, p 2)$ and $\left(q 1, q^{2}\right)$ are interchanged.

- If both edges $(p 1, p 2)$ and $(q 1, q 2)$ lie in the shared face $(f 1, f 2, f 3)$, then they do not intersect in their interiors as defined by proposition SSNI3D above.

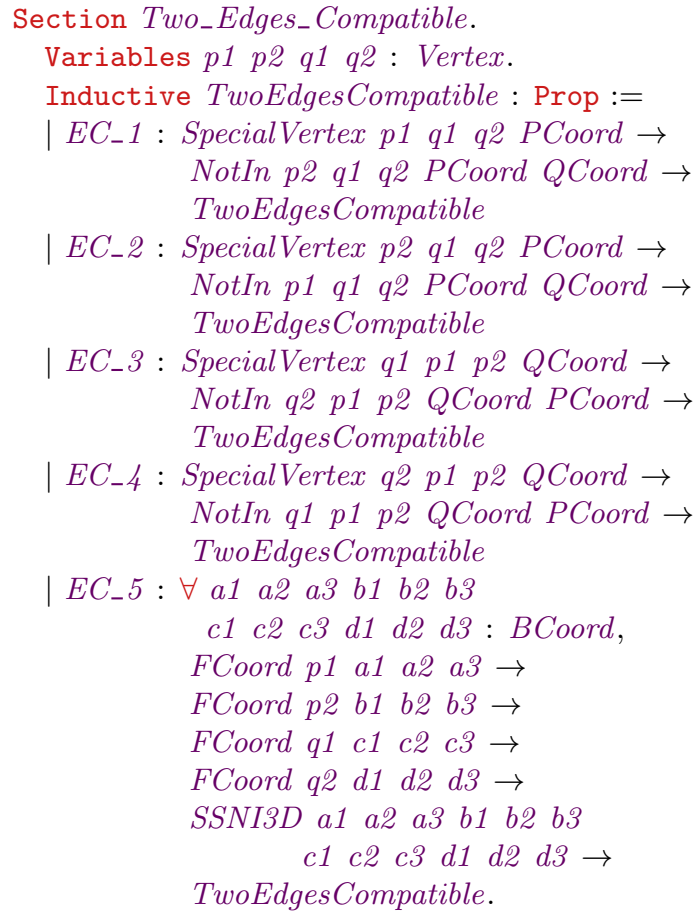

End Two_Edges_Compatible.

The special status of vertex $v$ with respect to edge ( $u 1$, u2) here means that $v$ is equal to one of the vertices $u 1$ or $u 2$, or it is off the shared face as defined by the corresponding parameter proposition $V$ (equal to $P C o o r d$ or QCoord depending on the branch of TwoEdgesCompatible).

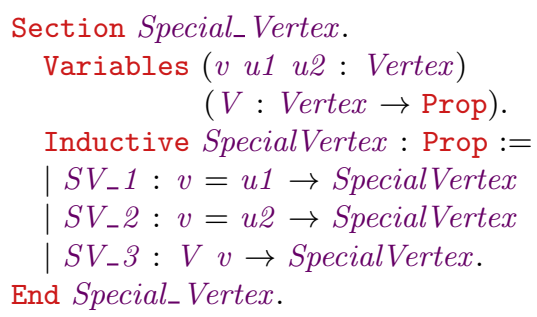

Vertex $v$ being NotIn edge $(u 1, u 2)$ holds if at least one of the following conditions is satisfied:

- $v$ is a special vertex with respect to $u 1, u 2$ as defined above.

- At least one of $u 1, u 2$ is off the shared face as defined by the appropriate proposition PCoord or QCoord.

- All three vertices $v, u 1$, u2 lie in the shared face and $v$ is not in the interior of edge $u 1$, $u_{2}$ as defined by proposition SPNI3D.

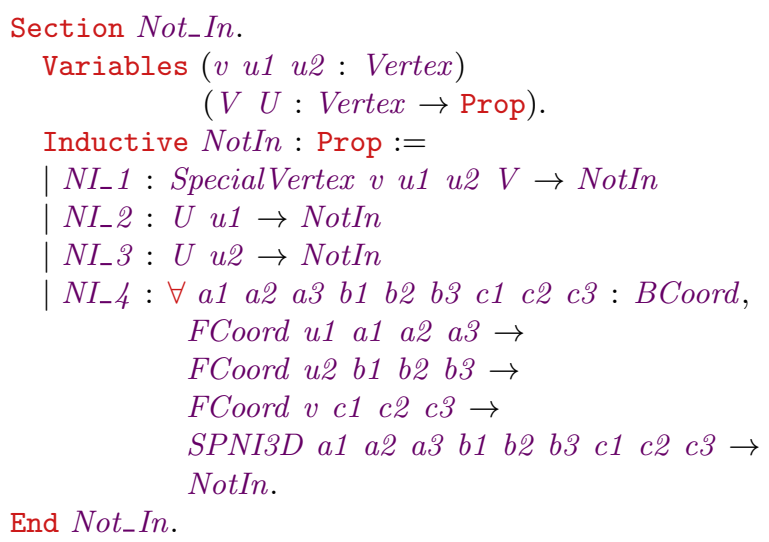

End Not_In.

Proposition AllEdgesCompatible below makes use of TwoEdgesCompatible by passing to it every pair of edges, each pair consisting of edges from both sides of the shared face.

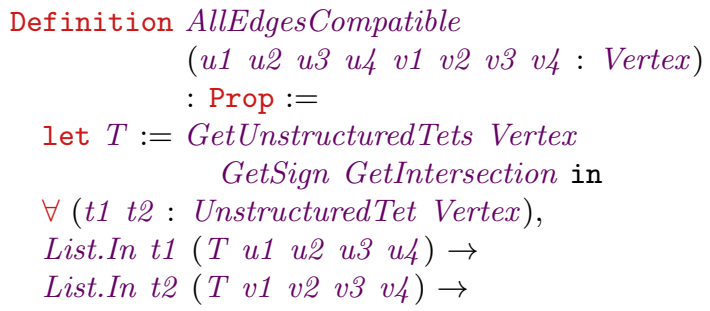


Forall EdgePairCompatible

(list_prod (Edges t1) (Edges t2)).

Given two regular tetrahedra, one defined by vertices $u 1, u 2, u 3, u_{4}$, and the other by $v_{1}, v_{2}, v_{3}, v_{4}$, function GetUnstructuredTets is called for each of these tetrahedra. This function returns a list of irregular tetrahedra for each regular tetrahedron. Let $t 1$ be an arbitrary tetrahedron in the list returned for $u 1, u 2$, $u 3, u_{4}$. Let $t_{2}$ be an arbitrary tetrahedron in the list returned for $v 1, v_{2}, v 3, v 4$. Six edges of $t 1$ and six edges of $t 2$ are collected into two respective lists using function Edges below:

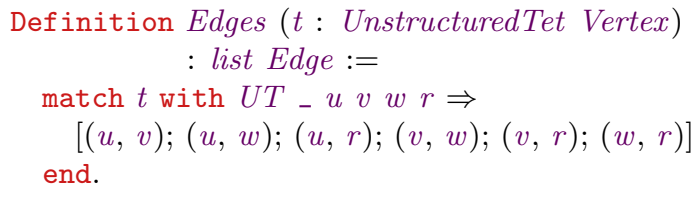

The standard library function list_prod returns a cartesian product of two lists, which are Edges t1 and Edges t2 in this development. Finally, the standard library proposition Forall asserts that a proposition supplied as its first parameter (EdgePairCompatible) holds for all elements of the list supplied as its second parameter. EdgePairCompatible simply unpacks the vertices of both edges and passes them to TwoEdgesCompatible:

Definition EdgePairCompatible ee := let ' $((p 1, p 2),(q 1, q 2)):=e e$ in

TwoEdgesCompatible p1 p2 q1 q2.

\subsection{Proof}

The following lemma proves that the proposition AllEdges Compatible holds for six arrangements of five vertices $f 1, f 2, f 3 p, q$ that define two tetrahedra with shared face $(f 1, f 2, f 3)$.

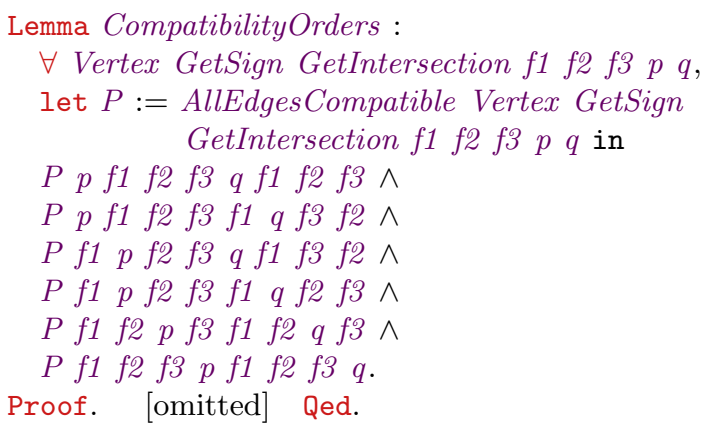

\section{CERTIFICATE FOR STRUCTURED AND UNSTRUCTURED TETRAHEDRA}

In this section, the correctness of the two functions, one returning structured and the other returning un- structured tetrahedra, is proven when they are used together. Function GetStructuredTet returns a structured tetrahedron which is then passed to GetUnstructuredTets.

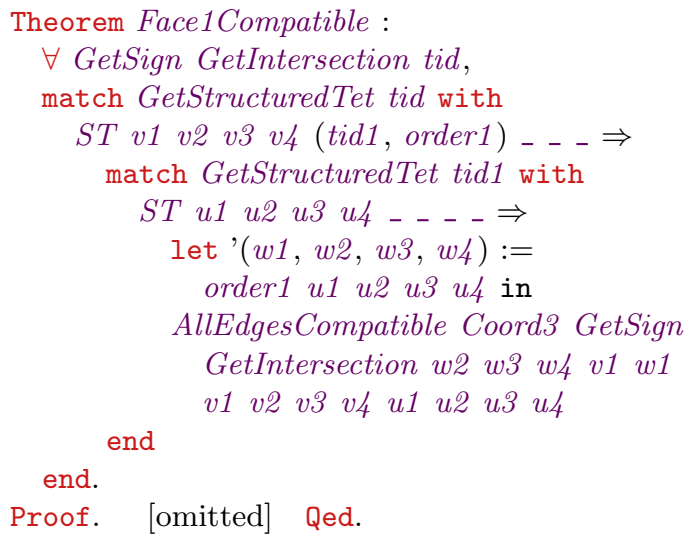

The statement of this theorem is somewhat similar to that of theorem FacesCorrect. Both theorems examine the result of two calls to function GetStructuredTet with respect to the face shared by the two returned structured tetrahedra. Theorem FacesCorrect proves that the shared face is identified correctly. Theorem Face1Compatible, on the other hand, proves that two sets of unstructured tetrahedra, each computed via function GetUnstructuredTets within the definition of proposition AllEdgesCompatible, satisfy this proposition. The proof of theorem Face1Compatible makes use of lemma CompatibilityOrders that requires one of six specific orderings of vertices of the two structured tetrahedra. The orderings of vertices returned by the calls to GetStructuredTet satisfy this requirement. Another three theorems are proven, Face2Compatible, Face3Compatible and Face4Compatible, that are identical to Face1Compatible except for the faces that are examined.

\section{DISCUSSION AND CONCLUSIONS}

Currently, major software projects consist of millions of lines of code, multiple subsystems, and are managed by large teams of developers (who tend to move between positions). With this level of complexity, formulating and maintaining the specifications of such systems, and ensuring that the implementation meets these specifications become a major challenge. The traditional approach to software specifications consists in writing textual descriptions in English (or other natural language) with a mix of mathematical statements, and maintaining them as code comments and/or as separate documents. There are two problem with such specifications. The first is that they tend to be highlevel and not necessarily reflective of the rich set of behaviors the code can exhibit. The second problem is that they are disconnected from the code, in the sense 
that updates to the code and to these specifications may be performed separately and not necessarily consistently with each other. The certified software development process illustrated in this paper solves both of these problems by virtue of the specifications referring to the actual implementation. It solves the first problem by using specifications stated in a precise logical language, and having these statements automatically checked for exhaustive coverage of all possible cases. It solves the second problem by having the specification directly reference the implementation. Appel et al. [9] coined the term deep specification to refer to specifications that are simultaneously rich (sufficiently detailed), two-sided (both implementable and useful), formal (stated in a formal language which supports automated tools), and live (connected to implementation). A number of model checking and design specification approaches based on formal languages like $\mathrm{Al}$ loy [5], AADL [19], VDM [20], and Z [21], operate on a high level of abstraction that is not connected with the implementation. An approach of annotating the routines with pre- and post-conditions, such as Design by Contract [22], relies on the developer's current understanding of these conditions and is also not live.

In addition to providing live specifications of software, the presented certification approach also guarantees that the proven properties hold for all input parameters that can be passed to the routines. In other words, it by design eliminates the need to test the resulting software for these properties. The caveat, however, is that the formally stated specifications have to correctly represent the expected properties.

Mesh generation is one of the application domains that is likely to benefit from the use of formal methods in general and of certified software development in particular. One reason behind this expectation is high complexity of mesh generation algorithms that need to balance multiple, often contradictory, requirements of element shape, boundary fidelity, mesh grading, number of elements, software running time and memory use, and others. These requirements can be thought of as a multidimensional design space, where each requirement represents one dimension of this space. Being able to a priori formally guarantee, or certify, certain properties of an algorithm's implementation allows for the reduction of the dimensionality of the remaining design space. An example of this phenomenon from the current work is the unfolding of the tetrahedral stencils: because the unfolded stencils have been automatically checked for consistency during a single certification event, the Parity Rule [12] does not need to be enforced at runtime during every execution of the software. Another reason behind the expectation of the usefulness of formal methods in mesh generation is the combinatorial nature of mesh stencils, or elements, which is suitable for automated enumeration and analysis of their possible arrangements.

The efficiency of the proof execution does not influence the performance of the final software product. Indeed, the proof is run at design time and then separated from the executable part by the process of extraction. This executable part, on the other hand, is the component which gets deployed and whose performance contributes to the efficiency of the resulting product. The main requirement on the proof efficiency is that it does not unreasonably slow down the design effort. The experience of the author of this work suggests that the proof design effort is dominated by the time spent on elucidating the properties that need to be proven, formalizing them, and discovering the appropriate proof strategies. The proof execution part appeared minor relative to these time investments. It was also noticed that the same result can be proven with different strategies that sometimes vary significantly in performance. The accompanying certificate [14] was run on a MacBook Pro equipped with an 8-core Intel Core i9 @ $2.4 \mathrm{GHz}$ processor and $32 \mathrm{~GB}$ of RAM memory. In the serial mode the proof completed in 46 minutes. Virtually all of this time was taken by the proof of lemma CompatibilityOrders. Experimentation with Coq's parallel proof modes revealed that this time can be reduced to 31 minutes with two proof threads. Increasing the number of proof threads to six, which is the number of independent proof goals in this lemma, did not yield further speedup.

The present work lays out a methodological foundation and a case study for developing certified mesh generation software. It demonstrates the use of the Calculus of Inductive Constructions for defining executable functions, stating their properties, and proving these properties via a direct examination of these functions (the property of liveness). These functions were extracted into OCaml code, supplemented with other OCaml functions needed for a working computer program, compiled, and executed. The evaluation of the performance of this program, as well as of the choices of the data structures, is out of the scope of this exposition and will be presented elsewhere.

It is anticipated that this work will be extended in the following directions. The first direction is the live proofs of the angle bounds reported in the original IS presentation [12]. The second direction is the addition of other shapes of structured tetrahedra that allow for more flexibility in filling in the octree leaves, leading to fewer resulting tetrahedra, as also originally reported [12]. The third direction is the study of stencils for multi-material interfaces, which can potentially be informed by previous work [23]. 


\section{References}

[1] Newcombe C., Rath T., Zhang F., Munteanu B., Brooker M., Deardeuff M. "How Amazon Web Services Uses Formal Methods." Communications of the ACM, vol. 58, no. 4, 66-73, Mar. 2015

[2] Lamport L. "TLA+ Hyperbook." http: //research.microsoft.com/en-us/um/people/ lamport/tla/hyperbook.html, 2015

[3] Eriksen M. "Functional at Scale." Communications of the ACM, vol. 59, no. 12, 50-55, Dec. 2016

[4] Avigad J., Harrison J. "Formally Verified Mathematics." Communications of the ACM, vol. 57, no. 4, 66-75, Apr. 2014

[5] Jackson D. Software Abstractions: Logic, Language, and Analysis. MIT Press, revised edn., 2012

[6] Chlipala A. "Ur/Web: A Simple Model for Programming the Web." Communications of the $A C M$, vol. 59, no. 8, 93-100, Jul. 2016

[7] Bertot Y., Castéran P. Coq'Art: The Calculus of Inductive Constructions. Springer, 2004

[8] INRIA France. "The Coq Proof Assistant, version 8.9.1." http://coq.inria.fr

[9] Appel A.W., Beringer L., Chlipala A., Pierce B.C., Shao Z., Weirich S., Zdancewic S. "Position Paper: The Science of Deep Specification." Philosophical Transactions of the Royal Society A: Mathematical, Physical and Engineering Sciences, vol. 375, no. 2104, 20160331, 2017

[10] Chernikov A., Xu J. "A computer-assisted proof of correctness of a marching cubes algorithm." International Meshing Roundtable, pp. 505-523. Springer, Orlando, FL, October 2013

[11] Lorensen W.E., Cline H.E. "Marching Cubes: A high resolution 3D surface construction algorithm." SIGGRAPH Comput. Graph., vol. 21, no. 4, 163-169, Aug. 1987

[12] Labelle F., Shewchuk J.R. "Isosurface Stuffing: Fast Tetrahedral Meshes with Good Dihedral Angles." ACM Transactions on Graphics, vol. 26, no. $3,57.1-57.10,2007$

[13] Dufourd J.F., Bertot Y. "Formal Study of Plane Delaunay Triangulation." M. Kaufmann, L.C. Paulson, editors, Interactive Theorem Proving, Lecture Notes in Computer Science, vol. 6172, pp. 211-226. Springer, 2010
[14] Chernikov A. "Coq Script: Certified Functions for Mesh Generation." https://github.com/ anchernikov/IMR2019, 2019

[15] Talos I., Jakab M., Kikinis R., Shenton M. "SPLPNL Brain Atlas." http://www.spl.harvard. edu/publications/item/view/1265, Mar. 2008

[16] Pierce B.C., et al. Software Foundations. 2019. https://softwarefoundations.cis. upenn.edu

[17] Chlipala A. Certified Programming with Dependent Types. 2019. http://adam.chlipala.net/ cpdt

[18] Ford N. Functional Thinking: Paradigm Over Syntax. O'Reilly Media, 2014

[19] Feiler P.H., Gluch D.P. Model-based engineering with $A A D L$ : an introduction to the $S A E$ architecture analysis $\& 5$ design language. Addison-Wesley, 2012

[20] Larsen P.G. "Ten Years of Historical Development "Bootstrapping" VDMTools." Journal of Universal Computer Science, vol. 7, no. 8, 692709, 2001

[21] Spivey M.J. The Z Notation: A Reference Manual. Prentice Hall, 1992

[22] Meyer B. "Applying "Design by Contract"." IEEE Computer, vol. 25, no. 10, 40-51, 1992

[23] Bronson J.R., Levine J.A., Whitaker R.T. "Lattice Cleaving: Conforming Tetrahedral Meshes of Multimaterial Domains with Bounded Quality." Proceedings of the 21st International Meshing Roundtable, pp. 191-209. Springer, San Jose, CA, 2013 


\title{
DISCRETE MESH OPTIMIZATION ON SURFACE AND VOLUME MESHES
}

\author{
Daniel Zint Roberto Grosso Florian Lunz \\ Chair of Computer Vision \\ Friedrich-Alexander-Universität, 91058 Erlangen, Germany \\ \{daniel.zint, roberto.grosso, flo.lunz\}@fau.de
}

\begin{abstract}
State of the art algorithms in surface mesh smoothing rely on computing new vertex positions on approximated shapes and re-projecting the results back onto the real surface or having no internal surface representation at all, which leads inevitably to suboptimal results. Discrete Mesh Optimization (DMO) is a greedy approach to topologyconsistent mesh quality improvement, which was initially designed to smooth triangle and quadrilateral meshes in two dimensions and tetrahedral meshes in three dimensions. We present a generalization of DMO which allows optimization on discretized surfaces, or more general d-dimensional manifolds. As the method is not bound to search directions, it is capable of finding the optimal vertex positions directly on a surface without any re-projection. Therefore, the proposed technique preserves the underlying surface or volume. We present examples for surface and volume meshes, showing the improvement-potential of considering boundary vertices in the smoothing process.
\end{abstract}

Keywords: mesh improvement, mesh smoothing, max-min optimization

\section{INTRODUCTION}

The discretization of some space $\Omega \subset \mathbb{R}^{d}$ in form of a mesh plays an important role in finite element based simulations and in computer graphics. For both cases a smooth mesh is preferred although the reasons may be different. Also the definition of a smooth mesh varies substantially depending on the field of application. As a consequence of this diversity a vast range of smoothing and optimization methods emerged within the last decades $[1,2,3]$. These methods aim to improve mesh quality by repositioning vertices while keeping the topology unchanged. In current research mesh smoothing is still a vividly discussed topic, especially for volume meshes $[4,5,6,7]$.

The method Discrete Mesh Optimization (DMO) [8] improves mesh quality iteratively while finding the optimal position for each vertex relatively to its neighborhood by evaluating a set of candidate positions. DMO does not rely on derivatives which allows quality metrics to be non-smooth or even discontinuous.
This also means that DMO can optimize for any quality criterion, e.g. roundness, anisotropy, or rectangularity. Furthermore, quality improvement is assured within each iteration.

Being independent of derivatives and search directions brings another advantage. The set of candidate positions can be mapped onto any parameterizable space. Thus, optimization of manifold meshes can be done precisely on the surface. Together with the assurance of quality improvement follows that DMO will converge to an optimal result. Many other methods tend to deform a mesh to something spherical.

We present a generalization of DMO to extend the area of application to meshes discretizing a domain $\Omega \subset \mathbb{R}^{d-k}$ with $d=2,3$ and $k \geq 0$, i.e. volume and surface meshes in 2- and 3-dimensional space. Furthermore, we introduce a smooth and vertex-interpolating surface estimation method. This enables applying DMO on surface meshes where the underlying shape is unknown. An important field of application is tetrahe- 
dral mesh improvement. Smoothing not just interior but also boundary vertices affects mesh quality significantly.

In this paper, we restrict ourselves to simplicial meshes. Just like DMO, this method can be easily adapted for other mesh types. As long as a quality metric for a mesh exists, the method can be applied.

In Section 1.1 we present the max-min problem (as presented in [8]) which we consider as the core issue of mesh smoothing. In Section 1.2 we display some widely-used smoothing methods. Section 1.3 gives a short introduction to DMO, according to [8]. It is followed by Section 2 where we generalize DMO and define the space of functions on which DMO is guaranteed to find the local optimum. Additionally, a surface estimation method is presented, which can be used if no surface description is available. In Section 3 we compare our method to other smoothing approaches. Conclusions are given in Section 4.

\subsection{The max-min Problem of Element Quality Improvement}

Assume for each element $e_{\mathrm{k}}$ of a mesh $M$ in $\mathbb{R}^{d}$ an element quality $q_{\mathrm{k}}^{(e)}$ is obtained by evaluating a quality metric $q^{(e)}\left(e_{\mathrm{k}}\right)$. The quality $q_{\mathrm{i}}^{(v)}$ of a vertex $v_{\mathrm{i}}$ that is positioned at $\mathbf{x}$ is defined as the minimal quality of its incident elements $e_{\mathrm{k}} \in N_{\mathrm{e}}\left(v_{\mathrm{i}}\right)$,

$$
q_{\mathrm{i}}^{(v)}(\mathbf{x})=\min _{e_{\mathrm{k}} \in N_{\mathrm{e}}\left(v_{\mathrm{i}}\right)} q_{\mathrm{k}}^{(e)}(\mathbf{x}) .
$$

From Equation (1) follows the local optimization problem for finding the maximum quality $q_{\mathrm{i}, \max }^{(v)}$ for a vertex $v_{\mathrm{i}}$,

$$
q_{\mathrm{i}, \max }^{(v)}=\max _{\mathbf{x}} q_{\mathrm{i}}^{(v)}(\mathbf{x})=\max _{\mathbf{x}} \min _{e_{\mathrm{k}} \in N_{\mathrm{e}}\left(v_{\mathrm{i}}\right)} q_{\mathrm{k}}^{(e)}(\mathbf{x}) .
$$

The optimal position $\mathbf{x}^{*}$ for $v_{\mathrm{i}}$ is given as

$$
\mathbf{x}^{*}=\arg \max _{\mathbf{x}} q_{\mathrm{i}}^{(v)}(\mathbf{x})=\arg \max _{\mathbf{x}} \min _{e_{\mathrm{k}} \in N_{\mathrm{e}}\left(v_{\mathrm{i}}\right)} q_{\mathrm{k}}^{(e)}(\mathbf{x}) .
$$

An iterative method which is meant to improve element quality should aim for finding $\mathbf{x}^{*}$ which is nontrivial as Equation (3) is only $C^{0}$ continuous, e.g. Figure 1.

Note that DMO actually improves vertex quality $q^{(v)}$ not element quality $q^{(e)}$. Using the definition in Equation (1) implies that by improving $q^{(v)}$ also the minimal element quality increases. Depending on the optimization goal, it might be reasonable to adjust Equation (1). This was done for example in [9] to adapt element size.

\subsection{Related Work}

Smoothing methods can be divided into three main groups, Laplacian-, physics-, and optimization-based.

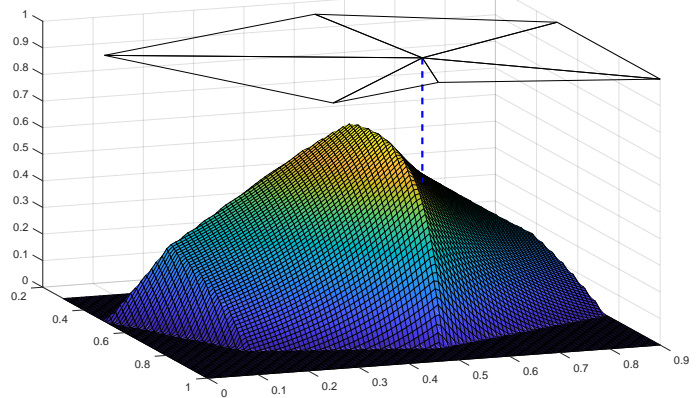

Figure 1: Vertex quality $q^{(v)}$ according to Equation (1) with $q^{(e)}$ defined as the mean ratio metric.

The classical Laplace-smoothing [10], developed for flat triangle meshes, is known to be fast but unstable in case of concave domains. A wide range of methods were proposed that modify the classical Laplace smoothing [11, 12, 13, 14, 15, 2, 3, 16, 17]. One representative is the "Smart" Laplacian Smoothing of Freitag [14] which only performs smoothing when mesh quality is increased. Using "Smart" Laplacian Smoothing without further processing steps does not lead to satisfying results as it does not improve mesh quality in concave regions. Freitag proposed to use it in combination with an optimization-based method. Laplacian-smoothers are fast but they also lead to suboptimal results. Many of these methods cannot guarantee that the quality will not decrease. Nevertheless, they are still used frequently.

Also for surface meshes Laplacian-based smoothing methods are standard. Here, they bring another disadvantage, namely they significantly change geometry when too many iterations are performed. Classical Laplace-smoothing drags the whole mesh towards its center of gravity, Figure 2(b). Applying cotangent weights makes the smoothing process unstable, Figure 2(c). A frequently used method is Taubin smoothing [18]. If only a few iterations are performed, the results look promising, as long as the parameters are chosen correctly. Changing the parameters or increasing the number of iterations also deforms the mesh, Figure 2(d).

Physics-based methods consider the mesh as a physical model. Some examples are spring-mass systems $[19,20]$, truss networks [21], or elasticity models $[22,23,24]$. Just like Laplace-based methods they do not provide any guarantee of mesh improvement.

Optimization-based methods are named after their approach of optimizing a quality metric. Some methods try to overcome the problem of non-differentiability by replacing Equation (2) with a smooth function 


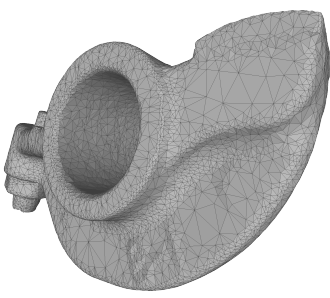

(a) Original

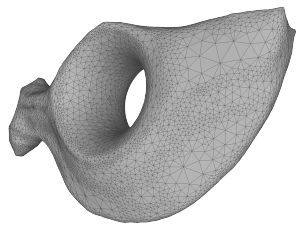

(b) Laplace, 100 iterations

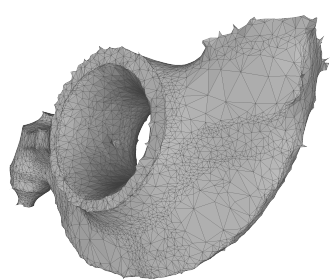

(c) Laplace with cotangent weights, 50 iterations

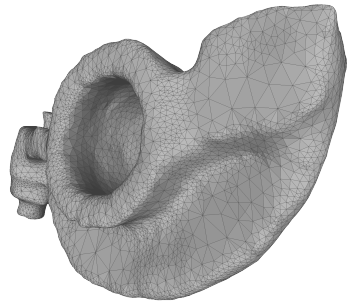

(d) Taubin, 500 iterations

Figure 2: Laplacian-based smoothing methods.

$[25,26,27,28,29]$. They run into the same problems as Laplacian-based methods when the domain is too complex.

To the authors knowledge, the currently most common smoothing algorithm is the method of Freitag. For example, it is implemented in the tetrahedral mesh improvement program Stellar [30]. While in [1, 14] the optimization is done with an analogue of the steepest descent method for smooth functions, later versions use the simplex algorithm to solve a linear programming problem [31, 32].

A derivative-free approach is done by Park and Shontz in [33]. They use pattern search in combination with backtracking line search to find a better vertex position. The convergence is suboptimal as it depends on search directions.

Rangarajan and Lew introduced the directional vertex relaxation (DVR) algorithm [7]. It solves the optimization problem by breaking it down to one dimension. This is achieved by providing a smoothing direction which can be chosen either randomly or by using previous knowledge. Within this one dimension the optimal solution can be found analytically. The major concern about this method is its randomness of relaxation directions as it leads to an inefficient smoothing with slow convergence.

Except Freitag's linear programming approach, all named optimization-based smoothing methods rely on linear search directions. For volume meshes this works fairly good, even though convergence is rather slow, but using search directions on surface meshes is not reasonable, because optimization is done along a line and not on the surface. A common way to deal with this problem is to perform the optimization on the tangent plane and re-project the vertex onto the surface. Finding the optimum cannot be guaranteed. Re-projection might even fail for complex geometries. Therefore, the search for optimal vertex positions should be restricted to the surface.

Zhang et al. [34] and Leng et al. [35, 36] present a geometric flow-driven approach for tetrahedral mesh smoothing. The methods use geometric partial differential equations to denoise the surface mesh and improve element quality while being volume preserving. For surface meshes emerging from geometry scanners, a wide range of feature preserving denoising methods exists $[37,38,39,40]$. All these methods are not in scope of this work as we concentrate on element quality optimization, not on surface denoising. We expect an input mesh that represents its geometry correctly.

\subsection{DMO}

DMO solves the max-min problem in Equation (2) by evaluating the vertex quality function with a greedy algorithm on a uniform grid. The grid's center of gravity is set to the vertex that should be optimized. The grid size is defined by the axis aligned bounding box for the one-ring neighborhood and a scaling factor $\omega$. Each grid-point is considered as candidate position where Equation (1) is evaluated, Figure 3. The vertex is repositioned at the best candidate if this increases its quality. After each iteration step the scaling factor $\omega$ is reduced such that the new grid size is twice the old grid spacing,

$$
\omega \leftarrow \omega \cdot 2 /(n-1),
$$

where $n$ is the number of grid points in one dimension. Furthermore, the grid is repositioned together with the vertex. Candidate evaluation and grid downscaling are repeated iteratively until the desired level of precision is reached.

\section{GENERALIZATION OF DISCRETE MESH OPTIMIZATION}

We reformulate DMO to cover surface and volume meshes by using an appropriate parameter space. We introduce the mapping of a uniform candidate grid from parametric domain $\Xi^{d-k}$ to real space $\mathbb{R}^{d}$, with $d=2,3$ and $0 \leq k<d$. The mapping function is denoted by

$$
\mathbf{x}(\xi): \quad \Xi^{d-k} \rightarrow \mathbb{R}^{d}
$$




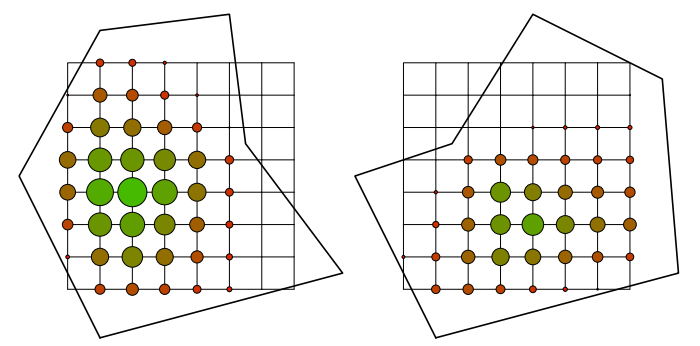

Figure 3: Uniform grid with quality metric evaluation for each candidate. A large green point represents good, a small red one bad quality

In general, the surface can be locally described as a smooth function,

$$
s(\xi): \quad \Xi^{d-k} \rightarrow \Xi^{d},
$$

where $\Xi^{d}$ is the local coordinate system with $\Xi^{d-k}$ being the parameter-subspace. The transformation from local to world coordinates is given as

$$
\Phi(\xi): \quad \Xi^{d} \rightarrow \mathbb{R}^{d},
$$

and its inverse as

$$
\Phi^{-1}(\mathbf{x}): \quad \mathbb{R}^{d} \rightarrow \Xi^{d} .
$$

DMO works best if $\mathbf{x}(\xi)$ is known, i.e. a parametrization of the surface is given. Nevertheless, a surface description is often missing. We present a local surface estimation which is interpolating and tangential in all vertices. The advantage of converging towards an optimal mesh remains also on estimated surfaces.

Section 2.1 introduces our way of estimating smooth surfaces. Features, such as sharp edges, cannot be presented by smooth surfaces but also need to be preserved. Thus, Section 2.2 adds a feature detection approach. The application of DMO on surface meshes is explained in Section 2.3. Section 2.4 defines the function space on which DMO is guaranteed to converge locally towards the optimum.

\subsection{Surface Estimation}

The generalization of DMO allows smoothing on surfaces but often the surface is unknown, e.g. for meshes from point cloud reconstruction. In such a case, surface estimation is required. We present an approach based on moving least squares which produces smooth, vertex-interpolating surfaces.

Much research was done in estimating surface quantities [41, 42, 43, 44]. Unfortunately, these methods do not construct smooth surfaces. The transition between local surface estimations is often discontinuous. Moving least squares methods are commonly used for point cloud estimation [45, 46, 47, 48]. As we already have a mesh, we can use simpler techniques to get a surface estimation.

We base our approach on [49]. The method fits truncated Taylor expansions to the nearest neighbors of vertices. Its objective is actually to estimate differential quantities. We extend the method with a moving least squares approach inspired by [45] for creating a smooth surface estimation. Furthermore, we intentionally break with the smoothness property at feature edges to represent them correctly.

The surface is approximated at each vertex $v_{k}$ with second order Taylor polynomials,

$$
T_{k}(\xi)=\left(\begin{array}{c}
\xi_{1} \\
\xi_{2} \\
\sum_{i=1}^{2} \sum_{j=i}^{2} a_{i j} \xi_{i} \xi_{j}
\end{array}\right)
$$

where we assume the normal and tangential vectors of the mesh in each vertex to represent the Monge coordinate system,

$$
\left(\begin{array}{l}
e_{1}^{\prime} \\
e_{2}^{\prime} \\
e_{3}^{\prime}
\end{array}\right)=\left(\begin{array}{l}
n \\
t_{1} \\
t_{2}
\end{array}\right)
$$

The transformations from local coordinate system $\left(e_{1}^{\prime}, e_{2}^{\prime}, e_{3}^{\prime}\right)$ to world $\left(e_{1}, e_{2}, e_{3}\right)$ and its inverse are

$$
\begin{aligned}
\Phi_{k}(\xi) & =\mathbf{o}_{k}+\xi_{1} e_{1}^{\prime}+\xi_{2} e_{2}^{\prime}+\xi_{3} e_{3}^{\prime} \\
\Phi_{k}^{-1}(\mathbf{x}) & =\left(e_{1}^{\prime}\left|e_{2}^{\prime}\right| e_{3}^{\prime}\right)^{\mathrm{T}}\left(\mathbf{x}-\mathbf{o}_{k}\right),
\end{aligned}
$$

where $\mathbf{o}_{k}$ is the origin of the local coordinate system.

We chose second order polynomials in Equation (9) because we can determine the parameters $a_{i j}$ with least squares using only the one ring neighborhood of a vertex. This results in a good approximation close to $v_{k}$ which degrades with distance. Always using the local surface estimation of the nearest neighbor would be an easy way to overcome this problem, but doing so introduces discontinuous transitions on the Voronoi edges, Figure 4(a). Instead, we perform an interpolation between the local surfaces of one-ring-neighborhoodvertices $N_{k}$, with the center vertex $v_{k}$,

$$
s_{k}(\mathbf{x})=\frac{\sum_{v_{i} \in N_{v}} \omega_{i}(\mathbf{x}) \Phi\left(T_{i}\left(\Phi_{k}^{-1}(\mathbf{x})\right)\right)}{\sum_{v_{i} \in N_{v}} \omega_{i}(\mathbf{x})},
$$

where the inverse squared distance weighting $\omega_{i}(\mathbf{x})=$ $1 /\left\|\mathbf{x}-v_{i}+\varepsilon\right\|^{2}$ with $\epsilon>0$ is applied to increase the influence of close vertices. The constant $\varepsilon$ prohibits $\omega_{i}\left(v_{i}\right) \rightarrow \infty$ and should be chosen as small as possible. Furthermore, $N_{k}$ also contains $v_{k}$ itself. Thus, we have a smooth, vertex-interpolating surface estimation, Figures 4(b) and 5.

Note that $s_{k}(\mathbf{x})$ is stated in world coordinates, not in local coordinates as in Equation (6). This is just a 


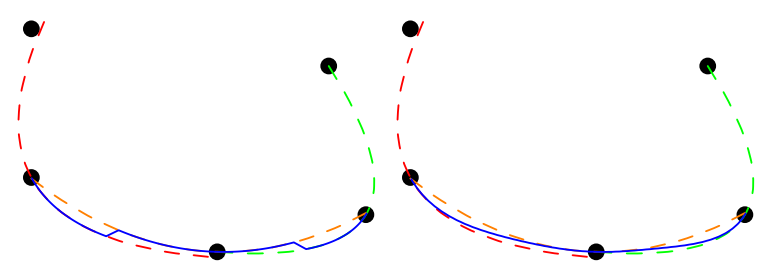

(a) Using nearest neighbor (b) Interpolating between surface estimation.

Figure 4: Surface estimation with second order polynomials (dashed lines) and different transitions(blue line).
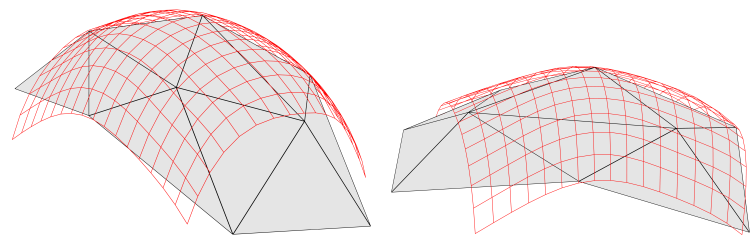

Figure 5: Surface estimation on a triangle mesh using interpolation between local surfaces.

matter of formulation. Equation (13) could also be stated in local coordinates but that would just make it more tedious to read.

The surface estimation is computed locally for each vertex of the original mesh. As vertices move during optimization, we always use the surface estimation of the closest vertex in the original mesh. In the beginning, each vertex will be its own nearest neighbor but due to mesh optimization, vertices might move quite far.

The surface estimation presented so far is only reasonable for smooth meshes. Sharp edges cannot be represented by second order Taylor polynomials. Therefore, we add a simple feature detection which takes care of boundaries and sharp edges, see Section 2.2. At feature vertices, a smooth surface estimation is not reasonable. Instead, the local surface estimation needs to be discontinuous. We estimate the surface at a feature vertex with tangential planes, separated by the feature edge, Figure 6. If a vertex is part of a feature edge, this edge splits the incident faces of the vertex in two sections. The normal of a tangential plane is the average normal of the incident faces on a section.

\subsection{Feature Detection}

In geometry processing, feature detection is a core issue and therefore well studied, [50, 51, 52, 53, 54]. Plenty of methods exist that can also handle noisy input data, $[55,56,57]$. We expect our input mesh to represent the geometry correctly and therefore do not require such sophisticated techniques.

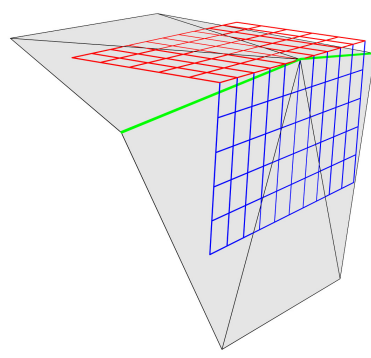

Figure 6: Surface estimation for a vertex on a feature edge (green).

We detect feature vertices by evaluating the angle between normals of two adjacent faces. If the angle is above a certain threshold, we consider the edge and its incident vertices as features. In our tests we used a threshold angle between 30 and 50 degrees, depending on the input mesh.

Geometric consistency does not necessarily require feature vertices to be static. A feature vertex $v_{k}$ can be smoothed if it has exactly two incident feature edges which are not shared by one face. For example, in the mesh cube, Figure 7(a), only the corner vertices need to remain fixed. All other feature vertices can move along the edges without changing the geometry. Feature edge estimation is analogue to the surface estimation method in Section 2.1 but with $k=2$, and the Taylor polynomial

$$
T_{k}(\xi)=\left(\begin{array}{c}
\xi_{1} \\
0 \\
a \xi_{1}^{2}
\end{array}\right),
$$

where $e_{1}^{\prime}$ and $e_{3}^{\prime}$ must lie in the plane described by $v_{k}$ and its adjacent feature vertices. With feature edge estimation, smoothing is also possible on more complex feature edges, e.g. Figure 7(b).

In the special case that one face contains only feature vertices, none of these vertices may be repositioned. This special case appears when two feature edges intersect, e.q. the faces in the corners of cube, Figure 7(a).

\subsection{DMO on Surface Meshes}

The local surface around a vertex $v_{k}$ is assumed to be either given or estimated, e.g. with the method in Section 2.1. Vertex $v_{k}$ is positioned at $\mathbf{x}_{k}$ and has the local coordinates $\xi_{k}$, Figure 8(a). The candidate points for DMO are positioned around $v_{k}$ in parametric space on a uniform grid with its center at $\xi_{k}$, Figure 8(b). For evaluating the quality metric the candidates are mapped onto the surface with Equation (5). The uniform grid is moved to the current optimum and scaled down, Figure 8(c). This is repeated until the desired level of precision is reached. Scaling down the grid 


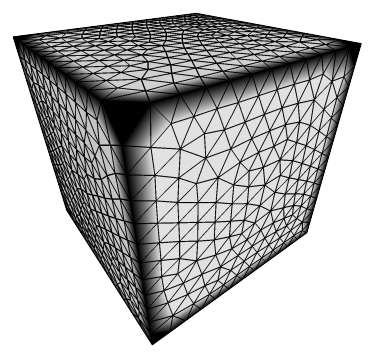

(a) cube

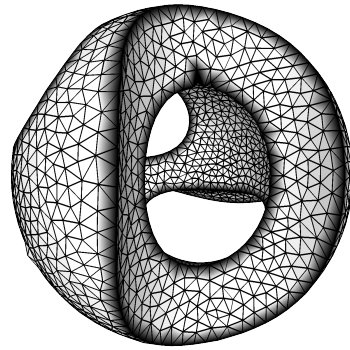

(b) sculpt
Figure 7: Feature edges for different meshes shaded in black.

more than two times did not have significant impact on quality in our test cases. Finally, the vertex is repositioned at the best candidate, Figure 8(d). Algorithm 1 states the vertex optimization routine adapted for surface meshes.

The resulting mesh highly depends on the chosen quality metric. We got satisfying results using the mean ratio metric, see Section 3, but for other cases alternate metrics might be more suitable. As DMO's implementation is independent of the quality metric, users can plug in a different quality metric in the algorithm to obtain more appropriate results for their specific application.

\subsection{Definition of Optimizable Function Space}

We define the function space $\mathcal{F}^{h}$ containing all functions that can be optimized by DMO using the initial grid size $h$.

Proposition 1. A function $f(\xi): \Xi^{d-k} \rightarrow \mathbb{R}$ with $0 \leq k<d$ is in $\mathcal{F}^{h}$ if it satisfies:

- $f(\xi)$ contains exactly one maximum $f_{\max }=$ $f\left(\xi_{\max }\right)$ and is strictly decaying from it in all directions.

- Given nested balls

$$
S_{i}=\left\{\xi \in \mathbb{R}^{d}:\|\xi-\mathbf{o}\|=r_{i}, \quad i=1,2\right\}
$$

with $r_{1}=h / \sqrt{d}, r_{2}=3 r_{1}$, and their shared origin o satisfying

$$
\left\|\xi_{\max }-\mathbf{o}\right\| \leq r_{1} .
$$

Assume a grid cell defined by its set of vertices $G_{h}$, with each vertex lying on the ball $S_{1}$, Figure 9. If for any orientation of the cell it holds,

$$
\max _{\xi \in G_{h}} f(\xi) \geq \max _{\xi \in S_{2}} f(\xi),
$$

then DMO will reach the optimum.
Proof. Assume $f_{\max }$ inside a grid cell defined by its set of vertices $G_{h}$, then $G_{h}$ must contain the maximal value of all grid vertices to ensure convergence towards $f_{\text {max }}$. Equation (17) states that the maximal value of $G_{h}$ is larger than all values on $S_{2}$. Considering, that $f(\xi)$ is strictly decaying, it follows that no vertex $\|\mathbf{v}-\mathbf{o}\| \geq r_{2}$ has a value greater than the maximum of $Q_{\max }$.

For the practical application of DMO it is not reasonable to check a function to satisfy Proposition 1 each time an optimization should be performed. Instead we include an additional step in the DMO algorithm. Prior to reducing the grid size it is checked, if the candidate node is on the grid-boundary. In this case, the grid size is kept unchanged and the greedy search is repeated but the center of the grid is moved to the last found optimum, Algorithm 1 Line 19-20. Thus, if the initial grid size was too large, the grid is iteratively moved towards the optimum. This heuristics produced good results in all cases we have applied our method.

As vertices never leave the estimated surface, DMO is point-wise surface preserving. In extreme cases, it might be possible that an edge "cuts" through the surface but this behavior was never experienced in any example. The same holds for volume preservation.

\section{RESULTS}

We compare the generalized DMO to other smoothing methods. In Section 3.1 the method is applied to triangle surface meshes as they are common in computer graphics. In Section 3.2 generalized DMO is compared to Stellar on tetrahedral meshes.

A quality comparison is given in terms of the mean ratio metric $[58,59,60,61,31,7]$, which is defined for triangles,

$$
q_{\mathrm{m}_{\mathrm{tri}}}=4 \sqrt{3} \frac{A}{\sum_{i=1}^{3} l_{i}^{2}},
$$

and tetrahedrons,

$$
q_{\mathrm{m} \text { tet }}=12 \sqrt[3]{9} \frac{V^{2 / 3}}{\sum_{i=1}^{6} l_{i}^{2}},
$$

where $A$ is the signed area of the triangle, $V$ the signed volume of the tetrahedron, and $l_{i}$ is the length of their incident edges. Note that this quality measure does not depend on free parameters that have to be input by the user. Therefore, no user interaction is required. We display mesh-quality by lexicographically ordering the elements according to their quality. The element index is given in logarithmic scaling as the elements with lowest quality are the most interesting ones. 


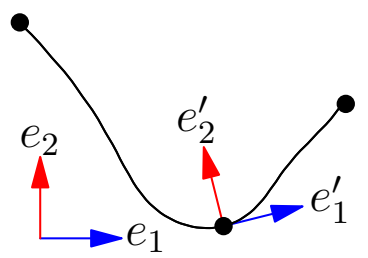

(a) Vertex with world and local coordinate system and estimated surface.

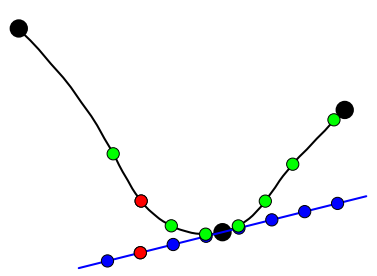

(b) Candidate positions in (c) Refinement around optiparametric space and on the mal candidate. surface.

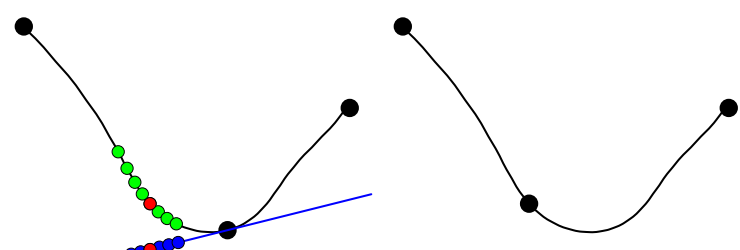

(d) Smoothed mesh.

Figure 8: DMO on surface mesh. The blue line represents the parameter space on which the vertices are distributed uniformly.

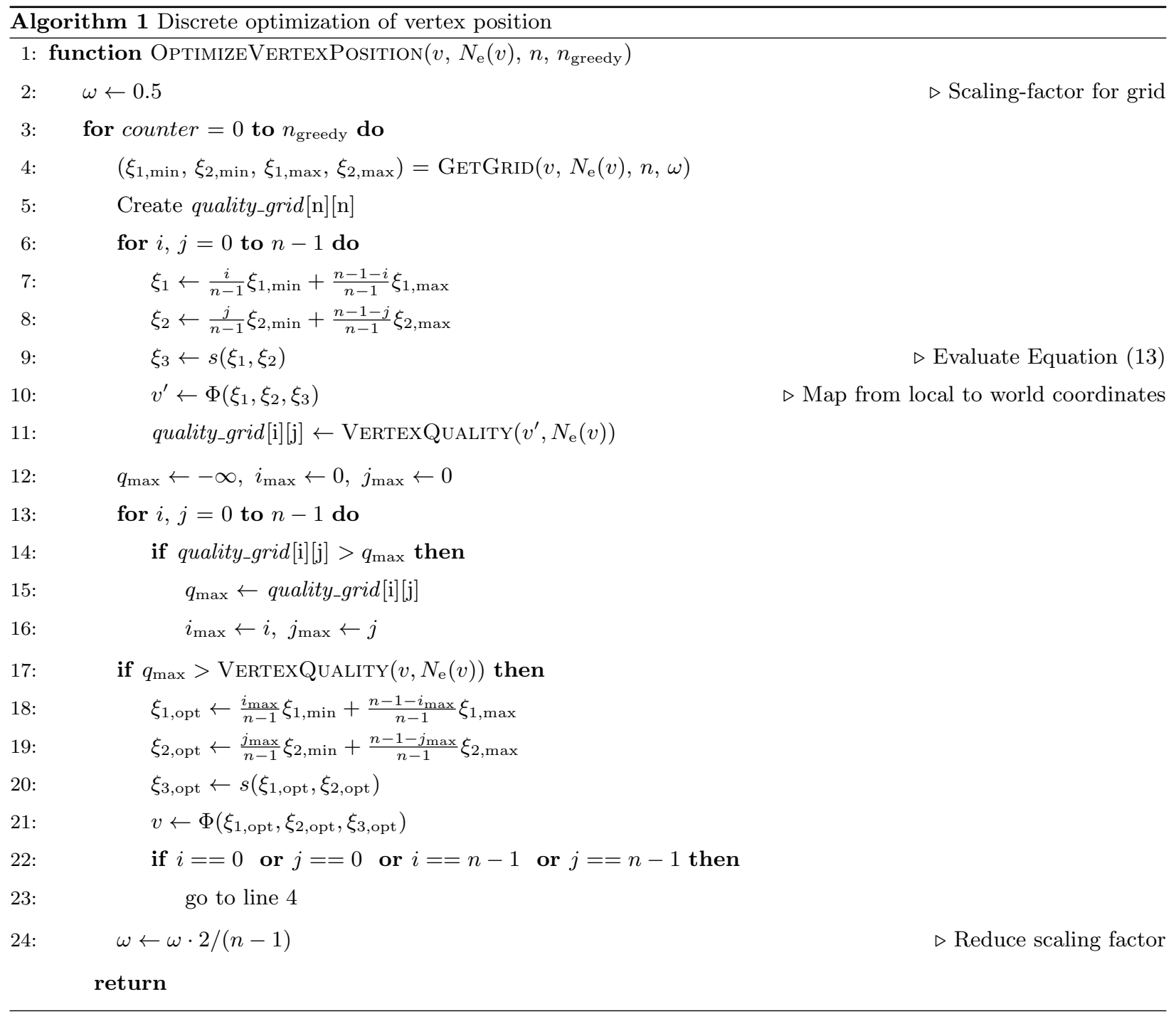




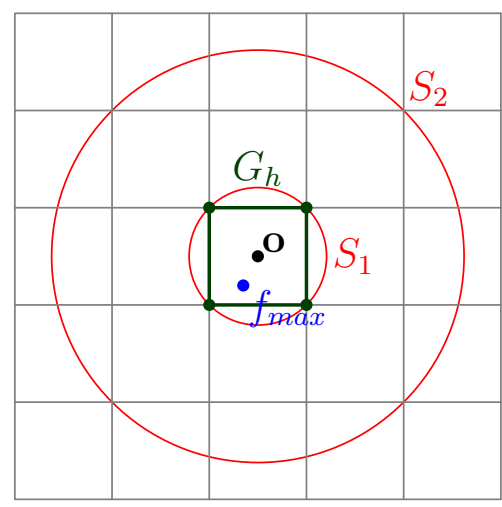

Figure 9: The best candidate of grid cell $G_{h}$ must have a larger value then any point on $S_{2}$ to guarantee convergence towards $f_{\max }$.

\subsection{Surface Meshes}

We compare our method to Taubin smoothing [18]. We use the implementation in Meshlab [62] with default parameters. Other smoothing methods were also tested in Meshlab but they either did not improve quality or changed the geometry so significantly that a comparison is pointless. For the presented examples the real surface is unknown. Therefore, DMO uses surface estimation of Section 2.1.

The first comparison is done on mesh tweety with 6752 vertices and a minimal quality 0.048, Figure 10(a). Taubin and DMO show both visually good improvements, Figures 10(b) and 10(c). However, Taubin smoothing leaves a significant amount of bad shaped triangles with a minimal quality of 0.089. DMO instead improves quality considerably to 0.484 .

Similar behavior of the smoothing methods is observed on mesh rocker-arm with 10044 vertices and a minimal quality of 0.077 , Figure 11(a). Taubin smoothing improves quality overall but cannot get rid of low quality triangles resulting in a minimal quality of 0.241 , Figure 11(b). DMO eliminates bad shaped elements completely giving a minimal quality of 0.599 , Figure 11 (c). Also, even with default parameters, the mesh becomes visibly more round at corners when applying Taubin smoothing. The shape remains unchanged by DMO.

On the mesh hand with 50085 vertices, Figure 12(a), Taubin cannot deal with the more complex geometry, reducing minimal element quality from 0.343 to 0.033 , Figure 12(b). DMO improves quality to 0.468, Figure $12(\mathrm{c})$.

The mesh filigree with 514300 vertices results from marching cubes and therefore contains very thin triangles with a minimal element quality of 0.104, Figure 13. In this example, Taubin smoothing reaches a higher minimal element quality than DMO, namely

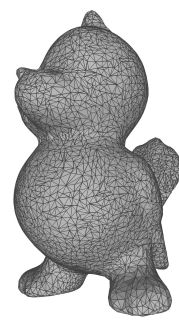

(a) Original

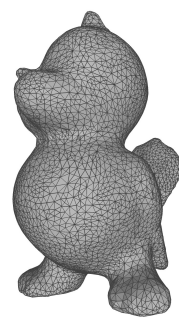

(b) Taubin

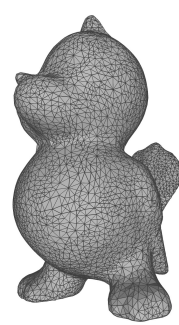

(c) $\mathrm{DMO}$

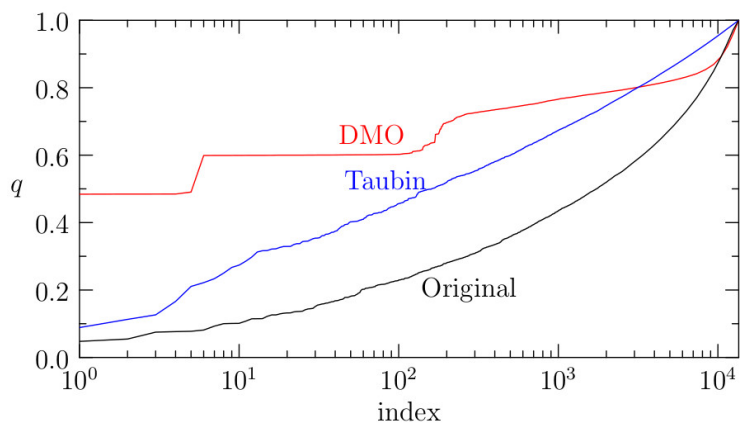

Figure 10: Comparison of Taubin smoothing and DMO on surface mesh tweety.

0.218 in comparison to 0.143 . Considering that Taubin smoothing may leave the surface, this is not a surprising result. The mesh is mostly regular and Laplacebased smoothers are highly efficient in such cases. DMO instead is bound to its surface estimation. If geometric consistency is not required, then Taubin smoothing is preferable here.

Also mesh shoe has some thin triangles which cannot be improved, Figure 14. Therefore, the minimal element quality remains 0.018. Also Taubin smoothing does not improve quality. Instead it reduces it to 0.017. Besides the worst elements, DMO has a significant impact.

The last surface mesh example is augustus with 9758 vertices. Again, DMO improves minimal element quality from 0.094 to 0.241 . Taubin also improves minimal quality to 0.145 . Taking a look at Figure 15 Taubin smoothing only improves the worst element but decreases quality of other bad shaped elements, whereas DMO increases quality of the worst 1000 elements.

\subsection{Volume Meshes}

We compare DMO to Stellar [30], a tetrahedral mesh improvement software. We only allow topologyconsistent optimizations to create a reasonable comparison. Stellar can also smooth boundary vertices. A quadric error metric is added to the optimization problem to keep the boundary mostly unchanged. 

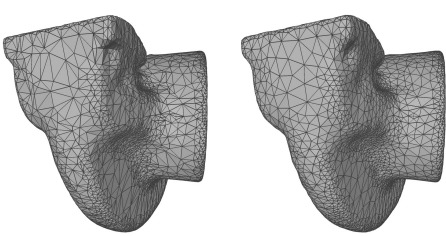

(a) Original

(b) Taubin

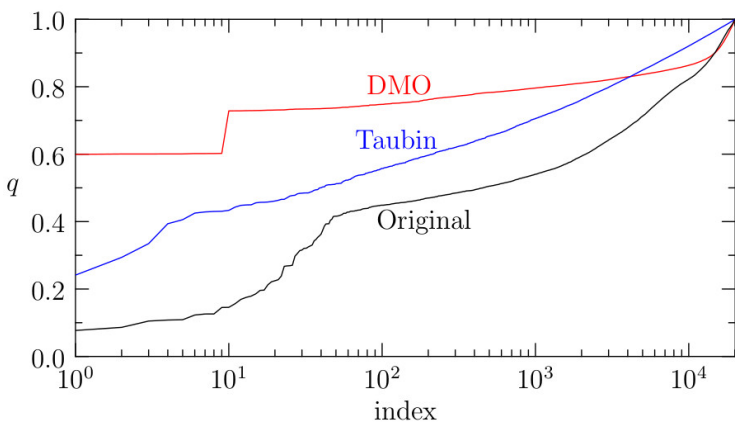

Figure 11: Comparison of Taubin smoothing and DMO on surface mesh rocker-arm.

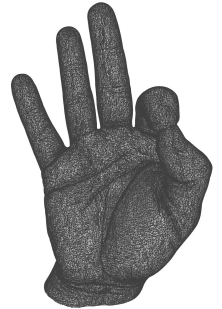

(a) Original

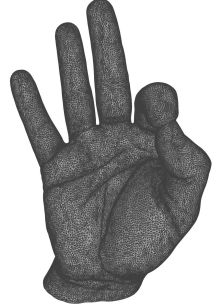

(b) Taubin

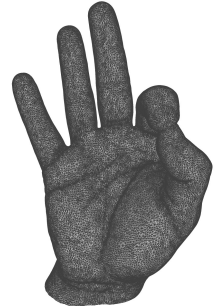

(c) $\mathrm{DMO}$

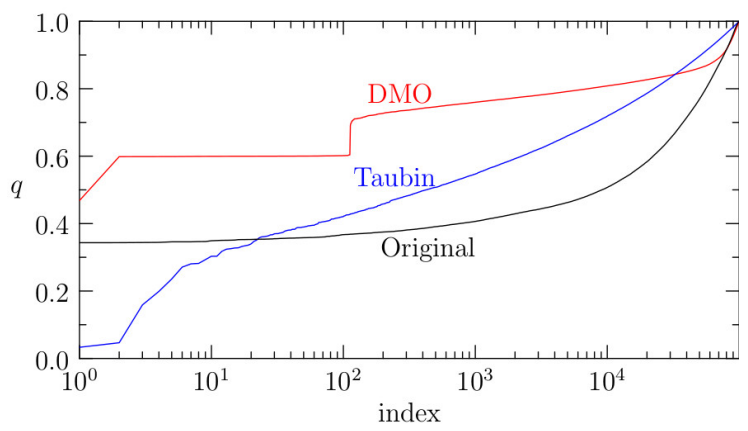

Figure 12: Comparison of Taubin smoothing and DMO on surface mesh hand.

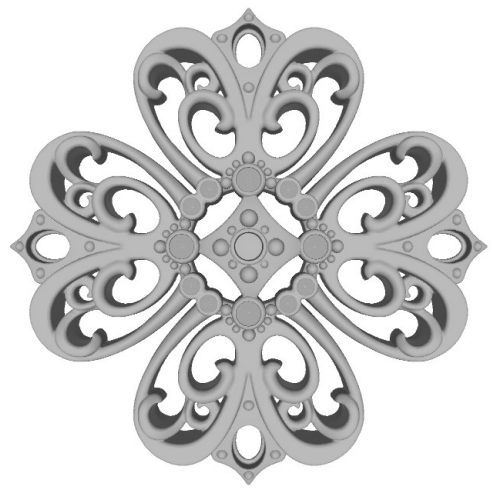

(a) Filigree

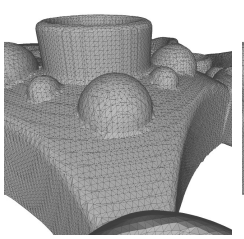

(b) Original

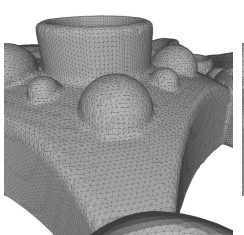

(c) Taubin

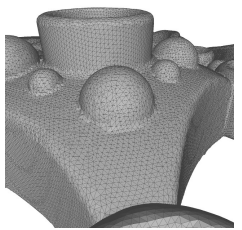

(d) DMO

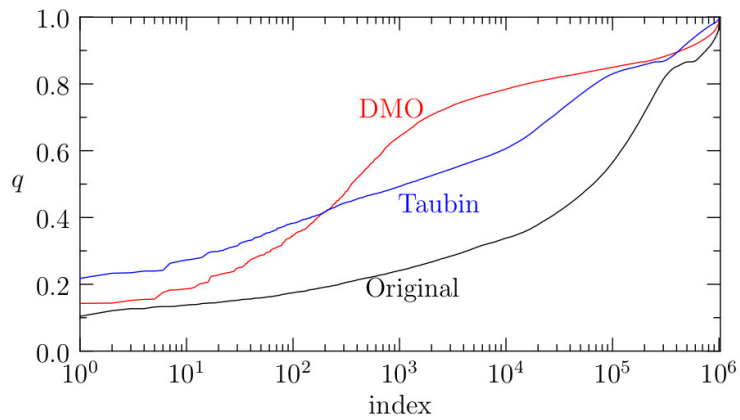

Figure 13: Comparison of Taubin smoothing and DMO on surface mesh filigree. 


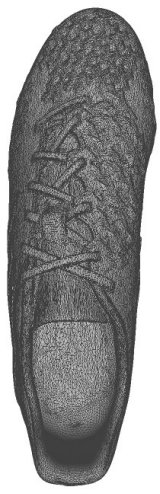

(a) Original

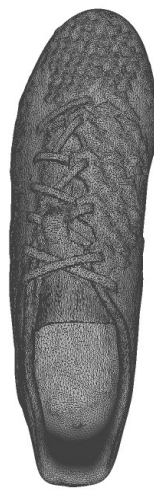

(b) Taubin

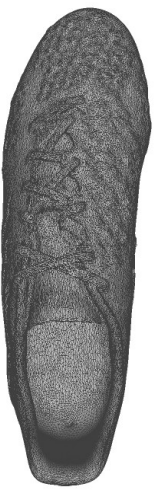

(c) $\mathrm{DMO}$

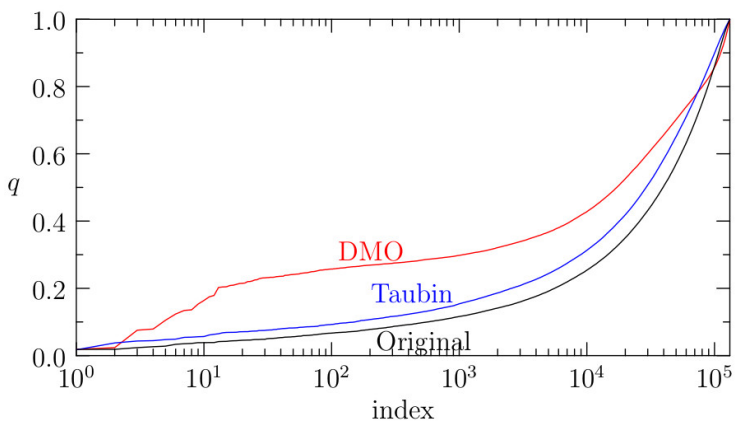

Figure 14: Comparison of Taubin smoothing and DMO on surface mesh shoe.

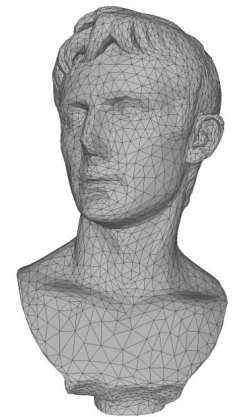

(a) Original

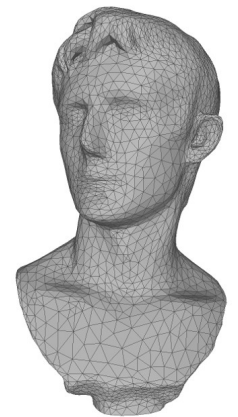

(b) Taubin

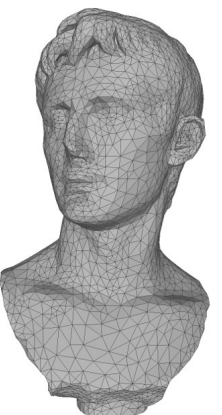

(c) $\mathrm{DMO}$

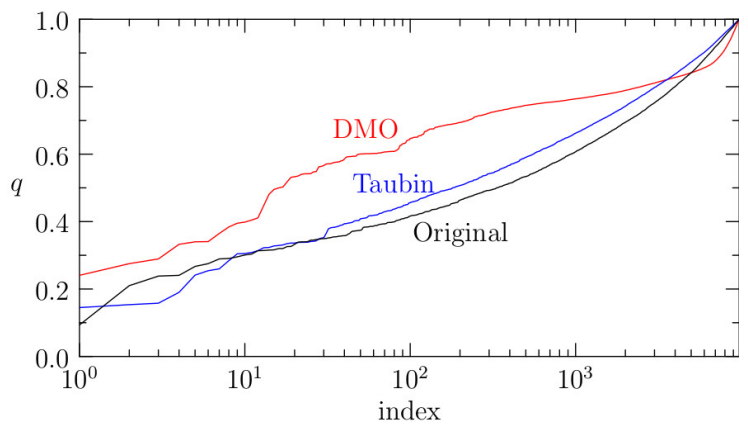

Figure 15: Comparison of Taubin smoothing and DMO on surface mesh augustus.
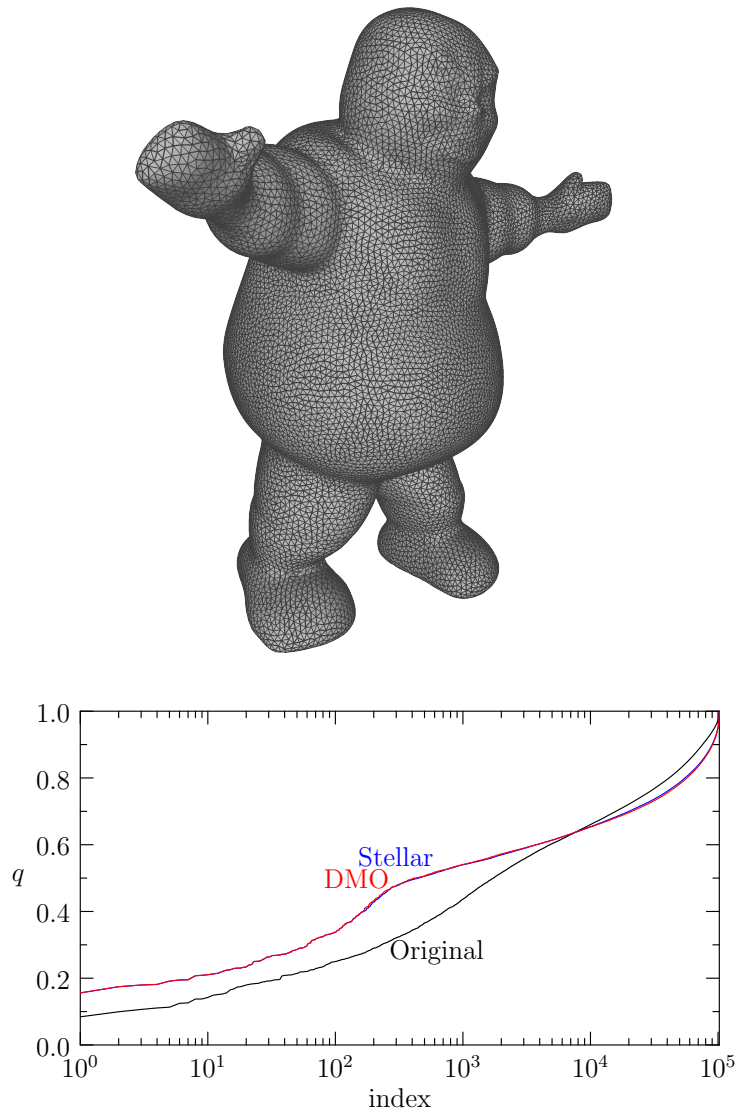

Figure 16: Volume mesh staypuft [30].

When deactivating boundary smoothing, DMO and Stellar converge towards the same result, Figure 16. This is expected as both solve the same optimization problem. The mesh staypuft from [30] was generated by Adam Bargteil's implementation of variational tetrahedral meshing [63].

Including boundaries, DMO has more freedom to move vertices along the boundary. Stellar penalizes boundary movement. Instead, DMO uses boundary information to put hard constraints on the optimization. This leads to much better quality, e.g. on mesh cube, Figure 17(a), from [30] using NETGEN [64]. Stellar can only slightly improve the minimal element quality from 0.628 to 0.635 in comparison to fixed boundaries. DMO reaches a minimal quality of 0.758 . Furthermore, Stellar changed the boundary, e.g. the corner vertices. For numerical simulations this might be a critical issue.

The tetrahedral mesh sphere, Figure 18(a), created with TetGen [5], benefits also from boundary smoothing. Even though, the surface meshes of Stellar, Figure 18(b), and DMO, Figure 18(c) look disturbed, both improve tetrahedral mesh quality significantly, 


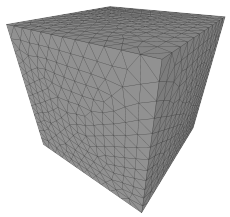

(a) Original

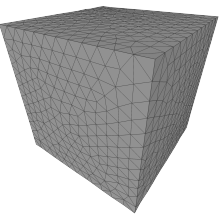

(b) Stellar

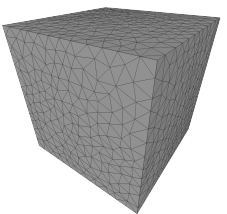

(c) $\mathrm{DMO}$

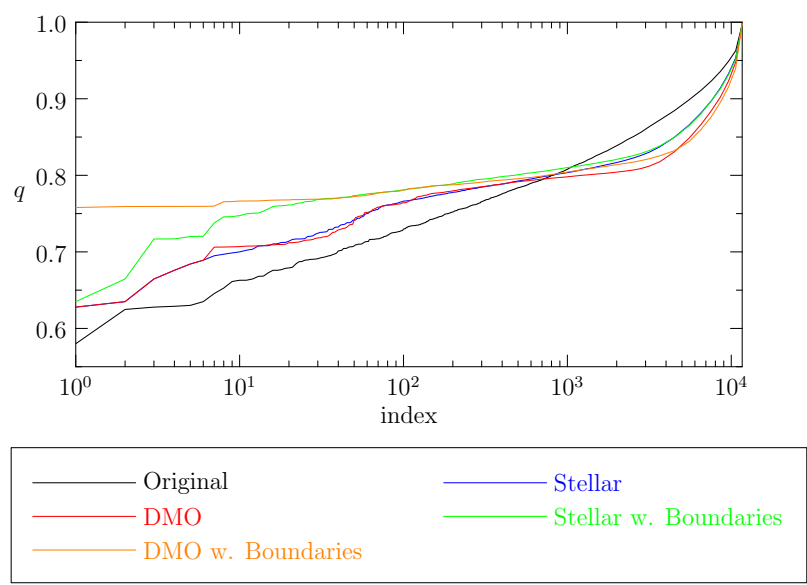

Figure 17: Volume mesh cube.

Figure 18. Without boundary smoothing Stellar and DMO reach a minimal quality of 0.361 . With boundary smoothing Stellar creates a minimal quality of 0.509, DMO of 0.545. Again, it has to be considered that some boundary vertices of Stellar do not lie precisely on the sphere anymore.

Observing Figures 18(b) and 18(c) one might realize that the surface meshes of DMO and Stellar look worse than the original. This is caused by optimizing for tetrahedral quality instead of triangle quality. Low quality elements are improved by decreasing the quality of their surrounding elements. Therefore, a former good looking surface mesh becomes disturbed, even though the minimal element quality increases.

Surface preservation constraints optimization. The mesh sculpt, Figure 19, from [30] was generated by Pierre Alliez's implementation of variational tetrahedral meshing [63]. On this mesh, Stellar creates a minimal element quality of 0.518, DMO 0.386. Slight movements on feature edges may have severe impact on element quality. DMO restricts movement to the feature edge, whereas Stellar has more freedom.

More complex shapes are presented with mesh fandisk, bracket-2, and bracket-3, Figures 20 to 22 . The surface meshes are provided by and generated with JIGSAW [65]. The tetrahedral meshes were generated with TetGen. For uncertain reasons, Stellar cannot handle these meshes well. DMO performs as expected and

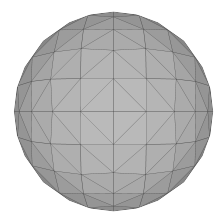

(a) Original

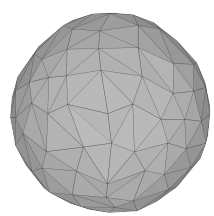

(b) Stellar

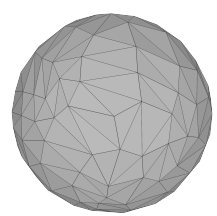

(c) $\mathrm{DMO}$

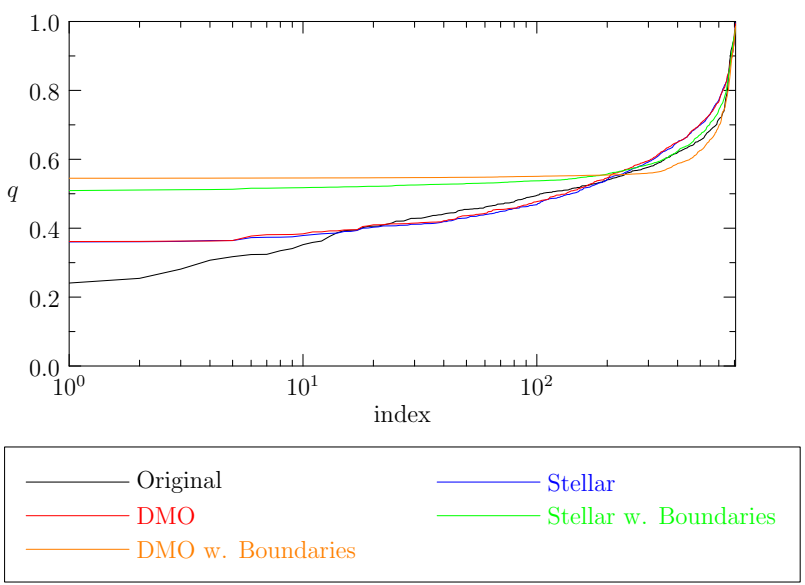

Figure 18: Volume mesh sphere.

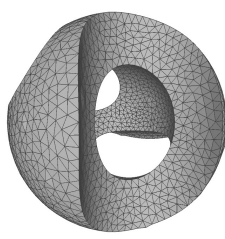

(a) Original

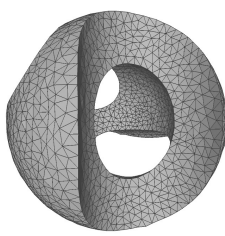

(b) Stellar

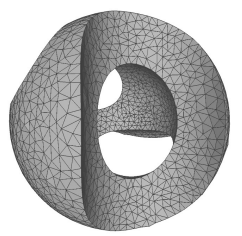

(c) DMO

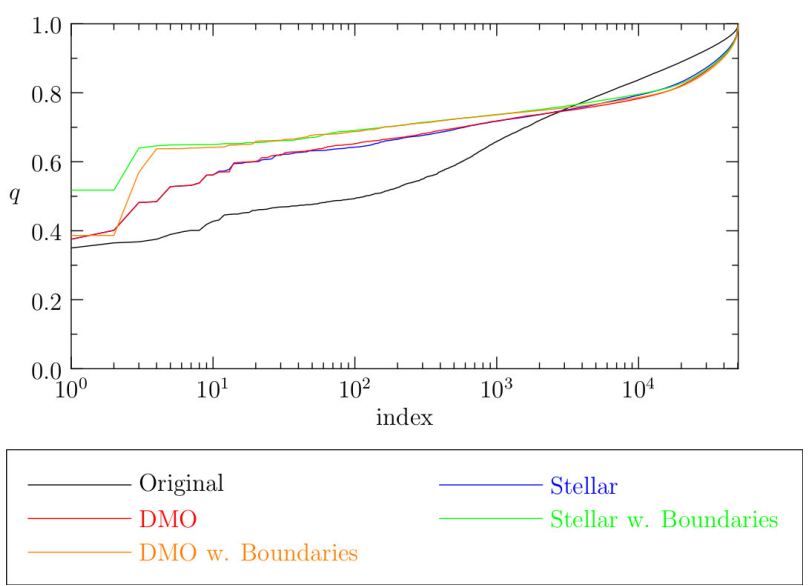

Figure 19: Volume mesh sculpt. 


\begin{tabular}{l|r|r} 
& DMO / Original & Stellar / Original \\
\hline cube & 1.0000 & 0.9995 \\
sphere & 0.9926 & 1.0044 \\
sculpt & 1.0002 & 0.9995 \\
fandisk & 1.0040 & 0.9998 \\
bracket-2 & 1.0034 & 1.0000 \\
bracket-3 & 1.0043 & 1.0000
\end{tabular}

Table 1: Relative volume of optimized meshes.

improves quality, when boundary vertices are also optimized. For fixed boundaries, DMO cannot improve minimal element quality.

Our method preserves the described volume very well. Table 1 compares the mesh volume before and after optimization. The volumes never differ more than $1 \%$.

DMO was developed for being executed on a GPU, which allows smoothing in the range of a few seconds. For the optimization of only interior vertices in tetrahedral meshes, DMO repositions about $1.1 \cdot 10^{5}$ vertices per second, e.g. DMO requires one second to perform 10 iterations on a mesh with 10000 vertices. In most cases, 10 iterations are enough to converge towards the optimum. Unfortunately, so far the surface estimation is only implemented on CPU. Therefore, a valid performance statement cannot be done for surface optimization. We expect the performance to be comparable as surface estimation is mostly a pre-processing step. On $\mathrm{CPU}$ the performance for tetrahedral meshes drops to 230 vertices per second. Therefore, using a GPU is highly recommended.

\section{CONCLUSION}

We presented a method to improve surface and volume meshes. Combining DMO's geometry- and topologyconsistency with its local optimality, results in a method which produces high quality meshes. Surface mesh quality can be significantly improved without the need of user interaction as it is required in other methods. This is due to the mean ratio metric used for the examples presented above. We demonstrated the effectiveness of our method for different representative examples including surface and volume meshes. Furthermore, we showed that smoothing boundary vertices of tetrahedral meshes has significant impact on their quality.

In future work we want to extend DMO with topological optimizations [30], [4]. DMO is perfectly suited for running on GPUs. We plan to develop a GPU implementation and compare performance to other smoothing techniques.

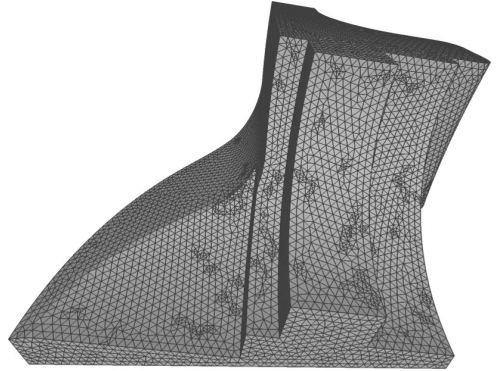

(a) Original

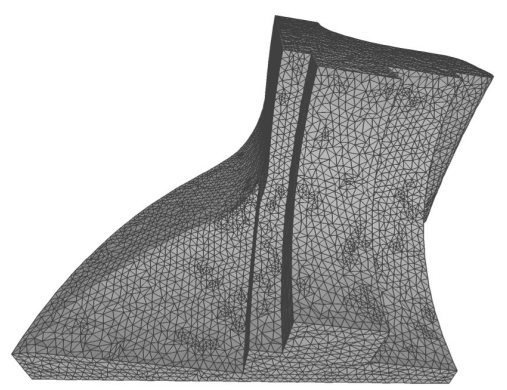

(b) Stellar

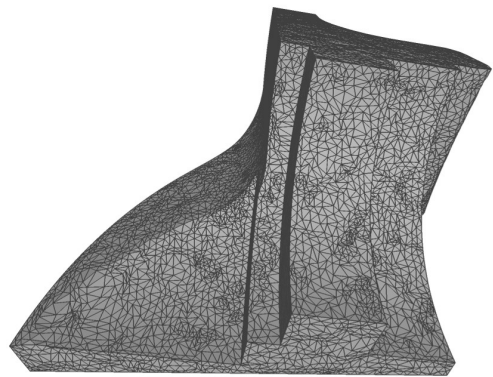

(c) $\mathrm{DMO}$
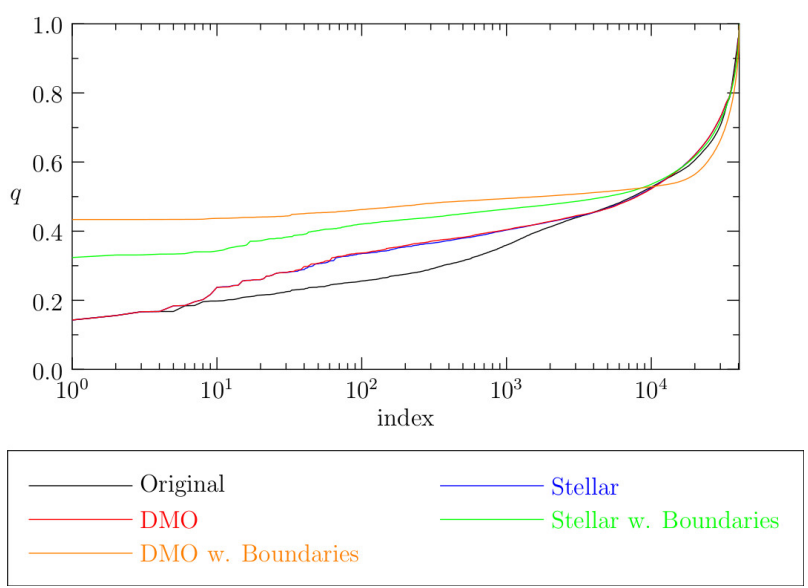

Figure 20: Volume mesh fandisk. 


\section{ACKNOWLEDGMENT}

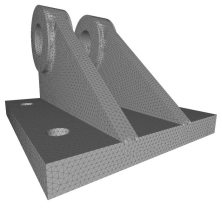

(a) Original

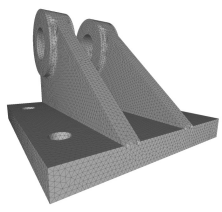

(b) Stellar

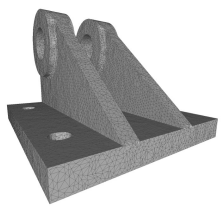

(c) $\mathrm{DMO}$
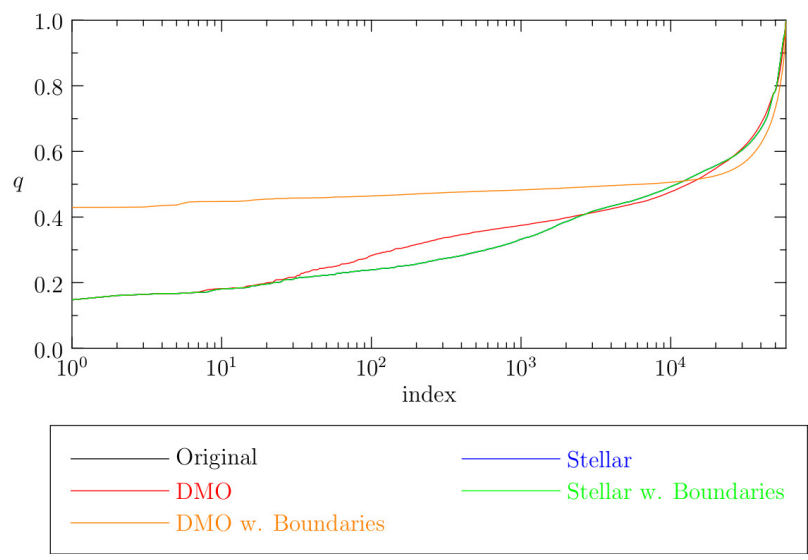

Figure 21: Volume mesh bracket-2.

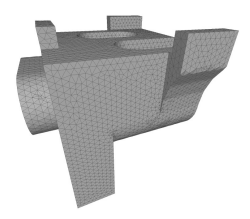

(a) Original

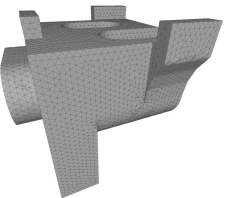

(b) Stellar

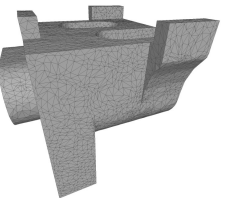

(c) $\mathrm{DMO}$
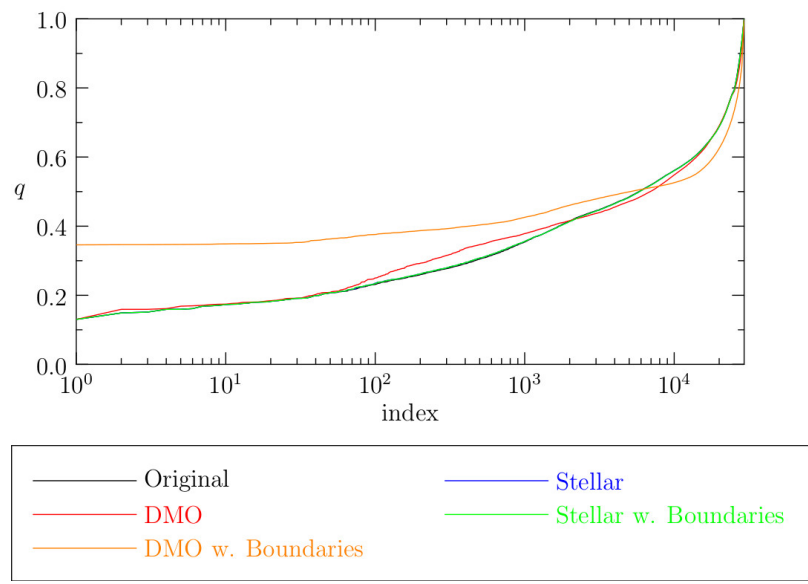

Figure 22: Volume mesh bracket-3.
This work has been supported by the DFG grant 'Rechenleistungsoptimierte Software-Strategien für auf unstrukturierten Gittern basierende Anwendungen in der Ozeanmodellierung' (GR 1107/3-1).

\section{References}

[1] Freitag L., Plassmann P., Jones M. "An efficient parallel algorithm for mesh smoothing." Tech. rep., Argonne National Lab., IL (United States), 1995

[2] Herrmann L.R. "Laplacian-isoparametric grid generation scheme." Journal of the Engineering Mechanics Division, vol. 102, no. 5, 749-907, 1976

[3] Jones R. "QMESH: A self-organizing mesh generation program." Tech. rep., Sandia Labs., Albuquerque, N. Mex.(USA), 1974

[4] Dassi F., Kamenski L., Si H. "Tetrahedral mesh improvement using moving mesh smoothing and lazy searching flips." Procedia engineering, vol. $163,302-314,2016$

[5] Si H. "TetGen, a Delaunay-based quality tetrahedral mesh generator." ACM Transactions on Mathematical Software (TOMS), vol. 41, no. 2, 11,2015

[6] Wicke M., Ritchie D., Klingner B.M., Burke S., Shewchuk J.R., O'Brien J.F. "Dynamic local remeshing for elastoplastic simulation." $A C M$ Transactions on graphics (TOG), vol. 29, no. 4, 49, 2010

[7] Rangarajan R., Lew A.J. "Provably Robust Directional Vertex Relaxation for Geometric Mesh Optimization." SIAM Journal on Scientific Computing, vol. 39, no. 6, A2438-A2471, 2017

[8] Zint D., Grosso R. "Discrete Mesh Optimization on GPU." 27th International Meshing Roundtable, 2018

[9] Zint D., Grosso R., Aizinger V., Köstler H. "Generation of Block Structured Grids on Complex Domains for High Performance Simulation (accepted)." Numerical Geometry, Grid Generation and Scientific Computing, 2019

[10] Field D.A. "Laplacian smoothing and Delaunay triangulations." International Journal for Numerical Methods in Biomedical Engineering, vol. 4, no. 6, 709-712, 1988 
[11] Blacker T.D., Stephenson M.B. "Paving: A new approach to automated quadrilateral mesh generation." International Journal for Numerical Methods in Engineering, vol. 32, no. 4, 811-847, 1991

[12] Blacker T.D., Stephenson M.B., Canann S. "Analysis automation with paving: a new quadrilateral meshing technique." Advances in engineering software and workstations, vol. 13, no. 5-6, 332-337, 1991

[13] Canann S.A., Liu Y.C., Mobley A.V. "Automatic 3D surface meshing to address today's industrial needs." Finite Elements in Analysis and Design, vol. 25, no. 1-2, 185-198, 1997

[14] Freitag L.A. "On combining Laplacian and optimization-based mesh smoothing techniques." ASME applied mechanics division-publicationsamd, vol. 220, 37-44, 1997

[15] George P., Borouchaki H. Delaunay Triangulation and Meshing: Application to Finite Elements. Hermès, 1998

[16] Knupp P.M. "Winslow smoothing on twodimensional unstructured meshes." Engineering with Computers, vol. 15, no. 3, 263-268, 1999

[17] Zhou T., Shimada K. "An Angle-Based Approach to Two-Dimensional Mesh Smoothing." IMR, pp. 373-384. 2000

[18] Taubin G. "A signal processing approach to fair surface design." Proceedings of the 22nd annual conference on Computer graphics and interactive techniques, pp. 351-358. ACM, 1995

[19] Blom F.J. "Considerations on the spring analogy." International journal for numerical methods in fluids, vol. 32, no. 6, 647-668, 2000

[20] Farhat C., Degand C., Koobus B., Lesoinne M. "Torsional springs for two-dimensional dynamic unstructured fluid meshes." Computer methods in applied mechanics and engineering, vol. 163, no. 1-4, 231-245, 1998

[21] Persson P.O., Strang G. "A simple mesh generator in MATLAB." SIAM review, vol. 46, no. 2, 329-345, 2004

[22] Baker T.J. "Mesh movement and metamorphosis." Engineering with Computers, vol. 18, no. 3, 188-198, 2002

[23] De Almeida V.F. "Domain deformation mapping: application to variational mesh generation." SIAM Journal on Scientific Computing, vol. 20, no. 4, 1252-1275, 1999
[24] Rumpf M. "A variational approach to optimal meshes." Numerische Mathematik, vol. 72, no. 4, 523-540, 1996

[25] Freitag L.A., Knupp P.M. "Tetrahedral mesh improvement via optimization of the element condition number." International Journal for $\mathrm{Nu}$ merical Methods in Engineering, vol. 53, no. 6, 1377-1391, 2002

[26] Kim J. "A Multiobjective Mesh Optimization Algorithm for Improving the Solution Accuracy of PDE Computations." International Journal of Computational Methods, vol. 13, no. 01, 1650002, 2016

[27] Knupp P. "Updating meshes on deforming domains: An application of the target-matrix paradigm." International Journal for Numerical Methods in Biomedical Engineering, vol. 24, no. 6, 467-476, 2008

[28] Xu K., Gao X., Chen G. "Hexahedral mesh quality improvement via edge-angle optimization." Computers \& Graphics, vol. 70, 17-27, 2018

[29] Zavattieri P.D., Dari E.A., Buscaglia G.C. "Optimization strategies in unstructured mesh generation." International Journal for Numerical Methods in Engineering, vol. 39, no. 12, 2055-2071, 1996

[30] Klingner B.M., Shewchuk J.R. "Aggressive tetrahedral mesh improvement." Proceedings of the 16th international meshing roundtable, pp. 3-23. Springer, 2008

[31] Freitag L., Jones M., Plassmann P. "A parallel algorithm for mesh smoothing." SIAM Journal on Scientific Computing, vol. 20, no. 6, 2023-2040, 1999

[32] Freitag L.A., Plassmann P., et al. "Local optimization-based simplicial mesh untangling and improvement." International Journal for $\mathrm{Nu}$ merical Methods in Engineering, vol. 49, no. 1, 109-125, 2000

[33] Park J., Shontz S.M. "Two derivative-free optimization algorithms for mesh quality improvement." Procedia Computer Science, vol. 1, no. 1, 387-396, 2010

[34] Zhang Y., Xu G., Bajaj C. "Quality meshing of implicit solvation models of biomolecular structures." Computer Aided Geometric Design, vol. 23 , no. $6,510-530,2006$

[35] Leng J., Zhang Y., Xu G. "A novel geometric flow-driven approach for quality improvement 
of segmented tetrahedral meshes." Proceedings of the 20th international meshing roundtable, pp. 347-364. Springer, 2011

[36] Leng J., Zhang Y., Xu G. "A novel geometric flow approach for quality improvement of multicomponent tetrahedral meshes." Computer-Aided Design, vol. 45, no. 10, 1182-1197, 2013

[37] Fleishman S., Drori I., Cohen-Or D. "Bilateral mesh denoising." ACM transactions on graphics (TOG), vol. 22, no. 3, 950-953, 2003

[38] Jones T.R., Durand F., Desbrun M. "Noniterative, feature-preserving mesh smoothing." ACM Transactions on Graphics (TOG), vol. 22, no. 3, 943-949, 2003

[39] Yu Y., Zhou K., Xu D., Shi X., Bao H., Guo B., Shum H.Y. "Mesh editing with poisson-based gradient field manipulation." ACM Transactions on Graphics (TOG), vol. 23, no. 3, 644-651, 2004

[40] Choudhury P., Tumblin J. "The trilateral filter for high contrast images and meshes." ACM SIGGRAPH 2005 Courses, p. 5. ACM, 2005

[41] Boissonnat J.D., Flototto J. "A Local Coordinate System on a Surface." Proceedings of the Seventh ACM Symposium on Solid Modeling and Applications, SMA '02, pp. 116126. ACM, New York, NY, USA, 2002. URL http://doi.acm.org/10.1145/566282.566302

[42] Goldfeather J., Interrante V. "A novel cubicorder algorithm for approximating principal direction vectors." ACM Transactions on Graphics (TOG), vol. 23, no. 1, 45-63, 2004

[43] Kalogerakis E., Nowrouzezahrai D., Simari P., Singh K. "Extracting lines of curvature from noisy point clouds." Computer-Aided Design, vol. 41, no. 4, 282-292, 2009

[44] Demarsin K., Vanderstraeten D., Volodine T., Roose D. "Detection of closed sharp edges in point clouds using normal estimation and graph theory." Computer-Aided Design, vol. 39, no. 4, 276-283, 2007

[45] Lancaster P., Salkauskas K. "Surfaces generated by moving least squares methods." Mathematics of computation, vol. 37, no. 155, 141-158, 1981

[46] Levin D. "The approximation power of moving least-squares." Mathematics of Computation of the American Mathematical Society, vol. 67, no. 224, 1517-1531, 1998
[47] Fleishman S., Cohen-Or D., Silva C.T. "Robust moving least-squares fitting with sharp features." ACM transactions on graphics (TOG), vol. 24, no. 3, 544-552, 2005

[48] Guennebaud G., Gross M. "Algebraic point set surfaces." ACM Transactions on Graphics (TOG), vol. 26, no. 3, 23, 2007

[49] Cazals F., Pouget M. "Estimating differential quantities using polynomial fitting of osculating jets." Computer Aided Geometric Design, vol. 22, no. $2,121-146,2005$

[50] Darom T., Keller Y. "Scale-invariant features for 3-D mesh models." IEEE Transactions on Image Processing, vol. 21, no. 5, 2758-2769, 2012

[51] Ohtake Y., Belyaev A., Seidel H.P. "Ridge-valley lines on meshes via implicit surface fitting." $A C M$ transactions on graphics (TOG), vol. 23, no. 3, 609-612, 2004

[52] Watanabe K., Belyaev A.G. "Detection of salient curvature features on polygonal surfaces." Computer Graphics Forum, vol. 20, no. 3, 385-392, 2001

[53] Hubeli A., Gross M. "Multiresolution feature extraction for unstructured meshes." Proceedings of the Conference on Visualization'01, pp. 287-294. IEEE Computer Society, 2001

[54] Zaharescu A., Boyer E., Varanasi K., Horaud R. "Surface feature detection and description with applications to mesh matching." 2009 IEEE Conference on Computer Vision and Pattern Recognition, pp. 373-380. IEEE, 2009

[55] Kim H.S., Choi H.K., Lee K.H. "Feature detection of triangular meshes based on tensor voting theory." Computer-Aided Design, vol. 41, no. 1, 47-58, 2009

[56] Wang X.c., Cao J.j., Liu X.p., Li B.j., Shi X.q., Sun Y.z. "Feature detection of triangular meshes via neighbor supporting." Journal of Zhejiang University Science C, vol. 13, no. 6, 440-451, 2012

[57] Zhihong M., Guo C., Mingxi Z. "Robust detection of perceptually salient features on 3D meshes." The Visual Computer, vol. 25, no. 3, 289-295, 2009

[58] Amenta N., Bern M., Eppstein D. "Optimal point placement for mesh smoothing." Journal of Algorithms, vol. 30, no. 2, 302-322, 1999

[59] Bank R.E., Smith R.K. "Mesh smoothing using a posteriori error estimates." SIAM Journal on Numerical Analysis, vol. 34, no. 3, 979-997, 1997 
[60] Bank R. "A Software Package for Solving Elliptic Partial Differential Equations-Users' Guide 7.0." Frontiers in Applied Mathematics, vol. 15, 1998

[61] Canann S.A., Tristano J.R., Staten M.L., et al. "An Approach to Combined Laplacian and Optimization-Based Smoothing for Triangular, Quadrilateral, and Quad-Dominant Meshes." IMR, pp. 479-494. Citeseer, 1998

[62] Cignoni P., Callieri M., Corsini M., Dellepiane M., Ganovelli F., Ranzuglia G. "Meshlab: an open-source mesh processing tool." Eurographics Italian chapter conference, vol. 2008, pp. 129-136. 2008

[63] Alliez P., Cohen-Steiner D., Yvinec M., Desbrun M. "Variational tetrahedral meshing." ACM SIGGRAPH 2005 Courses, p. 10. ACM, 2005

[64] Schöberl J. "NETGEN An advancing front 2D/3D-mesh generator based on abstract rules." Computing and visualization in science, vol. 1, no. $1,41-52,1997$

[65] Engwirda D. "Conforming restricted Delaunay mesh generation for piecewise smooth complexes." Procedia engineering, vol. 163, 84-96, 2016 


\title{
COARSE QUAD LAYOUTS THROUGH ROBUST SIMPLIFICATION OF CROSS FIELD SEPARATRIX PARTITIONS
}

\author{
Ryan Viertel $^{1} \quad$ Braxton Osting ${ }^{2} \quad$ Matthew Staten $^{1}$ \\ ${ }^{1}$ Sandia National Laboratories, Albuquerque, NM, U.S.A. rvierte@sandia.gov, mlstate@sandia.gov \\ ${ }^{2}$ University of Utah, Salt Lake City, UT, U.S.A. osting@math.utah.edu
}

\begin{abstract}
Streamline-based quad meshing algorithms use smooth cross fields to partition surfaces into quadrilateral regions by tracing cross field separatrices. In practice, re-entrant corners and misalignment of singularities lead to small regions and limit cycles, negating some of the benefits a quad layout can provide in quad meshing. We introduce three novel methods to improve on a pipeline for coarse quad partitioning. First, we formulate an efficient method to compute high-quality cross fields on curved surfaces by extending the diffusion generated method from Viertel and Osting (SISC, 2019). Next, we introduce a method for accurately computing the trajectory of streamlines through singular triangles that prevents tangential crossings. Finally, we introduce a robust method to produce coarse quad layouts by simplifying the partitions obtained via naive separatrix tracing. Our methods are tested on a database of 100 objects and the results are analyzed. The algorithm performs well both in terms of efficiency and visual results on the database when compared to state-of-the-art methods.
\end{abstract}

Keywords: cross fields, quad partitioning, quad meshing, surface decomposition

\section{INTRODUCTION}

Block structured quad meshes are often desirable because of their numerical efficiency [1], low memory requirements [2], and high mesh quality [3]. Having such a block structure on a surface is also advantageous for tasks such as spline fitting and isogeometric analysis [4]. In the past, such meshes have been designed by hand or in an interactive environment [5]. More recently, researchers have made progress towards fully automating this process [4]. The problem of coarse quad layout generation can be summarized as determining the placement of irregular nodes of the layout, and determining the connectivity of those nodes in such a way that the resulting layout is topologically valid, coarse, and such that a mapping of the region to a quadrilateral in the plane results in low distortion.

Various approaches have been taken to generate coarse quad partitions of surfaces, each of which address these problems of irregular node placement and determining connectivity in different ways. These methods include medial axis subdivision $[6,7]$, computing the Morse-Smale complex [8], surface foliations [9], dual loops [10], simplifying an existing quad mesh $[11,12]$, and more recently, cross field based approaches $[3,13,14,15,16,17,18]$. While in this discussion we focus on cross field based streamline tracing approaches, Campen [4] provides an excellent literature review on quad patching algorithms.

In cross field based streamline tracing approaches, irregular node placement is determined by computing singular points of the cross field, where simple singularities of positive or negative index correspond to irregular nodes of valence 3 or 5 respectively. The connectivity of the layout is then determined by either tracing out raw separatrices of the cross field, or by using the isolines of an underlying parameterization. A common problem with separatrix tracing approaches is 


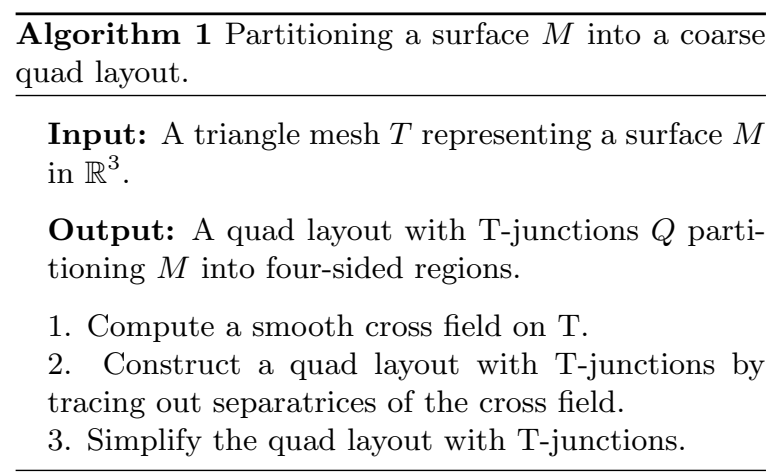

that on a discrete geometry, singularities are never perfectly aligned. In practice, this frequently causes limit cycles and very thin regions to occur within the quad layout. This is problematic for meshing because very small mesh elements are required. Further, the base complex of the mesh is often far more complicated than necessary, mitigating the benefits of a multi-block decomposition. Another problem is that despite the fact that in the continuum, streamlines can only cross each other orthogonally [19], numerical inaccuracies often lead to tangential crossings of streamlines. This is especially true near singularities, where large changes in direction occur over arbitrarily small lengths.

We make three primary contributions:

1. We extend the diffusion generated method for cross field design in [19] to curved surfaces. This method which has previously only been described in $2 \mathrm{D}$ tends to have good singularity placement in locations where singularities occur because of boundary curvature rather than Gaussian curvature of the surface, a common scenario for CAD surfaces.

2. We prove that near singularities, streamlines of a cross field are hyperbolic under a conformal map. This results in a simple method to compute streamlines in the neighborhood of a singularity that prevents tangential crossings.

3. We describe a novel algorithm for simplifying a quad partition with T-junctions such that the number of partition components strictly decreases and the number of T-junctions decreases monotonically.

To demonstrate these contributions, we include them in the pipeline described in algorithm 1, which takes as input a triangle mesh, and outputs a coarse quad layout, possibly with T-junctions.

\subsection{Related Work}

Several researchers have taken an approach to generating quad layouts that is similar to the pipeline in algorithm 1. Kowalski et al. [3] design a cross field by solving a PDE with a constraint applied via Lagrange multipliers. They numerically integrate streamlines from each interior singularity and boundary corner, and snap streamlines to singularities when they pass within a certain tolerance of the singularity in order to obtain a coarser quad layout. While streamline snapping works well on some examples, it is not robust in general because it can introduce tangential crossings when more than one streamline passes nearby a singularity. Fogg et al. [15] take a similar approach, but initialize the cross field by an advancing front method and then smooth it with the energy functional introduced by Hertzmann and Zorin [20]. Rather than snapping separatrices to singularities, they allow them to pass by singularities, resulting in thin regions throughout the partition. Ray and Sokolov [21] and Myles et al. [22] both implement robust streamline tracing algorithms based on edge maps [23], and then trace out streamlines in parallel until their first crossing with another streamline, forming a motorcycle graph [24]. This approach yields coarse quad patches because of the large number of T-junctions that appear in the decomposition. Because of the T-junctions, it is a nontrivial matter to assign globally consistent parametric lengths to each edge. Myles et al. [22] are able to achieve this via a heuristic method, which includes a collapse operation, similar to the one we describe in section 4, used to remove edges with zero parametric length, and 2-6 cone insertion to remove zero edges which cannot be collapsed. They subsequently generate a globally consistent seamless parameterization on each quad region. This method differs from our goal in that the parameterization is not quantized (cf. [16]) and so does not correspond to a quad mesh let alone a coarse quad decomposition. Campen et al. [16] compute quantized parameterizations on surfaces by solving a combinatorial optimization problem similar to [13] but leverage the structure of a motorcycle graph to determine a set of linear equality constraints which are applied to the final optimization problem, guaranteeing a valid solution which also outperforms previous parameterization methods, especially in cases with a large number of singularities or large target edge lengths. This enables them finally to extract a coarse quad mesh. Razafindrazaka et al. [14] trace out separatrices of a seamless parameterization to generate a graph of possible matchings between separatrices and singularities. They then formulate the problem of connecting singularities together as a minimum weight perfect matching problem. In a second paper [25] they extend their perfect matching method to work on an input quad mesh rather than a seamless parameteriza- 
tion. Zhang et al. [17] employ a similar strategy to [14], from an input seamless parameterization they identify candidate separatrices as monotone isolines of the parameterization in areas they call safety turning areas which are rectangular parameterizations between opposite singularities. From these candidate separatrices they choose an optimal set via a binary optimization problem. Pietroni et al. [18] trace out candidate curves connecting either two singularities or a singularity to a boundary which they call field-coherent streamlines, which can deviate from the cross field but never switch to a different direction of the field. They then select a valid subset of these candidate streamlines to form a quad layout with T-junctions by solving a binary optimization problem.

The pipeline we implement is most similar to $[14,17]$ and [18] because it attempts to generate a quad layout by directly manipulating the streamlines of an underlying cross field. It also shares similarities with the methods of Tarini et al. [11] and Bommes et al. [12], which attempt to simplify the base complex of an unstructured quad mesh via greedy application of grid preserving operators, the difference being that our simplification method is applied directly to the separatrices of a cross field rather than an input quad mesh.

\section{CROSS FIELD DESIGN}

Many different methods exist to design a smooth cross field on a curved surface $[20,26,27,28,29,30,31,32$, $33,34,35,36]$, any of which might be used in algorithm 1. In the interest of brevity, we will not offer a full review of the literature here but instead refer the reader to [34]. The diffusion generated method introduced in Viertel and Osting [19] is appealing in the context of meshing CAD surfaces because it is comparable in speed to the fastest methods such as those described in Knöppel et al. [31] and Jakob et al. [33], but also has an advantage over these methods on surfaces with boundary, which are common in CAD and meshing for FEM, because it tends to place singularities symmetrically even when the surface is flat (see Figure 1).
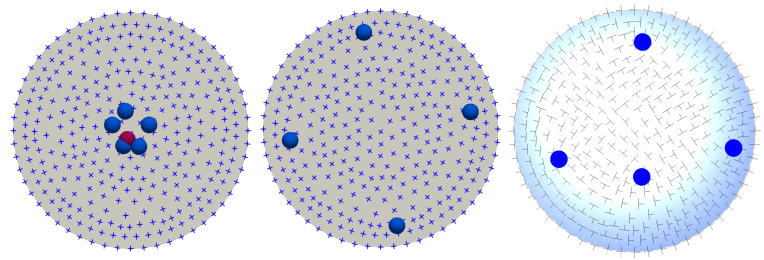

Figure 1: Left to right, a comparison of singularity placement from cross fields described in Knöppel et al. [31], Viertel and Osting [19], and Jakob et al. [33]. Singularities with positive and negative index are shown in blue and red respectively.
In this section, we extend the diffusion generated method for cross field design to curved surfaces. We use this method of cross field design as the first step in our pipeline (algorithm 1). In section 2.1, we first review some tools from the literature that have been used previously to develop cross field design methods on surfaces. In section 2.2 , we describe the implementation details of our method.

\subsection{Cross Fields on Surfaces}

The main difficulty in extending flat $2 \mathrm{D}$ methods to curved surfaces is the lack of a global coordinate system. In this section, we use concepts from differential geometry to formulate the cross field design problem on 2-manifolds. We consider a smooth, orientable 2manifold $M$ embedded in $\mathbb{R}^{3}$ and endowed with the Riemannian metric induced by the the Euclidean metric on $\mathbb{R}^{3}$. Let $\mathbb{T}=\{z \in \mathbb{C}:|z|=1\}$ be the circle group with group operation given by complex multiplication and let $\rho(4)$ be the set of the 4 th roots of unity. A cross is an element of $C=\mathbb{T} / \rho(4)$. There is a canonical group isomorphism $R: C \rightarrow \mathbb{T}$ called the representation map given by $R([c])=c^{4}$, where $c$ is any representative member of the equivalence class $[c] \in C$. We will refer to $u=c^{4}$ as the representation vector for $[c]$. The inverse representation map $R^{-1}: \mathbb{T} \rightarrow C$ assigns $u \in \mathbb{T}$ to $R^{-1}(u)=[\sqrt[4]{u}]$, the equivalence class of the 4 th roots of $u$.

Let $T_{p}$ be the tangent space of $M$ at a point $p$. The disjoint union of all tangent spaces on $M$ is called the tangent bundle and is denoted $T M$. For each tangent space $T_{p}$ we select a coordinate basis, $\left\{\left.\frac{\partial}{\partial x^{1}}\right|_{p},\left.\frac{\partial}{\partial x^{2}}\right|_{p}\right\}$. We also associate with each point $p$ of $M$ a space homeomorphic to $C$, which we call the cross space at $p$. We denote this space by $C_{p}$. The disjoint union of all cross spaces of $M$ defines a fiber bundle that we refer to as the cross bundle. A section of the cross bundle, or a choice of one cross per cross space, is called a cross field on $M$. We make the natural identification between $T_{p}$ and the complex plane by the map $(a, b) \mapsto a+i b$. In this way, we can identify a cross, $\left[c_{p}\right]$, in $C_{p}$ as an unordered set of four orthogonal unit vectors in $T_{p}$, which we call the cross component vectors. This also allows us to define a representation map $R_{p}$ at each point with respect to the local coordinate basis, and a representation vector $u_{p}=R_{p}\left(\left[c_{p}\right]\right)$, which we identify with the corresponding unit tangent vector in $T_{p}$. For simplicity, we will use complex notation for equations throughout the paper.

In $2 \mathrm{D}$ cross field design, the goal of designing a smooth cross field is often formulated as designing a harmonic representation vector field $u[19,34]$. That is, to minimize the Dirichlet energy

$$
E[u]:=\frac{1}{2} \int_{M}|\nabla u|^{2} d A
$$


with the constraint that $|u|=1$ at each point of the domain. We note that in general, this problem is illposed; however, generalized solutions exist if a finite number of singular points are removed from $M[19,37]$.

This strategy can be extended to surfaces by replacing the gradient operator $\nabla$ with the appropriate connection on the tangent bundle. The Levi-Civita connection provides a way to compare vectors on the tangent bundle that preserves the notion of inner product between tangent spaces. That is, if $P_{p q}$ is the parallel transport function for the Levi-Civita connection between $T_{p}$ and $T_{q}$, and if $v_{1}, v_{2} \in T_{p}$, then

$$
\left\langle v_{1}, v_{2}\right\rangle_{T_{P}}=\left\langle P_{p q}\left(v_{1}\right), P_{p q}\left(v_{2}\right)\right\rangle_{T_{q}} .
$$

Visually, the effect of using this connection in equation 1 is that a minimizing vector field appears smooth (see Figure 2).

In order to extend the strategy from $2 \mathrm{D}$, using equation 1 to design a smooth cross field, we seek a connection, $\nabla^{Q}$, on the tangent bundle with corresponding parallel transport function, $Q$, appropriate for transporting representation vectors. We choose $Q$ in such a way that the component vectors of the corresponding crosses are transported by $P$, the parallel transport function corresponding to the Levi-Civita connection. Let $\gamma_{p q}(t):[0,1] \rightarrow M$ be a Levi-Civita geodesic connecting points $p$ and $q$ such that $\gamma_{p q}\left(t_{p}\right)=p$, $\gamma_{p q}\left(t_{q}\right)=q$, and $t_{p}, t_{q} \in(0,1)$. Let $\phi_{p}$ be the signed angle from the velocity vector $\gamma_{p q}^{\prime}\left(t_{p}\right)$ to $\left.\frac{\partial}{\partial x^{1}}\right|_{p}$ and let $\phi_{q}$ be the signed angle from $\gamma_{p q}^{\prime}\left(t_{q}\right)$ to $\left.\frac{\partial}{\partial x^{1}}\right|_{q}$. Then if $\phi_{p q}=\phi_{p}-\phi_{q}, P_{p q}(v)=e^{i \phi_{p q}} v$ gives the Levi-Civita parallel transport function, $P_{p q}$, in coordinates with respect to the basis $\left\{\left.\frac{\partial}{\partial x^{1}}\right|_{p},\left.\frac{\partial}{\partial x^{2}}\right|_{p}\right\}$.

Then, $Q_{p q}$, the parallel transport function for representation vectors between $p$ and $q$, must satisfy

$$
Q_{p q}\left(R_{p}\left(\left[c_{p}\right]\right)\right)=R_{q}\left(\left[P_{p q}\left(c_{p}\right)\right]\right) .
$$

We can write $c_{p}=e^{i\left(\theta_{p}+2 k \pi / 4\right)}$ for some $k \in\{0,1,2,3\}$ where $\theta_{p}$ is the signed angle from $\left.\frac{\partial}{\partial x^{1}}\right|_{p}$ to one of the component vectors of $\left[c_{p}\right]$. We can now write equation 2 as

$$
Q_{p q}\left(e^{4 i \theta_{p}}\right)=e^{4 i\left(\theta_{p}+\phi_{p q}\right)} .
$$

It follows that $Q_{p q}(v)=e^{4 i \phi_{p q}} v$. In addition, we can define parallel transport on the cross bundle from $C_{p}$ to $C_{q}$ by $R_{q}^{-1} \circ Q_{p q} \circ R_{p}$. We can now use $\nabla^{Q}$ in equation 1 to define a smooth cross field. In the following section, we define the discrete Laplace equation corresponding to this energy and describe the diffusion generated method for cross field design in detail.

\subsection{Discrete Formulation}

On each node $n_{i}$ of the input triangle mesh, T, a normal vector, $\vec{n}_{i}$, is computed as an average of the vec-
Table 1: Boundary index assignment

\begin{tabular}{ll}
\hline Interior Angle & Index \\
\hline$\left(0, \frac{3 \pi}{4}\right)$ & $\frac{1}{4}$ \\
{$\left[\frac{3 \pi}{4}, \frac{5 \pi}{4}\right]$} & 0 \\
$\left(\frac{5 \pi}{4}, \frac{7 \pi}{4}\right]$ & $-\frac{1}{4}$ \\
$\left(\frac{7 \pi}{4}, 2 \pi\right)$ & $-\frac{1}{2}$ \\
\hline
\end{tabular}

tors normal to each adjacent face weighted by the tip angle at the node. This normal vector in turn defines the tangent space, $T_{i}$, at node $n_{i}$. We then arbitrarily select a vector in $T_{i}$, which we assign to be $\left.\frac{\partial}{\partial x^{1}}\right|_{i}$. Then $\left.\frac{\partial}{\partial x^{2}}\right|_{i}$ is the vector such that $\left.\frac{\partial}{\partial x^{1}}\right|_{i} \times\left.\frac{\partial}{\partial x^{2}}\right|_{i}=\vec{n}_{i}$.

Let $e_{i j}$ be the edge connecting nodes $n_{i}$ and $n_{j}$. We compute the value $\phi_{i}$ as the signed angle between the projection of $e_{i j}$ onto $T_{i}$ and $\left.\frac{\partial}{\partial x^{1}}\right|_{i}$. We then store the value $\phi_{i j}=\phi_{j}-\phi_{i}$, on the edge $e_{i j}$.

We now define the discrete parallel transport function $Q_{i j}: T_{i} \rightarrow T_{j}$ by $Q_{i j}(u)=e^{4 i \phi_{i j}} u$, which parallel transports representation vectors across $e_{i j}$. This allows us to compare two representation vectors $u \in T_{i}$ and $v \in T_{j}$ by

$$
\left|v-Q_{i j}(u)\right|^{2}
$$

In order to state a well-defined problem, we apply a Dirichlet condition. In the case of closed manifolds, we arbitrarily fix the orientation of a single cross. In the case of a bounded manifold, we apply a Dirichlet boundary condition by fixing $u_{i}$ on each boundary node. We make the convention that the cross field index of a boundary node (see [19]) is assigned according to Table 1.

For a boundary node $n_{i}$ of index 0 or $-\frac{1}{2}$, we compute the outward pointing unit normal vector of each boundary edge adjacent to $n_{i}$ lying in the plane of the adjacent boundary face. We then project these vectors into the tangent plane at $n_{i}$, and bisect them with a unit vector, $d_{i}$, which averages the directions of the facet normals. Since we want to align the cross to this vector, we set $u_{i}=d_{i}^{4}$. For nodes of index $\pm \frac{1}{4}, d_{i}$ is first rotated $\pi / 4$ radians about $\mathbf{n}_{i}$ in the positive direction before computing $u_{i}$.

\subsubsection{The Diffusion Generated Method for Cross Field Design}

Intuitively, we would like to solve until stationarity the time dependent problem

$$
\begin{aligned}
u_{t}(t, x) & =\Delta u(t, x) & & x \in M \\
u(t, x) & =g(x) & & x \in \partial M \\
u(0, x) & =u^{0}(x) & & x \in M
\end{aligned}
$$



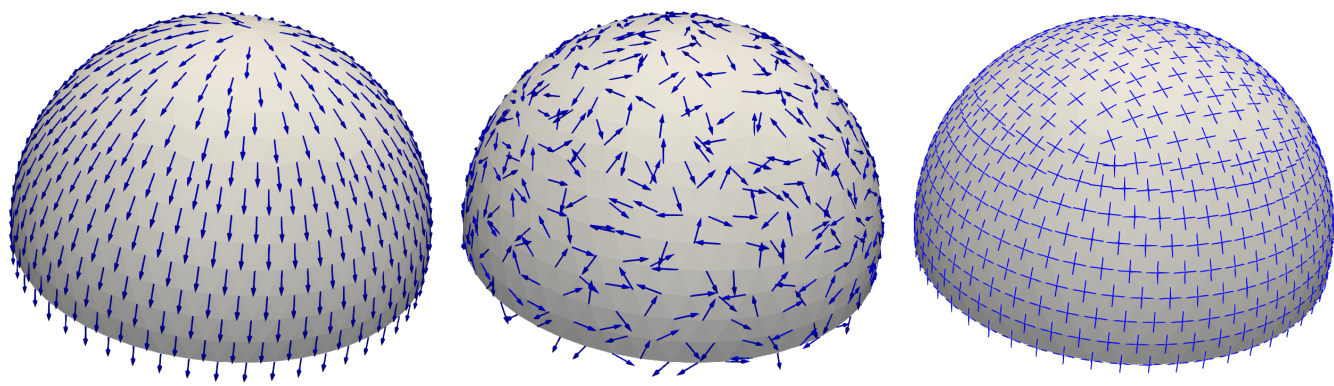

Figure 2: A comparison of fields and connections. Left: A smooth vector field as measured by the Levi-Civita connection. Center: A smooth vector field as measured by the connection $\nabla^{Q}$. Right: The cross field corresponding to the vector field in the center.

with the constraint that $|u(x)|=1$ pointwise, rather than directly solving the stationary problem with the same constraint. This key difference allows one to avoid the necessity of using a non-linear solver because the pointwise constraint can be enforced simply by normalizing the solution in between discrete time steps (see $[38,39]$ ). We proceed by defining a discrete Laplacian operator, $\Delta_{Q}$ corresponding to the connection $\nabla^{Q}$.

$$
\left.\Delta_{Q}(u)\right|_{i}=\frac{1}{\left|\mathcal{N}\left(n_{i}\right)\right|} \sum_{n_{j} \in \mathcal{N}\left(n_{i}\right)}\left(u_{j}-Q_{i j}\left(u_{i}\right)\right)
$$

where $\mathcal{N}\left(n_{i}\right)$ is the one-ring neighborhood of $n_{i}$ and $\left|\mathcal{N}\left(n_{i}\right)\right|$ is the area of that neighborhood. This discrete Laplacian operator allows us to assemble a discrete diffusion equation using a backward Euler time discretization;

$$
(I-\tau A) u=u_{0}+\tau b
$$

where $A$ is the matrix form of $\Delta_{Q}$. We iteratively solve this equation for a small time step $\tau$ and pointwise renormalize the solution between each iteration. The algorithm is described in detail in algorithm 2 .

\section{GENERATION OF A QUAD LAYOUT WITH T-JUNCTIONS}

After designing a cross field on a triangle mesh, the next step in algorithm 1 is to construct a quad layout with T-junctions. This is accomplished in two steps:

1. Determine singularity locations and ports.

2. Trace out separatrices of the cross field.

In section 3.1, we detail the first step of this process. In section 3.2, we describe our approach to streamline tracing, including our novel method for computing the trajectory of a streamline in the neighborhood of a

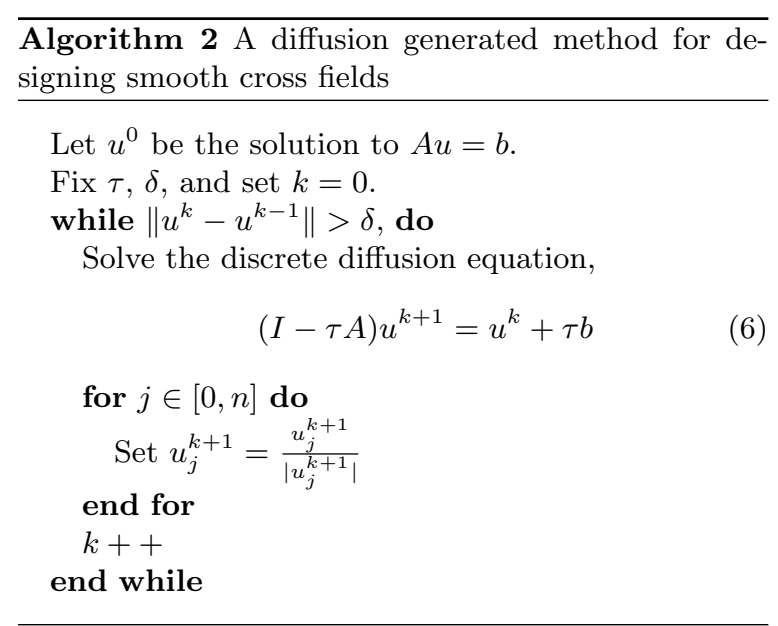

singularity. In section 3.3, we discuss stopping conditions for the streamline tracing algorithm as well as a heuristic method for handling tangential streamline crossings that occur commonly when tracing streamlines via numerical integration.

\subsection{Singularity and Port Detection}

Singularity and port detection in a cross field defined per node is well documented in the literature $[3,15,19,31,33]$. We use methods that have been developed previously, but include a description here for completeness.

\subsubsection{Matchings}

Across each edge, we assume that crosses make the smallest rotation possible. This is called the principle matching of the crosses. If $u_{i}=e^{4 i \theta_{i}}$, then the change in cross orientation between two nodes $n_{i}$ and $n_{j}$, denoted $\Delta_{i j}$, is the number between $-\frac{\pi}{4}$ and $\frac{\pi}{4}$ given by

$$
\Delta_{i j}=\left(\theta_{j}-\left(\phi_{i j}+\theta_{i}\right)\right)(\bmod \pi / 2)-\pi / 4 .
$$




\subsubsection{Singularity Detection}

The index of a triangle, $t_{i j k}$, with nodes $n_{i}, n_{j}$, and $n_{k}$ is the number given by

$$
\mathrm{I}\left(t_{i j k}\right)=\frac{\Delta_{i j}+\Delta_{j k}+\Delta_{k i}-\phi_{i j}-\phi_{j k}-\phi_{k i}}{2 \pi} .
$$

Practically, this is the number of turns that a cross makes while circulating the triangle, which is always an integer multiple of $\frac{1}{4}$. A triangle is singular if its index is non-zero. Summing the changes in cross orientation along each edge, we compute the total circulation of the cross around the triangle. On a flat surface, the total circulation must be a multiple of $\pi / 2$; however, on curved surfaces, this is not the case. In general, a vector in the tangent bundle of $M$ parallel transported along a closed curve does not always return to the same orientation after circulating the curve. To mitigate the effects of holonomy while transporting a cross around the triangle, we subtract from the total circulation the angle defect that occurs when parallel transporting a vector around the triangle via our discrete connection. This angle defect is given by $\phi_{i j}+\phi_{j k}+\phi_{k i}$. Subtracting the angle defect from the total circulation leaves us with a number that is a multiple of $\pi / 2$, from which we compute the index.

After we determine singular triangles, we approximate the location of the singularity within the triangle by taking the barycenter of the triangle. We choose an arbitrary node, $n$, on the triangle and rotate the cross component vectors at that node into the plane of the face. We use the ray starting at the barycenter and passing through $n$ as a reference axis, and compute the angle $\alpha$ that the nearest cross component vector makes with the reference axis. We then compute the angles where streamlines exit the singularity (ports) as $\alpha+\frac{2 \pi k}{4-d}$ where $d / 4$ is the index of the singularity (see [19]).

\subsection{Separatrix Tracing}

After designing a cross field on $T$, we build an initial quad partition by tracing separatrices of the cross field. Below, we describe the methods used for tracing separatrices in non-singular and singular triangles.

\subsubsection{Non-Singular Triangles}

In non-singular triangles $t_{i j k}$, we project the crosses on each corner of $t_{i j k}$ into the plane of the triangle. We define a reference coordinate axis in that plane, and compute a representation vector for each cross with respect to this reference coordinate axis. We interpolate the argument of the representation vector linearly over the triangle. This is possible because in a non-singular triangle, the argument is continuous as it circulates the boundary. Using this interpolation, we trace out the streamlines using Heun's method [3].

\subsubsection{Singular Triangles}

Previously, no streamline tracing methods have been suitable for accurately tracing streamlines in the neighborhood of a singular triangle. Numerical integration methods such as Heun's method and other Runge-Kutta methods [3, 15, 19, 40] are inaccurate in the neighborhood of a singularity since cross directions can change arbitrarily fast. They frequently compute discretizations of the streamline that "cut the corner" rather than traversing around the singularity as they should (see Figure 3). Streamline tracing methods based on edge maps [21, 22] are guaranteed to never result in tangential crossings, but since paths are discretized by straight lines through each triangle, they are limited in their ability to resolve the path of a trajectory around a streamline by the number of triangles that meet at a singular point.

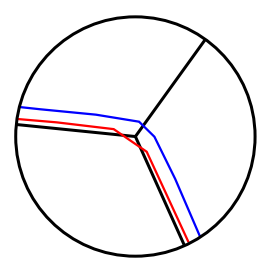

Figure 3: An illustration of a numerically traced streamline that "cuts the corner" and does not traverse around the singularity.

Here we develop a new method to accurately trace streamlines within the neighborhood of a singularity to any predefined resolution. This method guarantees that no tangential intersections of streamlines will occur within the neighborhood. We first prove that in $\mathbb{R}^{2}$, trajectories of streamlines through the neighborhood of a singularity are hyperbolas under a conformal transformation.

Let $f$ be a canonical harmonic cross field (see [19]) on a domain $D \subset \mathbb{R}^{2}$. Let $a \in \mathbb{R}^{2}$ be the location of a singularity of index $\frac{d}{4}$ where $d$ is an integer $\leq 1$. Consider the open ball $B\left(a, r_{0}\right)$ of radius $r_{0}>0$ centered at $a$. We seek an approximation for the trajectory of an arbitrary streamline passing through a point $q \neq a$ in $B\left(a, r_{0}\right)$.

The cross field, $f(z)$, partitions $B\left(a, r_{0}\right)$ into $4-d$ evenly angled sectors bounded by separatrices of the cross field [19]. In each sector, the cross field defines a local $(u, v)$ parameterization. Let $S$ be the open sector containing $q$, and let $s_{0}$ and $s_{1}$ be the separatrices bounding $S$ ordered counterclockwise. Because 
the cross field defines a local $(u, v)$ parameterization on $S$, there are two streamlines passing through $q$, one crossing $s_{0}$ orthogonally, the other crossing $s_{1}$ orthogonally. Without loss of generality, we consider $\gamma$, the streamline crossing $s_{1}$ orthogonally. This streamline crosses $s_{1}$ into $S^{\prime}$, the open sector adjacent to $S$ that is also bounded by $s_{1}$, and then exits $B\left(a, r_{0}\right)$ (see Figure 4).
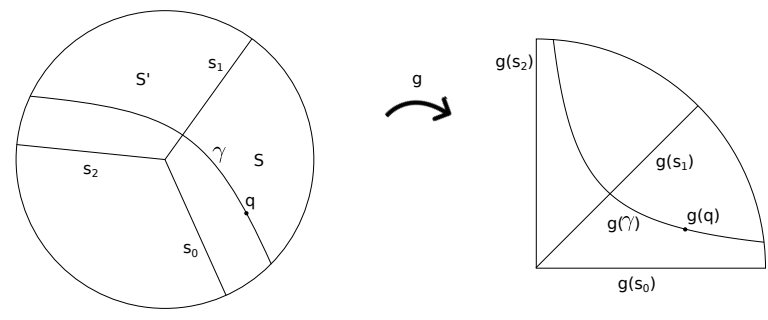

Figure 4: Streamlines in a neighborhood of a singularity become hyperbolas under a conformal map $g$.

By [19], the cross field in $B\left(a, r_{0}\right)$ can be written as

$$
f(z)=e^{i\left(\frac{d \theta}{4}+\frac{2 k \pi}{4}\right)}+o(r)
$$

where $d / 4$ is the index of the singularity, $z=r e^{i \theta}$, $k \in\{0,1,2,3\}$, and $\theta=0$ corresponds to $s_{0}$. For $r<r_{0}$ where $r_{0}$ is sufficiently small, we make the approximation

$$
f(z)=e^{i\left(\frac{d \theta}{4}+\frac{2 k \pi}{4}\right)} .
$$

Streamlines of the cross field are given by

$$
z^{\prime}=f(z)
$$

Since we are looking for the streamline crossing through $s_{1}$, we consider $k=0$. Thus, we are looking for the set $C=\left\{z(t) \in B\left(a, r_{0}\right) \mid t \in\left(t_{a}, t_{b}\right)\right\}$ where $z(t)$ on $\left(t_{a}, t_{b}\right)$ is the solution to the problem

$$
\begin{aligned}
z^{\prime} & =e^{i \frac{d \theta}{4}} \\
z(0) & =\left(r_{q}, \theta_{q}\right)
\end{aligned}
$$

in $D=\left\{z=r e^{i \theta} \mid r \in\left(0, r_{0}\right), \theta \in\left(0, \frac{4 \pi}{4-d}\right)\right\}$.

Proposition 1. $C=\left\{(x+i y)^{-(4-d) / 8} \mid x y=A, x \in\right.$ $\left.I_{x}\right\}$ for some constant $A$ on some interval $I_{x}$.

Proof. Consider a differentiable curve in $D$ given by $z(t)$ for $t \in(a, b)$. Consider the function $g(z)=$ $z^{(4-d) / 8}$ that maps $D$ to $\tilde{D}$, the sector of the upper right quadrant given by $\left\{w=\rho e^{i \varphi} \mid \rho \in\left(0, \rho_{0}=\right.\right.$ $\left.\left.r_{0}^{(4-d) / 8}\right), \varphi \in\left(0, \frac{\pi}{2}\right)\right\}$ (see Figure 4). Let $w(t)=$ $g(z(t))$ for $t \in(a, b)$. Taking the derivative of both sides, we have

$$
w^{\prime}(t)=g^{\prime}(z(t)) z^{\prime}(t)
$$

since $g^{\prime}(z) \neq 0$ in $D$, we have

$$
\begin{aligned}
\arg \left(g^{\prime}(z(t)) z^{\prime}(t)\right) & =\arg g^{\prime}(z(t))+\arg z^{\prime}(t) \\
& =\left(\frac{4-d}{8}-1\right) \theta+\arg \left(z^{\prime}(t)\right)
\end{aligned}
$$

In the case that $z(t)$ is a solution of equation 7 , we have

$$
\begin{aligned}
\arg w^{\prime}(t) & =\frac{d \theta}{4}+\left(\frac{4-d}{8}-1\right) \theta \\
& =-\frac{(4-d) \theta}{8}=-\varphi
\end{aligned}
$$

Thus $w^{\prime}(t)=\alpha(t) e^{-i \varphi}$ for some function $\alpha(t)$. Writing $w(t)=x(t)+i y(t)$, we have $x^{\prime}(t)=\alpha(t) \cos (\varphi), y^{\prime}(t)=$ $-\alpha(t) \sin (\varphi)$. Thus

$$
\frac{d y}{d x}=-\tan (\varphi)=-\frac{y}{x} \Longrightarrow y=\frac{A}{x}
$$

for some constant $A$. This equation describes the family of hyperbolas in the first quadrant with asymptotes at $\varphi=0$ and $\varphi=\frac{\pi}{2}$. The curve from this family passing through the point $g(q)=\rho_{q} e^{i \varphi_{q}}$ is given by $\left\{x+i y \mid x y=A_{q}\right\}$, where $A_{q}=\rho_{q}^{2} \sin \varphi_{q} \cos \varphi_{q}$. The curve $C$ can be recovered by taking the inverse image of this set under the mapping $g$, that is $C=\left\{(x+i y)^{-(4-d) / 8} \mid x y=A_{q}, x \in I_{x}\right\}$ where $I_{x}$ is an interval such that $(x+i y)^{-(4-d) / 8} \in B\left(a, r_{0}\right)$ for $x \in I_{x}$.

Proposition 1 provides a simple method for computing the trajectory of a streamline through a singular triangle. We make the assumption that within the triangle, the estimate

$$
f(z) \approx e^{i\left(\frac{d \theta}{4}+\frac{2 k \pi}{4}\right)}
$$

holds. Here again $\theta=0$ corresponds to the nearest separatrix clockwise from $q$. Making this assumption, we simply compute points along the hyperbola $x y=A_{q}$, and take the inverse image of each point. We use these points as discretization points of the streamline so long as they lay within the singular triangle. Since hyperbolas are convex, and $g^{-1}$ preserves the order of points along rays, in order to guarantee that two streamlines don't intersect tangentially, it is sufficient to evaluate the points of the hyperbola along predefined rays from the singular point.

\subsection{Partition Construction and Tangential Crossings}

By the Poincaré-Bendixson theorem for manifolds [41], streamlines of a cross field on a bounded manifold $M$ can do one of the following: 
1. Connect one or more singularities in a homoclinic or heteroclinic orbit

2. Exit the boundary

3. Approach a limit cycle

4. Approach a limit set that is all of $M$. In this case, $M$ must be a torus.

Because we are tracing out separatrices on a discrete mesh, in practice, they never line up perfectly with another singularity, so homoclinic and heteroclinic orbits will never occur. Thus streamlines in the discrete case can only either exit the boundary, or continue forever approaching either a limit cycle, or a limit set that is all of $M$. In order to generate a quad partition via separatrix tracing, separatrices that continue forever must be cut off after crossing some separatrix orthogonally. In practice, we use two stopping conditions: separatrices are traced until they either exit the boundary or cross the same separatrix more than once. The second condition is a simple way to eliminate the possibility of tracing out a separatrix forever, but can potentially create $\mathrm{T}$-junctions on separatrices that would eventually exit the boundary.

When tracing streamlines using a numerical method such as Heun's method, there is no guarantee that streamlines won't cross each other or exit the boundary tangentially. This becomes especially problematic along boundaries of meshes where the underlying geometry has high curvature but few triangles, resulting in few crosses that are actually aligned with the discrete boundary of the triangle mesh. Tangential crossings are problematic because the regions produced via separatrix tracing are no longer guaranteed to be foursided. Assuming a sufficiently fine triangle mesh along the boundary such that no separatrices exit tangentially, we observe in practice that tangential crossings on the interior typically occur in one of two cases. The first case is when one or more separatrices that approach a limit cycle are traced out for several rotations around the limit cycle. This problem is virtually eliminated by our approach of cutting off separatrices after they cross the same separatrix more than once.

The second case where tangential crossings occur is when there is a very small misalignment of singularities, such that two different separatrices follow virtually the same path. If the two separatrices are heading in opposite directions when the crossing occurs, then this problem can easily be fixed by cutting both separatrices off at the tangential crossing and combining them into a single separatrix, now connecting the two singularities in a heteroclinic orbit. If both separatrices are traveling in the same direction when they cross tangentially, there is no analogous simple operation to combine the two. However, we have observed that in practice, this almost always occurs when one of the separatrices passes very near the singularity where the other began. To mitigate the occurrence of tangential crossings when both separatrices are traveling in the same direction, we add a third stopping criteria for tracing separatrices: we cut off any separatrix inside of a singular triangle that orthogonally crosses one of the separatrices leaving the singularity. This third stopping condition greatly reduces the number of tangential crossings that occur when both streamlines are traveling in the same direction.

\section{PARTITION SIMPLIFICATION}

The misalignment of singularities when tracing out separatrices often results in small regions and limit cycles in the initial partition that would not exist if the separatrices coincided. In this section, we present a robust algorithm to simplify the partition obtained by naive separatrix tracing. The central step in the algorithm is an operation that extends the chord collapse operation for quad meshes $[42,43]$ to quad layouts with T-junctions. A similar collapse operation appears in Myles et al. [22].

A chord in a quad mesh is a maximal sequence of quads, $q_{1}, q_{2}, \ldots, q_{n}$ such that $q_{i}$ is adjacent to $q_{i+1}$, and $q_{i-1}$ and $q_{i+1}$ are on opposite sides of $q_{i}$. Figure 5 shows a chord of a quad mesh highlighted in blue. A partition obtained from streamline tracing is a quad layout with T-junctions, or a T-layout for short. We say that each component of a T-layout has four total sides, 2 pairs that are opposite each other. A side consists of at least one edge or more when T-junctions occur on that side. A chord of a T-layout is a maximal sequence of components, $c_{1}, c_{2}, \ldots, c_{n}$ such that $c_{i}$ is adjacent to $c_{i+1}, c_{i-1}$ and $c_{i+1}$ are on opposite sides of $c_{i}$, and no T-junction exists between $c_{i}$ and $c_{i+1}$. Figure 6 top shows various chords in a T-layout.

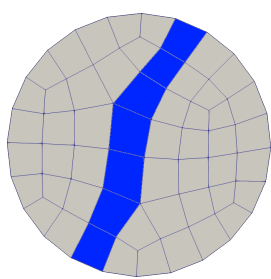

Figure 5: A chord in a quad mesh.

We call the set of edges shared by two partition components in a chord the transverse rungs of a chord. In the case that a chord begins or ends at a T-junction or on a boundary, we also include the first and last set of edges as transverse rungs of the chord. We also say that a chord has two longitudinal sides that consist 

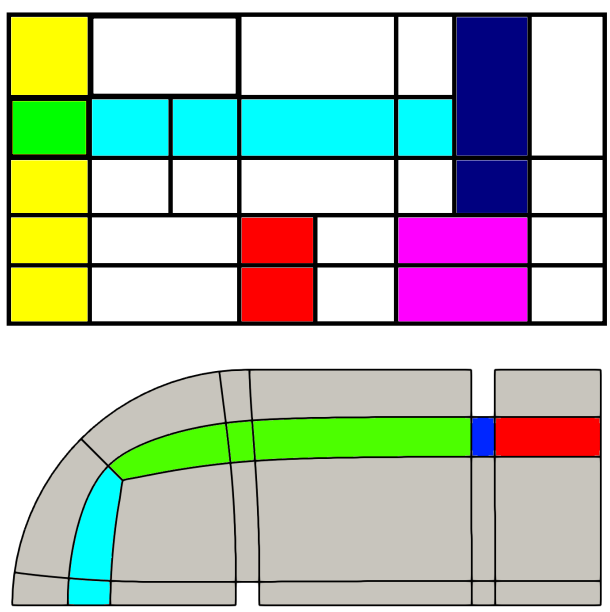

Figure 6: Illustrations of chords in two T-layouts. Top: Chords in a T-layout shown in various colors. The yellow and cyan chords overlap on the green component, illustrating how each component is part of two chords. Bottom: The four patches of a chord highlighted in cyan, green, blue, and red.

of all the edges of the partition components that are orthogonal to the transverse rungs.

A patch is a maximal subset of consecutive components of a chord such that singularities only occur on the first and last transverse rungs. A chord is partitioned into one or more patches, and singularities can occur only on the corners of patches. Figure 6 bottom shows the patches of a chord.

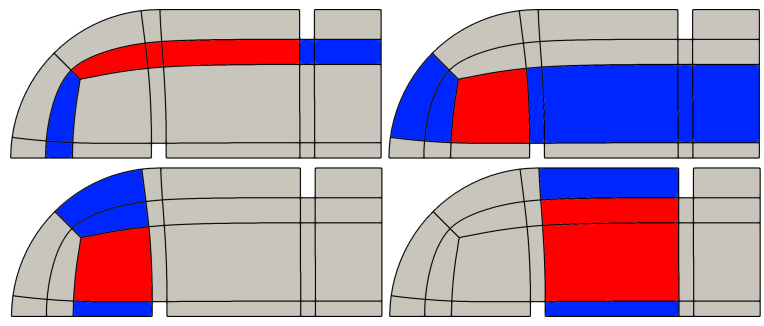

Figure 7: The four collapsible chords of a partition. Zip patches are highlighted in red and non-zip patches are highlighted in blue.

Our definition of chord collapse on a T-layout is motivated by the goal of simplifying the partition by removing one separatrix from each of two singularities, and then connecting singularities together by a single curve. We say that a patch of a chord in a T-layout is collapsible if it satisfies the following:

1. No singularities are connected across any trans- verse rung of the patch.

2. No singularity is connected to a boundary across any transverse rung of the patch.

3. If the patch starts or ends at a T-junction, then one of the following must be satisfied:

(a) The node opposite the T-junction on the same transverse rung is a singularity.

(b) The node opposite the T-junction on the same transverse rung is another T-junction with the same orientation.

(c) The node on the opposite corner of the patch from the T-junction is a singularity.

We say that a chord is collapsible if all of its patches are collapsible.

The first and second conditions prevent the possibility of having to combine two singularities into a single one or move a singularity to the boundary. They reflect an assumption that throughout the simplification process, we would like to keep the singularity set of the cross field and only modify the connectivity of the singularity graph. The third condition prevents the introduction of new T-junctions when collapsing chords or other invalid configurations such as a node with only two edges meeting at a corner. Figure 7 shows the collapsible chords for a given quad layout.

Given these assumptions, we can define a collapse operation on a collapsible chord. We define this operation by defining two sub-operations on patches. On a collapsible chord, any patch will either have singularities on opposite corners, or it will have one or two singularities only on one longitudinal side. We refer to the former as a zip patch, and the latter as a non-zip patch. The green patch in Figure 6 bottom is a zip patch and the other 3 are non-zip patches.

The collapse operation on a non-zip patch is to simply delete the edges on the longitudinal side without any singularities. The operation on a zip patch is to remove both longitudinal sides of the patch and replace them with a single line that connects the two singularities together. In practice, we take a weighted average of the two sides, figuratively "zipping" the two edges together to form the new line. If any T-junctions occur on a side that is deleted during a collapse, the hanging separatrix is simply extended after the collapse operation until it crosses the next separatrix. Figure 8 illustrates three consecutive chord collapses used to simplify a partition. The next chord to be collapsed in each frame has its zip patches highlighted in red and its non-zip patches highlighted in blue.

This collapse operation effectively replaces the two longitudinal sides of a chord with a single curve passing through each of the singularities on either side. 
It is easy to see that the resulting graph is still a $\mathrm{T}$ layout because the local connectivity at singularities is not changed and other crossings of separatrices are either unaffected or simply removed (see the proof of theorem 5.4 in [19]). We summarize this section with the following proposition,

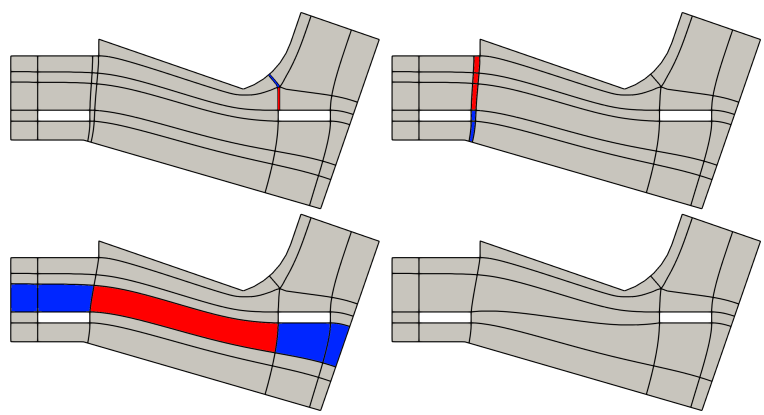

Figure 8: Three consecutive chord collapses simplify the quad layout. Zip patches to be collapsed at each step are colored in red and non-zip patches are colored in blue.

Proposition 2. Each chord collapse operation removes a chord from the T-layout, resulting in another T-layout with the same irregular nodes on the boundary and interior. A series of collapses monotonically reduces the number of T-junctions in the layout, and strictly decreases the number of partition components.

This simple operation forms the core of our partition simplification algorithm. As Figures 8 and 9 illustrate, repeated application of this operation has the potential to dramatically simplify a T-layout obtained from separatrix tracing. We take a greedy approach, collapsing first the thinnest chord that satisfies all conditions for collapse. The full loop is described in algorithm 3 .

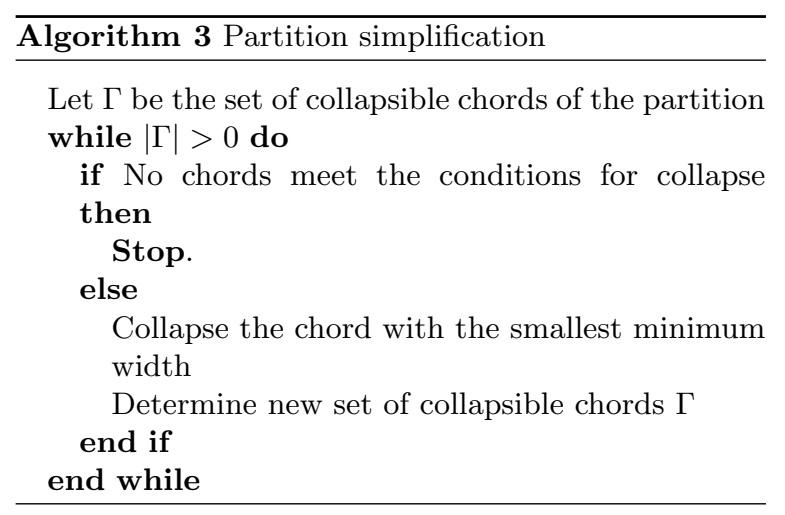

\subsection{Conditions for Collapse}

It is not always beneficial to collapse every collapsible chord. Figure 7 highlights four collapsible chords in

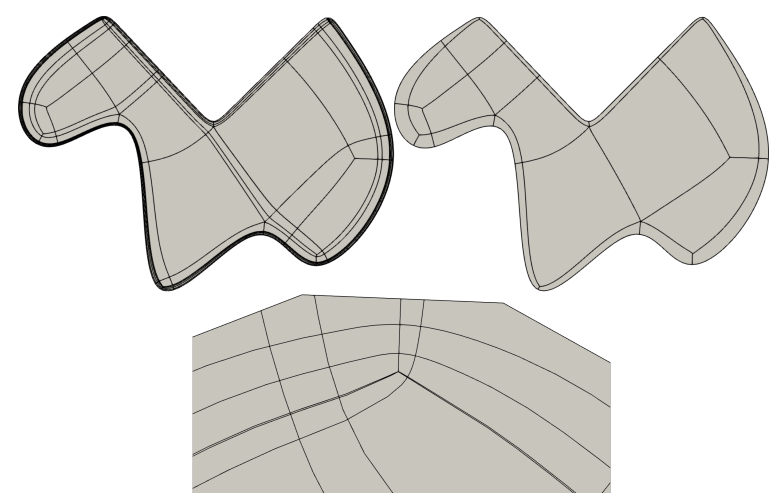

Figure 9: Before and after partition simplification. Top Left: The initial partition obtained by tracing separatrices. Top Right: A simplified partition after 10 chord collapses. Bottom: A close up of the top left corner of the geometry reveals extremely small components that occur because of misalignment in singularities in the initial partition.

a partition, but by most measures, it would only be beneficial to collapse the thinnest of the chords, since collapsing the others would lead to severe deformation in the newly created partition components adjacent to the zipped edge. The decision of whether to collapse is also application dependent. For example, in the final chord collapse in Figure 8, the difference in length on opposite sides of the regions adjacent to the zipped separatrix may outweigh the cost of a slightly more complex partition, depending on the application.

A complete exploration of how different applications might benefit from various collapse conditions will not be treated here, rather we only describe the conditions used in our examples. We define an energy for each patch and we subsequently define the energy of the chord as the minimum energy of any of its patches. The collapse condition evaluates to true if the energy is positive and false if the energy is negative.

For a non-zip patch, we set the energy to a positive constant value. The exact value is not important, this simply reflects the notion that collapsing a non-zip patch is not detrimental to the overall quality of the partition.

For zip patches, let $w$ be the mean of the length of each transverse rung of the patch. Let $l$ be the mean of the length of each longitudinal side of the chord. The energy for the patch is then defined as

$$
e=\frac{\pi}{8}-\arctan \frac{w}{l} .
$$

If the zip patch were perfectly rectangular, then $\arctan \frac{w}{l}$ would be equivalent to the angle that the diagonal makes with the base of the rectangle. In 
Table 2: Basic data for our pipeline on several models

\begin{tabular}{|c|c|c|c|c|c|c|c|c|c|}
\hline \multirow[b]{2}{*}{ Model } & \multirow[b]{2}{*}{$n$} & \multirow{2}{*}{$\begin{array}{c}\text { Cross Field } \\
\text { (s) }\end{array}$} & \multirow{2}{*}{$\begin{array}{l}\text { Tracing } \\
\text { (s) }\end{array}$} & \multirow{2}{*}{$\begin{array}{l}\text { Simp. } \\
\text { (s) }\end{array}$} & \multicolumn{2}{|c|}{ Components } & \multicolumn{2}{|c|}{ T-junctions } & \multirow{2}{*}{$\begin{array}{c}\text { Chord } \\
\text { Collapses }\end{array}$} \\
\hline & & & & & Before & After & Before & After & \\
\hline cognit & 7274 & 0.444 & 0.274 & 0.450 & 583 & 190 & 86 & 0 & 72 \\
\hline chainr5 & 4781 & 0.105 & 0.318 & 0.494 & 440 & 176 & 88 & 0 & 61 \\
\hline gluegun & 1842 & 0.074 & 0.124 & 0.254 & 725 & 189 & 36 & 0 & 45 \\
\hline sprayer & 954 & 0.037 & 0.027 & 0.011 & 29 & 12 & 6 & 0 & 4 \\
\hline faceplate & 47655 & 7.036 & 0.989 & 2.270 & 1500 & 227 & 120 & 0 & 101 \\
\hline part29 & 3265 & 0.121 & 0.051 & 0.023 & 66 & 22 & 3 & 0 & 6 \\
\hline test1 & 2703 & 0.115 & 0.044 & 0.027 & 47 & 19 & 1 & 0 & 5 \\
\hline engine2 & 564 & 0.025 & 0.053 & 0.057 & 194 & 63 & 11 & 1 & 15 \\
\hline pump & 2592 & 0.067 & 0.249 & 0.821 & 1014 & 303 & 88 & 4 & 80 \\
\hline
\end{tabular}

rough terms, this condition prevents chord collapses that result in a large deformation of the angles that separatrices make at singularities.

We found this particular collapse condition and the heuristic of collapsing thinnest chords first to produce quads with more rectangular corners than other collapsing strategies that we tried. Figure 10 shows a comparison between the results of collapsing a given initial partition using the strategy that we describe versus the strategy of greedily collapsing chords via our chord collapse operation, but using an energy analogous to that used in Tarini et al. [11] and Razafindrazaka et al. [14].

While we found the strategy of collapsing chords according to the conditions specified in this section to work well in our examples, it is a simple matter to substitute the sorting function and conditions for collapse in this algorithm with whatever is deemed appropriate for the application at hand.

\section{NUMERICAL EXPERIMENTS}

We tested our algorithm on 100 triangle meshes of surfaces with boundary derived from CAD models. All of the models except for the "faceplate" model are from a test suite used for development of the CUBIT software [44]. The "faceplate" model is the faceplate of the motor from the fan model at https://grabcad.com/ library/electric-fan-model-1. For the diffusion generated method, we used a time step $\tau=1 / \lambda_{1}$ where $\lambda_{1}$ is the first eigenvalue of the matrix $A$. We continued the iterations until $\left\|\vec{u}_{k}-\vec{u}_{k-1}\right\|<\sqrt{2 n} \times 10^{-6}$ where $n$ is the number of free nodes in the mesh. All examples were run on an Intel Core i5-2420m on a single thread.

In Table 2 and Figures 11 to 13, we present nine example models that are representative of the models used and results obtained in our experiment. Table 2 shows data for the number of nodes in the triangle mesh,

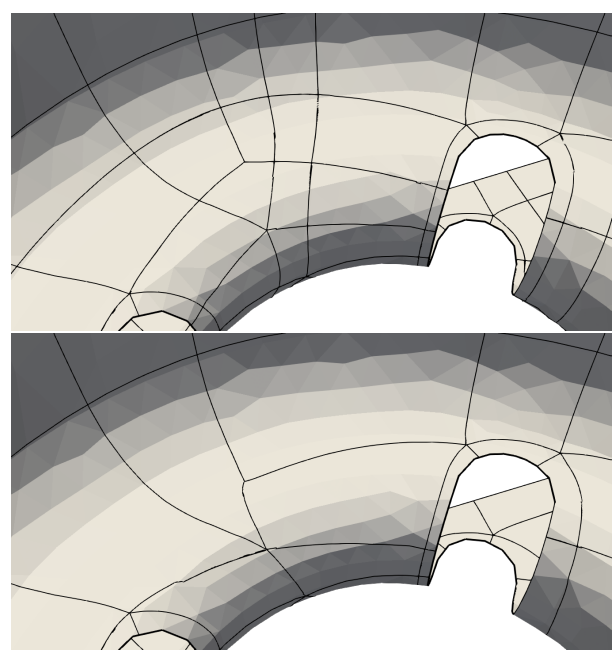

Figure 10: A comparison of collapse strategies. Top: The partition obtained by collapsing an initial partition according to the strategy defined in algorithm 3. Bottom: Result of collapsing the same partition using a greedy strategy collapsing chords in the order of highest to lowest energy using an energy analogous to that used in Tarini et al. [11] and Razafindrazaka et al. [14]. This strategy is over-aggressive in collapsing chords and we conclude that the energy does not work well with the chord collapse approach.

timing for the diffusion generated method, streamline tracing, and partition simplification methods, the number of components and T-junctions before and after simplification, and the total number of chord collapses performed. Figures 11 to 13 show the initial partition obtained via streamline tracing on the top and the final simplified partition on the bottom. The models in the table are shown in the same order as they appear in the figures, and the horizontal lines in Table 2 identify the cutoffs between figures.

Overall, we observe that our algorithm performs well 

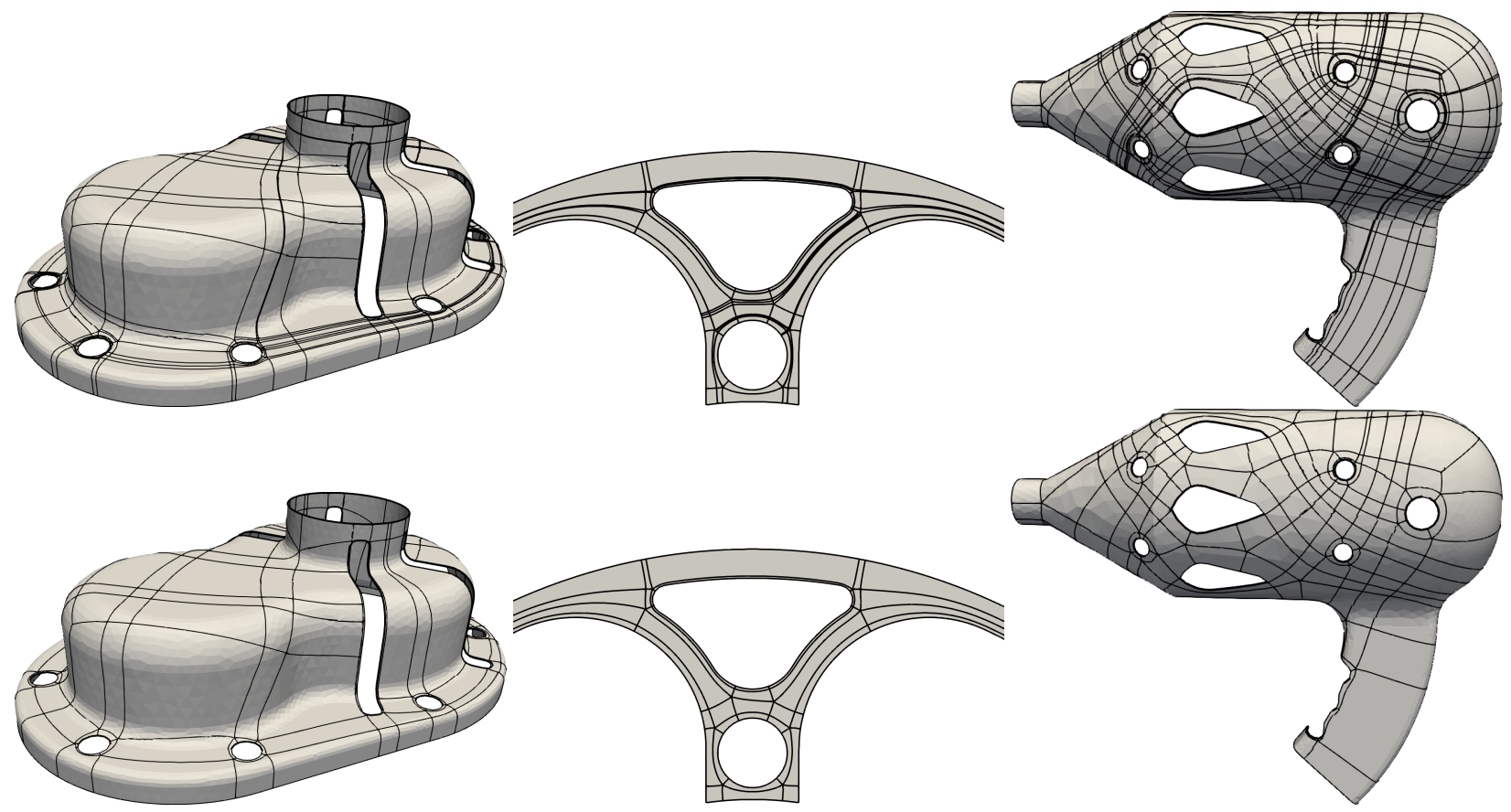

Figure 11: Examples of partitions simplified by our algorithm. The models from left to right are cognit, chainr5, and gluegun. Top: The initial partition obtained by tracing streamlines of the cross field obtained via the diffusion generated method. Bottom: A simplification of the partition on the top via our method.

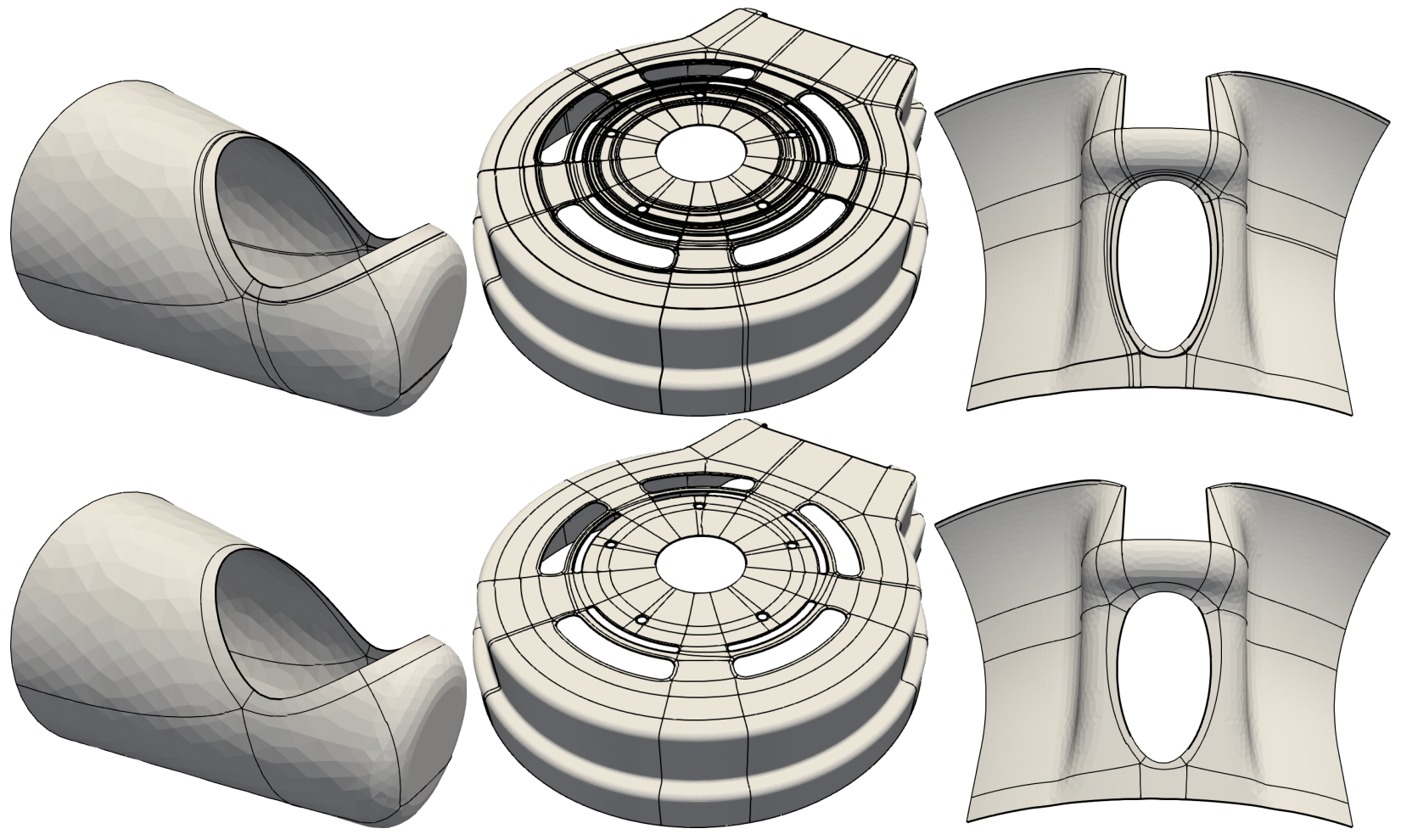

Figure 12: More examples showing the models sprayer, faceplate, and part29. See the caption for Figure 11. 

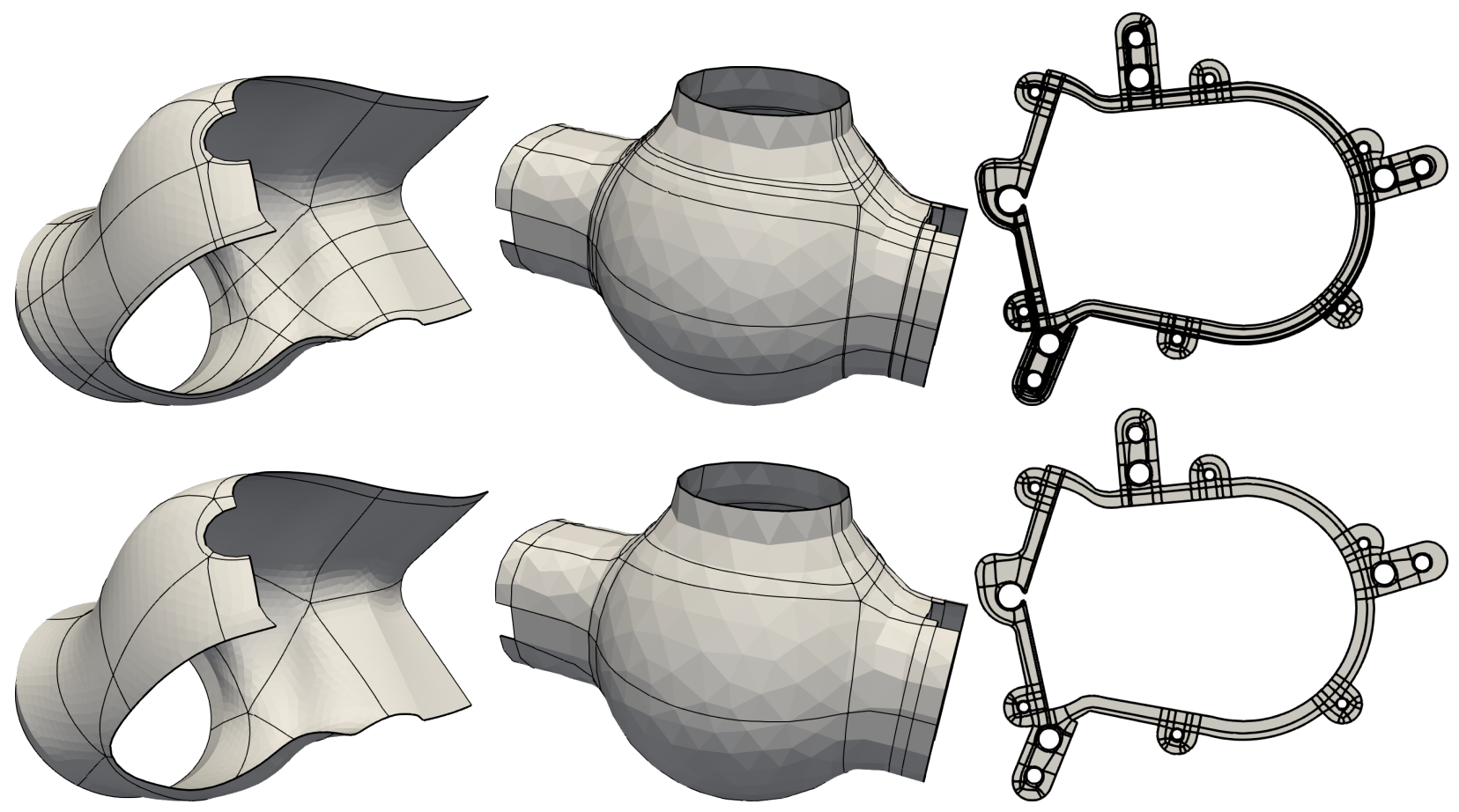

Figure 13: More examples showing the models test1, engine2, and pump. See the caption for Figure 11.

both in terms of efficiency and results. The timings reported for our cross field design method are comparable to those for the fastest cross field design methods [31, 33]. The timings for partition simplification reported in this paper are approximately an order of magnitude faster than those reported in [11] and [14] on similar sized models. Visually, the coarseness of the final quad layouts appear to be comparable across all three methods; however, a better comparison using the same models with each method is needed.

Out of the database of 100 models that we tested, eight models still had T-junctions after the simplification process. On four of those models, the T-junctions could be removed by simply continuing to trace the streamlines until they reached the boundary; see Figure 14 top. On the other four, there was at least one T-junction where the corresponding streamline approached a limit cycle. In each case that we observed, all T-junctions could have been removed from the initial partition by collapsing the chords in a different order (Figure 14 bottom), which suggests that perhaps a better collapse order would prioritize or even require collapsing chords that end in T-junctions.

\section{DISCUSSION}

In this paper, we have further developed three parts of the pipeline described in algorithm 1: an efficient method for high-quality cross field design, a method to accurately compute the trajectory of streamlines in the neighborhood of a singularity that avoids tangential crossings, and a robust partition simplification algorithm. We implemented a pipeline including these improvements, and executed our code on a database of 100 CAD surfaces. In all cases, the number of partition components and T-junctions was significantly reduced, and in 92 out of 100 cases we were able to generate a coarse quad layout with no T-junctions.

The diffusion generated method is well suited for cross field design on CAD surfaces because it results in smooth boundary aligned cross fields with good singularity placement near the boundaries. It is also comparable in speed to the fastest cross field design methods; however, a more in-depth analysis is needed to fully compare the results.

Our novel method for tracing the trajectories of streamlines near singular points is simple and allows for accurate computation while avoiding tangential crossings. Our implementation away from singularities is, however, limited by our choice of a node-based cross field representation, as we are not aware of any methods for such a representation that guarantee that streamlines in regular triangles will not cross tangentially. Our method could be improved by extending it to work in conjunction with streamline tracing methods such as Ray and Sokolov [21] and Myles et al. [22].

Our partition simplification algorithm is based on a 


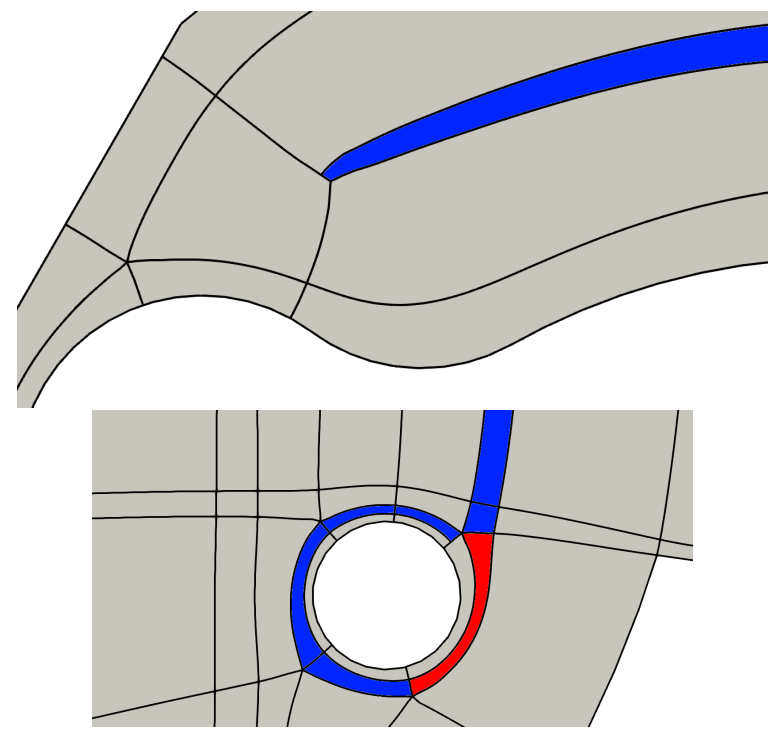

Figure 14: T-junctions not eliminated by our method. Top: A T-junction that can be removed simply by tracing the streamline until it reaches the boundary. The Tjunction occurred here because the separatrix intersected another separatrix while passing through a singular triangle. Collapsing the chord (highlighted in blue, full chord not shown) would remove the $\mathrm{T}$-junction, but the energy condition for collapse was not met. Bottom: A Tjunction resulting from a singularity approaching a limit cycle. Zip and non-zip patches of the chord adjacent to the $\mathrm{T}$-junction are marked in red and blue respectively. The colored chord is not collapsible because further up the chord a singularity is opposite a transverse rung (not shown). In the initial partition, this T-junction could have been removed by a chord collapse without this obstruction.

simple chord collapse operation and is guaranteed to strictly decrease the number of partition components at each step as well as monotonically decrease the number of T-junctions. While our collapse operation is similar to the operation for collapsing zero-chains in Myles et al. [22], the context in which the operation is applied is different. Perhaps the most important difference is that the T-mesh in [22] is a motorcycle graph, where separatrices are cut off after their first crossing with another separatrix, while in our method we trace out separatrices further, and cut them off according to conditions which allow for the collapse to have the effect of connecting two singularities together. This is illustrated in Figure 15. Our method also collapses chords much more aggressively than [22], as the primary goal of our algorithm is to generate a coarse quad layout (without T-junctions) whereas the reason for collapsing in [22] is to remove zero-chains which would result in a degenerate parameterization. The collapsing order in our algorithm prioritizes col-

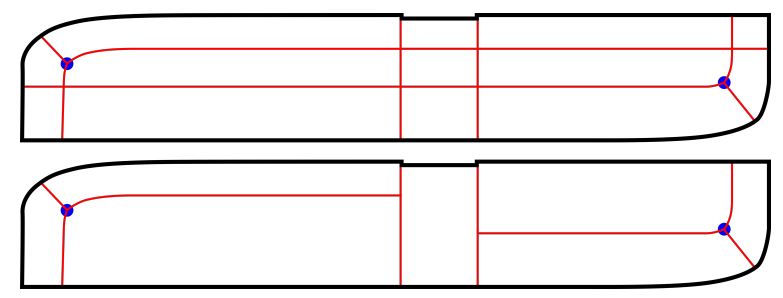

Figure 15: A simple geometry where a motorcycle graph can not be simplified via a chord collapse. Top: The partition traced out according to the conditions in section 3.3. The chord running lengthwise through the center can be collapsed. Bottom: The motorcycle graph for the same geometry. The collapsible chord from above is never formed because separatrices are cut off prior to it forming.

lapsing thin regions first whereas in [22] there is no discussion of order. There are also some subtle differences between the definitions of the operations themselves. For example, in [22], the definition of collapsible zero-chains depends on the assignment of parametric lengths to edges of the input T-layout, whereas our definition of a chord is strictly geometric. Further, the notion of a patch in our operation allows for zip operations spanning multiple quads where a zip like operation in [22] always occurs across a single quad. The cumulative effect of these differences is that we are able to demonstrate that the iterative collapse of chords can be an effective tool for generating coarse quad layouts, many times eliminating all T-junctions, whereas in [22], the collapse operation is used in a limited scope, with the purpose of ensuring global consistency of their parametric length assignment.

Our collapse method works directly on streamlines, so it does not require pre-meshing like methods such as Tarini et al. [11] and Bommes et al. [12], or prior computation of a seamless parameterization like Razafindrazaka et al. [14], Campen et al. [16], or Zhang et al. [17]. Since our method requires tracing streamlines accurately enough to avoid tangential crossings, the number of streamlines to be traced in our method scales linearly with the number of singularities as opposed to the method of Pietroni et al. [18], who allow computation of streamlines that cross tangentially at the cost that the number of streamlines to be drawn increases with combinatorial complexity as the number of singularities increases.

Each of $[14,16,17,18]$ formulate their problems as binary optimization problems, however, in order to achieve reasonable run times, they each significantly prune the search space by employing some clever method which leverages the structure of the T-layout generated by the separatrices of the underlying cross field or parameterization. While it appears that our 

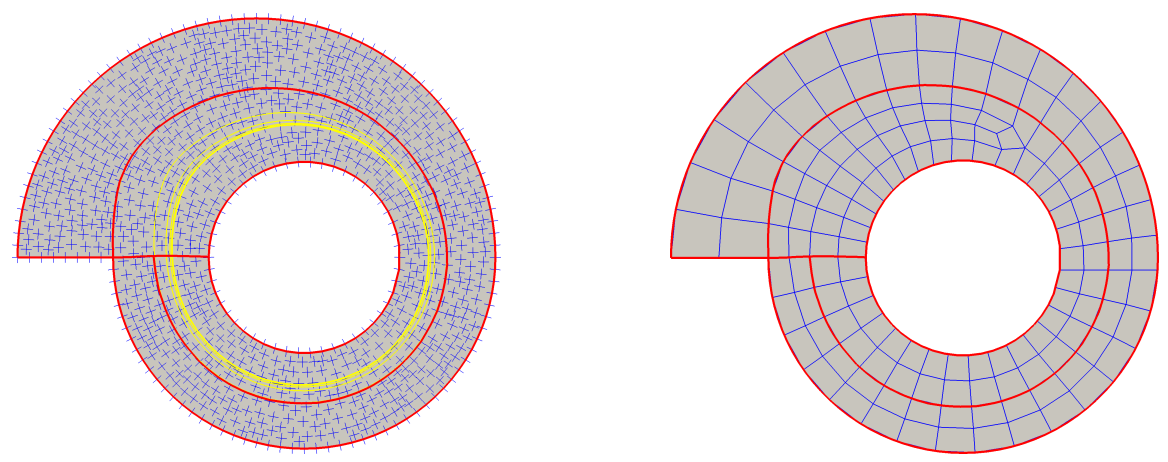

Figure 16: A surface where the cross field contains a limit cycle, resulting in a T-junction that cannot be removed without the introduction of additional singularities. Left: A boundary aligned cross field is shown in blue. The T-layout obtained by tracing out streamlines is shown in red. The yellow streamline begins at the geometric corner, follows the red curve of the partition, and continues on to converge to a limit cycle. Right: Because of the limit cycle, a pair of 3- and 5-valent nodes is needed to mesh the region adjacent to the $\mathrm{T}$-junction.

method is at a disadvantage because it takes a greedy approach, it is not clear to what degree the final results of each method are driven by heuristics or optimization. In addition, its not clear how well indirect objectives such as the total length of separatrices weighted by how far they drift from the underlying field, reflect objectives such as minimizing the number of quad components, or maximizing the minimal width of a chord which can be pursued directly via our method. A specific application with objectively stated goals and a common set of models is needed for a clear comparison between the quality of the quad layouts generated by all of the methods mentioned above.

The main downside of our partition simplification method is that it does not completely eliminate $\mathrm{T}$ junctions from the layout. It is not always possible to produce a quad layout with no T-junctions for a given set of singularities, so an important direction for future work is to develop a method that uses strategic insertion or removal of singularities in order to guarantee that the elimination all $\mathrm{T}$-junctions from a given input T-layout is possible. Such a result would be beneficial for applications in meshing for FEM, surface reconstruction into CAD from image data or geometries generated via topology optimization, and in constructing spline bases for isogeometric analysis.

\section{ACKNOWLEDGMENTS}

B. Osting is partially supported by NSF DMS 1619755 and 17-52202. R. Viertel and M. Staten are supported by Sandia National Laboratories. Sandia National Laboratories is a multi-mission laboratory managed and operated by National Technology \& Engineering Solutions of Sandia, LLC., a wholly owned subsidiary of Honeywell International, Inc., for the U.S. Department of Energy's National Nuclear Secu- rity Administration under contract DE-NA0003525. SAND2019-5668 C.

\section{References}

[1] Lindquist D.R., Gilest M.B. "A Comparison of Numerical Schemes on Triangular and Quadrilateral Meshes." 11th International Conference on Numerical Methods in Fluid Dynamics, pp. 369373. Springer Berlin Heidelberg, 1989

[2] Sandia National Laboratories. "Trilinos 12.12.1.", 2017

[3] Kowalski N., Ledoux F., Frey P. "A PDE Based Approach to Multidomain Partitioning and Quadrilateral Meshing." Proceedings of the 21st International Meshing Roundtable, pp. 137154. Springer Berlin Heidelberg, 2013

[4] Campen M. "Partitioning Surfaces Into Quadrilateral Patches: A Survey." Comput. Graph. Forum, vol. 36, no. 8, 567-588, 2017

[5] MegaCads. "MegaCads 2.5.", 2001

[6] Tam T.K.H., Armstrong C.G. "2D Finite Element Mesh Generation by Medial Axis Subdivision." Adv. Eng. Software, vol. 13, no. 5, 313-324, Sep. 1991

[7] Gould J., Martineau D., Fairey R. "Automated Two-Dimensional Multi-Block Meshing Using the Medial Object." Proceedings of the 20th International Meshing Roundtable, pp. 437-452. Springer Berlin Heidelberg, 2012

[8] Dong S., Bremer P.T., Garland M., Pascucci V., Hart J.C. "Spectral Surface Quadrangulation." 
ACM Trans. Graph., vol. 25, no. 3, 1057-1066, Jul. 2006

[9] Lei N., Zheng X., Si H., Luo Z., Gu X. "Generalized Regular Quadrilateral Mesh Generation based on Surface Foliation." Procedia Engineering, vol. 203, 336 - 348, 2017. 26th International Meshing Roundtable, IMR26, 18-21 September 2017, Barcelona, Spain

[10] Campen M., Bommes D., Kobbelt L. "Dual Loops Meshing: Quality Quad Layouts on Manifolds." ACM Trans. Graph., vol. 31, no. 4, 1-11, 2012

[11] Tarini M., Puppo E., Panozzo D., Pietroni N., Cignoni P. "Simple Quad Domains for Field Aligned Mesh Parametrization." ACM Trans. Graph., vol. 30, no. 6, 142:1-142:12, Dec. 2011

[12] Bommes D., Lempfer T., Kobbelt L. "Global Structure Optimization of Quadrilateral Meshes." Comput. Graph. Forum, vol. 30, no. 2, 375-384, Apr. 2011

[13] Bommes D., Campen M., Ebke H.C., Alliez P., Kobbelt L. "Integer-Grid Maps for Reliable Quad Meshing." ACM Trans. Graph., vol. 32, no. 4, 98:1-98:12, 2013

[14] Razafindrazaka F.H., Reitebuch U., Polthier K. "Perfect Matching Quad Layouts for Manifold Meshes." Comput. Graph. Forum, vol. 34, no. 5, 219-228, Aug. 2015

[15] Fogg H.J., Armstrong C.G., Robinson T.T. "Automatic Generation of Multiblock Decompositions of Surfaces." Int. J. Numer. Meth. Engng., vol. 101, no. 13, 965-991, 2015

[16] Campen M., Bommes D., Kobbelt L. "Quantized Global Parametrization." ACM Trans. Graph., vol. 34, no. 6, 192:1-192:12, Oct. 2015

[17] Zhang S., Zhang H., Yong J.H. "Automatic Quad Patch Layout Extraction for Quadrilateral Meshes." Comput. Aided Des. Appl., vol. 13, no. 3, 409-416, 2016

[18] Pietroni N., Puppo E., Marcias G., Roberto R., Cignoni P. "Tracing Field-Coherent Quad Layouts." Comput. Graph. Forum, vol. 35, no. 7, 485-496, Oct. 2016

[19] Viertel R., Osting B. "An Approach to Quad Meshing Based on Harmonic Cross-Valued Maps and the Ginzburg-Landau Theory." SIAM J. Sci. Comput., vol. 41, no. 1, A452-A479, Jan. 2019
[20] Hertzmann A., Zorin D. "Illustrating Smooth Surfaces." Proceedings of the 27th Annual Conference on Computer Graphics and Interactive Techniques, pp. 517-526. ACM Press/Addison-Wesley Publishing Co., 2000

[21] Ray N., Sokolov D. "Robust Polylines Tracing for N-Symmetry Direction Field on Triangulated Surfaces." ACM Trans. Graph., vol. 33, no. 3, 30:1-30:11, 2014

[22] Myles A., Pietroni N., Zorin D. "Robust FieldAligned Global Parametrization." ACM Trans. Graph., vol. 33, no. 4, 135:1-135:14, 2014

[23] Jadhav S., Bhatia H., Bremer P.T., Levine J.A., Nonato L.G., Pascucci V. "Consistent Approximation of Local Flow Behavior for 2D Vector Fields Using Edge Maps." Topological Methods in Data Analysis and Visualization II, Mathematics and Visualization, pp. 141-159. Springer Berlin Heidelberg, 2012

[24] Eppstein D., Goodrich M.T., Kim E., Tamstorf R. "Motorcycle Graphs: Canonical Quad Mesh Partitioning." Proceedings of the Symposium on Geometry Processing, pp. 1477-1486. Eurographics Association, 2008

[25] Razafindrazaka F.H., Polthier K. "Optimal Base Complexes for Quadrilateral Meshes." Comput. Aided Geom. D., vol. 52-53, 63-74, Mar. 2017

[26] Palacios J., Zhang E. "Rotational Symmetry Field Design on Surfaces." ACM Trans. Graph., vol. 26 , no. 3 , 55:1-55:10, Jul. 2007

[27] Ray N., Vallet B., Li W.C., Lévy B. "NSymmetry Direction Field Design." ACM Trans. Graph., vol. 27, no. 2, 10:1-10:13, 2008

[28] Ray N., Vallet B., Alonso L., Lévy B. "GeometryAware Direction Field Processing." ACM Trans. Graph., vol. 29, no. 1, 1:1-1:11, 2009

[29] Bommes D., Zimmer H., Kobbelt L. "MixedInteger Quadrangulation." ACM Trans. Graph., vol. 28 , no. 3 , 77:1-77:10, Jul. 2009

[30] Crane K., Desbrun M., Schröder P. "Trivial Connections on Discrete Surfaces." Comput. Graph. Forum, vol. 29, no. 5, 1525-1533, 2010

[31] Knöppel F., Crane K., Pinkall U., Schröder P. "Globally Optimal Direction Fields." $A C M$ Trans. Graph., vol. 32, no. 4, 59:1-59:10, 2013

[32] Panozzo D., Puppo E., Tarini M., SorkineHornung O. "Frame Fields: Anisotropic and Nonorthogonal Cross Fields." ACM Trans. Graph., vol. 33 , no. 4, 1-11, 2014 
[33] Jakob W., Tarini M., Panozzo D., SorkineHornung O. "Instant Field-Aligned Meshes." ACM Trans. Graph., vol. 34, no. 6, 189:1-189:15, 2015

[34] Vaxman A., Campen M., Diamanti O., Panozzo D., Bommes D., Hildebrandt K., Ben-Chen M. "Directional Field Synthesis, Design, and Processing." Comput. Graph. Forum, vol. 35, no. 2, 545-572, 2016

[35] Huang Z., Ju T. "Extrinsically Smooth Direction Fields." Comput. Graph., vol. 58, 109-117, 2016

[36] Beaufort P.A., Lambrechts J., Henrotte F., Geuzaine C., Remacle J.F. "Computing two dimensional cross fields - A PDE approach based on the Ginzburg-Landau theory." Proceedings of the 26th International Meshing Roundtable, pp. 219-231. Elsevier Ltd., 2017

[37] Bethuel F., Brezis H., Hélein F. GinzburgLandau Vortices, vol. 13 of Progress in Nonlinear Differential Equations and Their Applications. Birkhäuser Boston, 1994

[38] Ruuth S.J., Merriman B., Xin J., Osher S. "Diffusion-Generated Motion by Mean Curvature for Filaments." J. Nonlinear Sci., vol. 11, no. 6, 473, 2001

[39] Laux T., Yip N.K. "Analysis of Diffusion Generated Motion for Mean Curvature Flow in Codimension Two: A Gradient-Flow Approach." Arch. Rational Mech. Anal., vol. 232, no. 2, 11131163, May 2019

[40] Zhang E., Mischaikow K., Turk G. "Vector Field Design on Surfaces." ACM Trans. Graph., vol. 25, no. 4, 1294-1326, 2006

[41] Schwartz A.J. "A Generalization of a PoincaréBendixson Theorem to Closed Two-Dimensional Manifolds." Amer. J. Math., vol. 85, no. 3, 453458, 1963

[42] Borden M.J., Benzley S.E., Shepherd J.F. "Hexahedral Sheet Extraction." Proceedings of the 11th International Meshing Roundtable, pp. 147-152. Springer-Verlag, 2002

[43] Daniels J., Silva C.T., Shepherd J., Cohen E. "Quadrilateral Mesh Simplification." ACM Trans. Graph., vol. 27, no. 5, 148:1-148:9, Dec. 2008

[44] Sandia National Laboratories. "The CUBIT Geometry and Mesh Generation Toolkit 15.3.", 2017 


\title{
FEATURE-ALIGNED POLY-SQUARE MAPPING OF LARGE-SCALE 2D GEOMETRIES FOR SEMI-STRUCTURED QUAD MESH GENERATION
}

\author{
Celong Liu ${ }^{1,2} \quad$ Kelin $\mathrm{Hu}^{3} \quad$ Qin $\mathrm{Chen}^{4} \quad$ Xin $\mathrm{Li}^{2 *}$ \\ 1 OPPO US Research Center, Palo Alto, CA, U.S.A. \\ 2 School of Electrical Engineering and Computer Science, Louisiana State University, U.S.A. \\ 3 Tulane University, U.S.A. \\ ${ }^{4}$ Department of Civil Engineering, Northeastern University, U.S.A. \\ * Corresponding Author. Email: xinli@cct.lsu.edu.
}

\begin{abstract}
We develop a distributed algorithm to compute feature-aligned poly-square maps for large-scale 2D geometric regions and use it to construct low-distortion semi-structured quad meshes. Our proposed algorithm has two main components. The first is a feature-aware graph partitioning that considers workload balancing, minimal communication, geometric regularity, and feature-preserving. The second is a feature-preserved poly-square parameterization. We demonstrate that our algorithm is effective on meshing huge complex coastal/terrain data and can consequently benefit scientific simulations that run on such meshes using high-performance computer clusters.
\end{abstract}

Keywords: Large-scale Geometric Data Processing, Feature-aware Graph Partitioning, Semistructured Quad Mesh Generation

\section{INTRODUCTION}

Generating high-quality mesh is essential to numerical simulation tasks. While unstructured triangular meshes are commonly used due to their ease of generation and good adaptivity, structured or semistructured paralleletope meshes (e.g., quad meshes for $2 \mathrm{D}$, hex meshes for $3 \mathrm{D}$ ) are sometimes preferred due to their better support for efficient finite element analysis in many computation-intensive simulations in structural mechanics, fluid dynamics simulations [1]. Here, a structured mesh is one whose vertices all have constant valences and cells are arranged with regular connectivity; and a semi-structured mesh, also called block-structured mesh, is one that divides the domain into sub-regions, each of which is tessellated by a structured mesh.

This work developed an automatic semi-structured quad meshing algorithm for large-scale 2D planar geo- metric regions. To process such large geometric data, we develop a parallel algorithm to overcome the scale and performance issue. Parallel meshing strategies in existing literature are mainly on the generation of irregular simplex meshes, using either Delaunay-based methods or advancing front techniques to generate triangular or quad meshes [2-4]. Feature preserving is another important issue to consider in many scientific computing tasks. To enforce accurate evaluation or constraints on specific important points, curves, or regions, one would like to have an accurate sampling of these features. However, feature alignment is not always trivial during mesh generation.

For very big or complex geometric region, a divideand-conquer approach is a natural strategy for parallel processing. We follow the approach similar to [5]. First, we partition the data into solvable subregions, considering a trade-off among their size, ge- 
ometry, and feature sampling. Then, we map subregions onto canonical poly-square domains for mesh generation. Unlike [5] that constructs meshes locally using advancing front, which results in an unstructured mesh with potential artifacts along partitioning boundary and without supporting feature alignment, our new algorithm now (1) solves the local meshing through a mapping-based method and hence yields a locally structured (globally semi-structured) mesh, (2) integrates feature alignment in partitioning and mapping (mesh) computation, and (3) solves composition through a multi-pass global optimization and hence produces the smooth transition across partitioning boundary and allows flexible insertion of interior singularities to reduce mesh distortion.

The main contributions of this paper are as follows. (1) A feature-preserving large-scale semi-structured quad meshing algorithm is developed. Unlike existing large-scale quad meshing algorithms that often cannot handle feature preserving very well, our algorithm can resolve user-determined features effectively. (2) A new feature-aware graph partitioning model is proposed. It can effectively decompose complex geometric models into subregions by considering not just load balancing, minimal communication, but also geometric and feature constraints. (3) Meshes generated using our algorithm could lead to more accurate finite element simulations. Hence, this algorithm can benefit largescale scientific computing.

\section{RELATED WORK}

\subsection{Quadrilateral Mesh Generation}

Quadrilateral mesh generation algorithms can be generally classified into three types, based on the mesh regularity, namely, unstructured, structured, and semistructured meshes.

Structured Quad Meshing. Rigorously speaking, a fully-structured quad mesh is a tensor-product patch that deforms from an $N \times M$ grid. However, such a fully regular grid topology is often too restrictive: wrapping such a grid onto a region with a complex topology is infeasible and covering a shape with long and thin branches will result in severe distortion. Therefore it is essential to introduce some singularities or unstructured organization to a more flexible balance the trade-off between regularity and distortion.

Unstructured Quad Meshing. Commonly used and extensively studied quad meshing algorithms are on generating unstructured meshes. Classic algorithms include those based on triangle-to-quad conversion $[6,7]$, quad-tree projection $[8,9]$. A widely studied strategy is the advancing front algorithm that starts the construction of cells from the boundary, then propagates layer inwards by layer, until the propaga- tion fronts quench each other near media axes and form singularity curves. Notable advancing front algorithms include the classic paving [10], Q-Morph [11], H-Morph [12], to the recent frontal Delauney algorithm [13]. Another group of effective algorithms developed recently in the graphics field in constructing low-distortion surface parameterization and meshing is based on building a smooth cross-frame (2-rotational symmetry) field. This strategy has been very successfully in building low-distortion unstructured quad meshes, as demonstrated by multiple recent algorithms, such as QuadCover [14], Mixed-integer quadrangulation [15], Integer-grid maps [16], singularityrestricted field [17], QMorph Cross Field [18]. These methods can produce meshes with very desirable (i.e., low-distortion) element shape quality. However, the unstructured nature of these meshes sometimes still hampers their performance (compared with using more structured meshes) in scientific computing.

Semi-structured Quadrilateral Mesh Generation. Semi-structured or block-structured meshes divide the domain into sub-regions, call blocks, each of which is tessellated by a structured grid. The global layout of these blocks is often unstructured and can be encoded using a graph with irregular connectivity. The semi-structured mesh can be constructed on an arbitrary complex domain and offers a flexible trade-off between regularity and element distortion. The macro-unstructured-micro-structured hierarchy layout of a semi-structured mesh also naturally supports parallelization where the calculations on local blocks can be executed on parallel processors fully utilizing the numerical efficiencies of its regularity. On curved surfaces, recent frame-field approaches $[19,20]$ use rectangle patches to generate high quality semi-structured meshes, however they are hard to be extended to large scale geometric data. Polycube parameterizations have been used to generate semistructured meshes where each rectangular sub-patch is tessellated regularly, and the global layout is described by the topology of the polycube polyhedron. Multiple semi-automatic or automatic polycube parameterization algorithms have been developed for quad- or hexmeshing [21-26]. However, with polycube parameterization, the singularities locate at the polycube corners. Distortion is often significant near these singularities. Furthermore, solving optimal polycube parameterization is usually expensive and prohibitive to be directly applied to large-scale geometric data.

\subsection{Quad Meshing by Divide-and-Conquer}

To process large-scale geometric data, we adopt a domain decomposition approach that partitions the data into solvable subparts for distributed and parallel processing. Related to our approaches are var- 
ious divide-and-conquer algorithms in mesh generation. For example, medial axis decomposition was used to partition geometric regions $[27,28]$. The general ideas of these approaches are first to extract medial axis of regions then insert cuts on them to cut the region into smaller "shape atoms" which could be meshed using templates. Voronoi diagrams have also been used $[5,29]$ to generate coarse cells upon which quad meshes can be constructed. On curved surfaces, quad patch layout design has been studied to facilitate the construction of low-distortion quad meshing of surfaces. The Morse-Smale Complex has been used $[30,31]$ to design quadrilateral patches. More generally, the cross-frame field on the surface can be optimized to generate principal-direction-aligned quad layouts [19]. These geometry-adaptive quad patch layouts have been shown effective in generating highquality quad meshes for curved surfaces. The downside of these algorithms is their computational complexity. When dealing with large-scale geometric data, globally solving a non-linear (mixed) integer optimization would be infeasible.

\subsection{Feature Alignment Quad Meshing}

In some physical simulation problems, we often need to preserve the feature regions in the generated mesh for special study. T-mesh is a widely used approach to generate feature aligned quadrilateral mesh [32-34]. But T-junction is not acceptable in some largely scaled physics simulation system [35]. Another approach to generating feature aligned mesh is cross-field based parameterization [36,37]. They can generate highquality feature aligned quadrilateral mesh. However, the unstructured nature and their algorithm are not easy to be parallelized for largely scaled mesh generation. In this paper, we design a distributed and parallel meshing framework that can effectively generate high-quality feature aligned/preserved quadrilateral mesh.

\section{FEATURE-AWARE GRAPH PARTITIONING}

To effectively process a very big and complex geometric region, we first do a data partitioning to split the data into small, solvable subparts. In [38], a graph partitioning is solved to obtain subregions for quad mesh generation. An algorithm was proposed to optimize three criteria: (1) workload balancing where subregions should have similar sizes; (2) small communication cost where length of subregion boundary should be minimized; and (3) geometry regularity where each subregion should have corner angles close to $k \pi / 2$, $k \in\{0,1,2,3\}$. In our partitioning task, all these three criteria are still desirable, and we further consider one more criterion on feature preservation. To have features points or feature curves sampled in the final mesh, we can partition the geometry so that subregion boundaries go through these features since all the boundary elements will be exactly sampled in the final mesh. So we have the four criteria formulated as (4) the partitioning separators should go through feature curves as much as possible.

\subsection{Notation and Recap of Geometry- aware Partitioning}

We adopt the notations from [38] and recap that partitioning algorithm first before we explain our modification to the algorithm in the next section.

Given a triangulated 2D region $M=\left(V^{M}, E^{M}, F^{M}\right)$, where $V^{M}, E^{M}, F^{M}$ are the sets of vertices, edges, and faces (cells), respectively, let $G=\left(V^{G}, E^{G}\right)$ denote its dual graph, where $V^{G}, E^{G}$ are the sets of nodes and arcs. The weight of a node $v \in V^{G}$ is defined to be the area of its associated cell $f \in F^{M}$, and the weight of an arc is the length of its associated edge. A $k$-way partitioning on $M$ can be computed on $G$. Without losing generality, we explain its idea using the 2-way partitioning in the following (which can be directly generalized to $k$-way [5]) that partitions $G$ into two sub-graphs $G_{0}=\left(V^{G_{0}}, E^{G_{0}}\right)$ and $G_{1}=\left(V^{G_{1}}, E^{G_{1}}\right)$, where $V^{G_{1}}=V^{G} \backslash V^{G_{0}}$. An indicator variable $x_{i}$ is assigned to each node $v_{i}^{G} \in V^{G}$,

$$
x_{i}=\left\{\begin{array}{ll}
0, & \text { if } v_{i}^{G} \in V^{G_{0}} \\
1, & \text { if } v_{i}^{G} \in V^{G_{1}}
\end{array} .\right.
$$

Then for each $\operatorname{arc} e_{i j}^{G}=\left[v_{i}^{G}, v_{j}^{G}\right]$, we assign a variable $y_{i j}=x_{i}-x_{j}$ :

$y_{i j}= \begin{cases}0, & \text { if } v_{i}^{G}, v_{j}^{G} \text { in the same sub-graph } \\ 1 \text { or }-1, & \text { otherwise }\end{cases}$

We have $\mathbf{y}=\mathbf{U x}$, where $\mathbf{x}$ and $\mathbf{y}$ are node and arc variable vector respectively, and $\mathbf{U}$ is an $\left|E^{G}\right| \times\left|V^{G}\right|$ matrix.

Firstly, to balance the workload and avoid big area difference between subregions, we have the following constraint

$$
c_{1} \leq \mathbf{x}^{T} \mathbf{w}_{v}-c \leq c_{2},
$$

where $\mathbf{x}=\left(x_{1}, x_{2}, \ldots, x_{n}\right)^{T}$ is the variable vector, $\mathbf{w}_{v}=\left(w_{v_{1}}, w_{v_{2}}, \ldots, w_{v_{n}}\right)^{T}$ is the node weight vector, $c=\frac{1}{2} \sum_{i} w_{v_{i}}$, and $c_{1}, c_{2}$ are the constant thresholds. In our experiments, we set $c_{1}=c_{2}=0.1 c$.

Secondly, a smaller total separator length means less inter-process communication in parallel computing. Therefore, it is desirable to minimize the total separator length

$$
L_{S}=\mathbf{y}^{T} \mathbf{W}_{e} \mathbf{y}=\mathbf{x}^{T} \mathbf{U}^{T} \mathbf{W}_{e} \mathbf{U} \mathbf{x},
$$


where $\mathbf{y}=\left(y_{e_{1}}, y_{e_{2}}, \ldots, y_{e_{n}}\right)^{T}$ is the edge variable vector, $\mathbf{W}_{e}=\operatorname{diag}\left(w_{e_{1}}, w_{e_{n}}, \ldots, w_{e_{n}}\right)$ is a diagonal matrix composed of arc weights. Combining these above criteria one and two we have the common graph partitioning formulated on triangular meshes.

Thirdly, in quad meshing, it is desirable to have angles between separators close to $\frac{k \pi}{2}$. Ideally, we can use a separator angle term to penalize each such angle's deviation from $\frac{k \pi}{2}$. Consider two edges $e_{i}, e_{j} \in E^{M}$ of the original mesh $M$, we use $\operatorname{Inc}(i, j)=1$ to denote $e_{i}$ and $e_{j}$ are incident, and $\operatorname{Inc}(i, j)=0$ if they are not. If $\operatorname{Inc}(i, j)=1$, suppose they form an angle $\theta_{i, j}$. We define an angle deviation function

$$
\delta_{\theta_{i, j}}=\left\{\begin{array}{cl}
\min _{k \in\{1,2,3,4\}}\left|\theta_{i, j}-\frac{k \pi}{2}\right|, & \text { if } \operatorname{Inc}(i, j)=1 \\
0, & \text { if } \operatorname{Inc}(i, j)=0
\end{array}\right.
$$

to describe the deviation from angle $\theta_{i, j}$ to the nearest $\frac{k \pi}{2}$ angle. The accumulated separator angle deviation can then be formulated as

$$
D_{\theta}=\mathbf{y}^{T} \mathbf{W}_{\theta} \mathbf{y}=\mathbf{x}^{T} \mathbf{U}^{T} \mathbf{W}_{\theta} \mathbf{U} \mathbf{x},
$$

where $\mathbf{y}=\left(y_{e_{1}}, y_{e_{2}}, \ldots, y_{e_{n}}\right)^{T}$ is the edge variable vector, and

$$
\mathbf{W}_{\theta}=\left(\begin{array}{ccccc}
0 & \delta_{\theta_{1,2}} & \delta_{\theta_{1,3}} & \ldots & \delta_{\theta_{1, n}} \\
\delta_{\theta_{2,1}} & 0 & \ldots & \ldots & \delta_{\theta_{2, n}} \\
\ldots & \ldots & \ldots & \ldots & \ldots \\
\delta_{\theta_{n, 1}} & \ldots & \ldots & \ldots & 0
\end{array}\right)
$$

is an $\left|E^{G}\right| \times\left|E^{G}\right|$ matrix storing deviation angles $\delta_{\theta_{i, j}}$.

However, this dense angle deviation matrix $\mathbf{W}_{\theta}$ need to be computed from all the edge pairs of the tessellation $M$, and directly minimizing this angle deviation term is very expensive. In [38], a two-stage approximate algorithm is adopted. They first tessellate the $2 \mathrm{D}$ geometry using $L_{\infty}$-CVT whose cells have near-90 deg angles, then they perform a common graph partitioning on these cells to get an approximate partitioning.

\subsection{Feature Curve Preservation}

To preserve feature curves/points in the final mesh, one approach is to let the partitioning separators go through the features because the sampling of these separators (subregion boundaries) can directly and easily guarantee as boundary constraints in the subsequent parameterization step. In Fig. 1(a), a feature curve is indicated inside the mesh as red color and we want the final partitioning separator go through this curve. In Fig. 1(b), the separator (in blue color) roughly divides the region from the middle and does not pass the curve since the partitioning does not have a feature curve preservation mechanism. In Fig. 1(c), by applying the feature preservation term we will introduce in Eqn. 6, we can get the resultant separator pass the feature curve.

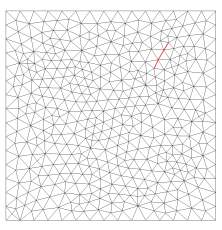

(a)

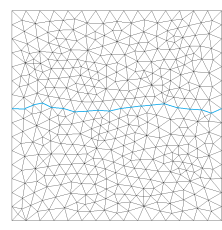

(b)

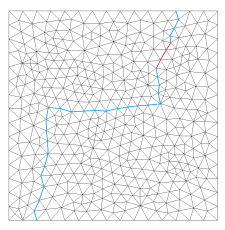

(c)
Figure 1: Partitioning results with or without feature preserving. (a) A rectangle region with feature lines highlighted, (b) partitioning without features preserved, (c) partitioning with features preserved.

Therefore, we incorporate a feature-preserving term into the graph partitioning algorithm as a soft constraint, to attract the partitioning boundary towards features as much as possible. As a soft constraint, some features may not be on subregions boundaries, and they will be handled in the next step during mapping computation.

A feature curve is discretely represented as a list of edges $\left\{e_{f_{1}}, e_{f_{2}}, \ldots, e_{f_{m}}\right\} \in E^{M}$, where $m$ is the total number of feature edges, $f_{i}$ is the index of the edge in $E^{M}$. We first define a feature edge indicator

$$
\sigma_{e_{i}}=\left\{\begin{array}{cc}
1, & e_{i} \in\left\{e_{f_{1}}, e_{f_{2}}, \ldots, e_{f_{m}}\right\} \\
0, & \text { otherwise }
\end{array}\right.
$$

then, define the feature-preserving term as

$$
D_{f}=\sum_{i=1}^{m} y_{e_{f_{i}}}^{2}=\mathbf{y}^{T} \mathbf{W}_{f} \mathbf{y}=\mathbf{x}^{T} \mathbf{U}^{T} \mathbf{W}_{f} \mathbf{U} \mathbf{x}
$$

where $\mathbf{y}=\left(y_{e_{1}}, y_{e_{2}}, \ldots, y_{e_{n}}\right)^{T}$ is the edge variable vector, and $\mathbf{W}_{f}=\operatorname{diag}\left(\sigma_{e_{1}}, \sigma_{e_{n}}, \ldots, \sigma_{e_{n}}\right)$ is an $\left|E^{G}\right| \times$ $\left|E^{G}\right|$ diagonal matrix. To get $\mathbf{W}_{f}$, we just need to traverse all the edges once.

If a feature line $e_{i}$ locates on a sub-region's boundary, then its contribution to $D_{f}$ is 1 since its edge variable $y_{e_{i}} \neq 0$ and $\sigma_{e_{i}}=1$. On the other hand, for a nonfeature edge or an edge that is not on the boundary, either its $y_{e_{i}}$ or $\sigma_{e_{i}}$ is zero, so its contribution to $D_{f}$ is 0 .

Thus, to make the separators align with most of the feature edges, we need the edge variables $\mathbf{y}$ which can minimize $-D_{f}$.

\subsection{Formulation of the Optimization}

Finally, the partitioning reduces to minimizing the separator angle deviation and feature-preserving penalty together with the total separator length, subject to the workload balance constraint, namely,

$\min _{\mathbf{x} \in\{0,1\}^{n}} E(\mathbf{x})=L_{S}+\lambda_{1} D_{\theta}-\lambda_{2} D_{f}$, subject to Eq. (1), 
where $\lambda_{1}, \lambda_{2}$ are weighting factors. In our experiments, we simply set $\lambda_{1}=1, \lambda_{2}=1$.

This is an NP-hard integer programming problem. Solving such an optimization problem accurately is prohibitively expensive for large graphs. So in scientific computing, where meshes often contain millions or billions of vertices, a more efficient approximate solving algorithm is needed.

\subsection{Approximate algorithm}

METIS [39] is a widely used open-sourced program for partitioning unstructured graphs. METIS using is a heuristic algorithm called Hill-Scanning, which can iteratively move vertices to corresponding groups and keep the balance of the partitioning by checking the energy gaining.

To have the feature-preserving term minimized, we modified the algorithm of METIS and developed a new solver incorporating the feature terms. We adopt the Metropolis-Hastings algorithm [40] which are widely used in stochastic optimization problems such as Monte-Carlo simulation, etc. For each possible moving of the vertices, we evaluate the change of energy, $\Delta E$, (Eqn. 7). If $\Delta E<0$, then we will accept this moving. Otherwise, if $\Delta E>0$, we will perform a random test:

- Generate a random number $p \in[0,1]$

- Test whether $p<e^{-\lambda_{M H} \Delta E}$, if so, accept the moving; otherwise reject it.

In our experiments, we set the $\lambda_{M H}$ to a small number 0.01 , so we can accept more energy increase move to avoid local minima.

The advantage of this stochastic optimization strategy is that it can be easily parallelized and give us very good partitioning results.

Fig. 2 shows an example of partitioning that incorporates feature curves. (a) shows a turtle shell region with feature curve highlighted. Without considering these features, the partitioning result is shown in (b). The feature-preserved partitioning result is shown in (c).

\section{FEATURE-PRESERVED POLY-SQUARE MAPS}

After partitioning, we can construct quad meshes on sub-regions in parallel. This section illustrates our local mesh construction. To ensure the element consistency across the subregion boundaries, we would select meshing algorithms that allow us to enforce a

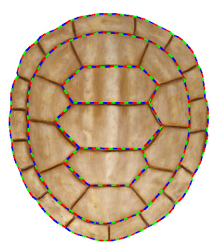

(a)

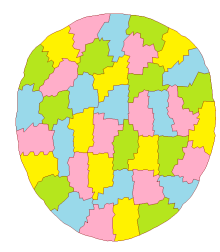

(b)

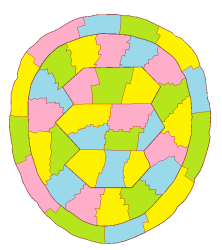

(c)
Figure 2: Partitioning results with or without feature preserving. (a) A turtle shell region with feature lines highlighted, (b) partitioning without features preserved, (c) partitioning with features preserved.

pre-determined boundary constraint, rather than using free-boundary parameterization algorithms, which is difficult to control and refine. One straightforward meshing strategy is to conduct an advancing front algorithm [10] to generate meshes on subregions from their consistently pre-sampled subregion boundaries. Despite its simplicity and efficiency, the advancing front algorithm has several limitations: (1) it only generates unstructured meshes; (2) the element quality near the media axes is hard to control and saliently worse than those near the subregion boundary; (3) for elements near subregion boundaries, their directional smoothness across the boundary could be undesirable, due to that the meshes are generated individually and in a one-pass manner. To overcome these three limitations to generate better-structured meshes and obtain a better control on mesh quality and boundary smoothness, we adopt a mapping based meshing strategy by computing a poly-square parameterization.

\subsection{Poly-square Parameterization}

A poly-square is a $2 \mathrm{D}$ orthogonal polygon whose edges are all parallel to coordinate axes. The 3D analogy of the poly-square parameterization is polycube parameterization, which has been extensively studied in graphics and computer-aided design literature $[23-26,41]$ and applied in texturing spline construction, and mesh generation (for 3D surfaces). Our partitioning produces 2D subregion with relatively simple geometry. So we follow the polycube parameterization algorithm of [41] and implemented it in 2D.

Each local subregion $M$ is described by its boundary $\partial M$. First, we tessellate it using a triangle mesh $\bar{M}=\{T, \bar{X}\}$ conforming to this boundary, where $T=\left\{t_{i}\right\}$ and $\bar{X}=\left\{\bar{x}_{i}\right\}$ are the sets of triangles and vertex coordinates, respectively. The output is a deformed triangle mesh $N=\{T, X\}$ where $X$ is the new corresponding vertex positions. $\partial N$ is a poly-square. This deformation is solved by minimizing an objective function consisting of two terms: (1) boundary normal alignment error, and (2) element distortion. 
Boundary Normal Alignment. The boundary vertices should be deformed to align with coordinate axes to make a poly-square. In Fig. 3(a), we have a pentagon region to be deformed. Only when we align its boundary normal to coordinate axes, the result will be a valid poly-square (Fig. 3(b)). Otherwise (Fig. 3(c)) it cannot become a poly-square.

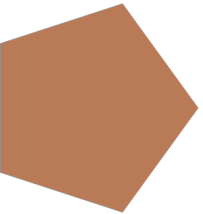

(a)

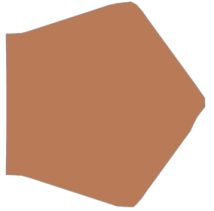

(b)

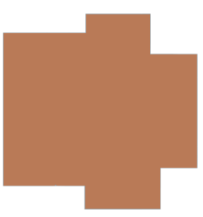

(c)
Figure 3: Boundary Normal Alignment for Poly-Square. (a) A pentagon before deformation; (b) deformation without boundary normal alignment; (c) deformation with boundary normal alignment.

This can be formulated by minimizing the $L_{1}$-norm deviation,

$$
d(\mathbf{n})=\|\mathbf{n}\|_{1}-1,
$$

where $\|\mathbf{n}\|_{1}=\sum_{i}^{k}\left|n_{i}\right|$ is the $L_{1}$-norm of vector $\mathbf{n}$. Given a boundary edge $e \in \partial N$, whose normal is $\mathbf{n}_{e}$, its deviation from axis coordinates is $d\left(\mathbf{n}_{e}\right)$. The accumulative boundary normal alignment error over the entire $\partial N$ can be written as:

$$
E^{B}(X)=\sum_{e_{i} \in \partial N} L\left(e_{i}\right)\left(\left\|\mathbf{n}_{e_{i}}\right\|_{1}-1\right) .
$$

where $L(e)$ is the length of $e$.

Deformation Distortion. To suppress the element's shape distortion during deformation, we shall also minimize the deformation distortion. In [41], the MIPS energy [42] is used to measure the distortion. On a triangle $t_{i}$, the MIPS distortion is defined as $\epsilon_{M I P S}\left(t_{i}\right)=\frac{\sigma_{i, 1}}{\sigma_{i, 2}}+\frac{\sigma_{i, 2}}{\sigma_{i, 1}}$, where $\sigma_{i, 1}, \sigma_{i, 2}$ are the two singular values of the Jacobian of transformation on triangle $t_{i}$. In [5], it is observed that having a heavier penalty on the severely distorted element is desirable to produce better shaped poly-square domain topology. Hence, they used an exponential form of MIPS, $\epsilon_{E M I P S}\left(t_{i}\right)=e^{\frac{\sigma_{i, 1}}{\sigma_{i, 2}}+\frac{\sigma_{i, 2}}{\sigma_{i, 1}}}$, to measure the distortion on triangle $t_{i}$. In [43], an Advanced MIPS energy (AMIPS) was developed, and it used the exponential terms to penalize the angle distortion as well. It also contains an area distortion term and allows us to find a balance between these two terms. AMIPS is formulated as

$$
\begin{gathered}
\epsilon_{A M I P S}\left(t_{i}\right)= \\
e^{\alpha s \cdot \frac{1}{4}\left(\frac{\sigma_{i, 1}}{\sigma_{i, 2}}+\frac{\sigma_{i, 2}}{\sigma_{i, 1}}\right)+(1-\alpha) s \cdot \frac{1}{2}\left(\operatorname{det}\left(J\left(t_{i}\right)\right)+\operatorname{det}\left(J\left(t_{i}\right)\right)^{-1}\right)}
\end{gathered}
$$

where $J\left(t_{i}\right)$ is the Jacobian matrix of transformation on $t_{i}, \alpha$ is a parameter controlling the weight of angle distortion and area distortion, and $s$ is a parameter controlling the level of penalty. This AMIPS energy allows us to control the area distortion better. A small $s$ has little effect on penalizing the maximal distortion, and a large $s$ will cause numerical instability. Therefore, we adopted AMIPS in this pipeline. In our experiment, we choose $\alpha=0.5, s=5$. Accordingly, the accumulative AMIPS distortion on the entire mesh can be computed as $E^{D}(X)=\sum_{t_{i} \in T} \epsilon_{A M I P S}\left(t_{i}\right)$.

Finally, combining these two terms, the poly-square deformation reduces to solving the following optimization problem:

$$
\phi=\operatorname{argmin}_{X} E^{D}(X)+\lambda E^{B}(X),
$$

where $\lambda$ is the weighting factor balancing the two different emphases of the Domain shape optimization. A small $\lambda$ indicates more emphasis on minimizing distortion and could result in a non-orthogonal and invalid poly-square. A big $\lambda$, in contrast, produces a valid poly-square by possibly introducing larger distortion and more singularities.

In all our experiments, we use $\lambda=5$ which is the balance point that can give a valid poly-square and least singularities.

We solve this problem using the Hybrid L-BFGS (Limited Memory Broyden-Fletcher-Goldfarb-Shanno Method) optimization framework [44].

\subsection{Preserving Feature Lines}

To ensure feature (lines) to be sampled in the final mesh, we require the feature points to be mapped to integer coordinates. In the data partitioning stage, feature lines are incorporated as a soft constraint. So some feature lines, not sampled as boundary region separators, need to be resolved here during parameterization.

To preserve feature lines in poly-square parameterization, we just need to force the sampled feature points to be deformed to integer coordinates.

To enforce this integer constraints, first, we add a soft constraint, to minimize the deviation of deformed feature lines' directions (or their normals) from axes coordinates. Namely, for feature edges $F=$ $\left\{e_{f_{1}}, e_{f_{2}}, \ldots, e_{f_{m}}\right\}$, we have the third penalty term,

$$
E^{F}(M)=\sum_{e_{i} \in F} L\left(e_{i}\right)\left(\left\|\mathbf{n}_{e_{i}}\right\|_{1}-1\right) .
$$

Then finally, after the optimization, we round the resultant coordinates of each feature line segment's end nodes to integers. Fig. 4 shows an example of featurepreserving in mapping computation. The red curve is the feature to sample. After deformation, they are 
mapped onto horizontal or vertical line segments with integer coordinates.

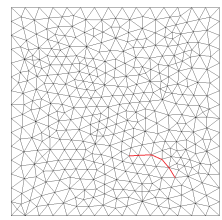

(a)

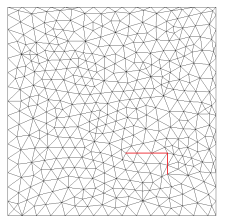

(b)

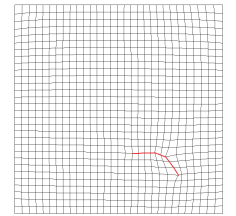

(c)
Figure 4: Poly-square mapping to mesh a square domain with features (red curve). (a) The original triangular mesh, (b) the deformed mesh, and (c) the final quad mesh.

\subsection{Initialization and Pre-orientation}

The deformation-based algorithm transforms each boundary edge to its nearest coordinate axis. So this deformation is affected by the shape's initial orientation. Optimizing a pre-orientation of $M$ before the deformation could provide a good initialization for the poly-square parameterization and lead to a better mapping result.

Adopting the strategy of [45], we select the preorientation that makes the directions of $M$ 's boundary line segments to have the smallest deviations from coordinate directions. More specifically, suppose we use a function $\Phi$ to measure the deviation of an edge's normal $\mathbf{n}$ from a coordinate axis, $\Phi(\mathbf{n})=n_{x}^{2} n_{y}^{2}$ where $n_{x}$ and $n_{y}$ are n's $x$ and $y$ components respectively.

Then an optimal pre-orientation, rotation $R$, minimizes the following function,

$$
\hat{R}=\operatorname{argmin}_{R} \sum_{e_{i} \in \partial M} \Phi\left(R \cdot \mathbf{n}_{i}\right) .
$$

The 2-dimensional rotation $R$ can be represented using an angle and the above problem can be solved using gradient descent method efficiently.

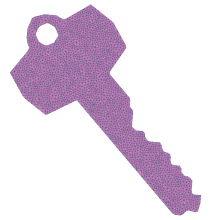

(a)

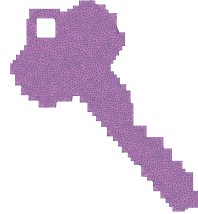

(b)

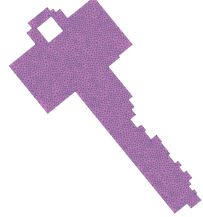

(c)
Figure 5: Pre-orientation for Poly-square Mapping. (a) The original mesh; (b) resultant poly-square without pre-orientation; (c) resultant poly-square with preorientation.

Fig. 5 shows an example of how pre-orientation improves the poly-square map. The direct poly-square deformation performed on the model (a), without preorientation, results in (b). With a pre-orientation, the deformation results in (c) which have fewer boundary corners, and also, smaller mapping distortion.

\section{CONQUERING LOCAL MAPS INTO GLOBAL PARAMETERIZATION}

\subsection{Global Poly-square Map (GPM)}

When processing a large or complicate region $M$, if we directly compute its poly-square parameterization, the optimization becomes too expensive. With the previous data partitioning, one simple strategy is to solve the poly-square parameterization distributedly.

Suppose the given domain $M$ is partitioned into subregions $M=\sum_{i} \cup M_{i}$. For each adjacent subregion pair $M_{u}$ and $M_{v}$, their separator is a common boundary curve $L_{u v}$, which is defined by a set of common vertices $\left\{X_{u v}\right\}=X_{u} \cap X_{v}$. Since each subregion will be mapped onto a poly-square domain on a separate processor. We need to enforce the following consistency constraint on common separators to keep boundary consistency:

$$
x_{I_{u, i}^{u v}}^{u}=x_{I_{v, i}^{u v}}^{v}, \forall i=1, \ldots, N_{u v},
$$

where $I_{u, i}$ is the index of the $i$-th vertex from $L_{u v}$ in $M_{u}, I_{v, i}$ is the index of the $i$-th vertex from $L_{u v}$ in $M_{v}, N_{u v}$ is the total number of vertices on $L_{u v}$. This constraint means each pair of corresponding vertices on the shared subregion boundary should have the same image after their deformations. Although this map is solved in a distributed way, this strict boundary constraint ensures the resultant parameterization is a globally continuous poly-square map. In the following, we denote this computed parameterization a Global Poly-square Map (GPM).

Fig. 6 demonstrate the GPM updating process for the sub-regions of the key, for the clearly visualization purpose, we use 8 subregions to do this demonstration. We can see that all the regions will continuously deform to a poly-square shape and the shared subregion boundaries will keep the same shape and orientation during all the time.

\subsection{Piecewise Poly-square Map (PPM)}

The poly-square map has all its singularities on the poly-square's corners. So usually, these regions have big distortion and cells near these singularities may be severely sheared (See Fig. 13 (b,e,h) for examples). To reduce the mapping distortion, designing a mechanism to allow placing interior singularities at suitable places is often very helpful. 


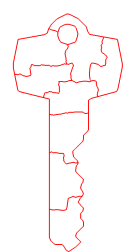

(a)

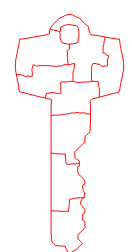

(b)

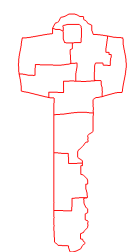

(c)

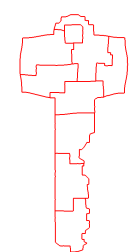

(d)

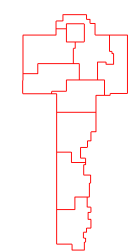

(e)
Figure 6: The deformation process of a GPM algorithm. (a) The partitioning before deform. (b) Iteration number $=1$. (c) Iteration number $=5$. (d) Iteration number $=$ 10. (e) Final poly-square result (Iteration number $=209$ )

In our computational pipeline, with data partitioning, we get subregions that have near perpendicular corner angles, which is desirable for poly-square parameterization. More importantly, we can build upon this partitioning a mechanism that could support the flexible insertion of interior singularities.

Following the previous notations, instead of using Equation 12, we enforce the following new consistency constraint on common separators:

$$
L\left(I_{u, 1}, I_{u, k}\right)=L\left(I_{v, 1}, I_{v, k}\right), \forall k=2, \ldots, N_{u v},
$$

where $I_{u, i}$ is the index of the $i$-th vertex from $L_{u v}$ in $M_{u}, I_{v, i}$ is the index of the $i$-th vertex from $L_{u v}$ in $M_{v}$, $L\left(I_{1}, I_{2}\right)$ is the curve length from point $I_{1}$-th vertex to $I_{2}$-th vertex, $N_{u v}$ is the total number of vertex on $L_{u v}$. This constraint means that for each pair of vertices on the common separator, their curve length on both subregions, after deformation, should be the same.

Interior Singularities. We do not require the parametric coordinates from two subregions to exactly overlap, and on the poly-square corners we We do not require the sum of its incident angles to be exactly $2 \pi$. For a corner whose surrounding angles do not sum up to $2 \pi$, we generate an interior singularity. We call this poly-square parameterization that allows interior singularities a Piecewise Poly-square Map (PPM).

Parallel Computation. Since different subregions are processed separately, during a subregion's deformation, its neighboring regions' deformation is unknown. If we process all the subregions simultaneously, the consistency constraint cannot be directly enforced. Therefore, we perform deformation in a multipass manner. All the non-adjacent subregions can deform simultaneously; then their new boundary geometry is propagated to adjacent subregions, which will be used as initial poses of their deformations in the next round. To do this, we perform a graph coloring algorithm [46] to divide all the subregions $\left\{S_{1}, S_{2}, \ldots, S_{p}\right\}$ into $k$ group $\left\{G_{1}, G_{2}, \ldots, G_{k}\right\}$. Each group consists of non-adjacent subregions and can be processed in parallel simultaneously.
Propagation on Atlas until Convergence. To support a more efficient propagation of boundary condition from a subregion to its neighbors, we construct a multi-layer buffer-zone around each subregion. From the boundary of subregion $\partial M_{i}$, we grow outwards to construct an offset contour $\partial B_{i}$, and call the region bounded by $\partial M_{i}$ and $\partial B_{i}$ the buffer zone $B_{i}$. In Fig. 7, we illustrate the relationship between a subregion $M_{i}$ and its buffer zone $B_{i}$. For simplicity, we can con-

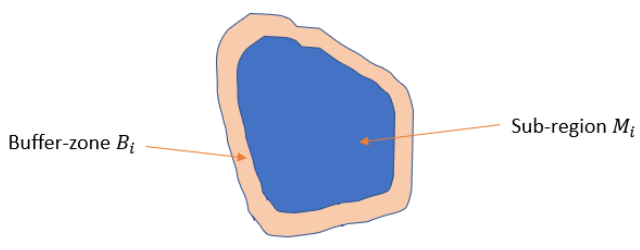

Figure 7: A subregion $M_{i}$ and its buffer zone $B_{i}$.

struct $B_{i}$ using the $k$-ring elements from $\partial M_{i}$. Then we call the enlarged patch $C_{i}=M_{i} \bigcup B_{i}$ the deformation chart. During iterative deformation, instead of only deforming $M_{i}$, we deform $C_{i}$ subject to two constraints (1) $\partial M_{i}$ should deform to an orthogonal polygon, and (2) the vertices on $\partial C_{i}$ should not move. Namely, we solve

$\phi_{i}=\operatorname{argmin} E^{D}\left(C_{i}\right)+\lambda E^{B}\left(M_{i}\right)$, s.t. $\partial C_{i}$ being fixed.

In the $k$-th iteration, $M_{i}$ deforms to make itself orthogonal while vertices on $\partial C_{i}$ are fixed to the positions from their last iteration. This ensures positional consistency across $\partial C_{i}$ with the neighboring regions. Vertices in the buffer zone $B_{i}$ deform together with $M_{i}$, and propagate to $M_{i}$ 's neighboring regions for their deformation in next iteration.

Atlas and Transitions among Local Charts. Deformation of $M_{i}\left(C_{i}\right)$ is performed under its local coordinate system $F_{i}$. Each vertex $v \in M_{i}$ stores its local coordinates with respect to $F_{i}$. If we denote the initial global coordinate system using a reference frame $F_{r}$, then $F_{i}$ can be described using a rotation from $F_{r}$ (No translation is introduced between coordinate systems). Hence, in the following, we also use the symbol $F_{i}$ to denote the coordinate transformation from $F_{r}$. For example, given a point $\mathbf{x}$ originally considered in $F_{r}, F_{i} \circ \mathbf{x}$ indicates its coordinates after being transformed into the coordinate system of $F_{i}$. During the $j$-th iteration, we first solve a re-orientation $\hat{R}_{i}^{j}$ for $M_{i}$, and update $F_{i}$ accordingly, $F_{i} \leftarrow \hat{R}_{i}^{j} \circ F_{i}$. Then, to construct $C_{i}$ in the coordinate system of $F_{i}$, we transform vertices in buffer zone $B_{i}$ from their own local coordinates into $F_{i}$, namely, $\forall \mathbf{x} \in M_{k}$ (represented under $F_{k}$ ), we get $\widetilde{\mathbf{x}}=F_{i} \circ F_{k}^{-1} \circ \mathbf{x}$. Now, $\widetilde{\mathbf{x}}$ is in the coordinate system of $F_{i}$. After local poly-square deformation, $\phi_{i}(\widetilde{\mathbf{x}})$ should be updated in its own coordinate system $F_{k}$ after being transformed back to it, 
i.e., $\mathbf{x} \leftarrow F_{k} \circ F_{i}^{-1} \circ \phi_{i}(\widetilde{\mathbf{x}})$. In Fig. 8, we demonstrate the pipeline of the updating of a local chart and its propagation.

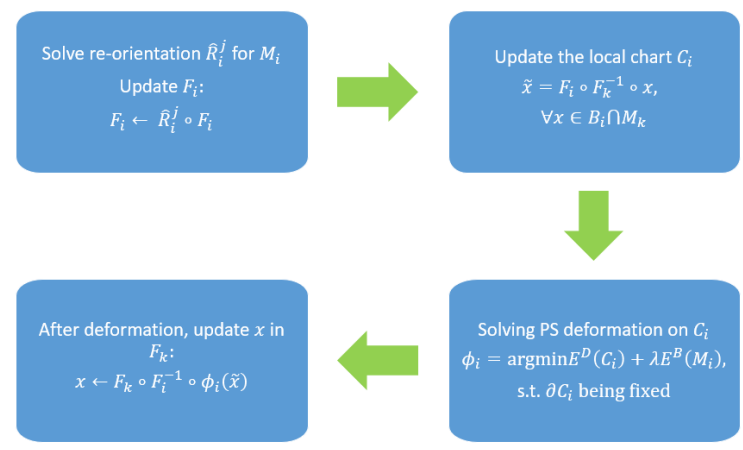

Figure 8: Update a local char and its propagation.

PPM Algorithm. Algorithm 1 summarizes our PPM algorithm. The deformations (Steps 5-12) are performed iteratively. In each iteration, we go through each subgroup $G_{k}$ and deform the independent subregions simultaneously. Deformations are performed on all the deformation charts $C_{j}=M_{j} \cup B_{j}$. After each deformation, $M_{j}$ is mapped to an updated polysquare and elements in $B_{j}$ is modified and updated in their patches. In Step 13, a threshold $T$ is used to check whether the deformation converges: when no vertex's movement is bigger than $T$ during the last iteration, the algorithm stops. In our experiments, we set $T=10^{-2}$, and the algorithm usually converges after around 15 iterations.

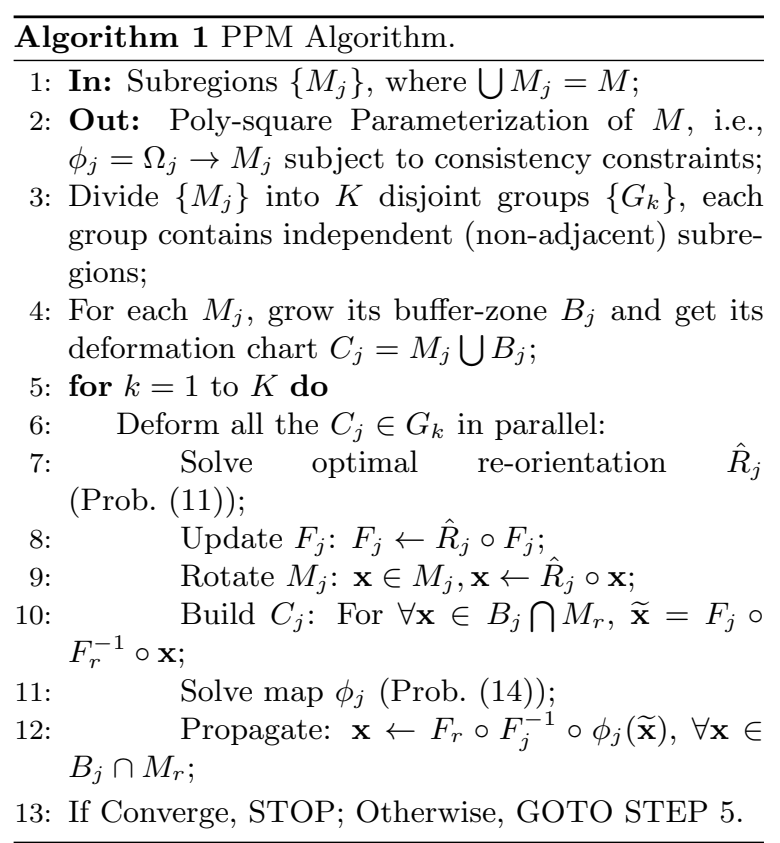

\subsection{Adaptive Singularity Refinement}

While PPM introduces some interior singularities, we can also further refine mesh locally when needed. Inspired by [47], we propose a new local singularity refinement method for regions that have large distortion or where singularities were inappropriately placed.

Poly-square maps generate two types of corners in the parametric domain, namely, $90^{\circ}$ and $270^{\circ}$ corners. If such corners are mapped to a flat boundary or feature curve, it will introduce big distortion and cannot be suppressed through variational relaxation. Fig. 12 (a) (blue points in pink patches) illustrates an example of such corners. We develop a set of topological strategies to modify such patches.

If we detect a vertex on boundary or feature curves which is 2 -valent, then it means a $90 \mathrm{deg}$ corner in the poly-square domain has been flattened here. Usually a Triangle Patch can be used to modify the patch surrounding such $90^{\circ}$ corner. See Fig. 12(a): each pink region contains a triangle patch. When large distortion is detected in this region, it is because a $90^{\circ}$ corner is mapped to a flat boundary region of $R$. Hence, such a region can be treated as a topological triangle (considering the arc on the boundary as one edge). We can quadrangulate this patch by the following algorithm.

As shown in Fig. 10, to quadrangulate a triangle patch, we just need to find a partition for the vertices on each edge. To form a valid quad tessellation, we need to assure the interval matching for this triangle patch. This leads to the following system:

$$
\begin{gathered}
{\left[\begin{array}{cccccc}
1 & 0 & 0 & 1 & 0 & 0 \\
0 & 1 & 0 & 0 & 1 & 0 \\
0 & 0 & 1 & 0 & 0 & 1 \\
1 & 0 & 0 & 0 & -1 & 0 \\
0 & 1 & 0 & 0 & 0 & -1 \\
0 & 0 & 1 & -1 & 0 & 0
\end{array}\right]\left[\begin{array}{l}
a_{0} \\
a_{1} \\
a_{2} \\
b_{0} \\
b_{1} \\
b_{2}
\end{array}\right]=\left[\begin{array}{c}
N_{0} \\
N_{1} \\
N_{2} \\
0 \\
0 \\
0
\end{array}\right]} \\
\leftrightarrow\left[\begin{array}{c}
a_{0}+b_{0}=N_{0} \\
a_{1}+b_{1}=N_{1} \\
a_{2}+b_{2}=N_{2} \\
a_{0}-b_{1}=0 \\
a_{1}-b_{2}=0 \\
a_{2}-b_{0}=0
\end{array}\right]
\end{gathered}
$$

If there exists an integer solution, then this triangle patch can be quadrangulated with a 3 -valent singularity inside that patch as shown in the left figure. If there is not an integer solution for it, we will quadrangulate it similar to the way we mesh the triangle region in Fig. 9, we can perform an extra partition in this local region. Specifically, we just need to run a 3 -way partitioning on this region, and by balancing loads (region size), separator lengths, and geometry, 


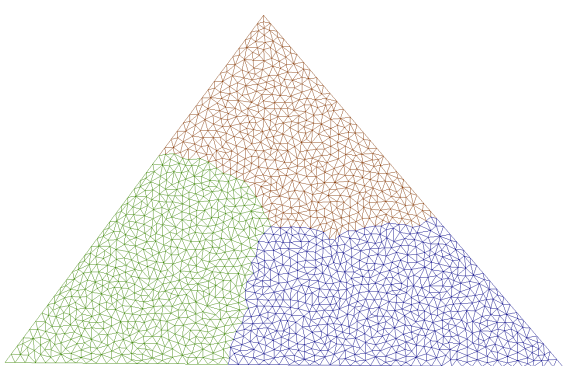

(a)

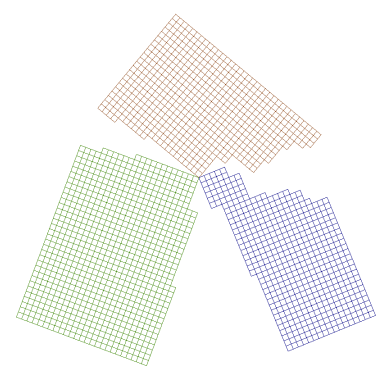

(b)

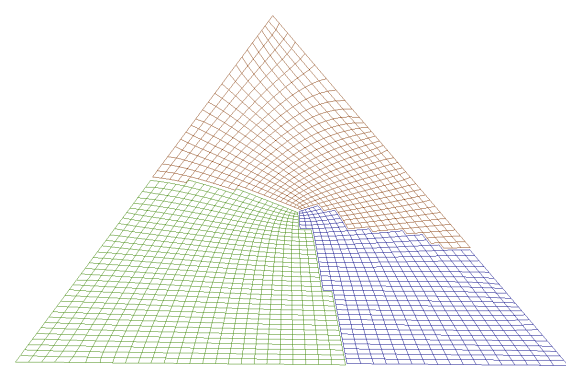

(c)

Figure 9: Quad Meshing of a Triangular Region. (a) The original mesh and the partitioning, (b) constructed poly-square of each sub-region, (c) meshing result.

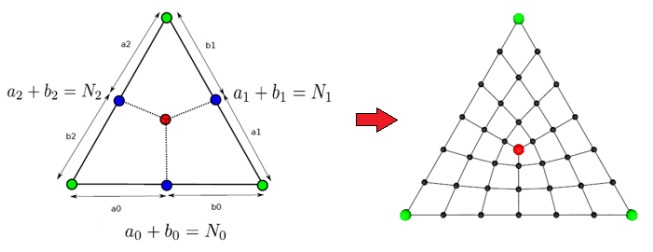

Figure 10: Quadrangulation a Triangle Patch

we will always get a partition like (a) in Fig. 9. These three newly obtained subregions can then be processed like all other subregions using the PPM algorithm. A singularity point will be generated in the middle while the boundary corner is removed. Fig. 12(b) illustrates the result of this operation applied to the two pink regions of (a). The distortion in this local area can be greatly reduced.

Another case is a vertex on boundary or feature curves, which is 4 -valent. Then it means a $270 \mathrm{deg}$ corner in the poly-square domain has been flattened here. Then a Pentagon Patch can often be traced around the $270^{\circ}$ corner. See Fig. $12(\mathrm{c})$ : each blue region contains a pentagon patch. Large distortion is detected in this region as well. This region can be treated as a topological pentagon. Similarly, with triangle patch, we can quadrangulate this patch by the following algorithm.

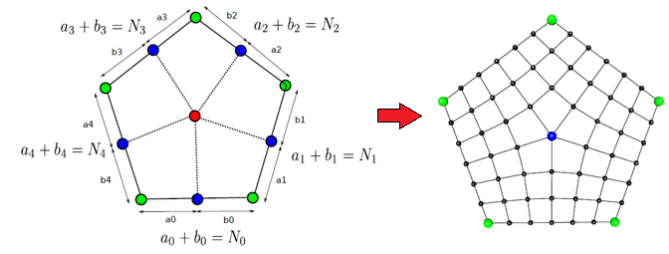

Figure 11: Quadrangulation a Pentagon Patch

As shown in Fig. 11, to quadrangulation a pentagon patch. We just need to find a partition for the vertices on each edge. And we need to assure the interval matching for this pentagon patch. This leads to the following system:

$$
\begin{gathered}
{\left[\begin{array}{cccccccccc}
1 & 0 & 0 & 0 & 0 & 1 & 0 & 0 & 0 & 0 \\
0 & 1 & 0 & 0 & 0 & 0 & 1 & 0 & 0 & 0 \\
0 & 0 & 1 & 0 & 0 & 0 & 0 & 1 & 0 & 0 \\
0 & 0 & 0 & 1 & 0 & 0 & 0 & 0 & 1 & 0 \\
0 & 0 & 0 & 0 & 1 & 0 & 0 & 0 & 0 & 1 \\
1 & 0 & 0 & 0 & 0 & 0 & 0 & 0 & -1 & 0 \\
0 & 1 & 0 & 0 & 0 & 0 & 0 & 0 & 0 & -1 \\
0 & 0 & 1 & 0 & 0 & -1 & 0 & 0 & 0 & 0 \\
0 & 0 & 0 & 1 & 0 & 0 & -1 & 0 & 0 & 0 \\
0 & 0 & 0 & 0 & 1 & 0 & 0 & -1 & 0 & 0
\end{array}\right]\left[\begin{array}{l}
a_{0} \\
a_{1} \\
a_{2} \\
a_{3} \\
a_{4} \\
b_{0} \\
b_{1} \\
b_{2} \\
b_{3} \\
b_{4}
\end{array}\right]} \\
=\left[\begin{array}{c}
N_{0} \\
N_{1} \\
N_{2} \\
N_{3} \\
N_{4} \\
0 \\
0 \\
0 \\
0 \\
0
\end{array}\right] \leftrightarrow\left[\begin{array}{c}
a_{0}+b_{0}=N_{0} \\
a_{1}+b_{1}=N_{1} \\
a_{2}+b_{2}=N_{2} \\
a_{3}+b_{3}=N_{3} \\
a_{4}+b_{4}=N_{4} \\
a_{0}-b_{3}=0 \\
a_{1}-b_{4}=0 \\
a_{2}-b_{0}=0 \\
a_{3}-b_{1}=0 \\
a_{4}-b_{2}=0
\end{array}\right]
\end{gathered}
$$

If there exists an integer solution, then this pentagon patch can be quadrangulated with a 5 -valent singularity inside that patch as shown in the left figure. If not, we can treat it with our more general Polygon patch quadrangulation algorithm.

A Polygon patch can always be traced as a $(n+2)$ sided topological polygon on the parametric domain (by iteratively performing $(n+2)$ times of moving forward then turning to the other iso-line counterclockwise). Then on this $(n+2)$-sided topological polygon we also perform a $k$-way partitioning, where we iteratively try integer $k$ from $\left\lceil\frac{n}{2}\right\rceil$ to $(n+2)$. The intuition behind this range is that we will try different combinatorial partitions on this $(n+2)$-sided polygon: the lower bound $\left\lceil\frac{n}{2}\right\rceil$ is the subregion number that we will get if we keep removing 4-sided polygons from the re- 


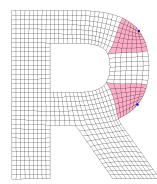

(a)

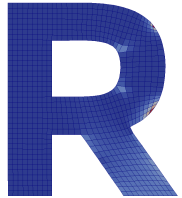

(e)

$0.97 / 0.32 / 0.07$

(b)

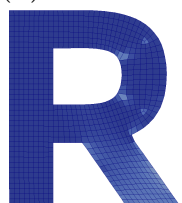

(f)

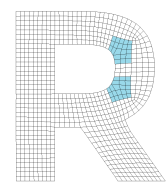

(c)

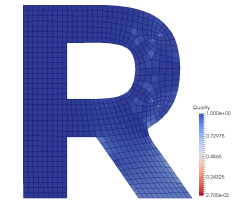

$(\mathrm{g})$

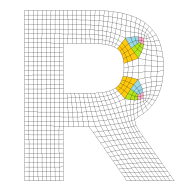

(d)
Figure 12: Adaptive refinement of boundary singularities. The quality shown in second row is scaled Jacobian (average/minimum of Scaled Jacobian/standard deviation). (a) The PPM result without any singularity modification: each pink patch contains a Type- 1 boundary singularity (Type-1 corner); (b) Refining the type- 1 singularity; (c) Blue patch contains a Type-2 boundary singularity (Type-2 corner). (d) Refining the type-2 singularity. (e) The meshing result of GPM. (f) The meshing result of PPM without singularity modification. (g) The meshing result of PPM after singularity modification.

gion until we can no longer do it; while $(n+2)$ indicate a topological Catmull-Clark subdivision which directly splits this $(n+2)$-gon into $n+2$ quads. For example, when $n=4$, we have a topological hexagon. We first try the $\left\lceil\frac{n}{2}\right\rceil=2$-way partitioning (a hexagon could split into 2 quad patches), then try 3, 4, till 6-way partitioning. When $n=3$ we get a pentagon, and we will try $k \in[2,5]$. Among all the partitioning results generated by different $k$, we can pick the one with the maximized minimal angle as our partition. Since $n$ is usually very small, this selection is very quick. Fig. 12(d) illustrates the result of the modification on Type- 2 corners in the blue regions in (c). In practice, users can get involved in this step to interactively select the template to repartition this local region and modify its singularity distribution. More complicated topological operations such as [47] can also be adopted.

\section{EXPERIMENTAL RESULTS}

We compare our algorithm with the advancing front algorithm [48] and the Global Poly-square Map (GPM) meshing discussed in Section 5.1. The advancing front generates unstructured meshes; GPM generates full structured meshes, while our algorithm, denoted as Feature-aligned Piecewise Poly-square Map (FPPM), generates semi-structured meshes.

The meshing distortion is measured using three metrics: Scaled-Jacobian, Skewness, and Edge Ratio.

The scaled Jacobian $\eta_{f}(Q)$ of each quad cell $Q$ is cal- culated based on [49]:

$\eta_{f}(Q)=\frac{1}{4}\left(\eta\left(x_{1}, Q\right)+\eta\left(x_{2}, Q\right)+\eta\left(x_{3}, Q\right)+\eta\left(x_{4}, Q\right)\right)$,

where $\eta(x, Q)$ is the scaled Jacobian distortion of vertex $x$ in cell $Q . \eta_{f} \in(0,1]$ for a valid quad cell, where 1 is ideal. $\eta_{f}<0$ indicates a flipped cell.

The Skewness $\kappa$ defined on a quad cell measures the deviation from the $90^{\circ}$ angle:

$$
\kappa(Q)=\max \left[\frac{\theta_{\max }-90}{90}, \frac{90-\theta_{\min }}{90}\right],
$$

where $\theta_{\max }$ and $\theta_{\min }$ are the maximal and minimal interior angles in quad $Q . \kappa \in[0,1]$ for a convex quad cell, where 0 is optimal. If $\kappa>1$ the quad cell becomes concave.

The Edge Ratio $r$ is the ratio of the longest edge length to the shortest one in cell $Q$, which measures the cell's deviation from a equilateral quad cell,

$$
r(Q)=\frac{l_{\max }}{l_{\min }}
$$

where $l_{\max }$ and $l_{\min }$ are the maximal and minimal edge lengths in cell $Q . r \in[1, \infty)$ where 1 is optimal.

Using either one of the above 3 metrics, we can measure all the quad cells, and document the average and worst values, and the standard deviation. For example, we use the triplet $\left(\bar{\eta}, \eta_{w}, \sigma_{\eta}\right)$ to indicate the average, minimum (worst), and standard deviation of the scaled Jacobian, and $\left(\bar{\kappa}, \eta_{\kappa}, \sigma_{\kappa}\right)$ to indicate the average, maximum (worst), and standard deviation of skewness.

\subsection{Comparing Meshing Quality on Small Geometries}

Meshing Letters "IMR". Fig. 13 shows the quad meshing on three letters, $I, M$ and $R$. The input is these letters' boundary contours extracted from images. The FPPM meshing results significantly outperform those generated by the other two algorithms.

Table. 1 reports the numerical measures computed on meshing results generated on small testing geometric regions. Besides the measure on scaled Jacobian $\left(\left(\bar{\eta}, \eta_{m}, \sigma_{\eta}\right)\right)$, we also report the triplets calculated on skewness and edge ratio, namely, $\left(\bar{\kappa}, \kappa_{m}, \sigma_{\kappa}\right)$ and $\left(\bar{r}, r_{m}, \sigma_{r}\right)$. Besides distortion, we also report the number of singularities $N_{s}$. Because the advancing front algorithm [48] also generates triangular cells in the final quad meshes, we also calculate the number of triangular elements and denote it as $N_{t}$. In all our GPM and FPPM results, $N_{t}$ are zero. From these statistics, we can see that our PPM algorithm generates significantly less distorted (especially on skewness and edge 
Table 1: Mesh Quality Comparison among the Advancing Front, GPM (Global Poly-Square Mapping), and PPM (Piecewise Poly-Square Mapping) algorithms. $N_{t}$ is the number of triangle cells; $N_{s}$ is the number of singularities; $k$ is the sub-regions number, for advancing front, we solve the whole region directly without paritioning; $t_{p} / t_{m}$ are the running time of partitioning and the running time of meshing in seconds; $\bar{\eta}^{*} / \eta_{w}^{*} / \sigma_{\eta}^{*}$ are the average, minimum, and standard deviation of Scaled Jacobian before applying Refinement; $\bar{\eta} / \eta_{w} / \sigma_{\eta}$ are the average, minimum, and standard deviation of Scaled Jacobian; $\bar{\kappa} / \kappa_{w} / \sigma_{\kappa}$ are the average, maximum, and standard deviation of Skewness; and $\bar{r} / r_{w} / \sigma_{r}$ are the average, maximum, and standard deviation of edge ratio.

\begin{tabular}{|c|c|c|c|c|c|c|c|c|c|c|}
\hline Model & Meshing Method & \# Cells & $N_{t}$ & $N_{s}$ & $k$ & $t_{p} / t_{m}$ & $\bar{\eta}^{*} / \eta_{w}^{*} / \sigma_{\eta}^{*}$ & $\bar{\eta} / \eta_{w} / \sigma_{\eta}$ & $\bar{\kappa} / \kappa_{w} / \sigma_{\kappa}$ & $\bar{r} / r_{w} / \sigma_{r}$ \\
\hline \multirow{3}{*}{ I } & Advancing Front & 407 & 15 & 19 & - & $-/ 0.323$ & $0.98 / \mathbf{0 . 7 2} / 0.04$ & $0.98 / 0.72 / 0.04$ & $0.18 / 0.61 / 0.10$ & $1.20 / 2.22 / 0.22$ \\
\cline { 2 - 12 } & GPM & 477 & 0 & 4 & 10 & $0.085 / 0.728$ & $0.99 / 0.71 / 0.02$ & $0.99 / 0.77 / 0.02$ & $\mathbf{0 . 1 6} / 0.73 / \mathbf{0 . 0 5}$ & $\mathbf{1 . 1 1} / 2.36 / 0.17$ \\
\cline { 2 - 12 } & PPM & 509 & 0 & 12 & 10 & $0.085 / 0.918$ & $\mathbf{0 . 9 9} / 0.69 / \mathbf{0 . 0 2}$ & $\mathbf{0 . 9 9} / \mathbf{0 . 9 1} / \mathbf{0 . 0 1}$ & $0.19 / \mathbf{0 . 5 7} / 0.10$ & $1.16 / \mathbf{1 . 9 2} / \mathbf{0 . 1 6}$ \\
\hline \multirow{3}{*}{$\mathrm{M}$} & Advancing Front & 550 & 27 & 37 & - & $-/ 0.141$ & $\mathbf{0 . 9 9} / \mathbf{0 . 8 4} / \mathbf{0 . 0 2}$ & $0.99 / \mathbf{0 . 8 4} / 0.02$ & $0.15 / \mathbf{0 . 5 4} / 0.11$ & $1.24 / 2.95 / 0.20$ \\
\cline { 2 - 12 } & GPM & 686 & 0 & 16 & 10 & $0.081 / 0.529$ & $0.95 / 0.31 / 0.07$ & $0.99 / 0.56 / 0.05$ & $\mathbf{0 . 1 0} / 1.00 / 0.14$ & $1.13 / 3.30 / 0.24$ \\
\cline { 2 - 11 } & PPM & 744 & 0 & 1 & 10 & $0.081 / 0.736$ & $0.97 / 0.48 / 0.09$ & $\mathbf{0 . 9 9} / 0.81 / \mathbf{0 . 0 1}$ & $0.13 / 0.75 / \mathbf{0 . 0 9}$ & $\mathbf{1 . 1 3} / \mathbf{1 . 7 2} / \mathbf{0 . 1 1}$ \\
\hline \multirow{3}{*}{$\mathrm{R}$} & Advancing Front & 677 & 42 & 71 & - & $-/ 0.141$ & $0.98 / \mathbf{0 . 6 0} / \mathbf{0 . 0 4}$ & $0.98 / 0.60 / \mathbf{0 . 0 4}$ & $0.18 / 1.00 / 0.17$ & $1.43 / 6.47 / 0.40$ \\
\cline { 2 - 11 } & GPM & 818 & 0 & 28 & 10 & $0.081 / 0.529$ & $\mathbf{0 . 9 8} / 0.42 / 0.07$ & $\mathbf{0 . 9 8} / 0.47 / 0.07$ & $\mathbf{0 . 1 2} / 1.00 / 0.28$ & $\mathbf{1 . 1 5} / 4.31 / 0.24$ \\
\cline { 2 - 10 } & PPM & 832 & 0 & 8 & 10 & $0.081 / 0.736$ & $0.97 / 0.32 / 0.07$ & $0.97 / \mathbf{0 . 7 4} / 0.05$ & $0.15 / \mathbf{0 . 4 7} / \mathbf{0 . 1 3}$ & $1.20 / \mathbf{4 . 0 2} / \mathbf{0 . 2 3}$ \\
\hline
\end{tabular}

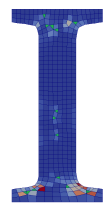

(a) $0.98 / 0.72 / 0.04$

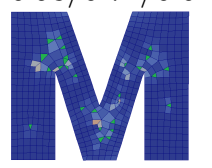

(d) $0.99 / 0.84 / 0.02$

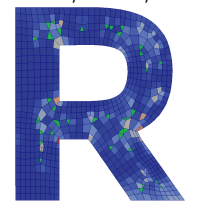

(g)

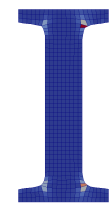

(b) $0.99 / 0.77 / 0.02$

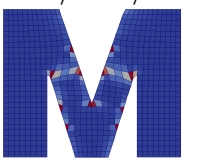

(e) $0.99 / 0.56 / 0.05$

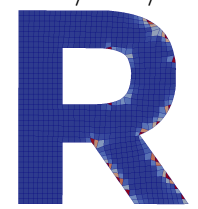

(h)

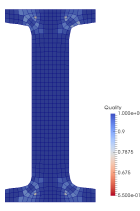

(c) $0.99 / 0.91 / 0.01$

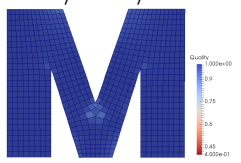

(f) $0.99 / 0.81 / 0.01$

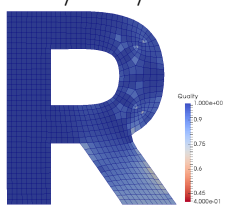

(i)
0.98/0.60/0.04 $\quad 0.98 / 0.47 / 0.07 \quad 0.97 / 0.74 / 0.05$

Figure 13: Meshing results of letters $I, M$ and $R$. Quad meshing results generated by the advancing front, GPM, and PPM algorithms are illustrated in the left, middle, right columns respectively. $\left(\bar{\eta}, \eta_{w}, \sigma_{\eta}\right)$ are reported in captions accordingly.

ratio) meshes than advancing front and GPM algorithms. For the generated quad meshes, we define a vertex to be a singularity if it is (1) a boundary vertex but is not valence-3, or (2) an interior vertex but is not valence-4. Note that FPPM places the singularities according to geometry, while the GPM constructs a big poly-square with many boundary singularities. Thus, FPPM often has fewer singularities.

\subsection{Meshing with Feature Alignment}

Meshing a Smile Face. Fig. 14 shows the meshing of a smiling face. The feature lines include the contours of the two eyes and the mouth curve, as shown in (a). The partitioning and meshing result without considering these features are shown in (b) and (c), while results preserving these feature lines are shown in (d) and (e). Here feature preserving is fully enforced during the partitioning stage.

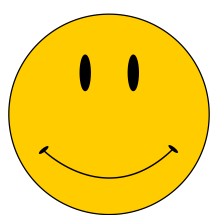

(a)

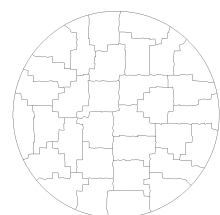

(b)

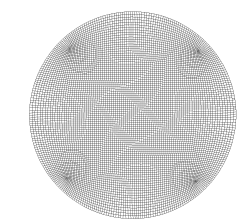

(c)

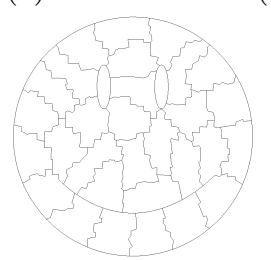

(d)

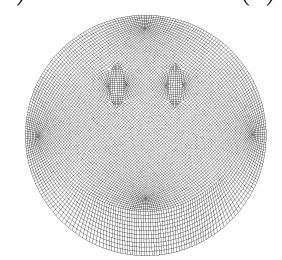

(e)
Figure 14: Meshing for a smiling face. (a) The smiling face image; (b) The partitioning result without feature; (c) Meshing without feature; (d) Feature aligned partitioning; (e) Feature preserved meshing

Meshing a Turtle Shell. Fig. 15 illustrates the meshing result of a turtle shell image. We extract a turtle shell's boundary contour and some feature curves from this image (a) and uniformly sample it using 400 points. The partitioning and meshing result without considering these features are shown in (b) and (c), while the feature-preserved results are shown in (d) and (e). Feature alignment is fully resolved.

Runtime Statistics. We run our algorithm in se- 


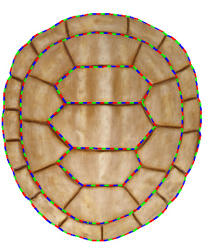

(a)

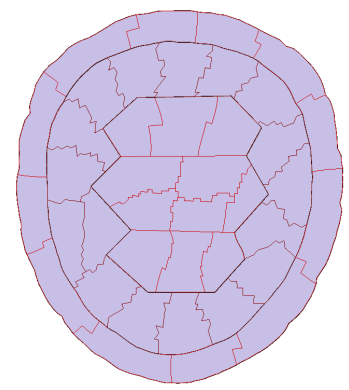

(d)

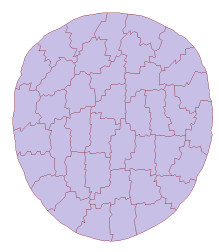

(b)

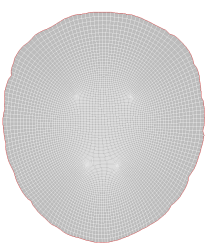

(c)

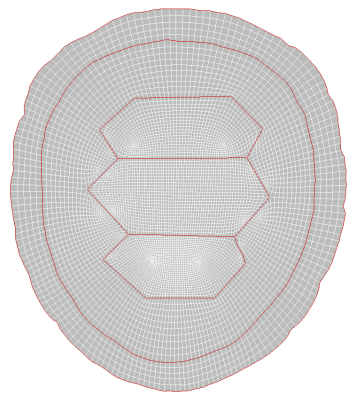

(e)
Figure 15: The quad meshing of a turtle shell model. (a) Features on the model, (b) data partitioning without considering features, (c) PPM meshing result without considering features, (d) data partitioning with feature considered, and (e) PPM meshing result with feature considered.

quential, 2-core, 5-core, 10-core, and 20-core scenarios to evaluate its scalability. The results are shown in Table 2 .

Table 2: Running Time in seconds. $t_{p} / t_{m}$ are the partitioning and meshing time, respectively.

\begin{tabular}{|c|c|c|c|c|c|}
\hline & Sequential & 2-core & 5-core & 10-core & 20-core \\
\hline Smile Face & $0.881 / 10.34$ & $0.491 / 5.807$ & $0.169 / 2.101$ & $0.094 / 1.162$ & $0.055 / 0.647$ \\
\hline Turtle Shell & $1.138 / 15.84$ & $0.624 / 8.024$ & $0.242 / 3.242$ & $0.126 / 1.638$ & $0.070 / 0.842$ \\
\hline
\end{tabular}

\subsection{Meshing for large-scale Geometry}

We evaluate the FPPM algorithm on big dataset obtained in practical modeling and scientific computing tasks. An example is a subsection of the Mississippi river as shown in Fig. 16(a). The levees and river boundary are treated as feature lines in the mesh generation. Using 50 cores, FPPM partitions this domain into 256 sub-regions in 113 seconds, and generates the quad mesh in 439 seconds. The final mesh contains $17,382,945$ elements with a minimum scaled Jacobian of 0.34. We can see the color-encoded result of the Scaled Jacobian in Fig. 16(b). In Fig. 16(c), a portion of the meshing result is zoomed in for closer visualization: the features are preserved very well.

\subsection{Simulation Experiments}

We solve partial differential equations on different meshes to compare these meshes' effectiveness in sci-

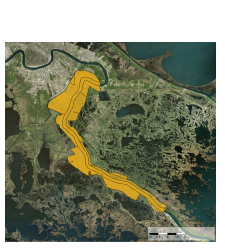

(a)

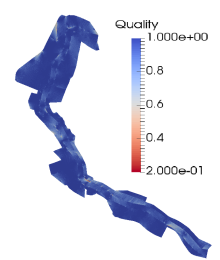

(b)

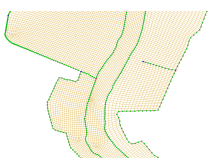

(c)
Figure 16: Quad meshing of the Mississippi river subsection and its levees. (a) The river subsection on Google Earth, (b) Color-encoded Scaled Jacobian of the mesh, and $(c)$ a zoom-in meshing result.

entific computing. We compare the simulation efficiency using the convergence of Error Residual on three types of meshes: (1) triangle meshes, (2) unstructured quad meshes generated by advancing front, (3) semi-structured quad meshes generated by our FPPM.

\subsubsection{Heat Diffusion Simulations}

We solve a heat diffusion (the Laplacian Equation) $\frac{\partial T}{\partial t}+\nabla\left(D_{t} \nabla T\right)=0$ where $T$ is the temperature, and coefficient $D_{t}$ is the thermal conductivity, on a mechanical part model as shown in Fig. 17(a): the red circle curve is the feature contour to be preserved in the final mesh. Initially, the outer boundary temperature is set as $273 K$ (Kelvin), the interior area is set as $0 K$. The temperature on the boundary and the center feature circle is fixed to $273 K$ and $0 K$ respectively. Fig. 17 shows the iteration-residual plot from the simulation. From the result statistics shown in Table. 3, we find that (1) The error residuals from the simulations performed on the quad meshes are one to two orders of magnitude smaller than that on the triangle meshes. (2) between the two quad meshes examined in this paper, our PPM algorithm produces significantly smaller error residuals in simulations.

Table 3: Simulation on mechanical part with the Delaunay triangle meshes, quad meshes generated by advancing front, and by our PPM algorithm.

\begin{tabular}{|c|c|c|c|c|c|}
\hline & \# Cells & $N_{s}$ & $\eta_{w}$ & $\epsilon(t=1)$ & $\epsilon(t=10)$ \\
\hline Triangle Mesh & $2.1 \mathrm{k}$ & - & - & $5.4 \times 10^{-4}$ & $1.3 \times 10^{-4}$ \\
\hline Advancing Front & $1.4 \mathrm{k}$ & 122 & 0.63 & $3.4 \times 10^{-4}$ & $0.5 \times 10^{-4}$ \\
\hline PPM & $1.9 \mathrm{k}$ & 12 & 0.66 & $1.9 \times 10^{-4}$ & $0.5 \times 10^{-4}$ \\
\hline
\end{tabular}

\subsubsection{Coastal Flow Simulations}

One application of this meshing technique is to generate high-resolution best-fitting meshes in riverine, coastal, and estuarine areas for hydrodynamic simulations. To improve model accuracy and avoid numerical errors, a qualified mesh needs to fit some special 


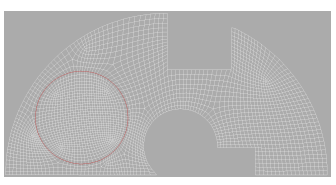

(a)

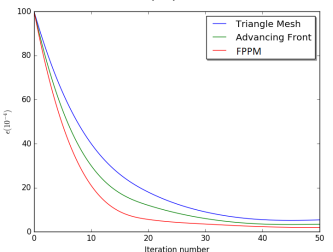

(c) $\epsilon(t=1)$

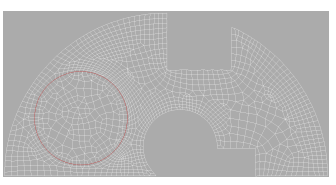

(b)

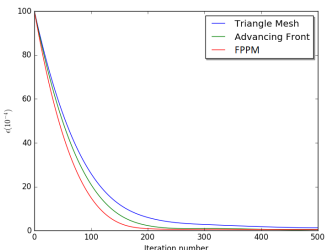

(d) $\epsilon(t=10)$
Figure 17: The iteration-residual plot of solving a Laplacian on three meshes generated from a Mechanical part model, at different $t$. (a) and (b) show show the meshing results from FPPM and the advancing front, respectively. (c) and (d) show the Iteration-residual at $t=1$ (c) and $t=10(\mathrm{~d})$.

features for the area of interest, such as coastlines, local bathymetries, hydraulic or marine structures. The normal structured (curvilinear) meshes are not suitable to fit sophisticated features in these areas, such as coastlines, river banks, small lakes, canals, levees, and breakwaters. There is a need to generate highquality feature-preserved meshes for effective coastal simulations.

We set up an idealized case to demonstrate the advantage of this meshing technique. The process-based numerical model package, the Delft3D model by Deltares (http://www.deltares.nl/en/), is utilized for this hydrodynamic simulation. The Delft3D model suite by Deltares [35], which has been widely used in the world, is capable of simulating flows, sediment transport, waves, water quality, morphological changes and ecological processes in coastal, riverine and estuarine areas [50].

In our experiment, there is a flow channel $(80 \times 200 \times 5$ $\mathrm{m})$ with double thin dams in it. The direction between the dam and the channel is 10 degrees. Two meshes with same sizes $(80 \times 200)$ are generated for comparison. One is a quad mesh that does not consider the feature of dams in Fig. 18(a). The other our generated quad mesh that fits double thin dams in Fig. 18(b). In our mesh, the double dams are sampled exactly by two straight lines in Fig. 18(d) and (f); while in the uniform mesh, the dams can only be represented approximately by two "zigzagged" lines in Fig. 18(c) and (e). Constant current conditions $(1 \mathrm{~m} / \mathrm{s})$ are applied at both ends of the flow channel. The result of flow velocity using our generated mesh in Fig. 18(d, f) which has the zoom-in details in Fig. 18(h) shows a smoothing distribution between double thin dams, which is reasonable. On the other hand, the result using the uniform mesh in Fig. 18(c, e) which has the zoomin details in Fig. 18(g) shows numerical errors at the corners of "zigzagged" lines, which is unreal. We can see some "velocity arrows" are blocked because of the "zigzagged" lines and their directions are distorted. They are fake results which will not occur in the real world. These indicate the advantage of our meshing technique for hydrodynamic simulations. We can see the results in Fig. 18.

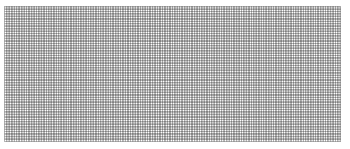

(a)

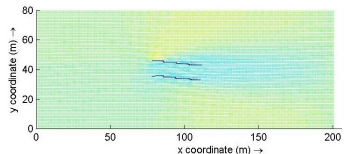

(c)

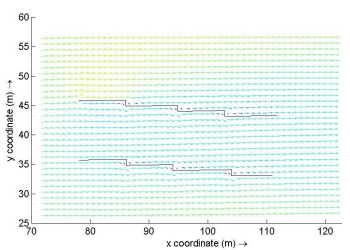

(e)

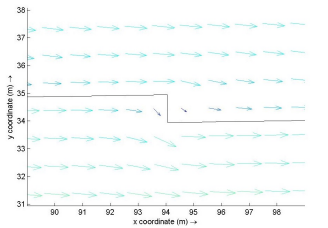

(g)

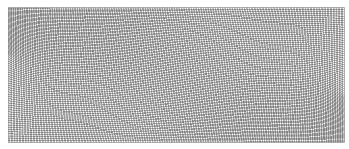

(b)

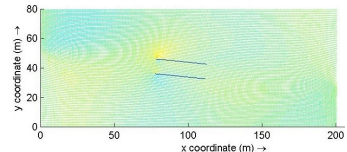

(d)

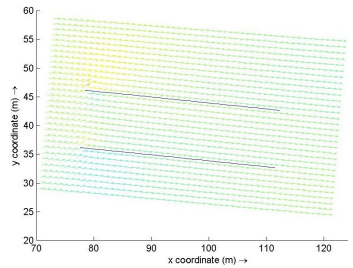

(f)

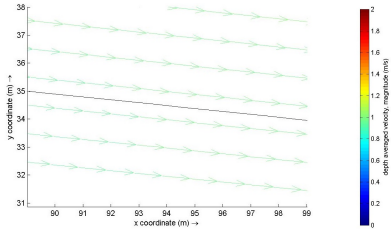

(h)
Figure 18: A flow simulation on a rectangle channel with double thin dams (features) in it. (a) The generated mesh without feature-preserving; (b) The featurepreserved mesh by considering the dam as the feature lines; (c, d) The simulated flow velocity fields using (a) and (b), respectively; (e, f) The zoom-in flow velocity fields around the dams from (c) and (d), respectively; $(g, h)$ The more close zoom-in flow velocity filed for a small region from (c) and (d), respectively.

\section{CONCLUSIONS}

We present a new distributed computational framework to generate semi-structured quad meshes on large-scale 2D regions in parallel. This framework supports feature-preservation and interior singularity insertion. As a result, it can produce high-quality, low distortion meshes. We demonstrate the effectiveness of this meshing algorithm and its application to largescale scientific simulations. 
Limitations. One limitation of our generated semistructured quad meshes is that the singularity placement and structured block layout are not globally optimized. Unnecessary singularities may be introduced during data partitioning. However, this cannot be easily solved due to the distributed nature of this algorithm, where each local processor is not aware of the global layout and distant subregions' geometry. In this work, such a limitation is partly remedied through singularity refinement. In the near future, we will explore distributed block layout optimization and singularity placement.

\section{ACKNOWLEDGMENTS}

This work is supported by National Science Foundation IIS-1320959 and CyberSEES-1539567. Part of this work was finished when the first three authors were in Louisiana State University. We thank IMR reviewers for their valuable suggestions.

\section{References}

[1] Benzley S.E., Perry E., Merkley K., Clark B., Sjaardema G. "A comparison of all hexagonal and all tetrahedral finite element meshes for elastic and elasto-plastic analysis." Proc. 4th Intl. Meshing Roundtable, pp. 179-191. 1995

[2] Linardakis L., Chrisochoides N. "Delaunay decoupling method for parallel guaranteed quality planar mesh refinement." SIAM JSC, vol. 27, no. $4,1394-1423,2006$

[3] Löhner R. "A 2nd generation parallel advancing front grid generator." Proc. 21st IMR, pp. 457474. Springer, 2013

[4] Panitanarak T., Shontz S.M. "Mdec: Metis-based domain decomposition for parallel $2 \mathrm{~d}$ mesh generation." $P C S$, vol. 4, 302-311, 2011

[5] Liu C., Yu W., Chen Z., Li X. "Distributed polysquare mapping for large-scale semi-structured quad mesh generation." Computer-Aided Design, vol. 90, 5-17, 2017

[6] Lo S. "Generating quadrilateral elements on plane and over curved surfaces." Comp. \& Structures, vol. 31, no. 3, 421-426, 1989

[7] Lee C.K., Lo S. "A new scheme for the generation of a graded quadrilateral mesh." Computers 83 structures, vol. 52, no. 5, 847-857, 1994

[8] Potyondy D.O., Wawrzynek P.A., Ingraffea A.R. "An algorithm to generate quadrilateral or triangular element surface meshes in arbitrary domains with applications to crack propagation."
I. J. Numerical Methods in Engineering, vol. 38, no. 16, 2677-2701, 1995

[9] Johnston B.P., Sullivan J.M., Kwasnik A. "Automatic conversion of triangular finite element meshes to quadrilateral elements." IJNME, 1991

[10] Blacker T.D., Stephenson M.B. "Paving: A new approach to automated quadrilateral mesh generation." I. J. NME, vol. 32, no. 4, 811-847, 1991

[11] Owen S.J., Staten M.L., Canann S.A., Saigal S. "Q-Morph: an indirect approach to advancing front quad meshing." I. J. Numerical Methods in Engineering, vol. 44, no. 9, 1317-1340, 1999

[12] Owen S.J., Saigal S. "H-Morph: an indirect approach to advancing front hex meshing." IJNME, vol. 49, no. 1-2, 289-312, 2000

[13] Remacle J.F., Henrotte F., Carrier-Baudouin T., Béchet E., Marchandise E., Geuzaine C., Mouton T. "A frontal Delaunay quad mesh generator using the $L_{\infty}$ norm." International Journal for Numerical Methods in Engineering, vol. 94, no. 5, 494-512, 2013

[14] Kaelberer F., Nieser M., Polthier K. "QuadCover - Surface Parameterization using Branched Coverings." CGF, vol. 26, 375-384, 2007

[15] Bommes D., Zimmer H., Kobbelt L. "Mixedinteger Quadrangulation." ACM Trans. Graph., vol. 28 , no. $3,77: 1-77: 10,2009$

[16] Bommes D., Campen M., Ebke H.C., Alliez P., Kobbelt L. "Integer-grid maps for reliable quad meshing." TOG, vol. 32, no. 4, 98, 2013

[17] Li Y., Liu Y., Xu W., Wang W., Guo B. "All-hex meshing using singularity-restricted field." $A C M$ TOG, vol. 31, no. $6,177,2012$

[18] Pellenard B., Orbay G., Chen J., Sohan S., Kwok W., Tristano J.R. "QMCF: QMorph Cross Field-Driven Quad-Dominant Meshing Algorithm." Procedia Engineering, vol. 82, 338-350, 2014

[19] Campen M., Kobbelt L. "Quad layout embedding via aligned parameterization." $C G F$, vol. 33, pp. 69-81. Wiley Online Library, 2014

[20] LYON M., CAMPEN M., BOMMES D., KOBBELT L. "Parametrization Quantization with Free Boundaries for Trimmed Quad Meshing." 2019

[21] Wang H., He Y., Li X., Gu X., Qin H. "Polycube splines." Computer-Aided Design, vol. 40, no. 6, 721-733, 2008 
[22] Lin J., Jin X., Fan Z., Wang C.C. "Automatic polycube-maps." International Conference on Geometric Modeling and Processing, pp. 3-16. Springer, 2008

[23] Gregson J., Sheffer A., Zhang E. "All-Hex Mesh Generation via Volumetric PolyCube Deformation." CGF, vol. 30, pp. 1407-1416. 2011

[24] Yu W., Zhang K., Wan S., Li X. "Optimizing Polycube Domain Construction for Hexahedral Remeshing." $C A D$, vol. 46, 58-68, 2014

[25] Hu K., Zhang Y.J. "Centroidal Voronoi tessellation based polycube construction for adaptive allhexahedral mesh generation." Computer Methods in Applied Mechanics and Engineering, vol. 305, 405-421, 2016

[26] Fang X., Xu W., Bao H., Huang J. "All-hex meshing using closed-form induced polycube." TOG, vol. 35 , no. $4,124,2016$

[27] Fogg H.J., Armstrong C.G., Robinson T.T. "Enhanced medial-axis-based block-structured meshing in 2-D." $C A D$, vol. 72, 87-101, 2016

[28] Rigby D. "Topmaker: A technique for automatic multi-block topology generation using the medial axis." ASME/JSME 2003 4th Joint Fluids Summer Engineering Conference, pp. 1991-1997. American Society of Mechanical Engineers, 2003

[29] Lévy B., Liu Y. " $L_{p}$ Centroidal Voronoi Tessellation and its applications." ACM Transactions on Graphics (TOG), vol. 29, p. 119. ACM, 2010

[30] Dong S., Bremer P.T., Garland M., Pascucci V., Hart J.C. "Spectral Surface Quadrangulation." ACM Trans. Graph., vol. 25, no. 3, 1057-1066, 2006

[31] Zhang M., Huang J., Liu X., Bao H. "A Wavebased Anisotropic Quadrangulation Method." TOG, vol. 29, no. 4, 118:1-118:8, 2010

[32] Myles A., Pietroni N., Kovacs D., Zorin D. "Feature-aligned T-meshes." ACM Transactions on Graphics (TOG), vol. 29, p. 117. ACM, 2010

[33] Jakob W., Tarini M., Panozzo D., SorkineHornung O. "Instant field-aligned meshes." TOG, vol. 34 , no. $6,189,2015$

[34] Liu L., Zhang Y., Liu Y., Wang W. "Featurepreserving T-mesh construction using skeletonbased polycubes." Computer-Aided Design, vol. $58,162-172,2015$

[35] Hydraulics D. "Delft3D-FLOW user manual." Delft, the Netherlands, 2006
[36] Tarini M., Puppo E., Panozzo D., Pietroni N., Cignoni P. "Simple quad domains for field aligned mesh parametrization." TOG, vol. 30 , no. 6,142 , 2011

[37] Myles A., Pietroni N., Zorin D. "Robust fieldaligned global parametrization." ACM Transactions on Graphics (TOG), vol. 33, no. 4, 135, 2014

[38] Yu W., Li X. "A Geometry-aware Data Partitioning Algorithm for Parallel Quad Mesh Generation on Large-scale 2D Regions." Proc. 24th IMR, vol. $124,44-56,2015$

[39] LaSalle D., Karypis G. "A parallel hill-climbing refinement algorithm for graph partitioning." Parallel Processing (ICPP), 2016 45th International Conference on, pp. 236-241. IEEE, 2016

[40] Roberts G.O., Rosenthal J.S., et al. "Optimal scaling for various Metropolis-Hastings algorithms." Statistical science, vol. 16, no. 4, 351367, 2001

[41] Huang J., Jiang T., Shi Z., Tong Y., Bao H., Desbrun M. " $l_{1}$-Based Construction of Polycube Maps from Complex Shapes." TOG, vol. 33, no. $3,25,2014$

[42] Hormann K., Greiner G. "MIPS: An efficient global parametrization method." Curve and Surface Design, pp. 153-162. 2000

[43] Fu X.M., Liu Y., Guo B. "Computing locally injective mappings by advanced MIPS." TOG, vol. 34 , no. $4,71,2015$

[44] Liu Y. "HLBFGS." 2009

[45] Fu X.M., Bai C.Y., Liu Y. "Efficient Volumetric PolyCube-Map Construction." CGF, vol. 35, pp. 97-106. Wiley Online Library, 2016

[46] Allwright J., Bordawekar R., Coddington P., Dincer K., Martin C. "A comparison of parallel graph coloring algorithms." Technical report, Northeast Parallel Architectures Center at Syracuse University, 1995

[47] Verma C.S., Suresh K. " $\alpha$ MST: A Robust Unified Algorithm for Quadrilateral Mesh Adaptation." Procedia Engineering, vol. 163, 238-250, 2016

[48] Autodesk-Inc. "Autodesk 3ds Max 2016." 2015

[49] Ruiz-Gironés E., Roca X., Sarrate J. "Optimizing mesh distortion by hierarchical iteration relocation of the nodes on the CAD entities." Procedia Engineering, vol. 82, 101-113, 2014 
[50] Hu K., Ding P., Wang Z., Yang S. "A 2D/3D hydrodynamic and sediment transport model for the Yangtze Estuary, China." Journal of Marine Systems, vol. 77, no. 1-2, 114-136, 2009 


\title{
ACCELERATING THE EXACT EVALUATION OF GEOMETRIC PREDICATES WITH GPUS
}

\author{
Marcelo de Matos Menezes ${ }^{1} \quad$ Salles Viana Gomes de Magalhães ${ }^{2}$ \\ Matheus Aguilar de Oliveira ${ }^{3} \quad$ W. Randolph Franklin ${ }^{4}$ \\ Rodrigo Eduardo de Oliveira Bauer Chichorro ${ }^{5}$ \\ ${ }^{1}$ Universidade Federal de Viçosa (MG) Brasil, marcelo.menezes@ufv.br \\ ${ }^{2}$ Universidade Federal de Viçosa (MG) Brasil, salles@ufv.br \\ ${ }^{3}$ Universidade Federal de Viçosa (MG) Brasil, matheus.a.aguilar@ufv.br \\ ${ }^{4}$ Rensselaer Polytechnic Institute, Troy NY, USA, mail@wrfranklin.org \\ ${ }^{5}$ Universidade Federal de Viçosa (MG) Brasil, rodrigo.chichorro@ufv.br
}

\begin{abstract}
This paper presents a technique for employing high-performance computing for accelerating the exact evaluation of geometric predicates. Arithmetic filters are implemented using interval arithmetic to reduce the necessity of exact arithmetic while ensuring the results of the predicates are still exact. Furthermore, the computation with interval arithmetic is offloaded to a CUDA-enabled GPU. If the GPU detects that some results cannot be trusted, the corresponding predicates are re-evaluated in parallel on the CPU using arbitrary-precision rational numbers. As a case study, a red-blue segment intersection algorithm has been implemented. Since the intervals are implemented using floating-point numbers, the parallel computing power of GPUs for processing these numbers led to a speedup of up to 289 times (when compared against a similar sequential implementation) in the evaluation of these predicates (and up to 40 times if the entire runnning-time of the algorithm is considered). The excellent performance associated to the exactness makes this technique suitable for accelerating geometric operations in fields such as CAD, GIS and VLSI design.
\end{abstract}

Keywords: computational geometry, exact computation, high-performance computing, GPU, CUDA

\section{INTRODUCTION}

A particular challenge in computational geometry problems is to address the errors caused by floatingpoint arithmetic. Inexact floating-point numbers violate most of the axioms of an algebraic field. For example, addition is not associative. Roundoff errors cause topological errors, such as causing an orientation predicate to report a point to be on the wrong side of a line segment. These errors may propagate to higherlevel operations (such as using orientation predicates to compute a convex hull), what makes the design of correct algorithms even harder.

While there are heuristics (such as epsilon-tweaking and snap rounding) that try to solve this, they are not guaranteed to always work.

A technique to guarantee computation will be free from round-off errors is representing the coordinates with exact arbitrary-precision rational numbers. The drawback is that in some applications the overhead associated to these numbers may be unacceptable. Also, the number of digits in the numerator and denominator of these numbers grow as arithmetic operations are performed (the size is typically the sum of the number of digits in the operands) and, thus, performance may degrade if the computation tree is deep.

Some techniques have been proposed to cope with this 
performance problem. Namely, arithmetic filters using interval arithmetic represent each exact number $e$ as an interval of floating-point values containing $e$. Thanks to guarantees of the IEEE-754 floating-point standard, for each arithmetic operation a new interval (which is guaranteed to contain the exact result of that operation) can be computed. Thus, predicates can be initially evaluated using intervals. If it is detected that the exact result of that predicate can be inferred from the bounds of the interval, this result is computed. Otherwise, the expression is re-evaluated using exact arithmetic (or intervals with more precise number types). As mentioned in [1], most of the time computation with intervals is enough to infer the exact result and, thus, predicates can be efficiently and exactly evaluated without the overhead of exact computation.

While recently the computing capabilities of desktop computers and workstations have increased due to multi-core processors and accelerators such as GPGPUs (General Purpose Graphics Processing Unit) and MICs (Many Integrated Core Architecture), many algorithms are still designed considering sequential architectures and, thus, they cannot take advantage of this computing power.

In this paper, we propose the use of a combination of GPUs and multi-core CPUs to accelerate the evaluation of exact predicates using arithmetic filters. During the parallel evaluation of predicates, the operations with intervals are offloaded to a GPU. Then, the (few) unreliable results are filtered and re-evaluated in parallel on the CPU using multiple-precision rationals. As a result, both high-efficiency and exactness are achieved.

To obtain performance, the algorithms being accelerated should be adapted so that the geometric predicates are evaluated in batch. For example, consider the problem of computing the intersection of two triangulated meshes. One critical step consists in, given a set of pairs of potentially intersecting triangles, determine which ones do intersect. Since the intersection of two triangles can be computed using orientation predicates, this algorithm could create a list of these predicates and offload their evaluation to the GPU in batch.

The performance and correctness make this technique suitable, for example, for processing large datasets (where the chance of failure in inexact algorithms is higher) in interactive applications such as GIS and CAD systems.

As a case study, we have developed a fast and exact algorithm for detecting red-blue intersections between two sets of edges in $2 D$. We intend to also apply these techniques to accelerate the solution of other important problems such as performing boolean operations on polygonal maps or polyhedral meshes.

\section{BACKGROUND}

\subsection{Roundoff errors}

Non-integer numbers are typically approximately represented in computers with floating-point values. The difference between the value of a non-integer number and its approximation is often referred as roundoff error. Even though these differences are usually small, these errors accumulate as sequences of arithmetic operations are performed. The presence of floating point errors in computer programs often creates serious consequences in diverse fields such as the failure of the first Ariane V rocket [2] and the failure of the Patriot missile defense system [3].

In geometry, roundoff errors can generate topological inconsistencies causing globally impossible results. For example, if the point of intersection of two lines segments is computed, the result may not lie in any of the two lines. Kettner et al. [4] presented some examples of failures caused by roundoff errors in computational geometry problems. In this study, they presented examples of how the evaluation of orientation predicates can be affected by floating-point errors. As a result, algorithms (such as one for computing convex hulls) relying on these predicates may fail.

The planar orientation predicate is the problem of finding whether three points $p=\left(p_{x}, p_{y}\right), q=\left(q_{x}, q_{y}\right)$, $r=\left(r_{x}, r_{y}\right)$ are collinear, make a left turn, or make a right turn. This predicate is computed by evaluating the sign of the following determinant:

$$
\left|\begin{array}{lll}
p_{x} & p_{y} & 1 \\
q_{x} & q_{y} & 1 \\
r_{x} & r_{y} & 1
\end{array}\right|
$$

Positive, negative and zero signs mean that $(p, q, r)$, respectively, make a left turn, right turn or are collinear. Roundoff errors may make the sign of this determinant to be evaluated wrongly, mis-classifying the orientation. To illustrate this problem, Kettner et al. [4] implemented a program to apply the planar orientation predicate (orientation $(p, q, r)$ ) on a point $p=\left(p_{x}+x u, p_{y}+y u\right)$ where $u$ is the step between adjacent floating point numbers in the range of $p$ and $0 \leq x, y \leq 255$. This results in a $256 \times 256$ matrix containing either blue, yellow and red points meaning that the corresponding point is detected to be above, on or below the line that passes through $q$ and $r$. Figure 1 shows the geometry of this experiment for $p=(0.5,0.5), u=2^{-53}, q=(12,12)$ and $r=(24,24)$. As it can be seen, several points have their orientation computed incorrectly. 


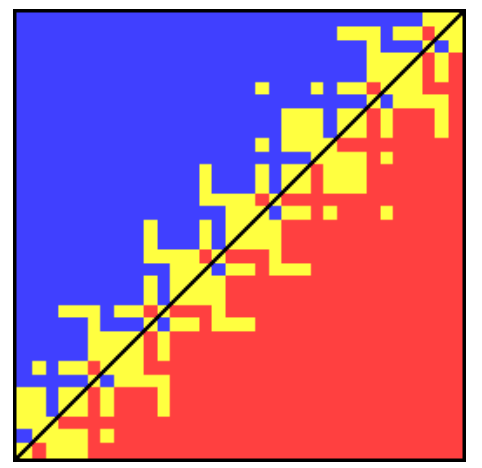

Figure 1: Roundoff errors in the planar orientation problem - Geometry of the planar orientation predicate for double precision floating point arithmetic. Yellow, red and blue points represent, respectively, collinear, negative and positive orientations. The diagonal line is an approximation of the segment $(q, r)$. Source: [4].

As shown by [4], these inconsistent results in the orientation predicates could make algorithms that use this predicate to fail.

Some techniques have been proposed to handle this problem. The simplest one, the epsilon-tweaking, consists of using an $\epsilon$ tolerance that considers two values $x$ and $y$ are equal if $|x-y| \leq \epsilon$. However this is a formal mess because equality is no longer transitive, nor invariant under scaling. Thus, in practice, epsilontweaking fails in several situations [4].

Snap rounding is another method to approximate arbitrary precision segments into fixed-precision numbers [5]. However, Snap rounding can generate inconsistencies and deform the original topology if applied consecutively on a data set. Some variations of this technique attempt to get around these issues $[6,7,8]$.

Shewchuk [9] presents the Adaptive Precision Floating-Point technique for exactly evaluating predicates. The idea is to perform this evaluation using the minimum amount of precision necessary to achieve correctness. As a result, it is possible to develop some efficient exact geometric algorithms. Geometric predicates can often be evaluated by computing the sign of a determinant and, thus, the actual value of this determinant does not need to be exactly computed as long as the sign of the approximated result is guaranteed to be correct. To determine if the sign of an approximation can be trusted, the approximation and an error estimate are computed and, if the error is big enough to make the sign possibly incorrect, the values are recomputed using higher precision. As mentioned by Shewchuk [9], this technique is not suitable to solve all geometric problems. For example, "a program that computes line intersections requires rational arithmetic; an exact numerator and exact denomina- tor must be stored" [9].

The formally proper way to effectively eliminate roundoff errors and guarantee algorithm robustness is to use exact computation based on rational number with arbitrary precision [10, 11, 4, 12]. Computing in the algebraic field of the rational numbers over the integers, with the integers allowed to grow as long as necessary, allows the traditional arithmetic operations, ,,$+- \times, \div$, to be computed exactly, with no roundoff error.

The cost is that the number of digits in the result of an operation is about equal to the sum of the numbers of digits in the two inputs. E.g., $\frac{214}{433}+\frac{659}{781}=\frac{452481}{338173}$. Casting out common factors helps, but that is rarely possible. However, this behavior is acceptable if the depth of the computation tree is small. Also, the performance penalty associated with rationals can be significantly reduced by employing techniques such as arithmetic filtering with interval arithmetic, as we will discuss in section 2.2.

\subsection{Arithmetic filters and interval arith- metic}

One technique to accelerate algorithms based on exact arithmetic is to employ arithmetic filters and interval arithmetic [13]. The idea is to use an interval of floating-point numbers containing each exact value. During the evaluation of predicates (which typically consists in the computation of the sign of an arithmetic expression), the arithmetic operations are initially applied to the intervals. After each arithmetic operation the result (an interval) is adjusted to guarantee that it will still contain the exact result of the operation (this is called the containment property). At the end, if the sign of the exact result can be safely inferred based on the sign of the bounds of the interval, its value is returned. Otherwise, the predicate is re-evaluated using exact arithmetic instead of the floating-point intervals. The term arithmetic filter derives from the process of filtering the unreliable results and recomputing them with exact arithmetic.

The key to the correct and efficient implementation of operations with interval arithmetic is the fact that the IEEE-754 standard for floating-point numbers explicitly define how the arithmetic operations are approximated: "the result of operations can be seen as if they were performed exactly, but then rounded to one of the nearest floating-point values enclosing the exact value" [13]. IEEE-754 also defines three roundingmodes (that can be selected at runtime): the results of the operations can be rounded to the nearest representable floating-point value, towards $-\infty$ or $+\infty$ (which selects, respectively, the previous or the next nearest representable floating-point numbers). 
These rounding modes are employed to adjust the intervals after each arithmetic operation, which guarantees that they always contain the exact value of the expressions. [13] illustrates this process with the addition operation. Suppose $x$ Interval $=$ $[x$.lower, $x$.upper $]$ and yInterval $=[$ y.lower, $y$.upper $]$ are, respectively, floating-point intervals containing the exact values $x$ Exact and yExact. The floatingpoint interval $[x$.lower \pm y.lower, $x$.upper $\mp$ y.upper $]$ (where \pm and $\mp$ represent, respectively, rounding towards $-\infty$ or $+\infty$ ) is guaranteed to contain the exact value of the expression $x$ Exact $+y$ Exact.

Since the intervals are computed in a way that the containment property is always preserved, if both bounds have the same sign then this sign is equal to the exact sign of the expression. Otherwise, the interval cannot be employed to infer the exact sign and thus, the expression will have to be re-evaluated with exact arithmetic (we refer to this as an interval failure). For example, if $x$ Exact is in the interval $[0.01,0.03]$, then $x$ Exact is certainly a positive number. However, if $x$ Exact is in the interval $[-0.0001,0.0001]$, then the sign of $x$ Exact can be either negative, zero or positive.

Since the roundoff errors accumulate, the width of the intervals increases as arithmetic operations are performed and thus, the deeper the computation tree is, the higher are the chances that computation with exact arithmetic will be necessary, which could slow down the algorithms. However, many practical algorithms do not present this problem [13].

While arithmetic filters can accelerate predicates, in some situations the exact computation cannot be avoided. For example, exact arithmetic would be necessary in operations where new geometric objects (e.g.: points) have to be computed (these types of operations are called geometric constructions). To illustrate this example, consider the problem of computing pairwise intersections of line segments: arithmetic filters could be employed to accelerate the orientation predicates employed to detect if two line segments do intersect, but exact arithmetic is necessary in order to output the (exact) coordinates of the vertices generated by the intersection of pairs of edges.

The excellent Computational Geometry Algorithms Library (CGAL) [14] supports exact computation through the use of arbitrary precision rational numbers (it also supports other number types) and arithmetic filters in its algorithms. Furthermore, this library provides a framework that allows programmers to easily develop algorithms with arithmetic filters.

There are multiple types of arithmetic filters [13]. Listing 1 illustrates one of the ways to develop an arithmetic filter using $\mathrm{C}++$ and CGAL: variables with the suffix _exact were created as GMP[15] (GNU Multiple Precision Arithmetic Library) arbitrary precision rationals (which are represented using the mpq_class type) while the ones with suffix _interval were defined using the interval arithmetic number type provided by CGAL. Arithmetic and boolean operators are overloaded for both the interval and arbitrary precision arithmetic types. If the comparison (line 8) cannot be evaluated safely, CGAL throws an unsafe_comparison exception. Once that exception is caught, the predicate can be re-evaluated using the exact version of the respective variables (line 14 ).

Listing 1: Using CGAL interval arithmetic framework

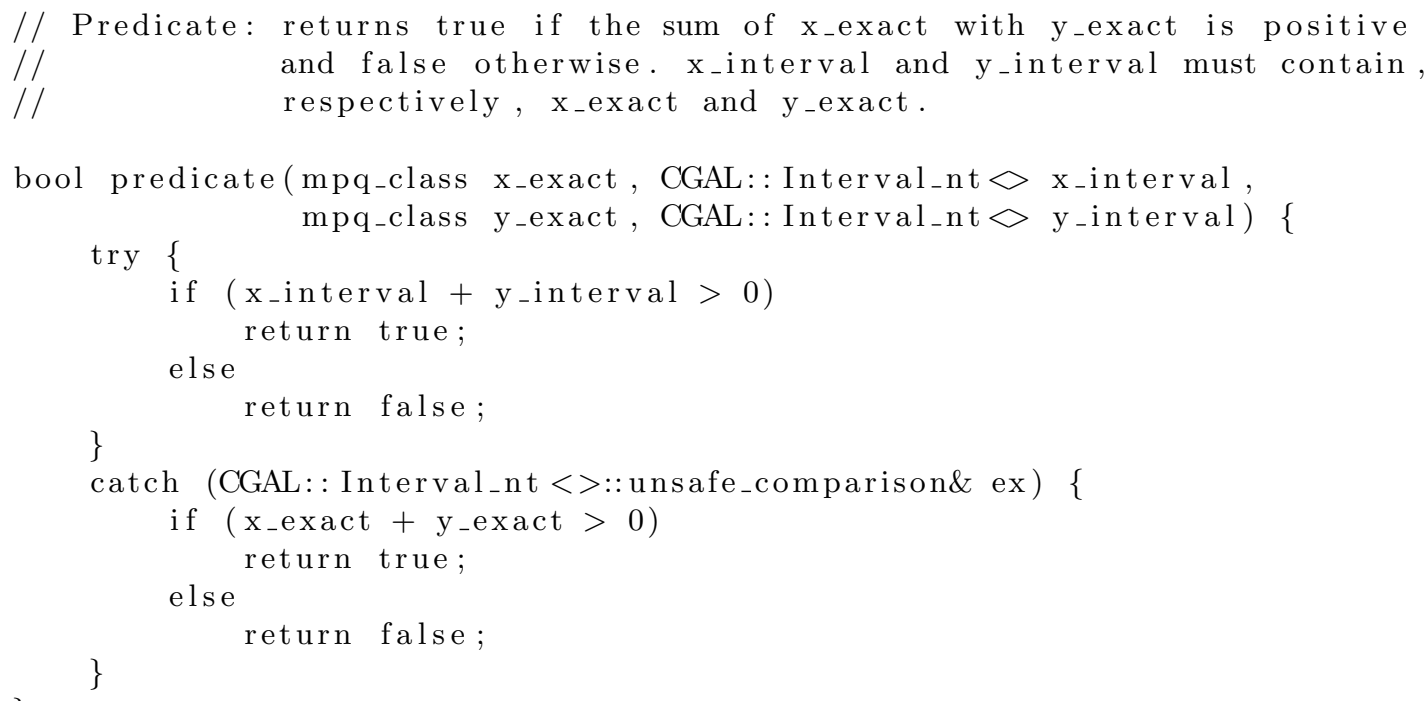


A challenge happens when a sequence of operations needs to be performed: in this situation, we may not know the exact value of the operands (since they were generated by several operations). CGAL provides a more generic and reusable type of filter that solves this by using a DAG (directed acyclic graph) to represent the history of operations employed to generate each geometric object.

This kind of filter is transparent to the user (not requiring an explicit try... catch block similar to the one shown above). For example, if the test if $(a+2 * b+c<$ $0)$ is performed, then intervals will be employed to try to evaluate the test without the necessity of computing with the rationals. Assume temp $=a+2 * b+c$ is the temporary value computed during the evaluation of if $(a+2 * b+c<0)$. If the sign of temp cannot be safely evaluated, its precision is increased (for example, by recomputing its value using rationals). This can be performed because the DAG associated to temp represents the history of operations that originated that value. I.e., temp knows it was computed by multiplying $b$ by 2 and adding the result to $a$ and $c$. This exact re-evaluation is lazily delayed until it is really needed ("as hopefully it won't be needed at all" [13]).

While these filters have some advantages (for example, they are efficient and can be easily and transparently used by developers), they also have some drawbacks. For example, the history DAG has a significantly high memory consumption, is hard to be maintained and is not thread-safe. Thus, even operations that do not modify the geometric objects (for example, "readonly" operations such as orientation predicates) often cannot be executed in parallel [16].

\subsection{High-performance computing and CUDA}

The advent of powerful multi-core CPUs and General Purpose GPUs (GPGPUs) with thousand of cores has increased the computing capability of relatively inexpensive computers. For example, currently (2019) a NVIDIA GeForce $1080 \mathrm{Ti}$ (a GPU with 3584 cores) can be purchased for $\$ 800$ USD and provide $11 \mathrm{Tflop} / \mathrm{s}$ of peak floating-point performance. Thus, it is important to design parallel algorithms able to use this computing power.

High-performance computing has been employed to accelerate some geometric algorithms. For example, Geometric Performance Primitives (GPP), the commercial product described in [17], performs (nonexact) map overlays using GPUs.

Zhou et al. [16] and Magalhães et al. [18] have developed parallel (for shared-memory multi-core CPUs) and exact algorithms for performing boolean operations on 3D meshes. Zhou et al. [16] uses CGAL routines (for example, to detect triangle-triangle intersections, to evaluate point-plane predicates, to perform Delaunay triangulations, etc) with an exact kernel with a lazy number type. Since these operations are not thread-safe, the authors have employed mutex locks to ensure correctness. Magalhães et al. [18], on the other hand, achieved thread-safeness by explicitly managing the exact arithmetic operations. For example, they implemented their own orientation predicates (using CGAL's interval arithmetic number type) and explicitly re-evaluated these predicates when the intervals were not reliable enough to ensure exactness (thus, CGALs' lazy evaluation using the history DAG was not employed in this algorithm).

While there have been exact and parallel algorithms for processing geometric data, porting these algorithms to GPUs is still a challenge, particularly when exact arithmetic operations with arbitrary-precision rationals is required. The algorithms employed in arbitrary-precision arithmetic "are not easily portable to highly parallel architectures, such as GPUs or Xeon Phi" [19]. One of the reasons for this is the typically non-trivial memory management required by this kind of computation [20].

Thus, libraries for performing higher-precision arithmetic on GPUs (such as CAMPARI [20] and GARPREC [21]) are typically designed to process extended-precision floating-point numbers.

However, thanks to arithmetic filters, floating-point operations can significantly reduce the frequency that rationals are required [1]. In this work, we combine the parallel computing capability of CPUs with GPUs for exactly performing geometric operations. The exact representation of the geometric objects is kept on the CPU, while approximate intervals (represented with floating-point numbers) are stored on the GPU. The combinatorial component of the geometric algorithms is executed on the CPU and the parallel evaluation of geometric predicates is offloaded to the GPU, which returns the exact result of each one or a flag indicating that a given predicate could not be safely evaluated with the intervals. The CPU, then, re-evaluates (also in parallel) these predicates that failed on the GPU.

While there has been research $[22,23]$ on the field of implementing interval arithmetic on GPUs, these works have focused on computer graphics applications (like ray tracing) and have not employed this technique to accelerate exact geometric computation using arithmetic filters.

\section{IMPLEMENTING EXACT PARALLEL PREDICATES}

As stated in section 2.2, a correct implementation of interval arithmetic relies on hardware compliance 
to the IEEE-754 standard. NVIDIA's GPUs double and single precision floating point implementations are in accordance with the standard since compute capabilities 1.3 and 2.0, respectively [24]. They adopt its newest version (IEEE-754:2008, as of June 2019), which allows the rounding criteria to be selected per machine instruction, completely removing the mode switching overhead [22].

In order to make interval arithmetic transparent during the evaluation of geometric predicates, we created a separate class, based on Collange et al. [22], to perform the calculations. Through operator overloading, the predicate code remains clean and concise, once the compiler intrinsics are hidden from the user.

For example, as mentioned by Collange et al. [22], the addition of two intervals $[a, b]$ and $[c, d]$ can be performed using the expression $[a, b]+[c, d]=$ $[a+c, \overline{b+d}]$ (where $\underline{a+c}$ and $\overline{b+d}$ indicate, respectively, the expression is rounded towards $-\infty$ and $+\infty)$. Listing 2 illustrates the implementation of the addition method, where the CUDA $\mathrm{C}$ functions _-_dadd_rd and _-_dadd_ru switches the double precision floating point rounding mode for additions to $-\infty$ and $+\infty$, respectively.

Listing 2: Some methods of our CudaInterval class

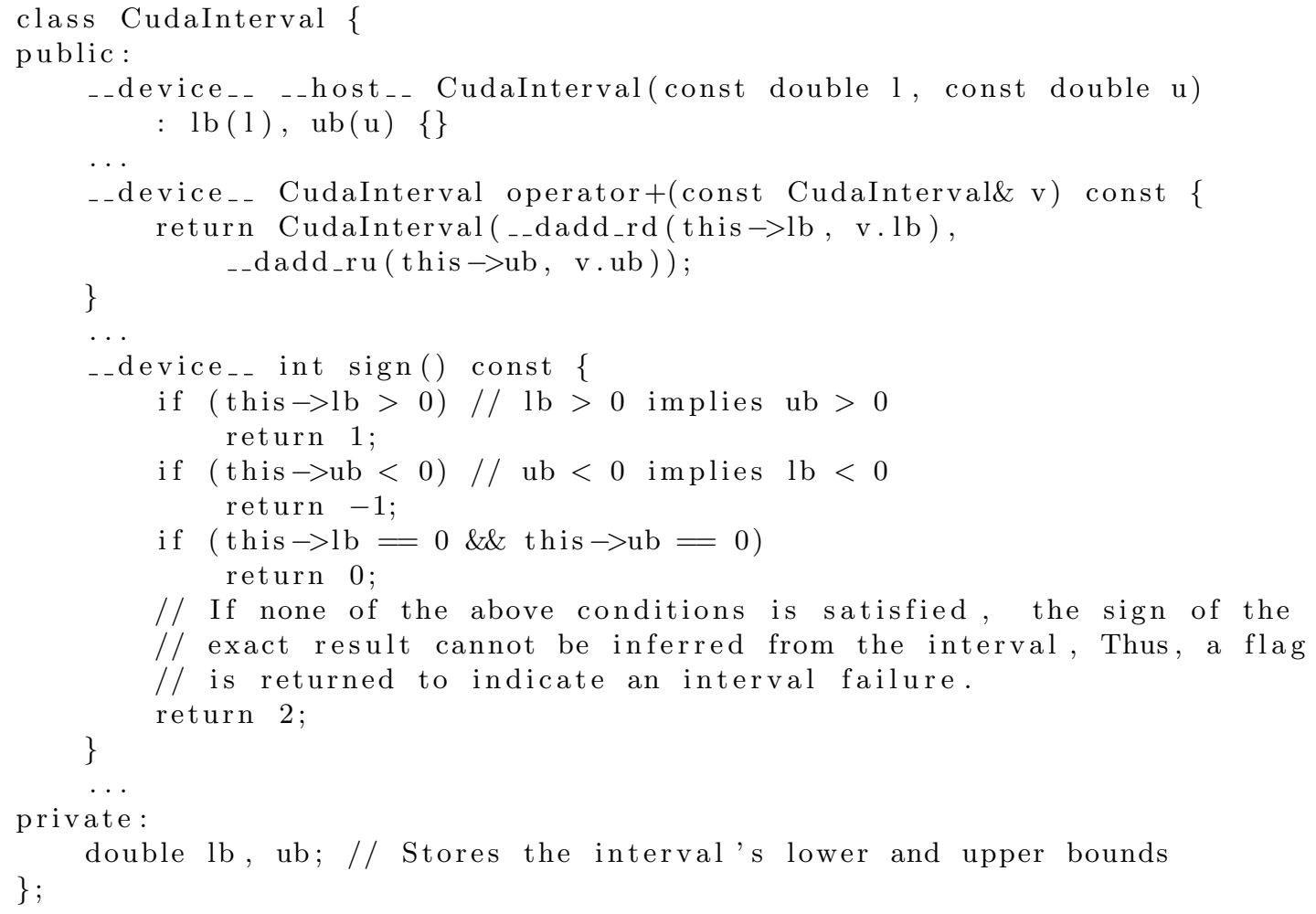

Besides the other arithmetic operators, whose implementations are similar to addition, our class has also the method sign, which returns 1,0 , or -1 if the interval's sign is guaranteed to be, respectively, positive, zero or negative. If the sign can't be inferred from the interval's bounds a special error flag is returned instead. The 2D orientation predicate, described in Section 2.2, can be easily implemented on the GPU side with interval arithmetic using our class, as shows listing 3. However, when an interval failure occurs during the sign evaluation, the responsibility to correctly handle the case is delegated to the CPU. Nonetheless, as shown by [1], and reinforced by our case study (sections 4 and 5) interval failures are rare and they usually do not affect the algorithms' overall performance.

Since GPUs are SIMT (Single Instruction, Multiple Threads) devices, its processing power can be explored by applying the same operation (for example, evaluating orientation predicates) on multiples triples of points in batch.

Even though this example is focused on 2D orientation predicates, it can be extended to other geometric operations using interval arithmetic. 
Listing 3: Orientation predicate on GPU

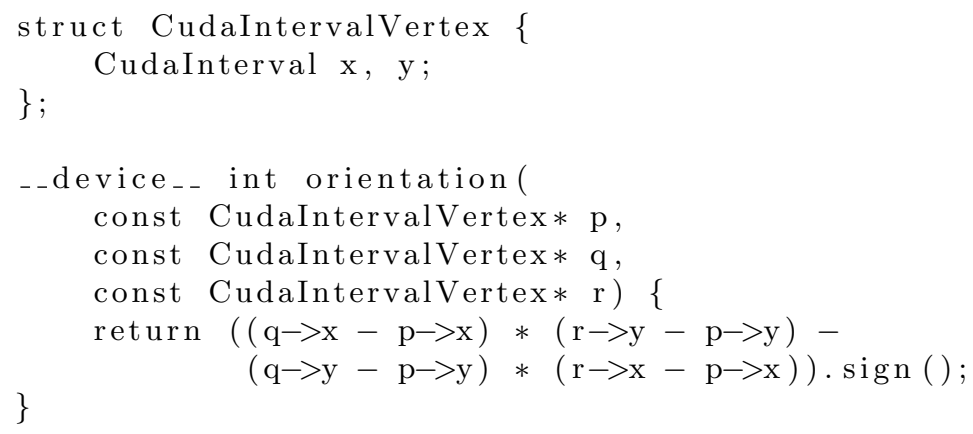

\section{FAST RED-BLUE INTERSECTION} TESTS

To evaluate the ideas presented in this paper, we have implemented a fast and exact algorithm for detecting red-blue intersection of line segments. Given two sets of segments $M_{1}$ and $M_{2}$ (assume the red and blue segments are from, respectively, $M_{1}$ and $M_{2}$ ), the objective is to find the pairs composed of red and blue segments that do intersect. This is performed by doing a pre-processing step with a uniform grid to cull pairs of segments that may intersect and, then, filtering the pairs that actually do intersect.

The uniform grid is typically employed in computational geometry to cull a combinatorial set of pairs of objects, generating a smaller subset containing elements that are more likely to coincide [25]. If the input is uniformly independently and identically distributed, the expected size of the resulting subset is linear on the size of the input plus the output [26, 27, 28]. Thanks to its simplicity and uniformity, it can be constructed and processed in parallel. For example, Audet et al. [17] employed a uniform grid on a GPU parallel algorithm for map overlay and Magalhães et al. [18] employed it to intersect 3D meshes in parallel.

Given the sets of segments $M_{1}$ and $M_{2}$, a grid $G$ with resolution $r$ (thus, containing $r \times r$ cells) and dimensions equal to the bounding-box containing both $M_{1}$ and $M_{2}$ is created. Then, for each segment $e$ from the two input sets, $e$ is inserted into the grid cells it intersects. The intersecting segments can be found by, for each grid cell $c$, testing all the pairs of red and blue segments from $c$ for intersection.

For performance and simplicity, as in Magalhães et al. [18], instead of rasterizing each segment $s$ in order to determine which cells $s$ intersects, the boundingbox $b$ of $s$ is computed and $s$ is considered to intersect all grid cells intersecting $b$. While this may increase the number of intersection tests that will have to be performed later, the correctness of the algorithm is maintained since the grid is employed only to find a set of edges that may intersect.

Similarly to Magalhães et al. [18], we have chosen to use a ragged array as the underlying data structure to implement the uniform grid. The ragged array stores a collection of arrays in a contiguous block of memory, by keeping track of each array's initial position. It can be easily constructed in parallel, with the cost of making two passes in the data to insert the edges, and has the advantage of being more cache friendly than storing one resizable array per cell, since it can represent the entire grid in contiguous memory [25]. Figure 2 illustrates these two data structures.

The creation of the ragged array storing in the grid the segments from each of the input sets $M_{i}(i=1$ or 2) is performed in two passes. First, the number of segments from $M_{i}$ in each cell is counted. Then, the array is allocated (with size equal to the sum of the number of edges in all cells) and the segments are scanned again and effectively inserted into the array.

In the first pass, the bounding-box of each segment $s$ in $M_{i}$ is initially computed on the GPU. This computation is performed in parallel and basically consists in determining the grid cells containing each of the two endpoints of $s$ (this is the only geometric operation performed during the construction of the uniform grid). Then, a counter cellSize[c] is created to compute the number of segments that will be inserted into each grid cell $c$ (we refer to this as the size of the cells). Finally, each segment $s$ is scanned (in parallel) and the counter of the cells the bounding-box of $s$ intersects is incremented (using atomic operations).

After the cell sizes are computed, a parallel exclusive prefix-sum operation is applied to the cellSize array. Assume cellStart is the content of cellSize after the prefix-sum. Thus, cellStart $[0]=0$, cellStart $[1]=$ cellStart $[0]+$ cellSize $[0]$ and, in general, cellStart $[c]=$ cellStart $[c-1]+$ cellSize $[c-1]$. Therefore, cellStart[c] represents the starting position of the edges of cell $c$ in the ragged array.

In the second pass, each segment $s$ in $M_{i}$ is pro- 
cessed again in parallel. For each cell $c$ intersecting the bounding-box of $s, s$ is inserted into the position cellStart $[c]+$ count $[c]$ of the ragged array, where count $[c]$ is a counter for the current number of segments inserted into $c$. Since count may be incremented in parallel, this operation is performed using an atomic increment and capture operation (which returns the current value in count and increments it).

Once the uniform grid is constructed, a list $L$ of the pairs of red and blue segments from all the grid cells is created. This list is generated in parallel using a strategy similar to the creation of the ragged-array. I.e., an initial pass is performed to count the number of pairs of edges in all grid cells and, then, a second one effectively inserts the pairs into the list.

The intersection between a pair of segments can be detected by evaluating $42 \mathrm{D}$ orientation predicates. Consider, for example, the segments $s_{1}$ (with endpoints $A$ and $B$ ) and $s_{2}$ (with endpoints $C$ and $D$ ). If the orientation of $(A, B, C)$ has a different sign than the one of $(A, B, D)$, then $C$ and $D$ are on opposite sides w.r.t. $s_{1}$ (the supporting line of $s_{1}$ intersects $s_{2}$ ). Similarly, if the orientation of $(C, D, A)$ has a different sign than the one of $(C, D, B)$, then the supporting line of $s_{2}$ intersects $s_{1}$. If both supporting lines intersect, then the segments do intersect. These 4 orientation predicates are performed in parallel on the GPU (see listing 3) for all pairs of segments in $L$.

Since edges may be inserted into multiple grid cells, a pair may be tested for intersection more than once (and, if they do intersect, multiple copies of them would be outputed by the algorithm). Preliminary experiments showed that a better performance is achieved when the duplicates are removed after the intersections are detected (instead of removing them before the intersection tests). This can be explained because, as it will be shown in section 5, detecting intersections using the GPU is a fast process.

All the geometric operations (determining the grid cells containing each segment endpoint and evaluating the orientation predicates for detecting intersections) are performed on the GPU in batch. However, since some of the operations performed with intervals (employed to determine the grid cells containing the endpoints of each segment) may return a failure code, after each batch of these operations the results are copied back to the CPU and the ones that failed are re-evaluated using arbitrary-precision rationals.

\section{EXPERIMENTS}

To evaluate the ideas proposed on this paper, the fast algorithm for intersecting edges was implemented on $\mathrm{C}++$ and evaluated on a AMD Ryzen 5 desktop with $63.2 \mathrm{GHz}$ cores (and 12 hyperthreads), $16 \mathrm{~GB}$ of RAM and a NVIDIA GeForce GTX 1070 Ti GPU.

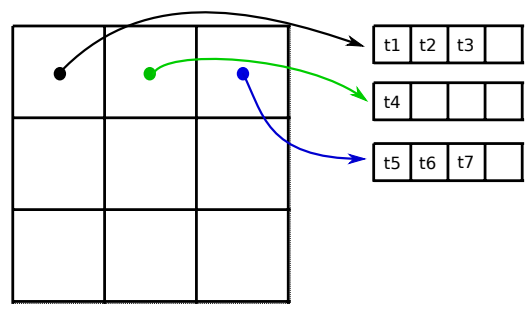

(a)

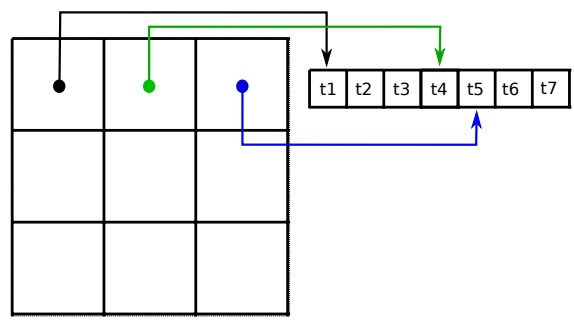

(b)

Figure 2: Dynamic array versus ragged array $-3 \times 3$ uniform grid using dynamic arrays (a) versus ragged array (b). Only the memory related to the first row of the grid is shown. Source: [25]

Arbitrary-precision arithmetic was provided by the GMP library [15] and the algorithm was parallelized with OpenMP (for the code targeted to the CPU) and CUDA (for the GPU code).

In all test cases a uniform grid with $2,500 \times 2,500$ cells has been created. However, there are heuristics for automatically choosing a grid resolution basing on statistics about the input datasets [25, 29]. For example, the grid size could be determined as a function of the input size in a way that the expected number of pairs of edges per cell is a given constant. As shown by Magalhães et al. [25], the range of grid configurations with reasonable performance optimum is broad.

Experiments have been performed using segments from four polygonal maps from two countries. The two maps from Brazil were obtained from the IBGE (the Brazilian geography agency) and represent the kinds of soil (BrSoil) and the counties (BrCounty) from Brazil. The two maps from the USA were obtained from the ESRI ArcGIS and the United States National Atlas web-pages. We also performed tests intersecting the largest dataset (UsCounty) with a version of itself (UsCountyRotated) rotated by $0.1^{\circ}$ (counterclockwise) around the center of the boundingbox of the original map. Experiments with UsCountyRotated are particularly hard for the uniform grid because it generates a high amount of potentially intersecting pairs of edges (thus, requiring more pairs of edges for being tested for intersection). 
Figure 3 illustrates four of the datasets and Table 5 present some statistics about the input maps and about the intersection computation process. As it can be seen, the size of the input datasets range from 200 thousand to 4 million segments. The average length of the segments is presented as a percentage of the diagonal of the bounding-box.

The last four rows of Table 5 present statistics about the pairs of evaluated input maps. In all cases, most of the uniform grid cells cover empty regions of the input datasets. Row Average \# pairs of segments/cell indicates the average number of pairs of red-blue segments per non-empty cell. Row Number of pairs of segments indicates the total number of pairs of red-blue segments in all cells (i.e., the number of pairs tested for intersection). As it can be seen in the last row, the actual number of intersections ranged from $6 \%$ to $0.005 \%$ of the number of intersection tests performed. Indeed, the dataset which generated the largest amount of intersection tests was the one with the smallest number of actual intersections.

We compared 5 versions of the algorithm (* marks a sequential implementation):

- Rational*: sequential implementation employing only arbitrary-precision rational arithmetic. This algorithm was evaluated in order to show the benefit obtained by the arithmetic filters in the other versions.

- Interval*: same as Rational*, but employing arithmetic filters with interval arithmetic.

- Rational: parallel (CPU) version of Rational*.

- Interval: parallel (CPU) version of Interval*.

- GPU: parallel (using the CPU and the GPU) version of Interval*.

Furthermore, as a baseline, we also implemented an algorithm using CGAL to detect intersections. This algorithm employs CGAL's method for intersecting dD Iso-oriented Boxes as a pre-processing step to initially cull the pairs of potentially intersecting segments. This culling process is sequential and employs a hybrid method composed of a sweep-line and a streaming algorithm to detect intersection between pairs of Axis-Aligned Bounding Boxes. Then, CGAL's do_intersect method is employed to check if each of the remaining pairs of segment do intersect. For exactness, the Exact_predicates_exact_constructions_kernel kernel has been employed (this CGAL kernel stores exact versions of the geometric constructors and employs arithmetic filters and lazy evaluation to accelerate the evaluation of predicates).

Table 5 presents the results obtained during the intersection of edges from pairs of input maps.
The pre-processing strategy performed by CGAL performs a better culling than the other methods, eliminating all pairs of edges whose bounding-boxes do not intersect (thus, the number of pairs of segments that really need to be checked for intersection is smaller in the CGAL algorithm). However, this happens at a cost of a more expensive pre-processing step (up to 5 times slower than Interval*). Besides having a faster pre-processing step, the Interval* method can be parallelized, while CGAL is sequential.

Indeed, while the total processing-time of Interval* was from 1.3 times faster to 7.7 times slower than CGAL, the parallel version using the GPU had a speedup ranging from 4 times to 10 times.

To better understand the influence of the GPU on the results, consider the intersection of segments from UsCounty with UsCountyRotated as example. The total time spent by the Interval* implementation for detecting intersections is $63.677 \mathrm{~s}(0.685 \mathrm{~s}$ to prepare the predicates and $62.992 \mathrm{~s}$ to evaluate them) and the time spent by the GPU implementation is $1.367 \mathrm{~s}(1.149 \mathrm{~s}$ to prepare the predicates and transfer the data to/from the GPU and $0.218 \mathrm{~s}$ to perform the evaluation). If only the time to evaluate the intersection predicates is considered, the achieved speedup is $289 \times$. This suggests that algorithms requiring a heavy usage of geometric predicates could benefit even more from the techniques presented in this paper.

As expected, the number of failures of the intervals was equal on the CPU and on the GPU. In the intersection of BrSoil with BrCounty, only 4 of the 877 thousand evaluated predicates $(0.0005 \%)$ evaluated failed, requiring an exact re-evaluation. In the intersection of UsCounty with UsAquifers, 3 of the 13 million predicates $(0.00002 \%)$ failed. Finally, in the intersection of UsCounty with UsCountyRotated, 4 of the 224 million predicates failed $(0.000002 \%)$.

\section{CONCLUSIONS AND FUTURE WORK}

We proposed the use of GPUs to accelerate the evaluation of exact geometric predicates filtered with intervals of floating-point numbers. The idea is to evaluate the predicates using interval arithmetic on the GPU. The (few) results that could not be guaranteed to be correct are, then, re-evaluated on the CPU using arbitrary-precision rationals.

As a proof of concept, a parallel algorithm for detecting intersections of red and blue line segments has been implemented. Because of the high computing power of the GPU for processing floating-point numbers, a speedup of up to 289 times (when compared against the sequential version) was obtained in the evaluation of the predicates (the speedup of the algorithm was up 


\begin{tabular}{|c|c|c|c|c|c|c|}
\hline \multirow[b]{3}{*}{ \# of seoments } & \multicolumn{6}{|c|}{ Pairs of maps evaluated } \\
\hline & BrSoil & BrCounty & UsCounty & UsAquifers & UsCounty & UsCountyRot. \\
\hline & 211,011 & 326,193 & $3,740,989$ & 352,924 & $3,740,989$ & $3,740,989$ \\
\hline Avg. segment length ( $\%$ of bb.) & $5 \times 10^{-4}$ & $4 \times 10^{-4}$ & $8 \times 10^{-7}$ & $1 \times 10^{-4}$ & $8 \times 10^{-7}$ & $8 \times 10^{-7}$ \\
\hline$\%$ of empty grid cells & & $86 \%$ & & $98 \%$ & & $98 \%$ \\
\hline Avg. \# pairs of segments/cell & & 0.3 & & 2.0 & & 34.7 \\
\hline \# of pairs of segments & & 300,039 & & $12,756,283$ & & $216,542,974$ \\
\hline \# of intersections & & 20,860 & & 11,948 & & 11,751 \\
\hline
\end{tabular}

Table 1: Statistics about the input datasets and about the intersection computation process.

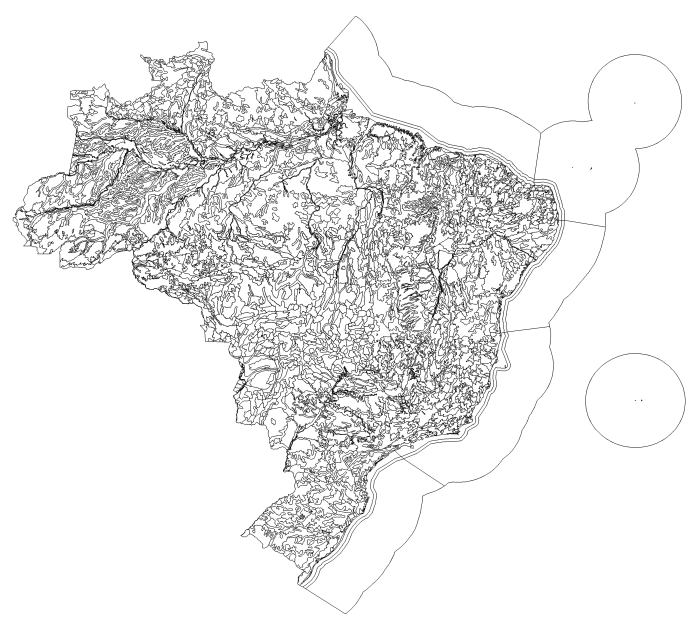

(a)

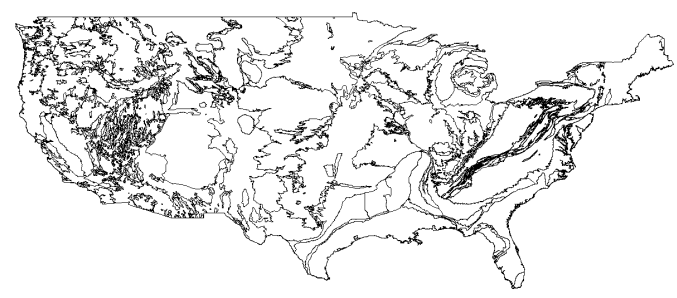

(c)

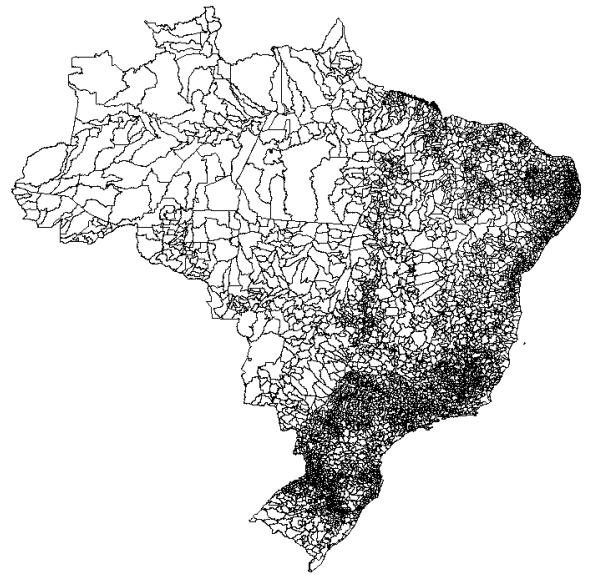

(b)

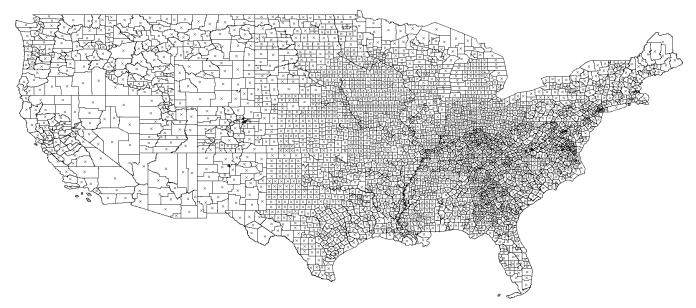

(d)

Figure 3: Maps employed in the experiments - BrSoil (a), BrCounty (b), UsAquifers (c), UsCounty (d) (these figures are not to scale). 


\begin{tabular}{|c|c|c|c|c|c|c|c|}
\hline \multirow{2}{*}{$\begin{array}{l}\text { Datasets } \\
\text { Method }\end{array}$} & \multicolumn{7}{|c|}{ BrCounty and BrSoil } \\
\hline & Rational* & Interval* & CGAL* & Rational & Interval & GPU & Speedup \\
\hline Pre-processing & 1.242 & 0.225 & 0.478 & 0.549 & 0.324 & 0.099 & 2 \\
\hline Inters. detec. & 1.444 & 0.152 & 0.015 & 0.385 & 0.040 & 0.018 & 9 \\
\hline Total time & 2.686 & 0.377 & 0.493 & 0.934 & 0.364 & 0.117 & 3 \\
\hline \# Inters. tests & 300,039 & 300,039 & 70,332 & 300,039 & 300,039 & 300,039 & - \\
\hline Datasets & \multicolumn{7}{|c|}{ UsCounty and UsAquifers } \\
\hline Method & Rational* & Interval* & CGAL* & Rational & Interval & GPU & Speedup \\
\hline Pre-processing & 7.884 & 0.812 & 2.628 & 1.710 & 0.392 & 0.164 & 5 \\
\hline Inters. detec. & 42.816 & 4.059 & 0.023 & 11.198 & 0.612 & 0.096 & 42 \\
\hline Total time & 50.700 & 4.871 & 2.651 & 12.808 & 1.004 & 0.260 & 19 \\
\hline \# Inters. tests & $12,756,283$ & $12,756,283$ & 158,653 & $12,756,283$ & $12,756,283$ & $12,756,283$ & - \\
\hline Datasets & \multicolumn{7}{|c|}{ UsCounty and UsCountyRotated } \\
\hline Method & Rational* & Interval* & CGAL* & Rational & Interval & GPU & Speedup \\
\hline Pre-processing & 14.532 & 1.422 & 7.482 & 2.798 & 0.454 & 0.251 & 6 \\
\hline Inters. detec. & 675.616 & 63.677 & 1.027 & 194.918 & 9.422 & 1.367 & 47 \\
\hline Total time & 690.148 & 65.099 & 8.509 & 197.716 & 9.876 & 1.718 & 40 \\
\hline \# Inters. tests & $216,542,974$ & $216,542,974$ & $11,254,031$ & $216,542,974$ & $216,542,974$ & $216,542,974$ & - \\
\hline
\end{tabular}

Table 2: Times (in seconds) spent by the different version of the algorithms for 3 pairs of datasets. Column Speedup shows the speedup of the GPU method when compared against the sequential implementation (Interval*).

to 40 times if the total running-time was considered).

The obtained performance and exactness makes this technique applicable for interactive applications (particularly on the fields of CAD, GIS and computational geometry).

As future work, we intend to apply this technique to other problems such as convex hull computation, $2 \mathrm{D}$ and $3 \mathrm{D}$ point location and boolean operations on meshes. Applications whose bottleneck is the evaluation of predicates could particularly present a better speedup.

Also, we intend to further improve the performance of the predicates. For example, a significant overhead is related to the communication between the CPU and the GPU. Reducing this communication (e.g., by moving the combinatorial part of the algorithms to the GPU) could lead to a performance improvement.

Finally, testing this technique in other architectures is also a future work: for example, high-end Xeon processors and MICs such as the Intel Xeon Phi are MIMD (Multiple Instruction, Multiple Data) processors (making it easier to port the combinatorial components of the algorithms to them). At the same time, these devices have a high parallel computing power for processing floating-point numbers (thanks to wide Single Instruction, Multiple Data - SIMD instructions in the individual cores). Thus, we believe both algorithms and exact geometric predicates could be accelerated on these devices using these instructions (keeping both in the same device would reduce the communication overhead).

\section{ACKNOWLEDGEMENT}

This research was partially supported by CAPES.

\section{References}

[1] Brönnimann H., Burnikel C., Pion S. "Interval arithmetic yields efficient dynamic filters for computational geometry." Discrete Applied Mathematics, vol. 109, no. 1-2, 25-47, 2001

[2] European Space Agency. "Ariane 501 inquiry board report.", $2015 . \quad$ URL ravel.esrin.esa.it/docs/esa-x-1819eng.pdf. (Retrieved on 06/15/2015)

[3] Skeel R. "Roundoff error and the Patriot missile." SIAM News, vol. 25, no. 4, 11, July 1992

[4] Kettner L., Mehlhorn K., Pion S., Schirra S., Yap C.K. "Classroom Examples of Robustness Problems in Geometric Computations." Comput. Geom., vol. 40, no. 1, 61-78, May 2008

[5] Hobby J.D. "Practical segment intersection with finite precision output." Comput. Geom., vol. 13, no. 4, 199-214, Oct. 1999 
[6] de Berg M., Halperin D., Overmars M. "An intersection-sensitive algorithm for snap rounding." Computational Geometry, vol. 36, no. 3, 159-165, Apr. 2007

[7] Hershberger J. "Stable snap rounding." Comput. Geom., vol. 46, no. 4, 403-416, May 2013

[8] Belussi A., Migliorini S., Negri M., Pelagatti G. "Snap Rounding with Restore: An Algorithm for Producing Robust Geometric Datasets." ACM Trans. Spatial Algorithms and Syst., vol. 2, no. 1, 1:1-1:36, Mar. 2016

[9] Shewchuk J.R. "Adaptive Precision FloatingPoint Arithmetic and Fast Robust Geometric Predicates." Discret. \& Comput. Geom., vol. 18, no. 3, 305-363, Oct. 1997

[10] Li C., Pion S., Yap C.K. "Recent progress in exact geometric computation." The J. Log. Algebr. Program., vol. 64, no. 1, 85-111, July 2005

[11] Hoffman C.M. "The Problems of Accuracy and Robustness in Geometric Computation." Comput., vol. 22, no. 3, 31-40, Mar. 1989

[12] Yap C.K. "Towards exact geometric computation." Comput. Geom., vol. 7, no. 12, 3 - 23, Jan. 1997

[13] Pion S., Fabri A. "A generic lazy evaluation scheme for exact geometric computations." Sci. Comput. Program., vol. 76, no. 4, $307-323$, Apr. 2011

[14] The CGAL Project. CGAL User and Reference Manual, 4.8 edn., 2016. http://doc.cgal .org/4.8/Manual/packages.html (Retrieved on 10/19/2017)

[15] Granlund T., the GMP development team. GNU MP: The GNU Multiple Precision Arithmetic Library, 6.0.0 edn., 2014. http://gmplib.org/ (Retrieved on $10 / 19 / 2017$ )

[16] Jacobson A., Panozzo D., et al. libigl: A Simple $\mathrm{C}++$ Geometry Processing Library, 2016. http://libigl.github.io/libigl/ (Retrieved on $10 / 18 / 2017$ )

[17] Audet S., Albertsson C., Murase M., Asahara A. "Robust and Efficient Polygon Overlay on Parallel Stream Processors." Proc. 21st ACM SIGSPATIAL Int. Conf. Advances Geographic Information Systems, SIGSPATIAL'13, pp. 304313. ACM, New York, NY, USA, Nov. 2013

[18] Magalhães S.V., Franklin W.R., Andrade M.V. "Fast exact parallel 3D mesh intersection algorithm using only orientation predicates." Proceedings of the 25th ACM SIGSPATIAL International
Conference on Advances in Geographic Information Systems, p. 44. ACM, 2017

[19] Popescu V. Towards fast and certified multipleprecision librairies. Ph.D. thesis, Université de Lyon, 2017

[20] Joldes M., Muller J.M., Popescu V., Tucker W. "CAMPARY: CUDA multiple precision arithmetic library and applications." International Congress on Mathematical Software, pp. 232-240. Springer, 2016

[21] Lu M., He B., Luo Q. "Supporting extended precision on graphics processors." Proceedings of the sixth international workshop on data management on new hardware, pp. 19-26. ACM, 2010

[22] Collange S., Daumas M., Defour D. "Chapter 9 Interval Arithmetic in CUDA." W. mei W. Hwu, editor, GPU Computing Gems Jade Edition, Applications of GPU Computing Series, pp. 99 - 107. Morgan Kaufmann, Boston, 2012

[23] Collange S., Flórez J., Defour D. "A GPU interval library based on Boost.Interval." 8th Conference on Real Numbers and Computers, pp. 61-71. 2008

[24] Whitehead N., Fit-Florea A. "Precision \& performance: Floating point and IEEE 754 compliance for NVIDIA GPUs." $r n(A+B)$, vol. 21, no. 1, 18749-19424, 2011

[25] Magalhães S.V.G., Franklin W.R. Exact and parallel intersection of $3 d$ triangular meshes. Ph.D. thesis, Rensselaer Polytechnic Institute, USA, 2017

[26] Akman V., Franklin W.R., Kankanhalli M., Narayanaswami C. "Geometric Computing and the Uniform Grid Data Technique." Comput. Aided Des., vol. 21, no. 7, 410-420, Sept. 1989

[27] Franklin W.R., Chandrasekhar N., Kankanhalli M., Seshan M., Akman V. "Efficiency of uniform grids for intersection detection on serial and parallel machines." N. Magnenat-Thalmann, D. Thalmann, editors, New Trends in Computer Graphics (Proc. Computer Graphics Int.'88), pp. 288-297. Springer-Verlag, Berlin, Germany, 1988

[28] Hopkins S., Healey R.G. "A Parallel Implementation of Franklin's Uniform Grid Technique for Line Intersection Detection on a Large Transputer Array." K. Brassel, H. Kishimoto, editors, 4th Int. Symp. Spatial Data Handling, pp. 95-104. Zürich, 23-27 July 1990

[29] Audet S., Albertsson C., Murase M., Asahara A. "Robust and efficient polygon overlay on parallel stream processors." Proceedings of the 21st 
ACM SIGSPATIAL International Conference on Advances in Geographic Information Systems, pp. 304-313. ACM, 2013 


\title{
BICAMERAL MESH ANISOTROPY
}

\author{
Nilanjan Mukherjee ${ }^{1}$, Jonathan Makem² and Jean Cabello ${ }^{1}$
}

\author{
Meshing \& Abstraction, Digital Factory, Simulation and Test Solutions, Siemens PLM Software, SIEMENS \\ 12000 Eastman Dr., Milford, Ohio 45150 USA mukherjee.nilanjan@siemens.com, \\ jean.cabello@siemens.com \\ ${ }^{2}$ Francis House, 112 Hills Road, Cambridge, UK. CB2 1PH jonathan.makem@siemens.com
}

\begin{abstract}
This paper discusses a new approach to controlling 2D local sizing in a bicameral anisotropic mesh. We define bicameral anisotropy as a mesh size variation of two distinctly different types in two separate chambers or subdomains. The first chamber is controlled by constant to linear local size functions. The second subdomain is governed by a nonlinear sizing function leading to transitioning meshes. A controlled advancing front approach is proposed for both triangular and quadrangular meshes with the singular goal of ensuring a high local quality metric in the first chamber. An H-shock sizing scheme governs the second chamber. Virtual mesh topology is constructed at the face boundary both at geometry and nodeloop levels to facilitate this type of bicameral meshing. Results clearly indicate the efficacy of the proposed approach leading to both a well controlled desired size field and high local element quality.
\end{abstract}

Keywords: bicameral anisotropy, soft-point, virtual-vertex, sub-edge, advancing front, loop-segment, paving

\section{MOTIVATION}

In a large number of industrial finite element analyses problems ranging from the toy industry to aerospace, structural designers and analysts have a need to focus on specific hotspots on the surface of engineering products and their components. Typically these hotspots represent local zones of high stress, impact loading, crack growth, areas of high double curvature or areas where the field parameters studied are overly sensitive. In the automotive industry, for example, these areas could represent fillet bends in engine blocks, valve seats, seam and spot weld locations on certain areas of the car-body. In the electronic industry, these hotspots are usually more abundant and can typically represent small areas of shock and impact in drop-test simulation (cell phones for example). In the aerospace industry from leading-edge blade modeling to many nooks and corners of engine components to tire modeling, there is a strong need to zoom in on the multi-physics of local areas.

Accordingly, the finite element model for these applications demand mesh anisotropy. Usually, the mesh anisotropy desired is bicameral in more than one way. At one level bicameral anisotropy can be defined as: a) size variation within the same mesh where some elements are very small while others may be ten to a hundred times larger and b) where an average element has a large size variation (some edges of the element are small while other edges are large). Typically, most examples of anisotropy in a 2D/3D mesh bear evidence of both types of size variation. The need for anisotropy often becomes bicameral in another different way. This type of size variation is zonal, where size in one subdomain of a face is either constant or linearly varied while in the second domain size varies/transitions according to non-linear functions.

\section{INTRODUCTION}

Mesh gradation or anisotropic mesh size control is a relatively new research problem. The first major investigations started appearing in open literature only in the late 1990s. A couple of the early investigations focused on mesh size variation during adaptive finite element analysis [1][2], where an initial isotropic mesh is used to perform an initial solve. Next, from the error estimates computed from 
the results [3][4] a size specification field is deduced (e.g. at the vicinity of each mesh vertex, the desired mesh size is specified) and the initial mesh is refined based on this field and re-solved. This process is iteratively repeated until solution convergence is achieved [5]. For surface meshes the geometric deviation or fidelity error is also computed apart from the solution error estimate which is a measure of the gap between the facets of the abstracted surface and the CAD surface. The problem faced is thus about the ways to control the mesh size variation or gradation. Borouchaki et al [6] proposed a two-way procedure to solve this problem of mesh size variation as a field problem.

Loseille et al [7] used an approximated Hessian-based metric field for anisotropic mesh adaption. The metric field approximates the interpolation error of the solution and is used to determine the local mesh density as well as the local element rotation and stretching. This was combined with an a-priori goal oriented error estimate for applications in the meshing of shock-dominated flows governed by Euler equations. The main disadvantage of this technique is that it cannot be applied to localized sub-division algorithms and therefore is of little practical use for problems of industrial complexity. Lecht et al [8] generalized a method for anisotropy detection based on the hessian of the Mach number to drive output-based adaptation for the compressible Navier-Stokes equations by using the derivative of the Mach number to guide anisotropy of the mesh. However, although the meshes are anisotropic, the value of the Mach number which governs the anisotropy is random.

Most mesh sizing algorithms reported in open literature refine meshes according to a size field computed from solution error estimates and the deviation of the mesh from CAD geometry. No particular zone of local refinement can be defined by the user and the areas of refinement do not reflect structured, layered, user-controlled meshes of high quality, especially with quadrilateral elements. The present paper proposes a novel method to address this problem.

\section{BICAMERAL MESHING}

To facilitate the localized zonal meshing of a region within the bounds of a larger global mesh exhibiting a different element size pattern/function, we enact the concept of Bicameralism, whereby a local and a residual global chamber ( $C_{1}$ and $C_{2}$ respectively) are meshed with different element size pattern/functions ( $S_{1}$ and $S_{2}$ respectively), as shown in figure 1 . The parent face, $F$, may be defined as the boolean union of the local and residual chambers given by

$$
F=C_{1} \cup C_{2}
$$

Similarly, the final mesh, $M$ on the parent face can be defined as the boolean union of the meshes in the local and residual chambers ( $M_{1}$ and $M_{2}$ respectively) expressed by

$$
M=M_{1} \cup M_{2}
$$

For tri meshing the bounds of the local chamber is circular and square for quad meshing, as shown in figure 1.

\section{PROPOSED ALGORITHM}

The problem to be addressed is defined by the following user specified input data data -

i) surface geometry (tessellated)

ii) a global size $\boldsymbol{s}_{\boldsymbol{g}}$ with which the entire surface is supposed to target

iii) mesh points or points on the surface, on edges and/or vertices that have a local size definition different from sg.

iv) a radius or square of influence ( $\mathbf{R}$ ) at each point in (iii) which denote the chamber of influence and

v) (optionally) a size variation function $g(r)$ for each chamber. If the function is not specified by the user, the mesher assumes constant size.

The problem at hand, is thus clearly bicameral, i.e. it is split into two separate chambers with clearly defined boundaries. Chamber I is called local chamber and is defined by the radius or square of influence specified by the user around certain points which are the origins of the local size field. The source or origin of a local chamber can be of three types of mesh points -

a) face interior mesh point

b) face vertex

c) edge-interior mesh point

Chamber II represents the rest of the geometry to be meshed and is called global-residual chamber. In the global-residual chamber the mesh size first transitions from the local size to global and then stabilizes to a field normal value which is the global size of mesh generation.

\section{VIRTUAL GEOMETRY CREATION FOR LOCAL SIZE CONTROL}

In order to bound the local mesh size chamber modifications to the physical domain of the problem, i.e., the boundary of the local chamber, need to be made. This is done by creating virtual geometry both on the boundary and interior of the face around the origin or source of the local chamber.

\subsection{Creation of OD and 1D Virtual Geometry}

OD and 1D virtual geometry are created using both finite element nodes and the parent geometry to efficiently expedite mesh sizing around mesh points with local size. This is done using soft-points, virtual-vertices, sub-edges and loop-segments. 
Key: Local Chamber, $\mathrm{C}_{1}$

Residual Chamber, $\mathrm{C}_{2}$

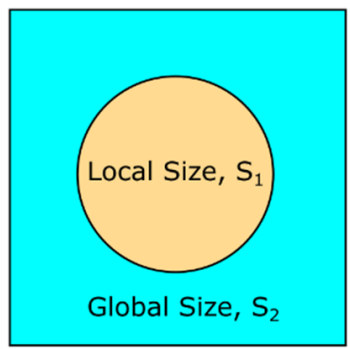

(a) circular zone

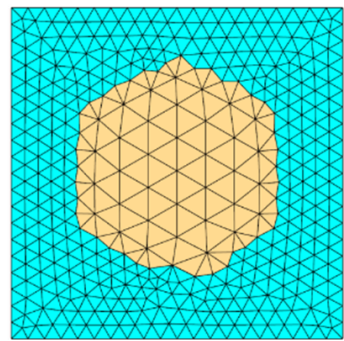

(c) tri meshing

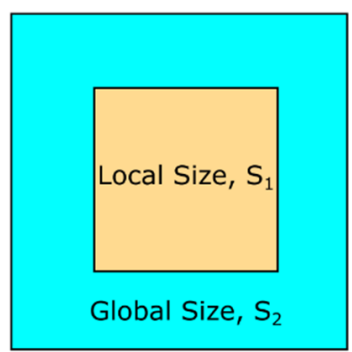

(b) square zone

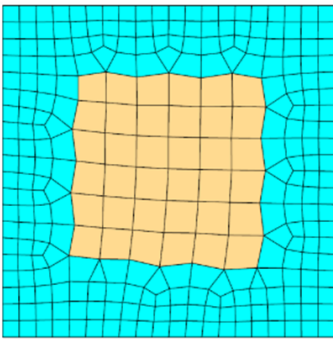

(d) quad meshing
Figure 1 Bicameral Mesh Sizing

\subsection{Creation of Local "Soft-Point" limiters}

If local size is defined at a vertex or on a mesh point created in the interior of an edge, we intersect the circle (of the radius of influence $\mathbf{R}$ ) with all edges meeting at the mesh point or vertex as shown in Figure 2. A node is created at the points of intersection prior to mesh generation. These nodes are called soft-points. They are "soft" in the sense they are not required to be created at these locations by the application. Instead, meshing algorithms create these nodes for the purpose of pre-seeding edges at specific locations, in this case as mesher-native vertices called virtual-vertices. A virtual-vertex is nothing but a soft-point which is a special type of node. The special property that distinguishes it from other nodes is that they are semi-permanent - they are not deleted if the mesh is deleted on the faces that share the edge, they remain similar to vertices. A virtual vertex can only be selectively deleted by a meshing function.

\subsection{Creation of Sub-Edges}

Figure 3 describes the next step, where the edge is virtually partitioned into segments called virtual sub-edges. In terms of node-geometry and face-edge association the edge owning the mesh point is still unique and undivided. For the purpose of mesh generation, however, it is sectioned into virtual sub-edges where each sub-edge is meshed as a separate entity.

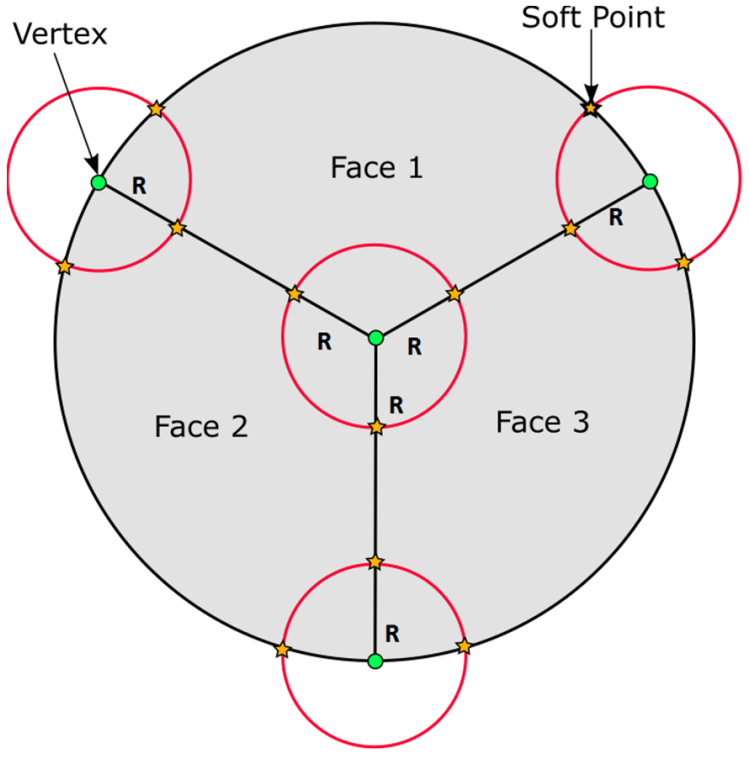

(a)

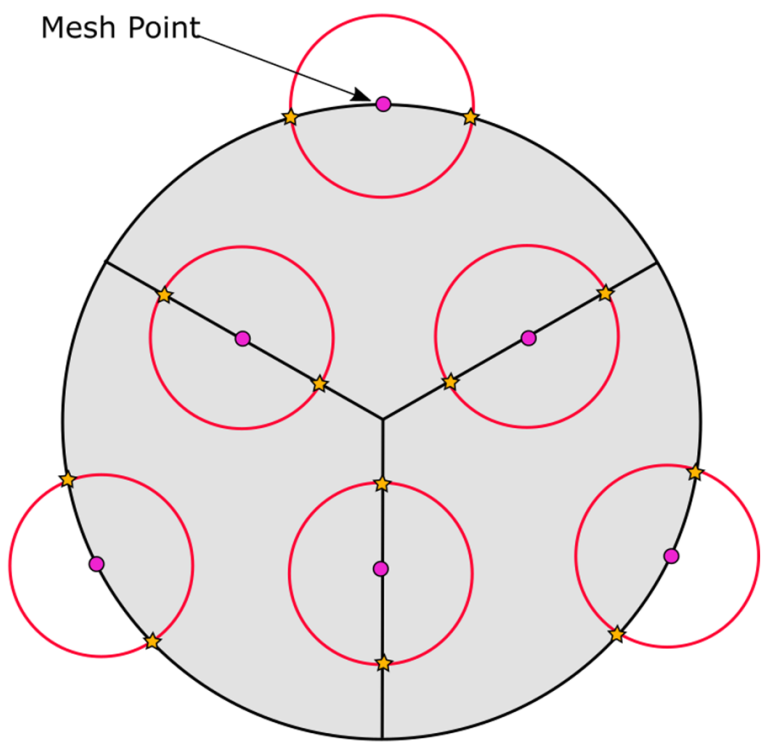

(b)

Figure 2. Local size on mesh point on edge (a) at the vertex, (b) in the interior.

This allows us to create different size distributions on the sub-edges. The sub-edge is a child of the geometry edge. Sub-edges can be deleted at any time if the virtual vertices on the parent edge are deleted, but the parent geometry edge will stay intact.

Two virtual-vertices can approach each other as shown in Figure 4 , if there are local size definitions at the two end vertices of an edge and the radii of influence of both are such that the circles come close or overlap. In such situations only one soft-point or virtual vertex is created at the mid-point of the overlap zone. 


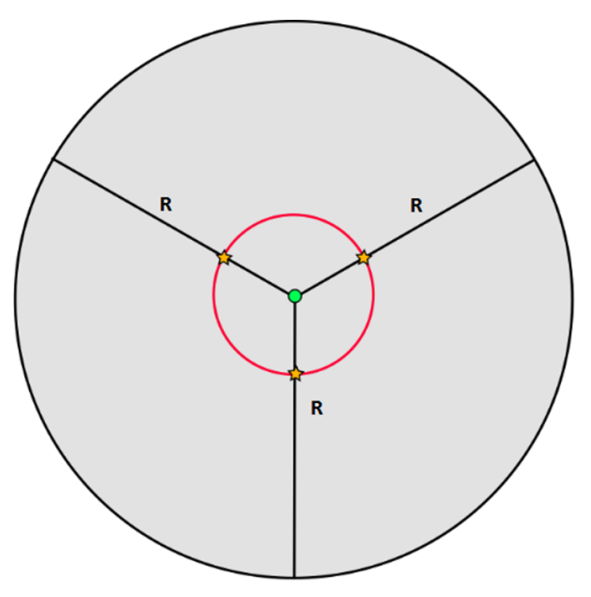

(a) soft points marking sub-edge extremeties

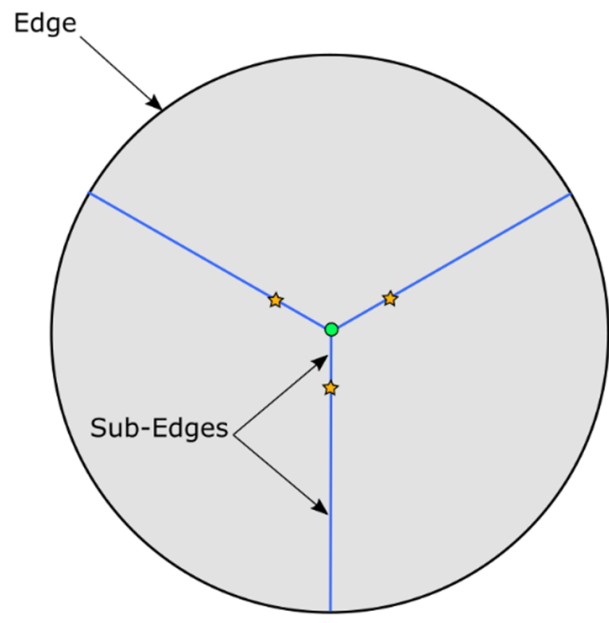

(b) sub-edge creation

Figure 3. Virtual sub-edges.

\subsection{Distribution of Local Size Information}

Once all mesh points with local size are processed creating OD and 1D virtual geometry, the faces are ready for mesh generation. The boundary edges of all faces are discretized according to the size information they own. Next, the discretized face-loops, now called node-loops are laid down in $2 \mathrm{D}$ for mesh generation. At this point, the local size information existing on the mesh point/virtual vertex - the size value and the radius of influence - need to be applied to certain boundary segments of the nodeloop. These segments of the nodeloop will be called loop-segments from here on.

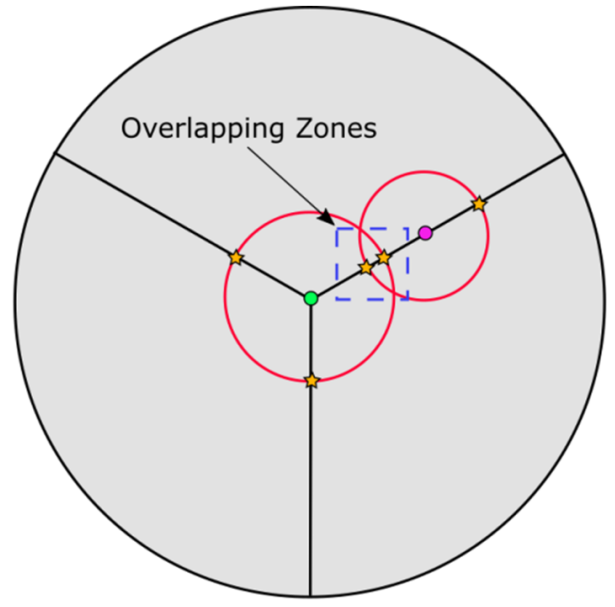

(a) zonal encroachment

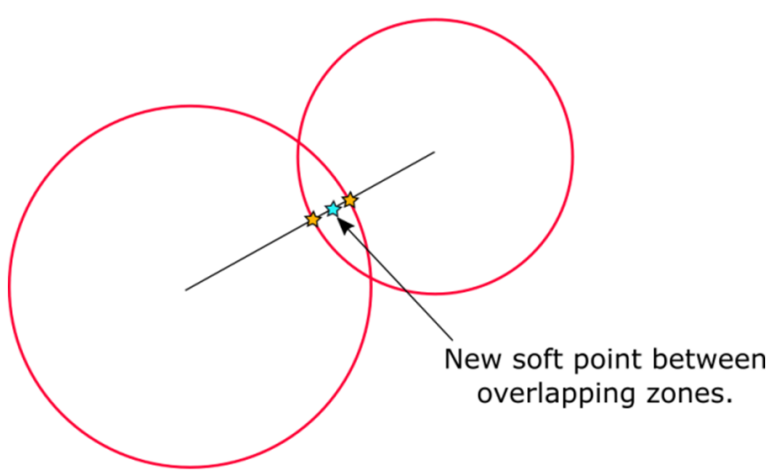

(b) soft point repositioning

Figure 4. Overlapping circles of influence

\section{LOOP SEGMENT GENERATION}

Once all virtual geometry is constructed, the face boundaries are completely discretized, the face is laid down or flattened into a 2D domain, node-loops defining the boundaries of the $2 \mathrm{D}$ area to be meshed are created. Certain segments or parts of such a node-loop are called loop-segment. A typical loopsegment is shown in Figure 5. The loop-segment $\mathbf{L}_{\mathbf{s}}$ basically defines the part or segment of the face boundary that falls within the radius of influence bound by soft-point limiters $\boldsymbol{s}_{\mathbf{0}}$ and $\boldsymbol{s}_{\mathbf{1}}$ (Fig.5) and can be expressed as

$$
L_{s}=\sum_{i=s_{0}}^{s_{1}} \boldsymbol{l}_{f i} \quad \text { in explicit domain }\left(s_{0}, s_{1}\right)
$$

and where $\mathrm{l}_{\mathrm{fi}}$ denotes the i-th loop-front as described by the CSALF $[9,10]$ algorithms.

It is important to note that the local chamber has a shape which is decided by the user. If the user does not specify the shape of a local chamber, circles are used for triangular 
elements and square shape for quadrangular. These two chamber shapes are used throughout the present paper. However, the overall algorithm is shape-independent as the chambers are filled with advancing front meshes which can cater to any general shape. For most industrial meshes analysts focus on either circular or square shaped chambers.

\subsection{Partial Paving/Advancing Front on Face Boundary}

Paving or Advancing Front $[9,10]$ have been around for the past three decades and are the most popular of triangular and quadrangular surface mesh generation algorithms today. The CSALF algorithms however [11,12], are largely improvised adaptations where more control is exercised on advanced/paved layers and the front advancing strategy is amalgamated with a mesh-area subdivision technique making it a rather hybrid approach. This hybrid paving/advancing front strategy is further specialized to create best quality elements in the local chamber within the radius of influence.

\subsection{Creation of Loop Segments}

Since the loop-segment is part of the face boundary that falls within the radius of influence $\mathbf{R}$, it must be discretized at the local size along with the circular arc between them. In Figure 5, the red segment on the face boundary defines the

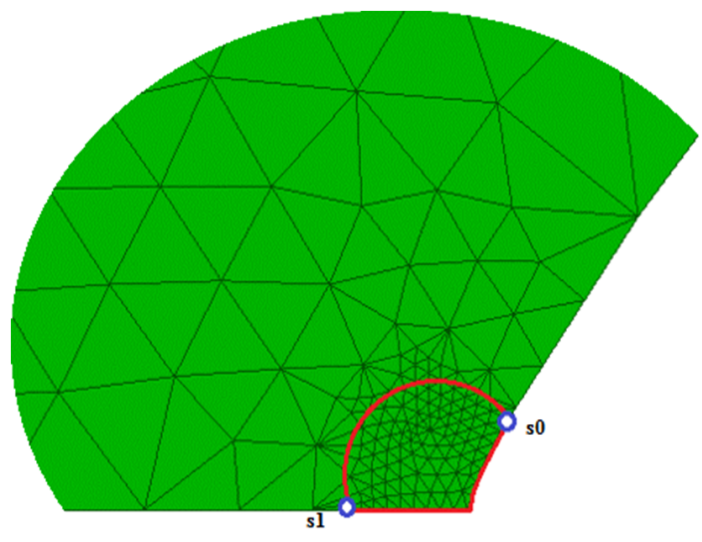

Figure 5. A typical loop-segment

loop-segment. As expected, the size of discretization along this segment reflects the local size defined at the vertex. The area inside the red circular arc defines the portion of the mesh area or nodeloop inside the radius of influence. The mesh size in this zone reflects the local size defined. The blue circles mark the soft-points or virtual vertices created on the edges incident at the corner vertices $s_{0}$ and $s_{1}$. These mark the start and end of the loop-segment and the intersection of the circle of local size influence and the edges joining the source or origin of the local chamber.

\subsection{Types of Loop Segments}

Three types of loop-segments are dealt with, as shown in figure 6 . Two of them are edge-based and the third one is in the interior of the face. These are :

\section{Vertex-based or edge-end loop-segment}

When the local size control is defined at a vertex, the loop segment covers all edges incident at that vertex. Figure 5 shows a classical vertex-based loop segment for a circular local chamber. Any edge-based loop-segment is defined by at least three points - the virtual vertex on connected edge 1 , the vertex of the edges or the origin of the local chamber where local size is defined and the virtual vertex on connected edge 2 . The loop-segment starts at virtual vertex $A$ and runs up to virtual vertex B as shown in Figure 6a.

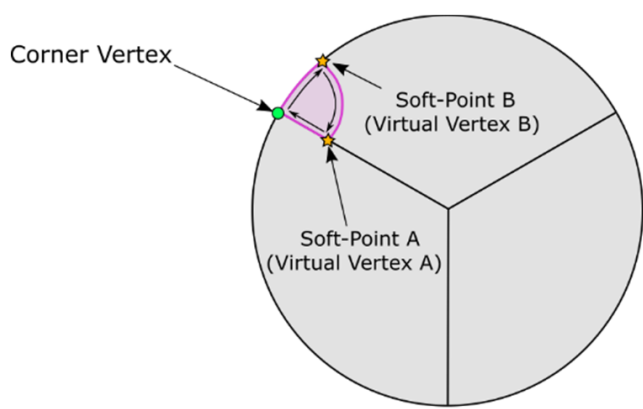

(a) corner type

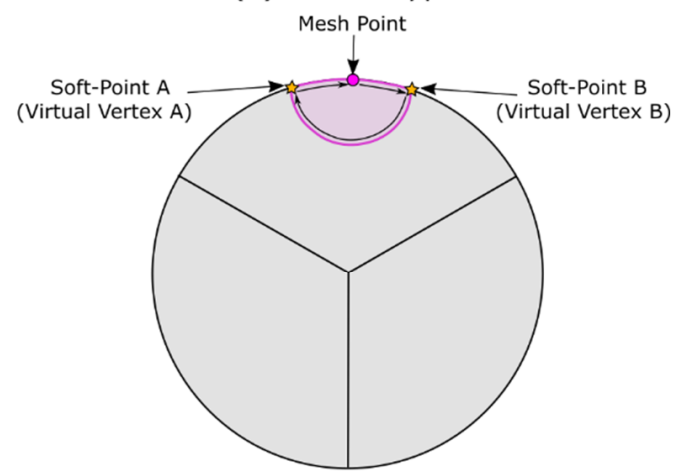

(b) edge type

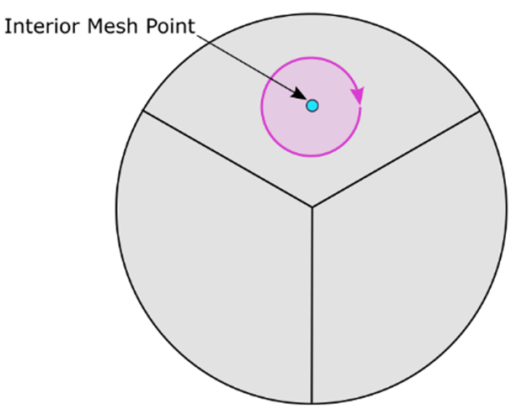

(c) face type

Figure 6. Loop segment types

\section{Edge interior loop-segment}

This second type of loop-segment is also defined by 3 points - starts at virtual vertex A, touches the origin of the local 
chamber, the mesh point on the edge and runs up to virtual vertex B (Figure 6b).

\section{One point loop-segment}

The third type of loop-segment is a one-point loop defined by the mesh point in the interior of the face (Fig.6c).

\subsection{Creation of Face-interior Point Loops}

Face interior mesh points with local size control are first appended to the face topology as new one-point loopsegments. Next, we pave around the point at local size gradually expanding the area covered by elements to the circle of influence. This is described in Figure 7,9 and 10.

Once paving stops as we reach the radius of influence $\mathbf{R}$, the free-edges of the cluster of elements form a closed node-loop as shown in Figure 10. The boundary of the white shaded area in Figure 10a and that of the orange shaded area in Fig. $10 \mathrm{~b}$ define the new loop.

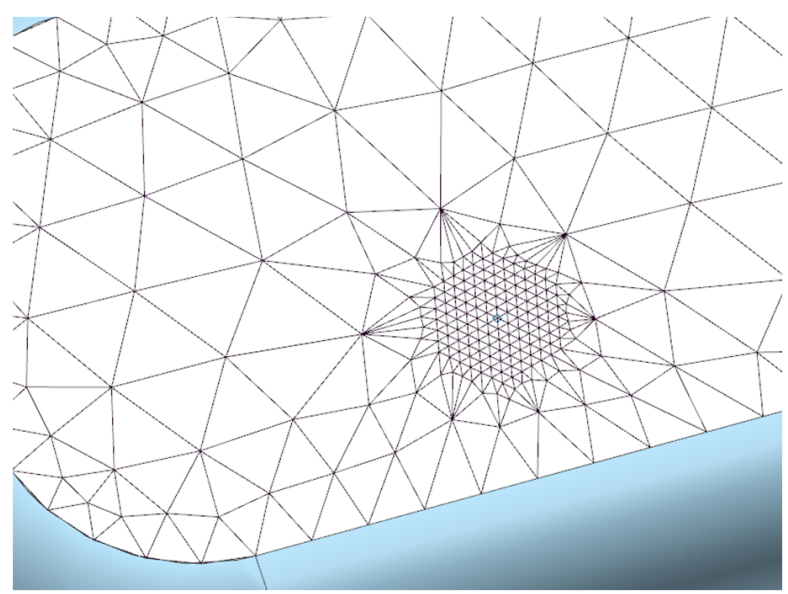

Figure 7 Face-interior point loop

This nodeloop replaces the single-point loop as part of the face topology. Therefore, in a nutshell, paving/advancing front of the single point-loop produces a new face interior loop as output which is added to the face thus altering its face topology for meshing the residual-global chamber. An example for how it is done is illustrated in Figure 12.

\subsection{Creation Partial Paving/Advancing Front Meshing of Edge Loop Segments}

The acronym CSALF stands for Combined Subdivision and Loop-Front mesher. The mesh area of the face is defined by node-loops. Every three adjacent nodes form what is called a "loop-front". Each loop-front is advanced based on an angular advancement template and terminated based on front-type pair based closure stencils $[11,12]$.

When all loop-fronts that form the node-loop have been advanced, a new nodeloop results. This is how the nodeloops are advanced - the outer moves inwards, the inners move outward. When they collide, loop-front advancement is arrested and the remaining mesh area is filled. CSALF meshers, therefore, are "symbiotic" in the sense they alloy two distinctly different mesh generation algorithms working recursively in tandem. Figure 8 shows two examples of loop- segment paving/advancing front with quadrilaterals and triangles.

In order to advance the fronts for the loop-segments the same CSALF meshers are used with more improvisation and tighter control. For edge-based loop-segments, we resort to partial advancement of the boundary in the sense elements are first created only along the length of the loop-segment $\boldsymbol{L}_{\boldsymbol{s}}$ defined in equation (3). When the circle or square of influence is all filled with these high quality elements, the mesher stops meshing chamber I. The free element edges of the cluster of elements created become a new node-loop which is inserted/appended to the face topology as shown in Figures 8-10 for both quadrilaterals (paving) and triangles (advancing front). The triangles created can be ideally equilateral or right-angled depending on the user's choice.

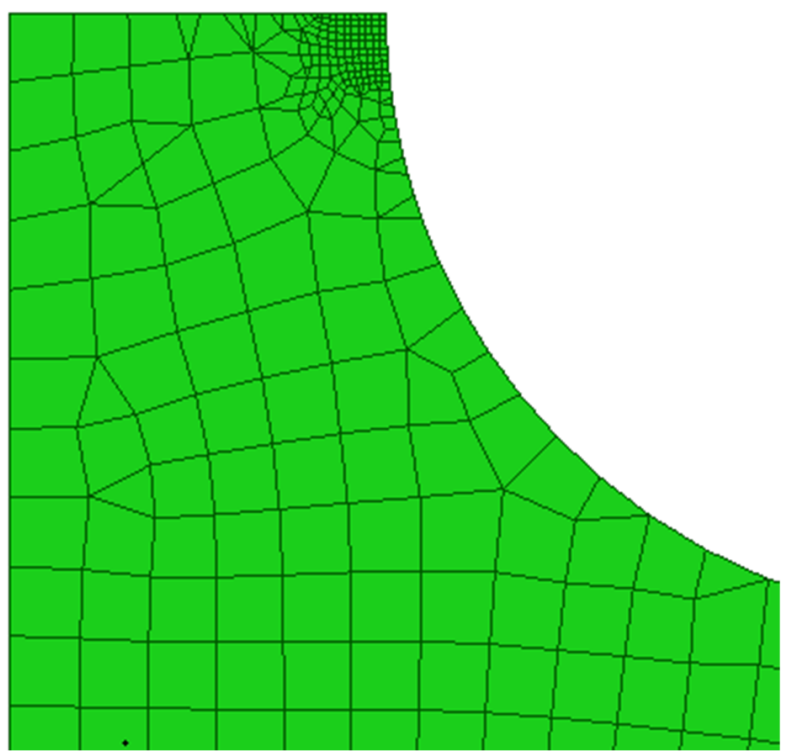

(a)

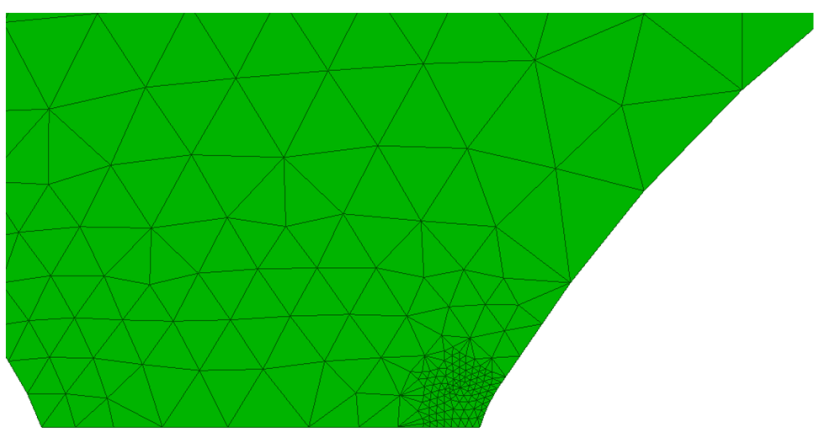

(b)

Figure 8. Partial paving (a)/ Advancing Front (b) on faces with local size on a boundary vertex.

The face-inserted loops are next joined up with other interior loops and the outer nodeloop to create a single connected nodeloop that represents the residual-global chamber II. The same CSALF mesher meshes chamber II next. 


\subsection{Partial Paving/Advancing Front Meshing of Face-interior Point-Loop}

For face-interior single point node-loops, the approach is different. In case of Advancing Front meshing with triangles, first six equilateral triangular elements are created around the point. Advancing loop-fronts continue and more layers of equilateral triangles result around the mesh point with local size until the circle of influence is covered as shown in Figure 10a. Loop-front advancement stops and the new nodeloop formed by the free element edges of the element cluster are appended to the mesh area.

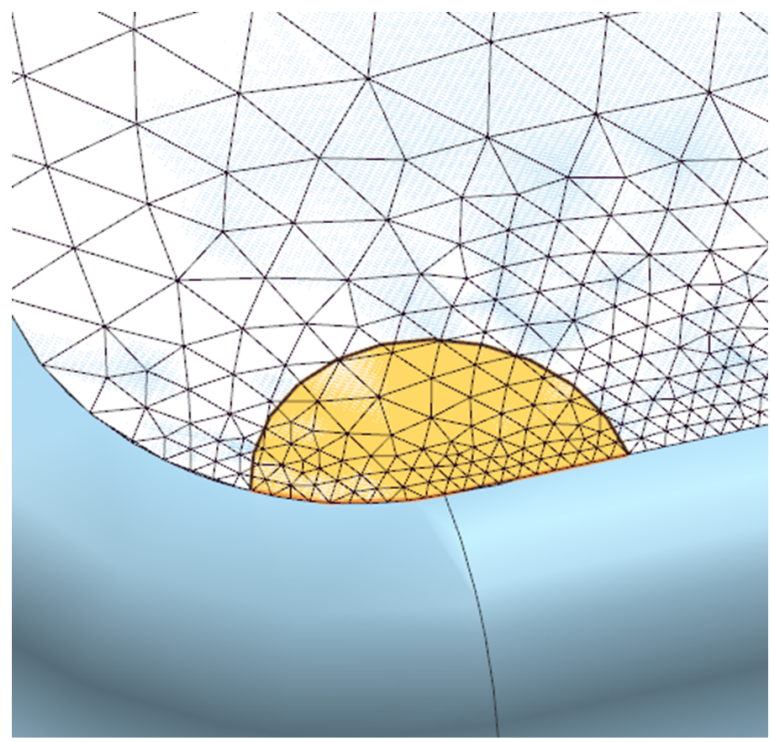

Figure 9. Paving/Advancing Front of an edgebased (vertex) loop-segment resulting in a new boundary loops.

In the case of paving with quadrilaterals, the question of direction of loop-front advancement arises, especially when the shape of the local chamber is expected to be rectangular. This is not an issue with advancing front meshing with triangles where the elements grow radially outward in all directions. Thus, for quads, if a direction vector is supplied by the user, four quadrilateral elements (2X2) created oriented along that direction. As paving continues, more layers of right-angled square elements are added until the circle of influence is entirely covered (Figure 10b). For quadrilaterals we create one or two additional layers beyond the circle of influence to allow for elegant mesh transitioning.

\subsection{Local Chamber Collision}

When the sources of local chambers are face-interior and the local chambers are in fair proximity, chamber collision is probable. Loop-front advancement is done one layer at time for each loop. Consequently, as loop-fronts advance from the origins of two face-interior local chambers as depicted in Figure 11, collision is detected. As soon as it happens, front advancement for the chamber in the collision zone is arrested as shown below.

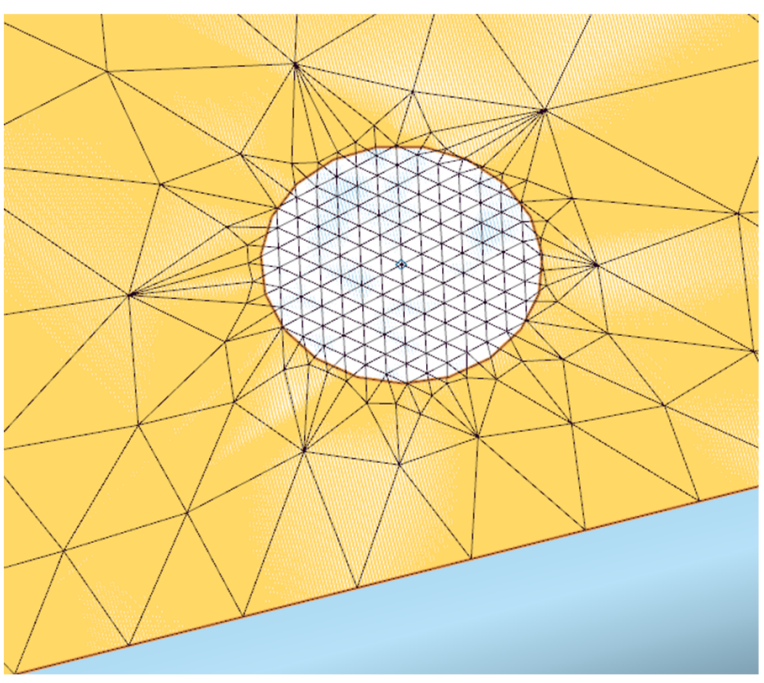

(a)

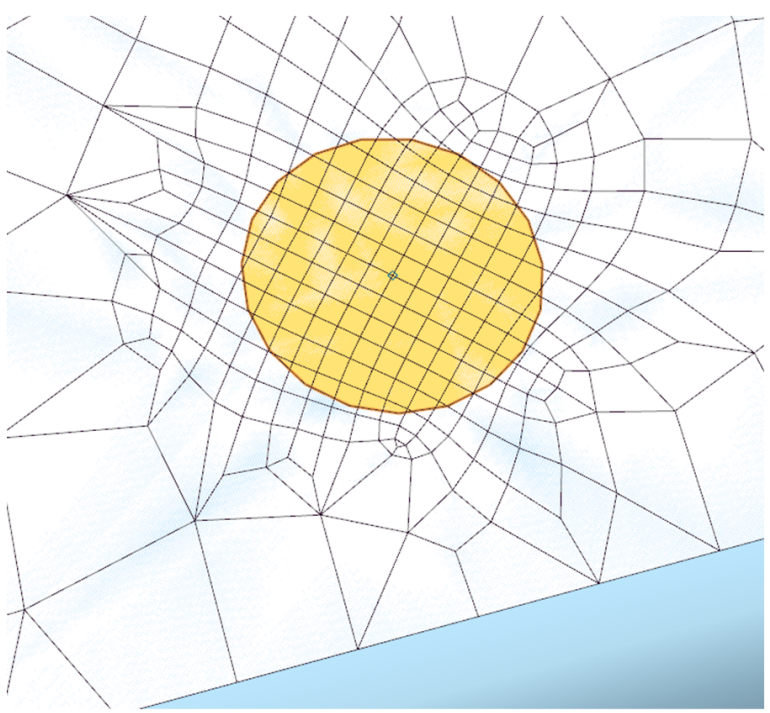

(b)

Figure 10. Partial paving (a)/ Advancing Front (b) on faces with local size on interior point.

Figure 12 depicts the nodeloop boundaries of the colliding local chambers when their growths are both arrested. These face-inserted loops are next joined with the outer nodeloop (and other inner nodeloop). Figure 13 shows the final mesh.

While paving/advancing front mesh generation in chamber I (as in case described by Figure 6a where three faces meet at one corner), the mesher first covers all local chambers (chamber I) of each face connected at the vertex. In case of the type of local chamber described in Figure $6 \mathrm{~b}$, the chamber is on the face-edge but not shared with other faces, whereas in Figure 6c, the local chamber is completely interior to the face. 


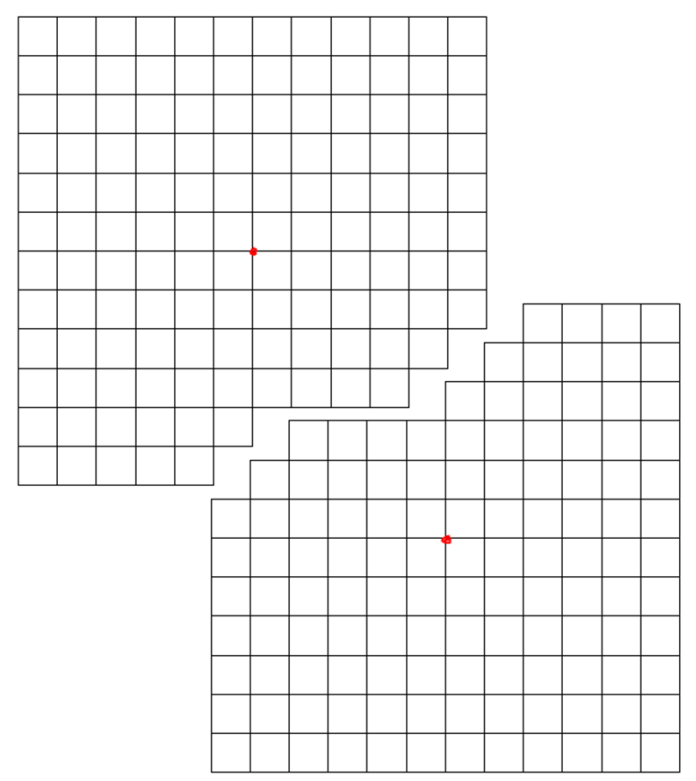

Figure 11. Chamber collision in the interior of the face.

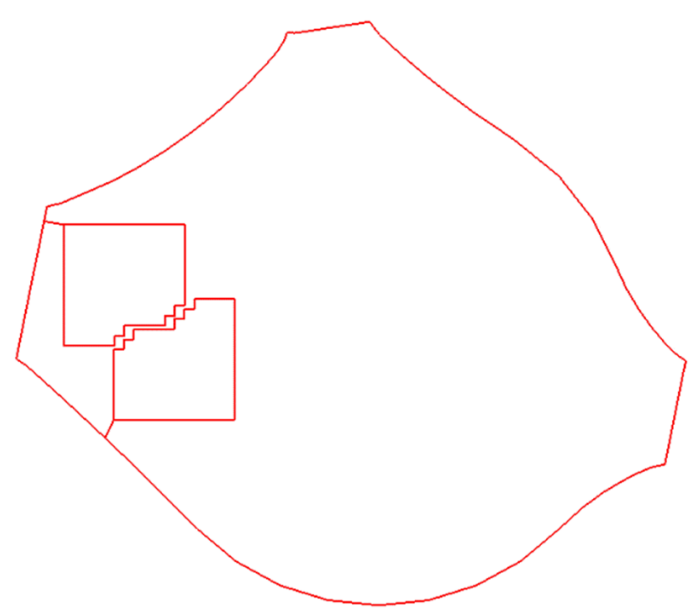

Figure 12. Truncated colliding local chambers joined with the outer face-boundary.

\section{BICAMERAL SIZE CONTROL}

Bicameral anisotropy can be variegated but at least binary in the limit. The size in each local ring or square of influence (local chamber) can vary according to a different local function. This paper only discusses constant to linear functions in the local chamber. Let us assume, the mesh size field in chamber I, is defined by F(I). The center or source of the chamber is point $\bar{P}$ from where the desired local size field originates. The field needs to be varied according to a userdefined function $\mathrm{g}$, whose domain for a circular shape is expressed in polar coordinate system as

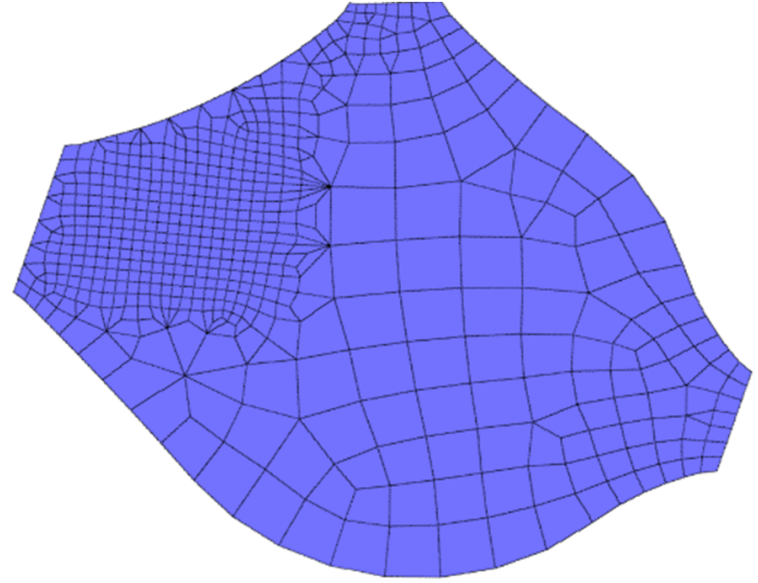

Figure 13. Final quadrilateral mesh on the face with interior local chamber origins.

$F(I)=g(r)$ in explicit domain $(0, R)$

where $\mathrm{R}=$ Radius of influence

and redefined for a square shape in a Cartesian coordinate system as

$F(I)=g(x)$ in explicit domain $(\bar{P}-R, \bar{P}+R)$

Figures 9-12 provide ample examples of such variations in local size. The sizing function in the residual-global chamber (also known as chamber II) is a $\mathrm{H}$-shock variation function expressed succinctly as

$F(I I)=\dot{h}_{A B}$

where $h_{A B}$ describes a shock type sizing function which is explained later in section 7.2.

\subsection{Various Aspects of Local Size Control}

In contrast with previously reported mesh sizing algorithms discussed in section 2, this algorithm also provides numerous user-driven ways to vary or regulate mesh size. Size variation can be controlled on edges connected to a vertex with local size even outside the radius of influence, at least two different triangular element types can be provided as option, local mesh size control can be made coarser (Figure 14) instead of finer and finally, within the radius of influence, thickness of layers can be varied. We provide a default behavior for each one of these options based on a best practice standard. 


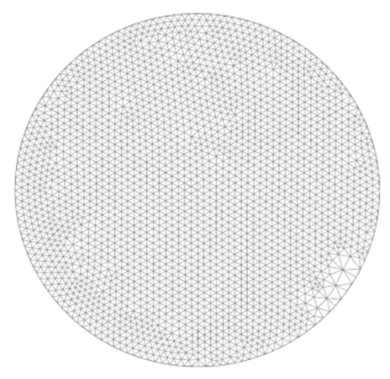

(a)

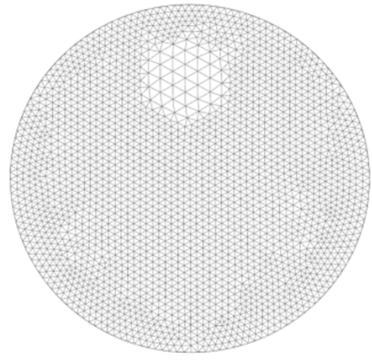

(b)
Figure 14. Coarser local size on the face boundary (a) and interior (b) in a finer size mesh.

\subsection{Mesh Sizing on Edges With Size Control}

To ensure better mesh size transition and element quality, our algorithm varies mesh size even outside the radius of influence on edges. Figure 15 explains size distribution scheme on edges incident on a vertex with local size control. The mesh size is kept constant at its local value $\mathrm{s}_{0}$ within the radius of influence $\mathbf{R}$. Over the next $\mathbf{2 R}$ length, on all edges connected to the vertex, the size is varied parabolically up to $50 \%$ of the global size $\mathrm{sg}$. Over the remaining length of the edge mesh size increases from $\mathrm{s} / \mathrm{g} 2$ to $\mathrm{sg}$ following the $\mathrm{H}$ shock size variation algorithm [6]. Equation family 7 describes the size function $\mathrm{g}(\mathrm{x})$ on the edge of length 1 as

$$
\begin{aligned}
& g(x)=s_{0} \quad \text { where } \quad R \geq x \geq s_{0} \\
& g(x)=s_{0}+b x^{2}+c x \quad \text { where } R+2 R \geq x>R \\
& \quad b=\frac{s_{g}-2 s_{0}}{12 R^{2}} \text { and } c=\frac{s_{g}-2 s_{0}}{12 R} \\
& g(x)=\frac{s_{g}}{2} f\left(H_{s}\right) \quad \text { where } l \geq x>(R+2 R)
\end{aligned}
$$

Figure 16 shows how controlled, multi-staged bicameral size variation avoids the local size from over-spilling too far into the edge and the connected faces. Figure 16a depicts a size transition with the H-shock method applied immediately outside the radius of influence on all edges connected to the vertex with local size. The finer size seems to over-penetrate into the edges and thus their owning faces. The effect of a multi-staged controlled size variation as described above reduces the "spill effect" as shown in Figure 16b. Beyond length $=\mathrm{R}$ on the meeting edges, the size is varied parabolically over $2 \mathrm{R}$ length and graded up to global size thereafter. Many users prefer the latter scheme while some might like a less drastic transition as in the former.

Furthermore, the size variation type in stage II (over the length of $2 \mathrm{R}$ ) is also optional. Users could use a linear or geometric progression variation instead of the default parabolic type. A contrast of geometric progression variation versus parabolic is demonstrated in Figure 17.

\subsection{Control of Triangular Element Types}

Two different triangular element types can be provided as option especially for mesh generation within the radius of influence on edges. A 90-degree triangle can be used (Figure 8 b) optionally although the best element perhaps is an equilateral triangle (Figure 7).
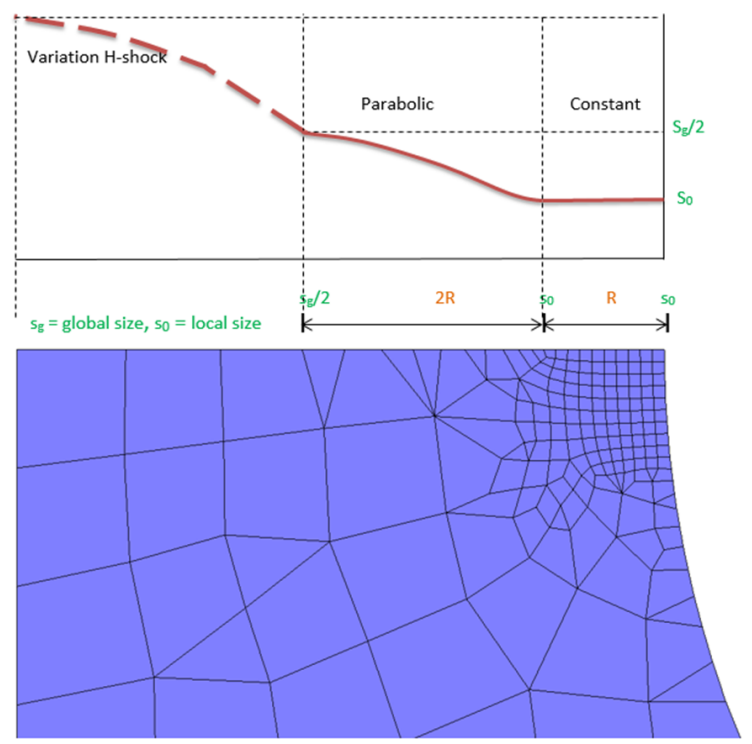

Figure 15. Mesh size distribution scheme on edges with local size on vertex.

\subsection{Coarser Mesh Size Local Control}

The local chamber mesh size does not necessarily have to be finer than the residual-global mesh size. Mesh size at mesh points or vertices can be made coarser instead of finer. Our approach handles such "reverse variation" systems with ease and elegance. Sizes get bigger within the radius of influence and reduce to global size outside the local zone. Figure $14 \mathrm{~b}$ shows examples of reverse size variation.

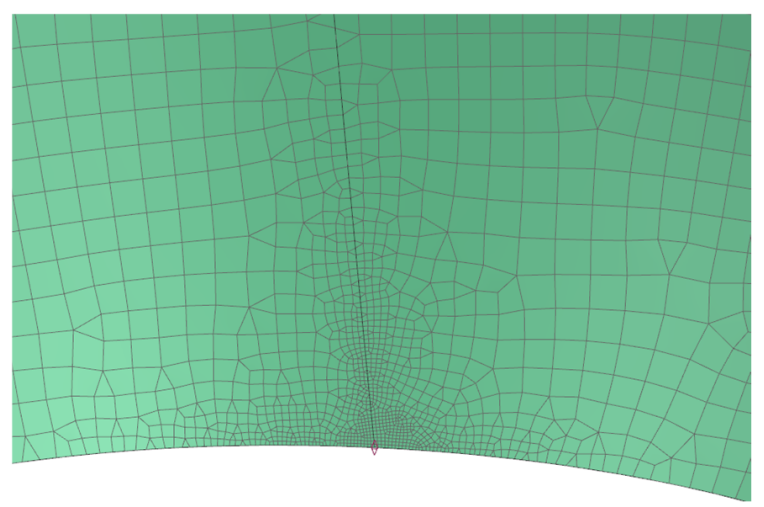

(a) 


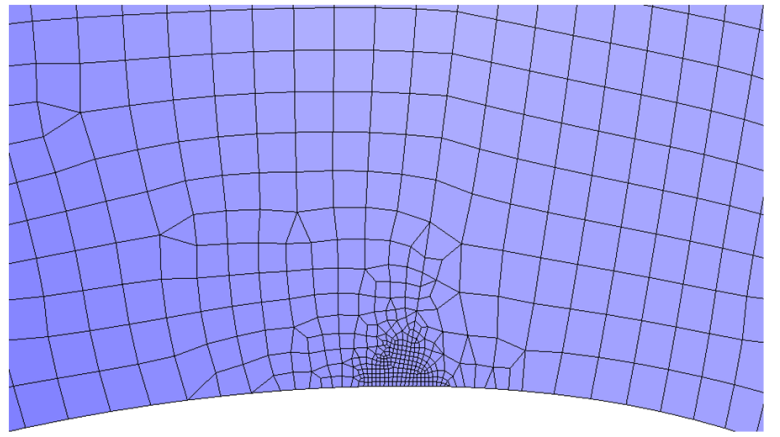

(b)

Figure 16. Size transition effect with controlled bicameral size distribution s on edges - H-shock based mesh size transition immediately beyond radius of influence (a) versus a staged constantparabolic-H-shock variation (b)

\subsection{Through-Layer Element Thickness Variation}

Bicameral mesh anisotropy by definition allows both controlled and uncontrolled mesh size variation in the local and global-residual chambers. For example, within the radius of influence, thickness of mesh layers can be varied by any function specified by the user. Figure 18 depicts a quad mesh on a fan casing (b) where a local box-size variation is applied at the center of the casing that follows a Fibonacci function.

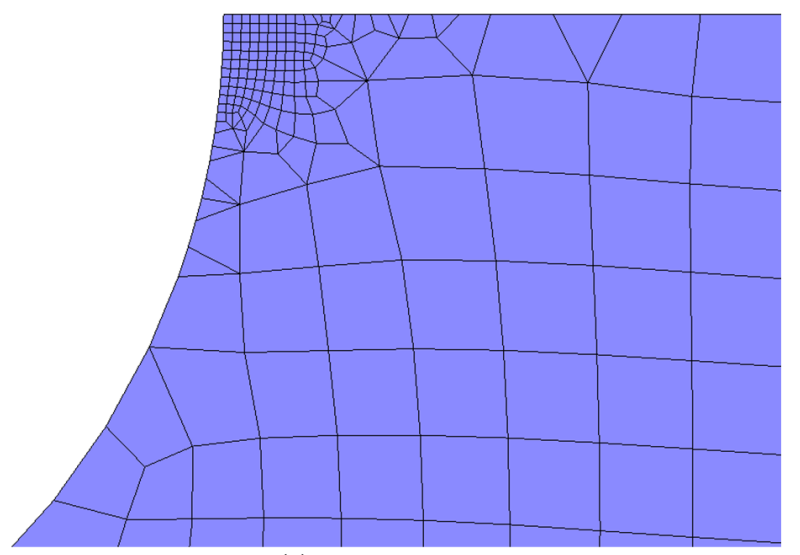

(a)

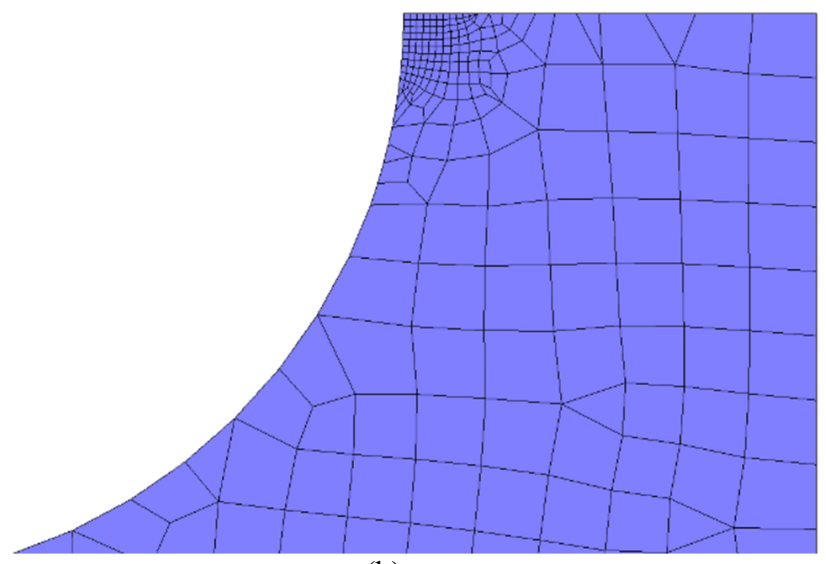

(b)

Figure 17. Size distribution schemes on edges with local size on vertex - geometric progression (a) versus parabolic (b)

A total box thickness (2L) of 20 is desired in 6 layers. The radii $R_{n}$ or half-thickness of each box layer variation can be expressed by the function family

$R_{n}=R_{n-1}+\left(F_{n} / \sum_{n=1}^{6} F_{n}\right) L$ where the Fibonacci sequence is expressed as $\left\{F_{n}\right\}_{n=1}^{6} ; R_{0}=0$ and the linear recurrence equation is

$F_{n}=F_{n-1}+F_{n-2}$ with $F_{1}=F_{2}=1$

The 6-layered local chamber mesh around the blue point of application on the fan cover is shown in Figure 18a.

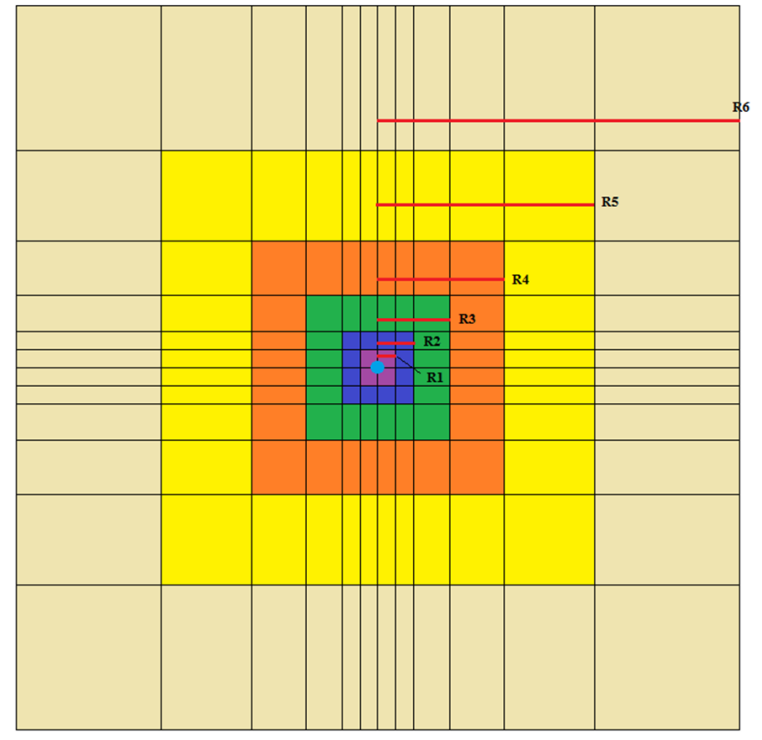

(a) 


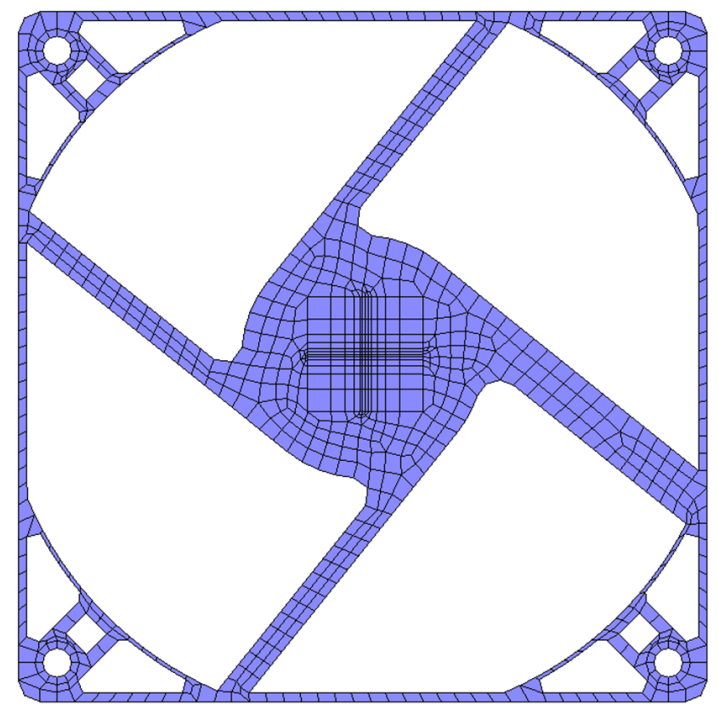

(b)

Figure 18. Six layers of Fibonacci size variation in a local chamber (a) on a fan casing (b)

\subsection{Mesh Size Transition in the Global- Residual Chamber}

Beyond the radius or boxes of influence of the local chambers lies the global-residual chamber, which is the rest of the surface area. In this chamber mesh size, on both edges and faces, is varied/transitioned following the previously published H-shock algorithm [6]. Borouchaki et al introduced notions of $\mathrm{H}$-variation and $\mathrm{H}$-shock associated with a control space and two correction procedures called $\mathrm{H}$ corrections. Size variation in a control space can be defined in two different ways - gradient of a size function $\mathrm{h}$, and the ratio of the Euclidean length of two adjacent edges. The Hvariation measures the gradient of the function $h$, while $H-$ shock represents the mesh gradation along an edge PQ and measures the distortion of interpolation function $h$ along it. The $\mathrm{H}$-correction factors tune the size variation factor at a point on the surface in all directions. An H-shock sizing function is developed based on the size-map or the background mesh used. For any edge $\mathrm{AB}$ of the triangular background mesh of length $\mathbf{I}_{\mathbf{A B}}$ in Euclidian space, the $\mathrm{H}$ shock size for the edge is expressed as

$h_{c}(A B)=\max \left(\frac{h(B)}{h(A)}, \frac{h(A)}{h(B)}\right)^{\frac{1}{l_{A B}}}$

which represents the mesh gradation along the background element edge $\mathrm{AB}$. This is a measure of the distortion of the sizing function $\mathrm{h}$ along the edge. This $\mathrm{H}$-variation can be defined at the vertices of the background mesh by considering the measures related to the adjacent edges. An edge shock (H-correction) correction is also used. The edge shock correction tries to guarantee that the size shock is bounded by a certain user defined value $\beta$.

We set out to assume a geometric variation of the size function

$h_{t}=h_{A}\left(\frac{h_{B}}{h_{A}}\right)^{t} \quad$ and

$l_{A B}=|A B| \int_{t=0}^{1} \frac{1}{h_{t}} \cdot d t$

Analytically equation (10) can be recast as

$l_{A B}=|A B| \frac{h_{A}-h_{B}}{h_{B} \cdot h_{A} \cdot \log \left({ }^{h_{B}} / h_{A}\right)}$

A dilemma arises when $h_{B}>h_{A}$ and $h_{c}>\beta$ around the decision about which one to keep. We vouch for the lower value. Under this condition, $h_{B}$ is modified as

$h_{\text {Bnew }}=\gamma h_{B}$ where $\gamma=\frac{\beta}{h_{c}(A B)}$

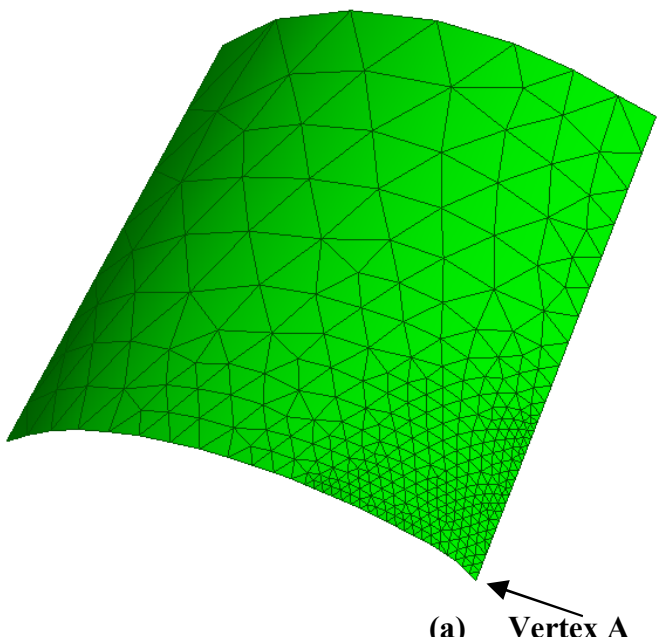

(a) Vertex $\mathrm{A}$

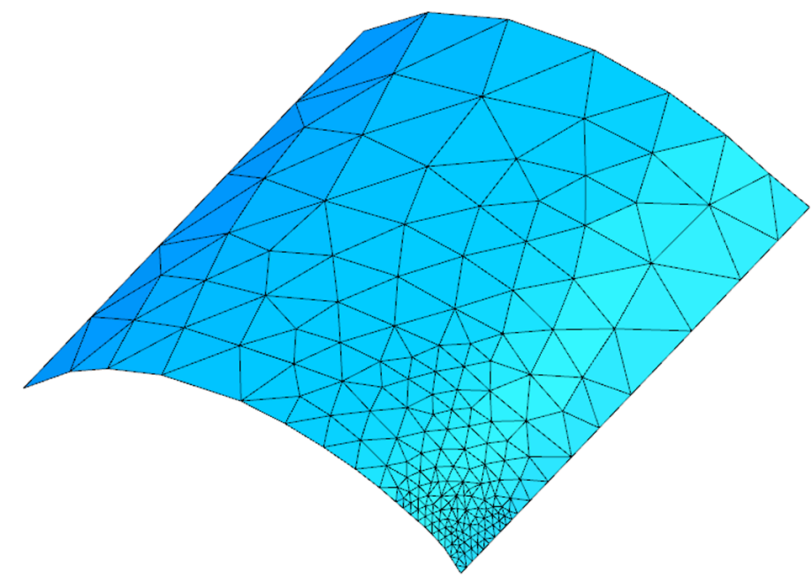

(b)

Figure 19. Comparison of size transition effect on a curved face with local size on vertex. H-shock transition (a) versus Bicameral anisotropy (b). 
Figure 19 shows two images of transitioning meshes on a curved plate with local size on one vertex. The global size $\mathbf{S g}=8$ and local size $\mathbf{s} \mathbf{l}$ at the lower right vertex $\mathrm{A}=0.5$ with a radius of influence $\mathbf{R}=5.0$. Figure 19 a shows the H-shock variation in comparison to bicameral anisotropy in 19b. In Figure 20. we compare the size variation along the diagonal line joining opposite vertices of the face starting at vertex A. With $\mathrm{H}$-shock type variation alone, the size field normalizes to global size at a much flatter gradient as is evident in the figure. In the case of bicameralism, the element size sharply grows to global size outside the zone of local influence.

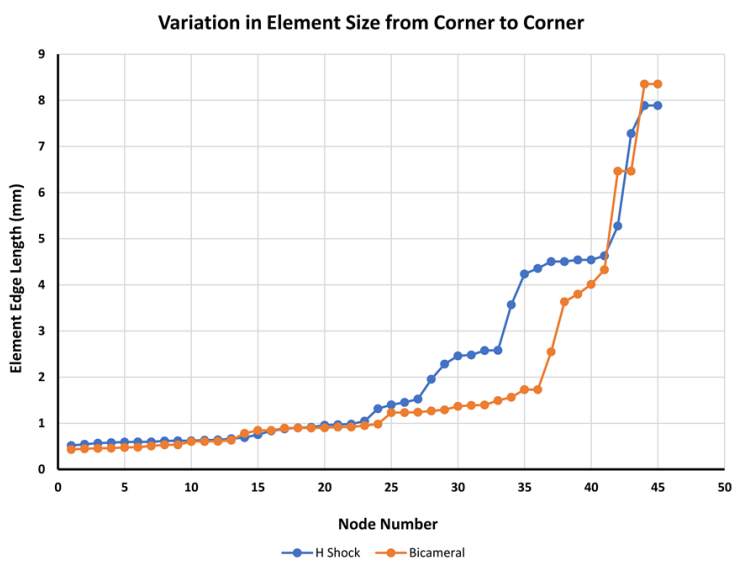

Figure 20. Comparison of variation in element size between H-Shock Variation and Bicameral Anisotropy.

\subsection{Weighted Half-Edge Method (WHEM) Flattening}

The present meshing algorithm is developed in $2 \mathrm{D}$ and is employed on the flattened 2D domain of the face. The Weighted Half-Edge Method for 2D parameterization of a tessellated 3D face has been developed at Simulation and Test Solutions, Siemens over the past decade by Beatty \& Mukherjee [13]. The method developed for both linear and nonlinear problems uses a compromise between conformal mapping (preserving triangle shape) and authalic mapping (preserving area or triangle altitude) to generate 2D domains with highly reduced transformational distortion. The meshes (both local and global) are generated in this 2D domain and transformed back to 3D space.

\subsection{Controlled Variational Smoothing}

The final mesh produced on the entire surface is smoothed by a variational smoother [14] which applies a variety of algorithms for node movement depending on the valency of the node (i.e. the number and types of elements connected to each node). For both triangular and quadrilateral meshing we create ideal or perfect quality elements within the radii of influence. Therefore, care is taken to not disturb them too much during mesh smoothing. The variational smoother is modified by inverse length-weighing to ensure mesh size gradients are not flattened out too much. Inverse length weighing constrains the smoother within the radii of influence so as not to disturb the elements created inside those zones or sub-areas.

\section{ADVANTAGES OF ADVANCING FRONT APPROACH IN BICAMERAL ANISOTROPY}

There are many ways of dealing with functional control of local and global anisotropy and their interaction, where the field equations varies. The use of a novel advancing front approach in such problems of bicameral anisotropy, as described in this paper, has many advantages and unique properties. Let us try to quantify the benefits in terms of its performance, efficiency and cost.

\subsection{Uniqueness}

State-of-the-art mesh sizing algorithms, firstly, are known to handle triangular meshes effectively. Not much is known about mesh size control of quadrilateral meshes. Secondly, the size variation in the mesh with existing algorithms is governed by a generic field-type or single function solution. This paper proposes a completely new approach of using a controlled advancing-front technique with both triangles and quadrilaterals to handle local size within a radius of influence. Outside the radius, a more generic field type approach is used. Furthermore, the local size control provided is point-based and thus offers size variation with more accuracy than existing algorithms.

\subsection{Flexibility}

The proposed mesh size control algorithm is designed to be flexible. It can handle mesh size control at vertices of edges and the interior of edges and faces. Being point and radius based, this size control algorithm is more pin-pointed than other algorithms both in terms of location and radius of influence. When zones of local sizing overlap, the algorithm resolves conflict is an elegant manner by giving proper weightage to the zones in proportion to the geodesic distance between them and their radii of influence. Additionally, since the mesh generated within the radius of control uses a specific algorithm - advancing front, irrespective of the global algorithm of mesh generation used, the local mesh conforms to a particular pattern. This is vastly unlike existing algorithms where mesh size or grading is expressed as a field function and the global meshing algorithm follows it. For different global meshing algorithms the type of mesh generated within the radius of influence, in such cases, will look and feel different. With the algorithm we propose, the mesh within the radius of influence will always look the same irrespective of the global meshing algorithm. 


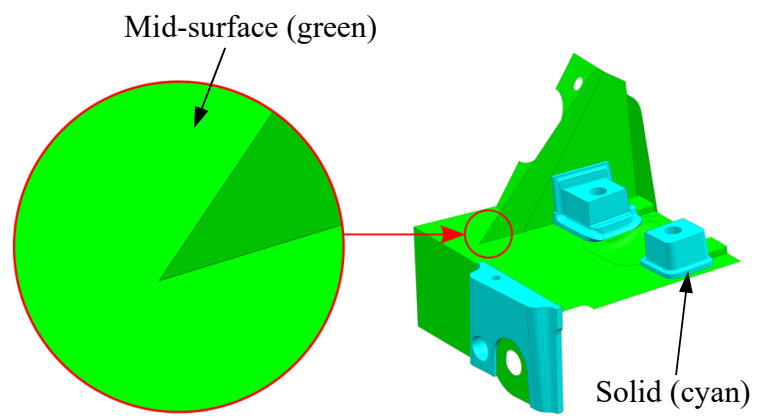

Sharp corner at mid-surface intersection

(a) Mixed dimensional model

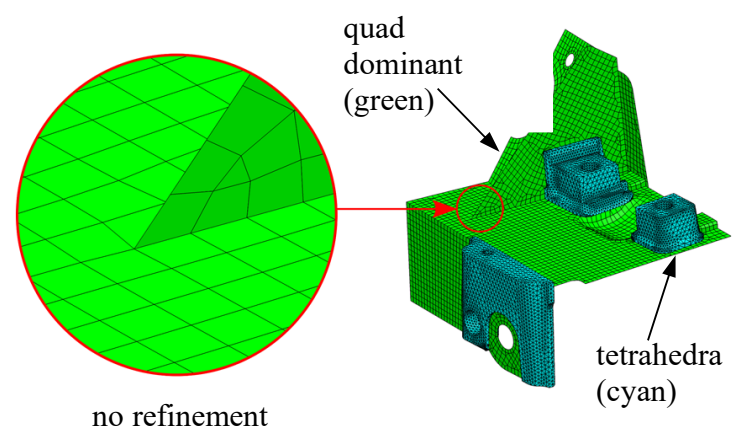

(b) Mixed dimensional mesh

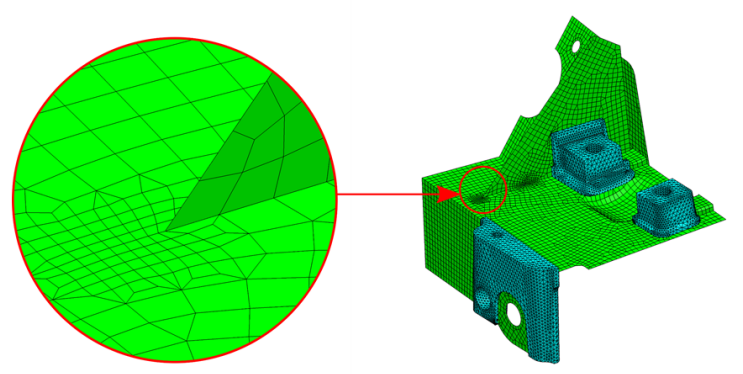

refinement to $0.125 \mathrm{x}$ global size

(c) Refined mixed dimensional mesh

Figure 21. Mesh refinement around sharp features in mixed-dimensional meshing

\subsection{User Control}

A great deal of user control is provided in a pin-pointed manner. Such accurate user-control also makes it stand apart from existing methods. First of all, the user gets to control local mesh sizing on a surface in three specific ways - a) at a vertex on its boundary $b$ ) in the interior of a boundary edge and c) in the interior of the face at specific locations. Figure 21 describes a typical example of how the aforementioned functionality is used in industry today. In mixed dimensional meshing where vast portions of thin-sheet volumes are represented by mid-surfaces, very often at $\mathrm{T}$-junctions, where the mid-sheets intersect sharp corners may occur. To improve the accuracy of the solution in this region it is often desirable to increase the density of the mesh, as shown in figure 21c. Secondly, the spread of the local size is controlled by a radius of influence. Thirdly, the local mesh produced is layered irrespective of the finite element type. This provides, as the fourth control option, a tool to the user to vary thickness of the layer in the first chamber according to certain standard functions like constant, ramp, sinusoidal or parabolic.

\section{CONCLUSION}

This paper introduces a new type of anisotropy called "Bicameral Anisotropy". Particularly prevalent in automotive, aerospace and electronic industries, bicameral anisotropy is about different size fields in local chambers versus the residual-global chamber that jointly make up the entire geometry meshed. The paper discusses, for a twodimensional problem, a new approach to controlling local variable sizing.

The first chamber is controlled by constant to linear local size functions. The second chamber, which is called the Residual-Global chamber making up the rest of the geometry, is governed by a nonlinear sizing function leading to transitioning meshes. We propose a controlled advancing front method, in both triangular and quadrangular meshes, that guarantees very high quality, well-controlled local quality metric in the first chamber. An H-shock sizing scheme governs the second chamber. Virtual mesh topology, and loop-segments are constructed at the face boundary both at geometry and nodeloop levels to facilitate this type of bicameral meshing. A wide range of user-control is provided which can allow for flexibility in varying the size-functions on both boundary and in the face-interior of the local chambers. Results clearly indicate the efficacy of the proposed approach leading to both a well controlled desired size field and high local quality in a completely automatic mode. 


\section{REFERENCES}

[1] M. J. Castro-Diaz, F. Hecht and B. Mohammadi, 'New progress in anisotropic grid adaptation for inviscid and viscid flows simulation', 4th Int. Mesh Roundtable, Albuquerque, New Mexico (1995).

[2] H. Borouchaki, P. L. George and B. Mohammadi,'Delaunay mesh generation governed by metric specifications. Part II: applications', Finite Elements Anal. Des., Vol.25, pp. 85 -109 (1997).

[3] M. Fortin, M. G. Vallet, J. Dompierre, Y. Bourgault and W. G. Habashi, 'Anisotropic mesh adaption: theory, validation and applications', Eccomas' 96, Paris, CFD Book, pp.174-199,(1996).

[4] R. Verfurth, A Review of a Posteriori Error Estimation and Adaptive Refinement Techniques, Wiley, Teubner (1996).

[5] J. Peraire, M. Vahdati, K. Morgan and O.

C.Zienkiewicz, 'Adaptive remeshing for compressible flow computations', J. Comput. Phys., Vol.72, pp.449 466 (1987).

[6] H. Borouchaki, F. Hecht, P.Frey, 'Mesh gradation control', Int. J. Numer. Meth. Engng. Vol.43, pp.11431165 (1998).

[7] A. Loseille, A Deriveux, F. Alauzet. 'Fully anisotropic goal-oriented mesh adaption for 3D steady state Euler equations. J. Comput. Phys., Vol 229. pp. 2866-2897 (2010).

[8] Leicht, T, Hartmann, R. Error estimation and anisotropic mesh refinement for $3 \mathrm{~d}$ laminar aerodynamic flow simulations., J. Comput. Phys. Vol 229. pp. 7344-7360 (2010).

[9] T. Blacker and M. Stephenson, 'Paving: A new approach to automated quadrilateral mesh generation', Int. J. Numer. Meth. Engng., Vol. 32, pp.811-847 (1991).

[10] R. Lohner, 'Progress in grid generation via the advancing front technique', Engng. With Computers, Vol.12, pp.186-210 (1996).

[11] N. Mukherjee, 'A Combined Subdivision and Advancing Loop-Front Surface Mesher (Triangular) for Automotive Structures', Int. J. Vehicle Struct. \& Systems, 2(1), pp.28-37 (2010).

[12] N. Mukherjee, 'CSALF-Q: A Bricolage Algorithm for Anisotropic Quad Mesh Generation', Proc. XXth International Meshing Roundtable, Paris, France, pp. 489-510, Springer (2011).

[13] K. Beatty, N. Mukherjee, 'A Transfinite Meshing Approach for Body-In-White Analyses'. Proc. 19 $9^{\text {th }}$
International Meshing Roundtable, Springer, pp.49-65 (2010).

[14] N. Mukherjee, 'System, method, and computer program product for smoothing', patent, US 9082220 B2, (July 14, 2015). 


\title{
PRE-CONDITIONING AND CONTINUATION FOR PARALLEL DISTRIBUTED MESH CURVING
}

\author{
Eloi Ruiz-Gironés Xevi Roca \\ Computer Applications in Science and Engineering, Barcelona Supercomputing Center - BSC, \\ 08034 Barcelona, Spain. \{eloi.ruizgirones, xevi.roca\}@bsc.es
}

\begin{abstract}
To generate large-scale meshes with highly stretched elements for complex geometries, we propose a distributed parallel Newton-GMRES penalty solver. For each non-linear iteration, we solve a pre-conditioned sparse linear problem. To choose the parallel pre-conditioner, we compare an algebraic multi-grid implementation with a restricted additive Schwarz domain decomposition with one level of overlap and local problems approximated with symmetric successive over-relaxation. We show that domain decomposition is faster and more energy efficient. Furthermore, to accelerate the penalty based solver, we propose a novel $p$-continuation technique with two unique features. First, it has an early termination criterion to stop the optimization of the initial polynomial degrees. Second, it estimates the initial penalty parameter for each polynomial degree. We conclude that this continuation can reduce four times (eight times) the wall clock time (energy per core) required to curve a whole boundary layer quartic mesh using the chosen domain decomposition.
\end{abstract}

Keywords: High-order mesh curving, parallel, pre-conditioner, $p$-continuation

\section{INTRODUCTION}

Curved high-order meshes are required for unstructured high-order methods to keep their advantages [1-5]. These advantages come in the form of geometric flexibility, high accuracy, and low numerical dissipation and dispersion. High-order methods feature exponential convergence rates and therefore, they have been proven to be faster than low-order methods in several applications [6-14], especially in those problems where an implicit solver is required [15].

Usually, to generate a curved high-order mesh an $a$ posteriori approach is used [16-26]. First, a linear mesh with elements of the desired shape and size is generated, and then, the mesh boundary is curved to match the target geometry. This step may introduce low-quality and inverted elements that have to be repaired using a high-order mesh curving technique. There are several manners to formulate the mesh curving problem: PDE-based methods like solid mechanics analogies [21,24,27-30] or the Winslow equation [25], and optimization-based methods [23, 31-34]. However, none of these methods can run in a distributed parallel environment while taking into account virtual geometry to generate large curved high-order meshes of complex domains, as we detail in Section 2. This capability, is critical to perform in parallel unsteady (fine graded meshes) or steady state (fine meshes with stretched elements) flow simulations for large Reynolds numbers with unstructured high-order methods.

To generate fine graded and stretched curved highorder meshes in parallel, we parallelize a high-order mesh curving formulation that takes into account virtual geometry [35]. The previous method poses the mesh curving problem as a constrained optimization problem in which the mesh distortion is minimized constrained to a non-linear boundary condition. This non-linear constraint allows different types of boundary conditions such as fixed boundary nodes, nodes sliding on the geometry, and nodes sliding on top of 
virtual entities. The constrained optimization is solved using a penalty method in which the boundary condition is introduced into the objective function using a penalty parameter. Then, several optimization problems are solved while increasing the penalty parameter to enforce the boundary condition. he optimization process is devised to favor that at each optimization step, a valid mesh is deformed to a valid mesh. To this end, two main ingredients have been considered. The first one is a functional that penalizes inverted elements by taking an infinite value. The second ingredient is a backtracking line search to Newton's method. Thus, if a Newton full step were deforming a valid mesh to an invalid mesh, leading to an infinite value of the functional, the backtracking line-search would decrease the step length until a valid mesh is obtained, leading to a finite value.

To solve the large linear problems arising in the global optimization of large curved high-order meshes, we need a pre-conditioned distributed parallel linear solver. The pre-conditioner is required since fine graded meshes with highly stretched elements hamper the condition number of the linear systems. The distributed parallel linear solver allows accommodating the large linear systems across the memory of different machines.

To select the parallel pre-conditioner, we have to consider the influence of the number of elements, element stretching, and the number of cores used by the linear solver. This consideration is so since when higher is the number of elements and their stretching more illconditioned is the linear system and thus, greater is the number of linear iterations. Moreover, increasing the number of cores might reduce the capacity of the parallel pre-conditioner to propagate the information across the mesh boundaries.

The first contribution of this work focuses on the selection of pre-conditioners for the linear problems that arise during the curving of high-order meshes. We consider two pre-conditioners, and we compare the time to solve the linear problems, the number of iterations of the linear solvers, and the energy consumption to generate the high-order curved mesh. As the method uses more computational resources during more time, the economic cost of generating the high-order mesh also increases. Therefore, it is important to devise efficient mesh curving methods in terms of computational resources like time and memory. This consideration is especially important when curving large-scale meshes in supercomputers where the computational resources are directly translated into energy consumption and economic costs.

In the second contribution, we develop a $p$ continuation technique to increase the robustness and computational efficiency of the mesh curving process.
The main idea is to use the converged solution of a given polynomial degree as the initial condition for the next polynomial degree. Thus, the $p$-continuation technique can be interpreted as a methodology to compute the initial condition for the final polynomial degree.

We propose two unique features in the $p$-continuation technique to reduce the number of linear and nonlinear problems to solve, and to improve the robustness of the optimization. The first novelty is to introduce an early-termination criterion to finalize the optimization of the initial polynomial degrees. Using this criterion, we avoid solving additional non-linear problems, and therefore, the use of computational resources is reduced. The second novelty is to estimate the value of the penalty parameter in the first iteration of each polynomial degree. The value of the penalty parameter is critical to converge the mesh curving process. If the penalty parameter is too high, the linear problems become more difficult, and the linear solver may not be able to solve the linear problem.

The rest of the paper is structured as follows. Section 2 reviews the literature related to the presented work. Section 3 presents the formulation of the proposed high order mesh curving methodology. Section 4 presents several examples to show the capabilities of the proposed formulation. Finally, Section 5 details the conclusions of this work.

\section{RELATED WORK}

Mesh curving methods can be divided into global and local methods. Global methods move all the nodes at the same time, while local methods move one node at a time. Global methods can be further divided into implicit methods that need to solve a sparse linear system, and explicit methods that move the nodes using an explicit formula. Note that herein, global (local) methods do not refer to obtain the global (local) minimum of an objective function.

One of the main bottlenecks in global implicit methods is the solution of a sparse linear problem to relocate all the mesh nodes at the same time. Therefore, efficient sparse linear solvers and pre-conditioners are necessary to curve high-order meshes composed of a large number of elements. In several works, the linear systems are solved using sparse direct solvers for morphing linear meshes [36], and curving highorder meshes [29,37]. Nevertheless, when the number of elements in the mesh increases, it is necessary to use iterative solvers. In general, the linear problems are solved either with MINRES, GMRES or conjugated gradients $[25,34,38-40]$. To improve the computational efficiency, the linear problems can be pre-conditioned in different manners. For instance, 
diagonal pre-conditioners [38], incomplete factorizations $[25,39]$, or especially designed pre-conditioners for the mesh curving problem [41]. Global methods need to assemble a sparse matrix and solve the associated linear problem. Therefore, the parallelization of these methods need to assemble the matrix in parallel and apply a parallel sparse iterative linear solver, see $[34,35,40]$.

To avoid solving a sparse linear system, reference [42] relocates the nodes using a first order steepest descent minimization method. That is, the method relocates the nodes using a multiple of the objective function gradient. Although the convergence rate is lower than using Newton's method, the iterations are performed faster and the memory requirements are reduced.

Instead of solving a fully coupled linear problem to move all the nodes at the same time, local approaches move one node at a time. This approach is both applied for linear meshes $[43,44]$ and high-order meshes $[22,26,45]$. Although the local approach uses less memory and each iteration is faster than in the global approach, the convergence rate to the optimal solution can be hampered. In local approaches, nodes that do not belong to the same element can be relocated at the same time. Thus, several authors propose to color the nodes of the mesh in such a manner that nodes of the same color can be relocated at the same time $[26,43,44]$. Moreover, in [44], they propose a mesh partitioning method to ensure that the cost to relocate the nodes in each sub-domain is roughly the same.

In the proposed work, we propose a distributed memory implementation that moves all the nodes at the same time. To accomplish this, we solve a sparse linear system of equations derived from Newton's method. Therefore, we expect faster convergence rates than first-order methods or local methods.

\section{PARALLEL MESH CURVING SOLVER}

We first briefly summarize the proposed mesh curving solver and non-linear solver, as presented in [35], and then introduce the new $p$-continuation technique to increase the robustness and the efficiency of the method.

\subsection{Mesh Curving Problem}

Given an initial linear mesh, $\mathcal{M}_{I}$, we want to characterize a curved high-order one, $\mathcal{M}_{P}$, in terms of a diffeomorphism $\phi^{*}[45,46]$. The optimal diffeomorphism presents optimal point-wise distortion, and satisfies a prescribed boundary condition. That is, $\phi^{*}$ is the minimizer of

$$
\begin{aligned}
& \min _{\boldsymbol{\phi} \in \mathcal{V}} E(\boldsymbol{\phi})=\|M \boldsymbol{\phi}\|^{2} \\
& \text { subject to: } \\
& \boldsymbol{T} \boldsymbol{\phi}=\mathbf{g}_{D}(\boldsymbol{T} \boldsymbol{\phi}),
\end{aligned}
$$

where $\boldsymbol{T}$ is the trace operator, $\mathbf{g}_{D}(\boldsymbol{T} \boldsymbol{\phi})$ is a non-linear Dirichlet boundary condition on $\partial \mathcal{M}_{I}$ that depends on the values of $\phi$, and

$$
M \phi(\mathbf{y})=\eta(\mathbf{D} \phi(\mathbf{y}))=\frac{\|\mathbf{D} \phi(\mathbf{y})\|^{2}}{n \sigma_{0}(\mathbf{D} \phi(\mathbf{y}))^{2 / n}}
$$

is a regularized point-wise distortion measure [23] defined in terms of the shape distortion measure for linear simplices [47], where $\|\cdot\|$ is the Frobenius norm for matrices, and

$$
\sigma_{0}=\frac{1}{2}(\sigma+|\sigma|)
$$

being $\sigma(\cdot)$ the determinant function. The regularized distortion measure takes a value of infinity when the determinant is negative or equal to zero, and takes finite values when the determinant is positive.

The non-linear boundary condition allows integrating a geometric model in the mesh curving process. In this work, we define the boundary condition as

$$
\mathbf{g}_{D}(\boldsymbol{T} \boldsymbol{\phi})=\sum_{i=1}^{N_{b}} \Pi\left(\mathbf{x}_{i}\right) N_{i}^{b},
$$

where $\mathbf{x}_{i}$ are the coordinates of the mesh nodes, $N_{b}$ is the number of boundary nodes, $\left\{N^{b}\right\}_{i=1, \ldots, N_{b}}$ is a Lagrangian basis of shape functions continuous between adjacent boundary faces, and $\Pi(\cdot)$ is a geometric orthogonal projection onto the CAD model. The boundary condition can be interpreted as in interpolation of the geometric model, in which the interpolation points are the projection of the boundary nodes. This boundary condition is non-linear and depends on the orthogonal projection of the boundary nodes.

\subsection{Mesh Curving Non-Linear Solver}

To solve the constrained optimization problem in (1), we use a penalty approach, see [48], in which we introduce the boundary constraint into the objective function in a weak sense as follows

$$
\min _{\phi \in \mathcal{V}} E_{\mu}(\phi)=\frac{E(\phi)}{\|1\|_{\mathcal{M}_{I}}^{2}}+\mu \frac{\left\|\boldsymbol{T} \boldsymbol{\phi}-\mathbf{g}_{D}(\boldsymbol{T} \phi)\right\|_{\partial \mathcal{M}_{I}}^{2}}{\|1\|_{\partial \mathcal{M}_{I}}^{2}}
$$

where $\mu$ is a penalty parameter that enforces the validity of the constraint when it tends to infinity. We have introduced the measures of the initial mesh and its boundary in order to balance the two contributions of the new functional. 
The main idea is to solve several unconstrained optimization problems with increasing penalty parameter in order to enforce the boundary condition. Nevertheless, the boundary condition depends on the actual solution of the problem. Thus, we apply a fixed-point iteration as

$$
\begin{aligned}
\mathbf{g}_{D}^{k} & =\mathbf{g}_{D}\left(\boldsymbol{T} \boldsymbol{\phi}^{k}\right), \\
\phi^{k+1} & =\underset{\phi \in \mathcal{V}}{\arg \min } E_{\mu}\left(\phi ; \mathbf{g}_{D}^{k}\right),
\end{aligned}
$$

being $k$ the $k$-th iteration of the proposed fixed-point solver.

We optimize each non-linear problem of the proposed penalty method using a backtracking line-search method in which the advancing direction is computed using Newton's method and the step-length is set using the Armijo's rule, see [48] for more details.

\section{$3.3 p$-Continuation Technique}

To improve the robustness of the proposed solver and to compute an initial condition for the non-linear solver, we propose to apply a $p$-continuation technique. Instead of directly computing the optimal mesh for a given polynomial degree, we iterate through the polynomial degrees and optimize them. The initial condition for each polynomial degree is the optimized mesh of the previous one. There are two main new contributions in our $p$-continuation technique.

The first contribution is an early termination criterion to stop the optimization of the initial polynomial degrees. The proposed early termination criterion reduces the computational cost of the full optimization process. To this end, we consider the mesh of the current polynomial degree, $\phi^{p}$, and let $\phi^{p+1}$ be the interpolation of $\phi^{p}$ using element-wise polynomials of degree $p+1$. The early termination criterion is defined using the boundary condition error of both meshes as

$$
\alpha\left\|\boldsymbol{T} \phi^{p}-\mathbf{g}_{D}\left(\boldsymbol{T} \phi^{p}\right)\right\|<\left\|\boldsymbol{T} \phi^{p+1}-\mathbf{g}_{D}\left(\boldsymbol{T} \phi^{p+1}\right)\right\| .
$$

That is, the optimization process of the current polynomial degree is finished when the error of the boundary condition is comparable to the error of the boundary condition of the next polynomial degree. In this work, we take $\alpha=2$.

The second contribution is the calculation of the initial penalty parameter for the optimization of each polynomial degree. A correct value of the penalty parameter facilitates the solution of the linear and non-linear problems and therefore, increases the robustness of the optimization process. For a sufficiently large penalty parameter, after optimizing the functional in Equation (4), the Lagrange multipliers of the associated constraint are approximated as

$$
\lambda^{p} \simeq-2 \mu^{p} \frac{\left\|\boldsymbol{T} \boldsymbol{\phi}^{p}-\mathbf{g}_{D}\left(\boldsymbol{T} \boldsymbol{\phi}^{p}\right)\right\|}{\|1\|_{\partial \mathcal{M}_{I}}} .
$$

When the early termination criteria is satisfied, we interpolate $\phi^{p}$ using polynomials of degree $p+1$, and compute the associated boundary condition of $\phi^{p+1}$. Assuming that the associated Lagrange multipliers approximated using Equation (6) are similar for both polynomial degrees, we consider

$$
\lambda^{p}=\lambda^{p+1} \text {. }
$$

Therefore, we obtain that

$$
\mu^{p+1}=\mu^{p} \frac{\left\|\boldsymbol{T} \phi^{p}-\mathbf{g}_{D}\left(\boldsymbol{T} \phi^{p}\right)\right\|}{\left\|\boldsymbol{T} \phi^{p+1}-\mathbf{g}_{D}\left(\boldsymbol{T} \phi^{p+1}\right)\right\|} .
$$

\subsection{Parallel Pre-Conditioned Linear Solver}

At each iteration of the non-linear solver we solve the following linear system derived from Newton's method:

$$
\mathbf{H} E_{\mu}(\mathbf{u}) \delta \mathbf{u}=-\nabla E_{\mu}(\mathbf{u})
$$

Since in this work we are dealing with large-scale meshes, we assemble and solve the linear system in a parallel framework. We use the FEniCS software to automatically generate the derivative expressions required to evaluate the Hessian and the gradient. Since we solve this problem in parallel, we need to distribute the mesh elements and degrees of freedom. To this end, we use the ParMETIS library. The resulting distribution allows computing the element contributions in a distributed manner. Specifically, we use the FEniCS software, where each elemental contribution to the residual and the residual Jacobian are computed in parallel. To this end, non-blocking communication is performed to communicate to send and receive the required data from adjacent processors. Then the contributions of the inner processor elements are computed and overlapped with the previously non-blocking communication. Then, the contributions from the processor boundary elements are computed. Finally, the residual and the residual Jacobian are assembled.

We have used the linear solvers and pre-conditioners implemented in the PETSc library [49]. We solve the linear systems using a GMRES method restarted every 20 iterations. To accelerate the convergence of the linear solver, we consider two pre-conditioners. The first one is a restricted additive Schwarz method with one overlap level. The local problems are approximated using two iterations of a symmetric successive over-relaxation method with $\omega=1$. The second preconditioner is an algebraic multi-grid method in which the coarsest level is solved using an LU decomposition. The number of coarsening levels is automatically computed by the PETSc implementation. 


\subsection{Algorithm Description}

Algorithm 1 describes the proposed penalty method with $p$-continuation technique for mesh curving. The input of the algorithm is an initial linear mesh $\mathcal{M}_{I}$, the final polynomial degree, $p_{\max }$, and the required tolerances for the boundary condition and the non-linear problem, $\varepsilon^{*}$ and $\omega^{*}$, respectively. In Lines $2-4$, we set the initial polynomial degree, and we initialize the quadratic mesh, $\phi^{2}$, to the identity mapping. That is, we introduce the additional nodes on the edges of the mesh, and we keep the straight-edged elements. The identity mapping is optimal with respect of the mesh quality however, it does not satisfy the boundary condition. The initial penalty parameter is set to 10 . In Line 5 we start the $p$-continuation loop, in which we iterate through all the polynomial degrees. Then, in Line 7 , we start the penalty method for the given polynomial degree. In Lines 8 and 9 we perform the fixed-point iteration. First, we update the boundary condition and then, we optimize the functional in Equation 4 to compute the new approximation of the optimal mesh. In Lines 10-16 we select the convergence criterion according to the current polynomial degree. If the convergence check passes and we are optimizing the last polynomial degree, the algorithm ends, Line 19. If the convergence check passes and we are not in the last polynomial degree, we compute the new penalty parameter, Line 21, according to Equation (7), and we perform the optimization of the next polynomial degree. If the convergence check fails, Line 24 , we increase the penalty parameter and tighten the tolerances.

\section{EXAMPLES}

This section presents several examples that show the capabilities of the presented high-order mesh curving method. Specifically, we show two three-dimensional weak scaling examples, and a large-scale example of a complex geometry with boundary layer.

To generate the initial linear meshes, we have used Pointwise [50]. The mesh curving solver has been implemented in Python [51] using the FEniCS [52] and the petsc4py [49] libraries. To project the boundary high-order nodes we have used both the geode [53] and the Open CASCADE [54] libraries interfaced with an in-house python wrapper developed using swig [55].

The optimization process has been performed in the MareNostrum4 super-computer located at the Barcelona Supercomputing Center. It is composed of 3456 nodes, connected using an Intel Omni-Path network. Each node contains two Intel Xeon Platinum $8160 \mathrm{CPU}$ with 24 cores, each at $2.10 \mathrm{GHz}$, and 96 GB of RAM memory. To obtain the total energy consumed by all tasks of the optimization job, we have

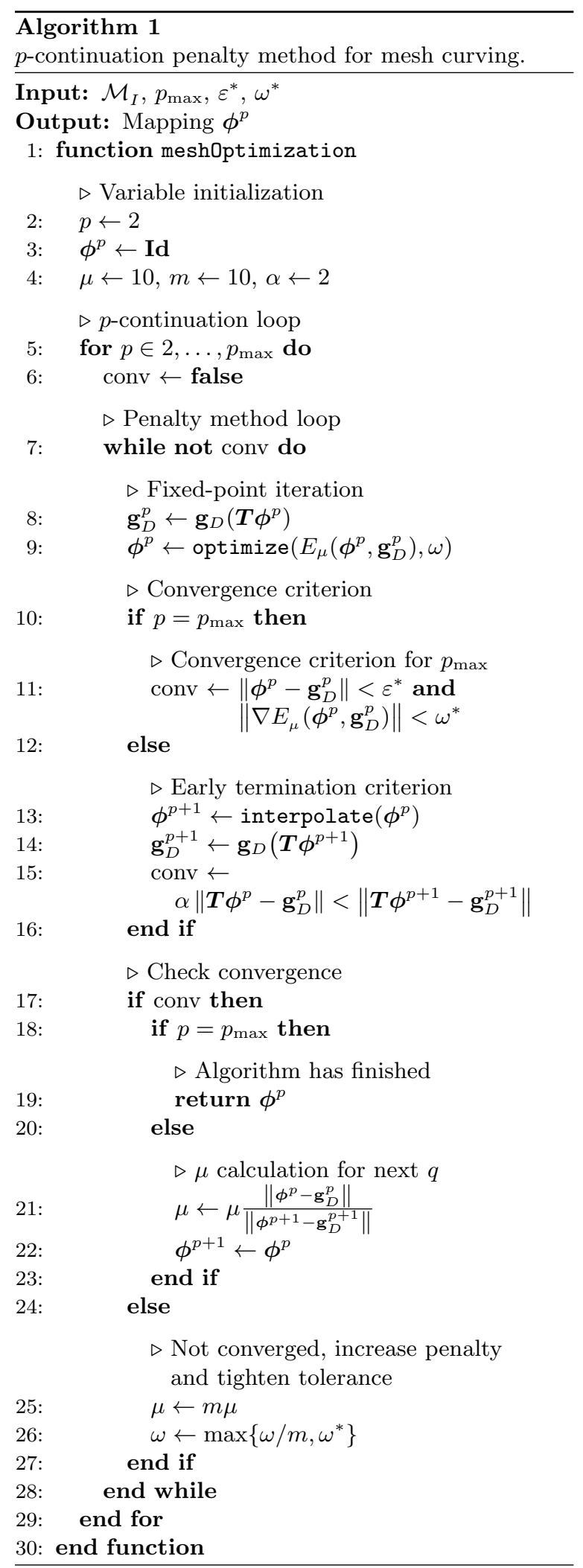


used the sacct command of the SLURM Workload Manager. Note that only in case of exclusive job allocation, this value reflects the real energy consumption.

The mesh visualization has been performed using Paraview 5.5.2 [56] in parallel in the MareNostrum4 supercomputer. We have used the high-order mesh visualization implementation of Paraview that subdivides each element in a given number of sub-elements. Note that the mesh partition to perform the visualization does not need to coincide with the mesh partition to perform the optimization. In general, for visualization purposes, less cores are needed since no global matrices are assembled and no linear systems are solved.

We compute the elemental quality relative to the initial mesh as [23]

$$
q_{e_{P}}=\frac{1}{\eta_{e_{P}}}, \quad \text { where } \eta_{e_{P}}=\left(\frac{\int_{e_{I}}(M \phi)^{2} \mathrm{~d} \Omega}{\int_{e_{I}} 1 \mathrm{~d} \Omega}\right)^{1 / 2} \text {. }
$$

The relative element quality takes values in the interval $[0,1]$. An ideal element has quality equal to one, and an inverted or tangled element has a quality of zero. In all the examples, we color the elements according to $1-q_{e_{P}}$ in logarithmic scale to check how close is the element quality to one. Thus, lower values denote higher quality elements.

In the examples, we compare the two pre-conditioners presented in Section 3.4. To faithfully compare both pre-conditioners, we solve the linear systems with a relative tolerance of $10^{-9}$. Thus, in all the cases, the evolution of the non-linear solver is not affected by the selection of the pre-conditioner, since the solution of the linear systems is numerically equal when different pre-conditioners are used.

In the examples we compare the two pre-conditioners presented in Section 3.4. To faithfully compare both pre-conditioners, we solve the linear systems with a relative tolerance of $10^{-9}$. Thus, in all the cases, the evolution of the non-linear solver is not affected by the selection of the pre-conditioner, since the solution of the linear systems is numerically equal when different pre-conditioners are used. Although using both pre-conditioners the optimal meshes are numerically equivalent, the figures show the optimal highorder meshes optimized using the additive Schwarz pre-conditioner.

\subsection{Weak Scaling: Isotropic Elements}

In this example, we perform a weak scaling analysis on the number of elements. We generate five isotropic meshes increasing the number of elements and the used cores in such a way that the number of elements per core remains constant. The domain is a sphere of radius four with a spherical hole in the center of radius one. The element sizes are chosen such that there are around 1500 elements per cores, and we have used 480, 960, 1440, 1920 and 2400 cores. This leads to meshes that are composed of $0.72 \cdot 10^{6}, 1.44 \cdot 10^{6}, 2.16 \cdot 10^{6}$, $2.88 \cdot 10^{6}$ and $3.60 \cdot 10^{6}$ elements of polynomial degree four. Figures 1a to 1e show the five curved high-order meshes generated for this example.

Figure 2 show the evolution of the constraint norm during the iterations of the non-linear optimization for the five meshes. At each non-linear iteration, we optimize the functional in Equation (4) and, if needed, the penalty parameter is increased in order to enforce the boundary condition. Dark blue circles denote the initial iteration of each polynomial degree in the $p$ continuation technique. The evolution of the constraint norm in all cases follows a similar pattern, even though the boundary mesh is not the same. Thus, we show that the proposed formulation presents a mesh independent behavior at the non-linear level. Specifically, the number of iterations to perform the whole optimization process is the same in all the cases and moreover, the number of non-linear iterations at each polynomial degree is also the same. During the first iterations of the quadratic mesh, the constraint norm decreases slowly. Nevertheless, from iteration three onwards, the constraint norm decreases geometrically with the non-linear iterations. When the constraint norm is of the same order as the constraint norm of the next polynomial degree, the early-termination criterion is activated. Thus, the norm of the boundary condition is similar to the norm of the boundary condition of the next polynomial degree. Then, we compute the new penalty parameter for the next polynomial degree. Note that the constraint norm also decreases geometrically with the non-linear iterations. Therefore, this shows that we have correctly selected the value of the penalty parameter.

In Figure 3 we present the time to solve all the linear problems, the total number of linear solver iterations, the energy consumption, and the energy consumption per core for the two pre-conditioners. We use the red, green and blue colors to denote the quantities of interest for the meshes of polynomial degree two, three and four of the $p$-continuation technique. Using both pre-conditioners, the time to curve the finer meshes is larger than the time to curve the coarser meshes, even when the number of elements per core is the same. The number of linear iterations to curve the quadratic mesh is significantly higher than the rest of the curving process, while the time spent on curving the initial polynomial degrees is significantly lower than the rest of the curving process.

While in the case of the additive Schwarz pre- 


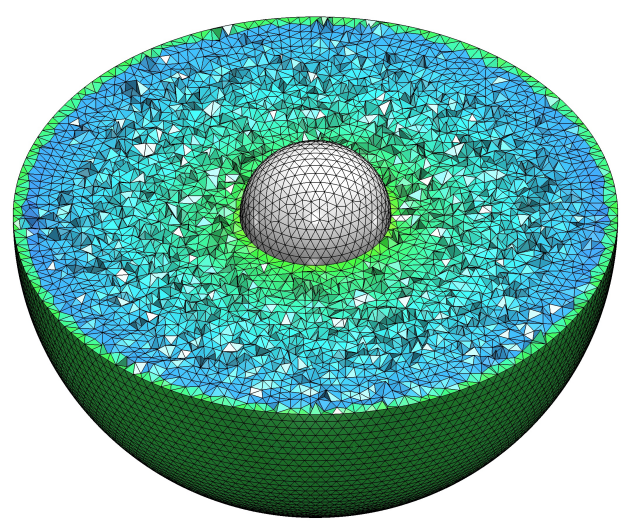

(a)

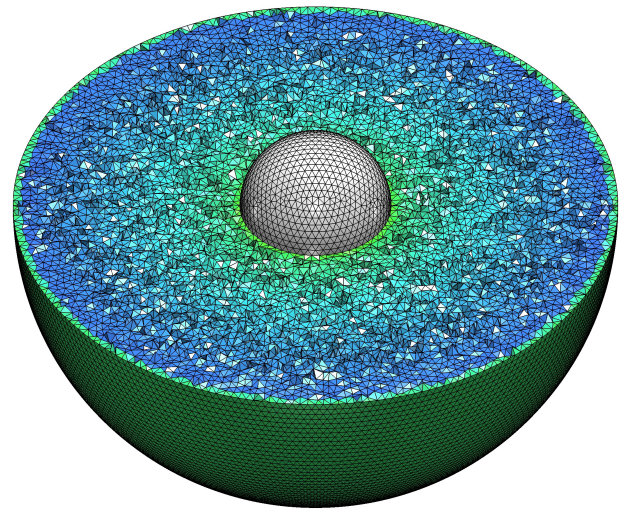

(c)

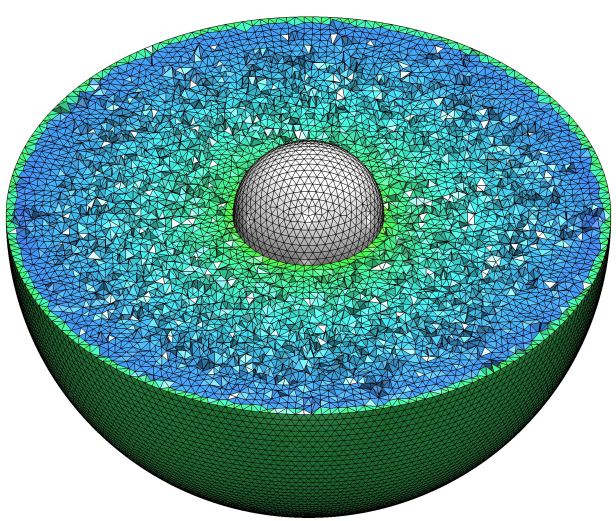

(b)

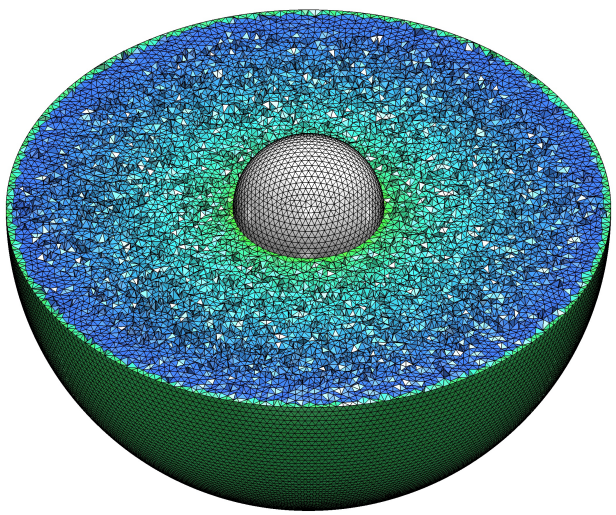

(d)

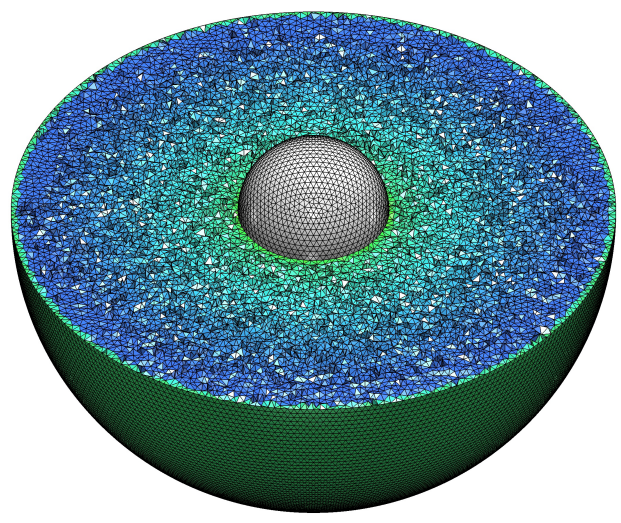

(e)

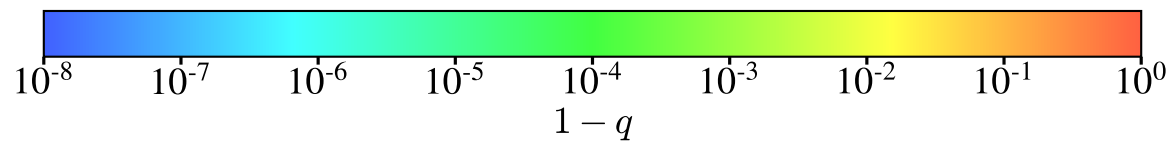

Figure 1: Optimized meshes of polynomial degree four using the proposed mesh curving solver, approximately composed of: (a) $0.72 \cdot 10^{6}$ elements on 480 cores; (b) $1.44 \cdot 10^{6}$ elements on 960 cores; (c) $2.16 \cdot 10^{6}$ elements on 1440 cores; (d) $2.88 \cdot 10^{6}$ elements on 1920 cores; and (e) $3.60 \cdot 10^{6}$ elements on 2400 cores.

conditioner the number of iterations of the linear solver increases, this is not the case when using the multi-grid pre-conditioner. However, the cost of each iteration is higher when using the multi-grid pre- 


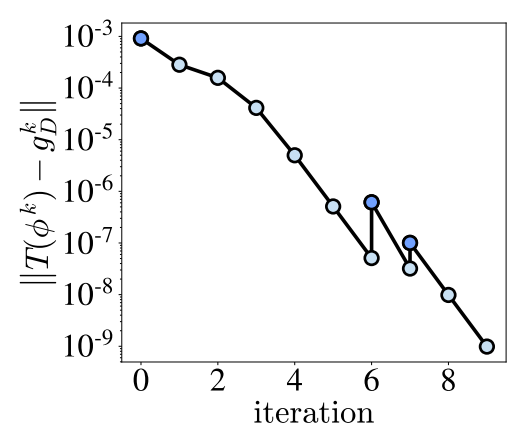

(a)

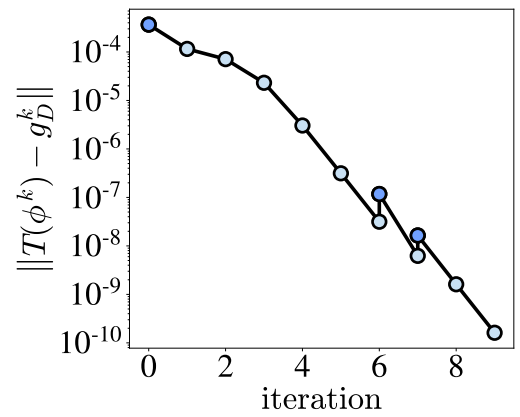

(d)

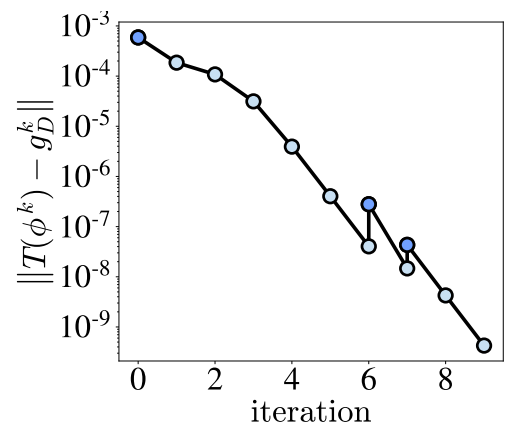

(b)

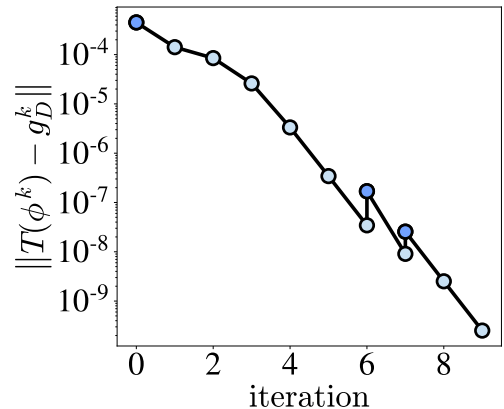

(c)

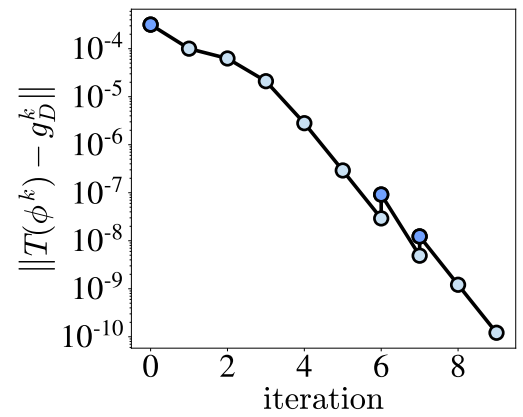

(e)

Figure 2: Evolution of the norm of the constraint through the optimization process for the mesh optimized with boundary layer stretching of: (a) 480 cores; (b) 960 cores; (c) 1440 cores; (d) 1920 cores; and (e) 2400 cores.

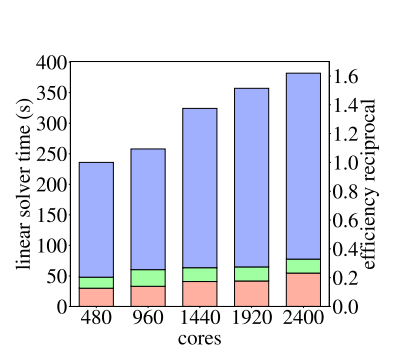

(a)

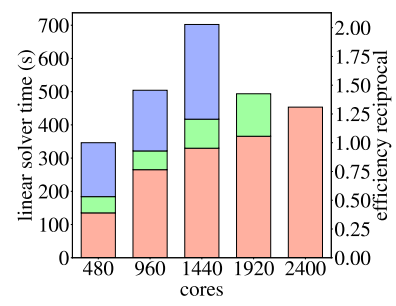

(e)

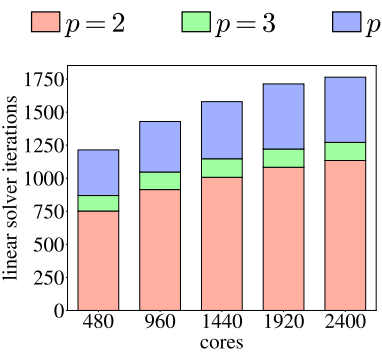

(b)

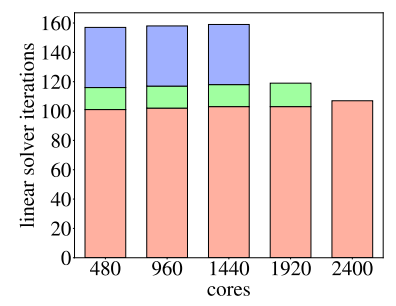

(f)

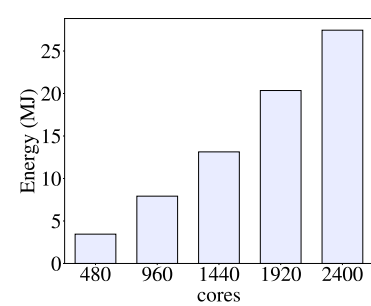

(c)

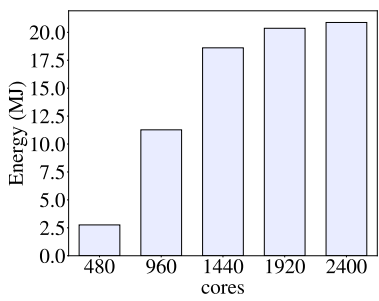

(g)

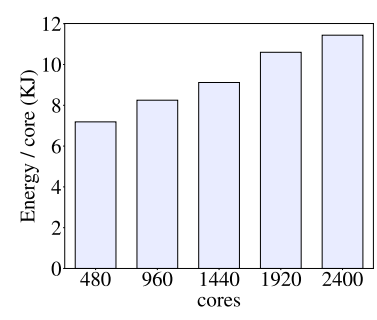

(d)

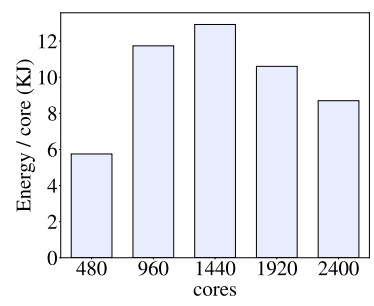

(h)

Figure 3: In rows, different pre-conditioners: (a), (b) (c) and (d) additive Schwarz; (e), (f), (g) and (h) multi-grid. In columns, in terms of the number of cores: (a) and (d) linear solver time; (b) and (e) linear solver iterations; (c) and (f) total energy consumption; and (d) and (h) energy consumption per core. 
conditioner, and so is the total time of solving the linear systems. The main reason is that the multigrid pre-conditioner uses more memory per core as the mesh becomes finer. Thus, using the multi-grid pre-conditioner we were not able to curve the last two meshes because of the high amount of used memory. Specifically, when curving the mesh with 1920 cores, we only obtained the cubic mesh, and when curving the mesh using 2400 cores, we reached the quadratic mesh.

Using both pre-conditioners, the total amount of consumed energy increases as the number of cores increase. This is expected because when curving finer meshes we are using more cores during more time. In addition, the memory consumption per core increases as the number of elements in the meshes increases. That is, when curving finer meshes, each core consumes more energy than when curving coarser meshes. Since multi-grid uses more computational resources than the additive Schwarz pre-conditioner, the multigrid pre-conditioner leads to higher energy demands than the additive Schwarz pre-conditioner.

\subsection{Weak Scaling: Boundary Layer Stretching}

In this example, we perform a weak scaling analysis in terms of the stretching of the boundary layer and the number of elements. The domain is a sphere of radius four with a spherical hole in the center of radius one. We generate five meshes increasing the stretching and the number of layers of a boundary layer generated around the inner sphere. In all the meshes, the boundary mesh is the same. The growth factor of all the boundary layers is $10^{0.1} \simeq 1.259$, that ensures that every ten layers, the width of the layer is multiplied by ten. The maximum stretching of each mesh is $1: 10^{1}$, $1: 10^{2}, 1: 10^{3}, 1: 10^{4}, 1: 10^{5}$. The number of layers in the boundary layer has been chosen to obtain roughly 1500 elements per processor, and we have used 96, 192, 288, 384 and 480 cores. Specifically, the number of layers of each mesh is $10,23,37,47$ and 57 . This leads to meshes that are composed of $135 \cdot 10^{3}$, $291 \cdot 10^{3}, 460 \cdot 10^{3}, 581 \cdot 10^{3}$ and $702 \cdot 10^{3}$ elements. Figures $4 \mathrm{a}$ to $4 \mathrm{e}$ show the five curved high-order meshes of this example.

Figure 5 show the evolution of the constraint norm over the non-linear iterations for the five cases. In this example, the boundary mesh is the same for all the cases and therefore, the evolution for the five meshes is practically the same. That is, the proposed solver exhibits mesh independence at the non-linear level. Moreover, in this example, the constraint norm decreases geometrically with the non-linear iterations. This is especially important during the first iterations of each polynomial degree in the $p$-continuation tech- nique. The main reason is that we compute a correct value of the penalty parameter. Thus, the non-linear solver can perform the continuation of the solution when increasing the polynomial degree.

Figure 6 shows the time to solve the linear problems, the total number of linear solver iterations, the energy consumption, and the energy consumption per core for the two pre-conditioners. In both cases, the time to solve the linear systems becomes larger as the boundary layer stretching increases. The problem becomes more difficult to solve because the high-stretched elements increase the condition number of the linear systems. That is, the number of linear solver iterations increases with the boundary layer stretching. Similarly as in the previous example, the mesh curving process spends most of the linear solve iterations in the curving of the quadratic and cubic meshes. Nevertheless, when using the additive Schwarz pre-conditioner, the curving of the quadratic and cubic meshes is a small fraction of the total time. While the multi-grid preconditioner uses fewer linear solver iterations than the additive Schwarz one, the time to solve the linear systems is one order of magnitude lower when using the additive Schwarz pre-conditioner.

As we increase the stretching of the boundary layer, the energy consumption increases with both preconditioners. Nevertheless, the energy consumption when curving the meshes using the multi-grid preconditioner is roughly twenty times larger than when using the additive Schwarz one. Moreover, the energy consumption per core also increases when we increase the element stretching. The main reason is that the cores are computing during more time because the linear solver needs more iterations to converge. In this example, as expected, we show with numerical evidence that the problem of high-order mesh curving becomes more difficult as the elements become more stretched.

\section{$4.3 p$-continuation Influence: Complex Geometry with Boundary Layer}

We apply the proposed mesh curving solver to curve a large-scale mesh generated for a falcon aircraft, and we show the advantages of the proposed $p$-continuation technique. The initial linear mesh contains $3.91 \cdot 10^{6}$ elements, and a boundary layer around the aircraft with a maximum stretching of $1: 400$. We perform the high-order mesh curving process with and without using the proposed $p$-continuation technique. Specifically, we apply the proposed solver to curve a mesh of polynomial degree four that contains $42 \cdot 10^{6}$ nodes using 2400 cores. Figure 9 shows the decomposition that we have used to perform the parallel mesh curving optimization process. We obtain the same solution whether we apply the proposed $p$-continuation tech- 


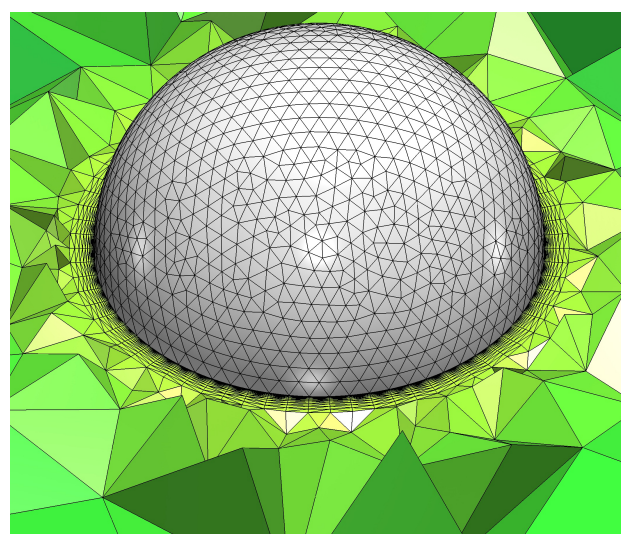

(a)

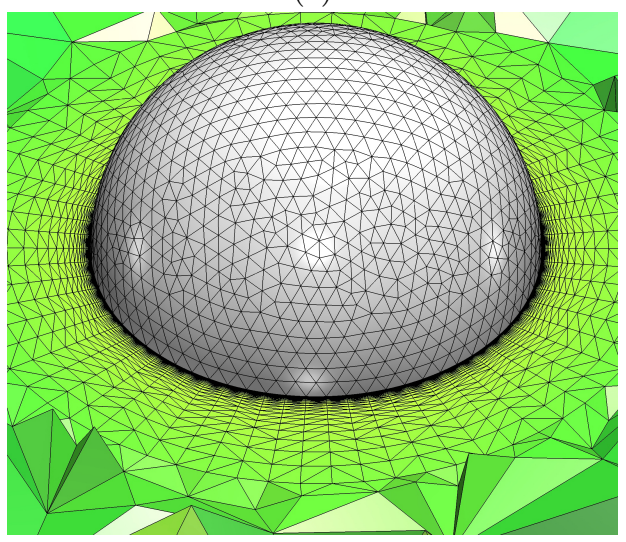

(c)

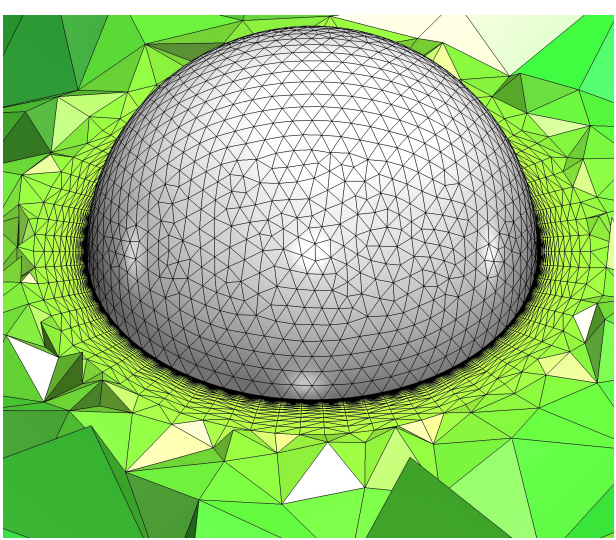

(b)

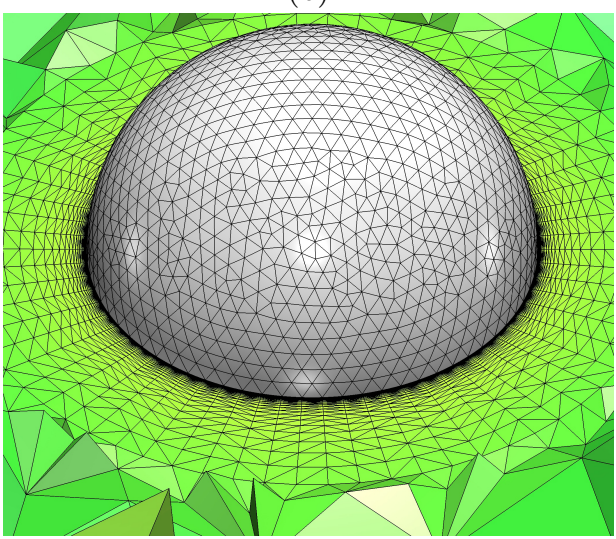

(d)

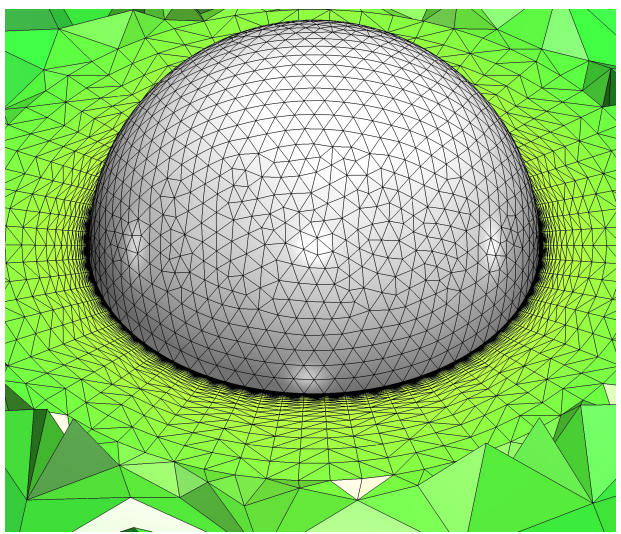

(e)

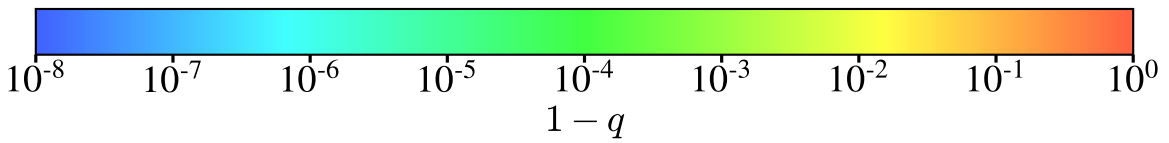

Figure 4: Optimized meshes using the proposed mesh curving solver with boundary layer stretching of: (a) $1: 1 \cdot 10^{1}$; (b) $1: 1 \cdot 10^{2}$; (c) $1: 1 \cdot 10^{3}$; (d) $1: 1 \cdot 10^{4}$; and (e) $1: 1 \cdot 10^{5}$.

nique or not. Figure 7 shows two global views of the curved high-order mesh, while Figures $8 \mathrm{a}$ and $8 \mathrm{~b}$ show detailed views of the mesh at the nose and at the wing- fuselage structure. Note that just the first layers close to the aircraft are curved and that the majority of the mesh contains straight-edged elements. 


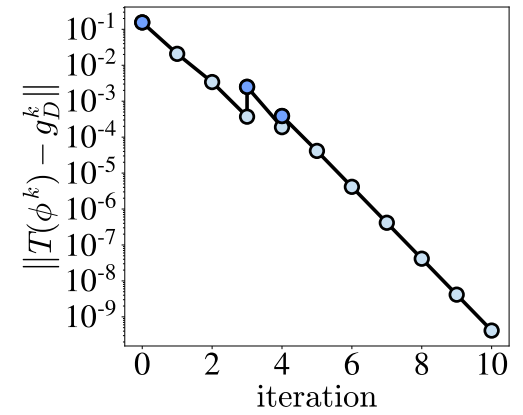

(a)

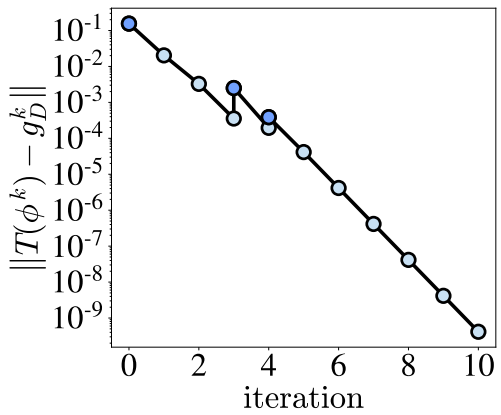

(d)

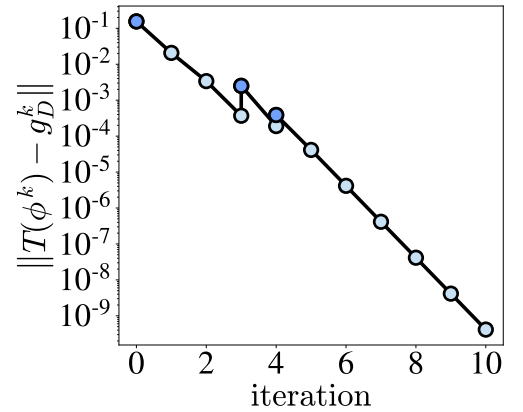

(b)

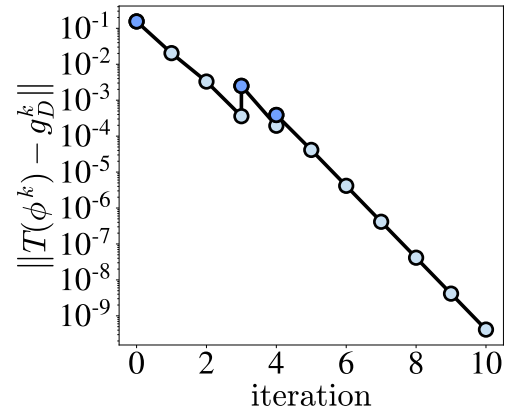

(c)

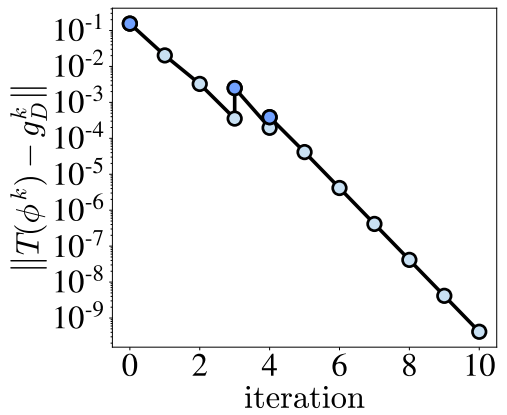

(e)

Figure 5: Evolution of the norm of the constraint through the optimization process for the mesh optimized with boundary layer stretching of: (a) $1: 10^{1}$; (b) $1: 10^{2}$; (c) $1: 10^{3}$; (d) $1: 10^{4}$; and (e) $1: 10^{5}$.

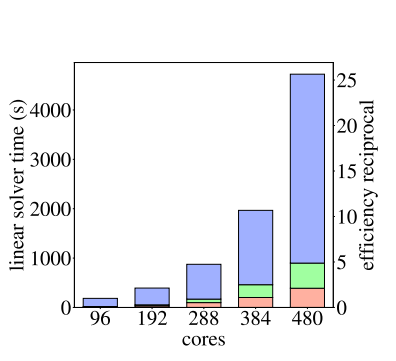

(a)

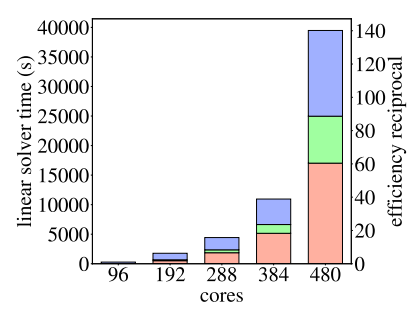

(e)

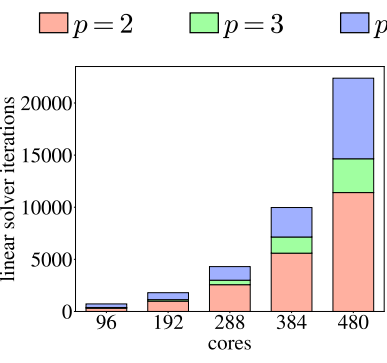

(b)

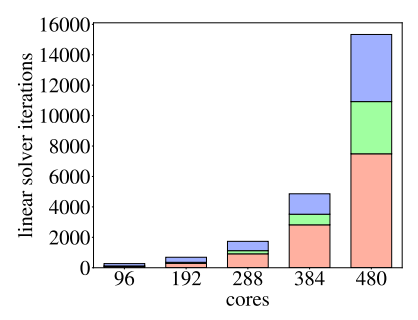

(f)

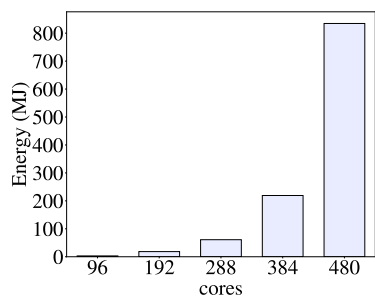

(g)

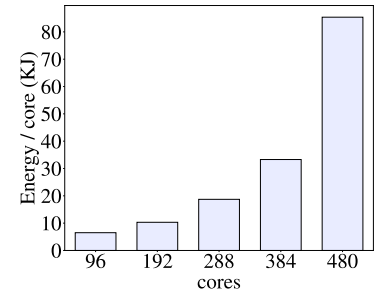

(d)

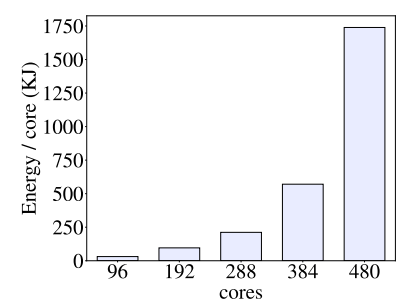

(h)

Figure 6: In rows, different pre-conditioners: (a), (b) (c) and (d) additive Schwarz; (e), (f), (g) and (h) multi-grid. In columns, (a) and (d) linear solver time; (b) and (e) linear solver iterations; (c) and (f) total energy consumption; and (d) and (h) energy consumption per core. 


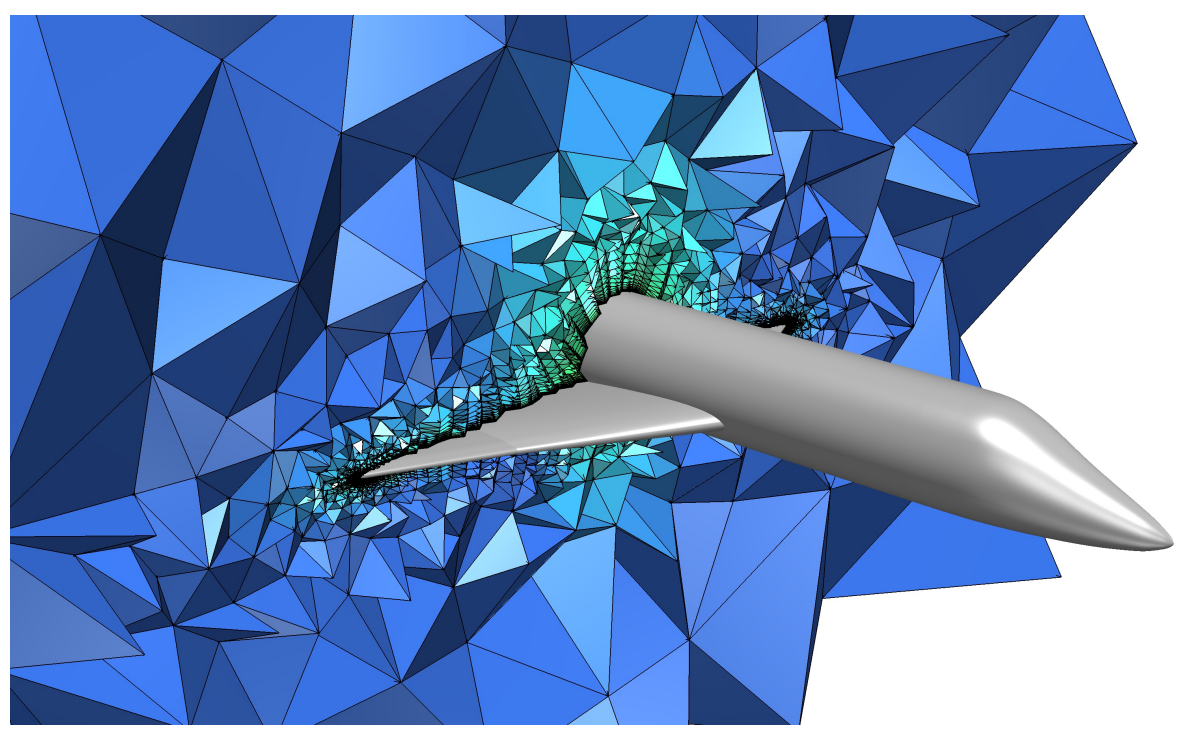

(a)

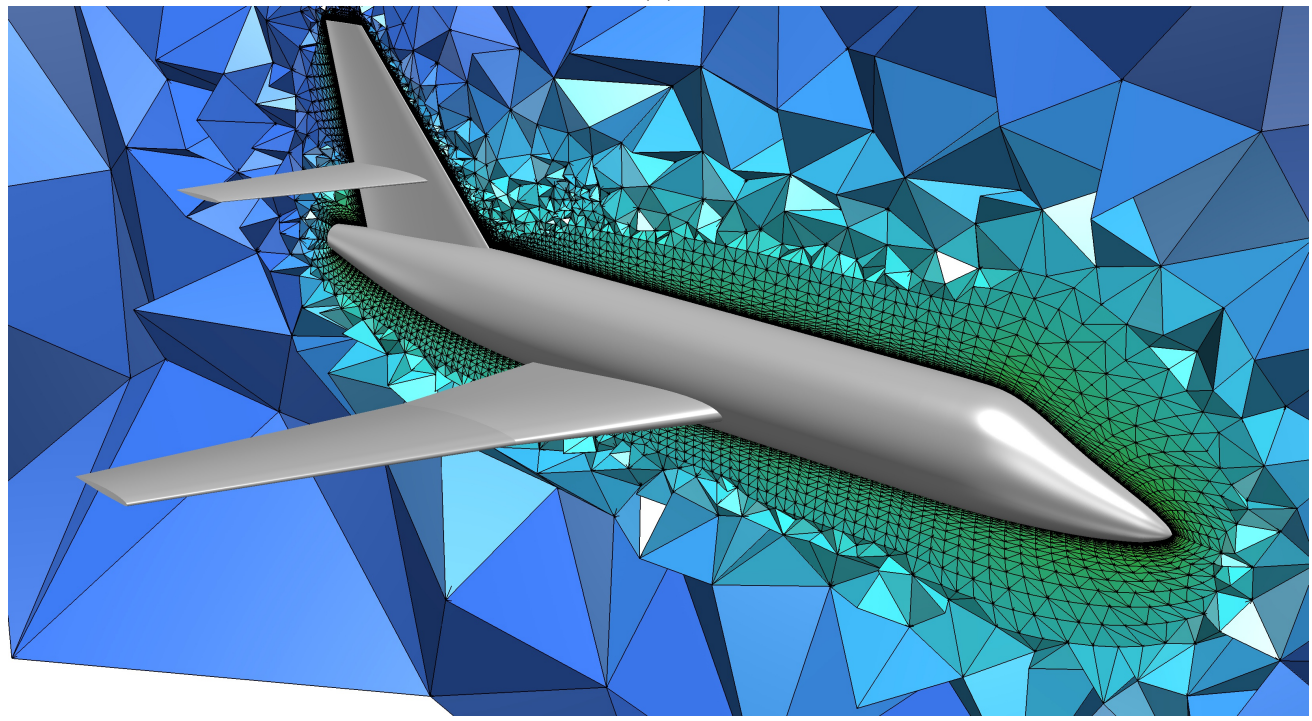

(b)

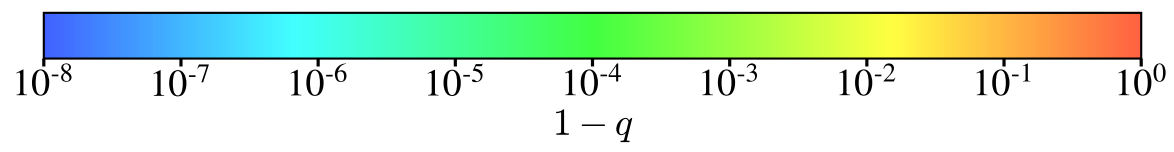

Figure 7: Optimized mesh of polynomial degree four of a falcon aircraft using the proposed mesh curving solver: (a) slice along the $x$ axis; and (b) slice along the $y$ axis.

Figures $10 \mathrm{a}$ and $10 \mathrm{~b}$ show the evolution of the constraint norm through the non-linear iterations of the penalty method with and without using the proposed p-continuation technique, respectively. The dark blue circles denote the initial iteration of each polynomial degree. When using the $p$-continuation technique, the whole process takes nine iterations to converge, while the case of directly optimizing the mesh of polyno- mial degree four takes 7 iterations. In both cases, in the last iterations of each polynomial degree, the constraint norm decreases geometrically with the number of iterations. Note that when using the $p$-continuation technique the estimation of the penalty parameter ensures that in the first iterations of each polynomial degree the constraint norm also decreases geometrically. 


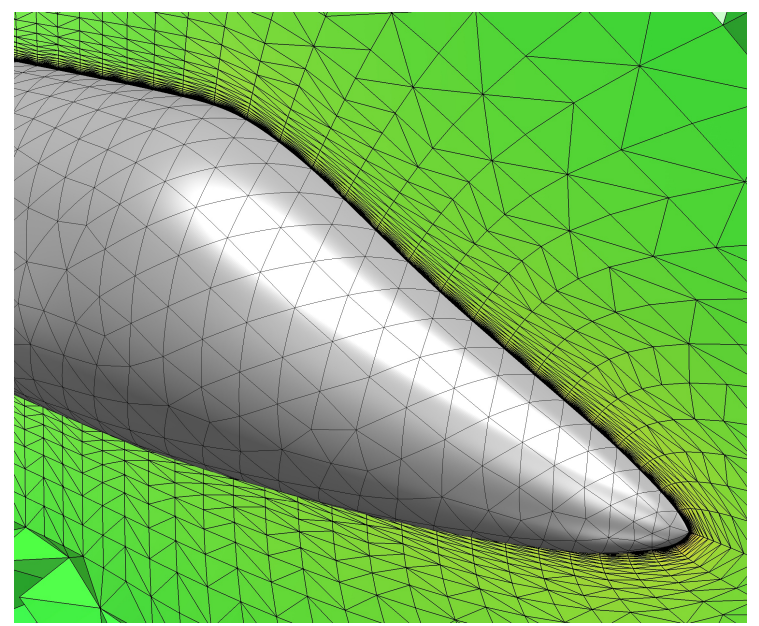

(a)

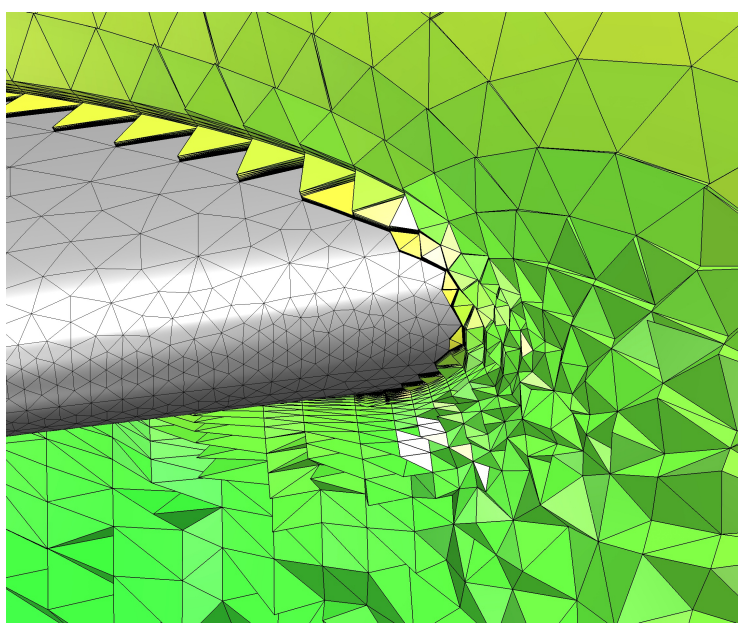

(b)

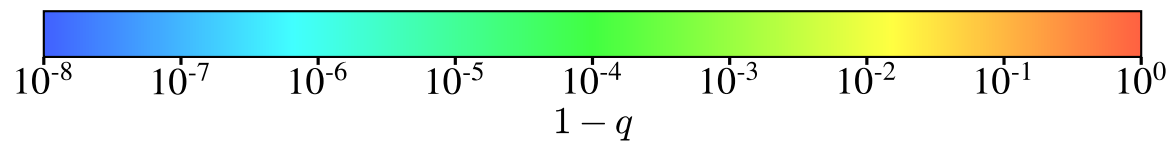

Figure 8: Detailed view of the optimized mesh of polynomial degree four of a falcon aircraft using the proposed mesh curving solver: (a) at nose; and (b) at the wing-fuselage.

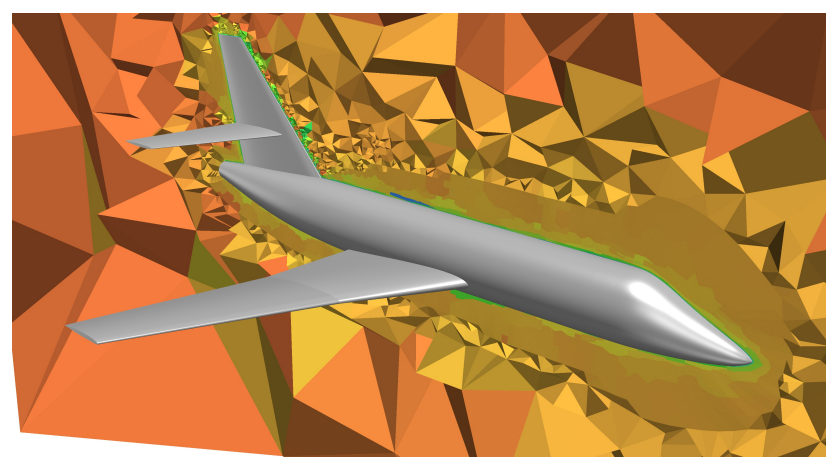

Figure 9: Parallel decomposition of the high-order mesh for a Falcon aircraft, where each color denotes a different processor.

Figure 11 shows the total time to solve the linear problems, the number of linear solver iterations, and the energy consumption for both cases. Note that the $p$-continuation technique reduces the time to solve the linear systems fourfold. The main reason is that we reduce in half the number of linear solver iterations and that only a fraction of the linear iterations are performed in the mesh of polynomial degree four. Since meshes of lower polynomial degree lead less unknowns and to matrices with fewer non-zero entries, each iteration of the linear solver is performed faster. For this reason, we have reduced the computational time and memory requirements with the proposed $p$ - continuation technique and therefore, the energy consumption is reduced five times.

Table 1 presents the time breakdown of the optimization process with and without the proposed $p$ continuation technique. In both cases, the time to solve linear systems is the major contribution of the total time. The assembly time is lower when using the $p$-continuation technique because the elemental matrices are smaller. The rest of the time contains the mesh reading and writing, the nodal projection, and the control flow of the algorithm. In both cases, this time is similar. Thus, the main advantage of the proposed $p$-continuation technique is that we reduce the time to construct the linear systems and to solve them.

\section{CONCLUDING REMARKS}

\subsection{Summary}

We have presented a mesh curving solver to generate curved high-order meshes. Specifically, we have extended our high-order meshing solver to take advantage of a distributed parallel environment. The proposed formulation shows mesh independence at the non-linear level. Nevertheless, the obtained linear systems do not show this behavior. As the number of elements increase and the elements become more stretched, the linear systems are harder to solve because the condition number of the matrices increases. It is especially important to highlight the difficulty 


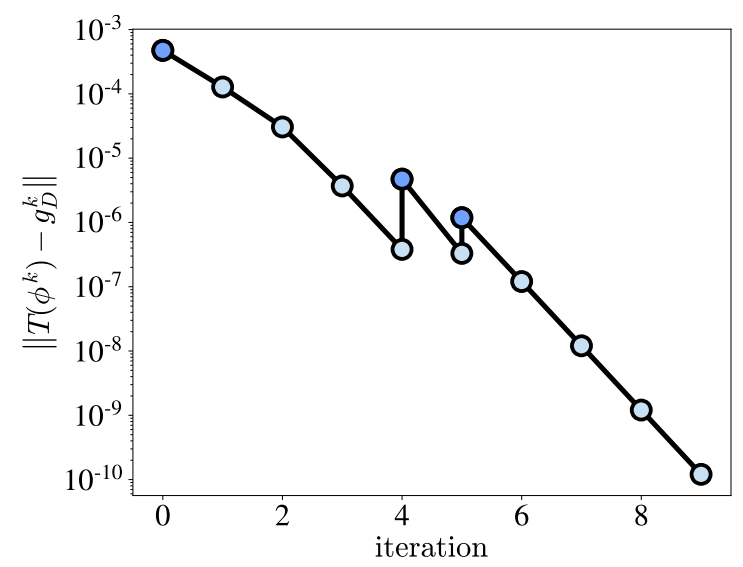

(a)

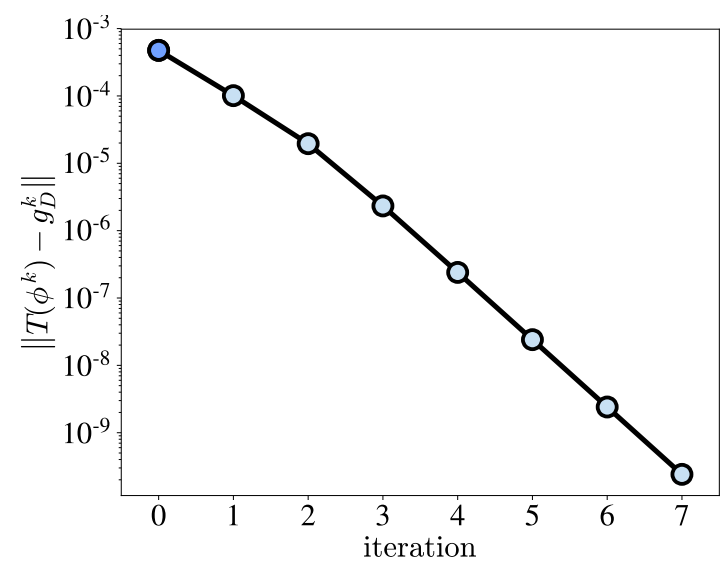

(b)

Figure 10: Evolution of the norm of the constraint through the high-order mesh curving process of a falcon aircraft.

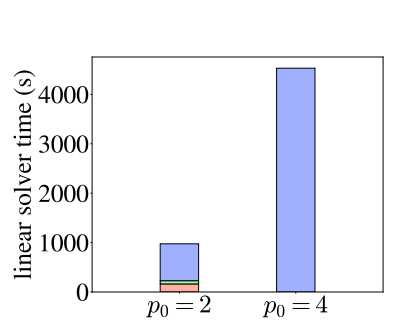

(a)

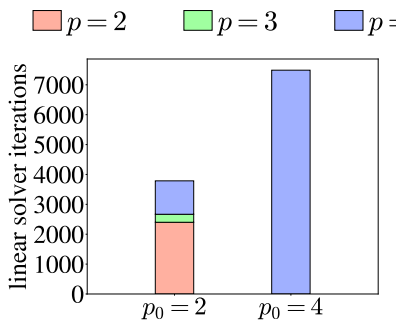

(b)

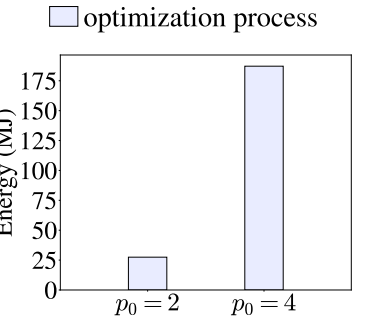

(c)

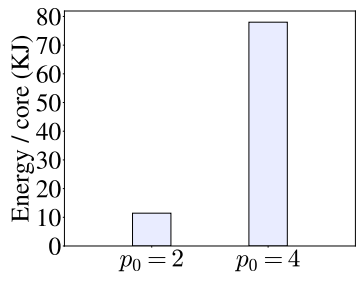

(d)

Figure 11: Curved high-order mesh generation for a falcon aircraft: (a) linear solver time; (b) linear solver iterations; and (c) energy consumption

Table 1: Time breakdown in seconds for the meshes generated of the Falcon aircraft

\begin{tabular}{rrrrr} 
Case & Total time $(\mathrm{s})$ & Assembly time $(\mathrm{s})$ & Linear solver time $(\mathrm{s})$ & other time $(\mathrm{s})$ \\
\hline$p_{0}=2$ & 1296 & 289.5 & 975.2 & 31.19 \\
$p_{0}=4$ & 4921 & 355.7 & 4531 & 34.48
\end{tabular}

increase when the elements become more stretched. Thus, the main difficulty of the mesh curving process is solving the linear systems, especially when stretched elements appear in the mesh.

In this work, we have analyzed two pre-conditioners in order to compare their performance in terms of the number of linear solver iterations, the time to solve the linear systems, and the energy consumption of the mesh curving process. The first pre-conditioner is an additive Schwarz method with one level of overlap in which the local problem is approximated using two iterations of a symmetric successive over-relaxation method. The second pre-conditioner is an algebraic multi-grid pre-conditioner in which the coarsest problem is solved using an LU decomposition. The PETSc implementation of the algebraic multi-grid method automatically computes the optimal number of levels to solve the linear problem.

The additive Schwarz pre-conditioner leads to a higher number of linear iterations than the algebraic multigrid. Nevertheless, each iteration of the algebraic multi-grid pre-conditioner takes more time than one iteration of the additive Schwarz one. Thus, the total time to solve the linear systems is lower when using the additive Schwarz pre-conditioner. Moreover, the algebraic multi-grid technique has to store the different coarsening levels and therefore it uses more memory than the additive Schwarz. Specifically, as the mesh becomes finer and the number of cores increases, the required memory per core increases. Moreover, even 
when keeping the same number of elements per core, the memory requirements make the algebraic multigrid method not usable for meshes with a high number of elements. Therefore, since the algebraic multi-grid method requires more memory and takes more time than the additive Schwarz method, the energy consumption is higher. Specifically, the economic cost of generating a curved high-order mesh is lower when using the additive Schwarz pre-conditioner.

We have proposed a $p$-continuation technique to reduce the computational resources of the mesh curving solver while increasing its robustness. The main idea is to use the solution of a polynomial degree as a starting position for the next polynomial degree. Therefore, the $p$-continuation technique can be interpreted as a methodology to compute an initial position of the mesh for the last polynomial degree. Since the initial position of the nodes for the last polynomial degree are close to the optimal position, the computational time to optimize the mesh is reduced and the robustness of the optimization process is increased.

We have devised two key ideas to define the $p$ continuation technique. The first one is an earlytermination criterion to finalize the mesh curving of the initial polynomial degrees. Therefore, we solve less linear problems and we improve the computational efficiency. The second idea is to compute an estimation of the penalty parameter for the initial iteration of each polynomial degree. In our experience, the selection of the penalty parameter is crucial for the efficiency and convergence of the mesh curving process. On the one hand, if the penalty parameter is too low, the boundary condition is not enforced enough, and the mesh curving process needs additional iterations of the penalty method. On the other hand, if the penalty parameter is too high, the condition number of the linear systems increase and therefore, the linear systems become harder to solve. Thus, the mesh curving process may not finalize because the linear problem could not be solved.

We have shown that the $p$-continuation technique does not spend much time curving the initial polynomial degrees, although most of the iterations are performed in the initial polynomial degrees. Therefore, we accelerate the mesh curving solver because the linear solver iterations of lower polynomial degree are faster since the number of unknowns and the number of non-null entries in the matrix are lower. Moreover, in the presented examples, we have shown that the number of linear solver iterations is also reduced. Therefore, the necessary energy to generate a curved high-order mesh is reduced because we are using the computational resources during less time, and the process is less memory intensive.

\subsection{Discussion}

In our solver, we have tested additional preconditioners. For instance, when using an additive Schwarz pre-conditioner without overlap levels, the linear systems do not converge. The main reason is that it takes more linear solver iterations to transfer the information of one processor to the others. This is solved by increasing the overlap levels of the additive Schwarz method. Additionally, the local problem can be approximated using an incomplete LU factorization without fill-in levels, ILU(0). In this case, the linear solver does not converge because the local problem is not well approximated. Although we can increase the fill-in levels, this would lead to higher memory requirements. Finally, to reduce the memory requirements of the algebraic multi-grid it is possible to limit the number of coarsening levels to reduce the memory requirements. Nevertheless, since the coarsest level solver is a direct decomposition solver, its memory requirements increase. Therefore it is needed to find a balance between the number of coarsening levels and the memory requirements of the coarsest level solver.

In this work, we are performing a full optimization process of the whole mesh. Although this approach may seem expensive, we ensure that the final curved high-order mesh is optimal in all the elements. This is an important point because non-optimized elements may introduce spurious oscillations in the solution of a simulation process. Nevertheless, there are implementations that only optimize the worst quality elements. Specifically, they optimize the elements with quality lower than a given threshold, and some additional elements around to increase the feasible locations of the high-order nodes. Therefore, these methods require to select the threshold value in such a manner that the optimized mesh is good enough for the simulation, and how many elements to additionally optimize in order to obtain a feasible solution. By optimizing the whole mesh, we ensure the optimality of all the elements without additional parameters.

The optimization of the whole mesh is especially important in cases with a highly-stretched boundary layer. In these cases, it is of major importance to obtain optimal elements in the boundary layer and therefore the whole boundary layer should be optimized. Since the number of elements in the boundary layer can be a significant fraction of the total elements, it is not clear the reduction in computational resources of optimizing only a fraction of the elements.

Although the existent literature comparing local and global mesh optimization does not deal with piecewise polynomial curved meshes and highly stretched elements, it suggests that for mesh curving a specificpurpose global optimization method might be preferred. That is, existent literature in local and global 
optimization methods for linear meshes shares a common conclusion, when highly optimized and accurate meshes are required, especially in isotropic meshes featuring high gradations of the element size, a specificpurpose global feasible Newton method outperforms local optimization methods. This setting also corresponds to the general mesh curving problem where we need to exploit the quadratic convergence of Newton's method since we want high-precision to both approximate the curved geometries and deal with highly stretched elements. Furthermore, we want to apply our mesh curving method to meshes featuring smaller size close the objects immersed in the fluid stream and bigger size in the far-field, and thus, we need to deal with high gradations of the element size. Nevertheless, in the near future, it could be interesting to compare our global parallel method featuring global quadratic convergence rate with a local parallel method featuring local quadratic convergence rate.

Note that we have only studied weak scaling results, but not strong scaling results, since we are interested in proposing a global method to generate large curved meshes in parallel. In our formulation, we need to store the Hessian pre-conditioner in a distributed fashion, and thus, these memory requirements determine the number of computing nodes. This need is so since each computing node has a fixed amount of main memory to deal with a maximum number of non-zero entries of the Hessian matrix, determined by the polynomial degree and the number of elements per processor. We prefer to use all the memory available in each computing node and thus, use a small number of processors for coarse meshes and a larger number of processors for fine meshes. This setting demands a weak scaling study, where instead of fixing the problem size and using more computing resources, we increase both the problem size, number of elements to curve, and the number of processors.

The process of generating a curved high-order mesh given a CAD model is decomposed in three stages. The first stage is the geometry healing and defeaturing of a given CAD model. The second stage is the generation of an initial linear mesh with elements of the desired shape and size. Finally, the third stage is the actual high-order mesh curving by optimizing the mesh distortion. In our particular case, we spend most of the time in the first two stages. Specifically, the first two stages require the work of trained personnel and specialized software.

Usually, if the initial linear mesh is good enough, the optimization process is performed without incident. In our experience, the high-order mesh needs enough resolution to represent the underlying CAD geometry. In addition, to avoid invalid elements, there should not be any elements with all the nodes on a smooth sur- face. Such elements have two high-order triangles that approximate the surface. Since the surface is smooth, this would lead to null Jacobians in the common edge.

\subsection{Conclusion}

The proposed $p$-continuation approach reduces four times (eight times) the total time (energy) used to curve a whole boundary layer mesh using 2400 cores. The $p$-continuation accelerates a Newton-GMRES penalty solver equipped with the chosen parallel preconditioner, a restricted additive Schwarz domain decomposition with one level of overlap and local problems approximated with two iterations of symmetric successive over-relaxation.

\section{ACKNOWLEDGMENTS}

This project has received funding from the European Research Council (ERC) under the European Union's Horizon 2020 research and innovation programme under grant agreement No 715546. This work has also received funding from the Generalitat de Catalunya under grant number 2017 SGR 1731. The work of Xevi Roca has been partially supported by the Spanish Ministerio de Economía y Competitividad under the personal grant agreement RYC-2015-01633.

\section{References}

[1] Szabó B., Babuška I. Finite Element Analysis. John Wiley \& Sons New York, 1991

[2] Schwab C. $p$-and hp-finite element methods: Theory and applications in solid and fluid mechanics. Clarendon Press Oxford, 1998

[3] Deville M., Fischer P., Mund E. High-order methods for incompressible fluid flow, vol. 9. Cambridge University Press, 2002

[4] Hesthaven J., Warburton T. Nodal Discontinuous Galerkin Methods: Algorithms, Analysis, and Applications. Texts in Applied Mathematics. Springer, 2007

[5] Karniadakis G., Sherwin S. Spectral/hp element methods for computational fluid dynamics. Oxford University Press, 2013

[6] Vos P.E., Sherwin S., Kirby R. "From $h$ to $p$ efficiently: implementing finite and spectral $/ h p$ element methods to achieve optimal performance for low- and high-order discretisations." J. Comput. Phys., vol. 229, no. 13, 5161-5181, 2010

[7] Cantwell C., Sherwin S., Kirby R., Kelly P. "From $h$ to $p$ efficiently: strategy selection for operator evaluation on hexahedral and tetrahedral 
elements." Comput. Fluids, vol. 43, no. 1, 23-28, 2011

[8] Cantwell C., Sherwin S., Kirby R., Kelly P. "From $h$ to $p$ efficiently: selecting the optimal spectral/ $h p$ discretisation in three dimensions." Math. Model. Nat. Phenom., vol. 6, no. 3, 84-96, 2011

[9] Löhner R. "Error and work estimates for highorder elements." Int. J. Numer. Meth. Fluids, vol. 67 , no. $12,2184-2188,2011$

[10] Yano M., et al. An optimization framework for adaptive higher-order discretizations of partial differential equations on anisotropic simplex meshes. Ph.D. thesis, Massachusetts Institute of Technology, 2012

[11] Kirby R., Sherwin S., Cockburn B. "To CG or to HDG: a comparative study." J. Sci. Comput., vol. 51, no. 1, 183-212, 2012

[12] Huerta A., Roca X., Angeloski A., Peraire J. "Are High-order and Hybridizable Discontinuous Galerkin methods competitive?" Oberwolfach Rep., vol. 9, no. 1, $485-487,2012$

[13] Löhner R. "Improved error and work estimates for high-order elements." Int. J. Numer. Meth. Fluids, vol. 72, 1207-1218, 2013

[14] Wang Z., Fidkowski K., Abgrall R., Bassi F., Caraeni D., Cary A., Deconinck H., Hartmann R., Hillewaert K., Huynh H., et al. "High-order CFD methods: current status and perspective." Int. J. Numer. Meth. Fluids, vol. 72, no. 8, 811845,2013

[15] Huerta A., Angeloski A., Roca X., Peraire J. "Efficiency of high-order elements for continuous and discontinuous Galerkin methods." Int. J. Numer. Meth. Eng., vol. 96, 529-560, 2013

[16] Dey S., Shephard M., Flaherty J. "Geometry representation issues associated with p-version finite element computations." Comput. Meth. Appl. M., vol. 150, no. 1-4, 39-55, 1997

[17] Dey S., O'Bara R., Shephard M.S. "Curvilinear mesh generation in 3D." Comput. Aided Design, vol. 33, 199-209, 2001

[18] Luo X., Shephard M.S., Remacle J.F., O'Bara R., Beall M., Szabó B., Actis R. "P-version mesh generation issues." Proc. 11th Int. Meshing Roundtable, pp. 343-354. Springer Berlin Heidelberg, 2002
[19] Luo X., Shephard M.S., O'Bara R., Nastasia R., Beall M. "Automatic p-version mesh generation for curved domains." Eng. Comput., vol. 20, no. 3, 273-285, 2004

[20] Shephard M.S., Flaherty J.E., Jansen K., Li X., Luo X., Chevaugeon N., Remacle J.F., Beall M., O'Bara R. "Adaptive mesh generation for curved domains." Appl. Numer. Math., vol. 52, no. 2-3, 251-271, 2005

[21] Persson P.O., Peraire J. "Curved Mesh Generation and Mesh Refinement using Lagrangian Solid Mechanics." Proc. 47th AIAA. 2009

[22] Moxey D., Green M., Sherwin S., Peiró J. "An isoparametric approach to high-order curvilinear boundary-layer meshing." Computer Methods in Applied Mechanics and Engineering, vol. 283, no. $0,636-650,2015$

[23] Gargallo-Peiró A., Roca X., Peraire J., Sarrate J. "Optimization of a regularized distortion measure to generate curved high-order unstructured tetrahedral meshes." International Journal for Numerical Methods in Engineering, vol. 103, no. 5, 342-363, 2015. URL http://dx.doi.org/10.1002/nme.4888

[24] Moxey D., Ekelschot D., Keskin Ü., Sherwin S., Peiró J. "High-order curvilinear meshing using a thermo-elastic analogy." Computer-Aided Design, vol. $72,130-139,2016$

[25] Fortunato M., Persson P. "High-order unstructured curved mesh generation using the Winslow equations." Journal of Computational Physics, vol. $307,1-14,2016$

[26] Eichstädt J., Green M., Turner M., Peiró J., Moxey D. "Accelerating high-order mesh optimisation with an architecture-independent programming model." Computer Physics Communications, vol. 229, 36-53, 2018

[27] Sherwin S., Peiró J. "Mesh generation in curvilinear domains using high-order elements." Int. J. Numer. Meth. Eng., vol. 53, no. 1, 207-223, 2002

[28] Xie Z., Sevilla R., Hassan O., Morgan K. "The generation of arbitrary order curved meshes for 3D finite element analysis." Comput. Mech., vol. 51, 361-374, 2012

[29] Poya R., Sevilla R., Gil A.J. "A unified approach for a posteriori high-order curved mesh generation using solid mechanics." Computational Mechanics, vol. 58, no. 3, 457-490, 2016 
[30] Sevilla R., Rees L., Hassan O. "The generation of triangular meshes for NURBS-enhanced FEM." International Journal for Numerical Methods in Engineering, vol. 108, no. 8, 941-968, 2016

[31] Toulorge T., Geuzaine C., Remacle J.F., Lambrechts J. "Robust untangling of curvilinear meshes." J. Comput. Phys., vol. 254, 8-26, 2013

[32] Karman S.L., Erwin J.T., Glasby R.S., Stefanski D. "High-Order Mesh Curving Using WCN Mesh Optimization." 46th AIAA Fluid Dynamics Conference, p. 3178. 2016

[33] Stees M., Shontz S.M. "A high-order log barrierbased mesh generation and warping method." Procedia Engineering, vol. 203, 180-192, 2017

[34] Panitanarak T., Shontz S.M. "A parallel log barrier-based mesh warping algorithm for distributed memory machines." Engineering with Computers, vol. 34, no. 1, 59-76, 2018

[35] Ruiz-Gironés E., Roca X. "Imposing boundary conditions to match a CAD virtual geometry for the mesh curving problem." Proceedings of the 27th International Meshing Roundtable, pp. 343361. Springer, 2018

[36] Staten M.L., Owen S.J., Shontz S.M., Salinger A.G., Coffey T.S. "A comparison of mesh morphing methods for 3D shape optimization." Proceedings of the 20th International Meshing Roundtable, pp. 293-311. Springer, 2011

[37] Ruiz-Gironés E., Sarrate J., Roca X. "Generation of Curved High-order Meshes with Optimal Quality and Geometric Accuracy." Procedia Engineering, vol. 163, 315-327, 2016

[38] Toulorge T., Lambrechts J., Remacle J. "Optimizing the geometrical accuracy of curvilinear meshes." Journal of Computational Physics, 2016

[39] Ruiz-Gironés E., Gargallo-Peiró A., Sarrate J., Roca X. "Automatically imposing incremental boundary displacements for valid mesh morphing and curving." Computer-Aided Design, 2019

[40] Dobrev V., Knupp P., Kolev T., Mittal K., Tomov V. "The Target-Matrix Optimization Paradigm for High-Order Meshes." SIAM Journal on Scientific Computing, vol. 41, no. 1, B50-B68, 2019

[41] Garanzha V., Kudryavtseva L. "Hyperelastic springback technique for construction of prismatic mesh layers." Procedia engineering, vol. 203, 401-413, 2017

[42] Karman S. "Curving for Viscous Meshes." Proceedings of the 27th International Meshing Roundtable, pp. 303-325. Springer, 2018
[43] Sastry S.P., Shontz S.M. "A parallel log-barrier method for mesh quality improvement and untangling." Engineering with Computers, vol. 30, no. $4,503-515,2014$

[44] Benítez D., Escobar J., Montenegro R., Rodríguez E. "Parallel Performance Model for Vertex Repositioning Algorithms and Application to Mesh Partitioning." Proc. 27th Int. Meshing Roundtable, 2018

[45] Ruiz-Gironés E., Roca X., Sarrate J. "Highorder mesh curving by distortion minimization with boundary nodes free to slide on a $3 \mathrm{D}$ CAD representation." Computer-Aided Design, vol. 72, 52-64, 2016

[46] Gargallo-Peiró A., Roca X., Peraire J., Sarrate J. "Distortion and quality measures for validating and generating high-order tetrahedral meshes." Engineering with Computers, vol. 31, no. 3, 423437, 2015

[47] Knupp P.M. "Algebraic mesh quality metrics." SIAM J. Numer. Anal., vol. 23, no. 1, 193-218, 2001

[48] Nocedal J., Wright S. Numerical optimization. Springer Verlag, 1999

[49] petsc4py. "PETSc for Python.", 2018. URL https://bitbucket.org/petsc/petsc4py

[50] Pointwise Inc. "Mesh Generation Software for CFD - Pointwise, Inc." http://www.pointwise.com, 2018

[51] Python Software Foundation. "Python." http://www.python.org, 2018

[52] Alnæs M.S., Blechta J., Hake J., Johansson A., Kehlet B., Logg A., Richardson C., Ring J., Rognes M.E., Wells G.N. "The FEniCS Project Version 1.5." Archive of Numerical Software, vol. 3, no. 100, 2015

[53] Geode. "Project Geode: Geometry for Simulation." http://www.pointwise.com/geode/, 2018

[54] CASCADE O. "Open CASCADE Technology, 3D modeling and numerical simulation." www. opencascade.org, 2012

[55] swig. "Simplified Wrapper and Interface Generator.", 2018. URL https://swig.org

[56] Ahrens J., Geveci B., Law C. "Paraview: An end-user tool for large data visualization." The visualization handbook, vol. 717, 2005 


\title{
NATURALLY CURVED QUADRILATERAL MESH GENERATION USING AN ADAPTIVE SPECTRAL ELEMENT SOLVER
}

\author{
Julian Marcon $^{1} \quad$ David A. Kopriva ${ }^{2,3} \quad$ Spencer J. Sherwin ${ }^{4} \quad$ Joaquim Peiró $^{5}$ \\ ${ }^{1}$ Imperial College London, London, U.K. julian.marcon14@imperial.ac.uk \\ 2 The Florida State University, Tallahassee, FL, U.S.A. kopriva@math.fsu.edu \\ ${ }^{3}$ San Diego State University, San Diego, CA, U.S.A. \\ ${ }^{4}$ Imperial College London, London, U.K. s.sherwin@imperial.ac.uk \\ ${ }^{5}$ Imperial College London, London, U.K. j.peiro@imperial.ac.uk
}

\begin{abstract}
We describe an adaptive version of a method for generating valid naturally curved quadrilateral meshes. The method uses a guiding field, derived from the concept of a cross field, to create block decompositions of multiply connected two dimensional domains. The a priori curved quadrilateral blocks can be further split into a finer high-order mesh as needed. The guiding field is computed by a Laplace equation solver using a continuous Galerkin or discontinuous Galerkin spectral element formulation. This operation is aided by using $p$-adaptation to achieve faster convergence of the solution with respect to the computational cost. From the guiding field, irregular nodes and separatrices can be accurately located. A first version of the code is implemented in the open source spectral element framework Nektar ++ and its dedicated high order mesh generation platform NekMesh.
\end{abstract}

Keywords: cross field, quadrilateral meshing, high order, spectral element method, adaptation

\section{INTRODUCTION}

Finite element [1], finite volume, (block structured) finite difference [2] and spectral element methods [3, 4] all benefit from using quadrilateral meshes. Yet fully automatic quadrilateral mesh generation is still difficult [5], especially for high order (curved) elements used in spectral element methods.

A method was described in [6] that generates large block structures with naturally curved boundaries that can be used as is, say for spectral element or block structured finite difference methods, or further subdivided into small quadrilaterals for finite element or finite volume methods. The idea behind the approach came from the computer graphics community, which used cross fields $[1,5]$. The procedure in [6] used a high order approach to compute a guiding field, accurately locating the irregular nodes, and accurately integrating the separatrices used to subdivide the domain. It used a high order continuous or discontinuous Galerkin spectral element method (SEM or DGSEM) on a base triangular mesh, the approximation depending on the regularity of the boundary conditions needed to solve Laplace problems that determine the guiding field. Unlike previous work, accuracy was preserved because all operations were performed on the guiding field rather than a cross field. That new procedure was implemented in the open source spectral $/ h p$ element framework Nektar ++ and its high order mesh generation utility NekMesh.

One of the significant costs in the high resolution field guided approach is the solution to the Laplace problems on the triangular mesh. As the polynomial order of the approximation gets high, the condition number of the matrices that must be solved (either directly or 
iteratively) becomes large, leading to increased cost to get the field solution. The approximation order was chosen ad hoc, and there was no way to tell whether or not the chosen approximation order was sufficient.

One can avoid the ad hoc nature of the choice of approximation order, and the slow convergence rates associated with choosing one that is higher than is necessary, by using an adaptive solver that adjusts the approximation to satisfy a desired error tolerance. With a spectral element solver, adaptation can be done either by subdividing the underlying triangular elements ( $h$-adaptation) and/or by increasing the polynomial order ( $p$-adaptation). In this work, we employ $p$ adaptation because of its best accuracy, compared to $h$-adaptation, with respect to the computational cost for smooth fields without discontinuities [7]. We use a dynamic $p$-adaptive strategy previously devised for compressible flow simulation $[8,9]$ and implemented in Nektar++.

The methodology consists of three main steps. First, a guiding field is computed. This requires the generation of a high order triangular mesh of the domain and the solution to the Laplace problem using a $p$-adaptive SEM or DGSEM solver. Then, analysis of the guiding field must be carried out to construct a separatrix graph. This construction process includes the location of interior critical points, the calculation of their valence as well as the valence of corners, and then the integration of streamlines and their possible manipulation. Finally, a high order quadrilateral mesh can be generated. To achieve this, streamlines are splined and used to split the CAD model into quadrilateral blocks, which can then be trivially meshed and refined.

This paper is structured as follows. Section 2 gives an outline of the mathematical formulation of the problem, including a definition of crosses, the guiding field and the partial differential equations to solve, a summary of the SEM/DGSEM formulation, and a overview of the $p$-adaptation strategy. Section 3 covers some of the implementation details and Section 4 illustrates the methodology with examples.

\section{FORMULATION}

The goal is to subdivide any simply or multiply connected two dimensional domain, $\Omega$, with piecewise smooth boundaries into quadrilateral subdomains. The decomposition is called regular if all the corners of the quadrilaterals have valence four, that is, each corner node connects four edges. Especially in multiply connected domains, there will be irregular nodes, where the valence will be larger or smaller than four. The problem is how to generate a quadrilateral decomposition that has a minimum number of irregular nodes.

\subsection{The Guiding Field}

An approach that has been used in the computer graphics community to generate quadrilateral meshes over surfaces relies on the idea of a cross field. For full discussion of cross fields and methods based on them, see, for example, references $[1,10,11,12]$. We will present the basic idea.

A cross has four way rotational symmetry and can be represented by the four vectors

$$
C(\psi)=\left\{\vec{c}_{k}=\left(c_{x}, c_{y}\right)_{k}=\left(\cos \left(\psi_{k}\right), \sin \left(\psi_{k}\right)\right)\right\},
$$

where

$$
\psi_{k}=\psi+k \frac{\pi}{2}
$$

for, $k=0,1,2,3$. In turn, the tangent angle, or phase, $\psi$, can be computed from the four quadrant inverse tangent,

$$
\psi=\frac{1}{4} \operatorname{atan} 2(\mathrm{v}, \mathrm{u}) \in\left[-\frac{\pi}{4}, \frac{\pi}{4}\right] .
$$

In other words, a cross is represented by four unit vectors $\vec{c}_{k}$ at any point $\vec{x} \in \Omega$ in a vector field $\vec{v}=(u(\vec{x}), v(\vec{x}))$ at which $\vec{v} \neq 0$. A cross rotated by an angle $\pi / 2$ is still the same cross.

The function $\psi$ is either tangent or orthogonal (due to the jump in the atan 2 function) to the streamlines of the field $\vec{v}$. It is parallel to one of the branches of a cross and is undefined at a critical point of the field, i.e. where $\vec{v}=(0,0)$. Given the way $\psi$ is computed from the arctangent, there will be a jump in value $\pi / 2$ in $\psi$ depending on the signs of $u$ and $v$ even if $\vec{v}$ is smooth. We will call lines in the field across which $\psi$ jumps jump lines.

The method rests on finding an appropriate guiding field $\vec{v}$. The only known values are on the boundary $\partial \Omega$ of $\Omega$. There, the field is aligned with the boundary to ensure that the mesh to be generated is aligned there. Along the boundary,

$$
\vec{v}_{b}=\left(u_{b}, v_{b}\right)=\left(\cos \left(4 \theta_{b}\right), \sin \left(4 \theta_{b}\right)\right),
$$

where $\theta_{b}$ is the tangent angle of the boundary. The vector $\vec{v}_{b}$ at any point along the boundary defines a cross (1) at that point. The factor of four in eq. (4) ensures the same $u, v$ values for each $90^{\circ}$ rotation of the angle $\theta_{b}$, and hence the rotational symmetry.

Boundary values defined with (4) are smoothly propagated to the interior. Solving a Laplace problem for $u$ and $v$

$$
\left\{\begin{array}{l}
\nabla^{2} \vec{v}=0, \quad \vec{x} \in \Omega \\
\vec{v}=\vec{v}_{b}, \quad \vec{x} \in \partial \Omega
\end{array}\right.
$$

guarantees that the field $\vec{v}$ is smooth in the interior of the domain. 


\subsubsection{Critical Points}

To obtain a valid block decomposition of an arbitrary simply or multiply connected domain, irregular nodes must be found where the valence $\mathcal{V} \neq 4$. In the cross field literature, these correspond to singular points of the cross field where crosses are undetermined. A similar phenomenon is observed in this guiding field method. We return to Eq. (3) where $\psi$ is undefined when $u=v=0$. Points where $\vec{v}=\overrightarrow{0}$ are called critical points of the guiding field and are a direct analogy of singular points of cross fields.

Critical points are tied to null isocontours in the domain. Zeroes in $u$ and $v$ emerge from boundary conditions and propagate into the interior of the domain due to the Laplace equation. This is shown in Fig. 1 where null isocontours are shown intersecting at critical points. Although $\psi$ is undefined at critical points, $\vec{v}$ remains smooth across the domain

We further need to calculate the valence of these irregular nodes, which will correspond to the number of quads connected to them. We categorize them analytically, as recognizable topologies in the $\vec{v}$ field [13], by the Poincaré index

$$
i_{\gamma}=\frac{1}{2 \pi} \oint_{\gamma} d \phi
$$

where $\gamma$ is a simple arbitrary closed curve around a critical point and

$$
\phi=\operatorname{atan} 2(\mathrm{v}, \mathrm{u})=4 \psi
$$

A regular node in the domain would yield an index of zero while irregular nodes have an index of \pm 1 . The index can, in turn, be used to calculate the number of separatrices connected to this irregular node, and therefore its valence. The index $i_{\gamma}=-1$ leads to $\mathcal{V}=5$ and $i_{\gamma}=+1$ to $\mathcal{V}=3$ [1, Lemma 5.1], [14].

We adapt the Poincaré index (6), using relation (7) to the guiding field to obtain

$$
I_{c}=\frac{1}{\pi / 2} \oint_{c} \frac{d \psi}{d \theta} d \theta
$$

where $c$ is a (small) counter-clockwise circular contour centered on the critical point. From this, the valence $\mathcal{V}$ of any point in the domain is defined as

$$
\mathcal{V}=4-I_{c} .
$$

The contour radius $c$ must be chosen so that the closed curve encloses only one critical point. If $c$ encloses multiple critical points, an incorrect combined valence will be computed. For example, two enclosed $\mathcal{V}=5$ critical points will produce a combined valence of 6 , while two opposite (one $\mathcal{V}=5$ and one $\mathcal{V}=3$ ) enclosed critical points will cancel out and produce a combined
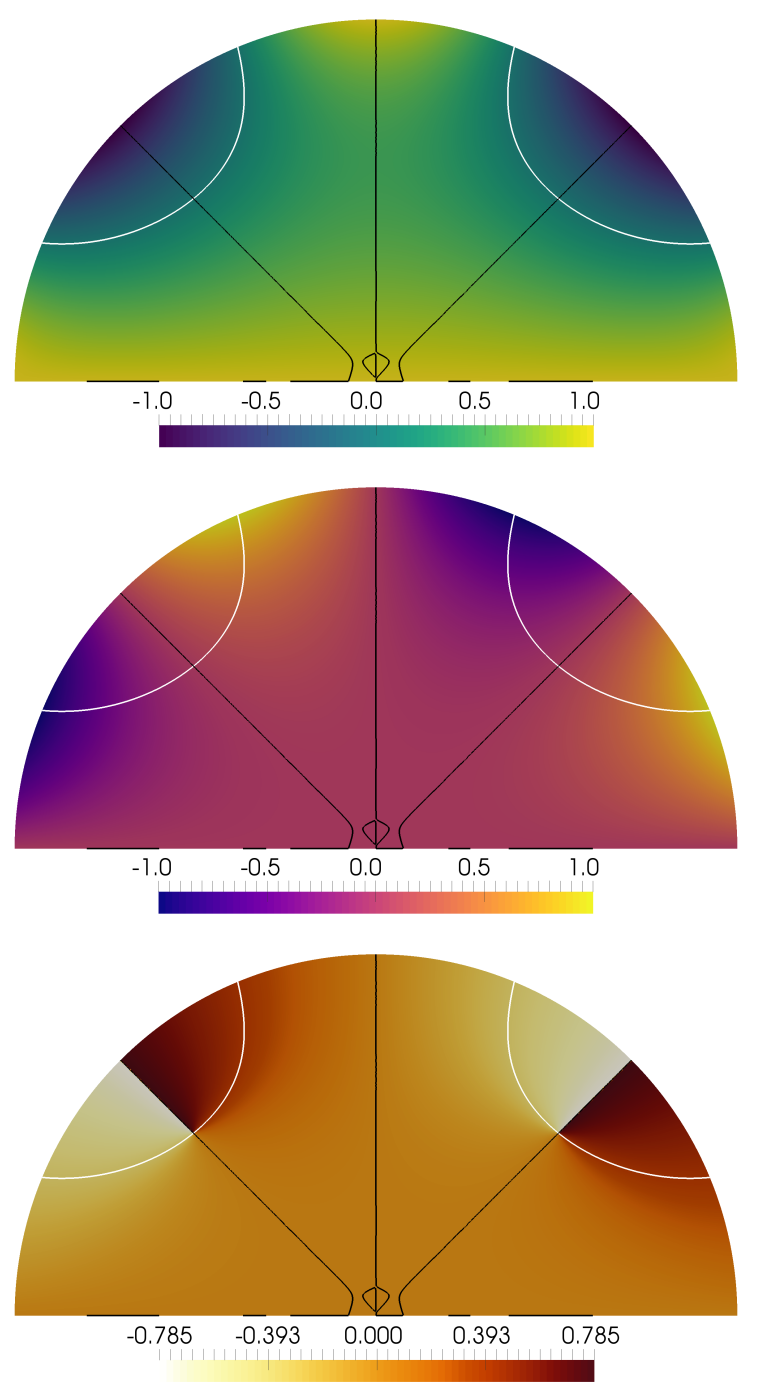

Figure 1: Solution $u$ (top) and $v$ (middle) fields and computed $\psi$ field (bottom) on the half disc geometry. Isocontours of $u=0$ and $v=0$ shown in white and black respectively.

regular node $(\mathcal{V}=4)$. In practice, critical points are far enough from each other that such problems do not arise, and one can always compute the radius around any critical point from the distance to the nearest of the remaining critical points.

Eq. (9) can be generalized to an open line circle integral to handle boundary points too [12], leading to

$$
I\left(\theta_{0}, \theta_{f}\right)=\frac{1}{\pi / 2} \int_{\theta_{0}}^{\theta_{f}} \frac{d \psi}{d \theta} d \theta
$$

such that $I_{c}=I(0,2 \pi)$. Similarly, the valence of any 
boundary point is defined as

$$
\mathcal{V}=\frac{\Delta \theta}{\pi / 2}-I\left(\theta_{0}, \theta_{f}\right)
$$

where $\Delta \theta=\theta_{f}-\theta_{0} . \quad I\left(\theta_{0}, \theta_{f}\right)$ can be viewed as a correcting factor for the valence towards an integer value.

\subsubsection{Streamline Integration}

Separatrices of the block decomposition, in the form of streamlines of the guiding field, must be traced from irregular nodes and boundary corners throughout the domain. The guiding field, akin to cross fields, can be used for this purpose too. We define a streamline as $\vec{x}(t)$ such as

$$
\frac{d \vec{x}}{d t}=\tilde{v}\left(\psi^{\prime}(\vec{x})\right)
$$

where $t$ is the integration parameter and $\tilde{v}\left(\psi^{\prime}(\vec{x})\right)$ is the adjusted guiding field vector.

Because $\psi$ is discontinuous, defined on $\left[-\frac{\pi}{4}, \frac{\pi}{4}\right]$ by (3), whereas crosses are invariant of rotations of $\pi / 2$, we use an adjusted guiding direction $\psi^{\prime}$. The guiding direction must indeed be adjusted by a multiple of $\pi / 2$ every time a streamline crosses a jump line, defined in Sec. 2.1. This allows us to retain the consistent directionality of a smooth cross field.

Eq. (12) can be integrated with respect to $t$ given appropriate initial conditions

$$
\vec{x}\left(t_{0}\right)=\vec{x}_{0},
$$

where $t_{0}$ is the initial integration time and $\vec{x}_{0}$ the location of the start of the streamline. The start of the streamline may either be an irregular node or a boundary corner. The ordinary differential equation (12) can be integrated using any traditional numerical scheme. Sec. 3.2.3 will more specifically cover the integration strategy.

\subsection{The Spectral Element Method}

We compute the BVPs (5) with either a continuous Galerkin (CG) or a discontinuous Galerkin (DG) spectral element method on a triangular mesh [3]. Spectral element methods are spectrally accurate, meaning that the convergence rate depends only on the smoothness of the solution. They are high resolution in that they use a large number of degrees of freedom within an element. Unlike traditional low order finite element methods, the high order polynomial expansion of the solution inside each element allows us to locate critical points, identify their valence, and finally trace streamlines with high order accuracy.

In this work, we use the spectral $/ h p$ element methods formulation described in detail in reference [3] and implemented in Nektar $++[15]$. We briefly survey the fundamentals of this method here for the numerical solution of PDEs, $\mathcal{L} u=0$, over a domain $\Omega$. The domain $\Omega$ is covered by a mesh of triangular finite elements, $\Omega_{e}$ so that $\Omega=\cup \Omega_{e}$ and $\Omega_{e_{1}} \cap \Omega_{e_{2}}=\partial \Omega_{e_{1} e_{2}}$ is either an empty set or the interface between two elements, and is of one dimension less than the mesh. We solve the PDE problem weakly and require that $\left.u\right|_{\Omega_{e}}$ is in the Sobolev space $H^{k}\left(\Omega_{e}\right) k=0,1$. In the CG formulation, we require the solution to be in $H^{1}, H^{0}$ for DG.

We formulate the solution to (5) in weak form: find $\vec{v} \in H^{k}(\Omega)$ such that

$$
a(\vec{v}, \vec{w})=l(\vec{w}) \quad \forall \vec{w} \in H^{k}(\Omega),
$$

where $a(\cdot, \cdot)$ is a symmetric bilinear form, $l(\cdot)$ is a linear form and $H^{k}(\Omega)$ is the Sobolev space formally defined as

$$
H^{k}(\Omega):=\left\{\vec{w} \in L^{2}(\Omega)\left|D^{\alpha} \vec{w} \in L^{2}(\Omega) \forall\right| \alpha \mid \leq k\right\} .
$$

We solve problem (14) numerically and therefore consider solutions in a finite dimensional subspace $V_{N} \subset$ $H^{k}(\Omega)$. In the finite dimensional subspace, the problem becomes: find $\vec{v}^{\delta} \in V_{N}$ such that

$$
a\left(\vec{v}^{\delta}, \vec{w}^{\delta}\right)=l\left(\vec{w}^{\delta}\right) \quad \forall \vec{w}^{\delta} \in V_{N},
$$

augmented with boundary conditions, (4). In the CG formulation, we also enforce the condition $V_{N} \subset C^{0}$.

To represent $\vec{v}^{\delta}(\vec{x})=\sum_{n} \hat{u}_{n} \Phi_{n}(\vec{x})$, we take a weighted sum of $N$ trial functions $\Phi_{n}(\vec{x})$ defined on $\Omega$. This transforms the problem of finding the subspace solution to one of finding the coefficients $\hat{u}_{n}$ that define $\vec{v}^{\delta}(\vec{x})$ within an element. To obtain a unique choice of coefficients $\hat{u}_{n}$, we require that the residual $R=\mathcal{L} \vec{v}^{\delta}$ be orthogonal to the polynomial space, i.e. that its $L^{2}$ inner product, with respect to the test functions $\Psi_{n}(\vec{x})$, is zero. In the Galerkin approximation, the projection the test functions are chosen to be the same as the trial functions, i.e. $\Psi_{n}=\Phi_{n}$.

The contributions of each element in the domain must be combined to construct the global basis $\Phi_{n}$. A parametric mapping $\mathcal{X}_{e}: \mathcal{E} \rightarrow \Omega_{e}$ exists to each element $\Omega_{e}$ from a standard reference element $\mathcal{E} \subset[-1,1]^{d}$. This mapping is given by $\vec{x}=\mathcal{X}_{e}(\vec{\xi})$, where $\vec{x}$ is the vector of the physical coordinates and $\vec{\xi}$ defines the coordinates in the reference space. In Nektar ++ , triangular, tetrahedral, prismatic, and pyramidal elements are created by collapsing one or more of the coordinate directions to create singular vertices. This allows it to support, for this work, more easily generated triangular meshes. 
We use a local polynomial basis on the reference element to represent the solution. A one-dimensional order- $P$ basis is a set of polynomials $\Phi_{p}(\xi), 0 \leq p \leq P$, defined on the reference segment $-1 \leq \xi_{1} \leq 1$. A tensor basis is used in two dimensional reference regions, where the polynomial space is constructed as the tensor product of one dimensional bases on segments and quadrilaterals.

Finally, the discrete solution in a physical element $\Omega_{e}$ can be expressed as

$$
\vec{v}^{\delta}(\vec{x})=\sum_{n} \hat{v}_{n} \phi_{n}\left(\mathcal{X}_{e}^{-1}(\vec{x})\right)
$$

with $\hat{v}_{n}$ being the coefficients computed by the Galerkin procedure. We therefore restrict the solution space to

$$
V:=\left\{\vec{v} \in H^{k}(\Omega)|\vec{v}|_{\Omega_{e}} \in \mathcal{P}_{P}\left(\Omega_{e}\right)\right\}
$$

where $\mathcal{P}_{P}\left(\Omega_{e}\right)$ is the space of order $P$ polynomials on $\Omega_{e}$.

An assembly operator assembles the element contributions to the global solution. In the CG formulation, elemental contributions of neighbours are summed to enforce $C^{0}$-continuity. In the DG formulation, flux values are transferred from the element interfaces into the global solution vector. Nektar ++ supports both discretizations, allowing us to trivially switch from one to the other at any time. The DG formulation is necessary for the method to work with arbitrary corner angles in the geometry, as will be explained in Sec. 3.1.

\subsection{1 p-Adaptation}

Spectral element methods also offer the freedom to locally increase or decrease the resolution of the solution according to an estimated local error. Adaptation is achieved by adding or removing modes - i.e. increasing or decreasing the polynomial order of individual elements. The $p$-adaptive strategy employed in this paper follows the procedure laid out in references [8] and [9]. The idea is simple: where local error is high, we increase local resolution; where local error is low, we decrease resolution. The procedure is described in Algorithm 1, where $e$ is an individual element, $S_{e}$ and $P_{e}$ are its associated error indicator and polynomial order, $\epsilon_{u}$ and $\epsilon_{l}$ are the upper and lower error thresholds, and $P_{\max }$ and $P_{\min }$ are the maximum and minimum polynomial orders allowed.

We use an estimate of the discretization error from the formulation described in reference [16] for the error indicator (sometimes called sensor). We only require to compute the solution $u_{P}$ once at order $P$, which we then project onto polynomial space $P-1$ to obtain

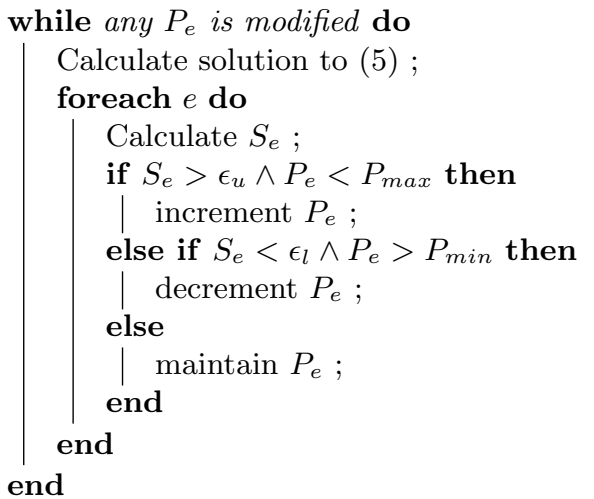

Algorithm 1: The $p$-adaptive procedure.

$u_{P-1}$. From these solutions at different polynomial orders, we calculate our sensor:

$$
S_{e}=\frac{\left\|u_{P}-u_{P-1}\right\|_{2, e}^{2}}{\left\|u_{P}\right\|_{2, e}^{2}},
$$

where $\|\cdot\|_{2}$ is the $L_{2}$-norm. For a modal expansion, this sensor is cheap to compute. It phyically represents the ratio between the energy of the highest mode and the rest of modal energy spectrum. The sensor $S_{e}$ can be computed for either component of the solution $\vec{v}$ in $(5)$.

\section{IMPLEMENTATION OF THE PROCEDURE}

The process is split into three main steps, each associated with different libraries and utilities of the Nektar ++ platform $[15,17]$ :

1. Computation of a guiding field by solving the Laplace problem (5). Carried out in the solver.

2. Analysis of the guiding field, including locating critical points, calculating their valence and the valence of corners, and the integration of streamlines. Carried out in the post-processing utility FieldConvert.

3. Generation of a quadrilateral mesh by splitting the CAD model into blocks and splitting these further into a fine mesh. Carried out in the meshing utility NekMesh.

This section provides an overview of the process, illustrated on the geometry of a half disc geometry (see Fig. 2), commonly used in the cross field literature $[14,10]$. The reader should refer to reference [6] for implementation details. 


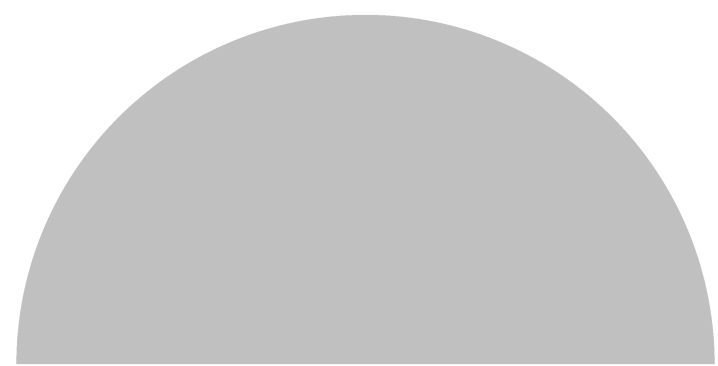

Figure 2: Geometry of a half disc for illustration of the quadrilateral meshing method.

\subsection{Computation of a Guiding Field}

A standard Laplace solver for the boundary value problem (5) is used in the open source spectral/hp element framework Nektar $++[15,17]$ with boundary conditions (4). The geometry is passed onto the guiding field solver in the form of a high order triangular mesh previously generated in NekMesh [17] following the procedure laid out in reference [18]. For the reference geometry, such a mesh is illustrated in Fig. 3.

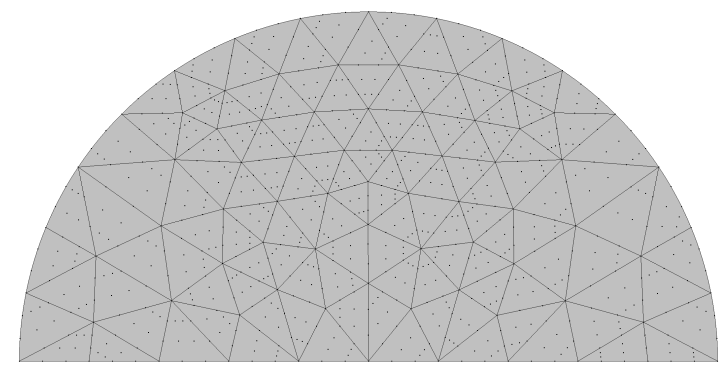

Figure 3: Third order triangular spectral element mesh for the half disc geometry.

For performance purposes, the triangular mesh should be coarse for fast computation of the guiding field. Resolution will be added in areas of interest by use of $p$-adaptation, as described in Sec. 2.2.1. A coarse mesh also proved to be lighter to process in the analysis of the guiding field, as will seen in Sec. 3.2.

Notice that the boundary conditions (BCs) (4) depend on the geometry and may therefore be discontinuous where the geometry is not smooth. To be exact, BCs are continuous if the boundaries satisfy one of two conditions:

- The curve at each point is $C^{1}$-continuous, i.e. a smooth curve; or
- The boundary curve is only $C^{0}$-continuous, i.e. a corner, and the angle is a multiple of $\pi / 2$.

Otherwise, they are discontinuous.

A discontinuous Galerkin formulation, available in Nektar ++ , offers a discretization consistent way of handling discontinuous BCs without the need for ad $h o c$ smoothing of a corner's BCs as used in traditional low order techniques [1].

As an example of a geometry that requires a DG solve, we present the polygon geometry shown in Fig. 4, which shows the solution and $\psi$ fields. We note that the discontinuous BCs are naturally imposed with the Dirichlet BCs enforced through fluxes when needed. Considerations beyond the scope of this paper are made to address the discontinuous nature of the field solution, but they however do not affect the rest of the procedure.

Following the calculation of a solution, $p$-adaptation can be applied iteratively to locally increase or decrease the local polynomial order inside each element. Using the approach described in Sec. 2.2.1, a finer solution is obtained without the need to uniformly increase the resolution and therefore the cost of the calculation. Fig. 5 shows the final distribution of local number of modes when adapting based on either $u$ (top) or $v$ (bottom). Higher polonomial orders are found near the boundaries where the gradient of the solution is stronger.

\subsection{Analysis of the Guiding Field}

The mesh generation procedure continues with an analysis of the guiding field, in a way where crosses are never generated. The analysis is carried out in FieldConvert, Nektar++'s post-processing utility [17], and consists of three main parts:

1. Location of interior critical points.

2. Calculation of the valence of interior critical points and corners.

3. Integration of streamlines.

The goal of this analysis is to construct a separatrix graph to pass on to NekMesh for the generation of a coarse high order quadrilateral mesh.

\subsubsection{Location of Interior Critical Points}

As described in Sec. 2.1.1, interior critical points are located at $\vec{v}=\overrightarrow{0}$. To locate all of them we take a simple narrowing down approach. We first identify elements 

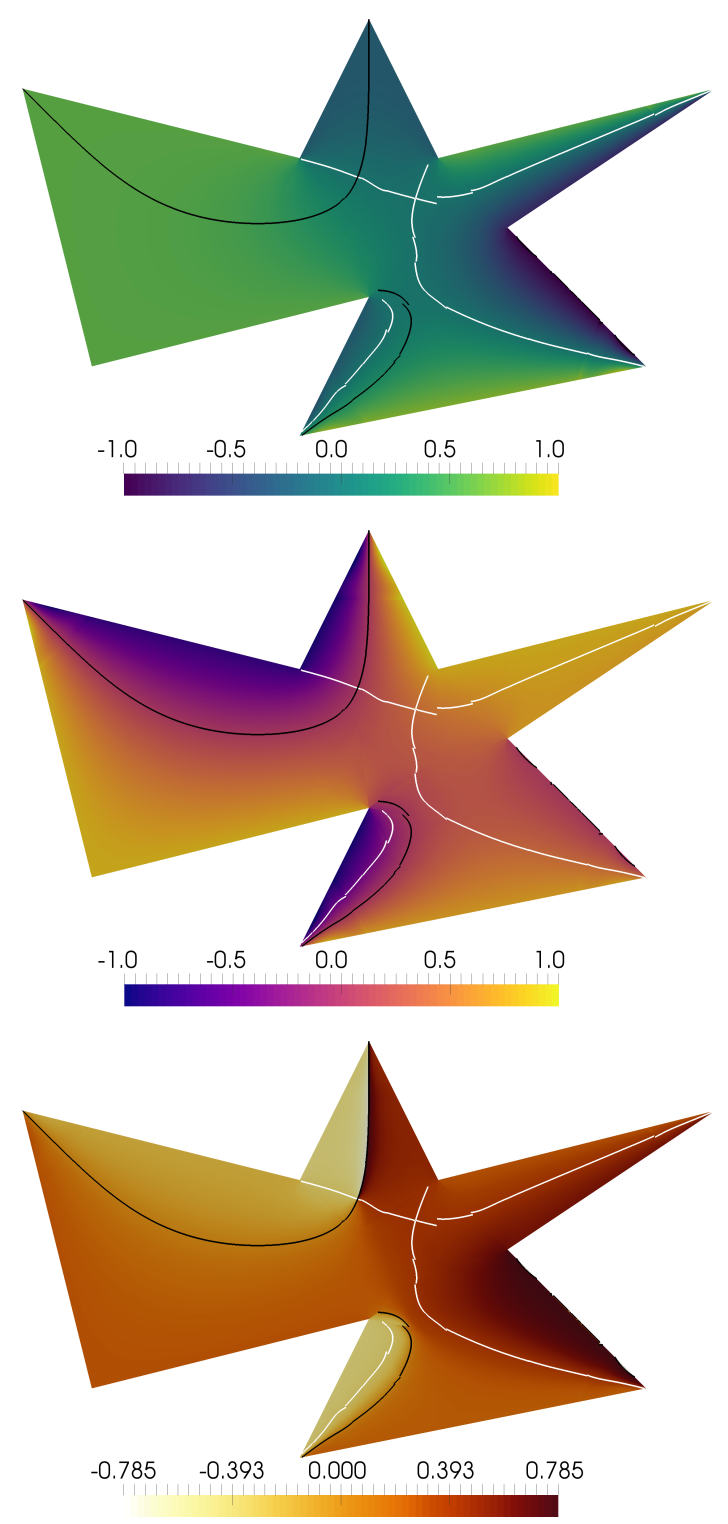

Figure 4: Solution $u$ (top) and $v$ (middle) fields and computed $\psi$ field (bottom) on a polygon with acute and obtuse corners using a DG discretization. Isocontours of $u=0$ and $v=0$ are shown in white and black respectively.

that are likely to contain a $\vec{v}=\overrightarrow{0}$ point. These elements should have at least one quadrature point with each of the values $u \leq 0, u \geq 0, v \leq 0$ and $v \geq 0$. These elements either contain a critical point or are close to an element that does. For each of these elements, we perform a Newton search for a point satisfying $\vec{v}=\overrightarrow{0}$. A critical point may be found outside of the element boundaries, in which case we ignore it. Fig. 1 shows the location of the two interior critical points of
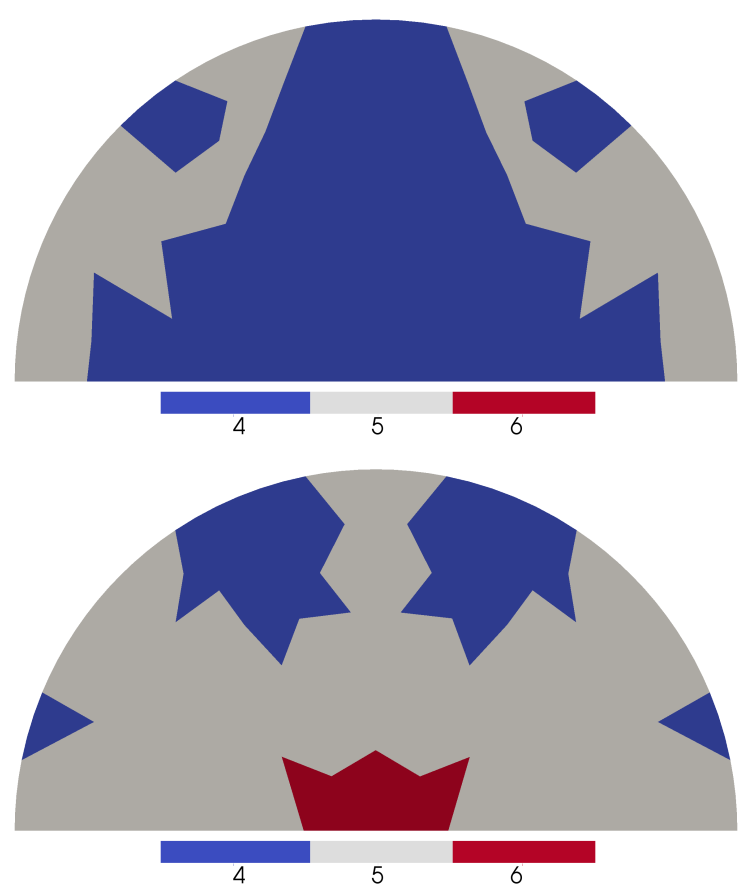

Figure 5: Distribution of local number of modes (= $P+1)$ for half disc geometry. Adaptation based on $u$ (top) and $v$ (bottom).

the reference geometry as the crossing of the $u$ and $v$ zero contours, consistent with the literature [14].

We accelerate the search inside each element by performing Netwon's method in reference space. Along with the initial identification of likely elements, all operations are performed in reference space at negligible computational cost.

\subsubsection{Calculation of Valences}

After the interior critical points are located, valences of these as well as of corners can be computed using (9) and (11) respectively.

Here again, crosses are not generated and valences can be computed from the guiding field alone. We note that the closed curve circle integral $I_{c}$ in (8) evaluates to zero when $\psi$ is continuous. This is the case of regular nodes in the domain. Where a critical point appears, lines of $\psi$ jumps appear and $I_{c}$ can be evaluated based on the number and direction of these. In a counter-clockwise manner, a positive jump (i.e. from $-\frac{\pi}{4}$ to $\frac{\pi}{4}$ ) leads to a negative index $I_{c}=-1$, and vice versa. The sign of the jump can itself be determined solely by values of $\vec{v}$, without the need to compute $\psi$ or the cross field.

Using this knowledge, we can calculate the valence of 
interior critical points from (9) by marching on a circle of small radius $c$ around each critical point. The same reasoning is applied to the calculation of corner valences with (11) where $\theta_{0}$ and $\theta_{f}$ must also be taken into account and are found trivially. From this logic, we find that the two critical points of the reference geometry are characterized by a single negative jump line each, and therefore have a valence $\mathcal{V}=3$. Likewise, the two corners have a trivially evaluated valence $\mathcal{V}=1$ due to $\Delta \theta=\pi / 2$ and the absence of any jump line in $\psi$. See Fig.1 (bottom) for reference.

Although these evaluations are performed in physical space at a negligible computational cost, they can further be accelerated by performing them in reference space.

\subsubsection{Integration of Streamlines}

Streamlines emanate from the interior irregular nodes and corners. Because $\vec{v}=\overrightarrow{0}$ at irregular nodes, $\psi$ is undefined. Likewise, $\psi$ at corners may be ambiguous if BCs are discontinuous. It is therefore necessary to identify the initial direction of each streamline by looking at the value of $\psi$ nearby the irregular nodes, at a small distance $c$ from them. The initial direction can be found iteratively by progressively refining an initial guess, akin to a fixed point search. For the first streamline of an interior irregular node, any initial guess may be taken. For subsequent streamlines as well as for streamlines coming from corners, an initial guess can be take at angles multiples of $\frac{\Delta \theta}{\mathcal{V}}$ where $\Delta \theta=2 \pi$ for an interior irregular node.

Subsequently, all streamlines are synchronously advanced throughout the domain by integrating (12). At any point, two streamlines may meet head on. For that purpose, we check the proximity of all streamline front points as well as their direction. If two front points are within merging distance and if they are pointing in opposite directions, their respective streamlines are merged. This merging distance is typically taken as the integration step size but it can also be artificially increased to perform aggressive merging, therefore reducing the total number of streamlines and simplifying the separatrix graph. Fig. 6 illustrates the difference between normal (top) and aggressive (bottom) merging. When two streamlines are flagged for merging, they are advanced up to each other's starting point and merged pointwise using trigonometric weight functions to retain an optimal streamline direction. These trigonometric weight functions deflect streamlines as is illustrated in Fig. 6 (bottom). Reference [6] contains more technical details regarding the procedure.

A $4^{\text {th }}$ order multi step Adams-Bashforth integrator is used in physical space. This integrator requires a search for the element that contains every new point,
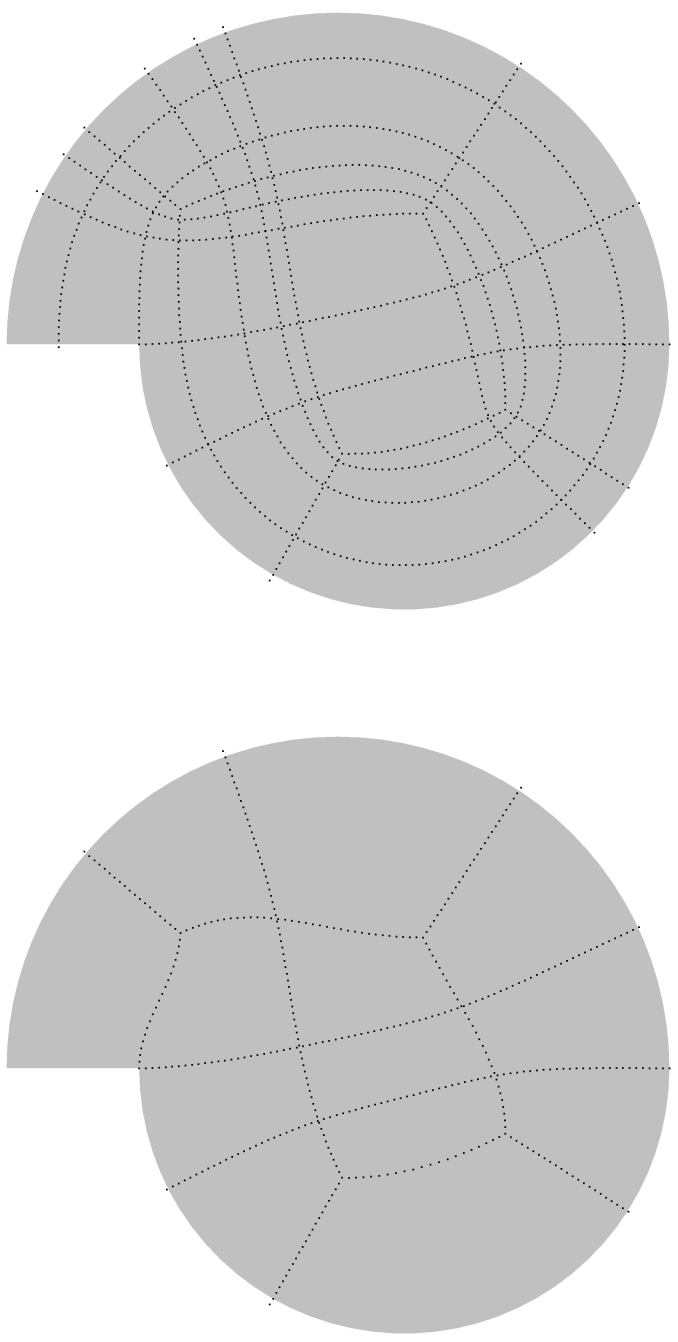

Figure 6: Example of normal (top) and aggressive (bottom) merging. A distance threshold of 5 times the step size was used for aggressive merging.

an operation that is expensive on large meshes. An inverse mapping is used to transform to parametric coordinates before the high order interpolation fo $\vec{v}$ is computed via (17). This process can be accelerated by integrating streamlines in reference space in a similar fashion to the work of Coppola et al. [19]. A standard Runge-Kutta scheme can then used while retaining the speed of reference space based integration.

\subsubsection{Midpoint Division}

A note should be made about corners with very shallow angles. Eq. (11) can lead to a valence of zero 
when $\Delta \theta$ and $\Delta \psi$ cancel each other. This represents the situation of all streamlines converging towards a corner. We call this corner degenerate as it will produce a triangular block, i.e. a collapsed quadrilateral block. This topology is not valid for the purpose of quadrilateral-only mesh generation, so we resort to $a d$ hoc manipulations. The triangular block can easily be split into three quadrilateral blocks using a midpoint division approach [20]. Practically, a 3-valence irregular node is introduced at the center of the triangular block a posteriori. Two of the three streamlines can be extended towards the domain boundaries in a straight line while the third streamline is integrated away from the degenerate corner towards the inside of the domain. An example of an ad hoc midpoint division manipulation is shown in Fig. 7.

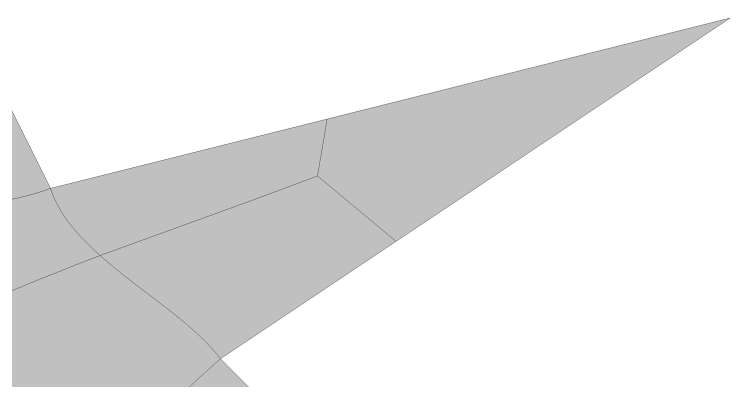

Figure 7: Detail of a domain showing a midpoint division of a degenerate quadrilateral caused by a sharp angled corner. The full domain is shown in Fig. 11.

\subsection{Generation of a Quadrilateral Mesh}

Once a separatrix graph has been generated, it is passed to NekMesh, Nektar++'s high order mesh generation utility [17]. NekMesh uses OpenCASCADE [21] as its CAD system for high order mesh generation. The OpenCASCADE kernel offers not only CAD query capabilities but also CAD modification tools. The discrete separatrices computed by FieldConvert are converted into interpolating splines. These splines can then be used to split the original CAD domains into the desired quadrilateral blocks. This results in a CAD model of topologically connected quadrilateral faces. A coarse high order quadrilateral mesh can then be trivially generated by NekMesh.

Such meshes are typically too coarse for spectral element analysis; it is often necessary to split them further. We take advantage of an isoparametric splitting approach described in [22] to refine strings of blocks, extended in references [23, 24] for bidirectional splitting. The method leverages the mapping between physical and reference space coordinates by splitting elements in reference space to obtain valid high order elements in physical space. In this procedure, it also preserves optimal alignment of blocks. Fig. 8 shows the coarse and fine meshes obtained on the reference geometry.
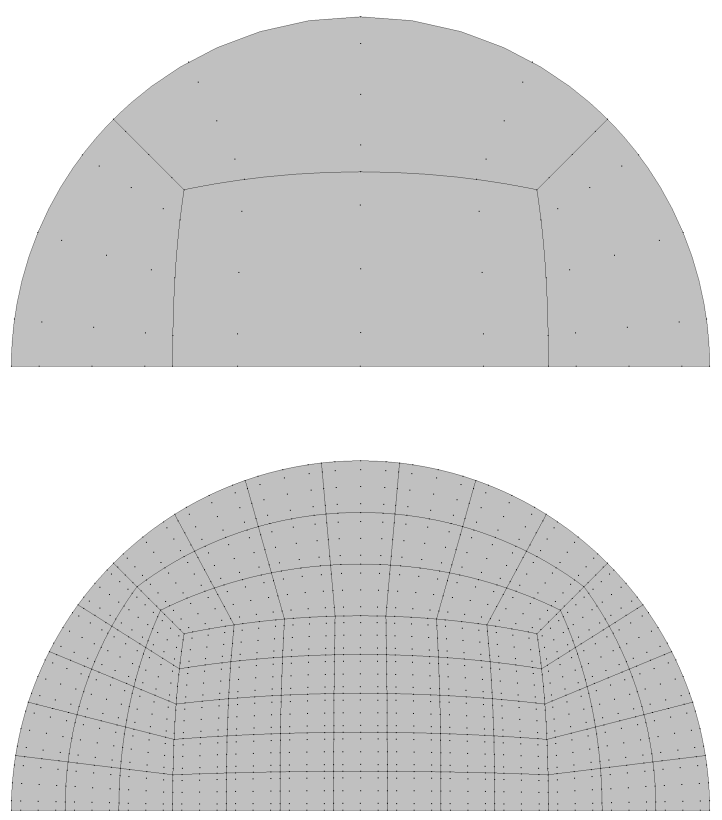

Figure 8: Coarse (top) and subdivided (bottom) quadrilateral meshes obtained on the half disc geometry based on detected critical points and traced and merged streamlines.

\section{ADVANTAGES OF THE METHOD}

We first illustrate advantages of the method with examples before analysing the use of $p$-adaptation in the procedure. These example geometries, e.g. the reference geometry of Fig. 2, are typically used as examples in the cross field literature $[1,14,10]$. The advantages that we want to illustrate in this section include minimal number of irregular nodes and possibility to aggressively merge streamlines (Geometry I), validity of the block decompositions and absence of limit cycles (Geometry II), a discretization consistent way of handling corners (Geometry III), the preservation of symmetries and patterns (Geometry IV), and finally the flexibility in generating finer meshes (Geometry $\mathrm{V})$.

Geometry I (Fig. 9) is a multiply connected domain previously meshed using cross fields in [14]. While some methods generate spurious irregular nodes in the upper central part of the domain, the approach described here generates only two 3 -valence nodes points to obtain an optimal block decomposition of the do- 
main. Aggressive merging can also be applied to connect the two irregular nodes. The reader will observe that optimal angles are preserved at the irregular nodes thanks to the trigonometric weights for merging.
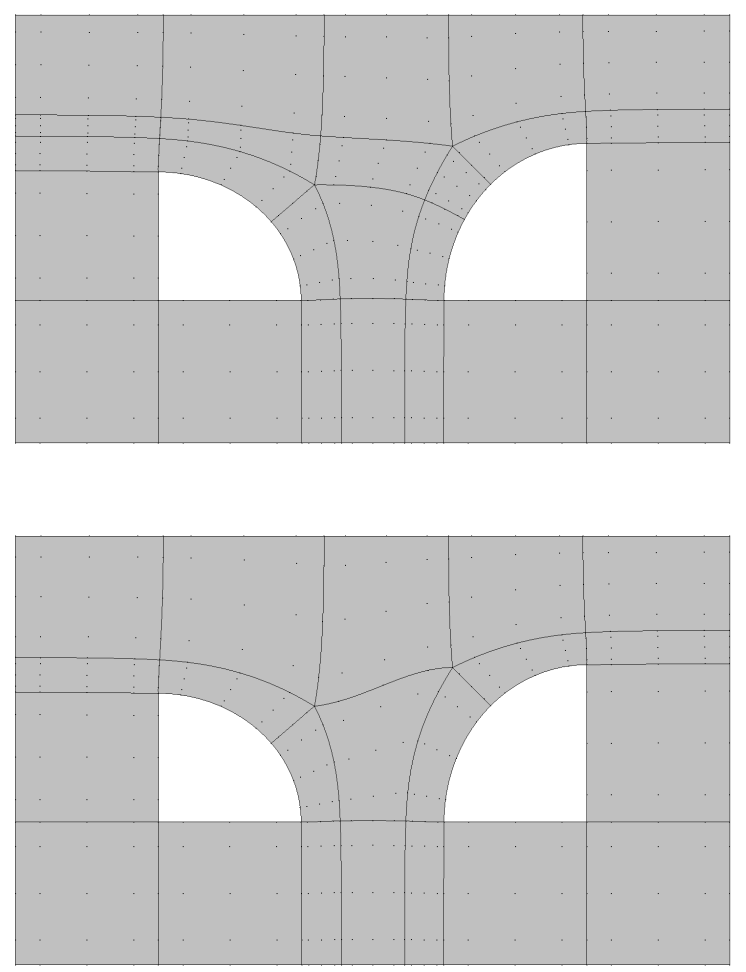

Figure 9: Coarse quadrilateral mesh obtained on Geometry I with normal (top) and aggressive (bottom) merging.

Geometry II (Fig. 10), sometimes called the nautilus, is typically prone to a limit cycle of the streamlines in low order methods leading to invalid block decompositions without ad hoc manipulations $[1,10]$. The formulation of the problem generates a guiding field in which no limit cycle appears. In fact, the irregular node placement is similar to that of a simple disc, with four 3-valence irregular nodes the optimal number for a valid block decomposition. As noted in Sec. 3.2.3, this improved separatrix graph was subject to aggressive streamline merging, for a visually more pleasing coarse mesh.

Geometry III (Fig. 11) illustrates the use of a DG discretization to handle angles that are not multiples of $\pi / 2$ without the need for ad hoc smoothing of corners. The rest of the procedure remains however unchanged for easier code implementation and maintenance. This example also features a sharp corner with a valence of zero whose connected degenerate block is split using the midpoint division method.

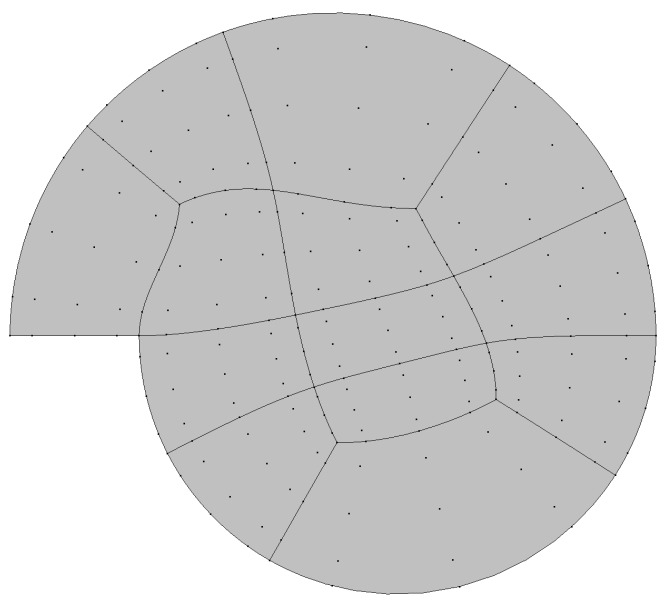

Figure 10: Coarse quadrilateral mesh obtained on Geometry II, a nautilus.

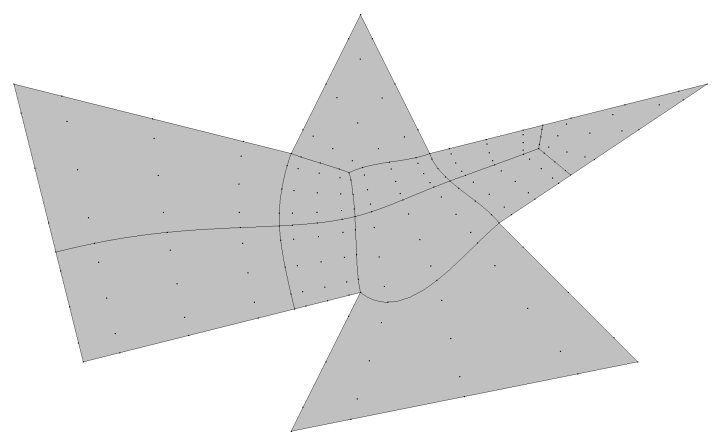

Figure 11: Coarse quadrilateral mesh obtained on Geometry III, a polygon.

Geometry IV (Fig. 12) illustrates the preservation of symmetries and patterns. The geometry is that of a simple gear and we observe the same irregular node pattern as seen in the cross field literature [1].

Whereas Geometries I-III illustrate the generation of naturally curved very coarse meshes, finer meshes are typically required for SEM analysis with user defined element size requirements. Geometry V (Fig. 13) illustrates the use of the isoparametric splitting to obtain a finer mesh with a certain distribution of elements. The geometry is that of NACA 0012 aerofoil, a typical geometry used in academic CFD studies.

We next demonstrate the use of $p$-adaptation. For Geometry I, we show the distribution of the local number of modes $(=P+1)$ after $p$-adaptation in Fig. 14 . The local polynomial order is higher mostly near bound- 


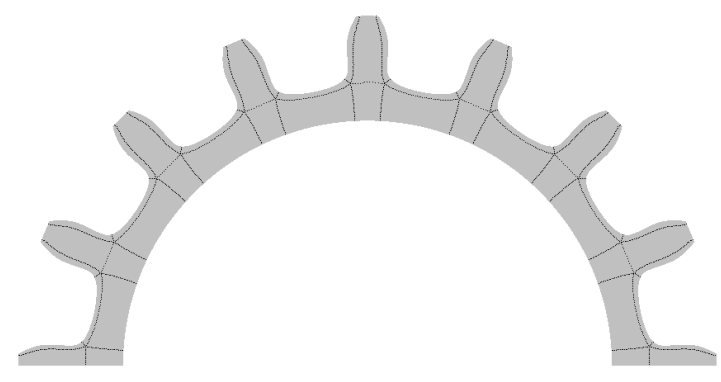

Figure 12: Block decomposition obtained on Geometry IV, a gear.
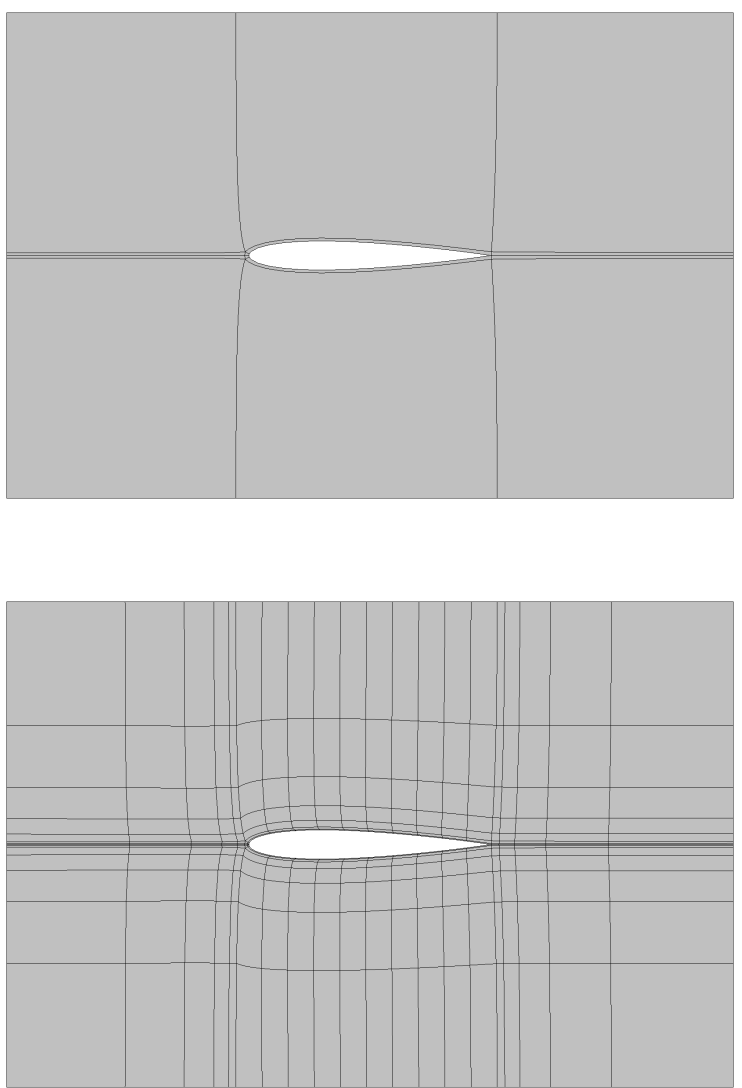

Figure 13: Coarse (top) and split (bottom) quadrilateral meshes obtained on Geometry V, a NACA 0012 profile. High order interior degrees of freedom have been hidden for clearer visualization.

aries and in the central area where rapid change in the solution is observed. The local polynomial order also seems unexpectedly high in the $v$-based case but this can be easily explained by the actual solution. Be- cause the geometry is dominated by Cartesian-aligned straight-sided boundaries, a large part of the field (especially the areas in the bottom left and right) has values close to $\vec{v}=(1,0)$. This means that the low order modes have no energy for the $v$ component. Therefore, for the $v$ based sensor, any energy appearing in the higher order modes will trigger an increase in the local polynomial order. For such examples dominated by Cartesian-aligned boundaries, it is therefore best to base the sensor on $u$ since $u_{b}=1$ in Cartesian directions. The same phenomenon is retrospectively observed for the half disc geometry in Fig. 5.
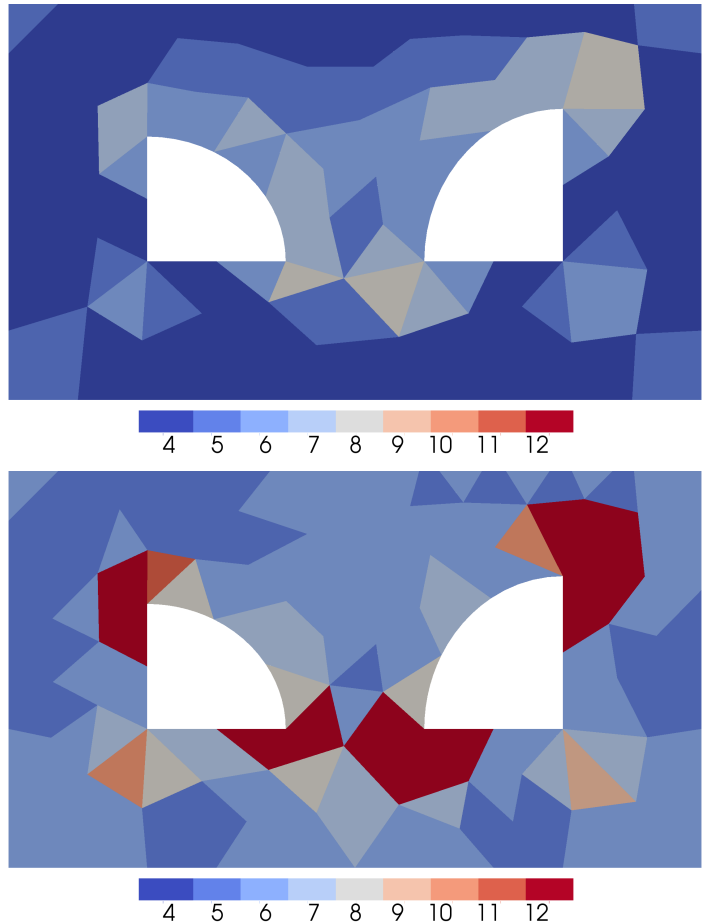

Figure 14: Distribution of local number of modes $(=P+1)$ for Geometry I. Adaptation based on $u$ (top) and $v$ (bottom).

The use of high order methods might intuitively sound prohibitively expensive for this method. We find, however, that the computational time remains low, with all examples in this paper taking $\mathcal{O}\left(10^{0}\right)$ seconds to process. The main cost in NekMesh is the computation of the streamlines because that is currently done in physical, not reference, space. Streamline integration currently takes between roughly $50 \%$ and $90 \%$ of the time of the whole procedure, depending on the size of the domain and of the base mesh, the integration step size, and how far streamlines have to travel. All other tasks - each solution of the Laplace problem, the analysis of the critical points and corners and the generation of a quad mesh - take about the same share of the remaining $10 \%$ to $50 \%$ of computational 
time.

\section{SUMMARY AND CONCLUSIONS}

We have demonstrated the benefits of a field guided method to generate quadrilateral block decompositions, and meshes, of multiply connected two dimensional domains. The guiding field is inspired by cross fields but crosses are, in fact, never generated. This method generates naturally curved elements suitable for spectral element methods, and other related methods, and it does so by using a spectral element solver. The guiding field is obtained from a boundary value problem using a Laplace solver, with boundary conditions determined by local geometrical information. This information can be discontinuous, in which case a discontinuous Galerkin formulation is used for a discretization consistent way of imposing these discontinuous boundary conditions. The separatrix graph, obtained from the analysis of the guiding field, is later used to split the two dimensional model. From this block decomposed geometry, a coarse, valid, curved mesh is generated that can be refined to users' needs. The procedure is implemented in the open source spectral/ $h p$ element framework Nektar ++ and its high order mesh generation tool NekMesh.

We emphasize some of the benefits of this method including some advantages over traditional cross field based approaches as well. Whereas high order mesh generation techniques typical suffer from invalid elements, the method presented here creates coarse a priori curved meshes that are naturally valid. It does so by using a spectral element solver, which not only benefits from spectral convergence but is also suitable to $p$-adaptation for better computational resources. This creates a high resolution guiding field, due to the high order information of the solution, used for the creation of an optimal separatrix graph: small number of irregular nodes, preservation of symmetries, absence of limit cycles, etc. The analysis of the guiding field is performed in reference space at a low computational cost. The refinement of the coarse mesh is later performed using an isoparametric splitting approach that preserves the high order validity of the mesh.

The authors envision two improvements of this proof of concept for better relevance in the CFD community. On the one hand, an extension to three dimensional hexahedral block decompositions must be sought. This extension requires a reformulation of the problem based on three dimensional frame arithmetic. Promising work of Ray et al. [25] is paving the way in that direction. On the other hand, the generation of metric aligned quadrilateral blocks, as was done in reference [26], seems possible through the use of a forcing term. The solution of a Poisson problem with adequate forcing would enable modifications of the sep- aratrix graph with respect to a metric field. Such a solution would also be appropriate for the relocation of irregular nodes away from boundaries with strong curvature (see for example Fig. 13).

\section{ACKNOWLEDGEMENTS}

This project has received funding from the European Union's Horizon 2020 research and innovation programme under the Marie Skłodowska-Curie grant agreement No 675008. This work was supported by a grant from the Simons Foundation (\#426393, David Kopriva). The first two authors would also like to thank Prof. Gustaaf Jacobs of the San Diego State University for his hospitality. Finally, JM would like to thank the committee of the $28^{t h}$ International Meshing Roundtable for their travel financial support.

\section{References}

[1] Viertel R., Osting B. "An Approach to Quad Meshing Based on Harmonic Cross-Valued Maps and the Ginzburg-Landau Theory." 2017

[2] Kozdon J., Dunham E., Nordström J. "Simulation of Dynamic Earthquake Ruptures in Complex Geometries Using High-Order Finite Difference Methods." Journal of Scientific Computing, vol. 55, no. 1, 92-124, 2013

[3] Karniadakis G.E., Sherwin S.J. Spectral/hp Element Methods for Computational Fluid Dynamics (Second Edition). Oxford University Press, 2005

[4] Kopriva D.A. Implementing Spectral Methods for Partial Differential Equations. Scientific Computation. Springer, May 2009

[5] Bommes D., Lévy B., Pietroni N., Puppo E., Silva C., Tarini M., Zorin D. "Quad-Mesh Generation and Processing: A Survey." Computer Graphics Forum, vol. 32, no. 6, 51-76, 2013

[6] Marcon J., Kopriva D., Sherwin S., Peiró J. "A High Resolution PDE Approach to Quadrilateral Mesh Generation." Journal of Computational Physics, 2019. In press

[7] Li Y., Premasuthan S., Jameson A. "Comparison of Adaptive h and p Refinements for Spectral Difference Methods." 40th Fluid Dynamics Conference and Exhibit. American Institute of Aeronautics and Astronautics, Reston, Virigina, jun 2010

[8] Ekelschot D., Moxey D., Sherwin S., Peiró J. “A p-adaptation method for compressible flow problems using a goal-based error indicator." Computers $\&$ Structures, apr 2016 
[9] Moxey D., Cantwell C., Mengaldo G., Serson D., Ekelschot D., Peiró J., Sherwin S., Kirby R.M. "Towards p-Adaptive Spectral/hp Element Methods for Modelling Industrial Flows." Spectral and High Order Methods for Partial Differential Equations ICOSAHOM 2016, pp. 63-79. Springer, Cham, 2017

[10] Viertel R., Staten M., Osting B. "Toward a paver replacement." 26th International Meshing Roundtable, IMR26. 2017

[11] Bunin G. "A Continuum Theory for Unstructured Mesh Generation in Two Dimensions." Tech. rep., sep 2006

[12] Bunin G. "Towards Unstructured Mesh Generation Using the Inverse Poisson Problem." ArXiv e-prints, Feb. 2008

[13] Tricoche X., Scheuermann G., Hagen H. "Continuous Topology Simplification of Planar Vector Fields." Proceedings of the Conference on Visualization '01, VIS '01, pp. 159-166. IEEE Computer Society, Washington, DC, USA, 2001

[14] Kowalski N., Ledoux F., Frey P. "A PDE based approach to multi-domain partitioning and quadrilateral meshing." 21th Int. Meshing Roundtable. 2012

[15] Cantwell C., Moxey D., Comerford A., Bolis A., Rocco G., Mengaldo G., De Grazia D., Yakovlev S., Lombard J.E., Ekelschot D., Jordi B., Xu H., Mohamied Y., Eskilsson C., Nelson B., Vos P., Biotto C., Kirby R., Sherwin S. "Nektar++: An open-source spectral/hp element framework." Computer Physics Communications, vol. 192, 205-219, 2015

[16] Persson P.O., Peraire J. "Sub-Cell Shock Capturing for Discontinuous Galerkin Methods." 44th AIAA Aerospace Sciences Meeting and Exhibit. American Institute of Aeronautics and Astronautics, Reston, Virigina, jan 2006

[17] Moxey D., Cantwell C., Bao Y., Cassinelli A., Castiglioni G., Chun S., Juda E., Kazemi E., Lackhove K., Marcon J., Mengaldo G., Serson D., Turner M., Xu H., Peiró J., Kirby R., Sherwin S. "Nektar++: enhancing the capability and application of high-fidelity spectral $/ h p$ element methods." Computer Physics Communications, 2019. Under review

[18] Sherwin S., Peiró J. "Mesh generation in curvilinear domains using high-order elements." International Journal for Numerical Methods in Engineering, vol. 53, no. 1, 207-223, jan 2002
[19] Coppola G., Sherwin S., Peiró J. "Nonlinear Particle Tracking for High-Order Elements." Journal of Computational Physics, vol. 172, no. 1, 356386 , sep 2001

[20] Li T., McKeag R., Armstrong C. "Hexahedral meshing using midpoint subdivision and integer programming." Computer Methods in Applied Mechanics and Engineering, vol. 124, no. 1-2, 171-193, jun 1995

[21] Open Cascade SAS. "Open Cascade.", 2018

[22] Moxey D., Green M., Sherwin S., Peiró J. "An isoparametric approach to high-order curvilinear boundary-layer meshing." Computer Methods in Applied Mechanics and Engineering, vol. 283, 636-650, jan 2015

[23] Marcon J., Turner M., Peiró J., Moxey D., Pollard C., Bucklow H., Gammon M. "High-order curvilinear hybrid mesh generation for CFD simulations." 2018 AIAA Aerospace Sciences Meeting, January. American Institute of Aeronautics and Astronautics, Reston, Virginia, jan 2018

[24] Marcon J., Peiró J., Moxey D., Bergemann N., Bucklow H., Gammon M. "A semi-structured approach to curvilinear mesh generation around streamlined bodies." AIAA Scitech 2019 Forum. American Institute of Aeronautics and Astronautics, Reston, Virginia, jan 2019

[25] Ray N., Sokolov D., Lévy B. "Practical 3D frame field generation." ACM Transactions on Graphics, vol. 35, no. 6, 1-9, nov 2016

[26] Fogg H.J., Armstrong C.G., Robinson T.T. "Multi-Block Decomposition Using CrossFields." J.P.M. de Almeida, P. Díez, C. Tiago, N. Pares, editors, VI International Conference on Adaptive Modeling and Simulation. 2013 


\title{
UNTANGLING HIGH-ORDER MESHES BASED ON SIGNED ANGLES
}

\author{
Mike Stees $^{1} \quad$ Myra Dotzel $^{2} \quad$ Suzanne M. Shontz ${ }^{3}$ \\ ${ }^{1}$ Department of Electrical Engineering and Computer Science, Information and Telecommunication \\ Technology Center, University of Kansas, 1520 W. 15th Street, Lawrence, KS, 66045, USA, \\ mstees@ku.edu \\ ${ }^{2}$ Department of Electrical Engineering and Computer Science, Department of Mathematics, \\ Information and Telecommunication Technology Center, University of Kansas, 1520 W. 15th Street, \\ Lawrence, KS, 66045, USA, myradotzel@ku.edu \\ ${ }^{3}$ Department of Electrical Engineering and Computer Science, Bioengineering Program, Information \\ and Telecommunication Technology Center, University of Kansas, 1520 W. 15th Street, Lawrence, KS, \\ 66045, USA, shontz@ku.edu
}

\begin{abstract}
One challenge in the generation of high-order meshes is that mesh tangling can occur as a consequence of moving the new boundary nodes to the true curved boundary. In this paper, we propose a new optimization-based method that uses signed angles to untangle invalid second- and third-order triangular meshes. Our proposed method consists of two passes. In the first pass, we loop over each high-order interior edge node and minimize an objective function based on the signed angles of the pair of triangles that share the node. In the second pass, we loop over face nodes and move them to the mean of the high-order nodes of the triangle to which the face node belongs. We present several numerical examples in two dimensions with second- and third-order elements that demonstrate the capabilities of our method for untangling invalid meshes.
\end{abstract}

Keywords: high-order mesh untangling, optimization, curvilinear triangular meshes

\section{INTRODUCTION}

One appealing aspect of high-order methods for solving partial differential equations is their ability to obtain more accurate solutions with a lower computational overhead than the corresponding low-order methods. One barrier to the adoption of these methods in the presence of curved domains is the lack of software capable of robustly generating high-order meshes [1]. In particular, to achieve the full potential of high-order methods in the presence of curved domains, these methods need to be paired with a highorder mesh that conforms to the curved domain [2,3].

The typical approach used by high-order mesh generation methods is to apply a transformation to a coarse low-order mesh $[4,5,6,7,8,9,10,11,12,13,14,15$, $16,17,18,19]$. The main difficulty in applying the transformation is obtaining a valid high-order mesh as the result. In general, these methods consist of the following three steps: (1) additional nodes are added to the low-order mesh; (2) the newly-added boundary nodes are projected onto the curved domain, and (3) the interior nodes are moved as a result of the boundary deformation. There are generally two approaches which are especially popular for transforming the low-order mesh. The first approach transforms the mesh based on optimization of an objective function $[4,6,7,11,12,14,15,16,17,20,21]$. Many of the proposed objective functions include a measure of element validity, which allows the methods to address 
invalid elements. While not all of the methods guarantee successful untangling, many of them are robust $[4,6,7,11,12,17]$. The second approach transforms the mesh based on the solution of a partial differential equation $[5,8,10,19]$. More specifically, Xie et al. [19] employed a linear elasticity approach, while Persson and Peraire [10] considered a nonlinear elasticity approach. Moxey et al. [8] used a thermoelastic model, and Fortunato and Persson [5] expressed the problem in terms of the Winslow equations.

In this paper, we describe a new two-pass method for untangling invalid second- and third-order triangular meshes. The first pass is an optimization-based approach that minimizes an objective function based on signed angles for each high-order interior edge node. The second pass is a smoothing step for the face nodes. The main focus of this work is to untangle invalid meshes that result from the boundary curving step of a typical high-order mesh generation method. Toward that end, we apply our method to several second- and third-order meshes that have invalid elements following the boundary curving process. The remainder of this paper is organized as follows. In Section 2, we present our new two-pass method for high-order mesh untangling. In Section 3, we demonstrate the performance of our method on several two dimensional examples. Finally, in Section 4, we offer concluding remarks and discuss some directions for our future work.

\section{UNTANGLING HIGH-ORDER CURVILINEAR MESHES}

In this section, we propose a two-pass local nodebased method for untangling high-order curvilinear triangular meshes. The first pass is based on the signed angles of curvilinear triangles, where a negative angle indicates tangling. For each iteration of the problem, we consider a high-order interior edge node. Then, we identify the two triangles that share the node and examine the four angles made by the tangent vectors adjacent to that edge. Our algorithm then moves the high-order edge node with the goal of making these angles positive. In our first pass, we solve the following unconstrained optimization problem:

$$
\begin{aligned}
f(x) & =(1-\beta)\left\|\mathbf{x}-\mathbf{x}_{\mathbf{I}}\right\|_{2}+\beta \sum_{i=1}^{4} \mathbf{e}^{-\mathbf{1 0} * \alpha_{\mathbf{i}}(\mathbf{x})}, \\
x^{*} & =\underset{\mathbf{x}}{\operatorname{argmin}} f(x) .
\end{aligned}
$$

where $\alpha_{\mathbf{i}}$ is the $i^{\text {th }}$ entry of the vector of the four signed angles adjacent to a given interior edge; $\mathbf{x}$ is the nodal position of the high-order edge node to be moved; $\mathbf{x}_{\mathbf{I}}$ is the initial position of the node at the start of the optimization, and $\beta$ is a user-defined weighting param- eter. By changing the value of $\beta$, more emphasis can be applied to the angles or the displacement of the node from its initial position. Note, if too much emphasis is placed on the displacement of the node, then the norm will dominate the objective function values, and the mesh will not be untangled.

To better understand the behavior of the objective function, consider the examples shown in Fig. 1 and the corresponding values shown in Fig. 2(a). The $\beta$ value in this example was 0.35 . In Fig. $1(\mathrm{a})$, we show the initial tangled mesh. At this point, the first term of $f(x)$ is zero because the interior node has not been moved. The second term will thus dominate the value of $f(x)$. In Fig. 1(b), we show the mesh after applying two iterations of the optimization method. As we see in the first two rows of Fig. 2(a), in both examples, the exponential term is the primary contributor to $f(x)$ because of $\alpha_{3}$, the negative angle. In Fig. 1(c,d), we see that the impact of the exponential term decreases as the values of the angles increase (e.g., from negative to positive) after four and nine iterations, respectively. In other words, the second term in $f(x)$ acts as a penalty function to enforce positive angles (i.e., an untangled mesh). Once the angles become sufficiently positive, then the first term in $f(x)$ becomes a larger contributor to the overall value of $f(x)$. The goal of this term is to reduce the amount of displacement for a given node by minimizing the node's distance from its initial location.

To find a local minimum of our unconstrained optimization problem, we use a derivative-free method. We do so because of the complexity of evaluating $f(x)$, specifically, the signed angle calculations. In particular, to solve our optimization problem, we use the Nelder-Mead simplex method [22]. For the motivational example in Fig. 1, a relaxed convergence tolerance of 0.01 was used for the Nelder-Mead simplex method. For all of our examples in the next section, the tolerance and maximum number of iterations for Nelder-Mead were 0.0001 and 400, respectively. Convergence is reached when the change in function values and the step size both satisfy the tolerance.

As described in [22], the Nelder-Mead simplex method is a direct search method that maintains a simplex at each step of the method. This simplex is defined by $n+1$ vertices and the corresponding function values, where $n$ is the dimension of the problem space. Before moving forward, let us introduce the following notation for the description of the $2 \mathrm{D}$ method. Let the vertices of the current simplex be represented as $v_{1}, v_{2}$, and $v_{3}$. In addition, denote their corresponding function values $f\left(v_{1}\right), f\left(v_{2}\right)$, and $f\left(v_{3}\right)$. Given these definitions, each iteration of a typical Nelder-Mead method consists of the following steps. First, the vertices are ordered from the lowest function value, say $f\left(v_{1}\right)$, to 
their highest function value, say $f\left(v_{3}\right)$. Second, the midpoint $m$ of the best side of the simplex is computed, i.e., the side opposite $v_{3}$. Third, a new simplex is computed from the current one using reflection, expansion, or contraction steps. In Fig. 3, we show examples of the reflection, expansion, and contraction steps, denoted by $r, e$, and $c_{o} / c_{i}$, respectively. We also illustrate the current simplex with a solid black line and the simplices computed via the operations in dashed black lines. To compute the new simplex, an attempt is made to replace $v_{3}$ by reflecting the vertex about the best side. If the reflected vertex $r$ leads to a decrease in the objective function, then an attempt is made at further reduction by computing an expansion vertex $e$. If $f(e)<f(r)$, then $v_{3}$ is replaced with $e$. Otherwise, $v_{3}$ is replaced with $r$. If the reflected vertex does not lead to a decrease in the objective function, then $r$ is contracted back to $c_{o}$, and the function values are compared again. If this step fails to decrease the function, then $c_{o}$ is reflected about the best edge to get $c_{i}$. If all of these steps are unsuccessful, then the simplex is shrunk toward vertex $v_{1}$, and a new simplex is formed with $v_{1}, m$, and the midpoint between $v_{1}$ and $v_{3}$

After minimizing the objective function for every highorder interior edge node (i.e., completing the first pass of our untangling algorithm), we perform a second pass to move the non-edge nodes. In this pass, for each non-edge node, we move the node to the mean of the high-order nodes of the triangle to which it belongs. To better motivate the need for two passes, we have included an example in Fig. 4. In Fig. 4(a), we show the initial tangled mesh. In Fig. 4(b), we show the mesh after completing the first pass of our method. Since the objective function applied in the first pass is formulated in terms of angles between edges, which do not apply to face nodes, the first pass neglects to improve the quality of these elements with respect to their face nodes. Thus, a face node's close proximity to the edge of its element could result in an invalid element. To address this kind of situation, we have included the second pass as shown in Fig. 4(c). This pass moves the face nodes toward the interior of the elements to which they belong. These two passes are performed until a tolerance is satisfied. In Alg. 1, we provide a pseudocode description of our untangling method. In the next section, we discuss how the signed angles $\alpha_{i}(x)$ of the curved elements are calculated.

\subsection{Computing the signed angles of curvi- linear triangles}

To compute the angle between two curves at a given node, we compute the derivatives of the curves, evaluate the derivatives at the given node, and then compute the angle between the resulting tangent vectors.

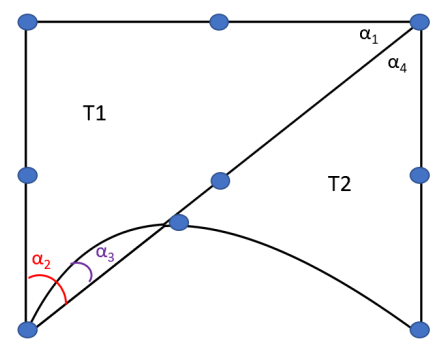

(a)

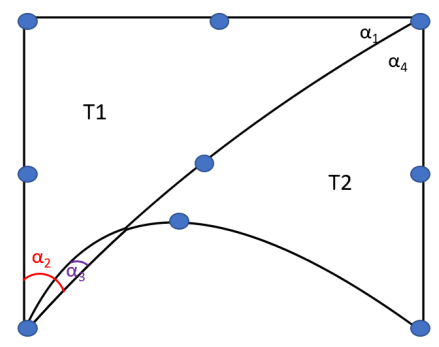

(b)

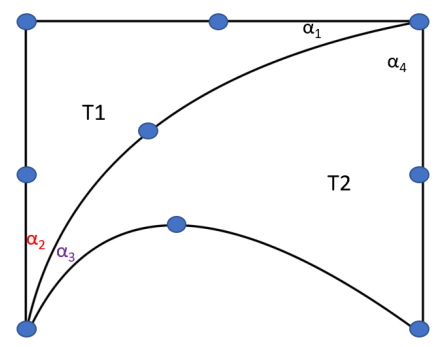

(c)

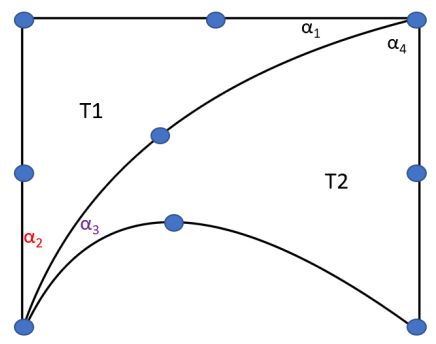

(d)

Figure 1: Motivating example: (a) the initial tangled mesh, (b) the mesh resulting from our method after two iterations, (c) the mesh resulting from our method after four iterations, and (d) the final mesh resulting from our converged method. 


\begin{tabular}{||c|r|r|r||}
\hline \hline $\begin{array}{l}\text { Iteration } \\
\text { Number }\end{array}$ & Term 1 & Term 2 & $f(x)$ \\
\hline 0 & 0.0000 & 29.1931 & 29.1931 \\
\hline 2 & 0.0812 & 4.9445 & 5.0257 \\
\hline 4 & 0.3096 & 0.1620 & 0.4716 \\
\hline 9 & 0.2538 & 0.1963 & 0.4501 \\
\hline
\end{tabular}

(a)

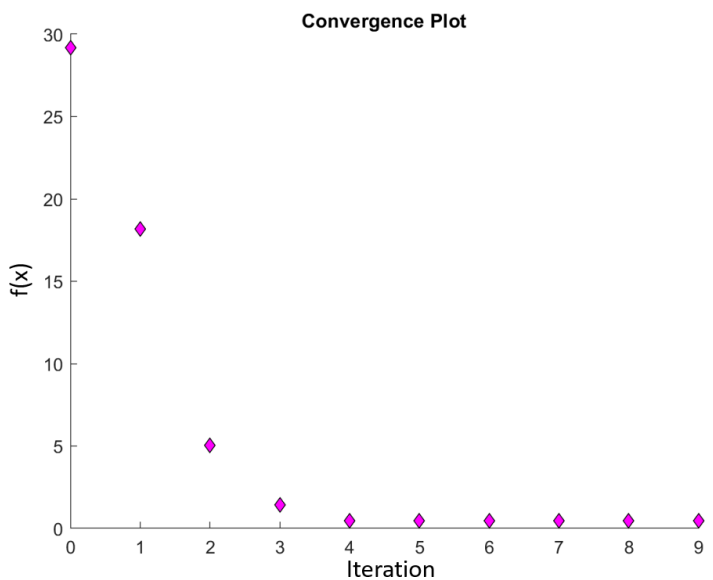

(b)

Figure 2: Figure showing (a) the contributions of each term in $f(x)$ during different iterations of the optimization method, and (b) a convergence plot of our method applied to the example in Fig. 1.

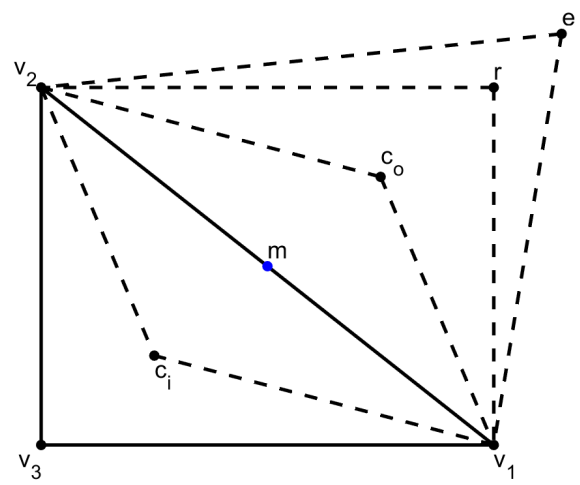

Figure 3: The current simplex marked by a solid line, and the simplices computed using the reflection, expansion, and contraction inside/outside operations during a single iteration of a typical Nelder-Mead method.

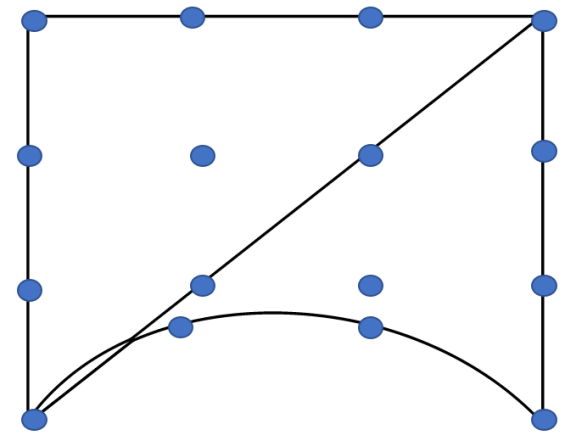

(a)

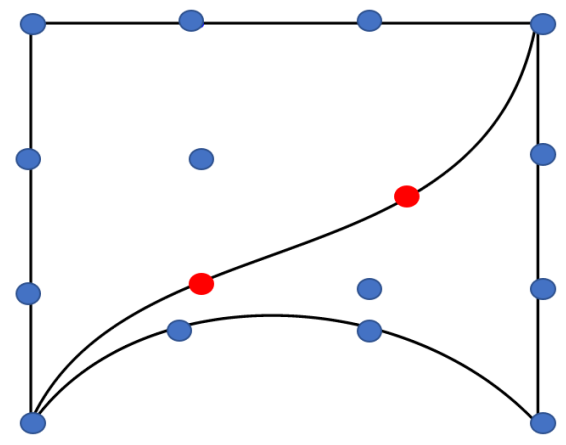

(b)

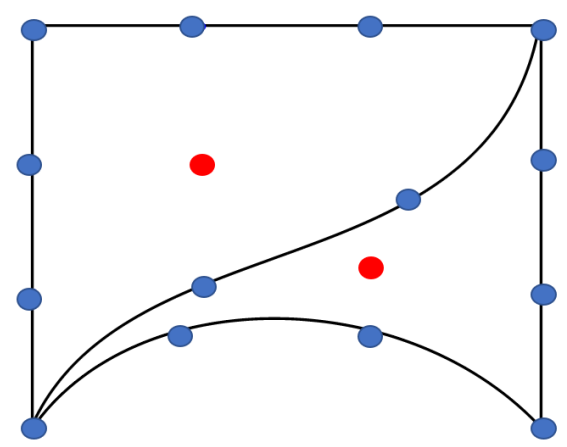

(c)

Figure 4: Motivating example for two pass approach: (a) the initial tangled mesh, (b) the mesh after completing the first pass with impacted nodes shown in red, and (c) the mesh after completing the second pass with influenced nodes shown in red. 


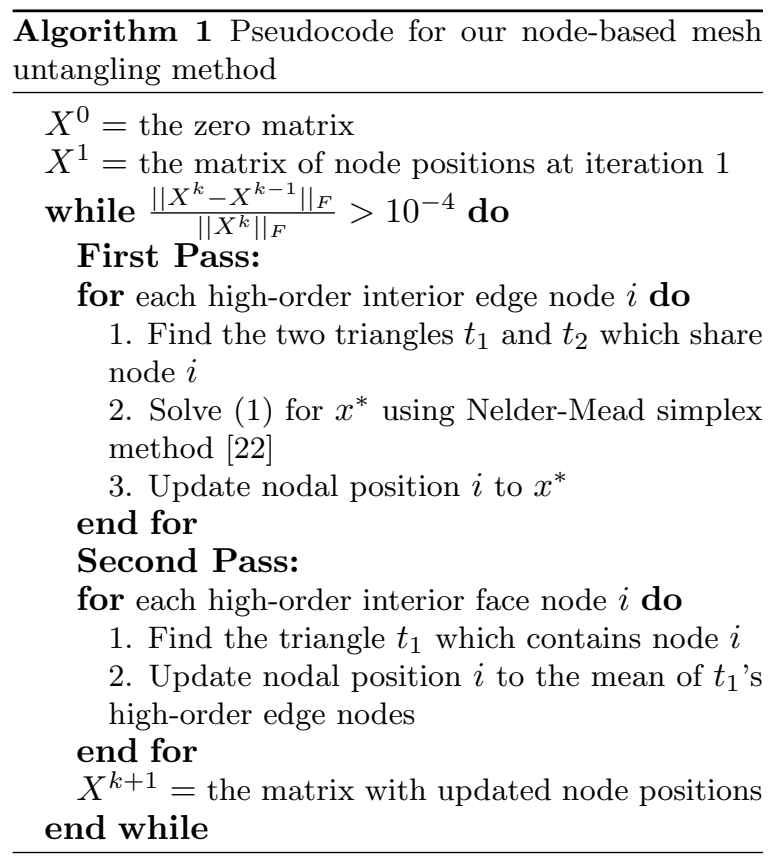

Using this approach, we compute the angles between each pair of edges of curvilinear triangles. For the following derivation, we use the third-order Lagrange element. The derivation for other orders is similar.

Consider the third-order Lagrange triangle shown in Fig. 5. To compute the angles between each pair of edges, we need to define mappings from each node on the edges of the reference element to the corresponding node on the edges of the physical element. Each edge corresponds to a third-order Lagrange element in one dimension. The shape functions associated with these elements are defined as:

$$
\begin{aligned}
& n_{1}(t)=\frac{9}{2}(1-t)\left(\frac{2}{3}-t\right)\left(\frac{1}{3}-t\right), \\
& n_{2}(t)=\frac{27}{2}(1-t)\left(\frac{2}{3}-t\right)(t) \\
& n_{3}(t)=\frac{27}{2}(1-t)\left(\frac{1}{3}-t\right)(-t) \\
& n_{4}(t)=\frac{9}{2}\left(\frac{2}{3}-t\right)\left(\frac{1}{3}-t\right)(t) .
\end{aligned}
$$

The derivatives of these shape functions with respect to $t$ are given by:

$$
\begin{aligned}
& n_{1}{ }^{\prime}(t)=\frac{1}{2}\left(-11+36 t-27 t^{2}\right), \\
& n_{2}{ }^{\prime}(t)=\frac{1}{2}\left(18-90 t+81 t^{2}\right), \\
& n_{3}{ }^{\prime}(t)=\frac{1}{2}\left(-9+72 t-81 t^{2}\right), \\
& n_{4}{ }^{\prime}(t)=\frac{1}{2}\left(2-18 t+27 t^{2}\right) .
\end{aligned}
$$

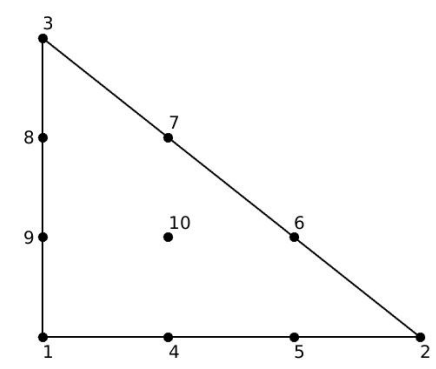

Figure 5: Third-order Lagrange reference unit triangle

Using these shape functions, we can define the mappings from each edge in the reference element to each edge in the physical element as:

$$
\begin{aligned}
& \mathbf{f}_{\mathbf{1 2}}(\mathbf{t})=\mathbf{x}_{\mathbf{1}} n_{1}(t)+\mathbf{x}_{\mathbf{4}} n_{2}(t)+\mathbf{x}_{\mathbf{5}} n_{3}(t)+\mathbf{x}_{\mathbf{2}} n_{4}(t), \\
& \mathbf{f}_{\mathbf{2 3}}(\mathbf{t})=\mathbf{x}_{\mathbf{2}} n_{1}(t)+\mathbf{x}_{\mathbf{6}} n_{2}(t)+\mathbf{x}_{\mathbf{7}} n_{3}(t)+\mathbf{x}_{\mathbf{3}} n_{4}(t), \\
& \mathbf{f}_{\mathbf{3 1}}(\mathbf{t})=\mathbf{x}_{\mathbf{3}} n_{1}(t)+\mathbf{x}_{\mathbf{8}} n_{2}(t)+\mathbf{x}_{\mathbf{9}} n_{3}(t)+\mathbf{x}_{\mathbf{1}} n_{4}(t) .
\end{aligned}
$$

The notation $f_{i j}$ denotes the edge between nodes $i$ and $j$ in Fig. 5. Now that we have the mappings, we need to compute the derivatives of our functions. Taking the derivative with respect to $t$ results in the following:

$\mathbf{f}_{\mathbf{1 2}}{ }^{\prime}(\mathbf{t})=\mathbf{x}_{\mathbf{1}} n_{1}{ }^{\prime}(t)+\mathbf{x}_{\mathbf{4}} n_{2}{ }^{\prime}(t)+\mathbf{x}_{\mathbf{5}} n_{3}{ }^{\prime}(t)+\mathbf{x}_{\mathbf{2}} n_{4}{ }^{\prime}(t)$,
$\mathbf{f}_{\mathbf{2 3}}{ }^{\prime}(\mathbf{t})=\mathbf{x}_{\mathbf{2}} n_{1}{ }^{\prime}(t)+\mathbf{x}_{\mathbf{6}} n_{2}{ }^{\prime}(t)+\mathbf{x}_{\mathbf{7}} n_{3}{ }^{\prime}(t)+\mathbf{x}_{\mathbf{3}} n_{4}{ }^{\prime}(t)$,
$\mathbf{f}_{\mathbf{3 1}}{ }^{\prime}(\mathbf{t})=\mathbf{x}_{\mathbf{3}} n_{1}{ }^{\prime}(t)+\mathbf{x}_{\mathbf{8}} n_{2}{ }^{\prime}(t)+\mathbf{x}_{\mathbf{9}} n_{3}{ }^{\prime}(t)+\mathbf{x}_{\mathbf{1}} n_{4}{ }^{\prime}(t)$.

Given these derivatives, we can return to the problem of calculating the angles between edges. As an example, suppose that we want to calculate the angle between edge $e_{12}$ and edge $e_{31}$ in Fig. 5 . To calculate the unsigned angle in radians, we could use the following formula:

$$
\theta=\arccos \left(\frac{-f_{12}{ }^{\prime}(0) \cdot f_{31}{ }^{\prime}(1)}{\left\|f_{12}{ }^{\prime}(0)\right\|\left\|-f_{31}{ }^{\prime}(1)\right\|}\right)=\frac{\pi}{2} .
$$

In order to calculate the signed angle in radians, we need to modify our calculations. First, we need to include an orientation unit vector $\mathbf{n}$. Then we need to modify our tangent vectors by adding a third component with a value of zero so that the cross product is defined, as well as normalize them. With these modifications, we can compute the signed angle using the following formula:

$$
\text { signed angle }=\operatorname{sgn}(\mathbf{n} \cdot(v 1 \times v 2)) \cdot \arccos (v 1 \cdot v 2)
$$

where

$$
\begin{aligned}
\mathbf{v 1} & =\frac{\left[f_{12}{ }^{\prime}(0), 0\right]}{\left\|\left[f_{12}^{\prime}(0), 0\right]\right\|_{2}}, \\
\mathbf{v 2} & =\frac{\left[-f_{31}{ }^{\prime}(1), 0\right]}{\left\|\left[-f_{31}{ }^{\prime}(1), 0\right]\right\|_{2}}, \\
\mathbf{n} & =[0,0,1]
\end{aligned}
$$




\section{NUMERICAL RESULTS}

In this section, we demonstrate the results from applying our method to untangle several high-order meshes. In each example, the nodes are processed in the order in which they occur in the original mesh. While we have explored other node orderings and found that the order does impact the number of outer iterations required for convergence, we note that this ordering does not influence the final resulting mesh. For each example, we provide a description of the mesh, the initial mesh (with tangled elements shown in red), the mesh which results from applying our untangling method, and the mesh element distortion as measured by the scaled Jacobian:

$$
\text { scaled Jacobian }=\frac{\min J(\xi)}{\max J(\xi)},
$$

where $J(\xi)$ is the Jacobian determinant. When reporting the mesh distortion, we list the minimum distortion and maximum distortion values. We also list the execution times for our untangling method (excluding I/O) in Table 1. The method was implemented in $\mathrm{C}++$, and the wall-clock execution times were measured on a machine with $16 \mathrm{~GB}$ of RAM and an AMD Ryzen 71700 CPU. All mesh visualizations and distortion evaluations were done using Gmsh $[23,24,25]$.

In our first example, we use a simple annulus geometry consisting of 30 elements to show the impact of different values of $\beta$ on the result. In Fig. 6(a), we show the initial mesh with two tangled elements. In Fig. 6(bd), we show the meshes resulting from $\beta$ values of 0.1 , 0.5 , and 0.9 , respectively. In Fig. 6(e), we show the min and max element distortions and execution times for each of the three values of $\beta$. As expected, higher values of $\beta$ place more emphasis on the angular component of the objective function which tends to result in larger displacements of the edge nodes. Initially, increasing the value of $\beta$ from 0.1 to 0.5 led to better elements with respect to distortion. Beyond 0.5, additional emphasis on the angles resulted in increased element distortion. For the remaining examples in this section, we report the value of $\beta$ that resulted in the mesh with the least distortion. We also plot histograms of the mesh element distortion in addition to reporting the maximum and minimum values.

In the second example, we applied our method to a simple 2D mechanical part consisting of 295 secondorder elements. Curving the boundaries resulted in two tangled elements near the innermost boundary. The initial tangled mesh and resulting untangled mesh are shown in Fig. 7(a,b). The minimum and maximum distortion values for these meshes are shown in Fig. 7 (c). Finally, we plot histograms for these distortion values in Fig. $7(\mathrm{~d}, \mathrm{e})$. In this case, our solution raised the minimum distortion value of the mesh from -0.178 to 0.228 .

In our third example, we use a simplified bike gear with 672 second-order elements. In contrast with our previous examples, this mesh has several stretched elements near the boundaries which increase the potential for tangled elements after curving the boundaries. The initial tangled mesh and untangled mesh are shown in Fig. 8(a,b). Close-up views of the top third of the mesh are depicted in Fig. 8(c,d). The minimum and maximum distortion values for this mesh are recorded in Fig. 8(e). Lastly, histograms of the distortion values are plotted in Fig. 8(f,g). In this case, our method increased the minimum distortion value from -1.730 to 0.211 , thus untangling the initial mesh.

As our last simplified example, we use a pressure plate consisting of 529 second-order elements. After curving the boundaries, six tangled elements were created along the holes in the top and bottom of the geometry. Fig. 9(a,b) show the original tangled mesh and the untangled mesh resulting from our method. We show detailed views of the center of $(\mathrm{a}, \mathrm{b})$ in Fig. 9(c,d), respectively. In Fig. 9(e) we give the minimum and maximum mesh element distortion values. Finally in Fig. 9(f,g) we plot histograms of the distortion values. For this example, our method increased the minimum distortion value from -0.178 to 0.345 .

For our remaining examples, we progress to more realistic meshes with a larger number of elements. The first example is a third-order gear composed of 1340 elements, eight of which are tangled. In Fig. 10(a,b) we show the initial tangled mesh and the final mesh produced by our untangling algorithm. In Fig. 10(c,d) we show detailed views of the center holes in $(a, b)$, respectively. The minimum and maximum mesh element distortion values are listed in Fig. 10(e). Finally in Fig. 10(f,g) we plot histograms of the distortion values. After applying our method, the minimum distortion value increased from -0.122 to 0.092 .

Our next example is a brake rotor composted of 7015 second-order elements, thirty-four of which are tangled. In Fig. 11(a,b) we show the initial tangled mesh and the final mesh produced by our untangling algorithm. In Fig. 11(c,d) we show detailed views of the center holes in $(a, b)$, respectively. The minimum and maximum mesh element distortion values are listed in Fig. 11(e). Finally in Fig. 11(f,g) we plot histograms of the distortion values. After applying our method, the minimum distortion value increased from -0.156 to 0.346 .

Finally, our last example is an anisotropic boundary layer mesh of an airfoil. This example is a modified version of an example taken from the $2 \mathrm{D}$ benchmarks 


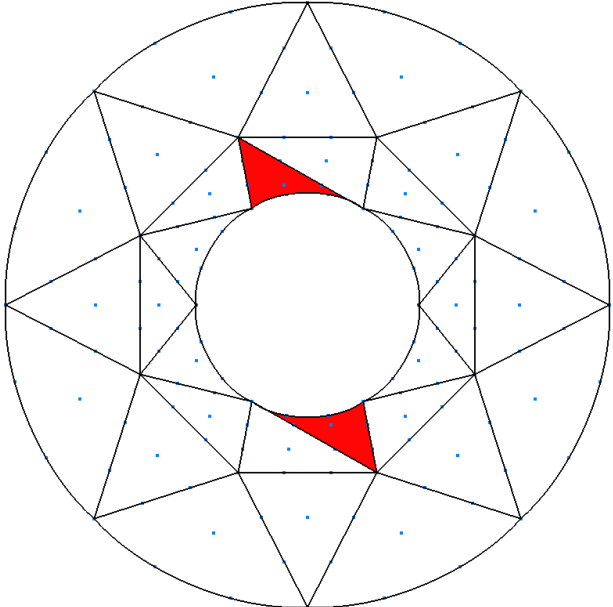

(a)

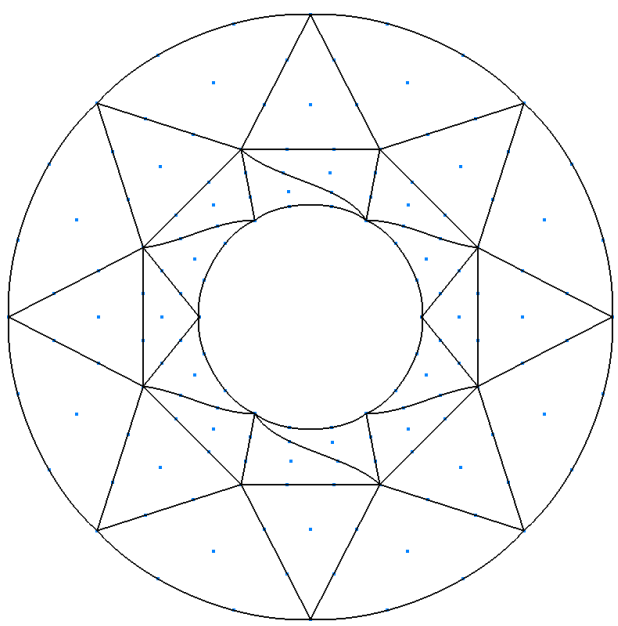

(c)

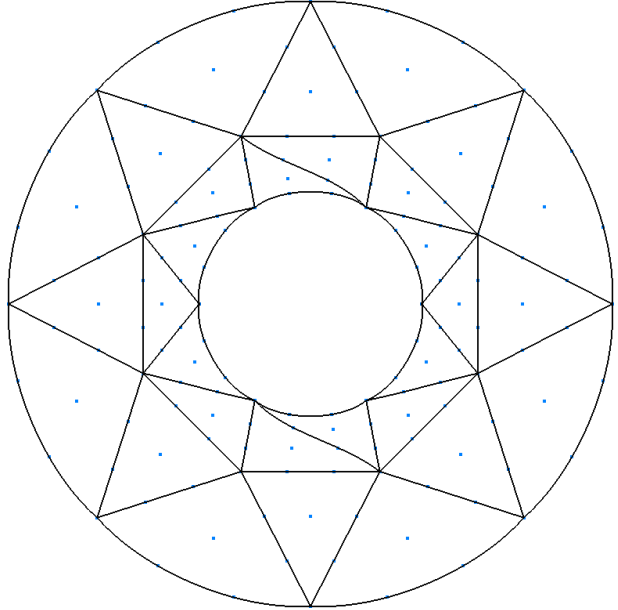

(b)

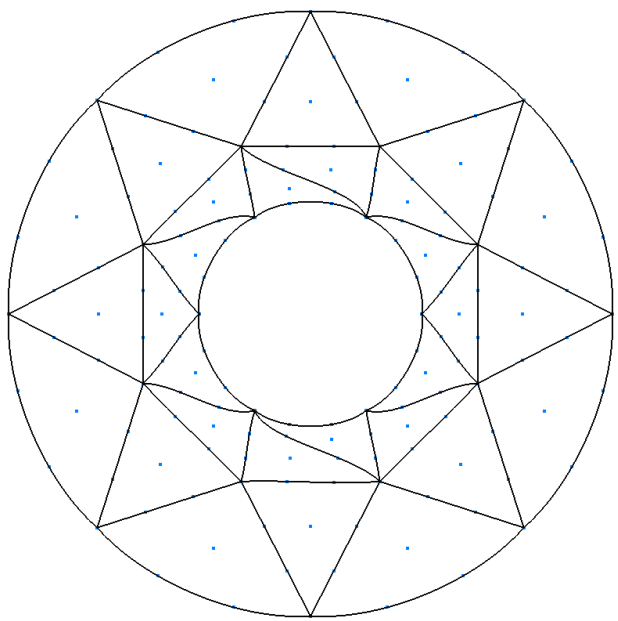

(d)

\begin{tabular}{||c|c|c|c||}
\hline & \multicolumn{2}{|c||}{ Distortion } & \\
\hline Beta & Min & Max & Runtime (s) \\
\hline 0.1 & 0.208 & 1.000 & 0.004 \\
\hline 0.5 & 0.472 & 1.000 & 0.005 \\
\hline 0.9 & 0.348 & 1.000 & 0.014 \\
\hline
\end{tabular}

(e)

Figure 6: Annulus example with three different $\beta$ values: (a) the initial mesh with two tangled elements; (b) to (d) untangled meshes for $\beta$ values of $0.1,0.5$, and 0.9 , respectively, and (e), shows the minimum and maximum element distortions and runtimes for each value of $\beta$. 


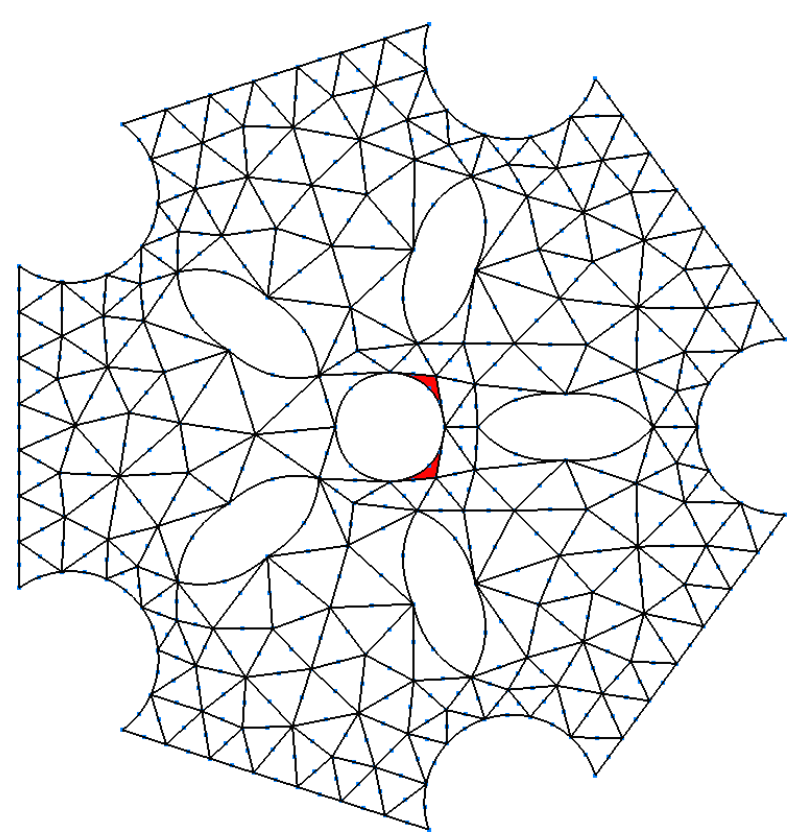

(a)

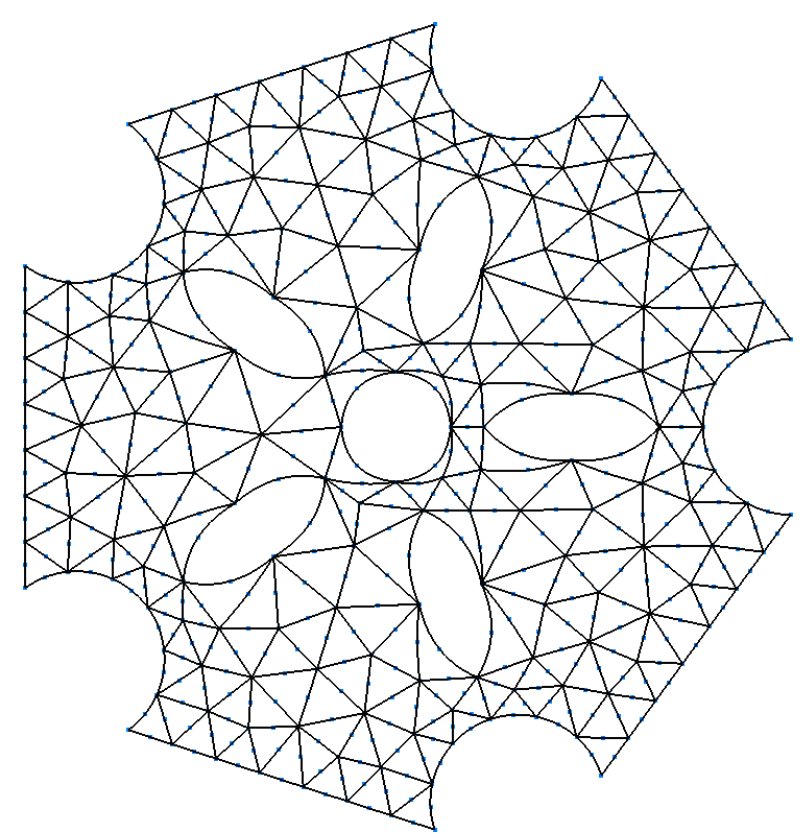

(b)

\begin{tabular}{||c|r|c||}
\hline & \multicolumn{2}{|c||}{ Distortion } \\
\hline Example & Min & Max \\
\hline original mesh & -0.178 & 1.000 \\
\hline resulting mesh & 0.228 & 1.000 \\
\hline
\end{tabular}

(c)

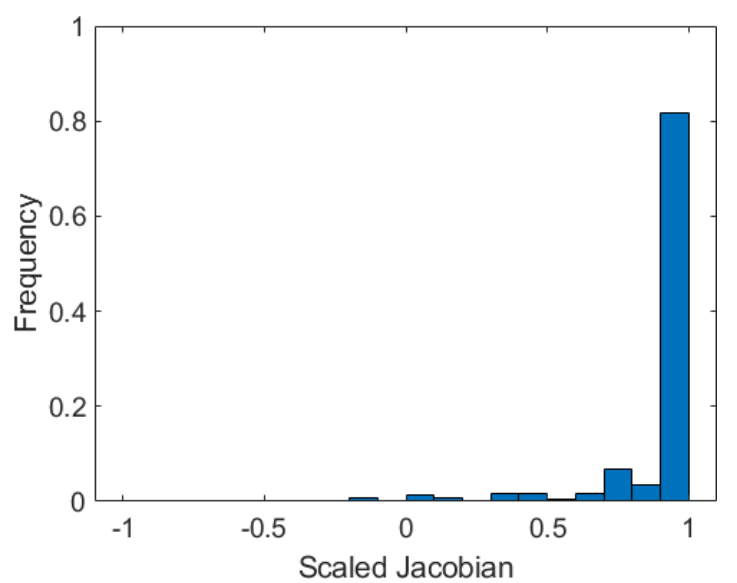

(d)

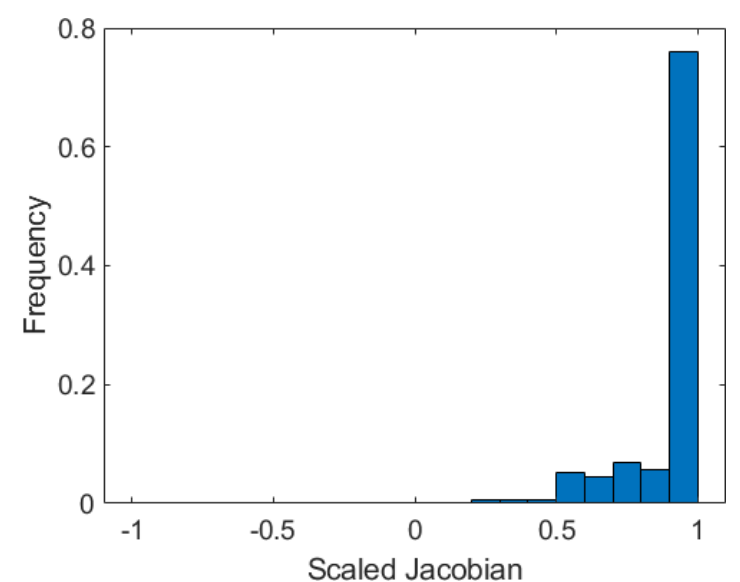

(e)

Figure 7: Mechanical part example: (a) the initial second-order mesh with two tangled elements; (b) the untangled mesh resulting from our method; (c) the minimum and maximum element distortion, and (d,e) histogram plots of the distortion metric for each mesh. 


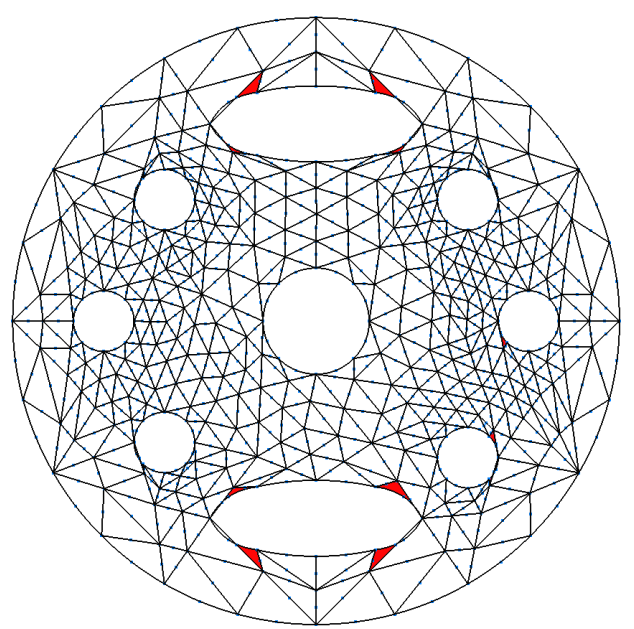

(a)

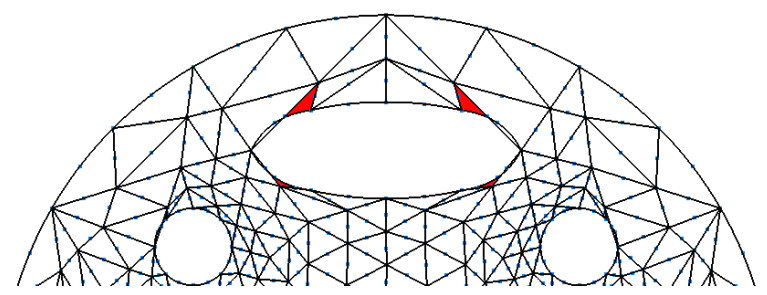

(c)

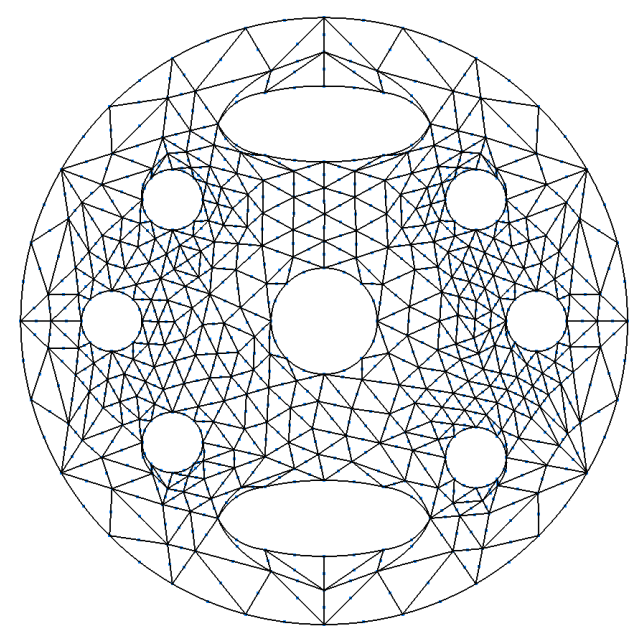

(b)

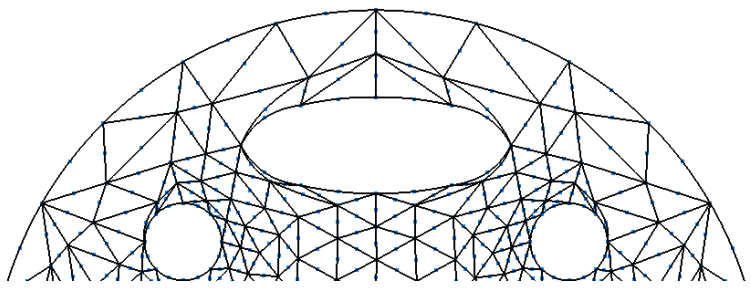

(d)

\begin{tabular}{||c|c|c||}
\hline & \multicolumn{2}{c||}{ Distortion } \\
\hline Example & Min & Max \\
\hline original mesh & -1.730 & 1.000 \\
\hline resulting mesh & 0.211 & 1.000 \\
\hline
\end{tabular}

(e)

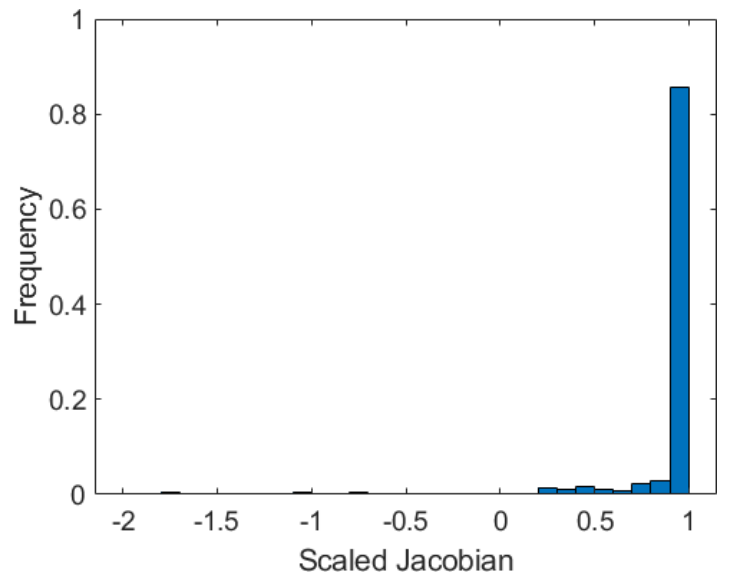

(f)

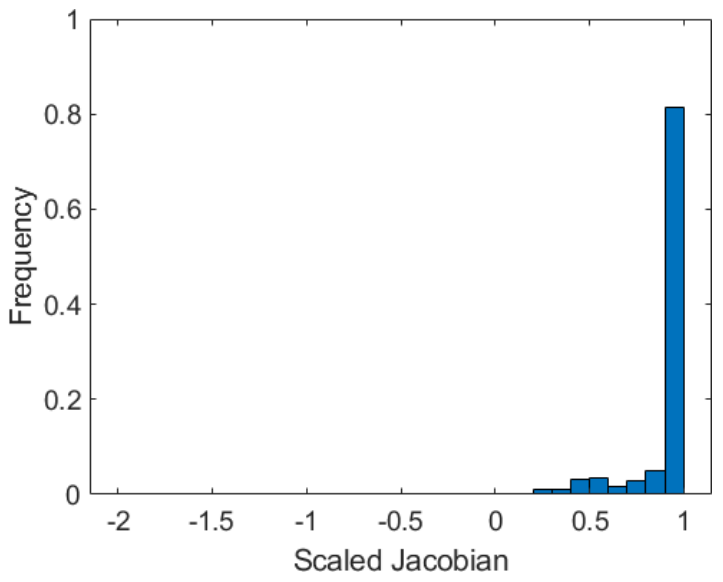

(g)

Figure 8: Bike gear example: (a) the tangled second-order mesh with fourteen tangled elements; (b) the mesh resulting from our method; $(c, d)$ detailed views of $(a, b)$, respectively; (e) the minimum and maximum element distortion, and $(f, g)$ histogram plots of the distortion metric for $(a, b)$, respectively. 


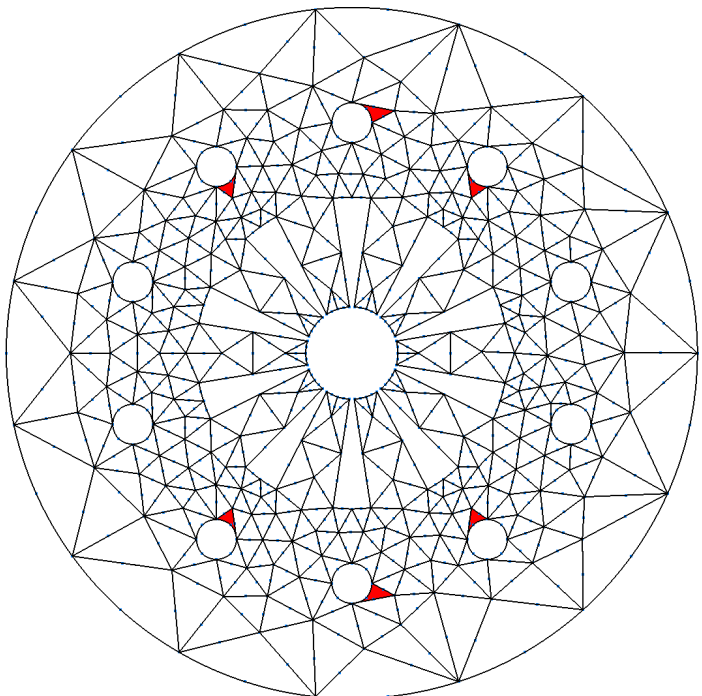

(a)

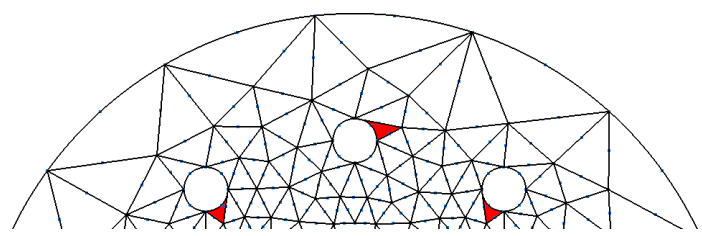

(c)

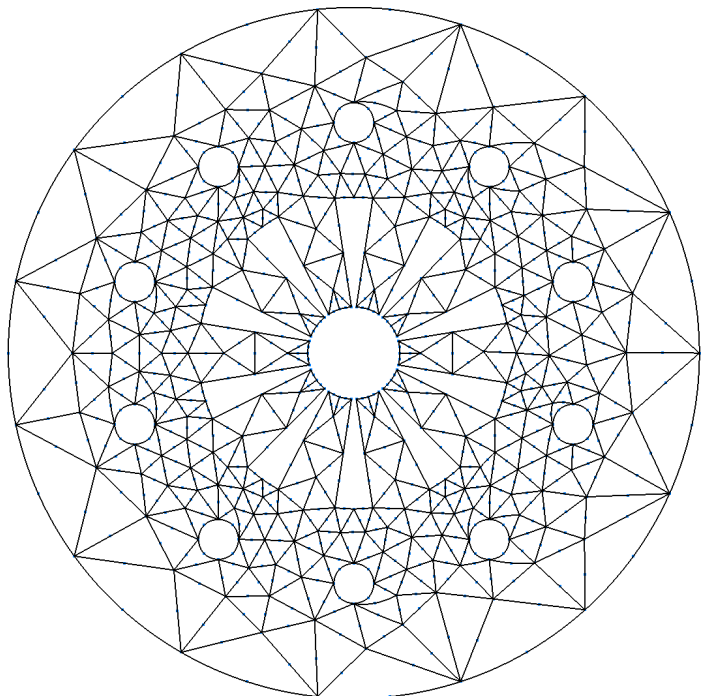

(b)

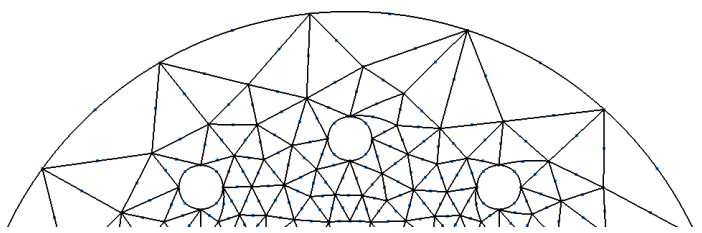

(d)

\begin{tabular}{||c|c|c||}
\hline & \multicolumn{2}{|c||}{ Distortion } \\
\hline Example & Min & Max \\
\hline original mesh & -0.178 & 1.000 \\
\hline resulting mesh & 0.345 & 1.000 \\
\hline
\end{tabular}

(e)

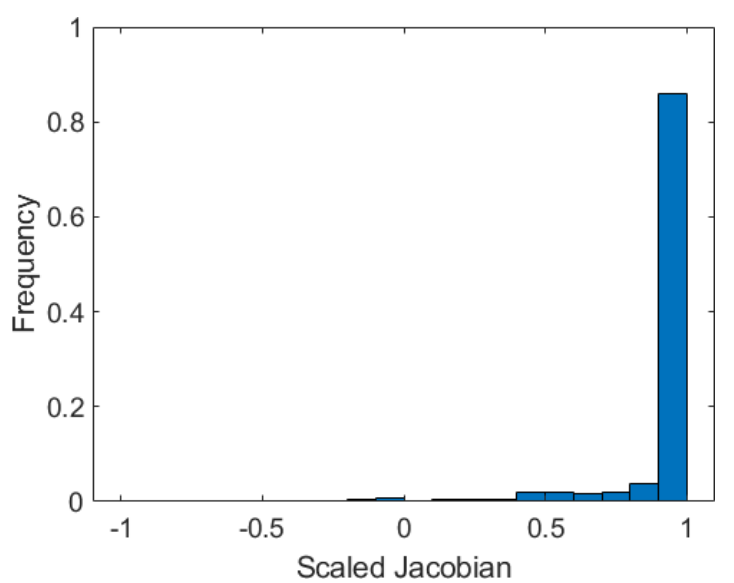

(f)

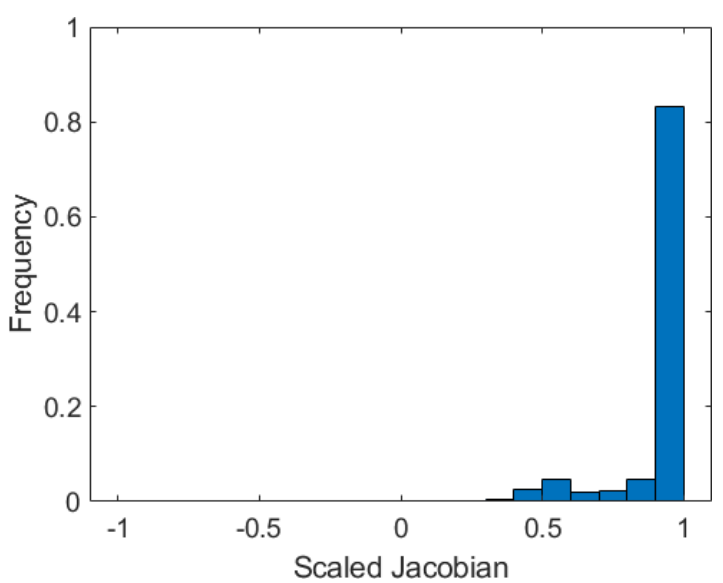

(g)

Figure 9: Pressure plate example: (a) the tangled second-order mesh; (b) the mesh resulting from our method; (c,d) detailed views of $(\mathrm{a}, \mathrm{b})$, respectively; (e) the minimum and maximum element distortion, and (f,g) histogram plots of the distortion metric for each mesh. 


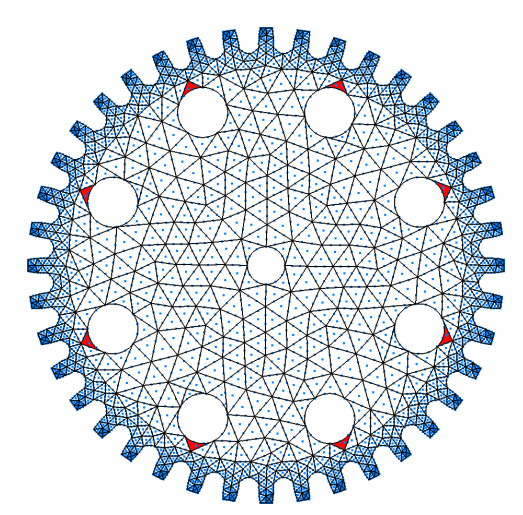

(a)

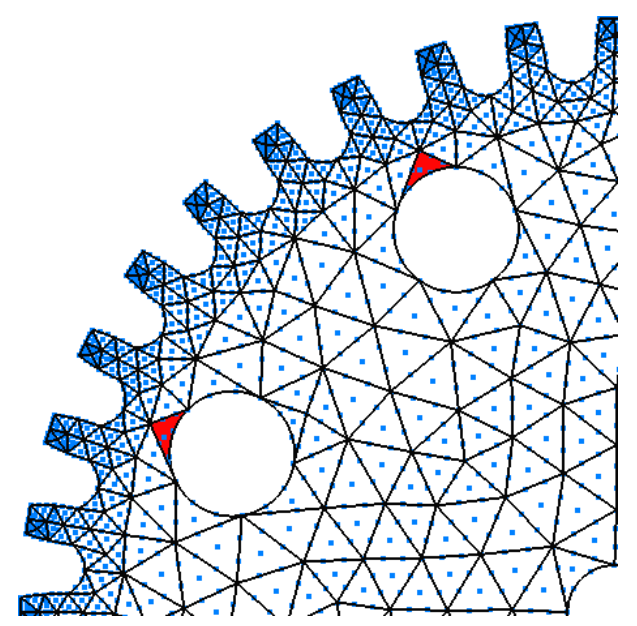

(c)

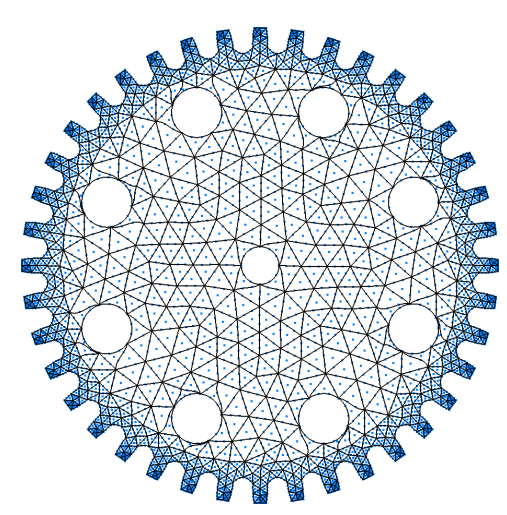

(b)

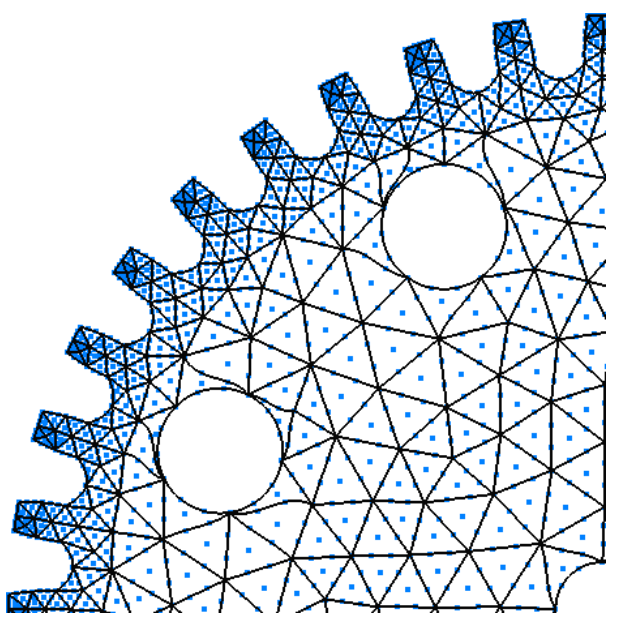

(d)

\begin{tabular}{||c|c|c||}
\hline & \multicolumn{2}{|c||}{ Distortion } \\
\hline Example & Min & Max \\
\hline original mesh & -0.122 & 1.000 \\
\hline resulting mesh & 0.092 & 1.000 \\
\hline
\end{tabular}

(e)

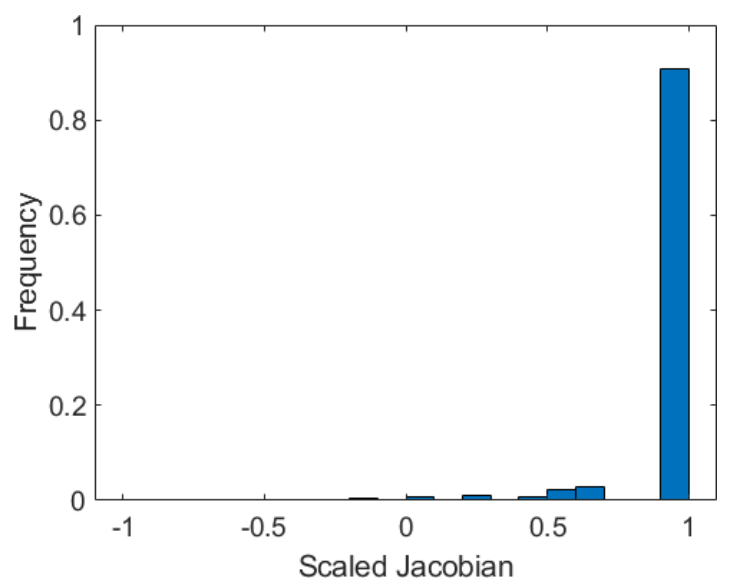

(f)

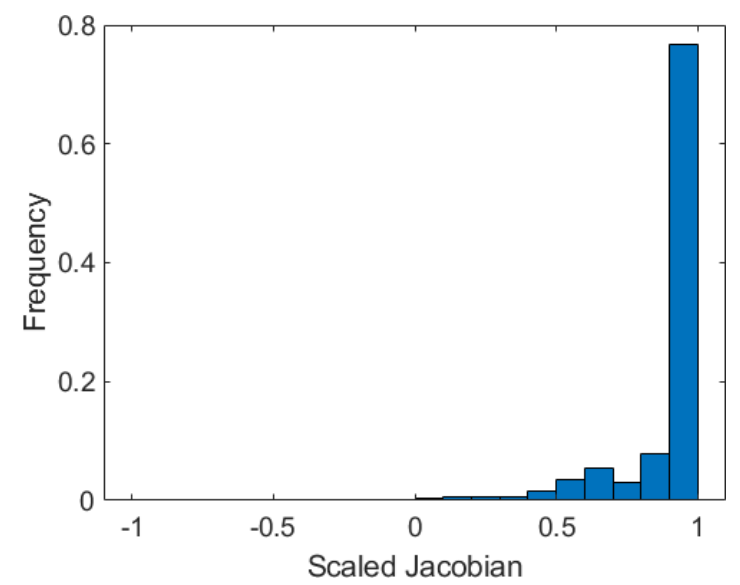

(g)

Figure 10: Gear example: (a) the initial third-order mesh with eight tangled elements; (b) the mesh resulting from our method; (c,d) detailed views of (a,b), respectively; (e) the minimum and maximum element distortion, and (f,g) histogram plots of the distortion metric for $(\mathrm{a}, \mathrm{b})$, respectively. 


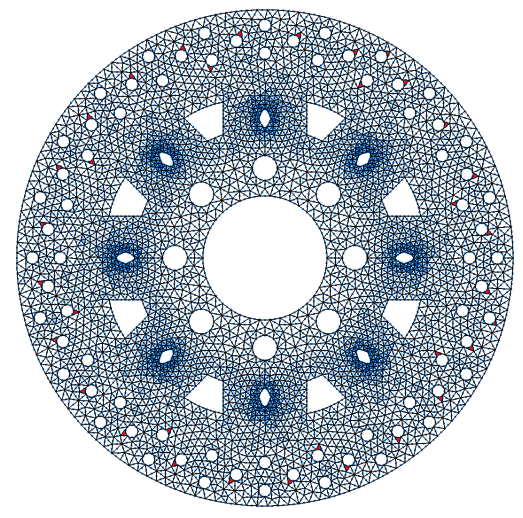

(a)

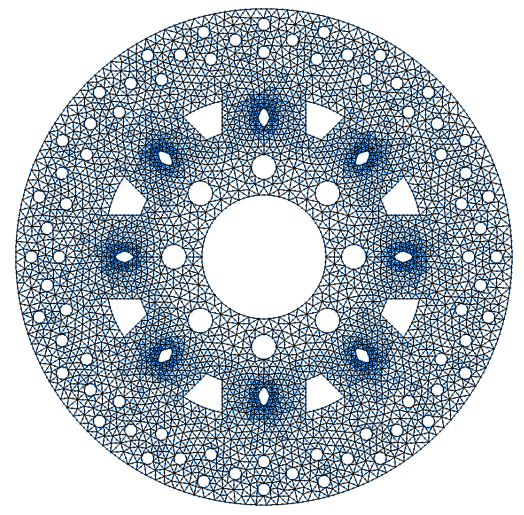

(b)

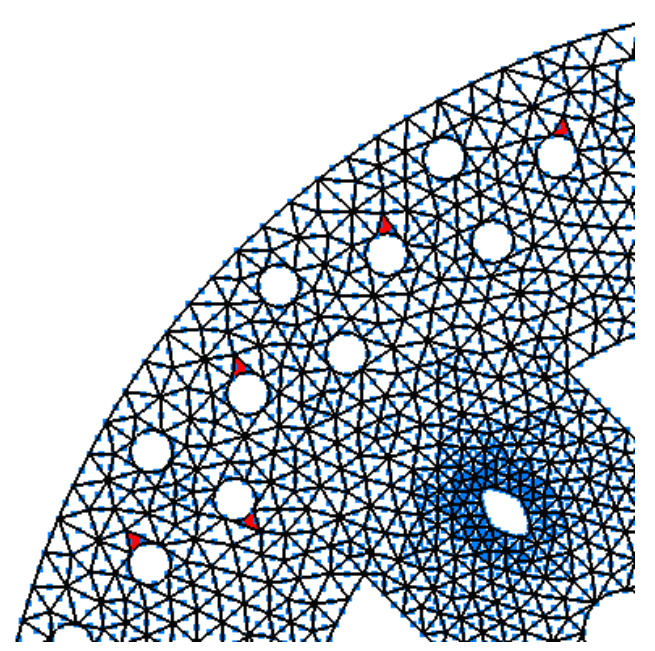

(c)

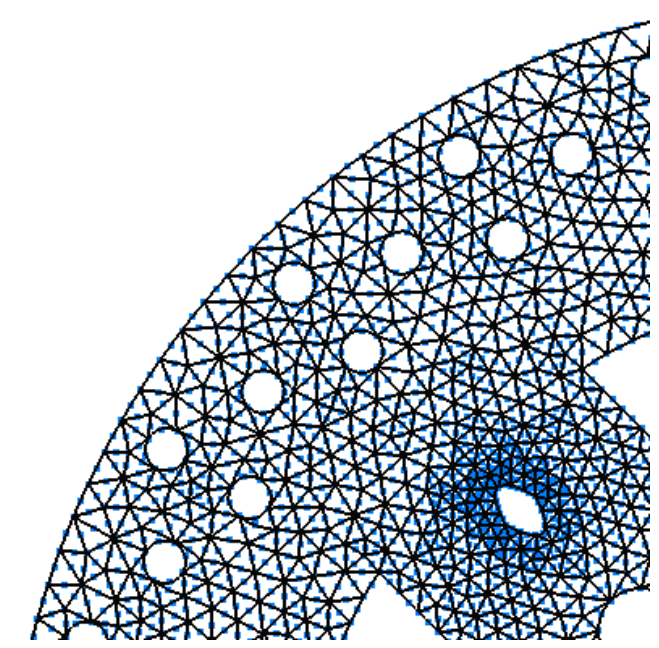

(d)

\begin{tabular}{||c|c|c||}
\hline & \multicolumn{2}{c||}{ Distortion } \\
\hline Example & Min & Max \\
\hline original mesh & -0.156 & 1.000 \\
\hline resulting mesh & 0.346 & 1.000 \\
\hline
\end{tabular}

(e)

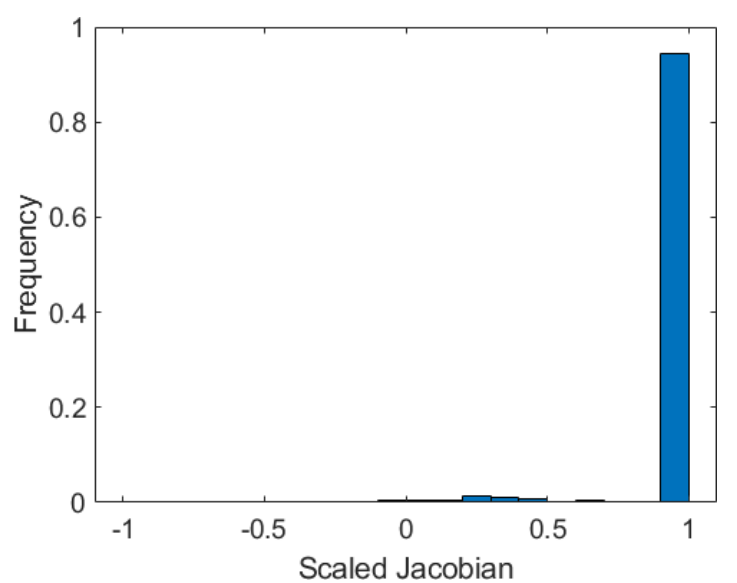

(f)

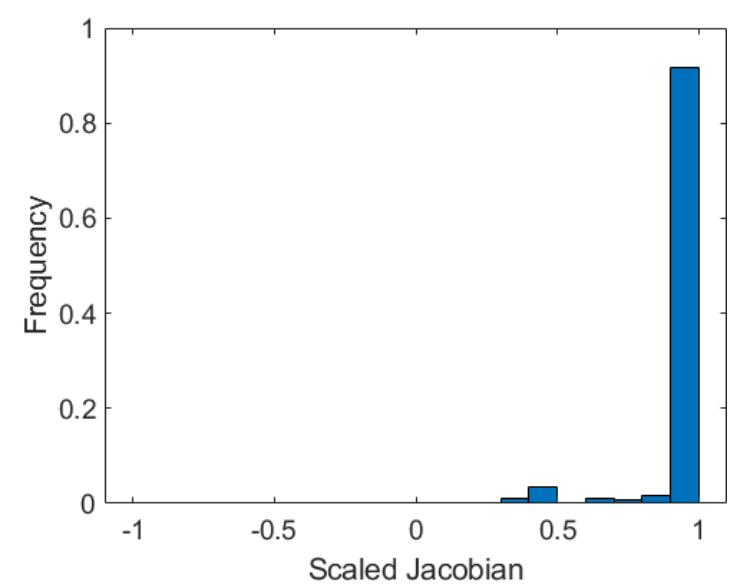

(g)

Figure 11: Brake rotor example: (a) the initial second-order mesh with thirty-four tangled elements; (b) the mesh resulting from our method; $(c, d)$ detailed views of $(a, b)$, respectively; (e) the minimum and maximum element distortion, and $(\mathrm{f}, \mathrm{g})$ histogram plots of the element distortion metric for $(\mathrm{a}, \mathrm{b})$, respectively. 


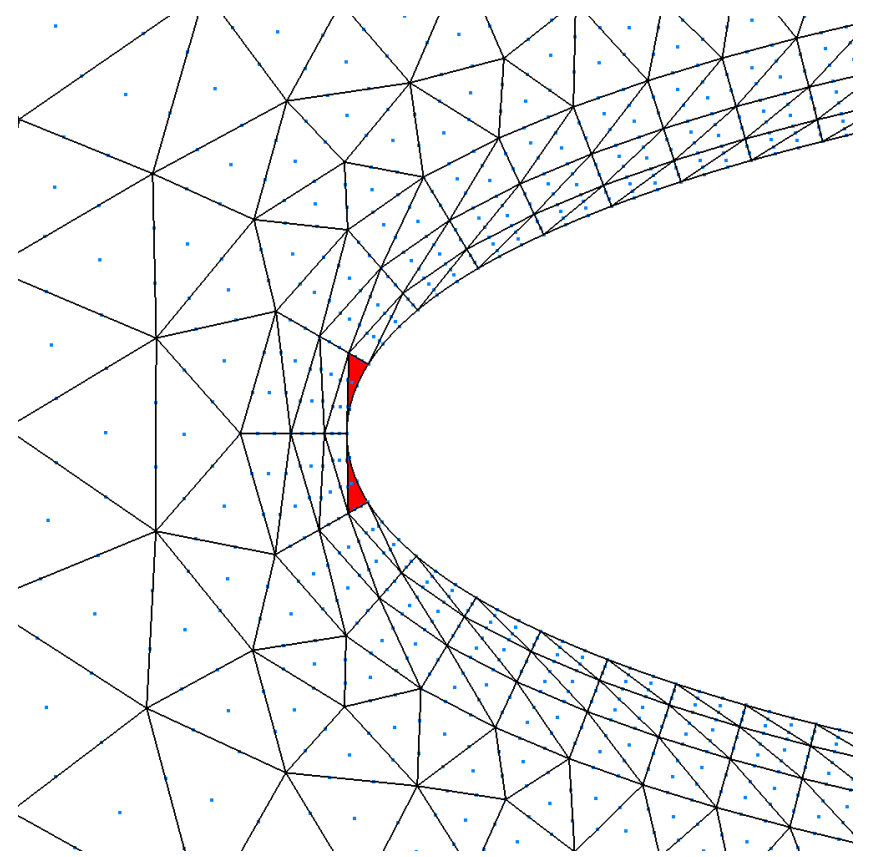

(a)

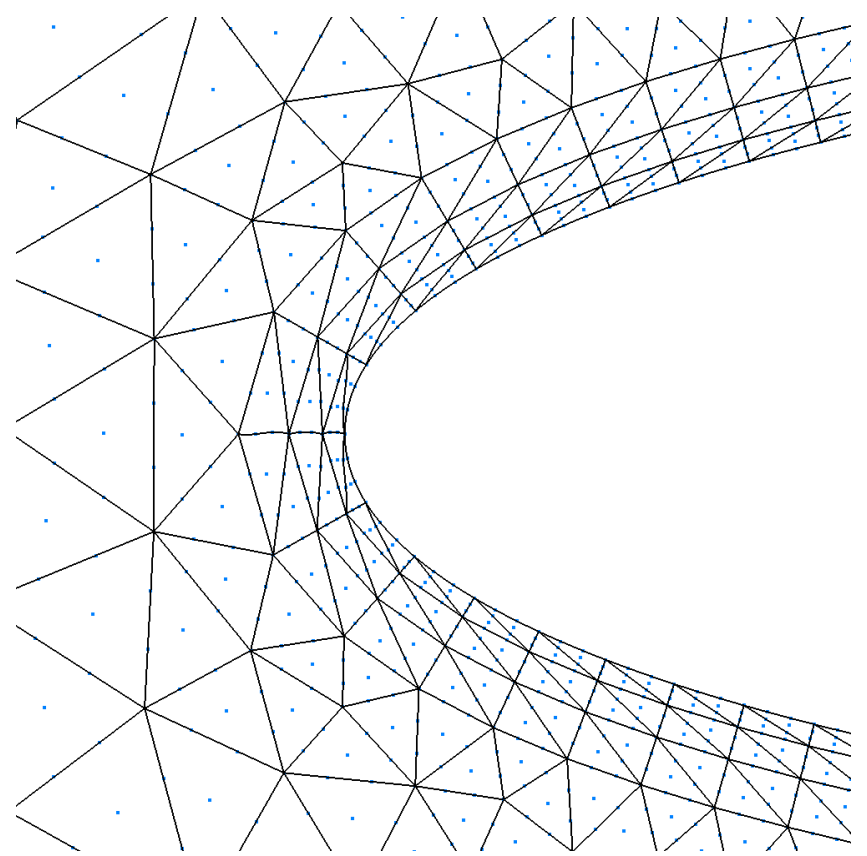

(b)

\begin{tabular}{||c|c|c||}
\hline & \multicolumn{2}{|c||}{ Distortion } \\
\hline Example & Min & Max \\
\hline original mesh & -0.109 & 1.000 \\
\hline resulting mesh & 0.053 & 1.000 \\
\hline
\end{tabular}

(c)

Figure 12: Airfoil example: (a) the initial third-order mesh with two tangled elements near the leading edge; (b) the mesh resulting from our method, and (c) the minimum and maximum element distortion. 


\begin{tabular}{||c|r|r|r|r|r|r||}
\hline Example & $\begin{array}{l}\text { Number of } \\
\text { Elements }\end{array}$ & $\begin{array}{l}\text { Mesh } \\
\text { Order }\end{array}$ & $\beta$ & $\begin{array}{l}\text { Number of } \\
\text { Iterations }\end{array}$ & First Pass & Second Pass \\
\hline annulus & 30 & 3 & 0.500 & 2 & 0.005 & 0.000 \\
\hline mechanical part & 295 & 2 & 0.500 & 2 & 0.041 & - \\
\hline bike gear & 672 & 2 & 0.035 & 5 & 0.321 & - \\
\hline pressure plate & 529 & 2 & 0.350 & 5 & 0.249 & -0.005 \\
\hline gear & 1340 & 3 & 0.950 & 8 & 11.323 & - \\
\hline brake rotor & 7015 & 2 & 0.850 & 2 & 19.643 & 0.005 \\
\hline airfoil & 5328 & 3 & 0.001 & 2 & 24.039 & - \\
\hline
\end{tabular}

Table 1: The number of elements, mesh order, beta value, number of outer iterations, and the wall clock times for each pass our untangling method (excluding I/O) for each example. Since the second-order meshes do not utilize the second pass, the columns are marked with a dash.

in the Gmsh repository. The mesh contains 5328 elements, with two tangled elements along the leading edge. In Fig. 12(a,b) we show the initial tangled mesh and the final mesh produced by our untangling algorithm. The minimum and maximum mesh element distortion values are listed in Fig. 12(c). The histogram plots for this example were omitted because there was minimal distinction between the two plots given the small percentage of tangled elements. After applying our method, the minimum distortion value increased from -0.109 to 0.053 .

As we illustrated in Fig. 6, there are usually several choices for the parameter $\beta$ that will result in an untangled mesh. As shown in Table 1, the trend we have observed thus far is that smaller values of $\beta$ perform better for meshes with stretched elements near the curved features like our examples in Figs. 8 and 12. In particular, a smaller value of $\beta$ was critical to maintaining the boundary layers in Fig. 12. Further experiments are necessary to determine what other factors influence the optimal value for $\beta$. The goal of these test cases was to explore the types of tangling that occur as a result of small deformations (e.g., moving the new boundary nodes onto the curved boundary during the typical high-order mesh generation process). With that in mind, our examples demonstrate that our method successfully addresses the typical types of tangling seen in this scenario. In addition to untangling the invalid patches, our method tends to reduce the amount of element distortion in all of our examples. In addition, there is potential to improve the performance of our method using parallel computing, as our local method can be applied to non-adjacent patches simultaneously.

\section{CONCLUDING REMARKS AND FUTURE WORK}

We have presented a new optimization-based method for untangling second- and third-order triangular meshes. The two-dimensional examples have shown that our proposed method based on signed angles is able to successfully untangle a variety of invalid second- and third-order meshes. Furthermore, our method tends to dramatically decrease the amount of element distortion present in the mesh. As our final example in Section 3 showed, the addition of the weighting parameter gives the user increased flexibility in defining the behavior of the objective function. One limitation of our method is that it does not move the low-order nodes. To address this, we plan to combine our untangling algorithm with a weight-based scheme like the one proposed in [14]. By combining these two approaches, we could use the weight-based scheme to move the low-order nodes, and the method we have proposed in this paper to move the high-order nodes.

Our future work will include exploring techniques for determining the ideal value of the weighting parameter $\beta$. We will also extend our implementation to untangle meshes composed of elements with $p>3$. In addition, we plan to extend the capabilities of our method to three dimensions by using signed solid angles between curved faces of high-order tetrahedral elements. We also plan to add support for additional element types (e.g., quadrilaterals, etc). Finally, we plan to explore examples with larger deformations that result in more complicated mesh tangling.

\section{ACKNOWLEDGMENTS}

The work of the first author was funded by NSF CCF grant 1717894. The work of the second author was funded by an REU supplement to NSF CCF grant 1717894. The work of the third author was supported in part by NSF grants CCF 1717894 and OAC 1808553. 


\section{References}

[1] Wang Z.J., Fidkowski K., Abgrall R., Bassi F., Caraeni D., Cary A., Deconinck H., Hartmann R., Hillewaert K., Huynh H.T., et al. "High-order CFD methods: Current status and perspective." International Journal for Numerical Methods in Fluids, vol. 72, no. 8, 811-845, 2013

[2] Bassi F., Rebay S. "High-order accurate discontinuous finite element solution of the 2D Euler equations." Journal of Computational Physics, vol. 138 , no. $2,251-285,1997$

[3] Luo X., Shephard M.S., Remacle J.F. "The influence of geometric approximation on the accuracy of high order methods." Rensselaer SCOREC report, vol. 1, 2001

[4] Dey S., Shephard M.S. "Curvilinear mesh generation in 3D." Proceedings of the 8th International Meshing Roundtable. 1999

[5] Fortunato M., Persson P.O. "High-order unstructured curved mesh generation using the Winslow equations." Journal of Computational Physics, vol. 307, no. 2016, 1-14, Feb. 2016

[6] Gargallo-Peiró A., Roca X., Peraire J., Sarrate J. "Distortion and quality measures for validating and generating high-order tetrahedral meshes." Engineering with Computers, vol. 31, no. 3, 423437, 2015

[7] Gargallo-Peiró A., Roca X., Peraire J., Sarrate J. "Optimization of a regularized distortion measure to generate curved high-order unstructured tetrahedral meshes." International Journal for $\mathrm{Nu}$ merical Methods in Engineering, vol. 103, no. 5, 342-363, 2015

[8] Moxey D., Ekelschot D., Keskin Ü., Sherwin S.J., Peiró J. "High-order curvilinear meshing using a thermo-elastic analogy." Computer-Aided Design, vol. $72,130-139,2016$

[9] Moxey D., Green M., Sherwin S., Peiró J. "An isoparametric approach to high-order curvilinear boundary-layer meshing." Computer Methods in Applied Mechanics and Engineering, vol. 283, 636 -650, 2015

[10] Persson P.O., Peraire J. "Curved mesh generation and mesh refinement using Lagrangian solid mechanics." Proceedings of the 47th AIAA Aerospace Sciences Meeting including The New Horizons Forum and Aerospace Exposition, p. 949. 2009

[11] Roca X., Gargallo-Peiró A., Sarrate J. "Defining quality measures for high-order planar triangles and curved mesh generation." Proceedings of the 20th International Meshing Roundtable, pp. 365383. Springer Berlin Heidelberg, 2012

[12] Ruiz-Gironés E., Sarrate J., Roca X. "Generation of curved high-order meshes with optimal quality and geometric accuracy." Proceedings of the 25th International Meshing Roundtable, vol. 163, pp. 315-327. Procedia Engineering, 2016

[13] George P.L., Borouchaki H. "Construction of tetrahedral meshes of degree two." International Journal for Numerical Methods in Engineering, vol. 90, no. 9, 1156-1182, 2012

[14] Stees M., Shontz S.M. "A high-order log barrierbased mesh generation and warping method." Proceedings of the 26th International Meshing Roundtable, vol. 203, pp. 180 - 192. Procedia Engineering, 2017

[15] Stees M., Shontz S.M. "An angular approach to untangling high-order curvilinear triangular meshes." Proceedings of the 27th International Meshing Roundtable, pp. 327-342. Springer, 2019

[16] Karman S.L., Erwin J.T., Glasby R.S., Stefanski D. "High-order mesh curving using WCN mesh optimization." 46th AIAA Fluid Dynamics Conference, p. 3178. 2016

[17] Toulorge T., Geuzaine C., Remacle J.F., Lambrechts J. "Robust untangling of curvilinear meshes." Journal of Computational Physics, vol. $254,8-26,2013$

[18] Turner M., Moxey D., Peiró J., Gammon M., Pollard C.R., Bucklow H. "A framework for the generation of high-order curvilinear hybrid meshes for CFD simulations." Proceedings of the 26th International Meshing Roundtable, vol. 203, pp. 206 - 218. Procedia Engineering, 2017

[19] Xie Z.Q., Sevilla R., Hassan O., Morgan K. "The generation of arbitrary order curved meshes for 3D finite element analysis." Computational Mechanics, vol. 51, no. 3, 361-374, 2013

[20] Sherwin S.J., Peiró J. "Mesh generation in curvilinear domains using high-order elements." International Journal for Numerical Methods in Engineering, vol. 53, no. 1, 207-223, 2001

[21] Dobrev V., Knupp P., Kolev T., Mittal K., Tomov V. "The Target-Matrix Optimization Paradigm for high-order meshes." SIAM Journal on Scientific Computing, vol. 41, no. 1, B50-B68, 2019

[22] Lagarias J.C., Reeds J.A., Wright M.H., Wright P.E. "Convergence properties of the Nelder-Mead simplex method in low dimensions." SIAM Journal on Optimization, vol. 9, no. 1, 112-147, 1998 
[23] Geuzaine C., Remacle J.F. "Gmsh: A 3-D finite element mesh generator with built-in pre-and post-processing facilities." International Journal for Numerical Methods in Engineering, vol. 79, no. 11, 1309-1331, 2009

[24] Johnen A., Remacle J.F., Geuzaine C. "Geometrical validity of high-order triangular finite elements." Engineering with Computers, vol. 30, no. $3,375-382,2014$

[25] Remacle J.F., Chevaugeon N., Marchandise E., Geuzaine C. "Efficient visualization of high-order finite elements." International Journal for $\mathrm{Nu}$ merical Methods in Engineering, vol. 69, no. 4, 750-771, 2007 


\title{
MULTIPLE APPROACHES TO FRAME FIELD CORRECTION FOR CAD MODELS
}

\author{
Maxence Reberol ${ }^{1 *} \quad$ Alexandre Chemin ${ }^{2} \quad$ Jean-François Remacle ${ }^{3}$ \\ ${ }^{1}$ Université catholique de Louvain, Avenue Georges Lemaitre 4, 1348 Louvain-la-Neuve, Belgium
}

\begin{abstract}
Three-dimensional frame fields computed on CAD models often contain singular curves that are not compatible with hexahedral meshing. In this paper, we show how CAD feature curves can induce non meshable 3-5 singular curves and we study four different approaches that aims at correcting the frame field topology. All approaches consist in modifying the frame field computation, the two first ones consisting in applying internal constraints and the two last ones consisting in modifying the boundary conditions. Approaches based on internal constraints are shown not to be very reliable because of their interactions with other singularities. On the other hand, boundary condition modifications are more promising as their impact is very localized. We eventually recommend the 3-5 singular curve boundary snapping strategy, which is simple to implement and allows to generate topologically correct frame fields.
\end{abstract}

Keywords: frame field, hexahedral meshing, block decomposition

\section{INTRODUCTION}

In the last decade, frame field based approaches $(\S 2)$ have become popular and promising for hexahedral and hex-dominant meshing. However, state-of-art frame fields are not guaranteed to have the right topology for full hexahedral meshing [1, 2, 3], especially when the CAD model contains feature curves on the boundary $(\S 3)$.

In $\S 3.3$, we show that the 3-5 singular curves frame field singularity graph issue, i.e. singular curve where the hexahedral valence smoothly transitions from three (index $+1 / 4$ ) on one extremity to five (index $-1 / 4$ ) on the other one, is due to the presence of concave and curved feature curves on the model. This 3-5 singular curve is the most common problem that arises in 3D frame field topologies.

Based on these observations, we investigate four approaches to automatically correct the frame field topology $(\S 4)$. We present the reasoning behind each approach as well as their pros and cons (frame fields corrected successfully, failures, and limitations). Two

\footnotetext{
*maxence.reberol@uclouvain.be
}

of the approaches rely on extrusion of objects inside the frame field: (i) the extrusion of boundary feature curves (§4.1) and (ii) the extrusion of singular curve extremities (§4.2). Extrusion based methods work on simple cases but fail when the extrusion process is perturbed by other singularities of the frame field.

Another possibility for correcting frame fields is to remove the concave feature curves, by transforming them into fillets (§4.3). In other words, irregular 3-5 transitions are much less present in smooth models. In the smooth case, frame fields are generally compatible with hex meshing, but the difficulty is to map the singularity graph onto the non-smooth original geometry. Instead, and it is what we consider to be our best approach, we propose to remove the 3-5 singular curves by snapping them on the boundary and adapting the frame field boundary conditions $(\S 4.4)$.

The presence of 3-5 singular curves in frame fields is one of the main issues that prevents frame-field based hexahedral meshing and automatic blocking. By removing them, our snapping correction extends the range of application of frame-field based meshing technology to a larger set of CAD models, where previous 
techniques would fail because of incorrect frame field topology. Nevertheless, this approach is limited to 3-5 singular curves close to the boundary, which are the most frequent in CAD, and cannot handle arbitrary cases of 3-5 singular curves.

\section{RELATED WORK}

The standard frame field based meshing approach consists in building a smooth and boundary-aligned frame field (§2.1) and using it as a guide to build a hexdominant $[4,5,6,7,8]$ or a fully hexahedral mesh $[9,10,11,3,12,13]$. When the frame field singularity graph does not match a feasible hexahedral mesh topology, mixed-integer parameterization approaches fail and hex-dominant ones produce lot of tetrahedra. Some heuristic-based attempts to correct the frame fields have been tried $(\S 2.2)$, but the problem remains largely unsolved.

\subsection{Frame field design}

In dimension two, crosses are objects made of two orthogonal directions, invariant by the four rotations of respectively 0, 90, 180 and 270 degrees. Cross fields are usually represented by $2 \mathrm{D}$ vector fields of the form $\mathbf{f}(\mathbf{x})=(\cos (4 \theta(\mathbf{x})), \sin (4 \theta(\mathbf{x})))$ [14]. To get a smooth vector field in a $2 \mathrm{D}$ domain $\Omega$, the natural way is to minimize the Dirichlet energy $\int_{\Omega}\|\nabla \mathbf{f}\|^{2}$ under Dirichlet boundary conditions. One issue with this approach is that the gradient of the frame field tends to infinity at singularities in the continuous setting. This is either ignored, as the energy stay finite after discretization, or addressed by using a scaling scalar field [15], or more recently by turning to the Ginzburg-Landau theory $[16,17]$. In practice, the frame fields obtained with these different approaches are similar, with singularities whose indices are compatible with quadrilateral meshing.

In dimension three, frames are made of three orthogonal directions and are invariant by the twenty-four rotations of the cube. For the generation of boundary aligned smooth frame field, the ideas are similar to the 2D case: find a convenient representation of the frame field and minimize the Dirichlet energy. However, finding a unique and continuous representation is more tricky. The current best candidates are spherical harmonics [18] and fourth-order tensors [19]. In both cases, the spaces are of dimension nine and the frames live on a manifold of dimension three. The Dirichlet energy is minimized while staying on the frame manifold, which in practice is done either by optimizing Euler angles associated to frames [20] or iteratively with a non-linear solver and by using recurrent projections on the frame manifold [19]. The resulting frame fields exhibit singularities made of internal curves, that is usually called the singularity graph. As in dimension two, the norm of the gradient tends to infinity at the singular curves, which can be seen as extrusions of the singular nodes of a boundary cross field.

Contrary to the $2 \mathrm{D}$ case, frame field singularity graphs do not always correspond to feasible hexahedral mesh connectivity. This issue has been extensively described in recent articles $[1,2,3]$. To our knowledge, there exist no frame field generation approach that has any kind of guarantee to provide a singular graph that is compatible with hex meshing.

\subsection{Frame field correction}

A simple 3D model exhibiting a fundamentally invalid singularity graph is the notch model $[1,2]$ (Figure 1), which is the boolean difference between a box and a cylinder. The singularity graph produced by all existing frame field methods is made of a single curve whose hexahedral valence is three on one extremity and five on the second one, that will be called a 3-5 singular curve in the rest of the paper. In a hexahedral mesh, it is not possible to have an interior vertex adjacent to only one valence three edge and one valence five edge (other adjacent edges being regular, i.e. valence four). The only valid vertex configurations are the ones which are topologically equivalent to sphere triangulations, as described in [3]. When a singularity graph contains a different configuration, e.g. a 3-5 singular curve, we say it is invalid or not hex-meshable.

Given a valid singularity graph, there exist methods that allow to compute a smooth frame field, e.g. $[3,21]$. Thus, correcting a frame field can be achieved by generating a valid singularity graph, i.e. the set of irregular edges of the associated block decomposition. But generating a valid singularity graph from scratch remains a totally open problem. An alternative approach is to start from an initial frame field, possibly non-meshable, and modify it in order to make it hexmeshable.

In [2], a valid frame field for the notch model is built by either manually extruding the concave feature curve inside the model (producing an internal surface) or by manually adding a fillet to the feature curve. In the present paper, we propose two approaches $(\S 4.1, \S 4.3)$ that aim at automatizing these manual interventions.

To avoid the block decomposition degeneracies caused by a 3-5 singular curve, Zheng and his co-authors [12] propose to replace it by two singular curves, one with a valence of three and one with a valence of five, whose geometries are determined by tracing streamlines starting at the extremities of the 3-5 curve. One of our correction approach $(\S 4.2)$ is based on this strategy. 
It is also worth mentioning that there can be local defects in the extracted singularity graph, e.g. the $z i g$ - zag issue. They can be corrected with local operations, as detailed in [22]. We are not interested in these issues in this paper as they are artifacts of discretization choices, and it is possible to avoid them by using a frame per vertex instead of one per tetrahedron.

\section{BOUNDARY-ALIGNED SMOOTH FRAME FIELD}

In this section, we first describe the energy minimization formulation of the boundary-aligned smooth frame field problem ( 33.1 ), which is common to most of the recent 3D frame field solvers. In this paper, the frame field is discretized on a tetrahedral mesh $\mathcal{T}$, by defining one frame per vertex (§3.2). More precisely, we use a continuous piecewise linear approximation of nine frame coefficients, which fully determine a 4-th order tensor representation of the frames [19]. The following section (§3.3) is a qualitative analysis of the associated frame field singularity graphs and of how boundary conditions can lead to configurations which do not correspond to hexahedral meshes.

As we do not enter into the details, readers unfamiliar with frame fields should refer to existing articles that explain extensively the theory and implementation of such fields, e.g. [18, 20, 23, 19].

\subsection{Continuous frame field formulation}

The goal is to compute a frame field as smooth as possible and that is aligned with the boundaries/features of the model. The natural approach is to translate these requirements into a Dirichlet energy minimization problem. Minimizing the Dirichlet energy (1) ensures the smoothness in the domain and the Dirichlet boundary conditions (2) enforce the alignment with the model boundary. Formally, the frame field $\mathbf{f}$ is the solution to the problem:

$$
\mathbf{f}=\underset{\mathbf{f}(\mathbf{x}) \in \mathcal{O}}{\operatorname{argmin}} \int_{\Omega}\|\nabla \mathbf{f}\|^{2}
$$

subject to boundary conditions:

$$
\left\{\begin{array}{l}
\mathbf{f}(\mathbf{x})=\mathbf{g}(\mathbf{x}) \text { for } \mathbf{x} \in \partial \Omega_{d} \\
\mathbf{f}(\mathbf{x}) \perp \mathbf{n}(\mathbf{x}) \text { for } \mathbf{x} \in \partial \Omega_{s}
\end{array}\right.
$$

with $\mathcal{O}$ the space of frames, $\partial \Omega_{s}$ a smooth subset of the model boundary $\partial \Omega$, where we want the frames to be tangent to the boundary, $\mathbf{n}$ the boundary normal, and $\partial \Omega_{d}$ another subset of $\partial \Omega$ where we impose the three directions of the frames. Usually, $\partial \Omega_{d}$ corresponds to the feature curves of the model, also called hard-edges or ridges. The symbol $\perp$ means that one of the three frame directions is parallel to a given vector (tangency constraint).

Before going further, one needs to choose a representation of the frames. Frames live in a space, denoted $\mathcal{O}$, which is the quotient space of the space of rotations $S O(3)$ with the octahedral group $O$ [23]. Unfortunately, there is no simple representation of these objects. To date, two representations have been proposed: spherical harmonics of degree four [18] and fourth-order tensors [19]. In both cases, frames live on manifolds of dimension three immersed in $\mathbb{R}^{9}$. There is an isomorphism between the two representations, so they are essentially equivalent. The continuity and uniqueness of these representations is a necessary condition to use directly their nine coefficients to compute distances and gradients, which is analogues to using the Euclidean distance on the unit circle instead of the circle arc length.

In the continuous setting, the Dirichlet energy (1) blows up because of the presence of singular curves and this singular behavior is the cause of many issues for the discretization.

\subsection{Discretization of the frame field prob- lem}

To find a numerical solution to the frame field problem introduced previously ( $\$ 3.1$ ), one needs to choose an approximation space for the frame field and a numerical scheme to solve the nonlinear problem (1).

We discretize the frame field on a tetrahedral mesh $\mathcal{T}$ and use a continuous piecewise linear approximation of the frame coefficients, which can be coefficients of either the spherical harmonics representation or of the 4-th order tensor one. The discretized frame field $\mathbf{f}_{h}$ is now entirely defined by its coefficients $\mathbf{f}_{i} \in \mathbb{R}^{9}$ at each vertex $v_{i} \in \mathcal{V}$ of the tetrahedral mesh. It is worth noting that inside a tetrahedron, the linearly interpolated coefficients do not correspond to a frame, but the closest frame can obtained by projection on the frame manifold $\mathcal{O}$. Compared to the one frame per tetrahedron discretization, the piecewise linear approximation is more efficient: less unknowns for the same mesh, better accuracy [20], possibility to use linear finite elements.

If each $\mathbf{f}_{i}$ is a frame, then the singularities, which correspond to infinite gradients in the continuous formulation, cannot be represented at vertices. With this approach, one possibility is to detect singularities by looking at axis permutations along edge loops. The smallest loops to look at are the internal faces of the tetrahedral mesh, which are simply triangles. The singularity graph is then made of connected chains of tetrahedra. In practice, it forces the singularity graph 

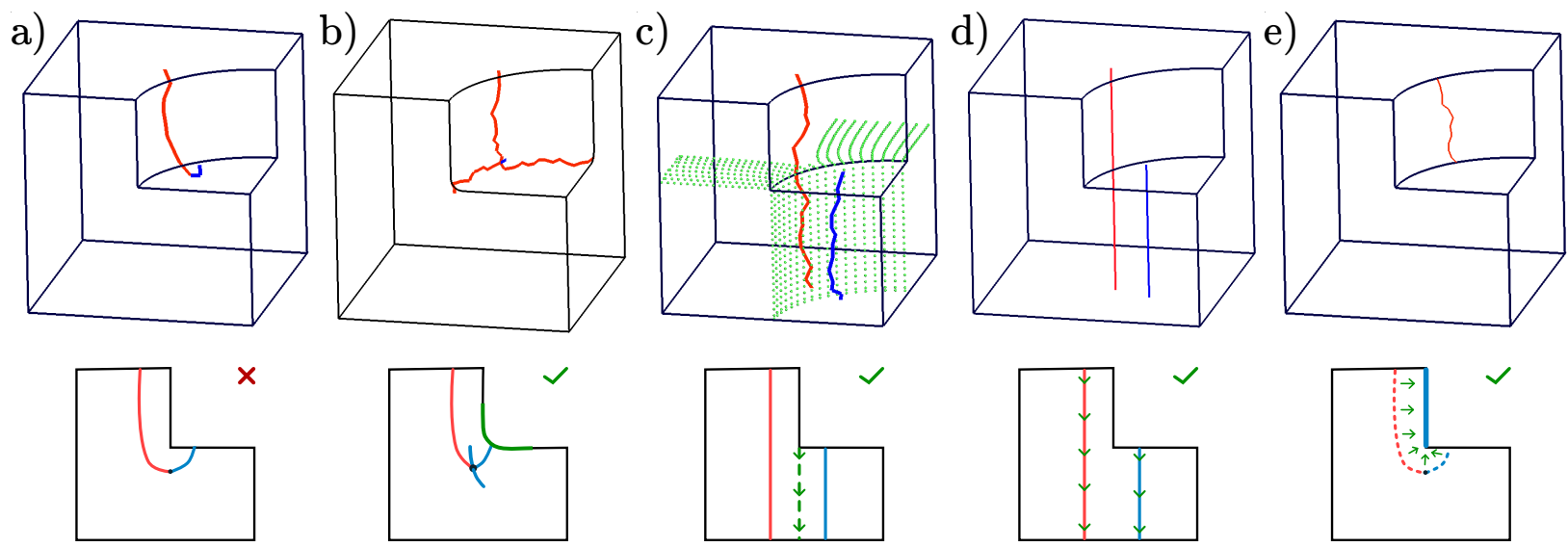

Figure 1: a) Natural frame field singularity graph, made of one non-meshable 3-5 singular curve. b) When replacing the concave feature curve by a fillet, the new singularity graph is made of three valence-five and one valence-three singularity curves, which are compatible with hexahedral meshing. c) Via the streamlines (green), the feature curve is extruded inside the volume and the resulting singularity graph is made of two singular curves (one valence five and one valence three). $d$ ) Tracing streamlines from boundary singular nodes generates two valid singular curves. e) The initial invalid 3-5 singular curve of a) is snapped to the boundary surface, creating a new valence three feature curve. Bottom row: schematic view of the corrections, in the diagonal cut view.

to be locally very distorted as it must be contained inside tetrahedra, whose facets are randomly oriented in the mesh.

Another way to deal with singularities is to allow the coefficients $\mathbf{f}_{i}$ to represent objects which are not frames. This is analogous to letting $2 \mathrm{D}$ crosses tend to zero at singularities instead of staying unit vectors. The advantage is that the singularities are smoother, because they are less affected by the tetrahedral mesh, but they are also more diffuse and there is no longer a clear localization of the singularities.

Frame field solvers usually work in two stages : initialization via Laplacian smoothing of the frame coefficients, without enforcing the frame constraint $f \in \mathcal{O}$, followed by a smoothing of the frames, where the frames $\mathbf{f}_{i}$ must lie on $\mathcal{O}$ or stay close to it. The process usually converges to a local minimum which is not far from the initialization [20].

The frame coefficients can be forced to stay on the frame manifold $\mathcal{O}$ by either recurring projections [19] or by optimizing the Euler angles of the associated rotations [20].

In any case, the Dirichlet energy associated to the frame field tends to infinity with mesh refinement, but stays finite because of the discretization. Consequently, these approaches only work on uniform meshes. On non-uniform meshes, the singularities move to areas of coarse elements as the same singularity graph topology can be represented while costing less energy.

We use 4th order tensors to represent the frames, and we allow the coefficients $\mathbf{f}_{i}$ to deviate from $\mathcal{O}$ at singularities. This particular choice is not important for the rest of the paper: the correction techniques we study can be applied to all energy-minimizing frame field solvers.

\subsection{Frame field singularity graph behavior}

Boundary aligned frame fields produced by energy minimizing methods are interesting because they exhibit singularities that form a graph, which is topologically similar to the singular edges of a hexahedral mesh. Unfortunately, as described in the literature $(\S 2.2)$, this graph is not always topologically equivalent to a valid hexahedral mesh.

As singular curves cost a lot of energy in (1), an amount tending to infinity with mesh refinement, the energy-based formulation of the frame field problem is asymptotically looking for solutions that minimize the length of the singular curves.

In CAD models, there are many feature curves on the boundary where we impose the frames, via the Dirichlet boundary conditions (2). These feature curves split the boundary in multiple patches, which become independent if we consider the associated surface cross field problems. This boundary splitting leads to the apparition of singular nodes on the surface, which are necessary to have coherent cross fields that respect the Poincaré-Hopf theorem. But from the point of view of the volume frame field, the boundary singular nodes must be the extremities of singular curves, as there are no isolated singular nodes in a 3D frame field. 
A simple example exhibiting this behavior is a box with a circular arc imprinted on one face, see Figure 2.a.. This example can be seen as a simplification of the notch model. In this example, the two patches on top must have singular nodes to accommodate their boundary conditions, but the frame field solver, which is minimizing the Dirichlet energy, do not propagate these singularities inside the model as this would cost a lot of energy, but merge them as soon as possible in the volume. Models with such configurations are, for example, the ones containing boolean operations involving spheres or cylinders that do not go through the whole model, which are common in CAD modelling.

Our interpretation is that the energy-minimization formulation ( $\S 3.1$ ) is similar to the Laplace equation $-\Delta \mathbf{f}=0$, even with the additional constraint $\mathbf{f}(\mathbf{x}) \in \mathcal{O}$. Hence, this is essentially a smoothing kernel that act locally when possible. Constraints from the boundary that cost energy (non-zero frame gradient) do not propagate far in the volume.

On the other hand, hexahedral mesh topological constraints (chords, sheets) propagate on arbitrary long distances. Thus, hexahedral meshes associated to frame fields are often different from the ones that a user would produce by manually building a block decomposition.

An ideal answer to this issue would be a new frame field problem formulation that allows better propagation of boundary constraints, but unfortunately none has been successfully developed up to now.

Another important observation is that even if the frame field have a wrong topology, one of the frame directions is not affected (the vertical ones in the notch and box with arc examples). At the transition from valence three to valence five in the 3-5 singular curve, the frame field stable direction along the singularity is no longer tangent to the curve. Exploiting this stable direction is the basis of the feature curve extrusion (§4.1) and singularity extrusion (§4.2) correction approaches. It also worth noting that [2] tried to penalize the incoherency between the frame field stable direction and the singular curve tangents, in order to iteratively correct the frame field, but it leads to numerical instability and this approach was eventually unsuccessful.

\section{FRAME FIELD CORRECTION TECHNIQUES}

We present and discuss various strategies to automatically correct frame fields, for them to be suitable for full hexahedral meshing. We focus on removing the 3-5 singular curves.
Feature curve extrusion (§4.1) and transformation of feature curves into fillets $(\S 4.3)$ are automation of the manual corrections applied to the notch model in [2]. Singularity extrusion ( $\$ 4.2$ ) has been introduced by [12] in the context of dual surface construction. Snapping of 3-5 singular curves to the boundary ( $(4.4)$ has not been used previously, to our knowledge. The four corrections are illustrated side-by-side on Figure 1 and Figure 3.

We show failure cases for the extrusion approaches $(\S 4.1, \S 4.2)$, where the extruded objects interact badly with other singular curves (Figure 2). The transformation of feature curves to fillet $(\S 4.3)$ is difficult to apply in practice because it requires to map the new singularity graph back to the initial geometry, which we did not automate. The snapping of 3-5 singular curves $(\S 4.4)$ seems the most promising approach in our opinion, but it leads to hexahedra with invalid geometry (zero jacobian at some corners) and a postprocessing (insertion of sheets) is required to have hexahedral meshes suitable for numerical simulation.

\subsection{Feature curve extrusion}

To recap the previous frame field observations ( $(3.3)$ : a concave feature curve can cause 3-5 singular curves, but when it happens there is a stable direction in the frame field which is not affected. We propose to use the stable direction of the frame field to extrude the concave feature curves inside the model. Once the new internal surface (extrusion of the curve) is generated, we add internal constraints to the frame field formulation and compute a new one. While this approach works for some models, it does not when the extrusion process (streamline tracing) is perturbed by other singularities.

Tracing streamlines from feature curves The simplest approach would be to propagate the feature curves straight into the model, following the directions of the initial boundary normals. It would work for simple blocky models, but not when the boundaries are curved, because the new internal surfaces would not follow the curvature of the model boundaries.

Instead, we extrude the concave feature curves by using certain directions in a initial frame field. In practice, we achieve this extrusion by tracing streamlines, that start from the feature curves and end on the first reached boundaries. In our case, the tracing is done by using a standard fourth order Runge-Kutta explicit scheme, very similar to the one used with vector fields. The only difference is that at each new position, we extract the frame direction which is closest to the previous direction. The reader can refer to Algorithm 1 for a more detailed description of the streamline tracing 
a

A

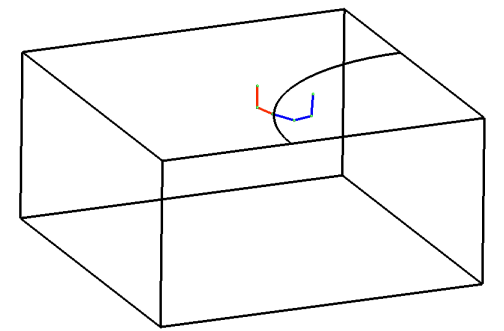

$\mathrm{B}$

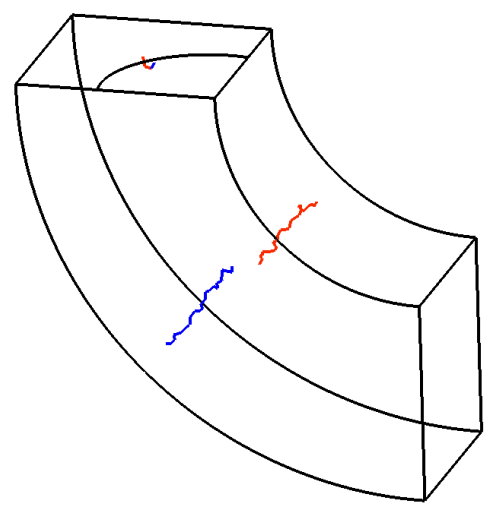

b
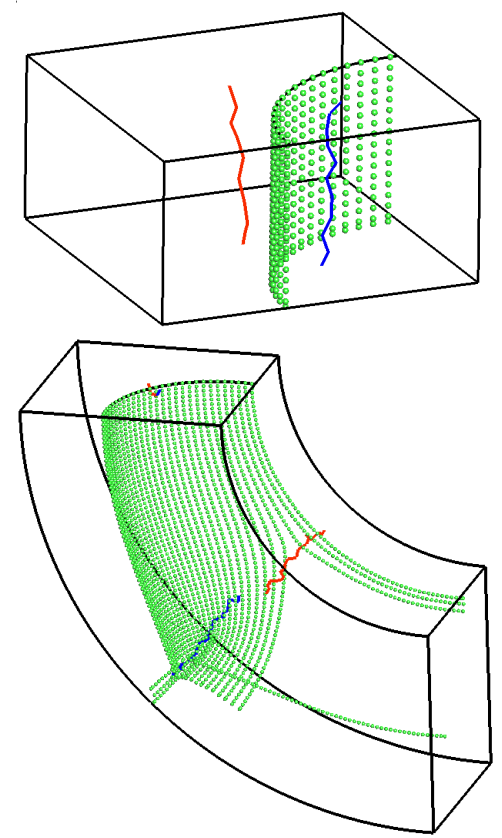

C
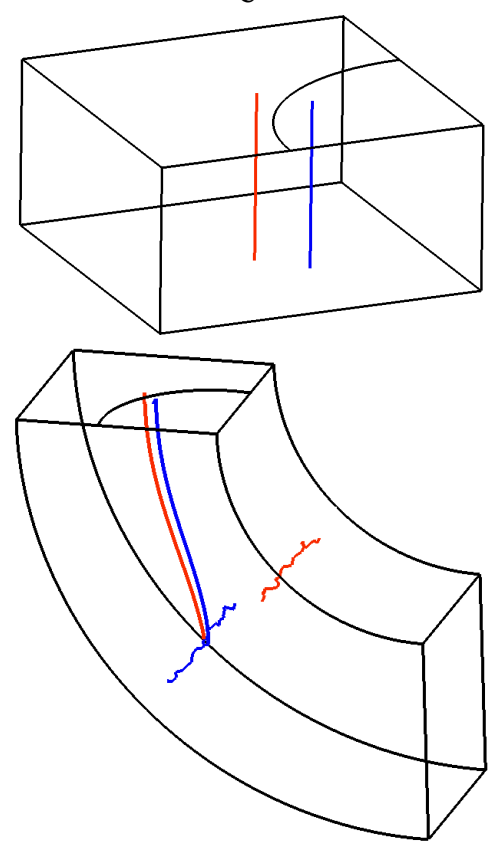

Figure 2: Model A: box with imprinted circle arc, simplified version of the notch model. Model B: same model but the box has curvature, which causes two additional singular lines. Col. a: singularities of the initial frame field. Col. b: feature curve extrusion, works for A but fails for B because of interaction with other singularities, which split the streamline trajectories. Col. c: singularity extrusion, works for A but fails for B because the streamlines hit another singularity.

a)

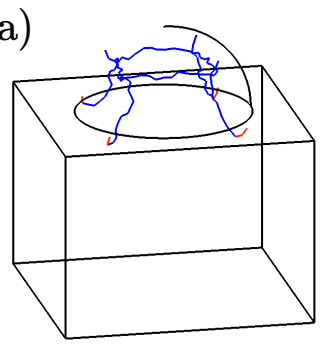

b)

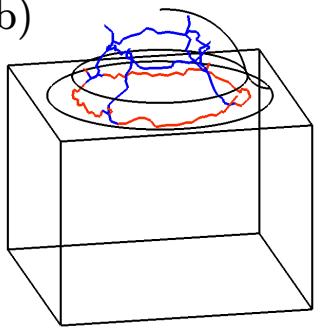

c)

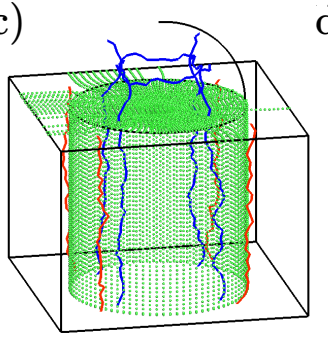

d)

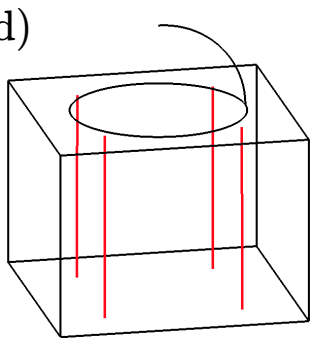

e)

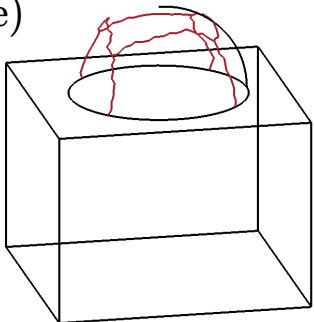

Figure 3: Half-sphere on top of a box. a) Natural frame field singularity graph, containing four non-meshable 3-5 singular curves. b) When replacing the concave feature curve by a fillet, the new singularity graph contains four additional singular curves of valence five, making the graph compatible with hexahedral meshing. c) With internal constraints (green), the feature curve is extruded inside the volume and the resulting singularity graph is valid. d) Tracing streamlines from boundary singular nodes replace the four 3-5 singularities by four singularities of valence five, but four valence three singular lines are missing. These ones should be traced from the internal singular nodes. e) The four 3-5 singular curves are snapped to the boundary, forcing snapping of the other singularities. 


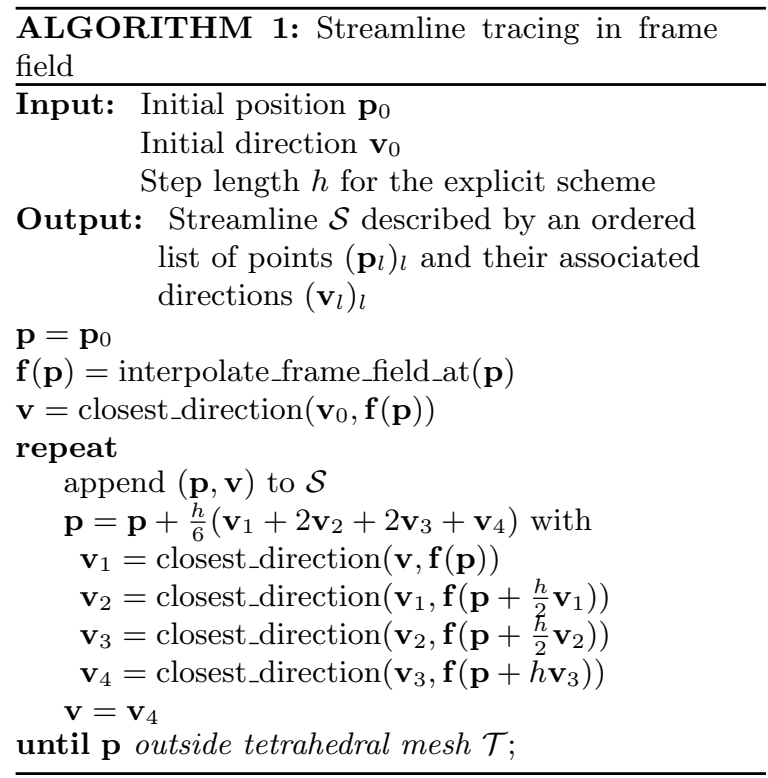

process.

To determine the initial directions for the propagation, we look at the appropriate hexahedral valence associated to the feature curve. It is usually one or three, and occasionally two for user-inserted curves on smooth surfaces, such as the circular arc on the Figure 2, or valence four for model with cuts whose dihedral angles are close to 360 degrees. For valence one, there is no need to propagate the curves as the frame field directions are already imposed by the surface normals at both sides of the curve. For valence two, three and four, we respectively extend the curve in one, two and three directions.

Successful applications of the streamline tracing process can be seen on Figures 1.c., 2.A.b and 3.c., where the extruded curves are shown with green points.

Internal constraints for frame field To compute a new frame field that respects the extruded curve, we add internal constraints in the boundary conditions (2) of the frame field formulation (§3.1). The new constraints (3) are tangential conditions, forcing one direction of the frames, similar to the boundary conditions on the smooth parts of the boundary.

Consider a streamline $\mathcal{S}=\left\{\left(\mathbf{p}_{k}, \mathbf{v}_{k}\right)\right\}_{k}$, made of $\left(\mathbf{p}_{k}\right)_{k}$ the points, $\left(\mathbf{v}_{k}\right)_{k}$ the associated directions, started from the edge $e$, of tangential direction $\mathbf{t}_{e}$. Then the associated internal conditions are:

$$
\forall k, \quad \mathbf{f}\left(\mathbf{p}_{\mathbf{k}}\right) \perp\left(\mathbf{t}_{e} \times \mathbf{v}_{k}\right)
$$

To apply these internal conditions precisely, we com- pute a new tetrahedral mesh containing the points $\left(\mathbf{p}_{\mathbf{k}}\right)_{k}$ associated to all the streamlines traced from the concave feature curves.

In our examples, the singularity graphs of the new frame fields are displayed on Figures 1.c., 2.A.b and 3.c.. They correspond to valid hexahedral meshes, similar to the ones a user would generate by manually building the block decompositions.

Failure cases and limitations Extruding a feature curve inside a frame field is equivalent to tracing a sheet. If the sheet encounters another singularity orthogonally, then it is sheared in multiple parts. When the multiple parts can stay inside a future valid sheet of the hexahedral mesh, as for the horizontal sheet in Figure 1.c., then our approach still works. But if the sheet is sheared into parts that are sent in different arbitrary directions, then the new internal surface makes no sense and should not be used to constrain the frame field, as it would definitely produce an incoherent frame field. This failure case is illustrated on Figure 2.B.b..

The feature curve extrusion is also not applicable to models where the frame field contains limit cycles, such as the Nautilus example in Figure 1 of [2]. In such cases, which are rare, the streamlines will spiral indefinitely without reaching any boundary. They can be detected by monitoring the streamline lengths.

Regarding the model on Figure 2.B., other frame field solvers could produce a frame field without the pair of valid singularities that accommodates the curvature. In that case, the feature curve extrusion correction would work. However, it is still possible to add features to the model, e.g. boolean difference with a cylinder, that would introduce singularities to perturb the extrusion of the feature curve.

The initial 3-5 singular curve is a local solution of the frame field solver to the boundary constraints. By extruding the feature curve using the stable directions, we are trying to generate global constraints, as they typically propagate through the whole model. We think this approach cannot work in the general case, as there is no reason that the feature curve conserves its shape during the extrusion process in the frame field.

\subsection{Boundary singular node extrusion}

Another possible extrusion approach is to propagate the boundary singular nodes via the frame field stable directions, instead of propagating the concave feature curves. This approach has been implemented in [12] to separate the contour of dual sheets, in the context of block decomposition. The additional step in our case is to compute a new frame field that respect these 
forced singularities.

The hope with this approach, compared to the feature curve extrusion ( $\$ 4.1)$, is that node extrusion produce curves that would not interact with other singularities, as they are much smaller than surfaces.

To extrude the singular node extremities of 3-5 singular curves, we use the same streamline tracing method (Algorithm 1) that was used for curve extrusion (§4.1). For each extremity, we trace a streamline in the direction of the stable direction, until it reached a boundary.

The streamline vertices are added in a new tetrahedral mesh and specific internal conditions are applied on them: forcing them be singular frames with a unique imposed direction. Another alternative could be to use frame field generation methods with fixed singularity graph $[3,21]$, but it would require imposing an entire singularity graph and we prefer to use a more flexible frame field solver that still has the possibility to displace the previous valid singularities, and potentially to spawn new ones.

Successful applications are shown on Figures 1.d., 2.A.c and 3.d.. They correspond to the same valid hexahedral meshes as the ones generated by the previous approach (§4.1). On Figure 3.d., the four internal valence three singularities are missing because we did not implement the tracing from an extremity which is an internal node, but it would work.

Limitations This approach suffers from the same failure case than the feature curve extrusion, which is shown on Figure 2.B.c.. Both streamlines hit an existing singularity during the tracing process, leading to an incoherent singularity graph.

The singularity extrusion correction technique may have a better success rate than the feature curve extrusion on specific models (less risk of collision), but it suffers from the same fundamental flaw (interaction with other singularities) and does not work in the general case.

\subsection{Transforming concave feature curves into fillets}

In the frame field analysis section ( $§ 3.3)$, we explained that non hex-meshable 3-5 singular curves are caused by feature curves, at least in the cases we studied. We can try to remove all hard-edges and compute frame fields on smooth models, assuming that energyminimizing frame fields on smooth models are less likely to contain 3-5 singular curves.

Even if smooth models can contain invalid 3-5 singular curves, e.g. the rockerarm in Figure 10 of [3], they are much less widespread than with CAD models in our experience. We think it is worth trying to exploit the smoothing of feature curves to correct frame fields. A feature curve (made of hard-edges) can be replaced by a smooth transition between the two surface patches adjacent to the curve. In terms of CAD modeling, this is equivalent to placing a fillet on the curve, as illustrated on Figure 1.b., Figure 3.b. and Figure 5.b..

Smoothing a convex feature curve (hex-valence of one) induces an internal singular curve of valence three in the frame field and smoothing a concave feature curve (hex-valence of three) induces an internal singular curve of valence five. If the feature curve that is replaced by a fillet was responsible for a 3-5 singular curve, the newly introduced singular curves join the invalid curve at its transition between valence three and five, creating a hex-meshable singular node. In the case of the notch model (Figure 1.a. and b.), the new singular node connects one valence-three and three valence-five singular curves after the insertion of the fillet. The associated block decomposition is shown on Figure 4.a.. Another application is illustrated on Figure 3.b., where the frame field of the smoothed model contains four additional valence-five singular curves, making it suitable for full hexahedral meshing.

From the point of view of the block decomposition, the fillet correction introduces a new hexahedral layer (the colored blocks on Figure 4.a.). This layer was partially present in the initial frame field, which contained the 3-5 singular curve, but it was pinched and it did not correspond to topological blocks (six quadrangular faces). The smoothing of the hard-edges allows to recover valid blocks.

Another example of singularity graph obtained after transforming the feature curves into fillet is illustrated on Figure 5. This model is a volume version of the box with arc model, but with the arc replaced by a groove. As with the notch model, the smoothing of the feature curves introduces new singular curves that connect with the 3-5 singular curves, making all the singular nodes hex-meshable.

This approach is interesting because it is a local approach, which implies local modifications of the frame field, well in accordance with the spirit of the energyminimizing frame field formulation.

Limitations Unfortunately, it is not straightforward to implement it in an automatic way. Once a hex-meshable frame field, or equivalently its singularity graph, is computed on the model with fillets, it must be brought back to the initial model with hardedges. Before trying to develop an automatic technique for building such mapping, it is interesting to do it manually and observe the result. 
a)

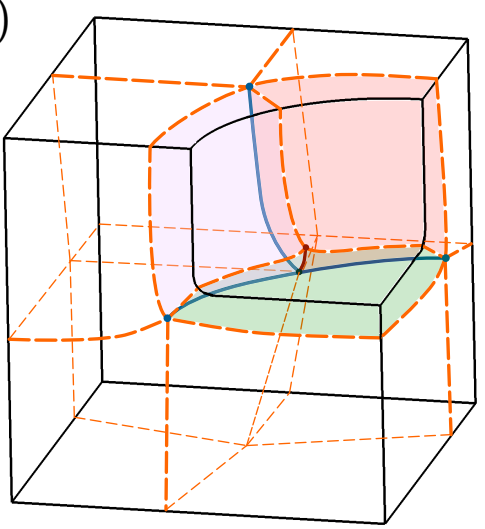

b)

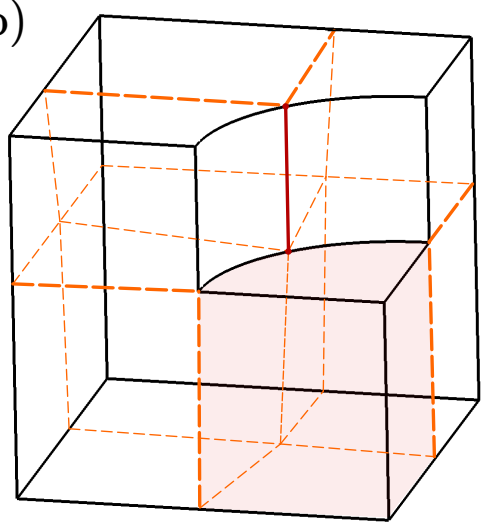

c)

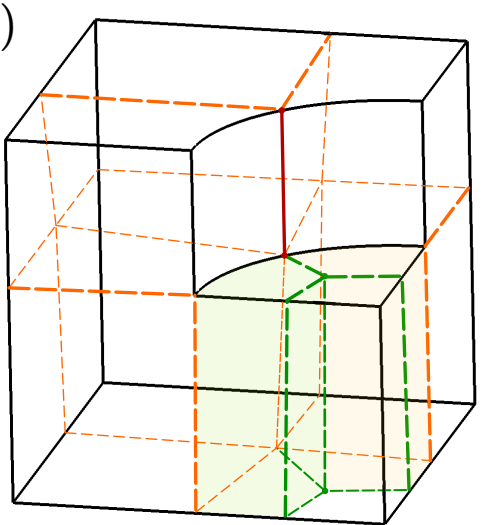

Figure 4: Block decomposition of the notch model after correction. a) Feature curve transformed to a fillet. The block decomposition is valid. b) 3-5 singular curve snapped to boundary. The block decomposition is topologically valid but the colored block has a flat corner. c) Refinement of the block with a flat corner. The decomposition is topologically and geometrically valid.

a)
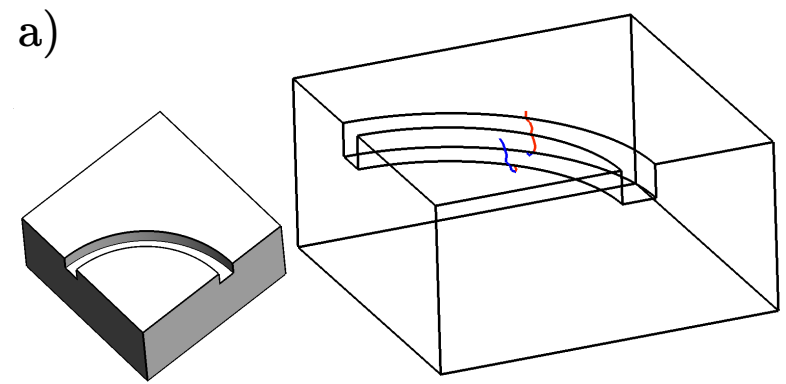

b)

Figure 5: a) Box with a circular rectangular groove on the top. The singularity graph is made of two 3-5 singular curves. b) The feature curves of the rectangular groove are transformed into fillets, making the geometry smooth. This change introduces singular curves along the groove, that connect with the previously invalid 3-5 singular curves. The new singularity graph, which contains four internal nodes, is compatible with hex meshing. 
By carefully looking at the block decomposition on the smoothed notch model on Figure 4.a., we can see that the valence-three boundary singular node has been positioned at the center of the fillet, where the curvature is maximal. In the initial model, this node has to be mapped on the center the concave feature curve. This implies that the topological block in green will have two adjacent edges on this curve, forming a flat angle. This geometry is not valid from a numerical analysis point of view, as the jacobian of the hexahedra would be zero on this corner.

Instead of pursuing this approach, which would require lot of engineering, we focus on a similar but simpler one: the boundary snapping of 3-5 singular curves $(\S 4.4)$.

\subsection{Boundary snapping of 3-5 singular curves}

The previous fillet approach $(\S 4.3)$ corrects the frame field singularity graph by adding singular curves in order to make all junctions hex-meshable. Another way to deal with the 3-5 singular curves is to make them disappear, instead of enriching them. By observing the block decomposition associated to the fillet correction on Figure 4.a., we can see that there is a layer of blocks close to the fillet (the colored ones). If we remove this layer, we get a new block decomposition where the singular curve is on the boundary, see Figure 4.b.. We propose to mimic this process by directly snapping 3-5 singular curves to the boundary, skipping the fillet correction (Figure 4.b.). A drawback is the resulting block decomposition has blocks with zero jacobian at some corners, but it was already the case with the fillet correction, and the final geometry can be improved by refining some blocks in a postprocessing phase (Figure 4.c.).

Singularity snapping Our snapping strategy is simple. For each 3-5 singular curve, we snap both extremities. If an extremity is a boundary singular node, it is snapped to the closest feature curve, if it is an internal singular node, it is snapped to the closest point of the boundary surface. Other singularities (initially valid) may have only one of their extremity snapped, if so the other is also snapped. This process is applied iteratively until all necessary singularity extremities have been snapped.

Once the extremities are snapped, the geometry of the curve on the boundary must be recomputed. A simple approach is to take the shortest path between both extremities on the boundary triangle edges, e.g. Figure 1.e. and Figure 3.e., but a more accurate one is to build a new triangular mesh of the boundary that includes smooth curves joining the snapped extremities.
Corrected frame field To generate a frame field that respect the snapped curves, the boundary conditions (2) of the frame field formulation (§3.1) must be adapted. Close to the snapped curves, which can be seen as new feature curves, the corrected frame field should not be aligned with the boundary normals. From the hexahedral mesh point of view, we would like to have edges of valence one or of valence three on parts of the surface boundary that are smooth, e.g. locally flat.

On the snapped curves, instead of imposing tangency to the boundary normal, we impose frames (Dirichlet boundary conditions) that are tangent to the curve and 45-degrees rotated from the boundary normal (along the curve axis).

Feature curves that received a snapped extremity are split and new boundary conditions are obtained by linearly interpolating the frames at both extremities of the split curves.

To avoid incompatible boundary conditions, we also remove the boundary alignment constraints on the vertices close to the snapped curves. These frames become free, allowing a smooth transition from the frames of the snapped curves to the frames on the rest of the model boundary.

While the new frame field is no longer boundary aligned everywhere, the affected areas remain localized and the resulting frame field is still similar to the initial one, minus the 3-5 singular curves that have been snapped.

Applications The singularity snapping correction is applied successfully on the notch model, see Figure 1.e., on the union of a cube and a half-sphere, see Figure 3.e. and on three more complicated CAD models, see Figure 6.

On the boxes with imprinted arcs (models A and B on Figure 2), the 3-5 singular curve would be reduced to a single node on the feature curve and the frame field would be as if there were no imprinted curves.

Geometry and block refinement One drawback of this approach is that it produces hexahedral blocks with an invalid geometry (zero jacobian at some corners), e.g. the bottom right block on Figure 4.b.. We propose to refine the affected blocks in a postprocessing step, as shown on Figure 4.c.. For more complex cases, the post-processing refinement can follow a template-based strategy, such as [25]. To preserve the topology of a hexahedral mesh, the refinement must be propagated to adjacent blocks when they share a refined quadrangular face. This is equivalent to sheet insertion. 
a
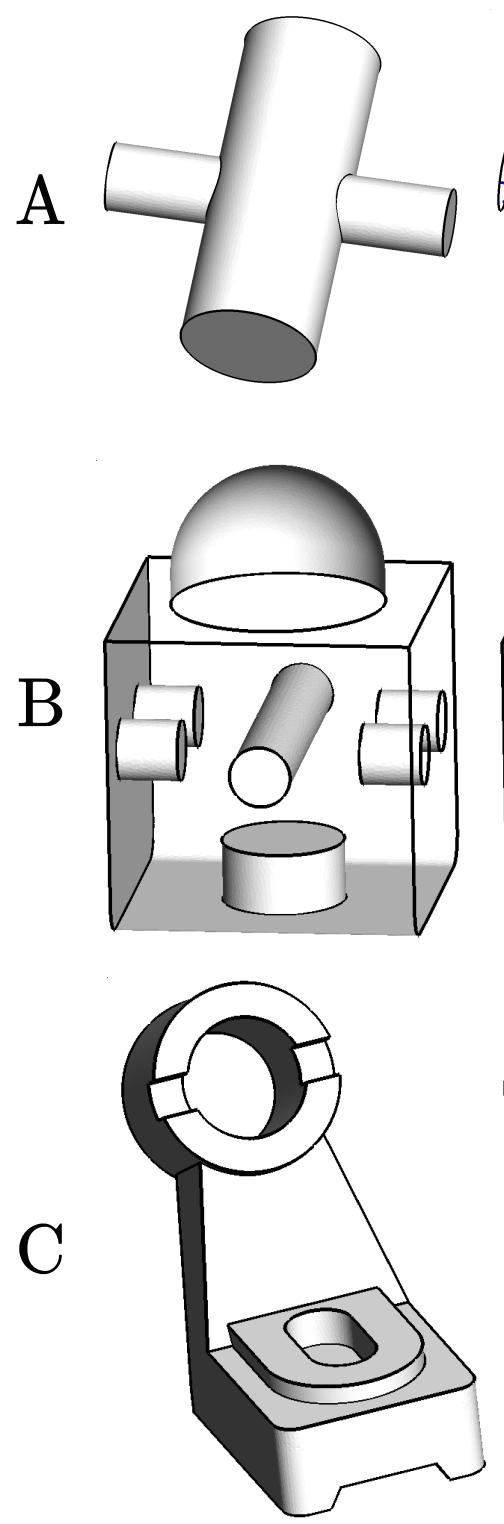

$\mathrm{b}$
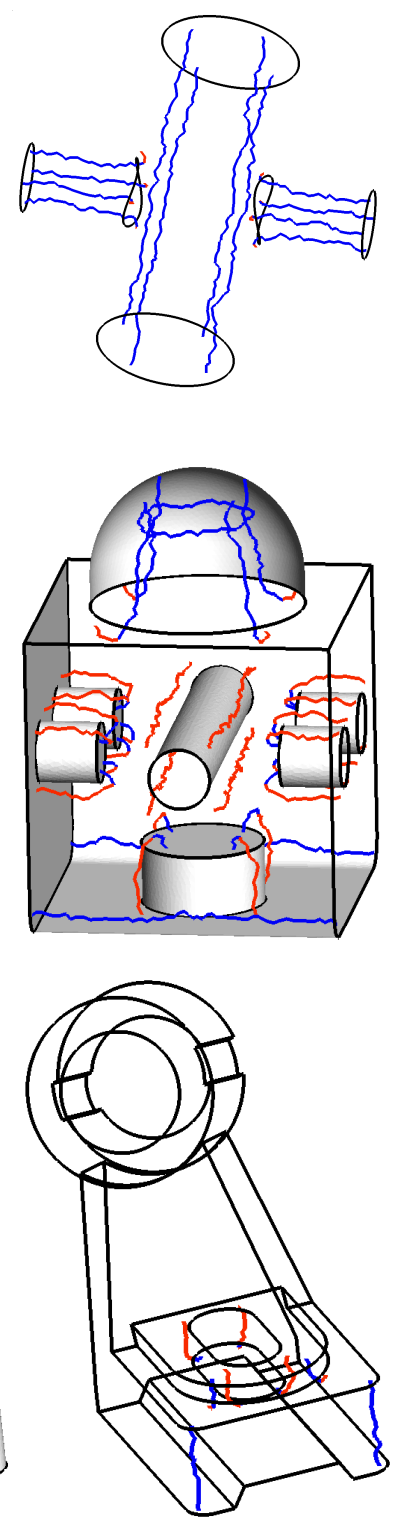

C
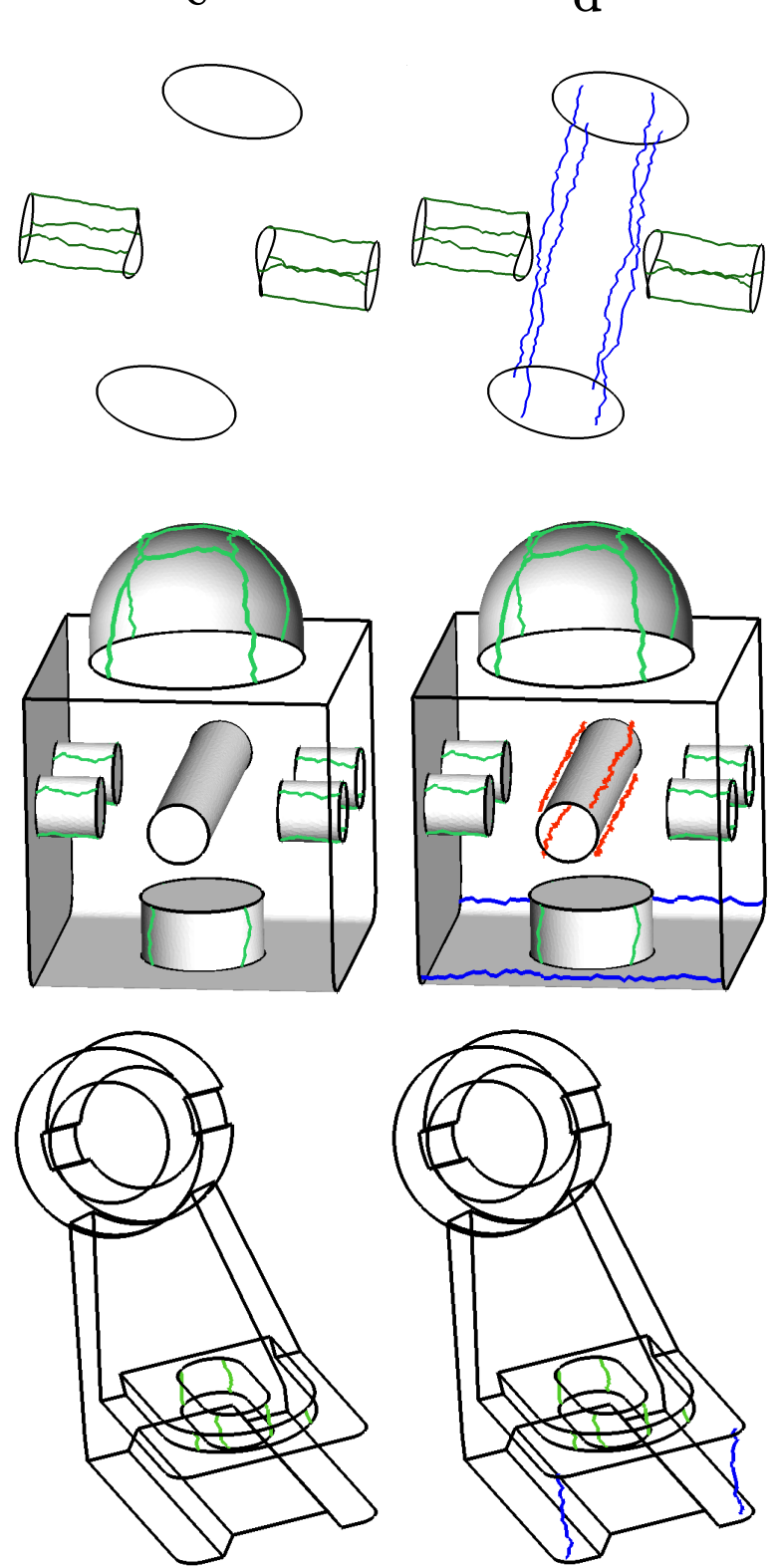

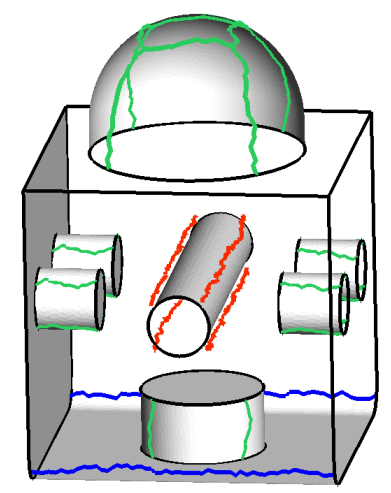

d

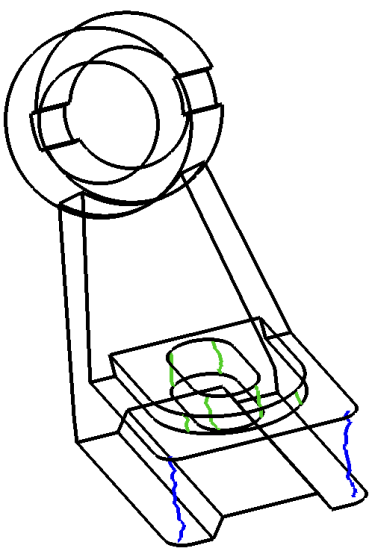

Figure 6: Model A: model built from the boolean union of two cylinders. Model B: model built from boolean operations between cylinders and a sphere, with two fillets on the bottom. Model C: model with various CAD features, from [24]. Col. a: initial models. Col. b: singularity graphs of the initial frame fields, which contain many 3-5 singular curves. Col. $c$ : snapping of the 3-5 singular curves, the snapped curves are shown in dark green. Col. $d$ : valid singularity graphs of the corrected frame fields, whose boundary conditions have been changed according to the snapped curves (in green). 
Limitations This approach is only applicable when the 3-5 singular curves are close to the model boundary, as they are snapped on it. When dealing with CAD models, this is often the case because the invalid singularities are mostly caused by curved surface patches, e.g. from a boolean operation with a cylinder.

That being said, there are 3-5 singular curves that lie far inside the volume and whose cause is global, e.g. the non-meshable singularity graph of the rockerarm model shown in the Figure 10 of [3]. Our snapping technique does not handle such case.

\section{CONCLUSIONS}

To deal with non-meshable 3-5 singular curves, which are induced by CAD feature curves, we studied four heuristic-based frame field correction strategies.

The feature curve extrusion (\$4.1) and the boundary singular node extrusion ( $\$ 4.2)$ techniques try to change globally the frame field topology in the same way a human user would naively proceed. This approach does not work when there are interactions with singularities produced by other features of the model.

A more local and promising approach is to remove the feature curves that induce the 3-5 singular curves, by transforming them into fillets $(\S 4.3)$. It allows additional singular curves that make the frame field singularity graph valid for hexahedral meshing. However, this approach is quite impractical because the mapping of the corrected frame field back to the initial geometry (without fillets) is not straightforward.

The last approach we explored is to remove the 3-5 singular curves by snapping them on the model boundary (§4.4). The resulting block decomposition associated to the corrected frame field is similar to the one obtained by using fillets, minus boundary layers. This technique, which is also local, is quite efficient and simpler to implement than the fillet correction. However, as the frame field is no longer aligned with the boundary everywhere, some blocks of the associated decomposition may have flat corners. Fortunately, these geometric defects can be removed via a post-processing block refinement procedure.

Short of having a better frame field formulation that do not produce 3-5 singular curves, we recommend using the singular curve snapping correction (\$4.4), when applicable.

Acknowledgement This research is supported by the European Research Council (project HEXTREME, ERC-2015-AdG-694020).

\section{References}

[1] Ray N., Sokolov D. "On smooth 3d frame field design." arXiv preprint arXiv:1507.03351, 2015

[2] Viertel R., Staten M.L., Ledoux F. "Analysis of Non-Meshable Automatically Generated Frame fields." Tech. rep., Sandia National Laboratories (SNL-NM), Albuquerque, NM (United States), 2016

[3] Liu H., Zhang P., Chien E., Solomon J., Bommes D. "Singularity-constrained Octahedral Fields for Hexahedral Meshing." ACM Transactions on Graphics (TOG), vol. 37, no. 4, 93:1-93:17, Jul. 2018

[4] Baudouin T., Remacle J.F., Marchandise E., Henrotte F., Geuzaine C. "A frontal approach to hex-dominant mesh generation." Advanced Modeling and Simulation in Engineering Sciences, vol. 1 , no. $1,8,2014$

[5] Bernard P.E., Remacle J.F., Kowalski N., Geuzaine C. "Frame field smoothness-based approach for hex-dominant meshing." ComputerAided Design, vol. 72, 78-86, Mar. 2016

[6] Gao X., Jakob W., Tarini M., Panozzo D. "Robust hex-dominant mesh generation using fieldguided polyhedral agglomeration." Transactions on Graphics (TOG), vol. 36, no. 4, 1-13, Jul. 2017

[7] Sokolov D., Ray N., Untereiner L., Lévy B. "Hexahedral-Dominant Meshing." Transactions on Graphics (TOG), vol. 35, no. 5, 1-23, Jun. 2016

[8] Ray N., Sokolov D., Reberol M., Ledoux F., Lévy B. "Hex-dominant meshing: mind the gap!" Computer-Aided Design, vol. 102, 94-103, 2018

[9] Nieser M., Reitebuch U., Polthier K. "CubeCover- Parameterization of 3D Volumes." Computer Graphics Forum, vol. 30, no. 5, 1397-1406, Aug. 2011

[10] Li Y., Liu Y., Xu W., Wang W., Guo B. "All-hex meshing using singularity-restricted field." Transactions on Graphics (TOG), vol. 31, no. 6, 1, Nov. 2012

[11] Lyon M., Bommes D., Kobbelt L. "HexEx: robust hexahedral mesh extraction." ACM Transactions on Graphics (TOG), vol. 35, no. 4, 123, 2016

[12] Zheng Z., Wang R., Gao S., Liao Y., Ding M. "Dual Surface Based Approach to Block Decomposition of Solid Models." 2018 
[13] Livesu M., Pietroni N., Puppo E., Sheffer A., Cignoni P. "Loopy Cuts: Surface-Field Aware Block Decomposition for Hex-Meshing." arXiv preprint arXiv:1903.10754, 2019

[14] Palacios J., Zhang E. "Rotational symmetry field design on surfaces." Transactions on Graphics (TOG), vol. 26 , no. 99,55 , Jul. 2007

[15] Knöppel F., Crane K., Pinkall U., Schröder P. "Globally optimal direction fields." ACM Transactions on Graphics (ToG), vol. 32, no. 4, 59, 2013

[16] Beaufort P.A., Lambrechts J., Henrotte F., Geuzaine C., Remacle J.F. "Computing cross fields A PDE approach based on the GinzburgLandau theory." Procedia engineering, vol. 203, 219-231, 2017

[17] Viertel R., Osting B. "An Approach to Quad Meshing Based on Harmonic Cross-Valued Maps and the Ginzburg-Landau Theory." SIAM Journal on Scientific Computing, vol. 41, no. 1, A452A479, 2019

[18] Huang J., Tong Y., Wei H., Bao H. "Boundary aligned smooth $3 \mathrm{D}$ cross-frame field." Proceedings of the 2011 SIGGRAPH Asia Conference on - SA '11. ACM Press, 2011

[19] Chemin A., Henrotte F., Remacle J.F., Van Schaftingen J. "Representing threedimensional cross fields using 4th order tensors." 2018

[20] Ray N., Sokolov D., Lévy B. "Practical 3D frame field generation." Transactions on Graphics (TOG), vol. 35, no. 6, 1-9, Nov. 2016

[21] Corman E., Crane K. "Symmetric Moving Frames." ACM Transactions on Graphics (TOG), vol. 38, no. 4, 87:1-87:16, Jul. 2019

[22] Jiang T., Huang J., Wang Y., Tong Y., Bao H. "Frame field singularity correctionfor automatic hexahedralization." IEEE Transactions on Visualization and Computer Graphics, vol. 20, no. 8, 1189-1199, 2014

[23] Solomon J., Vaxman A., Bommes D. "Boundary element octahedral fields in volumes." $A C M$ Transactions on Graphics (TOG), vol. 36, no. 3, 28,2017

[24] Ledoux F. "Paving the Path Towards Automatic Hexahedral Mesh Generation." invited talk at the 27th International Meshing Roundtable, 2018
[25] Schneiders R. "Refining Quadrilateral and Hexahedral Element Meshes." 5th International Conference on Grid Generation in Computational Field Simulations, pp. 679-688. CRC Press, 1996 


\title{
A REGULARIZATION APPROACH FOR AUTOMATIC QUAD MESH GENERATION
}

\author{
Julia Docampo-Sánchez \\ Robert Haimes \\ docampo@mit.edu \\ haimes@mit.edu \\ Aerospace Computational Design Laboratory, Department of Aeronautics \& Astronautics,
Massachusetts Institute of Technology
}

\begin{abstract}
Pure quadrilateral meshes are preferred when using shell-based structural analysis solvers since they provide more accurate results if compared to triangular or mixed meshes. Triangulations of complex trimmed surfaces (as constructed in $\mathrm{CAD}$ ) can be always generated and any triangle can be subdivided into three quadrilaterals by splitting the sides and introducing a new vertex at the centroid. Therefore, the conversion of a triangular mesh into a fully quadded conformal mesh is straightforward, and if the source triangulation is watertight, the resultant quad mesh maintains that property. However, triangle splitting implies that the quads inherit the original triangle shapes and the resulting mesh presents a very large number of irregular vertices. This paper describes a technique that recovers a significant amount of irregular vertices by performing iterative topological changes on the mesh and employs a modified Laplacian method for adjusting the vertex coordinates. The algorithm is robust, fast and produces a surface mesh of a BRep (where all vertices are on the geometry) that it is completely quadrilateral and semi-regular suitable for structural analysis and possibly other surface-based PDE solvers.
\end{abstract}

Keywords: Surface Mesh Generation, Computational Geometry, Quadrilateral, Laplacian

\section{INTRODUCTION}

Automatic mesh generation plays a key role in design systems when converting CAD output for use within various discipline analyses. For quad meshes, this can be done by constructing quadrilaterals directly or transforming triangular meshes into quads. The advantages of using triangular meshes as input is that for general surfaces, as for many other geometries, the mesh can always be produced using triangles. For applications in numerical simulations, a suitable mesh represents the surface geometry correctly (where all the vertices live on the surface or trimming curve) and has the appropriate element shape ensuring geometric accuracy and stability for the underlying solver.

In addition, the mesh should be as regular as possible. That is, most of its vertices should have valence four. 3 -valence vertices often produce flat elements. Higher valences on the other hand, imply having highly multivalued surface points (mesh vertices) since a vertex belongs to many elements. In both cases, this has a negative effect on the performance of the numerical scheme and should be addressed.

When using a triangle splitting approach for producing quads, the new mesh has an increased number of elements as well as irregular vertices. Further, depending on the triangle anisotropy the resulting quads shape becomes unsuitable. Hence, it is necessary to perform topological changes on the mesh targeting these problems and ensuring that the final tessellation becomes suitable for the desired application.

The methodology presented here produces pure quadrilateral meshes from a given triangulation. Starting with the triangle splitting procedure [1], it applies systematic topological operations that enable a significant improvement in the mesh quality. Our 
results suggest that in general we obtain semi-regular meshes with less than $5 \%$ irregular vertices. Since all the topological changes are performed in the parametric space of the geometry, its applications extend to general surfaces in 3-space. This procedure has been integrated into part of the ESP software suite [2]. The results presented here employed the software's internal tessellator, but the algorithm is designed to convert triangular meshes in general.

\section{QUAD MESH GENERATION}

Generating quad meshes can be done directly using advancing front techniques $[3,4,5]$ or defining quad partitions on the domain $[6,7,8,9,10,11]$. On the other hand, one can start with a triangular mesh and transform it into quads by merging or splitting the triangles. When merging triangles, this process requires appropriate triangle pairing $[12,13,14,15]$ since the resulting quadrangulation might leave several triangles that have to be subsequently eliminated [16].

Alternatively, any triangle can be split into three quads directly using its medians and inserting an extra vertex at the centroid [1] (see Figure 1). This approach is computationally efficient and produces pure quad conformal meshes. However, it naturally increases the total number of elements and the resulting mesh inherits the source triangle quality which in many regions generates quads that are too sharp or too flat. On top of that, the splitting step adds irregularity to the mesh. To overcome these limitations, mesh coarsening techniques $[17,18]$ can be applied as well as performing topological changes in the mesh. For example, the Quad Mesh Simplification [19] technique which uses poly-chords and the fundamental operation of quad collapsing. Or combining several fundamental operations, namely: swapping, splitting and collapsing [20, 21, 22, 23, 24].

Finally, most automated mesh processes require a post-processing step which ensures a suitable vertex distribution. That is, there are no sharp or flat elements and the quad's aspect ratio as well as the internal angles are within an admissible range. For quad meshes derived from triangles this is a requirement considering that the quad conversion step usually produces meshes of very poor quality. In addition, changing the mesh topology further deforms the quad shapes. Therefore, once the desired quad configuration is obtained, the final stage in the mesh generation process is to readjust the vertex coordinates. This can be cast as an optimization problem solving for the quad internal angles [25], orientation or side sizes [26]. Alternatively, one can solve variations of the Discrete Laplacian [25] or the elliptic operator [27] through an iterative scheme. Although element metrics such as skewdness, aspect ratios and the Jacobian matrix are better enforced through optimization [28, 29], iterative schemes are computationally cheaper.

The mesh regularization technique proposed in this paper builds upon the clean-up strategy from [20]. Unlike in $[20,22]$, the initial mesh comes directly from an unstructured triangulation consisting of nearly $50 \%$ irregular vertices. Using information from a vertex and its neighbors, we extend the set of possible two (three) step local topological operations from [20]. Each operation involves removing (adding) at most two quads and always reduces the total number of irregular vertices. Further, we adopt the approach from [22] to move irregular vertex pairs along the mesh whenever this operation preserves the number of elements.

As the algorithm evolves, vertex coordinates are recomputed using a modified Laplacian solver. At each iteration, the quad aspects ratios and relative angles about the moving vertex are used to decide the new vertex coordinates. Physical coordinates are computed from the underlying surface parametrization ensuring that mesh points remain on the surface. This technique has been tested over a wide range of complex geometries including surface cut-outs and surfaces with singular points. Although it is a heuristic approach, the algorithm consistently produces all valid meshes with isolated irregular vertices. The computational cost associated to this mesh processing step is less than a minute even for meshes consisting of $10 \mathrm{k}$ elements.

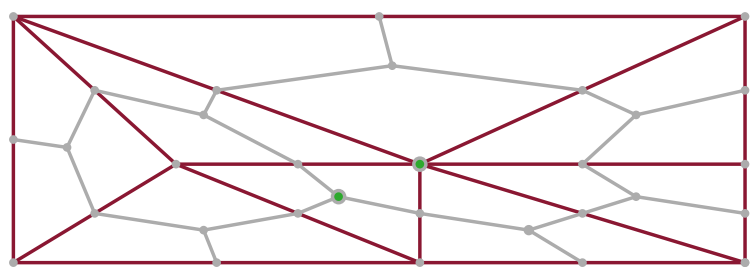

Figure 1: Catmull-Clark algorithm [1]: each triangle is split into three quads by inserting a new vertex at the centroid and creating three new sides (medians). The thicker lines (red) show the original triangles and the highlighted vertices indicate high/low valences.

\section{MESH REGULARIZATION}

Let us begin by briefly describing the Catmull - Clark [1] algorithm. Given a triangular tessellation, we split each triangle's side using the medians and insert a new vertex at all the triangle centroids. Then, as shown in Figure 1, three new quads are produced by linking the centroid location where the median intersects each side. The result is a fully quadded mesh but with many irregular vertices (valence $\neq 4$ ): the original vertices whose valence does not change, and the new vertices added at the centroids, which end up all having valence 
three. On the other hand, note that the new vertices that were inserted along triangle sides nicely end up with a valence of 4 ( 2 from the side split and 2 from the connection of the centroids of the triangle neighbors).

Hence, once the initial quadrangulation has been produced, we apply a regularization technique that recovers as many valence 4 vertices as possible. To motivate the discussion, in Figure 2 we have produced an idealized mesh showing the mesh manipulation pipeline. The first step (local operations) which is discussed in Section 3 consists of applying the basic element operations of swapping, collapsing and splitting (and combinations of the same such as swap-collapse or double splitting) systematically. As it will be discussed later, it is necessary to have at least 3 irregular vertices for an overall improvement. Hence, for pairs of irregular vertices surrounded by regular quads, a type of vertex translation is applied allowing quads from far regions to interact with each other. As [22] demonstrated, it is possible to identify the shortest path between two isolated irregular vertices and bring them together by inserting a number of elements proportional to their graph distance. For complex geometries, it is not always possible to generate space for inserting new vertices whilst preserving mesh validity so the vertex translation is restricted to vertex pairs with valences 3,5 and in a particular configuration. This is discussed at the end of this section.

Once there are no more possible operations (or the mesh is fully regular), we apply a Laplacian based iterative scheme for recomputing the vertex locations (in the parameter space of the surface) and produce the final mesh.

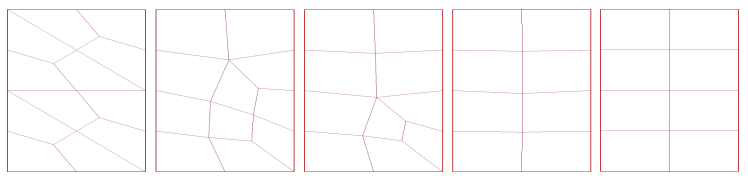

Figure 2: A simple quadded mesh obtained by triangle splitting and the evolution of the regularization algorithm including a final vertex coordinates computation.

We will start by detailing the basic operations followed by examples of compositions that allow for recovering regular vertices within a neighborhood (not all of them necessarily connected). We will use the following notation: $v_{i}$ are quad vertices, $\operatorname{val}\left(v_{i}\right)$ their valences and the pair $(a, b)$ means vertices with valences $a$ and $b$ respectively. Similarly, the group $\{a, b, c, d\}$ denotes the valences of an ordered quad.

Swapping: this process allows for exchanging high and low valence pairs by changing the vertex pair forming the common side of any two adjacent quads. If $\left(v_{1}, v_{2}\right)$ was the common side to both quads which has now become $\left(v_{3}, v_{4}\right)$, then:

$$
\begin{aligned}
& \operatorname{val}\left(v_{i}\right)=\operatorname{val}\left(v_{i}\right)-1, i=1,2, \\
& \operatorname{val}\left(v_{j}\right)=\operatorname{val}\left(v_{j}\right)+1, j=3,4 .
\end{aligned}
$$

Hence, as shown in Figure 3, the perfect swap occurs for a $(5,5)$ and $(3,3)$ pair, producing two regular quads. In practice, we don't require them to be perfect since, for example, swapping a $(5,5)$ pair with a $(3,4)$ gives $(4,4)$ and $(4,5)$ pairs, thus improving the overall mesh regularity.

Collapsing: eliminating a quad by merging two of its opposite vertices (see Figure 3). Given an ordered quad with vertices $\left\{v_{1}, v_{2}, v_{3}, v_{4}\right\}$, collapsing $v_{1}$ with $v_{3}$ results in:

$$
\begin{aligned}
\operatorname{val}\left(v_{2}\right) & =\operatorname{val}\left(v_{2}\right)-1, \quad \operatorname{val}\left(v_{4}\right)=\operatorname{val}\left(v_{4}\right)-1 \\
\operatorname{val}\left(v_{13}\right) & =\operatorname{val}\left(v_{1}\right)+\operatorname{val}\left(v_{3}\right)-2
\end{aligned}
$$

Therefore, the ideal collapse occurs for a $\{3,5,3,5\}$ quad since $\operatorname{val}\left(v_{2}\right)=\operatorname{val}\left(v_{4}\right)=5-1$ and $\operatorname{val}\left(v_{13}\right)=$ $3+3-2=4$. Like for the previous case, this operation is suitable as long as there are three irregular vertices.

Splitting: inverse operation to collapsing and it is applied whenever there are high valence vertices linked to low valence vertices. Figure 3 shows a split which goes from three irregular vertices to one. In this case, the quad distance between the vertices dictate the final valence distribution. In Figure 4 we illustrate a split for a valence 6 vertex which can result perfect or not depending on the $(3,3)$ pair configuration.

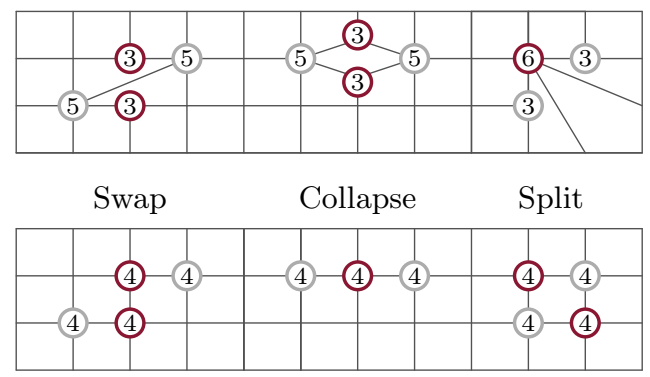

Figure 3: The three basic vertex operations showing how vertices gain / lose valences and become regular $(=4)$.

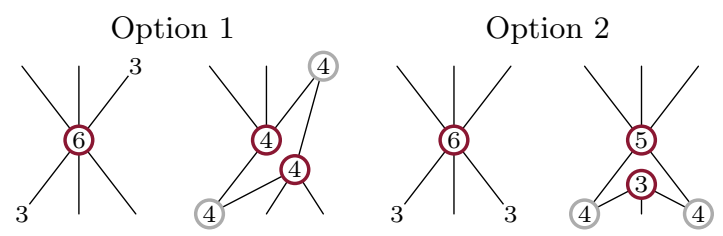

Figure 4: Splits depending on quad distances. Option 1 is perfect since vertices are 3 quads away but Option 2 produced a $(3,5)$ pair because they are $2(4)$ quads away. 
Using these operations alone leaves many irregular vertices behind. This is due to the fact that the remaining irregular vertices are either too far away or in a configuration for which none of the basic operations are suitable. However, by combining several operations as well as translating vertices, it is possible to improve the mesh quality even further, finally resulting in few or no irregular vertices. To illustrate this Figure 5 shows two stages of the regularization process: without vertex translation $(b)$ and using vertex translations together with compositions $(c)$. Notice that the final mesh has fewer elements but this is acceptable since we started with $3 \times$ the number of triangles.

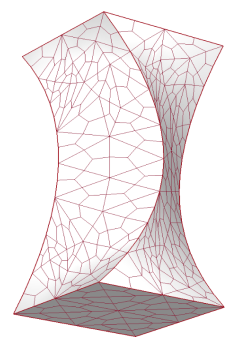

(a)

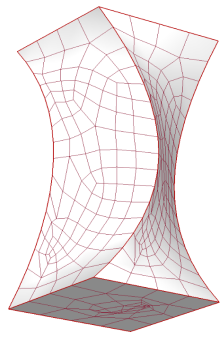

(b)

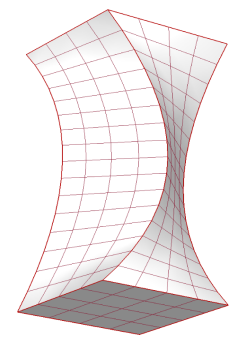

(c)
Figure 5: A twisted surface at the initial mesh $(a)$, without transfering the vertex valences $(b)$ and after applying full regularization with vertex translation $(c)$.

Composition: we have seen that three or more irregular vertices need to exchange valences effectively in order to improve the mesh overall. However, the basic operations require that all the irregular vertices have to be contained within a quad and its adjacent neighbors, and in a precise configuration; suitable collapses need a vertex distribution where opposite vertices have low-low and high-high valences respectively. Swaps on the other hand, need the vertex pairs to be three vertex counts apart (clockwise or counter-clockwise). In Figure 6 we illustrate a vertex star centered at $v_{0}$ with all the quads and vertices that are stored within this datatype. Star groups are employed to detect three or more irregular vertices around a particular vertex $(S)$. For example, the pair $\left(S, v_{2}\right)$ cannot see vertex $v_{6}$ from quad $q_{1}$ but star $S$ sees the three irregular vertices. This information is used to perform the operations shown in Figures 7, 8, 9 ,10, 11.

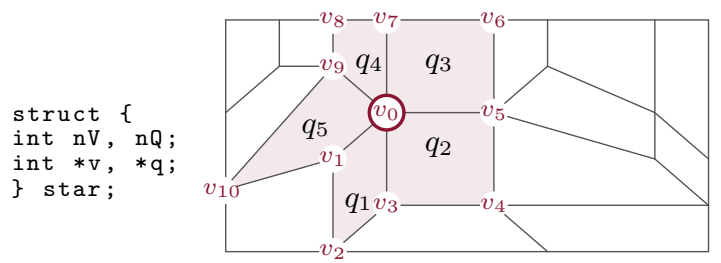

Figure 6: Vertex star $S\left(v_{0}\right)$ highlighting its surrounding quads and vertices stored clockwise in $* \mathrm{v}, * \mathrm{q}$.
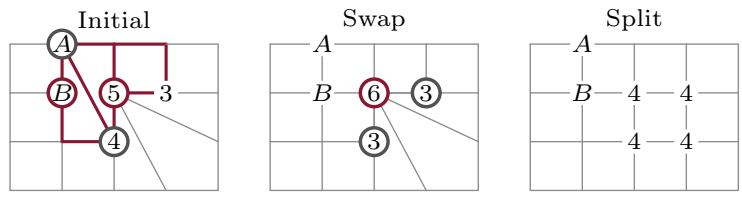

Figure 7: Swap-split process using three quads. Swapping increases the number of irregular vertices but produces an ideal scenario for a split. This operation is useful when $A=5$ and $B \leq 4$ or when $A \geq 4$ and $B=3$.
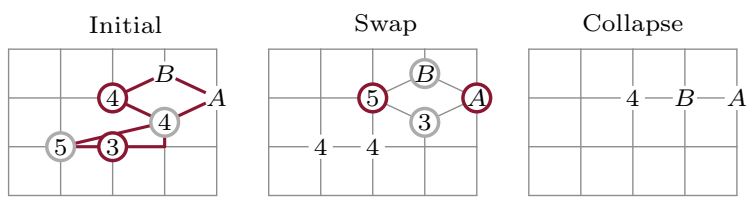

Figure 8: Swap-collapse using two irregular quads. We require that either $B=3$ and $A \geq 4$ or $A \geq 5$ and $B \leq 4$. Then, after swapping, the top quad is almost fully irregular and can be collapsed.
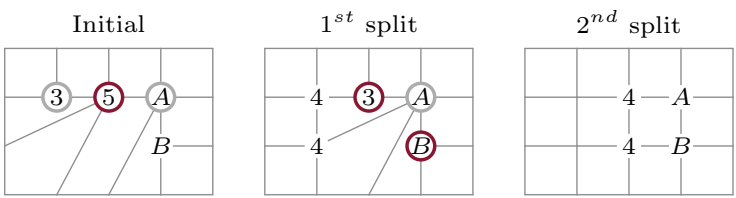

Figure 9: Double split: first split $(5)$ using $(3, A)$ as links. Then, perform a second split in $A$ through $(3, B)$ which are three quads distance. For this operation we need $A=5$ and $B \leq 4$ or $A=4$ and $B=3$.
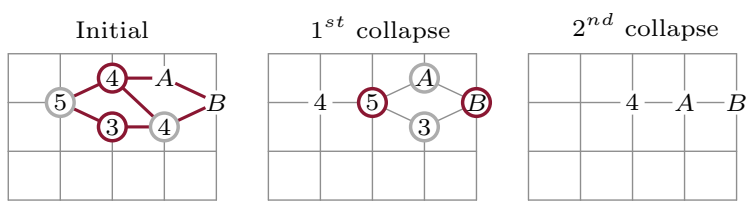

Figure 10: A double collapse: collapse the left quad $\{5,3,4,4\}$. Now the adjacent quad has $\{5,3, B, A\}$ valences and a second collapse is performed. We require that $A=3$ and $B \geq 4$ or $B \geq 5$ and $A \leq 4$.

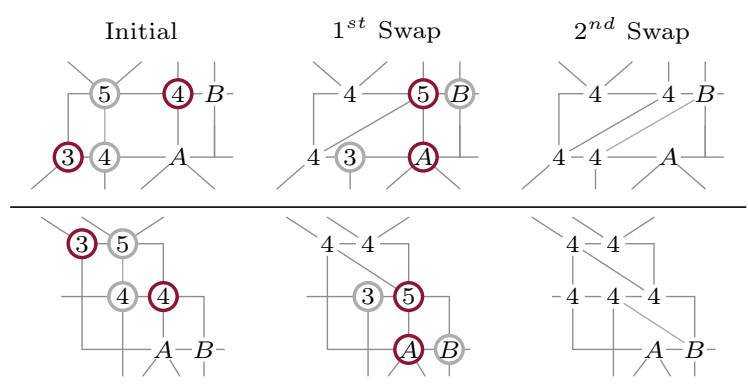

Figure 11: Double swaps. Adjacent quads (top) need $A=5$ and $B \leq 4$ or $B=3$ and $A \geq 4$. Diagonal swaps (bottom) need $A \geq 5$ and $B \leq 4$ or $B=3$ and $A \geq 4$. 
Translation: the swapping operation can be used to move irregular vertices along the mesh without varying the total number of elements (as opposed to collapse and split that remove/ add quads). For example, a $(4,5)$ pair swapped with a $(3,4)$ pair results in $(3,4)$ and $(4,5)$ valences. The total number of irregular vertices has not changed but their position has. In Figure 12 we show how we can take advantage of this operation to bring irregular vertices that were not in close proximity towards each other resulting in a regular region after two consecutive swaps. Vertex pairs of valences $(3,5)$ can be moved indefinitely along the mesh. If the vertices $(3,5)$ are linked, together they move forward (backwards). On the other hand, when they are opposite vertices (like in Figure 12) they move upwards (downwards). For pairs of vertices with valences $(3,3)$ or $(5,5)$, the swapping operation is more limited. It can be applied once to change their locations as shown in Figure 13. Unlike the $(3,5)$ pairs, a second swap of $(3,3)$ or $(5,5)$ pairs would return to the original configuration.
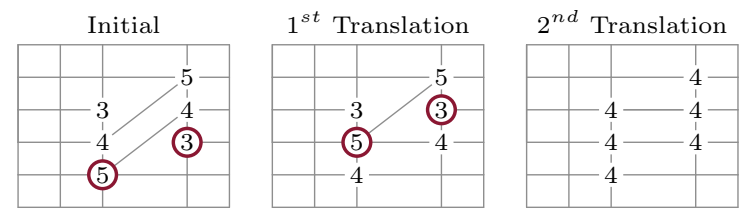

Figure 12: Translation of an irregular vertex pair $(3,5)$ upwards along the mesh. After two movements, a suitable swap was found producing a regular region.
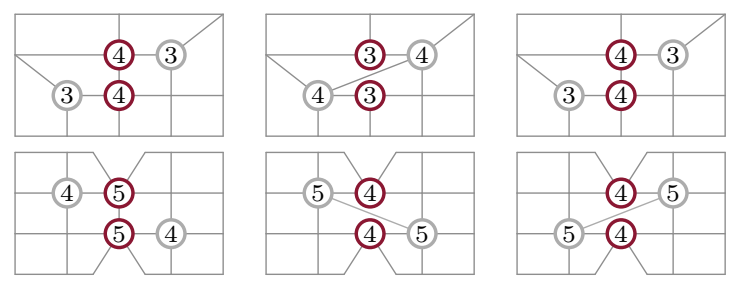

Figure 13: Possible swaps during vertex translation for vertex pairs with valences $(3,3)$ and $(5,5)$. The $(3,3)$ pair has only one option whereas the other one has two.

\section{MESH QUALITY}

Changing the mesh topology can lead to vertex configurations with unacceptable element shapes: too flat or sharp and even self-intersecting. Hence, vertex coordinates are recomputed during regularization ensuring that the entire affected local region remains valid. The new vertex locations are computed using a modification of the Laplacian operator which is detailed in Algorithm 2 and will be discussed later. Note that this procedure is applied after each topological operation.
Figure 14 illustrates a valid vertex split; in the first image we show the three vertices involved in this operation. The middle image shows the resulting split where the highlighted vertices will be (possibly) moved when considering the step valid. The local region in play are the vertices involved in the split directly together with all their neighbors. Notice that surface boundary vertices are not moving since we require the tessellation to be watertight. The quads affected by a movement of any of these vertices ares the ones that will be checked and are highlighted as well. By restricting the number of moving vertices to this group, the operation remains local ensuring that we don't create invalid quads that will be overlooked. The rightmost image shows the final vertex distribution after moving all the necessary vertices. In the event that invalid elements remain, the topological operation is rejected and the mesh is restored from its previous valid configuration. In the following, we discuss how to detect invalid elements along the surface.

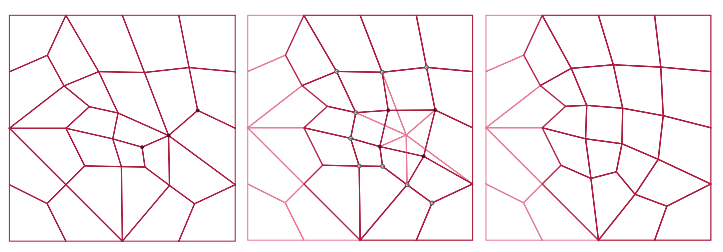

Figure 14: A vertex split highlighting the moving points and the quads that are checked when testing that the mesh remains valid.

\subsection{Detecting Invalid Elements}

Here we discuss how we ensure mesh validity throughout the regularization process. At the very least, a suitable mesh should be made of quads whose internal angles are less than $180^{\circ}$ [29]. Preserving this property ensures that there are no folds (self-intersecting quads). The idea is the following: use the underlying parametrization to obtain the surface normal an use it to generate the tangent plane at the centroid. Then, as shown in Figure 15, project all the quad coordinates onto that plane and calculate the vertex orientations relative to each other.

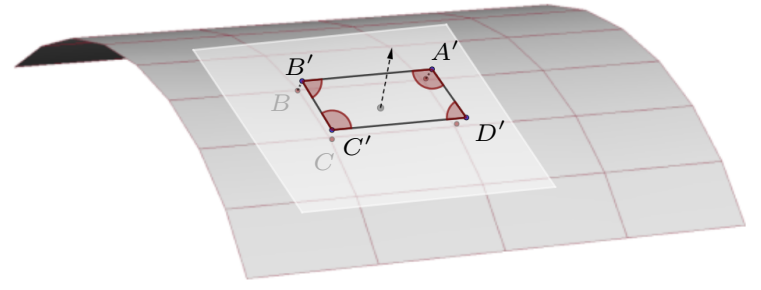

Figure 15: A cylindrical surface showing the tangent plane centered a particular quad and all its vertices projected onto such plane. 
By construction, vertices forming a quad are always stored clock-wise (positive) so we can detect obtuse or self-intersecting elements by looking at the orientations relative to every vertex. For every three consecutive vertices, the orientations are computed as follows:

$$
\operatorname{ori}(A B C)=\operatorname{sign}(<\overrightarrow{A B} \times \overrightarrow{A C}, \vec{n}>) .
$$

Here $<,>$ is the dot product and $\vec{n}$ the normal vector to the surface at the quad center (see Figure 15). All positive orientations indicate a valid quad. If there are at least two pairs with positive orientations, the quad is obtuse and finally when there are no vertices with positive orientation, then the quad is self-intersecting.

In Figure 16 we show an obtuse quad together with each vertex orientations. Notice that at $A$ and $C$ the orientations change sign $(+,-)(-,+)$ whereas at $B$ and $C$ we obtain $(+,+)$. For quads that have at least one boundary vertex, we also check that none of its sides have crossed the domain bounds. This happens frequently when performing operations on surfaces with internal holes that have sharp corners.

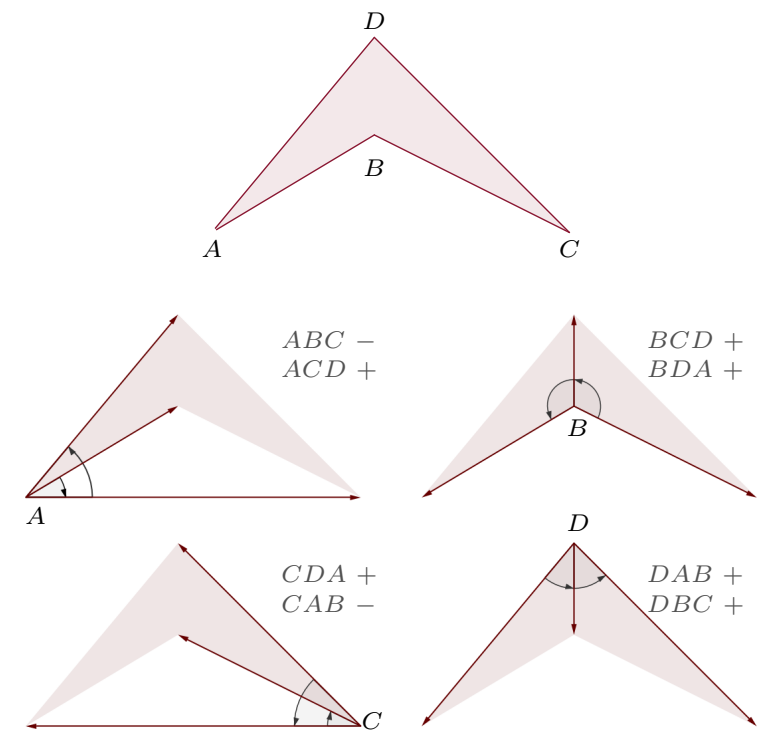

Figure 16: An obtuse quad showing each vertex orientations with respect the other vertices. Quads are valid only when the orientations are $(+,+)$ at every vertex.

\subsection{Laplacian using Angles and Ratios}

The new coordinates of a particular vertex are chosen depending on the quality of its surrounding quads which are categorized as shown in Figure 17. In addition, the vertex relative position to the mesh is also taken into account; vertices linked to boundary vertices (especially surface holes) have more restricted movement than those that are internal.

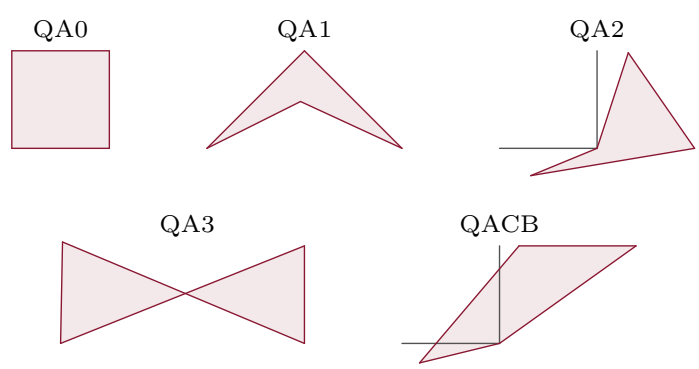

Figure 17: Possible quad configurations in descending order of validity: convex (QA0), concave (QA1), concave at an internal boundary (QA2), self-intersecting (QA3) and crossing the domain boundary (QACB).

For each vertex, we use its star to compute all the angles as well as the quad triangle ratios [30] which are illustrated in Figure 18. The vertex angles are computed at the normal plane using the same methodology described in Section 4.1 (see Figure 15) but in this case we use the surface normal at the central vertex to generate the plane to which all the other quads are projected. Irregular vertices (i.e. valence $\neq 4$ ) don't have an obvious orientation and the internal angles are computed just to detect detect flat or sharp elements. For regular vertices, we compute the angle distance between opposite vertices.
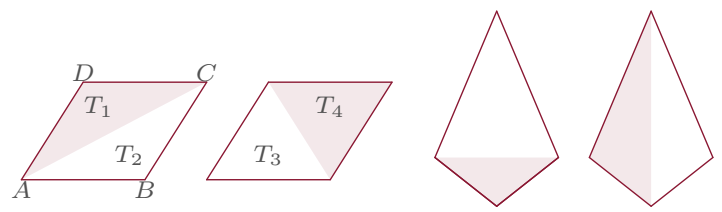

Figure 18: Two set of quads showing for each quad its two triangle ratios $\left(\left\{T_{1}, T_{2}\right\},\left\{T_{3}, T_{4}\right\}\right)$. Quads should have both ratios close (equal) to one like the left pair.

The triangle ratios (see Figure 18) are computed as follows. Let $Q_{A B C D}$ be an ordered quad, then:

$$
\left.\begin{array}{l}
T_{1}=\|A B \times A C\| \\
T_{2}=\|A C \times A D\|
\end{array}\right\} D_{12}(Q)=\min \left(\frac{T_{1}}{T_{2}}, \frac{T_{2}}{T_{1}}\right) .
$$

Similarly, defining $T_{3}=\|B C \times B D\|$ and $T_{4}=\| B D \times$ $B A \|$ gives $D_{34}(Q)$. The final quad ratio is given by

$$
R(Q)=\min \left(D_{12}, D_{34}\right)
$$

The vertex contribution of an obtuse quad is measured in the following way. Identifying $A$ with the vertex, if $T_{1}+T_{2}>T_{3}+T_{4}$ then vertex $A$ can improve / worsen the large angle and so we take $R_{A}(Q)=-D_{A}(Q)$. Otherwise $\left(T_{1}+T_{2}<T_{3}+T_{4}\right)$, we use the following:

$$
\begin{array}{ll}
R_{A}(Q)=-T_{4} / T_{3} & \text { if } \angle D A B>180^{\circ} \\
R_{A}(Q)=D_{12}(Q) & \text { otherwise. }
\end{array}
$$


In Algorithm 1 we provide details on how the vertex area function is implemented.

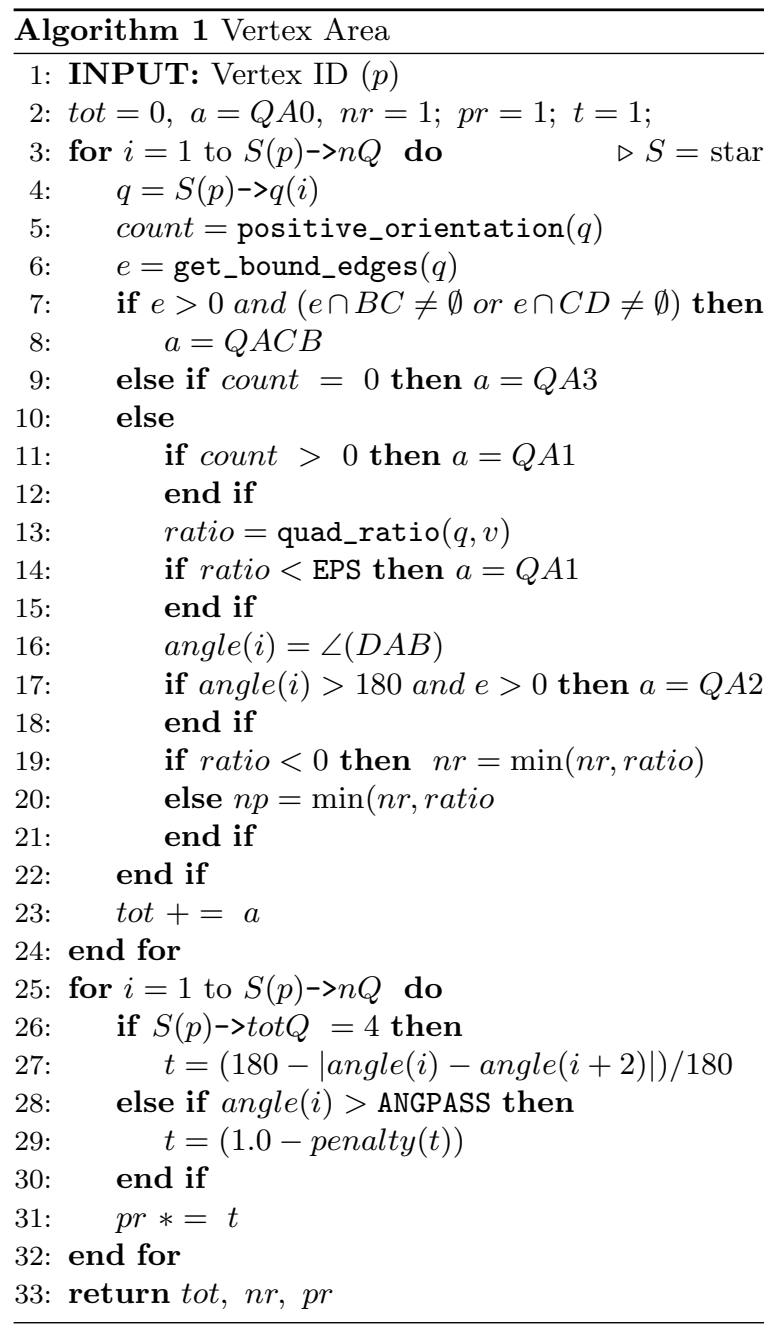

The actual vertex coordinates are computed using the centroid (Laplacian), midpoints between two opposite links and a biased Laplacian where a particular quad (two vertices) is excluded. This is useful for unfolding elements or reducing large angle sizes arising as a result of a swap, split, etc. Notice that the average surface parametric coordinates $u v$ does not necessarily result in the average physical coordinates. The centroid or mid points are computed in the following way. First evaluate the physical average (which may not live on the surface). Then, using an inverse evaluate function from the geometry kernel, obtain the closest surface point with its corresponding $u v$ pair.

Vertices linked to internal boundaries (surface holes) are treated differently. This is done in order to avoid the Laplacian element shrinking effect; a surface cutout that is convex (e.g., a circle) or a sharp corner is generally incompatible with an averaging method since such point may be driven towards the hole or create very large angles. Therefore, boundary vertices forming sharp angles are flagged and the Laplacian option is deactivated for any internal vertex linked to such vertices. This technique is described in Algorithm 2. Recall that $S(p)$ denotes the star at vertex $p$ and has the structure defined in Figure 6.

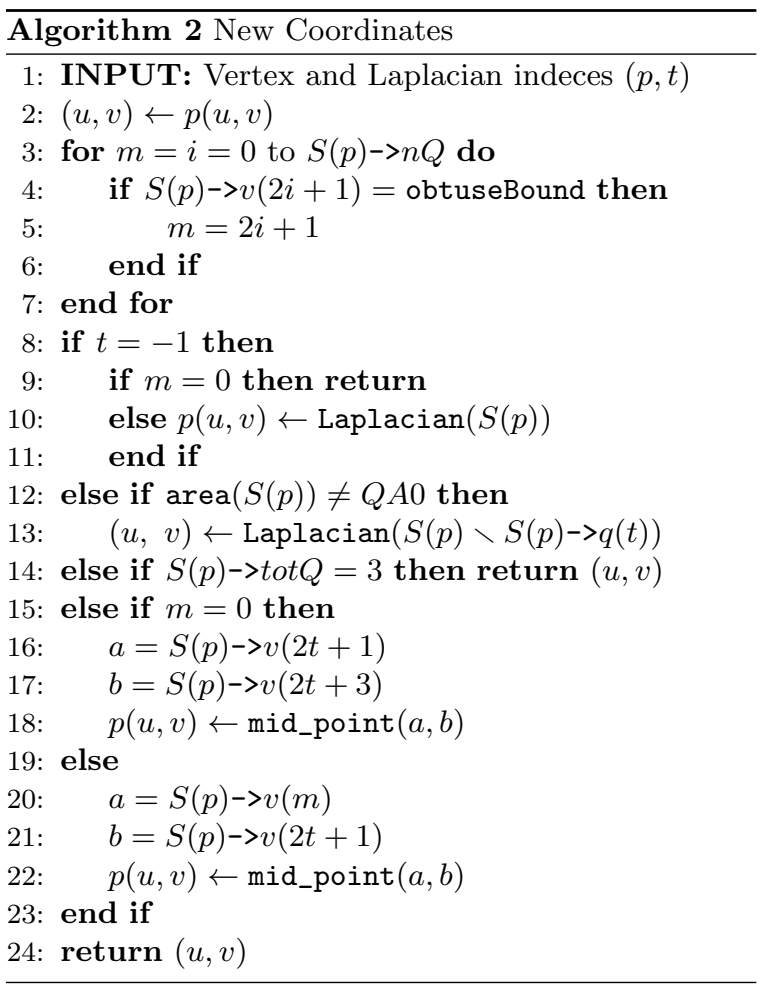

Finally, in Algorithm 3 we show how the actual vertex coordinates are chosen. This function is called iteratively at the highest level; during regularization, only few iterations are allowed since a vertex may undergo several manipulations and even get deleted. Once the regularization process is complete we invoke this function for 50 iterations to improve the final mesh.

The idea is the following: for each vertex, we compute all the possible vertex coordinates (Algorithm 2) and recompute the areas, triangle ratios and angles. Then we assess if the new coordinates have improved the ratios and angle differences and if so, the coordinates are stored as best candidate. Since unless the mesh is fully regular, it is impossible to obtain equal angles and ratios. Hence, we accept Laplacian coordinates whenever these values are above a certain tolerance which we have set to 0.25 . In Figure 19 we show two examples of surfaces before and after regularization using Algorithm 3 for recomputing coordinates. Surfaces with sharp internal corners like the star cut-out can produce large angles and even lead to element folding. In addition, the average coordinates may even pro- 
duce quads crossing the domain boundaries. Notice that the resulting mesh has quads with angles close to $90^{\circ}$ at each star vertex. Further, the sphere hole in the second image produces a circular edge where all the boundary vertices have angles $>180^{\circ}$ with respect to each other. When taking averages, this can lead to element shrinking and eventually move points outside the surface, i.e, towards the hole. Notice that the algorithm overcomes the element shrinking effect and produces a satisfactory result.

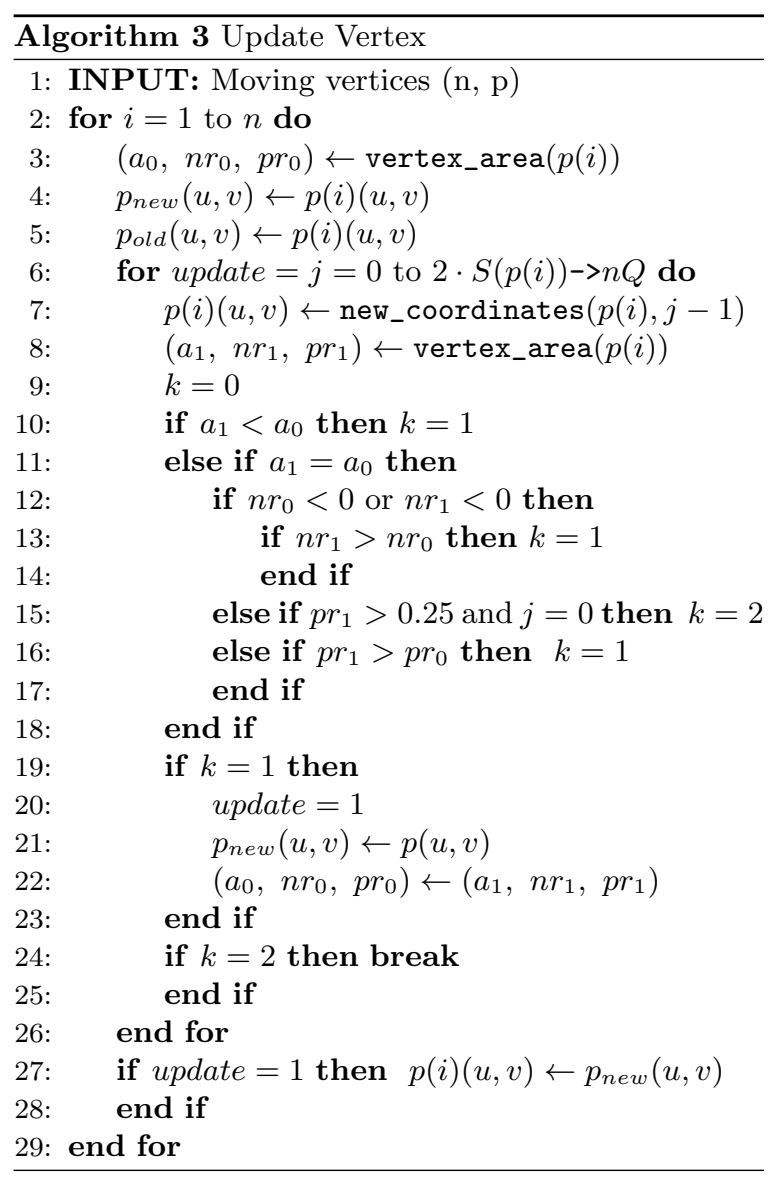

\section{VALIDATION}

The target for these quadrilateral meshes is structural analysis (specifically Built-up Element Models), and these results should not be viewed through a CFD lens. In a real sense, the task at hand is more difficult; it is harder to produce valid meshes for curved geometry when its applications require the element size to be coarser. We begin by looking at the algorithm performance over basic surfaces including surfaces with cut-outs. Figure 20 shows a cylinder and a composition of spheres. For the cylinder case, the final mesh that resulted was fully regular. We readily admit that such surface can be tessellated directly into fully regu-

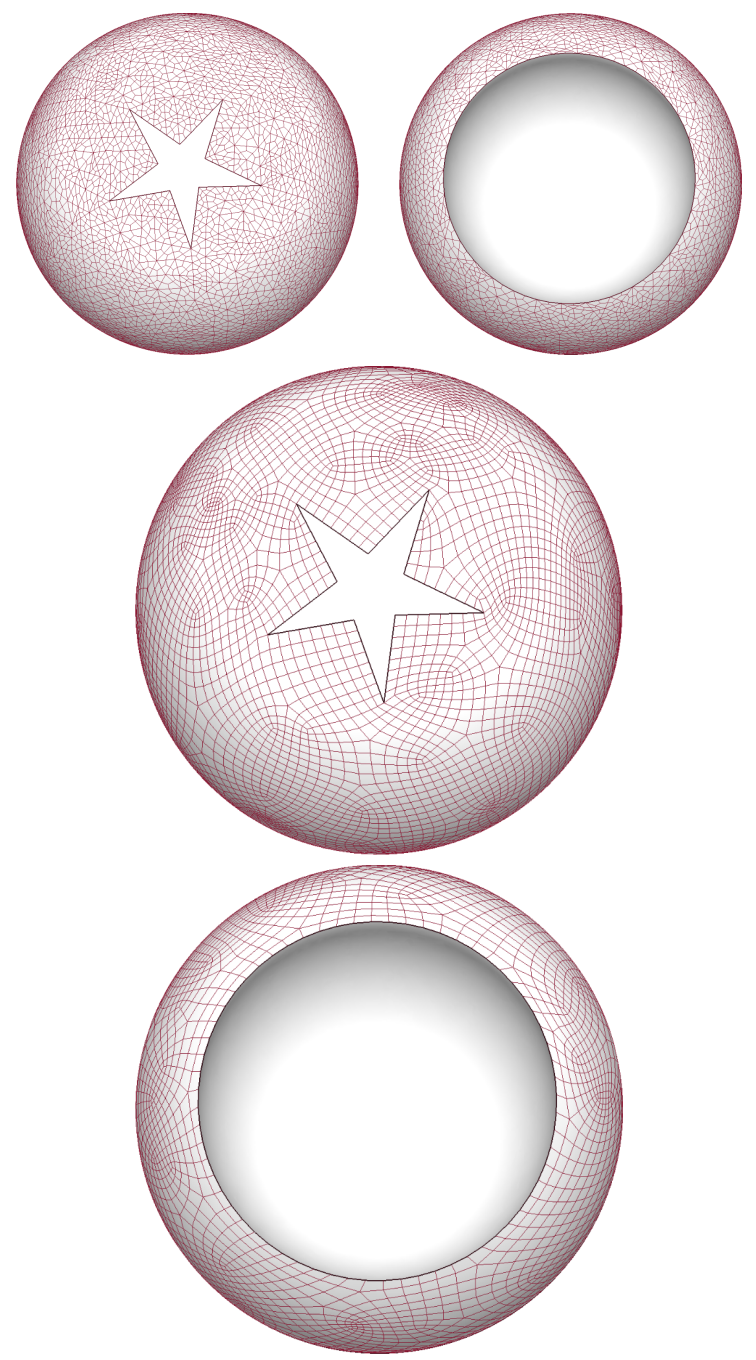

Figure 19: A sphere with two type of cut-outs, a star and a second sphere. Notice that all the angles at the star vertices are close to $90^{\circ}$ and that all the quads around the sphere hole have not collapsed towards the boundary. The top figures show the initial meshes.

lar quads and in this case, the triangulation was forced just for validation purposes. The spherical body is included to highlight the strength of the regularization technique: for example, the concave face contains a boundary vertex which has valence eighteen.

In Figure 21 we study several surfaces. The first two consist of a flat and a spherical surfaces with several cut-outs. The third one was produced by revolving a spline curve where the pole (top vertex) is singular. The coloring is based on the quad's largest angle. We haven't colored the initial mesh since we already know that these meshes are unsuitable. Notice that in all three cases, only a few angles are over $150^{\circ}$. 

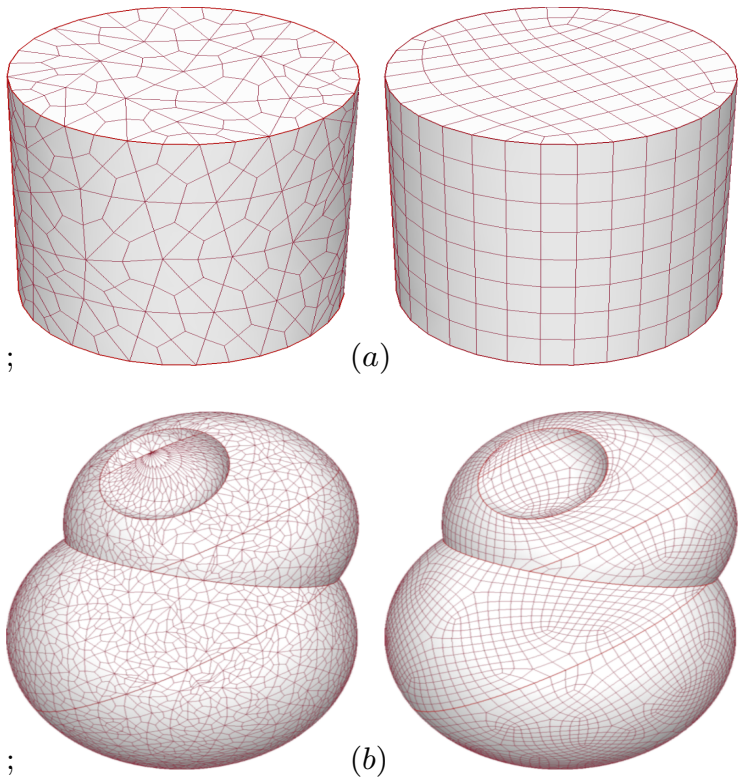

Figure 20: A cylinder and spherical body showing the meshes before and after regularization.
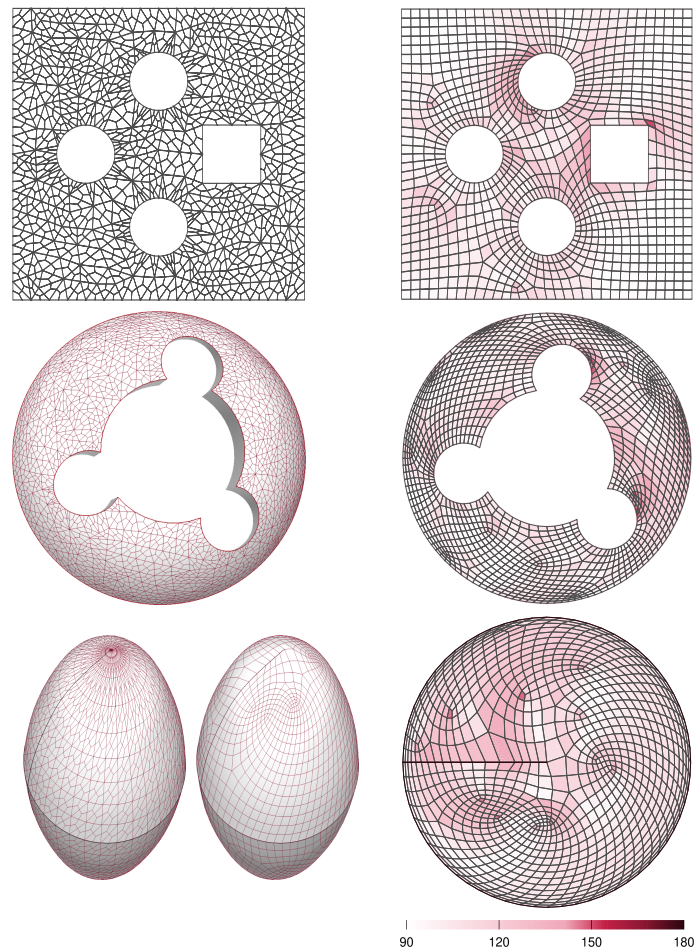

Figure 21: Three surfaces before and after regularization colored by the quads largest angles. The top (flat) and middle (spherical) present several cut-outs. The third is a surface of revolution which has a singularity at the pole. The highlighted lines denote the surface edges (fixed vertices) and the color map corresponds to the top face.
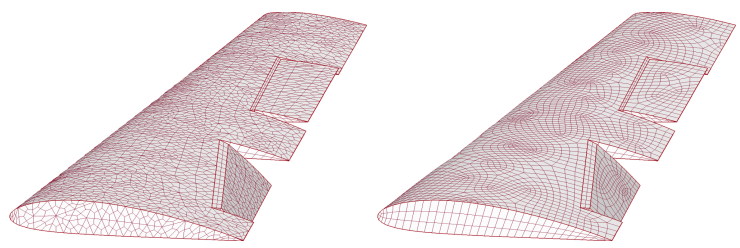

(a)

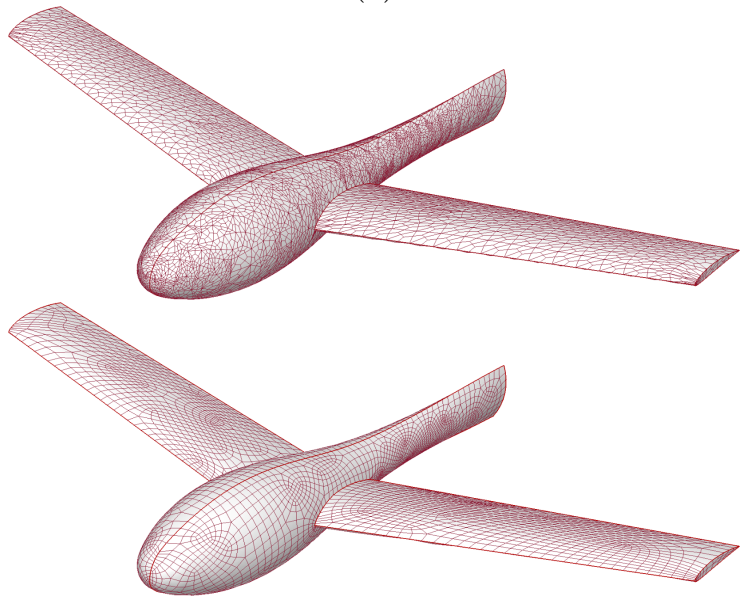

(b)

Figure 22: A wing body with two flaps $(a)$ and a full aircraft $(b)$ before and after regularization.

The wing and aircraft body shown in Figure 22 study the performance over more curved surfaces. The wing flaps are introduced to illustrate how the scheme works over trimmed surfaces. In both cases, the scheme is able to recover a large number of regular vertices producing good quality meshes. Notice that the mesh quality of the aircraft is especially high at the fuselage and at the nose where there is high curvature.

\subsection{A note on the Triangulation}

The performance of our technique clearly depends on the original tessellation. In particular, since boundary edges are fixed, the final mesh quality is strongly dictated by the side spacing at the bounds. The initial triangulations were generated by the tessellator in in EGADS [31].

It produces watertight triangulations of BReps by first discretizing the BRep Edges and then performing the trimmed surface (BRep Face) triangulations. There is no notion of grading of spacings because the tessellator is driven by being able to best represent the geometry with the fewest number of vertices/triangles. The technique used simply bifurcates regions that don't meet the user input criteria, which can obviously display abrupt spacing changes of a factor of 2 or more. In addition, large interior angle deviations between neighboring triangles as well as large side spacing are 
allowed (especially when using coarse tessellation parameters), thus producing triangulations that are far from equilateral.

In Figure 23 we show two wing profiles using the EGADS tessellator directly and applying the quadrilateral templating scheme described in [32]. Both tessellations used the same length criteria producing the same discretization at the surface bounds (BRep Edges). Observe that without templating (left), the initial tessellation has an excessive number of quads with highly irregular vertices at the leading edge. The result is that most of those quads are collapsed during regularization. The right images on the other hand, have a more realistic starting point and require much less mesh manipulation.

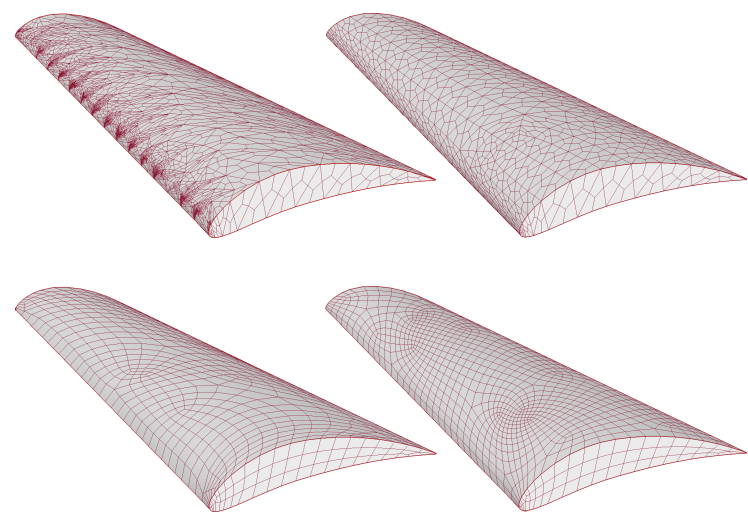

(a)

(b)

Figure 23: A comparison of two tessellations with $(a)$ and without $(b)$ the quadding template [32] and the resulting meshes after regularization (bottom).

\subsection{CPU Time and Regularity}

Finally, we focus on the actual performance both in terms of the number of recovered regular vertices as well as the computational costs. In Table 1 we show the results for some of the surface bodies used in the validation Section. The results are very consistent: the initial meshes start with $\sim 49 \%$ irregular vertices and after regularization, there are only $<4 \%$ left. Also it is important to note that the number of irregular vertices relative to the mesh doesn't grow as the mesh becomes finer nor is the performance affected as the surface geometry becomes more complex. In Table 2 we have gathered several surfaces and show results for the minimum quad triangle ratios [30] as well as minimum and maximum angles. It should be noted that angles are computed at the surface tangent plane and this may not always be accurate. In high curvature regions such as the wing leading edge, the projected coordinates can increase (reduce) the angle magnitude.

\begin{tabular}{l|cc|cccc} 
& \multicolumn{3}{c}{ Total Vertices } & \multicolumn{4}{c}{ Irregular Vertices } \\
\hline Figure & Initial & Final & Initial & $(\%)$ & Final & $(\%)$ \\
\hline $202^{\text {nd }}$ & 5799 & 5401 & 2843 & 49.0 & 188 & 3.5 \\
$22(a)$ & 4174 & 1029 & 1994 & 47.8 & 38 & 3.7 \\
$22(b)$ & 1876 & 1869 & 926 & 49.4 & 64 & 3.7 \\
$23(a)$ & 3259 & 3035 & 1631 & 50.0 & 108 & 3.6 \\
$23(b)$ & 10347 & 6169 & 5105 & 49.3 & 226 & 3.7 \\
\hline
\end{tabular}

Table 1: Results in terms of number of irregular vertices for several bodies considered in this manuscript.

The computational times are shown in Table 3 . It should be noted that the implementation operates on a BRep Face at a time and has been threaded providing scalability based on the number of cores available (and number of Faces to process). The results are very promising: even for the finer meshes the total simulation time remains in less than a minute.

\begin{tabular}{lccc} 
Figure & Min Ratio & Min Angle & Max Angle \\
\hline 21 top & 0.32 & 38 & 151 \\
21 middle & 0.29 & 35 & 160 \\
21 bottom & 0.36 & 41 & 147 \\
22-(a) top face & 0.31 & 18 & 162 \\
22-(b) fuselage & 0.18 & 34 & 150 \\
$23-(a)$ & 0.32 & 21 & 158 \\
\hline
\end{tabular}

Table 2: Global minimum ratios and minimum and maximum angles for several bodies shown in this paper.

\section{CONCLUSIONS AND ONGOING WORK}

We have presented an automatic mesh generation technique that produces almost regular quadrilateral meshes. Starting with a triangulation and using the splitting approach from [1], we produce a fully quadded mesh which then undergoes topological changes in order to recover regularity. Our results show that in general, it is possible to reduce the number of irregular vertices from around $49 \%$ to $<4 \%$.

The regularization process is coupled with a Laplacian based iterative scheme for computing the move-

\begin{tabular}{c|cc} 
Figure & Faces & Time (secs) \\
\hline $202^{\text {nd }}$ & 6 & 5 \\
$22(a)$ & 4 & 1 \\
$22(b)$ & 4 & 12 \\
$23(a)$ & 10 & 11 \\
$23(b)$ & 8 & 46
\end{tabular}

Table 3: Simulation times using a Intel(R) Xeon(R) CPU E5-2630 v3 @ $2.40 \mathrm{GHz}$ machine for the examples in Table 1 showing also the number of faces composing each body. 
ment of vertex coordinates. The resulting mesh is always valid but there is no notion of optimal sizing. Rather, we just attempt to reduce the angle deviation as well as produce quads with aspect ratio close to one and updating the new coordinates based on the "best candidate". This results in low scalar computational times (less than one minute) even for meshes starting with around $10 k$ quads. However, we understand the tradeoff in this approach and for specific applications, a quad distribution based on a specific metric should be introduced.

The initial triangulations were produced using the EGADS internal tessellator. This tool was designed for visualization purposes and therefore can produce poor quality meshes for simulations (see Figure $23(a)$ ) with excessive number of elements as a starting point. Since our technique is independent of the tesselator, it could use as input other triangulations designed specifically for applications in numerical simulations.

Finally, since the computational cost associated to our regularization scheme is rather low, our results suggest that the initial input mesh should be relatively coarse. In this case, the resulting mesh would consist on few irregular vertices. If a smaller sizing is desired, mesh refining can be applied directly by subdividing quads without introducing new irregular vertices.

\section{ACKNOWLEDGEMENTS}

This work was funded by the CAPS project, AFRL Contract FA8050-14-C-2472: "CAPS: Computational Aircraft Prototype Syntheses"; Dean Bryson is the Technical Monitor. We would also like to thank the anonymous reviewers for their feedback and useful suggestions.

\section{References}

[1] Catmull E., Clark J. "Recursively generated Bspline surfaces on arbitrary topological meshes." Computer-aided design, vol. 10, no. 6, 350-355, 1978

[2] Haimes R., Dannenhoffer J. "The Engineering Sketch Pad: A Solid-Modeling, Feature-Based, Web-Enabled System for Building Parametric Geometry." 21st AIAA Computational Fluid Dynamics Conference. 2003

[3] Talbert J.A., Parkinson A.R. "Development of an automatic, two-dimensional finite element mesh generator using quadrilateral elements and Bezier curve boundary definition." International Journal for Numerical Methods in Engineering, vol. 29, no. 7, 1551-1567, 1990
[4] Blacker T.D., Stephenson M.B. "Paving: A new approach to automated quadrilateral mesh generation." International Journal for Numerical Methods in Engineering, vol. 32, no. 4, 811-847, 1991

[5] White D.R., Kinney P. "Redesign of the paving algorithm: Robustness enhancements through element by element meshing." 6th International Meshing Roundtable, pp. 323-335. 1997

[6] Tam T., Armstrong C. "2D finite element mesh generation by medial axis subdivision." $A d$ vances in Engineering Software and Workstations, vol. 13, no. 5, $313-324,1991$

[7] Dong S., Bremer P.T., Garland M., Pascucci V., Hart J.C. "Spectral surface quadrangulation." Transactions on Graphics (TOG), vol. 25, no. 3, 1057-1066, 2006

[8] Tarini M., Puppo E., Panozzo D., Pietroni N., Cignoni P. "Simple quad domains for field aligned mesh parametrization." Transactions on Graphics (TOG), vol. 30, 142, 2011

[9] Kowalski N., Ledoux F., Frey P. "A PDE Based Approach to Multidomain Partitioning and Quadrilateral Meshing." X. Jiao, J.C. Weill, editors, 21st International Meshing Roundtable, pp. 137-154. 2013

[10] Bommes D., Campen M., Ebke H.C., Alliez P., Kobbelt L. "Integer-grid maps for reliable quad meshing." Transactions on Graphics (TOG), vol. 32 , no. $4,98,2013$

[11] Fogg H.J., Armstrong C.G., Robinson T.T. "Automatic generation of multiblock decompositions of surfaces." International Journal for Numerical Methods in Engineering, vol. 101, no. 13, 965-991, 2015

[12] Lo S., Lee C. "On using meshes of mixed element types in adaptive finite element analysis." Finite Elements in Analysis and Design, vol. 11, no. 4, $307-336,1992$

[13] Lee C.K., Lo S. "A new scheme for the generation of a graded quadrilateral mesh." Computers $\&$ Structures, vol. 52, no. 5, 847-857, 1994

[14] Borouchaki H., Frey P.J. "Adaptive triangularquadrilateral mesh generation." International Journal for Numerical Methods in Engineering, vol. 41, no. 5, 915-934, 1998

[15] Remacle J.F., Lambrechts J., Seny B., Marchandise E., Johnen A., Geuzainet C. "Blossom-Quad: A non-uniform quadrilateral mesh generator using a minimum-cost perfect-matching algorithm." 
International Journal for Numerical Methods in Engineering, vol. 89, no. 9, 1102-1119, 2012

[16] Owen S.J., Staten M.L., Canann S.A., Saigal S. "Q-Morph: an indirect approach to advancing front quad meshing." International Journal for Numerical Methods in Engineering, vol. 44, no. 9, 1317-1340, 1999

[17] Daniels II J., Silva C.T., Cohen E. "Localized quadrilateral coarsening." Proceedings of the Symposium on Geometry Processing, pp. 14371444. Eurographics Association, 2009

[18] Staten M.L., Benzley S., Scott M. "A methodology for quadrilateral finite element mesh coarsening." Engineering with Computers, vol. 24, no. 3, 241-251, 2008

[19] Daniels J., Silva C.T., Shepherd J., Cohen E. "Quadrilateral Mesh Simplification." Transactions on Graphics (TOG), vol. 27, no. 5, 148, 2008

[20] Kinney P. "Cleanup: Improving quadrilateral finite element meshes." 6th International Meshing Roundtable, pp. 437-447. 1997

[21] Tarini M., Pietroni N., Cignoni P., Panozzo D., Puppo E. "Practical quad mesh simplification." Computer Graphics Forum, vol. 29, pp. 407-418. 2010

[22] Peng C.H., Zhang E., Kobayashi Y., Wonka P. "Connectivity editing for quadrilateral meshes." Transactions on Graphics (TOG), vol. 30, 141, 2011

[23] Bozzo A., Panozzo D., Puppo E., Pietroni N., Rocca L. "Adaptive Quad Mesh Simplification." Eurographics Italian Chapter Conference, pp. 95102. 2010

[24] Verma C.S., Suresh K. "A robust combinatorial approach to reduce singularities in quadrilateral meshes." Procedia Engineering, vol. 124, 252-264, 2015

[25] Zhou T., Shimada K. "An Angle-Based Approach to Two-Dimensional Mesh Smoothing." 9th International Meshing Roundtable, pp. 373384. 2000

[26] Freitag L.A. "On combining Laplacian and optimization-based mesh smoothing techniques." Tech. rep., Argonne National Lab., IL, 1997

[27] Knupp P.M. "Winslow smoothing on twodimensional unstructured meshes." Engineering with Computers, vol. 15, no. 3, 263-268, 1999
[28] Knupp P.M. "Algebraic mesh quality metrics." SIAM journal on scientific computing, vol. 23, no. 1, 193-218, 2001

[29] Robinson J. "Some new distortion measures for quadrilaterals." Finite Elements in Analysis and Design, vol. 3, no. 3, 183 - 197, 1987

[30] Lo S. "Generating quadrilateral elements on plane and over curved surfaces." Computers $\mathscr{E}$ structures, vol. 31, no. 3, 421-426, 1989

[31] Haimes R., Drela M. "On the construction of aircraft conceptual geometry for high-fidelity analysis and design." 50th AIAA Aerospace Sciences Meeting Including the New Horizons Forum and Aerospace Exposition, p. 683. 2012

[32] Haimes R., Aftosmis M.J. "Watertight Anisotropic Surface Meshing Using Quadrilateral Patches." 13th International Meshing Roundtable, pp. 311-322. 2004 


\title{
GUARANTEED QUALITY-DRIVEN HEXAHEDRAL OVERLAY GRID METHOD
}

\author{
Nicolas Le Goff ${ }^{1} \quad$ Franck Ledoux $^{1} \quad$ Jean-Christophe Janodet ${ }^{2} \quad$ Steven J. Owen ${ }^{3}$ \\ ${ }^{1}$ French Alternative Energies and Atomic Energy Commission (CEA), CEA, DAM, DIF, F-91297 \\ Arpajon, France. nicolas.le-goff@cea.fr, franck.ledoux@cea.fr \\ ${ }^{2}$ IBISC, Univ. Evry, Université Paris-Saclay, 91025 Evry, France. jeanchristophe.janodet @univ-evry.fr \\ ${ }^{3}$ Sandia National Laboratories (SNL), 1515 Eubank SE Albuquerque, NM, U.S.A. sjowen@sandia.gov
}

\begin{abstract}
Hexahedral mesh generation using overlay grid methods has the benefit of being fully automatic, requiring minimal user input. These methods follow a mesh-first approach where an initial mesh, usually a grid, is used to overlay the reference geometry. Procedures to modify the initial mesh are then employed to best capture the geometry to get a conformal all-hex mesh [1]. One of the main drawbacks of those methods is the resulting mesh quality. While the interior of the mesh remains the same as the initial mesh, cells located at the material interfaces can end up quite deformed or even inverted, making the mesh totally useless for most numerical simulation codes. Considering an input mesh carrying volume fractions of the materials, the main purpose of the presented work is to ensure a minimal cell quality. Our method draws upon the overlay grid pipeline described in [2] where several steps (cell assignment correction, interface reconstruction, mesh adaptation) are altered to control cell quality.
\end{abstract}

Keywords: mesh generation, overlay grid, quadrilateral, hexahedral, guaranteed quality

\section{INTRODUCTION}

The finite element method (FEM) and the finite volume method (FVM) require to discretize the physical domain of interest with a mesh. Depending on the application field and the numerical scheme that is used, mesh properties can be totally different and can go from unstructured tetrahedral meshes to boundaryaligned block structured hexahedral meshes. In this work, we focus on hexahedral meshes and more specifically on the generation of unstructured hexahedral meshes starting from grid-like meshes.

This process is of interest particularly in an industrial context where a full simulation process is built by loosely coupling simulation codes acting on different mesh representations. In our case of study, we consider the "Euler to Lagrange" situation where (1) a first simulation code computes a solution to a physical problem onto a mesh (usually a structured grid) made of multi-materials cells, i.e a cell can contain different materials, then (2) a second simulation code acting on a pure unstructured hexahedral mesh is used (see Figure 1 for an illustration of the first stage), where every single cell contains a single material. The first code is said to be Eulerian, while the second one is said Lagrangian. In this context, the Eulerian code only needs a grid to work on, and the Lagrangian code requires a pure hexahedral unstructured mesh that can be created using an overlay grid method. Those methods $[3,4,5,2]$ developed in recent years have dramatically improved the ability to rapidly and automatically generate hexahedral meshes for complex geometries. They rely on a mesh-first approach to mesh generation where an initial base grid is used to overlay the reference geometry. Procedures to modify the base grid are also employed to best capture the geometry to define a conformal all-hex mesh.

As the generated mesh is used as an input to a nu- 

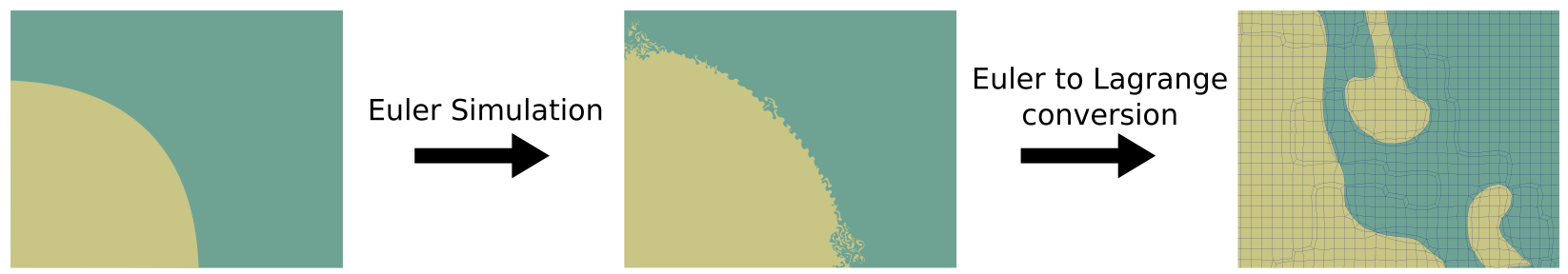

Figure 1: From left to middle, during an hydrodynamic simulation on a grid where two materials are defined (green and yellow) the interfaces between the two materials evolve. For running a Lagrangian code that requires a pure conformal mesh as an input, the mesh must be adapted along with material interfaces. Zoom is performed on the right on the final Lagrangian mesh.

merical simulation code, it must meet some quality requirements dictated by the numerical code. This quality can be defined by a quality metric and a minimum threshold that must be verified by every cell of the mesh. For instance, having non-inverted cells or cells whose scaled Jacobian measure is over 0.2 are possible criteria. The aim of this work is to control as much as possible the quality of the generated mesh. Considering the output of an Eulerian code, a selection of materials to consider, and a couple (quality metric, minimal threshold), we modify an existing overlay grid algorithm to control the mesh quality. For our purposes, the overlay grid algorithm we start from is based on Sandia's Sculpt [2] algorithm.

\section{$1.1 \quad$ Related works}

The overlay grid methods $[3,4,5]$ are all based on the same principle. Starting from a box $B$, which overlays the reference geometry $\Omega$ to be meshed, they discretize $B$ using a size-adapted grid $\mathcal{G}$, then adapt $\mathcal{G}$ both topologically and geometrically. Geometric adaptation mainly consists in projecting some nodes of $\mathcal{G}$ onto $\partial \Omega$, while topologic adaptation consists in applying refinement patterns in order to adapt the grid resolution to some local features of $\Omega$ (like a hole in a surface, a small detail compared to the overall geometry). The mesh to be deformed is then no longer a grid but an unstructured hexahedral mesh.

One of the main differences of [2] is to take as an input some volume fraction defined on $\mathcal{G}$. The reference geometry is then implicit and it can be used in our context to generate a pure Lagrangian mesh from a multimaterial Eulerian grid. Some other works $[6,7]$ share this type of inputs. In [7], the authors extended their interface reconstruction method [6] by iteratively moving the obtained interfaces, represented by triangular surface meshes in 3D, combining a Laplacian smoothing and a volume control contribution. Their method is dedicated to visualization purposes, and so one of their concerns is to obtain "good quality" triangles; they can also adapt the surface meshes, depending for example on a triangle edge length criteria threshold.
As our reference geometry is implicitly defined, interface reconstruction methods are relevant for us. Volume of fluids method [8], may be considered as related to our work. Starting from volume fractions living on any unstructured meshes (like an Eulerian grid for instance), those methods try to build interfaces with a strict volume preservation per cell. They do precisely control for volume inside each cell of the input. However, rather than producing pure computational elements, they can yield mixed elements in the output mesh where local interfaces are defined by discrete planar geometry. Such interfaces are globally non-conformal and aside from their usefulness for the simulation codes can only be used for simple visualization process or for initializing other numerical codes. On the contrary, overlay grid algorithms, such as Sculpt algorithm, require to get a smooth interface that approximates the interface surface. This smooth interface is then used to define a geometric model that can be a support for hex meshing.

\subsection{Main contributions}

Considering the "Euler to Lagrange" context, we propose a complete pipeline of steps to apply, where we try and control the mesh quality as much as possible at each step. This quality control is one of the main differences in the global process since most of the time mesh quality is ensured by post-processing where some mesh smoothing and untangling methods [9] are applied at the end of the pipeline, but these sometimes fail to improve the mesh quality.

Other contributions of our work are technical improvements on three steps of the pipeline. An overview of this pipeline is given in Section 2. The three improved steps are described in details from Sections 3 to 5 . In Section 3, we provide a new assignment correction process to avoid non-manifold topological configurations. In Section 4, we propose a new interface reconstruction algorithm. Compared to [2], it allows us to build an independent geometry model that can be used afterwards for performing node relocation and projection. This process mimics techniques described in $[6,7]$ but 
with the capability to better preserve volume fractions when $\mathcal{G}$ is an unstructured mesh. Section 5 introduces an adaptation loop that interleaves topological and geometrical operations in order to perform the grid movement. The main originality of this step is to define and use local pillowing operations to best control mesh modifications.

\section{OVERVIEW}

The method we propose adapts the pipeline defined in [2], where the initial input is $n$-dimensional grid $\mathcal{G}$, with $n=2$ or 3 . Each cell of $\mathcal{G}$ is a quadrilateral cell in $2 \mathrm{D}$ and a hexahedron in $3 \mathrm{D}$. We have the same context in this paper where every step of the algorithm can be applied to any unstructured quadrilateral or hexahedral mesh as an input without any algorithm and code modifications. As the input grid $\mathcal{G}$ is the output of an Eulerian simulation code, each cell $c \in \mathcal{G}$ can contain several materials. Let $\mathcal{M}$ be the set of materials defined on $\mathcal{G}$. Then, we also have as an input a family of material assignment functions $m a_{\mathcal{G}, m}$, simply noted $m a_{m}$ in the following, such that $m a_{m}: \mathcal{G} \rightarrow[0,1]$ and

$$
\sum_{m \in \mathcal{M}} m a_{m}(c)=1, \forall c \in \mathcal{G}
$$

A cell is said to be pure if it contains only one material and mixed otherwise. The material assignment function gives the volume fraction of materials of $\mathcal{M}$ in each cell of $\mathcal{G}$. For the sake of simplicity, we note $m a$ the family of material assignment functions, and we will call it the material assignment $m a$.

Let us now give a brief overview of the approach considering the 2D grid given in Figure 2- $a$ as an input. We have $|\mathcal{M}|=3$ and the volume fraction of each material is indicated in each cell.

1. Cell material assignment. The first step of the algorithm consists in assigning each cell of $\mathcal{G}$ to a single material of $\mathcal{M}$ (see Section 3.1). This is done by: First assigning a material to each cell $c$ considering only the volume fractions of materials in $c$ (see Figure 2-b); Secondly, correcting wrong topological configurations in the vicinity of every grid node (see Figure 2-c and Section 3.2.). Each cell of $\mathcal{G}$ is virtually assigned to a single material and we have a set of interfaces between cells virtually assigned to different materials. We call those virtual grid interfaces.

2. Material interface reconstruction. In every mixed cell of $\mathcal{G}$ containing materials $\left\{m_{1}, \ldots, m_{p}\right\}$, with $p>1$, we locally rebuild geometric interfaces between all those materials (see Section 4). This set of geometric interfaces will help us modify the grid $\mathcal{G}$ in Section 5.3.
3. Mesh adaptation. The final stage of the approach consists in modifying $\mathcal{G}$ in such a manner that the virtual grid interfaces fit geometric interfaces as much as possible while controlling grid cell quality. It relies on three main operations that we interleave in our process (see Section 5):

- First, we can move the nodes lying on a virtual grid interface towards the corresponding geometric interface (see Figure 2- $d$ ).

- Secondly, we can change the grid topology by performing pillowing ${ }^{1}$ [10] operations. It can be done in a traditional manner on each set of grid cells virtually assigned to the same material (see Figure 2-e) or by using local pillowing techniques (see Section 5).

- Thirdly, we can smooth all the grid nodes: inside each material and along the virtual grid interfaces (see Figure 2- $d$ ).

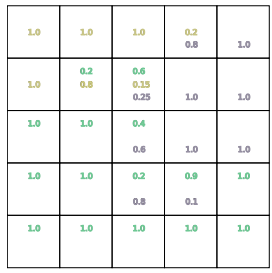

(a) volume fractions

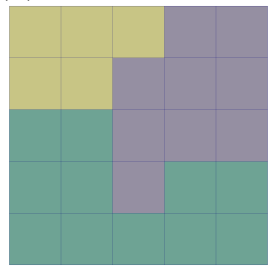

(c) assignment correction

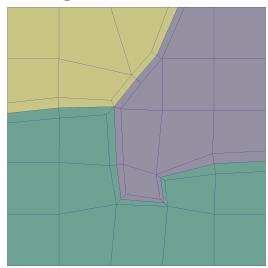

(e) pillowing

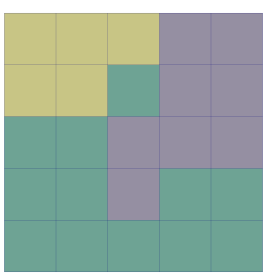

(b) local assignment

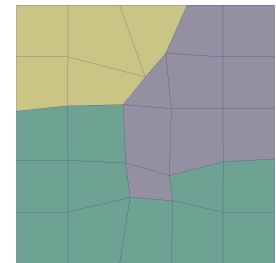

(d) interface movement

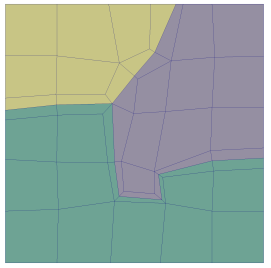

(f) smoothing
Figure 2: Overview of some operations involved in the proposed pipeline. Starting from the volume fractions given in $(a)$, grid cells are virtually assigned to each material in two stages ( $b$ and $c$ ) before being modified geometrically $(d$ and $f)$ and topologically $(e)$.

\footnotetext{
${ }^{1}$ The pillow operation consists in isolating a connex set of hexahedral cells $S_{H}$ by inserting a layer of hexahedral cells around it. Each cell of this layer can be created by inflating each quadrilateral cell of $\partial S_{H}$ into a single hexahedron.
} 


\section{CELL MATERIAL ASSIGNMENT}

The first stage of the pipeline consists in defining a new assignment to each cell of $\mathcal{G}$, where each cell is pure. This assignment is said virtual and is denoted va. The term virtual is used in opposition to the material assignment fraction, which is the reference assignment given as an input of the pipeline. As previously said, the material assignment is initialized locally at each cell then upated to resolve wrong topological configurations.

\subsection{Initialization via a local assignment}

In order to initalize the virtual assignment in each cell $c \in \mathcal{G}$, we simply select the material having the highest volume fraction in $c$; in case of equality the first material examined is selected. More formally,

$$
\forall c \in \mathcal{G}, v a(c)=m \text { with } m a_{m}(c)=\max _{p \in \mathcal{M}} m a_{p}(c) .
$$

It gives us the assignment as depicted in Figure 2- $b$ from the material assignment shown on Figure 2- $a$.

\subsection{Assignment correction}

It can be mandatory for the simulation codes or for mesh generation algorithms that each material be an assembly of disconnected manifold (when they need for instance to compute the normal to a material at all nodes of its interface) or for practical purposes (ease of implementation).

Informally, in $2 \mathrm{D}$, it means that a material is represented as a set of surface patches that do not overlap one another and do not touch along a single point. A single patch cannot also touch itself at a point (see Figure 3 on the left). Topologically, it corresponds to the fact that, in each point of a $d$-dimensional patch, one can define a single $d$-dimensional ball.
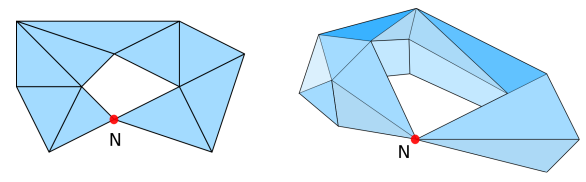

Figure 3: Illustration of non-manifold simplicial patches in 2D (left) and 3D (right). In both cases, the red node $\mathrm{N}$ induces that the patch is not a manifold.

In order to detect such situations for a material $m$ at a node $n$, we build a polyhedron made of the cells adjacent to $n$ assigned to $m$ and we use the EulerPoincaré relation for convex polyhedra. Considering a polyhedron having $N$ nodes, $E$ edges and $F$ faces, we have the formula

$$
N-E+F=2 .
$$

Let us apply this formula on a single hexahedral element made of 6 faces, 12 edges and 8 nodes, we have $N-E+F=8-12+6=2$ and relation 3 holds. Let us now consider the polyhedron build from the two cells shown on Figure 4- $a$. As they only share a node, we have $N=15, E=24$ and $F=12$ and so $N-E+F=3 \neq 2$. Relation 3 does not hold and the set of cells $C_{n, m_{i}}$ forms a non-manifold around point $n$.

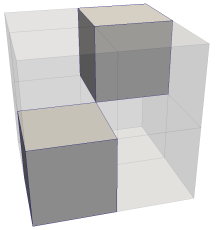

(a)

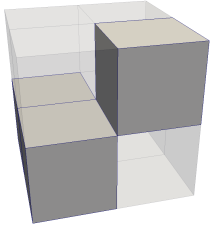

(c)

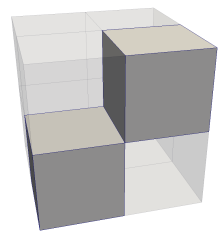

(b)

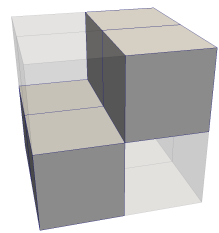

(d)
Figure 4: Example of non-manifold configurations for a material (non-transparent cells) around a node.

At the end of the mesh traversal, we have for each material a set of nodes indicating where non-manifold configurations occur. It is the input of the resolution procedure detailed by Algorithm 1. The resolution consists in modifying the virtual assignment initially done around the vicinity of each collected node. Note that solving the non-manifold issue locally to the vicinity of a node can lead to making non-manifold configurations appear at neighbouring nodes, which were originally not problematic (see for instance Figure 5- $b$ ). Hence, our resolution process is an iterative procedure where new nodes can be added to the set. As a consequence, we also must prevent a node to be treated several times leading to a livelock situation. We prevent such a situation from occuring by keeping track of local virtual assignments already performed around a node. If we treat a node again, we select a valid virtual assignment which was not already applied (see Figures $5-c$ and $d$ ). We iterate until there are no remaining non-manifolds.

Let us dive into the procedure we apply locally to every non-manifold node. This procedure is given in Algorithm 2. Let $n$ be a node where a non-manifold configuration occurs for adjacent cells $\mathcal{B}_{n}$. We get all 


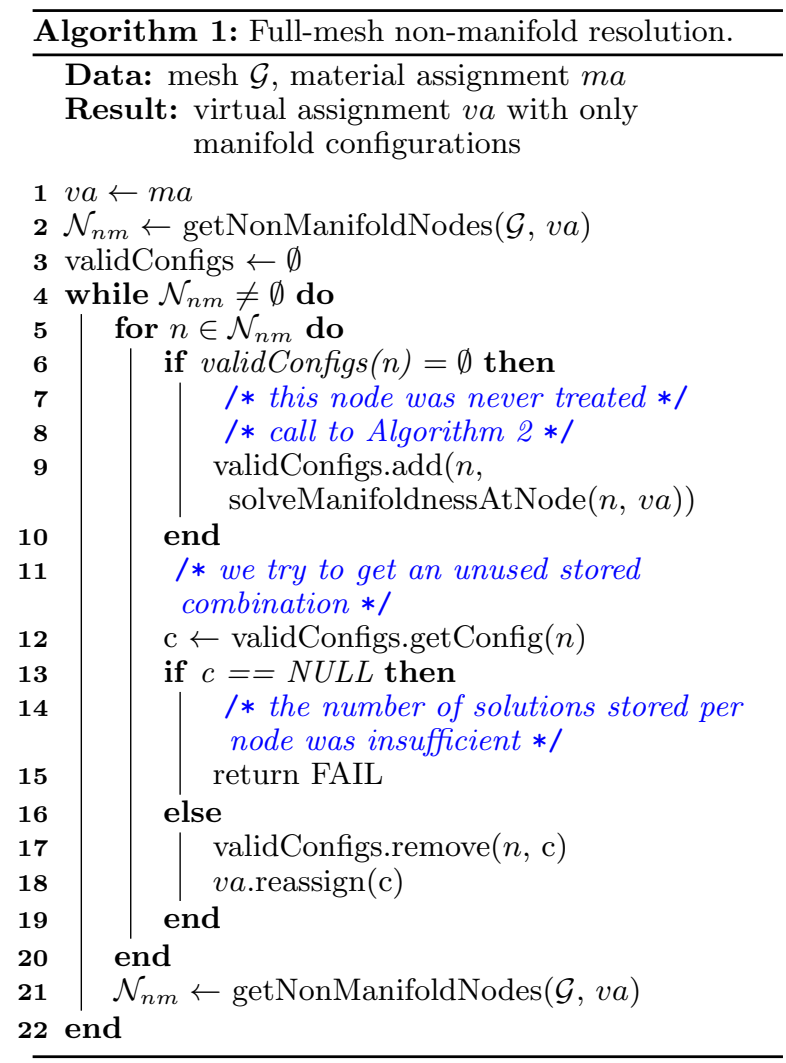

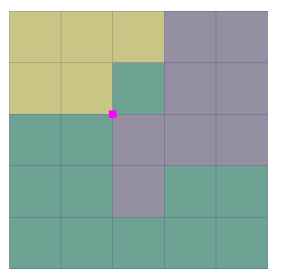

(a)

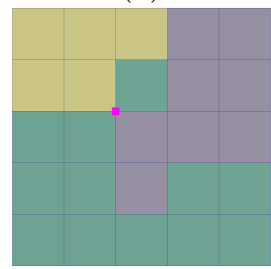

(c)

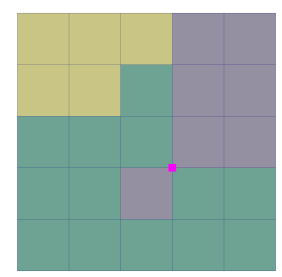

(b)

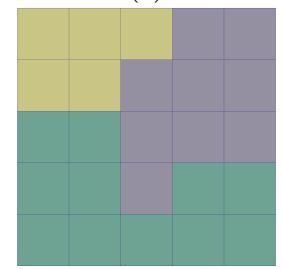

(d)
Figure 5: Example of non-manifold resolution and livelock avoidance strategy. (a) the initial material assignment with the non-manifold node highlighted; $(b)$ the new assignment (at Algo 1:7) solves the non-manifold at the first node but causes another to appear; $(c)$ the new non-manifold node is solved but we are back to the initial assignment; $(d)$ the non-manifold is solved again with another assignment (at Algo 1:10), thus avoiding an infinite loop between state $(a)$ and $(b)$. the possible assignments for any cell of $\mathcal{B}_{n}$ using the material assignment (line 2). They are gathered in the set $\mathcal{M}_{\text {possible. We then build the set that gathers all }}$ the possible configurations of virtual assignment where each cell of $\mathcal{B}_{n}$ can take any value in $\mathcal{M}_{\text {possible }}$ (line 3 ). In the loop (lines 4 to 16), we test those possible configurations and only keep the acceptable ones, i.e. configurations where we don't have non-manifold situations; we order them by cost and only keep a limited number of those (lines 14 and 15). The "best" configuration is the one that minimizes:

$$
\text { cost }=\sum_{c \in \mathcal{B}_{n}}\left|1-m a_{v a(c)}(c)\right| \operatorname{Vol}(c) .
$$

We have the following remarks about our procedure:

- The number of possible configurations is equal to $\left|\mathcal{M}_{\text {possible }}\right|^{\left|\mathcal{B}_{n}\right|}$. This number can far exceed the current hardware capabilities, so a limit to the potential materials $\left|\mathcal{M}_{\text {possible }}\right|$ is enforced, such as keeping only the four or five most preponderant materials that are in $\mathcal{B}_{n}$, based on material assignment;

- We keep the twenty (user's choice) best valid virtual assignments. Since we test every possible assignment and a node can be treated several times, we store the solutions when first treating the node and provide the next best not already used assignment (we allow the initial assignment to be tried twice) when asked again. This allows us to avoid infinite cycles, but if the upper limit is reached our code stops and the user has to rerun using a higher limit.

Note that additional assignment modifications can be dictated by other criterias, such as the expected final mesh quality as seen in [11] where cells forming isolated or thin chunks of one material are reassociated because these configurations usually lead to having bad quality cells, or sometimes the simulation code cannot handle small slivers of materials.

\section{INTERFACE RECONSTRUCTION}

This section focuses on building interfaces between materials that could help in the computation of a prospective new position (currently done similarly to [2]) for the interface nodes (the nodes adjacent to cells assigned to different materials). Additionally, extracting such interfaces will also bring the added benefit of producing a geometric model.

Material interface extraction poses two main issues: the desired interfaces should be smooth, typically when the goal is to visualize them, but at the same time they should also fit the input data as best as 


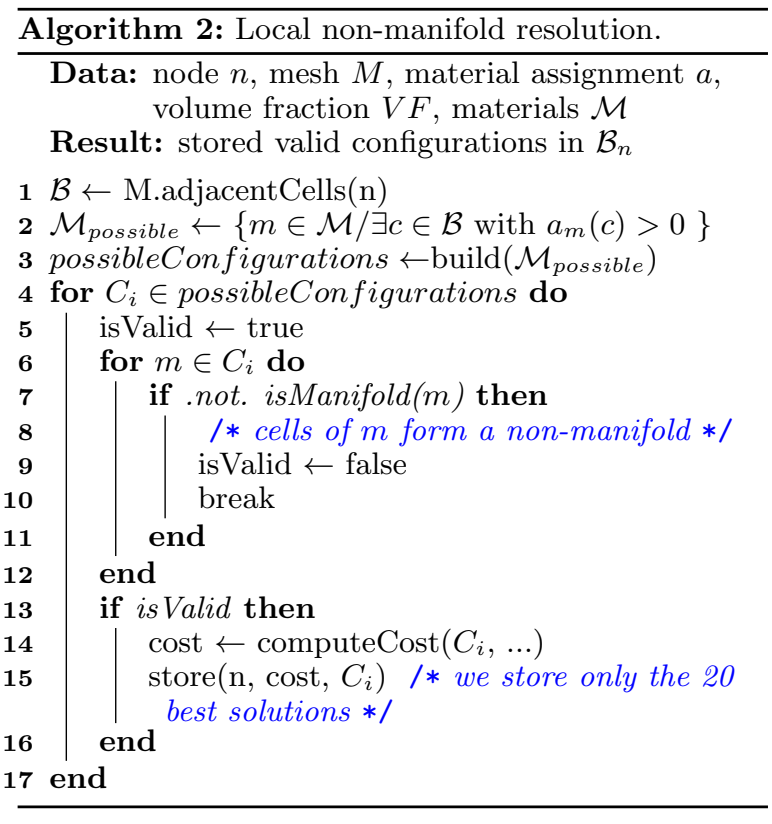

possible, namely the volume fractions; those two objectives can conflict with one another. Several methods exist, in different fields:

- ALE-simulation interface reconstruction.

A domain where material interface reconstruction is extensively studied and applied is CFD simulations. While those reconstructed interfaces have a built-in volume fractions preservation, they are not smooth, as they are discontinuous across cells [8]. Most of these methods also have the additional drawback of being material order-dependent;

- Sculpt

The position computed for the interface nodes in [2], and that we use in our implementation, depends on the volume fractions (and their gradient) and the virtual assignment. We have new positions for the nodes, but no geometric model;

- voxels

These methods decompose the mixed cells into sub-elements - typically a hexahedron will be refined into a grid - on which a partitioning is called with respect to the volume fractions inside each cell. The interfaces at the sub-elements level are aliased, and since these methods originated from visualization purposes the interfaces are usually simplified into smooth triangular surfaces. We will further study voxel-like methods in this section.

\section{1 problem under voxel terminology}

The discrete interface reconstruction techniques stems from the need to visualize the location of materials in the case where some of the cells are mixed and where the number of materials is greater than two. In the case where the number of materials equals two, classic iso-contouring methods provide an adequate solution but with more materials small gaps or artifacts can appear that are non-desirable. In [12] the authors introduced the decomposition (or refinement) of mixed cells into subcells (or pixels) which are in turn assigned to the materials present in the mixed cells they were spawned from; the work in $[6,7]$ extends it to cases with more than three materials per cell.

The voxel problem is the following: all the mixed cells and their adjacent pure cells are subdivided into pixels, and we want a virtual assignment on those pixels. Pixels spawned from pure cells are already assigned to the material of their corresponding pure coarse cell, leaving those spawned from mixed coarse cells as "free". We favor a pixel virtual assignment that has a low

- edgecut, defined as the sum of pairs of adjacent pixels assigned to different materials;

- discrepancy, defined as the sum over each coarse cell $c c$ of the absolute difference between the volume of each material present in $c c$ (for material $k$ it is $\left.m a_{k}(c c) \operatorname{Vol}(c c)\right)$ and the volume of the pixels of $c c$ assigned to $k$. It expresses whether the pixels assignment fit the volume fractions.

We compare four methods that solve the problem:

- mixed-integer linear programming

- simulated annealing

- graphcut

- greedy heuristic

\subsubsection{MIP}

We formulate the problem as a linear problem; since our variables are integers we in fact have a mixedinteger linear problem. In our implementation, we use the GLPK [13] library to solve the problem.

Problem formulation:

$$
\left\{\begin{array}{ll}
\min \sum_{p \in P, k \in M a t}\left|c_{p, k}-\frac{1}{|N(p)|} \sum_{q \in N(p)} c_{q, k}\right| & \\
\text { constrained to } & \forall p, k \\
c_{p, k} \in\{0,1\} & \forall p \\
\sum_{k \in M a t} c_{p, k}=1 & \forall k, \forall c c
\end{array}\right\}
$$


where $c_{p, k}$ returns 1 if pixel $p$ is assigned to material $k, 0$ otherwise (using the previous notations, it gives $v a(p)=k$ ). The first two constraints indicate that every pixel has an assignment and only one. The third constraint expresses that we want to have a discrepancy equal to zero (nbsub being the number of pixels in a coarse cell). The objective function that we want to minimize reflects the desire for pixels assigned to the same material to be clustered together, i.e. having a low edgecut.

\subsubsection{Simulated Annealing}

This method introduced in [6] consists in randomly assigning the pixels to materials with respect to the volume fractions data; pair of pixels spawned from the same coarse cell will then swap their virtual assignment, as described in Algo 3. Since the initial assignment to the pixels fit as best as possible the volume fractions and that only swaps are performed, the resulting pixels virtual assignment fits just the same. Note that this property does not hold true when the mesh is unstructured; more precisely when the pixels are of different size (see Figure 8).

\subsubsection{Graphcut}

Our problem can be seen as an energy minimization problem; in $[14,15,16]$ it was shown that expressing the energy function as the sum of a so-called data and smooth costs allows the problem to be solved using a graphcut.

Problem formulation, with $f_{p}$ being the virtual assignment of the pixel p and $E$ the pairs of adjacent pixels:

$$
E(f)=\lambda \sum_{p \in P} D_{p}\left(f_{p}\right)+\beta \sum_{\{p, q\} \in E} V_{p, q}\left(f_{p}, f_{q}\right)
$$

with basically the edgecut:

$$
V_{p, q}\left(f_{p}, f_{q}\right)= \begin{cases}0 . & \text { if } f_{p}=f_{q} \\ 1 . & \text { otherwise }\end{cases}
$$

and the cost of assigning a material to a free pixel (nearest is the nearest pixel issued from a pure cell assigned to $f_{p}$ ):

$$
D_{p}\left(f_{p}\right)=\left(1 .-m a_{f_{p}}(p)\right) p \text {.distance }\left(\text { nearest }\left(f_{p}\right)\right)
$$

We should note that the first term does not enforce matching the volume fractions. In order to apply the graphcut this term has to be dependent on only $p$; the expression that we chose tries to emulate that property but we will see in the results (Figure 7-c) that it is far from being efficient. The second issue comes from the energy function itself that is the sum of two terms not related to one another, and the values chosen for $(\lambda, \beta)$ might depend on the cases we run on.

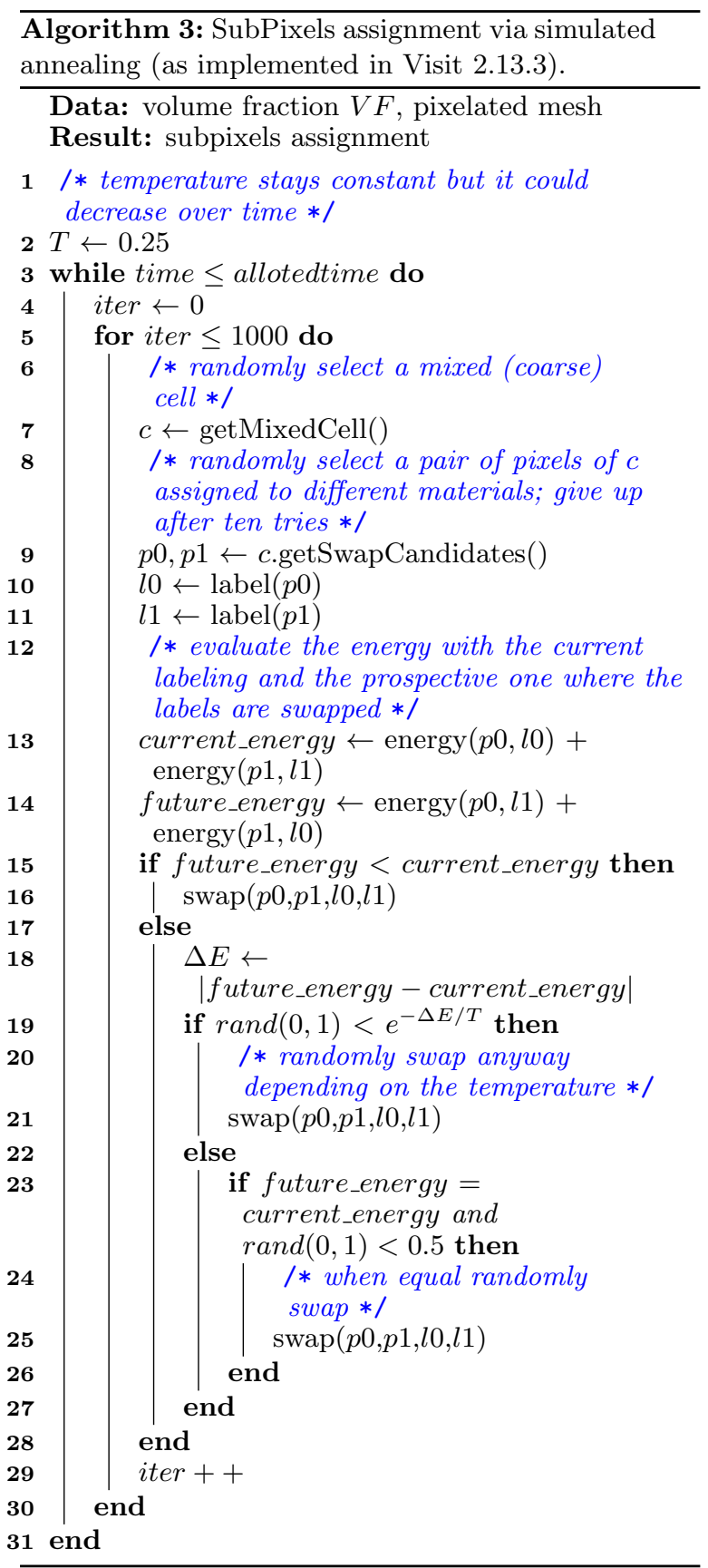




\subsubsection{Greedy Heuristic}

We have implemented a greedy heuristic (see Algo. 4) where at each iteration the free pixels are assigned volume fractions that depend on the values in their respective coarse cells adjusted to take into account the pixels that were already assigned (see Figure 6). $3 \mathrm{D}$ results are shown in Figure 9

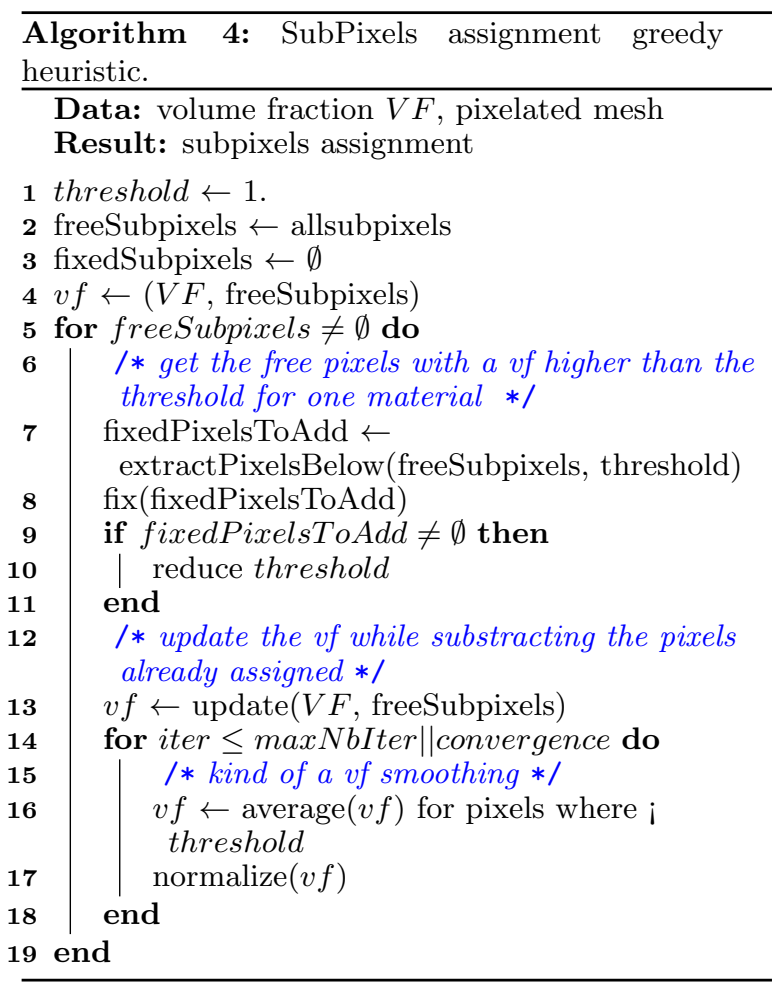

\section{2 results}

The results of the four methods can be seen applied on the $5 \times 5$ example in Figure 7 . The MIP implementation is impractical, as it does not return a solution in an acceptable time; it can sometimes return a valid (meaning that it fits the constraints) but not optimal solution, which is the case in Figure 7-a. The graphcut tends to return straight interfaces, resulting in a good edgecut, but as we have mentionned is quite bad when considering the discrepancy. That leaves us with the simulated annealing, which is better than our greedy heuristic in the case of a grid, but fares badly concerning the discrepancy in unstructured cases, as shown in Figure 8. All of those methods have the same memory limitation, as the submesh can be quite big. In practice, we will use our heuristic to build the pixelated interfaces, as it is a good compromise in structured and unstructured cases and does not rely on tuning parameters depending on the case.
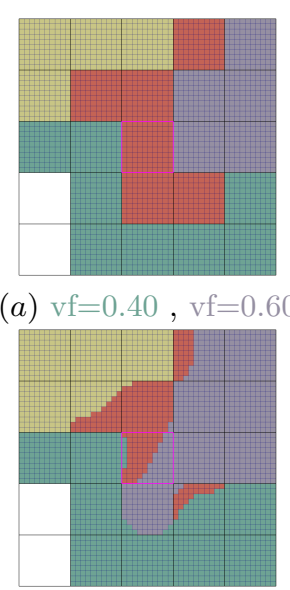

(c) $\mathrm{vf}=0.62, \mathrm{vf}=0.38$

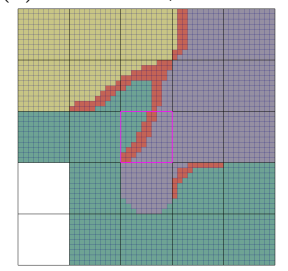

(e) $\mathrm{vf}=0.54, \mathrm{vf}=0.46$ (a) $\mathrm{vf}=0.40, \mathrm{vf}=0.60$

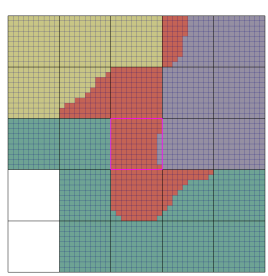

(b) $\mathrm{vf}=0.43, \mathrm{vf}=0.57$

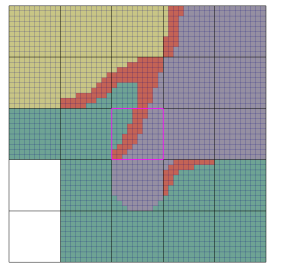

(d) $\mathrm{vf}=0.61, \mathrm{vf}=0.39$

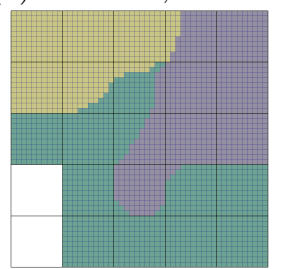

$(f)$
Figure 6: Greedy heuristic applied to the $5 \times 5$ example where we can see the evolution of the volume fractions (see Algo. 4:13) assigned to the free pixels of the central coarse cell below each figure. The wireframe black grid is the coarse mesh and the pixels colored in red are those not yet assigned.

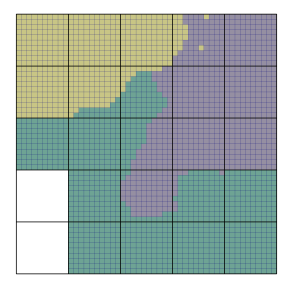

(a) MIP

$\mathrm{d}=0$, edgecut $=592$

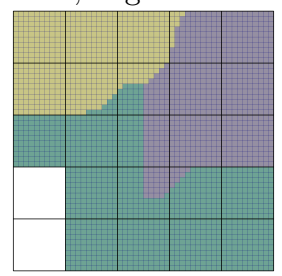

(c) graphcut

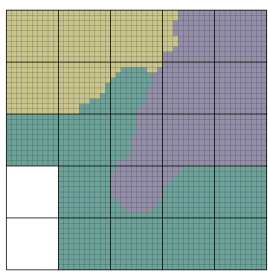

(b) simulated annealing $\mathrm{d}=0$, edgecut $=536$

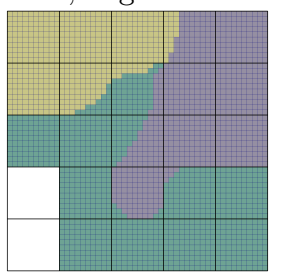

(d) greedy heuristic $\mathrm{d}=0.56$, edgecut $=558$
Figure 7: Comparison of the pixelated interfaces reconstruction methods. Note that we stopped the MIP implementation after 5 minutes. 


\section{MESH ADAPTATION}

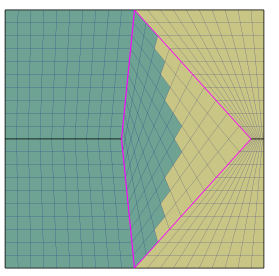

(a) $\mathrm{d}=0.0768$

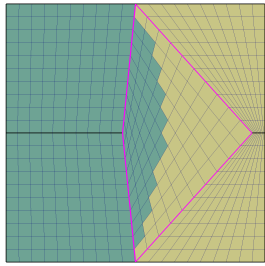

(c) $\mathrm{d}=0.264$

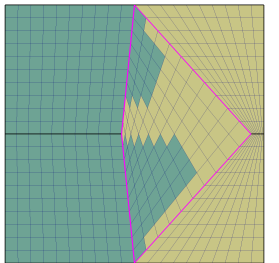

(e) $\mathrm{d}=0.1344$

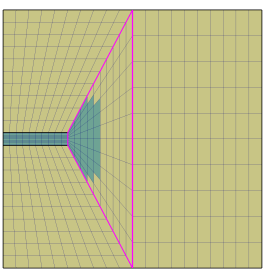

(b) $\mathrm{d}=0.024$

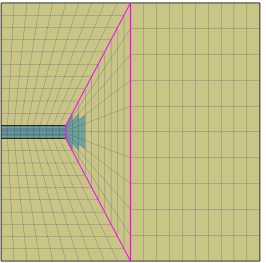

(d) $\mathrm{d}=0.1444$

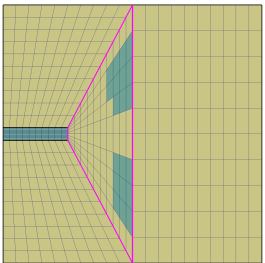

(f) $\mathrm{d}=0.1254$
Figure 8: Greedy heuristic (first row) versus simulated annealing (second and third rows) applied to unstructured cases. Only the highlighted cell is mixed, and the respective volume fractions are $(0.5,0.5)$ in the left, $(0.2,0.8)$ in the right. Two different results are shown for the simulated annealing method because some cluster of pixels can appear due to the randomness of the initial pixel assignment and the swaps.

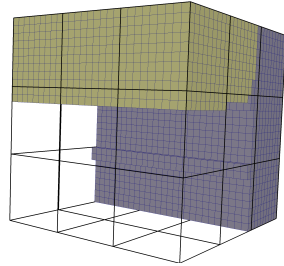

(a)

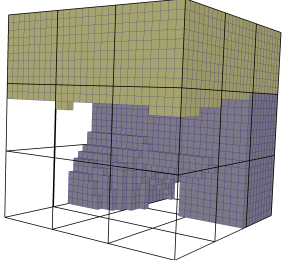

(b)
Figure 9: Greedy heuristic applied on two 3D cases with three materials. The third material is hidden. $(a)$ an extruded case; $(b)$ a "real" 3D case.
Up to now, the geometry and the topology of the grid $\mathcal{G}$ have not been modified. The aim of this section is to do so while offering some quality guarantees about the output mesh. From now on, we consider that the user provides a cell quality threshold $S_{q}>0^{2}$ in addition to the input mesh $\mathcal{G}$ carrying volume fractions data materialized by the material assignment functions $m a$. Assuming that all cells of $\mathcal{G}$ are initially above the user-input threshold $S_{q}, \mathcal{G}$ keeps satisfying the quality requirements at the beginning of this stage of the process. It is the operations that we are going to perform now that have an impact on the mesh geometry and/or its topology and where there is no longer any guarantee on cell quality. Those operations are: node movement, pillowing and smoothing. As a reminder, our starting point is the pipeline of operations given in $[2,11]$ and illustrated on Figure 10- $a$.

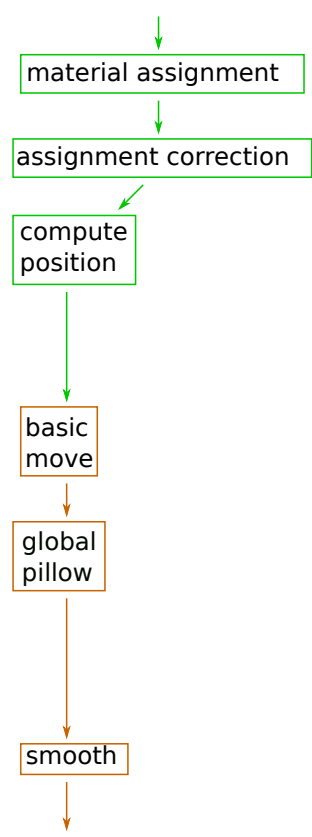

(a) base algorithm

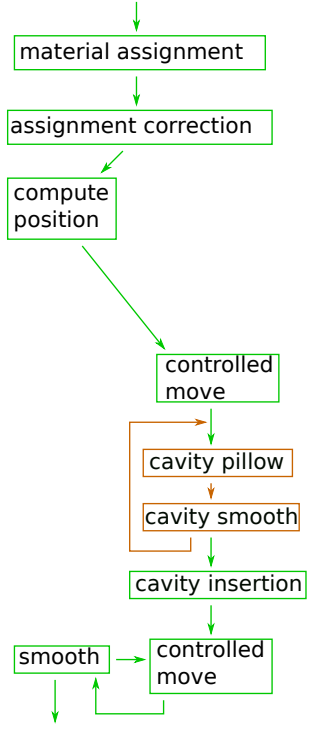

(b) our approach
Figure 10: Base algorithm diagram versus our modifications. In green the part where we have a guaranteed cell quality, and in orange the part where there is no control over this.

In this initial pipeline, after computing ideal locations for the nodes of $\mathcal{G}$ that are on virtual grid interfaces, we move the virtual grid interface nodes to these new locations in order to better capture the material interfaces. Such a direct movement tends to decrease the cells quality. Then a pillowing phase is applied,

\footnotetext{
${ }^{2}$ Typically, it can be a minimum scaled Jacobian [17] value below which no cell must be.
} 


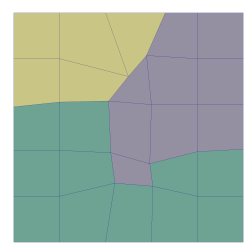

(a) basic move

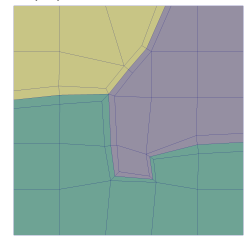

(c) pillow

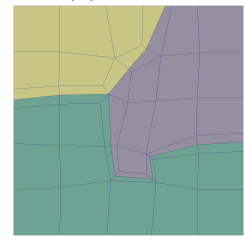

(e) smooth

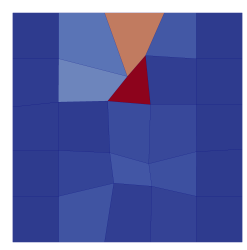

(b) $\operatorname{minSJ}=0.036$

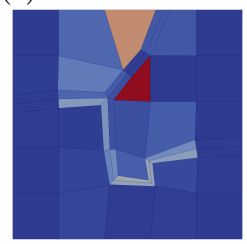

$(d) \operatorname{minSJ}=0.046$

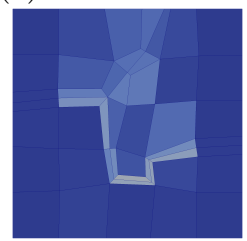

$(f) \operatorname{minS} \mathrm{J}=0.588$
Figure 11: Evolution of the cell quality during the base algorithm. In $(a)$ and $(b)$ the node movement causes a sharp decrease of the cell quality; in $(c)$ and $(d)$ the pillowing does not really improve the cell quality; in $(e)$ and $(f)$ the smoothing is efficient in this example.

where each material is wholly pillowed without taking cell quality into account, and eventually a final global smoothing stage is executed so as to improve the overall cell quality. The intermediate pillowing phase does not in itself improves quality, but it provides more degrees of freedom for the smoothing algorithm to work with. The example of Figure 11 illustrates that such a pipeline can lead to good results. While the cell quality is not strongly controlled, the mesh quality is improved during the process.

The example of Figure 12 shows the exact opposite. The mesh quality worsens because of a global pillowing technique that does not take into account some local geometric features. In this case, performing a pillowing around the right tip of the green area leads to strongly decreasing the inner angle of each quadrilateral cell around this node. Note that the worst cell after proceeding with the pillowing (in red in Figure 12- $d$ ) was the one hampering the displacement of the marked node. The subsequent smoothing applied does not improve the situation and the quality of the worst cell is eventually lower than at the beginning (0.237 agains 0.307$)$.

We consider that this process has two main drawbacks. First, node relocation is done without considering the quality of surrounding cells. We propose to control

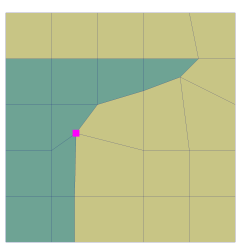

(a) controlled move

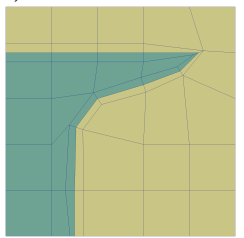

(c) pillow

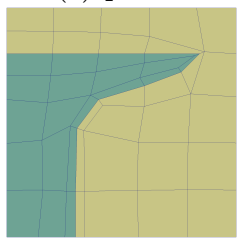

(e) smooth

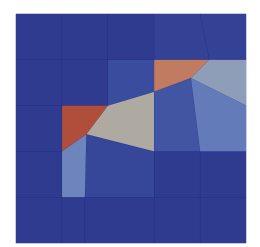

(b) $\operatorname{minSJ}=0.307$

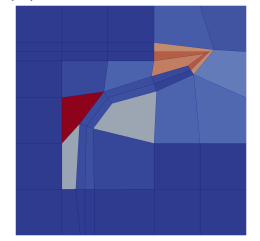

$(d) \operatorname{minSJ}=0.089$

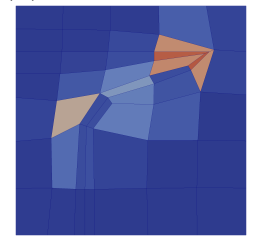

$(f) \operatorname{minSJ}=0.237$
Figure 12: Global pillowing quality problem on a $5 \times 5$ two materials case. $(a)$ and $(b)$ mesh after the controlled move with the marked node stopped at a distance of 0.04 from its desired position; $(c)$ and $(d)$ global pillowing; $(e)$ and $(f)$ the smoothing makes good use of the additional edge added to the marked node in the green material area to improve quality but cannot improve it at the tip.

this node movement and to forbid it if the cells quality get lower than $S_{q}$. Secondly, we propose to adopt a local pillowing strategy instead of a global one. Eventually we interleave those operations with a traditional smoothing step in our process (see Figure 10- $b$ ). It allows us to preserve quality above the threshold at all times in the new pipeline, which includes:

- a controlled node movement strategy;

- a localized cavity pillowing in place of a global indiscriminate one;

- a node position computation based on a geometric model extraction - the one given by the interface reconstruction in Section 4 - in order to smooth the target material interfaces, particularly necessary in $3 \mathrm{D}$.

In the subsequent examples used for illustration purposes, the threshold chosen for the minimum scaled Jacobian will always be $S_{q}=0.3$. 


\subsection{Controlled node movement}

As seen in Figure 10 and 11-b, the first phase that may decrease quality is the basic node movement. Considering that the input mesh meets the quality requirements, our strategy is to avoid moving the nodes when the quality is degraded below the threshold. For that purpose, we progressively move each node $n$ of the virtual grid interfaces towards their expected location $\mathbf{p}_{\text {ideal }}$ on the geometric interface. At each small movement of $n$, we check the quality of cells surrounding $n$. In our implementation, a small movement corresponds to $1 / 16$ of the distance between the location of $n$ and $\mathbf{p}_{\text {ideal }}$. The impact of this controlled movement can be seen in Figure 13, and the guarantee over the quality, represented in green in Figure 10-b, extends and reaches just before the pillowing stage.

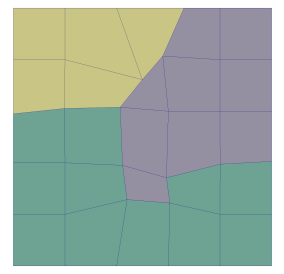

(a) basic move

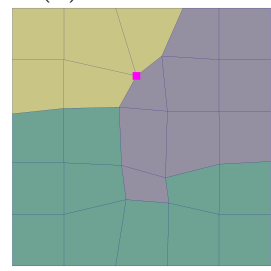

(c) controlled move

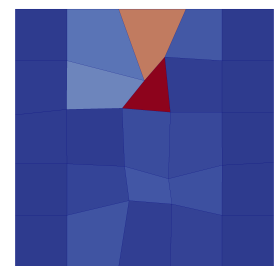

(b) $\operatorname{minSJ}=0.036$

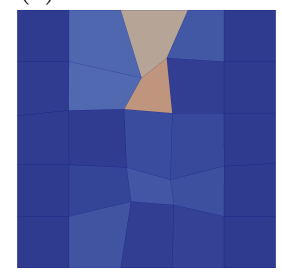

(d) $\operatorname{minSJ}=0.387$
Figure 13: Example of controlled movement. $(a)$ and $(b)$ basic move and the mesh quality; $(c)$ and $(d)$ controlled move, the marked node could not move all the way and was stopped at a distance of 0.118 from its expected location and the cell quality remains above 0.3 .

A direct side effect of this controlled movement is that some of the nodes did not reach their expected position. Let $\mathcal{N}$ be those nodes, we introduce an additional modification to the base algorithm to allow for those nodes to move further. After applying the topological modifications (the pillowing), the algorithm enters into a move-smooth loop (see Figure 10- $b$ ) so that the nodes of $\mathcal{N}$ can progressively keep moving towards the geometrical interfaces, eventually reaching it in the best cases. In this move-smooth loop, the smoothing stage has two prerequisites: it must not decrease the quality and nodes that have reached the geometric interfaces are fixed. At the end of this stage, the set $\mathcal{N}$ is not necessarily empty.

\subsection{Cavity pillowing}

As shown in Figure 12 , performing a global pillowing can lead to drastically decrease the mesh quality without any guarantee of successfully improving it during the final smoothing stage. In our pipeline, the pillowing is performed in a totally different manner. It aims to help moving nodes of $\mathcal{N}$ by providing more leeway for the smoothing algorithm to work with. The idea is then to apply pillowing operations in the vicinity of $\mathcal{N}$ while avoiding to change the mesh topology where the quality is already good.

The process we follow can be summarized as follows: for each node $n$ of $\mathcal{N}$ and each material $m$ adjacent to $n$, we extract cell groups that we are going to pillow. Each cell group is called a cavity. In theory, the idea would be to build many sets of cavities $\left\{c_{i}\right\}_{i>0}$ for $(n, m)$, pillow and smooth each cavity $c_{i}$ independently and keep the one that gives the best quality. If this quality is higher than the quality threshold $S_{q}$ then the pillowing of this "best" quality is published in the mesh.

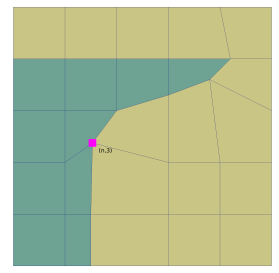

(a)

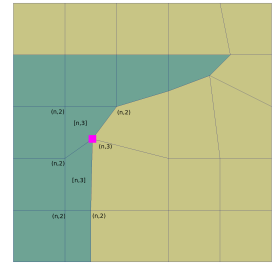

(c)

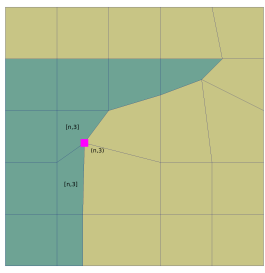

(b)

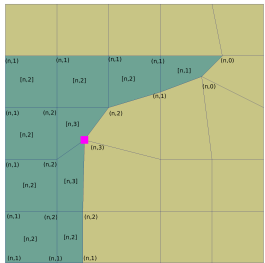

(d)
Figure 14: Cavity definition for the green material. (a) the node $n$ that could not reach its destination is marked and assigned a range (here it is 3 ); (b) the adjacent cells are also selected and a reference to $n$ is kept; $(c)$ the selection is extended to the adjacent nodes, a reference to $n$ is kept and the range is decreased; $(d)$ we continue until the range reaches zero or all the cells assigned to the material are selected.

As there is a huge number of potential cavities, we consider in practice a maximal distance to the node $n$ to build cavities. This distance corresponds to a vertex-based traversal of the mesh starting from $n$. For instance, for distance 1 , the cavity of $(n, m)$ contains all the cells of material $m$ that are adjacent to $n$. In all our examples, a maximal distance of 3 is chosen. As an illustration, Figure 14 shows our process 
to create cavities when $\mathcal{N}$ is reduced to a single node. Another implementation choice is to have a greedy approach where we do not perform the pilllowing for all the cavities. For a couple $(n, m)$, we publish the first one that provides a quality above $S_{q}$. This greedy algorithm is done by progressively decreasing the cavity distance. We made this choice because the cavities of several nodes of $\mathcal{N}$ are more likely to merge when they are big enough. Such merging reduces the number of topological changes. Figure 15 shows how we can end up with potentially several large-size cavities.

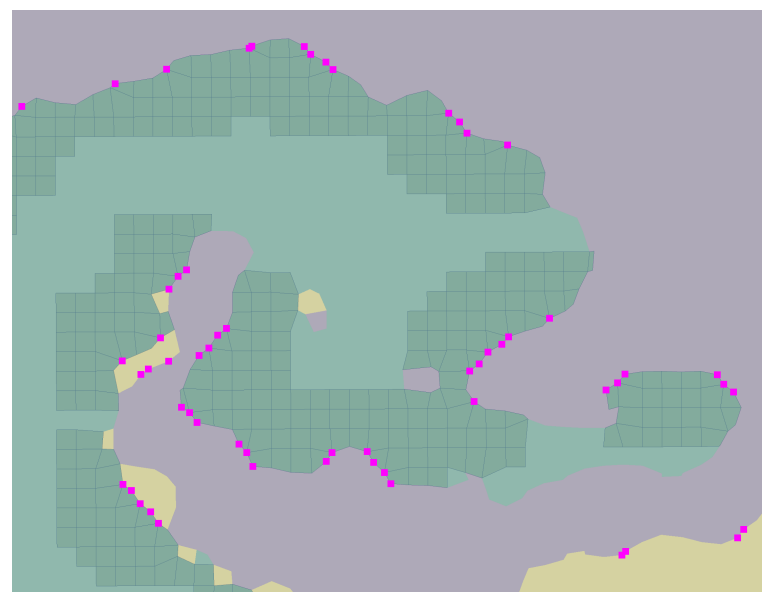

Figure 15: Cavity extracted for the green material in the triple point example (see Figure 19) where we can see the cavities spawned from different nodes merged to form bigger cavities. Note that marked nodes at the interface between the yellow and grey materials are ignored and do not spawn cavities, as we currently consider one material at a time.

As previously said, our greedy algorithm stops when we encounter a situation with a cavity quality that is above $S_{q}$. This is illustrated by Figures 16 and 17 . In Figure 16, we first extract a cavity of range 3 for the marked node (in $a$ ) and then we pillow and smooth it (in $b$ and $c$ ). As the quality is below the 0,3 threshold, we decrease the cavity range to 2 and start a second iteration (in $e, f, g$ and $h$ ) where we get the expected quality. In Figure 17, we see the local pillowing made on both materials adjacent to the marked node. Our overall algorithm is summarized by Algorithm 5).

\subsection{Geometric model extraction, projec- tion and smoothing}

So far we have tried to move the nodes towards a computed location that depends solely on the input volume fractions and the cells virtual assignment (as computed in [2]). No care was taken for the expected interfaces quality. It becomes relevant in $3 \mathrm{D}$ where the mesh entities forming the interfaces are no longer

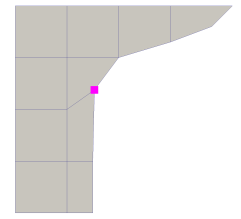

(a) cavity of range 3

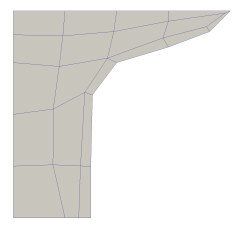

(c) smooth

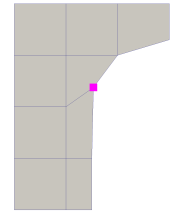

(e) cavity of range 2

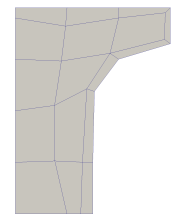

(g) smooth

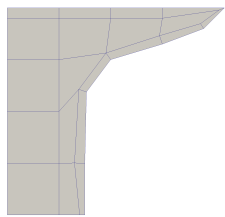

(b) pillow

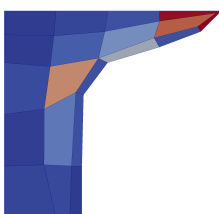

(d) $\operatorname{minSJ}=0.237$

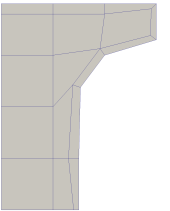

( $f$ ) pillow

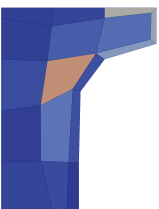

(h) $\operatorname{minSJ}=0.494$
Figure 16: Cavity pillowing and smoothing loop. ( $a, b$, $c$ and $d)$ A first iteration with a cavity of size 3 does not give the expected quality; $(e, f, g)$ and $h)$ a second iteration with size 2 meets the requirements. 


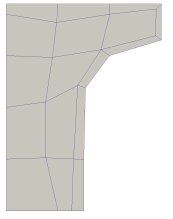

(a)

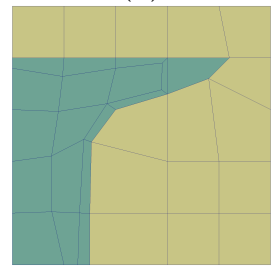

$(c)$

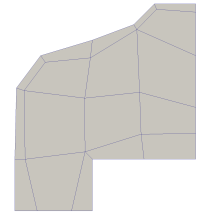

(b)

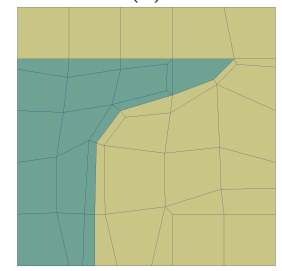

(d)
Figure 17: Cavity pillowing insertion back into the mesh. $(a)$ and $(c)$ the pillowed cavity for the green material and its insertion inside the mesh; $(a)$ and $(d)$ the same for the yellow material.

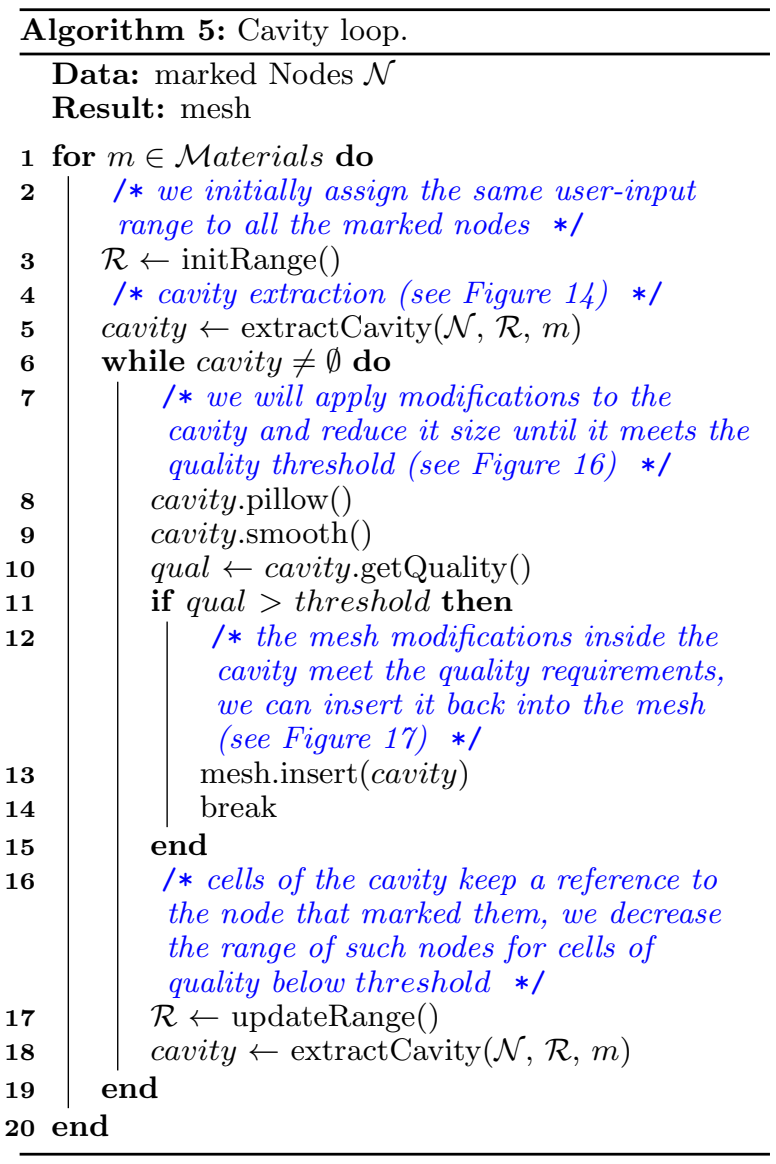

1-cells (or edges) but are now 2-cells (or faces). Moving the nodes to their computed ideal location can by-design leads to bad quality faces, hence severely limiting our nodes controlled movement. Figure 18- $a$ illustrates it in the asteroid case, the surface mesh we would obtain by moving the interface nodes at their expected locations has very bad-quality quadrilateral elements.

In order to counteract this we modify the computed nodes locations by applying a surface smoothing (see Figure 18- $d$ ). So as not to stray too far from the input volume fractions data and preserve the volume of the materials, the positions are constrained on a geometric model, which in our case is the model that can be extracted from interfaces reconstructed (see Figure 18b) as seen in section 4 . The resulting expected surface is shown in Figure 18-c. The benefits of this corrected new position computation appear in the asteroid 3D case where not applying this adjustment results in our algorithm being stuck after the first controlled move. Such a resulting mesh could be considered satisfactory, quality-wise, still we want the interface nodes to be located as much as possible close to where the material interfaces were determined to be.

\section{RESULTS}

In this section, we demonstrate and analyse the results of the proposed method on several cases, both $2 \mathrm{D}$ and $3 \mathrm{D}$; the metrics on the results are shown in Table 1 where dist $_{\text {init }}$ and dist $t_{\text {final }}$ are defined as the sum of the distances between the interface nodes and their expected position, respectively after the first controlled move and at the end of our algoritm. Admittedly those values heavily depend on the size of the grid, so comparisons between cases might not be relevant, but the ratio $\frac{\text { dist }_{\text {final }}}{\text { dist }_{\text {init }}}$ represents the improvements (the lower the better) our modified pipeline brings to counteract the drawback of the controlled node movement. We also compare our method with Sculpt on some examples regarding execution time and a discrepancy metric.

\subsection{Results of our method}

2D cases. We applied our approach in an intercode context, where our inputs are grid meshes carrying volume fractions data taken from a CFD simulation code at several time steps (see Figure 19) for two cases, the triple point and the double bar problems. They came from simulations run on grids of two different resolutions. The results in Table 1 highlight the motivations behind our approach: taking the triple point case at 1 second, we can see that for one grid resolution the base algorithm returns with a mesh containing no inverted cells (but still lower than the 0.3 minimum scaled ja- 


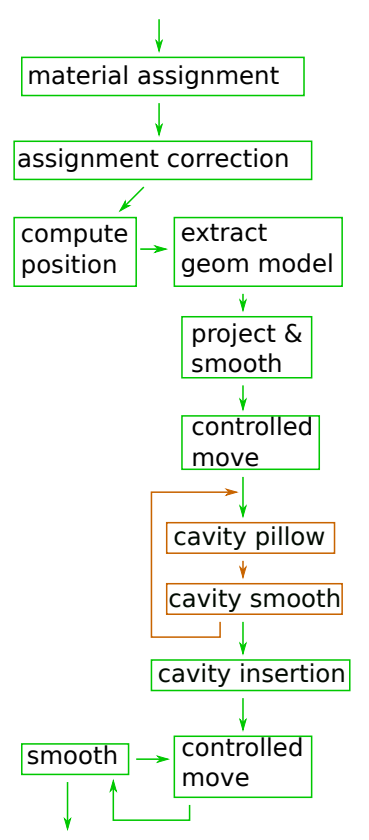

(d)

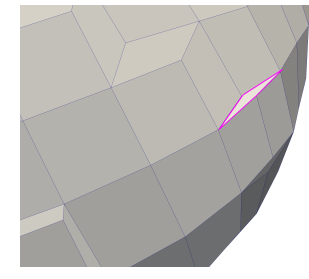

(a)

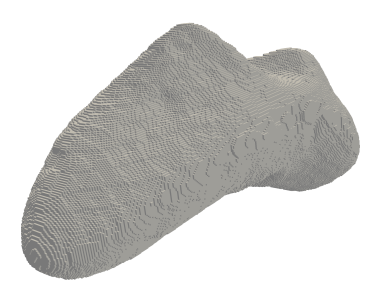

(b)

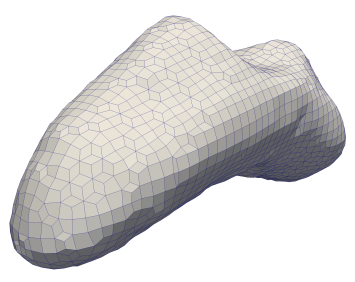

(c)
Figure 18: Motivation behind the adjustment to the computed node position, using the geometric model extraction and projection smoothing illustrated in the asteroid case in 3D. (a) close-up of the expected surface interface mesh where the marked quad has a quality of 0.068; (b) the pixelated interface between the asteroid and the exterior as obtained in section 4 and on which we will smooth the expected interfaces to remove problems such as those seen in $(a) ;(c)$ the smooth and projected expected node position; $(d)$ new pipeline that now includes the modifications to the computed positions. cobian threshold chosen by the user). That is not the case for the other resolution, making it unreliable. It is unrealistic to ask users to rerun their simulations with different resolutions at random, assuming it is even feasible, hence the need for our algorithm that consistently works.

Our method was applied on two additional hydrodynamics simulations issued from [18] (see Figure 20); in all those cases it improves the distance by at least an order of magnitude.

3D extruded cases. The same cases from Figure 19 were extruded ${ }^{3}$ and run in $3 \mathrm{D}$.

While our method does indeed result in meshes meeting the quality requirements the ratio of $d_{i s t_{\text {final }}}$ over dist $_{\text {init }}$ remains much higher than in the $2 \mathrm{D}$ cases.

"Real" 3D cases. Fully 3D cases were studied, one which input is a grid where the volume fractions data were computed by imprinting an asteroid model into the grid (see Figure 18) and the other one is an hydrodynamics simulation of a ball of fluid falling into a box computed using [19] and taken at several time steps (see Figure 21).

These cases make use of the improvements on the computed node position as described in Section 5.3 and shown in Figure 18.d. We should also note that in these cases, the first controlled move was executed with a threshold $S_{q}=0.3$ which was decreased to 0.2 for the remainder of the algorithm. Without this intermediate threshold the cavity adaptation never manages to produce a good enough one to insert back into the mesh, which means our output mesh would have been the mesh obtained after the first controlled move. The distance dist $_{\text {init }}$ and dist fina $_{\text {are }}$ illustrated in Figure 22, and a success of the decreasing cavity-size strategy is shown in Figure 23.

\subsection{Discrepancy and comparison}

Neither Sculpt nor our method preserve the volume fractions; in [20] the authors show that it is not an issue, depending on the application. Nevertheless, we are interested in measuring by how much our output deviates from the input; in [21] we define a per-cell discrepancy criteria for $c$ a cell of $\mathcal{G}$ as

$$
d_{c}=\sum_{m \in \mathcal{M}}\left|V\left(c \cap M_{\mid m}^{O}\right)-m a_{m}(c) V(c)\right|
$$

where $V(X)$ is the volume of any geometric space $X$ and $M_{\mid m}^{O}$ the output mesh restricted to the pure cells

\footnotetext{
${ }^{3}$ The 3D mesh is created from a $2 \mathrm{D}$ quad mesh, lying in the XY plane, by creating successive layers of hexahedral cells along the $\mathrm{Z}$ direction. Volume fractions are simply derived for each hexahedral cell from their origin quadrilateral cell.
} 
Table 1: Quality and distance metrics for the examples.

\begin{tabular}{|c|c|c|c|c|c|}
\hline case name & $\begin{array}{l}\text { minJS } \\
\text { base algo }\end{array}$ & $\begin{array}{l}\text { minJS } \\
\text { our algo }\end{array}$ & dist $_{i n i t}$ & dist $_{\text {final }}$ & $\frac{\text { dist }_{\text {final }}}{\text { dist }_{\text {init }}}$ \\
\hline \multicolumn{6}{|l|}{$2 \mathrm{D}$} \\
\hline triplepoint 1s 420x180 & 0.215 & 0.322 & 0.0676 & 0.0071 & 0.105 \\
\hline triplepoint $1 \mathrm{~s} 518 \times 222$ & -0.071 & 0.310 & 0.0856 & 0.0061 & 0.071 \\
\hline triplepoint $2 \mathrm{~s} 420 \times 180$ & -0.031 & 0.311 & 0.165 & 0.0138 & 0.084 \\
\hline triplepoint 2s 518x222 & 0.097 & 0.308 & 0.186 & 0.0163 & 0.088 \\
\hline doublebar $0.5 \mathrm{~s} 200 \times 100$ & 0.074 & 0.306 & 0.5915 & 0.0163 & 0.027 \\
\hline doublebar $0.5 \mathrm{~s} 214 \times 107$ & 0.091 & 0.301 & 0.5950 & 0.0121 & 0.020 \\
\hline doublebar 1s 200x100 & -0.177 & 0.300 & 0.5768 & 0.0319 & 0.055 \\
\hline doublebar 1s $214 \times 107$ & -0.109 & 0.301 & 0.6146 & 0.0411 & 0.067 \\
\hline hydro_toro_a & -0.104 & 0.300 & 116.70 & 6.0177 & 0.051 \\
\hline hydro_toro_b & -0.994 & 0.300 & 1902.3 & 104.34 & 0.055 \\
\hline \multicolumn{6}{|l|}{$3 \mathrm{D}$} \\
\hline triplepoint 1s 420x180x3 & 0.067 & 0.300 & 34.048 & 21.587 & 0.634 \\
\hline triplepoint $2 \mathrm{~s} 420 \times 180 \times 3$ & -0.157 & 0.300 & 74.5847 & 44.388 & 0.595 \\
\hline doublebar $0.5 \mathrm{~s} 200 \times 100 \times 3$ & 0.043 & 0.300 & 134.47 & 26.025 & 0.193 \\
\hline doublebar 1s 200x100x3 & -0.159 & 0.300 & 122.24 & 44.576 & 0.365 \\
\hline \multicolumn{6}{|l|}{ With our algorithm (Figure $18-d$ ) } \\
\hline asteroid & -0.13 & 0.200 & 319.874 & 31.148 & 0.097 \\
\hline balldrop_10 & & 0.274 & 41.426 & 5.5029 & 0.133 \\
\hline balldrop_15 & & 0.209 & 35.243 & 6.3432 & 0.18 \\
\hline balldrop_20 & & 0.221 & 35.824 & 18.149 & 0.506 \\
\hline balldrop_25 & & 0.200 & 75.346 & 39.089 & 0.519 \\
\hline
\end{tabular}
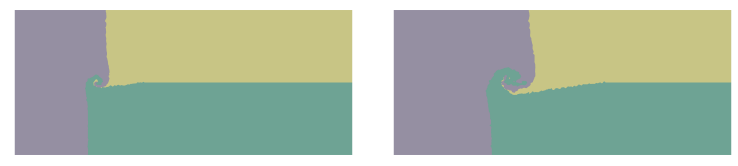

(a) $t=1 \mathrm{sec}$

(b) $t=2 s e c$
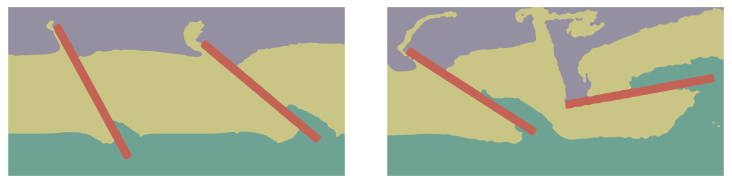

(c) $t=0.5 \mathrm{sec}$

(d) $t=1 \mathrm{sec}$

Figure 19: Examples of CFD simulations in 2D. Our algorithm was also applied to 3D cases extruded from the 2D. ( $a$ and $b$ ) triple point problem where three fluids of different densities lead to the formation of a vertex; ( $c$ and $d$ ) double bar problem where three fluids of different densities are stirred by two rotating blades.

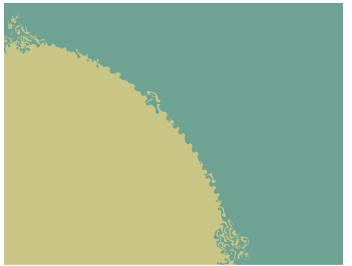

(a)

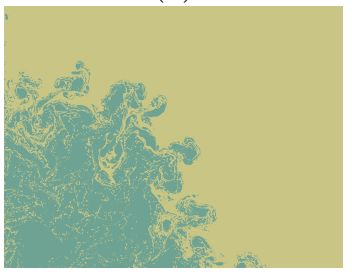

(c)

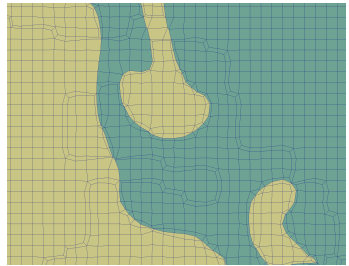

(b)

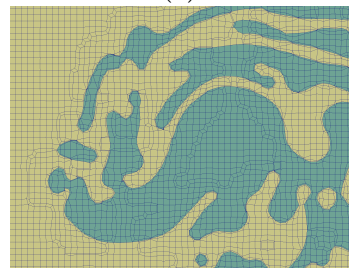

(d)
Figure 20: Other examples of hydrodynamics simulations in 2D [18]. $(a)$ and $(c)$ the two cases; $(b)$ and $(d)$ close-ups on our resulting meshes shown respectively. 


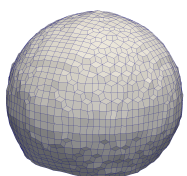

(a) time step 10

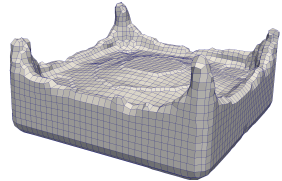

(c) time step 20

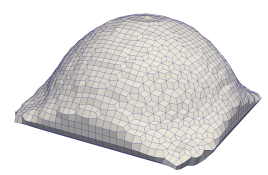

(b) time step 15

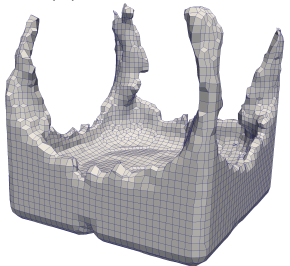

(d) time step 25
Figure 21: Resulting mesh from our algorithm applied to the balldrop case.

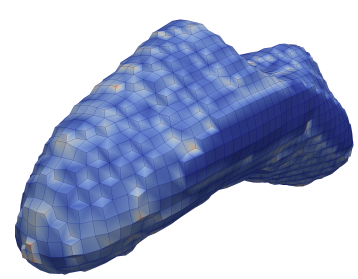

(a)

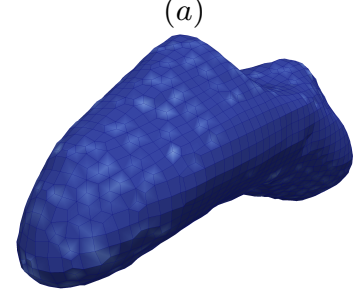

(c)

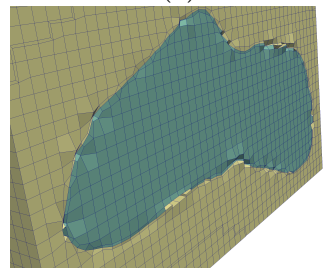

(e)

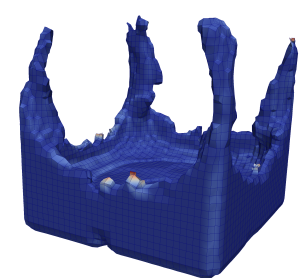

(b)

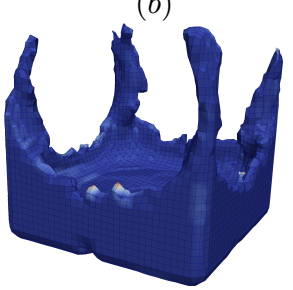

(d)

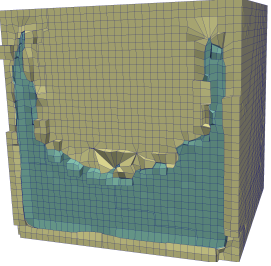

$(f)$
Figure 22: 3D cases measuring the impact of our algorithm. ( $a$ and $c$ ) the distance in the asteroid case between the interface nodes after the first controlled move and their computed position, and the same after applying our whole algorithm; ( $b$ and $d$ ) the same for the balldrop case at step 25; ( $e$ and $f$ ) a clipped view of both cases in order to exhibit the exterior.

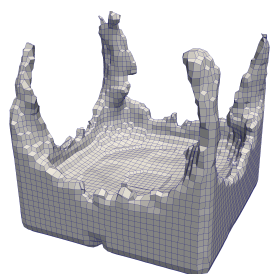

(a) $n$ bCells $=9548$

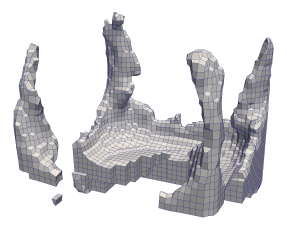

(b) $n b$ Cells $=4115$
Figure 23: Cavity downsizing for the pillow on the balldrop case at step 25. (a) first cavity on which the pillow was tried for the fluid material, it is practically the whole fluid; (b) cavity where the pillow and smoothing phases managed to produce a submesh of acceptable quality that was inserted back into the mesh.

of material $m$. It basically expresses the difference between the input volume fractions and the ones computed by imprinting our output mesh onto the initial grid $\mathcal{G}$. The discrepancy is simply the sum of the term computed in eq 5 for all the cells of $\mathcal{G}$.

We compared our approach with Sculpt (using Cubit $v 15.4 b)$ and Sculpt with an active contouring add-on developed in [21] aiming to reduce the discrepancy; the results can be seen in Table 2 . We do not claim to be exhaustive in testing Sculpt, which has many options to drive the mesh generation.

We can see that our method fits better the volume fractions, even after applying the add-on. It could be explained by the fact that in order for Sculpt to return an output mesh with no negative scaled Jacobian elements in those cases the defeaturing [11] option was activated, favoring mesh quality by sacrificing the volume fractions preservation (see the impact in Figure 24 where some small clusters of material were wiped out). Even with this option on, we were not able to obtain a mesh without inverted elements in the double bar $1.0 \mathrm{~s}$ example. In Figure 25 we can see that an agressive smoothing makes the rotating bar loose its shape.

We can also see that our robustness comes at a price, our method being approximately twenty times slower (Sculpt was run on a single MPI process, and our algorithm on a single thread). In Table 2 is shown that for the doublebar cases, which have the same grid size, the execution time varies significantly: our method is not only sensitive to the grid size, but also to the carried data.

\section{CONCLUSION AND FUTURE WORKS}

In this work, we have improved on a straightforward overlay grid algorithm to make it compatible with the requirements of the "Euler to Lagrange" context 
Table 2: Discrepancy and execution time comparison with Sculpt, Sculpt + add-on and our approach.

\begin{tabular}{lllll}
\hline case name & metric & Sculpt & Sculpt+ add-on & our algo \\
\hline triplepoint 1s 420x180x3 & discrepancy & 583.983 & 248.594 & 205.699 \\
& time (s) & 24.57 & 505.2 & 433.458 \\
triplepoint 2s 420x180x3 & discrepancy & 1204.86 & 513.38 & 540.6 \\
& time (s) & 26.5 & & 270.238 \\
doublebar 0.5s 200x100x3 & discrepancy & 852.687 & 351.924 & 187.9 \\
& time (s) & 9.14 & fail & 546.159 \\
doublebar 1.0s 200x100x3 & discrepancy & fail & & 334.8 \\
& time (s) & 84 & & \\
\hline
\end{tabular}

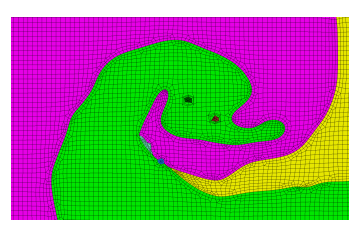

(a)

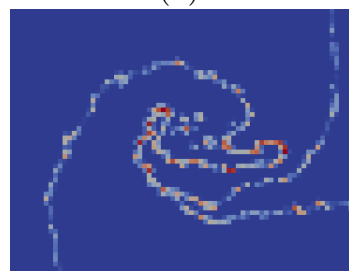

$(c)$

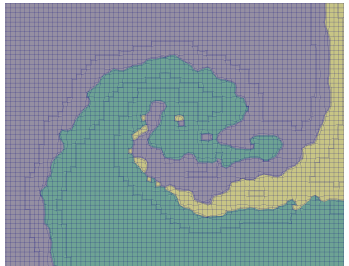

(b)

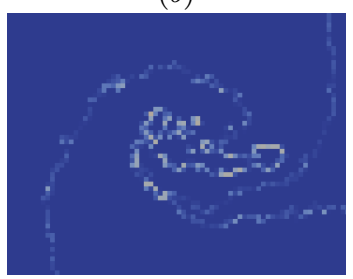

(d)
Figure 24: Comparison for the triplepoint 2sec case. (a) and $(b)$ output meshes from Sculpt and our method; $(c)$ and $(d)$ their respective per-cell discrepancy.

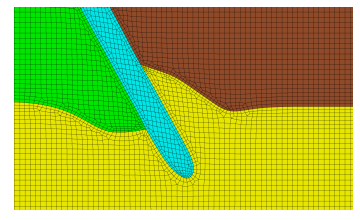

$(e)$

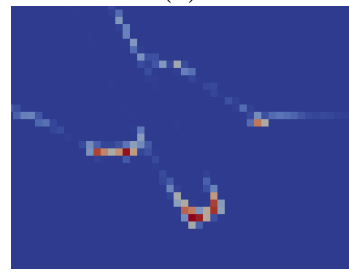

(g)

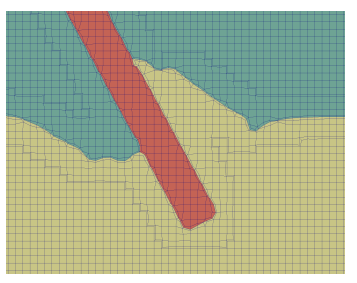

$(f)$

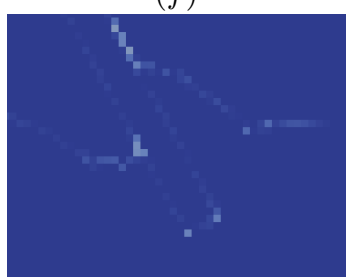

(h)
Figure 25: Comparison for the doublebar $0.5 \mathrm{sec}$ case. (a) and (b) output meshes from Sculpt and our method; $(c)$ and $(d)$ their respective per-cell discrepancy. by means of being able to control cell mesh quality to make the generated mesh a valid input for a Lagrangian mesh. While the method can still be improved, it reaches the aim of keeping the cell quality above an acceptable threshold. It was made possible by controlling node movement and introducing locality during pillowing.

Our pipeline is robust, but in order to improve the results further, the mesh adaptation pipeline will be simplified in the short term in order to be a more traditional loop adaptation process as it is done for triangular and tetrahedral mesh adaptation. The key point to be added in this process will be a new node movement based on successive simple operations put in an iterative process: node position computation, controlled movement towards the pixelated model, surface and volume smoothing. It will improve upon the "projection and smooth" step done at the beginning of the mesh adaptation for some 3D cases.

As seen on several examples throughout the paper, the pillowing operation can drastically decrease the mesh quality. Detecting configurations where no good pillowing can be applied could help offer a diagnostic on the pillow resulting quality and inform the user that his expected quality threshold will not be reachable, or at the cost of capturing the interfaces very poorly. More investigation of local pillowing will be done.

Eventually, our output mesh is a transformation of the input mesh, keeping the number of cells and their size quite similar. As it is usually expected of a Lagrangian simulation to require fewer cells than an Eulerian simulation for the same accuracy, we might need to coarsen and/or refine the output mesh. This process will use the material assignment and the interface reconstruction results to coarsen and refine the mesh locally before the mesh adaptation steps. Analyzing the inconsistencies between the mesh and the pixelated geometric model (see Figure 26) should provide hints to know where the grid could be refined. 


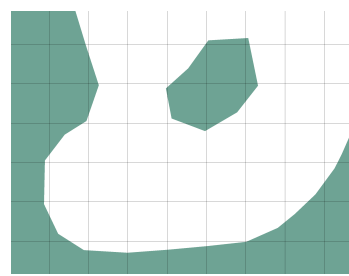

(a)

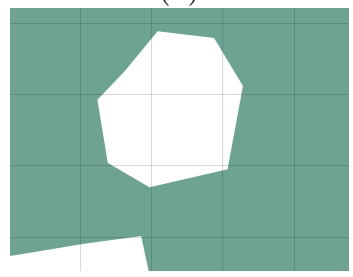

$(c)$

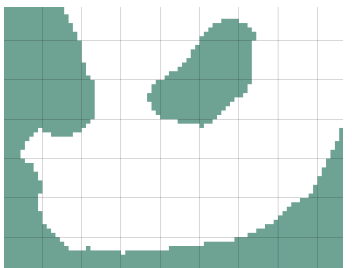

(b)

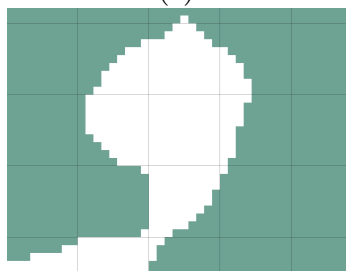

(d)
Figure 26: Topology similarities and differences between our final mesh and the pixelated interfaces on close-up of the hydro_toro_a case; the black wireframe is the original grid mesh carrying volume fractions data. $(a)$ and $(b)$ both return the same topology for the green material; $(c)$ and $(d)$ the topology is not the same.

\section{References}

[1] Schneiders R. "A grid-based algorithm for the generation of hexahedral element meshes." vol. 12 , no. $3,168-177,1996$

[2] Owen S.J., Staten M.L., Sorensen M.C. "Parallel Hex Meshing from Volume Fractions." Engineering with Computers, vol. 30, no. 3, July 2014

[3] Zhang Y.J. Geometric Modeling and Mesh Generation from Scanned Images. CRC Press, 2016

[4] "OpenFoam User Guide, SnappyHexMesh." https://cfd.direct/openfoam/, 2017

[5] Distene. "Volume Meshing: MeshGems-Hexa." http://www.meshgems.com, 2017

[6] Anderson J.C., Garth C., Duchaineau M.A., Joy K.I. "Discrete Multi-Material Interface Reconstruction for Volume Fraction Data." vol. 27, no. 3, 1015-1022, 2008

[7] Anderson J.C., Garth C., Duchaineau M.A., Joy K.I. "Smooth, Volume-Accurate Material Interface Reconstruction." vol. 16, no. 5, 802-814, 2010

[8] Kucharik M., Garimella R.V., Schofield S.P., Shashkov M.J. "A comparative study of interface reconstruction methods for multi-material ALE simulations." Journal of Computational Physics, vol. 229 , no. 7, 2432-2452, 2010
[9] Freitag L.A. "On Combining Laplacian And Optimization-Based Mesh Smoothing Techniques." Trends in Unstructured Mesh Generation, pp. 37-43. 1997

[10] Mitchell S.A., Tautges T.J. "Pillowing Doublets: Refining A Mesh To Ensure That Faces Share At Most One Edge." proceedings of the 4 th International Meshing Roundtable, pp. 231-240. Sandia National Laboratories, October 1995

[11] Owen S.J., Brown J.A., Ernst C.D., Lim H., Long K.N. "Hexahedral Mesh Generation for Computational Materials Modeling." vol. 203, 167-179, 2017

[12] Hege H.C., Seebass M., Stalling D., Zöckler M. "A Generalized Marching Cubes Algorithm Based on Non-Binary Classifications." Tech. Rep. SC-97-05, ZIB, Takustr. 7, 14195 Berlin, 1997

[13] GLPK. "GLPK (GNU Linear Programming Kit)." https://www.gnu.org/software/glpk/, 2018

[14] Boykov Y., Veksler O., Zabih R. "Fast approximate energy minimization via graph cuts." vol. 23, no. 11, 1222-1239, 2001

[15] Boykov Y., Kolmogorov V. "An experimental comparison of min-cut/max- flow algorithms for energy minimization in vision." vol. 26, no. 9, 1124-1137, 2004

[16] Kolmogorov V., Zabin R. "What energy functions can be minimized via graph cuts?" vol. 26 , no. 2 , 147-159, 2004

[17] Knupp P. "Algebraic mesh quality metrics." SIAM J. Sci. Comput., vol. 23, no. 1, 193-218, 2001

[18] Toro E. "Riemann Solvers and Numerical Methods for Fluid Dynamics: A Practical Introduction." Riemann Solvers and Numerical Methods for Fluid Dynamics. 2009

[19] Guy R. "A PIC/FLIP fluid simulation based on the methods found in Robert Bridson's "Fluid Simulation for Computer Graphics": rlguy/GridFluidSim3D." URL https://github.com/rlguy/GridFluidSim3D

[20] Owen S.J., Shelton T.R. "Evaluation of gridbased hex meshes for solid mechanics." Engineering with Computers, vol. 31, 5-29, 2015

[21] Le Goff N., Ledoux F., Owen S. "Hexahedral mesh modification to preserve volume." vol. 105, $42-54$ 


\title{
REVIVING THE SEARCH FOR OPTIMAL TETRAHEDRALIZATIONS
}

\author{
Célestin Marot $^{1} \quad$ Kilian Verhetsel $^{1} \quad$ Jean-François Remacle ${ }^{1}$ \\ ${ }^{1}$ Université catholique de Louvain, iMMC, Avenue Georges Lemaitre 4, bte L4.05.02, 1348 \\ Louvain-la-Neuve, Belgium
}

\begin{abstract}
This paper revisits a local mesh modification method known as the Small Polyhedron Reconnection (SPR) [1]. The core of the SPR operation is a branch and bound algorithm which computes the best 3D triangulation (tetrahedralization) of a polyhedron through an efficient exploration of the set of all its triangulations. The search can accomodate for additional geometric constraints and will inevitably find the highest quality triangulation of the polyhedron if a triangulation exists. This paper focuses on the design of an optimized SPR operator and its application to improving the quality of finite element meshes. Compared to the original algorithm, a speed-up of 10 million is obtained by changing the heuristics determining the search space exploration order. This enables the integration of the SPR operator into standard mesh generation procedures. We show quality improvements obtained by applying this operation to meshes that have already been optimized using smoothing and edge removal techniques.
\end{abstract}

Keywords: SPR, reconnection, mesh generation, topological transformation, optimal triangulation

\section{INTRODUCTION}

Unstructured tetrahedral mesh generation generally begins by creating a surface mesh of a given geometric model. Afterwards, a tetrahedral mesh constrained by the surface mesh is created using one of the few available 3D mesh generation algorithms: advancing front, Delaunay or octree-based techniques. Unfortunately, all of those 3D mesh generation methods tend to produce elements (tetrahedra) that are not readily suitable for finite element computations. For example, the Delaunay tetrahedralization doesn't exclude the creation of nearly flat tetrahedra, called slivers, that cause tremendous numerical errors. It is possible to detect slivers through various quality measures [2]. Any tetrahedral mesh generation procedure is followed by a so-called mesh improvement step that aims at optimizing the overall quality of the mesh, which is mainly driven by the quality of its worst element.

Mesh improvement methods can be classified into two categories: vertex relocation (or smoothing) methods and topological transformations. Smoothing is the act of modifying the coordinates of vertices without changing the connectivity of the mesh. On the other hand, topological transformations treat the mesh as a graph in which the vertex coordinates are fixed inputs.

Smoothing methods have been extensively studied in the past 25 years $[3,4,5,6]$. The objective of mesh smoothing is twofold: obtain a better overall quality and space out vertices harmoniously so that subsequent topological transformations improve the mesh. Indeed, smoothing and topological transformations are most effective when combined [7]. While most smoothing methods optimize the mesh directly and are thus dependent of the current triangulation, some methods are able to find good placement for points relatively independently of the triangulation, using moving mesh partial differential equations or central Voronoi tesselations $[8,6,9]$.

In this paper, a good distribution of points, obtained using one or a combination of these smoothing techniques, is assumed. The main focus of this paper is 
thus on the improvement of the efficiency of topological transformations. Topological transformations operates on cavities, which are the polyhedral holes formed by the removal of a face-connected subset of tetrahedra. In 3D, the most simple cavity has four vertices, and only one possible tetrahedralization. The most basic operations, called bistellar flips, operates on a cavity of five vertices formed by the removal of two or three tetrahedra. These are the 2-3 and 3-2 flips illustrated on Figure 1. These simple local reconnection schemes are effective at removing most lowquality tetrahedra from the mesh.

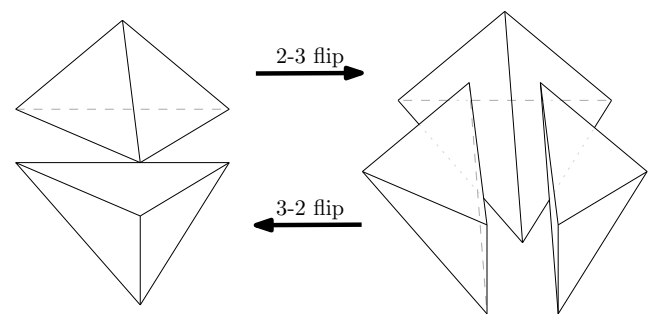

Figure 1: Two bistellar flips, namely the 2-3 and 3-2 flips, consisting in switching between two triangulations of a triangular bipyramid.

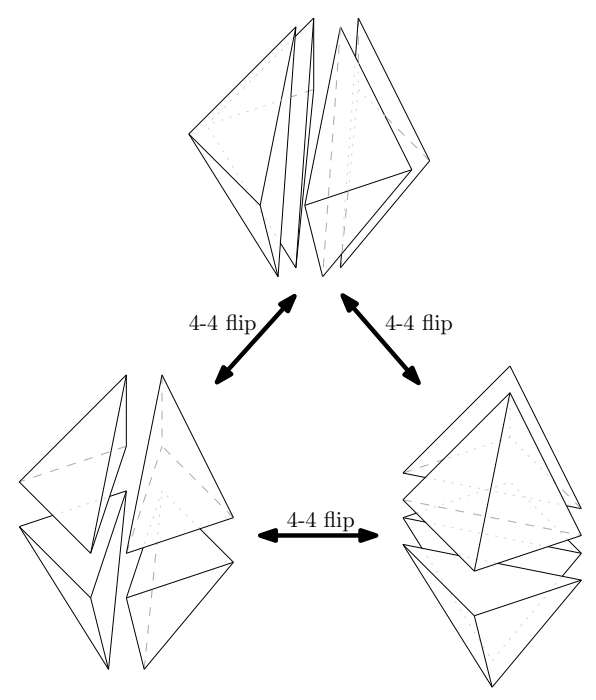

Figure 2: The 4-4 flip retriangulates an octahedron by changing the edge around which the four tetrahedra are placed.

When applying bistellar flips in a hill-climbing manner, meaning a flip is only performed if it improves the overall quality, one often reaches local minima where no bistellar flip can possibly improve the quality. To overcome this problem, increasingly complex methods have been implemented. A 4-4 flip (Figure 2) can be

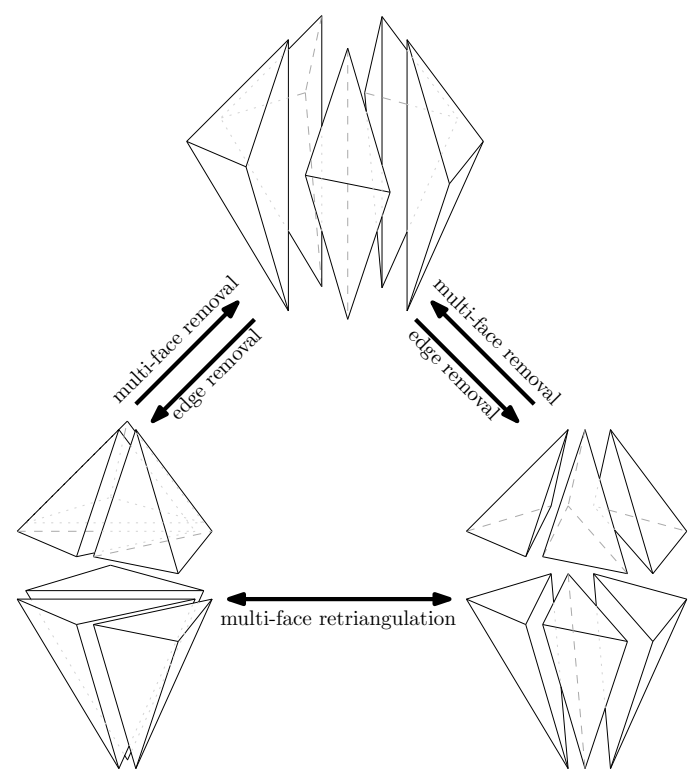

Figure 3: Edge removal, a more general operation than the 3-2 flip, replaces the cavity around an edge by choosing a triangulation of the vertices that are not on the edge.

obtained by a combination of a 2-3 and a 3-2 flip [10]. Therefore, adding this operation to the basic bistellar flips enables new optimizations that a hill-climbing technique would not find when the first 2-3 flip does not increase the mesh quality.

A more general transformation, called edge removal, edge swap or $n$-to- $m$ flip, operates on a cavity formed by the the set of tetrahedra surrounding an edge $[11,12]$ (Figure 3 ). The vertices that are not the endpoints of the edge form an annulus around that edge. For each $2 \mathrm{D}$ triangulation of that annulus, we can create a 3D triangulation of the cavity by linking each triangle to both end points of the edge. The edge removal operation considers all these possibilities and chooses the best one if it increases the quality of the current mesh. This operation supersedes the 3-2 and 4-4 flips and can also be implemented robustly as a series of bistellar flips $[12,10]$. The inverse of edge removal is called Multi-face removal [12]. It is less useful than edge removal in practice, and is particularly tedious to implement. Multi-face retriangulation, which is a combination of multi-face removal and edge removal can be used to overcome a valley in the objective function in some cases where multi-face removal cannot [13](Figure 3).

However, even with this large repertoire of topological operation, mesh improvement is still subject to 
local minima $[12,13]$. Instead of adding more and more operations to this zoo of topological transformation, it is possible to use an operation that generalizes all of them, called the small polyhedron reconnection (SPR) [1]. This operation considers the problem of finding the optimal triangulation of a cavity. A cavity, in this context, is a set of volumes defined by constrained closed surfaces, with possible interior constrained vertices, edges and triangles. The core idea of the SPR algorithm is to compute the best triangulation by searching the set of all possible triangulations using a branch and bound algorithm. The cavity is then replaced using its highest-quality triangulation to contain all constrained triangles and edges. This method is highly flexible and independent of the chosen quality measure. However, using the SPR operation in practice presents great technical challenges and subsequent mesh improvement procedures implemented in Tetgen [10], CGAL [14, 15] or MMG3D [16] do not use this method. Indeed, the performance of the SPR search algorithm varies dramatically based on the parameters and heuristics that it relies upon. A poor choice makes the algorithm completely inadequate for large meshes, which can now be created at a rate of several million tetrahedra per second [17]. The main contribution of this paper is to present efficient heuristics and implementation strategies that enable the use of SPR to optimize large meshes.

We propose improvements to the SPR algorithm by exploring the space of possible triangulations in a different order, aiming at a significant reduction to the number of triangulations that need to be considered during the search (Section 2.2). Repeated computations of expensive quality measures are also avoided by storing their results (Section 2.4). These choices affect the time taken to optimize cavities by several orders of magnitudes (Section 2.5). The optimized SPR procedure that is proposed has been integrated into our mesh improvement framework. The benefits of our approach are shown by applying it to large meshes (section 3).

\section{QUICKLY COMPUTING OPTIMAL TRIANGULATIONS}

The core of our method is an efficient algorithm to search for the best triangulation of a small polyhedral cavity $\mathcal{C}$. Let $\mathcal{T}(\mathcal{C})$ denote the set of all triangulations of this cavity. The best triangulation is defined as the triangulation of $\mathcal{C}$ that maximizes the quality of its worst element according to a quality measure $q$ :

$$
T_{\text {opt }}=\underset{T \in \mathcal{T}(\mathcal{C})}{\arg \max } \min _{t \in T} q(t)
$$

The set of triangulations considered by the algorithm

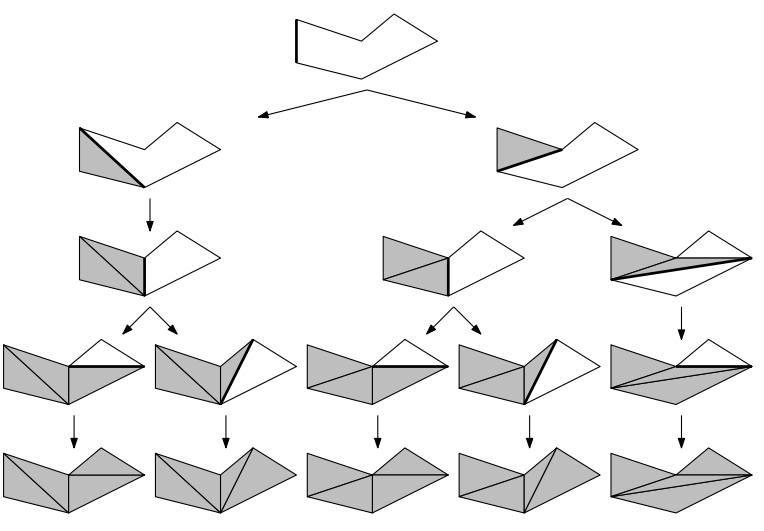

Figure 4: Enumerating the triangulations of a polygon. At each step, a boundary edge is picked, and a branch is created for each possible triangle that contains that edge. The untriangulated area remaining after a triangle insertion is filled by applying this procedure recursively.

may be restricted to only those that contain a given set of edges and triangles if certain features must be preserved.

An optimal triangulation is computed using a branch and bound algorithm (Algorithm 1). Starting from the boundary $\partial \mathcal{C}$ of the cavity $\mathcal{C}$ as input, a triangle $F \in \partial \mathcal{C}$ is selected. Any mesh of the cavity $\mathcal{C}$ must include a tetrahedron $t$ that contains the triangle $F$. Each possible tetrahedron is considered, by branching on all possible choices for its fourth vertex. After inserting a new tetrahedron, the boundary $\partial \mathcal{C}$ is replaced by the boundary of the part of the cavity which has not yet been filled with tetrahedra (see Figure 4). The best mesh of this new cavity is computed by recursively applying the algorithm. This corresponds to the exploration of a tree whose nodes correspond to triangulations and whose edges correspond to the insertion of tetrahedra.

Throughout this process, the best triangulation found so far is tracked, as well as its quality $q^{*}$. After each branch, an upper bound on the quality of the best solution that could be obtained is computed by finding the minimum quality element that has already been added to the solution. If the upper bound is worse than $q^{*}$, this part of the search tree is skipped. By the end of the algorithm, the optimal triangulation $T_{\mathrm{opt}}$ will have been found.

The rest of this section discusses important design choices in order to achieve an efficient algorithm:

1. for a given triangle, how to compute the set of tetrahedra that can be built on top of it;

2. the selection of the triangle $F$ on top of which the 
next tetrahedron should be built;

3. the order in which the tetrahedra containing $F$ are considered for insertion into the mesh;

4. how to avoid repeated evaluations of expensive geometric predicates.

Input: $B$ : the target boundary;

$T$ : a partial triangulation;

$T^{*}$ : the best triangulation found;

$q$ : a quality function

Output: The best triangulation of the cavity

if $B=\emptyset$ then return $T$;

$F \leftarrow$ some triangle of $B$;

foreach vertex $v$ do

$t \leftarrow$ the tetrahedron formed by joining $v$ to each vertex of $F$;

if $q(t)>\min _{t^{\prime} \in T^{*}} q\left(t^{\prime}\right)$ then

$$
T^{\prime} \leftarrow T \cup\{t\} ;
$$$$
B^{\prime} \leftarrow B-\partial t
$$$$
T^{*} \leftarrow \text { Optimize-Cavity }\left(B^{\prime}, T^{\prime}, T^{*}, q\right) \text {; }
$$

end

end

return $T^{*}$;

Algorithm 1: Optimize-Cavity: search for the best triangulation with a given boundary.

\subsection{Computing candidate tetrahedra}

At each branching step, the algorithm considers a triangular face $F$ before trying to add each tetrahedron $t$ that contains $F$ to the current triangulation. Because three of the vertices of $t$ must be the vertices of $F$, only one vertex needs to be chosen. Many of those choices need to be filtered out, because inserting the corresponding tetrahedra would result into an invalid triangulation. Below are the conditions used to eliminate such candidates.

Geometric validity Each candidate tetrahedron $t$ must have a positive orientation and a positive quality $q(t)$ greater than the quality $q^{*}$ of the best triangulation found so far.

Geometric intersections The solution must not include intersecting tetrahedra. The new faces and edges of each candidate $t$ are tested for intersection with the edges and triangles of the boundary of the unmeshed region as well as with constrained features. Tetrahedra that completely enclose a vertex are also rejected. All intersection tests are performed exactly by relying only on exact computations of the orientations of tetrahedra.

\subsection{Mesh construction order}

Changing the order in which tetrahedra are inserted into the mesh drastically affects the number of triangulations that need to be considered by the algorithm. This behavior is common in difficult optimization problems $[18,19]$. Heuristics are used to choose a favorable order for most cases.

First, a triangular face $F$ must be selected from the boundary of the unmeshed region. The algorithm then branches on the set of all tetrahedra containing $F$ that can be added to the current triangulation. Faces are selected by attributing a cost to each of them. This cost is computed by summing the number of candidate tetrahedra containing the face and their qualities. A lower cost means either fewer candidates, hence a smaller search tree, or worse candidates, hence a tighter upper bound allowing for more pruning.

\subsection{Ordering candidate tetrahedra}

Once the face $F$ has been selected, a second heuristic defines the order in which the different candidate tetrahedra are inserted into the mesh. If a good solution is found early, subtrees that provably cannot contain a better solution need not be explored. Hence, candidate tetrahedra are evaluated based on criteria used to determine how likely they are to be part of a good solution:

1. the number of faces shared with the boundary, since cavities with fewer boundary faces are generally easier to fill;

2. whether or not the candidate has a higher quality than the tetrahedra that have already been inserted into the mesh;

3. whether or not the candidate contains a new vertex, since any solution must contain all vertices present in the cavity.

Each tetrahedron is given a score based on the number of criteria that it meets. Candidates with a higher score are tested first.

\subsection{Reusing results of geometric predi- cates}

During the search, the orientations and qualities of many tetrahedra need to be evaluated. A robust algorithm requires these to be evaluated using adaptive precision in order to obtain consistent results despite numerical errors. As a result, these evaluations are computationally intensive. This effect is compounded by the need to evaluate the same tetrahedra many 
times, if it is considered as a candidate at multiple occasions during the search.

Computing the qualities of all $\left(\begin{array}{l}n \\ 4\end{array}\right)$ tetrahedra of an $n$-vertex point ahead of time would avoid repeated computations, but this solution is inadequate: the orientations and qualities of some tetrahedra are never needed. In many cases, the search ends early after only evaluating a small fraction of all possible tetrahedra.

Instead, our approach is to memoize the computation of quality values. To evaluate a tetrahedron $T$, it is first normalized as $T^{\prime}$ by sorting the indices of its four vertices. While sorting, the parity of the number of permutations that were performed is tracked. A table is then accessed to test whether or not the quality of $T^{\prime}$ is known. If not, it is computed and stored in the table. The quality of $T$ is the same as that of $T^{\prime}$ for an even number of permutations, and the opposite otherwise.

In addition, if the tetrahedron intersects a constrained edge or fully encloses a vertex, a null quality is stored in the look-up table. This prevents the insertion of these tetrahedra into the mesh without requiring the reevaluation of the intersection tests.

\subsection{Performance results}

The SPR algorithm can be used with any chosen quality measure. For the purpose of this analysis we use

$$
\gamma=\frac{\sqrt{24} 3 V}{\left|e_{\max }\right|\left(A_{1}+A_{2}+A_{3}+A_{4}\right)}=\frac{\sqrt{24} r_{i n}}{\left|e_{\max }\right|},
$$

where $V$ is the volume of the tetrahedron, $\left|e_{\max }\right|$ is the length of the longest edge, $A_{i}$ is the area of the $i$ th face and $r_{i n}$ is the inradius of the tetrahedron. The factor $\sqrt{24}$ is added such that the optimal tetrahedron, which is a regular tetrahedron, has a quality $\gamma=1$. This quality measure penalize all tetrahedra according to their associated interpolation error [2].

For each cavity featured in Figure 5, we measured the running time of the SPR operation (Table 1). All 5 cavities were extracted from real-world, non-optimized meshes. We measured the running time in two different settings:

1. with no initial lower bounds given to the algorithm, as is the case when SPR is used for boundary recovery and no initial triangulation is known;

2. with the quality of the initial triangulation $\gamma_{\text {orig }}$ as a lower bound, as is done for mesh optimization.

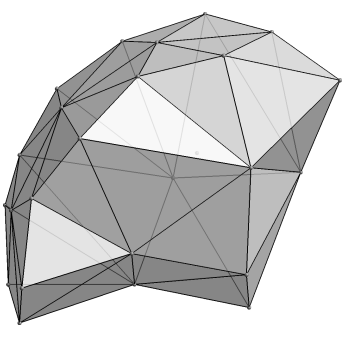

1

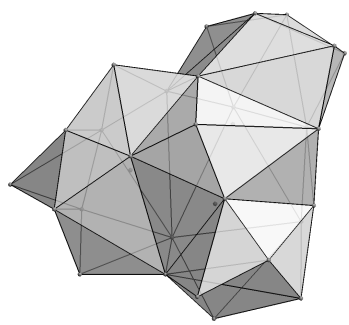

3

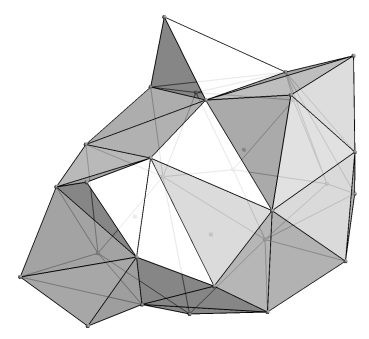

2

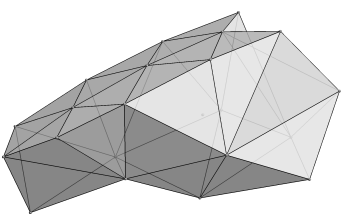

4

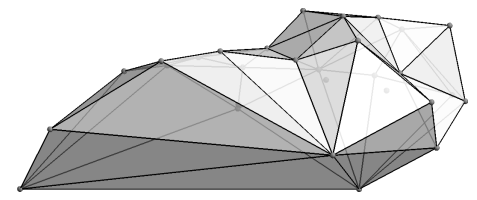

5

Figure 5: Different cavities on which the tests were conducted. № 1,3 and 4 were randomly extracted from the mesh improvement procedure of a torus or sphere. № 2 looks ordinary but is particularly slow without cleverly chosen heuristics, whereas № 5 comes from a mesh with bad elongated triangles on its surface.

The execution time in the second case is always strictly less than in the first, because the lower bound allows the algorithm to prune triangulations containing a tetrahedron with a quality worse than $\gamma_{\text {orig. All cav- }}$ ities were optimized within 3.6 milliseconds when the lower bound was used, and within 13.3 seconds otherwise.

We measured the speedups offered by the different improvements to the algorithm by disabling each optimization independently:

1. the face selection heuristic (Section 2.2);

2. the candidate ordering heuristic (Section 2.3);

3. the reuse of previously computed qualities of tetrahedra (Section 2.4).

The combined effect of these improvements was measured by disabling all of them simultaneously. The improved algorithm is between $10^{4}$ and $10^{7}$ times faster 


\begin{tabular}{lllllccllll} 
Cavity & $V$ & $|\partial C|$ & \multicolumn{2}{c}{ Quality } & \multicolumn{2}{c}{ Time $(\mathrm{ms})$} & \multicolumn{2}{c}{ Speedup } \\
& & & $\gamma_{\text {orig }}$ & $\gamma_{\text {after }}$ & $q(t)>0$ & $q(t)>\gamma_{\text {orig }}$ & Section 2.2 & Section 2.3 & Section 2.4 & Combined \\
\hline 1 & 25 & 44 & 0.28 & 0.52 & 3.1 & 2.5 & 25 & 1.4 & 0.68 & $4 \times 10^{4}$ \\
2 & 31 & 52 & 0.31 & 0.53 & 13.3 & 3.0 & $3.5 \times 10^{4}$ & 3.1 & $5 \times 10^{4}$ & $3 \times 10^{6}$ \\
3 & 31 & 52 & 0.25 & 0.51 & 9.6 & 3.6 & $10^{6}$ & 10 & 2072 & $>10^{7}$ \\
4 & 22 & 38 & 0.23 & 0.55 & 4.8 & 1.0 & 501 & 2.6 & 0.97 & $2 \times 10^{4}$ \\
5 & 29 & 48 & 0.29925 & 0.29938 & 7.3 & 1.3 & $5 \times 10^{4}$ & 1.1 & 1.5 & $8 \times 10^{4}$
\end{tabular}

Table 1: Summary of the results obtained by optimizing the cavities of Figure 5 using SPR. Speedups are given for $q(t)>\gamma_{\text {orig. }}$

than a naive implementation. In the case of the third cavity, the execution with all optimization disabled was stopped after 20 hours, and no improved solution found (Table 1).

All experiments were performed on an Intel ${ }^{\circledR} \mathrm{Core}^{\mathrm{TM}}$ i7-6700HQ CPU. Timings and speed ups are the average of 100 runs, or of two runs for entries that required more than one minute of computation time. Our implementation uses only a small amount of memory, although asymptotically proportional to $n^{4}$ were $n$ is the number of points of the cavity. For the cavities tested, the maximum RAM usage did not exceed $3 \mathrm{MB}$.

\section{APPLICATION TO MESH IMPROVEMENT}

Computing the optimal triangulation of a cavity is an expensive operation. When optimizing large meshes, this operator should be used alongside other mesh improvement techniques in order to achieve good performances. The optimization framework we proposes combines SPR with mesh smoothing and edgeremoval.

Mesh smoothing We use Laplacian smoothing, improved by a golden-section search on the segment between the original position and the average of neighboring vertices. This approach is effective in practice even though the objective function is not unimodal. Freitag et al. give a review of this combined Laplacian and optimization-based technique [4].

Edge removal The edge removal technique used here is based on tables containing all triangulations of the disk with up to seven points. We iterate through each triangulation in order to determine the most appropriate one.

Using SPR Throughout the optimization process, a list of low-quality tetrahedra is maintained. The threshold on the value of $\gamma$ that determines what is a "low-quality" tetrahedron is given as a parameter to our program. A threshold of 0.5 was used for all the results given in this paper. We first attempt to improve each bad tetrahedron using mesh smoothing and edge removal, as these techniques are much faster than SPR. SPR is then used to optimize cavities that surround the low quality tetrahedra that remain after applying other optimization techniques.

The cavity used to optimize a given tetrahedron is obtained by selecting all tetrahedra that contain at least one of its vertices ${ }^{1}$. There are about 50 tetrahedra in an average cavity (Figure 6a). To save time, the search only explores the first 2000 nodes of the search tree, since it is rarely beneficial to spend more time on individual cavities.

If the mesh quality is still insufficient after this step, the entire optimization process is repeated. Because of the modifications performed by applying the SPR operator, mesh smoothing and edge-removal may remove some of the remaining bad elements. This is done iteratively until the mesh can no longer be improved.

To avoid optimizing the same cavities multiple times, a modification flag is maintained for each tetrahedron. The SPR operator is only run on a cavity if at least one of its tetrahedra is marked as modified. The new tetrahedra obtained from any of the three optimization techniques are marked as modified. This modification flag is removed after running SPR if the solution has not improved.

SPR efficiency SPR is approximately $1000 \times$ slower than edge removal and smoothing, as Table 2 shows. The first SPR pass has a success rate above $85 \%$. In other words, the first time edge removal and smoothing failed to improve the mesh further, the SPR operation could improve $85 \%$ of bad tetrahedra. The efficiency of each operation decreases after each iteration of the mesh improvement process, until every operation fails for all bad tetrahedra. All iteration combined, SPR improved the mesh $63.1 \%$ of the times. This is particularly impressive considering that

\footnotetext{
${ }^{1}$ An other choice of cavity might be more efficient. We still need to investigate that.
} 


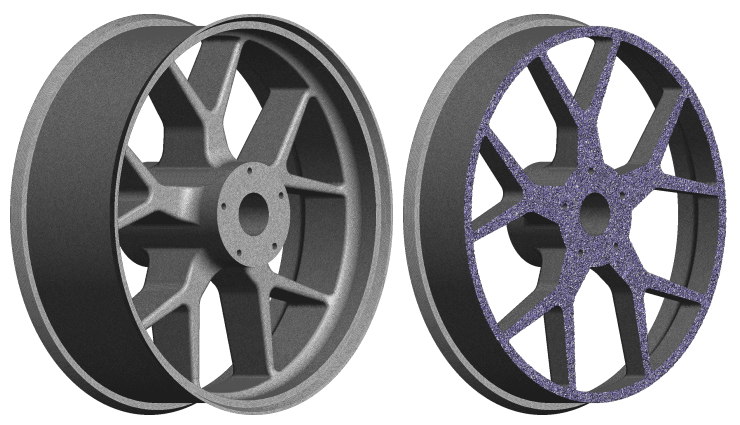

(a) rim mesh: 6285719 tet.
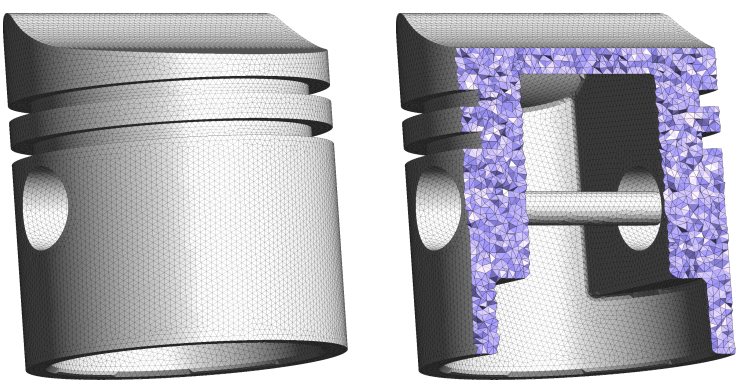

(b) piston mesh: 277648 tet.

Figure 6: Input tetrahedral meshes, both generated with our in-house 3D constrained Delaunay. The geometries come from the CAD models 00000040 and 00009733 from the ABC data set [20]. The surface meshes were generated with gmsh -2 -algo meshadapt -clcurv -clmax 1.5 and gmsh -2 -algo frontal -clscale 0.1 [21] respectively.

$$
\gamma<0.4
$$
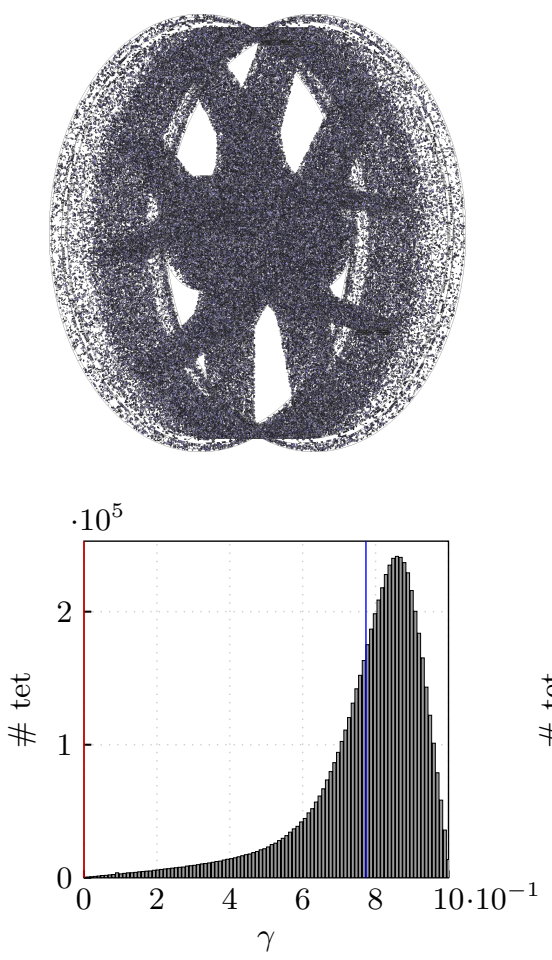

(a) unimproved mesh $\gamma<0.4$
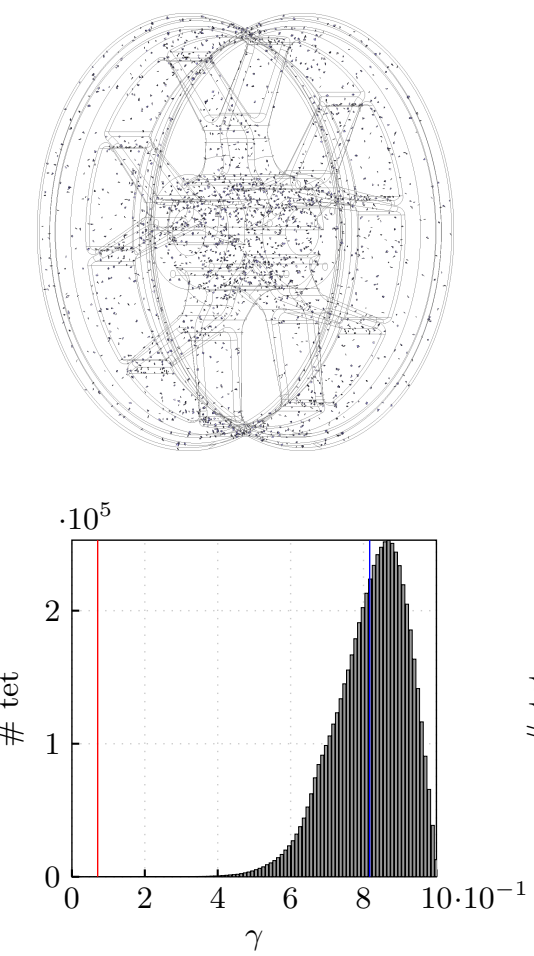

(b) ours without SPR $\gamma<0.4$
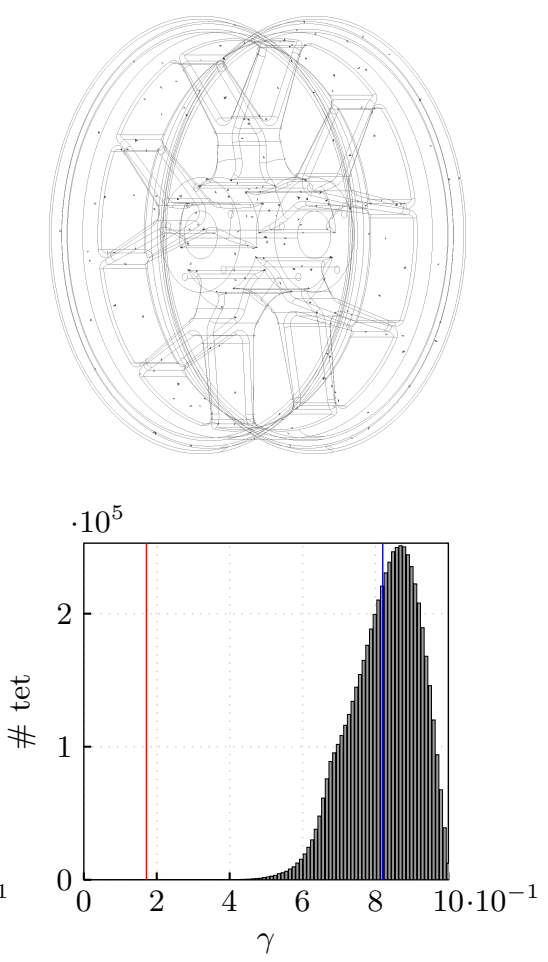

(c) ours

Figure 7: On the first row, tetrahedra with a quality $\gamma<0.4$ are displayed for the rim mesh (Figure 6a). On the second row is an histogram of qualities present in the rim mesh. The red and blue vertical lines indicate the minimum and average qualities respectively. First column correspond to the input mesh, without any mesh improvement. For the second column, only edge removal and smoothing were applied. For the last column, edge removal, smoothing and SPR were applied. 


$\begin{array}{lrrrrr} & \text { number of calls } & \text { total time } & \text { average time per call } & \text { success rate } & \text { cavity size (\# tet) } \\ \text { smoothing } & 5519647 & 33.0 & 6 \times 10^{-6} & 2.5 \% & 26.5 \\ \text { edge removal } & 6297417 & 6.3 & 1 \times 10^{-6} & 7.8 \% & 4.9 \\ \text { SPR } & 103602 & 397.9 & 3.8 \times 10^{-3} & 63.1 \% & 51.2\end{array}$

Table 2: Statistics on the mesh improvement procedure of the rim mesh (Figure 6a). The fourth column shows the proportion of calls that lead to a modification of the mesh. The last column shows the average number of tetrahedra in the cavity before the operation was executed.

\begin{tabular}{lrrrrrr} 
range of $\gamma$ & $0 . .0 .2$ & $0.2 \ldots 0.4$ & $0.4 \ldots 0.6$ & $0.6 . .1$ & total & time $[\mathrm{s}]$ \\
\hline unimproved mesh & 64097 & 197909 & 523801 & 5499912 & 6285719 & 0 \\
ours without SPR & 193 & 3794 & 172435 & 5726705 & 5903127 & 23.4 \\
ours & 14 & 344 & 95614 & 5718753 & 5814725 & 441.5 \\
Stellar & - & - & - & - & - & $>72000$
\end{tabular}

Table 3: Quality distribution of elements in the rim mesh, together with timings of the mesh improvement procedure.

the SPR operation is only used when both edge removal and smoothing are totally ineffective. However, the SPR operation still account for $90.1 \%$ of the total time.

In term of quality improvements, the SPR operation helps removing most bad tetrahedra as can be seen on Figure 7. An implementation based only on edge removal and smoothing still has 3987 tetrahedra with a quality below 0.4 when optimizing the large rim mesh. When using SPR, this number falls to 358 (Table 3). For the smaller mesh of a piston (Figure 6b), the results are similar (Table 4).

Comparison to Stellar Stellar is a tetrahedral mesh improvement program, elaborated by Klingner and Shewchuk in $2009^{2}$. The different methods used within this program are summarized in their paper [22] and best detailed in Klingner's Ph.D. dissertation [23]. To our best knowledge, no other program based on local modifications consistently optimizes meshes as far as Stellar does.

Indeed, Stellar implements many more operations than our simple edge removal and improved Laplacian smoothing [23]. First, Stellar uses a more sophisticated smoothing algorithm based on nonsmooth optimizations introduced by Freitag and Ollivier-Gooch [7]. Second, in addition to edge removal, it implements the inverse operation called multi-face removal ${ }^{3}$ and edge-contraction, also called edge-collapse, which is an operation that removes an edge from the mesh, replacing its two endpoints with a single vertex. The position of the remaining vertex is chosen using their advanced smoothing method. Finally, Stellar uses vertex insertion in combination with all other mesh op-

\footnotetext{
${ }^{2}$ http://people.eecs.berkeley.edu/ jrs/stellar/

${ }^{3}$ multi-face removal alone does not give Stellar any edge over our program because SPR supersedes it.
}

timizations techniques, forming a complex, huge composite operation. In comparison, our algorithm does neither add nor remove any vertex from the mesh. Thanks to edge-contraction and vertex insertion, Stellar gets significantly better results than our program for small meshes. However, while our simple mesh improvement procedure took 7 minutes to optimize the rim mesh shown in Figure 6a, Stellar was still in the first stages of its mesh improvement procedure after 20 hours. We were thus not able to improve large meshes with Stellar. Furthermore, the simplicity of our approach makes the code far simpler to maintain: our whole code is $\approx 2500$ lines long while Stellar is closer to 25000 .

For our tests, we disabled all operations of Stellar that were modifying the surface mesh, leaving only the operations explained previously. We can compare the results for the smaller piston mesh on the histograms given on Figure 8 and with the data in Table 4 . Stellar improves the mesh further but is slower than our program.

\section{CONCLUSION}

Thanks to a careful and efficient implementation, we have shown that the SPR operator can effectively be used to remove most of the low-quality tetrahedra that are left by other mesh improvement operations. Such an efficient implementation is important not only for better mesh improvement methods, but because it enables the use of SPR, a very flexible tool, for a wide range of other applications. Indeed, the SPR can replace any cavity-based topological transformation.

Its use for boundary recovery has already been studied in previous works $[24,25]$. Theoretically, the SPR operation provides a strong guarantee: if a triangulation of a polyhedron contains the required triangles and edges, the SPR operation executed on this polyhedron 


\begin{tabular}{lrrrrrr} 
range of $\gamma$ & $0 . .0 .2$ & $0.2 \ldots 0.4$ & $0.4 \ldots 0.6$ & $0.6 \ldots 1$ & total & time $[\mathrm{s}]$ \\
\hline unimproved mesh & 2758 & 8249 & 21028 & 245613 & 277648 & 0 \\
ours without SPR & 1 & 170 & 7904 & 254553 & 262628 & 0.8 \\
ours & 0 & 27 & 6269 & 253625 & 259921 & 14.5 \\
Stellar & 0 & 10 & 820 & 257193 & 258023 & 263.9
\end{tabular}

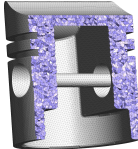

Table 4: Quality distribution of elements in the piston mesh, together with timings of the mesh improvement procedure.

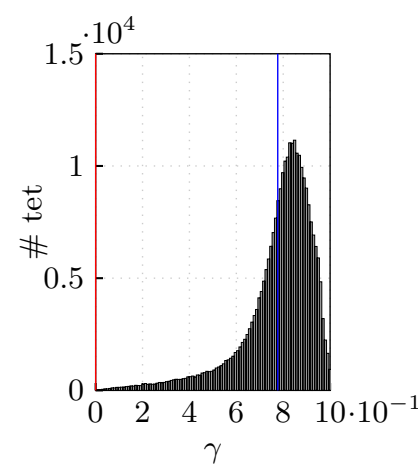

(a) unimproved mesh

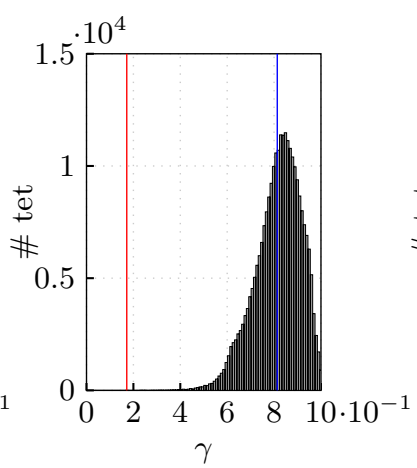

(b) ours w/o SPR

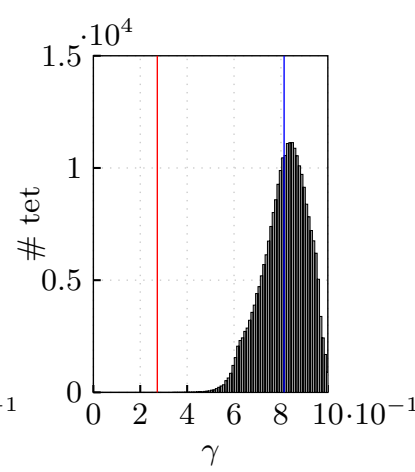

(c) ours

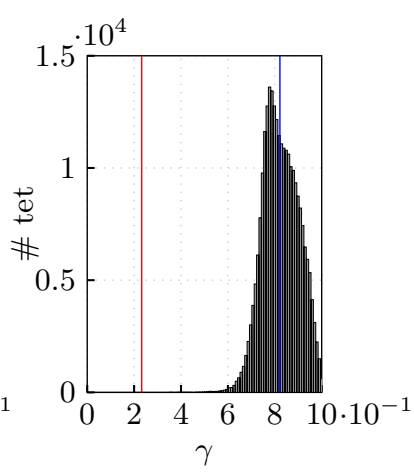

(d) Stellar

Figure 8: Histogram of qualities present in the piston mesh. The red and blue vertical lines indicate the minimum and average qualities respectively.

will inevitably find it. Therefore, using the SPR with a large-enough cavity that contains the missing edges and triangles will allow to recover the right geometry most of the time. The rest of the time, the cavity is not meshable as such. The algorithm can add one or more Steiner points inside the polyhedron in order to make it meshable. The number and the position of Steiner points is a difficult subject. which was, in our opinion, best studied by $\mathrm{Si}$ and Gärtner [26]. It may also be possible to enlarge the cavity, widening the panel of possible triangulations in order to make the polyhedron meshable.

Another valuable use of the SPR operation concerns the removal of vertices. Such an operation is useful for adaptive meshing where parts of the mesh must be dynamically coarsened. Vertex removal can also be used to improve mesh quality, in the same way that vertex insertion is used [22]. SPR makes it easy to find the best triangulation to fill the cavity left after removing a vertex. By comparison, a previous solution generates a suboptimal triangulation by applying the ear-clipping algorithm to the same cavity [27].

A fast implementation of the SPR operator is needed in order to use these techniques in practical meshing software.

\section{ACKNOWLEDGMENTS}

This project has received funding from the European Research Council (ERC) under the European Union's Horizon 2020 research and innovation programme (grant agreement HEXTREME, ERC-2015AdG-694020).

\section{References}

[1] Liu J., Sun S., Wang D. "Optimal Tetrahedralization for Small Polyhedron: A New Local Transformation Strategy for 3-D Mesh Generation and Mesh Improvement." p. 14, 2006

[2] Shewchuk J.R. "What Is a Good Linear Finite Element? - Interpolation, Conditioning, Anisotropy, and Quality Measures." Tech. rep., In Proc. of the 11th International Meshing Roundtable, 2002

[3] Amenta N., Bern M., Eppstein D. "Optimal Point Placement for Mesh Smoothing." Journal of Algorithms, vol. 30, no. 2, 302-322, Feb. 1999. URL http://linkinghub.elsevier.com/ retrieve/pii/S0196677498909841

[4] Freitag L., Jones M., Plassmann P. "A Parallel Algorithm for Mesh Smoothing." SIAM Journal on Scientific Computing, vol. 20, no. 6, 2023- 
2040, Jan. 1999. URL http://epubs.siam.org/ doi/10.1137/S1064827597323208

[5] Freitag L.A., Knupp P.M. "Tetrahedral mesh improvement via optimization of the element condition number." International Journal for $\mathrm{Nu}$ merical Methods in Engineering, vol. 53, no. 6, 1377-1391, Feb. 2002. URL http://doi.wiley. com/10.1002/nme.341

[6] Dassi F., Kamenski L., Farrell P., Si H. "Tetrahedral mesh improvement using moving mesh smoothing, lazy searching flips, and RBF surface reconstruction." ComputerAided Design, vol. 103, 2-13, oct 2018. URL http://www.sciencedirect.com/science/ article/pii/S0010448517302336

[7] Freitag L.A., Ollivier-Gooch C. "Tetrahedral mesh improvement using swapping and smoothing." International Journal for Numerical Methods in Engineering, vol. 40, no. 21, 3979-4002, Nov. 1997. URL http://doi.wiley.com/10.1002/\%28SICI\% 291097-0207\%2819971115\%2940\%3A21\%3C3979\% 3A\%3AAID-NME251\%3E3.0.CO\%3B2-9

[8] Huang W., Ren Y., Russell R. "Moving Mesh Partial Differential Equations (MMPDES) Based on the Equidistribution Principle." SIAM Journal on Numerical Analysis, vol. 31, no. 3, 709 730, Jun. 1994. URL https://epubs.siam.org/ doi/abs/10.1137/0731038

[9] Du Q., Wang D. "Tetrahedral mesh generation and optimization based on centroidal Voronoi tessellations." International Journal for Numerical Methods in Engineering, vol. 56, no. 9, 13551373, mar 2003. URL http://doi.wiley.com/ $10.1002 / \mathrm{nme} .616$

[10] Si H. "TetGen, a Delaunay-Based Quality Tetrahedral Mesh Generator." ACM Trans. Math. Softw., vol. 41, no. 2, 11:1-11:36, feb 2015. URL http://doi.acm.org/10.1145/2629697

[11] de L'isle E.B., George P.L. "Optimization of Tetrahedral Meshes." I. Babuska, W.D. Henshaw, J.E. Oliger, J.E. Flaherty, J.E. Hopcroft, T. Tezduyar, editors, Modeling, Mesh Generation, and Adaptive Numerical Methods for Partial Differential Equations, The IMA Volumes in Mathematics and its Applications, pp. 97-127. Springer New York, 1995

[12] Shewchuk J.R. "Two Discrete Optimization Algorithms for the Topological Improvement of Tetrahedral Meshes." p. 11
[13] Misztal M.K., Bærentzen J.A., Anton F., Erleben K. "Tetrahedral Mesh Improvement Using Multi-face Retriangulation." B.W. Clark, editor, Proceedings of the 18th International Meshing Roundtable, pp. 539-555. Springer Berlin Heidelberg, Berlin, Heidelberg, 2009. URL http://link.springer.com/10. 1007/978-3-642-04319-2_31

[14] Boissonnat J.D., Devillers O., Pion S., Teillaud M., Yvinec M. "Triangulations in CGAL." Computational Geometry, vol. 22, no. 1, 5-19, may 2002. URL http://www.sciencedirect.com/ science/article/pii/S0925772101000542

[15] Jamin C., Alliez P., Yvinec M., Boissonnat J.D. "CGALmesh: A Generic Framework for Delaunay Mesh Generation." ACM Transactions on Mathematical Software, vol. 41, no. 4, 1-24, Oct. 2015. URL http://dl.acm.org/citation.cfm? doid=2835205 .2699463

[16] Dobrzynski C. "MMG3D: User Guide." Technical Report RT-0422, INRIA, mar 2012. URL https : //hal.inria.fr/hal-00681813

[17] Marot C., Pellerin J., Remacle J.F. "One machine, one minute, three billion tetrahedra." International Journal for Numerical Methods in Engineering, vol. 117, no. 9, 967-990, 2019. URL https://onlinelibrary.wiley.com/ doi/abs/10.1002/nme.5987

[18] Gent I.P., Walsh T. "Easy Problems are Sometimes Hard." Artif. Intell., vol. 70, no. 1-2, 335-345, 1994. URL https://doi.org/10.1016/ 0004-3702 (94) 90109-0

[19] Gomes C.P., Selman B., Crato N. "Heavy-Tailed Distributions in Combinatorial Search." Principles and Practice of Constraint Programming - CP97, Third International Conference, Linz, Austria, October 29 - November 1, 1997, Proceedings, pp. 121-135. 1997. URL https://doi.org/ 10.1007/BFb0017434

[20] Koch S., Matveev A., Jiang Z., Williams F., Artemov A., Burnaev E., Alexa M., Zorin D., Panozzo D. "ABC: A Big CAD Model Dataset For Geometric Deep Learning." arXiv:1812.06216 [cs], dec 2018. URL http://arxiv.org/abs/1812. 06216. ArXiv: 1812.06216

[21] Geuzaine C., Remacle J.F. "Gmsh: a threedimensional finite element mesh generator with built-in pre facilities." p. 24, 2009

[22] Klingner B.M., Shewchuk J.R. "Aggressive Tetrahedral Mesh Improvement." M.L. Brewer, D. Marcum, editors, Proceedings of the 16th 
International Meshing Roundtable, pp. 3-23. Springer Berlin Heidelberg, Berlin, Heidelberg, 2008. URL http://link.springer.com/10 . 1007/978-3-540-75103-8_1

[23] Klingner B.M. Improving Tetrahedral Meshes. Ph.D. thesis, EECS Department, University of California, Berkeley, Nov 2008. URL http: //www2.eecs. berkeley.edu/Pubs/ TechRpts/2008/EECS-2008-145.html

[24] Liu J., Chen B., Chen Y. "Boundary recovery after 3D Delaunay tetrahedralization without adding extra nodes." International Journal for Numerical Methods in Engineering, vol. 72, no. 6, 744-756, 2007. URL https://onlinelibrary . wiley.com/doi/abs/10.1002/nme. 2044

[25] Chen J., Zhao D., Huang Z., Zheng Y., Gao S. "Three-dimensional constrained boundary recovery with an enhanced Steiner point suppression procedure." Computers \&S Structures, vol. 89, no. 5-6, 455-466, Mar. 2011. URL https://linkinghub.elsevier.com/retrieve/ pii/S0045794910002828

[26] Si H., Gärtner K. "3D boundary recovery by constrained Delaunay tetrahedralization." International Journal for Numerical Methods in Engineering, vol. 85, no. 11, 1341-1364, Mar. 2011. URL http://doi.wiley.com/10.1002/nme.3016

[27] Devillers O., Teillaud M. "Perturbations and Vertex Removal in a 3D Delaunay Triangulation." 2003. URL https://hal.inria.fr/ inria-00166710 


\title{
GMSH'S APPROACH TO ROBUST MESH GENERATION OF SURFACES WITH IRREGULAR PARAMETRIZATIONS
}

\author{
Jean-François Remacle ${ }^{1}$ and Christophe Geuzaine ${ }^{2}$ \\ 1 Université catholique de Louvain, Institute of Mechanics, Materials and Civil Engineering (iMMC), \\ Place du Levant 1, 1348 Louvain-la-Neuve, Belgium \\ 2 Université de Liège, Department of Electrical Engineering and Computer Science, Montefiore \\ Institute B28, Allée de la Découverte 10, 4000 Liège, Belgium
}

\begin{abstract}
This paper proposes a robust and effective approach to overcome a major difficulty associated to surface finite element mesh generation: the handling surfaces with irregular (singular) parametrizations such as spheres, cones or other surfaces of revolution produced by common Computer Aided Design tools. The main idea is to represent triangles incident to irregular points as trapezoids with one degenerated edge. This new approach has been implemented in Gmsh and examples containing thousands of surfaces with irregular points are presented at the end of the paper.
\end{abstract}

Keywords: Computer Aided Design, Mesh generation, Gmsh.

\section{INTRODUCTION}

Computer Aided Design (CAD) systems are used extensively for industrial design in many domains, including automotive, shipbuilding, and aerospace industries, industrial and architectural design, prosthetics, and many more. Engineering designs are encapsulated in such CAD models, which up to manufacturing tolerances exactly represent their geometry. While the engineering analysis process begins with such CAD models, the predominant method of analysis (the finite element method) requires an alternative, discrete, representation of the geometry: a finite element mesh. In such a mesh, the CAD model is subdivided into a (large) collection of simple geometrical shapes such as triangles, quadrangles, tetrahedra and hexahedra, arranged in such a way that if two of them intersect, they do so along a face, an edge or a node, and never otherwise.

Three-dimensional CAD models are represented on a computer using a "Boundary Representation" (BRep) [1]: a volume is bounded by a set of faces, a face is bounded by a serie of curves and a curve is bounded by two end points. The BREP is a discrete object: it is a graph that contains model entities together with all their topological adjacencies. Then a geometry is associated to each model entity. Figure 1 presents a moderately complex CAD model together with its 3D mesh generated using Gmsh [2].

As an example, consider a model face $F$ with its boundary

$$
\partial F=\left\{C_{1}, \ldots, C_{n}\right\} .
$$

Face $F$ is topologically closed, i.e. $\partial(\partial F)=\emptyset$ : each endpoint of the bounding curves $C_{j}$ is considered twice in $F$, one time positively and one time negatively. The geometry of a model face $F$ is its underlying surface $\mathcal{S}$ with its parametrization

$$
\mathbf{x}: A \mapsto \mathbb{R}^{3}, \quad(u, v) \mapsto \mathbf{x}(u, v)
$$

where $A \subset \mathbb{R}^{2}$ is a rectangular region $\left[u_{0}, u_{1}\right] \times\left[v_{0}, v_{1}\right]$. A parametrization is said to be regular if $\partial_{u} \mathbf{x}$ and $\partial_{v} \mathbf{x}$ are linearly independent:

$$
\partial_{u} \mathbf{x} \times \partial_{v} \mathbf{x} \neq \mathbf{0}
$$

for any $u, v \in A$. Points where $\partial_{u} \mathbf{x} \times \partial_{v} \mathbf{x}=\mathbf{0}$ are called irregular or singular points of the parametrization. We 


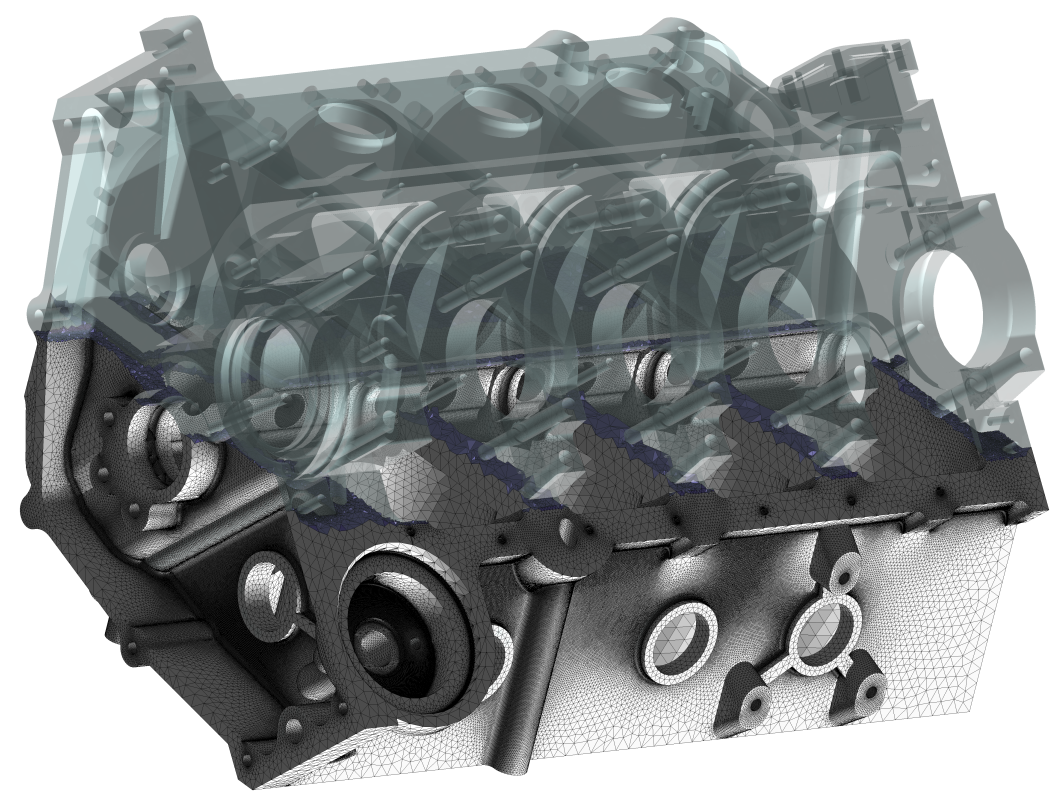

Figure 1: An Engine Block.

assume here that irregular points are isolated. Irregular points can occur for two possible reasons: (i) one of the partial derivatives $\partial_{u} \mathbf{x}$ or $\partial_{v} \mathbf{x}$ is equal to $\mathbf{0}$ or (ii) partial derivatives are non zero and are parallel. The second case (ii) where partial derivatives are non zero yet are parallel is not common in practice while case (i) appears quite often.

The underlying geometry of a face $F$ is thus a parametric surface $\mathbf{x}(u, v)$. Yet, its domain is often smaller than $A$ : $A$ is usually trimmed by boundaries $C_{j}$ and the geometry of the trimming curves are algebraic curves $\mathbf{c}_{j}(u, v)=0$ defined in the $(u, v)$ plane of $F$. Figure 2 shows an example of a trimmed surface.

Generating a triangular surface mesh of $F$ consists in generating a planar triangular mesh in its parameter plane whose map through $\mathbf{x}(u, v)$ is a valid mesh in $\mathbb{R}^{3}$ with triangles of controlled shapes and sizes.

A triangle is valid in the $(u, v)$ plane when it is properly oriented, i.e. when its area is strictly positive. It is indeed more complicated to assess that a triangle is valid in $\mathbb{R}^{3}$. Assume a triangle $(\mathbf{a}, \mathbf{b}, \mathbf{c})$ with its non unit normal $\mathbf{n}=(\mathbf{b}-\mathbf{a}) \times(\mathbf{c}-\mathbf{a})$ and the normal to the CAD surface at the centroid

$$
\left(u_{t}, v_{t}\right)=\frac{1}{3}\left(u_{a}+u_{b}+u_{c}, v_{a}+v_{b}+v_{c}\right)
$$

of the triangle:

$$
\mathbf{n}_{\mathrm{CAD}}=\partial_{u} \mathbf{x}\left(u_{t}, v_{t}\right) \times \partial_{v} \mathbf{x}\left(u_{t}, v_{t}\right) .
$$

We say that triangle $(a, b, c)$ is valid if $\mathbf{n}_{\mathrm{CAD}} \cdot \mathbf{n}>0$.

In the example of Figure 2, the depicted trimmed surface has no irregular points and the mesh generation procedure is usually straightforward. In this specific example, the anisotropic frontal-Delaunay approach that is implemented in Gmsh [2] was used based on the metric tensor

$$
\mathcal{M}=\left(\begin{array}{cc}
\left\|\partial_{u} \mathbf{x}\right\|^{2} & \partial_{u} \mathbf{x} \cdot \partial_{v} \mathbf{x} \\
\partial_{u} \mathbf{x} \cdot \partial_{v} \mathbf{x} & \left\|\partial_{v} \mathbf{x}\right\|^{2}
\end{array}\right)
$$

that is of full rank everywhere.

Surfaces with isolated irregular points are however very common in CAD systems: spheres, cones and other surfaces of revolution may contain one or two irregular points. Mesh generation procedures are known to be prone to failure close to irregularities. Consider for example the parametrization of a sphere as it is used to our best knowledge in every CAD system. A sphere of radius $R$ centered at the origin is parametrized as

$$
\begin{aligned}
& x(u, v)=R \sin u \cos v \\
& y(u, v)=R \sin u \sin v \\
& z(u, v)=R \cos u
\end{aligned}
$$




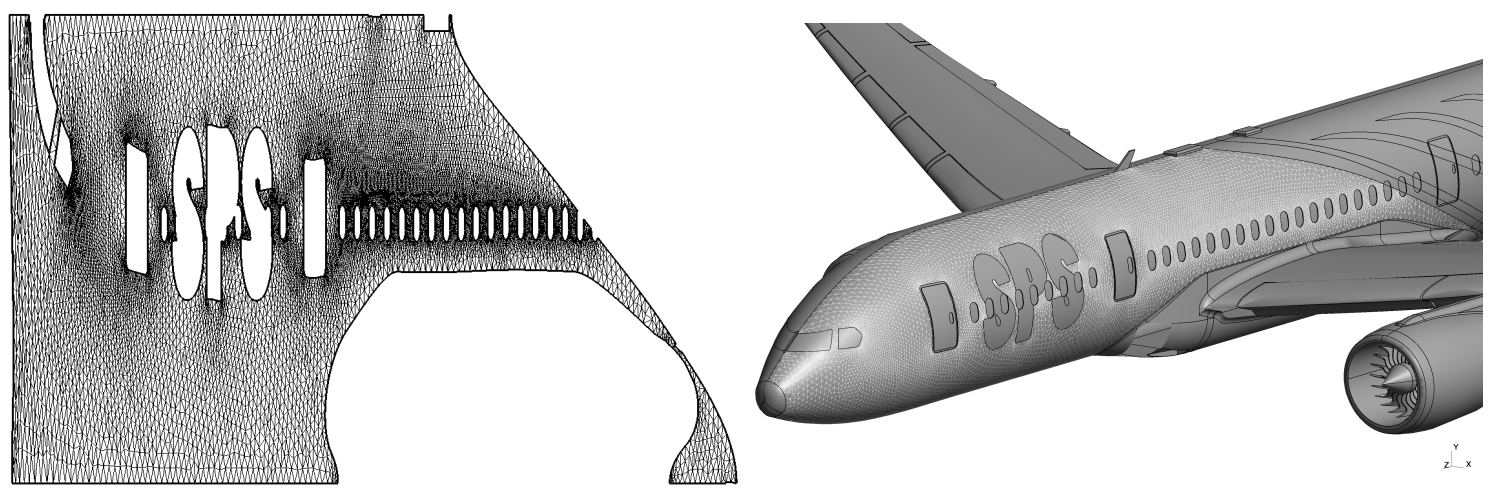

Figure 2: Surface mesh of a model face. View of the mesh in the parameter plane (left) and on $\mathbb{R}^{3}$.

where $u \in[0, \pi]$ is the inclination and $v \in[0,2 \pi[$ is the azimuth. At the poles, i.e. when $u=0$ or $u=\pi$,

$$
\partial_{v} \mathbf{x}=R(-\sin u \sin v, \sin u \cos v, 0)=(0,0,0)
$$

vanishes and this parametrization is irregular at the two poles of the sphere.

In this paper, a new approach is proposed that allows to generate meshes of surfaces with irregularities in an efficient and robust fashion. At first, we explain in $\S 2$ why indirect surface mesh generation procedures become fragile at the vicinity of irregular points. Then in $\S 3$ and $\S 5$, we present the critical modifications to standard meshing procedures that allow to address issues related to irregular parametrizations. Examples of CAD models with thousand of spheres and cones are finally be presented in $\S 7$.

\section{THE ISSUE OF MESHING SURFACES WITH IRREGULAR PARAMETRIZATIONS}

Two main approaches exist for surface meshing. The first approach, usually called the "direct approach" [3], consists in generating the mesh directly in $\mathbb{R}^{3}$. Different direct approaches have been proposed in the literature: advancing front methods $[4,5]$, octree based methods $[6,7]$, methods based on local mesh modifications $[8,9]$, methods based on restricted Voronoi diagrams $[10], \ldots$ Octree- and Voronoi- based methods have in common the need to intersect a $3 \mathrm{D}$ object (an octree or a Voronoi Diagram) with the surface that is to be meshed. When an octree is used, the intersection of the octree with the surface is usually irregular and local mesh modifications have to be performed in order to obtain a quality mesh. On the other hand, when the Voronoi diagram of the points is used, recovering edges (sharp features) of the surface is an issue. Other direct methods generate triangles on the surface without using any kind of 3D object. Advancing front methods and paving methods [11] add points and triangle on the surface using a frontal approach. Those methods handle sharp features without difficulties and allow to generate quality meshes. Yet, such methods are plaged with robustness issues (front colliding and 3D intersections of 2D objects). Some direct approaches $[8,9]$ start from a "CAD" mesh and modify it to produce a "computational" mesh with elements of controlled shapes and sizes. The main disadvantage of such an approach is that it requires an initial mesh. One may use STL triangulations provided by CAD modelers but those are not guaranteed to be watertight on a whole CAD model and a complex preprocessing step is usually required to fix holes and T-juntions. Another issue is related to what could be called an "isogeometric" argument: the final "computational" mesh and the inital "CAD" mesh are piecewise linear complexes that do not necessary cover the same geometry. Modifying an existing surface mesh using local mesh modification like vertex repositioning leads to vertices located outside of the input geometry i.e. the "CAD" mesh. While meshing procedures of this kind that actually ensure that the distance between the "CAD" and the "computational" mesh is bounded, those are based on complex datastructures and require to compute Haussdorff distances between triangulations [12].

When mesh generation procedures have access to parametrizations of surfaces, one can generate a planar mesh in the parametric domain and map it in 3D. This surface meshing approach is called "indirect". In Gmsh, surface meshes are generated in the parameter plane $(u, v)$ and standard "off the shelf" anisotropic 2D meshers are used for generating surface meshes. This is of course the main advantage of the indirect approach: a priori, no major coding effort is required to go from planar meshing to surface meshing. This last statement is of course a little bit too optimistic. En- 
suring that a planar mesh is valid is trivial: all triangles should be positively oriented. Now, if the surface parametrization $\mathbf{x}(u, v) \in \mathbb{R}^{3}$ is regular, then the mapping of the $(u, v)$ mesh onto the surface is itself valid because the composition of two regular mappings is regular. For example, the very simple mesh of the parameter plane of the whole sphere presented in Figure 3 maps exactly the sphere as depicted in the bottom part of Figure 3.

Here, the main issue is that we do not actually map entire triangles onto the surface but only their corners. The topology of the 2D mesh is simply "translated" in $3 \mathrm{D}$ : straight sided triangles in the $(u, v)$ plane become straight sided triangles in 3D. Another "isogeometric" issue thus appears in the indirect approach: the mapping $\mathbf{x}(u, v)$ of a triangle in the $(u, v)$ plane is not equal to the straight sided triangle in $\mathbb{R}^{3}$. So, a valid $2 \mathrm{D}$ triangle in the parameter plane does not necessary produce a valid 3D triangle on the surface. For example all the triangles in the parameter plane in Figure 3 are mapped onto zero-area triangles in $\mathbb{R}^{3}$. On the other hand, an invalid $2 \mathrm{D}$ triangle (i.e. with a negative area) may be perfectly valid in $3 \mathrm{D}$.

In ordre to illustrate those issues, Figure 4 shows the example of the parameter space of a complete sphere. An edge $(a, b)$ where $b$ is close to the north pole $p$ (in red) is considered. Edge $(a, b)$ is used to form a triangle $(a, b, c)$ where $c \in A=[0,2 \pi[\times[0, \pi]$. The iso-lines that are presented are iso-values of triangle qualities $^{1}$ : the particular point $c$ drawn on the Figure is the only one in the parameter plane leading to a valid equilateral triangle in $3 \mathrm{D}$. The grey zone in the Figure corresponds to the locations of points $c$ that form invalid elements in 3D. Invalid elements in the parameter plane $(u, v)$ correspond to points above the green line that passes through $(a, b)$. It can be seen that there exists a zone where triangles are valid in $2 \mathrm{D}$ but not in $3 \mathrm{D}$, and another zone where elements are valid in $3 \mathrm{D}$ but not in $2 \mathrm{D}$. Some interesting comments can be made with respect to Figure 4:

- The blue line is the 3D geodesic between $a$ and $b$. This geodesic is far from being a straight line in the parameter plane, especially when the edge $(a, b)$ is close to the pole (in red). Geodesics are straight lines in the parameter plane when the metric tensor $\mathcal{M}$ is constant (this is a sufficient condition). We will see in the next section that geodesics that are incident to an irregular point are also straight lines, even though the metric $\mathcal{M}$ has strong variations close to singularities.

\footnotetext{
${ }^{1}$ We use as quality metrric the ratio $2 \frac{r}{R}$ between the inner-radius $r$ and the circumradius $R$ multiplied by 2 in order to have a quality equal to one for the equilateral triangle.
}

- The point $c$ in Figure 4 that corresponds to an equilateral triangle $(a, b, c)$ is always in the valid zone i.e. the zone where triangles are both valid in $2 \mathrm{D}$ and $3 \mathrm{D}$. More generally, good quality triangles can always be formed in the parameter plane, even when the metric is very distorded.

- The zone that is valid in $2 \mathrm{D}$ but not in $3 \mathrm{D}$ is the most problematic for mesh generation algorithm that work in the parametric plane. Hopefully, this zone only contains points $c$ for which triangles $(a, b, c)$ are of bad quality.

It is difficult to generalize those three comments to general surfaces but our experience (through numerical experiments) shows that they do indeed hold.

The main question can thus be formulated as follows: assuming a surface with a parametrization that may contain isolated irregular points, can we always find a valid $2 D$ mesh that corresponds to a valid $3 D$ mesh?

When we started to think about version 4 of Gmsh, our answer to that question was tending to be no, at least using the current implementation of the surface mesh generators. The typical issue that was encountered at the time is illustrated on Figure 5. The right part of the Figure represents the mesh in the parametric plane $(u, v)$ while the right part of the Figure represents the surface mesh in $\mathbb{R}^{3}$.

In Figure 5, the surface $\mathcal{S}$ is a sphere. Points like $c$ or $g$ (in green) are classified on model face $F$. Points like $d$ or $f$ (in pink) are classified on regular model edges that bound $F$ while points like $b$ and $e$ are classified on the seam of $F$ (in order to have $\partial(\partial F)=\emptyset$ some CAD systems like OpenCASCADE close periodic surfaces with a seam). Point $b$ is a pole of the sphere: it is an irregular point. The parametric mesh is perfecly valid i.e. triangles cover exactly $A$ without overlap. Yet, even though triangle $(b, c, d)$ is correctly oriented in the parametric plane, it is invalid in $\mathbb{R}^{3}$. We are here in the situation of Figure 4 where point $a$ is above the geodesic between $c$ and $d$ in the parameter plane. One single edge flip could potentially make the $3 \mathrm{D}$ mesh valid and of better quality: exchanging edges $(c, d)$ and $(a, b)$ fixes all issues. Yet, doing so makes the parametric mesh invalid. With the set of points that is depicted in Figure 5, we found it impossible to build a quality mesh in $\mathbb{R}^{3}$ that is valid in the $(u, v)$ plane.

Contrary to what one might think, the main issue here is not the fact that the metric tensor (1) is of rank 1 at irregular points and very distorted around it. In the context of mesh generation, geometrical queries like the evaluation of the metric tensor $\mathcal{M}$ are never done at irregular points; and anisotropic mesh generators are able to generate meshes for smooth metric fields even though they are very distorted. The mesh gen- 

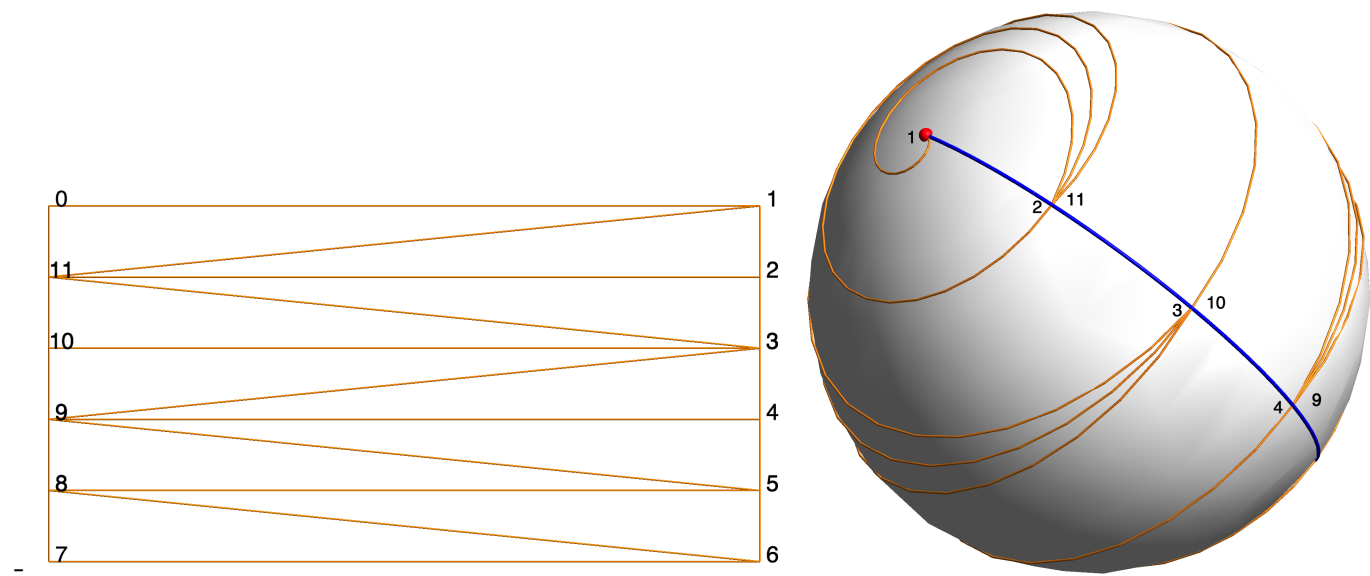

Figure 3: A very simple mesh (left) of the parameter plane of a sphere and (right) its mapping through spherical coordinates.

eration issue that arises here is essentially related to triangles (e.g. $(b, c, d)$ in the Figure) and edges that have one vertex like $b$ that corresponds to an irregular point of the parametrization.

Another minor issue will be fixed by our new approach. The existence of one degenerated mesh edge connecting points $b$ implies the existence of an irregular triangle $(d, b, b)$ that has one degenerated edge. This triangle can be eliminated in a post processing stage but its presence is quite annoying in the mesh generation process: computation of circumcircles, edge flips (flipping edge $(b, d)$ does not change the mesh), $\ldots$

\section{GEODESICS OF SURFACES OF REVOLUTION}

Most of the CAD surfaces that have irregular points are surfaces of revolution. Consider a surface of revolution with respect to the $z$-axis and suppose that the generating curve is

$$
\mathbf{c}(v)=(f(v), 0, g(v)), \quad v \in[0, T] .
$$

The parametrization of the surface is given by

$$
\mathbf{x}(u, v)=(f(v) \cos (u), f(v) \sin (u), g(v)),
$$

$(u, v) \in[0,2 \pi[\times[0, T]$. Geodesics of surface of revolution, even though their forms are not trivial (see for example the blue line of Figure 4), have specific properties [13]. One interresting property of surfaces of revolution is that meridian curves $u=$ cste are geodesics.

Surfaces of revolution may have irregular points: if $f(0)=g(0)=0$ in $(2)$, then $\mathbf{x}(u, 0)=(0,0,0)$ for every $u$. The origin of the axis belongs to the surface and is thus an irregular point as defined above. Let us now look at the parameter plane $(u, v)$ corresponding to a surface of revolution with a irregular point at $u=0$. Figure 6 gives an illustration of that situation. The thick red line $u=0$ is mapped onto one single point $\mathbf{x}=(0,0,0)$. Thus, edges $(g, b)$ and $\left(g, b^{\prime}\right)$ have the same end-points in $\mathbb{R}^{3}$ but the only geodesic between those two points is the meridian $(g, b)$.

This simple result allows us to critically examine Figure 5: edges like $(c, b),(g, b)$ or $(d, b)$ are far from being geodesics and are thus far from the their corresponding straight edges in $\mathbb{R}^{3}$, as depicted in Figure 7. On the other hand, edge $(b, f)$ is close to be a geodesic and its $3 \mathrm{D}$ representation is close to the corresponding straight line.

Coming back to the mesh generation problem, it should be interresting to replace all the edges that are incident to irregular points by meridians. The new representation of the mesh in the $(u, v)$ plane is depicted in Figure 8.

With this representation, the unique edge flip that allows to have a valid mesh in $\mathbb{R}^{3}$ is permitted. Edge $\left(a, b_{a}\right)$ (in dashed lines) can replace edge $(c, d)$ without creating invalid triangles in the parameter plane (edge $(c, g)$ could be flipped as well even though it is not required). Note here that triangles incident to irregular points are now right trapezoids with one degenerated edge, which means that no degenerated triangles exist in that new representation.

\section{MODIFICATIONS OF THE INITIAL MESH}

Our surface mesh generation procedure starts with an initial "empty mesh" i.e. a mesh in the parameter space that contains only vertices of the surface boundaries. Then, in this new procedure, edges that are ad- 


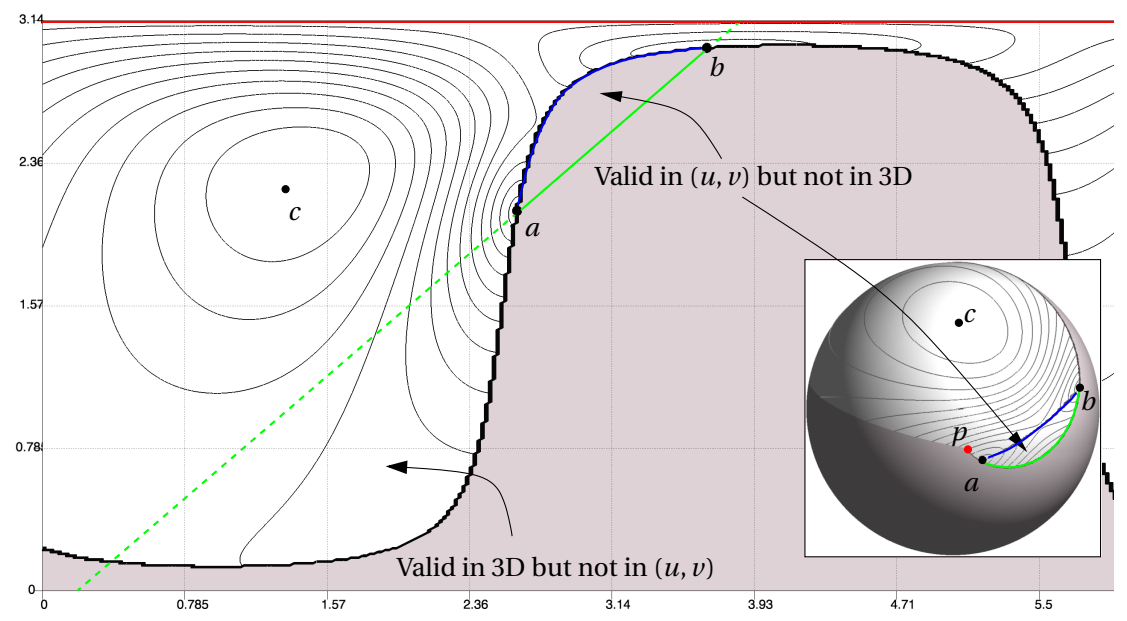

Figure 4: Parameter plane of a sphere. We consider an edge $(a, b)$. Figures depict the quality of a triangle $(a, b, c)$ with $c$ positioned anywhere in the parameter plane. The grey zone corresponds to invalid triangles in 3D.

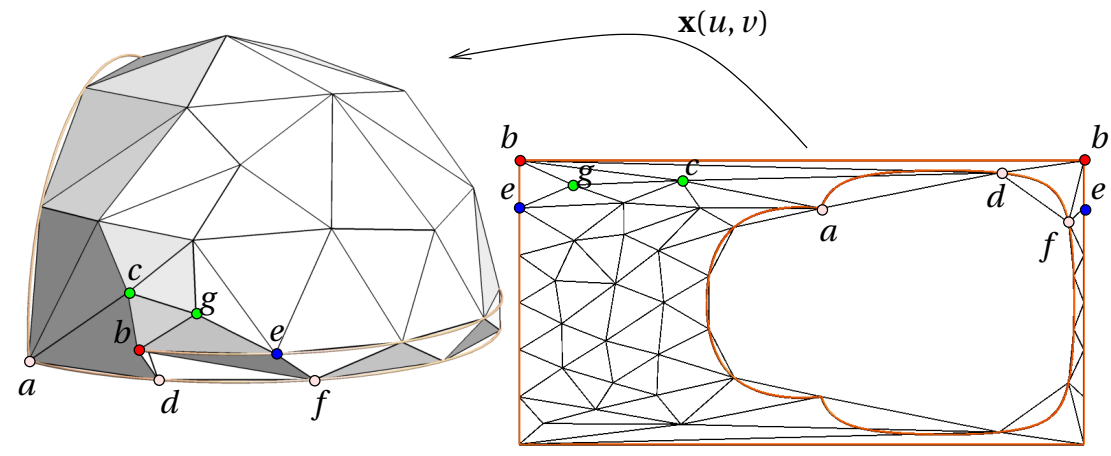

Figure 5: A valid mesh in the parameter space that is invalid in the real space.

jacent to singularities are transformed onto geodesics. The question that is addressed in this section is the validity of this initial transformation.

Consider the surface presented in Figure 9 together with a mesh generated using the new version of Gmsh's MeshAdapt surface mesher (see $\S 5$ below).

The initial mesh that contains all boundary points is presented in Figure 10. Again, a seam and two irregular points are present in the surface plus a trimming curve.

In our new procedure, all edges that are adjacent to irregular points are transformed onto geodesics. Figure 11 shows the result of that transformation.

It is actually easy to figure out that the initial transformed mesh of Figure 11 is actually wrong (inverted) in the parameter space. Figure 12 shows a zoom of the three problematic edges that make this mesh invalid. The problem comes from the fact that, in Figure 9, three edges of the internal boundary are initially connected to the point on the bottom right of the external rectangle. Yet, those edge normals are pointing upward so the proposed correction creates edgdes that intersect other edges of the internal boundary.

Addressing this problem is indeed quite simple. All problematic geodesic edges are split along their original path (not along the geodesic of course) up to the point when no intersection occurs. The resulting initial mesh is presented in Figure 13. 


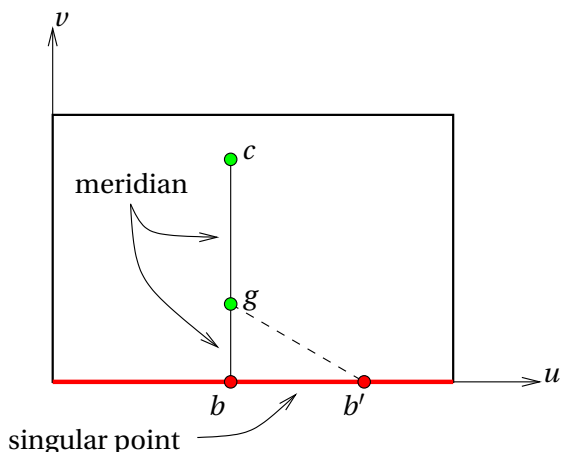

Figure 6: Parameter space corresponding to a surface of revolution with an irregular point at $u=0$.

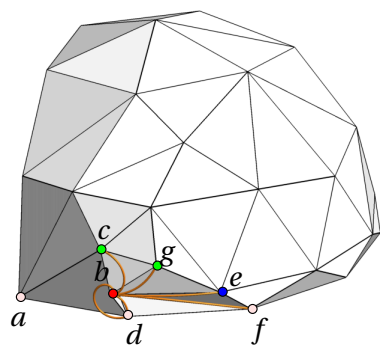

Figure 7: True mapping of straight lines in the parameter space onto $\mathbb{R}^{3}$ close to an irregular point.

\section{LOCAL MESH MODIFICATIONS: GMSH'S MESHADAPT ALGORITHM REVISITED}

Gmsh's most basic surface mesher is called $\mathrm{Me}$ $s h A_{d a p t^{2}}$. MeshAdapt's surface meshing strategy is based on the concept of local mesh modifications $[14,15,16]$. The algorithm works as follows. First, an initial mesh containing all the mesh points and edges of the model edges that bound a face is built in the parametric space $(u, v)$ (see $\S 4)$. Then, local mesh modifications are applied to the mesh in the parameter plane:

1. Each edge that is too long is split;

2. Each edge that is too short is collapsed;

3. Edge flips are performed in order to increase mesh quality;

4. Vertices are re-located optimally after steps 1,2 and 3.

\footnotetext{
${ }^{2}$ gmsh - algo meshadapt is the commandline that forces gmsh to use that algorithm.
}

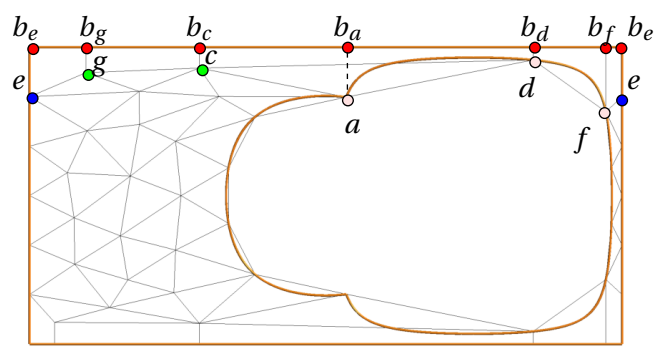

Figure 8: New representation in the parameter space where every edge connected to an irregular point is a meridian. Point $b_{i}$ 's all belong to edge $\left(b_{i}, i\right)$ even though all $b_{i}$ 's 3D locations are equal.

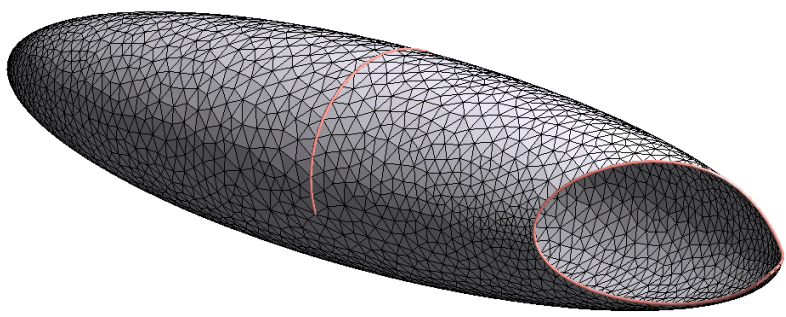

Figure 9: A surface.

Figure 14 illustrates local mesh modifications applied to edges that are in the vicinity of a irregular point $b$. When edge $(a, c)$ is flipped, a new instance of point $b_{c}$ is created on the degenerated edge and point $c$ becomes connected to $b_{c}$. The operation can be reversed as depicted in Figure 14. When an edge like $(a, d)$ is split at point $e$, a new point $b_{e}$ is created on the degenerated line. When an edge like $\left(c, b_{c}\right)$ that is connected to the irregular point is split, $b_{c}$ is replaced by $b_{e}$. Note that when a point like $e$ is relocated, point $b_{e}$ is relocated as well.

All four local mesh modifications of our algorithm involve details of implementation that are too specific to be described in a paper but that are critical for robustness. Interrested readers can download the source code of Gmsh 4.3.0 that implements the algorithm that exactly corresponds to the examples of the paper. Nevertheless, the the most critical part of the $\mathrm{Me}$ shAdapt surface mesher is the vertex relocation, both in term of the final mesh quality and of CPU time (it actually takes about $60 \%$ of the total mesh generation time). When parametrizations are very distorded, simple smoothing strategies do not actually produce improvements of the mesh, espacially close to singularities. In this new version of the algorithm, advanced optimization procedures have been used [17] for vertex relocation. Figure 15 show the mesh of the surface 


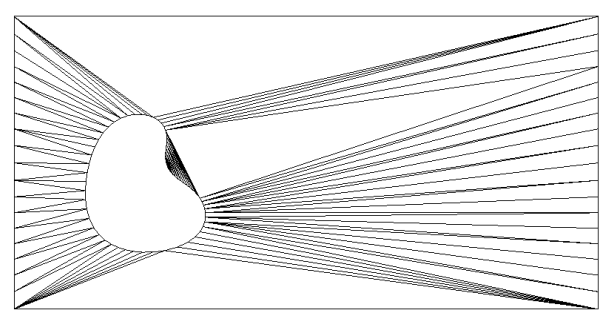

Figure 10: The initial mesh for the surface presented in 9 represented in the parameter plane.

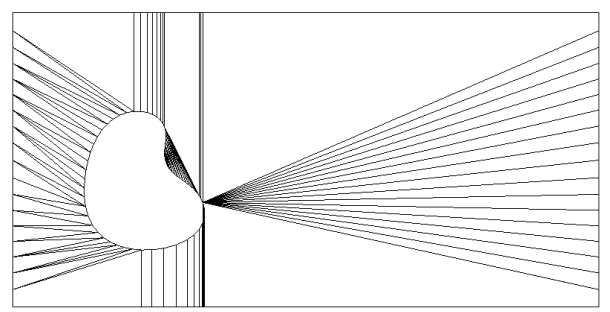

Figure 11: Intial mesh of surface 9 where all edges adjacent to irregular points are transformed into geodesics.

of Figure 9 at different stages of the MeshAdapt algorithm.

\section{DELAUNAY MESH GENERATION CLOSE TO IRREGULAR POINTS}

Gmsh's frontal-Delaunay algorithm is an extension to surface meshing of the planar frontal-Delaunay mesher described in [18]. Points are inserted in the domain in a frontal fashion while always keeping a valid mesh during the process. The mesh is generated in the $(u, v)$ plane which means that an anisotropic Delaunay criterion is required to produce isotropic meshes in 3D.

The most critical operation involved in that algorithm is the edge flip. Consider Figure 16: we would like to figure out wether edge $(a, d)$ should be flipped or not. In order to apply Delaunay's empty circle criterion, we actually work in the tangent plane and compute a unique metric tensor $\mathcal{M}$ at location $\left(a+b_{c}+c+d\right) / 4$ that is symmetrical with respect to points $a, b_{c}, c$, and $d$. This allows to avoid "unstable flips". In this tangent plane, circle $\mathcal{C}_{\mathcal{M}}(a, c, d)$ is an ellipsis. The new representation that is proposed here allows to provide a robust way of computing Delaunay flips.

In the example of Figure 16, edge $(a, d)$ should be flipped to $\left(c, b_{c}\right)$ because $b_{c}$ is inside $\mathcal{C}_{\mathcal{M}}(a, c, d)$. Other occurences of $b$ like $b_{a}$ or $b_{d}$ may be located outside $\mathcal{C}_{\mathcal{M}}(a, c, d)$ but the only edge that should be considered in the circle test is the geodesic $\left(c, b_{c}\right)$.

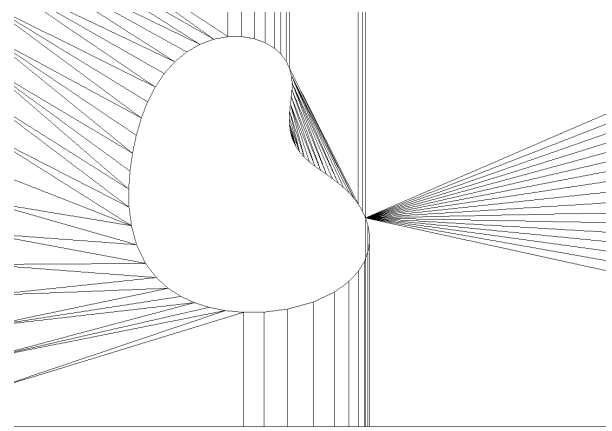

Figure 12: Zoom on the problematic geodesic edges that intersect internal edges of the trimming curve.

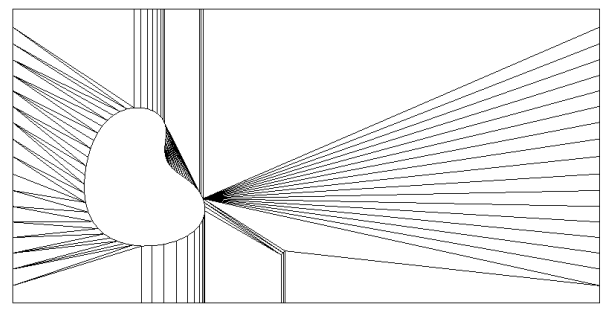

Figure 13: Corrected initial mesh.

\section{EXAMPLES}

We have chosen two examples that were invariably creating invalid elements in all previous versions of Gmsh.

\subsection{Many spheres}

One of the nastier parametrization that is constantly used in CAD systems is the sphere, with its two irregular points at the poles. As a first example, we have generated a CAD model that consist in a unit cube $B$ containing 5000 spheres with (pseudo-)random centers and radii $S_{i}, i=1, \ldots, 5000$. The final CAD model $C$ is computed as

$$
C=B \backslash S_{1} \backslash S_{2} \cdots \backslash S_{5000} .
$$

The final model $C$ is depicted in Figure 18. It consist in 3 volumes, 4971 surfaces (mainly trimmed spheres resulting from the boolean operations) and 18112 curves. Gmsh's actual script that was used to generate that model is given by

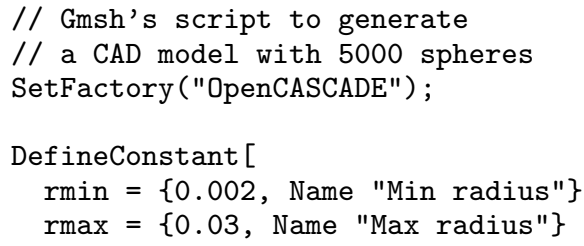



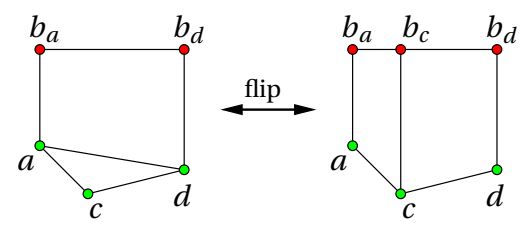

split $\uparrow$ collapse

split $\uparrow$ collapse
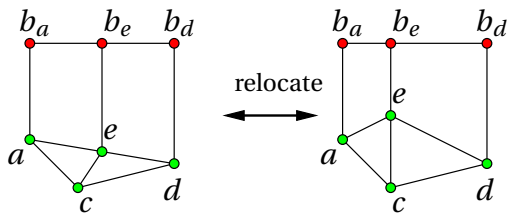

Figure 14: Local mesh modifications with irregular points.
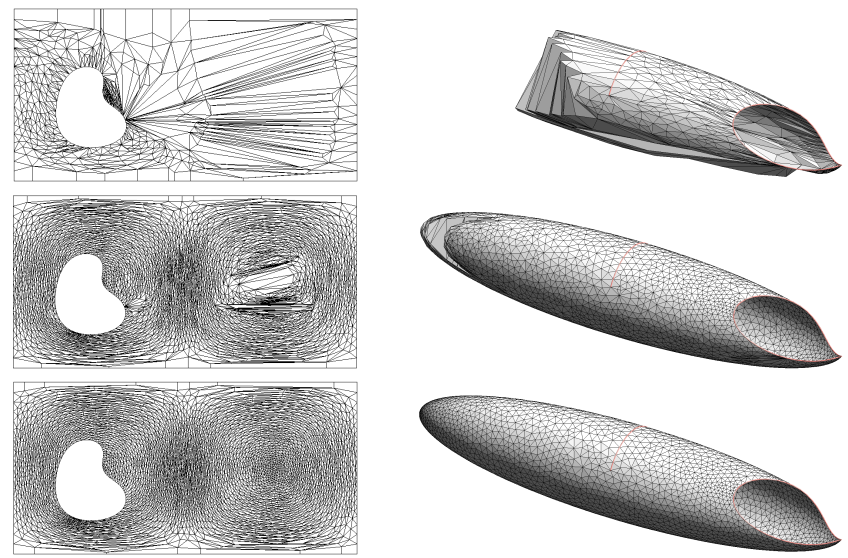

Figure 15: Different stages of the MeshAdapt algorithm ];

$\mathrm{n}=\{5000$, Name "Number of spheres" $\}$

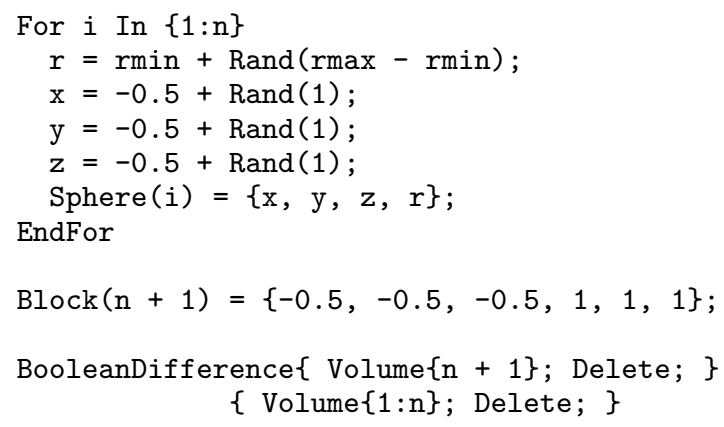

Some of the surfaces of the 5000 spheres model are really complex to mesh, especially when a trimmed

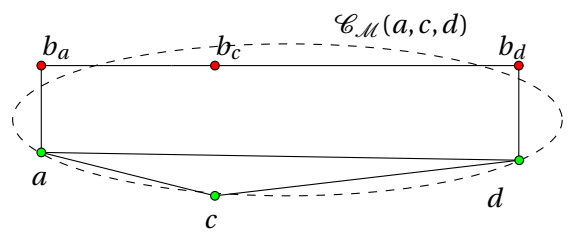

Figure 16: Illustration of the application of the circle criterion close to an irregular point.
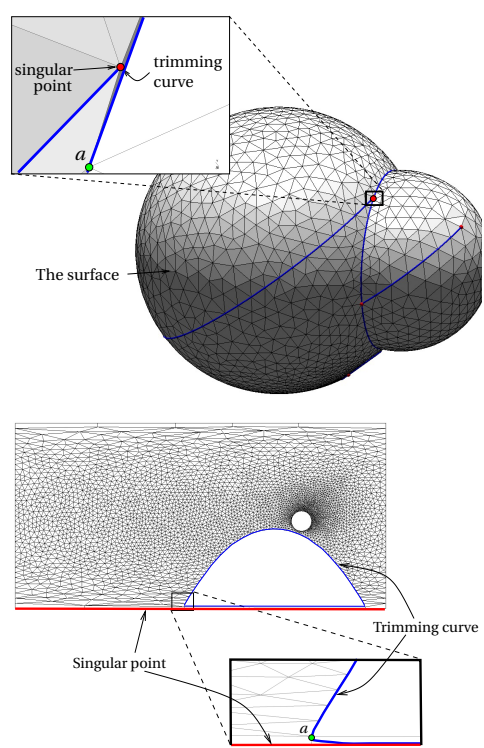

Figure 17: One surface of the 5000 sphere model that exhibit a complex configuration close to one of the poles of the sphere.

curve is very close to an irregular point. Figure 17 shows a complicated situation.

\subsection{Many ellipsoids}

Generating an ellipsoid can be done by applying an affine transformation to a sphere followed by a rotation. Using Gmsh's built-in scripting language, this can be achieved as follows:

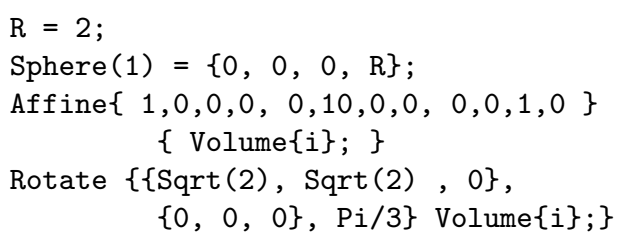

Ellipsoids have parametrizations that are even more distorded than spheres. In the following example, 450 

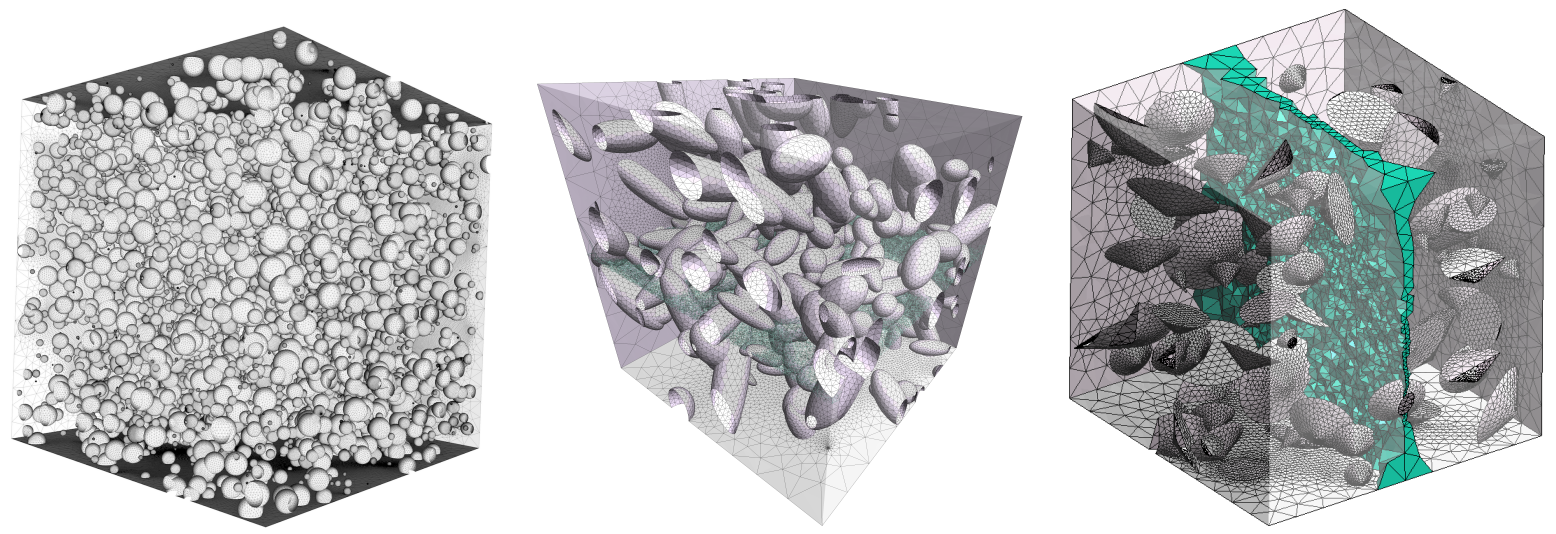

Figure 18: A complex model made of 5000 spheres (left), another model made of 450 ellipsoids (center) and a third model made of 100 intersecting cones (right).

ellipsoids $E_{i}, \quad i=1, \ldots, 450$ have been inserted into a unit cube, with random orientantions and random sizes. The final CAD model is again built as the unit cube "minus" all ellipsoids. In OpenCASCADE, ellipsoids are encoded as B-spline surfaces and their intersections takes way more effort than intersecting spheres: it actually took about 7 minutes to generate the CAD model while only 3 minutes were required to generate the surface mesh (295 surfaces for a total of 220,523 triangles) and only 14 seconds were required to generate the $3 \mathrm{D}$ mesh ( 55 volumes and 7,35 million tetrahedra). Figure 18 show a picture of the resulting mesh.

\subsection{Many cones}

Apex of cones are singular points of their parametrizations. We have generated a geometry with 100 intersecting cones in a box. Figure 18 show an image of the mesh.

\section{CONCLUSIONS}

Generating in a reliable manner a quality surface mesh for arbitrary CAD models entails dealing with various CAD systems idiosyncrasies. In this paper, we have presented a crucial modification of Gmsh's surface meshing algorithms that is an important step forward towards this goal, by handling surfaces with a finite number of irregular points. The two test cases that are presented are only indicative: hundered of other examples were successfully tested during the writing of this paper, all with surfaces that have singularities.

The lack of a structure of proof for surface meshing that is briefly explained in the introduction is one of the curses mesh generation people have to live with. Surface meshers that are reasonably reliable are all based on heuristics and their disfunctions and bugs can only be found through extensive testing. For example, the issue that has been explained in $\S 4$ has only been encountered twice in all our test cases. Yet, it has to be addressed because the rare conditions of apparition of the bug will definitively happen at some point in a software like Gmsh that is used by a large community.

In conclusion, we are aware that other issues will show up in the long term (maybe impossible) goal of $100 \%$ reliability. Yet, the improvements that are presented in this paper definitively make Gmsh's surface meshers more reliable on a large numer of test cases that were failing in previous versions. The method that is proposed does not require deep modifications of existing surface meshing algorithms. Yet, it allows to produce quality meshes for all test cases that we encountered.

\section{References}

[1] Weiler K. "The radial-edge structure: a topological representation for non-manifold geometric boundary representations." Geometric modeling for CAD applications, 1988

[2] Geuzaine C., Remacle J.F. "Gmsh: A 3-D finite element mesh generator with built-in pre-and post-processing facilities." International journal for numerical methods in engineering, vol. 79, no. 11, 1309-1331, 2009

[3] Borouchaki H., Laug P., George P.L. "Parametric surface meshing using a combined advancingfront generalized Delaunay approach." International Journal for Numerical Methods in Engineering, vol. 49, no. 1-2, 233-259, 2000 
[4] Hartmann E. "A marching method for the triangulation of surfaces." The Visual Computer, vol. 14 , no. $3,95-108,1998$

[5] Owen S.J., Staten M.L., Canann S.A., Saigal S. "Q-Morph: an indirect approach to advancing front quad meshing." International Journal for Numerical Methods in Engineering, vol. 44, no. 9, 1317-1340, 1999

[6] Maréchal L. "Advances in octree-based allhexahedral mesh generation: handling sharp features." Proceedings of the 18th international meshing roundtable, pp. 65-84. Springer, 2009

[7] Shephard M.S., Georges M.K. "Automatic threedimensional mesh generation by the finite octree technique." International Journal for Numerical methods in engineering, vol. 32, no. 4, 709-749, 1991

[8] Frey P. Yams a fully automatic adaptive isotropic surface remeshing procedure. Ph.D. thesis, Inria, 2001

[9] Béchet E., Cuilliere J.C., Trochu F. "Generation of a finite element MESH from stereolithography (STL) files." Computer-Aided Design, vol. 34, no. 1, 1-17, 2002

[10] Yan D.M., Lévy B., Liu Y., Sun F., Wang W. "Isotropic remeshing with fast and exact computation of restricted Voronoi diagram." Computer graphics forum, vol. 28, pp. 1445-1454. Wiley Online Library, 2009

[11] Blacker T.D., Stephenson M.B. "Paving: A new approach to automated quadrilateral mesh generation." International Journal for Numerical Methods in Engineering, vol. 32, no. 4, 811-847, 1991

[12] Borouchaki H., Frey P. "Simplification of surface mesh using Hausdorff envelope." Computer methods in applied mechanics and engineering, vol. 194, no. 48-49, 4864-4884, 2005

[13] Do Carmo M.P. Differential Geometry of Curves and Surfaces: Revised and Updated Second Edition. Courier Dover Publications, 2016

[14] Remacle J.F., Li X., Chevaugeon N., Shephard M.S. "Transient Mesh Adaptation Using Conforming and Non Conforming Mesh Modifications." IMR, pp. 261-272. 2002

[15] Li X., Shephard M.S., Beall M.W. "3D anisotropic mesh adaptation by mesh modification." Computer methods in applied mechanics and engineering, vol. 194, no. 48-49, 4915-4950, 2005
[16] Remacle J.F., Li X., Shephard M.S., Flaherty J.E. "Anisotropic adaptive simulation of transient flows using discontinuous Galerkin methods." International Journal for Numerical Methods in Engineering, vol. 62, no. 7, 899-923, 2005

[17] Knupp P.M. "Winslow smoothing on twodimensional unstructured meshes." Engineering with Computers, vol. 15, no. 3, 263-268, 1999

[18] Rebay S. "Efficient unstructured mesh generation by means of Delaunay triangulation and Bowyer-Watson algorithm." Journal of computational physics, vol. 106, no. 1, 125-138, 1993 


\title{
CAD DEFEATURING USING MACHINE LEARNING
}

\author{
Steven Owen ${ }^{1} \quad$ Timothy M. Shead ${ }^{2} \quad$ Shawn Martin ${ }^{3}$ \\ ${ }^{1}$ Sandia National Laboratories, Albuquerque, New Mexico, U.S.A. sjowen@sandia.gov \\ ${ }^{2}$ Sandia National Laboratories, Albuquerque, New Mexico, U.S.A. tshead@sandia.gov \\ ${ }^{3}$ Sandia National Laboratories, Albuquerque, New Mexico, U.S.A. smartin@sandia.gov
}

\begin{abstract}
We describe new machine-learning-based methods to defeature CAD models for tetrahedral meshing. Using machine learning predictions of mesh quality for geometric features of a CAD model prior to meshing we can identify potential problem areas and improve meshing outcomes by presenting a prioritized list of suggested geometric operations to users. Our machine learning models are trained using a combination of geometric and topological features from the CAD model and local quality metrics for ground truth. We demonstrate a proof-of-concept implementation of the resulting workflow using Sandia's Cubit Geometry and Meshing Toolkit.
\end{abstract}

Keywords: machine learning, mesh generation, tetrahedra, supervised learning, defeaturing

\section{INTRODUCTION}

An engineering analyst typically receives CAD models and assemblies that are developed based on manufacturing specifications which are not directly useful for analysis. For example, a CAD model may contain many small artifacts or irrelevant details that will have little effect on the outcome of a physics simulation, but dramatically slow the simulation by producing needlessly-complex meshes. Some automatic surface meshing techniques $[1,2,3]$ incorporate tolerant approaches that can ignore small geometric features and artifacts in the final mesh, but without careful user validation, fully automatic meshing methods run the risk of eliminating geometry that is required for simulation.

At the opposite end of the spectrum, fully manual defeaturing of a CAD model prior to meshing requires thorough inspection using advanced 3D CAD-based software tools such as $[4,5]$, after which the analyst

* Sandia National Laboratories is a multimission laboratory managed and operated by National Technology \& Engineering Solutions of Sandia, LLC, a wholly owned subsidiary of Honeywell International Inc., for the U.S. Department of Energy's National Nuclear Security Administration under contract DE-NA0003525. SAND2019-6988 C will devise a strategy for model preparation that is likely to include many complex, time-consuming geometric modifications. The defeaturing processs normally requires an expert user who can identify problematic geometry and select the appropriate tools to make local adjustments to the CAD model. These adjustments must be informed by sound engineering judgement based on knowledge of the physics to be simulated, along with an understanding of the mesh generation procedure and expected mesh quality outcomes.

Thus, we seek to significantly reduce the time and effort required for efficiently defeaturing CAD models while maintaining the ability of users to validate results and intervene in the process. Our goal is a system that permits users to graphically inspect a CAD model, efficiently guiding them to make modifications prior to automatic meshing that ensure quality meshing outcomes. Beginning with a solid design model composed of geometric entities (vertices, curves, and surfaces), the system should predict which entities will lead to suboptimal local mesh quality, presenting them to the user in prioritized order. For each entity, a set of suggested solutions should be presented, sorted based on their (predicted) ability to improve the lo- 
cal mesh quality outcomes. The user would then have the opportunity to preview, adjust, and perform the suggested operations as desired.

For this work, we are using machine learning to extend the framework described in [6], prioritizing suggested operations using predicted meshing outcomes to more effectively and efficiently guide the user.

\section{PRIOR WORK}

While machine learning is widely used in text, image, audio, and video analysis, there has been little research on the application of machine learning to model preparation for simulation. One notable work in this area is [7], which describes a limited environment for defeaturing CAD models where machine learning is driven by heuristic rule-based outcomes. While proposing several new criteria for evaluating defeauturing results from trained models, they rely on human interaction to judge the quality of results, making scalability problematic. In contrast, we use mesh quality metrics from an automatically generated FEA mesh as the training objective for defeaturing. This allows for automatic generation of training data, relying only on an embedded geometry and meshing environment. Other recent work has also demonstrated machine learning methods useful for shape recognition and classification of CAD models $[8,9,10]$. While related, these methods stop short of driving modifications to the CAD model such as those required for mesh generation and simulation.

\section{OVERVIEW}

Supervised machine learning is typically characterized as a problem where, given a training dataset $\left\{\left(\mathbf{x}_{\mathbf{1}}, \mathbf{y}_{\mathbf{1}}\right), \ldots,\left(\mathbf{x}_{\mathbf{n}}, \mathbf{y}_{\mathbf{n}}\right)\right\}$ with vector input features $\mathbf{x}$ and vector output features $\mathbf{y}$ (typically referred to as labels or ground-truth), it is assumed that there exists an unknown function $\mathbf{y}=f(\mathbf{x})$ that maps input features to output features. Using a learning algorithm, a model can be trained (or fit) to the data, so that the model approximates $f$. Once a model has been trained, it can be used to evaluate new, previouslyunseen input vectors to estimate (or predict) the corresponding output vectors. To apply supervised machine learning in a new problem area, the researcher must determine what the domain-specific outputs will be, identify the available domain-specific input features that can be used to predict them, and create a training dataset containing enough examples of each to adequately represent their distributions.

For this work, our first decision was to limit our scope to the defeaturing of individual parts. While operations correcting the interactions between parts to avoid gaps, overlaps and misalignments are of vital importance, we chose to save them for future work.
Next, we needed to define our machine learning model outputs. Since our goal, outlined in the introduction, was to rank geometric entities and solutions by their predicted local meshing outcomes, it followed that the outputs of our models $\mathbf{y}$ would be those outcomes, represented using mesh quality metrics. Similarly, the input features $\mathbf{x}$ for each model would be chosen to characterize the local CAD model geometry and topology that we presumed would drive those outcomes.

Given machine learning models that could predict mesh quality outcomes for a geometric entity or local region of a CAD model, we could use those predicted outcomes to present users with a sorted list of problem areas. Then, for a given problem area, we could use solution-specific machine learning models to present a sorted list of suggested solutions. A key insight during the design phase was the recognition that the set of local CAD model features that might influence the outcome for a given solution were themselves solution-specific. For example, there are at least two different strategies to resolve a sliver surface. One involves a composite operation that combines two adjacent surfaces (see Table 1(3)), while another is to remove the surface and extend the adjacent surfaces (see Table 1(1)). Describing the local geometry for these two distinctly different solutions requires distinctly different feature vectors. Because the size and definition of the input feature vectors $\mathbf{x}$ must be consistent for a given machine learning model, we necessarily trained multiple models, one per solution type.

At evaluation time, we can use our machine-learning models as follows:

- Predict the mesh quality outcomes for entities in a CAD model.

- Present the user with the list of entities, sorted from worst-to-best quality.

- For each entity in the list:

- Select a list of candidate operations for the entity.

- Predict the mesh quality outcome for each operation.

- Present the user with the list of operations, sorted from best-to-worst outcome.

Thus, the user is presented with a prioritized list of items to fix and operations to fix them. Inexperienced users can quickly defeature their model using a "greedy" approach by repeatedly choosing the first suggestion for every problem area, while users with greater experience are free to follow or ignore the suggested operations. We note that, while this greedy approach to defeaturing may not be optimal, it can 
provide inexperienced users with a principled, datadriven starting point for their work. We discuss alternatives to the greedy approach in Section 9.4.

\section{FEATURES}

To predict meshing outcomes with respect to local geometric entities requires characterization of the geometry and topology in the local neighborhood of each entity within the CAD model. For each entity $G_{R}(R=0,1,2)$ representing vertices, curves and surfaces respectively, a characteristic feature vector $\mathbf{x}^{G_{R}}$ was defined. In addition, local modification operations $O_{n}\left(G_{R}\right)$ that operate on $G_{R}$, were chosen. Since individual operations could involve modification of multiple nearby entities, a unique feature vector $\mathbf{x}^{O_{n}\left(G_{R}\right)}$ for each operation was also defined. While there are many possible choices for CAD operations, for purposes of this study we selected nine common operations available in the Cubit Meshing and Geometry Toolkit $[11,6]$ which are illustrated in Table 1.

Each of the nine operations $O_{n}\left(G_{R}\right)$ in Table 1 has a separate machine learning model with a distinct set of associated input features. In addition, three more models were created to characterize the unmodified entities $G_{R}$, making a total of twelve models used for this study. For each model, we tested several different types of input feature vectors.

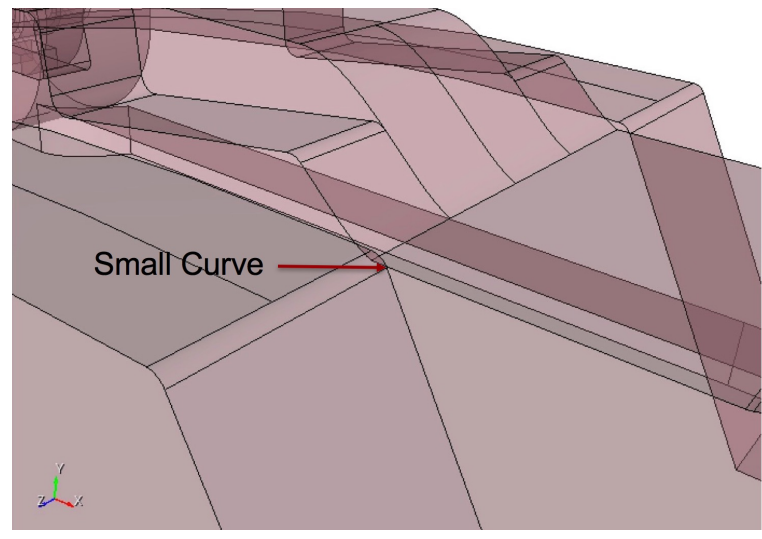

Figure 1: Small curve identified in CAD model

\subsection{Expert Features}

Expert features characterize $G_{R}$ and $O_{n}\left(G_{R}\right)$ based upon a fixed-length set of numerical values describing an entity and its relationships with its neighbors. For example, Figure 2 illustrates expert features for a given small curve from the CAD model shown in Figure 1. Table 2 describes the attributes used for expert features for vertices, curves and surfaces. Attributes in Table 2 are queried from a geometry engine

Geometry
operation name

(1) remove surface

(2) tweak replace surface

(3) composite surfaces

(4) collapse curve

(5) virtual collapse curve

(6) tweak remove topology curve

(7) tweak remove topology surface

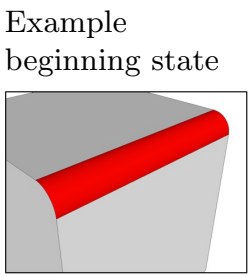

Example ending state
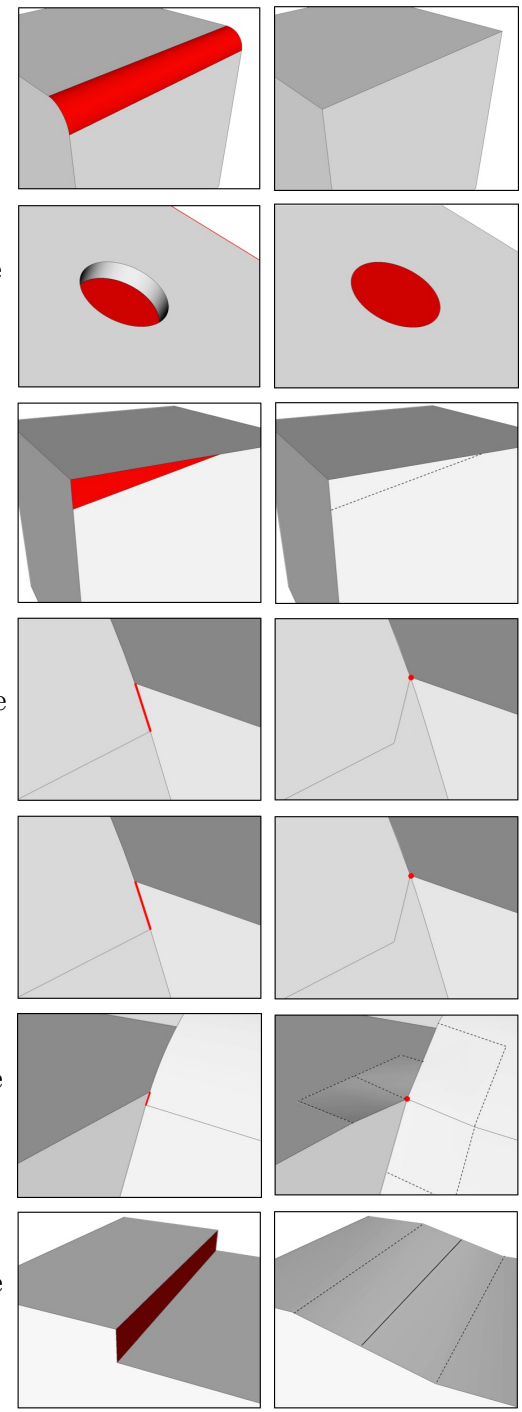

(8) blunt

tangency
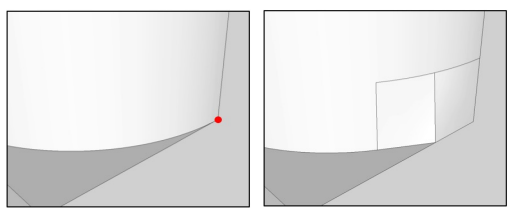

(9) remove cone
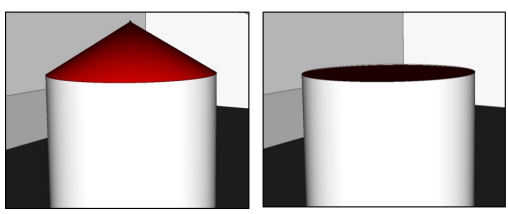

Table 1: Geometry modification operations $O_{n}\left(G_{R}\right)$. Example beginning and ending states of each operation are illustrated. 


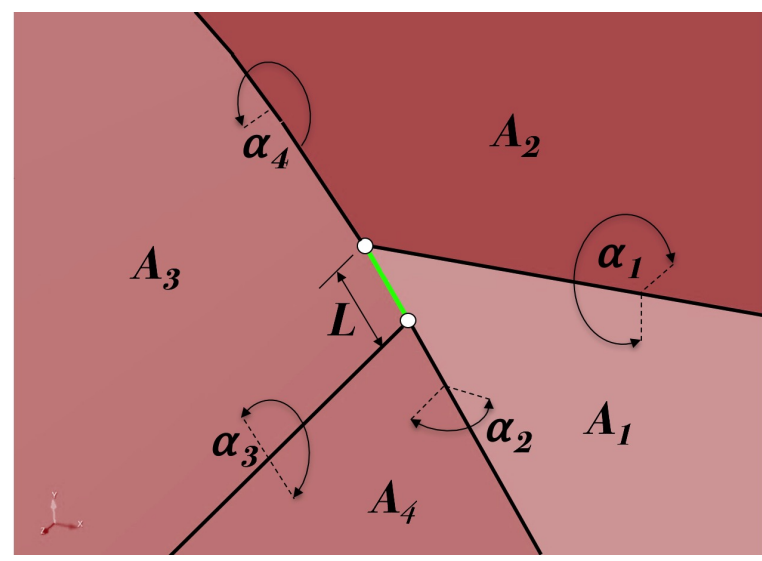

Figure 2: Sample expert features used for training data at a small curve

for each entity $G_{R}$ and used to construct $\mathrm{x}^{G_{R}}$. To create $\mathbf{x}^{O_{n}\left(G_{R}\right)}$, the vectors $\mathbf{x}^{G_{R}}$ for nearby entities were concatenated, so that the number of features used for each model is based upon the geometric entities involved in the operation. For instance, the composite operation $\mathrm{O}_{3}\left(G_{2}\right)$ includes attributes describing the two surfaces $G_{2}$ involved in the operation, as well as attributes from the neighboring curves and surfacess. In contrast, the collapse curve operation $O_{4}\left(G_{1}\right)$ includes features describing the curve to be collapsed, $G_{1}$ and its adjacent surfaces.

Since each machine learning model requires a constant size input feature vector $\mathbf{x}$ and local topology arrangements could include varying numbers of adjacencies, we truncate or extend the size of $\mathbf{x}$ to ensure a constant size. For example, the feature vector $\mathbf{x}^{G_{2}}$ for a surface includes attributes from surface $G_{2}$ as well as attributes from up to 4 adjacent curves and surfaces, where the adjacent surfaces are chosen from the two shortest and two longest surrounding curves. For surfaces with less than 4 curves, zeros are used to fill the remaining indices in $\mathbf{x}^{G_{2}}$.

\subsection{Geometric Features}

We introduce surflets and curvelets as complementary approaches for computing feature vector, $\mathbf{x}^{G_{R}}$ and $\mathbf{x}^{O_{n}\left(G_{R}\right)}$. Figures 3 and 4 illustrate surflet pairs developed by Wahl et. al.[12]. A surflet $S=(\alpha, \beta, \gamma, \delta)$ is a function of distance $\delta$ and angles, $\alpha, \beta, \gamma$ between two points with oriented normals on a surface as illustrated in Figure 5. Surflet pairs can be computed for any unique pair of points on the surface of a geometry. To convert an arbitrary number of surflets into a constant size vector $\mathbf{x}_{R}^{G}$, a four-dimensional histogram with dimensions defined by $\alpha, \beta, \gamma$ and $\delta$ is constructed. The values $\alpha, \beta, \gamma$ and $\delta$ for each surflet are computed and assigned to a discrete bucket $[I(\alpha), I(\beta), I(\gamma), I(\delta)]$. For our application we choose five intervals in each

\begin{tabular}{|c|c|c|}
\hline vertex & curve & surface \\
\hline $\begin{array}{l}\text { largest angle } \\
\text { between attached } \\
\text { curves }\end{array}$ & arc length & $\begin{array}{l}\text { surface type (pla- } \\
\text { nar, cylindrical, } \\
\text { parametric) }\end{array}$ \\
\hline $\begin{array}{l}\text { smallest angle } \\
\text { between attached } \\
\text { curves }\end{array}$ & $\begin{array}{l}\text { distance between } \\
\text { end points }\end{array}$ & number of loops \\
\hline tangency type & $\begin{array}{l}\text { distance from } \\
\text { mid-point to } \\
\text { segment }\end{array}$ & number of curves \\
\hline $\begin{array}{l}\text { number attached } \\
\text { curves }\end{array}$ & $\begin{array}{l}\text { tangent angle at } \\
\text { start }\end{array}$ & area \\
\hline \multirow[t]{6}{*}{ is convex } & $\begin{array}{l}\text { tangent angle at } \\
\text { end }\end{array}$ & perimeter \\
\hline & $\begin{array}{l}\text { exterior angle on } \\
\text { volume }\end{array}$ & $\begin{array}{l}\text { longest } \\
\text { curve/perimeter } \\
\text { ratio }\end{array}$ \\
\hline & $\begin{array}{l}\text { angle on surface } 0 \\
\text { at start }\end{array}$ & $\begin{array}{l}\text { shortest } \\
\text { curve/perimeter } \\
\text { ratio }\end{array}$ \\
\hline & $\begin{array}{l}\text { angle on surface } 0 \\
\text { at end }\end{array}$ & hydraulic radius \\
\hline & $\begin{array}{l}\text { angle on surface } 1 \\
\text { at start }\end{array}$ & $\begin{array}{l}\text { u principal curva- } \\
\text { ture at mid-point }\end{array}$ \\
\hline & $\begin{array}{l}\text { angle on surface } 1 \\
\text { at end }\end{array}$ & $\begin{array}{l}\mathrm{v} \text { principal curva- } \\
\text { ture at mid-point }\end{array}$ \\
\hline
\end{tabular}

Table 2: Expert features computed for individual geometric entities

dimension resulting in a total of 625 unique buckets. Our feature vector, $\mathbf{x}^{G}$ is therefore a vector of 625 integers that record the number of surflet pairs classified within each bucket $[I(\alpha), I(\beta), I(\gamma), I(\delta)]$.

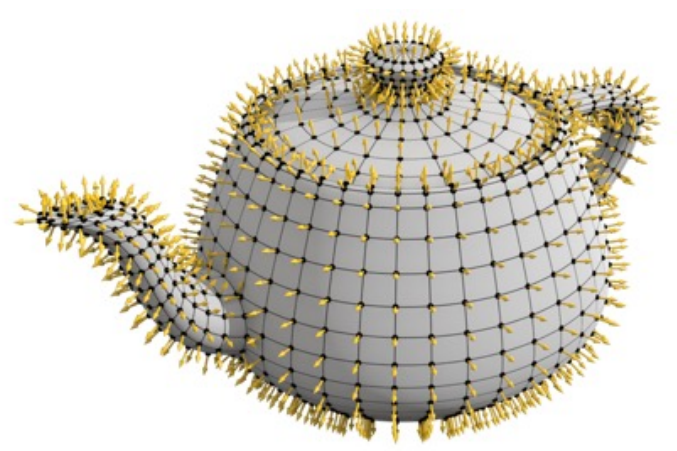

Figure 3: Example model showing points and normals used for computing surflets

Our implementation of surflet-based features requires first triangulating the surfaces of the CAD model to obtain a discretization. We limit the number of points that influence $\mathbf{x}^{G_{R}}$ and $\mathbf{x}^{O_{n}\left(G_{R}\right)}$ to those falling within a bounding box surrounding entity $G_{R}$ or $O_{n}\left(G_{R}\right)$. For computational efficiency, we also limit the number of points contributing to $\mathbf{x}^{G_{R}}$ and $\mathbf{x}^{O_{n}\left(G_{R}\right)}$ to 1000 when the local triangulation is dense, sampling the points at random from within the bounding box. 


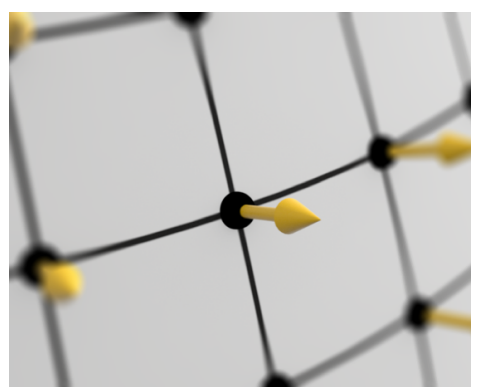

Figure 4: Close-up of a point and normal used for surflet calculation
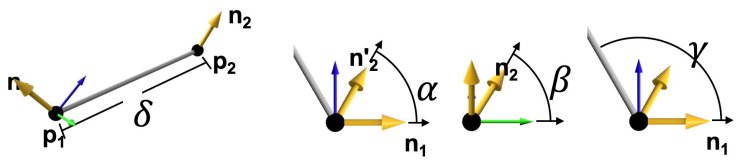

Figure 5: Surflet $\mathrm{S}$ is a function of the distance, $\delta$ and angles $\alpha, \beta, \gamma$ between two point / normal pairs on a surface

We note that surflet pairs can be computed from any point on the surface of the geometry near $G_{R}$ or $O_{n}\left(G_{R}\right)$. While providing an accurate representation of the nearby geometry, it tends to neglect any influence from the local topological arrangement of curves and surfaces. Since our objective in defeaturing is to identify changes to local topology through operations $O_{n}\left(G_{R}\right)$, we also explored an alternate method for constructing $\mathbf{x}^{G_{R}}$ and $\mathbf{x}^{O_{n}\left(G_{R}\right)}$ which we call "curvelets". In contrast to surflets, curvelets limit selection of points to those on the geometric curves that are topologically adjacent to $G_{R}$ or $O_{n}\left(G_{R}\right)$. Instead of limiting selection to a bounding box, we include points on all adjacent curves at $G_{R}$ or $O_{n}\left(G_{R}\right)$. In addition, rather than using the normal vector at a point, curvelets use the tangent vector on its associated curve. Surflets and curvelets can be used in combination or independently. We examine the characteristics and accuracy of surflets and curvelets and their combined effect in Section 7.

\section{GROUND TRUTH}

For each machine learning model associated with entity $G_{R}$ and operation $O_{n}\left(G_{R}\right)$, we needed to provide a ground truth output vector, which we do by generating a tetrahedral mesh and evaluating the local mesh quality. Automatic mesh generation methods often provide a variety of built-in algorithms to automatically improve or mitigate dirty geometry and we wish to take advantage of these capabilities. However, this means that the choice of meshing tool will have a significant effect on the resulting mesh quality, depending on the local topology, geometric modifications, and meshing parameters selected. Consequently, while our proposed methods are general and could be applied to using any automatic meshing tool, the ground truth values generated for this study are specific to the meshing tool and would not be transferable to another tool without re-training. In this work, we used the tools described in $[1,13]$ to generate our ground truth.

\subsection{Mesh Quality Metrics}

While any mesh quality metric [14] could be used to evaluate a mesh, we select three specific metrics based upon their representative characteristics. These include scaled Jacobian, in-radius and deviation.

Scaled Jacobian: The scaled Jacobian, $M_{s j}$ is defined as the minimum Jacobian at any of the four vertices of a tetrahedron divided by the lengths of its three adjacent edges. $M_{s j}=1$ represents a perfectly shaped equilateral tetrahedra, while $M_{s j}<0$ defines an inverted element. We utilize $M_{s j}$ as a ground truth as it is independent of mesh size and is representative of the Jacobian mapping function used in finite element methods.

In-Radius: The in-radius, $M_{i r}$ is defined as the radius of an inscribed sphere within a tetrahedra. Since this value is an absolute distance, we utilize a scaled in-radius value $M_{s i r} . M_{s i r}$ is defined as $M_{i r} / M_{i r}\left(S_{T}\right)$, where $M_{i r}\left(S_{T}\right)$ is the in-radius of an equilateral tetrahedra with edge length equal to target mesh size $S_{T}$. A value of $M_{\text {sir }}=1$ represents a perfectly sized element, while $M_{\text {sir }}<1$ is smaller than $S_{T}$ and $M_{\text {sir }}>1$ is larger than $S_{T}$. For training purposes we generate data at multiple target mesh sizes. We include $M_{\text {sir }}$ as a ground truth to learn characteristics that will avoid small elements that may result in long run-times for explicit FEA codes.

Deviation: The deviation, $M_{d}$, metric is defined as the distance from the centroid of a surface triangle to its closest point on the geometry. Unlike $M_{s j}$ and $M_{s i r}$ that describe characteristics of a tetrahedra, $M_{d}$ is a triangle metric that measures how closely the boundary of the mesh conforms to the prescribed geometry. For this metric we also compute a scaled deviation $M_{s d}=M_{s d} / S_{T}$. A value of $M_{s d}=0$ represents a triangle that perfectly matches the geometry. Values of $M_{s d}>0$ will be necessary for any geometry with curvature, however minimizing $M_{s d}$ is beneficial to ensure the mesh adequately represents the input geometry. In this case, the maximum value for $M_{s d}$ defines the worst quality.

Success/Failure: We note that candidate operations are identified for each small entity in a CAD model from a generic set of options. As a result, the particular local arrangement of curves and surfaces for a selected operation may not be valid. In most cases, the success or failure of an operation can only 
be determined by actually performing the operation and recording the result. Whether the CAD operation, $O_{n}\left(G_{R}\right)$ is successful and its subsequent meshing is successful, is also recorded and used as a label $M_{\text {success. }}$. This information is useful for identifying and eliminating solutions that would not be effective for defeaturing.

\subsection{Locality of Mesh Quality Metrics}

Assuming the mesh generation is successful following a CAD model modification, nearby tetrahedra and triangles at $G_{R}$ can be evaluated and a controlling minimum $M_{s j}, M_{s i r}$ and maximum $M_{s d}$ returned as a representative ground truth for operation $O_{n}\left(G_{R}\right)$. For this study we define the locality of the mesh near $G_{R}$ using two methods: bounding boxes, and local topology.

Bounding Box: We identify a set of tetrahedra, $\mathbf{T}_{B}$ falling within a Cartesian aligned bounding box $\mathbf{B}\left(G_{R}\right)$ surrounding entity $G_{R}$. The extent of $\mathbf{B}\left(G_{R}\right)$ is computed by adding the target mesh size, $S_{T}$ to all sides of a tight bounding box surrounding $G_{R}$. For operations $O_{n}\left(G_{R}\right)$, the set $\mathbf{T}_{B}$ includes a bounding box surrounding all entities involved in the operation. Figure 6 illustrates the set of tetrahedra, $\mathbf{T}_{B}$ falling within $\mathbf{B}\left(G_{1}\right)$ defined by a small curve, $G_{1}$. Only those tetrahedra in $\mathbf{T}_{B}$ falling within $\mathbf{B}\left(G_{R}\right)$ are considered when computing the controlling metrics for $M_{s j}, M_{s i r}$ and $M_{s d}$.

To compute $\mathbf{T}_{B}$ for an operation $O_{n}\left(G_{R}\right)$, the entities involved in the operation are identified prior to performing the operation and their combined bounding box computed. Once $O_{n}\left(G_{R}\right)$ is performed and a mesh generated, the controlling metrics can be computed. While simple to implement, depending on the arrangement of topology, $\mathbf{T}_{B}$ may encroach on other nearby entities where the controlling metric may conflict. We also note that the bounding box method is sensitive to orientation of the CAD model, as $\mathbf{B}\left(G_{R}\right)$ will be aligned with the global coordinate axis. To overcome these issues, we also introduce a method based upon the local topology at $G_{R}$.

Local Topology: We can identify the set of tetrahedra, $\mathbf{T}_{T}$ that share at least one mesh node on $G_{R}$ as well as those tetrahedra immediately attached to those at $G_{R}$. The local topology method for computing the controlling metrics is based only upon those tetrahedra in $\mathbf{T}_{T}$. Figure 7 illustrates the local set of tetrahedra $\mathbf{T}_{T}$ associated with a small curve. Since $\mathbf{T}_{T}$ includes only those tetrahedra in contact with $G_{R}$ and those immediately adjacent, it is less likely that $\mathbf{T}_{T}$ will encroach on neighboring entities. It also has the advantage of being insensitive to geometry orientation.

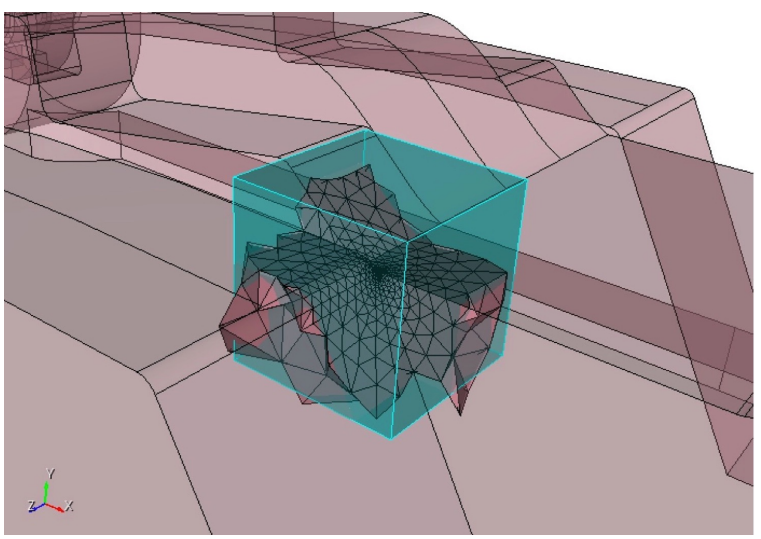

Figure 6: Example set of tetrahedra $\mathbf{T}_{B}$ defined by bounding box $\mathbf{B}\left(G_{1}\right)$ surrounding small curve $G_{1}$

We note that $\mathbf{T}_{T}$ is convenient to compute for $G_{R}$ prior to performing geometry operations as we can easily query for the set of nodes associated with $G_{R}$. However following operation $O_{n}\left(G_{R}\right)$, entity $G_{R}$ may no longer exist. For example, following the remove surface operation illustrated in Table 1 , the surface $G_{2}$ no longer exists, but is instead replaced by a curve defined by the intersection of two extended surfaces. As a consequence, it is necessary to identify one or more surviving entities for each operation $O_{n}\left(G_{R}\right)$ on which the set of tetrahedra $\mathbf{T}_{T}$ can be discerned. For the remove surface example, the surviving entity would be a single curve. $\mathbf{T}_{T}$ in this case would be defined by the set of nodes associated with the surviving curve. A similar set of surviving entities is also identified for each operation $O_{n}\left(G_{R}\right)$.

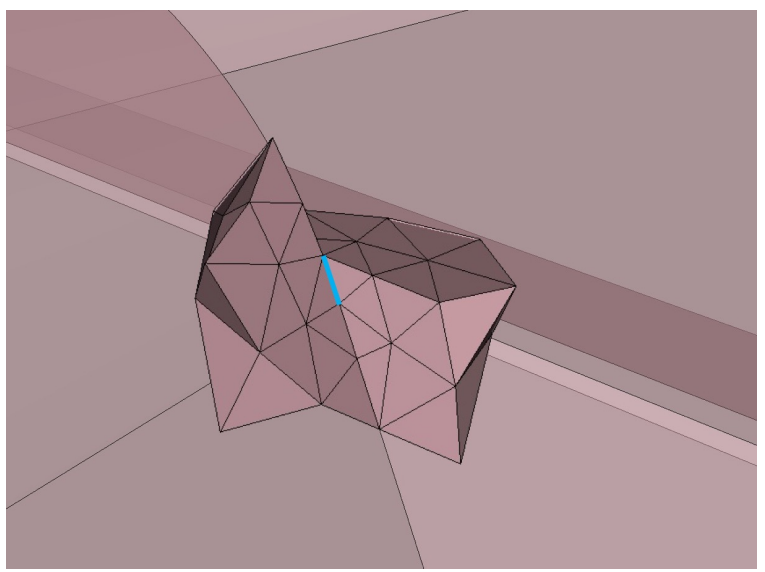

Figure 7: Example set of tetrahedra $\mathbf{T}_{T}$ defined by nodes associated with small curve $G_{1}$

\section{MACHINE LEARNING MODELS}

To generate training data for our study, we used a small sampling of 94 single-part CAD parts obtained 
from the open internet resource GrabCAD [15]. Figure 8 illustrates a few of these CAD parts used for training in this study. For each part, we generated training data for the twelve geometric operations described above, including 3 no_op models. This involved identifying small entities, where small was a function of a range of four target mesh sizes. We also identified bad vertices based on tangent or sharp angle conditions at the vertex. Relevant CAD operations selected for each small entity or bad vertex were identified from a pre-defined set of operations for each entity type. Some culling of relevant operations was initially accomplished to reduce the number of operations that needed to be trained. For example, the remove_cone operation was trained only for entities where the underlying CAD kernel identified it as a conic surface type. Similary blunt_tangency was only selected for vertices with adjacent curves forming an angle less than 10 degrees. For this reason the number of observations varied widely for each operation type. Table 3 shows the number of observations extracted from the CAD parts used for this study for each of the 12 operations trained.

\subsection{Training Data}

To generate training data, we used the following procedure:

\section{Import CAD part}

2. Compute a range of four target auto-sizes $S_{T}$ based on characteristics of the part. Do steps 3 to 10 for each $S_{T}$

3. Identify a list of the small entities and sharp vertices, $G_{R}$ based on $S_{T}$. Do steps 4 to 10 for each $G_{R}$.

4. Identify a list of relevant operations $O_{n}\left(G_{R}\right)$ for entity $G_{R}$. Do steps 5 to 10 for each $O_{n}\left(G_{R}\right)$

5. Compute fixed-length vectors of features $\mathbf{x}^{O_{n}\left(G_{R}\right)}$ for operation $O_{n}\left(G_{R}\right)$, including expert features, geometric features, and combinations of both.

6. Perform CAD operation $O_{n}\left(G_{R}\right)$

7. Mesh the part with size $=S_{T}$

8. Record success or failure of the geometry operation and meshing as label $M_{\text {success }}$

9. If geometry and meshing are successful, compute metrics, $M_{s j}, M_{s i r}$ and $M_{s d}$ based on locality $\left(\mathbf{T}_{T}\right.$ and $/$ or $\left.\mathbf{T}_{B}\right)$

10. Write one row to a .csv file containing features $\mathbf{x}^{O_{n}\left(G_{R}\right)}$, label $M_{\text {success }}$, and ground truth $M_{s j}$, $M_{\text {sir }}, M_{s d}$
We note that in some cases there were failures in this process, either because an operation failed, or meshing failed. This is indicated in step 8 of the above procedure. Table 3 also lists the number of failures for each operation in the study. When an operation failed, it was occasionally the result of a software defect. More often, the geometric kernel could not resolve the topology for the given input. For instance, a remove_surface is attempted on all surfaces identified as small. The local topology may not be resolvable for all cases where a small surface is to be removed. As a result, for our specific study, over half of the remove_surface operations failed. We did not distinguish between those that failed due to a software defect and those that failed due to topology. Instead, our models were trained to predict for any case of failure.

Failures in the subsequent meshing step happened when an operation succeeded, but its modifications affected the local topology so badly that the mesher was unable to proceed. In either case, we kept track of a categorical ground truth metric $M_{\text {success }}$ capturing whether the combination of operation and meshing succeeded or failed. Only operations that completed successfully recorded values for ground truth $M_{s j}, M_{s i r}$ and $M_{s d}$.

\begin{tabular}{|l|r|r|r|}
\hline & $\begin{array}{l}\text { Num } \\
\text { Obs. }\end{array}$ & $\begin{array}{l}\text { Num } \\
\text { Failed }\end{array}$ & $\begin{array}{l}\text { Num } \\
\text { Trained }\end{array}$ \\
\hline vertex_no_op & 1348 & 0 & 1348 \\
\hline curve_no_op & 9842 & 0 & 9892 \\
\hline surface_no_op & 5842 & 0 & 5842 \\
\hline remove_surface & 17,624 & 10,026 & 7598 \\
\hline tweak_replace_surface & 2569 & 1152 & 1417 \\
\hline composite_surfaces & 43,551 & 5020 & 38,531 \\
\hline collapse_curve & 13,830 & 2113 & 11,717 \\
\hline virtual_collapse_curve & 14,955 & 14,743 & 212 \\
\hline remove_topology_curve & 7056 & 5175 & 1881 \\
\hline remove_topology_surface & 3890 & 3102 & 788 \\
\hline blunt_tangency & 8059 & 3982 & 4077 \\
\hline remove_cone & 232 & 20 & 212 \\
\hline Totals & $\mathbf{1 2 8 , 4 8 4}$ & $\mathbf{4 5 , 3 3 3}$ & $\mathbf{8 3 , 5 1 5}$ \\
\hline
\end{tabular}

Table 3: Numbers of observations extracted from the 94 CAD parts used in this study.

\subsection{Training}

We used Python [16] and the scikit-learn library [17] to train machine learning models for each geometric operation. This included twelve classification models to predict whether an operation was likely to succeed or fail (see Section 7.1), and twelve regression models to predict our six per-operation mesh quality metrics 

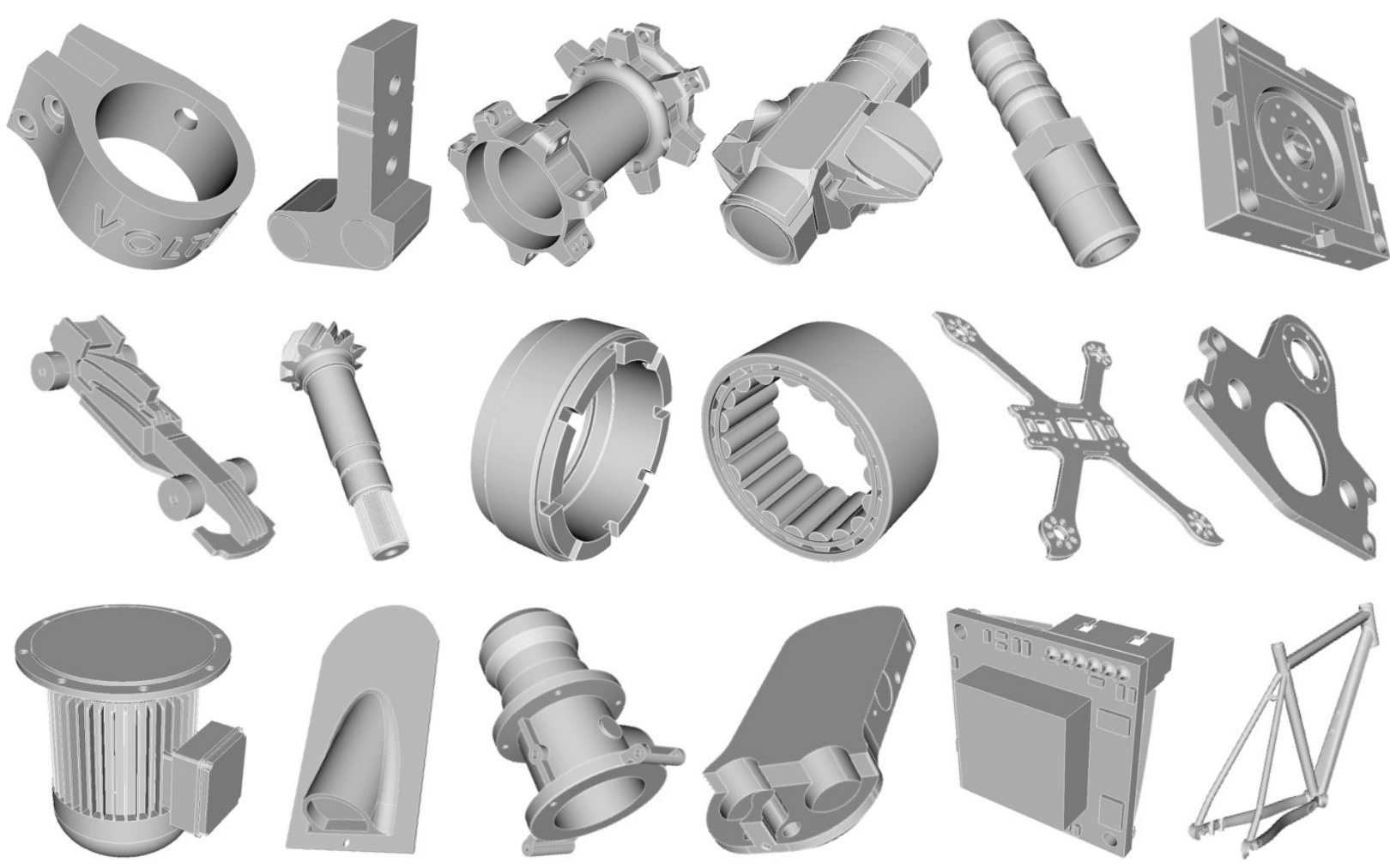

Figure 8: Examples of the $94 \mathrm{CAD}$ models used for training in this study.

(see Section 7.2). Thus, we created a total of 24 models.

Due to the novelty of our problem, there was great uncertainty over which (if any) of the features in our training data would be useful for generating machine learning models. For some model types, redundant, irrelevant, or misleading features can negatively impact the performance of the model. Thus, feature selection - including the removal of correlated features - is common in these cases.

In our case, we choose to use ensembles of decision trees (EDTs) instead [18]. An EDT is a collection of individual decision trees, each of which is trained on a subset of the full training data using a technique called bagging [19]. At evaluation time, the EDT's prediction is a weighted sum of the predictions of each of its individual trees. Colloquially, EDTs capture the "wisdom of crowds" by allowing each tree to become an expert on a subset of the data, using that localized wisdom to vote for a final result.

The beauty of EDTs is in their proven ability to exploit weak signals, even in features that are only slightly less than perfectly correlated. Thus, we did not perfom any feature thinning before training, finding it to be actively harmful to model accuracy.

Further, EDTs make popular general purpose machine learning models due to their easy interpretability (each tree contains a set of branching boolean tests that are applied to the input features, with output predictions stored in the leaf nodes) and their ability to compute feature importance metrics that capture how often a given feature is useful when arriving at a decision (see Figure 22 for sample feature importance outputs). It is worth noting that EDT feature importance metrics capture a more nuanced view of the input features than a simple correlation matrix, as they are a reflection of the rich, nonlinear representation space of the individual trees in the ensemble.

An important hyperparameter affecting the performance of an EDT is the number of trees that are contained within it. When training EDTs, we look to see that the performance of the ensemble converges asymptotically as the number of trees increases. Figure 9 illustrates this behavior for one of our models (note that the error is decreasing, which is synonymous with increasing performance). Using plots like Figure 9, we chose by inspection to use 75 trees each for the ensembles in the following experiments.

\section{RESULTS}

To evaluate the performance of a machine learning model, we typically split the available training data 


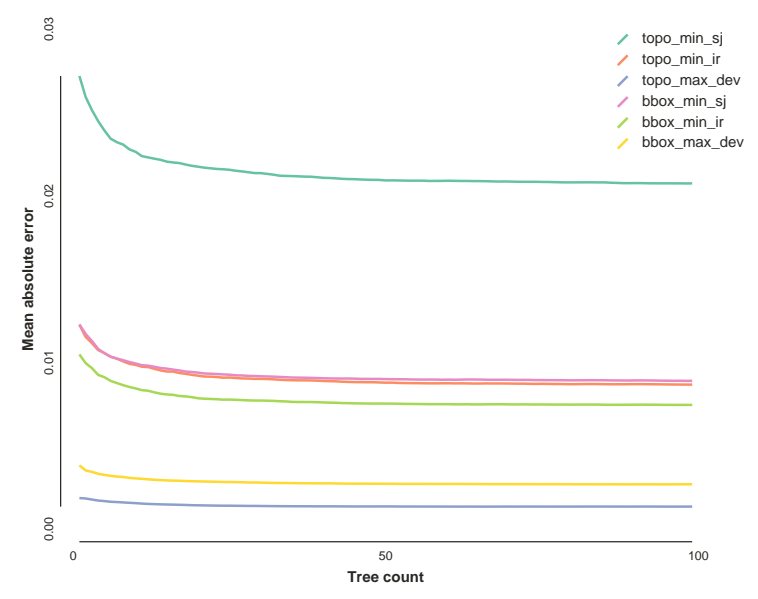

Figure 9: Performance of an EDT model versus the number of trees, for our six metrics.

into randomly-chosen partitions: one containing data used to train the model, and one containing data held back to test performance after training is complete. This makes it possible to identify models that have overfit or memorized their training data, by evaluating them solely on the unseen test data.

However, a single partitioning by itself complicates performance evaluation, since the partitions might, through random chance, lead to models with unrealistically high (or low) performance. This problem is compounded when comparing models (for example: comparing two models to see whether expert or surflet features produce better outcomes), since a model with an "unlucky" partitioning might be penalized unfairly when compared against a model with a "lucky" set of partitions.

To combat this effect, all of the following results were computed using $5 \times 2$ cross validation, which involves randomly partitioning the training data into two equal size sets; training a model on the first set and testing it on the second; training a model with the second set and testing it on the first; repeating this process four more times for a total of ten models. The resulting cross validated results are the averaged results from the individual models. Using $5 \times 2$ cross validation thus provides an extremely conservative estimate of a model's performance, and is widely used in the machine learning community when comparing two models to see which is best.

\subsection{Failure Prediction Models}

First, we evaluated the performance of our twelve peroperation failure prediction models. Because the failure prediction models are classification models that produce a single categorical "succeed" or "fail" out-

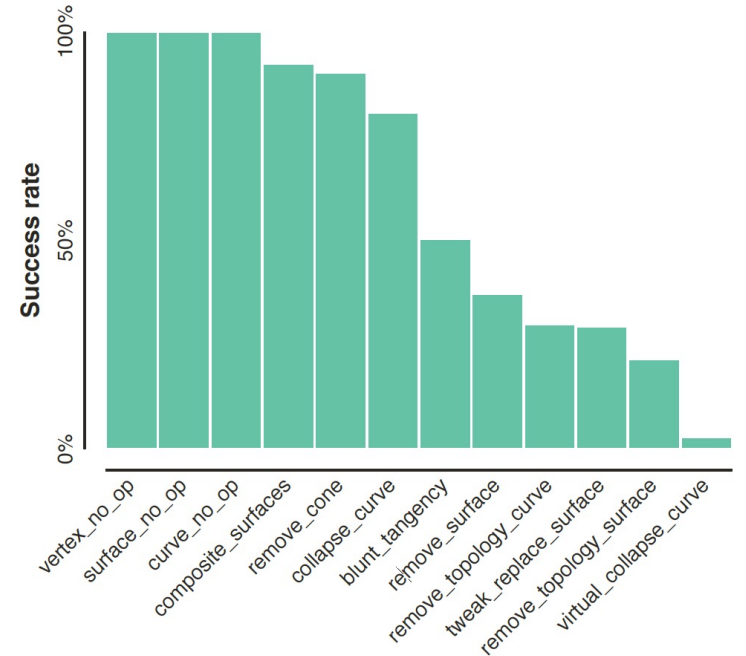

Figure 10: Percentage of meshing operations that succeeded, by type, sorted by success rate. The first three operations always succeed because they are do-nothing placeholders.

put, we evaluated their performance using precision and recall metrics. In this case precision is the percentage of operations predicted to fail that actually failed, while recall is the percentage of actual failures that were predicted to be failures. An ideal model should balance high recall (avoiding false negatives) with high precision (avoiding false positives).

From Table 3 and Figure 10, we see that there were many failures encountered during training data generation, and that some operations failed more often than they succeeded for the local arrangement of curves and surfaces. This suggests that the failure prediction models could play a significant role in avoiding problems for the end user, by steering them away from operations that are unlikely to succeed for a given region within the geometry.

Figures 11 and 12 show the precision and recall scores respectively for failure prediction models trained using four sets of features: expert, surflet, curvelet, and a combination of all three. The results are grouped by operation, and the groups are sorted using the scores for models trained using only expert features. In all cases, larger values are better.

Figure 12 shows that all of our models achieve excellent (nearly 100\%) recall, aggressively identifying all of the actual failures in the training data. However, Figure 11 paints a more complex picture, with the models achieving a wide range of precision scores. The models with low precision scores are too aggressive, since low precision in this case means that the model is predicting failures for operations that actually succeeded in real life. Models with a precision lower than 0.5 


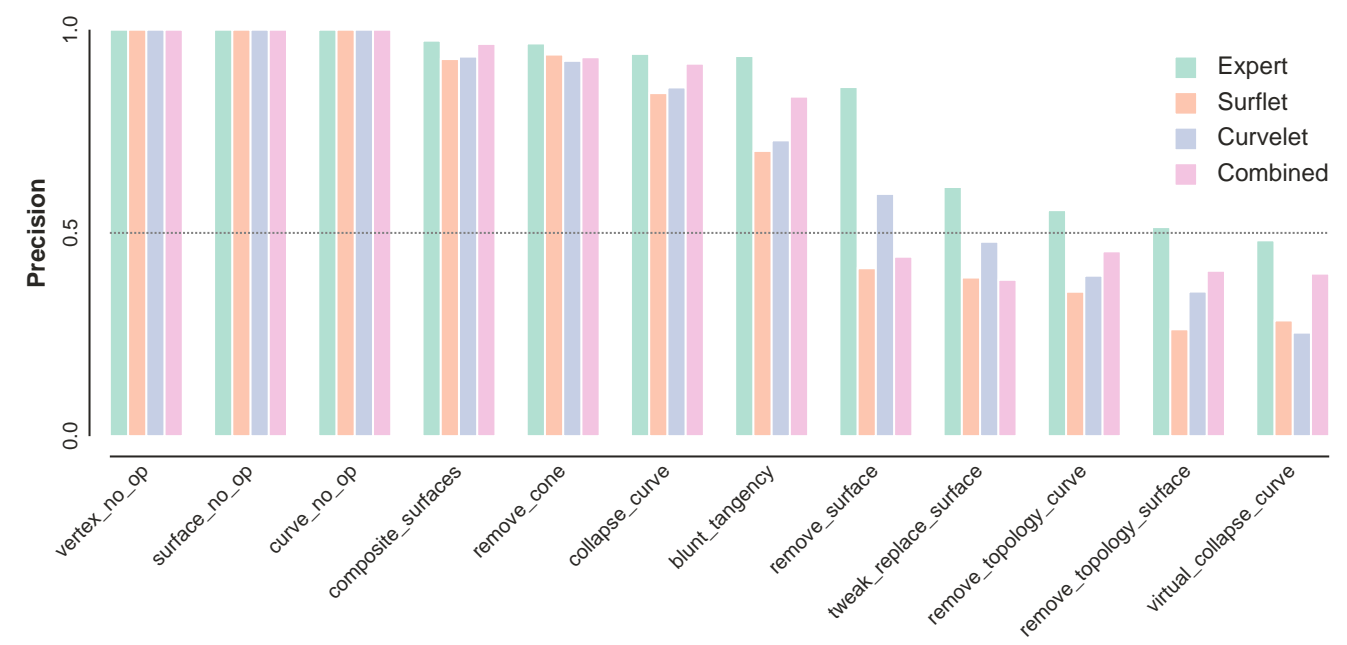

Figure 11: Failure prediction model precision. Larger values are better.

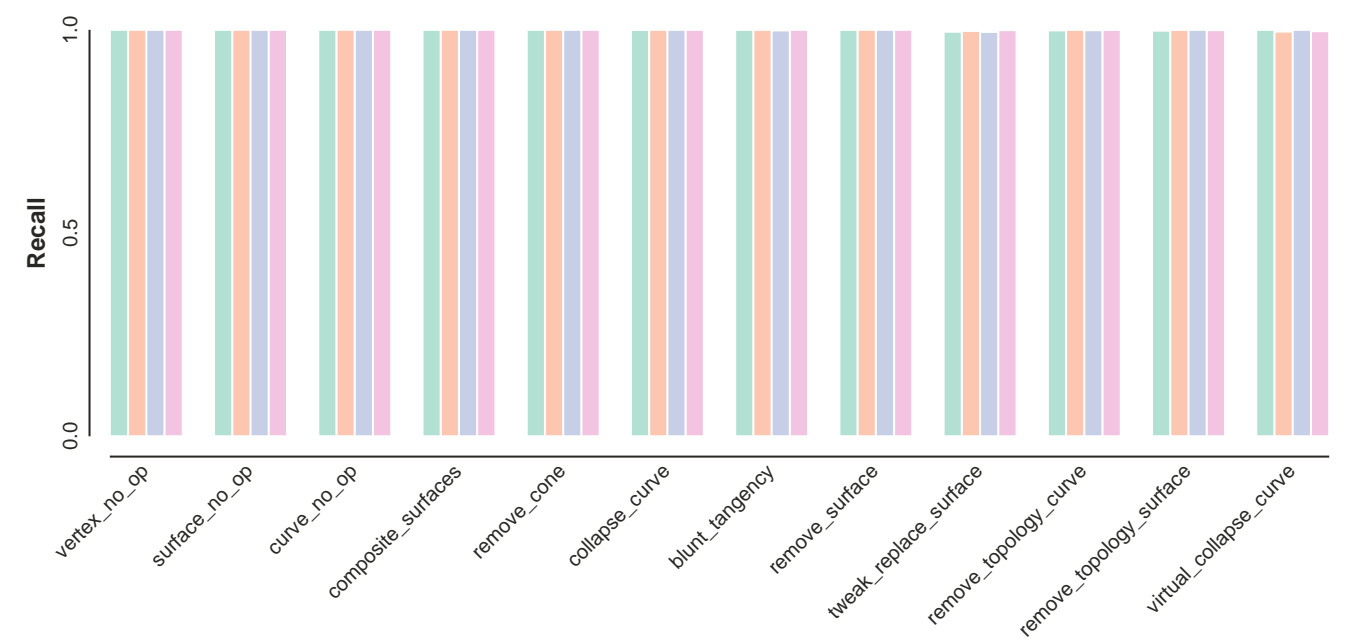

Figure 12: Failure prediction model recall. Larger values are better.

(marked with the dashed line in Figure 11) are wrong more often than right, and would not be useful in practice. It is interesting to note that the performance of the models in Figure 11 correlates closely with the failure rate in Figure 10, suggesting that unskewing the data may improve performance. Regardless, it is clear from the figures that the models trained using expert features have considerably higher performance than those trained with the other features in every case, even models trained with a combination of all three feature types. Further, we see that curvelet features produce slightly better results than surflets in most cases.

\subsection{Mesh Quality Models}

Next, we evaluated the performance of our twelve peroperation mesh quality models. As in the previous section, the models were evaluated using the expert, surflet, curvelet, and combined features. Since these models produced regression outputs predicting the scaled Jacobian $M_{s j}$, scaled in-radius $M_{s i r}$, and scaled deviation metrics $M_{s d}$, computed using bounding-box $\mathbf{T}_{B}$ and local topology regions $\mathbf{T}_{T}$, there were a total of six outputs for each model. Because these metrics are continuous rather than categorical, the model results are reported using mean absolute error (MAE), the 


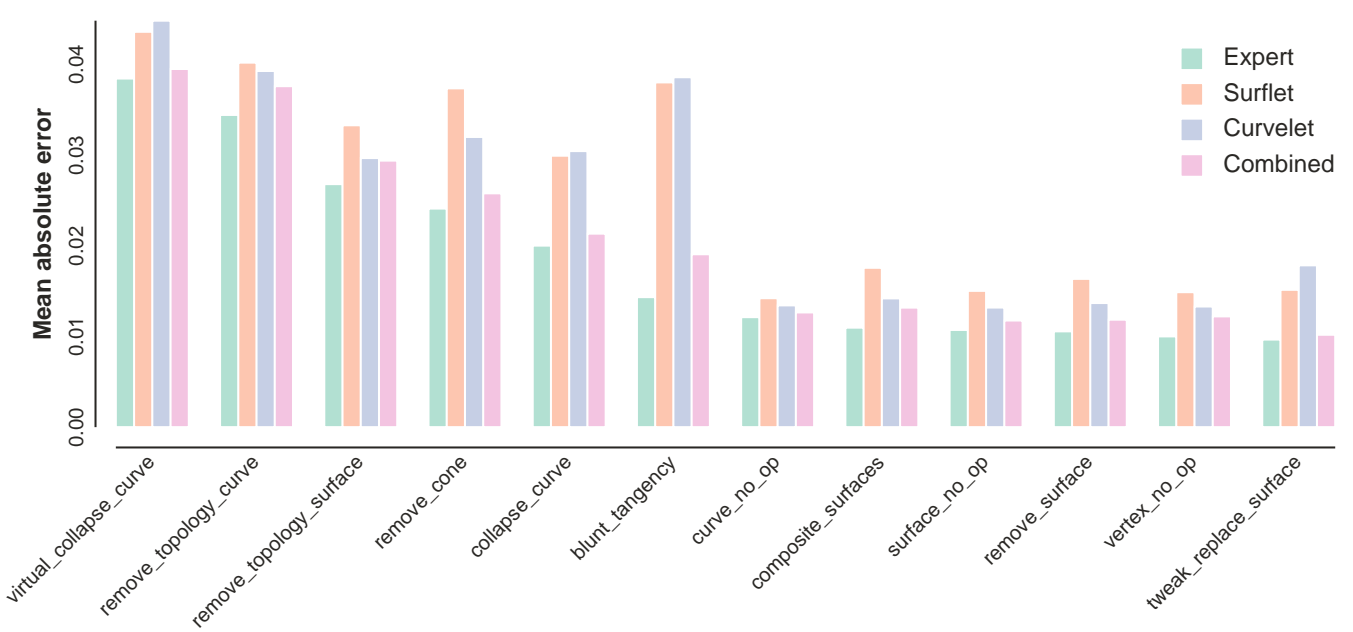

Figure 13: Mesh quality model MAE averaged across mesh quality metrics. Smaller values are better.

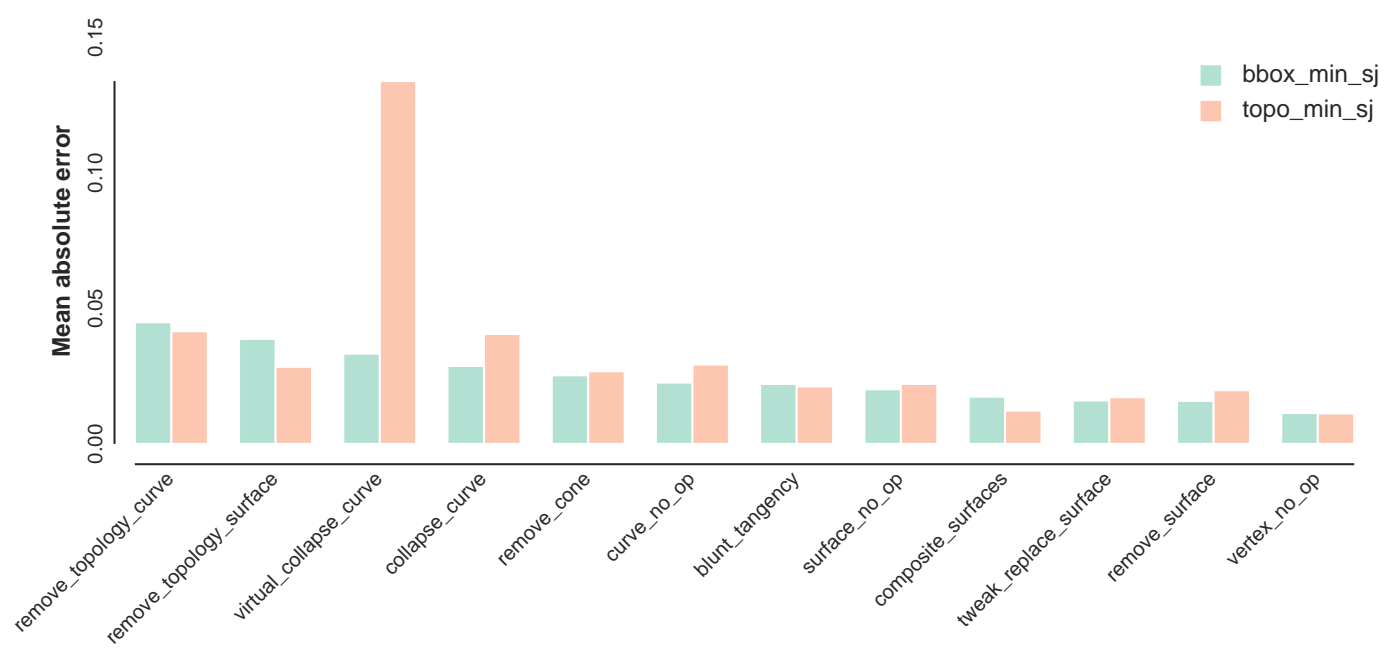

Figure 14: Comparing bounding-box and local topology scaled Jacobian metrics. Smaller values are better.

average of the absolute differences between the predicted and actual post-meshing quality metric values. Note that, in contrast to the failure prediction results, smaller MAE values are better.

Since the expert features performed so strongly in our failure prediction models, we began by looking for similar patterns with our mesh quality models. As seen in Figure 13, expert features once again perform significantly better than the geometric features, and curvelets continued to perform better than surflets in a majority of cases.
These results lead us to assert that geometric or shape characteristics by themselves are insufficient to accurately inform a model of the effects of CAD operations on a mesh. Instead, our results illustrate that the topological characteristics identified by our expert features are needed in order to more precisely predict meshing outcomes, and that more topological information is better than less.

Note that these results illustrate general trends among the different feature types, and that the MAE in Figure 13 is an average of the MAE for all six mesh qual- 


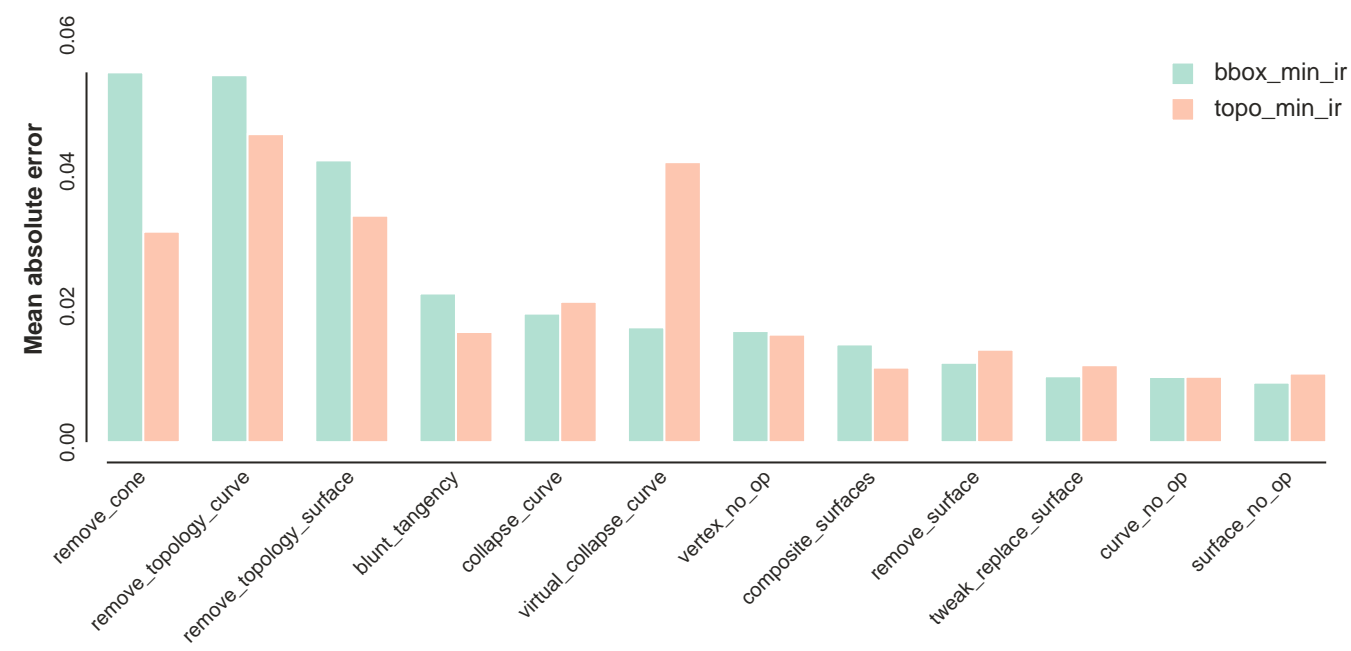

Figure 15: Comparing bounding-box and local topology in-radius metrics. Smaller values are better.

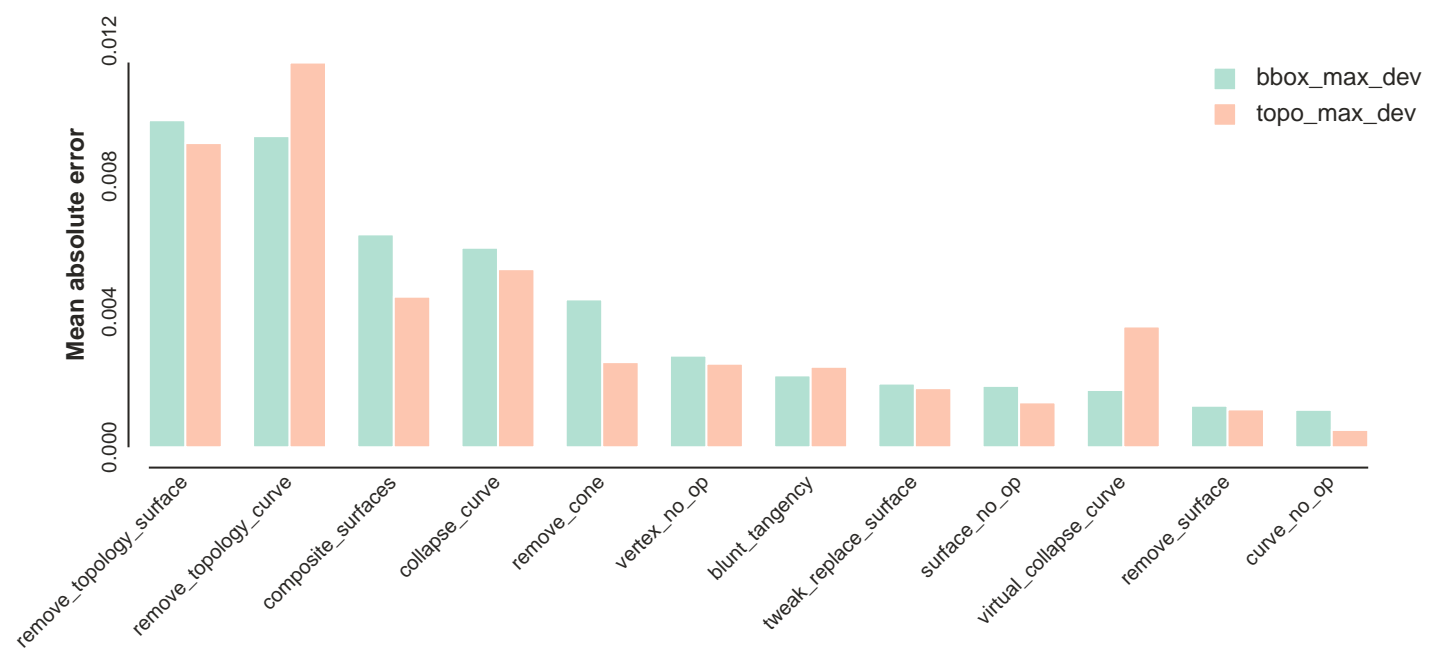

Figure 16: Comparing bounding-box and local topology deviation metrics. Smaller values are better.

ity metrics for each operation. Results for each of the mesh quality types $M_{s j}, M_{s i r}$ and $M_{s d}$ are illustrated separately in Figures 14, 15 and 16 respectively.

Since expert features proved more accurate in characterizing our CAD operations, we limit demonstration of performance of each of our three target metrics in Figures 14 through 16 to expert features. In these figures, we compare the performance of the two locality choices, bounding box $\mathbf{T}_{B}$ and topology $\mathbf{T}_{T}$. We observe that for both scaled Jacobian (Figure 14) and scaled in-radius (Figure 15) most operations had an MAE in the range of 0.05 or less. That means that predictions of $M_{s j}$ and $M_{s i r}$ from the proposed machine learning models can be expected to be less than 0.05 on average. For $M_{s d}$ (Figure 16) the MAE was less than 0.01 in most cases.

When comparing $\mathbf{T}_{B}$ and $\mathbf{T}_{T}$ the results were much more nuanced, with bounding-box locality providing a small-but-consistent performance boost for the scaled Jacobian metric, roughly identical performance for the in-radius metric, and consistently lower performance for the deviation metric. Based on the ambiguity of these results, we would likely choose to use local topology based regions in production, since they select sig- 
nificantly fewer tetrahedra, tend not to encroach on adjacent geometric features, and are orientation invariant, as described in Section 5.2.

We also note that for all operations trained, we computed performance only for those operations predicted to succeed as indicated by our failure models (see Figure 10). This tended to limit our sample size for some of the operations such as virtual_collapse_curve and remove_topology_surface. This may account for reduced accuracy in some of our predictions as illustrated by the outlier virtual_collapse_curve performance in Figure 14 .

\section{APPLICATION}

Each model was serialized to disk for use with interactive or automatic defeaturing. To demonstrate the proposed ML-based defeaturing environment both an interactive graphical tool and an automatic greedy algorithm were implemented.

\subsection{Interactive GUI}

A graphical user interface panel was implemented for the Cubit geometry and meshing toolkit $[11,6]$. Figure 17 illustrates the modified panel that we used to manage and drive defeaturing. In this environment, a list of entities $G_{R}$ shown in Figure 17(h) predicted to cause poor quality are listed ordered from worst to best based upon the selected metric (Figure 17(e)). Only those entities with predicted quality below a user-defined threshold shown in Figure 17(g) are displayed.

Selecting entity $G_{R}$ will reveal a list of possible solutions $O_{n}\left(G_{R}\right)$ shown in Figure 17(i) prioritized based on predicted mesh quality. Predicted quality outcomes are shown in parentheses next to each operation. The user can preview the operation, accept it, modify the solution, or ignore it all-together.

A simple illustration of how this GUI might be used is shown in Figure 18 and Table 4. In this example, a tangency condition at vertex 143 is predicted to result in nearby tetrahedra with a minimum $M_{s j}$ of approximately 0.0725 . To improve the quality, the operation composite create surface $\mathbf{7 7} \mathbf{4 2}$ is suggested which is predicted to result in $M_{s j}$ of approximately 0.1435 . In this example, the the machine learning models predict that combining or compositing the two adjacent surfaces 77 and 42 will improve the local mesh quality. The user can choose to accept this suggestion, or choose an alternative. To evaluate the accuracy for this one example, Figure 19 shows a mesh generated before and after performing the composite operation. The local mesh quality at vertex 143, illustrated in Figure 19 (a) results in $M_{s j}$ of about 0.1025 , an error

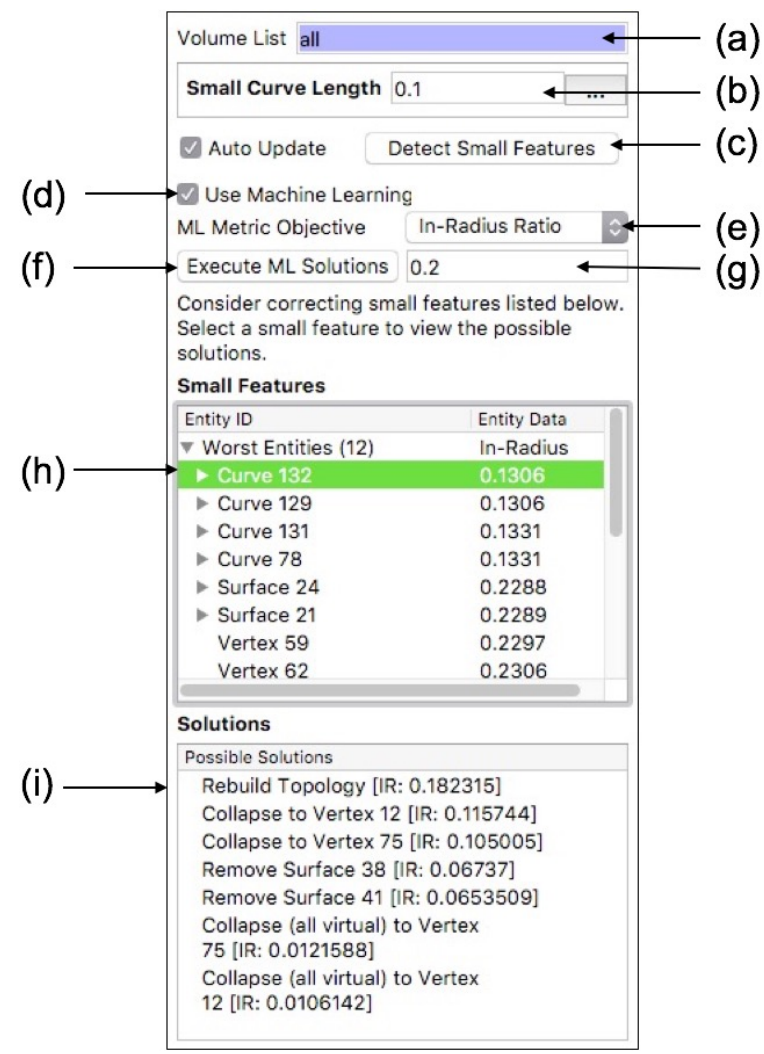

Figure 17: A proposed graphical interface for driving ML-based defeaturing. (a) List of volumes to be defeatured. (b) User defined size considered small. (c) Button to detect and populate panel with small features. (d) Option to use ML. Loads ML models. (e) User selects target metric for criteria (minimum $M_{s j}, M_{s i r}$ or maximum $M_{s d}$ ) (f) Button to execute automatic defeaturing using greedy criteria. (g) Limit for display of predicted worst quality. (eg. entities with predicted $M_{s j}$ less than 0.2 are listed) (h) Ordered list of entities showing predicted mesh quality. (i) Ordered list of CAD operations to correct the selected entity in $(\mathrm{h})$, prioritized by predicted quality.

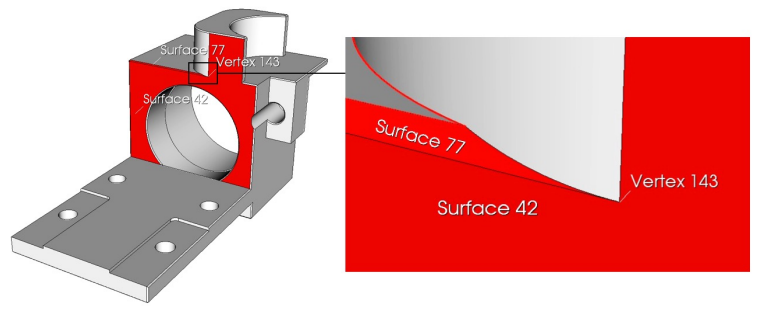

Figure 18: Defeaturing example where tangency condition exists at vertex 143. Machine learning models predict that a composite operation between neighboring surfaces 42 and 77 will improve mesh quality at this vertex.

of about 0.03. Similarly we show the mesh following the operation in Figure 19(b) where the local mesh 


\begin{tabular}{|l|l|l|l|l|l|l|l|}
\hline Operation & $\begin{array}{l}\text { Num } \\
\text { tets }\end{array}$ & $\begin{array}{l}\text { Global } \\
\text { min } M_{s j}\end{array}$ & $\begin{array}{l}\text { Num tets } \\
M_{s j}<0.2\end{array}$ & $\begin{array}{l}\text { Predicted } \\
\text { no-op }\end{array}$ & $\begin{array}{l}\text { Actual } \\
\text { no-op }\end{array}$ & $\begin{array}{l}\text { Predicted } \\
\text { op }\end{array}$ & $\begin{array}{l}\text { Actual } \\
\text { op }\end{array}$ \\
\hline Initial & 269957 & 0.1025 & 5 & & & & \\
\hline composite create surface $\mathbf{7 7} \mathbf{4 2}$ & 268528 & 0.1511 & 3 & 0.0725 & 0.1025 & 0.1435 & 0.1935 \\
\hline
\end{tabular}

Table 4: Example of the effect on scaled Jacobian $M_{s j}$ from a single operation performed on CAD model shown in Figures 18 and 19. Comparison to actual mesh quality is also shown.

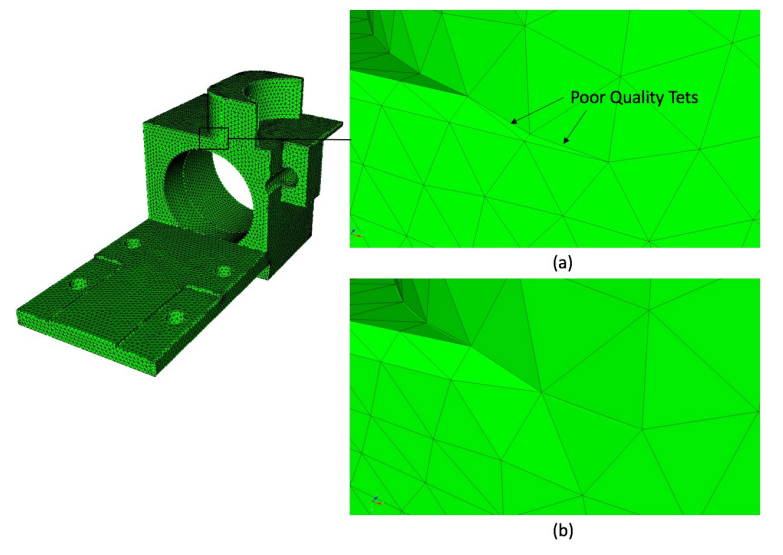

Figure 19: Tetrahedral meshes based on geometry in Figure 18 (a) Mesh at vertex 143 before suggested defeaturing operation. (b) following defeaturing operation. Mesh quality for this example is illustrated in Table 4.

quality resulted in $M_{s j}$ of 0.1935 , an error of about 0.05 .

\subsection{Greedy Algorithm}

The user also has the option to accept the best predicted solutions without having to manually execute each one individually. The button shown in Figure $17(\mathrm{f})$ runs a greedy algorithm as follows: the predicted worst quality entities $G_{R}$ are successively modified using the best predicted operations $O_{n}\left(G_{R}\right)$. This continues until all entities have a predicted quality exceeding the user specified threshold at Figure 17(g).

We illustrate a simple greedy procedure in Table 5 and Figures 20 and 21. In this example, seven operations are automatically selected based upon minimum scaled in-radius $M_{\text {sir }}$ predictions. In this case, Table 5 shows the number of small elements falling below a user defined threshold of $M_{\text {sir }}=0.2$ reduced from over 10,000 to 1 and the minimum $M_{\text {sir }}$ increased from 0.0078 to 0.1628 . The CAD operations composite and tweak remove topology are used to defeature the model as illustrated in Figures 20 and 21.

We note that the proposed machine learning models automatically identify the conical surfaces illustrated in Figure 20(a) as those that will produce an unfavorable $M_{\text {sir }}$ metric. In this case, the surface mesh generator used for this study [1] will characteristically identify and refine to the apex of the conical surfaces. Training has identified this characteristic and our models correctly predict the mesh quality outcome. Figure 20(b) shows a portion of the surface mesh at the conical surfaces if the suggested composite operation had not been applied. Figure 20(c) shows the same surfaces once the composite is applied. Note that the proposed models correctly identified the composite operation as the best method for increasing the target mesh quality, $M_{\text {sir }}$.

Similarly, the machine learning models have predicted that the small curve 218 illustrated in Figure 21(a) will produce mesh quality $M_{\text {sir }}$ less than the userprescribed threshold of 0.2 . The best choice for improving this condition was predicted to be the CAD operation, tweak remove_topology curve, illustrated in Figure 21(a). The surface mesh without applying this operation is shown in Figure 21(b) and the resulting mesh, if the operation is applied, is shown in Figure $21(\mathrm{c})$.

Although the principal purpose of the proposed machine learning models is to predict and correct the worst quality artifacts in a CAD models without meshing, to evaluate the accuracy of our methods, Table 5 compares the predicted metrics to the actual mesh quality from meshes produced before and after the operation is performed. For example, Table 5 shows that the predicted quality at curve 218 would change from $M_{\text {sir }}=0.1039$ to 0.2935 as a result of performing the indicated operation. We compare that with the actual mesh quality values of 0.1070 and 0.2813 respectively.

\section{CONCLUSIONS}

A new application of modern machine learning technologies to prepare models for simulation has been introduced. We have demonstrated the ability to accurately predict mesh quality based on local topology of a CAD part without having to generate a mesh. We have also introduced methods for identifying CAD operations to effectively defeature a CAD model by predicting meshing outcomes for a range of selected operations. A study based on a limited set of 94 opensource CAD parts was used to generate training data for 24 separate models that predict failure and mesh quality metrics. New methods for computing features and ground truth were introduced and their accuracy 


\begin{tabular}{|l|l|c|c|c|c|c|c|}
\hline Operation & $\begin{array}{l}\text { Num } \\
\text { tets }\end{array}$ & $\begin{array}{l}\text { Global } \\
\text { min } M_{\text {sir }}\end{array}$ & $\begin{array}{l}\text { Num tets } \\
M_{\text {sir }}<0.2\end{array}$ & $\begin{array}{l}\text { Predicted } \\
\text { no-op }\end{array}$ & $\begin{array}{l}\text { Actual } \\
\text { no-op }\end{array}$ & $\begin{array}{l}\text { Predicted } \\
\text { op }\end{array}$ & $\begin{array}{l}\text { Actual } \\
\text { op }\end{array}$ \\
\hline Initial & 269957 & 0.0078 & $10000+$ & & & & \\
\hline composite create surface 18 17 & 263957 & 0.0070 & 9477 & 0.0236 & 0.0086 & 0.4103 & 0.3139 \\
\hline composite create surface 15 14 & 256093 & 0.0072 & 5917 & 0.0236 & 0.0095 & 0.4103 & 0.2982 \\
\hline composite create surface 12 11 & 249603 & 0.0065 & 2020 & 0.0236 & 0.0080 & 0.4103 & 0.2962 \\
\hline composite create surface 9 8 & 245917 & 0.1069 & 54 & 0.0241 & 0.0065 & 0.4103 & 0.3147 \\
\hline tweak remove_topology curve 218 & 244754 & 0.1934 & 2 & 0.1039 & 0.1070 & 0.2935 & 0.2813 \\
\hline tweak remove_topology curve 176 & 244620 & 0.1628 & 4 & 0.1237 & 0.1935 & 0.2539 & 0.1628 \\
\hline tweak remove_topology curve 178 & 245172 & 0.1628 & 1 & 0.1352 & 0.1770 & 0.2381 & 0.1628 \\
\hline
\end{tabular}

Table 5: Example of CAD operations performed automatically from greedy predictions of minimum scaled in-radius $M_{s i r}$. Operations and mesh illustrated in Figures 20 to 21

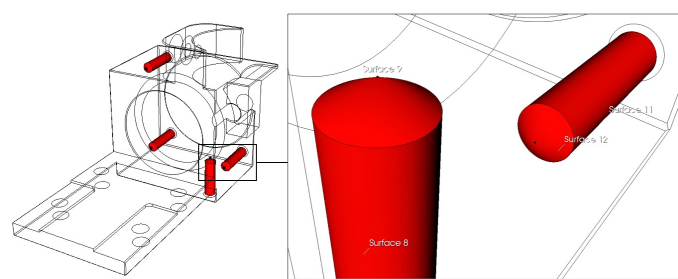

(a)

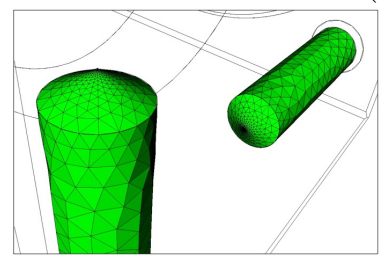

(b)

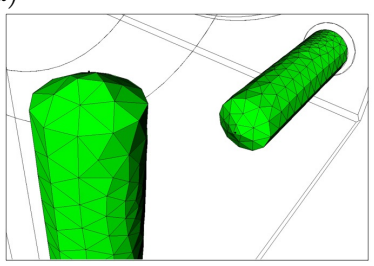

(c)
Figure 20: Illustration of initial 4 composite operations from Table 5 generated from the greedy method. Operations were automatically identified based on predictions of minimum scaled in-radius $M_{\text {sir }}$. (a) 4 holes with conical shafts are automatically identified. (b) Mesh produced on surfaces of holes without applying composite operations. Note that mesh generator automatically refines to cone apex. (c) Resulting mesh after composite operations applied.

assessed.

\subsection{Feature Importance}

We introduced new methods for defining features for our machine learning methods. We found that mesh quality predictions based on expert features were more accurate than geometric features that used surflets and curvelets. Expert features selected for each entity type and operation included local attributes such as arc lengths, angles, areas, curvatures and other characteristics. Although results indicated that the selected attributes in this implementation led to reasonably accurate predictions, additional study would be warranted to identify additional features of value.

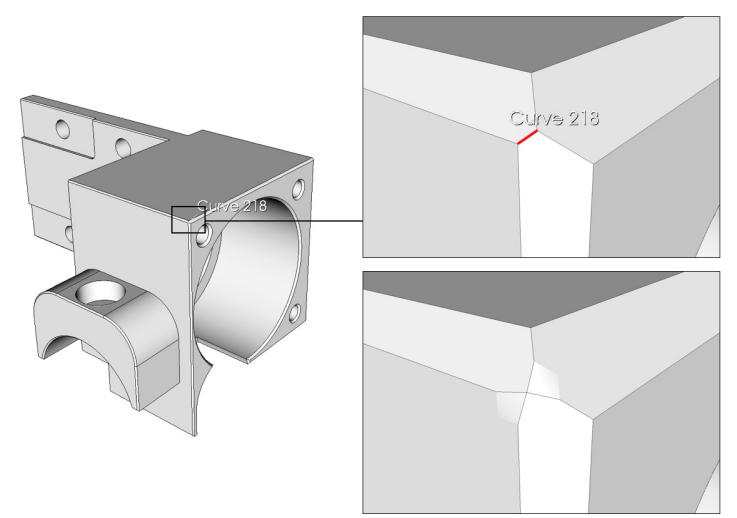

(a)

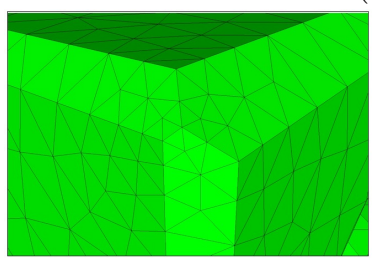

(b)

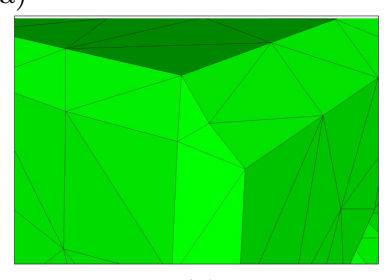

(c)
Figure 21: Example of tweak remove_topology curve operation used in the greedy procedure from Table 5. (a) (Top) Curve 218 is predicted to have poor quality, $M_{\text {sir }}$ (Bottom) tweak remove_topology curve operation applied to curve 218. (b) Surface mesh at curve 218 without applying operation. (c) Surface mesh after applying operation.

Figure 22 illustrates the relative importance of some of the expert features for 8 of the 12 operations used in this study. A higher importance for a specific feature indicates its relative influence on the mesh quality predictions produced by the machine learning models. While there is no consistent pattern in feature importance, it is worth noting that mesh_size tends to show up among the top four features for all models. The target mesh size $S_{T}$ is included as a feature for all models. Intuitively, these results indicate that mesh quality predictions are heavily dependent upon 
the user prescribed mesh size.

Although a definitive optimal set of features for the prescribed operations is beyond the scope of this study, understanding which features are most useful can help in understanding the problem domain and improving future implementations. For example, our training models for this study currently incorporate a range of four automatically selected mesh sizes. The importance that the mesh size feature plays in our results suggests that further training with additional mesh sizes is warranted.

\subsection{Ground Truth}

Ground truth for this application was defined by a set of mesh quality metrics including scaled Jacobian, Inradius and deviation. These were selected based on common requirements for analysis codes that require well-shaped, isotropic elements of consistent size and grading that conform well with the domain. For other applications that may require anisotropic elements or prescribed minimum elements through the thickness, additional or alternative metrics would need to be used as ground truth.

In this study, we also proposed two different methods for characterizing the locality of mesh quality metrics. The bounding box and topology-based methods tended to yield similar predictions, but we concluded that the use of topology-based locality was preferred since it represented the local environment of the operation better than an orientation insensitive bounding box.

We note that this study limits ground truth to mesh quality metrics that can be computed from an automatically generated tetrahedral mesh. Although mesh quality is an important factor in preparing a simulation model, there are many other factors that are not as easily characterized. For example, known loads, boundary conditions and other physics-based properties may influence the defeaturing performed by an analyst or engineer. These physically-based characteristics may also need to be considered when identifying features and ground truth for future implementation.

\subsection{Software Considerations}

For this study we selected a set of CAD operations for training from the Cubit geometry and meshing tool suite [11]. We also selected a commercial mesh generation tool, Meshgems [13] that served as the basis for training and defining our ground truth metrics. It is worth pointing out that the methods introduced are not specific to our implementation environment. Indeed, further work should be enlisted to identify an improved set of operations that can take advantage of a more comprehensive, robust and flexible CAD modeling environment. Additionally, alternative automatic mesh generation tools could be enlisted to train and identify ground truth metrics.

This study limited the number of input training models to a set of open-source CAD parts obtained from GrabCAD [15]. This was done to ensure reproducibility and provide a baseline for subsequent studies. For deployment in a practical defeaturing environment, a tool for selecting and building training data based on common analysis use cases would be preferable. Serialized data that can be queried at run-time from a defeaturing tool would be updated and customized as new CAD models are encountered.

We also recognize that in practice, depending on the CAD tool in use, an experienced analyst may identify a single CAD operation that involves multiple nearby geometric entities (meta-entities) to accomplish a single defeaturing task. For example, defeaturing metaentities such as a boss or blend could be accomplished by applying a single remove operation. While this can sometimes result in a preferred outcome, for purposes of this study, because of limitations in our CAD tool, we currently limit operations to single entities, leaving meta-entities for future work. Parametric modeling environments that maintain meta-entities or featurerecognition procedures could identify groupings of geometric curves and surfaces from which single operations could be identified and trained in a similar manner to that introduced in this work.

\subsection{Reinforcement Learning}

We have implemented a supervised machine learning approach to assist in CAD defeaturing. The proposed automatic greedy method suggests the best CAD alteration at each step, given the current state of the associated mesh. It may happen, however, that this approach could become mired in a local minimum, such that multiple coordinated actions are required to remove a particular undesired feature.

In addition to our greedy approach, we are also considering the use of Reinforcment Learning (RL). RL is an approach which can consider multiple coordinated actions, even arbitrary length sequences of actions leading to global minimums $[20,21]$. It is, however, computationally expensive and can be difficult to generalize from one context to another.

We will describe our work using RL in a later report, but we have so far achieved promising initial results on a simple CAD model using Q-learning. We have discovered global minimums using short sequences of CAD operations. We have also enumerated statistics showing that the defeaturing problem can be very difficult with many potential actions that can be detrimen- 


\begin{tabular}{ll} 
Feature & \multicolumn{1}{c}{ Importance } \\
\hline mesh_size & 1.65771 \\
exterior_angle 0 & 1.23113 \\
curvature2 1 & 0.524542 \\
largest_angle & 0.425724 \\
short_curve_ratio 1 & 0.414681 \\
shortest_curve_length 0 & 0.381628 \\
long_curve_ratio 0 & 0.353482 \\
long_curve_ratio 1 & 0.220527 \\
max_angle 1 & 0.210075 \\
short_curve_ratio 0 & 0.197236
\end{tabular}

(a) vertex_no_op

\begin{tabular}{ll} 
Feature & \multicolumn{1}{c}{ Importance } \\
\hline arc_length 2 & 2.96364 \\
max_angle 3 & 1.07613 \\
exterior_angle 2 & 0.833845 \\
mesh_size & 0.510938 \\
min_angle 3 & 0.472651 \\
num_loops 1 & 0.40401 \\
long_curve_ratio 1 & 0.227898 \\
long_curve_ratio 0 & 0.211206 \\
hydraulic_radius 0 & 0.186013 \\
short_curve_ratio 0 & 0.176082
\end{tabular}

(c) surface_no_op

\begin{tabular}{ll} 
Feature & \multicolumn{1}{c}{ Importance } \\
\hline long_curve_ratio 0 & 2.6299 \\
mesh_size & 0.763937 \\
num_curves 0 & 0.728801 \\
angle & 0.699596 \\
shortest_curve_length 2 & 0.325328 \\
hydraulic_radius 0 & 0.291079 \\
arc_length 0 & 0.261672 \\
shortest_curve_length 1 & 0.244802 \\
shortest_curve_length 0 & 0.240083 \\
area 2 & 0.231626
\end{tabular}

(e) blunt_tangency

\begin{tabular}{ll} 
Feature & \multicolumn{1}{c}{ Importance } \\
\hline mesh_size & 4.07086 \\
curvature2 0 & 0.978725 \\
hydraulic_radius 0 & 0.541933 \\
area 0 & 0.525383 \\
perimeter 0 & 0.431603 \\
hydraulic_radius 1 & 0.372828 \\
hydraulic_radius 4 & 0.349455 \\
shortest_curve_length 4 & 0.256454 \\
shortest_curve_length 1 & 0.255247 \\
exterior_angle 4 & 0.207114
\end{tabular}

(g) remove_cone

\begin{tabular}{ll} 
Feature & \multicolumn{1}{c}{ Importance } \\
\hline max_angle 0 & 2.39602 \\
mesh_size & 0.864585 \\
face_angle11 0 & 0.698811 \\
rel_mid_deviation 0 & 0.650515 \\
exterior_angle 0 & 0.484078 \\
shortest_curve_length 1 & 0.476223 \\
shortest_curve_length 0 & 0.269208 \\
exterior_angle 3 & 0.217465 \\
hydraulic_radius 1 & 0.152276 \\
rel_arc_length 0 & 0.133412
\end{tabular}

(b) curve_no_op

\begin{tabular}{ll} 
Feature & \multicolumn{1}{c}{ Importance } \\
\hline mesh_size & 2.51458 \\
short_curve_ratio 1 & 0.461274 \\
area 0 & 0.439399 \\
shortest_curve_length 0 & 0.336728 \\
shortest_curve_length 1 & 0.274238 \\
hydraulic_radius 2 & 0.189091 \\
short_curve_ratio 0 & 0.17918 \\
hydraulic_radius 1 & 0.168206 \\
min_angle 0 & 0.158146 \\
perimeter 1 & 0.152295
\end{tabular}

(d) composite_surfaces

\begin{tabular}{ll} 
Feature & \multicolumn{1}{c}{ Importance } \\
\hline mesh_size & 1.81267 \\
arc_length 0 & 0.65054 \\
shortest_curve_length 0 & 0.600115 \\
hydraulic_radius 0 & 0.37986 \\
hydraulic_radius 4 & 0.3305 \\
longest_curve_length 4 & 0.308023 \\
short_curve_ratio 0 & 0.248207 \\
short_curve_ratio 2 & 0.247287 \\
short_curve_ratio 1 & 0.20956 \\
short_curve_ratio 3 & 0.196559
\end{tabular}

(f) remove_topology_curve

\begin{tabular}{ll} 
Feature & \multicolumn{1}{c}{ Importance } \\
\hline mesh_size & 1.58561 \\
long_curve_ratio 0 & 0.794654 \\
hydraulic_radius 1 & 0.581031 \\
hydraulic_radius 2 & 0.496592 \\
exterior_angle 1 & 0.298324 \\
curvature2 0 & 0.238511 \\
area 4 & 0.220314 \\
hydraulic_radius 3 & 0.191892 \\
long_curve_ratio 1 & 0.165949 \\
rel_arc length 3 & 0.159437
\end{tabular}

(h) remove_surface

Figure 22: Feature importance: Top 10 expert features ranked by their importance for 8 of the 12 operations used in this study. Feature importance was computed as a by-product of the ensemble of decision trees (EDT) method used to generate our machine learning models. Note: values do not sum to one due to truncation and cross validation. 
tal to the overall improvement of the resulting mesh. In the future, we plan to generalize our approach to arbitrary CAD models that incorporate geometric features and potentially textual descriptions of CAD operations, all using a deep Q-learning framework.

\section{References}

[1] Distene, S.A.S. "An introduction to MeshGems-CADSurf V1.0, A fast, robust, high quality CAD surface mesher." marketing document, Bruyères le Châtel France, 2019. http://www.meshgems.com

[2] Ansys, Inc. "Ansys Meshing Solutions." marketing document, 2014. http://ansys.com

[3] Guo J., Ding F., Jia X., Yan D. "Automatic and High-quality Surface Mesh Generation for CAD Models." Computer-Aided Design, vol. 109, 49-59, 122018

[4] International TechneGroup (ITI). "CADFix, CAD Translation, repair and simplification." https://www.iti-global.com/cadfix

[5] Ansys SpaceClaim. "De-Featuring model for CAE." http://spaceclaim.com

[6] Owen S., Clark B., Melander D., Brewer M., Shepherd J., Merkley K., Ernst C., Morris R. "An Immersive Topology Environment for Meshing." Proceedings, 16th International Meshing Roundtable, pp. 553-577, 2008

[7] Danglade F., Pernot J.P., Philippe V. "On the use of Machine Learning to Defeature CAD Models for Simulation." Computer Aided Design and Application, vol. 11(3), 2013

[8] Ip C.Y., Regli W.C. "A 3D object classifier for discriminating manufacturing processes." Computers \& Graphics, vol. 30, 903916, 2006

[9] wei Qin F., Lu-ye Li S.m.G., ling Yang X., Chen $\mathrm{X}$. "A deep learning approach to the classification of 3D CAD models." Journal of Zhejiang University-SCIENCE C, vol. 15(2), 91-106, 2014

[10] Niu Z. Declarative CAD Feature Recognition An Efficient Approach. Ph.D. thesis, Cardiff University, 2015

[11] Quadros W.R., Owen S.J., Staten M.L., Hanks B.W., Ernst C.D., Stimpson C.J., Meyers R.J., Morris R. "Cubit Geometry and Meshing Toolkit, Version 15.4." Tech. Rep. SAND2019-3478, Sandia National Laboratories, Albuquerque, NM, 42019. https://cubit.sandia.gov/
[12] Wahl E., Hillenbrand U., Hirzinger G. "Surflet-Pair-Relation Histograms: A Statistical 3D-Shape Representation for Rapid Classification." Proceedings 4th International Conference on 3-D Digital Imaging and Modeling, pp. 474-481, 2003

[13] Distene, S.A.S. "Volume Meshing: Meshgems-Tetra." website, Bruyères le Châtel France, 2019. http://www.meshgems.com

[14] "The Verdict Geometric Quality Library." Sandia National Laboratories, Sandia Report SAND200\%-1751, 2007

[15] "GrabCad." URL https://grabcad.com

[16] "Python." URL https://python.org

[17] "scikit-learn, Machine Learning in Python." URL http://scikit-learn.org

[18] Breiman L. "Random forests." Machine learning, vol. 45, no. 1, 5-32, 2001

[19] Breiman L. "Bagging predictors." Machine learning, vol. 24, no. 2, 123-140, 1996

[20] Kaelbling L.P., Littman M.L., Moore A.W. "Reinforcement Learning: A Survey." CoRR, vol. cs.AI/9605103, 1996. URL http://arxiv.org/abs/cs.AI/9605103

[21] Arulkumaran K., Deisenroth M.P., Brundage M., Bharath A.A. "A Brief Survey of Deep Reinforcement Learning." $C o R R$, vol. abs/1708.05866, 2017. URL http://arxiv.org/abs/1708.05866 


\title{
BUILDING DIRECTION FIELDS ON THE MEDIAL OBJECT TO GENERATE 3D DOMAIN DECOMPOSITIONS FOR HEXAHEDRAL MESHING
}

\author{
Dimitrios Papadimitrakis ${ }^{1}$, Cecil G. Armstrong ${ }^{1}$, Trevor T. Robinson ${ }^{1}$, Alan Le Moigne ${ }^{2}$, \\ Shahrokh Shahpar ${ }^{3}$ \\ ${ }^{1}$ School of Mechanical and Aerospace Engineering, The Ashby Building, Queen's University of Belfast, \\ UK, BT95AH, dpapadimitrakis01@qub.ac.uk \\ ${ }^{2}$ Group Business Services - IT, Product Development System, Rolls-Royce plc, Derby, England, UK, \\ DE248BJ \\ 3Innovation Hub, Central Technology, Future Methods, Rolls-Royce plc, Derby, England, UK, DE248BJ
}

\begin{abstract}
In this work, a novel method for creating decompositions of general 3D domains, suitable for hexahedral mesh generation is presented. To accomplish this, frames and cross-fields are generated on top of the medial object of the domain. The geometrical and topological information carried by the medial object, together with the directional information of the frames/crosses help analyze the domain. By generating frames and cross fields on medial vertices, edges and faces based on touching vectors, a directional field is constructed on top of the medial object. Based on it, critical lines in the domain called, singularity lines, are identified. Starting from these, a complete line network is created on the interior of the domain. This network is extruded to the boundary in order to create the boundary of high-quality partitioning surfaces that are used to decompose the domain into regions appropriate for a high-quality hexahedral mesh. Examples are given to validate the method.
\end{abstract}

Keywords: Hex mesh, Medial Object, Frame fields, Cross fields, Singularity, Decomposition

\section{RELATED WORK}

Generating a structured mesh comprised of hexahedral elements has been a topic of research since the 1970s. Mapping methods were the first approach to be investigated for simple geometries. Either by solving partial differential equations [1] or by using algebraic interpolation techniques [2], [3], they aim to create the mesh by mapping a regular mesh in the parametric space to the physical space. Nowadays these methods are mostly used after the computational domain has been decomposed into mappable sub-regions or blocks.

Plastering [4], is the 3D equivalent of the paving method in 2D [5]. Starting from a boundary quad mesh it gradually constructs hex elements in an advancing front. While good element quality could be achieved close to the boundary, voids are created inside the volume which, often, cannot be meshed with hex elements. To avoid additional constraints, Staten et al. [6] proposed an extension which does not rely on a boundary mesh. By advancing fronts inwards and generating meshes on inner voids that define the boundary mesh, they created hexahedral meshes for rather simple geometries.

One of the most popular hex mesh generation algorithms is sweeping. The basic concept of this algorithm is that a quad mesh on a source face is extruded to a target face along a specified direction [7]. This one to one sweeping algorithm has been further improved through the years [8] to handle complex shapes and even cases where there is more than one source and/or target faces [9], [10]. However, when dealing with many to many type sweeps, there remains a level of domain decomposition/imprinting required to generate the meshable regions. 
Price et al. [11] used the 3D medial object to create a decomposition of a solid into sub regions that can be easily meshed by the midpoint subdivision technique. Using the topological and adjacency information the medial object provides, they came up with a set of 13 meshable solid primitives, each with at most 8 faces, with each face having between three and six sides. These primitives are placed along medial vertices, edges and surfaces to create the decomposition of the solid. In their second work [12] the authors extend their work so that geometries with medial surface degeneracies, shallow and concave edges can also be analyzed. However, problems such as $\mathrm{N}$-valent vertices and objects with two sided faces still need to be investigated. LayTracks3D [13] is a more recent work which also relies on the 3D medial object. By combining the medial object and the advancing front method hex-dominant meshes are created. Using medial axis junction curves together with the medial radii, simpler regions called corridors are created. After meshing medial surfaces inside the corridors, a further subdivision is provided with the creation of the so-called tracks. The advancing front method is then used to form the final hex dominant mesh. The author also gives information on how this method can be extended to create all-hex meshes and how it can be used for the meshing of assemblies with the creation of imprints on the medial object. The main drawbacks of these technique are a) the robust computation of the medial surface is still challenging $b$ ) a small change in object geometry can radically change the topology of the medial surface and, therefore, the resulting mesh. Finally, one more algorithm that uses skeletons of models to generate hexahedral meshes is that described by Livesu et al. in [14]. In this work high quality hexahedral meshes are generated after a tubular structure that resembles the initial geometry is constructed based on a curve skeleton. However, the method described is limited in models that admit a skeletal representation.

Nieser et al. [15] first proposed a method for generating a hexahedral mesh of a solid subjected to boundary alignment constraints by finding a volume parameterization of a manually created block decomposition of the domain. The notion of a frame field built upon a tetrahedral mesh that guides the parameterization was introduced and several conditions of the so called singularities and the gradient of the frame field where given. The automatic, fast and robust generation and correction of frame fields for general domains was the focus of many works to follow [16]-[20]. Liu et al. [21] recently proposed a method to generate frame fields with manually prescribed singularity graphs, thus giving the opportunity to manually correct topological invalid singularity graphs and then generate a correct frame field. The fundamental properties of hex meshes were first studied by Price et al. [11], [12]. In [21] these properties were used from a slightly different perspective to derive local and global conditions that are necessary for a hexmeshable frame field. Fogg et al. [22] also proposed conditions that a network of singularities must respect. However, the topology of the singularities of the frame fields that can be automatically computed by current methods are still not guaranteed to imply a valid hexahedral mesh even for simple geometries [23].
Having obtained a high-quality frame field, Kowalski et al. [24] suggested that the singularity graph of the frame field can be immediately used to create a domain partitioning and then a mappable block structure to avoid the expensive calculation of a volumetric parameterization. Shang et al. [25] described a different way of using a frame field on a tetrahedral mesh. In their approach, it is used not to guide a volumetric parameterization or a domain partitioning, but to drive the generation of mesh sheets in terms of the spatial twist continuum. This results in a more robust and parallelizable method that does not depend in heavy numerical libraries. However, the final mesh is boundary dependent since it relies on an initial surface quadrilateral mesh. Wang et al. [26] used frame fields in order to guide the creation of dual surfaces using an underlying hex mesh generated by a hex-to-tet method. By isolating singularities and boundary features, these surfaces generate block decompositions that respect the geometry and topology of the domain. However, many of their steps are heuristic and not guaranteed to work in all cases.

Another successful technique for producing all-hex meshes with block structure is that of Polycubes. In such methods, a solid formed from a union of cubes (Polycube) is created and represents the initial model. A hexahedral mesh can be easily created in the Polycube and then mapped back to the model to produces the final hexahedral mesh. High quality meshes are produced robustly even for complex models. However singularities merge close to the boundary reducing the quality in the regions that are important from a simulation perspective. The main challenge in these approaches is the robust generation of the Polycube structure, which remains an open problem. State of the art methods for generating Polycubes are [27] and [28].

Finally, in [29] Lim et al. propose generating multi-block decompositions for 2D domains based on an evolutionary algorithm. In order to generate blocks of high quality a set of fixed boundary points that capture all important geometric features is created. Based on this set, a new set of candidate points on the interior of the domain is generated. Quad meshes are generated based on those points and evaluated in an evolutionary fashion until the best block is derived. The results obtained are comparable to the state-of-the-art. The authors also discuss the extension of the method to 3D.

In this work, a method for the automatic decomposition of a general domain is proposed which combines the merits of the medial object and frame field approach. Continuing the work of [30], it is explained how frames and cross-fields can be constructed on top of the medial object in order to create an internal line network attached to singularity lines. This line network is then extended to guide the generation of partition surfaces that decompose the domain.

\section{PRELIMINARIES}

Before the method is explained some definitions are given in order to familiarize the reader with the concepts related to the work. 


\section{Medial object}

The medial object is the locus of the center of an inscribed sphere of maximal diameter as it rolls around the interior of an object. A sphere is maximal if there is no other inscribed sphere that contains it. The medial object is made up of medial surfaces, edges and vertices. In non-degenerate cases each medial surface is constructed by centers of spheres that touch two faces of the object, each medial edge by centers of spheres that touch three faces of the object and each medial vertex by centers of spheres that touch four faces of the object. Another possible configuration is the so called finite contact where the inscribed sphere is in contact with a finite portion of the boundary, like when the sphere rolls along the axis of a cylinder. One more case is that of curvature contact, where the curvature of the inscribed sphere and the minimum curvature of the surface are the same as, for example, at the foci of an elliptical extrusion. The vector that starts from a point on the medial object and ends at a position of contact of the corresponding maximal sphere with the boundary is called touching vector. The medial object has some important properties which are important for mesh generation. These are

- One to one correspondence with the domain.

- Dimensional reduction: the medial object is a $2 \mathrm{D}$ object.

- It is orientation independent.

- It identifies parts of the object boundary in geometric proximity.

\section{Mesh singularities}

In a quad mesh, singularities are called the nodes of the mesh on which more or less than four mesh edges are connected. The most common situations are those where three or five edges are incident to the node. These are referred to as positive and negative singularities accordingly. Similarly, in a hexahedral mesh, mesh edges where more or less than four hexahedral elements are connected are called singular edges. These edges connect to each other to form singularity lines. The number and the position of singularities affect the quality and the "flow" of the mesh. In general, a small number of singularities is preferred. Singularity lines also describe how the decomposition will look. In this work, two different types of categorization of singularities are used. The first one to describe the type of the singularity regarding the number of mesh elements connected to it. The second one to describe the nature of the singularity with respect to the medial object. It is important to note that the definition of a singularity is different from the work in [31]. Here, boundary edges and vertices where the number of attached elements corresponds to that implied by the dihedral angle are not considered to be singular. For example, a concave boundary edge of 270 degrees where three hex elements join is not considered to be singular.

Positive singularity: Five partition surfaces emanate from the singularity, e.g. Figure 1e. They are highlighted in blue hereafter.

Negative singularity: Three partition surfaces emanate from the singularity, e.g. Figure 9 left. They are highlighted in red hereafter.

Type-1 singularity: The singularity lies on the medial object.

Type-2 singularity: The singularity runs perpendicular to a medial surface.

Figure 1 shows the medial object, the mesh singularities, the partition surfaces and the singularity lines for a block with a hole. In (b) the medial object of the solid in (a) can be seen. In (c) a high-quality block structured mesh is generated. The points on the top surface from which the yellow lines emanate are mesh singularities. The yellow lines are the wireframe of the base-complex of the domain. In (d) the partition surfaces are given in yellow. Finally, in (e) the singularity lines on the interior of the domain are depicted. Partition surfaces emanate from these lines. Although in Figure 1 partition surfaces and singularity lines are depicted as part of an already existing hexahedral mesh with a block structure, in this work we present a method to identify singularities, build partition surfaces based on them, and decompose the domain into regions that can be used to generate a hexahedral mesh.

\section{Partition surfaces}

Partition surfaces are surfaces that emanate from singularities. These surfaces imply a partitioning of the domain in smaller regions and are bounded by singularities and by boundary curves. The wireframe of this partitioning is referred in the literature as the base complex of the domain. This is essential to generate a high-quality hexahedral mesh. Five partition surfaces emanate from each positive singularity and three partition surfaces emanate from each negative.

\section{Frames}

A frame consists of 3 mutual perpendicular unit vectors together with their opposite vectors $\{\boldsymbol{u}, \boldsymbol{v}, \boldsymbol{w},-\boldsymbol{u},-\boldsymbol{v},-\boldsymbol{w}\}$. These vectors represent the orientation in $3 \mathrm{D}$ space of a cube that has its faces normal to them. In terms of a mesh, the orientation of each mesh element can be thought to be approximately represented by such a frame or a cube. Figure 2 shows this representation. 


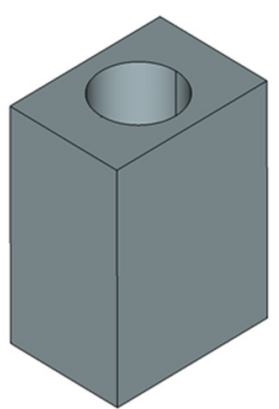

a

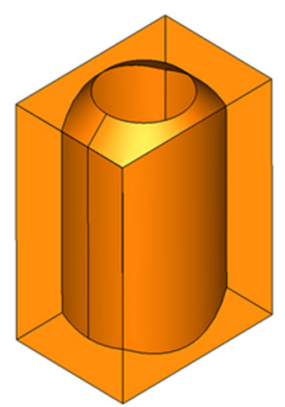

b

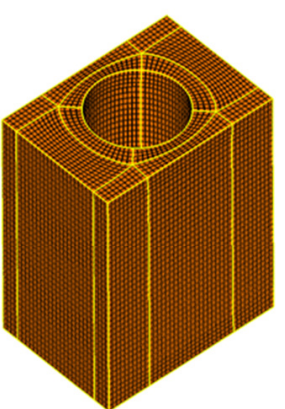

C

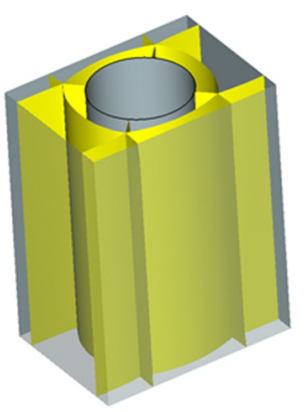

d

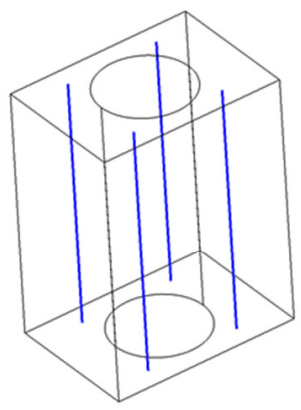

e

Figure 1: Object (a), medial object (b), Hex mesh (c). Yellow lines define the boundary of the base complex. Partition surfaces (d). Singularity lines (e).
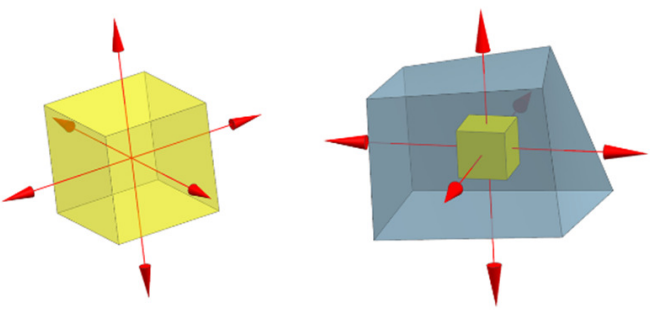

Figure 2: Cube represented by a frame (a). Mesh element represented by a cube and the corresponding frame (b).

\section{OVERVIEW OF THE METHOD}

Decomposing a general domain into blocks is a tedious task which requires both geometrical and topological information. Although many methods have been proposed in the literature, they all have their strengths and weaknesses. Inspired by previous research on medial object and frame fields, in this work a method is proposed that uses the directional information of a frame along with the structure that the medial object provides in order to decompose a domain into simple regions. Generating high-quality partition surfaces that define those regions is crucial. To achieve that, high-quality singularity lines and boundary lines are identified to form the boundary of the partition surfaces. These lines are produced after a line network is constructed on the interior of the domain based on an analysis that uses the medial object and directions defined through the touching vectors. Contrary to other methods, this work builds the boundaries of partition surfaces from the interior of the domain and is not constrained by a boundary cross-field. The following are the main steps of the method. Figure 3 shows the results after each step.

1) Generate frames along medial edges and medial vertices based on touching vectors.

2) Generate cross-fields on medial surfaces based on those frames.

3) Identify singularity lines lying on the medial object and being normal to it.

4) Trace streamlines that emanate from singularity lines on the medial object to construct a complete line network on the interior of the domain.

5) Project the line network to the boundary to generate boundary lines.

6) Define partition surfaces with the aid of singularity lines and boundary lines.

7) Decompose the domain based on the partition surfaces.

The input of the method is the medial object of the domain with a triangular mesh on each medial surface and the output is the internal line network and a set of partition surfaces. This work focuses mainly on the steps 1-6 aiming in the creation of the interior line network and on the generation of partition surfaces. Based on these surfaces, a domain decomposition is created with the use of the commercial software CADfix. 


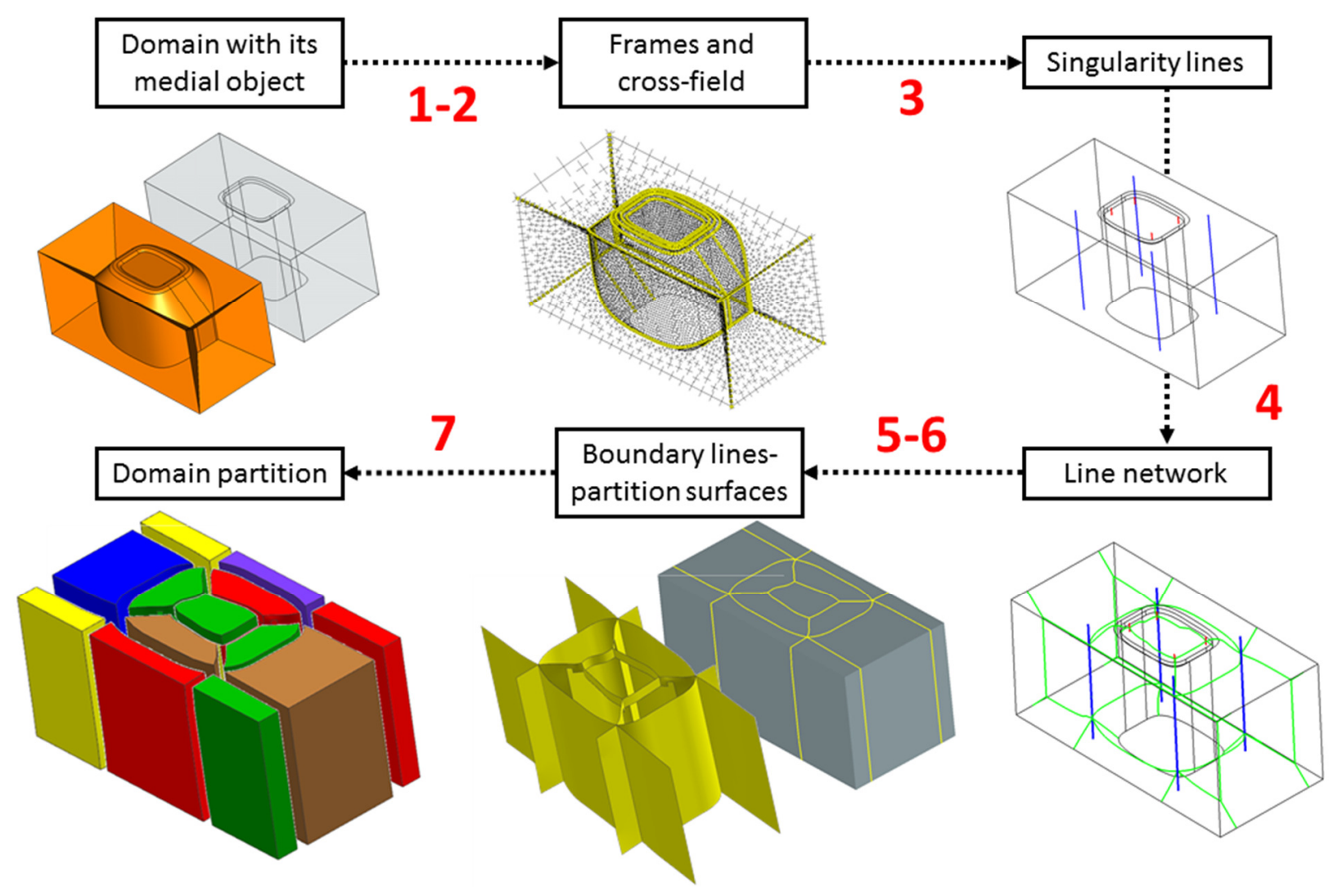

Figure 3: Steps of the method.

\section{GENERATING FRAMES}

\section{Functional representation of frame:}

To generate frames on the medial object, a functional representation of frames restricted to the unit sphere is used which exhibits their 24 symmetries. As described by [Ray et al], this function can be decomposed onto the basis of nine spherical harmonics namely $B=\left(Y_{4,-4}, Y_{4,-3}, \ldots, Y_{4,4}\right)$. Such a decomposition gives the opportunity to describe the function of each frame as $F=B a$ where the representation vector $a$ has length nine and describes the influence of each harmonic. To find the difference between two frames $i$ and $j$ with different orientations, the integral $\int_{S^{2}}\left(F_{i}(x)-\right.$ $\left.F_{j}(x)\right)^{2} d x$ is calculated on the unit sphere $S^{2}$. Since the function basis $B$ is orthonomal, this integral can be further simplified as $\left\|\left(a_{i}-a_{j}\right)\right\|^{2}$. The representation vector can be expressed by Euler angles that orient it in three-dimensional space relative to a global reference frame. The proximity of two frames can now be simply described by the difference of two vectors.

\section{Frames on medial edges and vertices:}

Orienting frames on medial edges and vertices depends on the touching vectors together with the boundary entities that they are associated with. Both the number of the touching entities and their type needs to be considered. Each touching vector represents a boundary entity. If this entity is a face, then the touching vector represents a mesh element on this face which has a rotational degree of freedom. If the entity is an edge or a vertex, then the touching vectors represents a mesh element on this edge or vertex which has no degrees of freedom. Since at each point of a medial edge/vertex, at least three touching vectors exist, a frame must be constructed that represents all the corresponding boundary entities. In order to accomplish that, firstly, a frame must be constructed for each boundary entity. Below it is explained how a frame that fits $n$ vectors can be created.

\section{Frames based on vectors}

Let $\vec{t}_{i}, i=(1, \ldots, n)$ be $n$ vectors in $3 \mathrm{D}$ space. Each pair of vectors $\left(\vec{t}_{i}, \vec{t}_{j}\right),\left\{i \neq j\right.$ and $\left.\vec{t}_{i} \cdot \vec{t}_{j} \neq 0\right\}$ defines a plane $P_{i j}$ with normal vector $\vec{t}_{n}=\vec{t}_{i} \times \vec{t}_{j}$. Vectors $\vec{t}_{n i}=\vec{t}_{n} \times$ $\vec{t}_{i}$ and $\vec{t}_{n j}=\vec{t}_{n} \times \vec{t}_{j}$ are sufficient to create two frames $\left\{\vec{t}_{i}, \vec{t}_{n}, \vec{t}_{n i}\right\}$ and $\left\{\vec{t}_{j}, \vec{t}_{n}, \vec{t}_{n j}\right\}$ both of which lies on the plane $P_{i j}$. The first frame corresponds to a representation vectors $a_{i j}$ and the second one to a representation vector $a_{j i}$ 
based on the functional representation of frames described before. By doing the same for each pair of non-collinear vectors, $l_{i},\left(l_{i} \leq n-1\right)$ frames are created for each vector $\vec{t}_{i}$, by solving n minimization problems,

$\min \sum_{j=1}^{l_{i}}\left\|a_{i}-a_{i j}\right\|^{2}, i=1, \ldots, n$

Each representation vectors represents a frame that best fit all frames that were created based on vector $\vec{t}_{i}$. A set of frames $F=\left\{a_{1}, \ldots, a_{n}\right\}$ is created.

By solving one more minimization problem described by the equation

$\min \sum_{i=0}^{n}\left\|a-a_{i}\right\|^{2}$

a frame that fits all frames on the set $F$ can be identified. This is the frame that fits all vectors $\vec{t}_{i}$.

Having defined a way to calculate a frame that best fits $n$ vectors, it can now be explained how frames are generated on medial edges and vertices. For all touching vectors that correspond to a boundary face a frame can be calculated based on equation (1), where the vectors that are fitted are the touching vectors. For each touching vector that corresponds to boundary edge/vertex, a frame can be created again based on equation (1), where the vectors that are fitted are the normal vectors of the boundary faces that are topological parents of the boundary edge/vertex. Having identified a frame for each touching vector, a single frame can be calculated by solving equation (2). This optimization problem can be relaxed if only the more constrained entities are taken into account each time. If for example a medial edge is associated with two boundary faces and one boundary edge the frame that corresponds to the boundary edge can directly be used. Figure 4 shows three examples of a cube/frame that is generated to fit three touching vectors (indicated in red). In (a), a cube perfectly fits the vectors since they are all normal to each other. In this special case the planes defined by each pair of touching vectors corresponds to the dual face of the cube. In (b), two of the touching vectors are collinear with opposite directions while the third one is normal to them. Only two of them are required to generate the frame. In (c), a more general case where the vectors are neither collinear nor normal to each other is depicted.

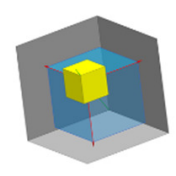

a

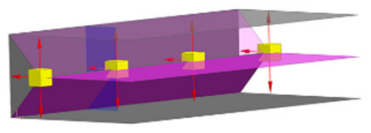

b

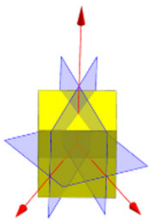

Figure 4: Cube/frame generated by touching vectors for three different configurations. When all touching vectors are normal to each other (a), when two of them are collinear (b), for a general configuration (c).

Frames calculated by this procedure are not forced to be aligned with medial edges. The only thing that constrains them is the orientation of the planes defined by the touching vectors.

\section{GENERATING CROSS-FIELDS}

In order to create a complete line network on the interior of the domain that will be used as a skeleton to construct partition surfaces, cross-fields are generated on medial surfaces. Such cross-fields define orientations along medial surfaces in the interior of the domain. Methods to generate cross-fields are vast in the literature. In the current work, crosses are generated by a propagation procedure described in [32]. The generation of the cross field will depend on boundary crosses on medial edges and vertices. Such crosses should lie on planes that are tangent to the medial surface at each point. Since boundary frames are generated to approximate all touching vectors there is no guarantee that they will lie on the medial surface. Frames are modified on medial edges and vertices so that they are normal to the medial surface. Let $a$ be the representation vector of a frame $F$ on a medial edge or vertex. Let also $\vec{n}$ be the normal vector of the medial surface. To find the frame $F_{n}$ with representation vector $a_{n}$ that is aligned with the normal vector $\vec{n}$ and is closest to the frame $F$ in terms of the functional representation, the function $E=\left\|a_{n}-a\right\|^{2}$ is minimized. An example of such frames is shown in Figure 5 on the left. The orientation of boundary crosses depends on the information carried by touching vectors. Such information may include hard boundary constraints due to edges or vertices that restrict frame orientation. While crosses are forced to lie on medial surfaces, they are not forced to be tangential on medial edges. This is expected to result in a simpler block topology than that of [13] where meshes on medial surfaces are forced to align with medial edges. Figure 5 shows an example of a 2D cross field generated on a medial surface. When boundary crosses are aligned with medial edges (right), a negative singularity emerges which, in the current method, is avoided (left).

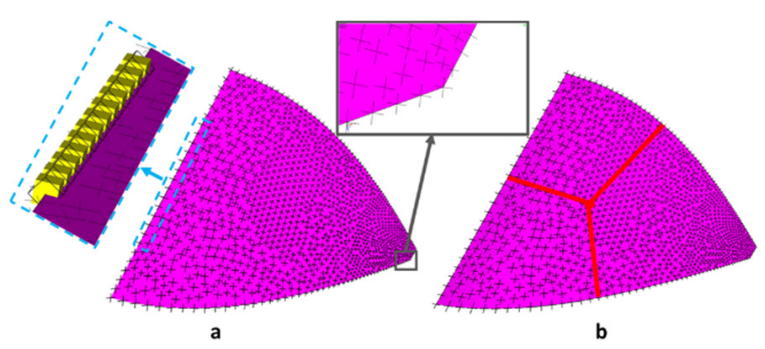

Figure 5: Frames are rotated to be normal to the medial surface. The cross field is not constrained to align with medial edges (a). When it is, more singularities are identified (b). 


\section{GENERATING LINE NETWORK}

\section{Singularities}

As discussed in a previous section, singularities can be distinguished by whether they lie on the medial object (Type-1) or they pass through a medial surface (Type-2). This section explains how such singularities can be identified.

Type-1: The procedure of generating singularities that lie on the medial object consists of two steps. In the first step, positions on medial edges where singularities enter the medial object are identified. In the second step, these lines are traced along the medial surfaces, based on the directional information of the cross-field, until they meet another singularity, the boundary, or they connect back to themselves.

By considering a medial edge as being part of one of its parent medial surfaces, touching vectors can be organized in groups of two. Each of them is associated with one boundary entity. By calculating the rotation of adjacent frames calculated by (1), the positions where a singularity is needed to create a well-structured decomposition that respects only those two boundary entities is identified. If, for example, those two touching vectors are associated with two boundary faces, then the singularity will enter the body through the medial edge and run across the medial surface which lies between those two boundary faces. Figure 6 shows how frames that correspond to the touching vectors along two neighboring points $a$ and $b$ on a medial edge can be compared. Here, one combination of three frames out of the four that correspond to each touching vectors is depicted. A similar analysis can be made for all combinations of three touching vectors. If the best fitting frame is calculated on many points along the medial edge based on the touching vectors, the position of the singularity can be visualized as a cube that suddenly "flips" (Figure 7). This "flip" occurs when the angle of the touching vectors passes through 45 or 135 degrees. Each time, a singularity that lies on the corresponding medial surface is sought.

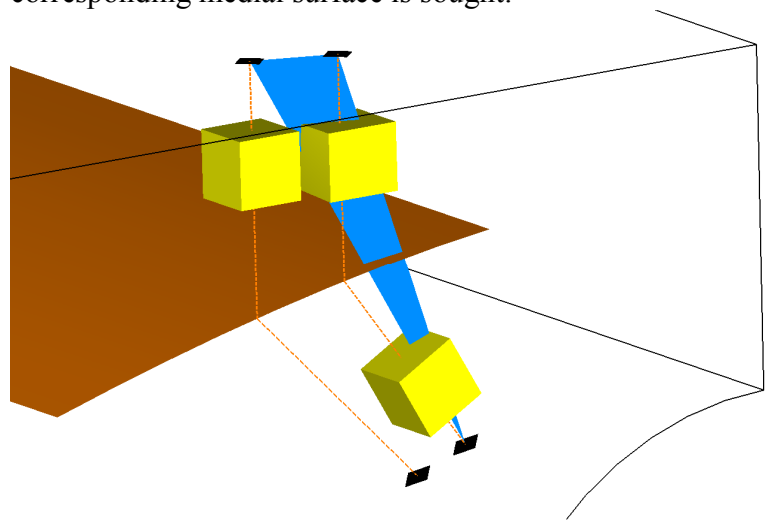

Figure 6: Identifying singularities entering medial surfaces by analyzing neighboring frames.

Having found the position where the singularity enters the medial face, then the singularity is created by tracing along the cross-field on the medial surface. Figure 7 shows an example of an elongated 5 sided block with a curved side edge. A positive singularity is traced along the medial surface.

Type-1 singularities are associated with the boundary of the domain through the medial object. Each end point of the singularity can be projected to a certain boundary entity in order to form a line that starts from the boundary and finishes on the boundary. The only exception is that of singularities that form loops and connect to themselves. The projection depends on the medial edge or the medial vertex on which the end point lies. From all the boundary entities with which this medial edge/vertex is associated with the one whose touching vector forms the smallest angle with the direction of the singularity line is chosen. This guarantees that the singularity will be connected to the boundary smoothly. On Figure 7(left) it can be seen how the end points of a Type-1 singularity are connected to the boundary. Furthermore, a Type-1 singularity is also associated with the boundary entities of the medial surface, on which it lies. If the singularity line is projected to these boundary entities, boundary lines or points are created. These lines/points will be used to generate partition surfaces as will be described in the following section.

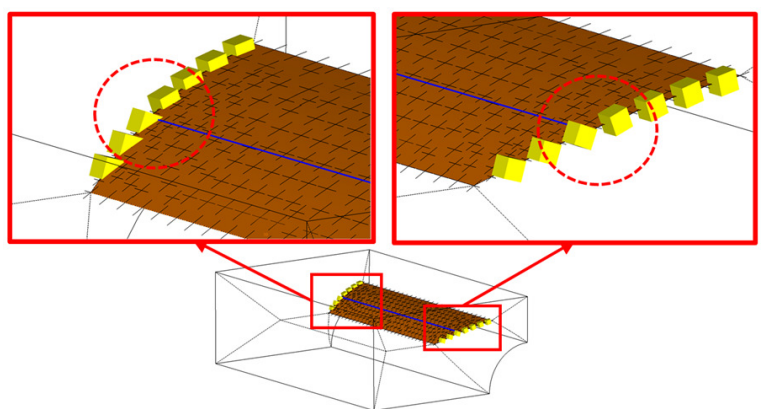

Figure 7: Example of a positive singularity traced on a medial surface. The singularity enters where frame orientations flips.

In a similar way, medial vertices can indicate positions where a singularity will enter the medial object through a medial edge. In this case touching vectors on the medial vertex are organized in groups according to the medial edge that is analyzed. Figure 8 shows an example of an elongated pentagonal prism where the medial edge in the middle carries a positive singularity line. A front view is also given where the frames can be seen to rotate around the medial edge.

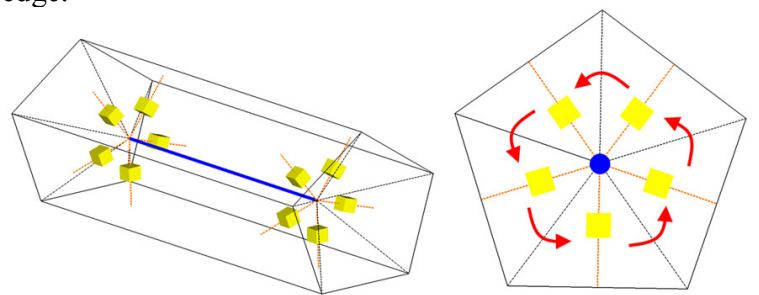

Figure 8: Identifying singularities carried by medial edges. Frames for each touching vector are analyzed. 
Type-2: Since crosses on medial surfaces are placed on nodes of an underlying triangular mesh, singularities are identified by analyzing rotations of crosses on each triangular mesh element. Singular points are identified on medial surfaces. Singularity lines are then created by extruding these points to the boundary entities associated with this medial surface. Since singularity lines should end on boundary faces and not on boundary edges or vertices, only medial surfaces that lie between two boundary faces are considered. Figure 9 shows an example of two short prisms. On the left, a negative singularity lies on the interior of the triangular prism. On the right, a positive singularity lies on the pentagonal prism.

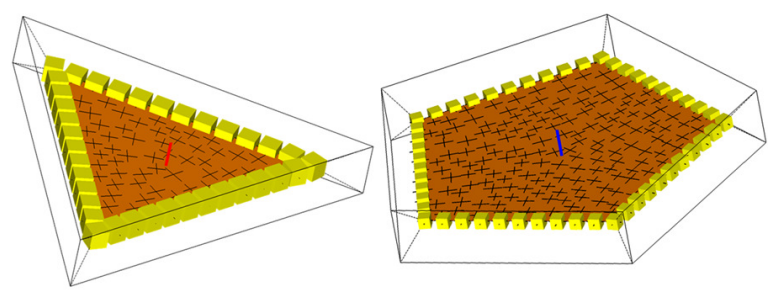

Figure 9: Singularities normal to medial surfaces are identified by cross fields. The simple examples of a triangular and pentagonal prism are illustrated.

\section{Streamlines}

In $2 \mathrm{D}$, five/three streamlines emanate from each positive/negative singularity. These streamlines follow the cross-field and form the decomposition of the domain by connecting to other singularities or to the boundary. In 3D, five partition surfaces emanate from each positive singularity line and three from each negative. In this work, instead of directly generating these surfaces, their bounding lines are first created. These lines can then be used to generate the surfaces. Similarly to $2 \mathrm{D}$, streamlines are emanated from each singularity. In Figure 18, these streamlines are depicted in green. Again, Type-1 and Type2 singularities are treated in a different way. Streamlines are traced on top of the medial object. On Figure 10 it is depicted how partition surfaces on semicircular plate intersect with the medial object of the domain defining lines that emanate from singularities and travel across different medial entities. Since it is the partition surfaces that are to be created it is logical to think reversely and first try to generate the green lines and then, based on them, define the partition surfaces.

\section{Streamline types}

Type-1: Each end of a Type-1 singularities can be treated locally as a $2 \mathrm{D}$ singularity and five/three streamlines can be initiated there, depending on whether the singularity is positive or negative. Thus, from each Type-1 singularity ten/six streamlines will be traced. These traces can lie on medial faces or medial edges and are traced until they join to a singularity or they meet a boundary edge. The cross-field on the medial object provides the directional information to guide these traces. The initial direction of the traces depends on the local structure of the medial object. Figure 11 shows the directions in one end of a positive singularity. Traces $\operatorname{tr} 1$ and tr4 follow the touching vectors and connect to boundary faces $\mathrm{BF} 1$ and BF2 respectively. Traces tr 2 and $\operatorname{tr} 3$ on the other hand, run across medial faces parallel to the boundary faces to create high-quality blocks. Finally, trace tr5 runs across the medial edge that lies between BF1 and BF2. A similar configuration would exist on the other end of the singularity line. It is also depicted how tracing along the medial object's entities provides a more global view. Trace tr5 from the top singularity line connects to the bottom one after travelling along medial edges.

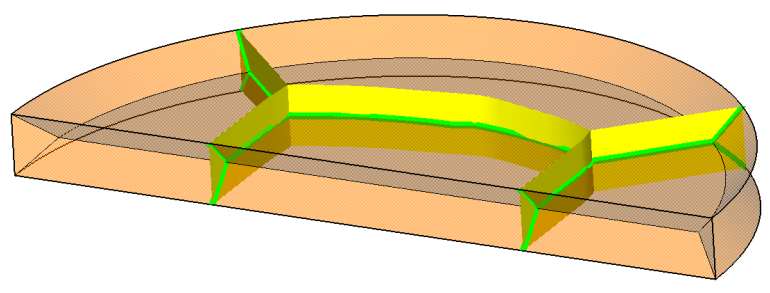

Figure 10: Partition surfaces (yellow) intersecting with the medial object to define streamlines.

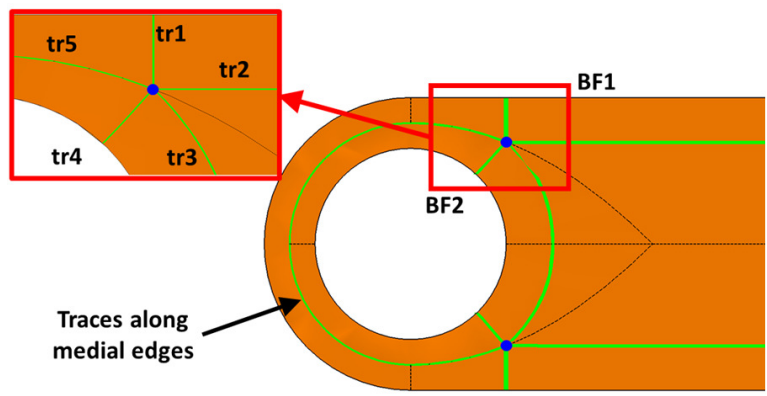

Figure 11: Streamlines are initiated based on the structure of the medial object. They can lie on medial surfaces and medial edges. The structure of the medial object can be crucial in connecting singularities together.

Type-2: Type-2 singularities are treated like in 2D. Thus three/five new traces will be initiated from each negative/positive singular point on a medial surface. The directions of the traces depend on the cross-field, as described in [32], and are traced until they join to another singularity or they meet a boundary edge. In this case, all traces start on the same medial surface. An example is given in Figure 16 which shows streamlines from four negative singularities.

\section{Boundary association}

In order to create the boundary lines that will support the partition surfaces, streamlines, like singularities, must be associated with the boundary. These associations depend on the type of the singularity and on the connectivity of the medial object with the boundary.

Each streamline that emanates from a Type-1 singularity will support the generation of one partition surface. The local nature of this partition surface (in the region of the endpoint 
of the singularity) can be described by its normal vector $\vec{n}=$ $\overrightarrow{t_{1}} \times \vec{t}$, where $\overrightarrow{t_{1}}=\overrightarrow{t_{v b}}$ is the vector that connects the end point of the singularity, to which the streamline is connected to, to the boundary of the domain and $\vec{t}$ is the tangent vector of the streamline at this endpoint. Since a streamline lies on a medial surface or a medial edge, it can be associated with, at least, two boundary entities. Regarding that, one partition surface will be created based on each streamline, choosing to which boundary entities the streamline will be associated to, depends on maintaining a smooth partition surface. If $\overrightarrow{t_{v}}$ is the touching vector that connects the starting point of the streamline with a boundary entity, then, if $\overrightarrow{t_{v}} \times \vec{t} \cong \vec{n}$, this boundary entity is associated with the streamline. This condition will ensure that the associations will result in smooth partition surfaces. If, on the other hand, this condition does not hold, then the partition surface will form a dihedral angle along the streamline. In Figure 12 the blue positive singularity can be seen to follow the direction of $y$ axis. Two of the five streamlines emanating from this singularity are shown in green. Figure 13 shows how streamline tr2 cannot be associated with both boundary entities. Only when projected to the top is the singularity parallel to the yellow surface generated. When it is projected to the right a surface perpendicular to the singularity is created which does not correspond to a partition surface that emanates from the singularity. On Figure 14, on the other hand, $\operatorname{tr} 1$ can be associated with both boundary entities since both projections generate surfaces that are parallel to the singularity.

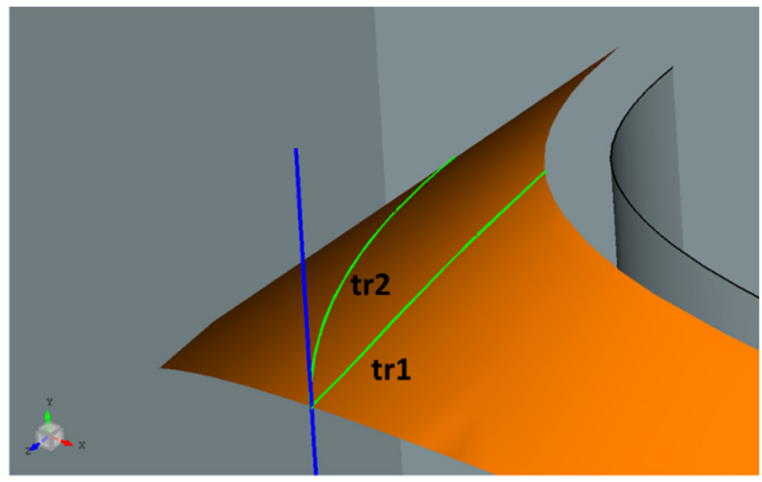

Figure 12: Two of the five streamlines that emanate from the blue positive singularity lie on the same medial surface and thus can be associated to two boundary entities. The association must be done so that the partition surfaces will be parallel to the singularity.

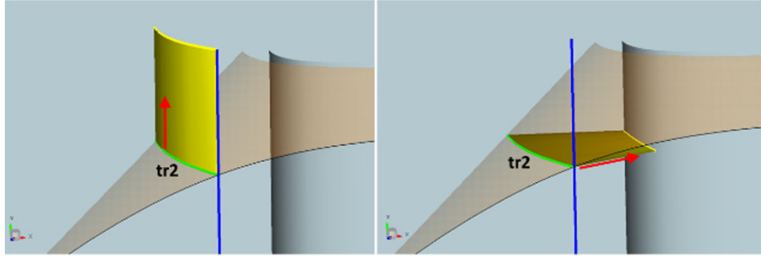

Figure 13: When tr2 is projected on the top, the corresponding surface respects the singularity line (left). When projected to the right the corresponding surface does not. Only projections that respect the singularity are kept so that the resulting partition surfaces will emanate from singularities.

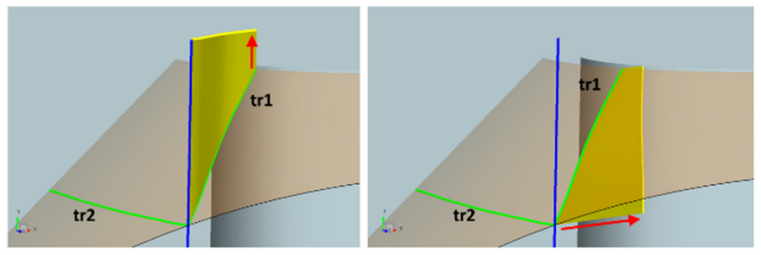

Figure 14: When tr1 is projected on both boundaries, the resulting surfaces are parallel to the singularity line and thus they can both assist on the creation of a partition surface that respect the singularity line. Tr1 is associated with both boundary entities.

Each streamline that emanates from a Type-2 singularity on the other hand is associated with both boundary entities that the medial surface, on which it lies, is associated with.

Tracing streamlines depends on the frames on medial edges and vertices and on the cross fields on medial surfaces. However, since the medial object consists of many different surfaces, edges and vertices, a trace might have to travel along many of them until it connects to another singularity or it meets the boundary. Medial edges and vertices indicate positions where a trace "jumps" from one medial entity to another. The way this transition will take place is important since these lines will form the structure to create partition surfaces. If a transition is smooth, then that will result in generating high-quality partition surfaces.

When a streamline passes from a medial entity to another the association to the boundary must be identified again. From all the boundary entities of the new medial entity, those that maintain the smoothness of the partition surface are chosen. A new trace is then initiated on the new medial entity. It is also important to note that when this transition occurs, more than one new traces might be initiated on different medial entities. For example on a non-degenerate medial edge three medial surfaces are connected. When a trace lying on one of them meets the medial edge, two new traces will be initiated. The association with the boundary must be derived for each new trace. On Figure 15, a trace from a negative singularity can be seen to break into two new traces when it meets a medial edge. It can also be seen how the direction of the new traces are such that the partition surface (light yellow) will continue smoothly. 


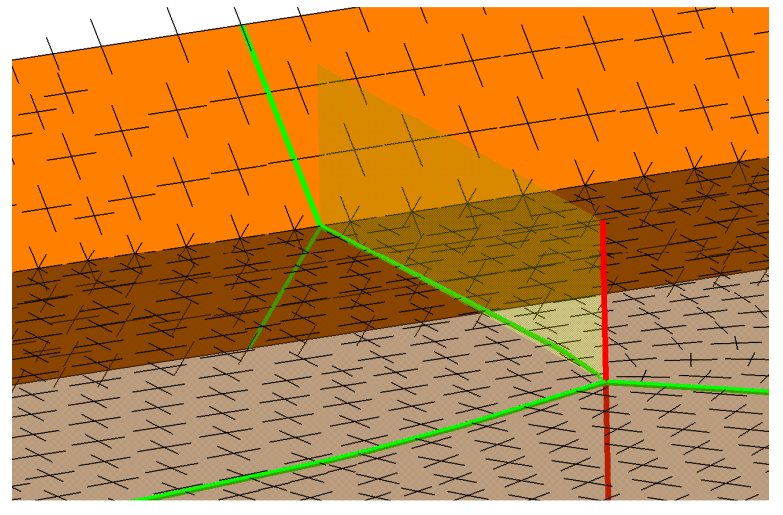

Figure 15: A streamline meeting a medial edge breaks into two new traces.

\section{Local control}

Tracing Type-1 and Type-2 singularities and their streamlines on the medial object gives the flexibility to handle different regions separately. Adjustments, corrections and simplifications of the line network can be accomplished separately on each medial surface/edge. If, for example, two streamlines on the same medial surface pass close to each other Figure 16 (a), they can be connected by manipulating only the traces on this medial surface before they propagate to different medial surfaces. The sudden jump on the streamlines in Figure 16 (b) appears because streamlines where forced to join. Lines like those can then be smoothed in order to increase the quality of the final block decomposition. By joining such lines spiral effects can be avoided. Increasing the cross-field density would force streamlines to pass closer to each other and thus produce a smaller step when joined in the cost of a more expensive cross-field computation. Joining lines can guided by a distance parameter that depends on the local radius of the maximum inscribed sphere of the medial object. Here the value of $r / 3.0$ was heuristically chosen. Since this radius is a direct measure of the local thickness of the domain, it is a good candidate to decide whether streamlines need to be joined to simplify the final decomposition.

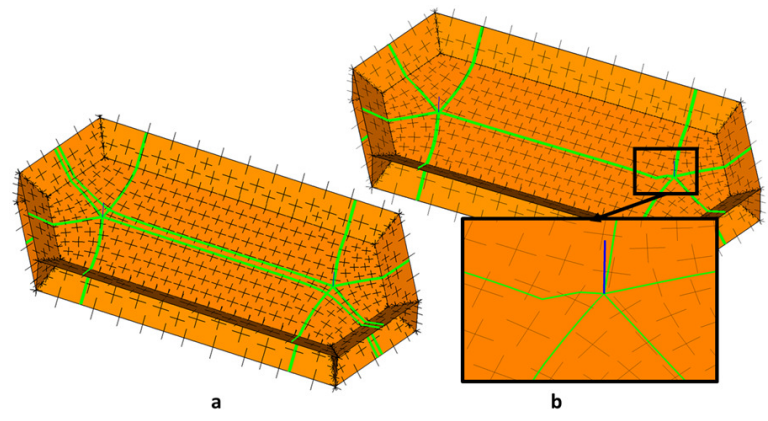

Figure 16: Traces from four Type-2 negative singularities are connected to create a simple topology on the interior. The line network can be locally adjusted by analyzing a selected medial surface.

\section{Line network}

After all singularity lines have been identified and all their streamlines have been traced, a complete line network is generated on the interior of the domain. This line network is strongly related to the medial object and each of the lines is associated with certain boundary entities. The network consists of the singularity lines and all the streamlines that emanate from them. Moreover, since the medial object is connected to the boundary, all streamlines are guaranteed to be connected to the boundary, or to another streamline, or to a singularity line. In Figure 17, an example of a model with a tip clearance is given. The tip is not flat and a cavity sits inside the solid tip. Four positive and four negative singularities are identified. The complete internal line network for this geometry is depicted in Figure 18 . Streamlines are indicated with green color. All lines are smoothed to support the generation of a high-quality partitioning.

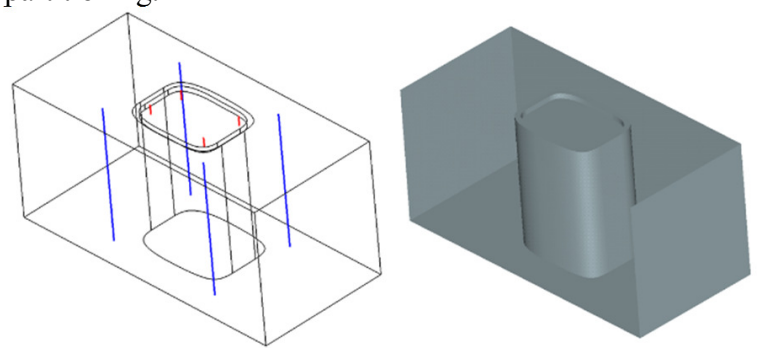

Figure 17: Singularity lines. View of the cavity.

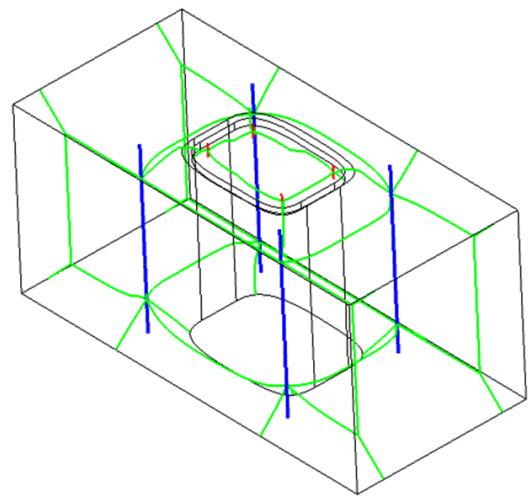

Figure 18: Line network consisting of singularity lines (red and blue) and streamlines (green). Streamlines lie on the medial object.

\section{BUILDING DECOMPOSITIONS}

\section{Boundary curves}

The line network consists of streamlines that lie on the medial object and of singularity lines. Furthermore, since each medial entity is associated with parts of the object's boundary, this association is inherited to the line network as described in section 6 . Through this association, boundary curves can be created by projecting each streamline and each 
singularity line to the boundary. Boundary curves that correspond to the same streamline will support the construction of one partition surface with the assistance of the singularity line. In Figure 19 an example is given for a positive singularity. Green lines represent trace lines that lie on the medial object. When projected to the boundary they produce the yellow boundary lines. On the right, a detail is given for the region around the concavities. The medial object structure captures such features and, as a result, the boundary lines take them into account too. These boundary lines start from singularities and, either meet the other end of the singularity or join to another singularity. Boundary lines together with singularities create loops of lines to define partition surfaces. In Figure 20, all boundary lines are depicted in yellow. For good quality surfaces to be generated it is important that singularities and boundary curves are smooth and thus a smoothing step is important. During smoothing the connectivity with the boundary should be maintained.

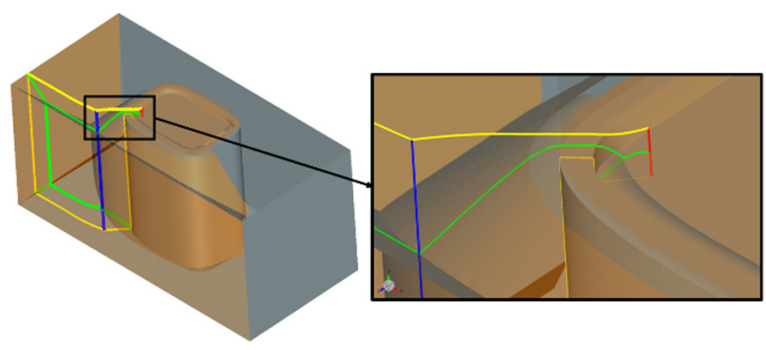

Figure 19: The green streamlines that lie on the medial object are extruded to form the yellow boundary lines (a). The medial object captures all features of the domain and so do the streamlines and the boundary lines. An example of two concavities is given in (b).

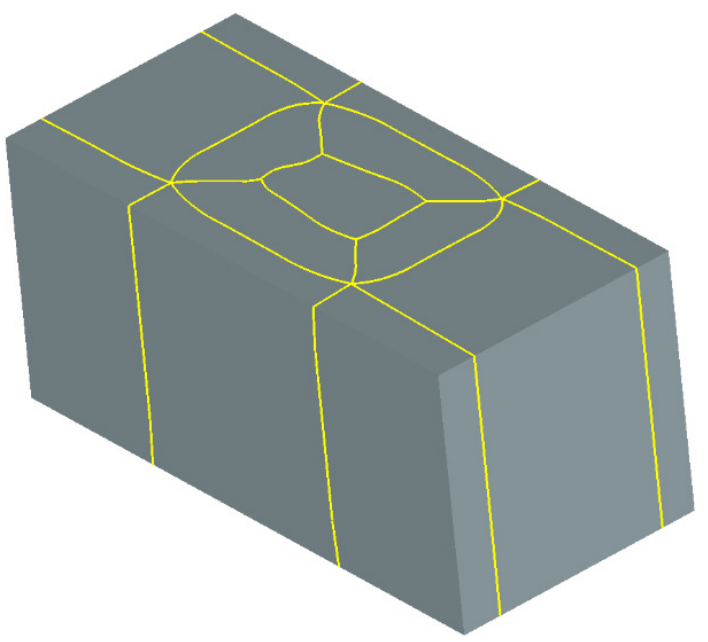

Figure 20: Boundary loops for the model of Figure 17.

\section{Partition surfaces}

The boundary curves and singularity lines define the boundaries of high-quality partition surfaces. Since boundary lines were generated using all the features of the domain it is expected that the partition surfaces will respect them too. Figure 21(a) shows partition surfaces on the interior of the model of Figure 17. Figure 21(b) depicts how the partition surface respects the two concavities. Since the medial object captures such features of the domain, so do the streamlines and consequently, the partition surfaces too. In Figure 23(a) the partition surfaces can be seen separately.

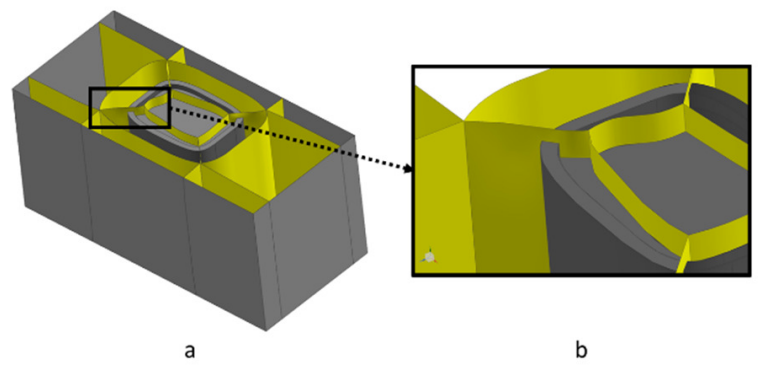

Figure 21: High-quality partition surfaces are generated (a). These surfaces respect features of the domain (b).

\section{Generate regions}

The partition surfaces that were generated in the previous step are used to decompose the domain. At this stage, the generation of regions suitable for hexahedral meshing is not fully automated. After automatically generating the line network and the partition surfaces, regions are constructed with the aid of the commercial software CADfix. In order to do that, the intersections between partition surfaces are identified. In Figure 22, an example of such intersections is given. In (a), two partition surfaces from two positive singularities on the left are highlighted in yellow. In (b), partition surfaces from two positive singularities on the right are also shown. In (c) the intersections with the yellow partition surfaces are given in purple. In (d) a top view of the intersection is given. These curves, together with the boundary curves and the singularity lines, define the boundaries of the regions that will be created and hexmeshed. These regions have no further singularities. The generation of two such blocks can be seen on the right of Figure 22. After the regions have been defined, the user can prescribe the density of the mesh through the meshing environment of CADfix and generate a hexahedral mesh. Figure 23(b) shows the decomposition implied by the partition surfaces for the model of Figure 17. It is important to note that although no singularities exist in the regions after decomposition, however, not all of the regions have a simple block structure. Due to concavities, some of the regions need to be decomposed further in order to have only simple blocks. An example of such a region is given in Figure 24(a). Although this region is not mappable, a good quality mesh can be created by sweeping (b). 


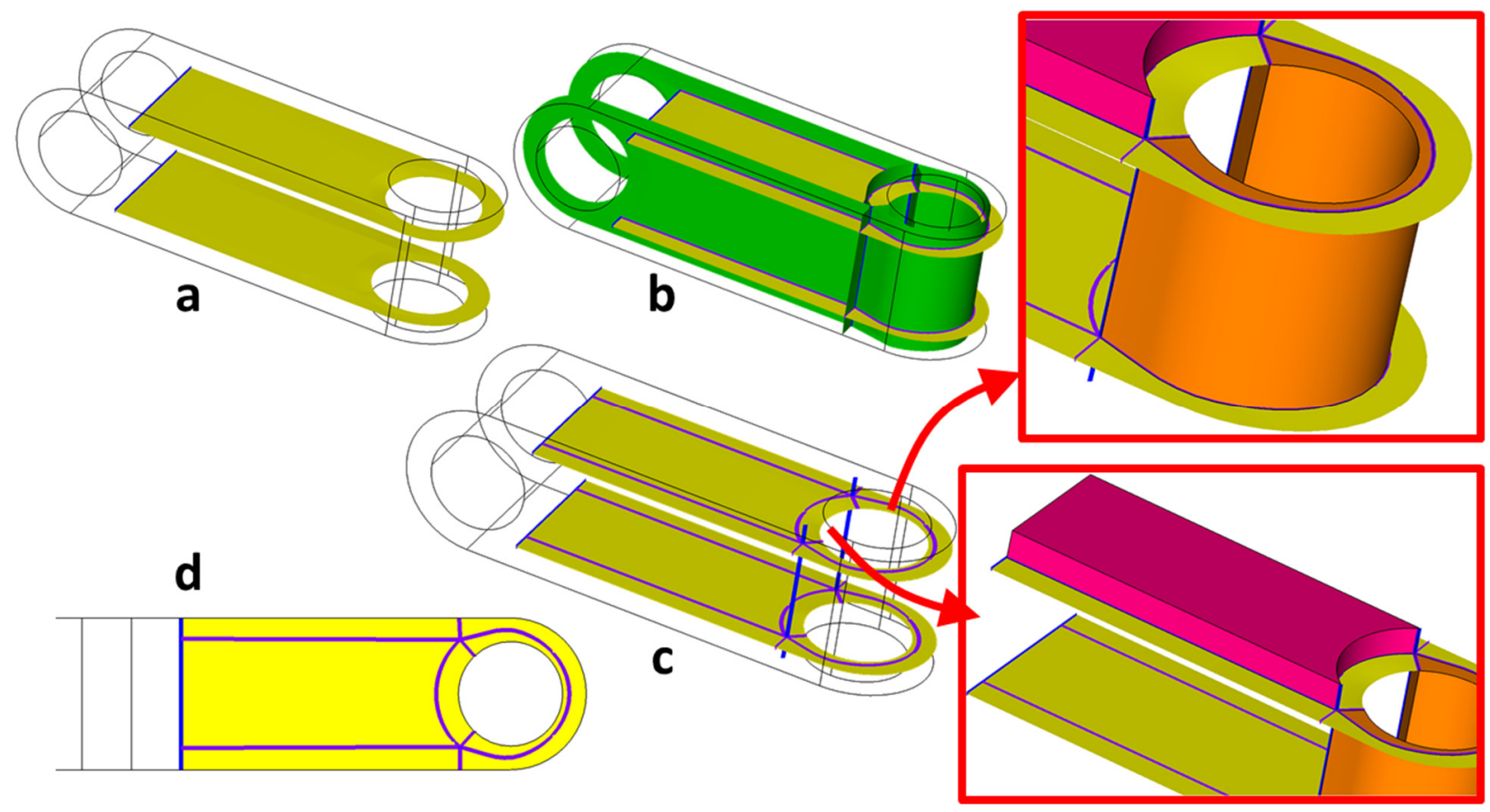

Figure 22: Partition surfaces from two positive singularities (a). Partition surfaces from two other positive singularities are highlighted in green (b). Intersections between them are shown in purple (c) and (d). In the right, two blocks are given. These are bounded by singularity lines, intersections between partition surfaces and boundary lines.
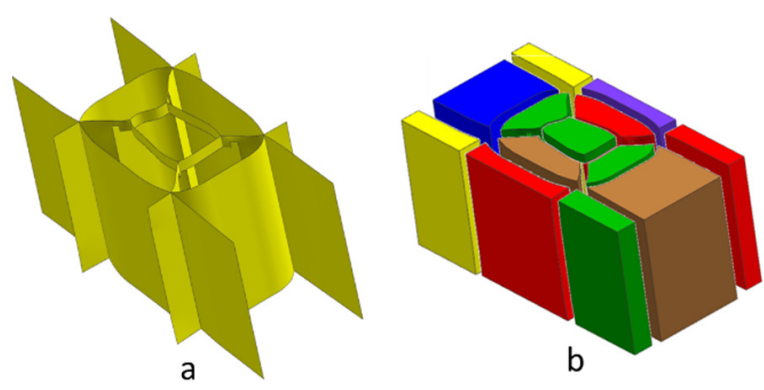

Figure 23: Partition surfaces (a). Decomposition

(b).
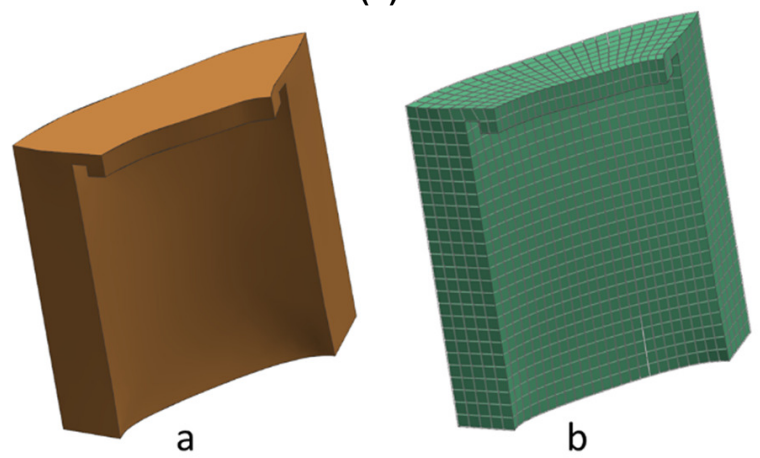

Figure 24: Region that is not a simple block (a). A high-quality mesh generated via sweeping (b).

\section{RESULTS}

The proposed method has been tested in a series of models and produced decompositions suitable for the generation of all hexahedral meshes. The medial object and all the meshes are created using the commercial software CADfix. Using the provided API the method has been implemented in Python. The first model is a simple thin semicircular plate shown together with its medial object in Figure 25.

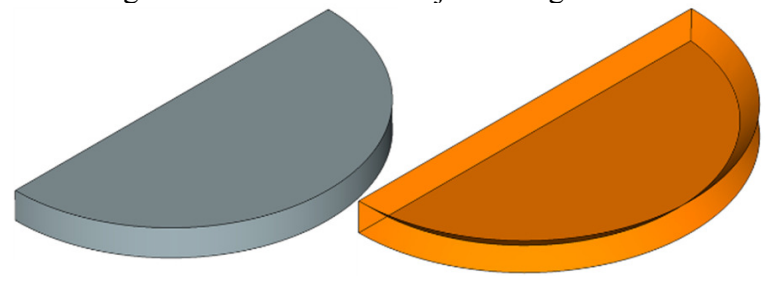

Figure 25: Thin semicircular plate with its medial object

Two negative singularities are identified on the central medial surface. The streamlines are traced until they meet the boundary or they connect to other singularities. Boundary lines are formed by extruding to the boundary these lines and, together with the singularities they form the partition surfaces. A block decomposition is generated and a high-quality mesh is created. Figure 26 shows these steps. Figure 27 shows the second model, a thin circular plate with two holes, together with its medial object. In this model, six positive and two negative singularities are identified. Figure 
28 shows the streamlines and the crosses generated on top of the medial object.
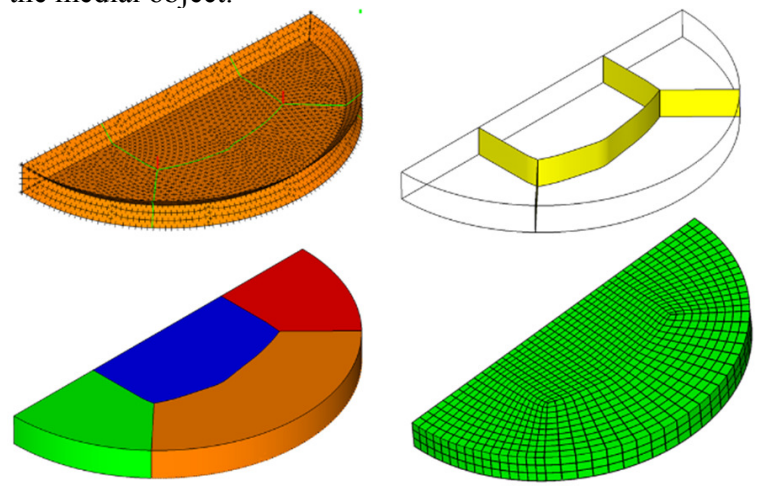

Figure 26: Streamlines (top left), partition surfaces (top right), block decomposition (bottom left) and hex-mesh (bottom right) for the thin semicircular plate.

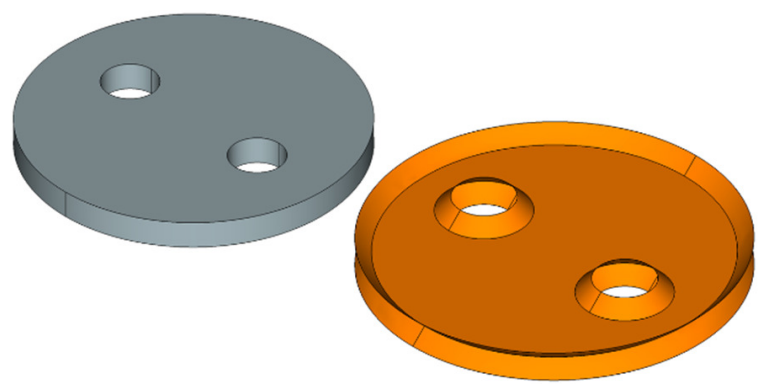

Figure 27: Circular plate with two holes together with its medial object.

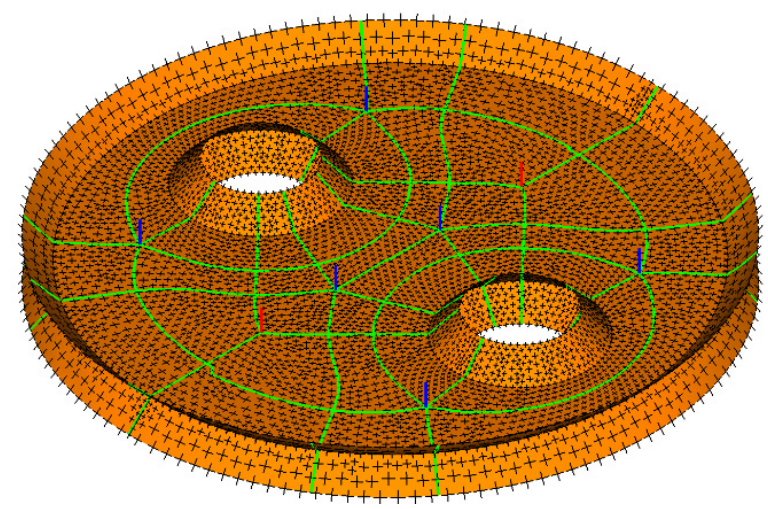

Figure 28: Cross-fields on the medial object and streamlines traced. Six positive and two negative singularities are identified.

The boundary lines that are created based on these streamlines are depicted in Figure 29.

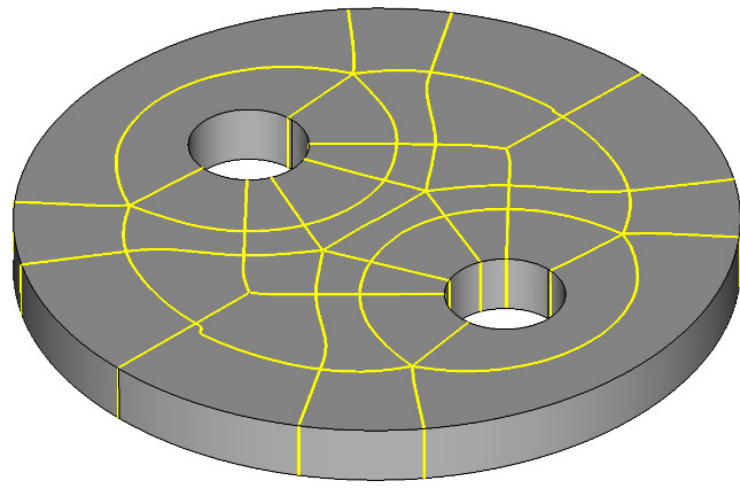

Figure 29: Boundary lines generated for the plate with the two holes.

A more complex model is shown in Figure 30. The concave features of this model make the decomposition quite challenging for a non-expert.

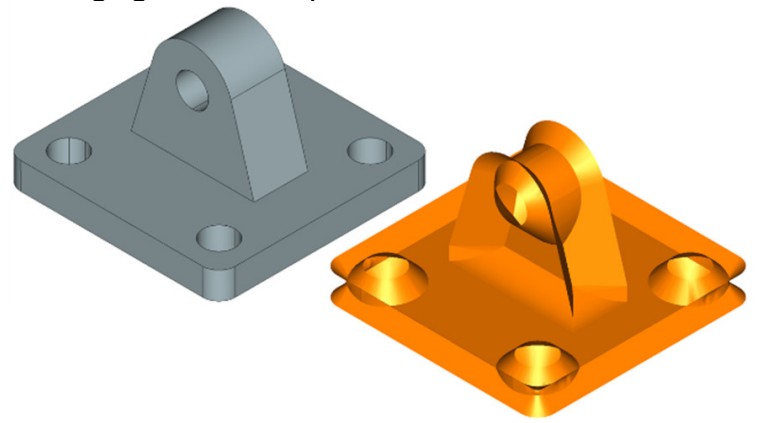

Figure 30: Solid model together with its medial object.

Two details of the medial object are given in Figure 31 to understand how the concavities affect the medial object. In Figure 31(a), the medial surface that maps the top to the bottom boundary face is highlighted. The loop formed by the four concave boundary edges results in a rectangular hole on the medial surface. As it can be seen in Figure 31(b), the medial object curves around the concavities. This proves to be really helpful since it will allow streamlines to curve around the concavities.

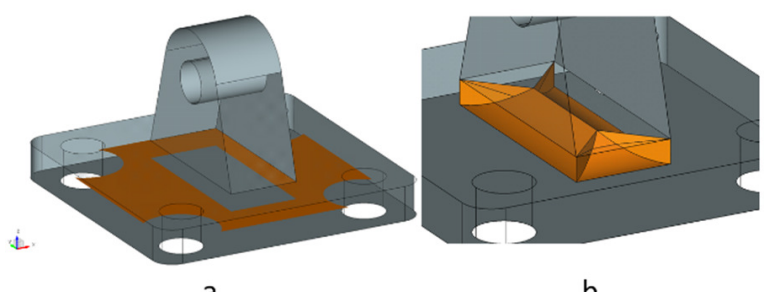

a

b

Figure 31: Concavities result in a hole in a medial surface (a). A detail of the medial surfaces around the concave boundary edges (b).

Figure 32 shows the cross-field on the medial surface of Figure 31(a). Four positive singularities are identified and the corresponding streamlines are shown in green. Although 
the streamlines belong to the same medial surface, the hole created by the concavity separates the singularities on the left from the singularities on the right. However, the medial object's connectivity around the concavities, shown in Figure 31(b), allows the streamlines to be connected on different medial surfaces.

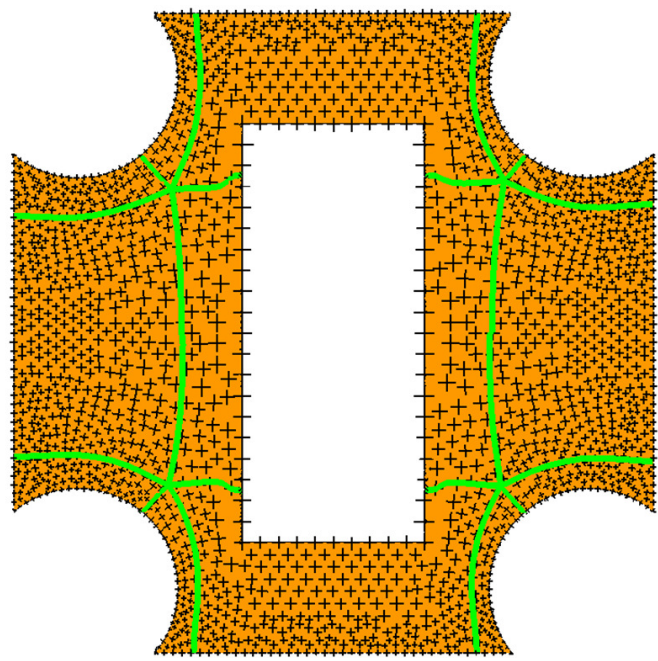

Figure 32: Concave edges result in a hole on the medial surface. The streamlines on the left side are not aware of those on the right.

In Figure 33 it can be seen how the structure of the medial object around the concavity makes it possible for the streamlines to connect to each other. It can also be seen how they connect to another blue positive singularity on the top. A detail is also given which shows how the streamline breaks into two near the concavity. One continues and connects to the other streamline coming from the right while the second one turns 90 degrees following the concave boundary until it connects to the positive singularity on the top.

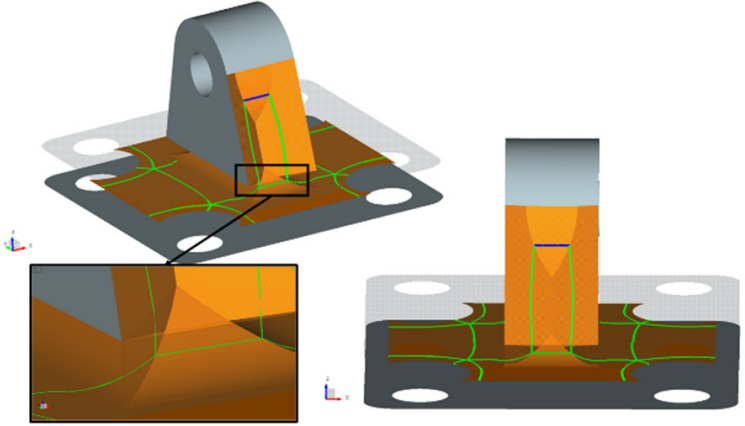

Figure 33: Streamlines traced from medial surface to medial surface on the medial object connect to each other although the concavity separated them. A streamline can be seen to "break" in two around the concavity.

Streamlines like these are "geometry aware" and help in generating a decomposition that captures all important features of the domain. In Figure 34 the partition surface that corresponds to the streamlines of Figure 33 is given. The positive singularities on which it is attached are shown in blue.

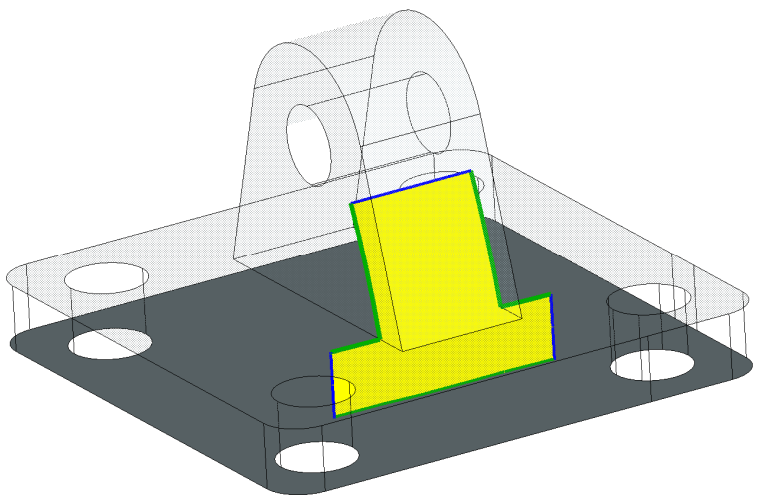

Figure 34: Partition surface attached to three positive singularities respecting the concave boundary edges.

The final decomposition of this model is given in Figure 35. Most regions are simple blocks. However, around the concavities the regions are a bit more complex (Figure 36(a)), and were created based on surfaces like the one depicted on Figure 34. A good quality mesh can still be created by sweeping like that shown in Figure 36(b) or alternatively the region could be further decomposed as shown in Figure 36(c). However, no further singularities exist in these regions. At this stage, such regions are not treated automatically. It is part of on-going research to automatically detect concave features and construct extra partition surfaces.

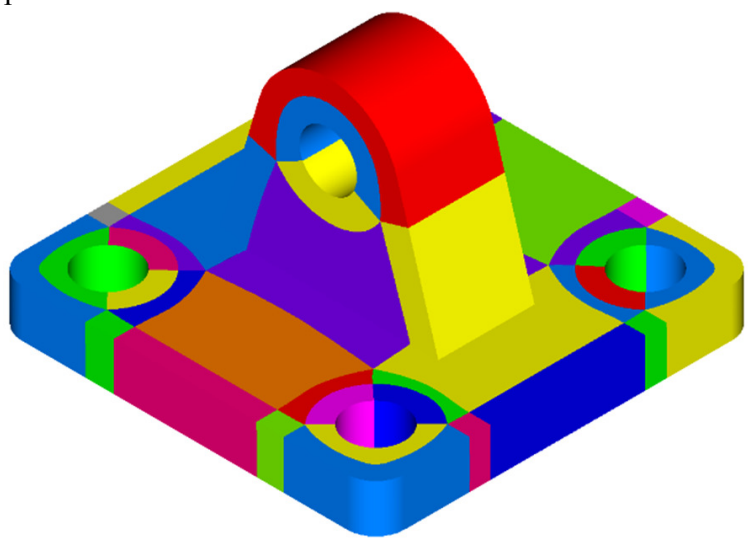

Figure 35: Decomposition of the model of Figure 27.

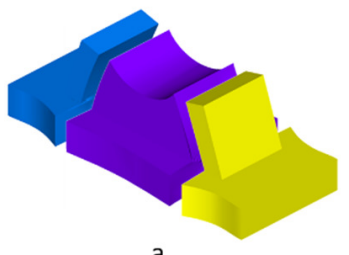

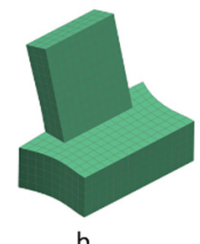

b

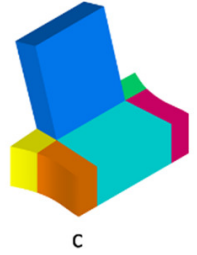

Figure 36: Non-simple block regions (a), mesh of yellow region created by sweeping (b), further decomposition into blocks of yellow region (c).

The mesh for the model of Figure 30 is given in Figure 37. 


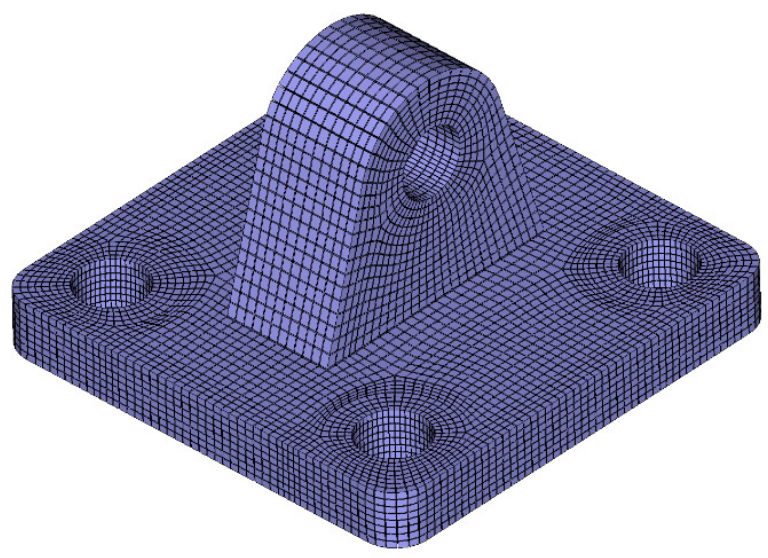

Figure 37: Mesh for the model of Figure 30. (Min. I Avg. Scaled Jacobian: $0.68 / 0.97$ )

Finally streamlines for one more example are given in Figure 38 (b). Partition surfaces for this geometry where shown in Figure 22. Four positive singularity lines are identified. The block decomposition that was generated based on the partition surfaces and the final hexahedral mesh are given in Figure 39 .

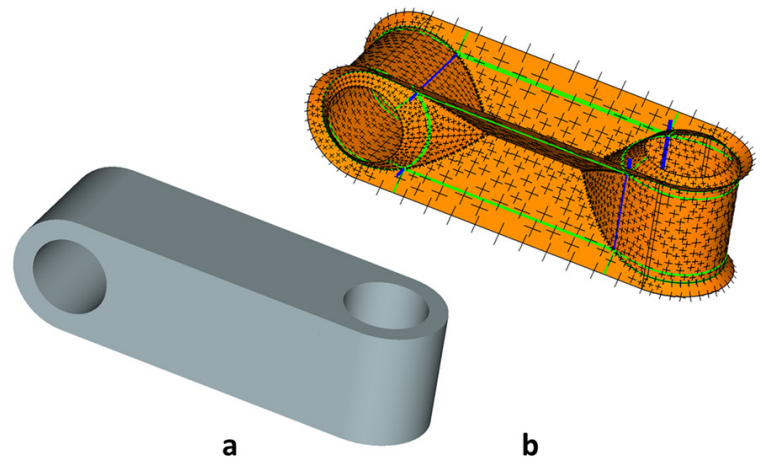

Figure 38: Model with two through holes in orthogonal directions (a). The cross-field on the medial object together with singularity lines and streamlines (b).

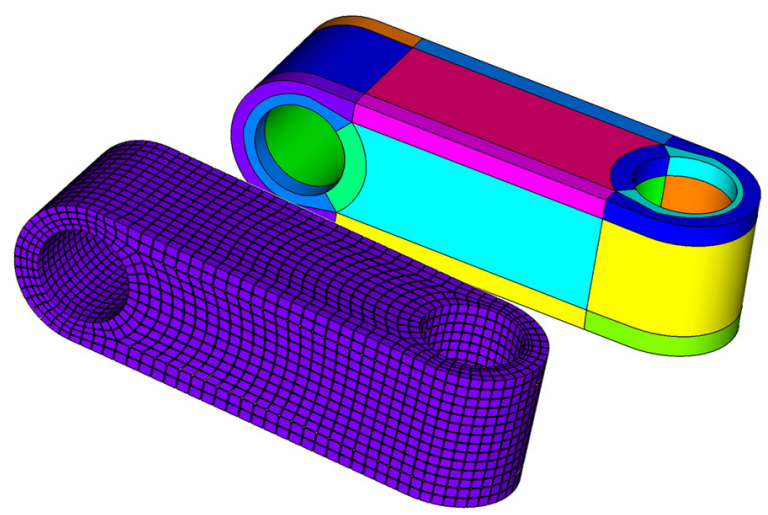

Figure 39: Final block decomposition and hexahedral mesh for the model of Figure 38. (Min. I Avg. Scaled Jacobian: 0.69 / 0.94)

\section{DISCUSSION}

Generating high-quality partition surfaces to create decompositions sufficient for all-hexahedral meshing can be proven to be difficult. Capturing all geometrical and topological features of the domain is challenging. In the current work, this issue was addressed by first generating a set of boundary curves and singularity lines which bound those surfaces. These lines are generated by projecting on the boundary a line network created on top of the medial object of the domain. Since the medial object captures all geometrical and topological features of the domain, the partition surfaces will respect them too. Furthermore, since the medial object by itself separates the domain into regions, a line network with simple topology can be created by modifying it locally on each medial surface. This simplifies the decomposition. Instead of manipulating partition surfaces, changes can now be done on curves on the medial object. Furthermore, by placing singularities on the medial object, they are pushed to the interior of the domain, far from the boundary. This ensures that the decomposition and the final mesh will have high quality close to the boundary. This is, in general, preferable for numerical simulations. Moreover, since singularity lines do not depend on an underlying tetrahedral mesh, noisy patterns that are common in frame-field methods are avoided and in general smooth lines are created. By generating frames based on touching vectors and not by aligning them with medial edges, unnecessary singularities are avoided. In general, compared to the current state of the art where the fixed cross-field topology of the boundary restricts the decomposition process, an attempt is made to construct it on cross-fields built on the interior and the project it to the boundary.

The medial object of the domain proves to be a really helpful framework on which singularities can be traced. At the same time, it captures efficiently all geometric features of the geometry and thus can provide important information in reasoning a high-quality decomposition. However, the medial object by itself is difficult to construct and no method exists that can guarantee a robust and efficient computation of the medial object of an arbitrary complex domain.

This work aims, not only on describing a method by which arbitrary domains can be decomposed for hexahedral meshing, but also to bring together the merits of two different methods. This can help to gain further knowledge regarding the long-standing problem of blockdecomposition. The medial object provides a topological and geometrical connectivity on the interior of the domain that could be beneficial. Having understood what exactly is needed, then a, more easily generated, imprecise medial object could be used to assist existing methods that rely on frame-fields.

The method was tested on a number of models and produced good quality partitioning of the domains. However, there are still issues that need to be addressed. Concave features can result in decompositions that are not simple blocks like those shown in Figure 24 and Figure 36. Extra partition surfaces are needed to fully decompose the domains into blocks. Generating such surfaces by exploring the imprints of the concavities on the medial object is a topic of future research. 
At this stage, the lack of a robust method to generate the exact medial object for every possible domain is the main drawback of the method as it relies on it. Handling concavities to generate pure block regions is another issue that needs to be addressed. Finally, degenerate cases where multiple points on the boundary map to a single point/line on the medial object (like for example a sphere, a cylinder or a blended convex edge) need to be further examined to make the method more complete.

\section{ACKNOWLEDGEMENTS}

The authors wish to acknowledge the financial support provided by Innovate UK via the GEMinIDS (project 113088), a UK Centre for Aerodynamics project. The authors acknowledge Rolls-Royce for granting permission to publish this paper.

\section{REFERENCES}

[1] J. Thompson, Z. U. a Warsi, C. W. Mastin, and J. F. Thomson, Numerical grid generation: foundations and applications, vol. 1. NorthHolland, 1985.

[2] W. J. Gordon and C. A. Hall, "Construction of curvilinear co-ordinate systems and applications to mesh generation," Int. J. Numer. Methods Eng., vol. 7, no. 4, pp. 461-477, Jan. 1973

[3] R. E. Smith and L. E. Eriksson, "Algebraic grid generation," Comput. Methods Appl. Mech. Eng., vol. 64, no. 1-3, pp. 285-300, Jan. 1987.

[4] S. Cannan, "Plastering - A new approach to automated, 3-D hexahedral mesh generation," in 33rd Structures, Structural Dynamics and Materials Conference, 1992.

[5] T. D. Blacker and M. B. Stephenson, "Paving: A new approach to automated quadrilateral mesh generation," Int. J. Numer. Methods Eng., vol. 32, no. 4, pp. 811-847, Jun. 1991.

[6] M. L. Staten, R. A. Kerr, S. J. Owen, T. D. Blacker, M. Stupazzini, and K. Shimada, "Unconstrained plastering-hexahedral mesh generation via advancing-front geometry decomposition," Int. J. Numer. Methods Eng., vol. 81, no. 2, pp. 135-171, Jan. 2010.

[7] P. M. Knupp, Next-Generation Sweep Tool: A Method for Generating All-Hex Meshes on Twoand-One-Half Dimensional Geometries. 1998.

[8] S. Cai and T. J. Tautges, "One-to-one sweeping based on harmonic S-T mappings of facet meshes and their cages," Eng. Comput., vol. 31, no. 3, pp. 439-452, Jul. 2015.

[9] M. A. Scott, S. E. Benzley, and S. J. Owen, "Improved many-to-one sweeping," Int. J. Numer. Methods Eng., vol. 65, no. 3, pp. 332-348, Jan. 2006.

[10] E. Ruiz-Gironés, X. Roca, and J. Sarrate, “A new procedure to compute imprints in multi-sweeping algorithms," in Proceedings of the 18th International Meshing Roundtable, 2009, Berlin,
Heidelberg: Springer Berlin Heidelberg, 2009, pp. 281-299.

[11] M. A. Price, C. G. Armstrong, and M. A. Sabin, "Hexahedral mesh generation by medial surface subdivision: Part I. Solids with convex edges," Int. J. Numer. Methods Eng., vol. 38, no. 19, pp. 3335-3359, Oct. 1995.

[12] M. A. Price and C. G. Armstrong, "Hexahedral mesh generation by medial surface subdivision: Part ii. solids with flat and concave edges," Int. J. Numer. Methods Eng., vol. 40, no. 1, pp. 111-136, Jan. 1997.

[13] W. R. Quadros, "LayTracks3D: A new approach for meshing general solids using medial axis transform," Compuer-Aided Design., vol. 72, pp. 102-117, 2016.

[14] M. Livesu, A. Muntoni, E. Puppo, and R. Scateni, "Skeleton-driven Adaptive Hexahedral Meshing of Tubular Shapes," Comput. Graph. Forum, vol. 35, no. 7, pp. 237-246, 2016

[15] M. Nieser, U. Reitebuch, and K. Polthier, "CUBECOVER - Parameterization of 3D volumes," Comput. Graph. Forum, vol. 30, no. 5, pp. 1397-1406, Aug. 2014.

[16] J. Huang, Y. Tong, H. Wei, and H. Bao, "Boundary aligned smooth 3D cross-frame field," in ACM Transactions on Graphics, 2011, vol. 30, no. 6, p. 1 .

[17] J. Huang, T. Jiang, Y. Wang, Y. Tong, and H. Bao, "Automatic Frame Field Guided Hexahedral Mesh Generation, Technical Report," 2012.

[18] Y. Li, Y. Liu, W. Xu, W. Wang, and B. Guo, "Allhex meshing using singularity-restricted field," ACM Transactions on Graphics, vol. 31, no. 6, p. 1, Nov. 2012.

[19] N. Ray, D. Sokolov, and B. Lévy, "Practical 3D Frame Field Generation," ACM Trans. Graph., vol. 35, no. 6, p. 233:1--233:9, Nov. 2016.

[20] J. Solomon, A. Vaxman, and D. Bommes, "Boundary Element Octahedral Fields in Volumes," ACM Transactions on Graphics, vol. 36, no. 3, May 2017.

[21] H. Liu, P. Zhang, E. Chien, J. Solomon, and D. Bommes, "Singularity-constrained Octahedral Fields for Hexahedral Meshing," ACM

Transactions on Graphics, vol. 37, no. 4, p. 93:1-93:17, Jul. 2018.

[22] R. T. Fogg HJ, Sun L, Makem JE, Armstrong CG, "Singularities in structured meshes and crossfields," Computer-Aided Design, vol. 105, pp. 11$25,2018$.

[23] F. L. Ryan Viertel, Matthew L Staten, “Analysis of Non-Meshable Automatically Generated Frame Fields.," Albuquerque, NM (United States), 2016.

[24] N. Kowalski, F. Ledoux, and P. Frey, "Smoothness driven frame field generation for hexahedral meshing," CAD Computer-Aided Design., vol. 72, pp. 65-77, Mar. 2016.

[25] F. Shang, Y. Gan, and Y. Guo, "Hexahedral mesh generation via constrained quadrilateralization," PLoS One, vol. 12, no. 5, p. e0177603, May 2017. [26] R. Wang, C. Shen, J. Chen, H. Wu, and S. Gao, 
"Sheet operation based block decomposition of solid models for hex meshing.," Computer-Aided Design., vol. 85, pp. 123-137, 2017.

[27] J. Gregson, A. Sheffer, and E. Zhang, "All-hex mesh generation via volumetric polycube deformation," Eurographics Symp. Geom. Process., vol. 30, no. 5, pp. 1407-1416, Aug. 2011.

[28] X. Fang, W. Xu, H. Bao, and J. Huang, "All-hex Meshing Using Closed-form Induced Polycube," ACM Transactions on Graphics, vol. 35, no. 4, p. 124:1--124:9, Jul. 2016.

[29] S. (2018). A. blocking of S. using E. A. Lim, C.W., Yin, X., Zhang, T., Goh, C.K., Alejandro, L.B., Moreno, \& Shahpar, "Automatic blocking of Shapes using Evolutionary Algorithm.," in Proceeding of the 27th International Roundtable, 2018.

[30] D. Papadimitrakis, C. G. Armstrong, T. T. Robinson, S. Shahpar, and A. Le Moigne, "A Combined Medial Object and Frame Approach to Compute Mesh Singularity Lines," in 27th International Meshing Roundtable., 2018.

[31] J. S. and D. B. Liu, Heng, Paul Zhang, Edward Chien, "Singularity-constrained octahedral fields for hexahedral meshing," ACM Transactions on Graphics 37, p. 93:1-93:17, 2018.

[32] H. J. Fogg, C. G. Armstrong, and T. T. Robinson, "Automatic generation of multiblock decompositions of surfaces," Int. J. Numer. Methods Eng., vol. 101, no. 13, pp. 965-991, Mar. 2015. 


\title{
DUAL-BASED USER-GUIDED HEXAHEDRAL BLOCK GENERATION USING FRAME FIELDS.
}

\author{
Simon Calderan ${ }^{1,2} \quad$ Guillaume Hutzler $^{2} \quad$ Franck Ledoux ${ }^{1}$ \\ ${ }^{1}$ CEA, DAM, DIF, F-91297, Arpajon, France. simon.calderan@ocre.cea.fr \\ 2 IBISC, University of Evry, Paris-Saclay, France
}

\begin{abstract}
Block structured hexahedral meshing is required for many applications but remains unreachable in an automatic manner for realistic simulation models. In practice, true industrial cases are handled using complex and rich interactive software, and generating a block structure for a mechanical CAD part can require several days to weeks for an expert engineer. For many years now, several scientific works have demonstrated that 3D frame fields are a very powerful tool for hexahedral meshing. These works remain mainly theoretical and their application is limited to simple 3D models. In this paper, we propose to provide the necessary components to build an interactive tool using frame fields. The principle of the approach is to build a valid dual structure made of 3D dual sheets, which are aligned along a 3D frame field, and then to convert this dual structure into its primal hexahedral block structure.
\end{abstract}

Keywords: blocking, hexahedral mesh generation, frame field, user-guided approach, dual structure

\section{INTRODUCTION}

For many application fields, such as high deformation mechanics, hydrodynamics or fluid dynamics, hexahedral meshes are preferred over tetrahedral meshes by physics scientific codes and the underlying numerical methods. More specifically, boundary-aligned hexahedral block-structured meshes are required. But such meshes are known to be very hard to create in practice. And despite the active research done in the last three decades, the generation of such meshes in an industrial context remains a manual task that can need several days to weeks depending on the CAD model complexity. It is clearly unbearable in the production life cycle and it is known that generating meshes in general will be keeping to be an important bottleneck in the near future [1].

In the past few years, many research teams working on hexahedral meshes have studied the usage of frame fields to reveal the expected block structure. They proposed different algorithms and heuristics but all of them were only able to get acceptable results on sim- ple CAD models. Complicated ones are only meshed using mixed meshes ${ }^{1}$ or hex-dominant meshes [2, 3, 4]. We think that frame fields are a relevant and strong tool for the generation of hexahedral meshes but they encounter at least two issues up to now: (1) no heuristics used to generate frame fields guarantees that their structure fits the hexahedral block structure; (2) even if the frame field is generated, the extraction of a block structure is not so automatic and robust as it should be. From this observation, we propose to consider a hybrid approach where the engineer will guide the algorithm in the generation process. Instead of providing a fully automatic procedure, we intend to provide useful tools to interactively generate hexahedral blocks starting from an input frame field. This way, we expect to integrate frame fields technology into industrial tools in a short time and so to contribute to diminish the global time to simulation.

\footnotetext{
${ }^{1}$ In 3D, a mixed mesh can contain any type of 3dimensional cells: tetrahedra, hexahedra, prisms, pyramids and polyhedra.
} 


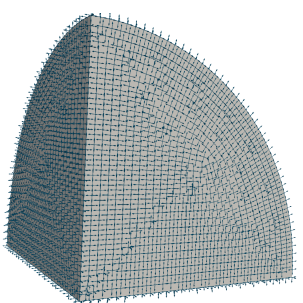

(a)

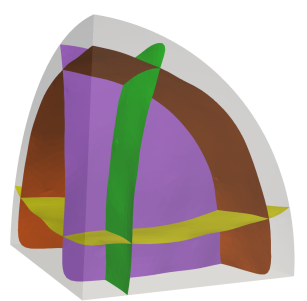

(b)

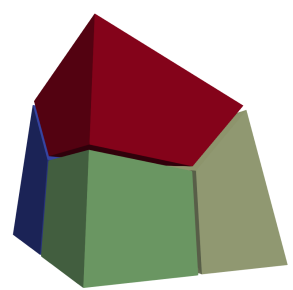

$(c)$

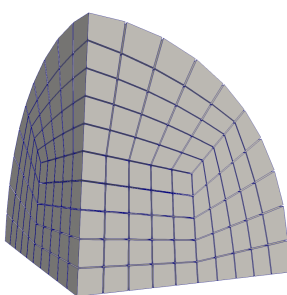

$(d)$

Figure 1: Starting from a tetrahedral mesh $\mathcal{T}$ where a frame is assigned at every node $(a)$, we first select a set of dual sheets $(b)$ that are used to create a block structure $(c)$, which is eventually refined to get a hexahedral mesh with the expected size properties $(d)$.

\section{$1.1 \quad$ Related works}

Our approach consists in extracting 3D dual sheets from a frame field. Using frame fields to interactively generate blocks was very recently proposed by [5] in a quite similar way. In their work, the authors provide an interactive environment to design dual sheets starting from boundary loops that the user can define in different manners. We follow the same path but instead of using an indirect approach where the user builds boundary loops using a surface cross field or some surface interactors disconnected from cross fields, we directly build 3D dual sheets. Authors of [6] provide an automatic algorithm based on the same concept, which is to build boundary loops starting from a surface cross field. In both cases, 3D dual sheets are deduced from boundary loops by using an implicit definition of surfaces [5] or a volume partitioning technique based on the min-cut, max-flow algorithm [6].

Using frame field for producing hexahedral meshes has received much interests during the past few years $[7$, $8,9,10]$. Frame fields provide directional information in the whole domain to be meshed. It can be used to drive tetrahedral mesh generation before applying a tet-to-hex recombination algorithm to generate mixed meshes, to directly extract the hexahedral block structure $[8,9]$, or to generate a parameterization that will then give the final mesh $[11,12]$. Frame field technology seems to have a high potential to get quality allhex meshes, but there remains a lot of work to do since the topology of generated frame fields does not always fit the topological structure of hexahedral meshes. As a consequence, frame fields are also used for generating high-quality hex-dominant meshes $[2,4]$. In the present work, frame fields are only an input and we do not focus on the way to generate it.

The dual structure of hexahedral meshes has been studied for its peculiar properties $[13,14,15,16]$ that are global to the mesh. Comparing to tetrahedral meshes where it is quite easy to perform local modifications as removing a node or swapping an edge, modify- ing hexahedral meshes in a safe manner ${ }^{2}$ is more global using operations on sheets or chords. The topological structure of hexahedral meshes has so been used to generate hexahedral meshes considering topology first via the Whisker-weaving algorithm [17, 18, 19] or the dual cycle elimination [20,21]. Either modifying meshes by inserting fundamental sheets to get a block-structure hexahedral mesh [22] or matching two hexahedral meshes along a contact surface [23] to get a full conformal hexahedral mesh for a CAD assembly model has been observed to be useful.

\subsection{Method overview}

Our method can be seen as similar to $[5,6]$ on many aspects, but is fundamentally different on one point. We build 3D dual sheets directly and not as enclosed by dual surface loops. Building a 3D sheet is more challenging on many points but it has the benefit of avoiding some side-effects: (1) a dual sheet can be enclosed by several dual boundary loops and finding the set of dual boundary loops that corresponds to the same dual sheet is not an obvious task; (2) it takes advantage of the whole geometrical information carried on the $3 \mathrm{D}$ frame fields inside the domain. The approach we propose consists in a pipeline that is made of three main steps (see Figure 1). Starting from a tetrahedral mesh $\mathcal{T}$ where a discrete frame field is given on every node of $\mathcal{T}$, we adopt the following process:

1. First, we interactively build a set of dual sheets that splits the volume into several dual zones (see Figure 1-b). A dual sheet is built from a picked tetrahedral cell of $t \in \mathcal{T}$ and a normal direction, which is one of the three frame directions linearly interpolated at the center of mass of $t$.

2. Second, we extract a block structure from the dual structure previously built (see Figure 1-c). This stage can fail if the dual structure provided

\footnotetext{
${ }^{2}$ In the meaning that the mesh always remains full-hex during the whole modification process.
} 
at stage 1 is incomplete. In this case, we go back to stage 1 to add and/or remove some dual sheets.

3. Eventually, we build a refined hexahedral mesh by using a simple transfinite interpolation in each block (see Figure 1- $d$ ).

The last stage of this pipeline being straightforward, we focus on the two first stages in the remainder of the paper. Section 3 is dedicated to stage 1, while Section 4 gives details about the properties a dual structure must ensure to generate a valid block structure. Section 5 shows how to generate hexahedral blocks from the dual structure. But beforehand, we introduce several useful notions in Section 2.

\subsection{Main benefits and drawbacks of our method}

A main difference between our works and [5, 6, 24] is that we directly build $3 \mathrm{D}$ sheets to decompose the geometric domain $\Omega$ we work on. It induces a main disadvantage, which is that our approach relies on tracing 3D stream surfaces along a 3D frame field defined in $\Omega$. Tracing such streamlines is more complex than tracing surface loops. It is due to the potential nonintegrability of the 3D frame field we start from and the numerical issues that are more difficult to control and handle in a robust programmatic way.

But we believe that the advantages of dealing with 3D sheets deserve to try and get a robust stream surface building process. Only considering surface loops as in $[5,6,24]$ imply several restriction and extra process. First, the underlying assumption is that a loop encloses a 3D sheet in the volume, and so corresponds to generating a $3 \mathrm{D}$ sheet. This assumption is very restrictive as a 3D sheet can be bounded by several surface loops (depending on the domain genus). A first issue is so to define a $3 \mathrm{D}$ sheets as bounded by several loops and not just one. By dealing with 3D sheets directly we avoid the heuristics proposed in $[5,6]$ for such situations. A second issue is that cross fields does not provide all the required information to build the $3 \mathrm{D}$ sheet bounded by one or several surface loops. As a consequence, [5] cut the volume following a constant direction, which is defined by the normal to the surface where the loop is defined (when it is possible), or provides interactive control to the users, who can add control points to deform the $3 \mathrm{D}$ sheets. In [6], the 3D surface is built automatically following a 3D field using a min-cut max-flow graph cutting algorithm. A third issue is that those approaches seem not be able to handle self-intersecting loop while a direct 3D approach allows it.

Considering now the full $3 \mathrm{~d}$ approaches that automatically build a 3D singularity graph and extract a block structure $[7,11,12,8,9,2,4]$, they are all of them limited to simple CAD examples and some CAD features are not possible to be handled (see examples of Fig. 24 and 26 for instance). It is also our case in this paper, but we consider that this work gives some mandatory components to design a new interactive tool with which the engineers we work with will be able to generate full-hex meshes more quickly than with their current tools.

\section{BACKGROUND}

Our approach relies on two main notions: frame fields and the hexahedral dual structure. In this section, we give the minimum of knowledge that is required for reading this paper. For more information about frame fields, please refer to $[10,12]$ and for the hexahedral dual structure, take a look at $[13,15]$.

\subsection{Notions of frame fields}

For the purpose of this work, we only consider discrete frame fields associated to a tetrahedral mesh. More specifically, given a tetrahedral mesh $\mathcal{T}$ that discretizes a CAD model $\Omega$, we consider that a frame field associates a frame to every node of $\mathcal{T}$.

A 3D frame $\mathbf{F}$ is defined as a 3 -tuple $\{\mathbf{u}, \mathbf{v}, \mathbf{w}\}$, where $\mathbf{u}, \mathbf{v}$ and $\mathbf{w}$ are three unit $3 \mathrm{D}$ vectors such that $\mathbf{u} . \mathbf{v}=0$ and $\mathbf{w}=\mathbf{u} \times \mathbf{v}$. In other words, $\mathbf{F}$ is defined by three unit vectors, that are orthogonal to each other forming an orthonormal right-handed basis of $\mathbb{R}^{3}$. $3 \mathrm{D}$ frames are naturally connected to the structure of hexahedral elements. Considering a

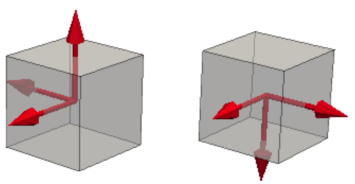
hexahedral cell, which has 3 main directions linking its pairwise opposite faces, 24 frames can thus represent it. This set of frames corresponds to the cubical symmetry group $\mathcal{G}$ (any map in $\mathrm{SO}(3)$ which maps coordinate axes to coordinate axes) and is invariant under rotations of $\frac{\pi}{2}$ around one of its three axis and forms an equivalence class.

A discrete frame field can be generated by many methods $[9,10,12]$. To our knowledge all of them try to get a smooth field while preserving the boundary alignment along $\Omega$. As a result, frames assigned to boundary nodes have one of their three directions aligned with the surface normal. Most of tetrahedral cells are called regular and a few ones are called singular. The frame field linearly interpolated in a regular tetrahedron does not contain any singularity, while a singular tetrahedron contains singularities (see Figures 2 and 3 ). We perform linear interpolation between frames into a tetrahedral cell by using a unit 
quaternion representation as done in [8]. In the context of discrete frame fields, we denote $S G(\mathcal{T})$ the set of singular tetrahedra of $\mathcal{T}$, and we name it singularity graph set. The set $S G(\mathcal{T})$ contains one-direction stable singular tetrahedra and unstable singular tetrahedra. The former are traversed by a single singularity line (see green tetrahedra on Fig. 3- $a$ ) while the latter contain a singularity point, i.e. a meeting point between several singularity lines (see red tetrahedra on Fig. 3-b).

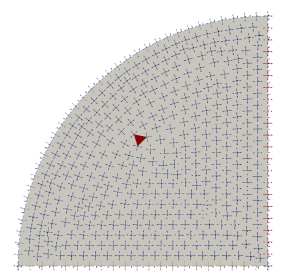

(a)

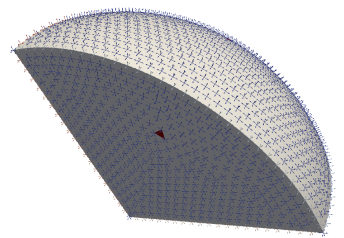

(b)

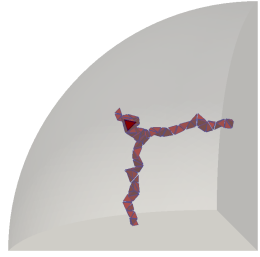

(c)

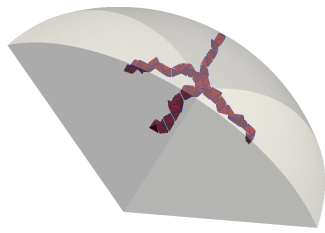

(d)
Figure 2: Several views of a discrete frame field. In $(a)$ and $(b)$, the boundary of the domain is visible with the frames at each node and singular tetrahedra in red. In $(c)$ and $(d)$, only the singular graph set is visible. It corresponds to the usual block decomposition we expect for such a geometric domain.

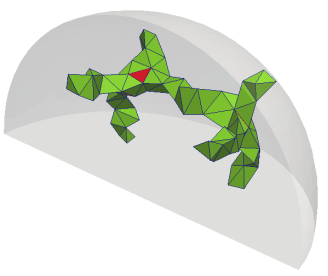

(a)

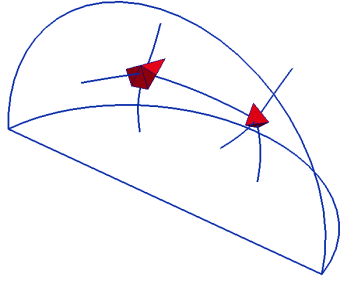

(b)
Figure 3: In $(a)$, the whole singularity graph set of a mesh $\mathcal{T}$, where green singular tetrahedra are traversed by a single singularity line (shown in $b$ ); In $(b)$, red singular tetrahedra contain a singularity point, which is the meeting point of several singularity lines.

\subsection{Primal and dual hexahedral mesh}

Building the dual of a mesh is a quite well-known process. To introduce it in the context of this work, we restrict our presentation to hexahedral meshes that are 3-dimensional meshes, or 3D meshes for short. Let us first introduce some terminology. A hexahedral mesh is made of hexahedral elements, which are 3 -dimensional cells or 3-cells, quadrilateral faces, or 2-cells, edges, or 1-cells, and nodes, or 0-cells. For a mesh $\mathcal{M}$, we denote $\mathcal{M}_{i}$, with $i \in[0 ; 3]$, the set containing all the $i$-cells of $\mathcal{M}$. We only consider conformal meshes. In other words, a 3-cell of a mesh $\mathcal{M}$ can only share an $i$-cell with another 3 -cell, with $i \in\{0,1,2\}$. The dual $\mathcal{D}_{\mathcal{M}}$ of a mesh $\mathcal{M}$ is also a $3 \mathrm{D}$ mesh, where:

- Each $i$-cell of $\mathcal{D}_{\mathcal{M}}$ corresponds to a $(3-i)$-cell of $\mathcal{M}$, with $i \in[0 ; 3]$. For any $i$-cell $c^{i}$ of $\mathcal{M}$, we denote $d_{c}^{n-i}$ the corresponding cell in $\mathcal{D}_{\mathcal{M}}$.

- If two 3-cells $a^{3}$ and $b^{3}$ of $\mathcal{M}$ share a face $f^{2}$, then nodes $d_{a}^{0}$ and $d_{b}^{0}$ are linked by edge $d_{f}^{1}$.

An example of a simple hexahedral mesh $\mathcal{M}$ and its dual mesh $\mathcal{D}_{\mathcal{M}}$ is given in Figure 4 .
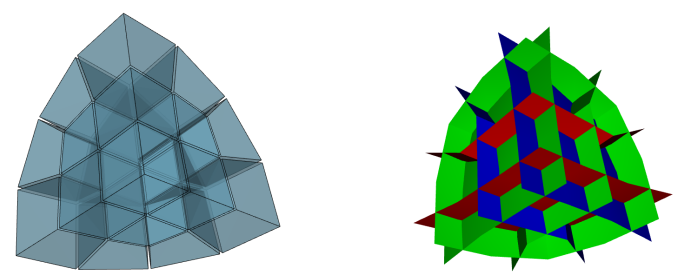

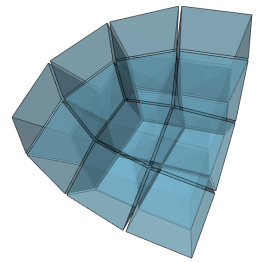

(a)

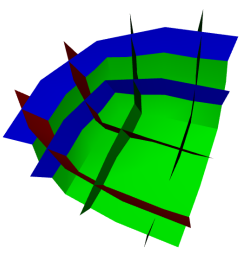

$(b)$
Figure 4: Example of a hexahedral mesh in $(a)$ and its dual in $(b)$. Note that the dual structure is represented as a set of surfaces intersecting each others.

Each dual node of a hexahedral mesh corresponds to a primal hexahedral cell. As a consequence, it is connected to six dual nodes and is adjacent to twelve dual faces. Such a dual node $n$ can so be seen as the intersection of exactly three dual surfaces made of four dual faces locally to $n$ (see Figure 5- $d$ ). From a broader point of view, the dual of a hexahedral mesh is structured as a simple arrangement of dual surfaces [13] (see Figure 5-b). We call each of these surfaces a dual sheet and the set of dual sheets split the initial domain into regions that we call dual zones (see Figure 5-c). 
This domain decomposition made of dual sheets and dual zones is the dual structure, also noted hex layout in [5].

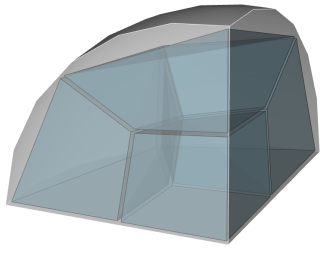

(a)

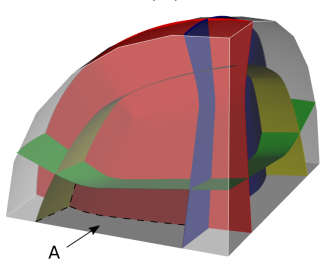

(c)

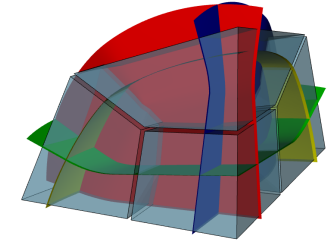

(b)

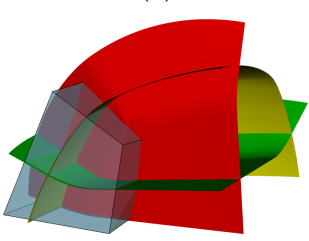

$(d)$
Figure 5: In $(a)$, the minimal block structure for a portion of sphere; In $(b)$ is shown the dual sheets and the block structure; On $(c)$, the dual zone A is delimited by the red; blue green ad yellow dual sheets; In $(d)$; a single hexahedral block corresponds to a dual node which is the intersection of 3 dual sheets: the green, yellow and red ones.

The notion of singularity graph $S G(\mathcal{T})$ introduced as the set of locations where frame fields are singular in Section 2.1 has also a significant meaning in the block structure, and more widely in hexahedral mesh topology. The singularity graph of a hexahedral mesh, and so a block structure, is the set of inner edges that are not adjacent to four hexahedral cells. In practice, those edges are mainly adjacent to three or five hexahedral cells.

\section{DUAL SHEETS CREATION}

The first stage of our approach consists in creating a set of dual sheets inside our domain $\Omega$. Our input is a tetrahedral mesh $\mathcal{T}$ that discretizes $\Omega$ and where frames are assigned at every node. The process we propose is the following one:

1. The user picks a tetrahedral cell $t \in \mathcal{T}$. Then we linearly interpolate in $t$ a frame $\mathbf{F}_{t}$ from the frames assigned to the corners of $t$. Note that we allow only regular tetrahedra to be picked, so that this interpolation is well-defined.

2. Then we select one of the 3 directions of $\mathbf{F}_{t}$. Let's call this direction $d$.
3. We try and extract a dual sheet starting from $t$ with normal direction $d$. This process is described below. It relies on two main concepts: sheet propagation (see Section 3.1) and control filters (see Section 3.2). It can fail if the extracted dual sheet encounters a tetrahedron of $S G(\mathcal{T})$ in an unacceptable configuration.

4. If the sheet extraction succeeds and satisfies the user expectations, the user repeats steps 1 to 3 until getting the desired set of dual sheets.

\subsection{Single sheet propagation}

We start by interactively selecting the input of our dual sheet extraction algorithm, which is summarized by Algorithm 1. Let $t_{0} \in \mathcal{T}$ be this first tetrahedron. Our extraction method consists in propagating a cutting surface named $\mathcal{S}_{c}$ in $\mathcal{T}$ using the frame field $\mathcal{F}$. In order to define $\mathcal{S}_{c}$, the user selects one of the $3 \mathrm{di}-$ rections of the frame $\mathbf{F}_{0}=\left(\mathbf{u}_{0}, \mathbf{v}_{0}, \mathbf{w}_{0}\right)$ defined in $t_{0}$. Let $\mathbf{d}_{0}$ be this direction vector. We call $\mathcal{E}_{t_{0}}$ the set of triplets $(e, \mathbf{n}, \beta)$, where $e$ is an edge of $t_{0}$ intersected by $\mathcal{S}_{c}, \mathbf{n}$ a locally normal vector of $\mathcal{S}_{c}$ at the point $p$ on $e$, with $p=(1-\beta) e_{0}+\beta e_{1}$, where $\left(e_{0}, e_{1}\right)$ are the end points of edge $e$ and $\beta \in[0,1]$. Tetrahedra adjacent to an edge of $\mathcal{E}_{\mathcal{W}_{i}}$ are the next tetrahedra to be cut. At each propagation step $i$ of the algorithm, they form the wave $\mathcal{W}_{i}$. They will be cut according to the information gathered in $\mathcal{E}_{t_{0}}$. And the process is repeated until reaching an iteration $j$ where $\mathcal{W}_{j}=\emptyset$.

Initialization of the sheet propagation. The first step of Algorithm 1 (line 1) consists in creating the initial cutting plane in $t_{0}$ (see Figure 7 ). To do this, we define the operation closest_component $\left(\mathbf{F}_{0}, \mathbf{v}\right)$ that finds the closest vector of $\mathbf{v}$ among the vectors $\left( \pm \mathbf{u}_{0}, \pm \mathbf{v}_{0}, \pm \mathbf{w}_{0}\right)$. We call $c_{0}$ the center of mass $t_{0}, \mathbf{F}_{c}$ the frame interpolated at $c$ from the frames defined at the nodes of $t_{0}$ and we note $\mathbf{n}_{0}=$ closest_component $\left(\mathbf{F}_{c}, \mathbf{d}_{0}\right)$. We then create the plane $\mathcal{P}_{0}$ going through $c$ and having $\mathbf{n}_{0}$ as a normal vector (see Figure $7(\mathrm{~b}))$. The set $\mathcal{E}_{t_{0}}$ is the set of triplets $(e, \mathbf{n}, \beta)$, where: $e$ is an edge of $t_{0}$ intersected by $\mathcal{P}_{0}$ at point $p_{0}$ (a red edge on Figure $7(\mathrm{~b})$ ); $\beta$ is the barycentric coordinate of $p_{0}$ along edge $e ; \mathbf{n}$ is equal to closest_component $\left(\mathbf{F}_{\beta}, \mathbf{n}_{0}\right)$, where $\mathbf{F}_{\beta}$ is the frame interpolated $^{3}$ at $p_{0}$ from the frames defined at the end points of $e$ (points $p_{i}$ are shown in blue on Fig. 7-b).

Propagation loop strategy. Then in order to create the sheet, we propagate the surface $\mathcal{S}_{c}$ in a breadth-first manner. We get all the

\footnotetext{
${ }^{3}$ Linear frame interpolation is done using a unit quaternion representation of frames, as done in [9].
} 

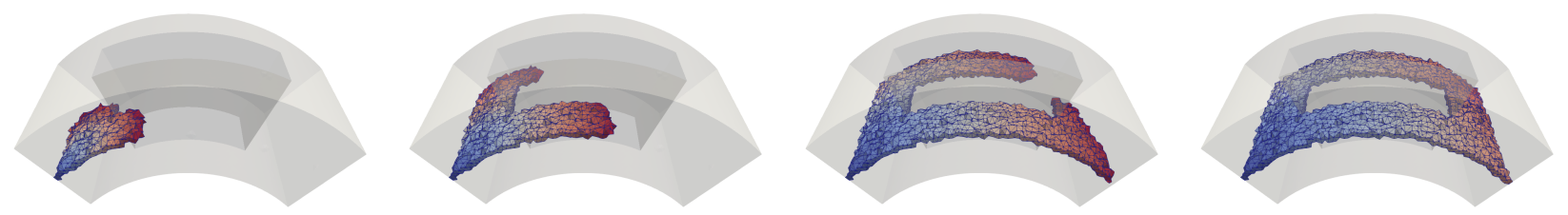

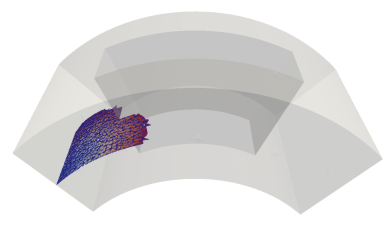

(a)

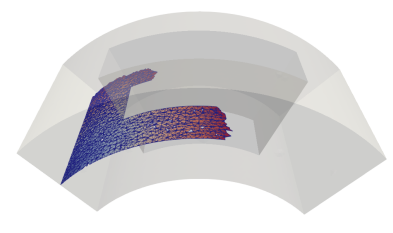

$(b)$

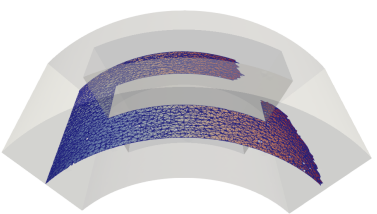

$(c)$

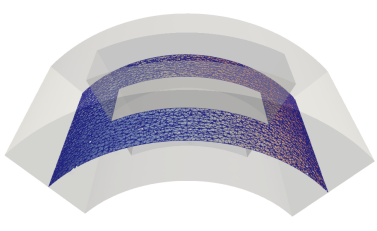

$(d)$

Figure 6: Illustration of the sheet creation into a regular field: In the top row, the set of intersected tetrahedra at different loop stages; in the bottom row, a surface-like representation of the advancing dual sheet. In $(a)$, we start from a single tetrahedron and then we advance in $(b)$ and $(c)$ into the domain following the frame field until reaching the boundary domain in all the directions in $(d)$. Color of an element (piece of surface, tetrahedron) is correlated to the wave number.

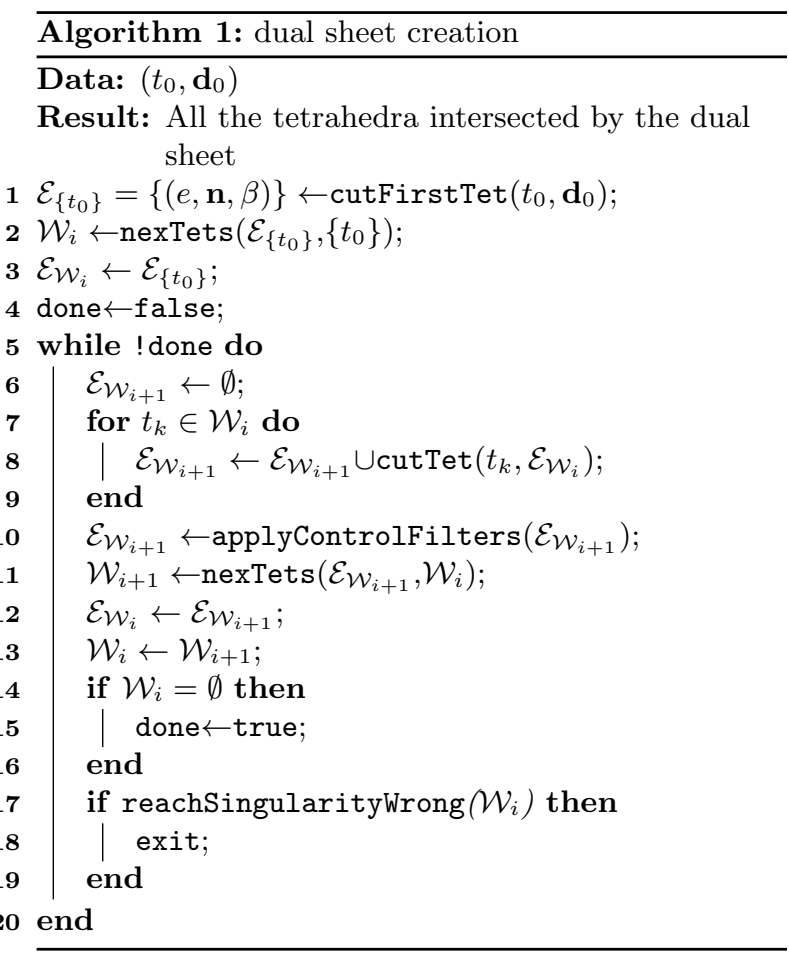

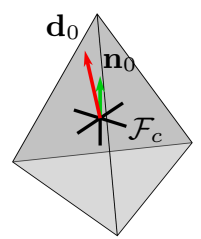

(a)

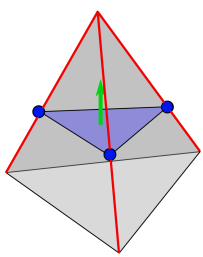

(b)

Figure 7: Edge cutting for the first tetrahedron $t_{0}$ in Algorithm 1. $\mathbf{F}_{c}$ is shown at the center of $t_{0}, \mathbf{d}_{0}$ is colored in red and $\mathbf{n}_{0}$, the closest vector of $\mathbf{d}_{0}$, is colored in green.

tetrahedra adjacent to an edge of $\mathcal{E}_{0}$ and not already traversed. These tetrahedra are the next wave to perform (denoted $\mathcal{W}_{i}$ at line 2). And we note

$\mathcal{E}_{\mathcal{W}_{0}}$ all the edges that were used to generated this wave (line 3). In the example on the right, the first tetrahedron $t_{0}$ is shown in dark transparency with its cutting plane, and the tetrahedra of $\mathcal{W}_{0}$ are shown in light grey. We then enter a loop process where surface $\mathcal{S}_{c}$ is propagated by successive waves (from line 5 to 19 in Algorithm 1). First we define $\mathcal{E}_{\mathcal{W}_{i+1}}$ the output intersection triplet of $\mathcal{S}_{c}$ in the tetrahedra of $\mathcal{W}_{i}$. By analogy, $\mathcal{E}_{\mathcal{W}_{i}}$ gathers all the input intersection points for the wave 
$\mathcal{W}_{i}$. Then we cut each tetrahedron $t_{k} \in \mathcal{W}_{i}$. The cutting method is different from the one used for $t_{0}$. While a local plane was created at the center of $t_{0}$, now each tetrahedron $t_{k}$ has already been cut along edges of $\mathcal{E}_{\mathcal{W}_{i}}$. At least one edge of $t_{k}$ is so already intersected by $\mathcal{S}_{c}$. The propagation scheme we use is based on face propagation. It is applied in function cutTet used at line 8 of Algorithm 1 and is detailed in Algorithm 2.

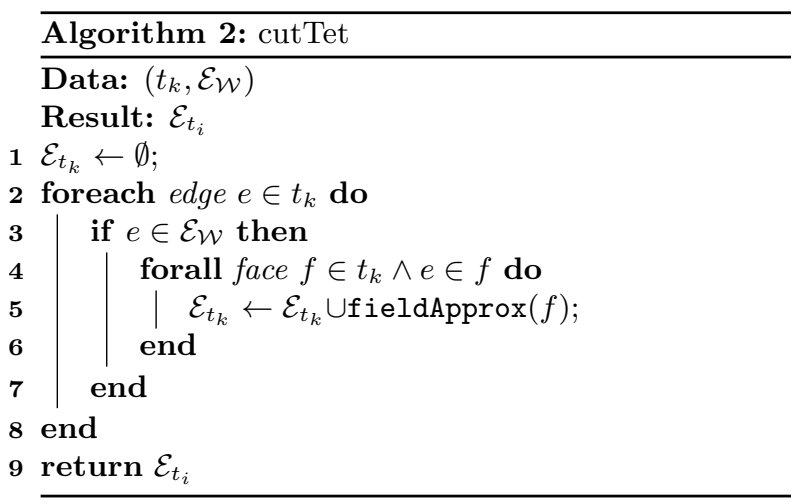

Propagation is done along each face of the current tetrahedron $t_{k}$ starting from edges that belong to $\mathcal{E}_{\mathcal{W}_{i}}$ (see Figure 8). Note that a face $f$ of a tetrahedron $t_{k}$ could have been already treated for the tetrahedron sharing $f$ with $t_{k}$, whether this tetrahedron belongs to the current wave or to a previous one. We start the cutting algorithm in $t_{k}$ with at least one intersected edge $e$ knowing that $e \in \mathcal{E}_{\mathcal{W}_{i}}$ with $(e, \mathbf{n}, \beta)$. For each face $f \in t_{k}$ that is incident to $e$ and not already treated, we compute an approximation of the cut in the frame field by using a $4^{\text {th }}$-order RungeKutta method (line 5 of Algorithm 2). It gives us an output intersection point in $f$. Let us note that if two edges of a face $f$ are cut while $f$ has not yet been traversed, we add $f$ to the set of treated faces in order to avoid inconsistencies ${ }^{4}$.

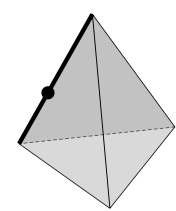

(a)

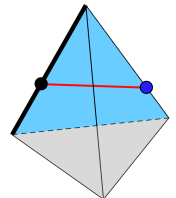

(b)

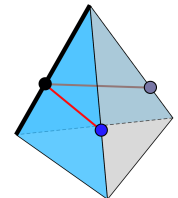

(c)
Figure 8: Simple illustration of a cut propagation into a single tetrahedron. In $(a)$, the initial state; in $(b)$ the first face cutting; and in $(c)$ the second face cutting.

As we cut geometrically through faces, it may hap-

\footnotetext{
${ }^{4} \mathrm{~A}$ third intersection must not be added, it would introduces unexpected configurations that could be difficult to handle.
}

pen that the output intersection point along an edge is located on a node $(\beta=0$ or 1$)$ of $\mathcal{T}$. In practice, we avoid such an intersection by randomly moving the intersected nodes. In our implementation, we store all the nodes that are intersected during a wave $\mathcal{W}_{i}$ in a set $\mathcal{N}_{i}$ and we move all of them simultaneously with small random perturbations. After that, we recompute the cut propagation in the tetrahedra of $\mathcal{W}_{i}$ that are adjacent to a node of $\mathcal{N}_{i}$. This process is repeated until having $\mathcal{N}_{i}=\emptyset$. In our different experiments, this set was always empty after a few number of iterations. Note that in our implementation, a node is considered intersected if the intersection point is located at a distance less or equal to $l=\alpha$.length $\left(e_{\min }\right)$ where $e_{\text {min }}$ is the shortest edge of $\mathcal{T}$ and $\alpha=0.1$. Figure 6 illustrates different stages of the dual sheet propagation for a regular grid-like frame field.

Singularity line traversal. During the wave propagation, we can reach some singular tetrahedral cells (line 17 of Algorithm 1). If the tetrahedral cell contains a singularity point, we stop the wave propagation. Otherwise, it means that the tetrahedron is traversed by a

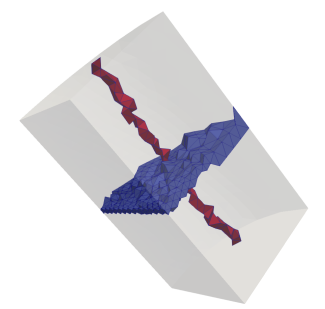

singularity line. In this case, we compare the normal vector to the dual sheet with the singularity line direction: if they are almost aligned (dot product evaluation), then the dual sheet can traverse the singularity line, else we stop the wave propagation. In our implementation, the normal to the dual sheet and the singularity line direction are considered aligned when their angle is less than $\pi / 4$.

\subsection{Control filters}

Our dual sheet creation process consists in creating successive waves. Each time we go through a tetrahedron, numerical approximations are done and small geometrical errors may appear. While they have a very little impact locally, their accumulation can lead to unexpected results. In particular the dual sheet can break and split (see Figure 9). In order to prevent such a situation to occur, we apply some filters to remove some data from $\mathcal{E}_{\mathcal{W}_{i}}$ at the end of each wave (line 10 of Algorithm 1).

We have two filters in our algorithm to control the wave propagation. The first one is based on the topology of $\mathcal{T}$; the second one considers the dual sheet geometry. During the dual sheet creation, we build successive waves. Each of these has a number, and all 

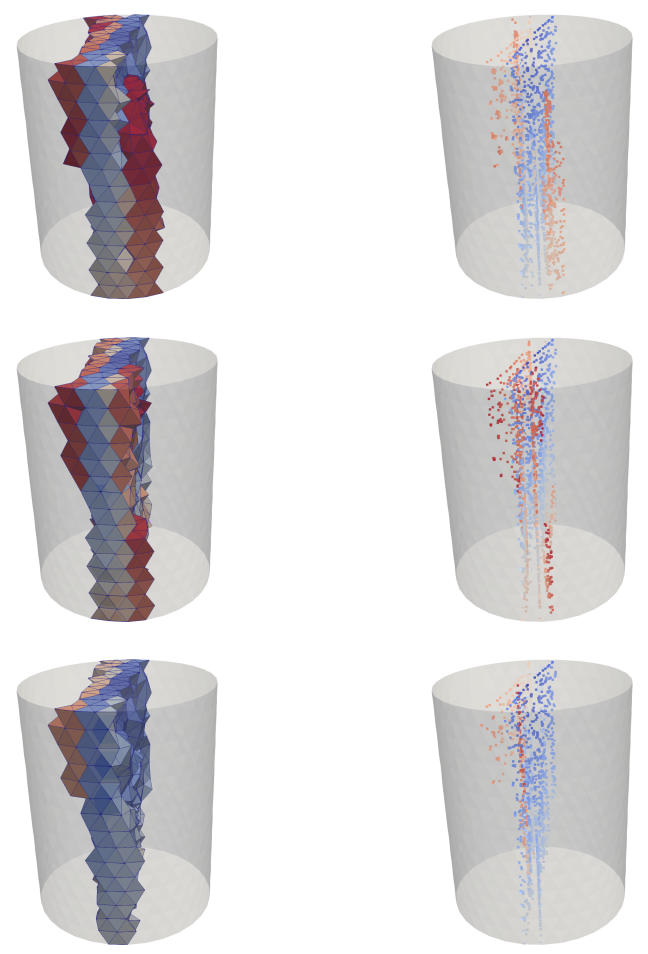

Figure 9: Impact of the geometric filter on the dual sheet propagation with the tetrahedra of the dual sheet on the left column and the created intersection points on the right. First row shows the result without applying the filter. Second and third rows respectively corresponds to $\lambda_{g}=0$ and $\lambda_{g}=\frac{\sqrt{2}}{2}$.

the tetrahedral cells that belong to a wave are labeled with this wave number. When we create the set $\mathcal{W}_{i+1}$, the topological filter removes from $\mathcal{W}_{i+1}$ all the tetrahedra that would share an edge, a face or a node with a tetrahedron belonging to $\mathcal{W}_{j}$, with $j \in\left[0, i+1-\lambda_{t}\right]$ and $\lambda \in \mathbb{N}^{*}$. In our implementation, we fixed $\lambda_{t}=2$.

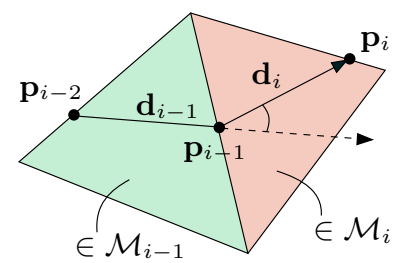

Figure 10: Geometric filtering based on predecessors.

The second filter controls the geometric propagation of each point in $\mathcal{E}_{\mathcal{W}_{i+1}}$. Let $\mathbf{p}_{i}$ be such a point (see Figure 10). We store for this point the two previous points used to generate it: the point $\mathbf{p}_{i-1}$ that belongs to the same tetrahedron of $\mathcal{M}_{i}$ as $\mathbf{p}_{i}$ and the point $\mathbf{p}_{i-2}$ that belongs to a tetrahedron of the previous wave $\mathcal{M}_{i-1}$. We then check the deviation between the previous direction $\mathbf{d}_{i-1}$ and the current direction $\mathbf{d}_{i}$. The edge $e$ is kept in $\mathcal{E}_{\mathcal{W}_{i+1}}$ if $\frac{\mathbf{d}_{i-1}}{\left\|\mathbf{d}_{i-1}\right\|} \cdot \frac{\mathbf{d}_{i}}{\left\|\mathbf{d}_{i}\right\|}>\lambda_{g}$, with $\lambda_{g} \in[0,1]$. Having $\lambda_{g}>0$ prevents the sheet from turning 90 degrees and eventually geometrically turning back in the domain. We can notice that this filter does not take the frame field $\mathcal{F}$ into account. This control and the topological control are mandatory near singularity lines where high curvature in the frame field induces much more difficulties to preserve the dual surface topology.

\section{DUAL BLOCKING PROPERTIES}

Applying the algorithm described in Section 3 allows us to partition the domain into blocks. But not all partition can produce a hexahedral block structure. This is why we have to check a minimal set of properties about the obtained block structure.

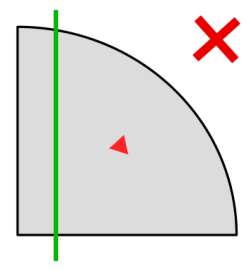

(a)

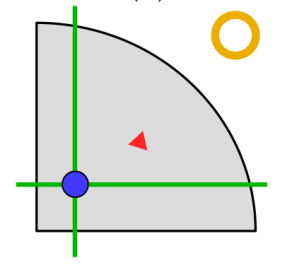

(b)

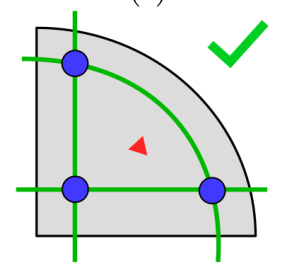

(d)

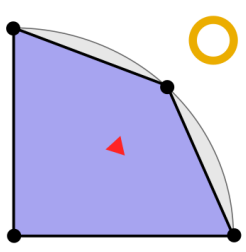

(c)

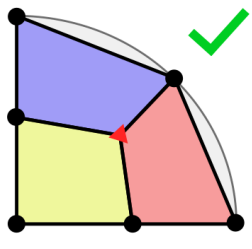

(e)
Figure 11: Successive dual curve insertions to build a quad block structure.

\subsection{Dual structure properties}

In order to explain our approach, let us consider the $2 \mathrm{D}$ example of Figure 11. In 2D, dual sheets are dual curves and the dual of a quad block structure can be seen as a simple arrangement of curves. In $(a)$, we consider that the user has only created one single dual curve. Obviously, this configuration does not correspond to a valid dual structure since at least one dual 
node, i.e. one primal face, must exist. In (b), a second curve is so added and the corresponding primal block structure is given in $(c)$. It is reduced to one quadrilateral block, which is a valid block structure. But it does not fit the frame field prescription. By construction, the input frame field has a singular triangle in the domain (shown in red on Fig. 11) and the usually expected block structure is the one given in $(e)$. In order to get this primal structure, one dual curve is missing: this line is added in $(d)$ in such a way that the singular red triangle is enclosed by dual curves into an inner-surface dual zone. In other words, a boundary dual zone should not contain a frame singularity.

In a similar way, a set of validity rules can be defined in 3D. In order to define this set of rules, let us first introduce the notion of classification. Let $\mathcal{T}$ be a tetrahedral mesh of a domain $\Omega$, where the boundary $\partial \Omega$ is made of a set $\partial \Omega_{2}$ of surfaces, a set $\partial \Omega_{1}$ of curves and a set $\partial \Omega_{0}$ of vertices. For the sake of simplicity,

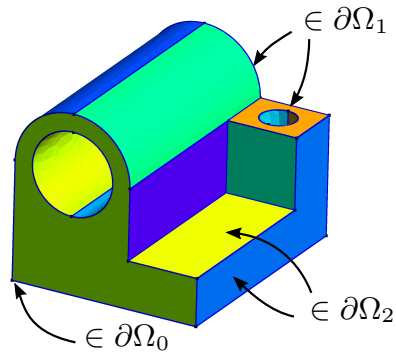
we consider that $\Omega$ is a single volume and not an assembly of volumes. Considering such a decomposition of $\Omega$ and a set of dual sheets that split $\Omega$ into dual zones $\mathcal{Z}_{\Omega}$, the following criteria must be checked:

1. At least one block exists, i.e. at least three dual sheets intersect in one single dual node;

2. A boundary dual zone can not contain more than one geometric point of $\partial \Omega_{0}$ or one boundary frame singularity point;

3. A boundary dual zone can not contain one boundary frame singularity point and being along a curve.

4. An in-volume dual zone can not contain more than one frame singularity point;

5. An in-volume dual zone that does not contain a frame singularity point can be traversed by one single frame singularity line at most.

First criterion is obvious and avoid peculiar cases where $\partial \Omega_{0}$ is empty (for totally smooth boundary). It is fulfilled by the example shown in Figure 12, where dual zones A, B, C and D are created. As each dual zone corresponds to a primal block node, we meet an issue with dual zone $\mathrm{C}$ that contains geometric vertices 1,2 and 3. We must split this dual zone so has to have each vertex in a different zone. It is the purpose of criterion 2 (see Figure 13). Criterion 3 prevents the situation depicted on Figure 11- $b$ to arise: considering the dual zone containing the red triangle (and so a boundary singularity point), this zone must not be bounded by a geometric curve. Criteria 4 and 5 are relative to in-volume dual zones. In our approach, a dual zone corresponds to a primal node, while a singularity point also corresponds to an expected primal node if you build the blocks from singularity points and lines [8]. If a dual zone would contain two singularity point, we would get an inconsistency. It is the purpose of criterion 4 . Criterion 5 is similar. A singularity line should correspond to an edge of the primal block structure. If a dual block $B$ contains two singularity lines $l_{1}$ and $l_{2}$ but no singularity point, it means that $l_{1}$ and $l_{2}$ do not intersect and it induces that the primal node relative to $B$ should be on two non-intersecting edges. That is not possible and it is the purpose of criterion 5 .
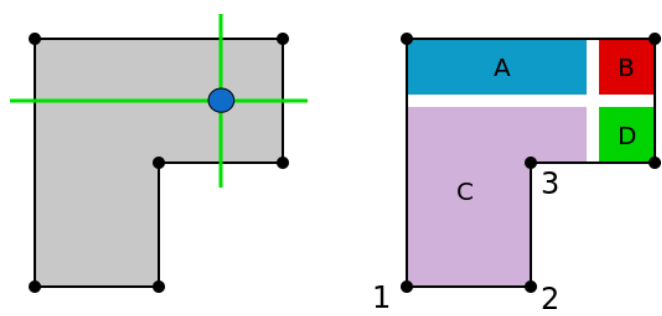

Figure 12: A 2D domain split in four boundary zones by inserting two dual curves. It does not allow to generate a valid primal quad structure.
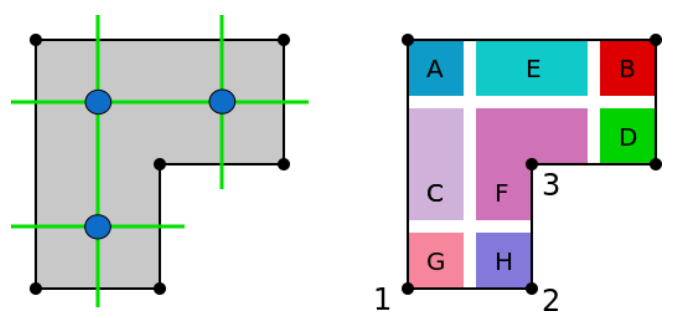

Figure 13: A 2D domain split in eight boundary zones that corresponds to a valid primal quad structure.
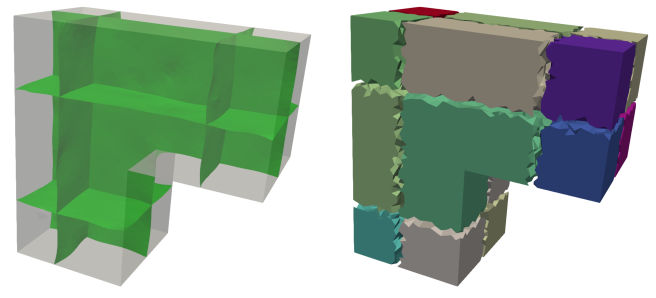

Figure 14: Equivalent 3D domain of the 2D examples shown in Figure 13. 


\subsection{Validity blocking algorithm}

Starting from the dual sheet extraction processed beforehand on tetrahedral mesh $\mathcal{T}$ we want to check the validity of the block decomposition. For each tetrahedron $t \in \mathcal{T}_{3}$, we know if it is traversed by $0,1,2$ or 3 dual sheets. Let $f_{s h}: \mathcal{T}_{3} \mapsto[0 ; 3]$ be the function giving this information. The algorithm we apply is the one provided in Algorithm 3. It gives as a result whether the induced block decomposition is valid or not and two functions $f_{b l}$ and $f_{c}$. Function $f_{b l}: \mathcal{T}_{3} \mapsto \mathbb{N}$ indicates for each 3-cell of $\mathcal{T}$ the block number it belongs to (value 0 means no block). Function $f_{c}: \mathbb{I N} \mapsto \mathbf{I N} \times \mathbf{I N}^{*}$ gives for each block $b_{i}$ the dimension and the id of the lowest geometric entity a node of $b_{i}$ is classified on.

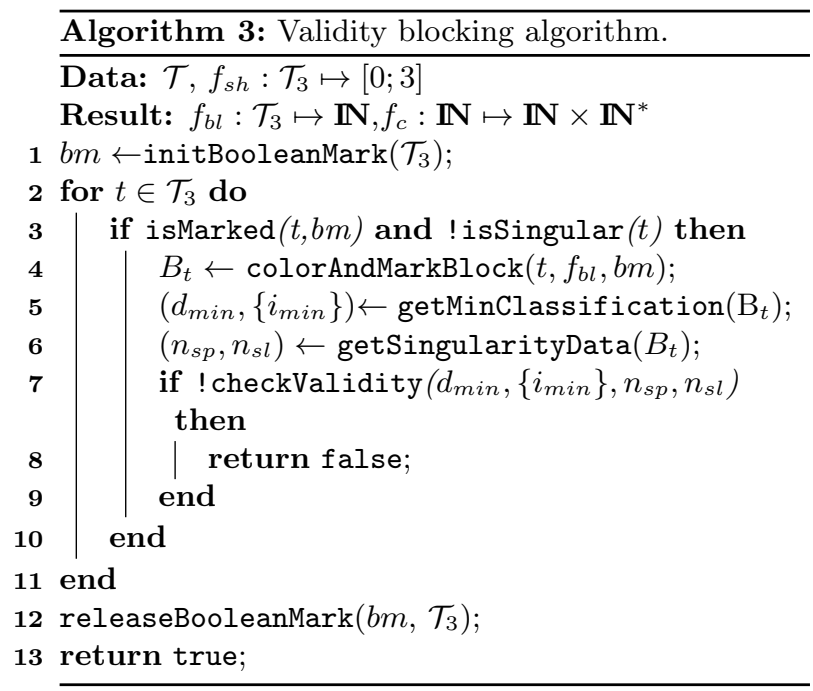

Algorithm 3 relies on a traversal of all the tetrahedra of $\mathcal{T}_{3}$. Let $t$ be a non-traversed and non singular tetrahedron, we color all the tetrahedra that belong to the same block as $t$ (line 4). This set of tetrahedra, denoted $B_{t}$, is built by a breadth-first traversal starting from $t$ and using edge connectivity. During the traversal, a tetrahedron $t^{\prime}$ is added to $B_{t}$ if $f_{s h}\left(t^{\prime}\right)=0$. Then we check the minimal classification $\left(d_{\min },\left\{i_{\min }\right\}\right)$ of the nodes of tetrahedra in $B_{t}$ (line 5$)$. Value $d_{\text {min }}$ gives the minimal dimension ( 0 for vertex, 1 for curve for instance); the set $\left\{i_{\text {min }}\right\}$ contains the id of the $d_{m i n}$-dimensional geometric entity. For instance, getting $(0,\{1,4,2\})$ would mean that some nodes of $B_{t}$ are classified on geometric vertices 1, 4 and 2. At line 6 , we get the number of singularity points $n_{s p}$ and the number of singularity lines $n_{s l}$ that contain tetrahedra of $B_{t}$. With data gathered at line 5 and 6 , we can check the validity of the rules previously given. For instance: $n_{s p}$ must be less or equal to 1 ; and if $d_{\min }=0$, $\left|\left\{i_{m i n}\right\}\right|$ must be equal to 1 .

\section{BLOCK EXTRACTION}

Once dual zones are validated and classified through function $f_{c}$, we have to create the primal nodes and the primal hexahedral blocks.

\subsection{Primal node creation}

Every tetrahedron $t \in \mathcal{T}_{3}$ is in a dual zone $\left(f_{b l}(t) \neq 0\right)$ or in a dual sheet $\left(f_{b l}(t)=0\right)$. By construction, a dual zone is discretized as a set of tetrahedra bounded by either dual sheets or domain boundary. From a topological point of view, a dual zone corresponds to a primal node in the block structure. In order to create this node, we use the classification data stored in $f_{c}$. Let's consider the dual zone $i$, and the corresponding primal node $n_{i}$, then $f_{c}(i)$ returns the dimension and id of the geometric entity to classify $n_{i}$. If the dimension is equal to $0, n_{i}$ is located on the corresponding geometric point. Otherwise, we initialize $n_{i}$ location at the center of mass of dual zone $i$ and if the dimension is equal to 1 , respectively 2 , we project $n_{i}$ onto the corresponding geometric curve, respectively the corresponding geometric surface.

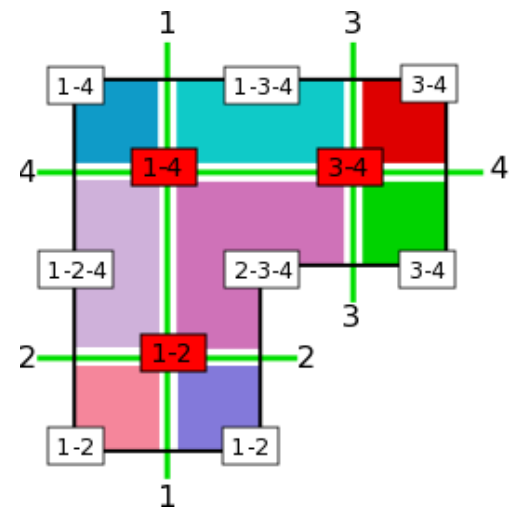

Figure 15: Dual zone numbering for building primal blocks. The label of a dual node $n$ is a couple of 2 dual sheet numbers (in red), which are the numbers of the dual curves that intersect each other at $n$. Dual zones are labeled by a $p$-uple of numbers, where each number corresponds to a bounding dual curve.

\subsection{Dual zone labeling}

We build primal blocks from the dual structure directly. More precisely, we identify each dual zone by a label that is a series of dual sheets numbers and we compare this series with the numbers of the dual sheets that intersect in a dual node. Let us consider the 2D case illustrated on Figure 15. Each dual node being the intersection of two dual curves in $2 \mathrm{D}$, we label it 
with two numbers ${ }^{5}$. For instance, the dual node intersected by dual sheet 1 and 2 has label $1-2$ (the dual node on the bottom left on Figure 15). Dual zones are labeled with the numbers of the dual sheets that surround them. As a consequence the label size of a dual zone is not fixed and this label is not necessary unique. For instance, the symmetry of the domain exhibited on Figure 16 induces that dual curve 1 intersects dual curve 2 twice. Two dual nodes have so the label $1-2$ and all the zone labels are carried by two dual zones. This labeling strategy extends to 3D where dual nodes are labeled with a triplet of dual sheets numbers ${ }^{6}$.

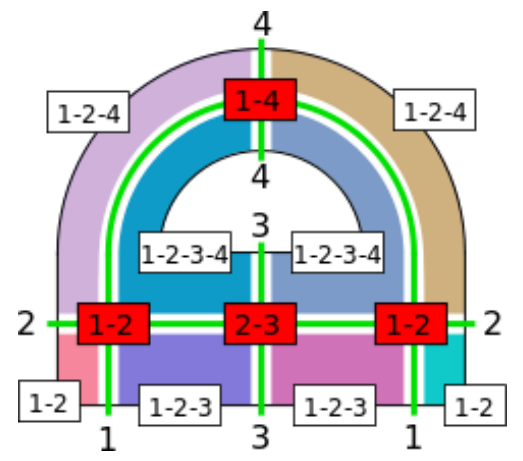

Figure 16: As two dual lines can intersect each other more that once, dual node and dual zone labeling is not unique.

Our method is quite similar to [5], where they label the dual zones, in a global way, according to their relative locations to all dual sheets, that are represented by implicit surfaces. They declare a dual zone as being on one side of a dual sheet (flag + ) or on the other side (flag -). If you have $n$ dual sheets, each zone is then labeled by a $n$-size vector of flags $(+,-)$. This strategy is not adapted to our algorithm since it does not allow us to handle self-intersecting dual sheets and it doesn't fit our inserting dual sheets for volumes that are not homeomorphic to a sphere. In the example of Figure 16, dual lines 3 and 4 should be merged into a single line to apply the strategy given [5] and get the right labeling for us. With our local strategy where we label each dual zone with the labels of the dual sheets it is enclosed in, we do not get such an issue.

\subsection{Primal block creation}

The label assignment previously done partially carries the topology of the dual structure and so of the primal hexahedral blocks. In order to create primal block, we adopt a two-stages procedure where: (1) First, we

\footnotetext{
${ }^{5}$ Note that it can be twice the same number when a dual curve intersects itself.

${ }^{6}$ The same number can occur twice in case of selfintersecting dual sheets.
}

build hex corners for every node $a$, which are 3-tuples of primal nodes $\mathcal{C}_{a}=\left(c_{1}, c_{2}, c_{3}\right)$ corresponding to a future block corner. In other terms, it means that $\left[a, c_{1}\right],\left[a, c_{2}\right]$ and $\left[a, c_{3}\right]$ will be three edges of a primal hexahedron; (2) Then, we build hexahedral blocks by combinatorial operations on hex corners.

Creation of hex corners. Each 3D dual node corresponds to a primal block. It is surrounded by 8 dual zones, each of them corresponding to a primal node that has been created at Section 5.1. We label each $3 \mathrm{D}$ dual node $n$, respectively a $2 \mathrm{D}$ one, with 3 numbers, resp. 2. These numbers correspond to the dual sheets, resp. line, that intersect at node $n$. Let $l_{n}$ be the label of $n$. In order to find out the dual zones surrounding $n$, we traverse all the dual zone labels and we keep those having $l_{n}$ as a sub-series of their own label. Let $Z_{n}$ be the set of dual zones surrounding the dual node $n$. If the dual node labeling is unique, i.e every dual node has a different label, then we have the standard configuration where $\left|Z_{n}\right|=8$ in $3 \mathrm{D}$, resp. 4 in $2 \mathrm{D}$, for all dual nodes.

But the dual node labeling can be not unique as soon as two dual sheets in $3 \mathrm{D}$, resp. dual lines in $2 \mathrm{D}$, intersect each other twice or more. It is the case on Figure 16 where two nodes have the label $1-2$ because lines 1 and 2 intersect twice. Both of those nodes gather all the dual zones of this simple model into their respective sets $Z_{n}$. And we get $\left|Z_{n}\right|>8$ in $3 \mathrm{D}$, respectively $\left|Z_{n}\right|>4$ in $2 \mathrm{D}$. In order to reduce $Z_{n}$ size to 8 in $3 \mathrm{D}$, resp. 4 in $2 \mathrm{D}$, we compute a distance between each zone of $Z_{n}$ and $n$ and we keep the 8 in 3D, resp. 4 in $2 \mathrm{D}$, closest ones. This distance is computed as follows. The dual node $n$ is located into a simplex $s_{n}$ of $\mathcal{T}$. We pick the center of mass of $s_{n}$ as an approximated location for $n$. Let $p_{n}$ be this location. Each dual zone being a set of simplices ${ }^{7} S$ in $\mathcal{T}$, we compute the Euclidean distance between $p_{n}$ and each center of mass of an element of $S$ and we keep the smallest distance.
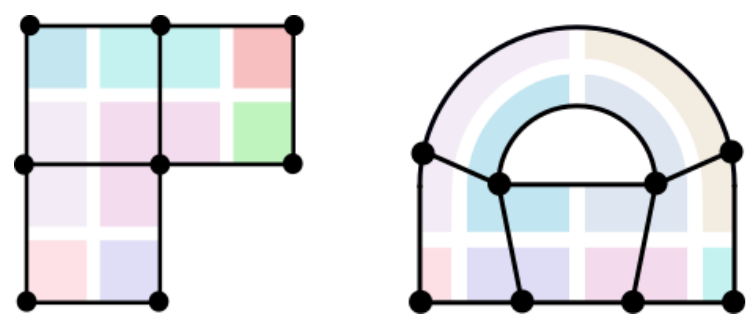

Figure 17: Final blocking for the models given in Figures 15 and 16.

At this stage, every primal node has a final geometric location and every primal block has the list of the pri-

\footnotetext{
${ }^{7}$ triangles in $2 \mathrm{D}$ and tetrahedra in $3 \mathrm{D}$.
} 


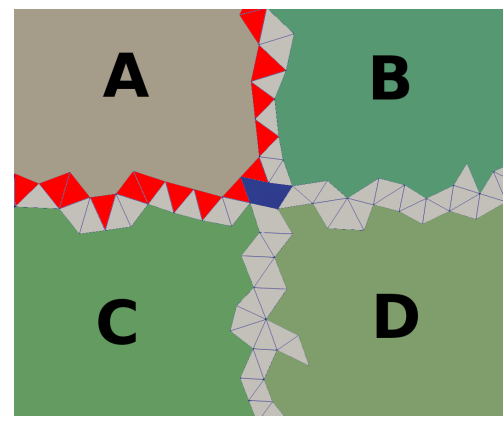

Figure 18: 2D example of dual sheet traversal in order to build corner data. Here, blue triangles belongs to 2 dual lines, while red ones are in a single dual line and in contact with dual zone $A$. Those red triangles allow our algorithm to connect dual zone $A$ to dual zones $B$ and $C$ to form a quad block corner

mal nodes it is built from. It remains to build those blocks in a non-inverted manner, as shown in Figure 19. Let $\mathcal{N}=\{A, B, C, D, E, F, G, H\}$ be the set of nodes of a block. For each primal node $n \in \mathcal{N}$ we have to create the hex corner $\mathcal{C}_{n}=\left(c_{1}, c_{2}, c_{3}\right) \in \mathcal{N}^{3}$, $c 1, c 2$ and $c 3$ are the other end points of the three edges starting from $n$ in the block (see Figure 19-b). By definition, a node of $\mathcal{N}$ corresponds to a dual zone, which is a subset $S$ of $\mathcal{T}$. Starting from a tetrahedron of $S$ we propagate through the tetrahedra of the dual sheets surrounding $S$ in order to touch tetrahedra of other dual zones. More specifically, we only propagate into tetrahedra that belong to a single dual sheet. Let us consider Figure 18 for a 2D example where we start from dual zone $A$. All the red triangles belong to a single dual sheet that surrounds $A$, while blue ones belong to two dual sheets. Red triangles, the ones we propagate through, are only in contact with dual zones $B$ and $C$. On the contrary, blue triangles stop the propagation and so prevent us to reach the dual zone $D$. In $2 \mathrm{D}$, it allows us to build the hex corner $\mathcal{C}_{A}=(B, C)$. We follow the same principle in $3 \mathrm{D}$.

Creation of primal blocks. Now that hex corner data is built, we build faces of blocks. On Figure 19-c, We start from node $A$ with hex corner $\mathcal{C}_{A}=(C, D, E)$. Considering the node $A$, two of the neighbor nodes are selected in $\mathcal{C}_{A}$, which are $C$ and $E$ here (see Figure 19$c)$. We can then obtain the first face by getting the node of $\mathcal{C}_{C} \cup \mathcal{C}_{E}-A$, which is $F$ (see Figure 19-d). The first face is so $A E F C$. We build two other faces adjacent to $A$ by selecting $D$ and $E$ in $\mathcal{C}_{A}$, then $C$ and $D$. Those faces are respectively $A E H D$ and $A C G D$ (see Figure 19-e). The final step is to create the last faces with the remaining node $B$. Such a process applied in 2D gives blocks of Figure 17 for the examples of Figures 15 and 16.

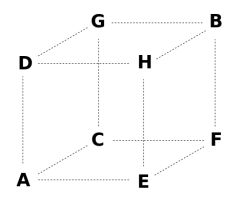

(a)

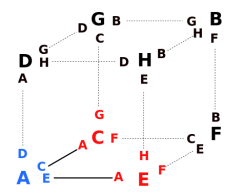

(c)

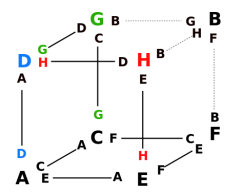

(e)

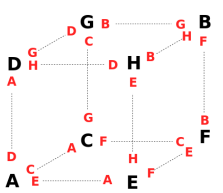

(b)

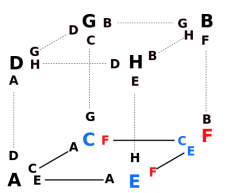

(d)

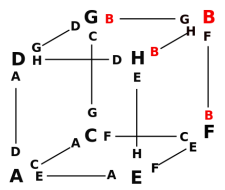

$(f)$
Figure 19: Creation of a primal block from its 8 primal nodes, where corner data is built first.

\section{EXPERIMENTAL RESULTS}

We applied our approach on several CAD models. They were imported into the gmsh software [25] using the step file format and gmsh was used to generate initial tetrahedral meshes. Discrete frame fields were then computed on each tetrahedral mesh by a method similar to the one described in [10]. Each tetrahedral mesh $\mathcal{T}$ enriched by a frame field defined at the nodes of $\mathcal{T}$ is then taken as the input of our software. The parameter $\lambda_{g}$ used in the dual sheet creation process is fixed to 0.2 for all the results shown in this section. Some results we obtained are shown on Figures 20,23, 27 and 28. For every model, we show the tetrahedral representation of dual sheets and dual zones and the final primal hexahedral blocks. Our pipeline of operations - dual sheet creation, block property checking, primal block generation - gives the expected results for those models when the input mesh is refined enough and the frame field is valid. Some statistics about the generation of all the presented results are given in Table 21.

\subsection{Impact of the mesh resolution}

Two steps are impacted by the mesh resolution: the dual sheet creation and the block extraction. Building a single dual sheet requires to propagate a dual surface into the tetrahedral mesh starting from a single point and direction. This propagation being done numerically by linearly integrating the field along each 

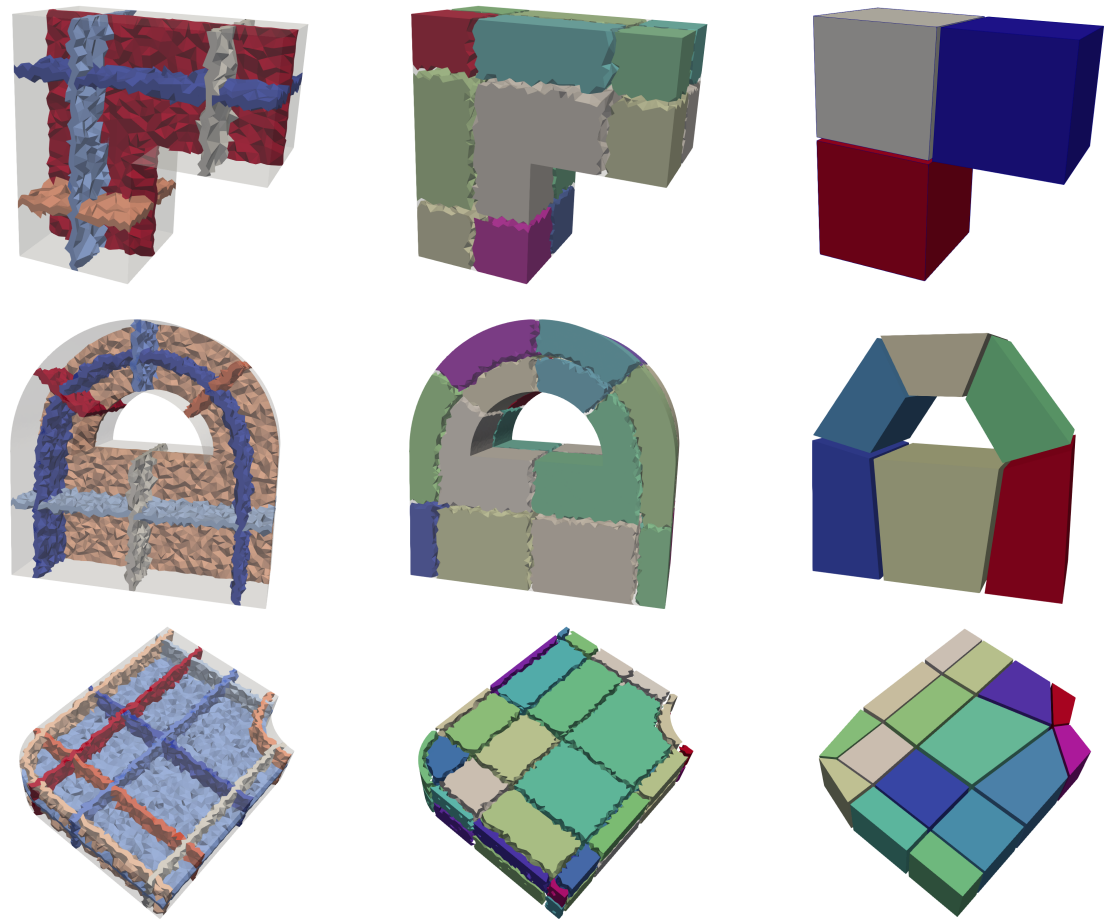

Figure 20: Dual structure (left), dual zones (middle) and final hexahedral blocks (right) for several models.

\begin{tabular}{|c|c|c|c|c|c|c|c|c|c|}
\hline Model & $|\mathcal{T}|$ & $\mid$ Sheets $\mid$ & $\mid$ Dual Zones $\mid$ & $|\mathcal{B}|$ & avg & min & max & validity & blocks \\
\hline B7 & 82879 & 4 & 15 & 4 & 0.3325 & 0.3 & 0.41 & 0.21 & 0.88 \\
B8 & 74112 & 7 & 33 & 10 & 0.2816 & 0.24 & 0.31 & 0.21 & 1.58 \\
B10 & 355048 & 10 & 40 & 12 & 1.8533 & 1.41 & 2.31 & 1 & 3.81 \\
B28 & 362531 & 24 & 136 & 49 & 1.3209 & 0.45 & 7 & 0.95 & 6.63 \\
B31 & 349598 & 15 & 96 & 37 & 1.7331 & 0.53 & 3.79 & 1.19 & 7.52 \\
B40 & 57984 & 5 & 16 & 3 & 0.2280 & 0.12 & 0.38 & 0.15 & 0.38 \\
B45 & 164904 & 7 & 24 & 6 & 0.6014 & 0.32 & 1.28 & 0.47 & 1.53 \\
S3 & 932672 & 19 & 184 & 97 & 7.3423 & 3.1 & 21.03 & 2.32 & 19.78 \\
S7 & 134494 & 13 & 96 & 51 & 0.5727 & 0.28 & 1.56 & 0.41 & 3.91 \\
S34 & 122399 & 10 & 51 & 17 & 0.6144 & 0.11 & 1.46 & 0.31 & 1.73 \\
S35 & 99829 & 9 & 50 & 16 & 0.3777 & 0.14 & 0.9 & 0.27 & 1.41 \\
S37 & 595953 & 6 & 39 & 14 & 4.5050 & 1.78 & 7.28 & 2.11 & 10.64 \\
S38 & 200245 & 18 & 112 & 44 & 0.70222 & 0.16 & 3.89 & 0.55 & 3.78 \\
S40 & 414542 & 23 & 164 & 74 & 2.1820 & 0.48 & 9.01 & 1.24 & 7.26 \\
\hline
\end{tabular}

Figure 21: Statistical results for our method. $|\mathcal{T}|$ is the number of tetrahedron in the mesh, $\mid$ Sheet $\mid$ the number of dual sheets created, $\mid$ Dual Zones $\mid$ the number of dual zone generated and $|\mathcal{B}|$ the number of blocks extracted. avg,min and max are respectively the average, minimal and maximal time for one sheet creation. validity is the time of our dual validation algorithm and blocks the time for the blocks extraction algorithm. All times are given in seconds. Experiments were made on a standard desktop computer. 
traversed tetrahedron, their size has an obvious impact on the result. For instance on Figure 22, the "same" dual sheet is created with two mesh resolutions. In $(a)$ with the coarser resolution the sheet creation process stops when it hits a singularity line. In $(b)$ with a refined resolution, the gap between singularity lines and the boundary is larger letting the dual sheet pass.

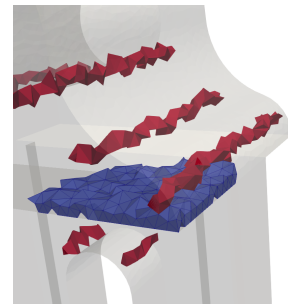

(a)

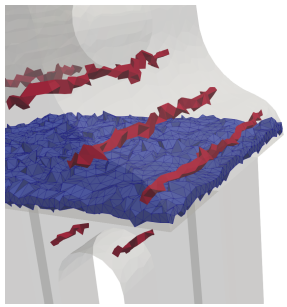

(b)
Figure 22: Extraction of a "same" dual sheet in a tetrahedral mesh of a geometric domain with two resolution levels: coarser in $(a)$ than in $(b)$.

Figure 23 illustrates another impact of the mesh resolution on our algorithm. Contrary to the examples of Figure 20, in both models of Figure 23, we have introduced boundary dual sheets by following both the frame field and the domain boundary. It is the case of the red dual sheets in the left column. A boundary dual sheet is created when we pick a tetrahedron along the domain boundary and we select as a sheet direction the normal to the surface. In this case, the dual sheet extraction algorithm presented in Section 3.1 may fail without adding this control. Indeed if the mesh resolution is too low, it may end up by hitting a singularity line that is too close to the boundary.

As our approach consists in directly creating 3D dual sheets, we can obtain 3D dual sheets bounded by several loops that have different topologies. Two examples are given on Figure 25. For both of them, we don't know how the 3D dual sheets would be recovered from the exhibited loops as done in $[5,6]$.

\subsection{Wrong frame field configurations}

The frame field is an input of our algorithm. As a consequence, if the frame field does not fit the requirement of hexahedral blocking, our algorithm fails to generate a block structure. We currently distinguish two main issues that limit the application of our algorithm ${ }^{8}$ : 3-5 singularity lines and "ski-ramp" configurations. The 3-5 singularity lines are known characteristics of frame fields that do not correspond to hexahedral block structure [26, 27]. Such a line

${ }^{8}$ This limitation is shared with other interactive approaches $[5,6]$ and automatic approaches that generate block structures. connects a 3 -valent boundary singular point $A$ to a 5 valent singular point $B$. It means that this line should correspond to a series of edges of the primal blocks such as they would be adjacent to 3 hexahedra at $A$ and to 5 hexahedra at $C$. Such a transition is not possible in a full-hex mesh without adding extra singularities. If you restrict the incident edges of a node in a hexahedral mesh to be 3,4 or 5 -valent, authors of [27] enumerate 11 topologically different interior node types. Among those configurations, there is no configuration with only one 3 -valent edge and one 5 -valent edge (others being regular 4 -valent).

As a consequence, our approach only allow the user to draw dual sheets far away from such a line (see Figure 24-left column), leading to an incomplete block solution. Looking at the dual sheet structure (on middleleft), we could expect to have more blocks generated (on the bottom-left). If we relax some validity rules, we can obtain a valid block structure that snaps the singularity line along the boundary surface.

The "ski-ramp" configuration corresponds to a part of geometrical models that where a surface is pinched forming a very narrow angle. In such a situation, the frame field is totally regular, i.e. has no singularity line. As a consequence and all the dual lines we could build coming from the left side of the ski jumps follow the parameterization direction (blue lines) and end up in the single point on the right.It induces that our meshing technique do not work in such a situation since two lines end up at on the same geometric curve (see dual structure on the top of Figure 26).

\section{CONCLUSION}

We have proposed an approach to build block hexahedral meshes in an indirect interactive manner. Starting from a 3D frame field, the user creates 3D dual sheets and the proposed algorithm build a dual structure that is eventually converted into hexahedral blocks. This process is iterative and guided by validity rules the dual structure must respect. It has been validated on several CAD models whose complexity is similar to the ones presented in Section 6. It fundamentally differs from recent interactive attempt to generate hexahedral blocks $[5,6]$ in the fact that we directly handle $3 \mathrm{D}$ dual sheets. This difference induces a totally different pipeline of algorithms and concepts: dual zones, validity rules for the dual structure, primal blocks creations. In order to go further, we will improve our approach following three main directions. 

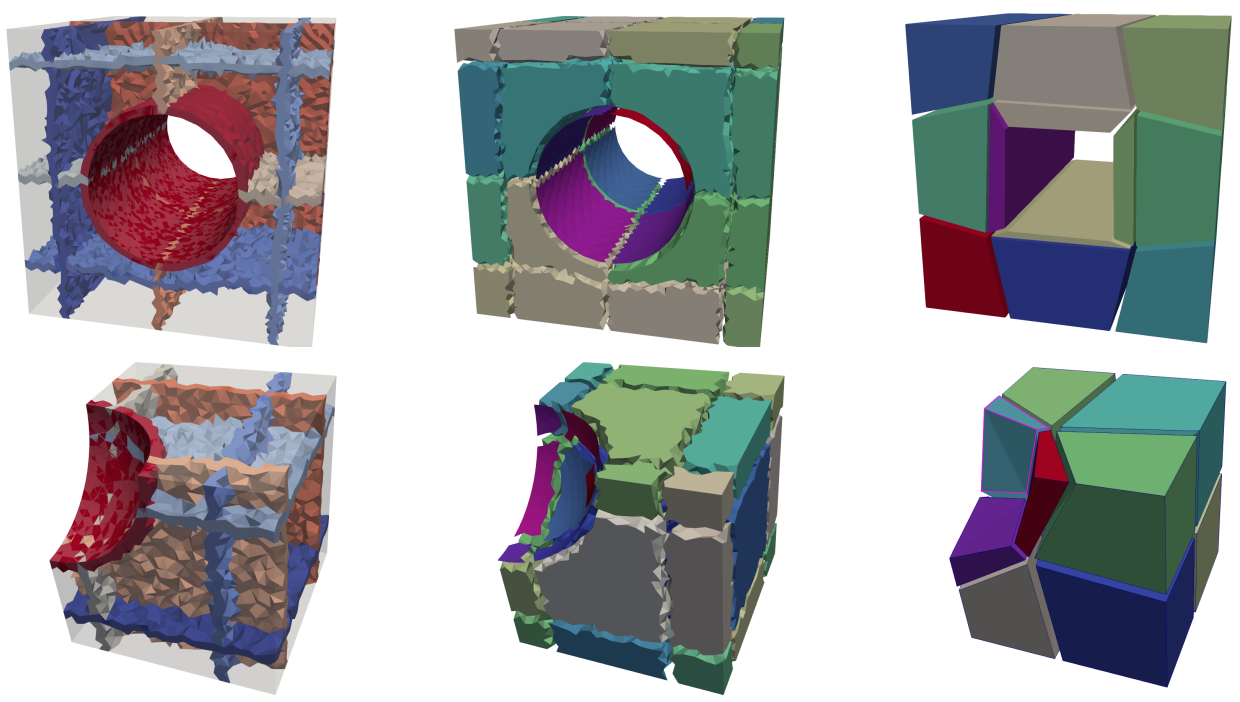

Figure 23: Dual structure (left), dual zones (middle) and final hexahedral blocks (right) for several models.
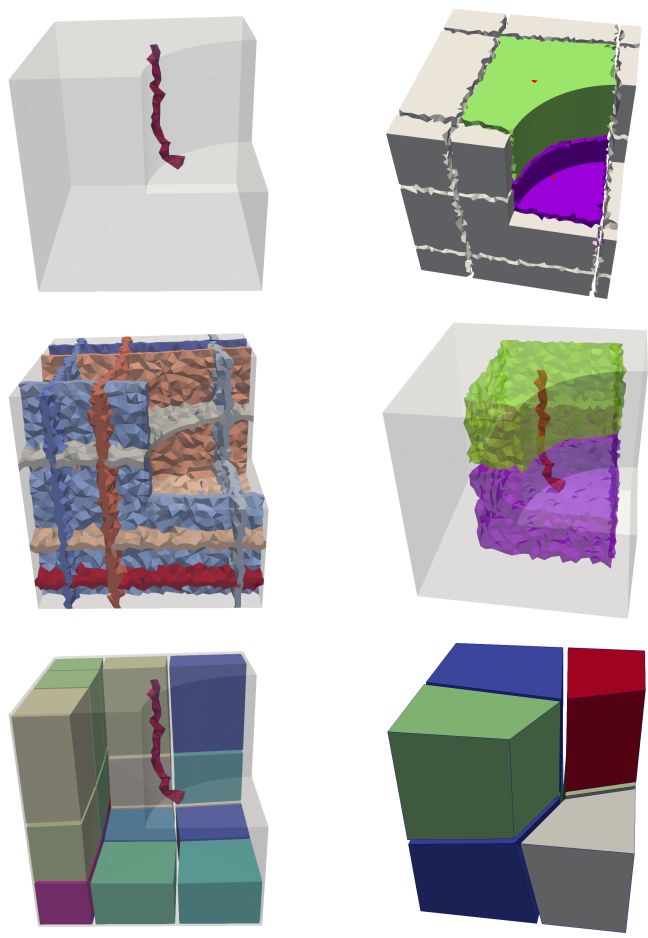

Figure 24: Because of a 3-5 singularity line (topleft), dual sheets are not generated near the singularity line (middle-left) and the block structure is incomplete (bottom-left). By relaxing our validity rules for this type of line, we can obtain a block structure that does not strictly follow the frame field topology (rigth column).
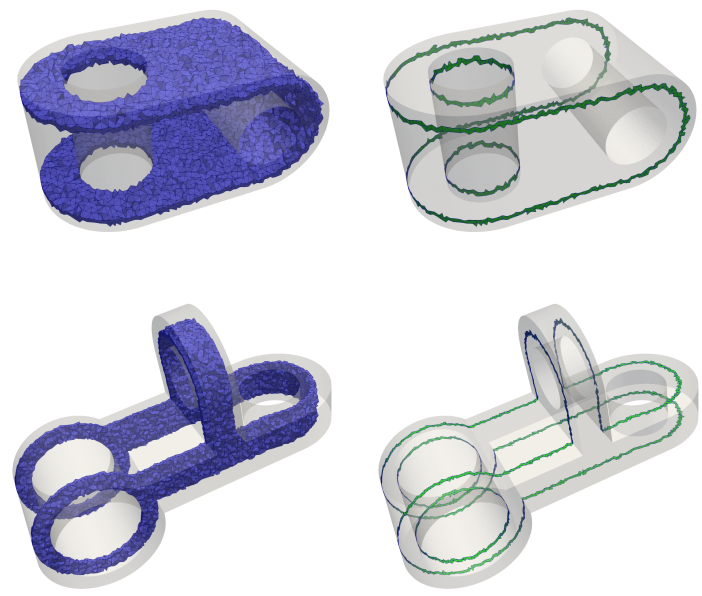

Figure 25: Examples of 3D dual sheets bounded by several boundary loops. 

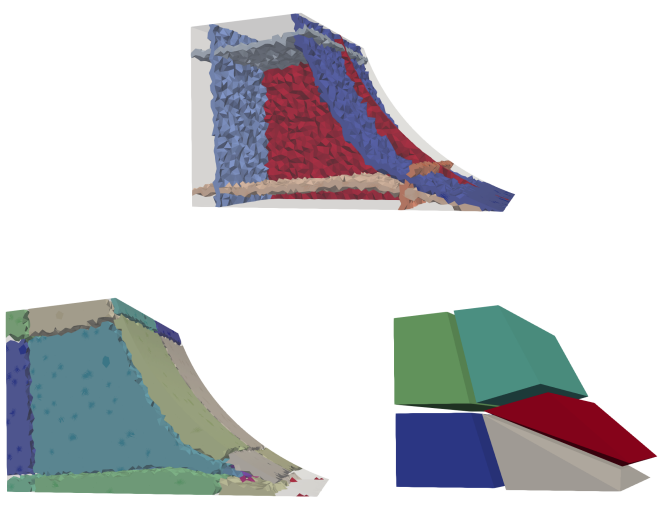

Figure 26: Example of a "ski-ramp" configuration. The dual structure is ill-formed with two dual sheets that almost merged together (top). Dual zones (bottom-left) and obtained hexahedral blocks (bottom-right) are finally incomplete.
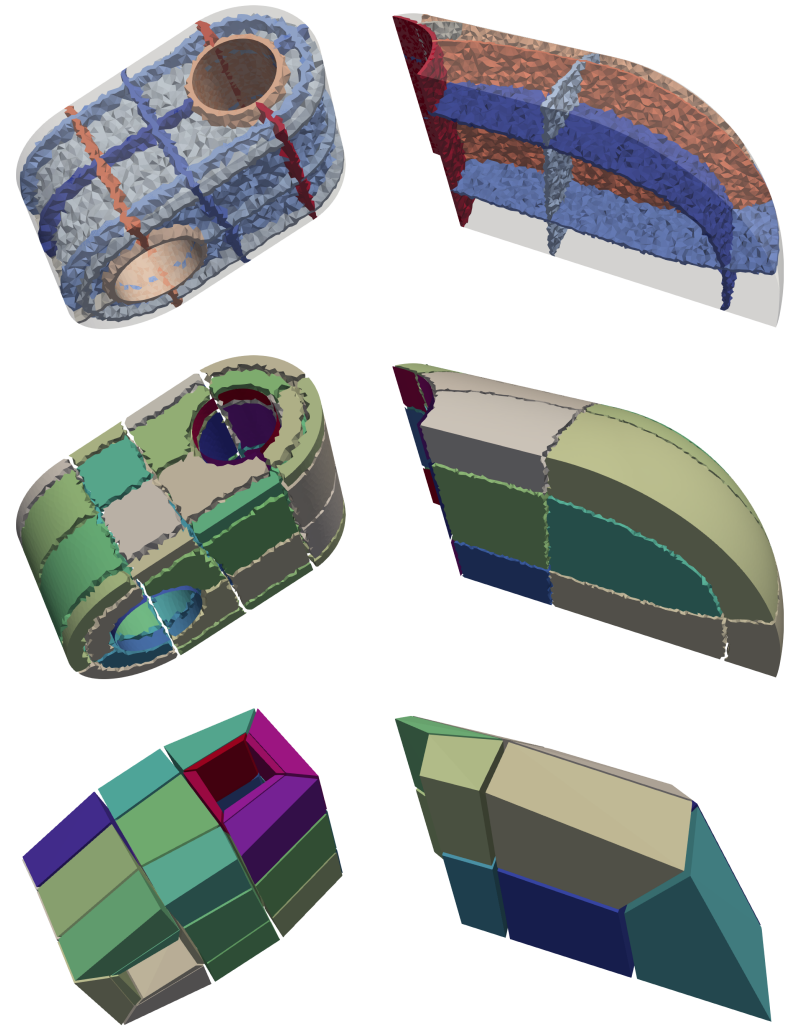

Figure 27: Dual structure (left), dual zones (middle) and final hexahedral blocks (right) for several models.

First, our dual sheet creation process requires a quite refined mesh. When two singularity lines are separated by only a few number of tetrahedra of $\mathcal{T}$, or when a singularity line is too close to the domain boundary, it may happen that the user is unable to insert a dual sheet in this area. We have provided a partial answer by introducing a boundary dual sheet insertion process, but we need a more global solution. Mesh adaption techniques on $\mathcal{T}$ with frame field interpolation seems reachable and would give the ability to adapt the mesh when problematic situations occur. Another option is to make the dual sheet creation algorithm more precise on coarse meshes.

The second direction to go through is relative to intrinsic frame field limitations. A frame field input may not corresponds to a valid hexahedral block structure. It happens at least in two situations: (1) very sharp regions with small angles (see Figure 26); (2) when a singularity line connects a 3 -type boundary singularity point to a 5-type one (see Figure 24). For the former case, we intend to add 3D interactors to "cut" these parts and replace them by an appropriate dual zone directly. For the latter case, 3D interactors coupled with a frame field modification algorithm should allow us to get a valid dual structure.

The last direction is about user-guidance and interactivity. We intend to provide support to the user when the block structure is detected as being invalid. We could also give to the user some suggestions of regions where to a tetrahedron should be picked for creating a dual sheet. Our current implementation can also be drastically improved to get timings compatible with a fluent interactive process (see Table 21 for our current results). 

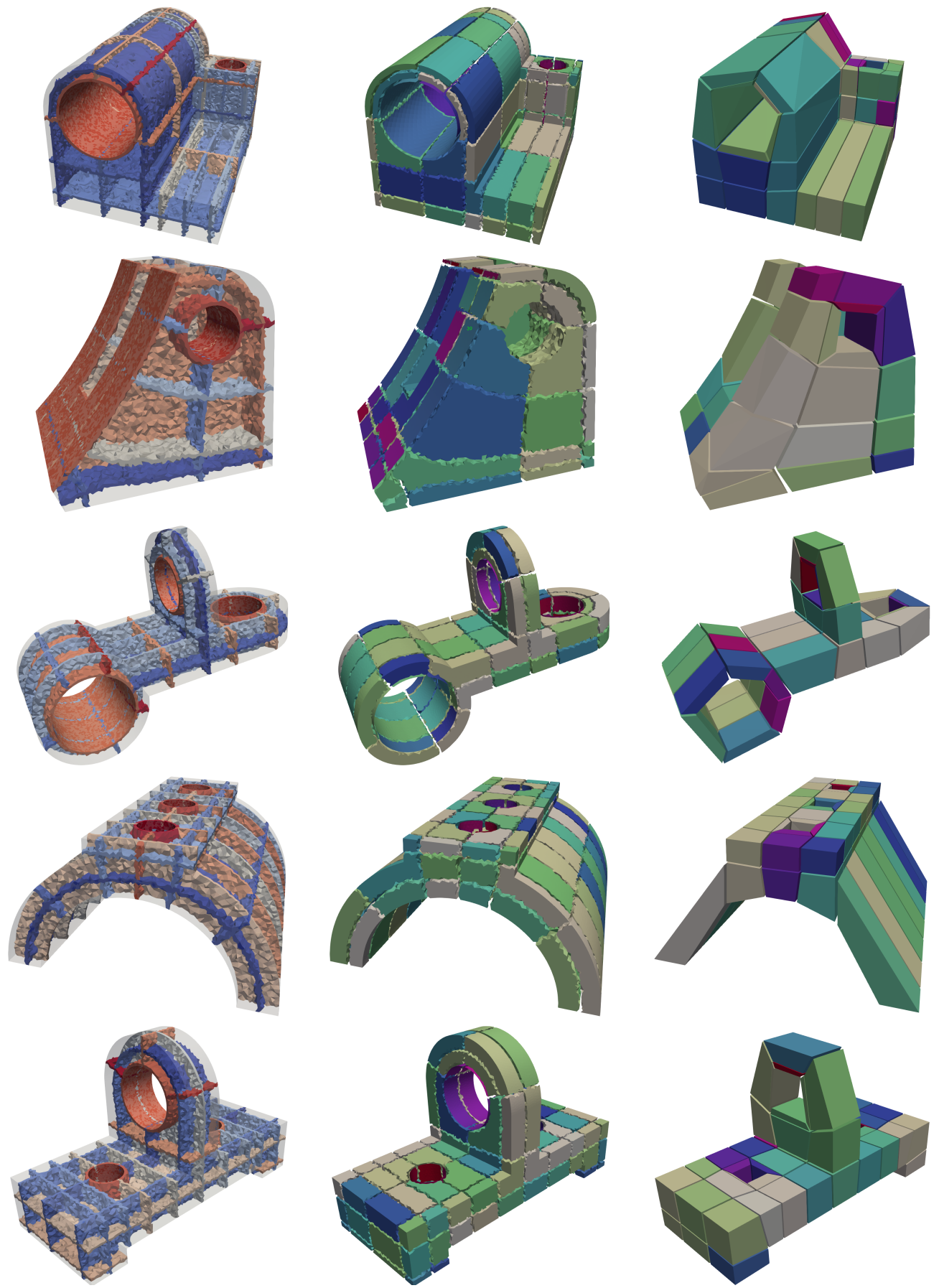

Figure 28: Dual structure (left), dual zones (middle) and final hexahedral blocks (right) for several models.

\section{References}

[1] Slotnick J., Khodadoust A., Alonso J., Darmofal D., William G., Elizabeth L., Mavriplis D. "CFD Vision 2030 Study: A Path to Revolutionary Computational Aerosciences, NASA/CR2014-218178, NF1676L-18332." 2014
[2] Sokolov D., Ray N., Untereiner L., Lévy B. "Hexahedral-Dominant Meshing." ACM Trans. Graph., vol. 36, no. 4, Jun. 2016

[3] Gao X., Jakob W., Tarini M., Panozzo D. "Robust Hex-dominant Mesh Generation Using Field-guided Polyhedral Agglomeration." ACM 
Trans. Graph., vol. 36, no. 4, 114:1-114:13, Jul. 2017

[4] Ray N., Sokolov D., Reberol M., Ledoux F., Lvy B. "Hex-dominant meshing: Mind the gap!" Computer-Aided Design, vol. 102, 94-103, 2018

[5] Takayama K. "Dual Sheet Meshing: An Interactive Approach to Robust Hexahedralization." Computer Graphics Forum, 2019

[6] Zheng Z., Wang R., Gao S., Liao Y., Ding M. "Dual Surface Based Approach to Block Decomposition of Solid Models." Proceedinds of the 26th International Meshing Roundtable, 2018

[7] Huang J., Tong Y., Wei H., Bao H. "Boundary aligned smooth 3D cross-frame field." ACM Trans. Graph., vol. 30, no. 6, 143:1-143:8, 2011

[8] Kowalski N., Ledoux F., Frey P. "Blockstructured Hexahedral Meshes for CAD Models Using 3D Frame Fields." Procedia Engineering, vol. $82,59-71,2014$

[9] Kowalski N., Ledoux F., Frey P. "Smoothness Driven Frame Field Generation for Hexahedral Meshing." Comput. Aided Des., vol. 72, no. C, 65-77, Mar. 2016

[10] Ray N., Sokolov D., Lévy B. "Practical 3D Frame Field Generation." ACM Trans. Graph., vol. 35, no. 6, 233:1-233:9, Nov. 2016. URL http://doi .acm.org/10.1145/2980179.2982408

[11] Nieser M., Reitebuch U., Polthier K. "CubeCover- Parameterization of 3D Volumes." Comput. Graph. Forum, vol. 30, no. 5, 1397-1406, 2011

[12] Li Y., Liu Y., Xu W., Wang W., Guo B. "Allhex Meshing Using Singularity-restricted Field." ACM Trans. Graph., vol. 31, no. 6, 177:1-177:11, Nov. 2012

[13] Timothy J. Tautges S.E.K., Rickmeyer T.J. "Local Topological Modifications of Hexahedral Meshes; Part I: A Set of Dual-Based Operations." ESAIM Proceedings Cemracs 2007, vol. 24, pp. 14-33. 2008

[14] Jurkova K., Ledoux F., Kuate R., Rickmeyer T., Tautges T.J., Zorgati H. "Local Topological Modifications of Hexahedral Meshes; Part II: Combinatorics and Relation To Boy Surface." ESAIM Proceedings Cemracs 2007, vol. 24, pp. 34-45. 2008

[15] Ledoux F., Shepherd J.F. "Topological and geometrical properties of hexahedral meshes." Engineering with Computers, vol. 26, no. 4, 419-432, 2010
[16] Ledoux F., Shepherd J.F. "Topological modifications of hexahedral meshes via sheet operations: a theoretical study." Engineering with Computers, vol. 26, no. 4, 433-447, 2010

[17] T.J Tautges T.B., Mitchell S. "The Whisker Weaving Algorithm: A Connectivity-based Method for Constructing All-Hexahedral Finite Element Meshes." International Journal For $\mathrm{Nu}$ merical Methods in Engineering, vol. 39, 33273349, 1996

[18] Folwell N., Mitchell S. "Reliable Whisker Weaving via Curve Contraction." proceedings of the $7^{\text {th }}$ International Meshing Roundtable, pp. 365378. 1998

[19] Ledoux F., Weill J.C. "An extension of the reliable whisker weaving algorithm." Proceedings of the 16th International Meshing Roundtable, pp. 215-232, 2008

[20] Muller-Hannemann M. "Shelling Hexahedral Complexes for Mesh Generation." Journal of Graph Algorithms and Applications, vol. 5, no. 5, 59-91, 2001

[21] Kremer M., Bommes D., Lim I., Kobbelt L. "Advanced Automatic Hexahedral Mesh Generation from Surface Quad Meshes." 22nd International Meshing Roundtable. Springer-Verlag, Berlin, 2013

[22] Kowalski N., Ledoux F., Staten M.L., Owen S.J. "Fun sheet matching: towards automatic block decomposition for hexahedral meshes." Engineering with Computers, vol. 28, 241-253, 2012

[23] Staten M.L., Shepherd J.F., Ledoux. F., Shimada K. "Hexahedral Mesh Matching: Converting non-conforming hexahedral-to-hexahedral interfaces into conforming interfaces." International journal for numerical methods in engineering, vol. 82, no. 12, 1475-1509, 2010

[24] Livesu M., Pietroni N., Puppo E., Sheffer A., Cignoni P. "Loopy Cuts: Surface-Field Aware Block Decomposition for Hex-Meshing." CoRR, 2019

[25] Geuzaine C., Remacle J.F. "Gmsh: A 3-D finite element mesh generator with built-in pre- and post-processing facilities." International Journal for Numerical Methods in Engineering, vol. 79, $1309-1331,2009$

[26] R. Viertel M.S., Ledoux F. "Analysis of Non-Meshable Automatically Generated Frame Fields." Research Note in the 25th International Meshing Roundtable. Springer-Verlag, Berlin, 2016 
[27] Liu H., Zhang P., Chien E., Solomon J., Bommes D. "Singularity-constrained Octahedral Fields for Hexahedral Meshing." ACM Trans. Graph., vol. 37 , no. 4, 93:1-93:17, Jul. 2018 


\title{
MULTI-BLOCK DECOMPOSITION AND MESHING OF 2D DOMAIN USING GINZBURG-LANDAU PDE
}

\author{
Jovana Jezdimirović Alexandre Chemin Jean François Remacle \\ Université catholique de Louvain, Louvain la Neuve, Belgium jovana.jezdimirovic@uclouvain.be
}

\begin{abstract}
An in-depth method to generate multi-block decomposition of the arbitrary $2 D$ domain using $2 \mathrm{D}$ cross fields solution of Ginzburg-Landau partial differential equation (PDE) is presented. It is relied on parameterization of multiblock decomposition of the domain, obtained by using particular PDE for the purpose of generating direction fields, appropriate number and localization of singular points and their separatrices. We have proved that solutions of particular PDE imply locally integrable vector fields and have adequate distribution of singularities, advocating its usage. Multi-block graph was generated by the separatrices and extraordinary vertices of the domain (singularities, corners and separatrices intersections) and obtained blocks were parameterized/remeshed. As a result, a mechanism to obtain multi-block structured all-quad mesh in automatic manner is developed.
\end{abstract}

Keywords: Ginzburg-Landau, cross fields, multi-block decomposition, all-quad mesh

\section{INTRODUCTION}

Multi-block structured meshes offer numerous advantages for mesh generation in general, as reported by an increasing number of authors $[1,2,3,4]$. Some of the crucial improvements refer to: numerical stability, quality of the solution and computational time, efficient use of advanced vector extensions (AVX) of modern microprocessors, development of efficient/optimal preconditioners, dramatically reduced memory footprint and the use for multi-structured domains.

The goal of the method presented here is to obtain automatic solution for the issue of multi-block decomposition and all quad meshing of $2 \mathrm{D}$ domains starting from a specific partial differential equation (PDE).

We consider as input a triangulated surface $\Omega$. Ginzburg-Landau PDE is then solved on $\Omega$ which allowed computation of a cross field $\tilde{\mathcal{C}_{\Omega}}$ [5]. The cross field is then used for computing a multi-block decomposition of $\Omega$ which is a global parameterization of the domain. In our approach, singularities are precisely located at first, lifting of the cross field is then computed and used to obtain domain separatrices. This stage leads to a block decomposition that is used to build a finite element quadrilateral mesh with the use of an elliptic smoother. Figure 1 presents an overview of the methodology.

Our method has specificities/advantages with respect to existing methods. First, we give a proof of integrability of unitary cross field, justifying its usage for domain partitioning. Multi-block decomposition is generated to be aligned with the cross field. Control over adding/reducing the number of blocks for creation/discarding of boundary layers is presented. The numerical methodology used avoids the problem of limit cycles in the reported examples from [6] and [7]. Finally, the true outcome of this paper is a fully functional multi-block mesh generator that is available in Gmsh [8], the open source finite element mesh generator.

\section{RELATED WORK}

Numerous methods for field designed parameterization/remeshing have been developed in the past decades, thorough overviews given in [9] and [10]. 

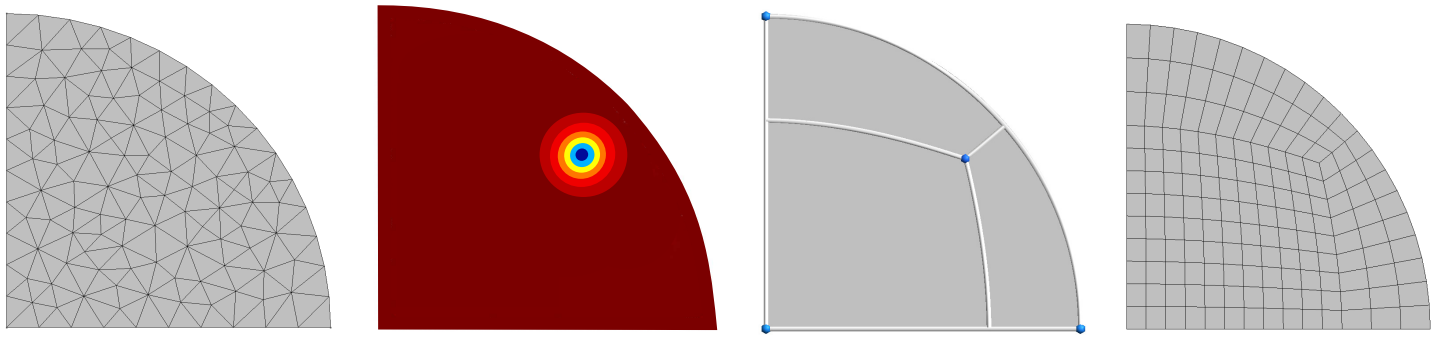

Crossfield

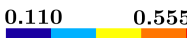

Figure 1: Simplicial mesh on $\Omega$, computed cross field, generated separatrices and final quad mesh (from left hand side to right hand side respectively)

One of the research lines generally relies on cut graph methodology, where cross field construction is followed by continuous parameterization, with differences on integer rounding $[11,12]$. When it comes to developments in correlation with our work, similarly to [3] and [6], we used a PDE based approach for multi-block decomposition purpose. In our case, more in depth developed procedures with extension to surfaces and specifities of the chosen PDE are shown. To avoid possible misalignments in the resulting parameterization, contrary to [13], multi-block decomposition remained aligned with the cross field. The techniques used for computing the cross field are from on [5] and they may seem to be similar to [14], although there are many differences. Some of the crucial divergencies are: the finite scheme relies on a Ginzburg-Landau PDE and not on the guidance field; the degrees of freedom are the real and imaginary parts of a vector field and not two angles defining the parameterization; the penalty factor is not constant, but it is governed by the mesh size. Following the idea of the importance of improving integrability of a cross field by [14] and [15], we have shown that, for a given cross field, it is always possible to find a scaling scalar function allowing to obtain a locally integrable cross field. This demonstration legitimates the usage of $2 \mathrm{D}$ cross fields to generate multiblock decompositions.

\section{PURPOSE OF 2D CROSS FIELDS FOR QUAD MESH GENERATION}

A $2 \mathrm{D}$ cross $\mathbf{c}$ is defined as a set of 4 orthogonal vectors of norm $l, \mathbf{c}=\left\{\mathbf{u}_{k}\right\}_{k \in[\mid 1,4]]}$. With a given 2D orthonormal basis $(\mathbf{x}, \mathbf{y}), \mathbf{u}_{k}=l \cos \left(\theta+k \frac{\pi}{2}\right) \mathbf{x}+l \sin \left(\theta+k \frac{\pi}{2}\right) \mathbf{y}$, represented in figure 2 .

On a $2 \mathrm{D}$ domain $\Omega$, for each point $\mathbf{x} \in \Omega$ it is possible to define a cross $\mathbf{c}(\mathbf{x})$. A $2 \mathrm{D}$ cross field on $\Omega$ is defined as the set $\mathcal{C}_{\Omega}=\{\mathbf{c}(\mathbf{x}), \mathbf{x} \in \Omega\}$.

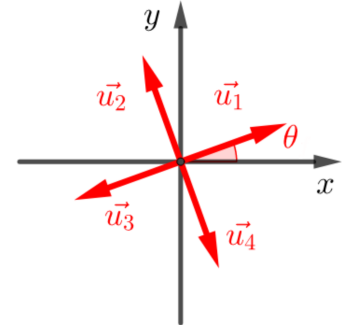

Figure 2: Cross definition

In order to highlight the use of $2 \mathrm{D}$ cross fields for quad mesh generation, we will focus on a $2 \mathrm{D}$ domain $\Omega$ conformal to the unit square. Let's $\mathcal{U}$ be the planar unit square, $\mathcal{F}$ a conformal transformation and $\Omega=\mathcal{F}(\mathcal{U})$ (figure 3).

Let's define $\mathcal{C}_{\mathcal{U}}$ as an uniform cross field of norm 1 aligned with the principal axis of $\mathcal{U}$ laying in the tangent space of $\mathcal{U}$, and $\tilde{\mathcal{C}}_{\Omega}$ the image of $\mathcal{C}_{\mathcal{U}}$ by $\mathcal{F}$. $\tilde{\mathcal{C}}_{\Omega}$ is a representation of the jacobian of $\mathcal{F}$ and in the following we will define $\mathcal{C}_{\Omega}$ as the normalized jacobian of $\mathcal{F}$.

It is possible to generate a quadrilateral multi-block decomposition of $\Omega$ by tracing integral lines of $\tilde{\mathcal{C}}_{\Omega}$, represented in figure 4 , which are identical to integral lines of $\mathcal{C}_{\Omega}$.

Unfortunately, the conformal transformation $\mathcal{F}$ is usually unknown, and computing it is a challenging process. Instead, we will focus on computing the normalized jacobian $\mathcal{C}_{\Omega}$ of $\mathcal{F}$, knowing that on the boundary $\partial \Omega$ one direction of the normalized jacobian has to be aligned with the normal of $\partial \Omega$ (figure 5 ). Therefore, we are looking for a normalized $2 \mathrm{D}$ cross field $\mathcal{C}_{\Omega}$ on $\Omega$, as smooth as possible and having one direction aligned to $\partial \Omega$ 's normal on the boundary. 

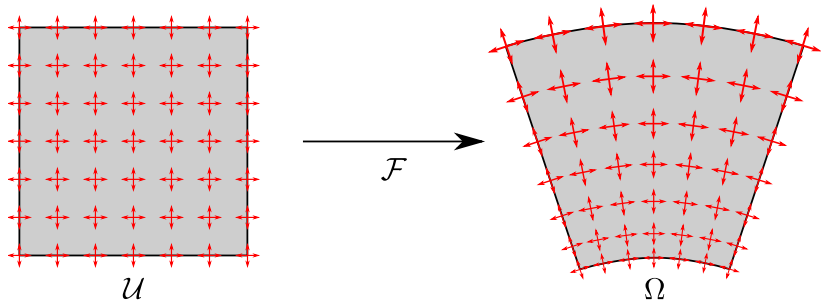

$\Omega$

Figure 3: Conformal transformation between unitary square and physical domain with associated cross fields
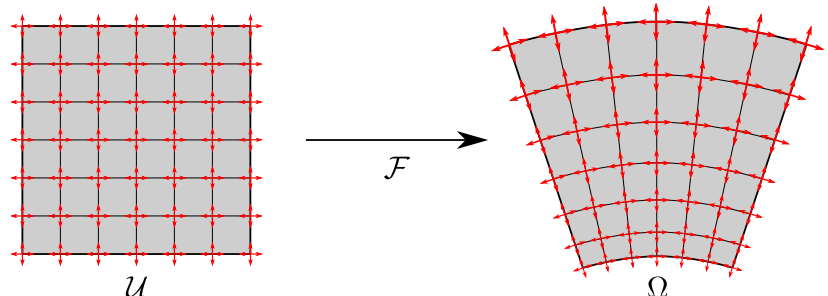

Figure 4: Quadrilateral multi-block decomposition of domain $\Omega$ obtained by propagating integral lines of $\mathcal{C}_{\Omega}$

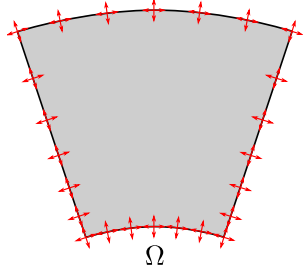

Figure 5: Reference problem for 2D cross field computation

The quadrilateral multi-block decomposition is then obtained by tracing $\mathcal{C}_{\Omega}$ 's integral lines. As we only computed a normalized cross field, which correspond to a normalized jacobian, one could wonder if it exists a conformal transformation $\mathcal{F}$ such as its normalized jacobian is equal to $\mathcal{C}_{\Omega}$. To show that it is the case, we will show that for a given normalized cross field $\mathcal{C}_{\Omega}$, it is easy to build a corresponding $2 \mathrm{D}$ cross field with the same orientation and non uniform norms $\tilde{\mathcal{C}}_{\Omega}$ which is integrable.

Assuming we know a normalized $2 \mathrm{D}$ cross field $\mathcal{C}_{\Omega}$, $\mathcal{C}_{\Omega}=\{\mathbf{c}(\mathbf{x}), \mathbf{x} \in \Omega\}$ with:

$$
\begin{aligned}
& \mathbf{c}=\left\{\mathbf{u}_{k}\right\}_{k \in[|1,4|]} \\
& \mathbf{u}_{k}=\left[\begin{array}{l}
\cos \left(\theta(\mathbf{x})+k \frac{\pi}{2}\right) \\
\sin \left(\theta(\mathbf{x})+k \frac{\pi}{2}\right)
\end{array}\right], \mathbf{x} \in \Omega
\end{aligned}
$$

which is completely defined by the function $\theta$. We are looking for $\tilde{\mathcal{C}_{\Omega}}=\{\tilde{\mathbf{c}}(\mathbf{x}), \mathbf{x} \in \Omega\}$ with :

$$
\begin{aligned}
\tilde{\mathbf{c}} & =\left\{\tilde{\mathbf{u}}_{k}\right\}_{k \in[|1,4|]} \\
\tilde{\mathbf{u}}_{k} & =l(\mathbf{x})\left[\begin{array}{l}
\cos \left(\theta(\mathbf{x})+k \frac{\pi}{2}\right) \\
\sin \left(\theta(\mathbf{x})+k \frac{\pi}{2}\right)
\end{array}\right], \mathbf{x} \in \Omega,
\end{aligned}
$$

where $\theta$ is known, such that $\tilde{\mathcal{C}}_{\Omega}$ is integrable.

A $2 \mathrm{D}$ cross field $\tilde{\mathcal{C}}_{\Omega}$ is integrable if and only if for $\forall \mathbf{x} \in$ $\Omega$, the Lie bracket of 2 orthogonal branches $\left(\tilde{\mathbf{u}}_{1}, \tilde{\mathbf{u}}_{2}\right)$ is equal to $\mathbf{0}$ :

$$
\left[\tilde{\mathbf{u}}_{1}, \tilde{\mathbf{u}}_{2}\right]=\nabla_{\tilde{\mathbf{u}}_{2}} \tilde{\mathbf{u}}_{1} \nabla_{\tilde{\mathbf{u}}_{1}} \tilde{\mathbf{u}}_{2}=\nabla \tilde{\mathbf{u}}_{1} \cdot \tilde{\mathbf{u}}_{2}-\nabla \tilde{\mathbf{u}}_{2} \cdot \tilde{\mathbf{u}}_{1}=\mathbf{0}
$$

As detailed computation in Appendix B shows, for $l \neq 0, \tilde{\mathcal{C}}_{\Omega}$ is integrable if $l$ verifies:

$$
\nabla(\log (l))=\left[\begin{array}{c}
\theta_{, y} \\
-\theta_{, x}
\end{array}\right] \text { on } \Omega .
$$

As $\theta$ is known, it is easy to compute $l$, with a multiplicative constant, and build an integrable cross field $\tilde{\mathcal{C}}_{\Omega}$. As crosses of $\mathcal{C}_{\Omega}$ and $\tilde{\mathcal{C}}_{\Omega}$ have the same orientation, integral lines of these 2 cross fields are identical. This justifies the usage of integral lines of a normalized cross field $\mathcal{C}_{\Omega}$ for generating multi-block decomposition.

Note that $H=\log (l)$ is defined in [16] as a Green's function that is proven, contrary to $\theta$, to be continuous everywhere in the domain except at singular points where $H$ blows up as $\log r$ which means that $l$ tends linearly to 0 at the vicinity of singularities. 
A multi-block decomposition of a domain $\Omega$ will be done in 2 steps. First, a normalized $2 \mathrm{D}$ cross field $\mathcal{C}_{\Omega}$ is generated on $\Omega$, then integral lines of $\mathcal{C}_{\Omega}$ are computed to generate the multi-block decomposition.

\section{GENERATING CROSS FIELD BASED ON GINZBURG-LANDAU PDE}

\subsection{D crosses representation}

As presented in the previous section, it is possible to completely define a cross with an angle $\theta$. However, symmetries of the cross lead to the fact that $\theta$ and $\theta+k \frac{\pi}{2}, k \in \mathbb{Z}$ define the same cross, thus this representation is not unique. It is possible to uniquely define a cross using the following representation:

$$
\vec{u}=(\cos 4 \theta, \sin 4 \theta), \quad \theta \in\left[0, \frac{\pi}{2}\right) .
$$

In this representation, $\vec{u}$ is invariant by a rotation of $\frac{\pi}{2}$, thus represents a cross with 4 orthogonal branches, as shown on figure 6 and figure 7 .

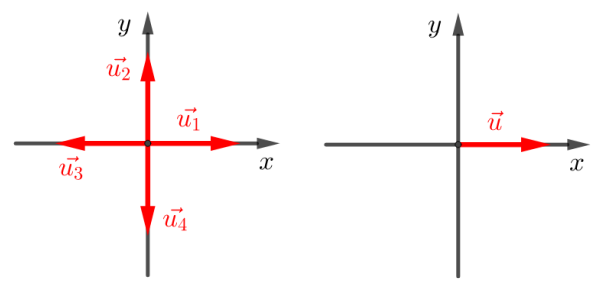

Figure 6: Reference cross (left) and its representation with $2 D$ vector $\vec{u}$ (right)

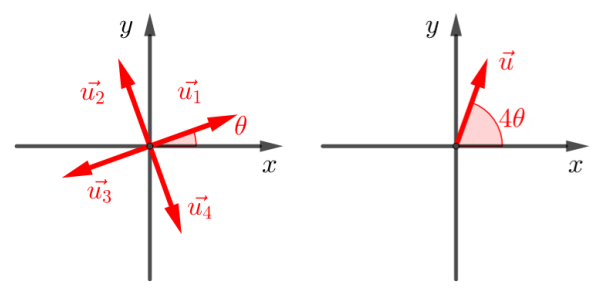

Figure 7: Rotation of reference cross by $\theta$ (left) and its representation with $2 D$ vector $\vec{u}$ (right)

\subsection{Computing 2D cross field through par- tial differential equations}

The idea of computing a cross field $\vec{u}=\left(u_{1}, u_{2}\right)$ is to force crosses to be aligned with the boundaries of $\Omega$ and to propagate those crosses inside $\Omega$ using a PDE that eventually produces smooth cross fields. In what follows, we propose a series of formulations that produce quite different results in practice.

The first approach is to choose $\vec{u} \in\left(H_{1}(\Omega)\right)^{2}$ and simply minimize the Dirichlet energy:

$$
E^{D}(\vec{u})=\frac{1}{2} \int_{\Omega}|\nabla \vec{u}|^{2} d v
$$

with appropriate boundary conditions. Minimizing $E^{D}$ is equivalent to solve $\nabla^{2} \vec{u}=0$. The main issue of that simple approach is that $u_{1}$ and $u_{2}$ are going to rapidly become equal to zero away from the boundaries. This is simply due to the mean value property of harmonic functions. When $u_{1}=u_{2}=0$, the cross direction $\theta=\frac{1}{4} \operatorname{atan} 2\left(u_{2}, u_{1}\right)$ is undefined. Even though cross fields issued from this naive approach are not suitable for block decomposition, they will be used as a starting point for other methods.

The main drawback of the first "naive" approach that has just been presented is that $\vec{u}$ actually leaves $S^{1}$ away from boundaries and is not a cross anymore. There are two possible options to force $\vec{u}$ to stay in $S^{1}$ : (i) choosing $\vec{u} \in S^{1}$ explicitly or (ii) choosing $\vec{u} \in\left(H_{1}(\Omega)\right)^{2}$ and penalize $\vec{u}$ away from $S^{1}$.

The first approach thus consists in choosing $\vec{u} \in S^{1}$ explicitly and writes $\vec{u}(\theta)=e^{i \theta}$. In this case, $\vec{u}$ is a complex number with $u_{1}$ and $u_{2}$ as its real and imaginary part and $u_{1}^{2}+u_{2}^{2}=1$. In this case,

$$
\min _{\vec{u} \in S^{1}} \int_{\Omega}|\nabla \vec{u}|^{2} d v
$$

is equivalent to

$$
\min _{\theta \in H^{1} / Q} E^{\theta}=\int_{\Omega}|\nabla \theta|^{2} d v .
$$

Here, $\theta$ lives in the quotient space $H^{1} / Q$ where $Q$ is the group of symmetries of the square. Angle $\theta$ thus does not live in a linear space and minimizing $E^{\theta}$ is not strictly equivalent to solve $\nabla^{2} \theta=0$. Solutions to that problem have already been provided by using a mixed integer approach [17]. It is also possible to write an explicit smoother that averages $\theta$ locally. This method provides exploitable results when the $\theta$ 's are initiated by minimizing $E^{D}$ using the first naive approach.

Another approach is to propose an alternative energy, namely the Ginzburg-Landay energy functional:

$E^{G L}(\vec{u})=E^{D}+E^{P}=\frac{1}{2} \int_{\Omega}|\nabla \vec{u}|^{2}+\frac{1}{4 \epsilon^{2}} \int_{\Omega}\left(|\vec{u}|^{2}-1\right)^{2}$,

where the parameter $\epsilon$ has a dimension of length and in literature is known as coherence length [16]. Energy (6) contains two terms: the standard Dirichlet energy and a penalization. The only minimizer of the Ginzburg-Landau functional is solution of:

$$
\nabla^{2} \vec{u}+\frac{1}{\epsilon^{2}} \vec{u}\left(|\vec{u}|^{2}-1\right)^{2}=0 .
$$


The only vector field that satisfies both $\nabla^{2} \vec{u}=0$ and $|\vec{u}|=1$ is the constant vector field. Consequently, whenever $\epsilon \neq 0$, the Ginzburg-Landau formulation cannot force $\vec{u} \in S^{1}$ everywhere and unit vectors can only exist if they don't actually "turn" i.e. if $\theta$ is constant.

A mixed approach consists in choosing $\vec{u} \in H_{1}\left(\Omega, S^{1}\right)$ i.e. choosing $\vec{u} \in S^{1}$ while keeping both its components. In this context, it can be shown [16] that the only minimizer of the Dirichlet energy $E^{D}$ is solution of

$$
\nabla^{2} \vec{u}+\vec{u}|\nabla \vec{u}|^{2}=0 .
$$

This formulation is strictly equivalent to $\nabla^{2} \theta=0$. Yet, it requires to choose $\vec{u} \in S^{1}$ a priori which is of course not easy. One can penalize $\vec{u}$ away from $S^{1}$ by using a penalization like in the Ginzburg-Landau case and solve

$$
\nabla^{2} \vec{u}+\vec{u}|\nabla \vec{u}|^{2}+\frac{1}{\epsilon^{2}} \vec{u}\left(|\vec{u}|^{2}-1\right)^{2}=0 .
$$

The smoothness term of this energy functional minimizes the gradient of the cross field and a penalty term makes its norm close to unity.

Further on, 2D crossfields will be computed by minimizing energy functional defined by the equation 6 . Crouzeix-Raviart finite elements are used for the interpolation and a Newton-Raphson scheme for solving the nonlinear problem (computational details in [5]), obtained result is shown in the figure 8 .

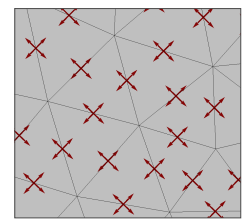

Figure 8: Generated crosses on triangulated surface $\Omega$

\subsection{Cross field topology}

For the orientable surface $\Omega$ with genus $g$ and $\mathrm{b}$ as the number of connected components of $\partial \Omega$, the Euler characteristic $\chi$ of $\Omega$ is an integer:

$$
\chi=2-2 g-b .
$$

Considering a mesh on $\Omega$ with $n$ nodes, $n_{e}$ edges and $n_{f}$ facets, the Euler formula states:

$$
\chi=n-n_{e}+n_{f} .
$$

According to (7) and (8) the mesh on the surface with Euler characteristic $\chi \neq 0$ will have irregular vertices. As shown in [5], these irregular vertices of the mesh are corresponding to singular points obtained by the cross field. Moreover, their number and type depend on Euler characteristic $\chi$. The type of critical point $x_{i}$ is defined by its index index $\left(x_{i}\right)$ and can be found directly by computing:

$$
\operatorname{index}\left(x_{i}\right)=\frac{1}{2 \pi} \oint_{C_{i}} d \theta
$$

where $\theta$ is an angular reference and $C_{i}$ is a closed curve on the surface $\Omega$ containing only one singularity: $x_{i}$. For a given quad mesh, a vertex $x_{i}$ with valance $v_{i}$, where $v_{i}$ represents the number of facets in the mesh adjacent to the $x_{i}$, the integral (9) is evaluated as:

$$
\operatorname{index}\left(x_{i}\right)=\frac{4-v_{i}}{4} .
$$

Therefore, vertices with index $0, \frac{1}{4},-\frac{1}{4}$ are respectively adjacent to four, 3 and 5 quadrangular elements. Dependency of number and type of singular points on $\Omega$ with Euler characteristic $\chi$ is given by PoincaréHopf theorem:

$$
\sum_{i} \operatorname{index}\left(x_{i}\right)=\chi(\Omega) .
$$

According to [16], the result of the minimization of the Ginzburg-Landau energy (6) in $2 D$ supports coexistence of $i n d e x\left(x_{i}\right)= \pm \frac{1}{4}$, as shown on figure 9 .

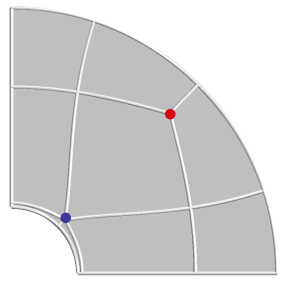

Figure 9: Coexistance of positive singularity (red) and negative singularity (blue) in $2 D$

In a special case, following [18], the result of (6) for $\vec{u} \in S^{1} / C_{N}$, corresponds to the elliptic Fekete points on a sphere (figure 10).
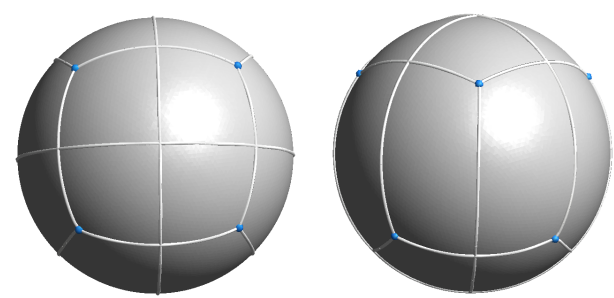

Figure 10: Singular points forming an anticube on the sphere 


\section{TRACING SEPARATRICES}

As already reported $[3,6,7,13]$ the tracing of separatrices of computed cross field accomplishes the goal of domain partitioning. This section describes procedures for generating the separatrices on arbitrary $2 \mathrm{D}$ domain $\Omega$. Our algorithm is divided into three stages: (i) the initiation of separatrices on a locally small neighborhood containing one singular point, (ii) the propagation of separatrices on the whole domain $\Omega$ and (iii) a post processing stage that allows to obtain the minimal number of separatrices.

\subsection{Initialization of separatrices inside the critical elements}

A triangular element $C_{i}, i \in[|1 ; n|]$ of the mesh is considered critical if a singular point is located at its vertex, edge or area, [3, 18]. For the sake of consistency with the numerical scheme used (i.e. Crouzeix-Raviart finite elements), singular points $S_{i}, i \in[|1 ; n|]$ will be located on the middle of the edges where cross field vanishes. By traversing all edges of the mesh and finding the locations with the smallest $\|\vec{u}\|$, the critical elements are marked and singular points extracted.

For the purpose of separatrices propagation on each critical element $C_{i}$, we iterated over each edge of $C_{i}$ to find points where the cross field is aligned with a singularity $S_{i}$, i.e. fulfilling 11 or 12 . Finding values of a cross field in these points is done by linear interpolation, similarly as in [3].

$$
\begin{aligned}
& \theta_{P_{i}}=\theta_{P_{i} S_{i}} \pm \alpha \\
& \theta_{Q_{i}}=\theta_{Q_{i} S_{i}} \pm \alpha
\end{aligned}
$$

with $\alpha$ representing the tolerance criteria.

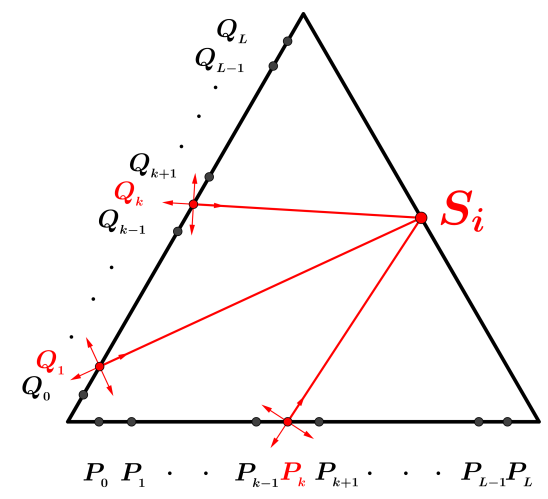

Figure 11: Obtaining separatrices on $C_{i}$

\subsection{Propagation of separatrices on the whole domain $\Omega$}

In order to obtain a decomposition of the domain $\Omega$, separatrices are propagated through a finite number of elements of triangulation $T=T_{i}-\left\{C_{1}, \ldots, C_{n}\right\}$ following the adequate direction of the cross field until stopping criteria is fulfilled. We used the propagation scheme described in [3] relying on Heun's (a variation of Runge-Kutta 2) numerical scheme:

$$
\begin{gathered}
P_{i+1}^{\prime}=P_{i}+h_{i}^{\prime} \cdot \overrightarrow{u_{i}}\left(P_{i}\right) \\
\vec{d}_{i}=\frac{\overrightarrow{u_{i}}\left(P_{i}\right)+\overrightarrow{u_{j}} P_{i+1}^{\prime}}{2} \\
P_{i+1}=P_{i}+h_{i} \cdot \vec{d}_{i}
\end{gathered}
$$

where $P_{i}$ is no-singular point on critical element $C_{i} ; \overrightarrow{u_{i}}$ and $\overrightarrow{u_{j}}$ are cross field directions; $h_{i}^{\prime}$ and $h_{i}$ represent the mesh size dependent step and $P_{i+1}$ is computed point. More detailed, as shown on figure 12, the algorithm aims to compute the point $P_{i+1}$, where $S_{i}$ represents a critical point, and $P_{i}$ is derived based on information about the direction $u_{i}$ which is the closest one to the input direction $\overrightarrow{p_{i}}=\overrightarrow{S_{i} P_{i}}$. Further on, the information about the direction $\overrightarrow{u_{j}}$, at the cross at point $P_{i+1}^{\prime}$ is used to obtain direction $\vec{d}_{i}$ generating point $P_{i+1}$ and allowing further propagation in the same manner.

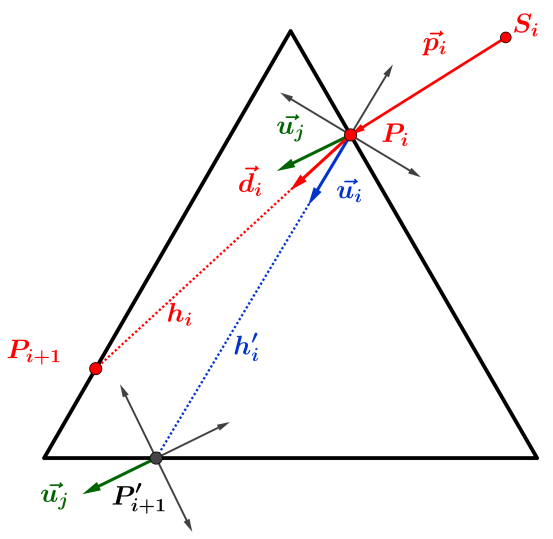

Figure 12: Separatrices propagation method

\subsection{Stopping criteria for separatrices prop- agation}

Separatrices generated in the manner described above are traced until they reached critical patch $E_{p i}$ (figure 13) or boundary $\partial \Omega$. For the computational purposes we defined a critical patch $E_{p i}$ around each singular point $S_{i}$, which represents a set of triangles with a locally small distance $r$ from $S_{i}$. 


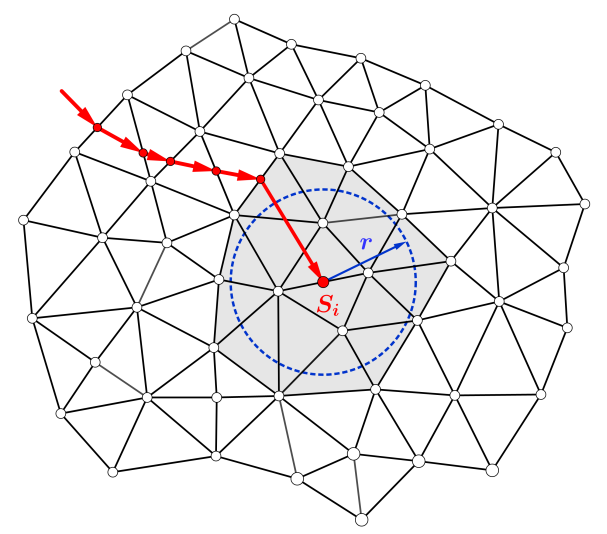

Figure 13: Critical patch

\subsection{Cleaning the redundant separatrices}

Presented method for separatrices propagation is not generating a minimal number of separatrices needed for multi-block decomposition of $\Omega$. This result is a direct consequence of allowing the propagation of the same separatrice from two different singular points (as shown in figure 14).
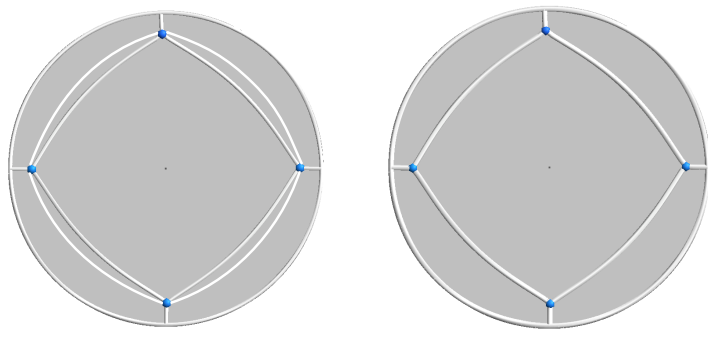

Figure 14: Multi-block decomposition of a circle: separatrices before (left) and after cleaning (right)

In order to obtain the minimal number of separatrices, we developed the procedure, detailed in algorithm 1 , to determine and discard redundant ones. Two or more separatrices are defined as redundant if they have: identical beginnings, identical endings and intersect the same set of separatrices. An example for two redundant separatrices is given in figures 15-16. In red are represented two singular points, in black generated separatrices and in blue and green two separatrices meeting the criterion for defining redundant separatrices exposed previously.

Figure 15 is a case where the set of separatrices intersected is empty. The block generated by redundant separatrices (in white) is made of two edges and can be removed by deleting one of the redundant separatrices without modifying the type of adjacent blocks (in grey)
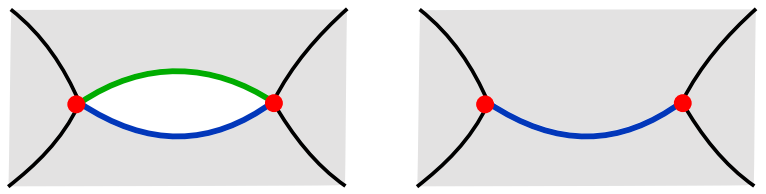

Figure 15: An empty set of separatrices' intersection (before and after)

Figure 16 is a case where the set of separatrices intersected is not empty. Redundant separatrices create the quadrangular blocks and two triangular blocks (in white). Deleting one of the redundant separatrices removes all blocks in white (including triangular blocks) without modifying the type of adjacent blocks (in grey).
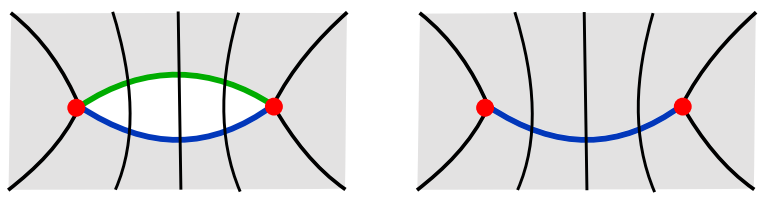

Figure 16: Not an empty set of separatrices' intersection (before and after)

Therefore, for each group of redundant separatrices a random one is chosen to be kept and all the other (redundant) separatrices are removed.

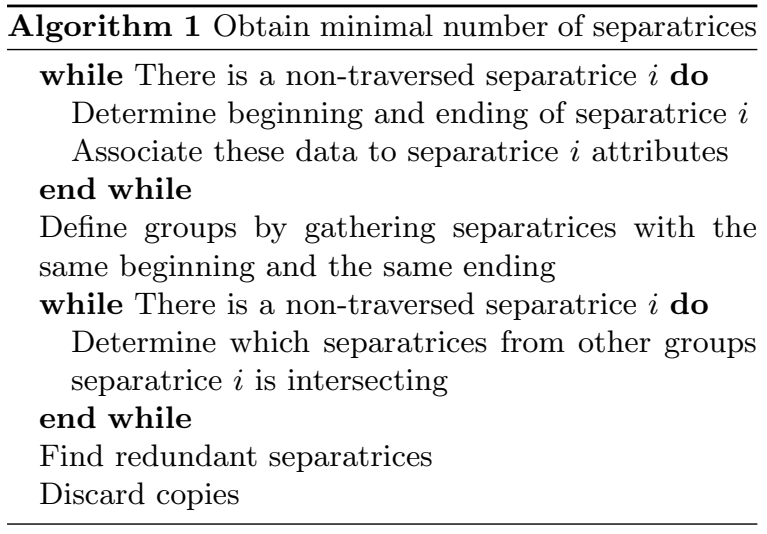

The reason for taking into account separatrices intersections is demonstrated in figures 17-18: separatrices number 1 and 2 have the same beginnings and endings (belong to the same group), but they are intersecting different sets of separatrices (as for separatrices 3 and $4)$. 


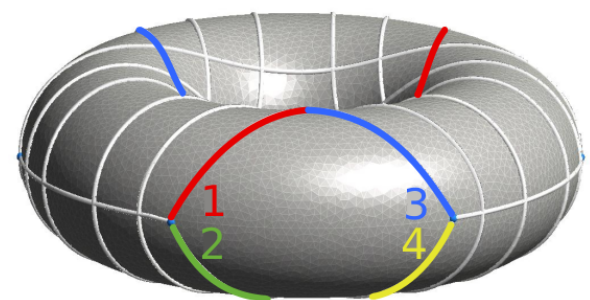

Figure 17: Multi-block decomposition of a torus
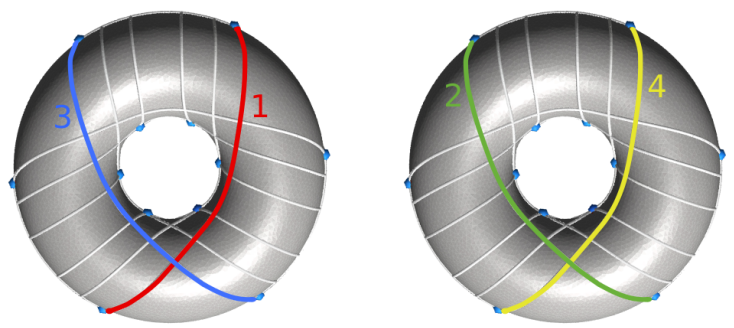

Figure 18: Overview of intersecting separatrices

\section{EXAMPLES OF MULTI-BLOCK DECOMPOSITION}

In this section we present the results obtained by applying Ginzburg-Landau PDE for the purpose of generating multi-block decomposition of the given domain $\Omega$. By the definition of separatrices, each block is smooth inside which allows meshing with quad elements. The quality of the mesh generated in this manner is expected to be high, due to the proven torsion free properties of a generated cross field.

In the following, we demonstrate the dependency of generated multi-block decomposition on:

- geometrical properties of the domain (figures 19/20)

- the type of boundary conditions imposed (figures 21/22)

- the value of coherence length parameter $\epsilon$ (figure 23).

Depending on requirements, we can add (remove) a boundary layer (as shown in figures $21 / 22$ ) by imposing weak or strong boundary conditions. Using different values for global coherence length $\epsilon$, we can generate different multi-block decompositions of the same domain. As shown on the figure 23, both of the decompositions are valid.

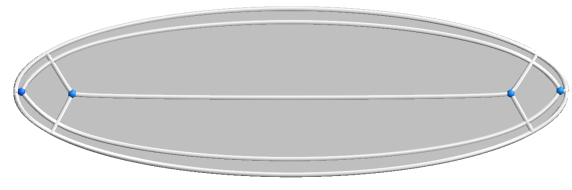

Figure 19: Eccentricity $e=0.71$

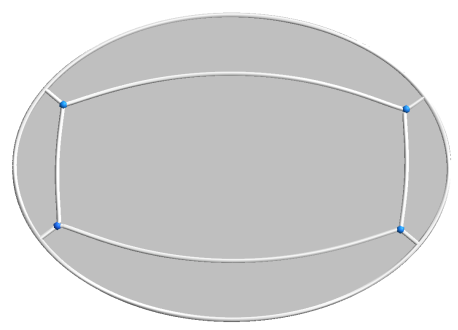

Figure 20: Eccentricity $e=0.95$

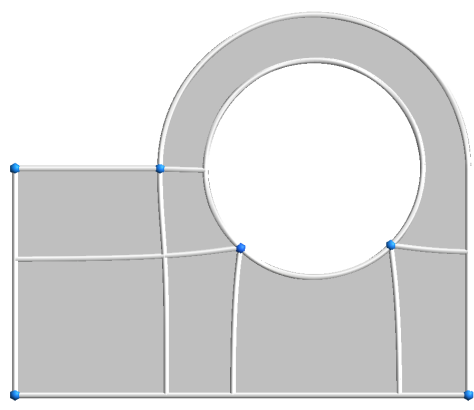

Figure 21: Imposed weak boundary conditions

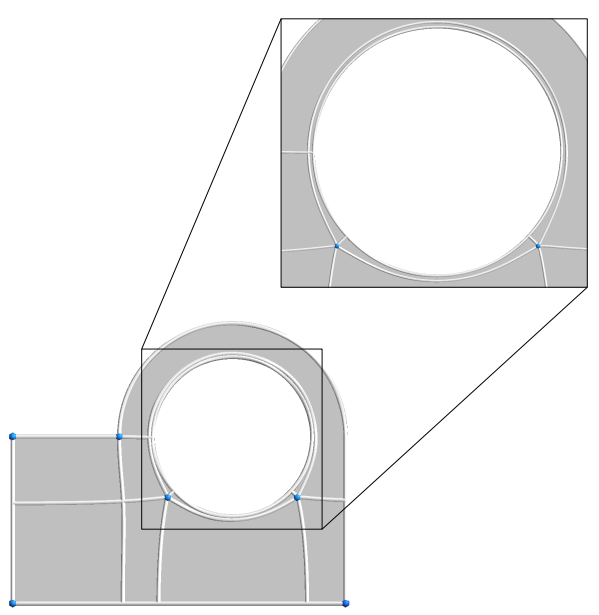

Figure 22: Imposed strong boundary conditions 

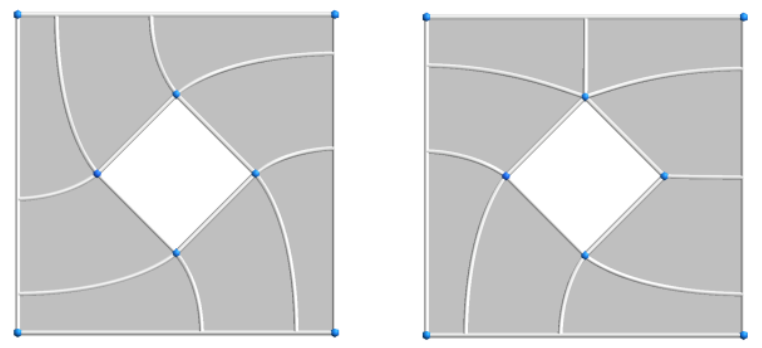

Figure 23: Coherence length $\epsilon=0.001$ (left) and $\epsilon=0.01$ (right)

We have chosen a few examples (figures 24-29) to demonstrate abilities of our algorithm for creating the multi-block decomposition of topologically and/or geometrically challenging domains.

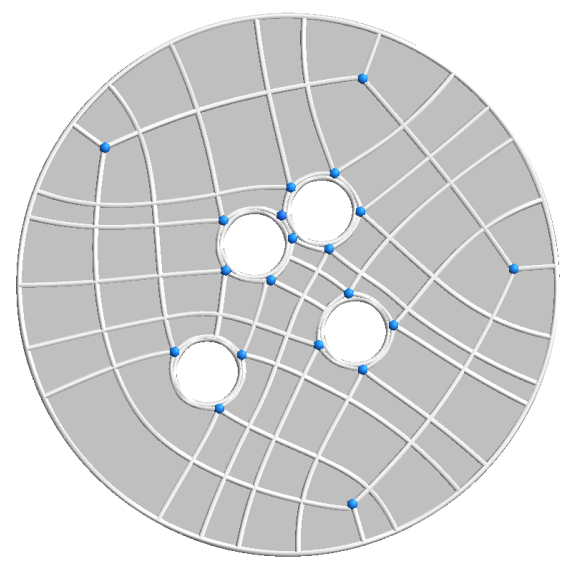

Figure 24: Euler characteristic $\chi=-3$

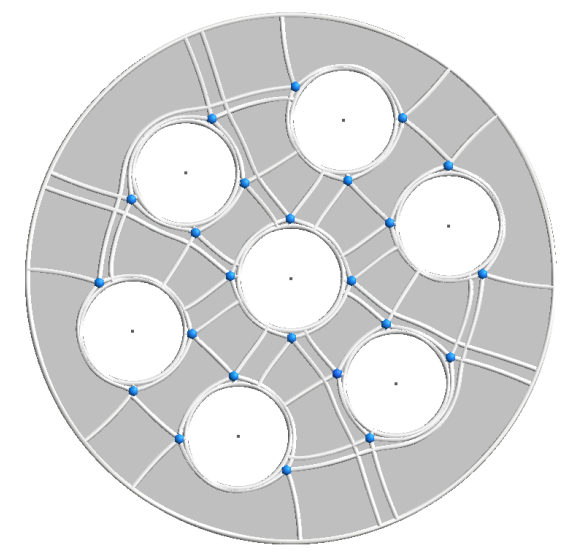

Figure 25: Euler characteristic $\chi=-6$
Following [5], generating cross field using GinzburgLandau functional has many desirable properties for meshing purposes. Recent reports on cross field generation $([6,7])$, pointed out the existence of the limit cycle, defined as one or more separatrices failure to converge to a singular point or a boundary, which prevents generating multi-block decomposition of the domain. The decompositions we obtained, on reported domains by [6] and [7], are shown in figures 30 and 31 .

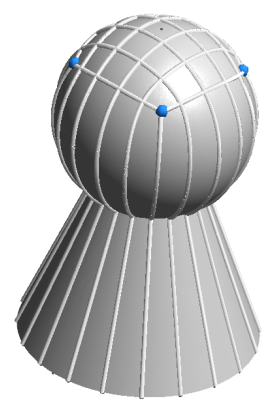

Figure 26: Closed manifold 1

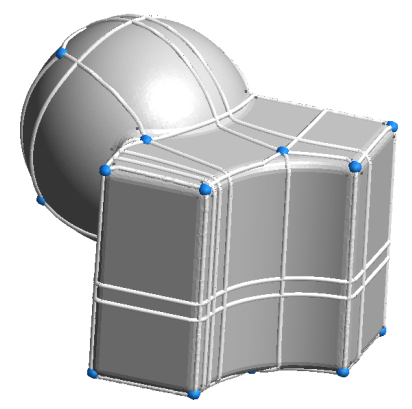

Figure 27: Closed manifold 2

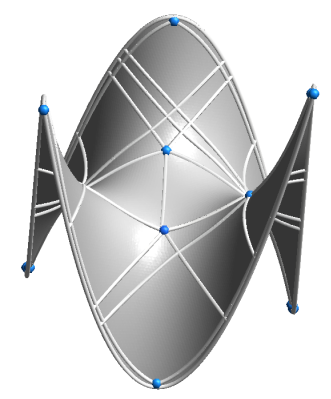

Figure 28: Open manifold 


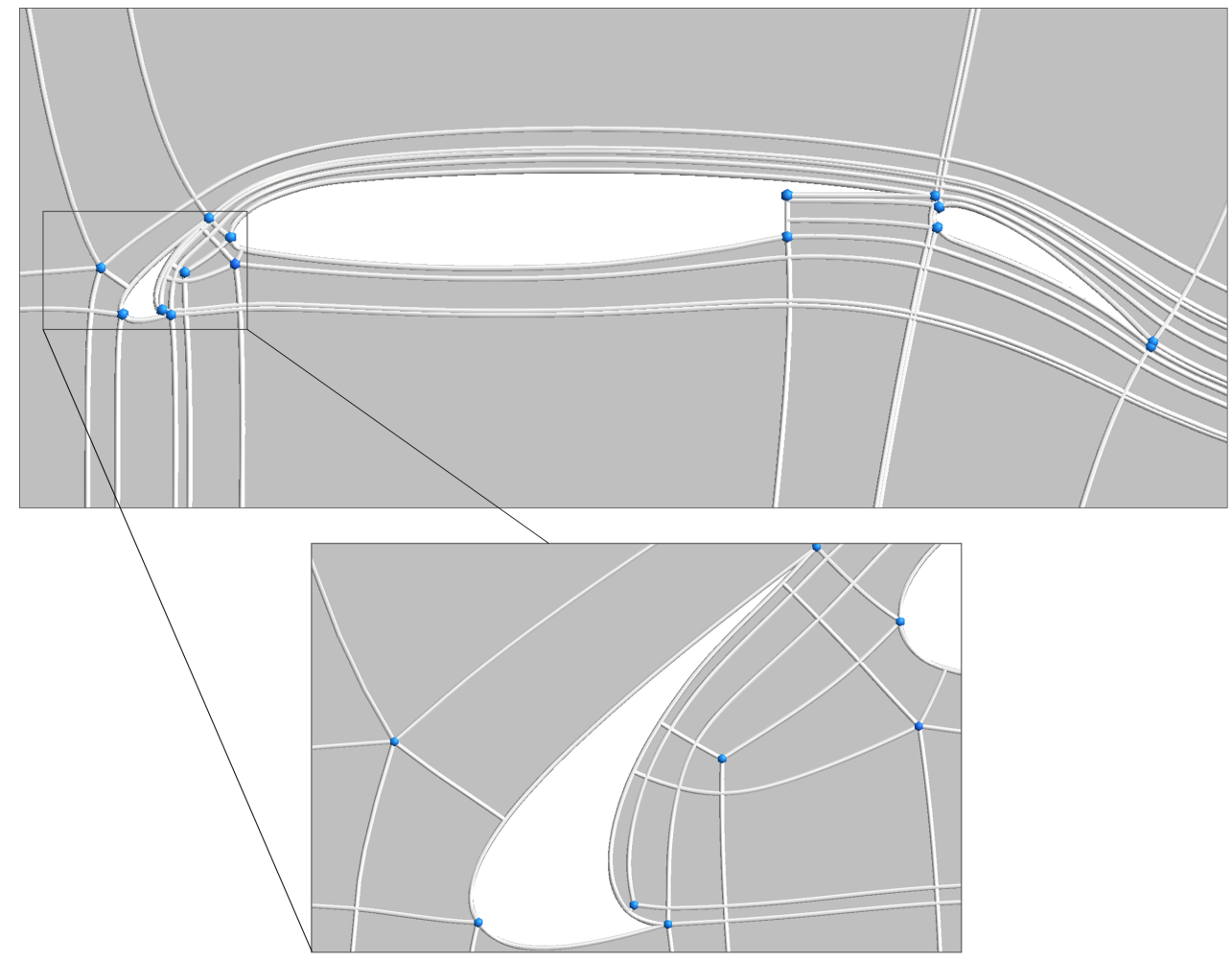

Figure 29: Multi-block decomposition of the wing
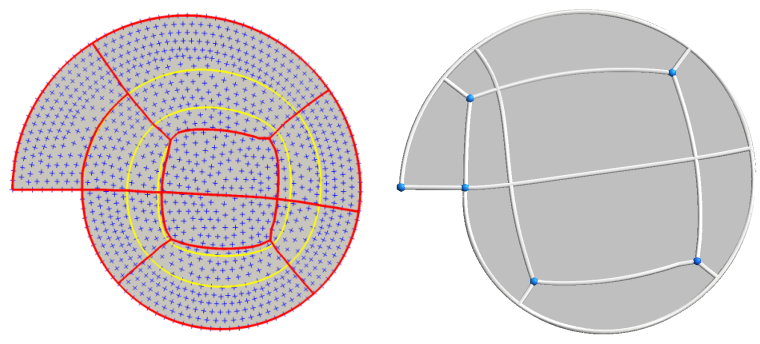

Figure 30: Multi-block decomposition by [6] (left) and by our approach (right)

\section{PLANAR GRAPH EMBEDDING OF THE MULTI-BLOCK DECOMPOSITION}

For the purpose of the efficient and practical representation of complex domains $\Omega$, according to [10], we will generate the planar graph embedding based on its multi-block decomposition (figure 32). To do so, we will use extraordinary vertices $T_{i}, i \in$ $\{1, \cdots, n\}$, defined as singular points, corners and intersections between separatrices and separatrices with $\partial \Omega$. These vertices are further on used for

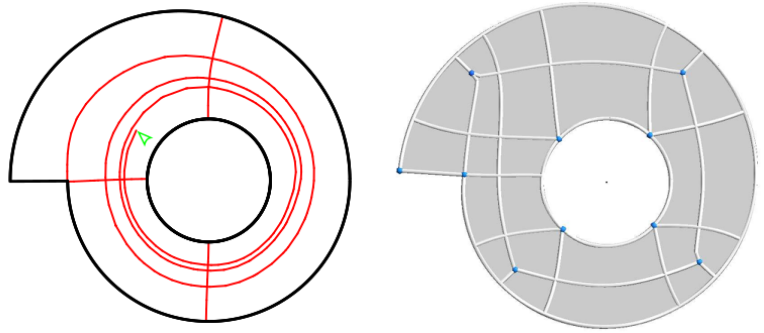

Figure 31: Multi-block decomposition by [7] (left) and by our approach (right)

creating the graph $\Gamma=\{V, E\}$ defined by vertices $V=\left\{T_{1}, T_{2}, \ldots, T_{n}\right\}$ and corresponding edges $E=$ $\left\{\left\{T_{1}, T_{2}\right\},\left\{T_{2}, T_{3}\right\}, \ldots,\left\{T_{j}, T_{n}\right\}\right\}$. For the purpose of meeting well-defined connectivity (figure 33 ) data information of orientation is associated with each extraordinary vertex $T_{i}: T_{m}, T_{n}, T_{p}$, e.g. listing of neighbors in counter-clockwise direction, where from graph edges $E$ are derived. Information on orientation and data structure ensured that each edge will be traversed only once and all quads $Q_{i}=\left\{T_{i}, T_{j}, T_{k}, T_{t}\right\}$ will be extracted. The used algorithm 2 , showed below, is the adaptation of work described by [19]. 

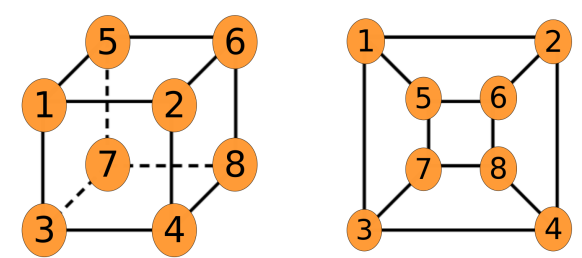

Figure 32: Illustration of a multi-block decomposition (left) and its planar graph embedding (right)
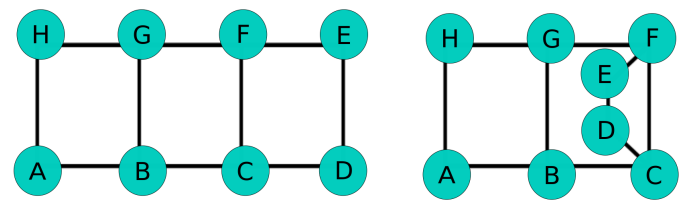

Figure 33: Importance of orientation data: connected (left) and disconnected graph (right)

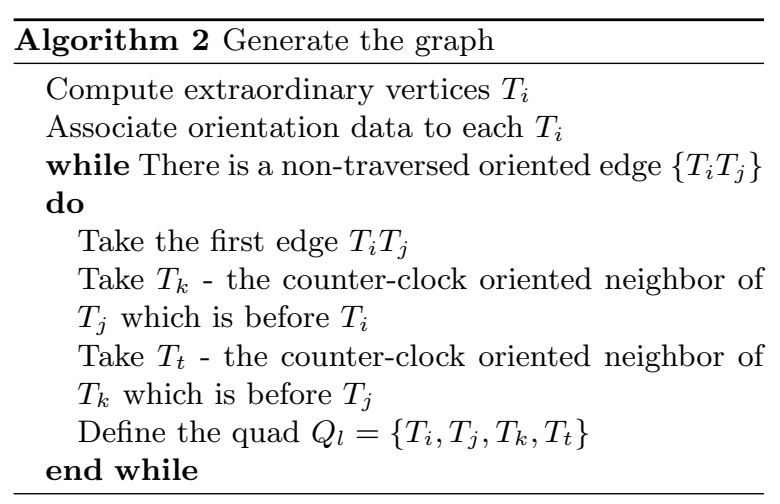

The obtained patches $Q=Q_{1} \cup Q_{2} \cup \ldots \cup Q_{n}$, by the definition of separatrices and singular points, have a smooth cross field inside, allowing further on parameterization/remeshing.

\section{WORKFLOW FOR PLANAR DOMAINS}

In case of planar domain $\Omega$, blocks defined by a planar graph embedding, are directly used for applying algebraic (1) and afterwards (2) elliptic grid generation (figure 34). The result obtained is all quad mesh. Theoretical and computational details on these methods are given in the following sections.

\subsection{Transfinite bilinear interpolation}

For the aim of refinement of each block, transfinite interpolation (TFI) is used as a, according to $[3,20,21]$, computationally efficient algebraic grid gen-

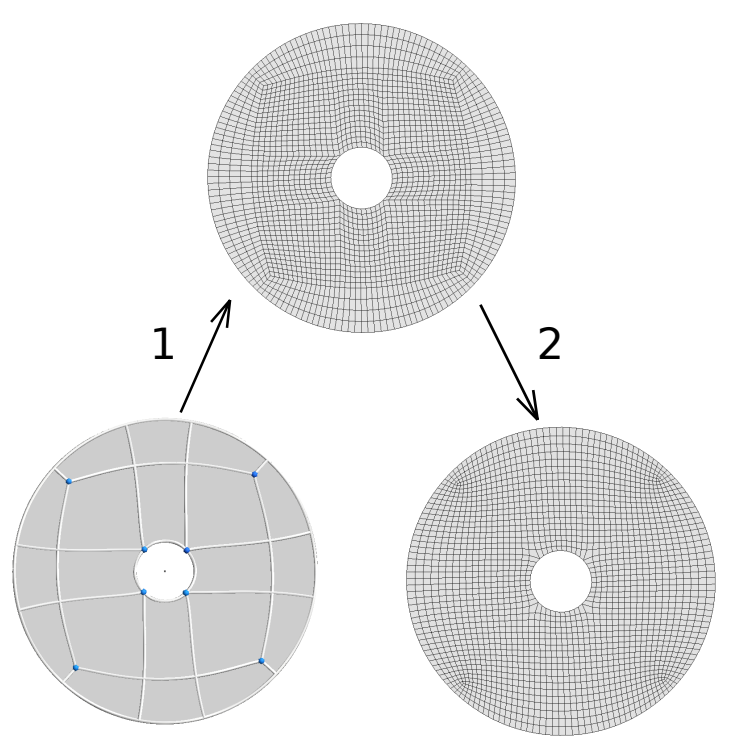

Figure 34: Illustration of the pipleine for the planar cases

eration technique. The grid obtained with this procedure is structured, conforming the $\partial \Omega$ and has controlled grid spacing. For a given physical domain $Q_{i}$, defined with parameterized curves $\vec{c}_{1}(u), \vec{c}_{3}(u), \vec{c}_{2}(v)$ and $\vec{c}_{4}(v)$ (shown in figure 35 ), the position of point $\vec{X}_{i}(u, v)$ in the given domain is defined by the equation 16:

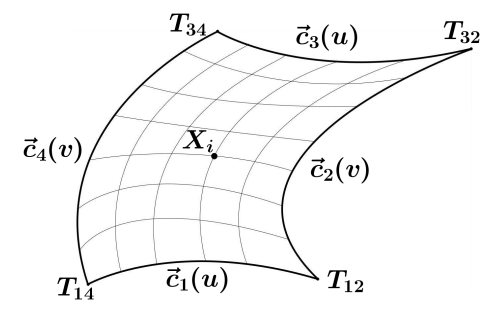

Figure 35: TFI grid points on physical domain $Q_{i}$

$$
\begin{aligned}
\vec{X}_{i}(u, v)= & (1-v) \cdot \vec{c}_{1}(u) \\
+\quad & v \cdot \vec{c}_{3}(u)+(1-u) \cdot \vec{c}_{2}(v)+u \cdot \vec{c}_{4}(v) \\
-\quad & {\left[(1-u) \cdot(1-v) \cdot \vec{T}_{12}\right.} \\
& +u \cdot v \cdot \vec{T}_{34} \\
& +u \cdot(1-v) \cdot \vec{T}_{14} \\
+ & \left.+(1-u) \cdot v \cdot \vec{T}_{32}\right] .
\end{aligned}
$$

As a result, a structured quad mesh $M_{t}=(V, E, Q)$, with vertices $V$, edges $E$ and corresponding quadrilaterals $Q$ is generated (figure 36 left). 

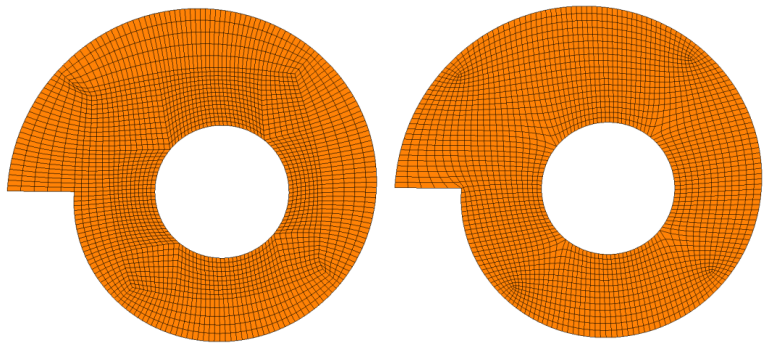

Figure 36: TFI mesh (left) and mesh smoothing (right)

In order to obtain better orthogonality and improve overall quality of elements in the quad mesh obtained, bilinear TFI has been used as a step towards implementing a PDE based meshing technique (figure 36 right).

\subsection{Grid smoothing}

To insure robustness and computational efficiency of a PDE based algorithm, which can be adopted for an unstructured mesh, our approach followed the work described in [22]. This work represents the Winslow smoothing [23] on 2D unstructured mesh and its based on solving the second-order nonlinear elliptic partial differential equations:

$$
\left\{\begin{array}{l}
g_{22} x_{\xi \xi}-2 g_{12} x_{\xi \eta}+g_{11} x_{\eta \eta}=0 \\
g_{22} y_{\xi \xi}-2 g_{12} y_{\xi \eta}+g_{11} y_{\eta \eta}=0
\end{array}\right.
$$

with $g_{11}, g_{12}$ and $g_{22}$ computed as:

$$
\left\{\begin{array}{l}
g_{11}=x_{\xi} x_{\xi}+y_{\xi} y_{\xi} \\
g_{12}=x_{\xi} x_{\eta}+y_{\xi} y_{\eta} \\
g_{22}=x_{\eta} x_{\eta}+y_{\eta} y_{\eta}
\end{array}\right.
$$

For the sake of completeness, the algorithm and its implementation are in detail explained in the Appendix A.

In order to determine the quality of a quad mesh, the measure of quadrilateral element quality $\eta(q)$ of the element $q$ with angles $\alpha_{i}$, is computed as in [24]:

$$
\eta(q)=\max \left(1-\frac{2}{\pi} \max \left(\left|\frac{\pi}{2}-\alpha_{i}\right|\right), 0\right) .
$$

Improvements of the mesh quality using Winslow smoother are shown on a few examples in the table below. Notations used for values of the minimum and average quality of elements are respectively $\eta_{\omega}$ and $\bar{\eta}$, where $\eta_{0.9}$ represents the percentage of elements in the mesh whose quality is greater than 0.9 and $h$ is the mesh size.

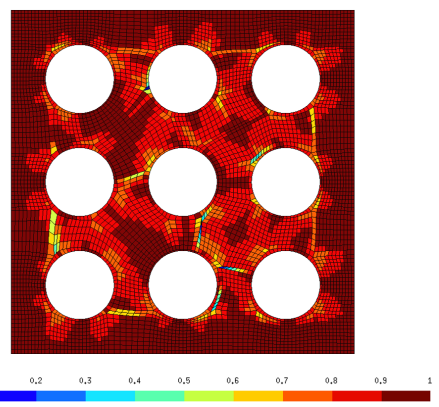

Figure 37: Quality of a mesh generated using TFI

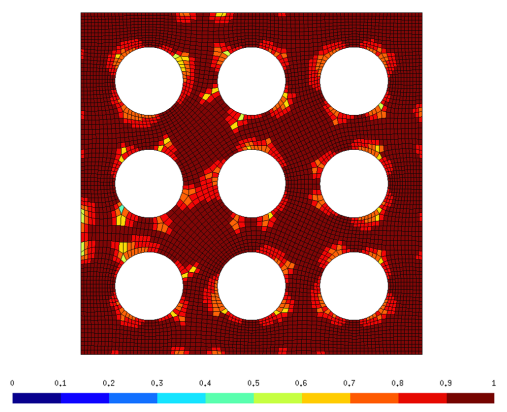

Figure 38: Quality of a mesh generated using Winslow smoother

\begin{tabular}{l|c|l|c|c|c}
\hline Figure & \multirow{2}{*}{$h$} & Algorithm & \multicolumn{3}{|c}{ Mesh quality } \\
& & & $\eta_{\omega}$ & $\bar{\eta}$ & $\eta_{0.9}$ \\
\hline Fig 37/38 & 0.01 & TFI & 0.01 & 0.90 & 0.59 \\
& & Winslow & 0.47 & 0.94 & 0.80 \\
\hline Fig 24 & \multirow{2}{*}{0.05} & TFI & 0.00 & 0.94 & 0.82 \\
& & Winslow & 0.52 & 0.93 & 0.79 \\
\hline Fig 25 & \multirow{2}{*}{0.01} & TFI & 0.14 & 0.92 & 0.73 \\
& & Winslow & 0.48 & 0.93 & 0.80 \\
\hline
\end{tabular}

Table 1: Comparison of TFI and Winslow mesh quality

\section{WORKFLOW FOR 2D MANIFOLDS}

We will now suppose that the domain $\Omega$ we are interested in is a $2 \mathrm{D}$ manifold. Workflow presented previously for planar domains has to be adapted for such domains. Indeed, the multi-block decomposition can be done in the same manner, but the algebraic grid generation and the elliptic grid smoothing will generate points not belonging to $\Omega$ in the general case. To be able to follow the grid generation procedure, first a parameterization of the domain $\Omega$ is needed. Figure 39 points out the main steps of the workflow. 

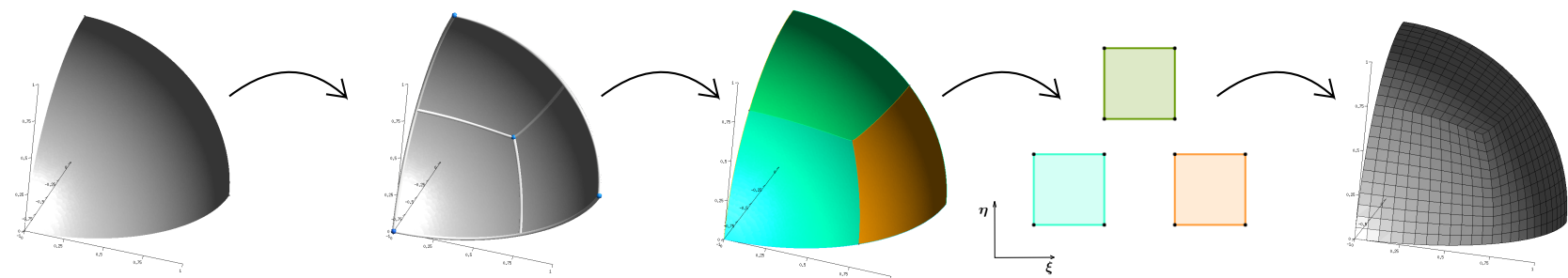

Figure 39: Illustration of the pipeline for the manifold cases

\subsection{Parametrization of the domain}

There are many ways to parameterize arbitrary 2D manifolds $\Omega[25,26,27]$ and they usually require to split $\Omega$ in a finite number $n$ of subdomains $\Omega_{i}, i \in$ $[|1, n|]$. Then each $\Omega_{i}$ is parametrized independently from each other. Such methods could be used to further generate grids only if boundaries of these subdomains correspond to the edges of the multi-block decomposition. The choice made here is to define each quad of the multi-block decomposition as a subdomain $\Omega_{i}$ and parameterize it independently following the method proposed in [27] (figure 40). The parameterization relies on mean value coordinates [28] which guaranties a one to one parameterization of each subdomain. For most of the multi-block decompositions obtained, each block can be parameterized with only one atlas, but it can happen that it is necessary to split a quad block in subdomains in order to get a proper parameterization. This kind of cases is handled by the methodology proposed in [27].

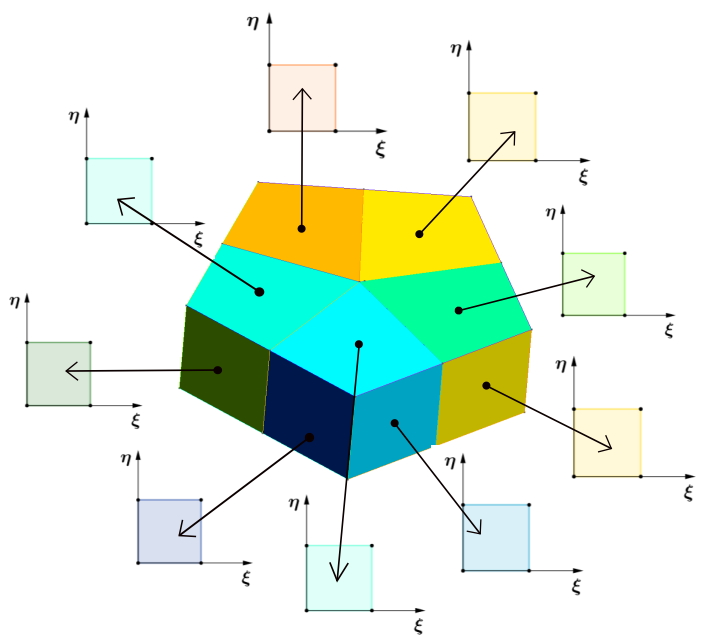

Figure 40: Illustration of the proposed parameterization

Once each subdomain $\Omega_{i}$ is parameterized, it is possi- ble to generate a first grid on each $\Omega_{i}$ with an algebraic method and then use an elliptic smoother in order to obtain a good quality all quad mesh.

It is important to note that, due to the choice of independent parameterization of each quad block, it is not possible anymore as in the planar case, to optimize the position of separatrices and extraordinary vertices with the elliptic smoother.

\subsection{Modification of original workflow}

The workflow for 2D manifold is obtained by modifying the workflow for planar cases through adding a parameterization step, as presented in figure 39. First, the mutli-block decomposition is generated. Then each block is parameterized independently. On each one of them, an initial grid is created in the parametric space, smoothed with Winslow smoother [22] and then mapped back to $\Omega$ in the physical space.

\section{DISCUSSION}

Due to the behaviour of the cross field at the vicinity of singular points, as well as our algorithm for creating critical patches, a triangular block can appear (figures $41-42)$. This issue is resolved by further propagation (figure 41), or, in cases when neither one of separatrices from the triangular block can not be further propagated, removal of the separatrice (figure 42).
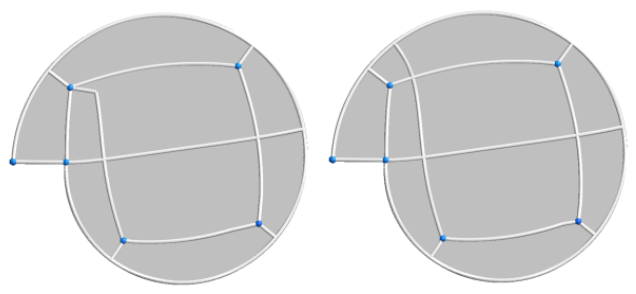

Figure 41: Before and after applying the algorithm for triangular block cleaning (repropagating) 

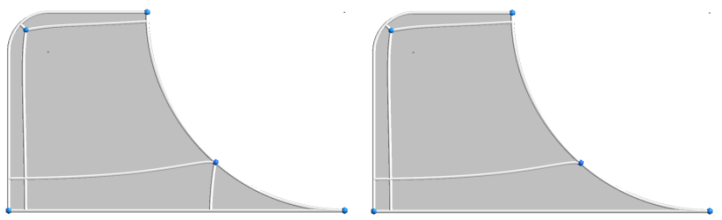

Figure 42: Before and after applying the algorithm for triangular block cleaning (removing)

When it comes to the computational cost of our method, it is subordinated to the number of elements of the triangulation. For most of the geometries, time for obtaining multi-block decomposition varied from less than one up to a few seconds with the same addition for parameterization/remeshing step. Concerning computational time for cross field generation, the current numerical scheme to solve the Ginzburg-Landau PDE described in [5] is not competitive with existing cross fields generators. Addressing this issue is the topic of the current work.

For the future directions of the work, the authors will address the thorough examinations of: optimization of performance time, robustness with large-scale numerical examples, reported problem of limit cycles existence and meshing with varying elements' sizes (figure 43).
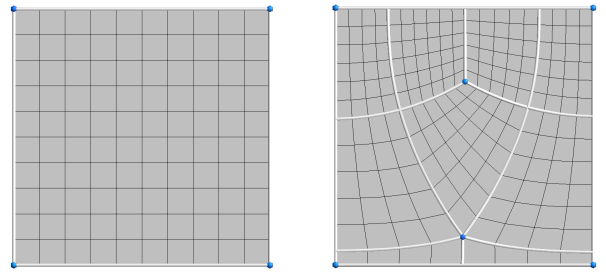

Figure 43: Uniform (left) and non-uniform quad mesh (right)

\section{CONCLUSION}

This paper presents a contribution in developing an algorithm for generating multi-block decomposition and all quad mesh of manifolds. The specific interest in using cross fields for these purposes is pointed out from a mathematical point of view. Choosing GinzburgLandau PDE is advocated by fair distribution of singular points - a crucial asset for a parameterization $[29,30]$. To the best of our knowledge, the algorithms to obtain the minimal number of separatrices and the proof of local integrability of $2 \mathrm{D}$ cross fields have not been exposed before. Last but not the least, we demonstrated how to use our method to create all quad meshes in an automatic manner and made it available in Gmsh, the open source finite element mesh generator.

\section{ACKNOWLEDGMENTS}

The present study was carried out in the framework of the research project "Hextreme", funded by the European Research Council (ERC-2015-AdG-694020) and hosted at the Université catholique de Louvain.

\section{Appendix A WINSLOW SMOOTHER}

Using the finite difference discretization, equations 17 can be written, for each node $n$ of the mesh, as:

$D_{n}(\mathbf{x})=G_{22} D_{\xi \xi}\left(\mathbf{x}_{n}\right)-2 G_{12} D_{\xi \eta}\left(\mathbf{x}_{n}\right)+G_{11} D_{\eta \eta}\left(\mathbf{x}_{n}\right)=0$,

where $G_{11}, G_{12}$ and $G_{22}$ are:

$$
\left\{\begin{array}{l}
G_{11}=D_{\xi}\left(\mathbf{x}_{n}\right) \cdot D_{\xi}\left(\mathbf{x}_{n}\right) \\
G_{12}=D_{\xi}\left(\mathbf{x}_{n}\right) \cdot D_{\eta}\left(\mathbf{x}_{n}\right) \\
G_{22}=D_{\eta}\left(\mathbf{x}_{n}\right) \cdot D_{\eta}\left(\mathbf{x}_{n}\right),
\end{array}\right.
$$

with values $D_{\xi}, D_{\eta}, D_{\xi \xi}, D_{\xi \eta}$ and $D_{\eta \eta}$ depending on the valance $v_{n}$ of the node $n$.

By defining logical space with: $\xi=\cos \theta_{m}, \eta=\sin \theta_{m}$ at each node, where

$$
\theta_{m}=\frac{2 \pi \cdot m}{v_{n}}
$$

the following equations (22 - 23) are derived: Approximations for $v_{n}=4$ :

$$
\left\{\begin{array}{l}
D_{\xi}\left(\mathbf{x}_{n}\right)=\frac{2}{v_{n}} \sum_{m=0}^{v_{n}-1}\left(\mathbf{x}_{m}-\mathbf{x}_{n}\right) \cos \theta_{m} \\
D_{\eta}\left(\mathbf{x}_{n}\right)=\frac{2}{v_{n}} \sum_{\substack{v_{n}-1 \\
v_{\xi \xi}}}^{v_{n}-1}\left(\mathbf{x}_{m}\right)=\frac{4}{v_{n}} \sum_{m=0}^{v_{m}}\left(\mathbf{x}_{m}\right) \sin \theta_{m} \\
D_{\xi \eta}\left(\mathbf{x}_{n}\right)=\frac{2}{v_{n}} \sum_{m=0}^{v_{n}-1}\left(\widehat{\mathbf{x}_{m}}-\mathbf{x}_{n}\right) \cos \theta_{m} \widehat{\theta_{m}} \sin \widehat{\theta_{m}} \\
D_{\eta \eta}\left(\mathbf{x}_{n}\right)=\frac{4}{v_{n}} \sum_{m=0}^{v_{n}-1}\left(\mathbf{x}_{m}-\mathbf{x}_{n}\right) \sin ^{2} \theta_{m}
\end{array}\right.
$$

where:

$$
\widehat{\theta_{m}}=\frac{2 \pi \cdot\left(m+\frac{1}{2}\right)}{v_{n}}
$$

and $\widehat{\mathbf{x}_{m}}$ are associated to the diagonal nodes, as shown in figures 44-45. 


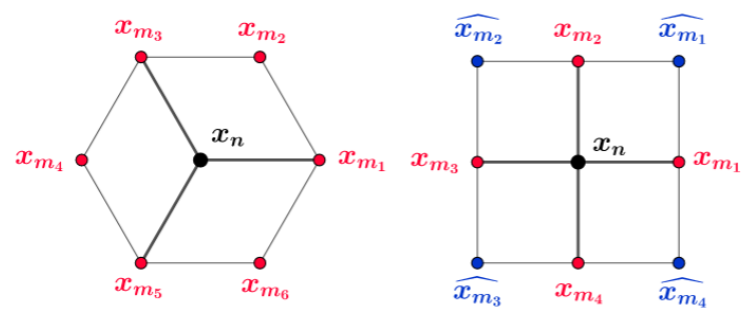

Figure 44: Dependency on $v_{n}$ of $\mathbf{x}_{n}$ used for approximation of 3 and 4 -valent nodes (left to right)

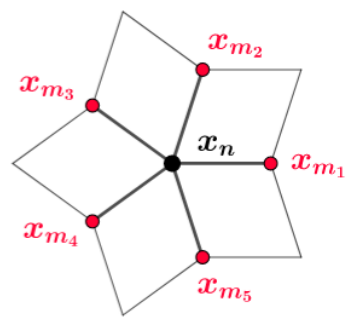

Figure 45: Dependency on $v_{n}$ of $\mathbf{x}_{n}$ used for approximation of 5 -valent nodes

Considering 3-valant nodes, equations demonstrated below are considering 6 neighbouring nodes (figure 44) and therefore the valant degree $v_{n}=3$ rises up to $v_{n}=6$, more detailed in [22].

Approximations for $v_{n}=3$ and $v_{n} \geq 5$ :

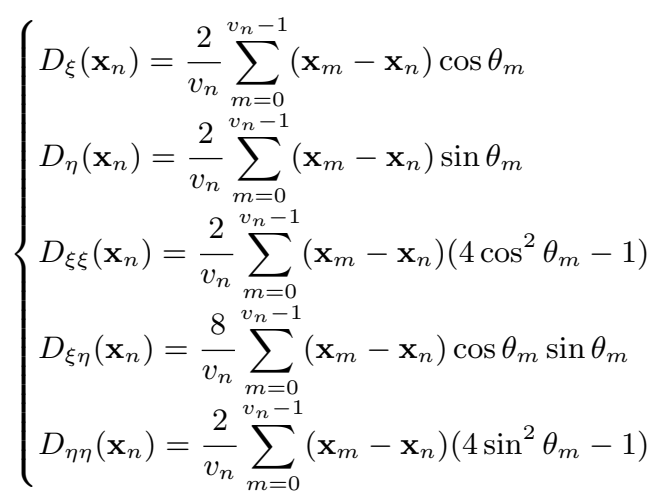

To solve the nonlinear problem $D_{n}(\mathbf{x})=0$, for each node $n$ from $M_{t}$ where boundary nodes are fixed, Picard iterations are performed. In order to do so, the following notations are defined: $M_{q}^{k}$ as the current quad mesh, $\mathbf{x}^{k}$ the coordinates of its nodes, $\mathbf{x}^{k+1}$ the coordinates of nodes after one smoothing iteration and $M_{q}^{k+1}$ the corresponding quad mesh. By computing the values $G_{11}^{k}, G_{12}^{k}$ and $G_{22}^{k}$ evaluated on every node of $M_{q}^{k}$, we can define:

$D_{n}^{k}(\mathbf{x})=G_{22}^{k} D_{\xi \xi}\left(\mathbf{x}_{n}^{k+1}\right)-2 G_{12}^{k} D_{\xi \eta}\left(\mathbf{x}_{n}^{k+1}\right)+G_{11}^{k} D_{\eta \eta}\left(\mathbf{x}_{n}^{k+1}\right)$

for all nodes $n$. This system of $n$ nonlinear equations is put under the form $D^{k} \cdot \mathbf{x}^{k+1}$, where $D^{k}$ depends only on $\mathbf{x}^{k}$. Finding $\mathbf{x}^{k+1}$ is done by solving $D^{k} \cdot \mathbf{x}^{k+1}=$ 0. Performed computational steps are described in algorithm 3.

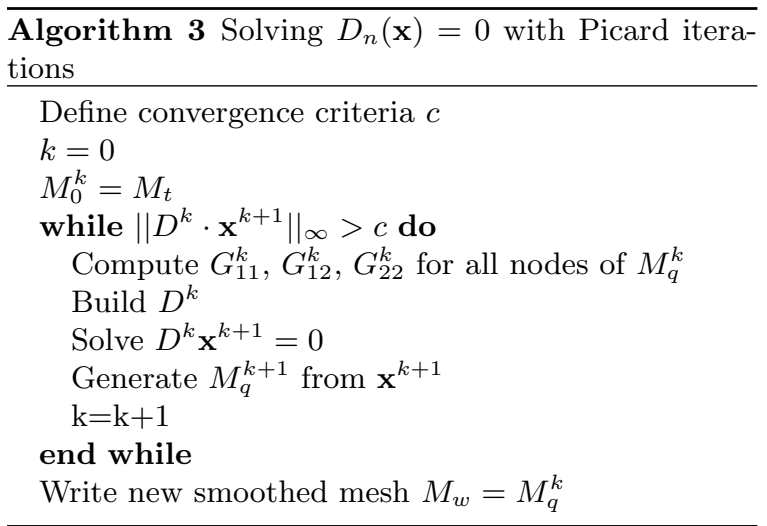

\section{Appendix B LOCAL INTEGRABILITY OF THE CROSS FIELD}

Starting from the definition of $2 \mathrm{D}$ cross field $\tilde{\mathcal{C}}_{\Omega}$ with the non uniform norms (equation 2) and replacing the corresponding values in equation 3 , we obtain the result computed in 24 , which shows that for $l \neq 0, \tilde{\mathcal{C}}_{\Omega}$ is integrable if $l$ verifies the condition 4 . 


$$
\begin{aligned}
& {\left[\tilde{\mathbf{u}}_{1}, \tilde{\mathbf{u}}_{2}\right]=\left[\begin{array}{ll}
l_{, x} \cdot \cos \theta-l \cdot \sin \theta \cdot \theta_{, x} & l_{y}^{\prime} \cdot \cos \theta-l \cdot \sin \theta \cdot \theta_{, y} \\
l_{, x} \cdot \sin \theta+l \cdot \cos \theta \cdot \theta_{, x} & l_{y}^{\prime} \cdot \sin \theta+l \cdot \cos \theta \cdot \theta_{, y}
\end{array}\right] \cdot\left[\begin{array}{c}
-l \cdot \sin \theta \\
l \cdot \cos \theta
\end{array}\right]} \\
& -\left[\begin{array}{cc}
-l_{, x} \cdot \sin \theta-l \cdot \cos \theta \cdot \theta_{, x} & -l_{y}^{\prime} \cdot \sin \theta-l \cdot \cos \theta \cdot \theta_{, y} \\
l_{, x} \cdot \cos \theta-l \cdot \sin \theta \cdot \theta_{, x} & l_{y}^{\prime} \cdot \cos \theta-l \cdot \sin \theta \cdot \theta_{, y}
\end{array}\right] \cdot\left[\begin{array}{l}
l \cdot \cos \theta \\
l \cdot \sin \theta
\end{array}\right] \\
& =\left[\begin{array}{l}
-l \cdot l_{, x} \cdot \sin \theta \cdot \cos \theta+l^{2} \cdot \sin ^{2} \theta \cdot \theta_{, x}+l \cdot l_{, y} \cdot \cos ^{2} \theta-l^{2} \cdot \sin \theta \cdot \cos \theta \cdot \theta_{, y} \\
-l \cdot l_{, x} \cdot \sin ^{2} \theta-l^{2} \cdot \sin \theta \cdot \cos \theta \cdot \theta_{, x}+l \cdot l_{, y} \cdot \sin \theta \cdot \cos \theta+l^{2} \cdot \cos ^{2} \theta \cdot \theta_{, y}
\end{array}\right] \\
& -\left[\begin{array}{c}
-l \cdot l_{, x} \cdot \sin \theta \cdot \cos \theta-l^{2} \cdot \cos ^{2} \theta \cdot \theta_{, x}-l \cdot l_{, y} \cdot \sin ^{2} \theta-l^{2} \cdot \sin \theta \cdot \cos \theta \cdot \theta_{, y} \\
l \cdot l_{, x} \cdot \cos ^{2} \theta-l^{2} \cdot \sin \theta \cdot \cos \theta \cdot \theta_{, x}+l \cdot l_{, y} \cdot \sin \theta \cdot \cos \theta-l^{2} \cdot \sin ^{2} \theta \cdot \theta_{, y}
\end{array}\right] \\
& =\left[\begin{array}{l}
l^{2} \cdot \theta_{, x}+l \cdot l, y \\
l^{2} \cdot \theta_{, y}-l \cdot l_{, x}
\end{array}\right]=l^{2}\left[\begin{array}{l}
\theta_{, x}+\frac{l, y}{l} \\
\theta_{, y}-\frac{l_{, x}}{l}
\end{array}\right]=\left[\begin{array}{l}
0 \\
0
\end{array}\right]
\end{aligned}
$$

\section{References}

[1] Shepherd J.F., Johnson C.R. "Hexahedral mesh generation constraints." Engineering with Computers, vol. 24, no. 3, 195-213, 2008

[2] Benzley S.E., Perry E., Merkley K., Clark B., Sjaardama G. "A comparison of all hexagonal and all tetrahedral finite element meshes for elastic and elasto-plastic analysis." Proceedings, 4th international meshing roundtable, vol. 17, pp. 179191. Sandia National Laboratories Albuquerque, NM, 1995

[3] Kowalski N., Ledoux F., Frey P. "A PDE based approach to multidomain partitioning and quadrilateral meshing." Proceedings of the 21st international meshing roundtable, pp. 137-154. Springer, 2013

[4] Chan J., Wang Z., Modave A., Remacle J.F., Warburton T. "GPU-accelerated discontinuous Galerkin methods on hybrid meshes." Journal of Computational Physics, vol. 318, 142-168, 2016

[5] Beaufort P.A., Lambrechts J., Henrotte F., Geuzaine C., Remacle J.F. "Computing cross fields A PDE approach based on the GinzburgLandau theory." Procedia engineering, vol. 203, 219-231, 2017

[6] Viertel R., Osting B. "An Approach to Quad Meshing Based on Harmonic Cross-Valued Maps and the Ginzburg-Landau Theory." SIAM Journal on Scientific Computing, vol. 41, no. 1, A452A479, 2019

[7] Fogg H.J., Armstrong C.G., Robinson T.T. "Automatic generation of multiblock decompositions of surfaces." International Journal for Numerical Methods in Engineering, vol. 101, no. 13, 965-991, 2015
[8] Geuzaine C., Remacle J.F. "Gmsh: A 3-D finite element mesh generator with built-in pre-and post-processing facilities." International journal for numerical methods in engineering, vol. 79, no. 11, 1309-1331, 2009

[9] Bommes D., Lévy B., Pietroni N., Puppo E., Silva C.T., Tarini M., Zorin D. "Quad Meshing." Eurographics (STARs), pp. 159-182. 2012

[10] Campen M. "Partitioning surfaces into quadrilateral patches: a survey." Computer Graphics Forum, vol. 36, pp. 567-588. Wiley Online Library, 2017

[11] Campen M., Bommes D., Kobbelt L. "Quantized global parametrization." ACM Transactions on Graphics (TOG), vol. 34, no. 6, 192, 2015

[12] Bommes D., Campen M., Ebke H.C., Alliez P., Kobbelt L. "Integer-grid maps for reliable quad meshing." ACM Transactions on Graphics (TOG), vol. 32 , no. $4,98,2013$

[13] Myles A., Pietroni N., Zorin D. "Robust fieldaligned global parametrization." ACM Transactions on Graphics (TOG), vol. 33, no. 4, 135, 2014

[14] Ray N., Li W.C., Lévy B., Sheffer A., Alliez P. "Periodic global parameterization." ACM Transactions on Graphics (TOG), vol. 25, no. 4, 14601485,2006

[15] Diamanti O., Vaxman A., Panozzo D., SorkineHornung O. "Integrable polyvector fields." $A C M$ Transactions on Graphics (TOG), vol. 34, no. 4, 38,2015

[16] Bethuel F., Brezis H., Hélein F., et al. GinzburgLandau Vortices, vol. 13. Springer, 1994 
[17] Bommes D., Zimmer H., Kobbelt L. "Mixedinteger quadrangulation." ACM Transactions On Graphics (TOG), vol. 28, no. 3, 77, 2009

[18] Jezdimirović J., Chemin A., Beaufort P.A., Remacle J.F. "Elliptic Fekete points obtained by Ginzburg-Landau PDE." Proceedings, 26th international meshing roundtable. Sandia National Laboratories Albuquerque, NM, 2017

[19] Brinkmann G., McKay B.D., et al. "Fast generation of planar graphs." MATCH Commun. Math. Comput. Chem, vol. 58, no. 2, 323-357, 2007

[20] Thompson J.F., Soni B.K., Weatherill N.P. Handbook of grid generation. CRC press, 1998

[21] Mukherjee N. "CSALF-Q: A bricolage algorithm for anisotropic quad mesh generation." Proceedings of the 20th International Meshing Roundtable, pp. 489-509. Springer, 2011

[22] Knupp P.M. "Winslow smoothing on twodimensional unstructured meshes." Engineering with Computers, vol. 15, no. 3, 263-268, 1999

[23] Winslow A.M. "Numerical solution of the quasilinear Poisson equation in a nonuniform triangle mesh." Journal of computational physics, vol. 1, no. 2, 149-172, 1966

[24] Remacle J.F., Lambrechts J., Seny B., Marchandise E., Johnen A., Geuzainet C. "Blossom-Quad: A non-uniform quadrilateral mesh generator using a minimum-cost perfect-matching algorithm." International journal for numerical methods in engineering, vol. 89, no. 9, 1102-1119, 2012

[25] Remacle J.F., Geuzaine C., Compere G., Marchandise E. "High-quality surface remeshing using harmonic maps." International Journal for Numerical Methods in Engineering, vol. 83, no. 4, 403-425, 2010

[26] Marchandise E., de Wiart C.C., Vos W., Geuzaine C., Remacle J.F. "High-quality surface remeshing using harmonic mapsPart II: Surfaces with high genus and of large aspect ratio." International Journal for Numerical Methods in Engineering, vol. 86, no. 11, 1303-1321, 2011

[27] Beaufort P.A., Geuzaine C., Remacle J.F. "Automatic surface mesh generation for discrete models. A complete and automatic pipeline." Submitted

[28] Floater M.S. "Mean value coordinates." Computer aided geometric design, vol. 20, no. 1, 1927,2003
[29] Vaxman A., Campen M., Diamanti O., Panozzo D., Bommes D., Hildebrandt K., Ben-Chen M. "Directional field synthesis, design, and processing." Computer Graphics Forum, vol. 35, pp. 545572. Wiley Online Library, 2016

[30] Nieser M., Polthier K. "Parameterizing singularities of positive integral index." IMA International Conference on Mathematics of Surfaces, pp. 265277. Springer, 2009 